\title{
FLORIDA HYDROGEN INITIATIVE
}

Final Report

\section{Florida Hydrogen Initiative (FHI)}

\author{
David L. Block, Director Emeritus \\ Florida Solar Energy Center/ \\ University of Central Florida \\ 1679 Clearlake Road \\ Cocoa, FL 32922 \\ Phone: (321) 638-1001 \\ E-mail: block@,fsec.ucf.edu
}

DOE Technology Development Manager:

John Garak, DOE HQ Technology Manager

Phone: (202) 586-1723

E-mail: john.garak@go.doe.gov

DOE Project Officer:

Greg Kleen, DOE Project Officer

Phone: (720) 356-1672

E-mail: gregory.kleen@go.doe.gov

Contract Number: DE-FC36-04GO14225

\section{Subcontractors:}

Rollins College, Winter Park, FL

EnerFuel, Inc., West Palm Beach, FL

Florida Atlantic University, Boca Raton, FL

Orlando Science Center, Orlando, FL

University of Central Florida, Orlando, FL

Florida Solar Energy Center, Cocoa, FL

Chevron Technology Ventures LLC (CTV), Houston, TX

SRT Group, Inc., Miami, FL

Electrolytic Technologies Corporation, Miami, FL

Florida State University, Tallahassee, FL

Bing Energy, Inc., Tallahassee, FL

Florida Institute of Technology, Melbourne, FL

University of South Florida, Tampa, FL

Project Start Date: October 1, 2004

Project End Date: June 30, 2013 


\title{
Florida Hydrogen Initiative (FHI) \\ Dr. David L. Block, PI \\ Florida Solar Energy Center/University of Central Florida
}

\author{
Executive Summary
}

The Florida Hydrogen Initiative (FHI) was a research, development and demonstration (RD\&D) hydrogen and fuel cell program funded by U.S. Department of Energy (DOE). The FHI program objectives were to develop Florida's hydrogen and fuel cell infrastructure and to assist DOE in its hydrogen and fuel cell activities by creating partnerships for demonstration projects, sponsoring research and development programs, facilitating technology transfer between the public and private sectors, developing industry support and establishing unique hydrogen/fuel cell university-level education programs. The FHI program funded 12 RD\&D projects. Each project was issued a sub-contract with specific tasks, deliverables and budget. The results of these projects are presented in this final report.

In this Executive Summary, a one paragraph description is given for each of the 12 projects conducted with FHI support. The 12 projects are presented in numerical order by task (Task 1 to Task 14). These include five projects (Tasks 1, 5, 7, 10 and 13) in the hydrogen technology areas, five (Tasks 2, 8, 9, 11 and 14) fuel cell related activities and two covering education and public understanding and information exchange (Task 3 and 12). Note that Tasks 4 and 6 were administrative tasks.

\section{Individual Project Summaries}

Task 1. Hydrogen Refueling Infrastructure and Rental Car Strategies -- L. Lines, Rollins College, M. Kuby, Arizona State University, R. Schultz and Z. Xie, Florida Atlantic University -- 2007

This project analyzes strategies for Florida's early stage adaptation of hydrogen-powered public transportation. In particular, the report investigates urban and statewide network of refueling stations and the feasibility of establishing a hydrogen rental-car fleet based in Orlando. These two scenarios are modeled and linked by the potential for a limited number of fueling stations, properly located, to serve the needs of a surprisingly high percentage of car renters in Orlando area. Although the study is 8 years old, the presented concept is still valid today and can form the basis of a new and an updated study.

\section{Task 2. Methanol Fuel Cell Vehicle Charging Station at Florida Atlantic University (Old Title - Hydrogen Technology Rest Area) - M. Fuchs, EnerFuel, Inc. - 2012}

This project was for a methanol fuel cell demonstration with the objective of promoting the use of renewable energy in Florida. The project objectives were to design, construct, and demonstrate a $10 \mathrm{~kW}_{\text {net }}$ proton exchange membrane fuel cell (PEMFC) stationary power plant operating on methanol, to achieve an electrical energy efficiency of $32 \%$ and to demonstrate transient response time of less than 3 milliseconds. The other project goal was to produce methanol from citrus waste which was not achieved. The project used a purchased Ida Tech PEM fuel cell system with integrated fuel processing capability. EnerFuel activities consisted of the complete system design, installation, operation, data collection and analysis. The project was not able to deliver methanol production from renewable resources and the Florida Atlantic University (FAU) demonstration site was found to be unsatisfactory for the data collection after the installation had been completed. 
Task 3 - Assessment of Public Understanding of the Hydrogen Economy Through Science Center Exhibits, J. Newman, Orlando Science Center, FL. - 2010

The primary objective of the Orlando Science Center (OSC) project was to design and build an interactive Science Center exhibit called: " $\mathrm{H}_{2}$ Now: the Great Hydrogen Xchange". The developed exhibit allowed OSC visitors to be able to visualize a community powered entirely by hydrogen and to discover that renewable energy sources are a viable alternative to other energy sources currently in use. The completed exhibit was so popular that exit surveys OSC regularly conducted revealed that visitors liked the exhibit and wished there was more to it. In response to its popularity, OSC added a video presentation produced by the H2Now exhibit designers, IDEAS, Inc., titled "I Am Hydrogen" and developed a panel exhibit on other alternative energy resources. The project is no longer active at OSC.

\section{Task 5. On-site Reformation of Diesel Fuel for Hydrogen Fueling Station Applications - A. Raissi, Florida Solar Energy Center, J. Stevens, Chevron Technology Ventures, LLC - 2010}

The objective of this project was to develop a new on-demand forecourt hydrogen production technology by catalytically converting high-sulfur hydrocarbon fuels to an essentially sulfur-free gas. The removal of sulfur from reformate is critical since most catalysts used for the steam reformation have limited sulfur tolerance. FSEC's responsibilities included building, operating and validating the performance of a diesel pre-reformer as well as developing and demonstrating a small scale desulfurization unit. Chevron Technology Ventures provided catalysts and know-how for the design and fabrication of the prereforming reactor. The pre-reformer was coupled with a regenerable $\mathrm{Fe}^{2+} / \mathrm{Fe}^{3+}$ redox/electrolysis system used for hydrogen sulfite removal from the pre-reformer effluent. Laboratory tests showed that electrolysis of acidic $\mathrm{FeSO}_{4}$ aqueous solution was highly efficient with a coulombic efficiency of $100 \%$ at a cell potential of $1.0 \mathrm{~V}$ and that the electrolytic process can be made to operate with a Pt-free anode to oxidize ferrous to ferric ions, thereby reducing the cost of the electrolytic scrubber. FSEC researchers have also developed a robust bi-functional catalyst for accomplishing the pre-reformation of the highsulfur fuels (sulfur content as high as 5,240 ppmw) to short chain hydrocarbons $\left(\mathrm{C}_{1}-\mathrm{C}_{4}\right.$, mostly propane) at an average yield of about $97 \%$. In summary, the project findings included the development of a novel process for converting high-sulfur diesel to a mixture of light molecular weight hydrocarbons that can be readily reformed with steam to fuel cell grade hydrogen gas.

\section{Task 7. Chemochromic Hydrogen Leak Detectors for Safety Monitoring - N. Mohajeri and N. Muradov, Florida Solar Energy Center - 2013}

The objective of this project was to develop and demonstrate a cost-effective and highly selective chemochromic (visual) hydrogen leak detector for safety monitoring at any facility engaged in transport, handling and use of hydrogen. The project evaluated two classes of chemochromic hydrogen sensors, an irreversible one and a reversible one. The irreversible sensor permanently changes color when exposed to hydrogen and the reversible sensor is one that changes color when exposed to hydrogen, but after the hydrogen contact has stopped, the sensor changes back to its original color. The project output will lead to a new generation of versatile chemochromic hydrogen detectors that employ "smart" materials that cost less, possess fast discoloration kinetics, are user-friendly, are reliable and have superior field worthiness. The project activities have evaluated the performance (sensitivity) of the sensors at different hydrogen concentrations in air (from 1 to $100 \mathrm{vol} \%$ ) and have determined the extent and found no interference with other reducing gases $\left(\mathrm{CO}, \mathrm{NH}_{3}, \mathrm{CH}_{4}, \mathrm{H}_{2} \mathrm{~S}\right)$. 
Task 8. Development of High Efficiency Low Cost Electrocatalysts for Hydrogen Production and PEM Fuel Cell Applications - M. Rodgers, Florida Solar Energy Center - 2013

The objective of this project was to decrease platinum (Pt) usage in fuel cells by conducting experiments to improve catalyst activity while lowering platinum loading through pulse electrodeposition. Optimum values of several variables during electrodeposition were selected to achieve the highest electrode performance, which was related to catalyst morphology. The type of catalyst support used was found to impact electrode performance, with reduced graphene oxide, Cabot Regal, and activated carbon resulting in the highest performances. A new electrodeposition method was developed where the carbon electrodes were seeded with nanosized Pt colloidal particles prior to electrodeposition. With the new method, it was possible to achieve the same performance as a commercial electrode using one-fifth the amount of Pt. Calcination resulted in improved ceria stability, although the ceria was not shown to affect performance of Pt-based electrodes. An electrodeposition flow cell was designed and built, and $25 \mathrm{~cm}^{2}$ electrodes were prepared. This was a 50 -fold scale up in size compared to the $0.5 \mathrm{~cm}^{2}$ electrodes. Platinum loading using the flow cell was low and, as a consequence, fuel cell performance was low. An accelerated stress protocol based on potential cycling showed that the electrode containing reduced graphene oxide had superior stability and did not degrade during testing.

\section{Task 9. Understanding Mechanical and Chemical Durability of Fuel Cell Membrane Electrode Assemblies - D. Slattery, Florida Solar Energy Center - 2013}

The objective of this project was to increase the knowledge base of the degradation mechanisms for membranes used in proton exchange membrane (PEM) fuel cells. The results show the addition of ceria (cerium oxide) has given durability improvements by reducing fluoride emissions by an order of magnitude during an accelerated durability test (fluoride emission is measure of membrane degradation). Ceria has also shown two-fold decrease in OCV decay (taken from accelerated durability test). Ceria is radical scavenging additive to Nafion membrane and has shown 5 fold reductions in fluoride emissions during liquid Fenton tests (commonly used to determine stability ex situ to fuel cell). Other results show that $\mathrm{PtCo} / \mathrm{C}$ (cobalt and platinum co-deposited on carbon support material) is better than $\mathrm{Pt} / \mathrm{C}$ and that the incorporation of a heteropolyacid sublayer reduces fluoride emission by a factor of 2-3. However, the sublayer is detrimental to cell performance and the process needs to include heteropolyacid as part of $\mathrm{Pt}$ layer to avoid performance degradation.

Task 10. Production of Low-Cost Hydrogen from Biowaste $\left(\mathrm{HyBrTec}^{\mathrm{TM}}\right)$ - R. Parker, SRT Group, Inc., Miami, FL -- 2013

This project uses a SRT Group developed hydrogen bromide $\left(\mathrm{HyBrTec}^{\mathrm{TM}}\right)$ process which produces hydrogen bromide $(\mathrm{HBr})$ from wet-cellulosic waste and co-produces carbon dioxide $\left(\mathrm{CO}_{2}\right)$. Next, electrolysis dissociates hydrogen bromide $\left(\mathrm{E}^{\mathrm{o}}=0.555 \mathrm{~V}\right)$ producing recyclable bromine and hydrogen (endothermic). A demonstration reactor and electrolysis vessel was designed, built and operated. Data was collected from bromination experiments at temperatures up to $250^{\circ} \mathrm{C}$, as well as electrolysis and combined bromination-electrolysis runs up to $200^{\circ} \mathrm{C}$. An economic model was also created to estimate the costs for a demonstration system. The final report presents the work undertaken, the results obtained, and the recommended next steps to further advance the technology.

Task 11. Development of a Low-Cost and High-Efficiency 500 W Portable PEMFC System - J. Zheng, Florida State University, H. Chen, Bing Energy, Inc. -- 2013

The objectives of this project were to develop a new catalyst structures comprised of highly conductive buckypaper and Pt catalyst nanoparticles coated on its surface and to demonstrate fuel cell efficiency improvement and durability and cell cost reductions in the buckypaper based electrodes. The project 
results showed that these objectives were achieved. Florida State University (FSU) activities were to optimize single cells with buckypaper supported catalysts while Bing Energy focused on the stack design, modeling, fixture, and tooling to accomplish the manufacture of the MEAs, components, and stack. Reported results imply that DOE performance and efficiency goals were achieved.

\section{Task 12. Development of an Interdisciplinary Hydrogen and Fuel Cell Technology Academic Program - J. Politano, C. Lockyer, M. McCay and K. Winkelmann, Florida Institute of Technology - 2013}

This project developed a hydrogen and fuel cell technology (HFCT) academic program at Florida Institute of Technology (FIT) in Melbourne, Florida. The developed FIT HFCT program allows students to follow hydrogen technology from introduction to long-term applications, to obtain a basic understanding of the technical fundamentals, to redirect their current technology focus as a means for new career options, to measure students' gains in knowledge of hydrogen, to interact with outside industries and to satisfy the industry need for hydrogen technology graduates. The project results were the development of hydrogen and fuel cell related education modules that have been implemented into courses in the General Chemistry laboratory and Mechanical and Sustainability Engineering and the offering of a HFCT master's degree level of specialization. The project also conducted R\&D in the areas of hydrogen and fuel cell technologies through senior level and graduate student projects. The project was completed as scheduled and a new university education program has been established.

\section{Task 13. Design and Development of an Advanced Hydrogen Storage System using Novel Materials - E. Stefanakos, D. Goswami, and A. Kumar, University of South Florida - 2013}

The goal of this project was to design and develop novel conducting polymeric nanomaterials for onboard hydrogen storage. The project approach was to examine synthesis of polyaniline (PANI) solid state hydrogen storage materials and to modify the synthesis parameters for optimized storage. Several novel materials such as polyaniline (emeraldine salt), ion doped polyaniline, carbon nanotube doped polyaniline, transition metal doped polyaniline, and graphene doped polyaniline, electrospun polyaniline, and hyper cross-linked polystyrene material were examined. The spillover enhancement for high surface area polymeric materials (i.e., hypercrosslinked polystyrene) was investigated for the first time. Spillover enhancement attracted the attention of the hydrogen storage community due to the very promising results reported (i.e. $\approx 5$ wt. $\%$ at room temperature and $10 \mathrm{MPa}$ ). However, spillover enhancement remains contentious subject due to contradictory results reported by different research groups. The results reported in this report showed that spillover enhancement in high surface area polymers has little practical value (i.e., 0.32 wt. $\%$. at room temperature and $10 \mathrm{MPa}$ ) for mobile applications.

\section{Task 14. Advanced HiFoil TM Bipolar Plates - J. Braun, M. Fuchs, EnerFuel, Inc. - 2013}

The goal of the Advanced HiFoil ${ }^{\mathrm{TM}}$ Bipolar Plates was to provide a durable, low cost bipolar plate for high temperature proton exchange membrane (HTPEM) fuel cells. The project results produced a durable, low cost bipolar plate with very high in-plane thermal conductivity. Stacks and systems using the advanced plate were operated as stand-alone, fully automated system using propane. All of the heat generated by the fuel cell was conducted through the edges of the bipolar plate and to an external 2-phase cooling circuit. Some of this heat was used to generate steam for use inside the fuel processor, while the additional waste heat is provided at $\sim 165^{\circ} \mathrm{C}$ to be put to work in combined heat and power applications. The net system electrical efficiency was $36 \%$ at $3 \mathrm{~kW}$ electric power output. When employed in a stationary CHP system, the bipolar plate technology is estimated to cost less than $\$ 500 / \mathrm{kW}$ at 50,000 units per year production volume, supporting the 2015 DOE target cost of $\$ 1,700 / \mathrm{kW}$ for the overall 
system. As a result of this project, EnerFuel has successfully developed and demonstrated a mass producible bipolar plate with unparalleled performance and physical attributes. 


\section{Table of Contents}

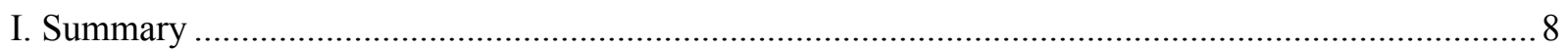

II. Comparison of Accomplishments with Program Objectives ......................................................... 8

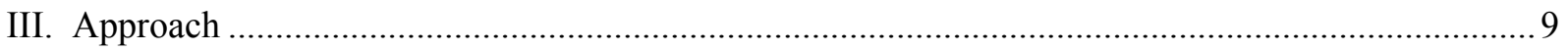

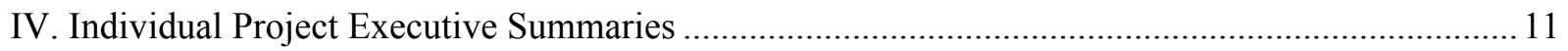

Task 1.Hydrogen Refueling Infrastructure and Rental Car Strategies .......................................... 11

Task 2. Methanol Fuel Cell Vehicle Charging Station (Old Title - Hydrogen Technology Rest

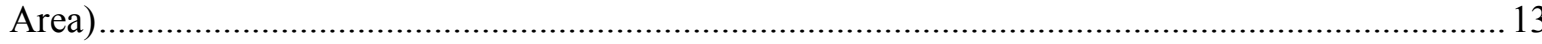

Task 3. Assessment of Public Understanding of the Hydrogen Economy Through Science Center

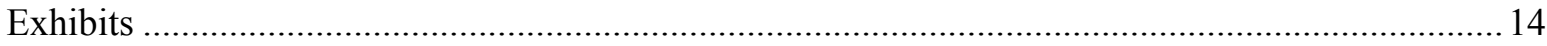

Task 5. On-site Reformation of Diesel Fuel for Hydrogen Fueling Station Applications ................ 16

Task 7. Chemochromic Hydrogen Leak Detectors for Safety Monitoring .................................... 17

Task 8. Development of High Efficiency Low Cost Electrocatalysts for Hydrogen Production and

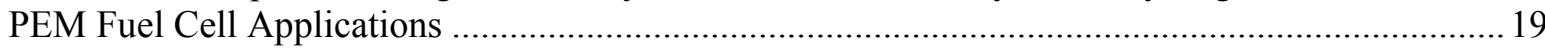

Task 9. Understanding Mechanical and Chemical Durability of Fuel Cell Membrane Electrode

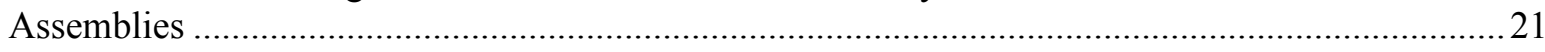

Task 10. Production of Low-Cost Hydrogen from Biowaste $\left(\mathrm{HyBrTec}^{\mathrm{TM}}\right)$................................. 23

Task 11. Development of a Low-Cost and High-Efficiency 500 W Portable PEMFC System ........24

Task 12. Interdisciplinary Hydrogen and Fuel Cell Technology Academic Program ....................25

Task 13. Design and Development of an Advanced Hydrogen Storage System using Novel

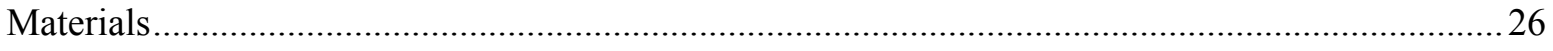

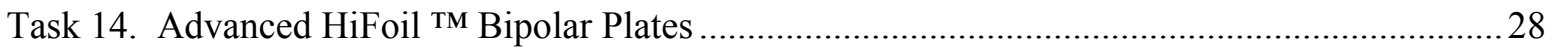

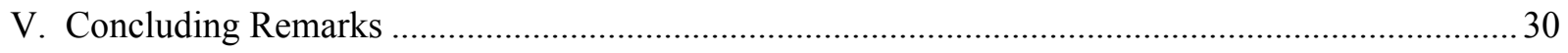

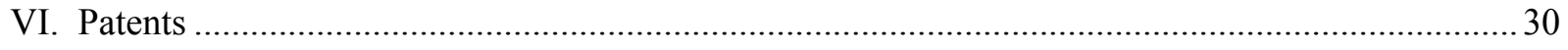

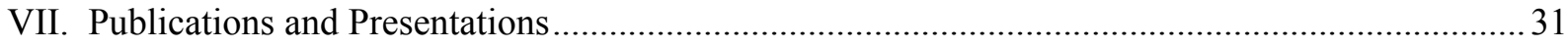

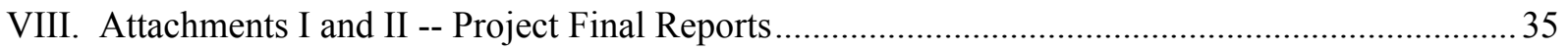




\section{Florida Hydrogen Initiative (FHI) \\ Dr. David L. Block, PI \\ Florida Solar Energy Center/University of Central Florida}

\section{Summary}

The Florida Hydrogen Initiative (FHI) was a research, development and demonstration (RD\&D) hydrogen and fuel cell program funded by U.S. Department of Energy (DOE). The FHI program objectives were to develop Florida's hydrogen and fuel cell infrastructure and to assist DOE in its hydrogen and fuel cell activities by creating partnerships for demonstration projects, sponsoring research and development programs, facilitating technology transfer between the public and private sectors, developing industry support and establishing unique hydrogen/fuel cell university-level education programs. The FHI program funded 12 RD\&D projects. Each project following peer review and DOE approval was issued a sub-contract with specific tasks, deliverables and budget. The results of these projects are presented in this final report.

\section{Comparison of Accomplishments with Program Objectives}

The FHI program objectives were to develop Florida's hydrogen and fuel cell infrastructure and assist the U. S. Department of Energy in its hydrogen and fuel cell program goals. The five objectives and the program results follow.

Objective 1 -- Creating partnerships for applied technology demonstration projects. The 12 funded FHI projects had 5 universities, 2 private firms and one public organization as PIs and 4 universities and 7 private firms as project partners. See Table 1 below.

Table 1. FHI Project PIs \& Partners

\begin{tabular}{|c|c|c|c|c|c|}
\hline & \multicolumn{3}{|c|}{ Principal Investigators } & \multicolumn{2}{|c|}{ Partners } \\
\hline TASK & UNIVERSITIES & PRIVATE & PUBLIC & UNIVERSITIES & PRIVATE \\
\hline 1 & Rollins & & & ASU, FAU & \\
\hline 2 & & EnerFuel & & FAU & AC,TRDA,GES \\
\hline 3 & & & OSC & & IDEAs \\
\hline 5 & UCF-FSEC & & & & $\begin{array}{l}\text { Chevron } \\
\text { Technology } \\
\text { Ventures }\end{array}$ \\
\hline 7 & UCF-FSEC & & & & \\
\hline 8 & UCF-FSEC & & & & \\
\hline 9 & UCF-FSEC & & & & \\
\hline 10 & & SRT & & & Electrolytic Tech \\
\hline 11 & FSU & & & & Bing Energy \\
\hline 12 & FIT & & & & \\
\hline 13 & USF & & & Tuskegee & \\
\hline 14 & & EnerFuel & & & \\
\hline Totals & 5 & 3 & 1 & 4 & 7 \\
\hline
\end{tabular}


Objective 2 -- Sponsoring research and development in the production, storage and use of hydrogen and in the use and application of fuel cells. Ten of the 12 projects were directly applied to this objective.

Objective 3 -- Facilitating technology transfer between the public and private sectors to create, build and strengthen high-growth potential, high technology companies. Five of the 12 projects were related to building high-growth potential, high technology companies.

Objective 4 -- Developing industry support or potential for widespread applications. Four of 12 projects are related industry support and widespread applications.

Objective 5 -- Developing unique hydrogen/fuel cell university-level education programs. This objective was met by one project.

\section{Approach}

The FHI program operated in two phases. In the Phase I program, activities began in 2004 and continued until 2009 during which the program was managed by the Florida Hydrogen Initiative, Inc., a not for profit corporation. During this period, four projects were funded. In Phase II, the DOE contract for the operation and management of the program was transferred to the University of Central Florida's Florida Solar Energy Center (FSEC). For the Phase II activities, the UCF Office of Research and Commercialization managed the contractual and financial aspects of the project and project management and technical oversight was provided by Dr. David Block of FSEC. During the Phase II period, eight projects were funded. This report presents the results for each of these 12 projects and covers both Phase I and Phase II activities.

The FHI program functioned by funding individual projects to conduct the research, development and demonstration activities. Each project was approved by DOE prior to issuing a sub-contract with tasks, deliverables and budget. For purposes of project management, each of the FHI sub-project activities was designated as a Task number within the program. The first six tasks, Tasks 1 to 6 , were part of the FHI Phase I activities as explained below. The next eight tasks, Tasks 7 to 14, were part of the Phase II activities. Tasks 2, 3 and 5 were carryover tasks and were completed in Phase II. The only exception to the Task rule was that Tasks 4 and 6 were management task awards to FHI, Inc. (Produced no results).

\section{A. Phase I}

In the Phase I program, a request for Letters of Interest (LOI) was used to select the initial four subprojects. These four projects were:

- Task 1. Florida Hydrogen Refueling Infrastructure -- Rollins College, Winter Park, FL

- Task 2. HyTech Rest Area: Demonstration of a Multi-kW Integrated Methanol Fuel Cell Power Plant for a Highway Rest Area -- EnerFuel Inc., West Palm, FL

- Task 3. Assessment of Public Understanding of the Hydrogen Economy through Science Center Exhibits-- Orlando Science Center, Orlando, FL

- Task 5. On-Site Reformation of Diesel Fuel for Hydrogen Fueling Station Applications -- Florida Solar Energy Center, jointly with the Chevron Technology Ventures, LLC.

In 2009, the FHI project was transferred by DOE to the University of Central Florida (UCF). This transfer marked the beginning of the Phase II program activities. 


\section{B. Phase II}

The Phase II activities began the process of allocating the remaining unobligated funds ( $\sim 2.5$ million) to new projects and to finish and/or complete the three Phase I projects. In October 2009, the funding allocation process began with a request for Letters of Interest (LOI). The LOI request resulted in five proposal submittals that were evaluated by a Proposal Evaluation Team of five distinguished members. The results of the Evaluation Team and discussions on each project's merit with DOE resulted in February 2010 in the selection of the following three new projects at FSEC:

- Task 7. Chemochromic Hydrogen Leak Detectors for Safety Monitoring - Dr. N. Mohajeri and Dr. N. Muradov, FSEC.

- Task 8. Development of High Efficiency Low Cost Electrocatalysts for Hydrogen Production and PEM Fuel Cell Applications - Dr. M. Rodgers, FSEC.

- Task 9. Understanding Mechanical and Chemical Durability of Fuel Cell Membrane Electrode Assemblies -Dr. D. Slattery, FSEC.

The next Phase II activity was the release to the public of a request for proposals (RFP) for the remaining funds of $\sim \$ 1.5$ million. The RFP was released in March, 2010 with applications due by April, 2010. The RFP was sent to a large list of potential responders. Following the receipt of 13 proposals, a six member review committee was established for proposal review and evaluation. The review committee met in May 2010 and recommended five new projects for funding. These 5 projects were submitted to DOE for approval. Approval was received and five projects were awarded in December 2010 as follows:

- Task 10. Production of Low-Cost Hydrogen from Biowaste (HyBrTec $\left.{ }^{\mathrm{TM}}\right)-\mathrm{Mr}$. R. Parker, SRT Group, Inc.

- Task 11. Development of a Low-Cost and High-Efficiency 500 W Portable PEMFC System Drs. J. Zheng, R. Liang, and W. Zhu, Florida State University, Mr. H. Chen, Bing Energy, Inc.

- Task 12. Interdisciplinary Hydrogen and Fuel Cell Technology Academic Program - Florida Institute of Technology, John Politano, Carolyn R. Lockyer, Mary Helen McCay, K Winkelmann

- Task 13. Design and Development of an Advanced Hydrogen Storage System using Novel Materials - E. Stefanakos, D. Goswami, and A. Kumar, University of South Florida

- Task 14. Advanced HiFoil ${ }^{\mathrm{TM}}$ Bipolar Plates - Mr. J. Braun, EnerFuel, Inc.

Thus, as of December 2010, all of the FHI funds were allocated and eight new RD\&D projects were under contract with work being conducted. In all, a total of 12 separate projects were conducted as part of the FHI program. The 12 projects include five involving hydrogen technologies (Tasks 1, 5, 7, 10 and 13), five involving fuel cell development (Tasks 2, 8,9,11 and 14) and two covering education and public understanding and information dissemination (Task 3 and 12). Note that Tasks 4 and 6 were administrative tasks and produced no results. The results for each of these 12 projects are presented in three ways in this final report as follows:

- A one paragraph Summary in the previous Executive Summary of this report,

- A two page Executive Summary for each of the projects which follows in the next section, and

- The complete final report of each project presented in the two Appendix sections at the end of this report.

The next sections will present each of the Executive Summaries of the above mentioned 12 projects. 


\section{Individual Project Executive Summaries}

The Executive Summaries are presented by task order starting with Task 1 and progressing through Task 14. The following Executive Summaries have been taken from each of the project final reports and shorten to two pages or less by the report author.

Task 1.Hydrogen Refueling Infrastructure and Rental Car Strategies, L. Lines, Rollins College, M. Kuby, Arizona State University, R. Schultz and Z. Xie, Florida Atlantic University -- 2007

A study by the National Renewable Energy Laboratory found that government fleets of hydrogen vehicles are not enough to jump-start the hydrogen industry. "Finding a way to transition from fleets to consumers," they concluded, "is equally important." This report addresses this critical need by developing strategies for Florida's early stages of hydrogen-powered consumer transportation. In particular, the report investigates urban and statewide networks of refueling stations and the feasibility of a hydrogen rental-car fleet based in Orlando. These two ideas are linked by the potential for a few stations, properly located, to serve the needs of a surprisingly high percentage of car renters in Orlando.

Hydrogen Rental-cars. The vision for a hydrogen rental-car business based at the Orlando International Airport (OIA) is compelling. Orlando is the country's \#1 tourist destination, but most of those tourists visit only a few local destinations. Central Florida has a reputation for innovation, with its unique theme parks and the hydrogen-powered space program at the Kennedy Space Center. Visitors could have a positive first exposure to hydrogen vehicles with no commitment, and become hydrogen advocates in their home regions and potential early adopters. The rental-car business combines the logistical advantages of a fleet operation while reaching large numbers of consumers. A hydrogen powered rentalcar fleet at OIA can provide guaranteed demand to the initial rollout of a hydrogen-refueling infrastructure in the area.

To investigate the feasibility of a hydrogen rental-car business at the Orlando International Airport, we surveyed rental-car customers to understand the demand side. Our survey of 435 renters found that half would be willing to pay more to rent a hydrogen car, and over $80 \%$ would be willing to detour a mile or more for a station. Most importantly, we found that three stations at the airport, theme parks, and downtown Orlando would enable $80 \%$ of car renters to complete trips to all of their destinations, increasing to $85 \%$ with a fourth station at Kennedy Space Center.

From the rental company's point of view, major barriers to a successful hydrogen rental-car business model include: viable consumer target markets; a steady revenue stream via rental of conventional vehicles; a means of identifying customers willing to upgrade to hydrogen; adequate range and refueling locations; breakdown and insurance coverage; the status value of hydrogen cars; the resale market for used vehicles; and most importantly, the availability of hydrogen cars to purchase at a reasonable (subsidized) price.

Modeling Hydrogen Refueling Infrastructure. Given the high cost of the initial refueling stations, locating a limited number of stations as efficiently as possible will be a key to early success of the industry in Florida. A model was developed that strategically locates stations to serve the maximum consumer demand possible, and quantifies the tradeoff between the number of stations and how much potential demand can be served. The foundation of the model is a road network and a set of traffic analysis zones within a geographic information system (GIS). Using GIS, we find the shortest path (in travel time) from every origin to every destination. Then, given the flow volume on each path, a driving range (in miles) that hydrogen vehicles can be reasonably driven between refueling, and the number of stations to build, the model uses operations-research (OR) techniques to solve for the optimal set of locations that maximize the amount of transport demand that can be served. Transport demand is 
measured either in number of trips or vehicle-miles traveled. The GIS and OR models are integrated in a spatial decision support system that researchers can use to develop data, enter assumptions, analyze various scenarios, evaluate tradeoffs, and map results. For the FHI, we used this model to investigate strategies for rolling out an initial refueling infrastructure in Florida at two different scales of analysis: a metropolitan Orlando network, and a statewide network.

Statewide Infrastructure. To maximize the potential for drivers to use the first set of stations, the model suggests clustering the first ten stations in the Miami-West Palm Beach corridor and the Tampa-Orlando 1-4 corridor, followed by filling in and connecting these clusters. In the statewide analysis, we assume a driving range for vehicles of 100 miles, which allows a large safety margin compared with the expected technical range of hydrogen vehicles of around 300 miles per fill-up. The 100-mile range should be treated as a maximum: it is important to place connecting stations where they can also serve crossing traffic flows and local traffic flows, rather than spacing them at regular 100-mile intervals. We also analyzed scenarios of 75- and 50 mile vehicle range, in which optimal station networks were progressively more clustered and less connected.

Orlando Infrastructure. To get the most benefit out of the initial refueling infrastructure in Orlando, the model locates stations at funnel points on the road network through which many trips pass, from many origins to many destinations. This strategy wins out over spreading the stations evenly across the metropolitan area. It is more important to locate the early set of stations along the routes people travel rather than locate them near their homes, especially because the first 10 stations can only truly be near a small fraction of Orlando residents' homes, but can be right on the route of over half of all trips.

Recommendations. We recommend developing a statewide network of 25 stations in five stages or tiers, so that as each tier is constructed, the clusters and the connections between them grow in a coordinated way. The first tier of three stations consists of the airport, downtown, and theme park stations in Orlando needed for the hydrogen rental-car business. Given Orlando's head start in the hydrogen industry with its existing station at the airport, we see it as the key to getting hydrogen moving in Florida. The second tier of seven stations rounds out the Top 10. It creates a hydrogen corridor from Miami Lakes to Ft. Lauderdale to Delray Beach, as well as a connected triangle between Tampa, Orlando, and Gainesville. The third tier fleshes out the Orlando, Tampa, and Miami clusters. The fourth tier completes the network up 1-95 from Palm Beach Gardens to Jacksonville. The fifth tier extends the 1-75 network north and south of Tampa-St. Petersburg, as well as adding to the Miami and Orlando clusters and shortening the distance between stations on Florida's Turnpike.

For Orlando, we recommend working towards an 11 - station network, which could capture $54 \%$ of intracity trips. The first tier or stage includes the airport, downtown, and theme park stations that are essential to the rental-car business, as well as two other stations on the northern side of town at traffic funnel points that capture heavy traffic flows not otherwise captured by the other three stations. The second tier of six stations includes stations in Kissimmee and Sanford that are recommended for the statewide network.

Next Steps. Future modeling research could focus on a more detailed analysis of the Miami and Tampa metropolitan areas similar to the Orlando network analysis, and an extension of the model to deal with drivers' deviations from their shortest paths. Next steps for the rental-car business plan are to begin discussions with rental-car companies, as well as energy companies that build stations, and possible partners at Disney and NASA. 


\section{Task 2. Methanol Fuel Cell Vehicle Charging Station (Old Title - Hydrogen Technology Rest}

Area), Michel Fuchs, EnerFuel, Inc.--2012

The HyTech project was a methanol fuel cell demonstration with the objective of promoting the use of renewable energy in Florida. The goal of the project was to produce methanol from citrus waste, thus demonstrating this application as a viable and inexpensive feedstock for generating electrical power. The project used a fuel cell system with integrated fuel processing capabilities and consisted of the complete system design, installation, operation and data collection. Over the course of the project, two system designs one for the Florida Turnpike Turkey Lake Service Plaza Demo, Orlando, and the other for an electric vehicle charging station at Florida Atlantic University, Boca Raton were completed and results were obtained.

Phase I -- Florida Turnpike Turkey Lake Service Plaza Demo, Orlando

The objective of the Phase I HyTech Rest Area project was to design, fabricate, install and demonstrate a grid-tied, multi-kW fuel cell system operating on citrus peel derived methanol. The fuel cell system was to be installed at the Florida Turnpike Enterprise's Turkey Lake service plaza in Orlando, Florida. The system would be used to offset a portion of the facility's grid power needs and to demonstrate the generation of electrical power from a waste product.

The goals and objectives of the Phase I project were as follows.

- Design, construct and demonstrate a grid-independent, multi-kW FC system operating on citrus peel derived methanol

- Produce sufficient methanol from citrus waste to support the demonstration

- Promote the project to the public using media coverage

The initial Phase I task was the design of the demonstration site that included the fuel cell systems, the 5,000 gallon above ground methanol tank and the inverters necessary to tie the system to the facility electric grid. The first part of the fuel cell system design was to evaluate two candidate fuel cell systems capable of being fueled with methanol. Based on the results of a study, indirect methanol fuel cells (IMFCs) were recommended for the program. This conclusion was arrived at through the evaluation of the current state of the art for IMFC and DMFC technologies.

The method of operation for the system assumes that the facility has a primary AC power source. Under normal conditions, the system only requires a single warm up event, after which the fuel processor is kept at temperature via system operation or via built-in resistive heaters. When the system is providing backup power, fuel is burned to provide the necessary heat for fuel processing. When the system is in standby mode, awaiting a backup power event, the built-in resistive heaters maintain the fuel processor at temperature. To account for any lag between the time backup power is needed and when the ElectraGen is able to respond, a $48 \mathrm{~V}$ battery system is electrically connected in parallel with the fuel cell system output.

To interface the IdaTech FCs into the Phase I program, SMA Sunny Island 5048U inverters were selected and incorporated into the design. The Sunny Island inverters are designed to manage a stand-alone grid system, with ability to utilize power from various sources, such as solar, fuel cell, wind, diesel generator or utility grid. Since $208 \mathrm{~V}, 3$ phase power was required to interface with the utility grid at the demo site, the inverters were arranged so that each powered a single phase. One of the inverters would be programmed as the "Master" and the other two set as "slaves". The "Master" automatically manages all three inverter outputs, as well as managing the tie-in/ synchronization of power into the facility mains. For redundancy, one set of three inverters would have been used with each two fuel cell systems. 
The basic configuration of the fuel cell system design and the layout of the demonstration site was completed and the resulting documents developed. From these documents, Gasoline Equipment Systems generated the civil and electrical plans necessary for site permitting and subsequent construction. These plans are provided in this report.

Phase II -- Electric Vehicle Charging Station (EVCS) at Florida Atlantic University (FAU) in Boca Raton

Due to cost constraints, EnerFuel performed a detailed evaluation of three new sites and then made a location change recommendation. EnerFuel presented the three options for changing the site and recommended the FAU location. This recommendation was accepted by UCF and DOE. Final approval was received in April 2012 to modify the project in order to design, construct and test a methanol fuel cell, electrical vehicle charging station, at Florida Atlantic University (FAU) in Boca Raton, FL.

The remainder of the project was directed at the Electric Vehicle Charging Station and the reconfiguring of FC system for the application. The charging station location was selected to be adjacent to FAU's existing vehicle charging complex in order to allow easy access to vehicles. FAU's mechanical engineering department was subcontracted to construct the station, as well as assist in system testing. Two vehicle charging spaces were set aside and devoted to the fuel cell charging station.

The fuel cell system was designed and constructed at the FAU site. The final results from the Phase II efforts were:

1. Demonstration of the EVCS was performed using the inverters.

2. Evaluation of the station's performance was based on FC test results at EnerFuel combined with the FAU results of using the inverters to charge the electric vehicles.

3. The charging station could not be operated as initially intended since no grid power was available to support the system startup.

4. The EVCS design successfully incorporated the FC system and inverters that were originally purchased for the Phase I project.

5. The project exposed engineering students with real world design issues by allowing several students to assist in the design and construction of the station.

In conclusion, the project showed the advantages of using a fuel cell, providing grid independent vehicle charging at high efficiency and the ability to use renewable fuels. Evaluation of the fuel cell charging station concept suggested that it could be used as a backup to "grid" charging, in locations that have no access to grid-power or in instances that require a rapidly deployable charging system. As an example, operations that rely on electric vehicles could continue to operate even during a major power outage, such as that brought on by a "Katrina"/ "Sandy" type event.

\section{Task 3. Assessment of Public Understanding of the Hydrogen Economy Through Science Center Exhibits, J. Newman, Orlando Science Center -- 2010}

The objective of this project was to design and build an interactive Science Center (OSC) exhibit called: " $\mathrm{H}_{2}$ Now: the Great Hydrogen Xchange". The exhibit would be used to satisfy the three objectives of creating awareness among the science center visiting public on hydrogen, assessing current public understanding about hydrogen science and engineering and increasing public understanding of hydrogen science and technology.

Finding sources of clean, renewable energy are one of today's most pressing issues. However, there is much information - and misinformation- about renewable energy, particularly regarding the use of hydrogen as a new energy carrier. Increasing the basic knowledge and awareness among the public about 
hydrogen as a viable energy vector, and correcting misconceptions of hydrogen and its properties, is necessary to create accurate and positive opinions about this substance as an important alternative to fossil fuels.

Key to this goal is the accurate and educationally effective translation of technical scientific and engineering concepts into a format that is easily understandable and accessible. To that end, $\mathrm{H}_{2} \mathrm{Now}_{\mathrm{N}}$ the Great Hydrogen Xchange exhibit will allow OSC visitors to be able to visualize a community powered entirely by hydrogen, and discover that the renewable energy is a viable alternative to other energy carriers currently in use.

Working with I.d.e.a.s at Disney MGM Studios, the Orlando Science Center set out to perform front end evaluation and subsequent design, fabrication, and installation of an interactive exhibition at OSC.

Beginning with the schematic design of concepts and educational objectives, the exhibit underwent design and prototyping of both physical components and interactive software. This exhibition, $\mathrm{H}_{2} \mathrm{Now}$, the Great Hydrogen Xchange, contains two main interactive areas. The first, the $\mathrm{H} 2$ Charging Station, is where the visitors will have the opportunity to express their opinions about alternative energy vectors, and then interact with increasing levels of content about hydrogen as an alternative fuel. As they answer questions correctly about hydrogen, they can earn Hydrogen Energy Units (HEU's) that they can then 'spend' at Hydropolis to power various interactive 'city' elements.

Software developers at I.d.e.a.s, working with OSC staff, incorporated several changes to the software design and interface based on critical analysis of visitor usage to test for mechanical effectiveness. These changes led to major modifications of both the software design and communication interface. OSC brought in a local multimedia design firm to complete this redesign at which time work concentrated on visitor evaluation to determine the extent to which the affective and cognitive goals of the exhibit were being met.

To evaluate the display, a total number of 65 visitors were observed and 66 interviewed for a total of 131 visitors. (Note that not all observed visitors took part in the interview). Since the exhibit was designed for visitors age 12 and up, participants were pulled from this age group and/or if a parent with children under age 12 was interviewed, the questions were posed to the parent. Additionally, children under age 12 were excluded from observation portion of the evaluation.

There were five learning objectives established for $\mathrm{H}_{2}$ Now. These objectives were done so that after participating in the exhibit, visitors would be able to:

1. Explain what hydrogen is, where it comes from, and some of its possible uses.

2. Distinguish between truth and myth about hydrogen as an alternative fuel, and give specific examples.

3. State a benefit and a challenge of hydrogen as an alternative energy vector.

4. Give an example of how hydrogen power is created, and how it can affect everyday life.

5. Comment on a possible impact of hydrogen power on the future state of the world.

The interview/questionnaire method was designed to glean visitor understanding of these five objectives. Clearly, the results indicate that the vast majority of visitors were able to achieve the first learning objective, and most visitors achieved the third learning objective. The results also indicate that, while the 'myth' of hydrogen as an alternative fuel was not specifically measured, it is clear that most visitors came away with a more clear understanding of hydrogen's use as a renewable energy carrier. Additionally, visitors were very clear on the benefits i.e., "clean, renewable, doesn't pollute', and as the results showed, also clear on its current challenges i.e., 'too expensive to produce'. Most visitors were also able to verbalize how hydrogen power is created as it is presented in the exhibit, and the extent that hydrogen can 
be used to effect everyday life. The only learning objective that was not clearly measured, but can be inferred from the questions posed, is the possible impact of hydrogen power on the world's future.

The $\mathrm{H}_{2}$ Now exhibit has completed software and hardware 'shakedown' that resulted in a streamlined interface and communication and is functioning fully at the Orlando Science Center. At the present time, the exhibit is not in use. In addition, exhibit enhancements have been made including a linear display on other sources of renewable energy and plans are in the works for an interactive 'video game' on renewable energy to complement the messages of $\mathrm{H}_{2} \mathrm{Now}$. The video game was received from a grant from Progress Energy Foundation.

Evaluations to determine the extent to which $\mathrm{H}_{2}$ Now meets educational objectives revealed that:

- $77 \%$ of the visitors surveyed liked the exhibit

- $64 \%$ indicated that they 'learned something' or found the exhibit 'informative' in the word choice section of the interview

- $\quad 32 \%$ found the exhibit 'interesting' indicating that both cognitive and affective learning has clearly taken place

- $65 \%$ indicated that they found out something they didn't know before. This finding is a clear indication of both cognitive gain and a change of attitude i.e., compare this to the $44 \%$ who, when given the choice, selected "I learned something new".

In addition, OSC has enhanced the experience with a short film produced by i.d.e.a.s called "I Am Hydrogen" and is exhibiting a linear display on additional information on renewable energy.

\section{Task 5. On-site Reformation of Diesel Fuel for Hydrogen Fueling Station Applications, A. Raissi, Florida Solar Energy Center, J. Stevens, Chevron Technology Ventures, LLC -- 2010}

The objective of this project was to research options leading to the eventual transformation from gasoline and diesel as the transportation fuels to that of hydrogen $\left(\mathrm{H}_{2}\right)$. One major research issue regarding the future use of hydrogen is how to deliver $\mathrm{H}_{2}$ fuel within the transportation sector. If $\mathrm{H}_{2}$ is to be derived from organic feedstocks, it may be desirable to deliver the feedstock to the local fueling station and then chemically convert or reform it to $\mathrm{H}_{2}$ on-site. Furthermore, it would be advantageous if the primary feedstock can be used as a fuel in the present engines (e.g., internal combustion engines, ICE, etc.). For example, diesel fuel could be delivered via tanker truck and converted on-site in a small reformer for generating and dispensing hydrogen at the fueling station. The advantages of this approach are:

- Existing delivery infrastructure (highway to fueling station) could be utilized.

- More energy can be delivered per trip when the tanker is filled with diesel instead of liquid hydrogen.

- The fueling station would be able to service both ICE and fuel cell powered vehicles simultaneously.

- The fueling station operation would be flexible and easily change fuel availability in the form of hydrogen or diesel fuel to meet the customer demand.

The aim of on-board reforming is to convert liquid fuels into a hydrogen-rich gas for use in ICE or fuel cell powered vehicles. The drawbacks of on-board reformers include their inherent complexity, added weight, high cost, and the need for an elaborate purification system to rid hydrogen of impurities that will harm proton exchange membrane fuel cells. It is noted that hydrogen purity requirements of the ICE power plants are much less stringent. 
Thus, instead of on-board reforming, the Florida Solar Energy Center (FSEC) jointly with Chevron Technology Ventures (CTV) developed a process for the on-site reforming of diesel fuel. The approach was to first pre-reform diesel to shorter chain hydrocarbons $\left(\mathrm{C}_{1}-\mathrm{C}_{6}\right)$ before further treatment in the main reformer unit. The removal of sulfur from the pre-reformate is a critical issue, as the reformer catalyst has limited sulfur tolerance. Another object of this project was to develop a new on-demand forecourt hydrogen production technology by catalytically converting high-sulfur hydrocarbon fuels to a sulfur-free gas. FSEC built, operated and validated the performance of a diesel pre-reformer, and also built and demonstrated a small scale desulfurization unit. CTV provided catalysts and know-how for the design and fabrication of the pre-reformer.

FSEC developed a novel process for converting high-sulfur diesel to a mixture of light molecular weight hydrocarbons that can be readily reformed with steam to fuel cell grade hydrogen gas. The process employs a catalytic pre-reformer coupled with a regenerable $\mathrm{Fe}^{2+} / \mathrm{Fe}^{3+}$ redox/electrolyzer system used for the scrubbing of $\mathrm{H}_{2} \mathrm{~S}$ from the pre-reformer effluent in the form of elemental sulfur. Results from laboratory tests showed that electrolysis of acidic $\mathrm{FeSO}_{4}$ aqueous solution was very efficient with a columbic efficiency of $100 \%$ at a cell voltage of $1.0 \mathrm{~V}$. It was also shown that the electrolytic process can be operated with a Pt-free anode to oxidize ferrous to ferric ions, thereby reducing the cost of the electrolytic system, considerably.

Furthermore, FSEC researchers developed a robust bi-functional catalyst for accomplishing the prereformation of the high-sulfur fuels (sulfur content as high as 5,240 ppmw) to short chain hydrocarbons $\left(\mathrm{C}_{1}-\mathrm{C}_{4}\right.$, mostly propane) at an average yield of $97 \%$. Results showed that after100 hours of continuous operation, the combined $\mathrm{Fe}^{2+} / \mathrm{Fe}^{3+}$ redox/electrolyzer $\mathrm{H}_{2} \mathrm{~S}$ scrubber and pre-reformer were able to achieve desulfurization efficiencies above $95 \%$ and remove sulfur down to less than 5 ppmv in the pre-reformer outlet gas.

\section{Task 7. Chemochromic Hydrogen Leak Detectors for Safety Monitoring - N. Mohajeri and N. Muradov, Florida Solar Energy Center -- 2012}

The ability to detect hydrogen leaks at storage, transmission and usage sites is crucial for hydrogen's safe handling and use. The output from this project is envisioned to lead to a new generation of versatile chemochromic hydrogen detectors that employ "smart" materials that cost less, possess fast discoloration kinetics, are user-friendly and reliable and have superior field worthiness. The project activities have evaluated the performance (sensitivity) of these visual sensors at different hydrogen concentrations in air (from 1 to $100 \mathrm{vol} \%$ ) and found no interference with other reducing gases $\left(\mathrm{CO}, \mathrm{NH}_{3}, \mathrm{CH}_{4}, \mathrm{H}_{2} \mathrm{~S}\right)$ at ambient temperatures.

In this project, two classes of chemochromic hydrogen sensors were examined, one that is irreversible and one that is reversible. The irreversible sensor permanently changes color when exposed to hydrogen and the reversible sensor changes color when exposed to hydrogen, but then after the hydrogen exposure has stopped, reverts back to its original color.

\section{Irreversible Sensors}

The objectives of the irreversible sensor program was to research, develop, and demonstrate a new generation of versatile chemochromic hydrogen detectors that employ "smart" materials and are fixed to a tape material which is placed on the exposure surface. The first generation of chemochromic hydrogen leak detectors; i.e. sensors, developed at and patented by FSEC, contained titanium dioxide particles doped with $\mathrm{PdO}$ encapsulated within a special silicone matrix. The process of coloration is irreversible i.e. once $\mathrm{PdO}$ pigments were exposed to hydrogen gas, the resulting color change would not revert back to the original color. In the presence of $5 \mathrm{vol} \% \mathrm{H}_{2}$ gas, the color change is discernible within about 5 min. 
The tape can be used at temperatures as low as $-30{ }^{\circ} \mathrm{C}$ and as high as $50{ }^{\circ} \mathrm{C}$ (higher temperatures have not been tested).

The focus of the irreversible sensor project was twofold: First, to develop the next generation chemochromic pigments that would have a shorter response time toward hydrogen gas and second, to lower the overall cost of the sensor materials. Three approaches were considered to address these challenges:

1. To find alternative materials to $\mathrm{TiO}_{2}$ for $\mathrm{PdO}$ support.

2. To dope the pigment with a noble or non-noble metal to improve the reduction kinetics of PdO particles.

3. To study alternative matrices for pigment encapsulation.

The results were:

1. Alternative support to replace $\mathrm{TiO}_{2}$ for $\mathrm{PdO}$ deposition - One of the most important discoveries of this project was the identification of barium sulfate as a new support for hydrogen chemochromic pigments. Use of barium sulfate has potential to reduce the pigment's cost and to heighten sensitivity toward hydrogen by reducing the response time by fourfold compared to the $\mathrm{TiO}_{2}$ based pigment. Finally, initial environmental testing of the barium sulfate based pigments has shown no effect on the performance when exposed to salt water, fresh water, or humidity. Exposure to sunlight, i.e. UV, has shown minor discoloration similar to $\mathrm{TiO}_{2}$ based pigments.

2. Noble or non-noble metal doping of $\mathrm{PdO} / \mathrm{TiO}_{2}$ pigments to improve the reduction kinetics of $\mathrm{PdO}$ particles -- During the course of this project, a new doping technique was developed that has shown to improve the hydrogen response time of the $\mathrm{PdO} / \mathrm{TiO}_{2}$ pigment by eightfold. It is noteworthy to mention that the same doping technique can be used with barium sulfate technology to further enhance the hydrogen sensitivity. Alternative matrices for pigment encapsulation - In pursuit of improving the membrane casting technique, a new flowable silicone matrix has been identified that is highly comparable to the original military grade RTV-3145 in terms of hydrogen permeability. The new matrix, Dow Corning 734, has not only simplified the casting process but also has reduced the waste of valuable pigment while casting and reduced curing time. Furthermore, the cost of 734 is about one quarter of the cost of RTV-3145.

The funding of this research activity has also resulted two filed US patent applications. One based on the new pigments (U.S. Application No. 13/588,779; Doped Palladium Containing Oxidation Catalysts) and the other, the application of chemochromic membrane in PEM fuel cells to check for pinhole and crack defects in PEM before or after fuel cell operation (U.S. Application No. 13/594,163; Chemochromic Membranes for Membrane Defect Detection).

\section{Reversible Sensors}

The second part of the chemochromic sensor research was the development of cost-effective, highly selective reversible hydrogen visual sensors that are reversible. Reversible sensors mean that the sensor changes color when exposed to hydrogen, but then after the hydrogen exposure has stopped, the sensor color reverts back to its original color.

The main objectives of the reversible sensor work have been successfully accomplished in that a series of active, highly selective and durable reversible hydrogen sensors have been developed. The reversible chemochromic pigments are based on the oxo-compounds of molybdenum (Mo) and tungsten (W) that have suitable redox potentials for the reduction reaction in the presence of $\mathrm{H}_{2}$ and bleaching reaction in 
the presence of air. These sensors show very high selectivity toward hydrogen and no interference with other reducing gases such as $\mathrm{CO}, \mathrm{CH}_{4}$ and $\mathrm{NH}_{3}$. They also exhibited resistance to sulfur poisoning.

By changing the chemical composition of the pigments, they can acquire slow (days-to-weeks) or fast (minutes -to-hours) bleaching characteristics. The developed pigment formulations showed excellent performance and sensitivity at a wide range of $\mathrm{H}_{2}$ concentrations in air (from 1 to 100 vol.\%). Although the pigments (especially, Mo-based pigments) demonstrated some UV sensitivity, they performed well indoor. Long-duration environmental testing of the reversible sensors exposed to the atmospheric elements for 5 months showed that W-based pigments had better durability than Mo-based pigments. More work is needed for improving UV-resistance of the reversible pigments.

\section{Task 8. Development of High Efficiency Low Cost Electrocatalysts for Hydrogen Production and PEM Fuel Cell Applications - M. Rodgers, Florida Solar Energy Center -- 2013}

The primary objective of this project was to decrease platinum (Pt) usage in fuel cells by increasing catalyst activity and broadening the knowledge base of structure-property relationships in catalysts. Proton exchange membrane fuel cells (PEMFCs) depend on the use of platinum-based electrocatalysts. Due to their high catalytic activity and their stability in the fuel cell environment, platinum or platinum alloys supported on carbon black are presently the catalysts of choice for PEMFCs to catalyze both the anodic hydrogen oxidation and cathodic oxygen reduction reactions. Fuel cell performance is usually limited by the slow kinetics of the cathode oxygen reduction reaction (ORR). A major factor limiting fuel cell usage is the cost, and current $\mathrm{Pt} / \mathrm{C}$ catalysts represent about $40 \%$ of the total cost.

Catalytic activity of platinum-based catalysts depends greatly on the dispersion and the size distribution of the platinum crystallites. Not only is small particle size desirable for obtaining large active surface areas, but for all the platinum in a PEMFC catalyst layer to be active, the fuel or oxidant must react at the interfacial region between the PEM and the platinum, known as the "three phase reaction zone". This zone is the three phase interface of catalyst, carbon, and electrolyte that allows effective gas and water diffusion and proton transport and electron transport to and from the catalyst sites.

Because contact between the electrolyte and the catalyst is a critical factor in obtaining high performance, current approaches aimed at lowering the platinum loading focus on localizing the catalyst to where the electrocatalytic reaction takes place. Pulse electrodeposition appears to be the most promising method of catalyst fabrication due to advantages such as controlled particle size, stronger adhesion, uniform electrodeposition, selectivity of hydrogen, and reduction of internal stress. Control of factors such as $t_{\text {off }}$, $\mathrm{t}_{\mathrm{on}}$, duty cycle, peak current density, catalyst precursor, co-catalyst, and stabilizing agent allow fine tuning of the catalyst structure. The structure can be further tweaked with the use of methods to change the mass transport rate of the catalysts in the electrodeposition solution.

In this work, catalysts were prepared by electrodeposition on carbon paper that had been loaded with carbon and Nafion $^{\circledR}$, resulting in a three phase reaction zone. Pulse electrodeposition was used with a rotating disk electrode as a first step, taking advantage of the benefits of pulse electrodeposition. The rotation of the working electrode in rotating disk experiments brought analyte molecules from the bulk solution towards the electrode, resulting in increased mass transport over diffusion-only experiments. Catalyst activity was also measured using a rotating disk electrode. After achieving high performance, the stability of the electrodes was investigated and the electrodeposition process was scaled up to form an electrode that is a practical size for use in a fuel cell.

These results led to the optimization of several variables (platinum precursor concentration, electrode rotation rate, peak current, number of cycles, on time, off time, stabilizer concentration) during electrodeposition. These values were then used in all future electrodepositions. The results showed that 
electrode morphology impacted performance in that improved performance was generally observed with higher electrode coverage, smaller particle size, and the formation of "feathery" or "flakey" particles.

The goal of the next task was to evaluate carbon supports used in the gas diffusion electrodes. Catalyst supports can affect both the activity and the stability of electrodes in fuel cells. Different carbon supports, including XC-72, Cabot Regal GP-47, activated carbon KB-B, and graphene oxide were investigated. Thermal pretreatment of XC-72 carbon had no effect on electrode performance. The performance of electrodes made with XC-72 carbon support was lower than that of Cabot Regal GP-47 and activated carbon KB-B. Graphene is promising as a support due to its high surface area and stability and it was shown that when graphene oxide was reduced, the electrode performance at high voltage was improved over that of the baseline electrode.

Next, three Pt alloy based colloidal particles were synthesized via a polyvinylpyrrolidone (PVP) protected process. The colloidal nano-particles of the Pt ternary system with presence of PVP were stable and the particle size distributions were uniform with an average size of 4 to $5 \mathrm{~nm}$. The nanoparticles were adsorbed onto the carbon electrode through immersion of the sprayed carbon paper into the colloidal solution followed by sonication, but the ORR was very low. This low performance was attributed to the low quantity of particles adsorbed.

Results showed that catalyst morphology is very important in electrode performance. In many cases in this project pulse electrodeposition led to less desirable catalyst morphologies, including Pt particle aggregation. To prevent Pt particle aggregation a new electrodeposition method was developed where the carbon electrodes were seeded with nanosized Pt colloidal particles prior to electrodeposition. This new electrodeposition method improved electrode morphology and, therefore, performance. By seeding the electrodes with catalyst nanoparticles prior to electrodeposition, it was possible to achieve the same performance as a commercial electrode with $1 / 5$ the amount of Pt. The improved performance of the seeded electrodes was attributed to improved catalyst coverage on the electrodes. It was also shown that carrying out successive plating rather than direct plating resulted in improved performance, which was attributed to smaller Pt agglomerate size.

Because lanthanide elements have shown surprising results in terms of catalytic activity and outstanding material properties, Pt- and Pd-lanthanide based catalysts were evaluated. Ceria was formed through thermal hydrolysis, adsorbed onto the carbon powder, and deposited onto GDLs. Pd was electrodeposited onto the GDLs. Although differences in performance were observed, SEM showed that the ceria disappeared through the electrodeposition and characterization process. The loss of ceria was attributed to the electrochemical reactions resulting in formation of $\mathrm{Ce}^{3+}$, which is soluble. To prevent loss of ceria, a new procedure was developed whereby the ceria was calcined prior to being exposed to any electrochemical conditions. It was clear that the calcination resulted in improved ceria stability, although the calcined ceria was not shown to affect performance of Pt-based electrodes.

Pd-based electrodes were prepared using catalyst seeding. The best performance was observed when Pt$\mathrm{Pd}$ was used as a seed, while the performance of the electrodes that used Pt-Ru and Pt-Pd-Ru as seeds were quite similar. Although their performances were lower than those of the commercial Pt-based catalyst, when normalized for PGM content, the performance of the Pd electrodes were improved over the commercial catalyst.

Scaling up of the electrode and electrode stability were investigated. For scaling up, an electrodeposition flow cell was designed and built and electrodes of $25 \mathrm{~cm}^{2}$ were prepared. This was a 50 -fold scale up in size compared to the $0.5 \mathrm{~cm}^{2}$ electrodes that were prepared with the rotating disk electrode.

Unfortunately, platinum loading using the flow cell was low and, as a consequence, fuel cell performance was poor. 
An accelerated stress protocol based on potential cycling was developed to investigate the stability of electrodes containing activated carbon and reduced graphene oxide as supports. It was shown that the electrode containing reduced graphene oxide had superior stability and did not degrade during testing.

\section{Task 9. Understanding Mechanical and Chemical Durability of Fuel Cell Membrane Electrode Assemblies - D. Slattery, Florida Solar Energy Center -- 2012}

The useful life and durability of the current proton exchange membrane fuel cell (PEMFC) falls short of that required for its commercialization, primarily due to combined chemical and mechanical degradation of the membrane. The objective of this project was to increase PEM membrane useful life through a better understanding of the degradation mechanisms and their mitigation. Durability targets for membranes in automotive and stationary fuel cell applications have been established by DOE to be 5,000 hours and 40,000 hours, respectively. The current fuel cell membrane useful life (i.e. 2,500 hrs, as of 2011) falls short of both DOE durability targets.

One of the main sources of membrane degradation is hydroxyl radical attack of the membrane polymer. These hydroxyls are formed where platinum, hydrogen, and oxygen are present. Once formed, the radicals diffuse into the membrane, and attack both the main- and side-chains of the membrane polymer, leading to pinhole and crack formation. This loss of membrane integrity results in large-scale hydrogen crossover, shortening the cell life and generating large performance losses. Mitigation of chemical degradation will significantly enhance the lifetime of membranes, making fuel cells more economical. However, in order to decrease chemical degradation, the chemical processes that contribute to membrane failure must be better understood.

This project consisted of four major tasks. The objectives for Task 1 were to identify five suitable additives for scavenging radicals in the membrane and then to characterize the membranes containing additives and determine the platinum particle size as a function of additive. Task 2 objectives were to locate and characterize the platinum band in the membranes and to investigate the impact of cell components and operation parameters on platinum band formation and membrane degradation. The goals of Task 3 were to select some heteropolyacids to be used for mitigation of platinum band formation and to incorporate these into a sublayer between the membrane and the electrode. And, finally, Task 4 was to combine the best performing additive from Task 1 with the most effective heteropolyacid from Task 3 to achieve a more durable cell.

The Task 1 objective was to identify additives for scavenging radicals. The results from experimentation established ceria's ability to mitigate membrane degradation through radical scavenging. Key results are as follows:

1. Five additives for scavenging radicals were identified, down selected to two candidates through exsitu gas and liquid Fenton testing; and then the best candidate, cerium oxide, was evaluated in-situ for both automotive and stationary applications.

2. Ex-situ testing on ceria's ability to stabilize a PEM membrane demonstrated that ceria particles reduced fluoride emission by up to one order of magnitude due to the ceria's ability to scavenge hydroxyl and hydroperoxyl radicals. The degradation mitigation was found to increase with increasing additive concentration, though it was independent of ceria formulation and therefore of particle size. End-group unzipping was confirmed as the main degradation mechanism for the liquid Fenton test by reaction product analysis and the gas Fenton test verified a reduced level of side-chain attack versus testing without cerium. 
3. Experiments showed that cerium oxide acts as a radical scavenger and successively protects the membrane, reducing both the OCV decay and fluoride emissions dramatically.

4. The analysis of the platinum particles in the membrane showed that Pt band formation is also influenced by the presence of this cerium additive. Cerium oxide incorporation resulted in the formation of fewer and larger particles platinum, with less platinum precipitation overall.

5. In 500 hour tests, the addition of $1.0 \mathrm{wt} \%$ of commercial ceria resulted in sevenfold decrease in the OCV decay rate versus the baseline while losing less than $1 \%$ of its fluorine inventory. It also showed no change in performance or hydrogen crossover, resulting in an essentially unchanged MEA.

The results from Task 2, experiments showed relative humidity $(\mathrm{RH})$, equivalent weight (EW), catalyst type, and amount of platinum deposited in the membrane each influenced degradation in varying degrees. For RH, degradation was higher for the 20 and 30\% RH and lower for 10 and 100\% RH. At high RH, decreased degradation is attributed to dilution of radicals, higher oxide coverage on $\mathrm{Pt}$, and lower partial pressures of reactant gases. At low $\mathrm{RH}$, decreased degradation is attributed to reduced Pt band formation due to low mobility of Pt in low RH conditions. With regard to the influence of EW, $950 \mathrm{EW}$ polymers exhibited the lowest durability. This has been explained in terms of degree of crystallinity, which increases with increasing EW and decreasing side chain length, and which in turn impacts the mechanical stability of the membrane.

The third parameter investigated, catalyst type, showed that fluoride emission was three times higher for $\mathrm{Pt} / \mathrm{C}$ than for $\mathrm{PtCo} / \mathrm{C}$ and the $\mathrm{OCV}$ decay was nearly twice as great for the $\mathrm{Pt} / \mathrm{C}$ as that for the $\mathrm{PtCo} / \mathrm{C}$. Transmission electron microscopy images showed that platinum particles were dispersed throughout the membrane of the catalyst coated membrane $(\mathrm{CCM})$ containing $\mathrm{PtCo} / \mathrm{C}$, while the $\mathrm{CCM}$ containing $\mathrm{Pt} / \mathrm{C}$ showed formation of a platinum band. The platinum band in the cell containing $\mathrm{Pt} / \mathrm{C}$ is cited as the cause for the increased degradation compared to the cell containing $\mathrm{PtCo} / \mathrm{C}$.

When Pt was incorporated into the membrane, the highest degradation was observed with the membrane containing $10 \mathrm{~mol} \% \mathrm{Pt}$, while membranes containing 0, 30, and $50 \mathrm{~mol} \% \mathrm{Pt}$ resulted in very low fluoride emission. Membrane degradation was only accelerated when the Pt particles were small and close together (average particle size $=2.4 \mathrm{~nm}$, average particle distance $=10 \mathrm{~nm}$ for $10 \mathrm{~mol} \% \mathrm{Pt}$ after testing). When the Pt particles in the membrane were larger and further apart, very little degradation was observed.

In Task 3, the addition of heteropoly acids (HPAs) to sublayers was investigated to determine their effect on platinum band formation. Results showed HPAs reduce the fluoride emission rate (FER) by as much as half, with phosophotungstic acid (PTA) showing the greatest reduction. The OCV loss with HPAs was significantly reduced, again with PTA showing the greatest improvement. Modeling of the voltage profile through the membrane and sublayers indicated that only PTA was capable of reducing platinum ions. The PTA-1V and STA-1V HPAs resulted in sublayer voltage profiles that were too high for platinum reduction. For all HPAs investigated, HPA mobility within the cell proved problematic, with the concentration of HPA in the sublayers decreasing after testing. Tethering the HPA to the surface of the carbon support through covalent bonding is possible, and could be employed in future efforts.

The Task 4 objective was to combine the best additive to a membrane (from Task 1 ) and combine it with a sublayer containing the most effective HPA (from Task 3). Using the experimental results from Task 1 and $3,1 \mathrm{wt} \%$ ceria was added to the membrane and PTA to a sublayer. The resulting system demonstrated a 5-fold reduction in degradation compared to a cell without either membrane additive or PTA sublayer. Additional improvements in performance and durability are expected by incorporating the PTA directly into the electrode. 


\section{Task 10. Production of Low-Cost Hydrogen from Biowaste $\left(\mathrm{HyBrTec}^{\mathrm{TM}}\right)$ - R. Parker, SRT Group,}

Inc., Miami, FL -- 2012

This project uses the SRT developed hydrogen bromide $\left(\mathrm{HyBrTec}^{\mathrm{TM}}\right)$ process to solve problems associated with conventional biowaste-to-fuel processing using anaerobic digester by exploiting two thermochemical advantages that reduce both the cost and energy of converting waste-to-fuel. The first advantage is that the SRT process occurs at moderate temperatures and pressures giving fast chemical reactions, high product yields, and the release of significant thermal energy. This advantage minimizes the size of equipment and allows for use of the co-produced heat. Second, the intermediate hydrogen carrier chemical bonds are weak, requiring less energy than water electrolysis to free hydrogen from the bromine solution.

This report presents the results from SRT's biowaste-to-hydrogen process (HyBrTec $\left.{ }^{\mathrm{TM}}\right)$ that was developed and tested from December 2010 through June 2012. The final report presents the work undertaken, the results obtained, and the recommended next steps to further advance the technology. The work is considered a success by verifying cellulose to $\mathrm{H}_{2}$ conversion efficiency of $85 \%$. The objective to establish ash-treatment procedures and confirm $99.9 \% \mathrm{Br}_{2}$ recovery rates will be met in future work.

A demonstration reactor and electrolysis vessel was designed, built and operated. Data was collected from bromination experiments at temperatures up to $250^{\circ} \mathrm{C}$, as well as electrolysis and joint brominationelectrolysis runs up to $200^{\circ} \mathrm{C}$. Learning occurred throughout this process to better define the requirements for a prototype system. An economic model was also created to estimate the costs for a demonstration system.

Pure cellulose was used for bromination. While full conversion was expected at $200^{\circ} \mathrm{C}$, it was found that only $150^{\circ} \mathrm{C}$ was required to achieve $100 \%$ conversion of cellulose to hydrobromic acid ( $\mathrm{HBr}$ ) and carbon dioxide $\left(\mathrm{CO}_{2}\right)$. It was also found that $80 \%$ of the cellulose was converted at the significantly lower temperature of $75^{\circ} \mathrm{C}$. Both these results were surprising, and suggest a glass reactor can be used instead of the high pressure metal one currently used to meet anticipated safety concerns.

The electrolysis experiments showed open circuit voltages below 1 volt at most conditions considered. For instance, a $20 \mathrm{wt} \% \mathrm{HBr}$ solution at $150^{\circ} \mathrm{C}$ yielded an open circuit voltage of 0.6 volts. Combination bromination and electrolysis runs were undertaken in which it was shown that hydrogen was produced as cellulose was brominated. This supports a process in which cellulose and electricity inputs are continuously converted to hydrogen.

The electrolysis of $\mathrm{HBr}$ solution inside a single reactor vessel was found to be inefficient and, thus, future work will separate bromination and electrolysis reactors to allow each to be optimized to its specific requirements and characteristics.

The results presented herein are very favorable for further development of the process. Next steps are to continue high temperature $\mathrm{HBr}$ electrolysis, perform more detailed analysis after bromination, and integrate a prototype system to operate continuously. Further economic details will follow in parallel to the above experimental work.

In addition, SRT acknowledges the participation provided by subcontractor, Electrolytic Technologies Corporation, North Miami Beach, FL, whose support contributed to the success of the program. 
Task 11. Development of a Low-Cost and High-Efficiency 500 W Portable PEMFC System - J. Zheng, Florida State University, H. Chen, Bing Energy, Inc. -- 2013

The objective of the buckypaper based Low-Cost and High Efficiency 500W Portable PEMFC System was to research and develop a proton exchange membrane fuel cell (PEMFC) in order to improve membrane manufacturing processes and improve cell efficiencies and lifetimes. PEMFC has been selected as the leading near-term technology candidate for fuel cell vehicles because of its high power density, quick start capability, and technology maturity. Advancements in fuel cell technology have reduced the size, weight and cost of fuel cell electric vehicles. Fuel cell electric vehicles have been produced with "a driving range of more than 250 miles between refueling". They can be refueled in less than 5 minutes. Deployed fuel cell buses have a $40 \%$ higher fuel economy than diesel buses. However, high cost and relative low performance of PEM fuel cell are still major barriers to hinder the widespread application of PEMFC power source for autos and the military.

Electrode structures and catalysts are critical for the determining performance of PEMFC and their products. Particularly, catalyst activity and utilization efficiency of platinum group metal (PGM) based electrode technology will directly determine the cost. To reduce PGM usage and improve cell performance, new technologies based on novel electrode materials and structures, understanding of the electrode structure-performance relationships and optimization of catalyst-support interactions need to be explored, characterized and demonstrated. This project has made technical breakthrough on catalyst usage and life, and cell power density by using the mixed carbon nanotubes (CNT) and carbon nanofibers (CNF) free-standing papers (buckypaper) as supporting network of catalytic electrodes.

In this project, a research and development team, from Florida State University (FSU) and Bing Energy International (BEI) have explored and demonstrated effective and scalable techniques to fabricate affordable Pt/buckypaper electrode with gradient-structured ready for fuel cell stack. The team has systematically investigated the effect of electrode microstructure on the performance of resultant fuel cells and optimized the electrode structure to greatly improve the catalyst efficiency and cell durability. A product prototype of a fuel cell stack used for portable power applications has been demonstrated.

The project results determined that Pt/double-layered buckypaper (DLBP) with tailored gradient structure demonstrated promising Pt utilization and stability of supports in spite of a relatively large Pt particle size. By applying this Pt/DLBP as a cathode catalyst layer, the MEA exhibited excellent power performance with a relative low Pt loading. The rated powers of $1.5 \mathrm{~W} / \mathrm{cm}^{2}$ and $0.8 \mathrm{~W} / \mathrm{cm}^{2}$ at $0.65 \mathrm{~V}$ were achieved with a cathode Pt loading of $0.2 \mathrm{mg} / \mathrm{cm}^{2}$ for oxygen and air, respectively. The Pt utilization was better than the current state-of-the-art value $\left(>0.4 \mathrm{mg} / \mathrm{cm}^{2}\right)$ achieved by the conventional $\mathrm{Pt} / \mathrm{C}$ catalyst at a comparable cell $\left(0.8-1.0 \mathrm{~W} / \mathrm{cm}^{2}\right)$ output.

The research also evaluated the durability of catalyst support for Pt/DLBP in a MEA according to DOE's suggested protocol. The SWNT/CNF buckypaper-based Pt catalyst has shown good durability under an accelerated degradation test in a cathode environment. This was due to the high corrosion resistance of $\mathrm{CNF}$ and its high degree of graphitization.

FSU and Bing Energy also developed an 11 cell stack. The MEA size of each cell is $50 \mathrm{~cm}^{2}$. The Pt loading is $0.2 \mathrm{mg} / \mathrm{cm}^{2}$ and $0.05 \mathrm{mg} / \mathrm{cm}^{2}$ at cathode and anode, respectively. The fuel cell stack was characterized using hydrogen at anode and oxygen or air at cathode. The rated powers $(0.65 \mathrm{~V} /$ cell $)$ of 670 and $358 \mathrm{~W}$ were obtained when oxygen and air were used, respectively. 


\section{Task 12. Interdisciplinary Hydrogen and Fuel Cell Technology Academic Program - J. Politano,}

C. Lockyer, M. McCay, K. Winkelmann, Florida Institute of Technology -- 2013

This project developed a hydrogen and fuel cell technology (HFCT) academic program at Florida Institute of Technology (FIT) in Melbourne, FL. The project results were the development of hydrogen and fuel cell related education modules that have been implemented into courses in the General Chemistry laboratory and Mechanical and Sustainability Engineering and the offering of a HFCT master's degree level of specialization. The project also conducted R\&D in the areas of hydrogen and fuel cell technologies through senior level and graduate student projects. The project was completed as scheduled and a new university education program has been established.

Task 1: Develop Master's Degree Area of Specialization under Existing MS Programs in Hydrogen and Fuel Cell Technologies.

Task 1 developed a Master of Science degree program with an area of specialization in Hydrogen or Fuel Cell Technology (HFCT). This program requires a bachelor of science or the equivalent in engineering, applied mathematics, chemistry or physics for entrance. The program specializations and new courses were approved by the Florida Tech College of Engineering Council and the Florida Tech Graduate Council. Changes were made to the School Catalog to reflect the new specializations and to include new courses.

A list of core courses was developed that comprise the academic elements necessary for this area of specialization. The student, in consultation with his or her advisor, selects three of the courses which constitute required courses for the specialization (typically comprising nine credit hours). Six credit hours are required of any 5000-level courses with a MTH prefix along with nine credit hours of elective courses at the 5000- or 6000-level with an MAE (for the Mechanical Engineering specialization) of CHE prefix (for the Chemical Engineering specialization). Courses from other departments may be used with department head approval. The remaining program requirements are met with thesis, six credit hours (minimum) of XXX5999 (depending on the department). Requirements for the non-thesis option are the same as for the thesis option except the thesis credits are replaced with two additional elective courses for a total of 15 elective credits.

Two new graduate courses were developed. (1) CHE 5250: Hydrogen Technology which presents the fundamental knowledge of hydrogen, the potential future development of hydrogen science and technology, the use of hydrogen as a fuel, its properties, production and storage and discusses hydrogen technology applications. (2) MAE 5330: Principles of Fuel Cells which presents fuel cell fundamentals.

\section{Task 2: Develop Modules for Core and Elective Courses.}

This project identified and developed modules to bring hydrogen and fuel cell technology into the traditional mechanical engineering and chemistry engineering undergraduate curriculum. Following module development, a select number of undergraduate engineering and sciences courses were identified to include the developed hydrogen and fuel cell related modules.

Undergraduate courses which included hydrogen modules are: Engineering Thermo-dynamics; MAE 3191, Introduction to Sustainability, ISC 1500; Applied Sustainability, ISC 4000; Introduction to Electrical Power Systems, ECE 4861; Power System Operation, ECE 5683; Physical Chemistry Laboratory I, CHM 3011; Introduction to Chemical Engineering, CHE 1101; Materials Science and Engineering, CHE 3260; Nanotechnology, CHE 5567/4592 and the Materials Characterization Lab. 


\section{Task 3: Student Design or Research Projects.}

Graduating seniors in the fields of engineering and science are required to undertake a capstone project which may take the form of a design or research project and these are guided by the relevant faculty member. The senior students prepare and exhibit these capstone projects at the end of the spring term along with a written report.

Two hydrogen related projects were a Metal Hydride Hydrogen/Thermal Energy Storage Senior Design Project whose objective was to design, build, and investigate a metal hydride hydrogen/ thermal energy storage system. A graduate level project was Hydrogen-based Residential Emergency Power Supply Capstone Project with the objective of developing cost estimates for a hydrogen based residential emergency power supply.

\section{Task 4: Develop Laboratory Experiments for General Chemistry Courses.}

This task is unique within the DOE education program. Dr. Winkelmann implemented hydrogen-themed, laboratory experiments within the General Chemistry II lab curriculum in order to:1) implement a set of general chemistry laboratory experiments that focus on issues related to hydrogen fuel research, and 2) measure students' gains in knowledge of hydrogen as a fuel source and their interest in and appreciation of chemistry as a subject. Students began performing these experiments in spring 2012 and will continue to perform them for the foreseeable future. In spring 2013, Dr. Winkelmann created video tutorials were implemented to help students understand the laboratory experiment procedures.

Students completed an attitudinal survey created at Florida Tech and the U.S. Department of Energy's Hydrogen Knowledge and Opinion Survey (HKOS). These surveys measured students' attitudes about chemistry and their understanding of issues related to hydrogen fuel technology. Students enrolled in General Chemistry II lab completed both surveys at the beginning and end of each semester of 2011 and 2012. An analysis of the surveys shows no change in students' attitudes about chemistry lab before or after adding the new hydrogen-themed experiments. This result was disappointing since it was hoped that the hydrogen-themed experiments would spark students' interest in chemistry. Students were more favorably inclined towards hydrogen technology and demonstrated more knowledge of hydrogen after completing the new experiments. This result shows that students can not only learn about chemistry topics in their lab course but can also learn about other important issues, such as hydrogen fuel technology.

Dr. Winkelmann supported two students to perform an extensive web and literature search to find other sources of hydrogen curriculum materials which other schools have created. The purpose of this search is to keep up to date with the growth of hydrogen technology in science and engineering education. The web and literature search were completed in December 2012.

\section{Task 13. Design and Development of an Advanced Hydrogen Storage System using Novel Materials - Drs. E. Stefanakos, D. Goswami, and A. Kumar, University of South Florida -- 2013}

The goal of this project was to design and develop novel conducting polymeric nanomaterials for onboard hydrogen storage with a system gravimetric capacity of $5.5 \mathrm{wt} . \%$ or greater and reversible hydrogen storage characteristics at moderate temperature $\left(<100^{\circ} \mathrm{C}\right)$. The project approach was to examine synthesis of polyaniline (PANI) solid state hydrogen storage materials and to modify the synthesis parameters for optimized storage capabilities. The major challenge in conducting the research was to develop polymer nanostructures that can store hydrogen at room temperature, and be reversible for many cycles. The preliminary work conducted at the University of South Florida (USF) used PANI nanostructures and studied the morphological effects of $\mathrm{H}_{2}$ cycling on PANI NF-ES. 
Several novel materials such as polyaniline (emeraldine salt), ion doped polyaniline, carbon nanotube doped polyaniline, transition metal doped polyaniline, and graphene doped polyaniline, electrospun polyaniline, and hyper cross-linked polystyrene material were examined. The spillover enhancement for high surface area polymeric materials (i.e., hypercrosslinked polystyrene) was investigated for the first time. Spillover enhancement attracted the attention of the hydrogen storage community due to the very promising results reported (i.e. $\approx 5 \mathrm{wt}$. $\%$ at room temperature and $10 \mathrm{MPa}$ ). However, spillover enhancement controversial due to contradictory results reported by different research groups. The results reported in this report showed that spillover enhancement in high surface area polymers has little practical value (i.e., $0.32 \mathrm{wt}$. \% at room temperature and $10 \mathrm{MPa}$ ) for mobile applications.

The original tasks of the projects were based on the promising results of previous USF work on electrospun polyaniline, and its modification by carbon nanostructures, which showed 3-10 wt. \% storage capacity at $25^{\circ} \mathrm{C}-125^{\circ} \mathrm{C}$. However, the previous results of the electrospun polyaniline were not reproducible. The actual hydrogen storage capacity of the electrospun polyaniline was found to be less than $0.1 \mathrm{wt} . \%$ at room temperature which could not support further work since the system level design utilizing a $0.1 \mathrm{wt}$ \% storage capacity material has no practical value.

Following this initial materials research, the project objectives were modified to address the difficulties associated with the hydrogen storage measurements, and shifted our research direction to develop novel porous, high surface area polymeric materials and to explore the spillover phenomenon in the hypercrosslinked polystyrene. The revised tasks and the proposed work therein are as follows:

- Task 1: Development of discrepancies in experimental results among various research groups because of inaccuracies in measuring procedures and equipment.

- Task 2: Development of a modified list of polymeric materials that may provide improved gravimetric densities.

- Task 3: Continue testing to understand the hydrogen storage physisorption and chemisorption processes in conducting polymers (i.e., polyaniline).

- Task 4: Conduct education and outreach.

In the first task, USF research presented an uncertainty analysis of hydrogen storage measurements and discussed the most important error sources. The errors in hydrogen sorption measurements are of major concern in the hydrogen storage community which impede the down selection process of candidate materials and set back progress in the field. Therefore, the design stage uncertainty analysis of the volumetric hydrogen storage measurements was studied in detail and guidelines are given for high accuracy hydrogen storage measurements. For instance, sample loading in volumetric hydrogen storage measurements are shown to be an important parameter that needs to be considered. The research presented in this study will help the experimentalists to spot the major error sources in volumetric hydrogen storage measurements and to improve the accuracy of their measurements.

In task two, the spillover enhancement in hypercrosslinked polystyrene was investigated and the results were that the bridge building technique is better than the wet impregnation method. However, the data showed that hydrogen storage capacity of the hypercrosslinked polystyrene is around $0.32 \mathrm{wt} \%$ at room temperature and 100 bar pressure which is not useful for practical applications. Contrary to other works published on spillover enhancement in activated carbon and metal organic frameworks which claimed a 10 fold increase in the hydrogen storage capacity at room temperature and $10 \mathrm{MPa}$ pressure, USF researchers did not observe significant enhancement, but only an increase of $10 \%$ in the hydrogen storage capacity of the hypercrosslinked polystyrene as compared to the base material. Thus, the conclusion was that, spillover enhancement in hydrogen storage materials is uncertain. 
More fundamental studies are required to elucidate the spillover enhancement mechanism and more collaborative work (i.e., round robin tests) are required to address the discrepancies in the hydrogen storage measurements among the different labs. Other work in this task successfully synthesized nitrogen enriched high surface area polymers by Yamamato coupling; however, synthesized boron enriched high surface area polymers were not achieved.

In task 3 work, polyemeraldine base, polyemeraldine salt, polyleucoemerldine and polypernigraniline materials were synthesized. However, the experimental investigations did not show more than $0.1 \mathrm{wt}$ \% hydrogen storage capacity.

Finally in task 4 on education, USF pursued the development of an energy course in materials for hydrogen and fuel cells, organized a seminar on hydrogen storage and fuel cells in order to educate researchers and students within USF and regularly held seminars on energy related research.

\section{Task 14. Advanced HiFoil ${ }^{\mathrm{TM}}$ Bipolar Plates - J. Braun, M. Fuchs, EnerFuel, Inc., -- 2013}

The goal of the Advanced "HiFoil" project, is to provide a durable, low cost bipolar plate for use in advanced high temperature proton exchange membrane (HTPEM) fuel cells for stationary, vehicle auxiliary power units and electric vehicle range extender (EVRE) applications. As a result of this project, EnerFuel successfully developed and demonstrated a mass producible bipolar plate with unparalleled performance and physical attributes.

EnerFuel's patent pending HiFoil ${ }^{\mathrm{TM}}$ laminate technology was used as the starting point for this development. HiFoil ${ }^{\mathrm{TM}}$ laminates consist of two layers of flexible graphite that are bonded to a thin metal foil using a proprietary high temperature conductive adhesive. As a baseline, these laminates provide the following attributes:

- Two orders of magnitude lower cost than gold coated stainless steel

- Greater corrosion resistance

- Excellent performance in 4-cell testing (using corrugated flow field inserts)

- HTPEM stack tested for 1,000 hours with no failure

- Ex-situ thermal cycled from room temperature to $200^{\circ} \mathrm{C}$ over 10,000 times with no delamination

Unfortunately these laminates were only capable of being formed into corrugations. They were used as flow field inserts in a complex air-cooled fuel cell that required eleven layers of materials to function as the bipolar plate. In addition, the corrugated laminate flow field design did not accomodate the use of a liquid coolant for recovery of fuel cell waste heat.

In order to accomplish the project goal of providing a durable, low cost bipolar plate for a wide range of HTPEM applications, and based on EnerFuel's existing technology, two generations of advanced bipolar plates were developed. The first generation bipolar plate (Gen1 HiFoil ${ }^{\mathrm{TM}}$ ) involved the use of a laminate with thicker and more thermally conductive flexible graphite outer layers. The intent was to emboss the flow field channels into these outer layers, and to use these layers to conduct heat to the outer edges of the cell. The thin metal layer at the core of the laminate was also modified to improve conductivity through the z-axis of the bonded layers.

However, the Gen1 efforts revealed that the advanced flexible graphite was already at near the maximum theoretical density, thus the material did not allow for embossing the required reactant channels. The flexible graphite vendor was willing to provide the material in a less dense form, yet this approach has additional drawbacks. Lowering the density of the foil, while allowing the channels to be imprinted, 
would have resulted in low and high density regions within the bipolar plate, resulting in high acid uptake from the MEA and low structural integrity at these low density regions. In addition, the modification to the metal core layer necessarily contained small amounts of thallium, a toxic substance. As a result of the shortcomings in the Gen1 approach, the design was reevaluated and an alternate approach was selected.

Although not quite as low in material cost as the Gen1 approach, the new Gen2 HiFoil ${ }^{\mathrm{TM}}$ approach is less challenging from a manufacturing standpoint, and is better suited for high volume bipolar plate production. The Gen 2 approach maintains the thicker layers of advanced flexible graphite from the previous generation, but instead of laminating the flexible graphite to a metal foil core, the flexible graphite is laminated between two outer layers of moldable graphite composite. In this arrangement, the flexible graphite's high in-plane thermal conductivity can still be exploited for conducting heat out to the edge of the cell, without the need to emboss any flow channels in this difficult to form material. Due to the moldable nature of the outer graphite composite material, the flow fields can be easily molded into the outer layers of the bipolar plate. In addition, the polymer binder in the outer composite layer acts as an effective barrier to acid, thereby mitigating issues associated with acid up-take from the MEA.

In situ evaluation of the Gen2 laminate bipolar plates consisted of 4, 36 and 132-cell fuel cell stack testing, as well as validation in a $3 \mathrm{~kW}$ net stationary power system. Electrical performance of the fuel cell stacks matched very closely with MEA manufacture performance curves. In addition, stack testing revealed a typical cell-to-cell temperature spread between $7^{\circ} \mathrm{C}$ and $10^{\circ} \mathrm{C}$. In system testing this spread increased, but still remained below $14^{\circ} \mathrm{C}$. Such temperature uniformity is critical in maintaining optimal fuel cell performance. This is especially important when utilizing a fuel processor, since cells operating at lower temperature are more susceptible to poisoning by the carbon monoxide contained in the hydrogen rich stream.

Along with the MEA, the bipolar plate makes up the core of the HTPEM fuel cell power system, and the cost of these two components comprise the bulk of the fuel cell system cost. Cost quotes for molded HiFoil ${ }^{\mathrm{TM}}$ bipolar plates yield a bipolar plate cost of $\$ 840$ per $\mathrm{kW}$ of net electrical power, at production volumes of 2000 systems per year. In yearly system volumes of 50,000, this cost would be expected to drop well below $\$ 500$ per $\mathrm{kW}$, in line with DOE 2015 DOE target cost of $\$ 1,700 / \mathrm{kW}$ for the overall system cost targets for stationary CHP fuel cell systems. Thus, the overall objectives of the program were successfully achieved.

In summary, a durable, low cost bipolar plate for use in HTPEM fuel cell systems was developed, having very high in-plane thermal conductivity. Stacks and systems using the advanced plate were operated in stand-alone, fully automated operation using propane from a portable tank. All of the heat generated by the fuel cell was conducted through the edges of the bipolar plate and to an external 2-phase cooling circuit. Some of this heat was used to generate steam for use inside the fuel processor, while the additional waste heat is provided at $\sim 165^{\circ} \mathrm{C}$ to be put to work in combined heat and power applications. The net system efficiency was $36 \%$ at $3 \mathrm{~kW}$ electric power output.

The development efforts supported by this program have been remarkably successful. EnerFuel's HiFoil ${ }^{\mathrm{TM}}$ bipolar plate and related fuel cell stack technology is currently patent pending under U.S. Patent Applications 13/566347, 13/566406, 13/566531, 13/566551, 13/566585, and 13/566629. This technology is being deployed in production ready 36-cell and 132-cell HTPEM fuel cell stacks. The 36-cell stacks are integrated into EnerFuel's commercially available 1.5kW Fuel Cell Power System (FCPS); while the 132cell stacks are integrated into EnerFuel's advanced 3kW stand-alone stationary power system (currently available to selected partners). 


\section{Concluding Remarks}

The Florida Hydrogen Initiative (FHI) was a research, development and demonstration (RD\&D) program funded by U.S. Department of Energy (DOE) for the purpose of developing a hydrogen and fuel cell infrastructure. The FHI program operated in two phases over a period of 2004 until 2013. The project was managed by the Florida Hydrogen Initiative, Inc. and by the University of Central Florida's Florida Solar Energy Center (FSEC). In Phase II, the project management and technical oversight was provided by Dr. David Block of FSEC. The program operated by funding 12 individual projects which then conducted the RD\&D program activities. All 12 of the projects were completed and results are presented for each project. The 12 projects include five involving hydrogen (Tasks 1, 5, 7,10 and 13), five involving fuel cells (Tasks 2, 8,9,11 and 14) and two covering education and public understanding and information (Task 3 and 12). The individual results for each of these 12 projects are presented in three ways: (1) as a one paragraph Summary in the Executive Summary of this report, (2) as a two page Executive Summary of each project in the report body and (3) as the complete final report for each project in the two Attachment sections. The majority of the projects produced significant results as presented in the final reports.

\section{Patents}

In this section, the patents from the FHI program are listed under each project.

Task 6. On-Site Reformation of Diesel Fuel for Hydrogen Fueling Station Applications, A. Raissi, Florida Solar Energy Center, J. Stevens, Chevron Technology Ventures LLC-January 2008 to December 2009

1. Huang, C., Smith, F., Linkous, C.A., Ramasami, K.K., T-Raissi, A., Muradov, N.Z. "Method and System for Hydrogen Sulfide Removal,” U.S. Patent Application No. 61/023,755.

Task 7. Chemochromic Hydrogen Leak Detectors for Safety Monitoring, N. Mohajeri and N. Muradov, Florida Solar Energy Center - April 2010 to December 2012.

1. N. Mohajeri, "Doped palladium containing oxidation catalysts", U.S. Patent Application No. $13 / 588,779$

2. P. Brooker, N. Mohajeri "Chemochromic membranes for membrane defect detection", U.S. Patent Application No. 13/594,163

Task 9. Understanding Mechanical and Chemical Durability of Fuel Cell Membrane Electrode Assemblies, D. Slattery, Florida Solar Energy Center - April 2010 to June 2012

1. R.P. Brooker and L.J. Bonville. "Electroactive Species in Layer Between Electrode and Membrane for Fuel Cells", UCF-32278 (10395) (Serial No. 13-647,073) filed October 8, 2012.

2. B. P. Pearman, M. Rodgers, N. Mohajeri, R. P. Brooker, D. K. Slattery, "Polymer Membranes with Rare Earth or Transition Metal Modifier", (2012) U.S. Patent Application No. 61/682,833.

Task 11. Development of a Low Cost and High Efficiency 500W Portable PEMFC System, J. Zheng, Florida State University, H. Chen, Bing Energy Inc. - December 2010 to December 2012

1. J.P. Zheng, W. Zhu, C. Zeng, Z.Y. Liang, B. Wang, C. Zhang, and W. Zhu, "A Method of making catalytic electrodes for fuel cells", U.S. Provisional Patent Application was filed. 
Task 14. Advanced HiFoil Bipolar Plates, J. Braun, M. Fuchs, EnerFuel, Inc.-December 2010 to March 2013

1. Graham. M., Braun, J., Pavlik, T. "Fuel cell stack having a structural heat exchanger," US Publication No. 20130034790 A1 filed Aug 3, 2012.

2. Pavlik, T., Braun, J., Graham, M. "Bipolar plate assembly for use in a fuel cell," US Publication No. 20130034789 A1 filed Aug 3, 2012.

3. Pavlik, T., Braun, J., Graham, M., Torres, M. "Bipolar plate assembly having a recess and an insert member for placement in the recess," US Publication US20130034799 A1 filed Aug 3, 2012.

4. Pavlik, T., Braun, J., Graham, M. "Bipolar plate assembly having an insert member," US Publication No. US20130034800 A1 filed Aug 3, 2012.

5. Pavlik, T., Braun, J., Graham, M. "Bipolar plate assembly having an encapsulated edge," US Publication No. US 20130034797 A1 filed Aug 3, 2012.

6. Pavlik, T., Braun, J., Graham, M. "Bipolar plate assembly having an adjustment member," US Publication No. US20130034801 A1 filed Aug 3, 2012.

\section{Publications and Presentations}

This section presents the publications and presentations from the $12 \mathrm{FHI}$ projects. If a project is not listed, then there were no publications and presentations for that project.

Task 5. On-Site Reformation of Diesel Fuel for Hydrogen Fueling Station Applications, A. Raissi, Florida Solar Energy Center, J. Stevens, Chevron Technology Ventures LLC - January 2008 to December 2009

\section{Publication}

Muradov, N.Z., Ramasamy, K.K., Linkous, C.A., Huang, C., Adebiyi, I., Smith, F., T-Raissi, A., Stevens, J. "Combined Pre-Reforming-Desulfurization of High-Sulfur Fuels for Distributed Hydrogen Applications," J. Fuel 89 (2010) 1221-9.

\section{$\underline{\text { Presentations }}$}

Muradov, N.Z., Ramasamy, K.K., Huang, C., Adebiyi, I., Smith, F., Linkous, C.A., T-Raissi, A., Stevens, J. "Combined Pre-Reforming-Desulfurization of High-Sulfur Diesel for Hydrogen Fueling Station Applications", Presented at the 2009 AIChE Spring Meeting, Tampa, Florida (2009).

Muradov, N.Z., Ramasamy, K.K., Huang, C., T-Raissi, A., Stevens, J. "Catalytic Processing of High-Sulfur Fuels for Distributed Hydrogen Production", Paper accepted for presentation at and inclusion in the proceedings of the 18 World Hydrogen Energy Conference, Essen, Germany, May, 2010. 


\section{Phase II Projects - Tasks 7 to 14}

Task 7. Chemochromic Hydrogen Leak Detectors for Safety Monitoring, N. Mohajeri and N. Muradov, Florida Solar Energy Center - April 2010 to December 2012.

\section{$\underline{\text { Publications }}$}

Nazim Muradov, Ali T-Raissi, Gary Bokerman, Monica Kowalczik, Errol Hincamp. Passive Chemochromic Hydrogen Leak Detectors. International Journal of Hydrogen Energy, in preparation.

$\underline{\text { Presentations }}$

N. Mohajeri, "FSEC's Irreversible Chemochromic Hydrogen Sensor Overview”, Fuel Cell Seminar, Orlando, Fl. November 3, 2011

N. Mohajeri, P. Kubiak, "Improved Titania Supported Palladium Oxide Pigments for Irreversible Chemocromic Hydrogen Sensor”, Fuel Cell Seminar, Orlando, Fl. November 3, 2011

Nazim Muradov, Ali T-Raissi, Gary Bokerman, Errol Hincamp. Passive Chemochromic Hydrogen Leak Detectors for Safety Monitoring. Proc. $19^{\text {th }}$ World Hydrogen Energy Conference, Toronto, Canada, 2012

Nazim Muradov, Nahid Mohajeri, Ali T-Raissi, Gary Bokerman, Errol Hincamp. Chemochromic Hydrogen Leak Detectors for Safety Monitoring. DOE-FSEC FHI Project Progress Review Meeting, October, 2012

Task 8. Development of High Efficiency Low Cost Electrocatalysts for PEM Fuel Cell Applications, $M$. Rodgers, Florida Solar Energy Center - April 2010 to June 2013

\section{$\underline{\text { Publications }}$}

C. Odetola, Master's Thesis, 2012, Development of High Efficiency, Low Cost Electrocatalysts for Proton Exchange Membrane Fuel Cell Applications, Florida Institute of Technology

C. Huang, C. Odetola, M.P. Rodgers, Nanoparticle modified electroplating for the preparation of low Pt loading high performance electrocatalysts, submitted to Chemical Communications, 2012.

C. Huang, C. Odetola, M.P. Rodgers, Seed-mediated pulse electrodeposition of Pt electrocatalysts for oxygen reduction reaction, submitted to Chemistry of Materials, 2012.

\section{$\underline{\text { Presentations }}$}

October 2011: M.P. Rodgers, C. Huang, C. Odetola, Development of electrocatalysts for PEM fuel cells by electrodeposition, Electrochemical Society Meeting, Boston, Massachusetts, USA.

September 2011: C. Huang, Preparation and Characterization of Nanophase Pt and Pt-Alloy Cocatalysts for Solar Hydrogen Production, Nano Florida, Miami, Florida

$>\quad$ Winner of outstanding oral presentation award 
September 2010: M.P. Rodgers, C. Huang, Electrodeposition of Catalyst for use in Proton

Exchange Membrane Fuel Cells, Florida Energy Systems Consortium Summit, Orlando, Florida, USA

Task 9. Understanding Mechanical and Chemical Durability of Fuel Cell Membrane Electrode Assemblies, D. Slattery, Florida Solar Energy Center - April 2010 to June 2012

\section{$\underline{\text { Publications }}$}

M.P. Rodgers, L.J. Bonville, D.K. Slattery, Evaluation of the Durability of Polymer Electrolyte Membranes in Fuel Cells Containing $\mathrm{Pt} / \mathrm{C}$ and $\mathrm{Pt}-\mathrm{Co} / \mathrm{C}$ Catalysts under Accelerated Testing, Electrochemical Society Transactions, 41(1) 1461, 2011.

M.P. Rodgers, N. Mohajeri, L.J. Bonville, D.K. Slattery, Accelerated testing of polymer electrolyte membranes in fuel cells containing $\mathrm{Pt} / \mathrm{C}$ and $\mathrm{PtCo} / \mathrm{C}$ catalysts, Journal of the Electrochemical Society, 159, B564, 2012.

R.P. Brooker, L.J. Bonville and D.K. Slattery, Decreasing Membrane Degradation through Heteropolyacid Sublayers, Journal of the Electrochemical Society, accepted 2012 M.P. Rodgers, B.P. Pearman, N. Mohajeri, L.J. Bonville, D.K. Slattery, Effect of Membrane Equivalent Weight on Degradation Under Accelerated Stress Conditions, submitted to Electrochimica Acta, 2012.

\section{Presentations}

March 2011, M.P. Rodgers, Fuel Cell Research, Women's International Research Engineering Summit (WIRES), Cocoa, Florida, USA.

March 2011, M.P. Rodgers, L.J. Bonville, D.K. Slattery, Evaluation of the Durability of Polymer Electrolyte Membrane Fuel Cells containing Pt/C and Pt-Co/C Catalysts under Accelerated Testing, 2011 Annual Joint Symposium \& Exhibition of the Florida Chapter of the AVS Science and Technology Society, Orlando, Florida, USA.

October 2011, R.P. Brooker, D.K. Slattery, L.J. Bonville and J.M. Fenton, Platinum Band Formation Mitigation through Heteropolyacid Sublayers, Presentation \# LRD42-1, Fuel Cell Seminar, Orlando, Florida, USA.

October 2011, R.P. Brooker, D.K. Slattery, L.J. Bonville and J.M. Fenton, Platinum Band Formation Mitigation through Heteropolyacid Sublayers, Electrochemical Society Meeting, Boston, Massachusetts, USA.

October 2011, M.P. Rodgers, L.J. Bonville, D.K. Slattery, Evaluation of the Durability of Polymer Electrolyte Membranes in Fuel Cells Containing Pt/C and Pt-Co/C Catalysts under Accelerated Testing, Electrochemical Society Meeting, Boston, Massachusetts, USA.

September 2012, M.P. Rodgers, B.P. Pearman, D. Cullen, L.J. Bonville, D.K. Slattery, The effect of Pt deposited in the membrane on membrane degradation, Carisma Medium and High Temperature Fuel Cell Conference, Copenhagen, Denmark. 
September 2012: M.P. Rodgers, B.P. Pearman, N. Mohajeri, L.J. Bonville, D.K. Slattery, Effect of membrane equivalent weight on degradation under accelerated stress conditions, Carisma Medium and High Temperature Fuel Cell Conference, Copenhagen, Denmark.

Task 11. Development of a Low Cost and High Efficiency 500W Portable PEMFC System, J. Zheng, Florida State University, H. Chen, Bing Energy Inc. - December 2010 to December 2012

\section{$\underline{\text { Publications }}$}

W. Zhu, C. Zeng, J.P. Zheng, R. Liang, C. Zhang, and B. Wang, "Preparation of Buckypaper Supported Pt Catalyst for PEMFC Using Supercritical Fluid Method", Electrochem. and SolidState Lett. 14, B81 (2011).

W. Zhu, R. Liang, and J.P. Zheng, "A 3-D Catalytic Electrode Structure for Ultra-low Platinum Loading and High Performance PEMFCs", accepted by ECS Transactions.

\section{$\underline{\text { Presentations }}$}

W. Zhu and J.P. Zheng, "Ultra-Low Platinum Loading High-Performance PEMFCs Using Buckypaper-Supported Electrodes", Small Fuel Cells 2011, Boston MA, Jun 9-10, 2011.

J.P. Zheng, W. Zhu, R. Liang, C. Zhang, and B. Wang, “A 3-D Catalytic Electrode Structure for Ultra-low Platinum Loading and High Performance PEMFCs", The 2012 Villa Conference on Energy and Environmental Research, Olando, FL, April 16-20, 2012.

J.P. Zheng, W. Zhu, R. Liang, C. Zhang, and B. Wang, “A 3-D Catalytic Electrode Structure for High Performance and Los Cost PEMFCs", the 243rd American Chemical Society National Meeting, San Diego, California, March 27, 2012.

J.P. Zheng, "Transformation of University Research to Commercialization”, Florida Energy Summit Orlando, Florida, August 17, 2012. (Panel Speaker)

J.P. Zheng, W.J. Cao, G.Q. Zhang, W. Zhu, P. Andrei, Rl Liang, and M. Zhang, "Carbon Nanotube composite electrodes for energy storage devices and fuel cells", IUPAC $8^{\text {th }}$ International Conference on Novel Materials and Synthesis (NMS-VIII) \& $22^{\text {nd }}$ International Symposium on Fine Chemistry and Functional Polymers (FCFP-XXII), Xi'An, China, October 16, 2012, (Keynote Speaker).

Task 13. Design and Development of an Advanced Hydrogen Storage System using Novel Materials, E. Stefanakos, D. Goswami, and A. Kumar, University of South Florida0)-December 2010 to March 2013

$\underline{\text { Publications }}$

DE Demirocak, PhD. Thesis on Hydrogen Storage Materials (Hydrogen storage in hypercrosslinked polystyrene and Li-Mg-N-H complex hydride), University of South Florida (Submission Date: 04/02/2013)

A D'angelo, MSc. Thesis on Hydrogen Storage Materials (Investigation and Synthesis of Novel Graphene-Based Nanocomposites for Hydrogen Storage), University of South Florida (Submission Date: 03/22/2012) 
DE Demirocak, S Kuravi, MK Ram, CK Jotshi, SS Srinivasan, A Kumar, DY Goswami, EK Stefanakos, Investigation of Polyaniline Nanocomposites and Cross-Linked Polyaniline for Hydrogen Storage, Advanced Materials Research, 2012, 445, p. 571-6.

DE Demirocak, MK Ram, SS Srinivasan, A Kumar, DY Goswami, EK Stefanakos, Spillover enhancement for hydrogen storage by Pt doped hypercrosslinked polystyrene, International Journal of Hydrogen Energy, 2012, 37(12), p. 12402-10.

DE Demirocak, SS Srinivasan, MK Ram, DY Goswami, EK Stefanakos, Volumetric Hydrogen Sorption Measurements - Uncertainty Error Analysis and the Importance of Thermal Equilibration Time, International Journal of Hydrogen Energy, 2012, 38(3), p. 1469-77.

DE Demirocak, MK Ram, SS Srinivasan, DY Goswami, EK Stefanakos, Hydrogen and Carbon Dioxide and Ammonia Adsorption in N doped High Surface Area Polymers. (in preparation)

SS Srinivasan, R Ratnadurai, AR Phani, PC Sharma, DY Goswami, EK Stefanakos, Study of the growth of PANI nanofibers by various methods and its effect on Hydrogen Storage,

Tatvanveshan, Volume 1, Issue 1, May-October 2012, Page 12-19, ISSN: 2319-5827.

\section{Attachments I and II -- Project Final Reports}

The following two attachments present the complete final reports from each of the 12 FHI projects. Because of size, the reports are presented as two attachments, the first being the projects from Phase I and the second are the projects from Phase II. Note that the only project completely finished in Phase I was the Task 1 project. All other projects were completed at different times during the Phase II activities. For convenience, each of the projects are identified as a task number beginning with 1 and ending with 14 . Tasks 4 and 6 were not projects giving the 12 projects. 


\section{Attachment I -- Phase I Projects - Tasks 1, 2, 3 and 5}

Task 1. Hydrogen Refueling Infrastructure and Rental Car Strategies, L, Lines, Rollins College, M. Kuby, Arizona State University, R. Schultz and Z. Xie, Florida Atlantic University - September 2005 to June 2007

Task 2. Methanol Fuel Cell Vehicle Charging Station at Florida Atlantic University, M. Fuchs, EnerFuel, Inc. - October 2007 to June 2012

Task 3. Assessment of Public Understanding of the Hydrogen Economy through Science Center Exhibits, J. Newman, Orlando Science Center - July 2006 to April 2009

Task 5. On-Site Reformation of Diesel Fuel for Hydrogen Fueling Station Applications, A. Raissi, Florida Solar Energy Center, J. Stevens, Chevron Technology Ventures LLC - January 2008 to December 2009 


\title{
FLORIDA HYDROGEN INITIATIVE
}

Sub-eontractor Finel Report

\author{
Hydrogen Refueling Infrastructure And \\ Rental Car Strategies For \\ Commercialization Of Hydrogen In Florida
}

\author{
Lee Lines (PI) \\ Department of Environmental Studies, Rollins College, 1000 Holt Ave., \\ Box 2753, Winter Park, FL 32789-4499, USA. (407) 628-6377. \\ Email: 1lines@Rollins.edu \\ Michael Kuby \\ School of Geographical Sciences, Arizona State University \\ Tempe, AZ 85287-0104, USA. (480) 965-7533. \\ E-mail: mikekuby@asu.edu \\ Ronald Schultz and Zhixiao Xie \\ Department of Geosciences, Florida Atlantic University, Boca Raton, FL \\ 33431, USA. (561) 297-3250. \\ E-mail: schultzr@fau.edu,xie@fau.edu
}

June 30, 2007

FHI Agreement No. 2005-01 - Task 1

US Department of Energy Grant Award No. DE-FC36-04G014225 


\title{
Hydrogen Refueling Infrastructure and Rental Car Strategies For Commercialization of Hydrogen in Florida
}

\author{
Lee Lines, Rollins College \\ Michael Kuby, Arizona State University \\ Ronald Schultz and Zhixiao Xie, Florida Atlantic University
}

Executive Summary

A recent study by the National Renewable Energy Laboratory found that government fleets of hydrogen vehicles are not enough to jump-start the hydrogen industry. "Finding a way to transition from fleets to consumers," they concluded, "is equally critical." This report addresses this critical need by developing strategies for Florida's early stages of hydrogen-powered consumer transportation. In particular, the report investigates urban and statewide networks of refueling stations and the feasibility of a hydrogen rental-car fleet based in Orlando. These two ideas are linked by the potential for a few stations, properly located, to serve the needs of a surprisingly high percentage of car renters in Orlando.

Hydrogen Rental-cars. The vision for a hydrogen rental-car business based at the Orlando International Airport (OIA) is compelling. Orlando is the country's \#1 tourist destination, but most of those tourists visit only a few local destinations. Central Florida has a reputation for innovation, with its unique theme parks and the hydrogen-powered space program at Cape Kennedy. Visitors could have a positive first exposure to hydrogen vehicles with no commitment, and become hydrogen advocates in their home regions and potential early adopters. The rental-car business combines the logistical advantages of a fleet operation while reaching large numbers of consumers. A hydrogen powered rental-car fleet at OIA can provide guaranteed demand to the initial rollout of a hydrogenrefueling infrastructure in the area.

To investigate the feasibility of a hydrogen rental-car business at the Orlando International Airport, we surveyed rental-car customers to understand the demand side. Our survey of 435 renters found that half would be willing to pay more to rent a hydrogen car, and over $80 \%$ would be willing to detour a mile or more for a station. Most importantly, we found that three stations at the airport, theme parks, and downtown Orlando would enable $80 \%$ of car renters to complete trips to all of their destinations, increasing to $85 \%$ with a fourth station at Kennedy Space Center.

From the rental company's point of view, major barriers to a successful hydrogen rental-car business model include: viable consumer target markets; a steady revenue stream via rental of conventional vehicles; a means of identifying customers willing to upgrade to hydrogen; adequate range and refueling locations; breakdown and insurance coverage; the status value of hydrogen cars; the resale market for used vehicles; and most importantly, the availability of hydrogen cars to purchase at a reasonable (subsidized) price. Solutions to each of these issues are proposed here. Synergies with NASA or Disney, refueling stations, and auto manufacturers have the potential to overcome these problems. The strongest evidence that a hydrogen rental-car business is a realistic possibility is the recent announcement by Hertz of a plan to rent a small number of hydrogen vehicles in Iceland. Given this new development and accelerated plans for producing hydrogen vehicles by major automobile manufacturers, planning for the hydrogen rental business and associated refueling infrastructure should begin now. A hydrogen rental-car start-up in the 2012-2015 time frame would place Florida at the forefront of the hydrogen industry. 
Modeling Hydrogen Refueling Infrastructure. Given the high cost of the initial refueling stations, locating a limited number of stations as efficiently as possible will be a key to early success of the industry in Florida. A model was developed that locates stations optimally to serve the maximum consumer demand possible, and quantifies the tradeoff between the number of stations and how much potential demand can be served. The foundation of the model is a road network and a set of traffic analysis zones within a geographic information system (GIS). Using GIS, we find the shortest path (in travel time) from every origin to every destination. Then, given the flow volume on each path, a driving range (in miles) that hydrogen vehicles can be reasonably driven between refuelings, and the number of stations to build, the model uses operations-research (OR) techniques to solve for the optimal set of locations that maximize the amount of transport demand that can be served. Transport demand is measured either in number of trips or vehiclemiles traveled. The GIS and OR models are integrated in a spatial decision support system that researchers can use to develop data, enter assumptions, analyze various scenarios, evaluate tradeoffs, and map results. For the Florida Hydrogen Initiative, we used this model to investigate strategies for rolling out an initial refueling infrastructure in Florida at two different scales of analysis: a metropolitan Orlando network, and a statewide network.

Statewide Infrastructure. To maximize the potential for drivers to use the first set of stations, the model suggests clustering the first ten stations in the Miami-West Palm Beach corridor and the Tampa-Orlando 1-4 corridor, followed by filling in and connecting these clusters. In the statewide analysis, we assume a driving range for vehicles of 100 miles, which allows a large safety margin compared with the expected technical range of hydrogen vehicles of around 300 miles per fill-up. The 1OO-mile range should be treated as a maximum: it is important to place connecting stations where they can also serve crossing traffic flows and local traffic flows, rather than spacing them at regular 1OO-mile intervals. We also analyzed scenarios of 75- and 50 mile vehicle range, in which optimal station networks were progressively more clustered and less connected.

Orlando Infrastructure. To get the most benefit out of the initial refueling infrastructure in Orlando, the model locates stations at funnel points on the road network through which many trips pass, from many origins to many destinations. This strategy wins out over spreading the stations evenly across the metropolitan area. It is more important to locate the early set of stations along the routes people travel rather than locate them near their homes, especially because the first $1 \mathrm{O}$ stations can only truly be near a small fraction of Orlando residents' homes, but can be right on the route of over half of all trips.

Recommendations. We recommend developing a statewide network of 25 stations in five stages or tiers, so that as each tier is constructed, the clusters and the connections between them grow in a coordinated way. The first tier of three stations consists of the airport, downtown, and theme park stations in Orlando needed for the hydrogen rental-car business. Given Orlando's head start in the hydrogen industry with its existing station at the airport, we see it as the key to getting hydrogen moving in Florida. The second tier of seven stations rounds out the Top 10. It creates a hydrogen corridor from Miami Lakes to Ft. Lauderdale to Delray Beach, as well as a connected triangle between Tampa, Orlando, and Gainesville. The third tier fleshes out the Orlando, Tampa, and Miami clusters. The fourth tier completes the network up 1-95 from Palm Beach Gardens to Jacksonville. The fifth tier extends the 1-75 network north and south of Tampa-St. Petersburg, as well as adding to the Miami and Orlando clusters and shortening the distance between stations on Florida's Turnpike. 
For Orlando, we recommend working towards an 11- station network, which could capture 54\% of intra-city trips. The first tier or stage includes the airport, downtown, and theme park stations that are essential to the rental-car business, as well as two other stations on the northern side of town at traffic funnel points that capture heavy traffic flows not otherwise captured by the other three stations. The second tier of six stations includes stations in Kissimmee and Sanford that are recommended for the statewide network.

Next Steps. Future modeling research could focus on a more detailed analysis of the Miami and Tampa metropolitan areas similar to the Orlando network analysis, and an extension of the model to deal with drivers' deviations from their shortest paths. Next steps for the rental-car business plan are to begin discussions with rental-car companies, as well as energy companies that build stations, and possible partners at Disney and NASA. 


\section{Table of Contents}

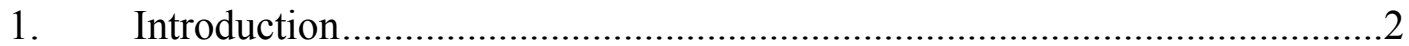

2. Hydrogen Refueling Infrastructure Developments

and Vehicle Production Plans: Domestic and Foreign ..................................4

2.1 Hydrogen Refueling Infrastructure Developments ..................................

2.2 Hydrogen Vehicle Production Plans ........................................................6

3. Hydrogen Rental Car Feasibility Study …………....................................

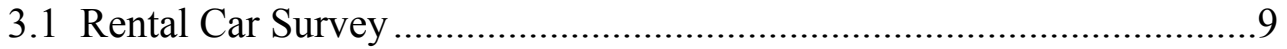

3.2 Barriers and Solutions from the Corporate Point of View........................15

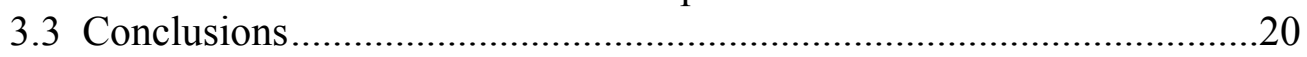

4. Optimal Refueling Station Location Analysis for Florida .............................22

4.1 Prior Research on Refueling Infrastructure Modeling ...........................22

4.2 Modeling Approach: The Flow Refueling Location Model and the Spatial Decision Support System..............................................23

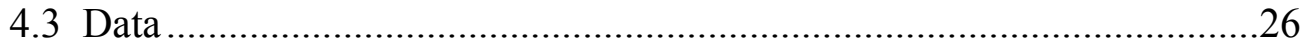

4.4 Florida Statewide Station Location Analysis..............................................37

4.5 Orlando Metropolitan Station Location Analysis ......................................47

5. Recommendations for the Hydrogen Refueling Station Infrastructure in Florida and Orlando …………………............................54

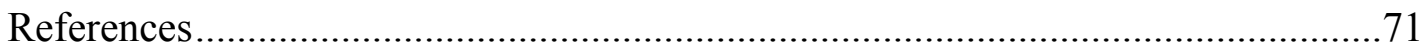

Appendix 1: Car Renter Survey Instrument...........................................................74

Appendix 2: Statistical Relationships Between Demographic

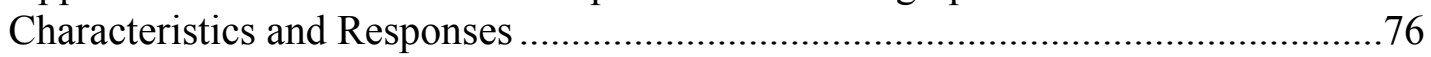

Appendix 3: Spatial Decision Support System for Locating

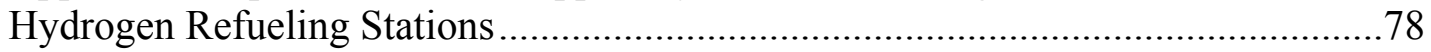

Appendix 4: Data Processing Steps ......................................................................91

Appendix 5: CNG Stations in Florida..........................................93 


\title{
Hydrogen Refueling Infrastructure and Rental Car Strategies For Commercialization of Hydrogen in Florida
}

\author{
Lee Lines, Rollins College \\ Michael Kuby, Arizona State University \\ Ronald Schultz and Zhixiao Xie, Florida Atlantic University
}

\section{INTRODUCTION}

Widespread problems associated with the fossil fuel economy, such as the availability of fossil fuels, reliance on oil imports from Middle-Eastern countries, and greenhouse gas emissions, have motivated the vision and effort to transition from a fossil fuel economy to a hydrogen economy. Barriers to the hydrogen economy, however, are high. These barriers include the cost of building the infrastructure, the maturity of hydrogen production, delivery, and storage technologies, and perceived safety risks [1,2].

As Florida considers the transition to a hydrogen economy, it would do well to learn from past experiences with other alternative fuels. A 2006 study by the National Renewable Energy Laboratory (NREL) [3] studied the lessons that can be learned from other alternative fuels and applied to hydrogen. The study ranked the various barriers in three ways: a comprehensive literature review, rankings by NREL scientists and engineers, and rankings by Clean Cities coordinators. All three rankings agreed that the \#1 barrier is the availability of alternative fuel infrastructure — or lack thereof. The high cost of purchasing alternative-fuel vehicles (AFVs) and the availability of AFVs were also identified as major barriers by NREL and Clean Cities coordinators.

These two sets of barriers - refueling infrastructure and available/affordable vehiclesare closely interrelated. It is often said that the transition to hydrogen faces a chickenand-egg quandary [1-5]. Manufacturers will not be able to sell mass-produced vehicles until consumers are able to refuel the cars when traveling between their desired origins and destinations. Likewise, hydrogen-fuel producers and distributors will have no market for hydrogen until manufacturers begin mass-producing vehicles.

The typical solution to this chicken-and-egg dilemma is (a) government-subsidized initial alternative-fuel infrastructure and (b) incentives or mandates for government and industry fleets of AFVs. This two-pronged approach is meant to provide guaranteed initial demand for both vehicles and stations. Yet, a major message from the NREL study of barriers [3] was that the fleet market, while undoubtedly important, "was not sufficient to generate significant sales for vehicle manufacturers." The study concluded that "finding a way to transition from fleets to consumers is equally critical."

To this end, our research focuses on a unique integration of two closely related solutions to these two related sets of barriers for the transition to consumer use of hydrogen vehicles in Florida. We analyze an optimal network of refueling stations for intra-city trips in the Orlando metropolitan area and for statewide, intercity travel. In addition, we evaluate the feasibility of a hydrogen rental-car business based at the Orlando 
International Airport. The rental-car business model has most of the logistical advantages of a fleet approach to introducing AFVs, with the additional benefit of reaching the consumer market. Orlando is the country's number one tourist destination, attracting millions of tourists from all over the US and the world. A hydrogen rental-car fleet based in Orlando would attract worldwide attention and introduce thousands of potential buyers to hydrogen vehicles in a fleet-like situation. The hydrogen rental-cars would provide a base level of guaranteed demand for the initial set of hydrogen stations in and around Orlando. Orlando's tourism industry is uniquely well-suited for a hydrogen rental-car business; in that most of these tourists visit only a handful of local destinations, making it possible to serve most visitors with a small number of well-placed hydrogen stations.

The first part of our report provides a brief overview of both sides of the chicken-and-egg problem in Florida, the US, and worldwide. Section 2.1 reviews the efforts underway worldwide to develop initial networks of hydrogen refueling stations, while Section 2.2 reviews the current timetables for hydrogen vehicle production by major car makers.

Section 3 analyzes the feasibility of a hydrogen rental-car business based at the Orlando International Airport. Section 3.1 presents the results of a survey conducted of $435 \mathrm{car}$ renters in Orlando, while Section 2.2 then uses these survey results and other sources of information to assess the barriers to a hydrogen rental-car operation and propose some practical solutions.

The final part of our study focuses on the optimal refueling infrastructure for hydrogenpowered vehicles in Florida. The emphasis here is on developing a coordinated system of multiple stations, rather than choosing an exact suitable parcel of land for a station site.

Site criteria have been studied elsewhere, and include accessibility, traffic, fleet operations, safety, host partners' experience with gaseous fuels, hydrogen supply, adequate space, logistical factors, energy sources, and zoning [6]. Section 4.1 reviews the literature on approaches to planning and modeling the best locations for a network of refueling stations. Section 4.2 introduces our approach using the Flow-Refueling Location Model (FRLM) to optimize and compare various strategies for developing an initial hydrogen-refueling infrastructure for Florida. The model uses operations research and GIS techniques, along with sophisticated and detailed transport data bases, to locate a coordinated set of stations so as to maximize the potential for Florida consumers to refuel as many trips, or replace as much gasoline consumption, as possible. Section 4.3

describes the data sources and data preparation steps. Sections 4.4 and 4.5 then apply the model to a statewide network and to the Orlando area, respectively. Section 5 integrates the two sets of results and the hydrogen rental-car analysis into a final set of recommendations for developing a refueling network. 


\section{HYDROGEN REFUELING INFRASTRUCTURE DEVELOPMENTS AND VEHICLE PRODUCTION PLANS: DOMESTIC AND FOREIGN}

\subsection{Hydrogen Refueling Infrastructure Developments}

As the State of Florida plans for a hydrogen future, it is useful to provide a brief overview of the efforts of other regions to develop networks of refueling stations. Many regions in the US and around the world have begun planning or deployment of an initial network of hydrogen refueling stations in order to break the well-known chicken-and-egg cycle, by which the lack of vehicles inhibits station development, and vice versa.

We begin this review in California, where due to air-quality problems, the state of California has forged ahead of any other region in the world in developing a hydrogenrefueling infrastructure. As of the end of 2006, 24 hydrogen stations were built and operating in California, with 13 more planned for 2007. Of these 24 stations, 15 are available for public use. Initially, in April, 2004, California proposed a statewide Hydrogen Highway (CAH2NET) with 200 refueling stations spaced 20 miles apart by 2010 at a cost of up to $\$ 100$ million [6]. Though California has backed off somewhat from this ambitious goal, current plans for Phase 1 call for 50-100 stations by 2010. The existing stations are clustered mainly in the Los Angeles and San Francisco-Sacramento regions, with a few others in San Diego, Palm Springs, and Lake Tahoe [7,8]. The idea is to provide a higher level of service to a smaller but densely populated and polluted area, thus maximizing the number of more-likely buyers who could refuel their hydrogen vehicles on most of their trips. Stations connecting these clusters along rural interstate highways are targeted for Phase 2. The State of California allocated $\$ 6.5$ million to CAH2NET to develop stations in both 2005 and 2006. The December, 2006 Report to the Legislature also identified 126 vehicles and 55 hydrogen-related businesses in California [9]. Supporting the development of the CAH2NET is the California Fuel Cell Partnership, a coalition of 31 auto and energy companies, government agencies, and technology companies that work together to promote commercialization of hydrogen vehicles.

Other regions are also developing or planning networks of hydrogen stations. There are over 140 stations worldwide, with $48 \%$ in the US, $14 \%$ in Japan, and $13 \%$ in Germany $[10,11]$. By the end of 2007, the number of stations worldwide will surpass 160 [12]. In the United States, New York's H2-NET hopes to have 20 stations by 2015-2020 to facilitate travel from New York City to Buffalo. They also plan to add hydrogen-fueling capability to 70 existing CNG stations, and replace $5 \%$ of conventional fuel demand with hydrogen by 2020. Illinois is planning a $2 \mathrm{H} 2$ Hydrogen Highway, and the Northern $\mathrm{H}$

Fuels Network in the Great Plains region of the US and Canada proposes 12 stations 200 $\mathrm{km}$ apart by 2012. Discussions are ongoing in South Carolina, in Connecticut, and for a Denver-to-Los Angeles network.

At the national scale, the National Renewable Energy Laboratory (NREL) analyzed the potential development of a national backbone network to make long-distance, interstate 
trips possible by 2020 [13]. They estimated that 284 stations are needed, at a total construction cost of $\$ 837$ million. The cost per station ranged from $\$ 248,000$ to $\$ 5.1$ million depending on the estimated demand and technology. They concluded that many of the stations could be economically self-sustaining in the near term based on "aggressive assumptions" of 1\% penetration of hydrogen light-duty vehicles in 2020 increasing to $20 \%$ in 2030 . Fourteen of these stations are suggested for Florida, including six at existing natural gas stations and eight at new sites. (The methods used in this study are reviewed in the following section.) At the same time, DOE is putting greater emphasis in 2007 on developing clusters of stations rather than corridors [14].

Outside of the US, many countries are seeding initial refueling infrastructures. In Canada, British Columbia hopes to have a Hydrogen Highway in place connecting Vancouver to Whistler Ski Resort in time for the 2010 Winter Olympics. Five stations are already open. The Canadian federal government has provided CDN\$33 million of funding to the Transportation Fuel Cell Alliance. The European Union has a Joint Technology Initiative with the stated goal to kick-start commercialization by 2015 [12]. The Scandinavian Hydrogen Highway Project consisting of HyNor (Norway), Hydrogen Link (Denmark) and HyFuture (Sweden) is trying to link Oslo with Copenhagen.

In the less-developed world, India has developed a National Hydrogen Roadmap, and both Singapore (SINERGY) and China have opened two stations each. In China, the Shanghai Hydrogen Lighthouse Program aims to build a small network of stations for fueling 90 taxis and ten fuel-cell buses by 2010 [12].

Most stations being developed recently provide gaseous hydrogen. Very few provide liquid hydrogen. A recent trend is to build more multi-fuel stations rather than dedicated hydrogen stations. Developing stations to sell hydrogen as well as CNG and other alternative fuels - and possibly even conventional gasoline - is a way to share investment costs and minimize technology risks [12]. One refueling infrastructure project in Europe, called HyChain MiniTrans, is investigating the possibility of selling hydrogen canisters from a dispenser.

\section{Recent Developments in Orlando and Florida}

There are currently two hydrogen refueling stations in the Orlando metropolitan area: the Boggy Creek Hydrogen Refueling Station near the Orlando International Airport and a mobile refueling station at Progress Energy's Jamestown Operations Center near Oviedo.

The Boggy Creek Hydrogen Refueling Station is the result of a collaborative project involving the State of Florida, Chevron Technology Ventures, Ford Motor Company, and Progress Energy. Built and operated by Chevron Technology Ventures, the Boggy Creek station (natural gas reformation) opened in May of 2007. Initially it will refuel four hydrogen-powered airport shuttle buses (Ford V-10, E-450). Four additional H2 shuttle buses will be in service by 2008. Under the current plan, the Boggy Creek station is scheduled for decommissioning in May of 2010. 
The mobile refueling station at Progress Energy's Jamestown Operation Center opened in September of 2005 and is scheduled for replacement by a fixed electrolysis station in late 2007. This test site is part of a US Department of Energy Demonstration Project: Ford and BP Controlled Hydrogen Fleet and Infrastructure Demonstration and Validation Project. The station currently provides power for six Ford Focus hydrogen fuel cell vehicles operated by Progress Energy and Florida's Department of Environmental Protection. Under the current plan, this station is scheduled for decommissioning in late 2008.

In 2004, the Florida Hydrogen Business Partnership was formed by 27 companies and government agencies with a mission to "establish Florida as a center of hydrogen technology commercialization for the Americas" [15]. The Partnership targeted Orlando as the site for five operating hydrogen stations by 2007. The State of Florida aims to develop the nation's first one-stop permitting standard for hydrogen fueling infrastructure by creating a single, uniform permitting standard and consistent safety codes across all jurisdictions in Florida, and its Energy Plan 2006 calls for sales and corporate income tax credits for hydrogen vehicles and fueling infrastructure [16].

\subsection{Hydrogen Vehicle Production Plans}

Lack of refueling infrastructure is only half of the chicken-and-egg problem for hydrogen. Florida must also account for the timetables for the manufacture of hydrogen vehicles. While researchers have developed a variety of models for predicting the coevolution of infrastructure and vehicles, the following section is based on announcements by auto manufacturers rather model simulations.

In their February 2007 survey of the light-duty vehicle market [12], Fuelcelltoday.com, an industry website that publishes annual market surveys, concludes that major manufacturers have in some cases accelerated their timetables for commercializing fuelcell vehicles (Table 2.1).

General Motors expects to begin production of a fuel-cell version of its Chevrolet Volt, possibly as early as 2010 , and they could hit showrooms before 2020 [17]. They are transferring 500 fuel cell engineers from the research department to production. According to Autocar.co.uk, "the world's second largest car company now views hydrogen fuel cell power as the 'the end game,' according to its director of advanced technology vehicle concepts Dr Christopher Borroni-Bird" [18]. GM predicts that FCVs could match conventional vehicles in affordability by the time the scale of production reaches about 1 million units.

Honda has announced it will launch a limited number of fuel-cell vehicles based on the FCX concept car by 2008 in Japan and the US [19]. Hyundai aims to market an affordable FCV by 2015, and boldly forecasts a $90 \%$ market share for FCVs by 2050 . Renault is reported to begin production of FCVs in India by 2010. Toyota recently announced they would be providing Hertz with Prius hybrids converted to run on hydrogen for a hydrogen rental car operation in Iceland by April, 2008 [20]. 
Several manufacturers are banking on the internal combustion engine (ICE). BMW advertises that they will begin selling cars using liquid hydrogen in ICEs by 2008. Mazda is developing a rotary engine that can run on gasoline or hydrogen - a development that could potentially remove major concerns about running out of hydrogen when not in the vicinity of a hydrogen station [21].

Table 2.1 Automobile Manufacturers' Hydrogen Vehicles [17-23]

\begin{tabular}{|c|c|c|c|}
\hline Company & Technology & Driving Range & Future Plans \\
\hline BMW & ICE & 240 & $\begin{array}{l}\text { Currently introducing series } \\
\text { production hydrogen-fueled } \\
\text { internal combustion engine } \\
\text { Hydrogen } 7 \text { vehicles. }\end{array}$ \\
\hline GM & $\begin{array}{l}\text { Fuel Cell } \\
\text { (Chevrolet Volt) }\end{array}$ & 200-300 miles & $\begin{array}{l}\text { Begin production of a fuel- } \\
\text { cell version of its Chevrolet } \\
\text { Volt, possibly as early as } \\
2010 \text {. }\end{array}$ \\
\hline Hyundai & & & $\begin{array}{l}\text { "Wants to sell a hydrogen } \\
\text { powered vehicle at an } \\
\text { affordable price by } 2015 . "\end{array}$ \\
\hline Honda & $\begin{array}{l}\text { Fuel Cell ("FCV, } \\
\text { FCX") }\end{array}$ & 235 miles & $\begin{array}{l}\text { "Plans to introduce a } \\
\text { limited number of second } \\
\text { generation FCX vehicles to } \\
\text { the Japanese and US } \\
\text { markets in 2008." }\end{array}$ \\
\hline Mazda & $\begin{array}{l}\text { Dual Fuel ICE } \\
\text { ("RX-8 } \\
\text { Hydrogen RE") }\end{array}$ & $\begin{array}{l}62 \text { miles on } \\
\text { hydrogen and } \\
341 \text { miles on } \\
\text { gasoline }\end{array}$ & $\begin{array}{l}\text { Currently leasing } \\
\text { prototypes. }\end{array}$ \\
\hline Nissan & ICE & & Early 2010 s \\
\hline THINK & $\begin{array}{l}\text { Fuel Cell ("city } \\
\text { vehicle") }\end{array}$ & 180 miles & \\
\hline $\begin{array}{l}\text { PSA Peugeot } \\
\text { Citroen }\end{array}$ & $\begin{array}{l}\text { Fuel cell concept } \\
\text { vehicle }\end{array}$ & 300 miles & \\
\hline
\end{tabular}

At its Scenario Analysis Meetings in January, 2007, Dr. Sigmund Gronich, Transition Strategy Manager of DOE's Hydrogen, Fuel Cells, and Infrastructure Technologies Program, presented three scenarios for hydrogen vehicle production in the United States, as shown in Table 2.2, with hundreds to thousands produced by 2012 [14].

Table 2.2 DOE Scenarios for Hydrogen Fuel Cell Vehicle Production

\begin{tabular}{clllll}
\hline Scenario & By 2012 & By 2015 & By 2018 & By 2021 & By 2025 \\
\hline 1 & $100 \mathrm{~s}-1000 \mathrm{~s}$ & & $10,000 \mathrm{~s}$ & & 2 million \\
2 & $1,000 \mathrm{~s}$ & $10,000 \mathrm{~s}$ & $100,000 \mathrm{~s}$ & & 5 million \\
3 & $1,000 \mathrm{~s}$ & & & $1,000,000 \mathrm{~s}$ & 10 million \\
\hline
\end{tabular}




\section{HYDROGEN RENTAL-CAR FEASIBILITY STUDY}

This section introduces and analyzes a new strategy for creating a base level of demand for an initial refueling infrastructure that may be uniquely well suited for the Orlando area-namely a hydrogen rental-car fleet based at the Orlando International Airport. Generating a base level of demand for hydrogen is a key to overcoming the chicken-andegg quandary outlined in the previous sections. The risk in rolling out the refueling infrastructure first to short-circuit the chicken-and-egg problem is that the stations may initially be underutilized. While government vehicle fleets will undoubtedly provide some underlying demand for the initial set of stations, the transition from government and industry fleets to consumer vehicles may stall without a strategy to get consumers into hydrogen vehicles. A hydrogen rental-car fleet has the potential to overcome this obstacle, especially one based in Orlando, the number one tourist destination in the United States. In addition, as Box 1 shows, we are not the only ones who think that a hydrogen rental-car fleet is a feasible idea whose time has come.

Orlando attracts millions of tourists each year from all over the US and the world. Furthermore, Orlando has a reputation of being on the cutting edge, with its innovative theme parks, the planned town of Celebration, and the nearby Kennedy Space Center.

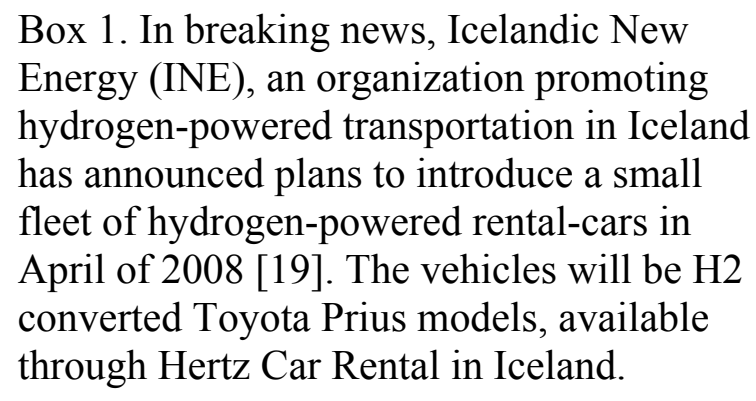
hydrogen-powered transportation in Iceland has announced plans to introduce a small fleet of hydrogen-powered rental-cars in April of 2008 [19]. The vehicles will be $\mathrm{H} 2$ converted Toyota Prius models, available through Hertz Car Rental in Iceland.

Visitors and Florida residents alike could be introduced to hydrogen-powered vehicles in Orlando with no long-term commitment on their part. Their first experience with hydrogen could be a positive one because of the worry-free availability of centralized service by rental companies and convenient location of refueling stations. In fact, a very small number of stations would be needed to serve most rental-car trips. Benefits would be felt locally, nationally, and worldwide, as renters would become hydrogen advocates in their home regions, and would be more likely to purchase hydrogen vehicles. Florida would benefit by developing a base level of demand for its infrastructure, as well as hydrogen expertise and a reputation for being on the cutting edge of hydrogen deployment.

To examine barriers to using hydrogen as a fuel source for the rental-car market and propose some possible solutions, we pursue two primary lines of research. First, to understand the consumer point of view, we conducted a personal intercept survey of 435 potential car renters. Second, we explored the feasibility of a rental-car business model from the corporate point of view by reviewing literature and conducting interviews with key people in the hydrogen and rental-car industries about barriers to a rental-car business and some possible solutions to those barriers. 


\subsection{Survey of Car Renters}

Consumer attitudes will clearly play a pivotal role in any effort to develop a hydrogenbased rental-car fleet at the Orlando International Airport (OIA). To better understand what is needed to develop a hydrogen rental-car option at OIA we conducted a personal intercept survey of 345 potential car renters at the OIA and 90 visitors to Orlando's theme park attractions at International Drive. The airport surveys were conducted in the OIA rental-car terminal between March and June of 2006. The International Drive visitor surveys were conducted in the shuttle bus waiting areas during August-September, 2005.

The survey instrument (Appendix 1) focused on each respondent's knowledge and awareness of hydrogen technology, reasons for and against renting a hydrogen vehicle, willingness to pay more to rent a hydrogen vehicle, willingness to drive out of their way to refuel, intended destinations in the Central Florida region, and socio-demographic characteristics.

Our typical survey respondent was 43 years of age and female, married with two children, and college educated (see Table 3.1). The majority of respondents were employed in either private industry/business or government/education, with an annual household income considerably higher than the US median household income of $\$ 46 \mathrm{~K}$. The survey included visitors from 14 different countries (most notably Canada and Great Britain); however, the majority of survey respondents $(80 \%)$ were from the United States.

Table 3.1 Socio-economic Characteristics of Survey Respondents $(n=435)$

Average age:

Gender

Marital status:

Number of children:

Education:

Occupation:

Household income:

Country of origin:
43

Female: $59 \% \quad$ Male: $41 \%$

Married: $80 \% \quad$ Single: $20 \%$

None: $14 \% \quad$ One: $13 \% \quad$ Two: $41 \%$

Three: $20 \% \quad$ Four: $7 \% \quad$ Five or more: $5 \%$

Less than high school: $\quad 0 \%$

High school graduate: $\quad 27 \%$

College graduate: $\quad 53 \%$

Post-graduate degree: $\quad 20 \%$

Student: 3\% Private industry/business: 39\% Government/education: $21 \%$ Military: 1\% Retired: $6 \%$ Other: $30 \%$

Under \$30K: 6\% \$30K-49K: $15 \%$

\$50K-99K: $43 \% \quad$ Over \$100K: $36 \%$

United States: $80 \% \quad$ United Kingdom: $10 \%$

Canada: $6 \% \quad$ Other 11 countries: $4 \%$ 
Within our group of survey respondents there was considerable variation in the general understanding of hydrogen vehicle and fuel-cell technology. Despite the wide variation in responses, a significant proportion of the respondents possessed some knowledge of the technology and a familiarity with how it works (see Figure 3.1). This moderate level of knowledge of hydrogen technology may reflect recent media coverage of alternative energy technologies. It may also reflect the high level of educational attainment for our survey respondents ( $73 \%$ are college graduates).

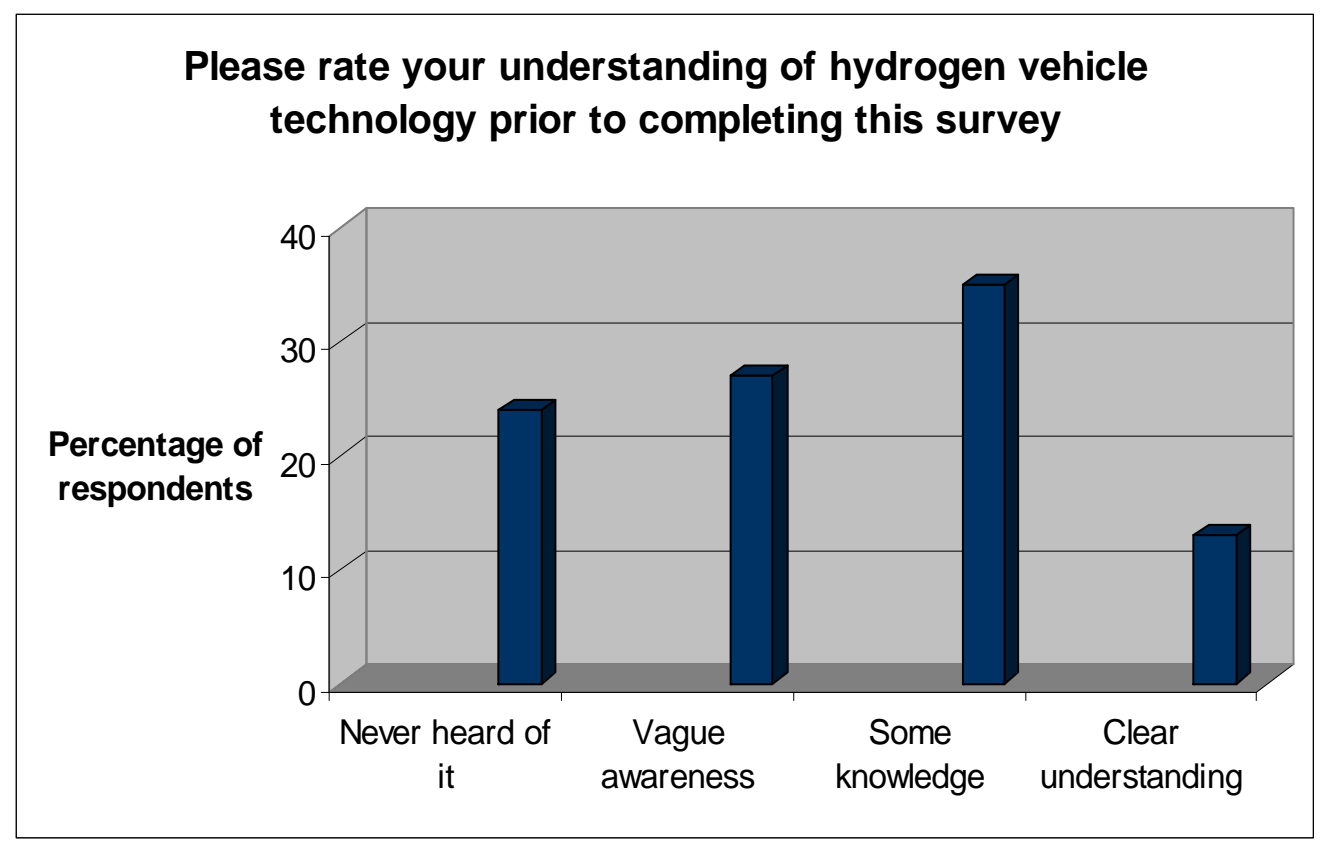

Figure 3.1 Consumer understanding of hydrogen fuel-cell technology

On the question of consumer willingness to pay more to rent a hydrogen vehicle, our survey respondents were evenly divided ( $49 \%$ responding positively). Of the total number of visitors surveyed, 21 percent indicated that they were willing to pay an additional $\$ 5$ to $\$ 10$ per day to rent a hydrogen car (see Figure 3.2). Roughly $6 \%$ of all respondents were willing to pay an additional $\$ 15$ to $\$ 20$ per day to rent a hydrogen vehicle. 


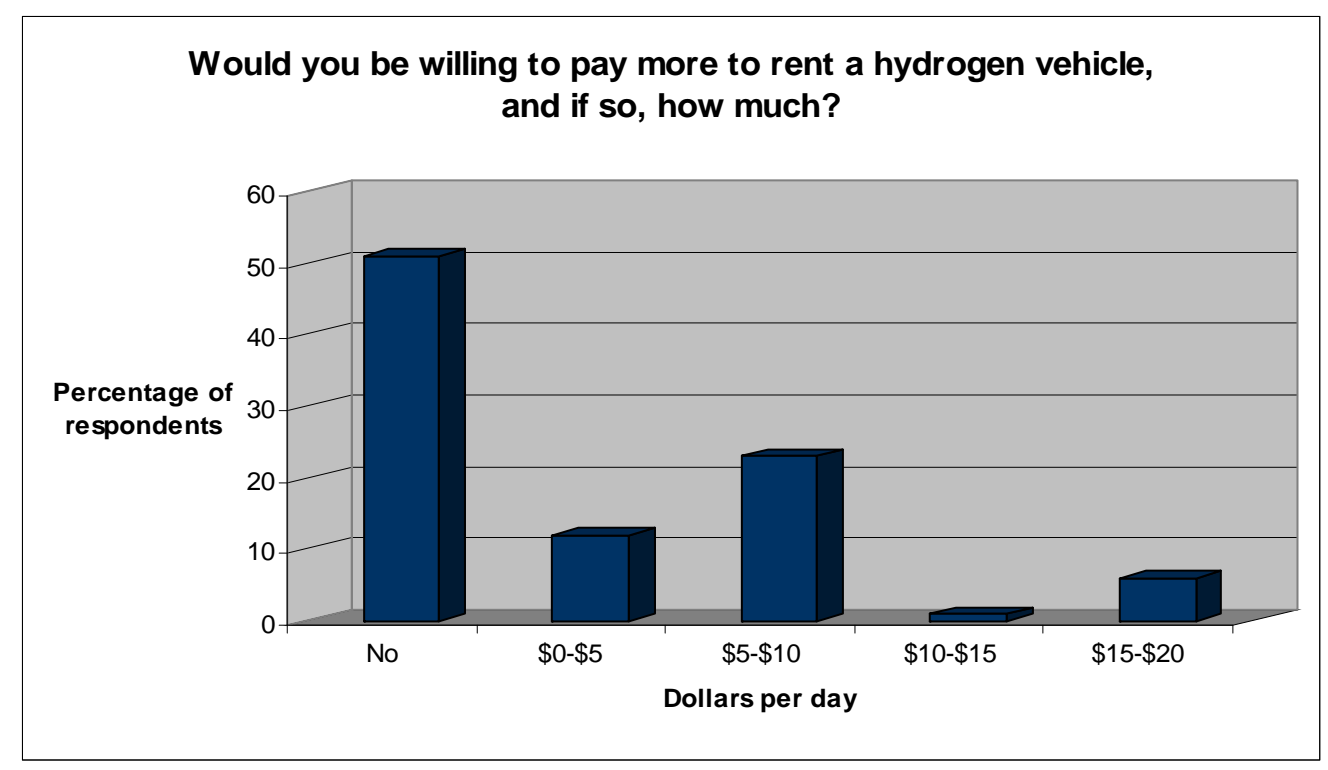

Figure 3.2 Consumer willingness to pay more to rent a hydrogen vehicle

The willingness of car renters to consider a hydrogen vehicle is clearly influenced by a number of different factors. To better understand these considerations in the consumer decision-making process, we asked our survey respondents to rate the importance of a number of features in convincing them to rent a hydrogen vehicle (see Table 3.2). These features reflect a wide range of potential renter concerns including vehicle performance, cost, convenience, customer support, and vehicle safety. Survey respondents rated the importance of each feature on a scale from $\mathbf{1}$ (not important) to $\mathbf{5}$ (very important).

Table 3.2 Features influencing consumer decision to rent a hydrogen vehicle

Feature

Average response

Scale: $\mathbf{1}$ (not important) to $\mathbf{5}$ (very important)

Map of refueling stations in Florida and Orlando

4.3

Ability to exchange for a gasoline car at no extra cost

4.0

Priority parking at theme parks

3.9

Using a pollution-free vehicle

4.4

Fuel cost per mile comparable to gasoline

4.4

Opportunity to experience a new technology

4.0

Opportunity to test drive before renting

3.8

Vehicle performance (e.g., acceleration, noise)

4.1

Driving range of vehicle

4.4

Availability of insurance

4.3

Full-service refueling by a trained attendant

4.0

On-call, roadside repair/refueling service

4.4 
The most interesting aspect of the results displayed in Table 3.2 is that respondents rated all features as important. Features considered most important included the driving range of the vehicle, fuel cost per mile, availability of an on-call roadside repair/refueling service, and the opportunity to use a pollution-free vehicle. Other important features were the availability of vehicle insurance and an obtainable map of refueling stations in Florida and Orlando. The responses to this question reflected an interesting mix of practical concerns (e.g., vehicle performance) and wider motivations (e.g., using a pollution-free technology) surrounding the decision to rent a hydrogen vehicle.

Perhaps most significant in our survey of potential car-renters are the questions centered on the intended destinations of Orlando visitors and their willingness to drive out of their way to refuel a hydrogen vehicle. The question on intended destinations - "Which of the following destinations did you (or will you) visit on your trip to Orlando"-provided each respondent with a list of twelve major destinations (see Table 3.3). The responses to this question provide a snapshot of where our potential car renters are driving, and perhaps more importantly, where they are not driving (note: since most respondents identified multiple destinations, summed percentage values exceed 100 percent).

Table 3.3 Intended destinations of potential hydrogen car-renters

\begin{tabular}{lc}
\hline Destination & $\begin{array}{c}\text { Percentage of respondents } \\
\text { identifying this destination }\end{array}$ \\
\hline Magic Kingdom & 63 \\
Universal Studios & 42 \\
EPCOT & 40 \\
Sea World & 35 \\
& \\
Other theme park & 21 \\
Downtown Orlando & 20 \\
Port Canaveral (cruises) & 10 \\
Kennedy Space Center & 9 \\
& \\
Tampa-St. Petersburg & 9 \\
Daytona Beach & 8 \\
Miami-Palm Beach area & 3 \\
Everglades region & 3 \\
\end{tabular}

As expected, a significant proportion of our respondents identified at least one of the major Orlando theme park attractions as an intended destination. Somewhat surprising were some of the low response rates for destinations beyond the Orlando area such as Daytona Beach ( 8 percent) and Tampa-St. Petersburg (9 percent). This immediately raised a number of follow-up questions. What percentage of visitors to Orlando are renting vehicles and driving beyond the Orlando area? What specific set or "bundle" of 
destinations is typical for a car renter at the Orlando International Airport? The answers to these questions have important implications for the initial planning and rollout of a hydrogen-refueling infrastructure in Orlando.

The average length of stay for the 435 Orlando visitors surveyed in our study is 7.9 days. If we assume that the majority of visitors renting a car will rent that car for the duration of their stay, it becomes important that the vehicle they are renting can get them to their entire bundle of destinations for the week. If the rented vehicle is hydrogen-powered, this bundle of destinations will place a specific set of demands on the refueling infrastructure. For example, a number of widely separated destinations will require a very different refueling infrastructure than a concentrated set of destinations in a confined geographic area. In fact, even a single trip to a more distant destination (e.g. Kennedy Space Center) during the course of an otherwise geographically concentrated week of driving will require a different set of stations to refuel that trip. The ability of the refueling network to refuel such a trip could be the pivotal factor in the consumer's decision to rent, or not rent a hydrogen-fueled vehicle.

To examine this issue, we analyzed bundles of visitor destinations both within and outside of the Orlando metropolitan area (Figure 3.3). Most significantly, 80 percent of the car renters surveyed did not identify an intended destination outside of the theme park areas or downtown Orlando. Only 20 percent of our respondents identified intended travel destinations beyond the Orlando area.

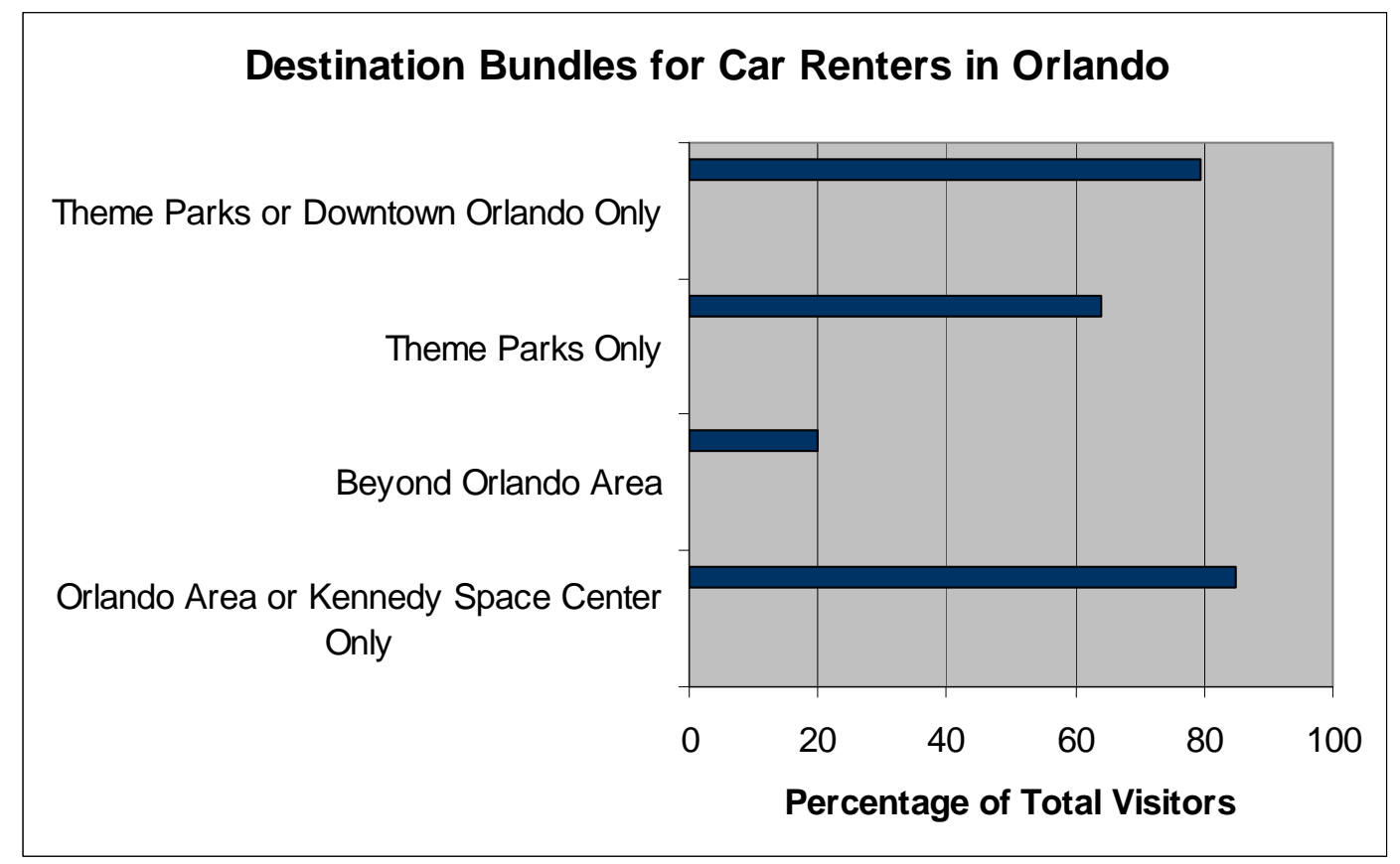

Figure 3.3 Destinations of car renters surveyed at the Orlando International Airport

These findings have important implications for the potential feasibility of a hydrogenpowered fleet of rental vehicles based at OIA. Given that the majority of airport car renters are concentrating their trips in the area of the theme parks and downtown 
Orlando, the initial refueling network required to support this fleet of cars could be modest. In fact, our survey data suggests that just three refueling stations (located at OIA, downtown Orlando, and Disney) could cover the refueling needs for roughly 80 percent of the car rentals originating from the Orlando International Airport.

We also asked potential car renters how far out of their way they would be willing to drive for a hydrogen refueling station without considering it a hassle. The survey results for this question also support the idea that three initial stations in the Orlando area will likely cover the majority of airport car rentals. More than $80 \%$ of our survey respondents expressed a willingness to drive more than one mile for a hydrogen refueling station, and $46 \%$ were willing to drive more than three miles out of their way (Figure 3.4). These visitors - many of whom are on vacation, and will only need to make this detour once or twice - may exhibit a greater willingness to divert from their optimal driving path than Orlando residents, making the rental-car network more flexible in the refueling of trips.

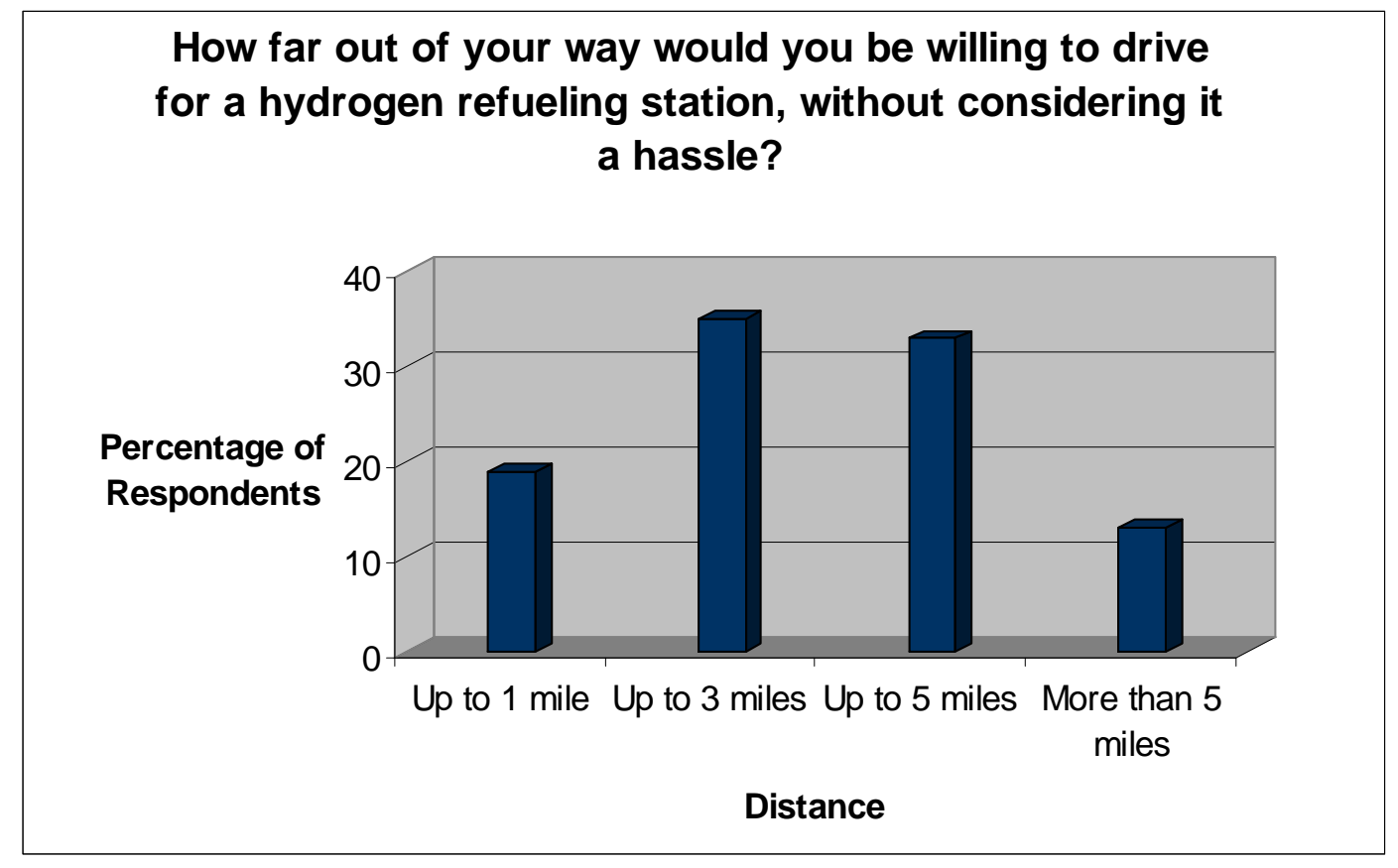

Figure 3.4 Willingness of car renters to go out of their way for a hydrogen refueling station

For all survey questions, we analyzed the results statistically to determine if the demographic characteristics of the renter significantly influenced their responses. We used analysis of variance (ANOVA) to search for relationships between the opinion variables and gender, marital status, education, and income. Our tests, summarized in Appendix 2, were conducted at the $95 \%$ confidence level or as alternatively stated, the $5 \%$ significance $(\alpha)$ level. We found that people with higher education levels are significantly less concerned about refueling hydrogen vehicles themselves and less concerned about the rental price. Women were significantly more concerned with roadside on-call service, the ability to exchange a hydrogen vehicle for a conventional one, the cost per mile, and the vehicle performance. Males, on the other hand, claimed a 
better understanding of hydrogen technology and were more concerned with having to detour to a station. Not surprisingly, those with higher education and male gender rented cars more frequently.

The survey results outlined in this section have important implications for the initial hydrogen vehicle refueling infrastructure in Florida. Most analysts agree that a major impediment to the introduction of hydrogen fuel-cell technology is the initial cost and individual consumer risk associated with early adoption. Car renters may provide an ideal market for the first wave of hydrogen vehicles in Central Florida. A fleet of hydrogenfueled rental-cars based at OIA could introduce consumers to hydrogen technology in a setting where their individual costs would be short-term and shared by the rental-car agencies. Since most car-rental trips are concentrated in the area of the theme parks and metro Orlando, the logistical costs associated with consumer support for this rental-car fleet would be quite reasonable.

\subsection{Barriers and Solutions from the Corporate Point of View}

To understand the barriers to a successful hydrogen rental-car business from the supplier's point of view, and assess solutions to overcoming those barriers, we studied various reports on the hydrogen economy and spoke with a number of key informants. In particular, we interviewed the manager of a rental-car company, EV Rentals, which currently rents hybrid vehicles in partnership with Fox Rent A Car, and previously had experience renting electric and Compressed Natural Gas (CNG) vehicles.

\section{Target Market}

Knowing your target market is an important starting point for any new business idea. In the case of renting hydrogen cars, a number of market segments stand out as potential renters of hydrogen vehicles:

1. Green Consumers. Ecologically minded consumers have shown a willingness to pay extra for goods and services that allow them to live a more sustainable lifestyle. Such services range from alternative-fuel vehicle purchases, organic foods, renewable electricity, and many others. In addition, EV Rentals has found that green companies, and companies that are marketing to other green companies, are willing to rent alt-fuel and hybrid cars. EV Rentals has found the status value of renting alt-fuel cars to be important to green consumers and companies. Therefore, the car must be easily identifiable by others as a sustainable form of transportation - and not just by a small decal.

2. Technophiles. Hydrogen cars capture the imagination of technophiles. Whether it is a fuel cell powering an electric motor, or simply an ICE running on the same fuel that blasts rockets into space, technophiles and car buffs may be very interested in trying out a hydrogen vehicle.

3. Alt-fuel Privileges. Some demand for AFVs derives from the privileges associated with them. Use of HOV lanes by single-occupant vehicles is a major incentive in congested areas. EV Rentals noted that some customers in Los Angeles enjoy free 
downtown parking with their AFV rentals. We think that priority parking for hydrogen cars at Disney theme parks or Kennedy Space Center might provide an important incentive to rent a hydrogen car, especially if the priority parking also included refueling by a trained technician while they visited the attraction.

In addition, EV Rentals has found it important to also rent conventional vehicles. Conventional vehicles provide a base revenue stream in case demand for alt-fuel cars fluctuates. Offering conventional vehicles would provide the agents with the opportunity to offer upgrades to hydrogen vehicles. Agents can be trained to ask a few key questions that may indicate willingness to upgrade, such as:

"Is this a business or tourist trip?"

"Are you planning to stay within the Orlando area?"

"Are you planning to visit Kennedy Space Center?"

"Would you like priority parking at the theme parks?"

\section{Customer Concerns}

While the idea of trying a hydrogen vehicle on a short-term rental basis may be attractive, various concerns may deter potential customers from doing so. Some main concerns, and solutions to overcome these, are listed below:

1. Safety. Given that most renters in our survey were middle-aged families with two children and not very knowledgeable about hydrogen, a key concern is likely to be safety. References to the Hindenburg or to "riding a hydrogen bomb" elicit fearful responses. Educational videos comparing the risks of hydrogen to gasoline may help overcome these fears. Videos could be shown on the Internet and at the rental counter. A brief instructional session may be necessary before customers depart with their rented vehicles, and safety brochures should also be placed in each car and on the rental counter and Internet.

2. Breakdowns. Advertising can emphasize the lower maintenance requirements of clean-burning hydrogen fuel, but renters are still likely to be concerned about breakdowns and the lack of knowledge and parts at most auto-repair shops. In the survey, on-call roadside repair service was rated as highest in importance to customers with a 4.4 average, and free replacement by a gasoline vehicle was slightly less important at 4.0. In the Orlando area, substitution of another hydrogen vehicle might be possible, but outside of Orlando, quick replacement by a conventional vehicle would seem to be the only viable solution. To make this goal achievable, it is important that the managers of the rental business have experience working with other companies at the Orlando airport, because rental companies often ask each other for help to deliver replacement cars quickly.

3. Refueling. In the survey, the driving range of vehicles tied for the highest average importance with 4.4, while a map of refueling stations averaged 4.3 , and refueling by a trained mechanic averaged 4.0. Many renters who visit only the theme parks and/or the convention center may not require even a single refueling. Offering customers the ability to return their car empty, without the usual penalty of high 
fuel fees, might be a major incentive. If refueling could be coupled with parking at Kennedy Space Center, Disney theme parks, or downtown Orlando, this would provide a large incentive. Ability to "practice" refueling when renting the car might also allay such concerns.

4. Insurance. Insurance companies are unfamiliar with hydrogen vehicles, and replacement cost of early vehicles will be high. Customers will undoubtedly be uncertain of whether their regular policy will cover damage to a hydrogen vehicle. The rental-car business must work out the insurance issues for the customer, and government subsidy of supplemental insurance undoubtedly will be necessary to begin with.

\section{Refueling Availability}

A customer's ability to rent a hydrogen vehicle hinges on the availability of refueling stations to serve not just one or several of the destinations they are planning to visit, but all of their destinations. A rental-car business based at OIA could be launched with the as few as three hydrogen stations:

1. At the Orlando International Airport (already exists);

2. Near the cluster of theme parks in southwest Orlando;

3. Downtown Orlando.

This group of stations, along with the airport station, could serve roughly $80 \%$ of car renters. A fourth station at Kennedy Space Center would raise this percentage of renters who could make all of their intended trips to around $85 \%$. Additionally, a station in Tampa would raise this percentage to around $90 \%$.

\section{Purchase of Hydrogen Cars}

The supply of hydrogen cars is the biggest barrier to overcome. Currently, all hydrogen cars made by major automakers are custom-made prototypes, with the BMW H7 series soon to be the first production-run hydrogen vehicle sold to the general public. A number of manufacturers, however, have plans to begin production in the 2010-2015 time period (see Section 2.2). The cost of vehicles may dictate when the rental-car business might become feasible. Options are discussed below:

1. FCVs. Fuel-cell prototypes currently cost up to $\$ 1$ million to produce. Automakers have carefully controlled these valuable investments to protect them for continued use and to gain as much information as possible from each user. Until very recently, automakers have been highly circumspect about placing FCVs in the hands of untrained users in uncontrolled situations. One manufacturer suggested to us that even the first generation of production-scale vehicles would be too valuable to use as rental-cars. However, with the recent announcement of Toyota's willingness to provide prototypes of a hydrogen-hybrid Prius for the Hertz/New Icelandic Energy hydrogen rental-car operation in Iceland, it now 
appears possible that a manufacturer would be willing to provide more-costly preproduction vehicles as rentals.

2. ICEs. ICE prototypes are also quite costly, whether hydrogen-only or dual-fuel. It is conceivable, however, that production runs of ICEs will reach the needed scale and cost to supply a subsidized rental business before FCVs.

3. Converted Conventional Cars. A number of small businesses currently convert conventional ICEs to burn hydrogen. Though still a custom procedure, a company in the Phoenix, Arizona area quotes $\$ 8,000$ per conversion. If each hydrogen car could be rented for $\$ 10$ more per day for 300 days per year, the conversion costs could be paid off in about three years. Liability issues for using converted cars might be a stumbling block.

While purchase costs are the most daunting hurdle facing hydrogen rental-cars, the barrier may not be insurmountable. The free mass media publicity to the manufacturer that supplies the vehicles could easily be worth millions of dollars of advertising. Crossmarketing with Disney could also create substantial buzz. Word of mouth from satisfied rental customers could promote sales throughout the world. In addition, it may be possible to begin with converted ICEs, then move to production ICEs, and eventually to FCVs. It may also be possible to start with just a few hydrogen vehicles added to EV Rental's fleet of other alt-fuel vehicles.

\section{Resale of Used Vehicles}

The car rental industry is a low-margin business. A key element of financial success is the ability to resell used vehicles, which are typically retired from rental service at around 30,000 miles. Cars that are in high demand may be kept in service longer, while cars in low demand may need to be sold sooner. Conventional vehicles are typically sold back to manufacturers for sale through their dealers or sold to wholesalers. No such used car market, however, is likely to exist for retired hydrogen rental-cars. EV Rentals had to make their own markets for used electric and CNG cars. Drawing on their experience, here are some solutions to the resale problem:

1. Environmental Groups. Used hydrogen vehicles could be marketed to members of large national environmental groups such as the Sierra Club, National Wildlife Society, Audubon Society, Resources for the Future, Nature Conservancy, etc.

2. Respiratory Health Groups. Marketing to members of the American Lung Association or the Asthma and Allergy Foundation of America could reach large numbers of potential buyers.

3. Car Clubs. Various hobbyist groups are interested in alternative-fuel cars.

4. State Vehicle Fleets. The State of Florida might be able to indirectly subsidize the rental-car business by promising to purchase used hydrogen vehicles for some of their fleets. This would keep the cars in use in state and promote continued use of Florida refueling infrastructure, and perhaps provide a reasonably-priced supply of zero emission vehicles (ZEVs) to the state government. 
5. Past Rental Customers. A database can be maintained of customers who rented hydrogen cars, and used cars could be offered to them first. They will have already test-driven the cars.

A potential unrecognized benefit of developing this business in Orlando is that, with most rental-cars never leaving the Orlando area, hydrogen rental-cars might not reach the 30,000 mile threshold for resale for 3-5 years, thus spreading the cost of cars over a longer time span.

\section{Operator Characteristics}

Though financially challenged, a small rental-car company such as EV Rentals might be best suited to run the first hydrogen rental-car business, if the cars were affordable or subsidized. A lean, flexible, and hungry company may be necessary to work around the barriers that are likely to arise. Such companies need to offer products or services that the large rental-car companies do not, and they are likely to test this market first.

Large companies, on the other hand, with their higher revenues, national advertising, and ties with automakers, would have more resources to support such a venture should they choose to do so. Hertz's decision to launch a hydrogen rental-car joint venture in Iceland certainly indicates their willingness to innovate in this direction. Hertz will add 3,400 Prius hybrids by 2008, while Avis is adding 1,000 Toyota Prius hybrid cars to their fleets in California; Portland, Oregon; Seattle, Washington; and Washington, D.C. by July, $2007[23]$.

\section{Synergies}

A hydrogen rental-car business based in Orlando could benefit from tremendous synergies with other hydrogen initiatives:

1. Added Demand for Stations. The rental-cars would provide an additional source of hydrogen demand for certain stations, especially those located near the theme parks, the airport, downtown Orlando, Kennedy Space Center, Daytona Beach, and Tampa. Several of these stations are also recommended stations for the statewide network (see Section 5).

2. Kennedy Space Center. Many people associate hydrogen energy with the space program. Hydrogen is burned to launch the shuttle into space, where hydrogen fuel cells generate electricity and drinking water for the shuttle astronauts. Crossmarketing with NASA could give a tremendous boost to the rental-car idea. Priority parking at Kennedy Space Center (KSC) and a highly visible refueling station, perhaps offering tours, would help promote the rental-car business. Officials at Kennedy have informed us that they bleed off approximately 1,000 gallons of hydrogen from their LH2 storage tanks each day. While there are no immediate plans to capture this hydrogen for transportation use, they have indicated to us their intention to do a feasibility study of a hydrogen station at the Visitor Center. There is already a CNG station at KSC. 
3. Airport Station. A station built and operated by Chevron Technology Ventures (Boggy Creek Hydrogen Fueling Station) opened at the Orlando International Airport in May, 2007. Initially it will refuel four hydrogen-powered airport shuttle buses (Ford V-10, E-450). Four additional H2 shuttle buses will be in service by 2008. Though located off-site on nearby Boggy Creek Road, rental-car employees could drive the cars there for refueling. Advertising on the hydrogen-powered shuttle buses could help promote the new business ("You're already riding in a hydrogen-powered vehicle - now drive one yourself!").

4. Disney. Disney theme parks (e.g., EPCOT) have always had a futuristic appeal. Disney might be willing to locate a station at one of their parks. In doing so, they could convert their parking lot trams - which are similar to airport baggage trams - to run on hydrogen. A highly visible station site that also offered priority parking to hydrogen vehicles would aid in cross promotion. Development of a hydrogen-powered space ride at one of their theme parks might someday be possible. Alternatively, a station could be located near I-4 on International Drive or EPCOT Center Drive.

5. Climate. Current FCV prototypes reportedly face operational and durability issues in cold weather. The warm Orlando climate is well suited to the large-scale use of an early generation of production vehicles.

6. Used Rental-cars. As mentioned earlier, state vehicle fleets could agree to purchase used rental-cars at a discount to the full cost to meet state ZEV goals. In turn, this guaranteed resale market could remove a significant uncertainty from the rental-car business.

7. Florida Hydrogen Business Partnership. This alliance of 27 companies and government agencies might find the hydrogen rental-car business in Orlando highly appealing as a way to put Florida and Orlando at the forefront of commercialization of hydrogen.

8. The Hydrogen Economy. The renters who completed our survey came from all over the United States and several foreign countries, renting their vehicles for an average of 7.9 days. Some 40-50 individuals a year could rent each hydrogen car, exposing thousands of potential customers to a positive, low-risk experience that could generate positive word-of-mouth and political support for hydrogen power.

\subsection{Conclusions}

1. A fleet of hydrogen-powered rental-cars based at the Orlando International Airport could play a key role in the initial rollout of a hydrogen refueling infrastructure in Central Florida.

2. Consumer attitudes reflect an interesting mix of practical concerns (e.g., driving range of the vehicle) and wider motivations (e.g., using a pollution-free technology) surrounding the decision to rent a hydrogen vehicle.

3. Our survey results suggest that just three refueling stations (located at OIA, downtown Orlando, and Disney) could cover the refueling needs of about $80 \%$ 
of car renters originating from OIA. With a fourth station at Kennedy Space Center, $85 \%$ of car renters could be served. The clustered tourist destinations and climate make Orlando the ideal location for a hydrogen rental-car business.

4. The cost of hydrogen vehicles for a rental fleet represents the most difficult barrier to a feasible rental-car business, but Hertz, Toyota, and New Icelandic Energy have recently shown a willingness to overcome that. Starting off with hybrids converted to burn hydrogen instead of gasoline seemingly can enable the business to begin in the early 2010s or as soon as refueling stations can be built. The publicity generated by the rental business, as well as the opportunity for consumers to experience $\mathrm{H}_{2}$ cars in a low-risk setting, may be an adequate incentive for automakers to subsidize cars to the rental business.

5. Most other hurdles - such as resale of used vehicles, maintenance, and targeting potential markets - should be easier to overcome.

6. Synergies would be plentiful between the rental business and NASA, Disney, energy companies, and other state hydrogen initiatives.

7. The next step for the rental-car business plan is to begin discussions with rental-car companies, as well as energy companies that build stations, and possible partners at Disney and NASA. 


\section{OPTIMAL REFUELING STATION LOCATION ANALYSIS FOR FLORIDA}

The lack of a hydrogen-refueling infrastructure has been pinpointed as the greatest challenge in the transition to a hydrogen economy [3]. Given the high cost of the initial set of refueling stations, locating them as efficiently as possible will be a key to the early success of the industry in Florida. To address this need, we developed a model that locates a given number of stations optimally to serve the maximum consumer demand possible, measured as origin-destination trips, or vehicle-miles traveled [25]. The model, called the Flow Refueling Location Model, takes into consideration the structure of the road network, the routes that people drive regularly from their origins to their destinations, the amount of traffic on those routes, and a reasonable or safe driving range between fill-ups for hydrogen cars. The model then locates the stations optimally and quantifies the tradeoff between the number of stations and how much demand can be served. Operations research techniques and geographic information systems are integrated in a spatial decision support system that researchers can use to develop data, enter assumptions, analyze scenarios, evaluate tradeoffs, and map the results. For the Florida Hydrogen Initiative, we used this model to investigate strategies for introducing an initial refueling infrastructure in Florida at two different scales of analysis: a metropolitan Orlando network, and a statewide network.

\subsection{Prior Research on Refueling Infrastructure Modeling}

In recent years, a large number of academic papers and government reports have addressed the infrastructure needs for the transition to hydrogen energy. While numerous studies have examined the entire supply chain from production to delivery to fueling stations [26], or the total number of initial hydrogen stations needed [4], our focus here is on methods used to solve optimally for the locations of a coordinated network of stations.

The problem of optimally locating refueling stations had attracted little attention in the published literature until the recent push for alternative fuels. In the operations research literature, the lack of earlier published research is likely due to the fact that, by the time the mathematical methods and computer hardware and software needed to address such problems became available, gasoline stations were already ubiquitous in more-developed countries. With the advent of alternative fuels and the need to develop a refueling infrastructure, researchers have recently published several new modeling approaches. We group these approaches into GIS models and operations research models.

Several researchers have been using Geographic Information Systems (GIS) to locate hydrogen-refueling stations. Melaina [27] proposed a GIS method based on the idea of condensing the existing network of gasoline stations into clusters, which yields exact station locations and station sizes. In the NREL study discussed in Section 2.1, Melendez and Milbrandt [13] used GIS to develop a national network of stations to enable longdistance trips on interstate. They selected heavily traveled interstates with over 20,000 vehicles per day, narrowed that set by choosing major north-south and east-west routes, and then subjectively placed stations along those routes using other GIS data layers as 
guides. The other GIS layers included existing hydrogen production plants, other alt-fuel stations, population, and US highway intersections. Stations were placed no more than 50 miles apart in the east and in urban areas, and no more than 100 miles apart in the west.

Although GIS is a powerful tool for integrating detailed spatial data layers, it is not ideal for "combinatorial optimization," in which the model must choose a combination of locations from a large set of candidate sites. Given the astronomical number of combinations that are possible for many real-world problems, many researchers turn to operations research (OR) techniques based on linear programming and heuristic solution. One of the earliest OR papers for locations of gasoline stations was by Goodchild and Noronha [28], who developed a model to open and close gasoline stations to maximize a company's market share. They based market share on two kinds of demand: home-tofacility trips, and traffic volumes on network links. Bapna et al. [29] used multiobjective programming to locate reformulated gasoline stations in India. One objective minimized the sum of travelers' costs and station investment costs, with costs based on the number of stations needed to make travel possible on each link given the driving range of vehicles. The second objective maximized the population on enabled links.

Several papers have used a variant of the $p$-median model —one of the most widely used OR models for facility location. The $p$-median model locates a given number $(p)$ of stations so as to minimize the total distance from residential nodes to their nearest open station [30,31]. Nicholas, Handy, and Sperling [32] used a p-median approach to locate hydrogen-refueling stations in Sacramento County, California. Their results show that when the number of hydrogen stations reaches about $30 \%$ of the number of existing gasoline stations, the average driving time to the nearest station could be similar to today. Nicholas and Ogden [33] used a $p$-median model to examine hydrogen station needs to achieve equivalent levels of convenience in the four major urban areas of California.

Beginning around 1990, researchers have been developing a new approach to facility location, known as "flow capturing" or "flow intercepting" models [34-42]. In flowcapturing location models (FCLM), demand consists of paths through a network instead of points of origin for trips to the facility and back. Flow-capturing models locate facilities conveniently on the origin-to-destination routes that drivers already use on trips they already make. The basic model locates $p$ facilities so as to intercept as many trips as possible. The literature suggests these models are ideal for locating "discretionary" facilities, such as ATMs, convenience stores, and fast food, at which people stop on the way to somewhere else rather than make a special trip from home to facility and back. They have also been applied to billboards and vehicle-inspection stations. In our opinion, the FCLM also provides a realistic behavioral basis for locating alternative-fuel stations.

\subsection{Modeling Approach: The Flow Refueling Location Model and the Spatial Decision Support System}

Kuby and Lim [25, 43-44] modified the basic FCLM for the purpose of locating hydrogen-refueling stations. Modifications were necessary because the FCLM assumes that a single station anywhere on a path is enough to capture that demand. For refueling 
longer trips, however, a single station may not be enough to complete a trip from an origin to a destination and back again. Currently, despite their high efficiencies, prototype hydrogen vehicles have a much shorter driving range than conventional vehicles due to the energy density of on-board storage systems. US DOE has set a target of 300 miles for the technical range of hydrogen vehicles, but the safe practical range may be far less. Thus, many intercity trips will require multiple refuelings at reasonable intervals. To deal with fuel limitations, Kuby and Lim adapted the FCLM by incorporating a driving range parameter that can be set by the user. The resulting Flow-Refueling Location Model (FRLM) is the basis for our analysis.

The FRLM maximizes the number of trips that can potentially be refueled with a given number of stations $p$, and can be stated as a mixed-integer linear programming problem:

$\operatorname{Max} \mathrm{Z}=\sum_{q \in Q} f_{q} y_{q}$

Subject to:

$$
\begin{array}{ll}
\sum_{h \in H} b_{q h} v_{h} \geq y_{q} & \forall q \in Q \\
a_{h k} x_{k} \geq v_{h} & \forall h \in H ; k \in K \\
\sum_{k \in K} x_{k}=p & \\
x_{k}, v_{h}, y_{q} \in\{0,1\} & \forall k, h, q
\end{array}
$$

where:

$$
\begin{aligned}
& q=\text { index of O-D pairs (and, by implication, the shortest paths for each pair) } \\
& Q=\text { set of all O-D pairs } \\
& f_{q}=\text { flow volume on the shortest path between O-D pair } q \\
& y_{q}=1 \text { if } f_{q} \text { is captured } \\
& 0 \text { otherwise } \\
& k=\text { potential facility location } \\
& K=\text { set of all potential facility locations } \\
& x_{k}=1 \text { if a facility is located at } k \\
& 0 \text { otherwise } \\
& p=\text { the number of facilities to be located } \\
& h=\text { index of combinations of facilities } \\
& H=\text { set of all potential facility combinations } \\
& a_{h k}=1 \text { if facility } k \text { is in combination } h \\
& 0 \text { otherwise } \\
& b_{q h}=\quad 1 \text { if facility combination } h \text { can refuel O-D pair } q \\
& 0 \text { otherwise } \\
& v_{h}=1 \text { if all facilities in combination } h \text { are open } \\
& 0 \text { otherwise }
\end{aligned}
$$


The objective function (1) maximizes the flow volume, in number of trips that can be refueled. Constraint (2) prevents a trip from being counted as refueled unless a valid combination of stations $h$ is open that can refuel that path given the safe vehicle range, which is a user input. Constraint (3) prevents a combination of stations $h$ from being open unless all the individual stations $j$ in that combination are open. Constraint (4) limits the number of stations to $p$, a user input. Finally, (5) stipulates that a station or a combination of stations is considered open or not, and likewise that any origin-destination flow can be considered either refuelable or not.

It is important to recognize that our concept of a "safe driving range" is related to, but not equivalent to, station spacing. If stations are spaced, say, 100 miles apart, it still may not be possible to complete some trips without exceeding a 100 mile driving range. For instance, in Figure 4.1, although stations are 100 miles apart on this highway, it is not possible to travel from A to B and back without exceeding a 100-mile limit.

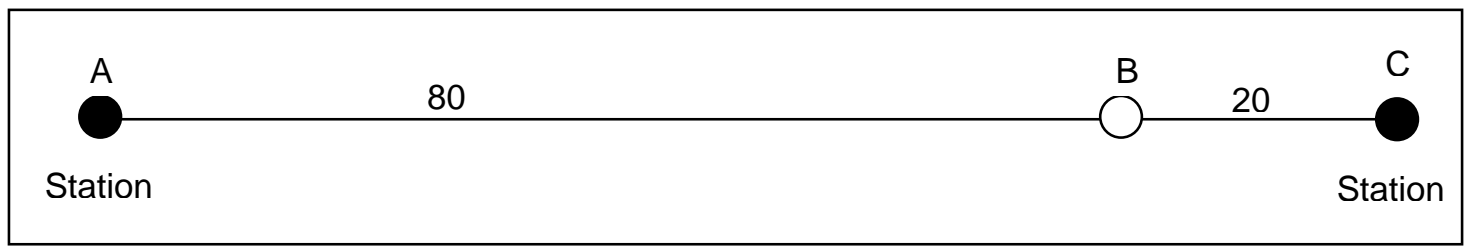

Figure 4.1 Station spacing $\neq$ safe driving range

For the Florida Hydrogen Initiative, we integrated the FRLM into a GIS interface using ArcGIS software and computer programming software Microsoft Visual Studio.net. The resulting Spatial Decision Support System (SDSS) performs three primary tasks:

1. Processing transport network data from GIS sources into a format suitable for the solution algorithms;

2. Solving the FRLM for a given driving range and number of facilities;

3. Displaying the outputs in map and graph form.

Appendix 3 provides additional details about the SDSS.

While linear programming software can solve the FRLM optimally, we developed two heuristic solution algorithms to solve larger, more realistic cases of the model. Heuristic algorithms are able to solve large problems efficiently, although they do not guarantee finding the absolute global mathematical optimum. The first heuristic solution method is the widely used greedy-adding-and-substitution (GAS) algorithm [45]. A greedy algorithm without substitution adds one facility at a time but does not look ahead to where subsequent facilities should be placed. At each iteration, a greedy algorithm places a facility at the site that increases the objective function the most - in this case, the site that refuels the most flow volume above and beyond what the previous facilities were able to refuel. The simple greedy algorithm, which had been used successfully with the FCLM [34], has been shown to be suboptimal for the FRLM [25]. Not looking ahead to where subsequent facilities should go can lead to a suboptimal solution, which the 
substitution algorithm addresses by allowing 1-4 swaps of unused candidate sites for chosen candidate sites. So, for instance, if after locating the $8^{\text {th }}$ facility at the next best site, the $3^{\text {rd }}$ site has become partly redundant, the GAS algorithm could swap another site for it, and then perform several additional substitutions before moving on to the $9^{\text {th }}$ site. The greedy algorithm with four substitutions generated the results presented in this report.

The second algorithm is a genetic algorithm, so-called because it mimics the process of evolution of genes toward a higher fitness level [46]. If the model is used to locate 25 facilities among 500 candidate sites, for instance, each possible solution consists of a string of 25 " $1 \mathrm{~s}$ " and 475 " $0 \mathrm{~s}$ " in some particular order The genetic algorithm creates a population of random solutions, and then mutates, crosses, and evaluates them in a process mimicking evolution towards a solution that is able to refuel a higher flow volume.

Both algorithms allow the user to set certain common parameters (see Appendix 3):

1. A safe working driving range for vehicles (a distance).

2. The number of stations to locate.

3. The objective function to be maximized. Choices include number of trips refueled, vehicle-miles traveled (VMT=trips*distance) refueled, and number of origin-destination pairs refueled. ${ }^{1}$ The VMT objective, first introduced in [47], weights longer trips more heavily than shorter trips, and is useful for maximizing the amount of gasoline potentially replaced by hydrogen. The trips objective, on the other hand, might maximize consumer adoption of hydrogen vehicles, assuming consumers are more likely to purchase a vehicle if they are able to complete more trips with the available stations.

4. The node ID number of any existing stations, or any stations the analyst wishes to force into the solution.

5. For the greedy algorithm, the analyst can set the maximum number of substitutions to perform at each iteration. For the genetic algorithm, the analyst can control a number of evolutionary parameters.

\subsection{Data}

The three universities worked closely together through an elaborate series of steps to build, check, and calibrate detailed and realistic GIS data bases for the Orlando and statewide case studies (Appendix 4). The process was similar for both case studies, with a few exceptions noted. In each case, we first obtained GIS road network data bases. We also obtained a table of trip volumes among more than 4000 traffic analysis zones (TAZs) from FDOT - though this table did not contain long-distance trips for the statewide analysis. We identified and corrected many topology errors in the raw network and simplified the road network by eliminating minor and duplicative streets while retaining needed connectivity. We then processed the remaining road segments into a topological

\footnotetext{
${ }^{1}$ The number of O-D pairs refueled simply sets the $f_{q}$ in the model to 1 for each O-D pair $q$. In the dialog box, this objective is called "unweighted routes." It is useful mainly for debugging purposes, and is not used for any of our results because it counts lightly traveled routes and heavily traveled routes the same.
} 
network consisting of junction nodes and arcs connecting them, which involves substantial editing to split and combine segments as needed and place junction points at intersections. Next we aggregated nearby TAZs together and selected a single origindestination (OD) point to represent each area considering the locations of major intersections and traffic generators. The OD points are a subset of the larger set of road junctions. We also aggregated the trips in the trip table corresponding to the aggregation of the TAZs. These two steps - simplifying the network and aggregating TAZs into OD points - had to be carefully coordinated with each other.

Once the network was built, we generated shortest paths minimizing travel time between each OD pair (see example in Figure 2). We checked many paths one by one to see if they followed realistic routes that Floridians would take. If necessary, to make the paths more realistic, we calibrated the speeds associated with different classes of roads, added missing road links, or changed OD locations. Because the FDOT trip table did not include long-distance intercity trips, such as Orlando to Miami, we had to estimate intercity flow volumes using a gravity model. Finally, we ran the models for Orlando and Florida and checked thoroughly for errors that might have caused the model to locate stations in unrealistic locations. Local knowledge by the three researchers based in Florida, including Dr. Lines in Orlando and Drs. Schultz and Xie in Boca Raton, was invaluable in developing and testing these databases. 


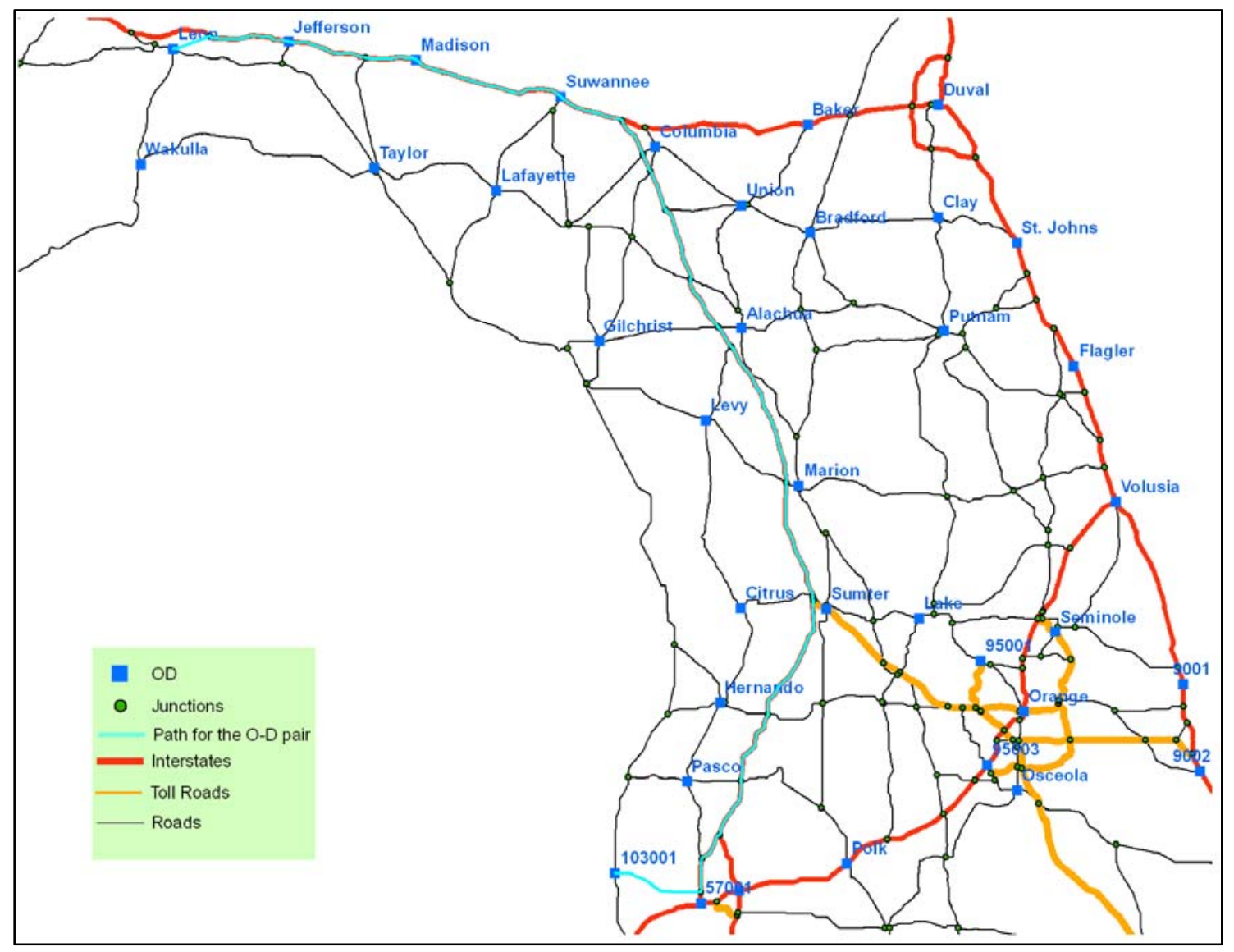

Figure 4.2 Sample path from Tallahassee (Leon County) to Clearwater (103001)

\section{Orlando Data}

The study area includes all of Orange and Seminole counties and the northwest region of Osceola including the cities of Kissimmee and Saint Cloud. We obtained highway networks and maximum speed data from FDOT, and detailed street networks from ESRI, Inc. The FDOT research division assisted us in obtaining TAZ to TAZ trips flows from their travel demand models. FDOT divides the study area into $358 \mathrm{TAZs}$, which we aggregated to 102 larger zones, being careful to ensure contiguity and compactness of the aggregated TAZs. Selection of OD points within each TAZ considered the geometric center of the aggregated TAZ, the spatial relationship between the OD points and the main road network, as well as population density. This resulted in aggregated TAZs that are closer together in denser and more central urban areas. Speeds on arterial streets were reduced from posted speed limits by $15 \%$ to reflect normal driving conditions. Figure 4.3 shows the network used for the Orlando case study. 


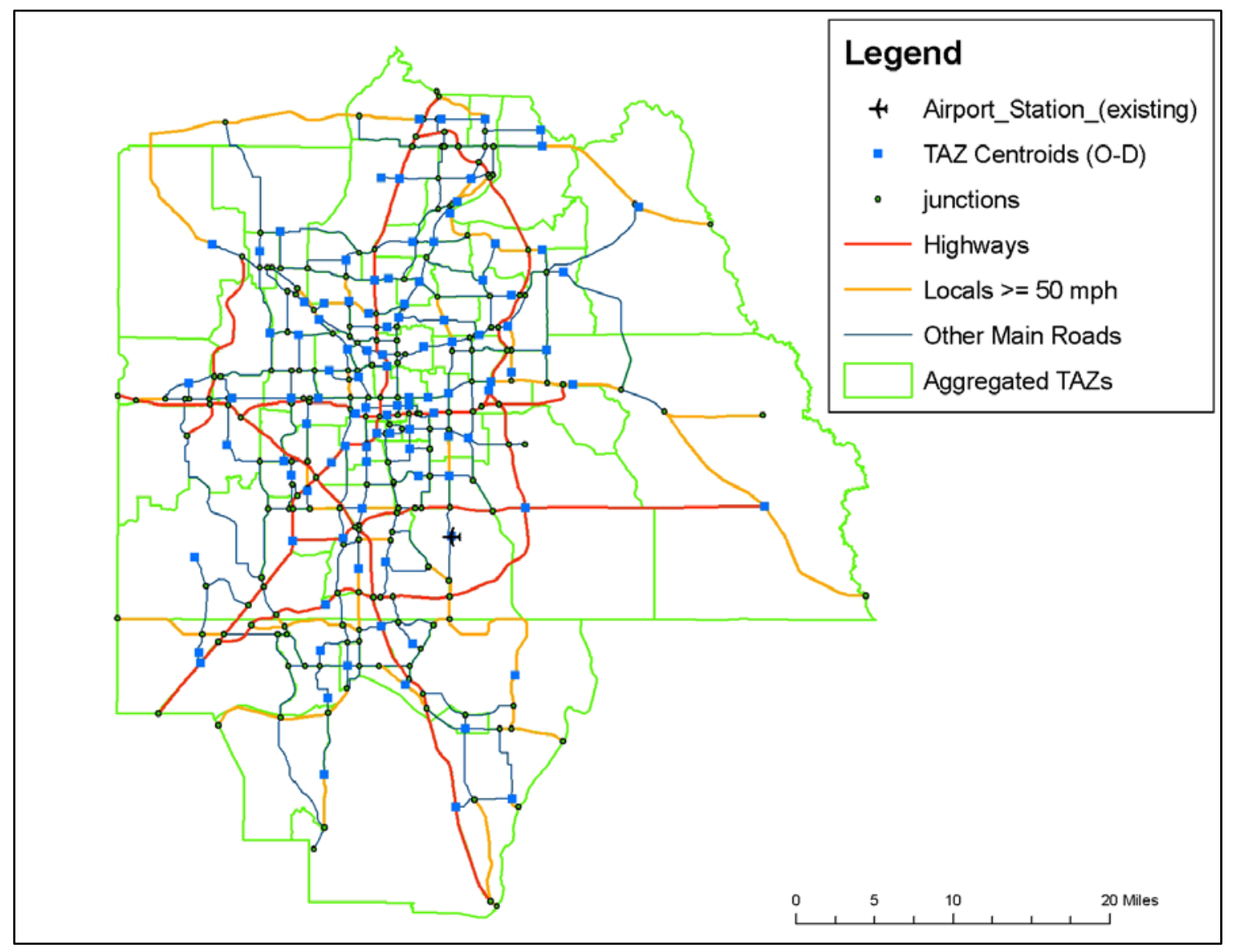

Figure 4.3 Network for the Orlando case study

To create the Orlando trip table, we extracted from the FDOT trip data the trips between 358 original TAZs in the study area. The trips were then aggregated to the 102 aggregated OD points.

Internal flows within each aggregated OD point were excluded from the analysis. These very short but frequent trips would otherwise dominate the trip volumes of the entire network and dictate station locations largely according to how we aggregated the 358 original TAZs into 102 OD points. While intra-zonal trips are sometimes included in transport planning because of their impact on local street congestion, we determined that including them would undermine our purpose of locating hydrogen stations to serve the Orlando metropolitan area.

\section{Florida Data}

For modeling stations for statewide, intercity travel, the study area included the entire state of Florida. Using ESRI and FDOT road networks, we included all interstate highways, toll roads, and US highways, as well as other major state highways used for intercity trips, as shown in Table 4.1. We reduced the speeds on all roads other than 
limited-access highways by $15 \%$ from their maximum speed limits to reflect slower driving conditions.

\section{Table 4.1. State Highways Included in the Statewide Network}

Florida Turnpike (from I-75 near Wildwood to Homestead)

Suncoast Parkway (State Road 589

Sawgrass Expressway

Beeline Expressway (State Road 528)

Central Florida Greeneway (State Road 417)

East-West Expressway (State Road 408)

State Road 429Lee Roy Selmon Expressway (State Road 618)

For modeling intercity trips, the basic level of spatial aggregation was by county. Large urban counties consisting of several distinct urban areas were further subdivided into separate ODs. Disaggregated counties included Dade, Broward, and West Palm in southeast Florida, Lake near Orlando, and Pinellas and Hillsborough in the Tampa-St. Petersburg area. Similarly, several small rural counties were aggregated into a neighboring county, such as Dixie into Gilchrist. In all, the State of Florida was aggregated into 74 ODs (Figure 4.4). 


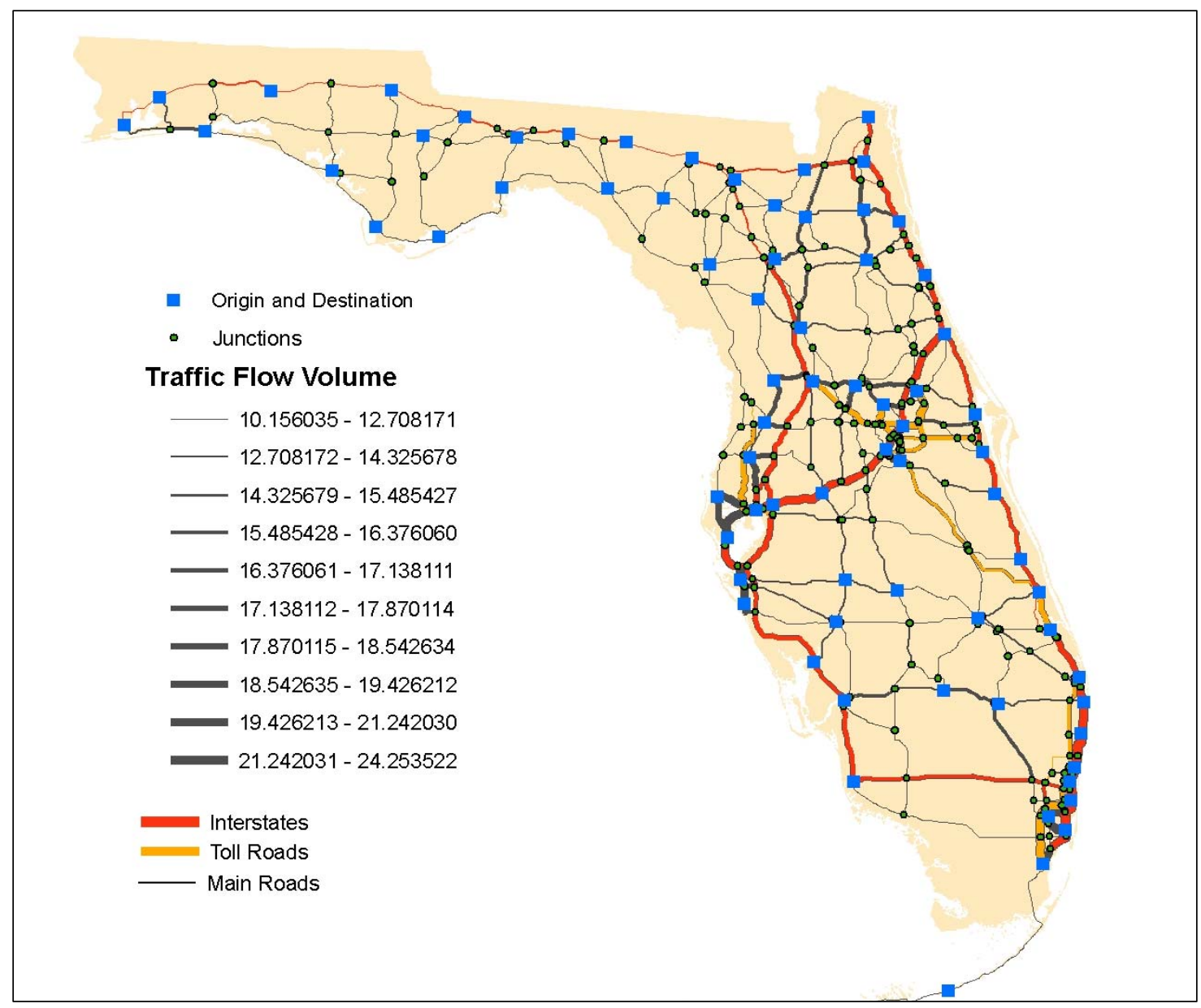

Figure 4.4 Network used for the statewide case study

Only a few US states collect data on long-distance intercity trips and model statewide flows. The FDOT trip data that we used for the Orlando study does not include intercity trips except between nearby cities. We therefore used a spatial interaction or "gravity" model to estimate intercity flows. Spatial interaction models are the most widely used approach in transportation planning to estimate how trips are spatially distributed across a network $[48,49]$. The formula used is shown in equation (6):

$T_{i j}=P_{i}^{*} P_{j} * F F_{i j}$

where:

$T_{i j} \quad=$ number of trips between zone $i$ and $j$

$P_{i}=$ population of zone $i$

$P_{j} \quad=$ population of zone $j$

$F F_{i j}=$ friction function between nodes $i$ and $j$

The shape and steepness of the friction (or impedance) function is a key input to a spatial interaction model. We used a friction function from Michigan's statewide travel forecasting model [50], which is touted by a Federal Highways Administration guidebook as an example of best practice in statewide travel forecasting [51]. We adopted the 
Michigan friction function developed for intercity home-based social-recreational and vacation trips (see Figure 4.5 and equation 7).

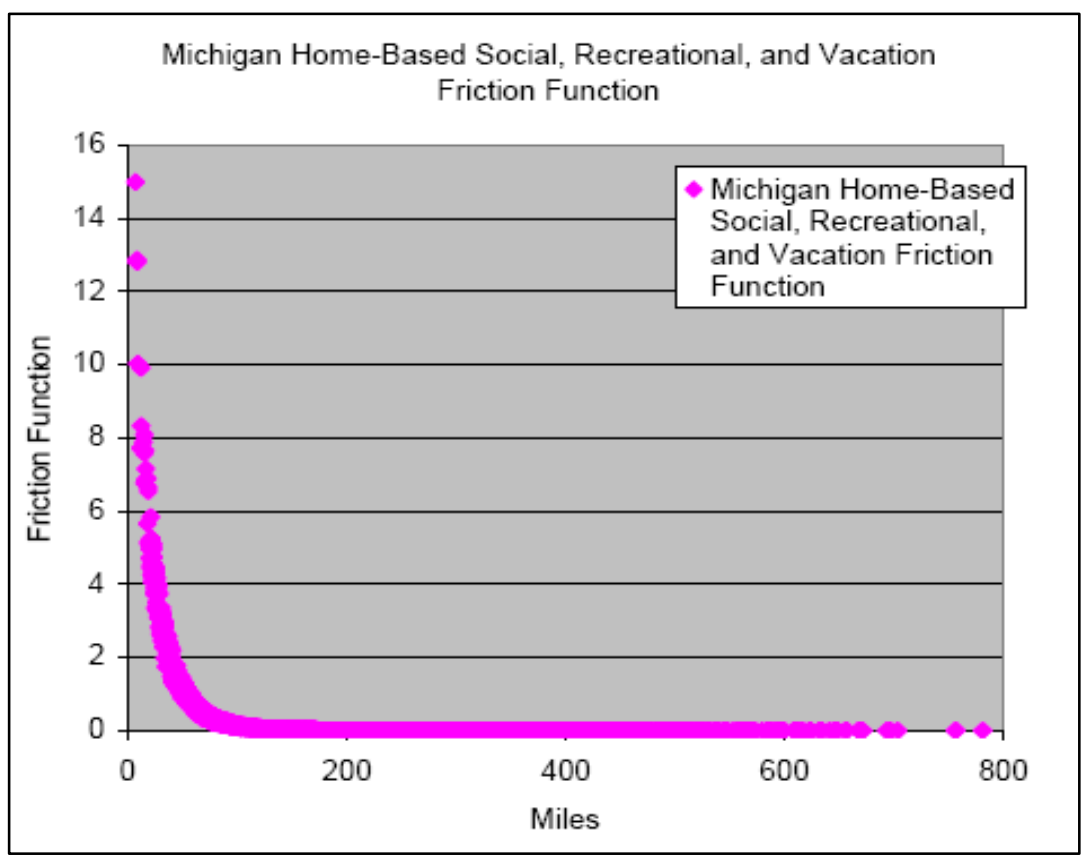

Figure 4.5. Michigan friction function shown as a distance-decay curve

$F F_{i j}=50 * G C_{i j}^{-0.114} * e^{-0.03 * G C_{i j}}$

where:

$G C_{i j}=$ generalized cost between zone $i$ and $j=0.75 *$ miles $+0.5 *$ minutes

To estimate trip volumes, we applied the equations in (6) and (7) to each OD pair, plugging the travel time and length of the shortest pair into the generalized cost formula and the aggregated populations of $i$ and $j$ into (6). The resulting $T_{i j}$ values were then standardized to a percentage of the total. This data set consisting of each OD pair's percentage of the statewide total of intercity trips was used as the trip volumes in the FRLM. Though it is impossible to make a map showing the trip volumes for each of the 2,701 OD paths in the model, the net result of the flows on all these paths can be mapped. Figure 6a aggregates all trip volumes on all paths using each link of the highway network, which can be compared to the actual traffic volume on major Florida roads in Figure 6b. Looking at the rural roads and interstates only-because urban road volumes are inflated by local traffic - the modeled traffic flows on the simplified network appear similar to the actual flows.

This study does not include out-of-state flows because surrounding states may not proceed at the same pace in the transition to a hydrogen economy. Stations justified partly by out-of-state trips could be underutilized if surrounding states transition more slowly. Likewise, trips by Florida residents to neighboring states might not be possible if those states do not develop an infrastructure on their side of the border. Given that out-of- 
state tourists make up a substantial share of the traffic in the Panhandle region, the number of resident trips there are not proportional to the traffic volumes on roads.

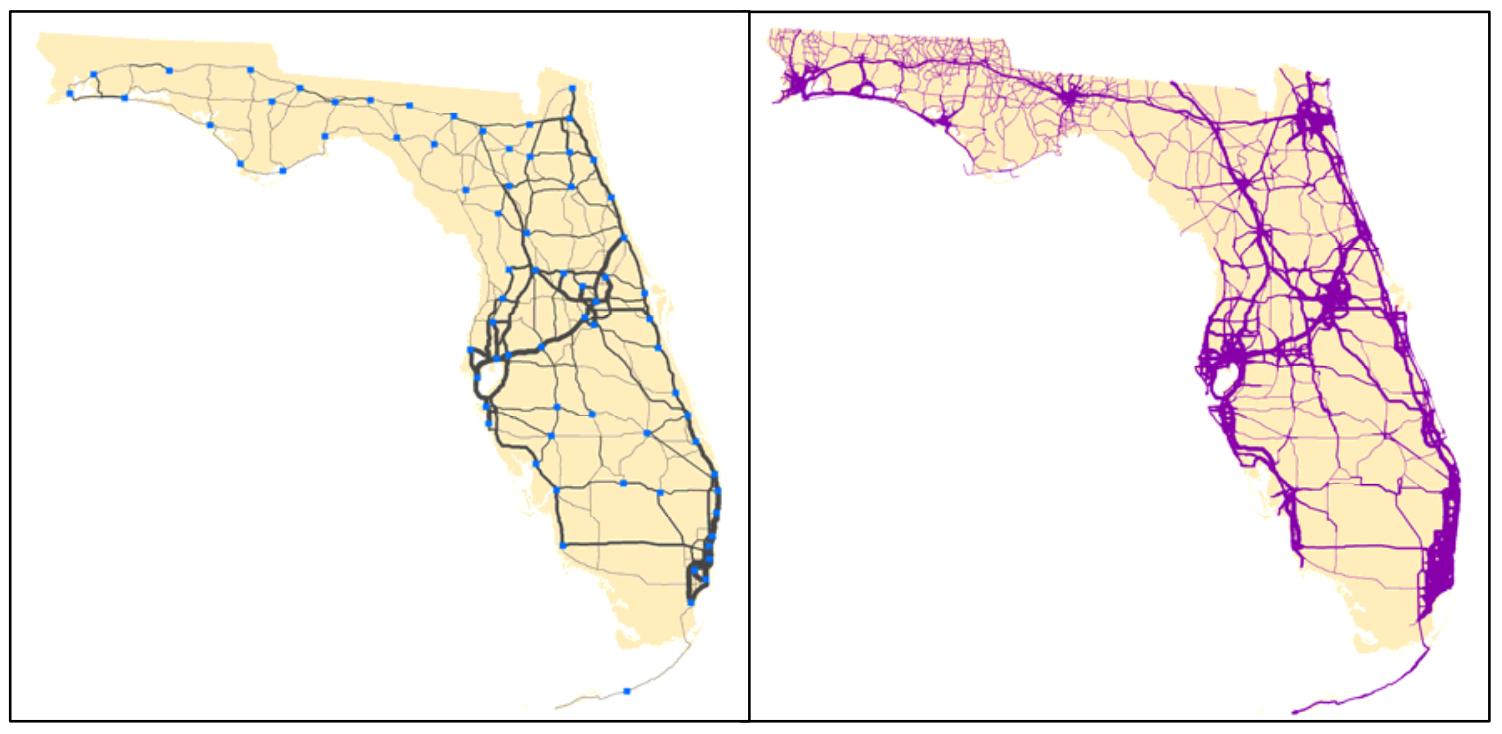

Figure 4.6

(a) Estimated intercity use of the statewide highway network (aggregated from spatial interaction model results). (b) Actual Florida traffic volumes on road segments (FDOT data)

\section{Hydrogen Demand Factor Scenarios}

The base case for both the Orlando and statewide models was based on the total number of trips made by Florida residents. All consumers, however, are not equally likely to purchase hydrogen vehicles in the early stages of commercialization. Therefore, we also ran the model with demand weighted by a hydrogen demand factor.

Our method followed the National Renewable Energy Laboratory (NREL) report on Geographically Based Hydrogen Consumer Demand and Infrastructure Analysis [52], adapted as needed to be consistent with our modeling approach. NREL developed a GIS model that divides the United States into a 20x20 mile grid and classifies each grid square into seven classes ranging from 7 or "very high" to 1 or "low" on various socioeconomic, transportation, and policy variables. Their model predicts higher total demand in areas with higher median income; more people with bachelor's degrees or higher; more two-car households; more workers over 16 years old who commute more than 20 minutes one way; air quality non-attainment (at the county level); participation in Clean Cities coalitions (county level); zero-emission mandates and number of state incentives (state level); and more hybrid vehicle registrations (state level). Scores from 1 to 7 are assigned to different levels of each variable. Each variable was assigned a weight (Table 4.2). For each grid square, the score on each variable was multiplied by the weight and summed across all variables, leading to an overall demand classification for each grid 
square, shown in Figure 4.7. Stakeholders, including car manufacturers and fuel companies, reviewed their choice of variables and the weights on each variable.

To adapt their model for use with our Flow Refueling Location Model in Florida, several changes were required:

1. The NREL model estimates total hydrogen consumer demand in each area. Our model, on the other hand, required a per

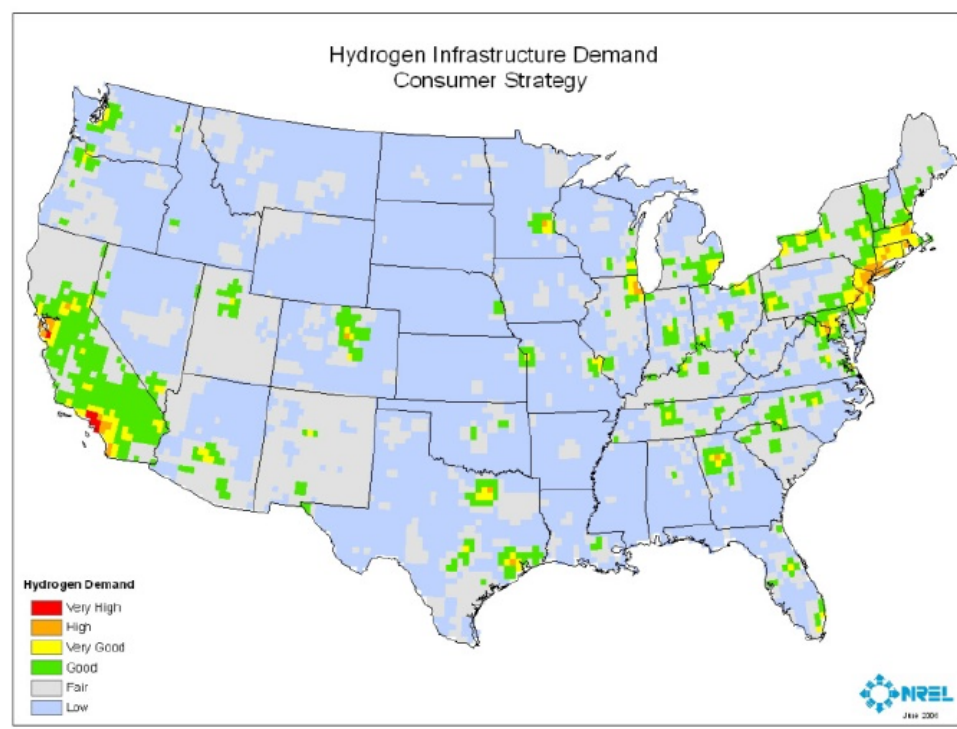

Figure 4.7 Consumer hydrogen demand classes from NREL's GIS model (Source: Melendez and Milbrandt 2006) capita estimate of consumer demand. Our data set already has the total trips from origin zone to destination zone, so what was needed was a method to weight the total number of trips according to the likelihood of individual consumers in the origins and destinations to purchase a hydrogen vehicle. This necessitated changing variables: from total number of people with bachelors degrees to percentage of people with bachelors degrees; from total households with $2+$ vehicles to a percentage of households; and so on.

2. Several variables used by NREL were state-level variables. State incentives, ZEV mandates, and hybrid registration data were by state and therefore did not vary within Florida. In addition, Florida has no counties in non-attainment status for air quality. These variables were dropped and their weights reassigned to other variables.

3. We used an equal-interval classification scheme. We took the range from the high value of each variable to the low value and divided it equally into seven classes. For percentage variables, the top class was extended up to $100 \%$ and the bottom class down to $0 \%$ to cover all possible values.

4. NREL used a 20x20 mile grid for the entire US. Because our study is at a much finer scale, we used census tracts. Using the weights, a score between 1.0 and 7.0 was calculated for each tract.

5. An average score for each aggregated OD zone (consisting of multiple census tracts or even entire counties) was then calculated from the scores of the constituent census tracts using a spatial weighting procedure similar to the NREL procedure. These average scores were not, however, rounded to integer values, but were treated as continuous variables from 1 to 7.

6. For each OD trip, the scores of the origin and destination zones were averaged.

7. The average score from 1 to 7 for the OD pair was then converted linearly to a weighting factor between 0 and 1 . This value was used as a multiplier on the number of trips from the origin to the destination. 
Table 4.2 Hydrogen Consumer Demand Scoring and Weighting System:

NREL Model and FHI Adaptation

\begin{tabular}{|c|c|c|c|c|c|}
\hline $\begin{array}{c}\text { NREL } \\
\text { Data Layer } \\
\text { (weight - \%) }\end{array}$ & $\begin{array}{l}\text { NREL } \\
\text { Classes }\end{array}$ & \begin{tabular}{|l|} 
NREL \\
Rank \\
Score \\
\end{tabular} & $\begin{array}{c}\text { FHI } \\
\text { Data Layer } \\
\text { (weight - \%) } \\
\end{array}$ & $\begin{array}{c}\text { FHI } \\
\text { Classes }\end{array}$ & $\begin{array}{l}\text { FHI } \\
\text { Rank } \\
\text { Score }\end{array}$ \\
\hline \multirow{7}{*}{$\begin{array}{c}\text { Median } \\
\text { Household } \\
\text { Income } \\
\text { (High - 15\%) }\end{array}$} & $54,955-86,901$ & 7 & \multirow{7}{*}{$\begin{array}{c}\text { Median } \\
\text { Household } \\
\text { Income } \\
\text { (High - 23\%) }\end{array}$} & $172,515-200,001$ & 7 \\
\hline & $43,109-54,954$ & 6 & & $145,029-172,514$ & 6 \\
\hline & $36,152-43,108$ & 5 & & $117,542-145,028$ & 5 \\
\hline & $30,673-36,151$ & 4 & & $90,056-117,541$ & 4 \\
\hline & $24,748-30,672$ & 3 & & $62,569-90,055$ & 3 \\
\hline & $15,405-24,747$ & 2 & & $35,083-62,568$ & 2 \\
\hline & $0-15,404$ & 1 & & $0-35,082$ & 1 \\
\hline \multirow{7}{*}{$\begin{array}{c}\text { Number } \\
\text { of people with } \\
\text { bachelor's degrees } \\
\text { (Medium }-10 \%)\end{array}$} & $943,877-1,770,650$ & 7 & \multirow{7}{*}{$\begin{array}{c}\text { Percentage } \\
\text { of people with } \\
\text { bachelor's degrees } \\
(\text { Medium }-18 \%)\end{array}$} & $75.7-100$ & 7 \\
\hline & $415,521-943,876$ & 7 & & $63.1-75.6$ & 6 \\
\hline & $228,465-415,520$ & 6 & & $50.5-63.0$ & 5 \\
\hline & $123,779-228,464$ & 5 & & $38.0-50.4$ & 4 \\
\hline & $51,563-123,778$ & 4 & & $25.5-37.9$ & 3 \\
\hline & $14,107-51,562$ & 3 & & $12.84-25.4$ & 2 \\
\hline & $0-14,106$ & 2 & & $0-12.83$ & 1 \\
\hline \multirow{7}{*}{$\begin{array}{c}\text { Number } \\
\text { of workers } \\
\text { age } 16+ \\
\text { who commute more } \\
\text { than } 20 \text { minutes } \\
\text { (Medium }-10 \% \text { ) }\end{array}$} & $908,659-1,572,668$ & 7 & \multirow{7}{*}{$\begin{array}{c}\text { Percentage } \\
\text { of workers } \\
\text { age } 16+ \\
\text { who commute more } \\
\text { than } 20 \text { minutes } \\
\text { (Medium }-18 \% \text { ) }\end{array}$} & $78.6-100$ & 7 \\
\hline & $418,740-908,658$ & 7 & & $66.3-78.5$ & 6 \\
\hline & $219,920-418,739$ & 6 & & $53.9-66.2$ & 5 \\
\hline & $109,577-219,919$ & 5 & & $41.5-53.8$ & 4 \\
\hline & $47,249-109,576$ & 4 & & $29.1-41.4$ & 3 \\
\hline & $12,529-47,248$ & 3 & & $16.8-29.0$ & 2 \\
\hline & $0-12,528$ & 2 & & $0-16.7$ & 1 \\
\hline \multirow{7}{*}{$\begin{array}{l}\text { Number of } \\
\text { Households with } \\
2+\text { Vehicles } \\
\text { (High }-15 \%)\end{array}$} & $179,419-312,470$ & 7 & \multirow{7}{*}{$\begin{array}{c}\text { Percentage of } \\
\text { Households with } \\
2+\text { Vehicles } \\
(\text { High }-23 \%)\end{array}$} & $80.8-100$ & 7 \\
\hline & $312,471-516,079$ & 7 & & $68.0-80.7$ & 6 \\
\hline & $118,941-179,418$ & 6 & & $55.2-67.9$ & 5 \\
\hline & $68,543-118,940$ & 5 & & $42.4-55.1$ & 4 \\
\hline & $30,240-68,542$ & 4 & & $29.6-42.3$ & 3 \\
\hline & $8,065-30,239$ & 3 & & $16.6-29.5$ & 2 \\
\hline & $0-8,064$ & 2 & & $0-16.5$ & 1 \\
\hline \multirow{2}{*}{$\begin{array}{c}\text { Clean Cities } \\
\text { Coalitions, by } \\
\text { county } \\
(\text { Medium }-10 \%) \\
\end{array}$} & Yes & 7 & \multirow{2}{*}{$\begin{array}{c}\text { Clean Cities } \\
\text { Coalitions, by } \\
\text { county } \\
(\text { Medium }-18 \%) \\
\end{array}$} & Yes & 7 \\
\hline & No & 1 & & No & 1 \\
\hline \multirow{4}{*}{$\begin{array}{c}\text { Air Quality } \\
\text { (Medium - 10\%) }\end{array}$} & Severe & 7 & \multirow{4}{*}{ Not applicable } & & \\
\hline & Moderate & 6 & & & \\
\hline & Marginal & 5 & & & \\
\hline & None & 1 & & & \\
\hline \multirow{2}{*}{$\begin{array}{l}\text { State Incentives } \\
\text { (Medium-10\%) }\end{array}$} & Yes & $5-7$ & \multirow{2}{*}{ Not applicable } & & \\
\hline & None & 1 & & & \\
\hline \multirow{2}{*}{$\begin{array}{c}\text { ZEV Sales } \\
\text { Mandate } \\
\text { (Medium - 10\%) }\end{array}$} & Yes & 7 & \multirow[b]{2}{*}{ Not applicable } & & \\
\hline & No & 1 & & & \\
\hline \multirow{7}{*}{$\begin{array}{l}\text { Registered Hybrid } \\
\text { Vehicles, by state } \\
\text { (Medium - 10\%) }\end{array}$} & $1,551-2,875$ & 7 & \multirow{7}{*}{ Not applicable } & & \\
\hline & $686-1,550$ & 6 & & & \\
\hline & $372-685$ & 5 & & & \\
\hline & $169-371$ & 4 & & & \\
\hline & $68-168$ & 3 & & & \\
\hline & $12-67$ & 2 & & & \\
\hline & $0-11$ & 1 & & & \\
\hline
\end{tabular}


Consumer hydrogen demand scores were calculated for every OD zone in both the statewide network and the Orlando network. The multiplier between 0 and 1 should not be interpreted literally as the percentage of consumers who will buy hydrogen vehicles in the early commercialization stage. Rather, the multipliers should be interpreted in relative terms. For instance, suppose that a given OD pair has demographic and policy variables that give it an average score of 5 on a scale from 1 to 7 , which translates to a 0.67 multiplier. Meanwhile, another OD pair has a score of 2 because of some combination of lower income and education, shorter commutes, less car ownership, or not being part of a Clean Cities Coalition. The multiplier for the second OD pair would be 0.167 . The interpretation of these two multipliers should be that four times as many consumers are likely to adopt hydrogen vehicles for trips between the first OD pair than for the second OD pair. Demand scores for areas in Florida are shown in Figure 4.8.

As the NREL authors admit, "there is no single best data classification method" [52, p4]. Likewise, different experts would assign weights differently. Our consumer demand weighted scenarios are not meant to be a definitive approach to estimating future hydrogen demand geographically. Rather, they represent one possible and relatively straightforward approach to accounting for the obvious fact that all consumers are not equally likely to adopt hydrogen technology. The main purpose of analyzing scenarios based on these trip multipliers is to determine how sensitive the optimal station locations are to assumptions about the hydrogen vehicle trip volumes between places.

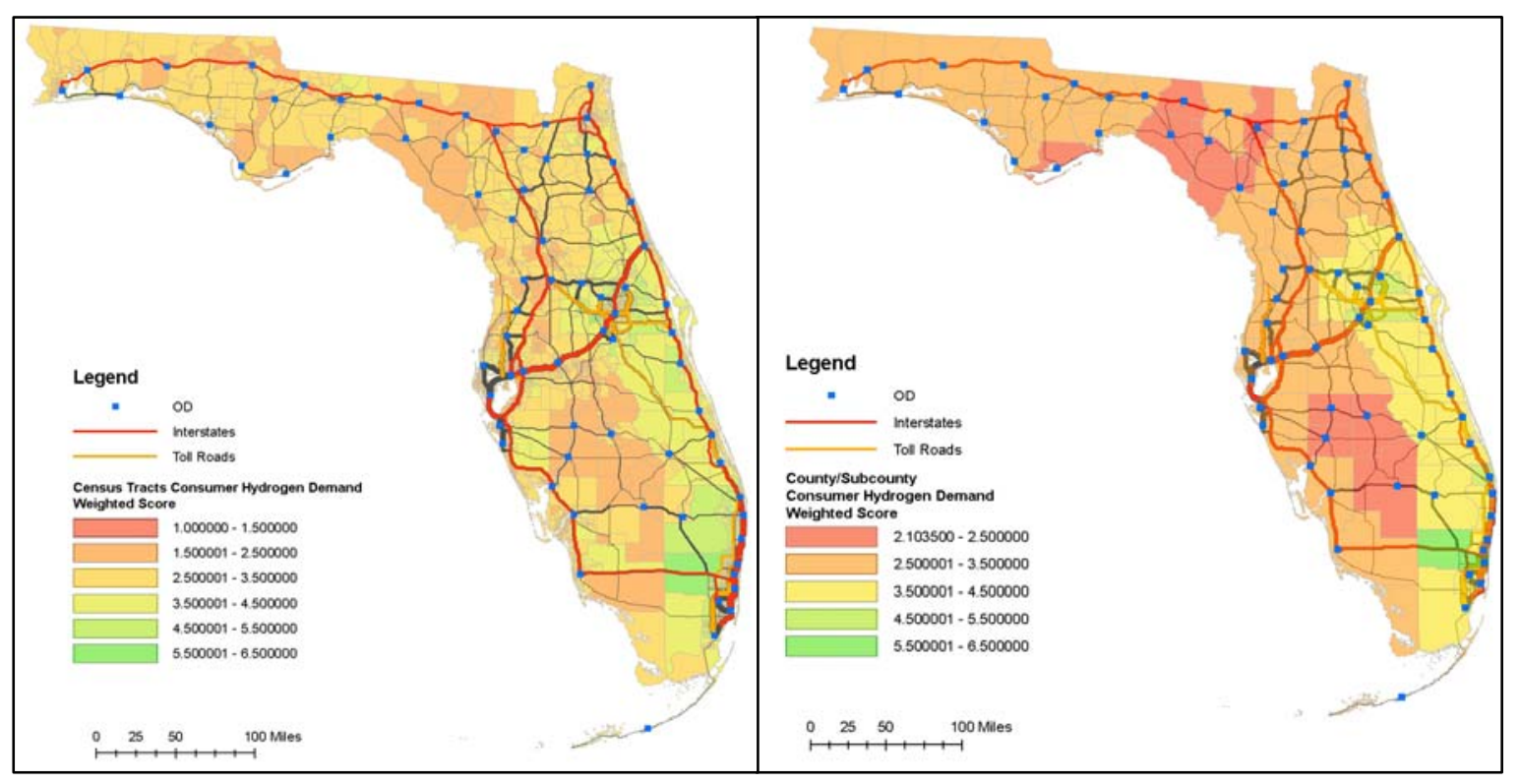

Figure 4.8 Hydrogen consumer demand scoring and weighting results at the (a) census tract level and (b) county level 


\subsection{Florida Statewide Station Location Analysis}

The base case scenario for the Florida statewide analysis uses raw unweighted trip volumes and 100 miles as a reasonable "safe" vehicle range. This number represents a distance drivers would feel comfortable traveling between stations on a round trip, rather than the technological maximum of the vehicle. The technological maximum is substantially higher: prototype vehicles are already being road-tested or planned with driving ranges between 180 and 300 miles (Table 2.1). The National Research Council Report on The Hydrogen Economy [2] assumes a 300-mile driving range for their infrastructure scenarios. The original California Hydrogen Highway plan was to space stations every 20 miles along interstates, though their current thinking about station spacing is closer to 50 miles. The NREL national hydrogen network analysis spaces stations 50 miles apart in the eastern US and urban areas, and 100 miles apart west of the Mississippi River [13]. NREL assumed 50 mile spacing in the east, however, because interstates in the east are "used extensively for short trips."

We adopt NREL's 100-mile assumption as a safe driving range of 100 miles that allows for a substantial margin of driver error, suboptimal performance, improper filling, side trips, detours, and stations that may be closed or inoperative. With our model, however, we do not have to assume NREL's more conservative 50-mile spacing as a proxy for capturing shorter trips, because we actually have trip data for both short and long intercity trips. If closer station spacing is justified by capturing the shorter trips that would otherwise fall between stations, then the model will space them more closely. In other words, the trips dictate the station spacing, with the assumed safe driving range of 100 miles being strictly a maximum distance between refuelings on each individual trip.

We also ran scenarios assuming a 50-mile and 75-mile working range. Any distance can be input into the model.

Base Case and Illustration of How the Model Works: 100 Mile Vehicle Range and Maximizing Unweighted Trips

Figure 4.9 shows optimal locations for five stations in Florida, and the routes they can refuel. The model locates four stations in SE Florida and one in Tampa. These five stations are capable of refueling $62 \%$ of the estimated intercity trips in Florida. Clustering stations in the greater Miami-Palm Beach area is a smart strategy for serving the largest number of intercity trips

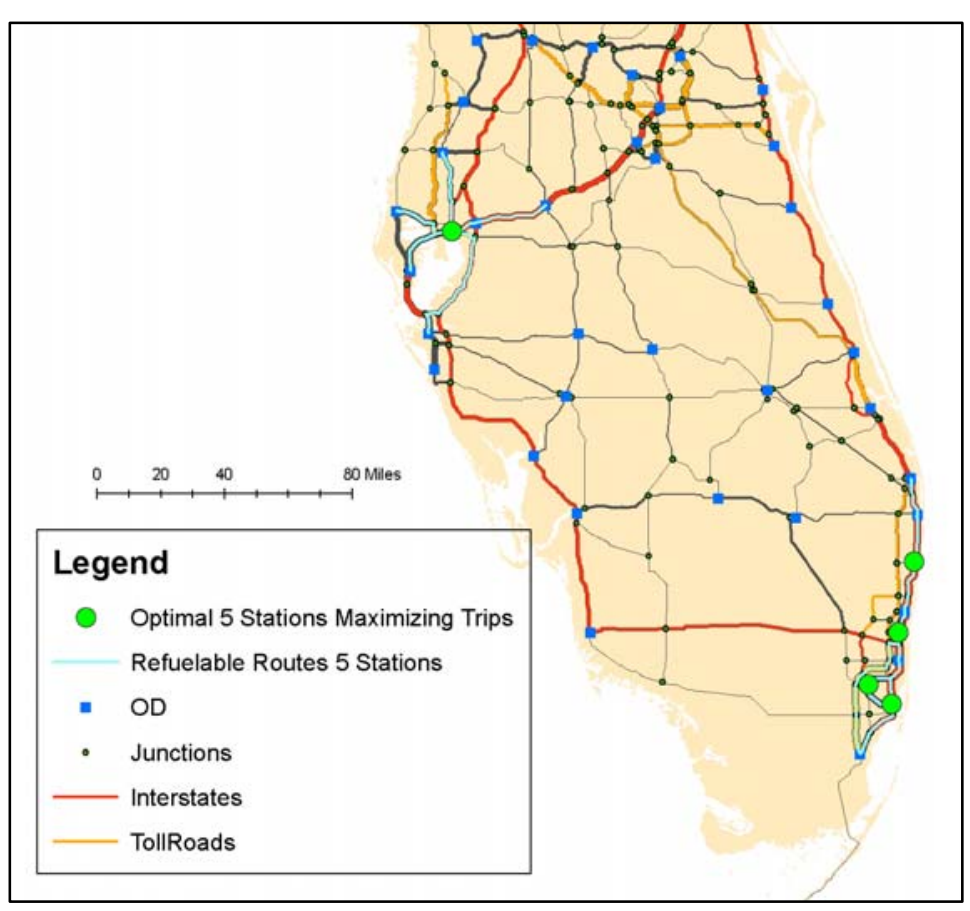

Figure 4.9 Optimal 5 stations maximizing trips with 100-mile working range 
with a few stations. The large number of intercity trips in this area of 5.4 million people is the result of large population nodes with short distances between them. (Keep in mind that trip volumes were estimated by a gravity model, and that the number of trips is proportional to the product of the origin and destination populations and inversely proportional to the distance between them). In addition, the linear arrangement of cities along the coast means that most intercity trips travel north and south on the main highways. Furthermore, by stringing the stations along the coast, the arrangement of these four stations allows trips to be made that are longer than 100 miles round trip. Outside of this cluster, the other station, in Tampa, facilitates round trips between the area's largest city and some large surrounding cities such as St. Petersburg, Lakeland, Sarasota, and Clearwater.

The optimal system of ten stations for maximizing unweighted trips includes two major clusters: a linear one in SE Florida and another connecting the Tampa-St. Petersburg (2.6 million people) and Orlando (1.9 million) metropolitan areas (Figure 4.10). Each cluster now has five stations, and the stations that were optimal with only five stations remain optimal with ten. These ten stations are able to refuel $77 \%$ of the intercity trips in Florida.

With 15 stations, the model adds three stations to the OrlandoTampa cluster, one station to southeast Florida, and one in

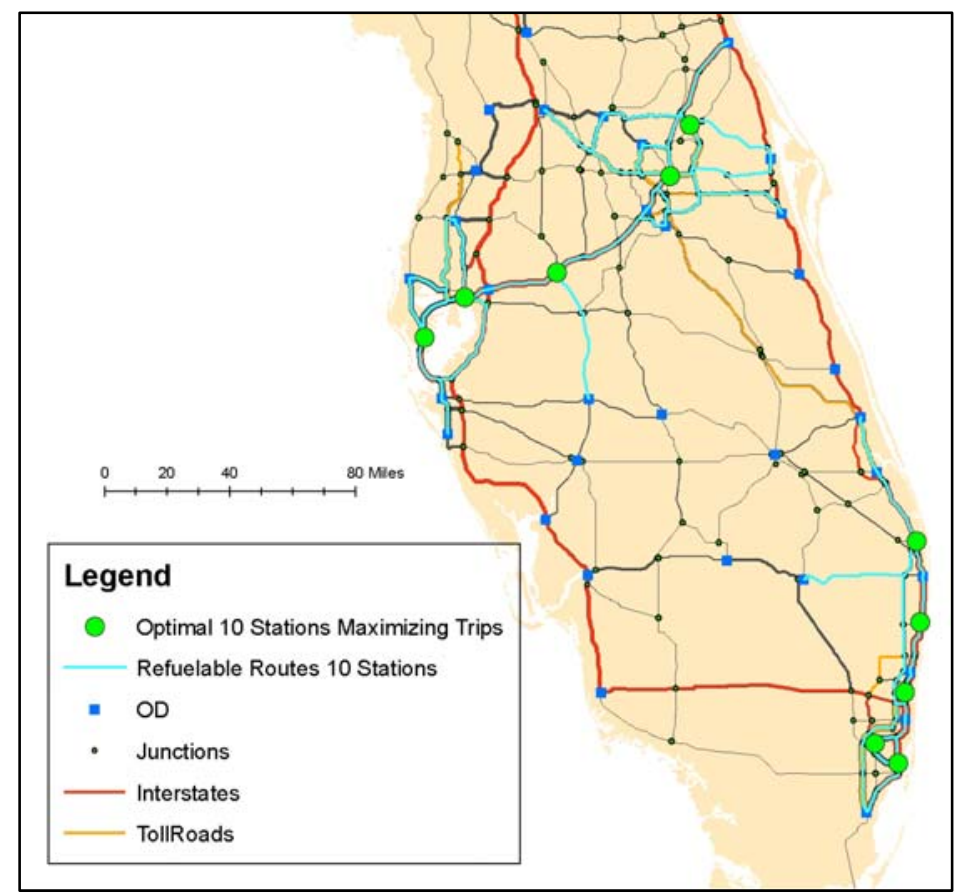

Figure 4.10 Optimal 10 stations maximizing trips with 100-mile working range

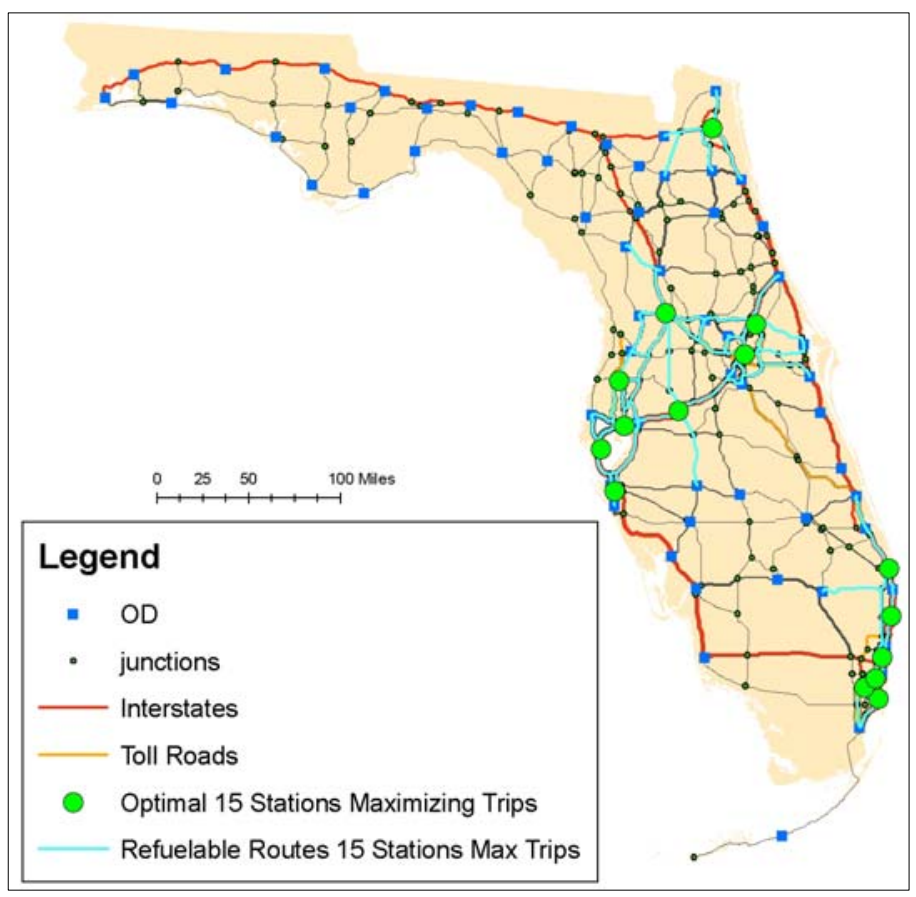

Figure 4.11 Optimal 15 stations maximizing trips with 100-mile working range 
Jacksonville (population 1.2 million). The stations that were optimal within the first five and the first ten remain optimal with 15 , which adds confidence to these recommendations. As we saw before in the case of Tampa, the single station in downtown Jacksonville potentially enables trips from central Jacksonville to the five surrounding counties. These 15 stations refuel $84 \%$ of intercity trips (Figure 4.11).

A new strategy emerges when expanding the network to 20 stations (Figure 4.12). The model begins adding some connecting or bridging stations. Stations are added on I-95 at Daytona, Cocoa Beach, and Vero Beach, facilitating north-south trips among nearly every pair of cities from Coral Gables in the south almost to the Georgia border in the north. Despite enabling trips up and down the east and further down the west coast, these five stations only increase the percentage of refuelable intercity trips from $84 \%$ to $89 \%$, because Florida residents make far fewer long-distance trips than shortdistance trips.

With 25 stations, the model adds stations as far north as Gainesville, home to the University of Florida, and adds to the clusters in the Orlando, Tampa, and Miami areas (Figure 4.13). One noteworthy aspect of this solution is that it serves neither the Florida Panhandle nor trips from Miami to Tampa or Orlando. Despite ignoring these trips, these five stations serve almost $93 \%$ of estimated intercity trips. Strictly from the point of

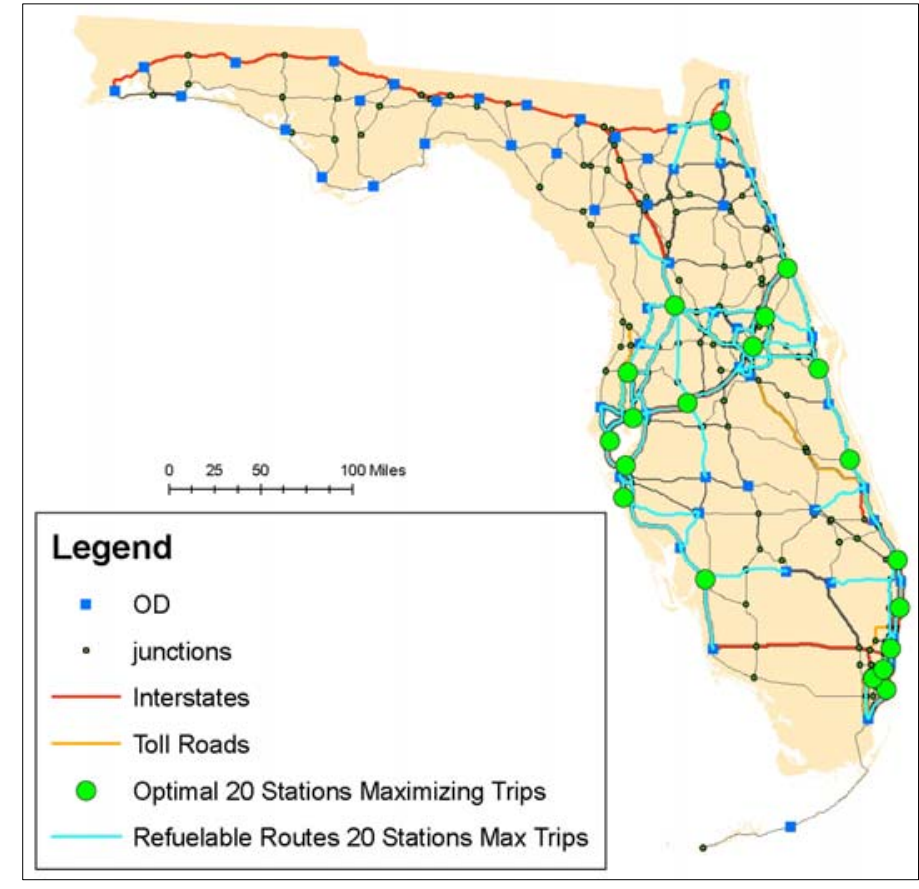

Figure 4.12 Optimal 20 stations maximizing trips with 100-mile working range

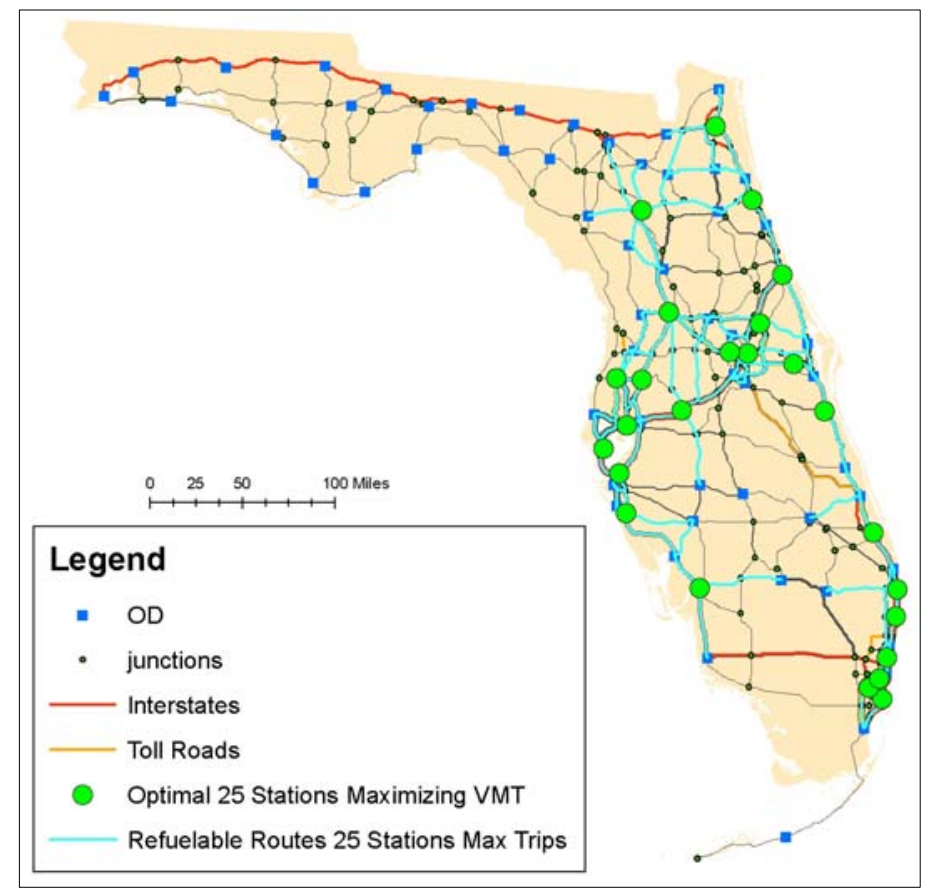

Figure 4.13 Optimal 25 stations maximizing trips with 100-mile working range 
view of maximizing the number of intercity trips that can be served with 25 stations, locating stations in the Panhandle or the Everglades simply does not pay off. It is worth pointing out, however, that other factors may justify stations there.

Note also that some stations that were optimal with 20 stations are no longer optimal with 25. For instance, the station that was near Cocoa Beach east of Orlando shifts off of I-95 to the junction point in the "Y" shape. This station is now able to serve trips from Orlando to both Cocoa Beach and Cape Kennedy. This shift is made possible by the addition of a station on I-95 south of Cocoa Beach near Melbourne. From Melbourne it is less than a 100-mile trip north to Daytona, so the ability to drive from one end of Florida to the other on I-95 is not compromised.

The base case scenario has been presented in some detail above to demonstrate how the model locates stations. Next, scenarios based on other assumptions are summarized in less detail.

\section{Maximizing VMT}

In this scenario, the model maximizes vehicle-miles traveled (VMT) instead of the number of trips. This VMT objective multiplies the number of trips on each shortest path by its distance in order to maximize the gasoline consumption potentially replaced by hydrogen. The results are surprisingly similar (Figure 4.14). Twelve of the locations that were optimal for maximizing trips remain optimal for maximizing VMT, and numerous others shift only slightly. There continue to be clusters of stations around Miami, Orlando, and Tampa, as well as a station in Jacksonville. The priority order, however, is different, with one of first five stations shifted to Orlando. The Orlando station enables ODs within 50 miles of Orlando to complete trips, and also makes the 85-mile Orlando-Tampa trip possible. Despite the increased emphasis on serving longer trips, there would still not be enough VMT served in the Panhandle to justify placement of scarce refueling resources in that region.

Two examples highlight the differences in the max-trips

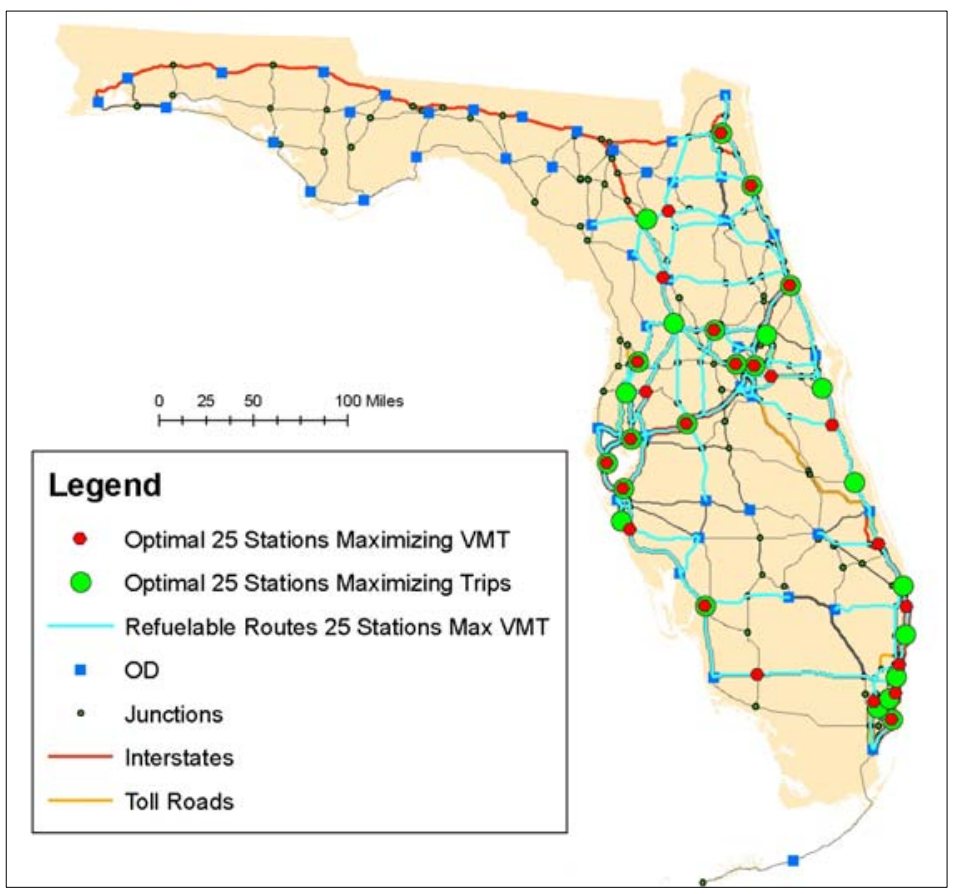

Figure 4.14 Comparison of optimal networks of 25 stations maximizing VMT and trips, for a 100-mile working range and max-VMT solutions. 
When maximizing VMT, the model locates a station on I-75 crossing the Everglades, because of the higher weight placed on the long-distance trips between the Miami and Tampa-St. Petersburg conurbations. To support this strategy, several stations in the Miami area shift location slightly in order to be at key junctions where trips funnel towards I-75 while still being able to serve short intercity trips within southeast Florida. The second notable change is that the station in Gainesville in the max-trips scenario shifts eastward onto US-301, the fastest route between Jacksonville and Tampa. While Gainesville has a larger population than this node, a station in Gainesville would serve primarily shorter trips, whereas the station on US-301 facilitates fewer but longer trips.

\section{Shorter Vehicle Range}

Figure 4.15 presents results for 25 stations maximizing trips, but assuming working ranges of 50 miles and 75 miles instead of 100 miles. The shorter range provides a larger margin for error in case of stations being inoperative, drivers refueling improperly, or drivers taking side trips. With a range of 75 miles, more stations are needed to link Miami with Jacksonville via I-95 because of the closer spacing required. The first ten stations continue to be placed in the Miami-Palm Beach region and the Tampa-Orlando I-4 corridor, and the network does not reach as far north as Gainesville. With a 50-mile range, the optimal network of 25 stations breaks up into 3 independent clusters. Stations are clustered even closer together because of the shorter range, and as a result, there are not enough

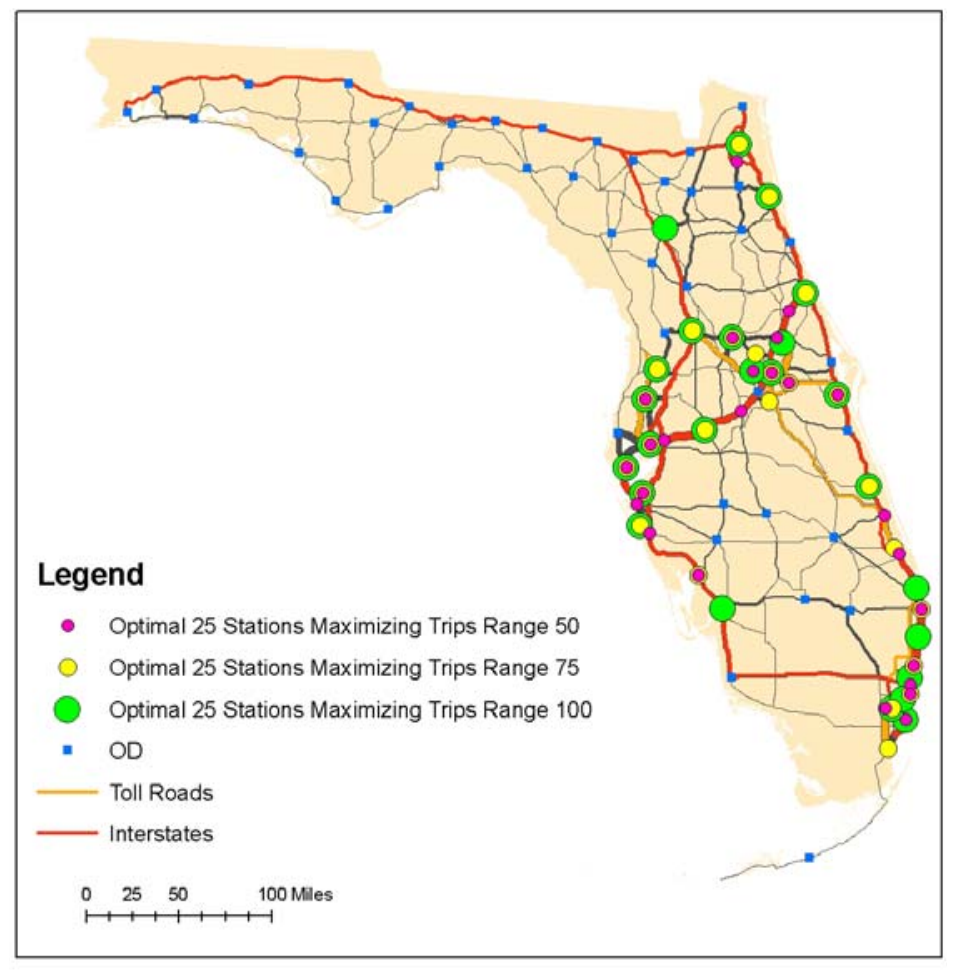

Figure 4.15 Comparison of optimal networks of 25 stations for different vehicle ranges stations to provide the linkages between the Miami-Palm Beach cluster, the Tampa-Orlando cluster, and the solo station in Jacksonville.

\section{Tradeoff Curves}

Figure 4.16 provides tradeoff curves for both the max-trips and max-VMT objectives for assumed safe driving ranges of 75 and 100 miles. First, as expected, all curves show a general pattern of diminishing marginal returns. Each subsequent station tends to add 
fewer trips than the previous station, as the best locations are used up. Previous research $[25,43]$ has shown, however, that returns are not strictly diminishing, because sometimes it takes two additional stations to be able to refuel a well-traveled but longer trip. Second, the curves for a 75 -mile range are lower than the curves for a 100-mile range. This is simply the result of the need for more stations spaced closer together to serve the same long-distance round trips. Third, a given number of stations can generally refuel a higher percentage of trips than VMT. A greater percentage of the total VMT consists of very long trips between smaller origins and destinations, which require more stations to serve.

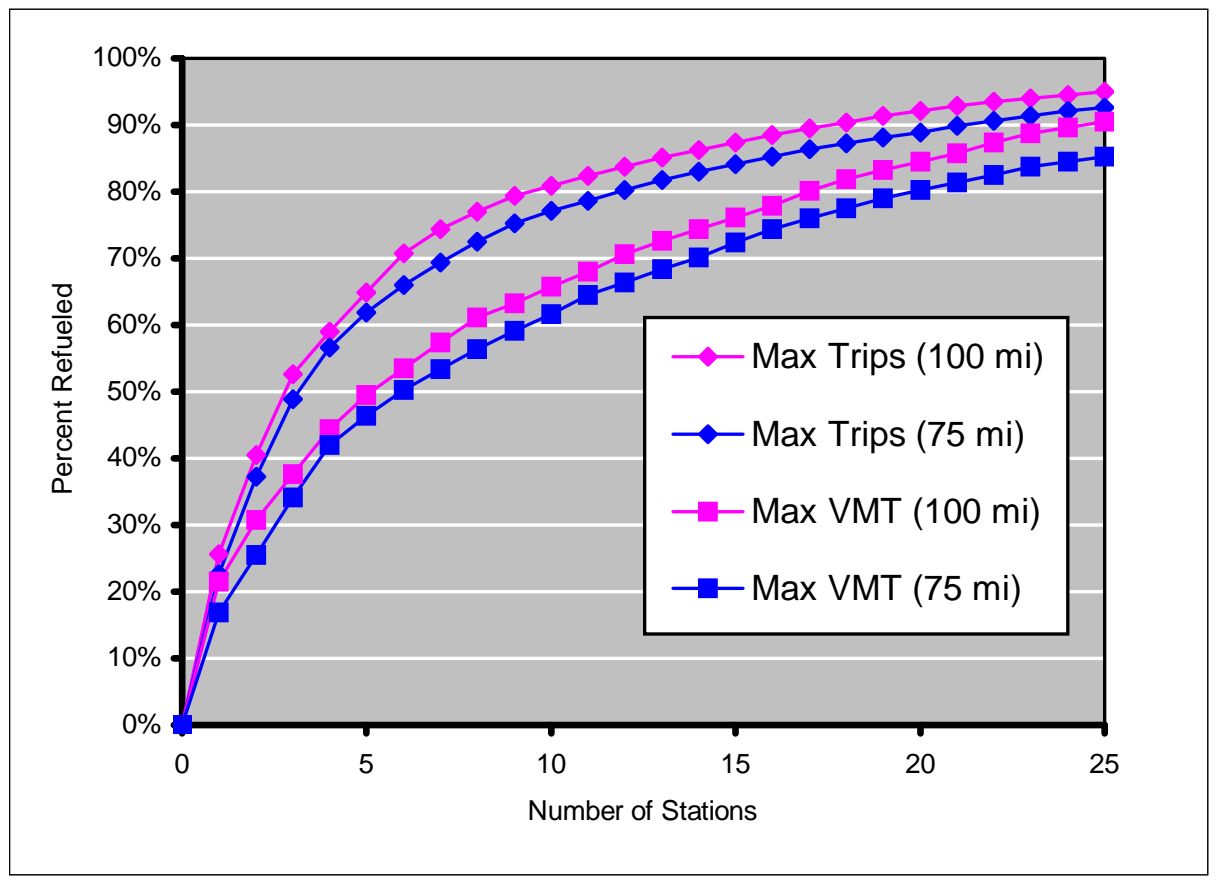

Figure 4.16 Tradeoff between number of stations and percent of demand able to be refueled for the statewide network

\section{Other Factors: Rental-cars, State Government, and Scientific Research}

The station networks in the previous scenarios were optimized with no a priori assumptions about any particular station being part of the network. There may be certain locations that should be included for tourism, political, or scientific reasons even though the model, based on intercity estimated trip demand, does not select them. To begin with, we found in the rental-car section of this study that the bundles of trips made by approximately $80 \%$ of car renters at the Orlando International Airport could be serviced by three hydrogen stations: at OIA, downtown Orlando, and the theme parks. Of these, only downtown Orlando was consistently chosen by the model based on intercity trips. Many trips to the theme parks are intra-city trips that are not modeled in the statewide network. In addition, OIA was not modeled as a destination for intercity trips based on the gravity model. Yet these stations play a key role in the rental-car business model. 
Likewise, no scenarios with 25 or fewer stations ever chose Tallahassee based on the intercity trips to, from, or through it. A station in Tallahassee, however, may be justified on other legitimate grounds. As the home of Florida State University, a station in Tallahassee may be important for scientific research and environmental education. Similarly, as the state capital, a station could be important for demonstrating hydrogen technology and for fueling governmental fleets. Finally, a station in Tallahassee may be justified by the intra-city trips of the highly educated workforce there. Similar considerations point to a station in Gainesville near the University of Florida near I-75. For this reason, we ran some scenarios in which these five stations are forced into the solution, and the rest of the station network was optimized around these five fixed points.

Forcing in these five stations involves a large sacrifice in terms of the number of intercity trips served - but only in the early stages of infrastructure development. As Figure 4.17 shows, the three rental-car stations in the Orlando area and the Gainesville/Tallahassee stations can serve only $7 \%$ of intercity trips estimated by the gravity model for our Florida statewide trip table, versus $65 \%$ for the first five stations optimally located in southeast Florida and Tampa. While this appears at first to be a costly tradeoff, it is important to keep several things in mind. First, one must consider the difference between potential trips and actual trips. A small network of stations in southeast Florida can potentially refuel a large number of intercity trips, but until consumers start buying hydrogen cars, the potential will remain unrealized. In contrast, the stations situated to serve a hydrogen rental-car fleet have a built-in source of demand. Likewise, the Tallahassee and Gainesville stations may serve very little intercity demand but might be heavily used by early adopters, such as professors and scientists, as well as university and government fleets making local trips not included in this intercity model. Second, none of these five required stations are in the Miami area, where the number of intercity trips is highest.

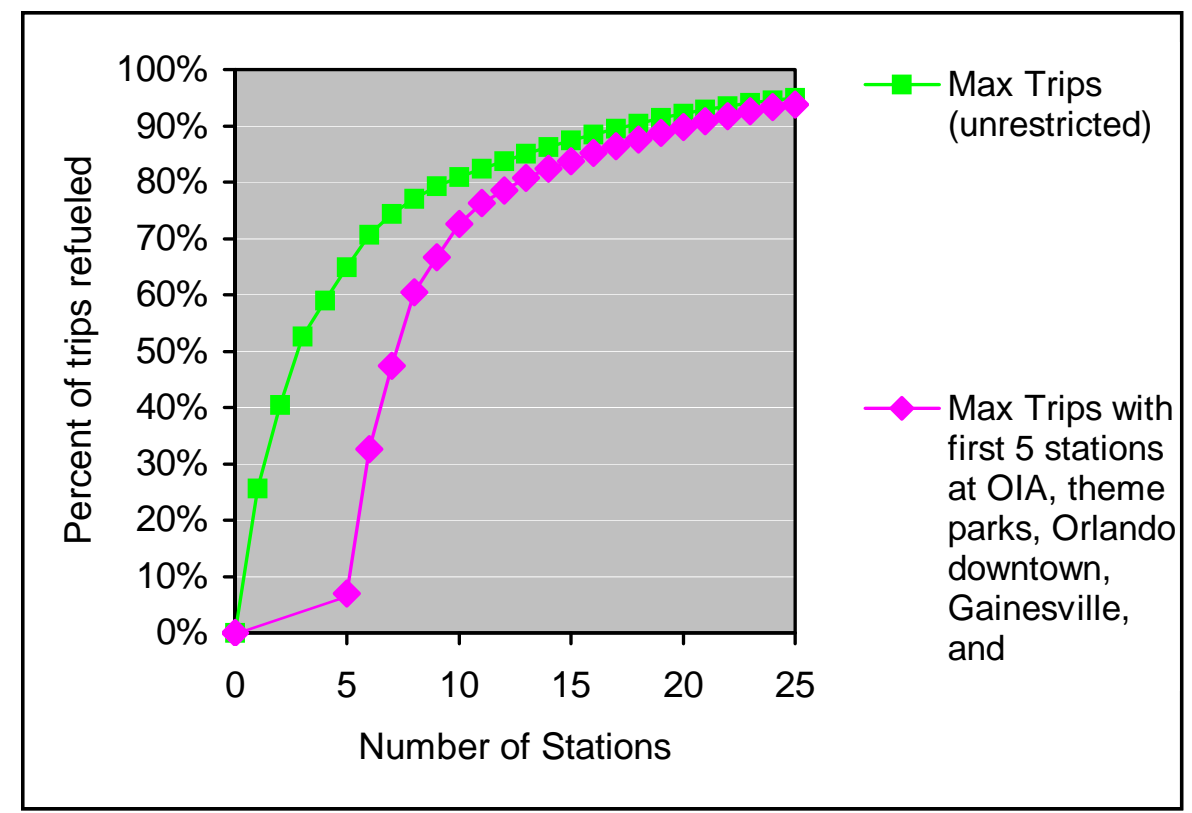

Figure 4.17 Tradeoff curves for max trips, 100-mile range scenarios. 
When we run the model to locate five optimal stations in addition to the five required stations, the model chooses the same four stations in SE Florida and one in Tampa to increase the percentage of refuelable trips to $73 \%$, compared with $81 \%$ for the unrestricted model. As we continue to add stations in both model runs, the gap continues to close between the two scenarios. With 15 stations the difference is $87 \%$ v. $84 \%$; closing to $92 \%$ vs. $90 \%$ for 20 stations; and $95 \%$ vs $94 \%$ for 25 stations. Thus, as the network grows, the sacrifice of locating stations specifically for the rental-cars and universities becomes negligible.

The downtown Orlando and Gainesville stations would be optimal in any case in the unrestricted network, entering in the top 10 and top 25 respectively. The question, then, is how does forcing in the stations at the theme parks, the Orlando airport, and Tallahassee change the optimal location of the other stations?

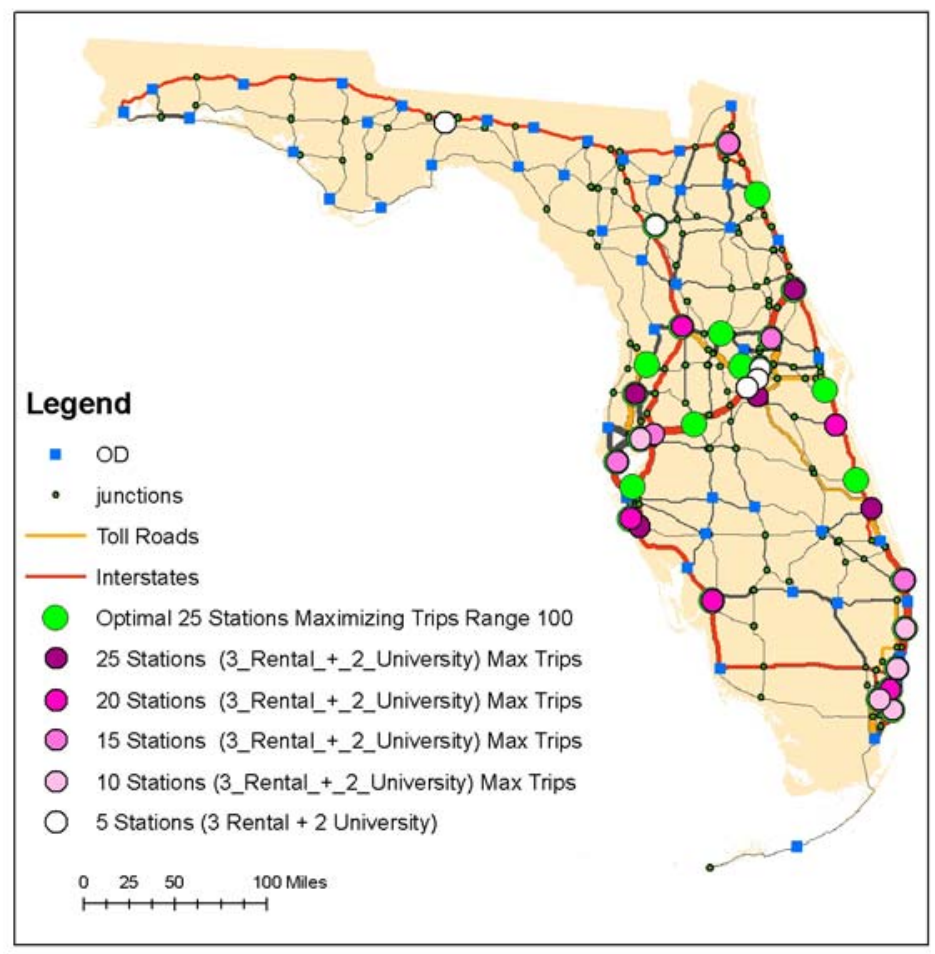

Figure 4.18 Optimal 25 stations for maximizing trips refueled, assuming 3 rental-related stations are built in Orlando and 2 university stations are built, color-coded by priority, and compared with optimal network without rental and university stations (green circles)

The main change is that these three stations replace some of the stations from the Orlando-Tampa-Ocala triangular cluster (in Figure 4.18, green circles show former optimal 25 stations without these required stations). Intermediate size cities in the less urbanized parts of the cluster - such as Ocala, Tavares, Brooksville, and Lakeland - drop out of the Top 25, as does the connecting station on I-75 across the Everglades in the max VMT scenario. Another important finding is that it is still not worthwhile to connect the Tallahassee station to Gainesville or Jacksonville by locating other stations on I-10 - at least not with the first 25 stations. There are simply not enough intercity trips along this corridor by Florida residents to justify putting other connecting stations on I-10. More trips or VMT could be served by locating those stations elsewhere.

Finally, many stations remain where they would otherwise have been placed. There are 17 stations in the maximizing trips scenario and 10 stations in the maximizing VMT scenarios that remain exactly the same, showing that these are good locations regardless of whether the rental-car stations and university stations are included in the top 25 . 
We ran a number of infrastructure scenarios using the weighted hydrogen consumer demand factors based on NREL's GIS-based model [52]. Using multipliers based on demographic and policy variables, we weighted the number of trips and the VMT by the likelihood of consumers at the origins and destinations to adopt hydrogen technology. We then ran a number of different scenarios for the statewide network, with and without forcing the three rental stations and two university stations into the solution, and maximizing weighted trips or weighted VMT. All weighted demand scenarios assumed a 100-mile safe vehicle driving range.

In the weighted demand scenarios, the Miami-Palm Beach and Orlando metropolitan area are predicted to have higher consumer demand than the Tampa and Jacksonville areas mainly because of their Clean Cities coalitions, but also because of demographics and longer commutes. As a

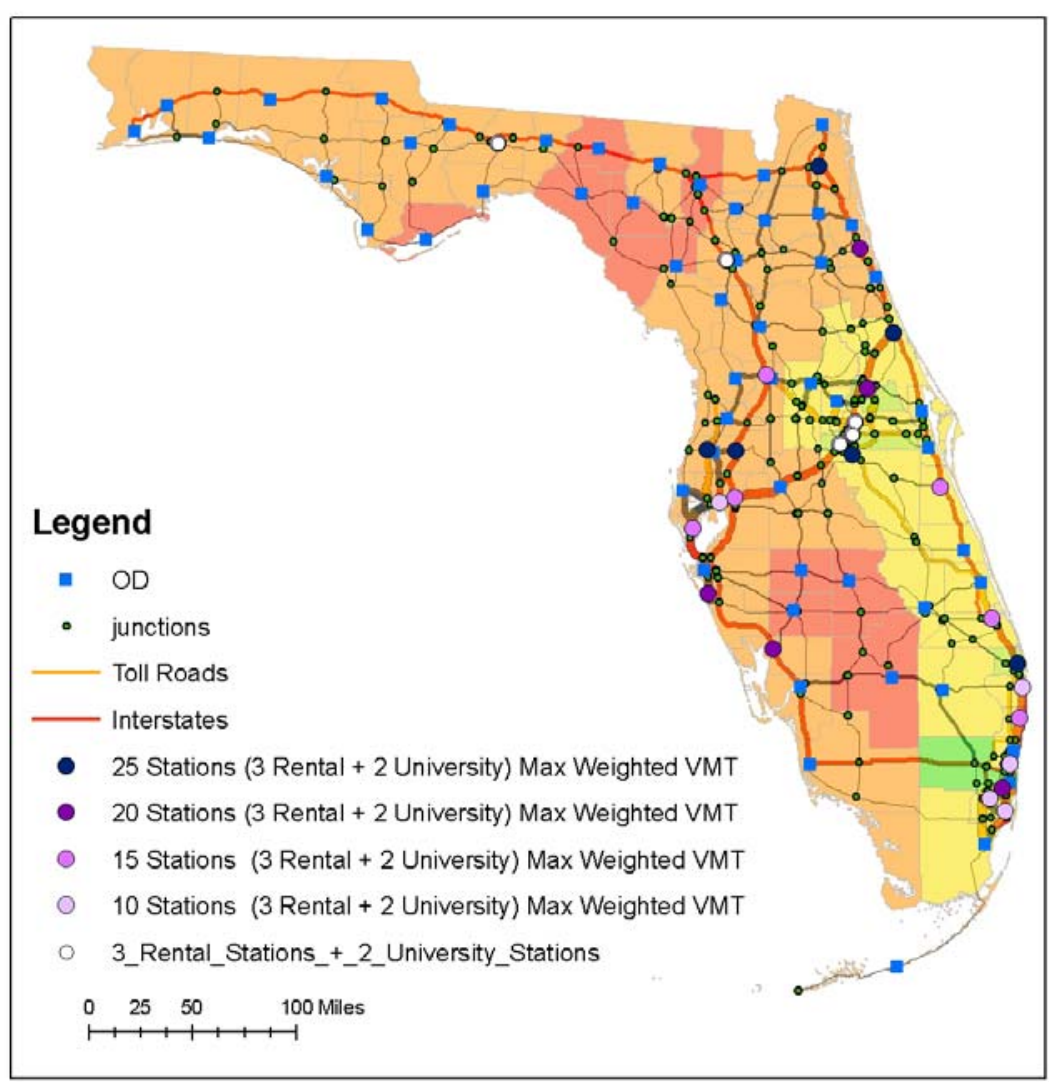

Figure 4.19 Optimal 25 stations maximizing VMT weighted by hydrogen consumer demand scores, assuming 3 rental-related stations in Orlando and 2 university stations result, there is slightly more clustering in these two urban areas and earlier connecting stations between them to maximize the demand that can be served with the same number of stations. In the max trips scenario with five rental-car/university stations, a station in Tavares in the medium-demand area northeast of Orlando replaces a station in mediumlow demand Sarasota. In the max VMT scenario with five rental-car/university stations (Figure 4.19), 24 of 25 stations remain the same, but a station in Palm Beach Gardens replaces one on I-95 near St. Augustine. Stations between Palm Beach and the Space Coast become a higher priority, moving up into the Top 15. Overall, however, many locations continue to be optimal both with and without geographically varying weighted consumer demand, which helps lead us to a robust final set of recommendations for the initial hydrogen refueling infrastructure in Florida. 


\section{General Conclusions}

A number of general policy conclusions can be drawn from these analyses regarding the development of a statewide network of stations for the early stages of the transition to hydrogen vehicles:

1. Although the model does not assume either a clustering or bridging strategy, the preliminary results appear to suggest a strategy somewhat similar to that being developed in California, beginning with clusters in the major cities and later building bridging stations between them to facilitate trips between urban regions.

2. In order to maximize both VMT and trips, the initial set of stations should be clustered in and around the largest metropolitan areas, where they can refuel the high trip volumes between heavily populated nodes that are close together. Clustering also enables the stations to work together to refuel medium-length trips that require multiple stations along the travel route.

3. The Miami-West Palm Beach region is ideal for the first cluster because of its high population, short distance among cities, and its linear arrangement.

4. The I-4 corridor consisting of Tampa and Orlando and the rapidly urbanizing area between them is the second-best cluster.

5. There is no consistent formula or guideline to determine when bridging stations should be introduced to connect the clusters. Given the high expense of hydrogen refueling stations, it does not make sense in Florida to roll out bridging stations on all interstate highways at once-something California has gradually come to realize. Connecting Tampa and Orlando along I-4 is the first priority, followed by connecting West Palm Beach to Daytona to Jacksonville on I-95. The I-75 route across the Everglades connecting Miami to Tampa-St. Petersburg-Fort Myers is less important, as is the Florida Turnpike connecting Miami to Orlando and Gainesville. The I-10 corridor connecting Jacksonville to Tallahassee might be justified by out-of-state traffic, but not by in-state, intercity traffic.

6. Careful thought and planning must be given to the spacing of bridging stations. Spacing stations too far apart could lead to emergency situations and stranding of vehicles that could endanger lives and generate bad publicity. On the other hand, spacing stations too closely sacrifices coverage of more trips and could lead to duplication and underutilization. Our choice of a 100-mile safe driving range is consistent with NREL's assumptions, and should be adequate for dealing with detours, getting lost, and incomplete refueling, and station closures. Assuming maximum spacing of 50 or 75 miles would lower the percentage of intercity trips and VMT that can be refueled by a given number of stations, perhaps unnecessarily. It would also further cluster the optimal 25 stations and reduce coverage for other well-populated parts of Florida.

7. Whatever the recommended station spacing, it should be enforced as a maximum spacing only. Strict regular spacing will waste scarce resources. It is important to place bridging stations where they can also serve crossing traffic flows and local traffic flows. 
8. Not all Florida residents are equally likely to purchase hydrogen vehicles when they become available. While difficult to estimate, NREL has provided a solid basis for estimating geographic differences in consumer demand [52], which we have adapted to weight our estimated trips between places. While use of this data introduces additional uncertainty, we think it provides a more reasonable estimate of early consumer demand than the raw trip estimates, and we place greater emphasis on these scenarios accordingly. That being said, the weighted consumer demand multipliers do not have a large effect on the optimal facility locations, inducing only minor shifts towards the Orlando and the southeastern counties.

9. Factors not included in the model's data must be taken into consideration. Unique synergistic opportunities with a possible OIA rental-car business, with Florida's flagship universities, Kennedy Space Center and Disney, and the state capital should be considered when locating stations.

10. Tradeoff curves are helpful for thinking about how many stations should be built. The results clearly show that while the greatest demand can be served by the first 10-15 stations, the potential demand continues to grow steadily through 25 stations.

\subsection{Orlando Metropolitan Station Location Analysis}

The Orlando area was chosen as the study area for analysis at the metropolitan scale. Orlando is the focus of our feasibility study on a hydrogen rental-car business, and it is also the site of Florida's first two hydrogen stations. One station is located at the Orlando International Airport to refuel airport shuttle vans. Although it is not officially a permanent station, we treat it as existing and permanent in these model runs, and it is therefore always the first station sited by the model. The second existing station is a mobile refueling unit currently located at 2801 State Road 426 in Oveido. This station is not open to the public and is scheduled for decommissioning in 2008 , and we do not treat it as existing in any scenarios.

The most important difference between analysis at the state scale and at the local scale is that the range of the vehicle becomes a non-factor. Hardly any round trips in Orlando exceed the 100-mile safe vehicle range in length. Therefore, one station anywhere on a path can refuel almost any O-D pair. ${ }^{2}$

\footnotetext{
${ }^{2}$ For those few round trips longer than 100 miles, the path is likely to follow a major highway, and a single station anywhere near the middle of the path could easily serve the trip by refueling it in both directions. For a round trip of, say, 110 miles (the longest in our Orlando network), the only way that a single station would not be able to refuel the vehicle before it reached the 100-mile mark is if the station were located within five miles of either the origin or destination.
} 


\section{Base Case}

The base case for the Orlando network is maximizing trips based on the FDOT trip table, with only the airport location required. Figure 4.20 shows the optimal locations for 25 stations, color-coded by priority. We generated these priorities by first solving the model for 5 stations, then 10 stations, and so on up to 25 stations. The color shown for each location is the highest order in which it was first chosen. If a location was chosen for a small number of stations but was then replaced by a different location for a larger number of stations, the swap is shown by an

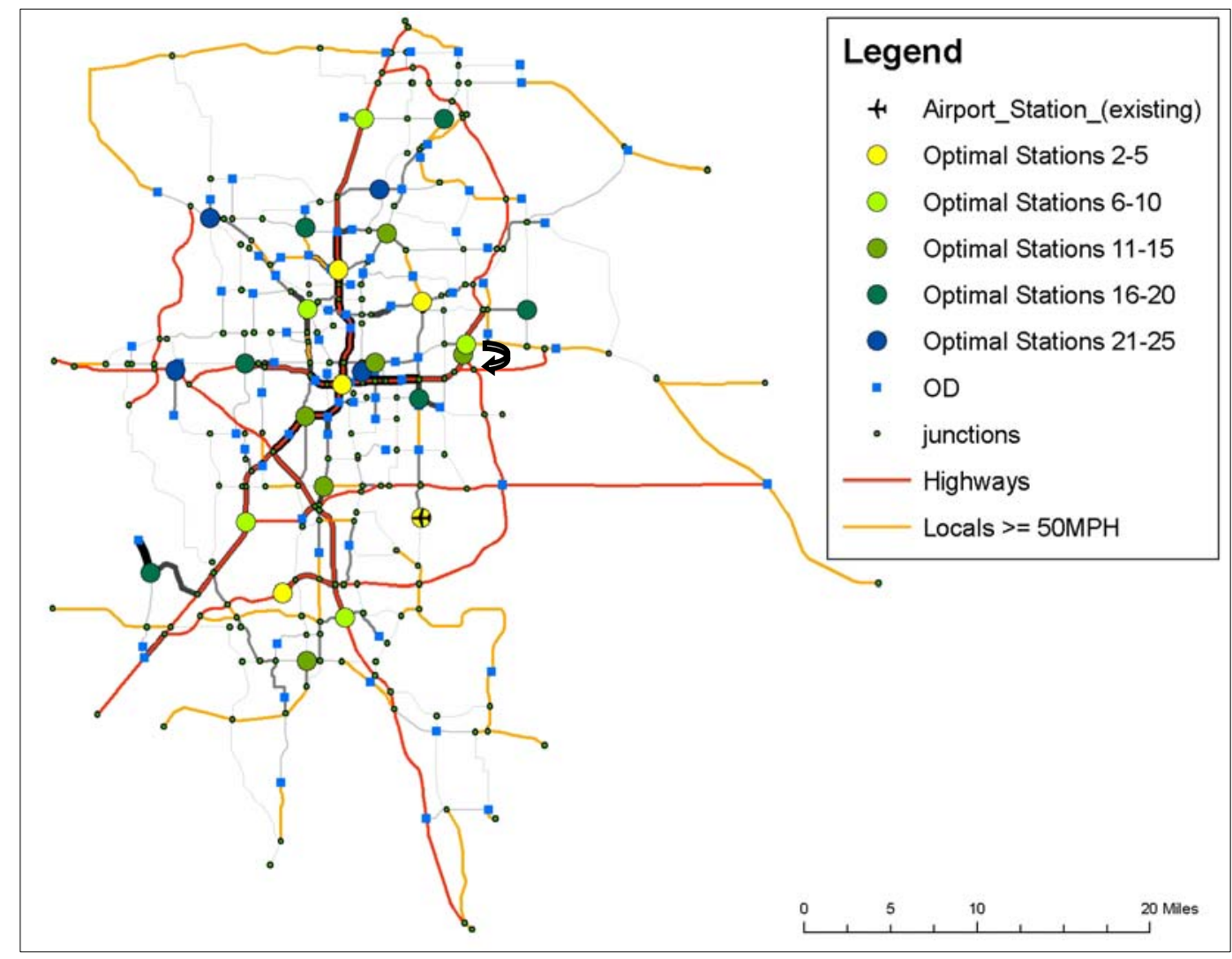

Figure 4.20 Optimal 25 stations in the Orlando area, maximizing trips with 100-mile working range arrow. The stability of this sequence of solutions is a positive outcome for planning stations in Orlando. It means that if stations are developed in this order, future stations are not likely to cannibalize the demand of the earlier stations substantially enough to make them suboptimal.

After the existing station at Orlando International Airport, the best location is in downtown Orlando, near the intersection of I-4 and the East-West Expressway. As can be seen in the tradeoff curve of Figure 4.21 , this $2^{\text {nd }}$ station intercepts and can potentially refuel $14 \%$ of the daily trips in Orlando. It is assumed that a suitable site could be found near this freeway intersection. The possibility of a feasible downtown site is not unrealistic, as evidenced by the hydrogen station in downtown Phoenix, Arizona operated by Arizona Public Service, which is used by APS vehicles and by taxis that run on CNG$\mathrm{H}_{2}$ blends. 


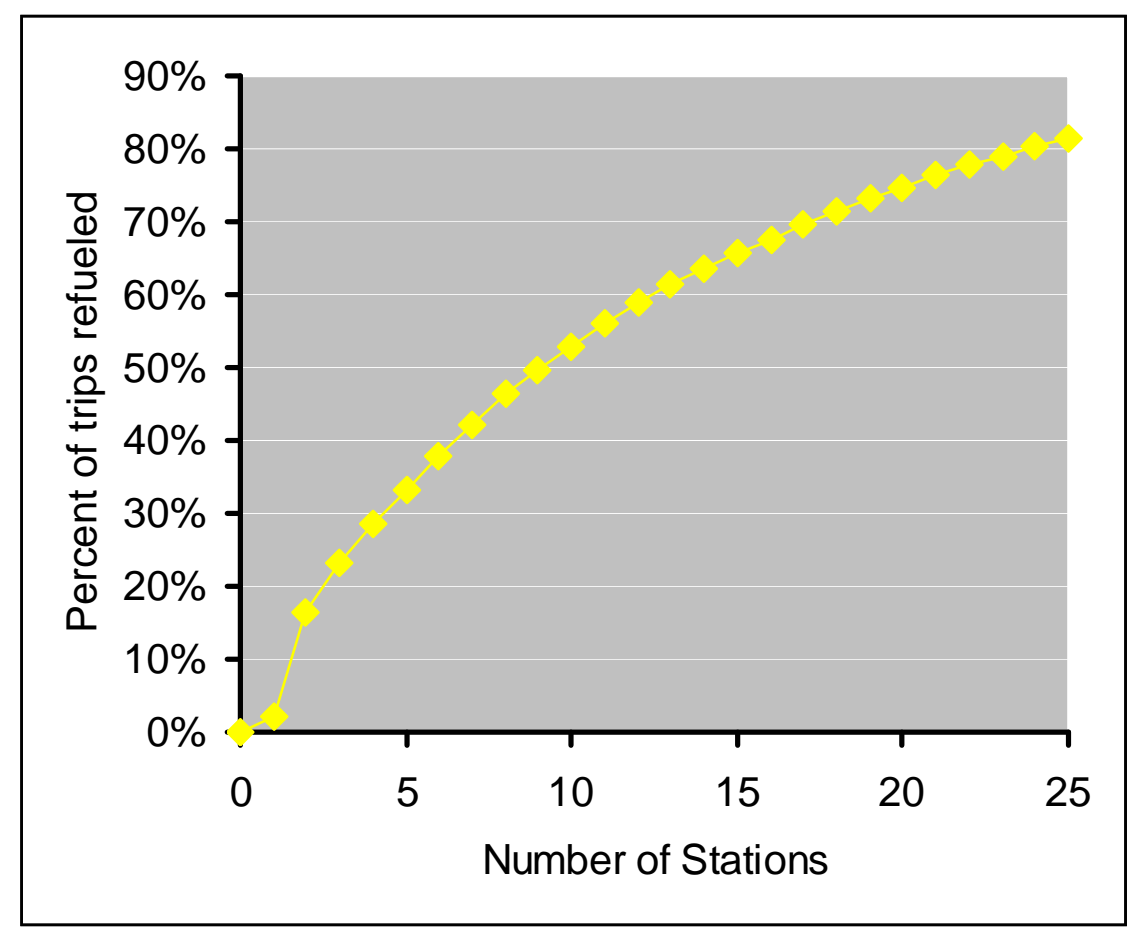

Figure 4.21 Tradeoff between number of stations and percent of inter-zonal trips refueled for Orlando

Of the eight next best station locations (Figure 4.20), six are on major freeways. These sites are located on freeways with high passing traffic volumes as well as high crossing traffic flows and/or originating and ending flows. While these freeway sites are on hightraffic roads, they are not necessarily at the next highest traffic sites in the network. What is more important is that they intercept high-traffic flows that are not captured by the other stations. Notice that the freeway stations in the top ten are located a substantial distance away from each other and from the downtown station. They each capture substantial flow volumes that are not otherwise captured by the other stations. A certain amount of cannibalization of demand is inevitable, but our modeling approach tries to maximize the unique flow volume that can be refueled with each additional station. The model also places two of the top ten stations at heavily trafficked intersections of major arterial streets: Aloma Ave. (SR 426) and Semoran Blvd. (SR 436) northeast of downtown, and John Young Parkway (SR 423) and US Highway 441 northwest of downtown.

As important as where the model locates stations is where the model does not locate stations. In contrast with other methods such as the $p$-median model, the FRLM does not necessarily try to spread the locations around to minimize the average distance from residential zones to their nearest stations. The top ten station sites are not spread evenly around metropolitan Orlando. Many O-D centroids remain far from any of the top ten sites, and large areas appear to be under-served. Appearances, however, can be deceiving. Many trips from these residential areas do in fact pass through at least one of 
the top ten station sites. In all, the top ten sites intercept $53 \%$ of daily inter-zonal Orlando trips.

As seen in the statewide results, the tradeoff curve indicates diminishing marginal returns for additional locations. While the first five stations capture $33 \%$ of trips, the subsequent sets of five add $20 \%, 13 \%, 9 \%$, and $7 \%$ respectively. Not surprisingly, as we move up the curve, fewer and fewer "freeway funnel" points remain to intercept large volumes of passing flows that were not already intercepted by previous locations. Thus, the model eventually begins to adopt a different strategy, spreading stations 16-25 around Orlando in more suburban locations on arterial streets (Figure 4.20). Even so, the overall spatial pattern of 25 stations selected by the FRLM is more concentrated than would be expected with a distance-minimizing, $p$-median approach.

There is some empirical evidence that consumers prefer to refuel near their homes $[32,53]$. This raises the question whether the FRLM or the $p$-median model is more behaviorally realistic. When presented with a choice of many stations along their driving route, we agree that most drivers would prefer a station near home, all else being equal. Stations near home are more familiar, and offer the opportunity to refuel when one is in less of a rush. Locating stations in each and every neighborhood, however, is a luxury that we cannot afford when building the first set of hydrogen stations at roughly $\$ 1$ million apiece. We would argue that when locating the initial refueling infrastructure, the rules must be different. It is most important in the early stages of the infrastructure rollout to locate on the routes of as many trips as possible. With only a handful of stations, we believe that early adopters will be willing to refuel far from home as long as the station is on their regular commuting or shopping route and does not require a detour. With only a handful of stations, a station that is far from home but on their route may in fact be more convenient than a station that is within a few miles of their home but requires a special trip to go there.

\section{Rental-oriented Stations and Weighted Demand Scenarios}

To optimize the refueling network around the three targeted rental-car stations, only one change is needed. Because we already treat the airport station as fixed, and the downtown station is always the first station added, only the station at the epicenter of the theme parks needs to be required. We designated the area near Epcot Center Drive and World Drive as the location.

The weighted demand scenarios account for the difference in the likelihood of consumers in different census tracts to purchase hydrogen vehicles. We ran two scenarios based on weighted trips and weighted VMT. Both assumed the airport station exists and the theme park station would be built, and both added 5 stations at a time from $p=5$ to $p=25$. The optimal stations for maximizing weighted trips is shown in Figure 4.22. 


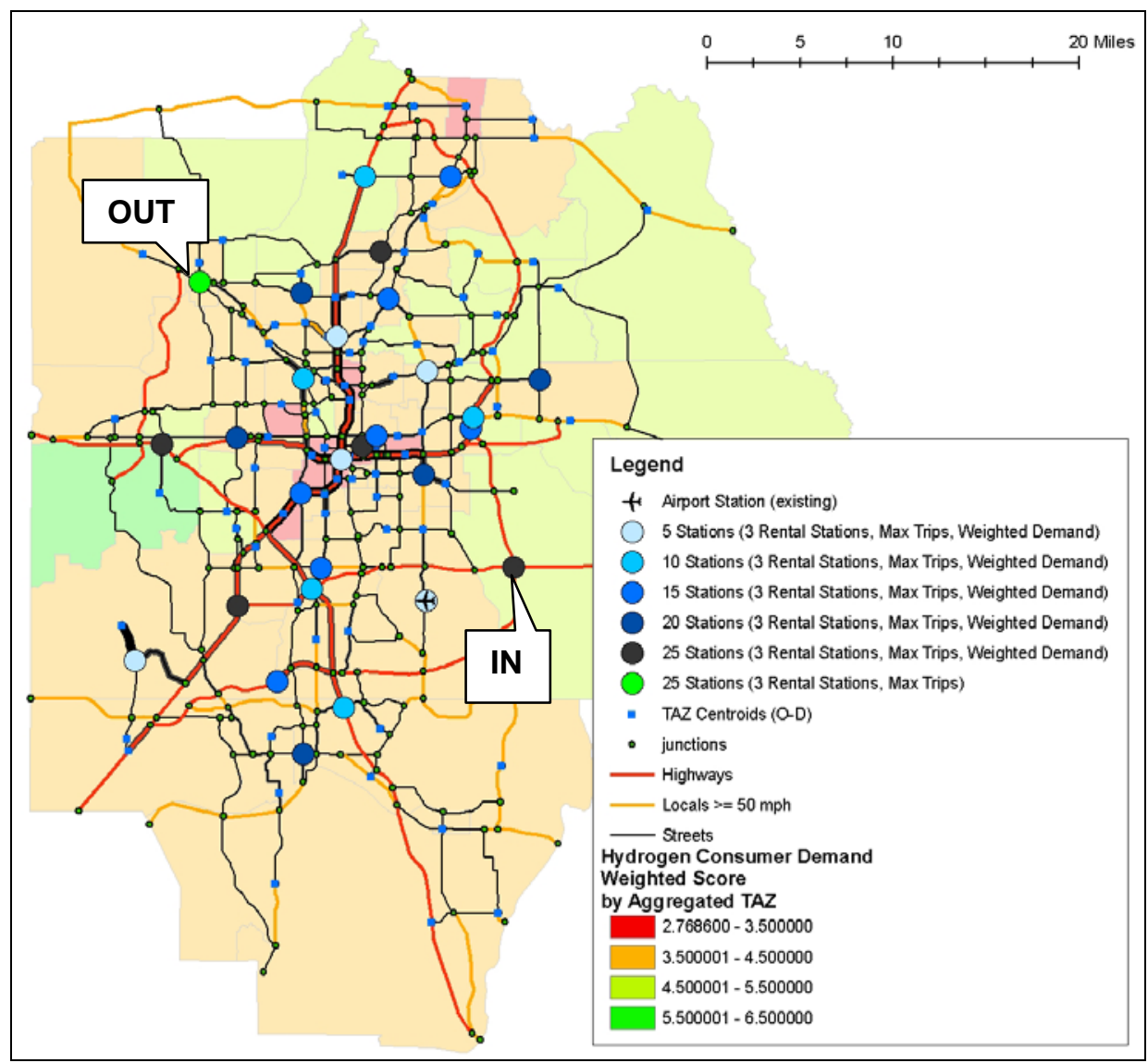

Figure 4.22 Optimal 25 stations maximizing trips refueled weighted by hydrogen consumer demand, assuming airport and theme park stations are built, compared with similar unweighted scenario (green circles)

Geographic differences in adoption rates had only a minor effect on the optimal station locations. Of the 25 optimal stations in the unweighted scenario, only one in northwest Orlando in an area with a demand score of around 4 drops out of the solution. It is replaced by a station in southeast Orlando in an area with a demand score of around 5.

\section{General Conclusions of the Orlando-Scale Analysis}

Several general conclusions emerge from this analysis:

1. To get the most benefit out of the initial refueling infrastructure rollout, it is important to locate stations at funnel points on the road network through which many trips pass, from many origins to many destinations. In Orlando, this is best achieved by locating most of the first ten stations on major freeways with high volumes of passing traffic, where they intersect with other freeways or with major arterials and high volumes of crossing traffic, and where many trips start and end.

2. It is equally important that these funnel points duplicate or cannibalize each other as little as possible. Thus, the major freeway locations within the first ten 
locations are spaced far apart on the network where they can capture different sets of freeway flows and different sets of crossing/starting/ending trips.

3. The highest priority locations, and the first to be built, should not necessarily be spread evenly across the landscape to minimize average distance from where people live to their nearest stations. As more stations are added beyond the first ten, however, optimal locations are increasingly spread around to smaller and smaller funnel points in suburban areas, and the overall network gradually begins to resemble an even distribution that would minimize average distance to stations.

4. While other empirical research has shown that consumers tend to refuel their conventional vehicles near their homes, we would caution against concluding that the initial set of stations should be located according to that principle. We believe it is more important to locate the early set of stations along the routes people travel rather than locate them near their homes, especially because the first 10 stations can only truly be near a small fraction of Orlando residents' homes, but can be right on the route of over half of their trips.

5. Diminishing marginal returns are less pronounced at the Orlando metropolitan scale, as can be seen in Figure 4.23. While each additional station serves less new demand, there is no particular number of stations at which the demand served begins to noticeably level off. Any of the optimal networks of 10 stations can refuel over $52 \%$ of the daily trips in the network. The next 10 stations add about $22 \%$ more weighted trips or $19 \%$ more weighted VMT. Beginning with a rollout of approximately 10 stations in the Orlando area would place stations on convenient routes for a large number of consumers with demographic profiles reflecting a greater likelihood of adopting hydrogen vehicles in the future. 


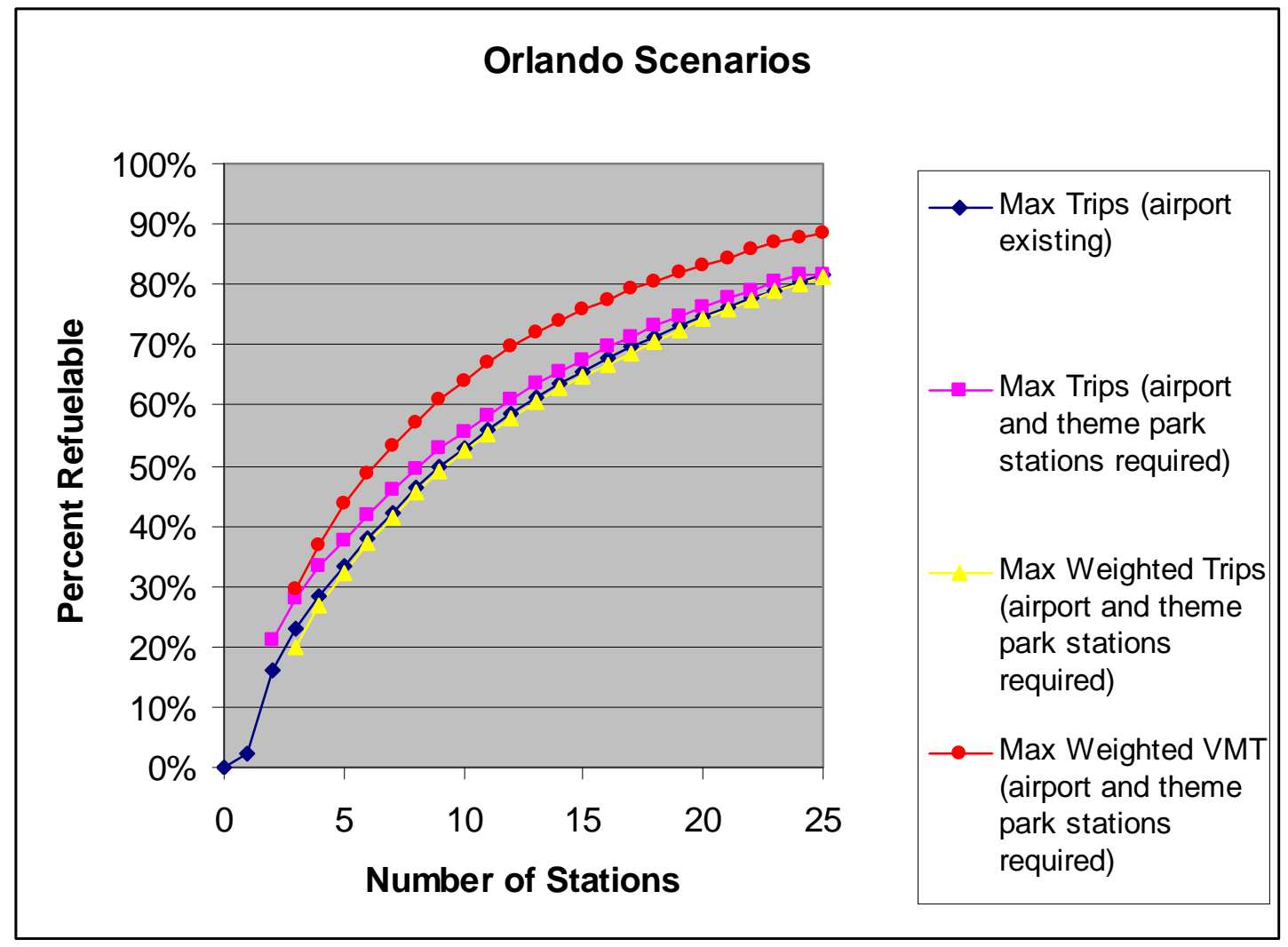

Figure 4.23 Tradeoff curves for the Orlando scenarios 


\section{RECOMMENDATIONS FOR THE HYDROGEN REFUELING STATION INFRASTRUCTURE IN FLORIDA AND ORLANDO}

In planning an initial hydrogen-refueling infrastructure in Florida, our team of researchers considered the results of a large number of model scenarios for both the statewide and Orlando networks, but ultimately employed our expert judgment and local knowledge to interpret the model results and arrive at our best recommendations. This approach recognizes that the Flow-Refueling Location Model for Florida is capable of analyzing tens of thousands of origin-destination pairs and sorting through trillions of possible station combinations in ways that are impossible without the benefits of operations research and GIS. At the same time, we recognize the limitations of the model and the datasets, and the importance of factors that are not included in the models. In addition, no single model scenario can determine the best hydrogen station network for refueling.

For this reason, we looked for station locations that consistently perform well across a variety of scenarios. The most robust locations can coordinate well with a variety of other stations in refueling short trips by themselves and longer trips that require multiple refuelings, regardless of vehicle range, demand weighting, and whether we are maximizing trips or VMT. For the Orlando area, we also analyzed which locations would perform well for intercity, intra-city, and rental-car trips. Finally, our team of four professors - with expertise in transport modeling, geography, and tourism and a combined 60 years living and driving in Florida - weighed the importance of various factors not included in the model to propose the station locations presented below.

\section{Recommended Statewide Network of Hydrogen Refueling Stations}

Table 5.1 lists all stations that were chosen as optimal in any scenario, and their highest rank in each scenario. Rows represent stations, and columns represent model scenarios, and the cells relate whether the station was optimal in the scenario. Some scenarios were solved separately for $5,10,15,20$, or 25 stations, which allowed us to prioritize the stations into Top 5, Top 10, and so on. Other scenarios were solved only for 10 or 25 stations. Based on all the model results and the expert judgment of the research team, we then sorted the stations (and the rows of the table) into tiers of Top 5, Top 10, and so on through Top 25. In ranking them, we considered synergies, spacing, timing, and local intra-city consumer demand. We do not attempt to rank stations within each tier of five.

In ranking the stations, we did not consider all scenarios equally. For the statewide network of intercity trips, we place greater emphasis on the scenarios that include the three stations in Orlando needed for serving rental-car trip and the two flagship university stations. We also put more emphasis on the weighted demand scenarios and the 100-mile range scenarios. Finally, because our focus in the statewide network is on facilitating trips long-distance trips, we placed slightly more emphasis on maximizing VMT. Our final recommendations for 25 stations are quite close to the Max VMT-Range 100Weighted Demand-Five Required Sites scenario in the last column of Table 5.1, with the substitution of Kennedy Space Center for the Shady Hills station, substitution of Fort Pierce for Palm City, some minor relocation of locations within towns, and some 
shuffling of stations among the tiers. As can be seen in Table 5.1, these 25 recommended stations generally perform very well across the board. However, through the modeling we are confident that this set of stations will coordinate well with each other for serving longer distance trips and cannibalize each other's demand as little as possible.

The locations are mapped in Figure 5.1, and it can be seen that the connecting stations outside of the clusters are nicely spaced. The spacing of stations along I-95 never exceeds 100 miles. The spacing on I-4 between the I-75 station at Mango and any of the Orlando area stations is also less than 100 miles, as is the distance from the Ft. Pierce station to the southernmost Orlando station at Osceola Parkway.

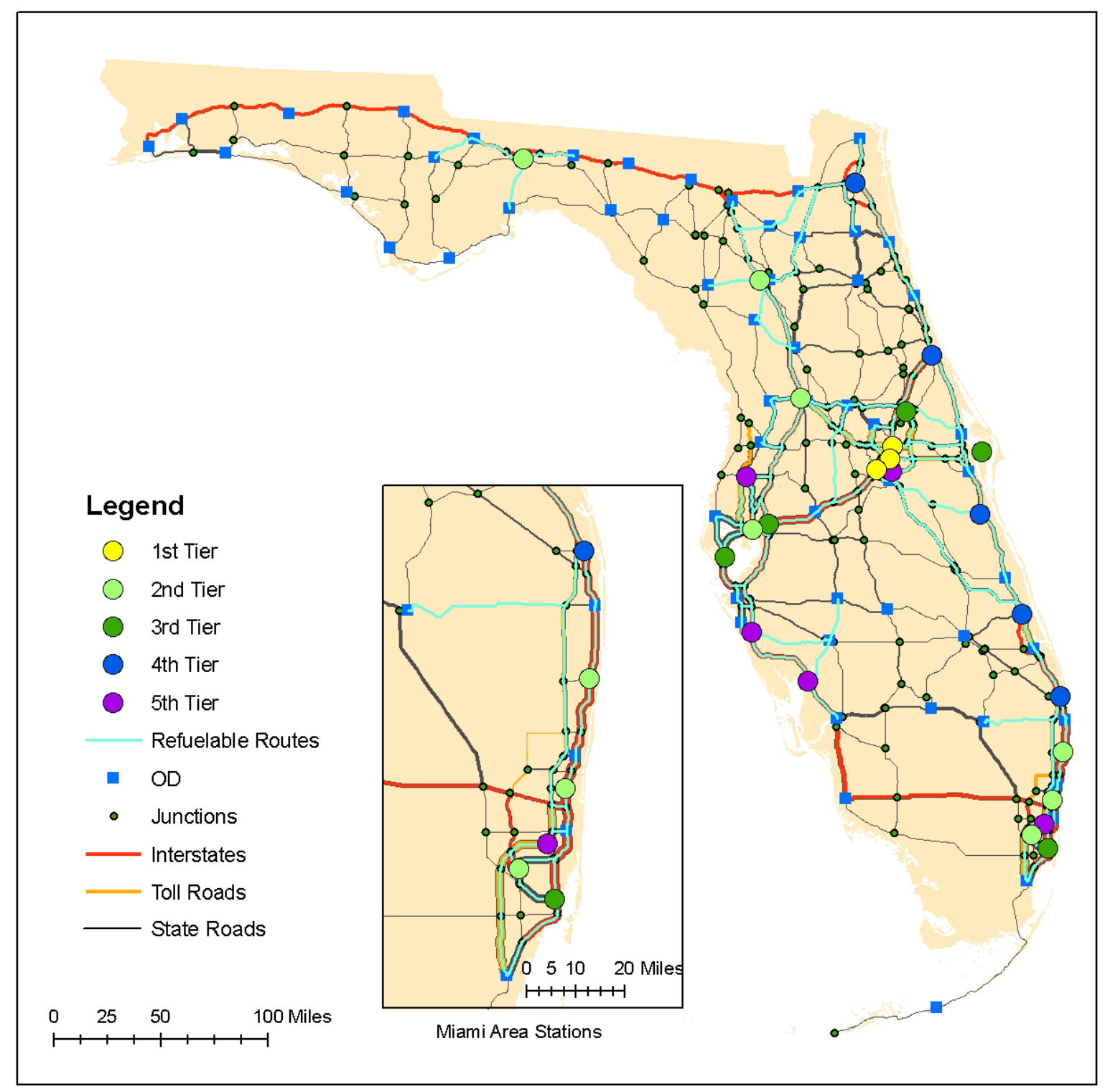

Figure 5.1 Recommended hydrogen stations for the statewide network 
As can be seen in Figure 5.1, we propose developing clusters of stations and connecting stations along major interstates in stages, so that as each tier is constructed, the clusters and the connections between them grow in a coordinated way. The first tier of three stations consists of the airport, downtown, and theme park stations in Orlando needed for the hydrogen rental-car business. Given Orlando's head start in the hydrogen industry with its existing station at the airport, we see it as the key to getting hydrogen moving in Florida. The second tier of seven stations rounds out the Top 10. It creates a hydrogen corridor from Miami Lakes to Ft. Lauderdale to Delray Beach, as well as a connected triangle between Tampa, Orlando, and Gainesville. The third tier fleshes out the Orlando, Tampa, and Miami clusters. The fourth tier completes the network up I-95 from Palm Beach Gardens to Jacksonville. The fifth tier extends the I-75 network north and south of Tampa-St. Petersburg, as well as adding to the Miami and Orlando clusters and shortening the distance between stations on Florida's Turnpike.

\section{Discussion}

We included the Orlando airport, downtown, and theme park stations in the Top 5 because the hydrogen rental-car business would be impossible without them. There are several factors that recommend them above the two university stations. First, a number of other universities around the US have hydrogen stations (e.g., Penn State, UC-Davis, UCLA, UC-Irvine, UC-Riverside, and CSU-LA), but the hydrogen rental-car company at the Orlando airport could be the first in the US. Second, these Orlando stations have more potential to do triple duty for intra-city trips, intercity trips, and rental-car trips than the university stations. Third, the rental-car business can provide guaranteed customers for these stations, unlike the university stations.

We ranked the two university stations in the second set of five stations. The Gainesville station was actually optimal in a number of scenarios, based on intercity trips to and from Gainesville and along I-75. It becomes an even higher priority considering the already thriving scientific research program at the University of Florida, as well as university vehicle fleets and the greater likelihood of professors and administrators to be early adopters. These same arguments apply to Florida State University in Tallahassee, minus the through-traffic benefits but plus the benefits to state government fleets and for purposes of political demonstrations and leadership. 
Table 5.1 Recommended Statewide Station Network*

\begin{tabular}{|c|c|c|c|c|c|c|c|c|c|c|c|}
\hline Jct & City & Intersection & $\begin{array}{l}\text { Max } \\
\text { Trips } \\
\text { Range } \\
100\end{array}$ & $\begin{array}{l}\text { Max } \\
\text { VMT } \\
\text { Range } \\
100\end{array}$ & $\begin{array}{l}\text { Max } \\
\text { Trips } \\
\text { Range } \\
50^{+}\end{array}$ & $\begin{array}{l}\text { Max } \\
\text { Trips } \\
\text { Range } \\
75^{\#}\end{array}$ & $\begin{array}{l}\text { Max } \\
\text { VMT } \\
\text { Range } \\
75^{+}\end{array}$ & $\begin{array}{l}\text { Max Trips } \\
\text { Range } 100 \\
\text { Five } \\
\text { Required } \\
\text { Sites }\end{array}$ & $\begin{array}{l}\text { Max Trips } \\
\text { Range } 100 \\
\text { Weighted } \\
\text { Demand }\end{array}$ & $\begin{array}{l}\text { Max Trips } \\
\text { Range } 100 \\
\text { Weighted } \\
\text { Demand } \\
\text { Five } \\
\text { Required } \\
\text { Sites }^{+}\end{array}$ & $\begin{array}{l}\text { Max VMT } \\
\text { Range } 100 \\
\text { Weighted } \\
\text { Demand } \\
\text { Five } \\
\text { Required } \\
\text { Sites }\end{array}$ \\
\hline \multicolumn{12}{|c|}{ First Tier - Top 3 Locations } \\
\hline 190 & $\begin{array}{l}\text { Orlando Int'l } \\
\text { Airport }\end{array}$ & Boggy Creek Rd. & & & & & & Existing & & Existing & Existing \\
\hline 183 & $\begin{array}{l}\text { Orlando } \\
\text { Downtown }\end{array}$ & $\begin{array}{l}\text { I-4 \& East West } \\
\text { Expwy }\end{array}$ & Top 10 & Top 5 & Top 25 & Top 10 & Top 25 & $\begin{array}{l}\text { Required } \\
\text { Rental } \\
\text { Station }\end{array}$ & & $\begin{array}{l}\text { Required } \\
\text { Rental } \\
\text { Station }\end{array}$ & $\begin{array}{l}\text { Required } \\
\text { Rental } \\
\text { Station }\end{array}$ \\
\hline 197 & $\begin{array}{l}\text { Orlando } \\
\text { Theme Parks }\end{array}$ & $\begin{array}{l}\text { Epcot Ctr Dr. } \\
\text { between I-4 \& } \\
\text { World Dr. }\end{array}$ & & & & & & $\begin{array}{l}\text { Required } \\
\text { Rental } \\
\text { Station }\end{array}$ & & $\begin{array}{l}\text { Required } \\
\text { Rental } \\
\text { Station }\end{array}$ & $\begin{array}{l}\text { Required } \\
\text { Rental } \\
\text { Station }\end{array}$ \\
\hline \multicolumn{12}{|c|}{ Second Tier - Top 10 Locations } \\
\hline 242 & Delray Beach & $\begin{array}{l}\text { I-95 \& W Atlantic } \\
\text { Ave } \\
\text { I-95 \& Sunrise }\end{array}$ & Top 5 & Top 15 & & & Top 25 & Top 10 & & & Top 15 \\
\hline 256 & Ft Lauderdale & $\begin{array}{l}\text { Blvd } \\
\text { I-75 \& Palmetto }\end{array}$ & Top 5 & Top 5 & * & & & Top 10 & & & Top 10 \\
\hline 266 & Miami Lakes & Expwy & Top 5 & Top 10 & & Top 10 & & Top 10 & Top 5 & Top 25 & Top 10 \\
\hline 107 & Tampa & $\mathrm{I}-4 \& \mathrm{I}-275$ & Top 5 & Top 5 & Top 25 & Top 25 & & Top 10 & Top 5 & Top 25 & Top 10 \\
\hline 155 & Gainesville & $\begin{array}{l}\text { I-75 \& W Newberry } \\
\text { Rd } \\
\text { I-75 \& Florida's }\end{array}$ & Top 25 & & & & & $\begin{array}{l}\text { Required } \\
\text { University } \\
\text { Station }\end{array}$ & Top 25 & $\begin{array}{l}\text { Required } \\
\text { University } \\
\text { Station }\end{array}$ & $\begin{array}{l}\text { Required } \\
\text { University } \\
\text { Station }\end{array}$ \\
\hline 163 & Wildwood & Tpke & Top 15 & Top 10 & & Top 25 & Top 25 & Top 20 & Top 20 & Top 25 & Top 15 \\
\hline 292 & Tallahassee & $\begin{array}{l}\text { Tennessee St \& } \\
\text { Monroe St }\end{array}$ & & & & & & $\begin{array}{c}\text { Required } \\
\text { Univ./Govt. } \\
\text { Station }\end{array}$ & & $\begin{array}{c}\text { Required } \\
\text { Univ./Govt. } \\
\text { Station }\end{array}$ & $\begin{array}{c}\text { Required } \\
\text { Univ./Govt. } \\
\text { Station }\end{array}$ \\
\hline
\end{tabular}

* indicates that another location within the same city was in the Top 25 for the scenario in question. The other location(s) can be found further down in the table. Does not apply to large cities such as Miami, Orlando, and Jacksonville, where different locations may be far apart.

${ }^{+}$Stations in these columns are not ranked. Only one scenario (for $\mathrm{p}=5$ ) was analyzed.

\# Stations in this column are either Top 10 or Top 25 . Other $\mathrm{p}$ values were not analyzed. 


\begin{tabular}{|c|c|c|c|c|c|c|c|c|c|c|c|}
\hline Jct & City & Intersection & $\begin{array}{l}\text { Max } \\
\text { Trips } \\
\text { Range } \\
100\end{array}$ & $\begin{array}{l}\text { Max } \\
\text { VMT } \\
\text { Range } \\
\mathbf{1 0 0}\end{array}$ & $\begin{array}{l}\text { Max } \\
\text { Trips } \\
\text { Range } \\
\mathbf{5 0}^{+}\end{array}$ & $\begin{array}{l}\text { Max } \\
\text { Trips } \\
\text { Range } \\
75^{\#}\end{array}$ & $\begin{array}{l}\text { Max } \\
\text { VMT } \\
\text { Range } \\
75^{+}\end{array}$ & $\begin{array}{l}\text { Max Trips } \\
\text { Range } 100 \\
\text { Five } \\
\text { Required } \\
\text { Sites }\end{array}$ & $\begin{array}{l}\text { Max Trips } \\
\text { Range } 100 \\
\text { Weighted } \\
\text { Demand }\end{array}$ & $\begin{array}{l}\text { Max Trips } \\
\text { Range } 100 \\
\text { Weighted } \\
\text { Demand } \\
\text { Five } \\
\text { Required } \\
\text { Sites }^{+}\end{array}$ & $\begin{array}{l}\text { Max VMT } \\
\text { Range } 100 \\
\text { Weighted } \\
\text { Demand } \\
\text { Five } \\
\text { Required } \\
\text { Sites }\end{array}$ \\
\hline \multicolumn{12}{|c|}{ Third Tier - Top 15 Locations } \\
\hline 109 & Mango & I-4 \& I-75 & & & Top 25 & & Top 25 & Top 15 & & Top 25 & Top 15 \\
\hline 270 & Miami & $\begin{array}{l}\text { I-95 \& I-195 } \\
\text { S Orlando Dr \& }\end{array}$ & Top 5 & Top 5 & Top 25 & & Top 25 & Top 10 & & & Top 10 \\
\hline 169 & Sanford & Eastern Beltway & Top 10 & Top 15 & * & & * & Top 15 & Top 10 & Top 25 & Top 20 \\
\hline 209 & $\begin{array}{l}\text { St.Petersburg } \\
\text { Kennedy } \\
\text { Space Center }\end{array}$ & $\begin{array}{l}\text { I- } 275 \& 5 \text { th Ave N } \\
\text { Visitor's Center } \\
\text { Not included in } \\
\text { statewide network. }\end{array}$ & Top 10 & Top 10 & Top 25 & Top 10 & Top 25 & Top 15 & Top 10 & Top 25 & Top 15 \\
\hline \multicolumn{12}{|c|}{ Fourth Tier - Top 20 Locations } \\
\hline 93 & $\begin{array}{l}\text { Daytona } \\
\text { Beach }\end{array}$ & $\begin{array}{l}\mathrm{I}-95 \& \mathrm{I}-4 \\
\text { St Hwy } 70 \\
\text { between I-95 and }\end{array}$ & Top 20 & Top 25 & & Top 25 & Top 25 & Top 25 & & Top 25 & Top 25 \\
\hline 217 & Fort Pierce & Florida's Turnpike & & & Top 25 & & & Top 25 & Top 15 & Top 25 & \\
\hline 82 & $\begin{array}{l}\text { Jacksonville } \\
\text { June Park } \\
\text { (near }\end{array}$ & $1-95 \& I-10$ & Top 15 & Top 25 & & Top 25 & Top 25 & Top 15 & Top 15 & Top 25 & Top 25 \\
\hline 149 & Melbourne) & I-95 \& Coast Hwy & & Top 15 & & & & Top 20 & Top 15 & Top 25 & Top 15 \\
\hline 232 & $\begin{array}{l}\text { Palm Beach } \\
\text { Gardens }\end{array}$ & I-95 \& PGA Blvd & Top 10 & & & & & Top 15 & Top 25 & & Top 25 \\
\hline \multicolumn{12}{|c|}{ Fifth Tier - Top 25 Locations } \\
\hline 201 & Kissimmee & $\begin{array}{l}\text { Osceola Parkway } \\
\text { between Forida's } \\
\text { Tpke and Orange } \\
\text { Blossom Trail }\end{array}$ & & & & Top 25 & & Top 25 & & Top 25 & Top 25 \\
\hline 263 & Miramar & $\begin{array}{l}\text { Florida's Tpke \& } \\
\text { County Line Rd }\end{array}$ & Top 15 & * & & & * & Top 20 & & & Top 20 \\
\hline
\end{tabular}

\footnotetext{
${ }^{+}$Stations in these columns are not ranked. Only one scenario (for $\mathrm{p}=5$ ) was analyzed.

\# Stations in this column are either Top 10 or Top 25 . Other $p$ values were not analyzed.
} 


\begin{tabular}{|c|c|c|c|c|c|c|c|c|c|c|c|}
\hline Jct & City & Intersection & $\begin{array}{l}\text { Max } \\
\text { Trips } \\
\text { Range } \\
100\end{array}$ & $\begin{array}{l}\text { Max } \\
\text { VMT } \\
\text { Range } \\
100\end{array}$ & $\begin{array}{l}\text { Max } \\
\text { Trips } \\
\text { Range } \\
\mathbf{5 0}^{+}\end{array}$ & $\begin{array}{l}\text { Max } \\
\text { Trips } \\
\text { Range } \\
75^{\#}\end{array}$ & $\begin{array}{l}\text { Max } \\
\text { VMT } \\
\text { Range } \\
75^{+}\end{array}$ & $\begin{array}{l}\text { Max Trips } \\
\text { Range } 100 \\
\text { Five } \\
\text { Required } \\
\text { Sites }\end{array}$ & $\begin{array}{l}\text { Max Trips } \\
\text { Range } 100 \\
\text { Weighted } \\
\text { Demand }\end{array}$ & $\begin{array}{l}\text { Max Trips } \\
\text { Range } 100 \\
\text { Weighted } \\
\text { Demand } \\
\text { Five } \\
\text { Required } \\
\text { Sites }^{+}\end{array}$ & $\begin{array}{l}\text { Max VMT } \\
\text { Range } 100 \\
\text { Weighted } \\
\text { Demand } \\
\text { Five } \\
\text { Required } \\
\text { Sites }\end{array}$ \\
\hline 98 & Pasco & I-75 \& St Hwy 52 & Top 15 & * & Top 25 & Top 25 & Top 25 & Top 25 & Top 15 & Top 25 & \\
\hline $\begin{array}{l}81 \\
50\end{array}$ & $\begin{array}{l}\text { Sarasota } \\
\text { Solana }\end{array}$ & $\begin{array}{l}\mathrm{I}-75 \text { \& St Hwy } 72 \\
\mathrm{I}-75 \text { \& Duncan Rd }\end{array}$ & * & Top 25 & $\begin{array}{l}\text { Top } 25 \\
\text { Top } 25\end{array}$ & $\begin{array}{c}* \\
\text { Top } 25\end{array}$ & $\begin{array}{c}* \\
\text { Top } 25\end{array}$ & Top 25 & * & * & Top 20 \\
\hline $\begin{array}{c}\text { Never } \\
176\end{array}$ & $\begin{array}{l}\text { Chosen by M } \\
\text { Lake City }\end{array}$ & $\begin{array}{l}\text { I, but Could be Imp } \\
\text { I-75 \& I-10 }\end{array}$ & rtant for & onnectin & Tallahas & to Jacl & onville, C & ainesville, an & Points Sou & , and for Int & tate Traffic \\
\hline Other & Erequently $\mathrm{O}_{\mathrm{B}}$ & nal Locations & & & & & & & & & \\
\hline $\begin{array}{l}176 \\
203\end{array}$ & $\begin{array}{l}\text { Brooksville } \\
\text { Cocoa West }\end{array}$ & $\begin{array}{l}\text { US Hwy } 41 \text { \& St } \\
\text { Hwy } 50 \\
\text { I-95 \& King St }\end{array}$ & $\begin{array}{l}\text { Top } 25 \\
\text { Top } 20\end{array}$ & Top 25 & Top 25 & $\begin{array}{l}\text { Top } 25 \\
\text { Top } 25\end{array}$ & $\begin{array}{l}\text { Top } 25 \\
\text { Top } 25\end{array}$ & & Top 25 & & \\
\hline 240 & Fort Myers & $\begin{array}{l}\text { I-75 \& Palm Beach } \\
\text { Blvd }\end{array}$ & Top 20 & Top 15 & & & & Top 20 & Top 20 & Top 25 & \\
\hline 257 & Hollywood & $\begin{array}{l}\text { I-95 \& Hollywood } \\
\text { Blvd }\end{array}$ & & Top 25 & Top 25 & Top 10 & Top 25 & & Top 5 & Top 25 & \\
\hline 88 & Lakeland & I-4 \& US Hwy 98 & Top 10 & Top 10 & & Top 10 & & & Top 10 & & \\
\hline 213 & Manatee & $\begin{array}{l}\text { I-75 \& I-275 } \\
\text { Central Florida } \\
\text { Greenway \& Bee }\end{array}$ & Top 20 & Top 15 & Top 25 & Top 25 & Top 25 & & Top 20 & & \\
\hline 202 & Orlando & $\begin{array}{l}\text { Line Expwy } \\
\text { Floridas Tpke \& } \\
\text { SW Martin Downs }\end{array}$ & & Top 25 & Top 25 & Top 25 & Top 25 & & & & \\
\hline 223 & Palm City & Blvd & & Top 15 & & Top 25 & & & & & Top 15 \\
\hline 225 & Palm City & I-95 \& Kanner Hwy & & & Top 25 & & & & & & \\
\hline 226 & Palm City & $\begin{array}{l}\text { I-95 \& Floridas } \\
\text { Tpke }\end{array}$ & & & & & Top 25 & & & & \\
\hline
\end{tabular}

\footnotetext{
${ }^{+}$Stations in these columns are not ranked. Only one scenario (for $\mathrm{p}=5$ ) was analyzed.

\# Stations in this column are either Top 10 or Top 25 . Other p values were not analyzed.
} 


\begin{tabular}{|c|c|c|c|c|c|c|c|c|c|c|c|}
\hline Jct & City & Intersection & $\begin{array}{l}\text { Max } \\
\text { Trips } \\
\text { Range } \\
100\end{array}$ & $\begin{array}{l}\text { Max } \\
\text { VMT } \\
\text { Range } \\
100\end{array}$ & $\begin{array}{l}\text { Max } \\
\text { Trips } \\
\text { Range } \\
\mathbf{5 0}^{+}\end{array}$ & $\begin{array}{l}\text { Max } \\
\text { Trips } \\
\text { Range } \\
75^{\#}\end{array}$ & $\begin{array}{l}\text { Max } \\
\text { VMT } \\
\text { Range } \\
75^{+}\end{array}$ & $\begin{array}{l}\text { Max Trips } \\
\text { Range } 100 \\
\text { Five } \\
\text { Required } \\
\text { Sites }\end{array}$ & $\begin{array}{l}\text { Max Trips } \\
\text { Range } 100 \\
\text { Weighted } \\
\text { Demand }\end{array}$ & $\begin{array}{l}\text { Max Trips } \\
\text { Range } 100 \\
\text { Weighted } \\
\text { Demand } \\
\text { Five } \\
\text { Required } \\
\text { Sites }^{+}\end{array}$ & $\begin{array}{l}\text { Max VMT } \\
\text { Range } 100 \\
\text { Weighted } \\
\text { Demand } \\
\text { Five } \\
\text { Required } \\
\text { Sites }\end{array}$ \\
\hline 92 & Pasco & I-75 \& St Hwy 52 & & Top 10 & & & & & & & Top 25 \\
\hline 98 & $\begin{array}{l}\text { Pasco } \\
\text { Pompano }\end{array}$ & $\begin{array}{l}\text { US Hwy } 41 \text { \& St } \\
\text { Hwy } 52\end{array}$ & Top 15 & & Top 25 & Top 25 & Top 25 & Top 25 & Top 15 & Top 25 & \\
\hline 248 & Beach & $\begin{array}{l}\text { I-95 \& Atlantic Blvd } \\
\text { US Hwy } 301 \& \text { US }\end{array}$ & & Top 25 & Top 25 & Top 10 & & & Top 5 & Top 25 & \\
\hline 301 & Sarasota & Hwy 41 & Top 20 & & & Top 25 & Top 25 & Top 20 & Top 20 & Top 25 & Top 20 \\
\hline 273 & $\begin{array}{l}\text { South Miami } \\
\text { Heights }\end{array}$ & $\begin{array}{l}\text { Dixie Hwy \& W } \\
\text { Dade Expwy }\end{array}$ & & & & Top 10 & & & Top 10 & Top 25 & \\
\hline 124 & St. Johns & I-95 \& St Hwy 16 & Top 25 & Top 25 & & Top 25 & Top 25 & & & Top 25 & \\
\hline 144 & St. Johns & I-95 \& St Hwy 206 & & & & & & & & & Top 20 \\
\hline 37 & Tavares & $\begin{array}{l}\text { US Hwy } 441 \text { \& St } \\
\text { Hwy } 19\end{array}$ & Top 25 & Top 25 & Top 25 & Top 25 & & & & Top 25 & \\
\hline 241 & $\begin{array}{l}\text { West Palm } \\
\text { Beach }\end{array}$ & $\begin{array}{l}\text { I-95 \& Southern } \\
\text { Blvd }\end{array}$ & & Top 5 & Top 25 & Top 10 & & & Тор 10 & Top 25 & $\begin{array}{l}\text { Top } 10 \text { (but } \\
\text { replaced in } \\
\text { Top } 25 \text { ) }\end{array}$ \\
\hline \multicolumn{12}{|c|}{ Occasionally in Top 25--Not Recommended } \\
\hline 4 & Alachua & $\begin{array}{l}\text { St Hwy } 26 \text { \& US } \\
\text { Hwy } 301 \\
\text { Orange Blossom } \\
\text { Trl \& Western }\end{array}$ & & Top 25 & & & & & & & \\
\hline 145 & Apopka & Expwy & & & & Top 25 & & & Top 20 & & \\
\hline 79 & Bradenton & $\begin{array}{l}\text { St Hwy } 64 \text { \& US } \\
\text { Hwy } 41\end{array}$ & & & Top 25 & & & & & & \\
\hline 58 & Celebration & $\begin{array}{l}\text { I-4 \& Irlo Bronson } \\
\text { Mem. Hwy }\end{array}$ & & & & & Top 25 & & & & \\
\hline 67 & Citrus Ridge & I-4 \& US Hwy 27 & & & Top 25 & & & & & & \\
\hline 249 & Collier & I-75 \& St Hwy 29 & & Top 25 & & & & & & & \\
\hline 139 & De Land & I-4 \& St Hwy 44 & & & Top 25 & & & & & & \\
\hline
\end{tabular}




\begin{tabular}{|c|c|c|c|c|c|}
\hline Jct & City & Intersection & $\begin{array}{l}\text { Max } \\
\text { Trips } \\
\text { Range } \\
100\end{array}$ & $\begin{array}{l}\text { Max } \\
\text { VMT } \\
\text { Range } \\
100\end{array}$ & $\begin{array}{l}\text { Max } \\
\text { Trips } \\
\text { Range } \\
\mathbf{5 0}^{+}\end{array}$ \\
\hline 258 & Lauderdale & I-95 \& SW 34th St & & & Top 25 \\
\hline 268 & $\begin{array}{l}\text { Hialeah } \\
\text { Gardens }\end{array}$ & $\begin{array}{l}\text { Okeechobee Rd \& } \\
\text { W Dade Expwy }\end{array}$ & & & Top 25 \\
\hline 43 & Jacksonville & US Hwy 17 \& I-295 & & & Top 25 \\
\hline 78 & Manatee & $\begin{array}{l}\text { US Hwy } 301 \& \text { St } \\
\text { Hwy } 70 \\
\text { I-75 \& Dade }\end{array}$ & Top 15 & & \\
\hline 267 & Miramar & Expwy & & Top 25 & \\
\hline 181 & Oakland & $\begin{array}{l}\text { W Colonial Dr \& } \\
\text { Floridas Tpke }\end{array}$ & & & \\
\hline 160 & Ocala & I-75 \& US Hwy 27 & & Top 25 & \\
\hline 182 & Ocoee & $\begin{array}{l}\text { Floridas Tpke \& } \\
\text { Maguire Rd } \\
\text { Floridas Tpke \& } \\
\text { Central Florida }\end{array}$ & Top 25 & Top 25 & Top 25 \\
\hline 201 & Orlando & Greeneway & & & \\
\hline 244 & Palm Beach & $\begin{array}{l}\text { Floridas Tpke \& } \\
\text { Southern Blvd } \\
\text { I-95 \& Palm Coast }\end{array}$ & & & \\
\hline 299 & Palm Coast & Pky & & & \\
\hline 166 & Sanford & St Hwy 46 \& I-4 & & & Top 25 \\
\hline 81 & Sarasota & $\begin{array}{l}\text { I-75 \& St Hwy } 72 \\
\text { I-4 \& Seminole }\end{array}$ & & Top 25 & Top 25 \\
\hline 164 & Seminole & $\begin{array}{l}\text { Blvd } \\
\text { St Hwy } 52 \text { \& }\end{array}$ & & & \\
\hline 100 & Shady Hills & Suncoast Pky & & & \\
\hline & Titusvill & $95 \& \mathrm{G}$ & & & \\
\hline
\end{tabular}

Max Trips Max VMT

Range $100 \quad$ Range 100

Weighted Weighted

Demand Demand

Five Five

Required Required

Sites $^{+} \quad$ Sites
Top 15

Top 25

Top 25

Top 25

Top 25

Top 20

Top 25

Top 25

Top 10

\footnotetext{
${ }^{+}$Stations in these columns are not ranked. Only one scenario (for $\mathrm{p}=5$ ) was analyzed.

\# Stations in this column are either Top 10 or Top 25 . Other $p$ values were not analyzed.
} 


\begin{tabular}{|c|c|c|c|c|c|c|c|c|c|c|c|}
\hline & & & & & & & & Max Trins & & $\begin{array}{l}\text { Max Trips } \\
\text { Range } 100 \\
\text { Weiahted }\end{array}$ & $\begin{array}{l}\text { Max VMT } \\
\text { Range } 100 \\
\text { Weighted }\end{array}$ \\
\hline & & & Max & Max & $\operatorname{Max}$ & $\operatorname{Max}$ & Max & Range 100 & Max Trips & Demand & Demand \\
\hline & & & Trips & VMT & Trips & Trips & VMT & Five & Range 100 & Five & Five \\
\hline & & & Range & Range & Range & Range & $\begin{array}{l}\text { Range } \\
75^{+}\end{array}$ & Required & Weighted & Required & $\begin{array}{l}\text { Required } \\
\text { Sites }\end{array}$ \\
\hline Jct & City & $\begin{array}{l}\text { Intersection } \\
\text { W Hillsborough }\end{array}$ & & & & & & Sites & Demand & & \\
\hline & Town 'n' & Ave \& Veterans & & & & & & & & & \\
\hline 102 & $\begin{array}{l}\text { Country } \\
\text { West Vero }\end{array}$ & Expwy & & & & & Top 25 & & & & \\
\hline 153 & Corridor & I-95 \& St Hwy 60 & Top 20 & & & Top 25 & Top 25 & & & & \\
\hline
\end{tabular}

\footnotetext{
${ }^{+}$Stations in these columns are not ranked. Only one scenario (for $\mathrm{p}=5$ ) was analyzed.

\# Stations in this column are either Top 10 or Top 25 . Other $p$ values were not analyzed.
} 
Stations on the southeastern coast provide the most bang for the buck because of the large populations with favorable demographics and the linear arrangement of cities. Compared with some other areas of Florida, however, there is less consistency across scenarios regarding which particular stations are optimal. The combination recommended here, consisting of Miami Lakes, Delray Beach, Ft. Lauderdale, Miami (I-95 \& I-195), Miramar, and Palm Beach Gardens, were grouped together in several scenarios, including the max VMT weighted demand scenario. Miami Lakes in particular entered every scenario with a 100-mile vehicle range, and was usually one of the first sites chosen.

In the Orlando area, we recommend two stations in addition to the downtown, theme park, and airport stations needed for hydrogen car rentals. In the $3^{\text {rd }}$ tier we propose a station near Sanford and Lake Mary that would capture long-distance trips that skirt around the beltway in northeast Orlando away from the three rental stations. The final Orlando station in the Top 25 would be located somewhere around Osceola Parkway between Orange Blossom Trail and Florida's Turnpike. This station would serve the Kissimmee area and daily commutes on the Turnpike, as well as enable drivers to connect to a station in Fort Pierce without exceeding the 100-mile safe driving range.

While there is no station on I-4 midway between Tampa and Orlando, long-distance commuting from the Lakeland area could conceivably be possible if the family had a hydrogen car for long-distance commuting and a second conventional car for local trips. Lakeland commuters could refuel near their jobs in Tampa or Orlando and be able to complete several long-distance commutes before refueling again near work. A similar logic applies to intercity commutes from West Palm Beach.

Daytona is another location of particular interest, which we place in the $4^{\text {th }}$ tier. It was rarely chosen in the Top 20 in the model, but it consistently appeared in the Top 25 as a key connecting station between I-4 and I-95. About 5\% of car renters included it in their bundle of trips with the Orlando theme parks and/or downtown Orlando. Like KSC, it may attract technology enthusiasts who are more likely adopters. Daytona is also a popular weekend destination from Orlando and the terminal point of the I-4 corridor.

Seven of these 25 stations are in towns where a publicly accessible CNG station exists (Appendix 5). These include Tampa, Fort Lauderdale, Delray Beach, Palm Beach Gardens, Fort Pierce, Kennedy Space Center, and Daytona Beach. Five of the other cities have privately owned CNG stations not open to the public, including Orlando, St. Petersburg, Miami, Jacksonville, and one at the Disney theme parks. While co-locating a hydrogen station with these CNG stations may save on investment and permitting costs, the CNG stations may not be located in high-traffic and high-visibility locations ideal for a consumer-oriented station. Most CNG stations were originally sited for serving industrial or governmental fleets.

Although not justified by any of our model runs, a station at the junction of I-10 and I-75 near Lake City should be considered at some point. This station will eventually be needed to connect Tallahassee with Jacksonville, Gainesville, and points south. It would also be important for out-of-state traffic coming from the north at some future time. 


\section{Recommended Orlando Area Network of Hydrogen Refueling Stations}

Because of the finer scale of the OD zones in the Orlando network, we feel that the consumer hydrogen demand multipliers are likely to be more reliable for the Orlando network than the statewide network. Therefore, we considered those scenarios to be the most important. Secondly, we consider maximizing trips more important than maximizing VMT for the urban network. In the urban network, in contrast to the statewide network, it is actually easier to refuel the longer trips, because the long trips are more likely to use freeways and more likely to encounter at least one station somewhere along the route. The emphasis, therefore, should be on refueling as many trips as possible, both short and long. The more trips that can be refueled, the more convenient it will be for consumers, more of whom will be able to purchase hydrogen vehicles. Compared with the statewide network, there is more consistency across scenarios in the Orlando network, leading to a robust plan with reduced uncertainty.

We recommend an initial network of 11 hydrogen-refueling stations that together can refuel about $54 \%$ of weighted trips (Figure 5.2). Development in coordinated stages is less important than in the statewide network because distances within the metro area are not long enough to require multiple refuelings on any given trip. The stations are grouped into tiers mainly according to their potential to add to the total trips that can be refueled. Five of the stations are also important in the statewide network.

\section{Discussion}

Eight of the 11 recommended stations were consistently in the Top 10 in every scenario. They are easy, robust recommendations. The three stations needed for the rental business are joined in the Top 5 by stations at I-4 and Maitland Blvd. and at Aloma Ave. and Semoran Blvd. These stations at funnel points of the network consistently maximize the number of trips that could not otherwise be refueled by the downtown, airport, and theme park stations. We propose placing the theme park station near I-4 so it would be accessible to through traffic for the statewide network. Alternatively, Disney might be interested in having a station at EPCOT or Magic Kingdom, or it could be placed on International Drive.

The second tier includes stations in Northwest Orlando at Orange Blossom Trail \& N John Young Parkway, in Heathrow at I-4 \& W Lake Mary Blvd, and in Union Park at Central Florida Greenway \& E Colonial Dr. (Hwy 50) that consistently place in the Top 10. A few points about these and the remaining stations are worth mentioning:

- The Union Park station trades in and out of optimal solutions with a site just to its south at the Greenway (SR 417) and Valencia College Lane. The site we recommend on Colonial Drive is more frequently optimal in networks of more than 15 stations, meaning it is likely to remain a good location as the network develops further. The Valencia College Lane site, in contrast, has complicated access problems if coming from the south on the Greenway. 
- The Florida Mall station would be somewhere near Orange Blossom Trail and Sand Lake Rd. This location trades in and out of solution with a station just to its south at the intersection of Florida's Turnpike and the Beach Line Expressway. A station anywhere in this vicinity, with high visibility and easy access to the freeways, would be ideal.

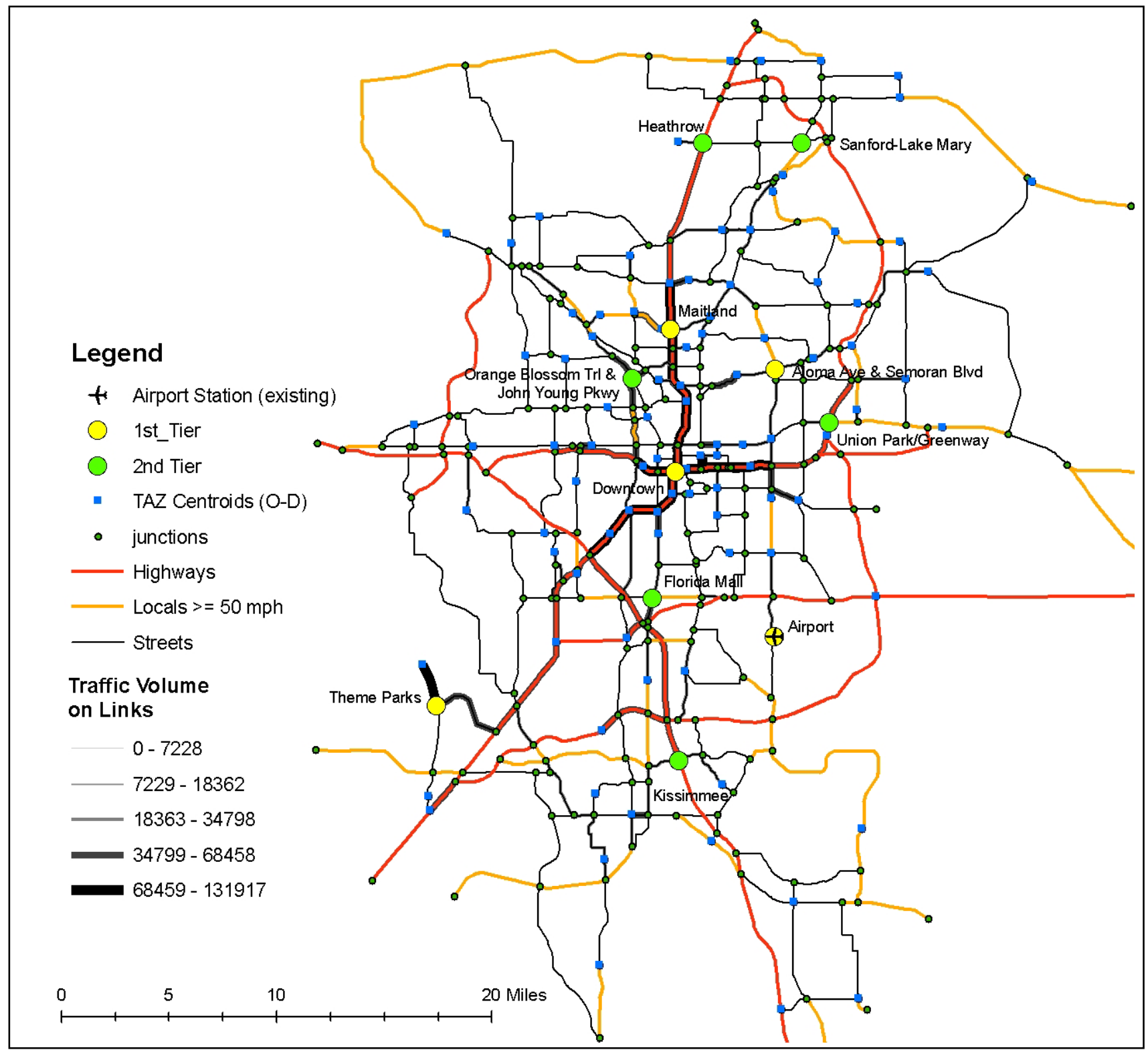

Figure 5.2 Recommended stations for the Orlando area 
Table 5.2 Recommended Orlando Station Network

\begin{tabular}{|c|c|c|c|c|c|c|}
\hline Jct & Intersection & Place & $\begin{array}{l}\text { Max } \\
\text { Trips } \\
\text { Airport }\end{array}$ & $\begin{array}{l}\text { Max } \\
\text { Trips } \\
\text { Airport + } \\
\text { Disney }\end{array}$ & $\begin{array}{l}\text { Max Trips } \\
\text { Airport + } \\
\text { Disney } \\
\text { Weighted } \\
\text { Demand }\end{array}$ & $\begin{array}{l}\text { Max VMT } \\
\text { Airport + } \\
\text { Disney } \\
\text { Weighted } \\
\text { Demand }\end{array}$ \\
\hline \multicolumn{7}{|c|}{ First Tier - Top 5} \\
\hline & Aloma Ave \& & East of Winter & & & & \\
\hline 29 & Semoran Blvd & Park & Top 5 & $\begin{array}{l}\text { Top } 5 \\
\text { Required }\end{array}$ & $\begin{array}{l}\text { Top } 5 \\
\text { Required }\end{array}$ & $\begin{array}{l}\text { Top } 10 \\
\text { Required }\end{array}$ \\
\hline 144 & $\begin{array}{l}\text { Epcot Ctr Dr between } \\
\text { I-4 \& World Dr }\end{array}$ & Theme Parks & Top 20 & $\begin{array}{l}\text { Rental } \\
\text { Station }\end{array}$ & $\begin{array}{l}\text { Rental } \\
\text { Station }\end{array}$ & $\begin{array}{l}\text { Rental } \\
\text { Station }\end{array}$ \\
\hline 176 & I-4 \& W Maitland Blvd & Maitland & Top 5 & Top 5 & Top 5 & Top 10 \\
\hline 202 & I-4 \& East-West Expwy & $\begin{array}{l}\text { Downtown } \\
\text { Orlando }\end{array}$ & Top 5 & Top 5 & Top 5 & Top 5 \\
\hline 305 & $\begin{array}{l}\text { S Access Rd \& } \\
\text { Airport Blvd }\end{array}$ & $\begin{array}{l}\text { International } \\
\text { Airport }\end{array}$ & Existing & Existing & Existing & Existing \\
\hline \multicolumn{7}{|c|}{ Second Tier - Top 11 Stations } \\
\hline 66 & $\begin{array}{l}\text { Orange Blossom Trail } \\
\& \text { N John Young Pky } \\
\text { S Orange Blossom }\end{array}$ & $\begin{array}{l}\text { Northwest } \\
\text { Orlando }\end{array}$ & Top 10 & Top 10 & Top 10 & Top 10 \\
\hline 126 & $\begin{array}{l}\text { Trail \& Sand Lake Rd } \\
\text { S Orlando Dr between } \\
\text { Cent Florida Greenway }\end{array}$ & $\begin{array}{l}\text { Florida Mall } \\
\text { Sanford - Lake }\end{array}$ & Top 15 & Top 15 & Top 15 & Top 20 \\
\hline 148 & $\begin{array}{l}\text { \& Lake Mary Blvd } \\
\mathrm{I}-4 \text { \& }\end{array}$ & Mary & Top 20 & Top 20 & Top 15 & Top 25 \\
\hline 168 & $\begin{array}{l}\text { W Lake Mary Blvd } \\
\text { Cent Florida Greenway }\end{array}$ & Heathrow & Top 10 & Top 10 & Top 10 & $\begin{array}{l}\text { Top } 5 \\
\text { Top }\end{array}$ \\
\hline 180 & $\begin{array}{l}\text { \& E Colonial Dr } \\
\text { Osceola Pky between } \\
\text { Florida's Tpke \& }\end{array}$ & Union Park & Top 10 & Top 10 & Top 10/25 & $10 / 15 / 25$ \\
\hline 210 & Orange Blossom Trail & Kissimmee & Top 10 & Top 10 & Top 10 & Top 20 \\
\hline \multicolumn{7}{|c|}{ Top 15 Stations } \\
\hline 71 & $\begin{array}{l}\text { US Hwy } 17 / 92 \& \\
\text { St Hwy } 436 \\
\text { E Colonial Dr \& }\end{array}$ & $\begin{array}{l}\text { Casselberry } \\
\text { Fashion }\end{array}$ & Top 15 & Top 15 & Top 15 & Top 15/25 \\
\hline 101 & $\begin{array}{l}\text { N Bumby Ave } \\
\text { Cent Florida Greenway }\end{array}$ & Square Mall & Top 15 & Top 15 & Top 15 & Top 20 \\
\hline 179 & $\begin{array}{l}\text { \& Valencia College Ln } \\
\mathrm{I}-4 \text { \& }\end{array}$ & $\begin{array}{l}\text { Valencia East } \\
\text { Southwest }\end{array}$ & Top 15 & Top 15 & Top 15 & \\
\hline 252 & S John Young Pky & Orlando & Top 15 & Top 15 & Top 15 & \\
\hline \multicolumn{7}{|c|}{ Top 20 Stations } \\
\hline & Town Loop Blvd \& & $\begin{array}{l}\text { Seminole } \\
\text { Towne Center }\end{array}$ & & & & \\
\hline 269 & $\begin{array}{l}\text { Town Center Blvd } \\
\text { South Sermoran Blvd }\end{array}$ & Mall & Top 5 & Top 15 & Top 15 & \\
\hline 44 & $\begin{array}{l}\text { \& Curry Ford Rd } \\
\text { St Hwy } 434 \text { \& }\end{array}$ & $\begin{array}{l}\text { East Orlando } \\
\text { Northwest }\end{array}$ & Top 20 & Top 20 & Top 20 & \\
\hline 57 & $\begin{array}{l}\text { St Hwy 438a } \\
\text { N Alafaya Trl \& }\end{array}$ & $\begin{array}{l}\text { Orlando } \\
\text { University of }\end{array}$ & Top 20 & Top 20 & Top 20 & \\
\hline 81 & $\begin{array}{l}\text { University Blvd } \\
\text { W Colonial Dr \& N }\end{array}$ & Central Florida & Top 20 & Top 20 & Top 20 & Top 20 \\
\hline 88 & Hiawassee Rd & Pine Hills & Top 20 & Top 20 & Top 20 & \\
\hline
\end{tabular}




\begin{tabular}{|c|c|c|c|c|c|c|}
\hline Jct & Intersection & Place & $\begin{array}{l}\text { Max } \\
\text { Trips } \\
\text { Airport }\end{array}$ & $\begin{array}{l}\text { Max } \\
\text { Trips } \\
\text { Airport + } \\
\text { Disney }\end{array}$ & $\begin{array}{l}\text { Max Trips } \\
\text { Airport + } \\
\text { Disney } \\
\text { Weighted } \\
\text { Demand }\end{array}$ & $\begin{array}{l}\text { Max VMT } \\
\text { Airport + } \\
\text { Disney } \\
\text { Weighted } \\
\text { Demand }\end{array}$ \\
\hline \multicolumn{7}{|c|}{ Top 25 Stations } \\
\hline & W Vine St \& & & & & & \\
\hline 132 & $\begin{array}{l}\text { S John Young Pky } \\
\text { E Robinson St \& }\end{array}$ & $\begin{array}{l}\text { Kissimmee } \\
\text { Downtown }\end{array}$ & Top 15 & Top 15 & Top 20 & Top 15 \\
\hline 102 & $\begin{array}{l}\text { N Mills Ave } \\
\text { Florida's Tpke \& }\end{array}$ & Orlando & Top 25 & Top 25 & Top 25 & \\
\hline 185 & Maguire Rd & $\begin{array}{l}\text { Ocoee } \\
\text { Orange } \\
\text { County } \\
\text { Convention }\end{array}$ & Top 25 & Top 25 & Top 25 & \\
\hline 219 & I-4 \& Bee Line Expwy & Center & Top 10 & Top 25 & Top 25 & Top 25 \\
\hline 272 & St Hwy 434 \& Wilma St & Longwood & Top 25 & Top 25 & Top 25 & \\
\hline \multicolumn{7}{|c|}{ Optimal in Some Scenarios but not Consistently - Not Recommended } \\
\hline & Cent Florida Greenway & Innovation & & & & \\
\hline 207 & $\begin{array}{l}\text { \& Bee Line Expwy } \\
\text { Florida's Toke \& }\end{array}$ & $\begin{array}{l}\text { Place } \\
\text { Near Florida }\end{array}$ & & & $\begin{array}{l}\text { Top } 25 \\
\text { Top } 10 \\
\text { but not }\end{array}$ & Top 15 \\
\hline 218 & $\begin{array}{l}\text { Florida's Tpke \& } \\
\text { Bee Line Expwy } \\
\text { E Main St \& }\end{array}$ & $\begin{array}{l}\text { Near Florida } \\
\text { Mall }\end{array}$ & & Top 10 & $\begin{array}{l}\text { but not } \\
\text { Top 15-25 }\end{array}$ & \\
\hline 63 & $\begin{array}{l}\text { Sand Lake Rd } \\
\text { S Park Ave \& }\end{array}$ & $\begin{array}{l}\text { Apopka } \\
\text { Lockheed- }\end{array}$ & Top 25 & Top 25 & & Top 20 \\
\hline 54 & $\begin{array}{l}\text { St Hwy } 435 \\
\text { N Apopka Vineland Rd }\end{array}$ & Martin & & & & Top 25 \\
\hline 62 & $\begin{array}{l}\text { \& Clarcona Ocoee Rd. } \\
\text { US Hwy } 17-92 \&\end{array}$ & Clarcona & & & & $\begin{array}{l}\text { Top } 25 \\
\text { Top } 20 \text { not }\end{array}$ \\
\hline 78 & $\begin{array}{l}\text { St Hwy } 434 \\
\text { N Hoagland Blvd \& }\end{array}$ & $\begin{array}{l}\text { Longwood } \\
\text { Osceola }\end{array}$ & & & & 25 \\
\hline 138 & $\begin{array}{l}\text { W Vine St } \\
\text { Red Bug Lake Rd \& }\end{array}$ & Square Mall & & & & Top 25 \\
\hline 172 & $\begin{array}{l}\text { Cent Florida Greenway } \\
\text { East-West Expwy \& }\end{array}$ & Oviedo & & & & Top 20 \\
\hline 186 & Hiawasee Rd. & $\begin{array}{l}\text { Pine Hills } \\
\text { Universal }\end{array}$ & & & & Top 15 \\
\hline 215 & $\begin{array}{l}\text { I-4 \& Florida's Tpke } \\
\text { Florida's Tpke \& Cen }\end{array}$ & $\begin{array}{l}\text { Studios } \\
\text { South Orange }\end{array}$ & & & & $\begin{array}{l}\text { Top } 10 \\
\text { Top } 5 \text { not }\end{array}$ \\
\hline 222 & Florida Greenway & $\begin{array}{l}\text { County } \\
\text { Disney's Wide } \\
\text { World of }\end{array}$ & & & & $\begin{array}{l}20 \text { not } 25 \\
\text { Top } 15 \text { but } \\
\text { not in Top }\end{array}$ \\
\hline 231 & $\begin{array}{l}\text { I-4 \& World Dr } \\
\text { South Sermoran Blvd }\end{array}$ & Sports & & & & 25 \\
\hline 241 & \& East-West Expwy & Azalea Park & & & & $\begin{array}{l}\text { Top } 20 \\
\text { Top } 15 \text { but }\end{array}$ \\
\hline 248 & $\begin{array}{l}\text { University Blvd \& Cen } \\
\text { Florida Greenway }\end{array}$ & $\begin{array}{l}\text { University of } \\
\text { Central Florida }\end{array}$ & & & & $\begin{array}{l}\text { not in Top } \\
20 \text { or } 25\end{array}$ \\
\hline
\end{tabular}


- The station near Kissimmee is an important station in the fifth tier for the statewide network. This station needs to be close to a Turnpike exit for drivers coming from the southeast part of the state. Otherwise it is over 100 miles from Fort Pierce. This station also needs to be close to the heavy traffic on South Orange Blossom Trail. A location on the newly developing Osceloa Parkway between the two highways would be ideal.

- We include an $11^{\text {th }}$ station in the second tier on South Orlando Drive between the Eastern Beltway and Lake Mary Blvd in Sanford. This station usually becomes optimal in the range of 15-25 stations, but being an important component of the statewide network, we moved it up for consistency between the state and metro networks.

\section{Future Research}

Additional research could improve on these results in a number of ways. To begin with, a more detailed analysis of the Miami and Tampa metropolitan areas, similar to the Orlando network analysis, should be performed. In the statewide network, the MiamiPalm Beach area is represented by nine OD points, while the Tampa area stretching from Sarasota to Pasco County to Lakeland is represented by eight points, compared with over 100 in the Orlando intra-city network. Modeling these two metropolitan areas at the same level of detail as Orlando was beyond the limited scope of this project. While adequate for modeling longer-distance intercity trips, the level of geographic detail used for Miami and Tampa should be improved for modeling their intra-city trips.

Future research could also incorporate out-of-state trips, which were omitted from these data, limiting the traffic in the Panhandle. Also, detouring from shortest paths is not considered in the model, although we have sometimes adjusted our recommendations based on the potential for minor detouring to move a site up in the rankings. Recent developments in flow-capturing research may make it feasible to incorporate detouring behavior [54].

The model and Florida data sets could be applied to other policy questions. Decisionmakers may want to test other assumptions and strategies. In addition, the model could be applied to other alternative fuels. A denser network of CNG stations would have to take into account the locations of existing CNG stations. There is little in the model that is specific to hydrogen. Demand patterns for other alternative-fuel vehicles might be similar to those for hydrogen. 


\section{Acknowledgements}

We would like to thank the Florida Hydrogen Initiative for their support of this project. We especially wish to thank Steve Adams and Ed Levine for their enthusiastic support of our work and for serving as Executive Directors of FHI. We also thank Florida Congressman Dave Weldon for spearheading the creation of FHI. Their leadership has been essential in advancing hydrogen energy in Florida.

We thank the National Science Foundation for their support of Dr. Kuby's basic scientific research that created the initial Flow Refueling Location Model. Their funding of "Extensions of Flow-Capturing Models for Refueling of Alternative Fuel Vehicles, under NSF Grant No. 0214630, laid the groundwork for our application of the model to Florida. We also acknowledge the support of Dash Ltd., who graciously provided us with their Xpress-MP linear programming software as part of their Academic Partnership Program.

We could not have done this work without the brilliant efforts of several students. Ph.D. candidate Seow Lim at Arizona State University (ASU) programmed the greedy and genetic algorithms that solve the FRLM. Ph.D. student Jong-Geun Kim at ASU worked closely with Seow Lim to develop the GIS components of the Spatial Decision Support System, and worked closely with the faculty team to build and test the networks and data bases. Ph.D. student James Clancy at ASU was instrumental in interviewing key people in the hydrogen and rental-car industry for understanding the barriers and solutions from the corporate point of view. Marissa Williams, who recently graduated from Rollins College, did a tremendous job interviewing car renters at the Orlando airport and compiling the data.

Several people in the industry have been extremely generous with their time. Yongqiang $\mathrm{Wu}$ of the Systems Planning Office at the Florida Department of Transportation, Roberto Miquel of Cambridge Systematics in Tallahassee, and Dennis Hooker of MetroPlan Orlando provided essential transportation data and GIS layers. Margo Melendez and Anelia Milbrandt of the National Renewable Energy Laboratory in Golden, Colorado have provided data, advice, and encouragement. Bob Kelley of EV Rentals in Phoenix, AZ generously shared his insights and experience regarding renting alternative-fuel vehicles. Karen Faussett of the Michigan DOT provided information about their statewide forecasting model. We also thank the following for information they shared: John Masiello of Progress Energy in Orlando; Nicole Barber of Florida DEP; Herman Everett of NASA; Gerry Paulus of City of Mesa; and Ray Hobbs of Arizona Public Service. We have learned much about the hydrogen industry from discussions with Gene Nemanich, former President of the National Hydrogen Association; about hydrogen station location from Michael Nicholas and Marc Melaina of UC Davis; and about flowcapturing models from M. John Hodgson and Weiping Zeng of University of Edmonton. Max Wyman, of Terragenesis, Inc. in Tempe, AZ provided the original inspiration for this work.

Ann Francis at Rollins College did a marvelous job of administering this project, and we also acknowledge the essential support of Toni Holbrook and Carol Wilson. We also 
thank Mike Pasqualetti, Libby Wentz, Bryan Landry, Barbara Trapido-Lurie, Tony Brazel, Jana Hutchins, Kathy Hermanson, Carole Sassatelli, Kay Pealstrom, Joyce Hartman-Diaz, Andrea Schmitt, Tara Barton, Rebecca Bliquez, and Fatemah Dili at ASU, and Chris Upchurch at University of Utah, for their help in various ways. At Florida Atlantic University, we wish to thank Kimberly Russo, Wendy Stephens, Tobin Hindle, and Russell Ivy for their assistance. 


\section{References}

1. US Department of Energy. National Hydrogen Energy Roadmap. US Department of Energy, Washington DC (2002).

2. National Research Council. The Hydrogen Economy: Opportunities, Costs, Barriers, and R\&D Needs. The National Academies Press, Washington D.C. (2004)

3 M. Melendez. Transitioning to a hydrogen future: learning from the alternative fuels experience. National Renewable Energy Laboratory. Technical Report No. NREL/TP-540-39423 (2006).

4. J. Ogden. Prospects for building a hydrogen energy infrastructure. Annual Review of Energy and the Environment 24, 227-279 (1999).

5. M. W. Melaina. Initiating hydrogen infrastructures: preliminary analysis of a sufficient number of initial hydrogen stations in the US. International Journal of Hydrogen Energy 28, 743-755 (2003).

6. California Environmental Protection Agency. California Hydrogen Blueprint Plan, Volumes 1 and 2, (May 2005): www.hydrogenhighway.ca.gov/plan/plan.htm (Last accessed June 29, 2007).

7. California Fuel Cell Partnership. Driving for the Future: www.fuelcellpartnership.org/. (Last accessed April 30, 2004).

8. California Fuel Cell Partnership. 2004-2007 Plans: The Next Four Years:

www.fuelcellpartnership.org/fact_sheets/factsheet_04-07.html. (Last Accessed March 31, 2007).

9. California Air Resources Board. California Hydrogen Highway Network: Year-End Report to the

Legislature (Dec. 2006): www.hydrogenhighway.ca.gov/facts/yepr_fa.pdf. (Last accessed March 31, 2007.)

10. K. A. Adamson and G. Crawley. Fuel Cell Today 2006 Worldwide Survey:

www.fuelcelltoday.com/FuelCellToday/FCTFiles/FCTArticleFiles/Article_1148_Fuel\%20Cell\%20Today\%20

World\%20Survey\%202006.pdf. (Last accessed March 31, 2007).

11. Fuel Cells 2000. Worldwide Hydrogen Fueling Stations:

www.fuelcells.org/info/charts/h2fuelingstations.pdf. (Last Accessed June 29, 2007).

12. K. A. Adamson and G. Crawley. Fuel Cell Today 2007 Automotive Infrastructure Survey:

www.fuelcelltoday.com/FuelCellToday/FCTFiles/FCTArticleFiles/Article_1187_Automotive\%20Infrastructure .pdf. (Last accessed June 19, 2007).

13. M. Melendez and A. Milbrandt. Analysis of the Hydrogen Infrastructure Needed to Enable Commercial Introduction of Hydrogen-Fueled Vehicles: Preprint. NREL Report No. CP-540-37903 (2005).

14. S. Gronich. Overview of 2010-2025 Scenarios. US Department of Energy, Office of Energy Efficiency and Renewable Energy, DOE 2010-2025 Scenario Analysis Meeting: January 31, 2007:

www1.eere.energy.gov/hydrogenandfuelcells/analysis/pdfs/scenario_analysis_gronich1_07.pdf

15. Florida Hydrogen Business Partnership. 2005. Florida's Accelerated Commercialization Strategy for

Hydrogen Energy Technologies. Florida Department of Environmental Protection (May 2005):

www.dep.state.fl.us/secretary/news/2005/03/0323_02.htm (Last Accessed June 28, 2007).

16. Breakthrough Technologies Institute, Inc. State Activities That Promote Fuel Cells and Hydrogen Infrastructure Development. Washington, DC (updated October 2006):www.fuelcells.org/info/StateActivity.pdf (Last Accessed June 30, 2007). 
17. $\mathrm{H}_{2}$ Daily. GM Moves Toward Production of Fuel Cell Vehicles (June 15, 2007):

www.h2daily.com/news/gm-moves-toward-production-of-fuel-cell-vehicles-20070615-459-50.html

18. AutoCar.co.uk. Petrol and Diesel are Dead, says GM (June 11, 2007):

www.autocar.co.uk/News/NewsArticle/AllCars/225989/. (Last Accessed June 30, 2007).

19. Fuel Cells 2000. Fuel Cell Vehicles (From Auto Manufacturers): www.fuelcells.org/info/charts/carchart.pdf.

20. Fuelcelltoday.com. Iceland to Introduce Hydrogen Rental Cars (June 21, 2007):

fuelcelltoday.com/FuelCellToday/IndustryInformation/IndustryInformationExternal/NewsDisplayArticle/0,160 2,9340,00.html (Last Accessed June 30, 2007).

21. Fox News. Com. Mazda Plans Dual-Fuel Car in Japan (February 15, 2006):

www.foxnews.com/story/0,2933,184980,00.html (Last Accessed June 30, 2007).

22. G. Crawley. Fuel Cell Today Market Survey: Light Duty Vehicles Fuel Cell Today (February 2007): www.fuelcelltoday.com/FuelCellToday/FCTFiles/FCTArticleFiles/Article_1158_ MarketSurvey2007.pdf (Last Accessed June 30, 2007).

23. B. D. Solomon and A. Banerjee. 2007. A global survey of hydrogen energy research, development and policy. Energy Policy 34: 781-792.

24. B. Fowler. Rental Car Companies Hertz, Avis Announce Plans to Boost Hybrid Fleets. Environmental News Network (June 15, 2007): www.enn.com/energy.html?id=1616 (Last Accessed June 30, 2007).

25. M. J. Kuby and S. Lim. The flow-refueling location problem for alternative-fuel vehicles. Socio-Economic Planning Sciences 39, 125-145 (2005).

26. G. Conzelmann, R. Cirillo, M. Mintz, C. Macal, and M. North. Modeling the Evolution of a New Hydrogen Infrastructure Using a Complex Adaptive Using a Complex Adaptive Systems Approach. National Hydrogen Association Conference Proceedings (April 26-30 2004).

27. M. W. Melaina. Estimating relative station sizes in early hydrogen station networks. Proceedings of the National Hydrogen Association Annual Conference, Washington, D.C. (2005)

28. M.F. Goodchild, V.T. Noronha. Location-allocation and impulsive shopping: the case of gasoline retailing. In Spatial Analysis and Location-Allocation Models (Edited by A. Ghosh, G. Rushton), pp. 121-136. van Nostrand Reinhold, New York (1987).

29. R. Bapna, L.S. Thakur, S.K. Nair. Infrastructure development for conversion to environmentally friendly fuel. European Journal of Operational Research 142, 480-496 (2002).

30. S.L. Hakimi. Optimum locations of switching centres and the absolute centres and medians of a graph. Operations Research 12, 450-459 (1964).

31. C.S. ReVelle and R. Swain. Central facilities location. Geographical Analysis 2, 30-42 (1970).

32. M. A. Nicholas, S. Handy, and D. Sperling. Hydrogen refueling network analysis using geographic information systems. The Proceedings of the National Hydrogen Association, Los Angeles (2004).

33. M. A. Nicholas and J. Ogden. Detailed Analysis of Urban Station Siting for California Hydrogen Highway Network. Transportation Research Record 1983, 129-139 (2006).

34. M.J. Hodgson. A flow capturing location-allocation model. Geographical Analysis 22, 270-279 (1990). 
35. O. Berman, M.J. Hodgson, D. Krass. Flow intercepting models. In Facility Location: A Survey of Applications and Methods (Edited by Z. Drezner), pp. 389-426. Springer-Verlag, New York (1995).

36. O. Berman, R.C. Larson, N. Fouska. Optimal location of discretionary service facilities. Transport. Science 26, 201-211 (1992).

37. M.J. Hodgson. Developments in flow-based location-allocation models. In Economic Advances in Spatial Modelling and Methodology: Essays in Honor of Jean Paelinck (Edited by D. Griffith, C.G. Amrhein, J.-M. Huriot), pp. 119-132. Kluwer Academic Publishers, Boston (1997).

38. M.J. Hodgson and O. Berman. A billboard location model. Geographical and Environmental Modeling 1, 25-45 (1997).

39. M.J. Hodgson and K.E.Rosing. A network location-allocation model trading off flow capturing and p-median objectives. Annals of Operations Research 40, 247-260 (1992).

40. M.J. Hodgson, K.E. Rosing, and A.L.G. Storrier, Applying the flow capturing location-allocation model to an authentic network: Edmonton, Canada. European Journal of Operational Research. 90, 427-443 (1996).

41. M.J. Hodgson, K.E, Rosing, and J. Zhang. Locating vehicle inspection stations to protect a transportation network. Geographical Analysis 28, 299-314 (1996).

42. W. Zeng, M.J. Hodgson, I. Castillo. The pickup problem: consumers' locational preferences in flow interception. Forthcoming in Geographical Analysis.

43. M. J. Kuby, S. Lim, and K. Wang. A model for optimal location of hydrogen refueling stations: An Arizona case study. Proceedings of the National Hydrogen Association Annual Conference, Los Angeles (2004).

44. M.J. Kuby and S. Lim. Location of alternative-fuel stations using the flow-refueling location model and dispersion of candidate sites on arcs. Networks and Spatial Economics 7, 129-152 (2007).

45. Daskin. Network and Discrete Location Analysis, John Wiley and Sons, New York (1995).

46. Alp, Erkut, E., and Drezner, Z. An efficient genetic algorithm for the p-median problem. Annals of Operations Research 122, 21-42 (2003).

47. C. Upchurch, M. Kuby, and S. Lim. A capacitated model for location of alternative-fuel stations. Forthcoming in Geographical Analysis.

48 W. R. Black. Transportation: A Geographical Analysis. Guilford, New York (2003).

49 S. Hanson and G. Giuliano. The Geography of Urban Transportation ( $3^{\text {rd }}$ ed.). Guilford, New York (2004).

50 Center for Urban Transportation Studies, University of Wisconsin-Milwaukee. Guidebook on

Statewide Travel Forecasting. Federal Highways Administration, US Department of Transportation (1999).

51 KJS Associates. Statewide Travel Demand Model: Update and Calibration, Phase II. 1996

52 M. Melendez and A. Milbrandt. Geographically Based Hydrogen Consumer Demand and Infrastructure Analysis. NREL/TP-540-40373 (2006).

53 R. Kitamura and D. Sperling. Refueling behavior of automobile drivers. Transportation Research $21 \mathrm{~A}(3)$, 235-245.

54. W. Zeng. GIS-Based Facility Location Planning with Different Types of Consumers. Ph.D. Dissertation, University of Alberta, Department of Earth and Atmospheric Sciences (2007). 


\section{Appendix 1: Car Renter Survey Instrument}

\section{Florida Hydrogen Initiative Car Rental Study Rollins College/Florida Atlantic University/Arizona State University/ US Dept. of Energy}

This survey will help us understand what is needed to develop a hydrogen rental-car option for the Orlando International Airport. Hydrogen, the most common and lightest element in the universe, is produced from water or natural gas. Hydrogen cars either use an internal combustion engine to burn hydrogen or a fuel cell to generate electricity for a quiet but powerful electric motor. The only emission from either process is water vapor. Hydrogen rental-cars would be refueled by trained professionals at a limited number of convenient locations.

Please circle the appropriate items or fill in the blanks. Please write any answer that cannot be adequately expressed by checking or filling in a blank.

1. Which of the following destinations did (will) you visit on your trip to Orlando?

\begin{tabular}{|c|c|c|c|}
\hline [1] Disney World & [2] Universal Studios & [3] Sea World & [4] EPCOT \\
\hline [5] Other theme park & $\begin{array}{l}\text { [6] Downtown } \\
\text { Orlando }\end{array}$ & $\begin{array}{l}{[7] \text { Kennedy Space }} \\
\text { Ctr. }\end{array}$ & [8] Daytona Beach \\
\hline $\begin{array}{l}\text { [9] Tampa-St. } \\
\text { Petersburg }\end{array}$ & $\begin{array}{l}{[10] \text { Miami-Palm }} \\
\text { Beach }\end{array}$ & [11] Everglades & $\begin{array}{l}\text { [12] Port Canaveral } \\
\text { (cruises) }\end{array}$ \\
\hline
\end{tabular}

2. How long was your stay in the Orlando area? days

3. Rank the importance of the following features in convincing you to rent a hydrogen car on a future trip to Orlando, on a scale from 1 (not important) to 5 (very important)?

Scale is: 1 (not important) to 5 (very important)
a. Map of refueling stations in Florida and Orlando
$[1] \quad[2] \quad[3] \quad[4] \quad[5]$
b. Able to exchange for a gasoline car at no extra cost
$[1] \quad[2] \quad[3]$
[4] [5]
c. Priority parking at theme parks
$[1] \quad[2] \quad[3] \quad[4] \quad[5]$
d. Using a pollution-free vehicle
$[1] \quad[2] \quad[3] \quad[4] \quad[5]$
e. Fuel cost per mile comparable to gasoline
$[1][2] \quad[3] \quad[4] \quad[5]$
f. Opportunity to test-drive first
g. Driving range of vehicle (miles between refuelings)
$[1] \quad[2] \quad[3] \quad[4] \quad[5]$
h. Vehicle performance (acceleration, noise)
$[1] \quad[2] \quad[3] \quad[4] \quad[5]$
i. Opportunity to experience a new technology
$[1] \quad[2] \quad[3] \quad[4] \quad[5]$
j. Availability of insurance
$[1][2] \quad[3] \quad[4] \quad[5]$
k. Full-service refueling by a trained attendant
$[1][2] \quad[3] \quad[4] \quad[5]$
1. On-call, roadside repair/refueling service

$[1][2] \quad[3] \quad[4] \quad[5]$
$[1][2] \quad[3] \quad[4] \quad[5]$ 
4. How far out of your way would you be willing to drive for a hydrogen refueling station, without you considering it a hassle?

[1] up to 1 mile [2] up to 3 miles [3] up to 5 miles [4] more than 5 miles

5. Would you be willing to pay more money to rent a hydrogen vehicle? [1] Yes [2] No

If yes, how many more dollars per day? [1] \$0-\$5 [2] \$5-\$10 [3] \$10-\$15 [4] \$15-\$20

If no, would you be willing to rent a hydrogen car at a lower price? [1] Yes [2] No

6. Please rate your understanding of hydrogen vehicle and fuel-cell technology prior to completing this survey:

[1] Never heard of it [2] Vague awareness [3] Some knowledge of how it works

[4] Clear understanding of how it works

7. Please tell us a little about yourself:

Age: [1] 20-29 [2] 30-39 [3] 40-49 [5] 50-59 [6] 60-69 [7] 70 and above

Education: [1] less than high school [2] high school [3] college graduate

[4] post-graduate degree

Marital Status: [1] Single [2] Married

Number of children: [0] [1] [2] [3] [4] [5] [6 and above]

Occupation: [1] student [2] private industry/business [3] military

[4] government/education [5] retired [6] other

Origin: (What state or country do you consider home?)

Household income: [1] under \$30K [2] \$30K-49K [3] \$50K-99K [4] over \$100K

Thank you very much for your cooperation. 


\section{Appendix 2}

\section{Statistical Relationships Between Demographic Characteristics and Responses}

We analyzed the survey results statistically to determine if the demographic characteristics of the renter significantly influenced their responses. We used analysis of variance (ANOVA) to search for relationships between the opinion variables and gender, marital status, education, and income. Our tests were done at the $95 \%$ confidence level or as alternatively stated, the $5 \%$ significance level (also known as the alpha level).

There are no statistically significant variations in survey response on all opinion questions BY Marital Status.

For Gender, there are seven (7) questions that have significant differences (M vs F). They are:

\begin{tabular}{|l|l|l|l|l|}
\hline & $\begin{array}{l}\text { Male } \\
\text { Mean }\end{array}$ & $\begin{array}{l}\text { Female } \\
\text { Mean }\end{array}$ & F-TestValue & Significance \\
\hline Ability to Exchange & 3.68 & 4.10 & 12.98 & .000 \\
\hline Cost Per Mile & 4.21 & 4.52 & 11.73 & .001 \\
\hline Vehicle Performance & 3.85 & 4.22 & 14.69 & .000 \\
\hline Road Service & 4.18 & 4.53 & 14.50 & .000 \\
\hline $\begin{array}{l}\text { How Often do You Rent } \\
\text { Cars }\end{array}$ & 1.57 & 1.37 & 6.96 & .009 \\
\hline How Far to Station & 2.52 & 2.33 & 4.28 & .039 \\
\hline $\begin{array}{l}\text { Understanding of } \\
\text { Technology }\end{array}$ & 2.72 & 2.13 & 37.92 & .000 \\
\hline
\end{tabular}

Conclusions: on first 4 opinion questions above females are more concerned than males. On frequency of rental, distance to station, and understanding of technology males indicate higher use, more concern with distance to station, and they say they understand the technology at a higher level.

For Education, there are four (4) significantly different responses.

\begin{tabular}{|l|l|l|l|l|l|}
\hline & $\begin{array}{l}\text { High } \\
\text { School } \\
\text { Mean }\end{array}$ & $\begin{array}{l}\text { College } \\
\text { Mean }\end{array}$ & $\begin{array}{l}\text { Grad } \\
\text { School } \\
\text { Mean }\end{array}$ & F-Test Value & Significance \\
\hline Pollution Free & 4.52 & 4.41 & 4.19 & 3.20 & .042 \\
\hline $\begin{array}{l}\text { Refueling by } \\
\text { trained agent }\end{array}$ & 4.28 & 3.88 & 3.99 & 4.69 & .010 \\
\hline $\begin{array}{l}\text { How Often do You } \\
\text { Rent Cars }\end{array}$ & 1.28 & 1.45 & 1.60 & 4.18 & .016 \\
\hline Lower Price & 1.10 & 1.02 & 1.00 & 5.13 & .007 \\
\hline
\end{tabular}

Conclusions: people with higher education levels are less concerned with renting a pollution-free car, refueling by trained agent, and lower price. Higher education persons rent cars more frequently. 
For Income there are two (2) significantly different responses.

\begin{tabular}{|l|l|l|l|l|l|l|}
\hline & $\begin{array}{l}\text { Less than } \\
\mathbf{\$ 3 0 K}\end{array}$ & $\begin{array}{l}\mathbf{\$ 3 0 K}- \\
\mathbf{\$ 4 9 K}\end{array}$ & $\begin{array}{l}\mathbf{\$ 5 0 K}- \\
\mathbf{\$ 9 9 K}\end{array}$ & $\mathbf{\$ 1 0 0 K}+$ & $\begin{array}{l}\text { F-Test } \\
\text { Value }\end{array}$ & Significance \\
\hline $\begin{array}{l}\text { Understanding } \\
\text { of Technology }\end{array}$ & 2.00 & 2.22 & 2.30 & 2.61 & 4.89 & .002 \\
\hline $\begin{array}{l}\text { How Often do } \\
\text { You Rent Cars }\end{array}$ & 1.00 & 1.41 & 1.32 & 1.65 & 7.31 & .000 \\
\hline
\end{tabular}

Conclusions: Higher income persons believe they understand the technology better, and in general higherincome persons rent cars more often. 


\section{Appendix 3}

\section{Spatial Decision Support System for Locating Hydrogen-Refueling Stations}

This appendix is based on a draft chapter from the future dissertation of Seow Lim

\section{Software Architecture of the FRLM-SDSS}

We implement the FRLM-SDSS by extending the ArcGIS Desktop interface using the ESRI ArcObjects and Microsoft .NET technologies. It provides an intuitive user interface for data input, data conversion, model execution options, and display results.

Workflow

Figure 1 shows the workflow normally performed when working with the FRLM-SDSS. Detailed information about each step is discussed in the next sections.

- Work with the input data using ESRI ArcGIS Desktop products, such as ArcMap and ArcCatalog to create GIS data in the ArcGIS personal geodatabase format. This includes generating the shortest path between origin and destination using ArcGIS Network Analysis tool.

- Run the ConvertData custom .NET ArcGIS command implemented as part of the SDSS to convert the input GIS data in the personal geodatabase to the FRLM native network data format in a Microsoft Access database.

- Invoke the greedy or genetic algorithms implemented as custom .NET ArcGIS commands. User can specify parameter values, such as number of facilities, fixed facilities, objective type, and vehicle range, before running the algorithm. The algorithm read in the data from the FRLM native network data in the Access database.

- After completing the run, the algorithm outputs the results to the ArcMap window, text file and the Access database. Results include the selected facilities, covered routes, time taken to solve the problem, and percentage of flow covered. 


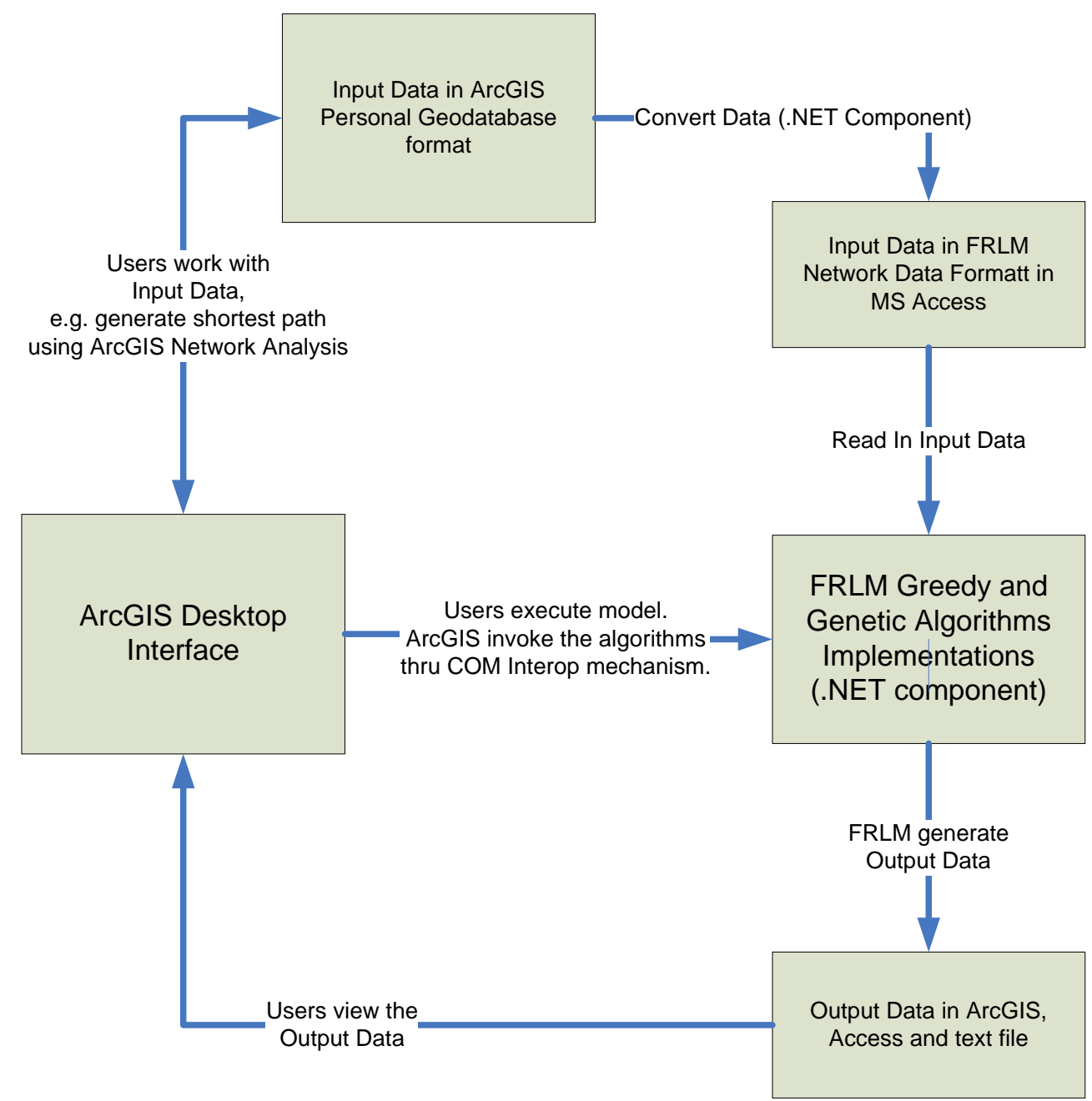

Figure 1: Workflow for the SDSS

Input

The inputs to the SDSS include spatial and non-spatial data. The spatial inputs to the SDSS are in the form of ESRI ArcGIS feature data class. Users build the input data by manipulating spatial data using ArcGIS Desktop. The five input layers for the SDSS are:

1. Population centers point layer. This layer can be derived from spatial manipulation of an underlying population data set. For instance, the user can define zones, and then the SDSS would find the centroids, automatically move them to the nearest road, and compute the populations within the zone. This layer forms the origin and destination (OD) pairs of the network.

2. Junctions point layer. This layer is derived from the junctions of the road network. Junctions are also important for turns in the shortest path algorithm.

3. Candidate facilities point layer. This layer defines the candidate facilities for hydrogenrefueling stations. The population centers and junctions layers form the basis of this layer. 
4. Road network line layer. This layer defines the road network of the region where hydrogen-refueling stations are to be allocated. All the population centers and candidate facilities must be connected to a line in the road network. We also use the length and road type (e.g. Interstate, tool road, us highway, local street) of the road segments to determine the shortest paths based on travel time. Travel time for every road segment is estimated using length divided by the driving speed corresponded to the road type.

5. Shortest path routes layer. This layer is obtained by using the closest facility feature of ArcGIS network analysis tool. By using the origin-destination pairs in the population centers layer, ArcGIS network analysis calculates the shortest path for every origindestination pair using the shortest path algorithm. We use travel time as the impedance of each road segment for calculating the path with the shortest travel time.

The non-spatial input data to the SDSS includes vehicle range, number of facilities to be built, and trip table for all origin and destination pairs.

\section{Data Conversion}

We implement a data conversion tool using .NET and ArcObject to convert data in ArcGIS format into tables in Microsoft Access. These tables in Access form the input data for the heuristic algorithms. The tables in Access are simplified representations of the data in ArcGIS format, as they only contain the connectivity information for the origin-destination, candidate facilities, road network segments, and shortest path routes. The real-world locations and shapes of the objects are not preserved when converting from ArcGIS format to Access format, because such information is not needed by the algorithms.

In the GIS, a road segment that connects two nodes can comprise of multiple lines to represent the real location and shape of the road in the real-world. The real-world location of the nodes, such as origins, destination, and candidate facilities, are also preserved. Such information is important in the GIS because both input and output data can be displayed on a map with a realistic looking road network.

The data conversion algorithm produces the simplified network model by performing spatial analysis on the GIS road network to determine the connectivity of the network. In the simplified network model, the link between two nodes is represented by a line associated with the corresponding impedance value, such as length or traveling time. The SDSS loads the simplified network from the database and initialize the network on the computer's memory before performing the computational search. Without the one-time data conversion, the SDSS would have to perform spatial analysis on the GIS network every time before executing the search algorithms. This would have introduced redundant computational time to the SDSS.

\section{Algorithms Execution}

After the input data layers are converted, the user can execute the algorithms. The user has the choices of selecting different algorithms to the problem. The algorithms include greedy, greedy substitution and genetic algorithms. These algorithms are implemented using the Microsoft .NET programming framework. The SDSS extends ArcGIS Desktop interface by allowing users to 
invoke the algorithms using customized buttons and dialogs from ESRI ArcMap application. Figure 2 shows the custom buttons on ArcMap that can be clicked by user to invoke the greedy and genetic algorithms, in addition to the data conversion tool.

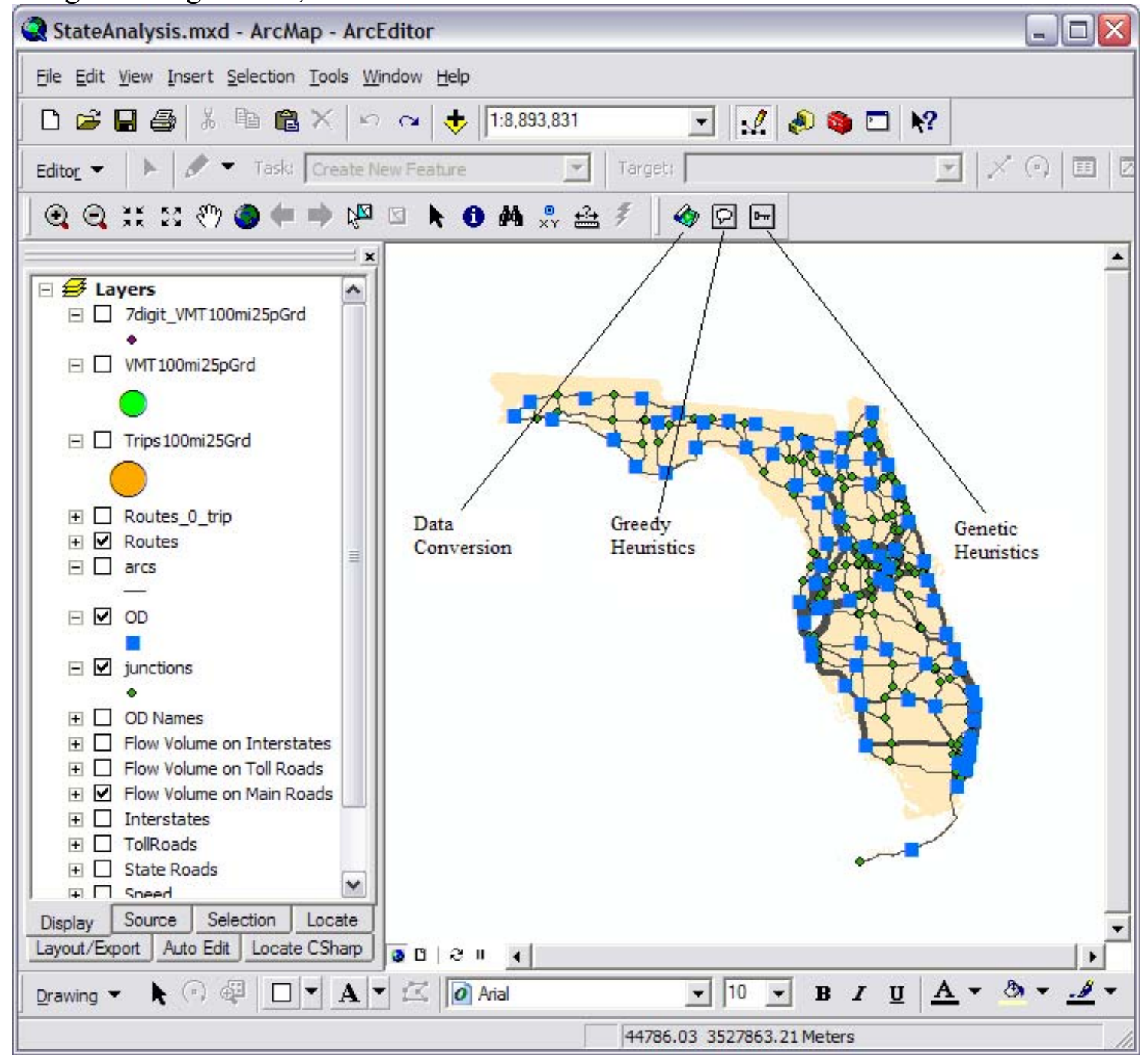

Custom FRLM-SDSS ArcMap buttons

Figure 2:

For each algorithm, users have the options to specify different parameters for the algorithms. For instance, users can specify the vehicle range, the number of facilities, and the objective to be maximized. Users can also specify refueling stations that already exist on the network. In this case, the algorithm will always include the specified facilities in the final selection of optimal refueling stations. This is useful in cases where existing hydrogen-refueling stations are already built within the network. These execution options enable the users to perform scenario analysis and study the results of different scenarios. Figure 3 and 4 show the execution options for running the greedy and genetic algorithms. 
The parameters that are common for both the greedy and genetic algorithms are as follow:

- Vehicle Range: The vehicle range of the hydrogen vehicle. The unit is based on the unit of the GIS road layers, usually in meter.

- Number of facilities: The total number of hydrogen-refueling facilities to be built, including the fixed facilities.

- Objective Type: The objective to maximize. For "Number of Trips", the model maximizes the total number of trips covered by refueling facilities. The number of trips for every route is stored in the trip table. For "Unweighted Routes", every route is considered to have the weight of one. This objective is useful for validating the model and data. For "Vehicle Mile Trip", the model maximizes the total number of vehicle miles times the number of trips for all the paths that are covered by the refueling stations. For example, if shortest path route A has 100 trips with length of 10000 meters, and shortest path route $\mathrm{B}$ has 100 trips with length of 5000 meters, the model will attempt to cover route A first since it has double the number of "Vehicle Mile Trip" of route B. "Unit Length Per Mile" is the number of length unit in a mile. For example, if the unit is meter, then "Unit Length Per Mile" is 1609.3. This value is used by the model to calculate the "Vehicle Mile Trip" for every path.

- Fixed Facilities: These are the existing hydrogen refueling stations in the network. User specifies the facility ID of these facilities ID from the network model. For example, the input parameter of "10 23154 " specifies that facility 10, 23 and 154 as fixed facilities. The "Number of Facilities" includes the facilities specified in this field. If the "Number of Facilities" is five, and three fixed facilities are specified in this field, the model will search for two more facilities to maximize the objective.

- Show selected facilities: Determine whether selected facilities will be output on the GIS after the execution of the algorithm.

- Show covered routes: Determine whether routes covered by the selected facilities will be output on the GIS after the execution of the algorithm.

Algorithm-specified parameters, such as iterations, number of generations, are covered in the later chapter specified to the algorithms. 


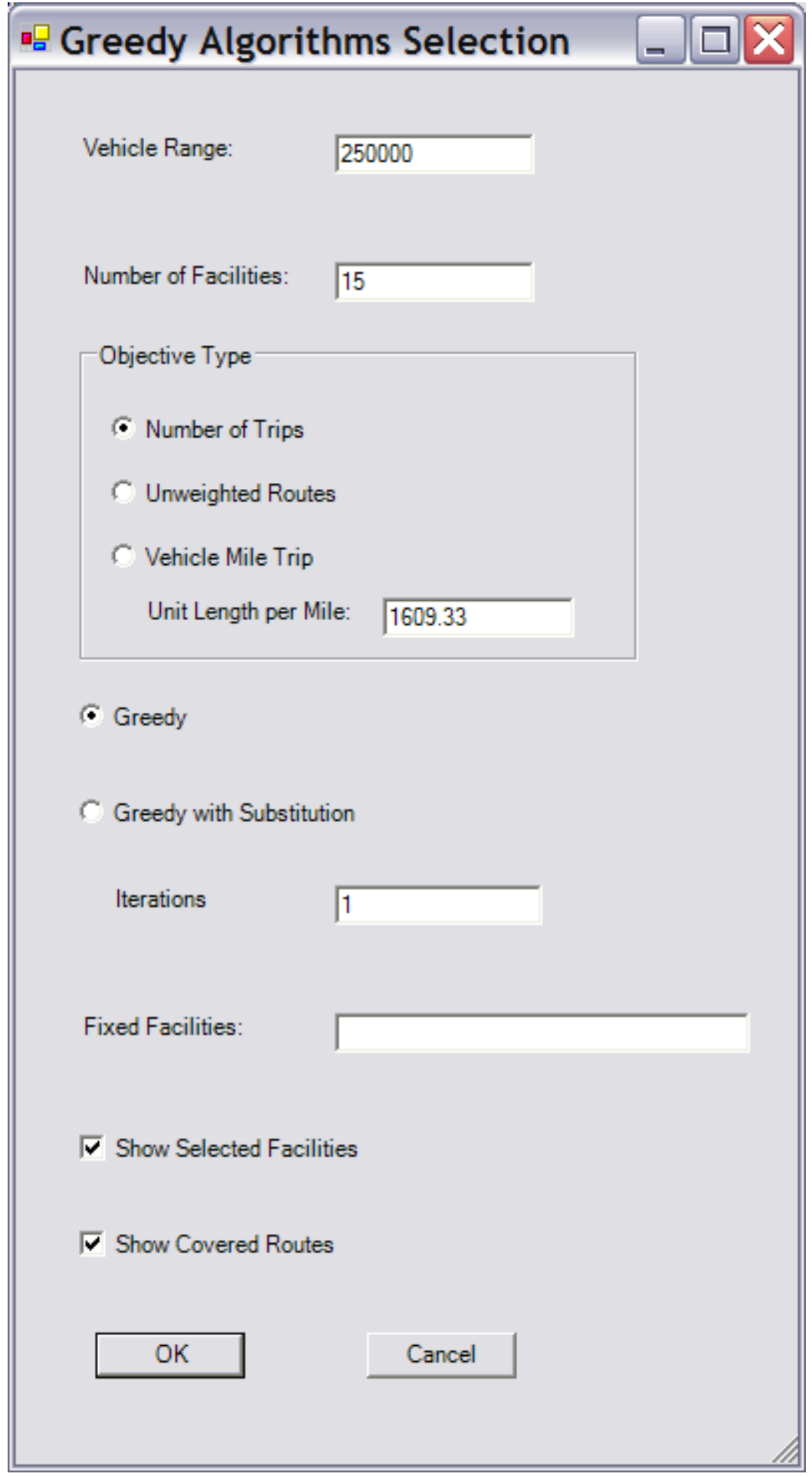

Figure 3: Execution Options for Greedy and Greedy Substitution Algorithm 


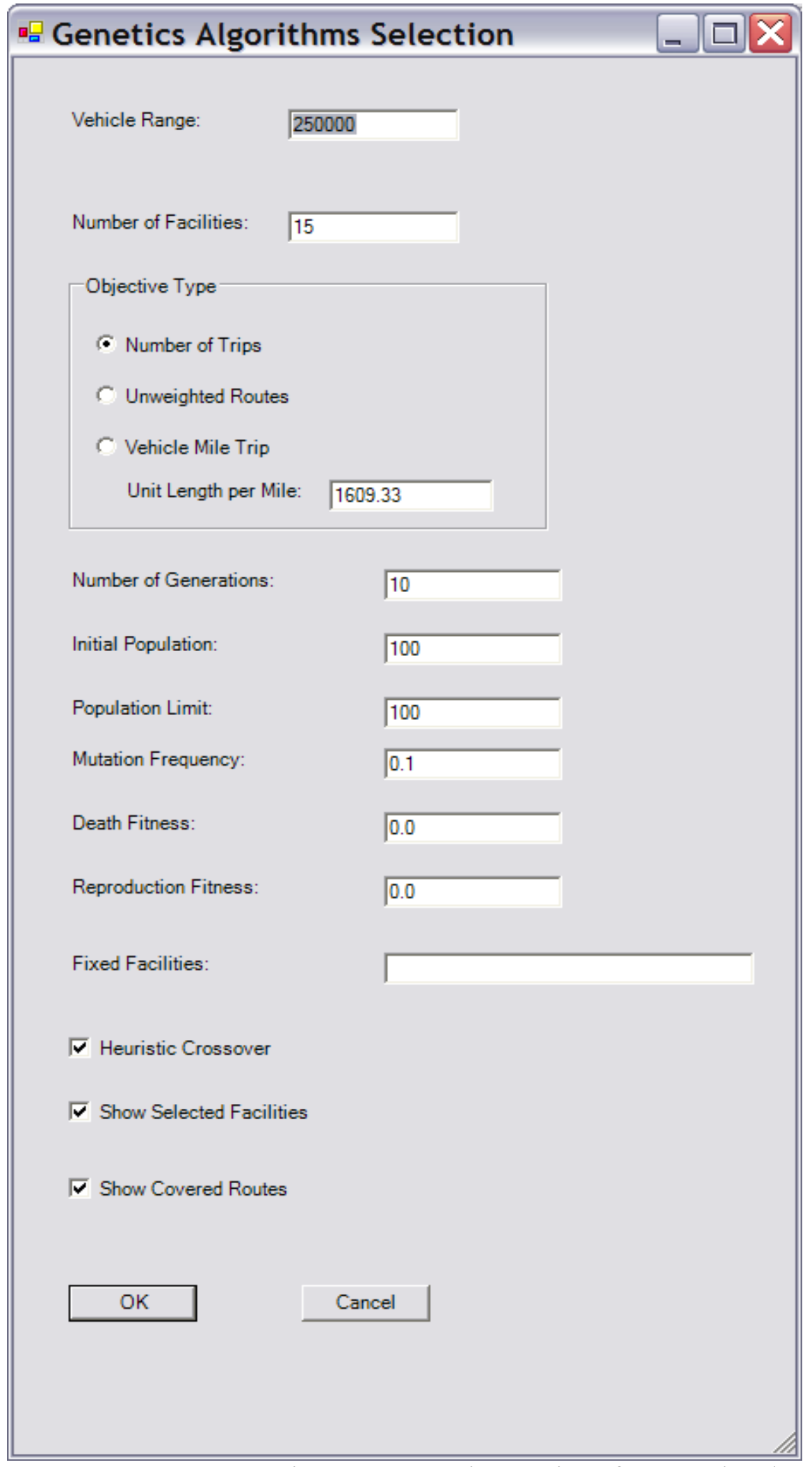

Figure 4: Execution Options for Genetic Algorithm 
Output

Once the algorithm completes its execution, it output the results to the map and a text file. The map output of the model consists of the following:

- Selected refueling stations are output as selected features in the junction layer.

- Covered routes are output as selected features in the shortest path route layer.

The user can easily export the selected features to separate feature data class using ArcGIS data export tool. The SDSS also output detailed results, such as percentage covered, paths refueled information, into a text file. Figure 5 shows the map output of an algorithm run. The gas stations represent the selected facilities, while highlighted routes represent covered routes. Note that the routes displayed on the map are different from road segments. Every route is comprised of one or more road segments. From the output map, what we see are essentially road segments that are part of at least one covered route. 


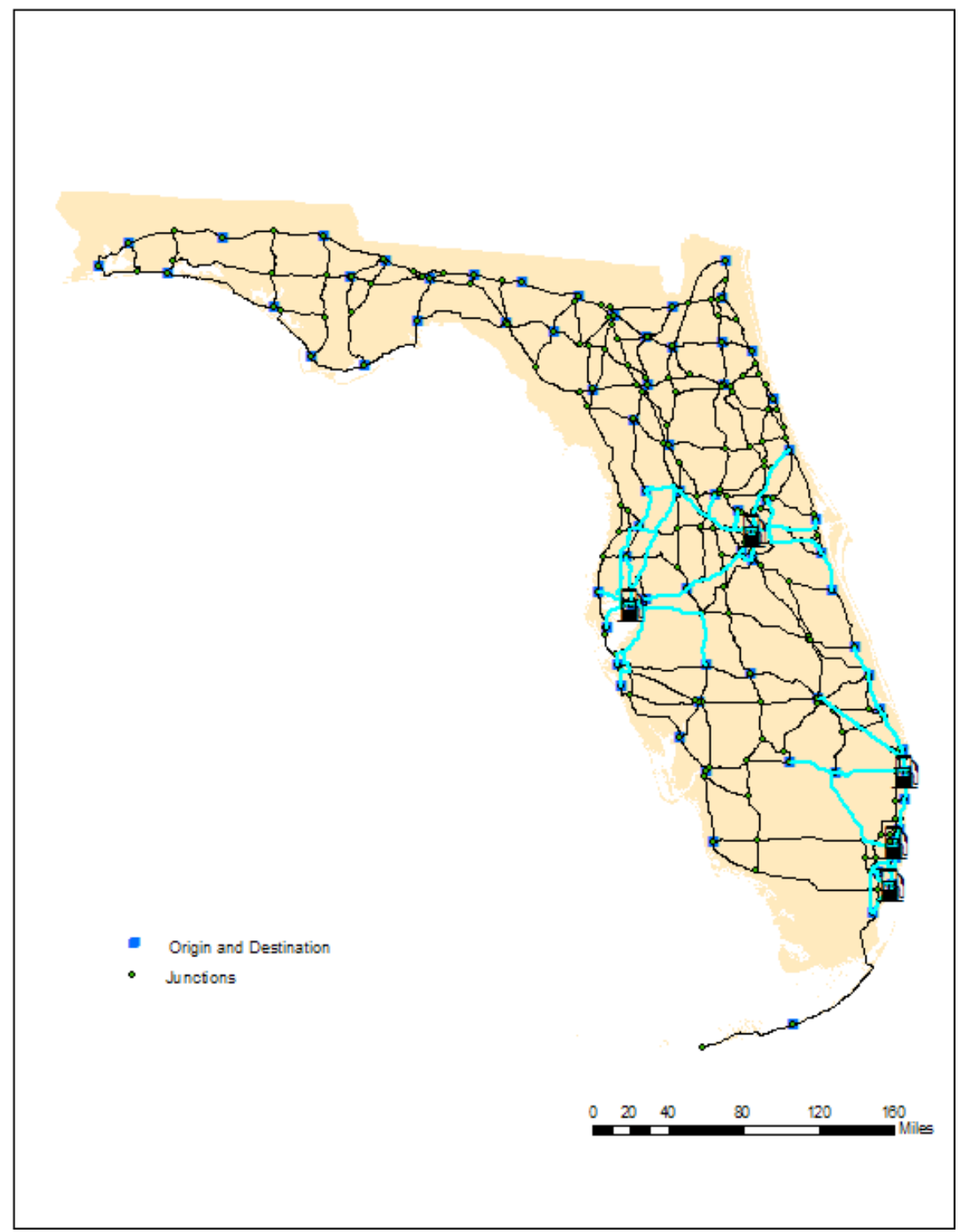

Figure 5: Sample output of an algorithm run 


\section{Solving the Flow Refueling Location Model}

Kuby and Lim (2005) originally solved the FRLM problem by using branch-and-bound algorithm. While branch-and-bound algorithm is able to obtain optimal solution for the problem, it would usually take too long to solve for a complex real-world complex problem. Thus, we implement heuristic algorithms, such as greedy and genetic, within the SDSS to help obtain solutions within a more reasonable timeframe.

To solve the FRLM using brand-and-bound, we develop a program to generate all the possible facility combinations for every path. These combinations are determined exogenous to the FRLM, and form the input to the model. The generation of these combinations can be very computational intensive, if the network has long paths with large number of facilities. With heuristic algorithms, we evaluate facility combination (i.e. solution to the problem) as part of the search algorithm, and thus we do not need to pre-generate the combinations of facilities for every path.

\section{Greedy and Greedy with Substitution Algorithms}

The greedy algorithm selects one facility at a time to maximize the additional flow covered. The selected facility is then fixed in the selected set. The algorithm repeats the selection of one facility at each iteration until it selects the total number of facilities it needs (Daskin 1995).

The greedy-with-substitution algorithm is based on the greedy algorithm. However, after each new facility is added, other facilities in the selected set are substituted with facilities in the nonselected set. If the substitution increase the total flow covered, the substitution that yields the most improved solution are preserved. The user can specify the number of iterations of substitution from one to four. The algorithm attempts to substitute one facility in the selected set with facilities from the non-selected set one at a time. If the substitution improves the solution, the algorithm will attempt to substitute one additional facility in the selected set with facilities from the non-selected set until the number of iterations is reached. If no substitution improves the solution, no additional substitution will be performed for that particular solution set. Refer to chapter three for more information regarding greedy and greedy substitution algorithms.

\section{Genetic Algorithms}

Genetic algorithms are heuristic search algorithms that iteratively evolve into better solutions, similar to the process of biological evolution (Alp, Erku and Drezner 2003). In genetic algorithms, solutions randomly interact with each other to produce new solutions. Just like the process of evolution, where the fittest survive, genetic algorithms tend to keep the good solutions while eliminating the poor solutions. One of the common applications of genetic algorithms is to perform combinatorial search. The FRLM is a combinatorial search problem, where we select $p$ facilities out of $n$ candidate facilities to maximize the flow covered. Thus, it would be valuable to investigate the performance and effectiveness of genetic algorithms in solving the FRLM. 
The implementation of genetic algorithms is unique in every problem. The encoding, fitness function, population size, new member generation, mutation frequency, replacement and termination need to be designed and fine-tuned for every problem.

Encoding is the strategy of representing the solutions of a search problem in terms of chromosomes. For location-allocation problem, a set of $p$ number of candidate facilities can form a chromosome in the implementation of genetic algorithms.

The fitness function is generally the same as the objective function, which determines how fit a particular chromosome or solution is. For maximization problem, such as the FRLM, the higher the value of the objective function or fitness function, the better the solution is.

Population size determines the number of solutions or chromosomes the genetic pool has at one time. Large population size provides higher solutions diversity, but also increases the computation time, as the genetic algorithms need to perform more operations and calculations.

New member generation is the crossover algorithm that determines how new solutions are produced from two old solutions. Mutation is the mechanism of introducing noise into the solutions set to help the algorithms escape from local optima situations. Replacement and termination determines how one generation of solutions is replaced by the next generation.

In this SDSS, we implement genetic algorithm to search for optimal solutions for the FRLM. The genetic algorithm is a lot more computing extensive than the greedy and greedy substitution algorithms. However, depending on the problem and conditions, the genetic algorithm may have a better chance to obtain better solution than the greedy algorithm. Refer to chapter four for more information about solving the FRLM using genetic algorithm.

\section{Application of the SDSS: The Florida Hydrogen Initiative}

The goal of the Florida Hydrogen Initiative (FHI) is to "move Florida to the forefront of the nation's hydrogen economy" (Florida Hydrogen Initiative 2007). Funded by the FHI, the FRLMSDSS is applied to the Orlando and Florida statewide road network to locate hydrogen-refueling stations. We collect and build the input spatial data for both case studies. Table 1 shows the spatial input layers for both the Orlando and Florida case studies.

\begin{tabular}{|l|l|l|}
\hline OD Centers & \multicolumn{1}{|c|}{ Orlando } & \multicolumn{1}{c|}{ Florida } \\
& $\begin{array}{l}\text { Aggregated from TAZs } \\
\text { defined by FDOT. }\end{array}$ & $\begin{array}{l}\text { County Centroids. Some } \\
\text { large urban counties are } \\
\text { disaggregated, while some } \\
\text { small rural counties are } \\
\text { aggregated. }\end{array}$ \\
\hline Junctions & $\begin{array}{l}\text { Defined by analysts at all } \\
\text { intersections of arcs. }\end{array}$ & $\begin{array}{l}\text { Defined by analysts at all } \\
\text { intersections of arcs. }\end{array}$ \\
\hline Candidate Facilities & $\begin{array}{l}\text { Combines the OD and } \\
\text { junctions layer. }\end{array}$ & $\begin{array}{l}\text { Combines the OD and } \\
\text { junctions layer. }\end{array}$ \\
\hline Road Network & FDOT and ESRI layers & FDOT layers \\
\hline
\end{tabular}




\begin{tabular}{|l|l|l|}
\hline Shortest Path Routes & $\begin{array}{l}\text { Generated using ArcGIS } \\
\text { Network Analysis Closest } \\
\text { Facilities, and specified the } \\
\text { population centers layer as } \\
\text { the facilities. }\end{array}$ & $\begin{array}{l}\text { Generated using ArcGIS } \\
\text { Network Analysis Closest } \\
\text { Fopulatios, and specified the } \\
\text { the facilities. }\end{array}$ \\
\hline
\end{tabular}

Table 1: Input Spatial Layers to FHI

The trip table for Orlando is derived from the aggregation of Florida Department of Transportation (FDOT) TAZ-TAZ weekday flows. The trip table for Florida statewide is generated from a modified gravity model formulation. We could not obtain the estimated statewide long-distance trip tables from Florida as it appears very few states collect those kind of information anymore.

We use the SDSS to locate hydrogen-refueling stations using different input parameters, such as the number of facilities. With the output capability of the SDSS, we can easily compare the results and visualize the locations of refueling stations generated by the algorithms.

\section{User's Comments}

We solicited some comments from the users of the SDSS regarding the system to help us determine what are the things that we did right, as well as the areas for improvements. Below are excerpts from some of the users.

\section{Zhixiao Xie - GIS Expert}

GIS was useful in several aspects for the FHI-SDSS: data preparation (network, nodes), network analysis (shortest distance, for example), visualization of model results. The visualization function is especially indispensable. It helped us effectively combine general transportation infrastructure, census data and local knowledge when simplifying network and determining appropriate nodes. Visualization was also essential for presenting and evaluating model results. To general users, it is the first impression of the model. For me, it was based on the location maps of the proposed stations that we were able to examine the differences between model results and intuition and identify some possible model issues for improvement. So GIS-based visualization is possibly one of components I like more and it was implemented very nicely. Some more details on model parameters and methods in the form of help file may be useful to end users, since it is the key for successfully utilizing the model, but not absolutely necessary if it is only intended for research purpose.

\section{Conclusions}

In order to transition to a hydrogen-based transportation infrastructure, one of the steps is to build a hydrogen-refueling infrastructure so that hydrogen fuel is easily accessible to consumers. The FRLM-SDSS is a powerful tool to help decision makers locate hydrogen-refueling stations in regions of different scales during the initial built-up of hydrogen-refueling infrastructure. By integrating GIS with the FRLM, the SDSS combines the mapping and spatial analysis of a GIS with the optimization capability of an operations research model. In the SDSS, we implement the 
greedy, greedy substitution, and genetic algorithms to solve the FLRM problem. This provides the users more options to solve the problem during the decision making process. It also allows us to study the effectiveness of these different heuristic algorithms in solving the FRLM problem. The SDSS has been utilized by the Florida Hydrogen Initiative to determine the optimal locations for hydrogen-refueling stations in the Orlando metropolitan and the state of Florida.

For future research, the FRLM-SDSS can be applied to larger scale areas, such as the U.S. or the continental Europe, for locating hydrogen or other alternative-fuel refueling stations. The SDSS can also be utilized to locate refueling stations used for different transportation modes, such as the railroad system. For theoretical type of research, different heuristic algorithms, such as tabu search and simulated annealing, can be implemented to solve the problem. This will allow us to compare the effectiveness of these algorithms with the greedy and genetic algorithms. The FRLM can also be extended to consider traffic flows that can detour from the shortest path to refuel. 


\section{Appendix 4 \\ Data Processing Steps}

1. Florida Atlantic University (FAU) obtains a database for a GIS road network.

2. FAU — consulting with Rollins College (RC) — combines FDOT traffic analysis zones (TAZs) into a set of aggregated TAZs that are the origins and destinations of trips in the model. These typically number over 100 .

3. FAU — consulting with RC — selects a single point, known as a centroid, to represent each aggregated set of TAZs. These centroids serve as the origin and destination points (ODs) of all trips in the model, as in most transport models.

4. FAU — consulting with RC - selects a subset of more important roads to include in the model network for serving these origin and destination points.

5. FAU and ASU obtain speed data for all road segments.

6. The teams obtain or generate a table of traffic volumes among TAZs.

7. The FAU team combines the traffic flows from the FDOT TAZ level to the aggregated ODs.

8. The FAU team sends these GIS databases to ASU for further processing.

9. ASU classifies OD nodes and junction nodes within the network.

10. Using SDSS software developed for this project, ASU combines smaller segments of roads into single arcs connecting nodes. These arcs are geographically accurate, not stylized straight lines as in many networks. Distances and travel times (based on speed data) of all subsegments of an arc are totaled.

11. ASU generates shortest travel-time paths for every OD pair. Paths for ij are considered identical to ji. With $100+$ OD nodes, this is typically $5000+$ paths $(100 \times 100 / 2)$.

12. ASU chooses a sample of OD pairs and makes maps of their shortest travel time paths. ASU sends these maps to FAU and RC to spot-check the realism of routes generated by the model.

13. Based on their local knowledge, FAU and RC check whether these paths follow a route likely to be used by real drivers. For unlikely paths, we determine the cause of the poor routing, such as missing roads, and unrealistic road speeds. After some experimentation, we lowered posted speed limits by $15 \%$ to estimate the actual average speed on all roads that are not limited-access highways. Steps 12 and 13 are repeated until satisfactory paths are generated. This is the first step of model calibration to reality.

14. Using all the inputs generated thus far, ASU solves the Flow-Refueling Location Model (FRLM) to optimally choose the 10 best refueling locations.

15. ASU maps the optimal locations, superimposed on maps of the traffic flow on each arc (created by our SDSS software by summing traffic over all paths using each arc).

16. ASU sends these maps and other outputs to FAU and RC to check whether the locations and traffic flows are realistic, and compare with known traffic flows. For unlikely locations and flow volumes, we study the network for causes, such as missing roads, missing access points, overaggregation of TAZs, and poor choice of centroids. Local knowledge of the main traffic generator points and the road usage is invaluable in this process. We then make changes to the network as far back as Step 9, and repeat Steps 916 until satisfactory results are achieved.

17. We then solve the model for 1-25 optimal new facilities to generate a base case.

18. Finally, we define and solve a variety of scenarios, such as: 
- Maximizing the number of trips that can be refueled

- Maximizing the VMT of trips that can be refueled

- Maximizing trips and VMT weighted by the NREL hydrogen demand model

- Experimenting with different vehicle ranges 


\title{
Appendix 5 \\ CNG Stations in Florida
}

\author{
Station Name \\ Energy Services Center/ Fort Pierce Utilities Authority \\ City Gas Company of Florida \\ NASA - Kennedy Space Center \\ City Gas Company of Florida \\ Okaloosa Gas District \#1 \\ City of North Miami \\ TECO Peoples Gas \\ Teco/Peoples Gas \\ City of Sunrise Mobile Station \\ Peoples Gas System, Inc. \\ Shalimar \\ Palm Beach County Fleet Mgmt. \\ City of North Miami Police Station \\ City of Sunrise \\ Palm Beach County \\ Space Coast Coalition - Titusville CNG Station \\ Motorfuelers, Inc. \\ TECO Peoples Gas \\ Cash's Amoco \\ Donnini Enterprises - Light House Shell
}

Address

1701 S 37th St

955 E. 25th St.

Bldg. M6-688

8150 N.W. 90th St.

136 Lewis St.

776 N.E. 125th St.

301 Maple Avenue

301 Maple Ave

4747 N. Nob Hill Rd.

1400 Channelside Dr.

1015 6th St SW

1250 Eglin Pkwy.

345 S Congress Ave

6869 Municipal Dr

1855 N.E. 142nd St.

700 NE 124th St

8300 NW 44th Street

14150 NW 8th Street

4401 NW 103rd Avenue

3700 Belvedere Rd

4235 Capron Road N

13790-B 49th St N

Kennedy Space Ctr

618 W Internat'l Speedway Blvd.

345 S.W. 24th St.

9730 Hwy. A-1-A
City

Fort Pierce

Hialeah

Medley

Niceville

North Miami

Panama City

Panama City

Sunrise

Tampa

Winter Haven

Shalimar

Delray Beach

Milton

North Miami

North Miami

Sunrise

Sunrise

Sunrise

West Palm Beach

Titusville

Clearwater

\section{Access}

Public - call ahead

Public - call ahead

Public - call ahead

Public - call ahead

Public - call ahead

Public - call ahead

Public - call ahead

Public - call ahead

Public - call ahead

Public - call ahead

Public - call ahead

Public - card key after hours

Public - card key at all times

Public - card key at all times

Public - card key at all times

Public - card key at all times

Public - card key at all times

Public - card key at all times

Public - card key at all times

Public - card key at all times

Public - credit card at all times

Public - see hours

Public - see hours

Fort Lauderdale Public-see hours

Palm Beach Gardens Public - see hours

Source: National Renewable Energy Laboratory, Alternative Fuels Data Center (April 12, 2007). 


\title{
FLORIDA HYDROGEN INITIATIVE \\ Sub-conticetor Find Report \\ Methanol Fuel Cell Vehicle Charging Station (Previously: HyTech Rest Area Project)
}

\author{
November 9, 2012
}

\section{Principal Investigator:}

Dr. Michel Fuchs

michel.fuchs@enerfuel.com, 561-868-6720, ext 239

\section{Sub-contractor:}

- Dr. Amir Abtahi, Florida Atlantic University, Boca Raton, FL

- Ryan Greenough, Technological Research and Development Authority, Melbourne, FL

- Robert Penoyer, Gasoline Equipment Systems, Melbourne, FL

- Robert Anderson, Anderson Consulting, Palm Harbor, FL

\section{Working Partners:}

- George Gurlaskie, Progress Energy, Orlando, FL

- The Florida Turnpike Enterprise, Orlando, FL

Project Period: October 4, 2007 to June 30, 2012

Delivered to:

\author{
Dr. David Block \\ Florida Solar Energy Center \\ University of Central Florida \\ 1679 Clearlake Road \\ Cocoa, Florida 32922 \\ Contract No. 20126050, PO: 191461
}

This report does not contain any proprietary, confidential or otherwise restricted information

\section{HHENERFUEL}

1501 Northpoint Pkwy, Suite 101

West Palm Beach, Florida 33407

561-868-6720

www.enerfuel.com

NC. 


\section{Final Report \\ FHI: HyTech Rest Area Project}

UCF Contract Number: 20126050, PO: 191461

November 9, 2012

\section{Executive Summary}

The HyTech Rest Area project was a methanol fuel cell demonstration with the objective of promoting the use of renewable energy in Florida. The initial goal of the project was to produce methanol from citrus waste, thus demonstrating this application as a viable and inexpensive feedstock for generating electrical power. The project was to use a fuel cell system with integrated fuel processing capabilities and consisted of the complete system design, installation, operation and data collection. Over the course of the project, the concept changed with the result being the conducting of two system designs due to the changing of fuel cell system locations. For this report, a Phase I and a Phase II set of activities will be reported to cover these two events. All of the project tasks were completed and results were obtained.

Phase I -- Florida Turnpike Turkey Lake Service Plaza Demo, Orlando, Florida

The objective of the Phase I HyTech Rest Area project was to design, fabricate, install and demonstrate a grid-tied, multi-kW fuel cell system operating on citrus peel derived methanol. The fuel cell system was to be installed at the Florida Turnpike Enterprise's Turkey Lake service plaza in Orlando, Florida. The system would be used to offset a portion of the facility's grid power needs and to demonstrate the generation of electrical power from a waste product.

The Phase I project began in October 2007 and was conducted until December 2009 when the location was re-evaluated. To assist in completing the project, the Technology Research and Development Authority (TRDA) and Anderson Consulting assisted EnerFuel as subcontractors. TRDA was responsible for site promotion, permitting and the conducting of the environmental impact study for the site. Anderson Consulting was responsible for developing and obtaining the citrus derived methanol. In addition, the Florida Turnpike Enterprise (FTE) provided the demonstration site and Progress Energy (now Duke Power) provided the technical assistance in connecting the system to the grid.

The goals and objectives of the Phase I project were as follows.

- Design, construct and demonstrate a grid-independent, multi-kW FC system operating on citrus peel derived methanol

- Produce sufficient methanol from citrus waste to support the demonstration

- Promote the project to the public using media coverage 
The initial Phase I task was the design of the demonstration site that included the fuel cell systems, the 5000 gallon above ground methanol tank and the inverters necessary to tie the system to the facility electric grid. The first part of the fuel cell system design was to evaluate two candidate fuel cell systems capable of being fueled with methanol. These two candidates were direct methanol fuel cells (DMFCs) and indirect methanol fuel cells (IMFCs). The DMFCs do not require a fuel processor and are able to electrochemically oxidize methanol, while IMFCs employ a fuel processor to generate hydrogen from methanol, which, in turn, fuels a PEM hydrogen fuel cell.

Based on the results of this study, IMFCs were recommended for the program. This conclusion was arrived at through the evaluation of the current state of the art for IMFC and DMFC technologies. Generally, DMFCs are outperformed by IMFCs due to the greater maturity of the IMFC technology, the relatively high power level at which the fuel cell will operate, and the operating conditions of the fuel cell.

For example, high power IMFCs have already been successfully demonstrated in stationary and residential power applications. On the other hand, DMFCs beyond the $1 \mathrm{~kW}$ level have seldom been demonstrated. This is because for higher power applications, the benefits of the system simplicity of DMFCs are diminished by their inherent inefficiencies and stack design complexity. Additionally, benefits of DMFCs, such as fast transient response, and relatively short start-up times are not major considerations for the HyTech Rest Area project. This is because the fuel cell system is expected to operate continuously, at average loads and will be grid parallel.

To arrive at these conclusions, models were developed to determine the size of the fuel cell stack, cost of the fuel cell stack, volume of fuel required, and efficiency of the fuel cell system for IMFC and DMFC systems. The results are summarized below:

- For a $10 \mathrm{~kW}$ system a DMFC stack would require at least 3 times the number of cells than an IMFC system using a hydrogen proton exchange membrane fuel cell (H-PEMFC) stack.

- High methanol dilution and low operating efficiency required higher fuel volume quantities. For example, most DMFC systems are run with methanol diluted to 3$4 \%$ weight in water. IMFCs are typically only diluted to $95-96 \%$ weight. Therefore to produce $10 \mathrm{~kW}$ per month a DMFC would require a fuel volume 20 times larger than an IMFC

- DMFC stack costs are much higher than H-PEMFC stack costs. This is due to the higher number of cells and high catalyst loading of a DMFC stack. Results of stack cost analysis show a DMFC cost at least an order of magnitude higher than for H-PEMFC

- It is estimated that a DMFC system for the HyTech Rest Area would have a cost that is $579 \%$ higher than an equivalent IMFC system 
- The overall system efficiency of the IMFC system was estimated to be approximately $30 \%$. The overall system efficiency of the DMFC system was calculated to be approximately $20 \%$

- Due to lower efficiency, methanol crossover, and anode $\mathrm{CO}_{2}$ release, DMFCs emit trace amounts of methanol. IMFCs could also emit trace methanol emissions from the reformer burner, especially during start-up. For this reason, whichever system is used should be installed in an outdoor, well ventilated area

Given that in almost every important metric, IMFC systems outperforms equivalent DMFC systems, the results showed that IMFCs would be the preferred choice. From this result, the fuel cell system was configured to utilize four (4), $5 \mathrm{~kW}$ fuel cell systems (FCS) purchased from IdaTech, LLC.

The IdaTech ElectraGen ${ }^{\mathrm{TM}}$ XTR systems are designed for telecommunication backup applications. However, it was determined that they could be operated in a continuous fashion for the duration of the demonstration period. Although two systems would have been sufficient to meet the requirements of the project, an additional two units were purchased for redundancy purposes.

The ElectraGen system requires 200-240 VAC power to bring the fuel processor/ reformer up to operating temperature. Once at operating temperature the reformer is able to generate hydrogen as needed by the fuel cell stack. The warm up process take between 3 to 4 hours.

The method of operation for the system assumes that the facility has a primary AC power source. Under normal conditions, the system only requires a single warm up event, after which the fuel processor is kept at temperature via system operation or via built-in resistive heaters. When the system is providing backup power, fuel is burned to provide the necessary heat for fuel processing. When the system is in standby mode, awaiting a backup power event, the built-in resistive heaters maintain the fuel processor at temperature. To account for any lag between the time backup power is needed and when the ElectraGen is able to respond, a $48 \mathrm{~V}$ battery system is electrically connected in parallel with the fuel cell system output.

To interface the IdaTech FCs into the Phase I program, SMA Sunny Island 5048U inverters were selected and incorporated into the design. The Sunny Island inverters are designed to manage a stand-alone grid system, with ability to utilize power from various sources, such as solar, fuel cell, wind, diesel generator or utility grid. Since 208V, 3 phase power was required to interface with the utility grid at the demo site, the inverters were arranged so that each powered a single phase. One of the inverters would be programmed as the "Master" and the other two set as "slaves". The "Master" automatically manages all three inverter outputs, as well as managing the tie-in/ synchronization of power into the facility mains. For redundancy, one set of three inverters would have been used with each two fuel cell systems.

The basic configuration of the fuel cell system design and the layout of the demonstration site was accomplished and the resulting documents developed. From 
these documents, Gasoline Equipment Systems generated the civil and electrical plans necessary for site permitting and subsequent construction. These plans are provided in this report.

An equally important part of the Phase I work was for Anderson Consulting to develop methanol from citrus waste and in particular ethanol made from citrus waste. When the project was first proposed the value of citrus waste, e.g. peels and pulp, was very low. Its main use was in the production of animal feed. However, the price of corn, shortly after the project commenced, rapidly began to increase. This prompted an increase in the demand for other types of animal/cattle feed, including those made from citrus waste. As a result, a low value waste stream suddenly increased in value and very much diminished the waste-to-fuel attractiveness of the project.

The second major issue was the relatively low methanol yield produced from the generation of citrus derived ethanol. The only distiller willing to provide the necessary methanol, Florida's Bartow Ethanol, ended up not being able to do so. Bartow produces citrus derived ethanol for use in beverages. During the process methanol is produced as a byproduct that is generally discarded. An upgrade in their distillation process enabled greater ethanol yields to be extracted from the peels and pulp. This had the effect of reducing the amount of methanol, in the leftover methanol/water mixture, from $45.8 \%$ to $4 \%$, by volume. Thus, processing the mixture to extract the methanol did not make any economic or efficiency sense.

Once it became evident a citrus waste derived methanol was no longer viable, EnerFuel looked into other bio derived methanol sources. The use of woody bio-mass was looked into. The University of Florida was found to have a program to investigate woody biomass ethanol production. Their pilot plant, although capable of producing methanol, was in the process of construction and was not expected to be able to generate more than a few gallons of methanol.

BioMCN, from the Netherlands, was identified to be producing methanol from the glycerin byproduct of bio-diesel production. BioMCN produces large quantities of methanol, but the cost associated in transportation and import fees did not make this a viable alternative.

Thus, the methanol source from bio or bio waste products waste was not pursued any further.

The results achieved from the Phase I efforts were as follows:

1. Two candidate fuel cell systems were evaluate -- direct methanol fuel cells (DMFCs) and indirect methanol fuel cells (IMFCs). Based on the results of this study, IMFCs were recommended for the program.

2. An indirect methanol fuel cell was selected and configured to utilize four (4), 5kW fuel cell systems (FCS) purchased from IdaTech, LLC. 
3. To interface the IdaTech FCSs, SMA Sunny Island $5048 \mathrm{U}$ inverters were selected and incorporated into the design.

4. The basic configuration of the fuel cell system design and the layout of the demonstration site was accomplished.

5. From the basic configuration design, Gasoline Equipment Systems generated the civil and electrical plans (provided in report) necessary for site permitting and subsequent construction.

6. The obtaining of methanol from citrus waste and a pilot plant operation were studied and evaluated. The results were not found to be favorable.

At this point, the project management changed and questions were raised about the continuation of the Phase I efforts. The major three items that caused this re-evaluation were the problems with obtaining a building code permit from Orange County, FL., the extensive cost that would be required for installation and safety of the Turnpike location and the difficulty for follow on activities at the Turnpike site. Thus, EnerFuel was requested to conduct an evaluation of a new site for the demo activity.

Phase II -- Electric Vehicle Charging Station (EVCS) at Florida Atlantic University (FAU) in Boca Raton, Florida

EnerFuel performed a detailed evaluation of three new sites and then made a location change recommendation. Two options were associated with the city of West Palm Beach, Florida and the third at FAU. The first of these options, consisted of setting up the FC system at West Palm Beach's water reclamation plant to offset a portion of the facility's power needs. Although the plant had its own 7500 gallon methanol tank, this option was deemed to be too similar to the Phase I effort and therefore rejected.

The second option would have used the FC systems to provide backup power to one of the several lift stations within the city limits. The purpose of the lift station is to maintain system pipe pressure and to assure sewage flows to the water reclamation plant and does not backup. This option was rejected since no grid power would be available for startup. In addition, the startup time was considered too lengthy.

The third option was to use the FC system as part of a electric vehicle charging station located at Florida Atlantic University (FAU) in Boca Raton, Florida. In addition, FAU's involvement in the project would successfully exposed engineering students to fuel cell technology and renewable fuels and, thus, promoting renewable energy technologies.

On February 10, 2010, EnerFuel presented the three options for changing the site and recommended the FAU location. This recommendation was accepted by UCF and DOE. Final approval was received on April 8, 2012 to modify the project to consist of the design, construction and testing of a methanol fuel cell, electrical vehicle charging station, at Florida Atlantic University (FAU) in Boca Raton, Fl. 
The remainder of the project was directed at the Electric Vehicle Charging Station and the reconfiguring of FC system for the application. The charging station location was selected to be adjacent to FAU's existing vehicle charging complex in order to allow easy access to vehicles. FAU's mechanical engineering department was subcontracted to construct the station, as well as assist in system testing. Two vehicle charging spaces were set aside and devoted to the fuel cell charging station.

The goals and objectives of the Electric Vehicle Charging Station (EVCS) project were as follows.

- Design, construct and demonstrate a grid-independent, methanol fueled, fuel cell powered, electric vehicle charging station

- Utilize FCs and inverters purchased for the Phase I project

- Provide opportunity to expose students to fuel cell technologies

- Promote the project to the public

The fuel cell system was designed and constructed at the FAU site. The final results from the Phase II efforts were:

1. Demonstration of the EVCS was performed using the inverters.

2. Evaluation of the station's performance was based on FC test results at EnerFuel combined with the FAU results of using the inverters to charge the electric vehicles.

3. The charging station could not be operated as initially intended since no grid power was available to support the system startup.

4. The EVCS design successfully incorporated the FC system and inverters that were originally purchased for the Phase I project.

5. The project exposed engineering students with real world design by allowing several students to assist in the design and construction of the station.

In conclusion, the project showed the advantages of using a fuel cell, providing grid independent vehicle charging at high efficiency and the ability to use renewable fuels. Evaluation of the fuel cell charging station concept suggested that it could be used as a backup to "grid" charging, in locations that have no access to grid-power or in instances that require a rapidly deployable charging system. As an example, operations that rely on electric vehicles could continue to operate even during a major power outage, such as brought on by a "Katrina"/ "Sandy" type event.

Additional details on the project and all the tasks are provided in the following sections. 
Table of Contents

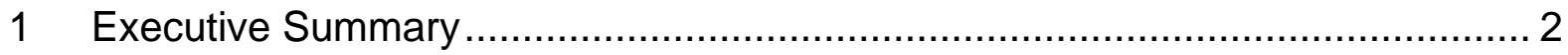

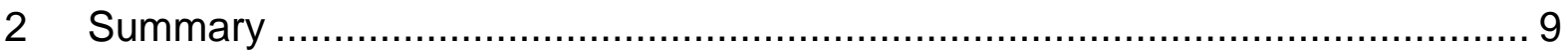

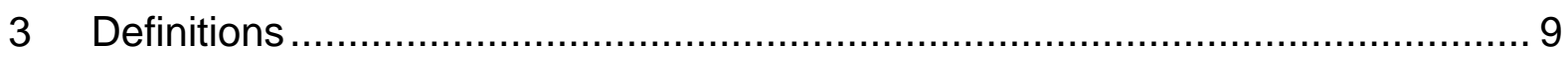

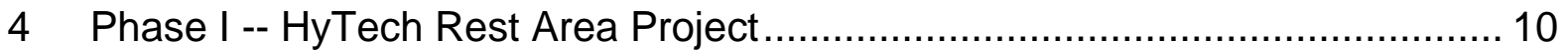

4.1 Phase I Project Goals and Objectives ………......................................... 10

4.2 Phase I Comparison of Objectives with Accomplishments .......................... 12

4.3 Phase I System and Demonstration Site Design .......................................... 14

4.3.1 Demonstration Site Design ..................................................................... 14

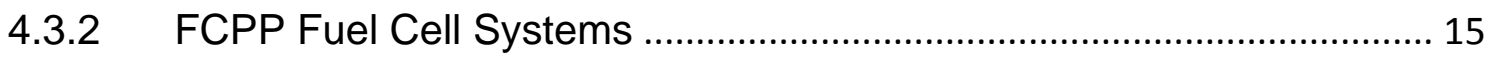

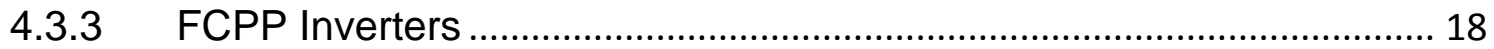

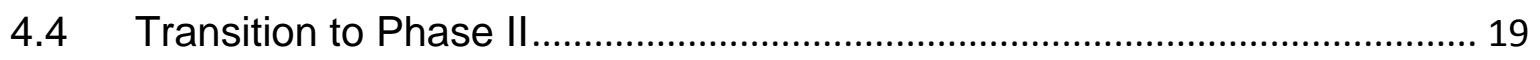

5 Phase II -- Methanol Fuel Cell, Electric Vehicle Charging Station Project ........ 22

5.1 Phase II -- Project Description .................................................................. 22

5.2 Phase II -- Objectives and Accomplishments ................................................ 25

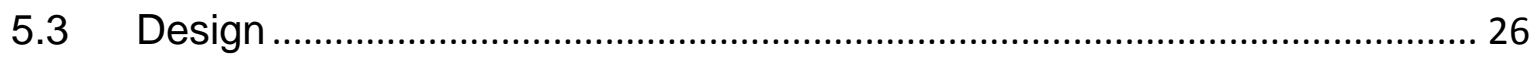

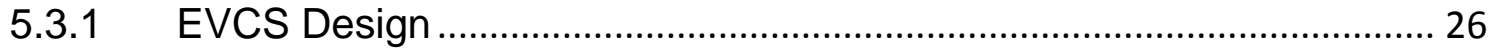

5.3.2 EVCS System Modeling ................................................................... 31

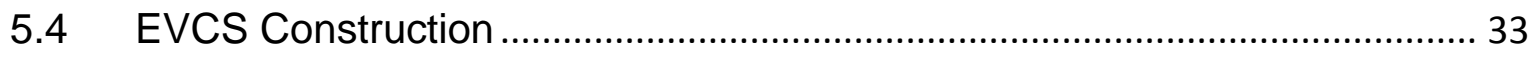

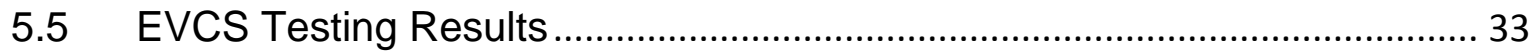

5.6 EVCS Project Accomplishments............................................................... 37

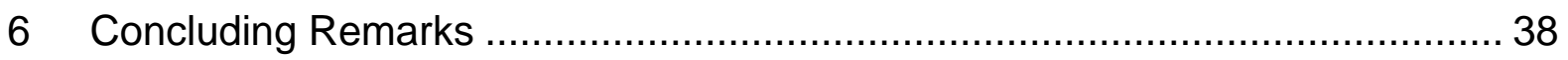




\section{Summary}

The HyTech Rest Area project was conceived to help promote the use of renewable energy in Florida. The original goal of the project was to demonstrate the use of citrus waste as a viable and inexpensive fuel feedstock for generating electrical power. A fuel cell system with integrated fuel processing capabilities was proposed to demonstrate the concept. Over the course of the project several developments required the project objectives to be modified. Although the scope was changed, the project was still successful in demonstrating the potential of using alternative energy to enhance the efficiency and cost savings of onsite power generation.

Phase I of the HyTech Rest Area project consisted of the design, fabrication, installation and demonstration of a grid-tied, multi-kW fuel cell system operating on citrus peel derived methanol. The fuel cell system was to be installed at the Florida Turnpike Enterprise's Turkey Lake service plaza in Orlando, Florida. The system would have been used to offset a portion of the facility's grid power needs and demonstrate the generation of electrical power from a waste product.

Due to the cost and difficulty of processing the citrus waste into methanol, as well as the subsequent increase in value of citrus waste for the production of animal/cattle feed, the attractiveness of using this waste source diminished. Alternate bio-feedstock sources were investigated but none were available or in sufficient quantity to satisfy the needs of the project. As a result the objective of the project was modified.

After evaluating various options for the project, it was decided that the best alternative would be to modify the project, but still utilize as much of the already purchased hardware as possible. The best alternative was selected to be the construction and demonstration of a grid independent, electric vehicle charging station at Florida Atlantic University in Boca Raton, Florida. The demonstration of the charging station constituted the second phase of the HyTech project.

This report covers the design, construction and accomplishments of the Phase I -HyTech Rest Area project and Phase II -- Electric Vehicle Charging Station demonstration.

Chapter 4 covers Phase I, while chapter 5 covers the Phase II objectives, accomplishments, project activities, design and test results. Chapters 6 covers the project financials and chapter 7 concludes the report.

\section{Definitions}

BOP: balance-of-plant

CHP: Combined heat and power

COTS: commercial-off-the-shelf 
DMFC: Direct methanol fuel cell

DOE: US Department of Energy

EV: Electric vehicle

EVCS: Electric vehicle charging station

FAU: Florida Atlantic University

FCPP: Fuel cell power plant (includes all four IdaTech fuel cell systems, inverters, batteries and methanol storage tank)

FCS: Fuel cell system (i.e. IdaTech 5kW ElectraGen fuel cell system)

FTE: Florida Turnpike Enterprise

HT-PEMFC: High temperature, proton exchange membrane fuel cell

$\mathrm{MeOH}$ : Methanol

PEMFC: Proton exchange membrane fuel cell

SOC: State of charge

SOW: Statement of work

TRDA: Technology Research and Development Authority

\section{Phase I -- HyTech Rest Area Project}

\subsection{Phase I Project Goals and Objectives}

The HyTech Rest Area project was active from October 2007 until December 2009. The main goal of the project was to demonstrate the use of citrus waste as a viable and inexpensive fuel feedstock for generating electrical power. To accomplish this EnerFuel's task was to design, build and demonstrate a grid-independent, multi-kW fuel cell power plant (FCPP) operating on citrus peel, methanol fuel.

The Technology Research and Development Authority (TRDA) and Anderson Consulting assisted in the project as subcontractors. The TRDA was responsible for site promotion and permitting, as well as conducting the environmental impact study of the site. Anderson Consulting was responsible for obtaining the necessary citrus derived methanol. In addition, the Florida Turnpike Enterprise (FTE) and, Orlando based utility company, Progress Energy (now Duke Power), respectively, provided the demonstration site and technical assistance in connecting the FCPP to the grid. The location of the demonstration site is shown in Figure 4.1. 


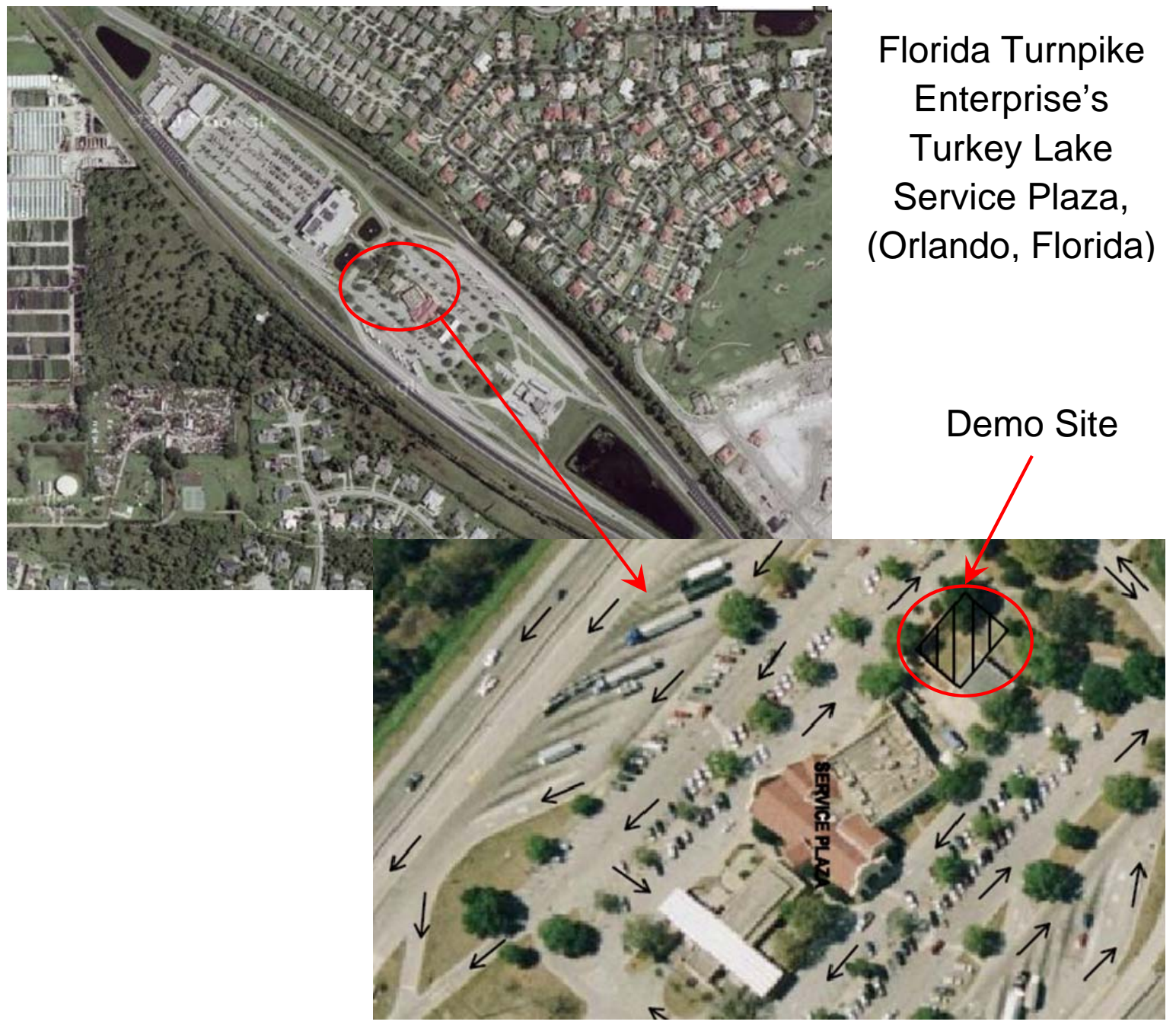

Figure 4.1 HyTech Rest Area project demonstration site

The goals and objectives of the HyTech project were as follows.

- Design, construct and demonstrate a grid-independent, multi-kW FCPP operating on citrus peel, methanol fuel

- Produce sufficient methanol from citrus waste to support the demonstration

- Promote the project to the public using media coverage 


\subsection{Phase I Comparison of Objectives with Accomplishments}

Not all of the Phase I objectives were achieved before the project was changed to the Methanol Fuel Cell, Electric Vehicle Charging Station. Table 4.1 summarizes the accomplishments achieved with respect to project objectives.

Table 4.1 Phase I -- HyTech Rest Area Project Objectives vs. Accomplishments

\begin{tabular}{|c|c|}
\hline Objective & Accomplishment \\
\hline $\begin{array}{l}\text { Design, construct, install } \\
\text { and demonstrate a grid- } \\
\text { independent, multi-kW } \\
\text { FCPP operating on citrus } \\
\text { peel, methanol fuel }\end{array}$ & 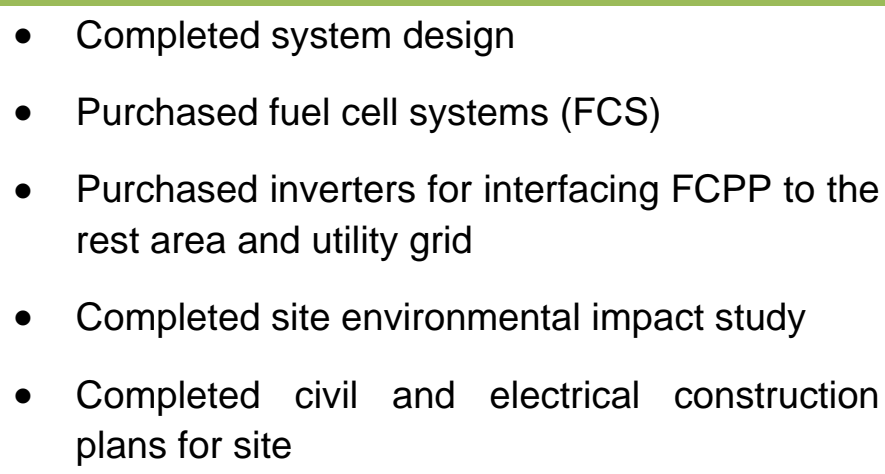 \\
\hline $\begin{array}{l}\text { Produce sufficient methanol } \\
\text { from citrus waste to support } \\
\text { the demonstration }\end{array}$ & Not pursued \\
\hline $\begin{array}{l}\text { Promote the project to the } \\
\text { public using media } \\
\text { coverage }\end{array}$ & Not pursued \\
\hline
\end{tabular}

Even with the subcontracting of Anderson Consulting, securing a citrus waste methanol source was not successful. Several issues arose during the course of the project that diminished the availability of the bio-methanol. The biggest contributors were the increase in the value of citrus waste and the lower methanol yield from processing the citrus peel.

When the project was first proposed the value of citrus waste, e.g. peels and pulp, was very low. Its main use was in the production of animal feed. However, the price of corn, shortly after the project commenced, rapidly began to increase (Figure 4.2). This prompted an increase in the demand for other types of animal/cattle feed, including those made from citrus waste. As a result, a low value waste stream suddenly increased in value and diminished the waste-to-fuel attractiveness of the project.

The second major issue was the relatively low methanol yield produced from the generation of citrus derived ethanol. The only distiller willing to provide the necessary methanol, Florida's Bartow Ethanol, could only do so in a low methanol/water concentration.

Bartow produces citrus derived ethanol for use in beverages. During the process methanol is produced as a byproduct that is generally discarded. An upgrade in their 
distillation process enabled greater ethanol yields to be extracted from the peels and pulp. This unfortunately had the effect of reducing the amount of methanol, in the leftover methanol/water mixture, from $45.8 \%$ to $4 \%$, by volume. Processing the mixture to extract the methanol was not a viable option. A previous quote for processing 6000 gallons from the $45.8 \%$ mixture was $\$ 40,000$. The cost for the $4 \%$ mixture would have been much higher.

Once it became evident a citrus waste derived methanol was no longer viable, EnerFuel looked into other bio derived methanol sources. The use of woody bio-mass was considered. The University of Florida was found to have a program to investigate woody bio-mass ethanol production. Their pilot plant, although capable of producing methanol, was in the process of construction and was not expected to be able to generate more than a few gallons of methanol.

BioMCN, from the Netherlands, was identified to be producing methanol from the glycerin byproduct of bio-diesel production. BioMCN produces large quantities of methanol, but the cost associated in transportation and import fees did not make this a viable alternative.

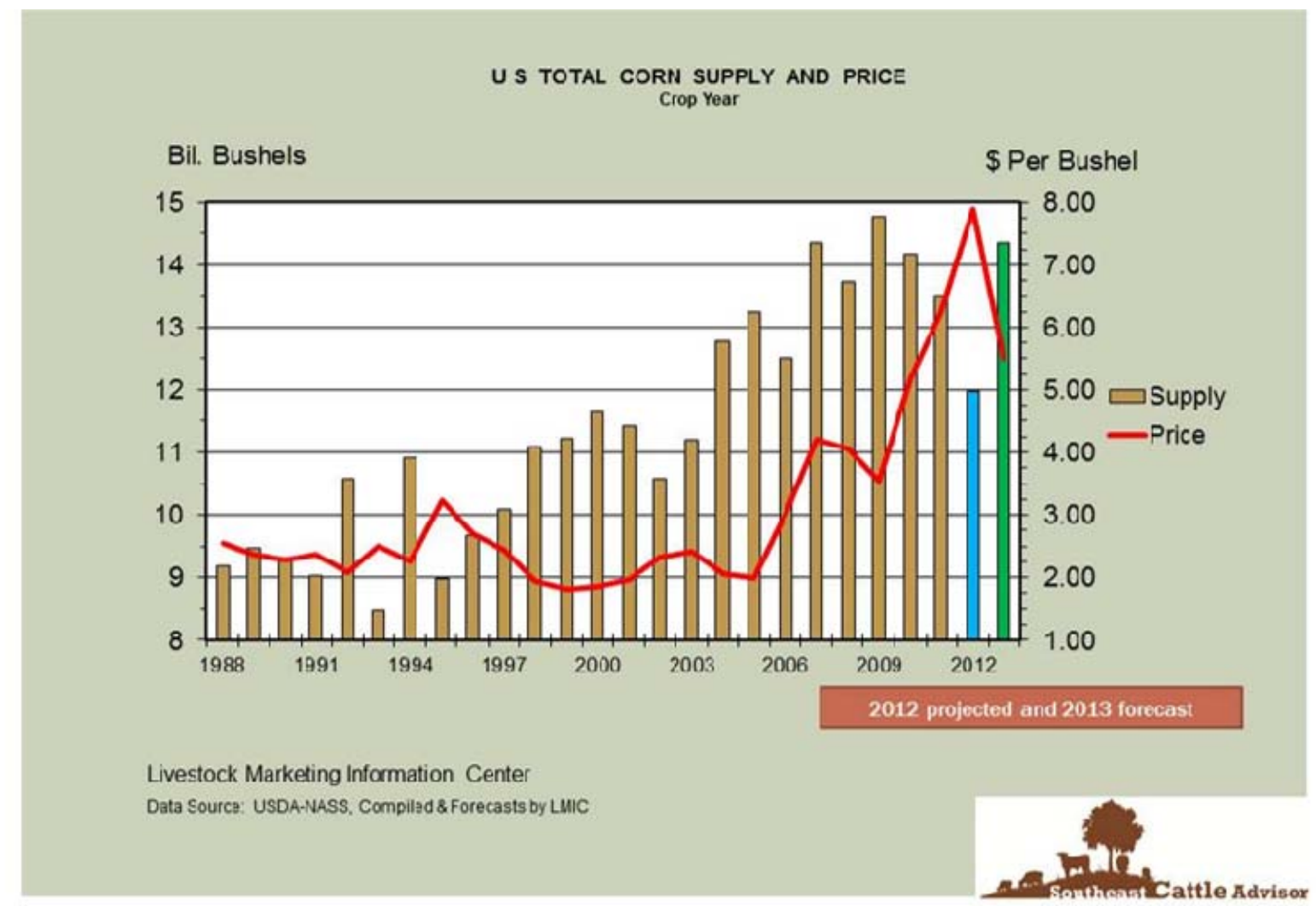

Figure 4.2 Price of corn since $1988^{1}$

${ }^{1}$ C. Lacy, “September 12 WASDE Provides Some Good News for Cattle Producers", COW - Sep 13, 2012, Southeast Cattle Advisor, www.secattleadvisor.com

ENERFUEL, INC. 


\subsection{Phase I System and Demonstration Site Design}

\subsubsection{Demonstration Site Design}

The design of the demonstration site included the fuel cell systems, 5000 gallon above ground methanol tank and the inverters necessary to tie the system to the facility mains. The power generated would be used to offset a part of the "grid" energy delivered to the food court portion of the service plaza. Figure 4.3 shows the basic configuration of the FCPP design, while Figure 4.4 shows the layout of the demonstration site.

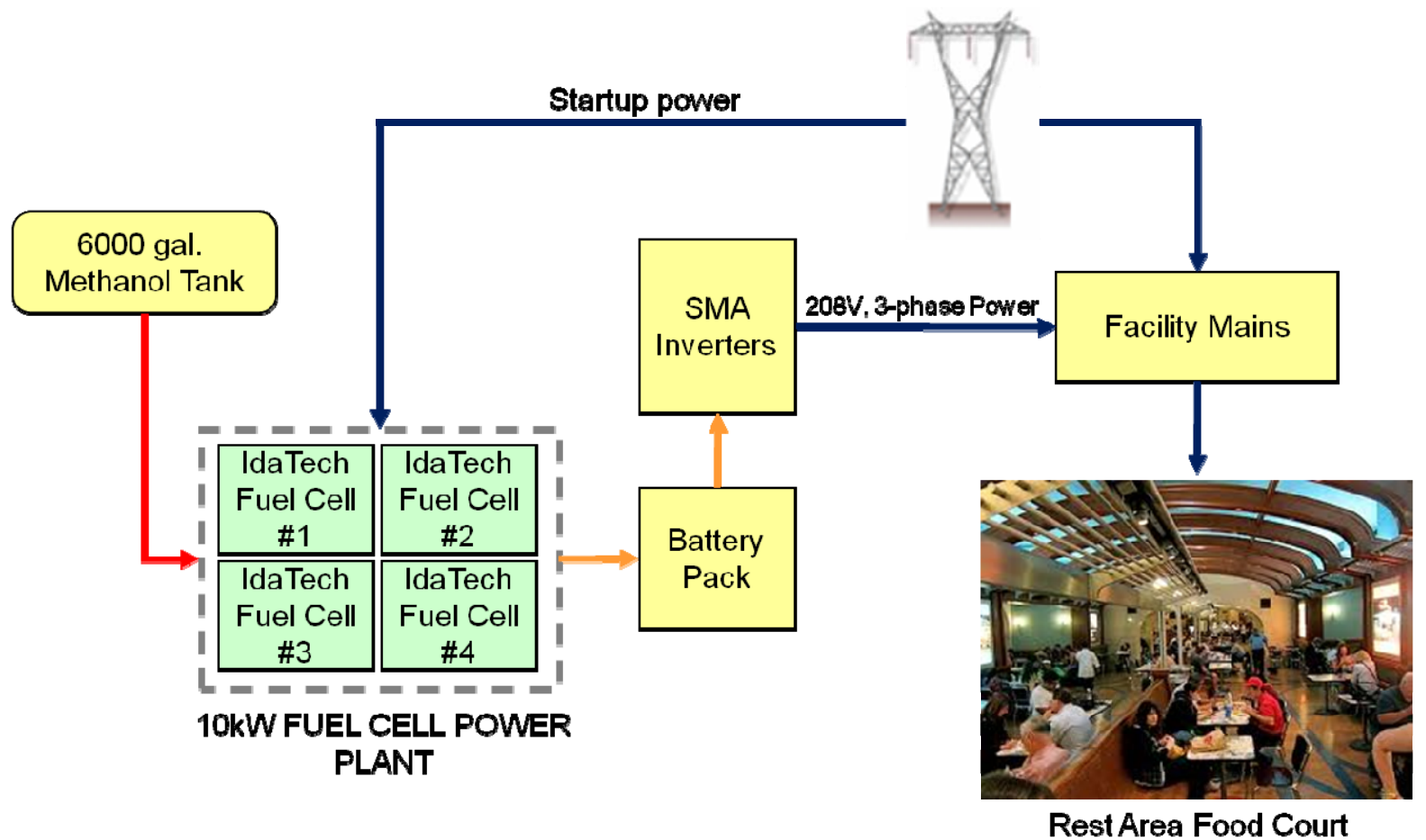

Figure 4.3 Conceptual design of the FCPP intended to provide citrus waste derived electrical power to the FTE Turkey Lake service plaza, food court

Gasoline Equipment Systems was subcontracted to generate the civil and electrical plans necessary for site permitting and construction ${ }^{2}$.

\footnotetext{
${ }^{2}$ For a copy of the civil and electrical plans please contact the report author
} 


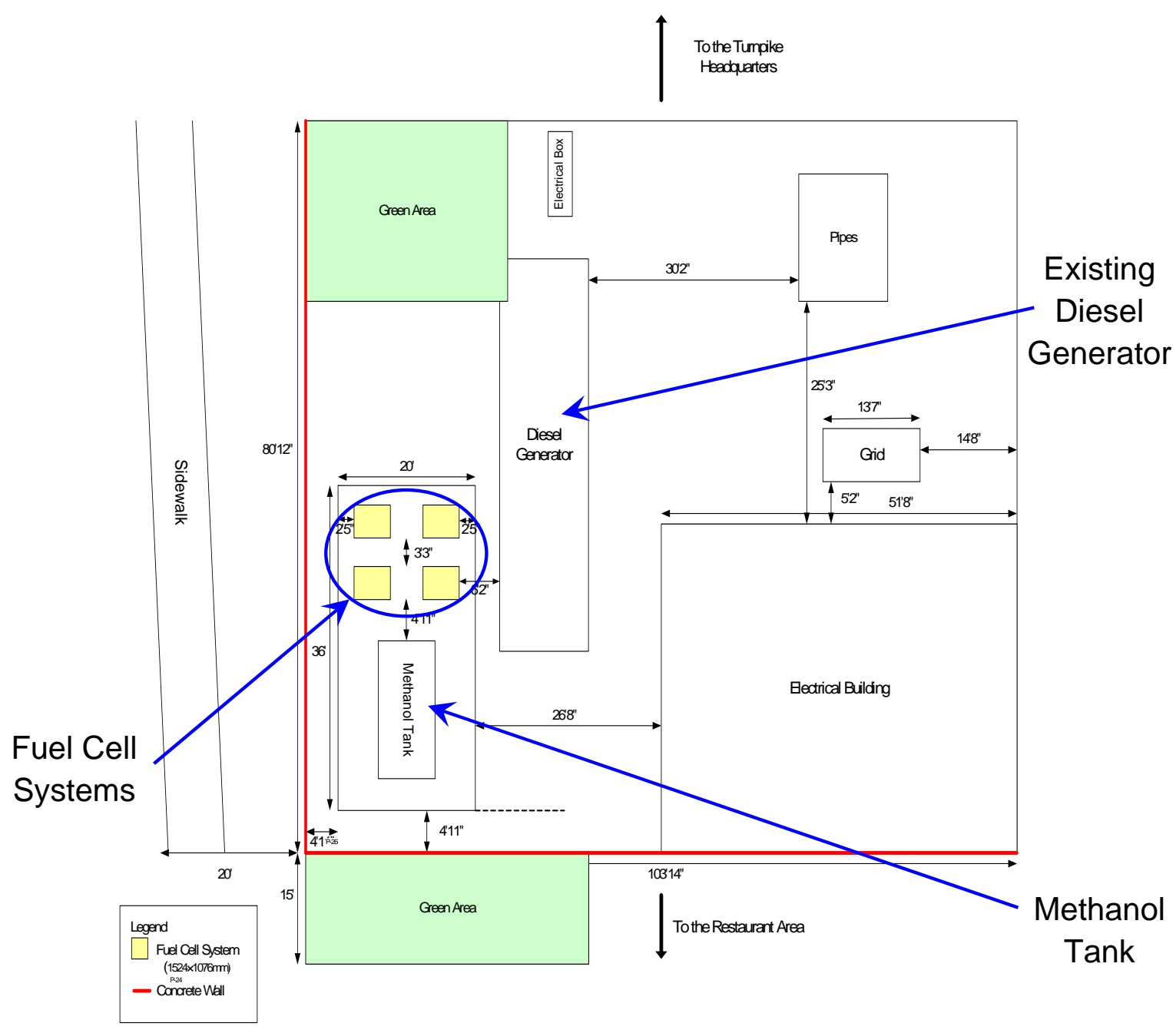

Figure 4.4 HyTech Rest Area demonstration site layout

\subsubsection{FCPP Fuel Cell Systems}

The design of the HyTech FCPP was configured to utilize four (4), 5kW fuel cell systems (FCS) purchased from IdaTech, LLC (Figure 4.5). Originally, EnerFuel had proposed building its own direct methanol fuel cell (DMFC) systems; however after performing a detailed trade analysis ${ }^{3}$, it was determined that purchasing the systems would be quicker and more cost effective.

The IdaTech ElectraGen ${ }^{\mathrm{TM}}$ XTR systems are designed for telecommunication backup applications, however it was determined that they could be operated in a continuous

\footnotetext{
${ }^{3}$ For a copy of the DMFC vs. Reformer Based PEMFC Trade Study please contact the report author
} 
fashion for the duration of the demonstration period. Although two systems would have been sufficient to meet the requirements of the project, an additional two units were purchased for redundancy purposes. Specifications for the FCSs are shown in Table 4.2 .

The ElectraGen system requires 200-240 VAC power to bring the fuel processor/ reformer up to operating temperature. Once at operating temperature, the reformer is able to generate hydrogen as needed by the fuel cell stack. The warm up process takes between 3 to 4 hours.

The method of operation for the system assumes that the facility has a primary AC power source. Under normal conditions, the system only requires a single warm up event, after which the fuel processor is kept at temperature via system operation or via built-in resistive heaters. When the system is providing backup power, fuel is burned to provide the necessary heat for fuel processing. When the system is in standby mode, awaiting a backup power event, the built-in resistive heaters maintain the fuel processor at temperature. To account for any lag between the time backup power is needed and when the ElectraGen is able to respond, a $48 \mathrm{~V}$ battery system is electrically connected in parallel with the fuel cell system output (Figure 4.6).

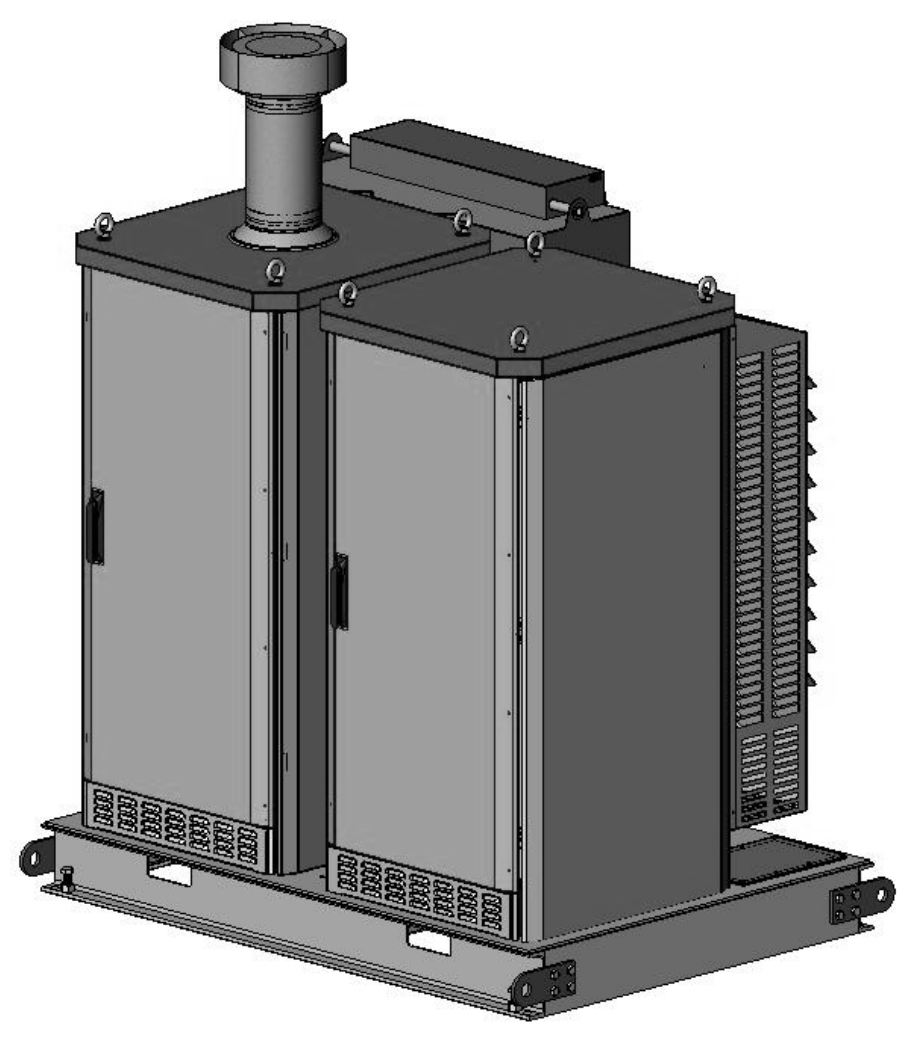

Figure 4.5 IdaTech fuel cell system showing the fuel cell cabinet on the right and the fuel processor/ reformer on the left 


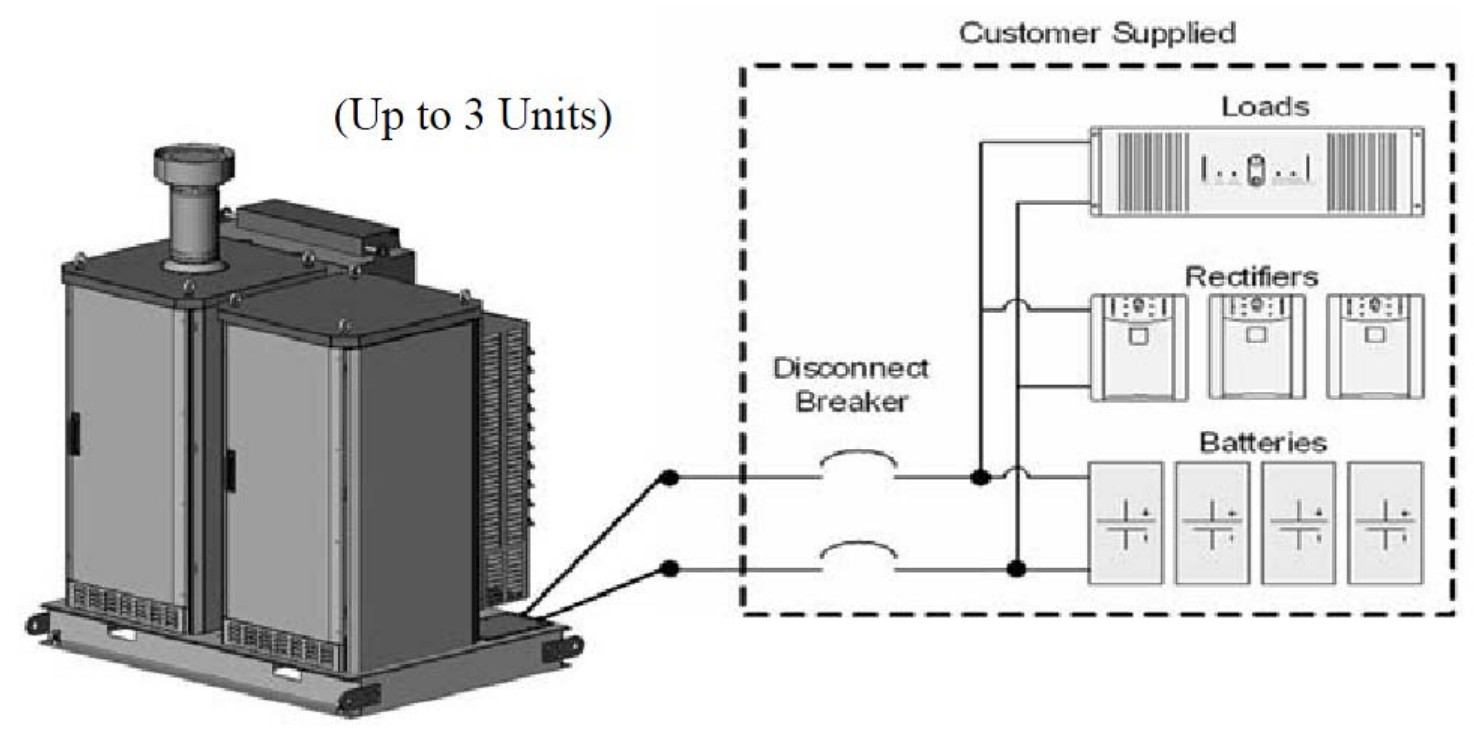

Figure 4.6 IdaTech ElectraGen electrical connection with loads and 48V battery system

Table 4.2 IdaTech Fuel Cell System Specifications

\begin{tabular}{|c|c|}
\hline Specification & Metric \\
\hline Application & $\begin{array}{l}\text { Telecommunication backup } \\
\text { power }\end{array}$ \\
\hline Operating environment & Laboratory \\
\hline Continuous power output & 500 - 5000 Watts \\
\hline Voltage & $48 \mathrm{VDC}$ \\
\hline Methanol consumption & 49 mL/min (max) @5kW \\
\hline Target temperature range & $-40^{\circ} \mathrm{C}$ to $50^{\circ} \mathrm{C}$ \\
\hline Fuel & $\begin{array}{l}\text { methanol/water premix ( } 62 \% \\
\text { methanol by weight) }\end{array}$ \\
\hline $\begin{array}{l}\text { Startup time to standby } \\
\text { mode (fuel processor cold) }\end{array}$ & 3-4 hours \\
\hline $\begin{array}{l}\text { Standby mode to power } \\
\text { output (fuel processor at } \\
\text { temperature) }\end{array}$ & $<1$ second \\
\hline $\begin{array}{l}\text { Startup power } \\
\text { requirements }\end{array}$ & $\begin{array}{l}\text { 200-240VAC, } 60 \mathrm{~Hz}, 1 \varnothing, 10 \\
\text { Amperes }\end{array}$ \\
\hline
\end{tabular}




\begin{tabular}{|l|l|}
\hline Specification & \multicolumn{1}{c|}{ Metric } \\
\hline Internal fuel tank capacity & 208 Liters \\
\hline Battery system & $\begin{array}{l}48 \mathrm{~V}, 100 \mathrm{AH} \text { (four (4) C\&D } \\
\text { Technologies TEL12-105 12V } \\
\text { batteries) }\end{array}$ \\
\hline Weight & $550 \mathrm{~kg}$ \\
\hline Volume & $\begin{array}{l}152 \mathrm{~cm} \mathrm{(W)} \times 107 \mathrm{~cm} \mathrm{(L)} \mathrm{x} \\
150 \mathrm{~cm}(\mathrm{H})\end{array}$ \\
\hline
\end{tabular}

\subsubsection{FCPP Inverters}

To interface the IdaTech FCSs into the rest area facility, SMA Sunny Island 5048U inverters (Figure 4.7) were incorporated into the design. The Sunny Island inverters are designed to manage a stand-alone grid system, with ability to utilize power from various sources, such as solar, fuel cell, wind, diesel generator or utility grid (Figure 4.8). Since $208 \mathrm{~V}, 3$ phase power was required to interface with the facility mains, the inverters were arranged so that each powered a single phase. One of the inverters would be programmed as the "Master" and the other two set as "slaves". The "Master" automatically manages all three inverter outputs, as well as managing the tie-in/ synchronization of power into the facility mains. For redundancy, one set of three inverters would have been used with each two fuel cell systems. Figure 4.9 shows the detailed electrical arrangement of the inverters, FCSs and batteries.

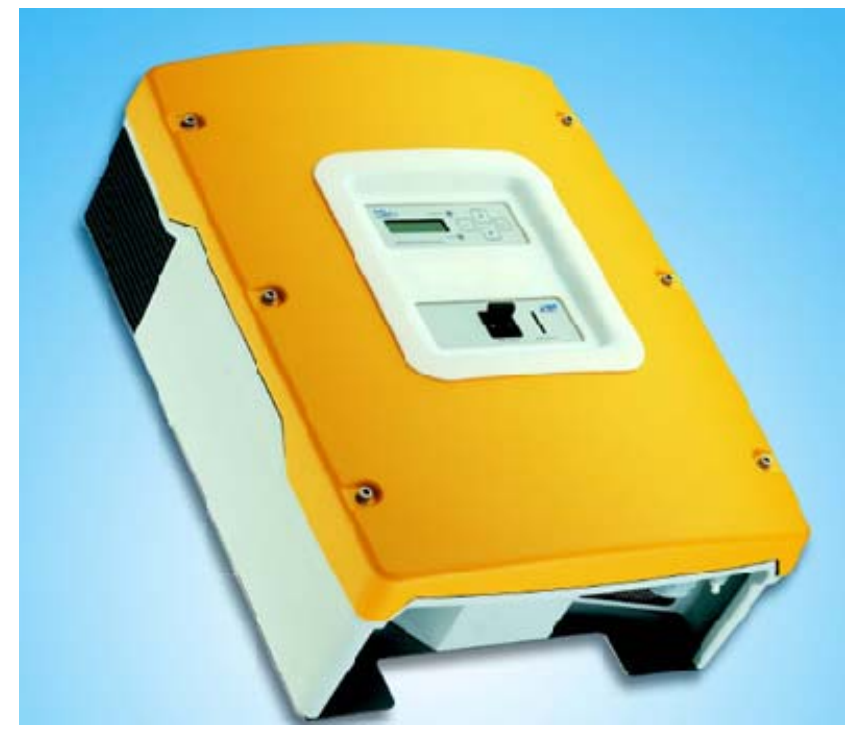

Figure 4.7 SMA Sunny Island 5048U, 5kW inverters 


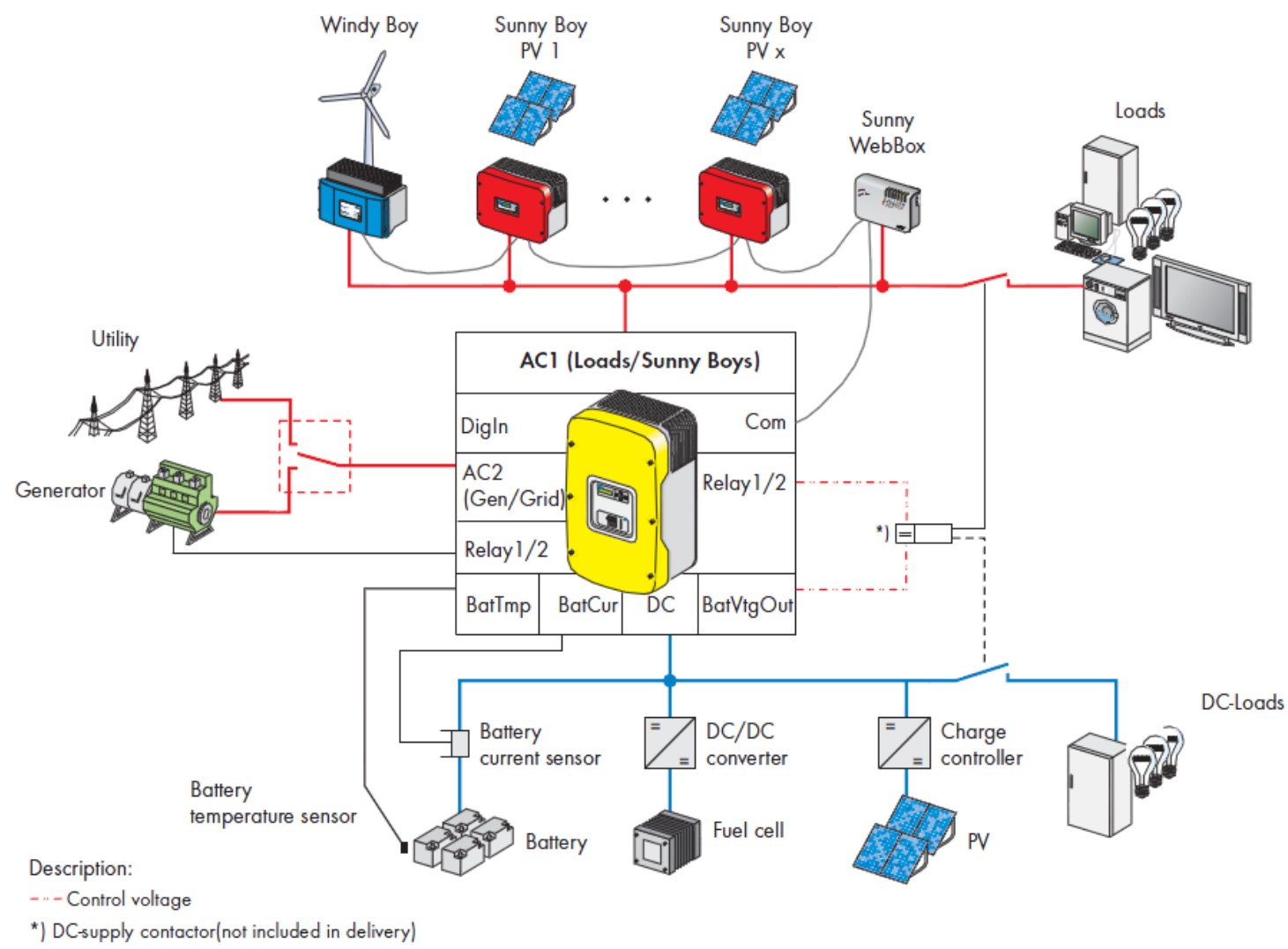

Figure 4.8 SMA Sunny Island 5048U stand-alone grid capability

\subsection{Transition to Phase II}

In 2009, the project management changed and questions were raised about the continuation of the Phase I site location efforts. The three major items that caused this re-evaluation were the problems with obtaining a building code permit from Orange County, FL., the extensive cost that would be required for installation and safety of the Turnpike location and the difficulty for follow on activities at the Turnpike site. Thus, EnerFuel was requested to conduct an evaluation of a new site for the demo activity.

Phase I Results

The results achieved from the Phase I efforts are as follows:

1. Two candidate fuel cell systems were evaluated -- direct methanol fuel cells (DMFCs) and indirect methanol fuel cells (IMFCs). Based on the results of this study, IMFCs were recommended for the program.

2. An indirect methanol fuel cell was selected and configured to utilize four (4), 5kW fuel cell systems (FCS) purchased from IdaTech, LLC. 
3. To interface, the IdaTech FCSs, SMA Sunny Island $5048 \mathrm{U}$ inverters were selected and incorporated into the design.

4. The basic configuration of the fuel cell system design and the layout of the demonstration site was accomplished.

5. From the basic configuration design, Gasoline Equipment Systems generated the civil and electrical plans (provided in report) necessary for site permitting and subsequent construction.

6. The obtaining of methanol from citrus waste and a pilot plant operation were studied and evaluated. The results were not found to be favorable. 


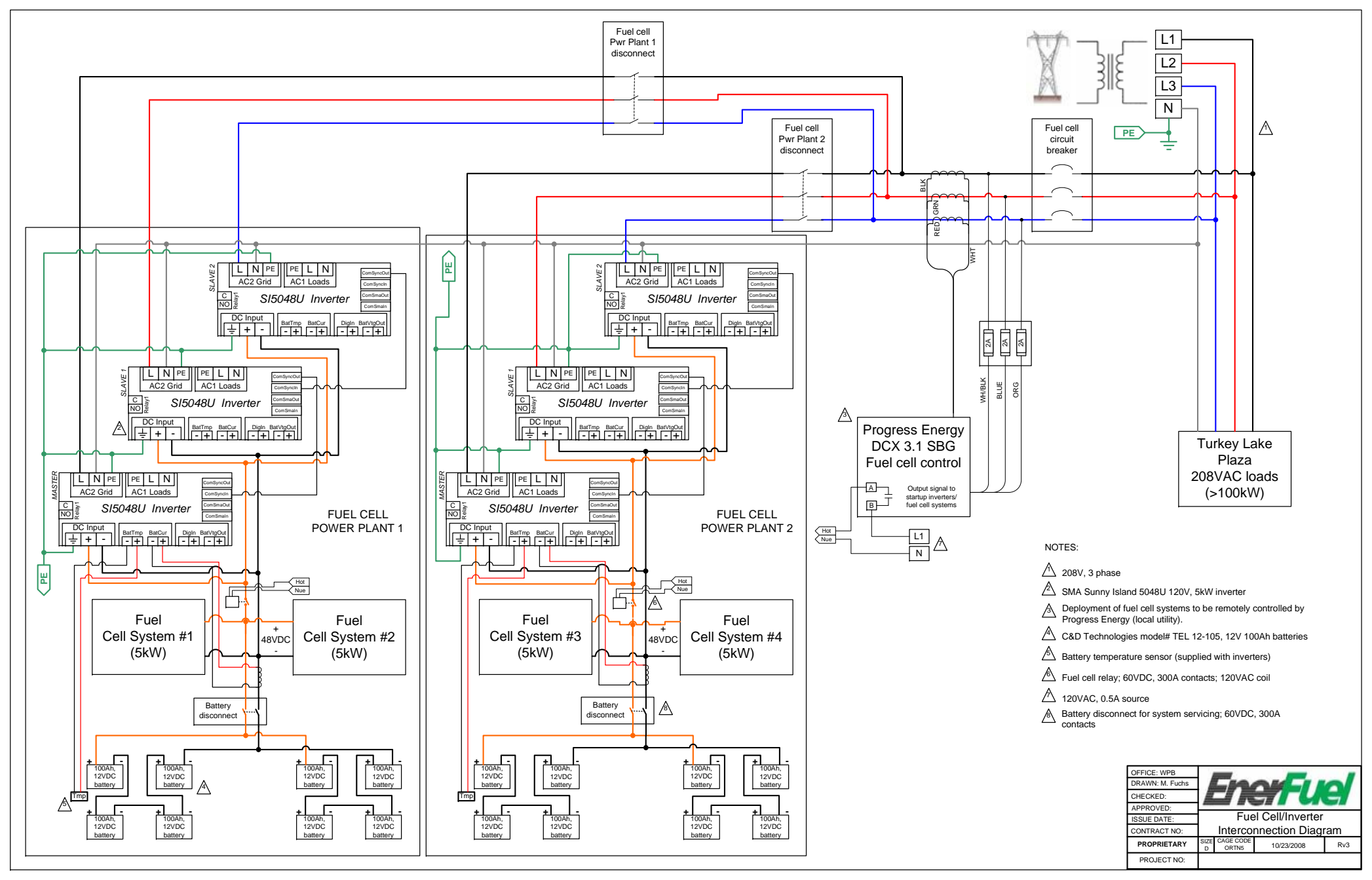

Figure 4.9 Detailed electrical arrangement of the inverters, FCSs and batteries

ENERFUEL, INC.

Page $\mathbf{2 1}$ of $\mathbf{3 8}$ 


\section{Phase II -- Methanol Fuel Cell, Electric Vehicle Charging Station Project}

Following a UCF request, EnerFuel performed a detailed evaluation of three possible new sites and then made a location change recommendation. On February 10, 2010 EnerFuel presented options for changing the scope of the HyTech Rest Area project. Final approval was received, from the DOE, on April 8, 2012 to modify the project to consist of the design, construction and testing of a methanol fuel cell, electrical vehicle charging station, at Florida Atlantic University (FAU) in Boca Raton, Florida (Figure 5.1).

Two other demonstration site options were explored but found not to be well suited for the existing FCPP or not compelling enough. Both options were associated with the city of West Palm Beach, Florida. The first of these options, consisted of setting up the FCPP at West Palm Beach's water reclamation plant to offset a portion of the facility's power needs. Although the plant had its own 7500 gallon methanol tank, this option was deemed to be too similar to the HyTech Rest Area effort and therefore rejected.

The second option would have used the FCPP to provide backup power to one of the several lift stations within the city limits. The purpose of the lift station is to maintain system pipe pressure, at critical points, to assure sewage flows to the water reclamation plant and does not backup. This option was rejected since no grid power would be available to startup the FCSs. In addition, the startup time of the FCSs, from cold, was considered too lengthy.

\subsection{Phase II -- Project Description}

The Methanol Fuel Cell, Electric Vehicle Charging Station project was configured to demonstrate the charging of electric vehicles using a bio-methanol capable fuel cell system. The charging station location was selected to be adjacent to FAU's existing vehicle charging complex to allow easy access to vehicles. See Figures 5.2 and 5.3, FAU's mechanical engineering department was subcontracted to construct the station, as well as assist in system testing. Two vehicle charging spaces were set aside and devoted to the fuel cell charging station. 


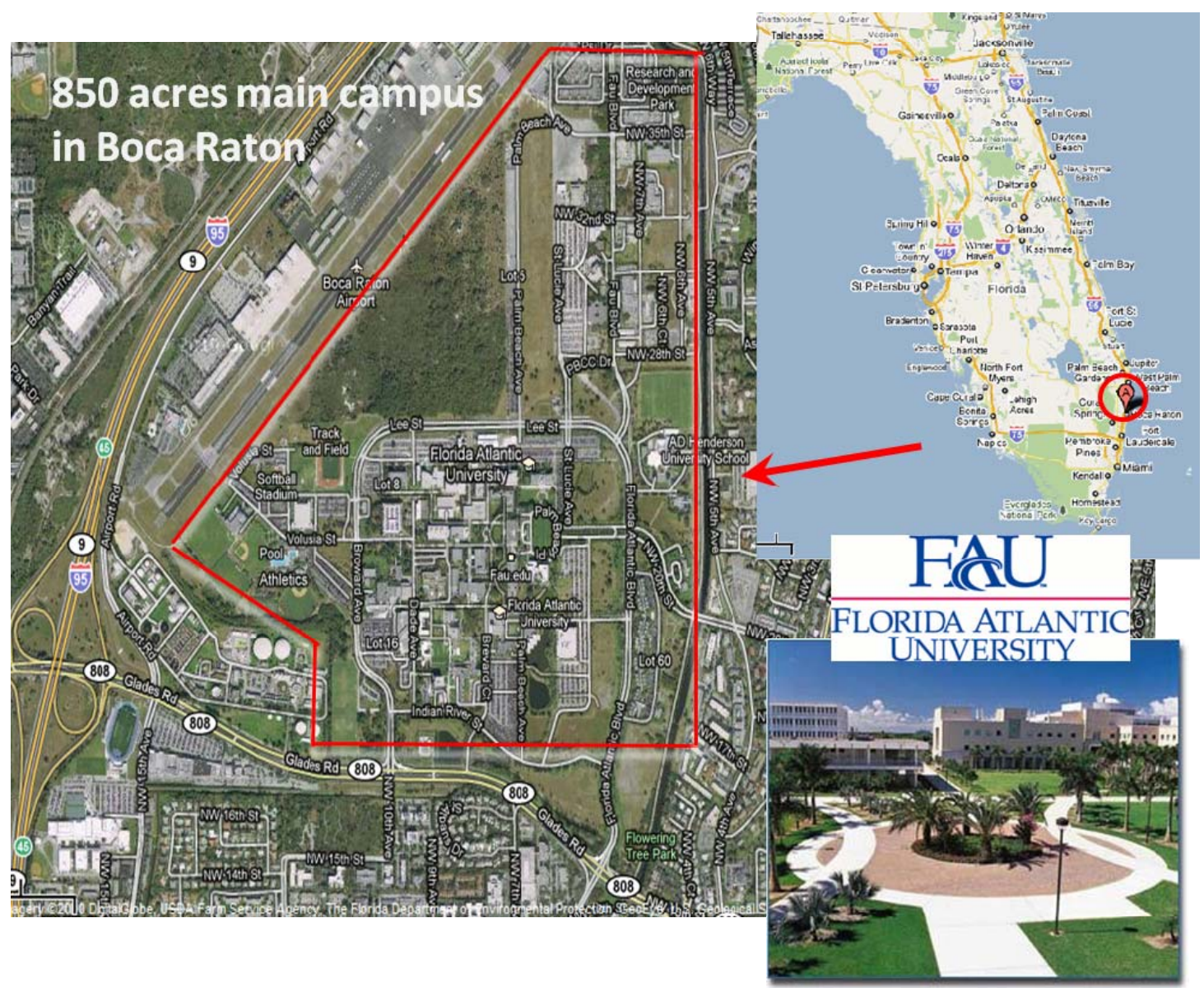

Figure 5.1 FAU campus

ENERFUEL, INC. 


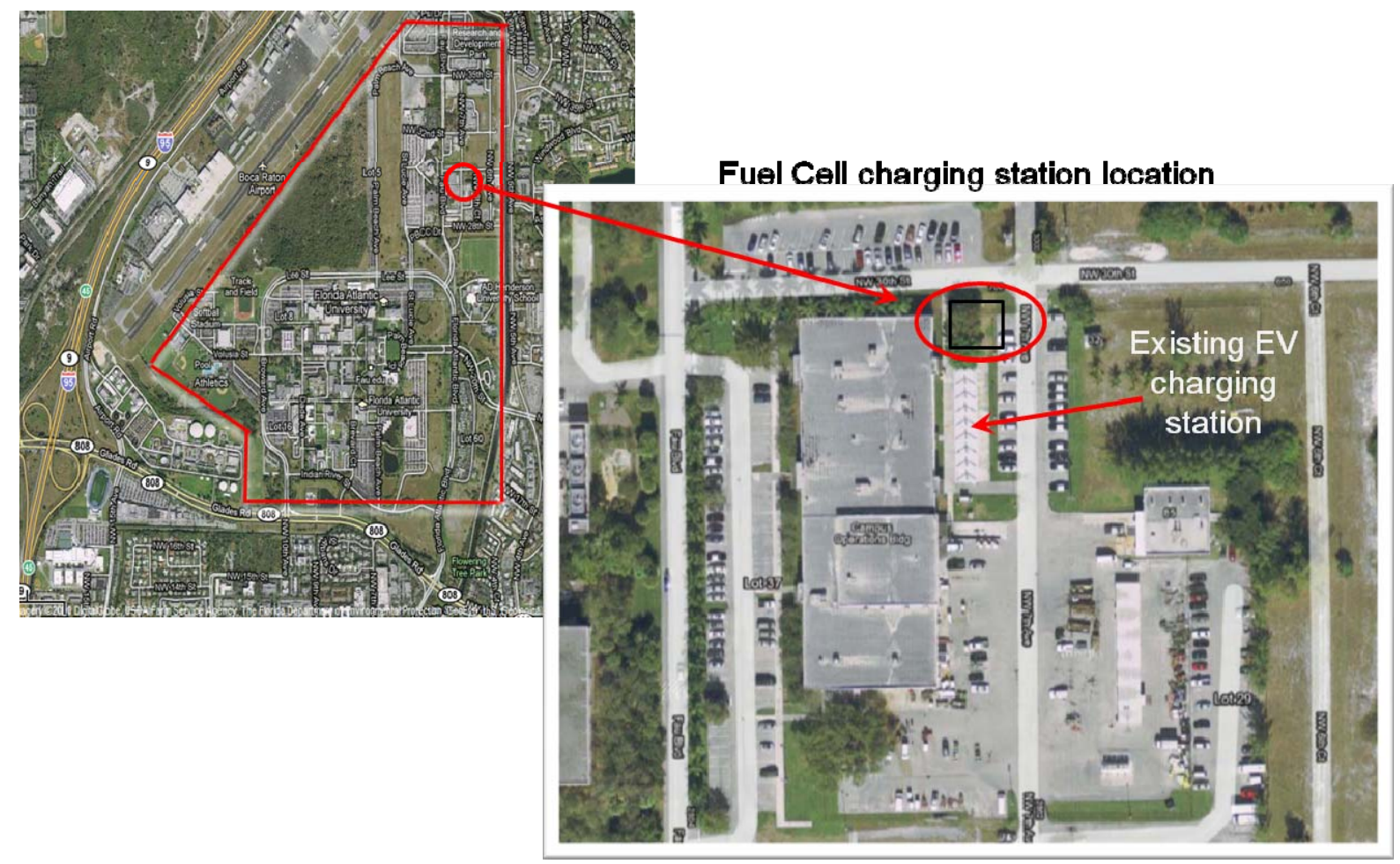

Figure 5.2 Fuel cell electric vehicle charging station location

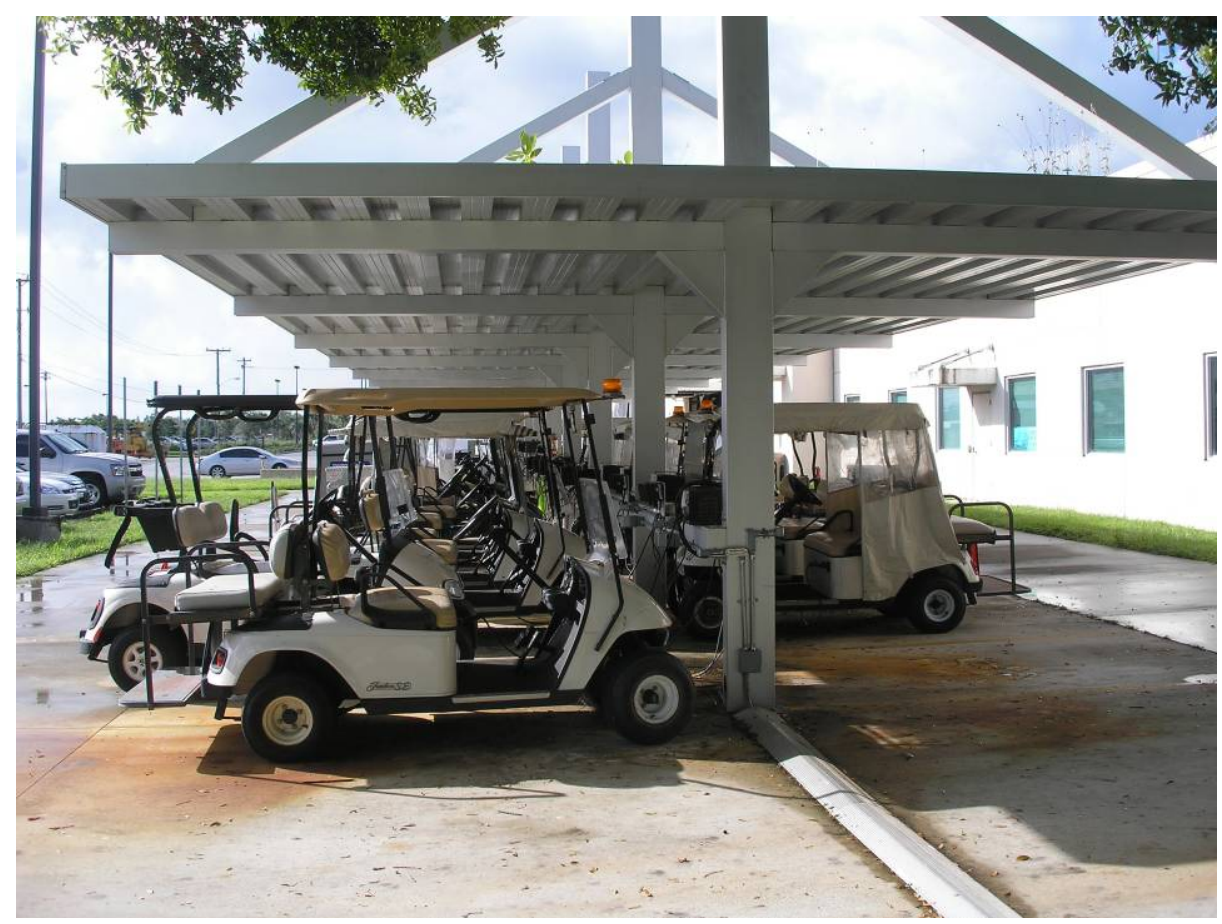

Figure 5.3 Existing FAU electric vehicle charging complex 


\subsection{Phase II -- Objectives and Accomplishments}

The goals and objectives of the Methanol Fuel Cell, Electric Vehicle Charging Station project were as follows.

- Design, construct and demonstrate a grid-independent, methanol fueled, fuel cell powered, electric vehicle charging station

- Utilize FCSs and inverters purchased for the HyTech rest area project

- Provide opportunity to expose students to fuel cell technology

- Promote the project to the public

Table 5.1 summarizes the accomplishments achieved with respect to the project objectives.

Table 5.1 MeOH Fuel Cell, EV Charging Station Project Objectives vs. Accomplishments

\begin{tabular}{|c|c|}
\hline Objective & Accomplishment \\
\hline $\begin{array}{l}\text { Design, construct and } \\
\text { demonstrate a grid- } \\
\text { independent, methanol } \\
\text { fueled, fuel cell powered, } \\
\text { electric vehicle charging } \\
\text { station }\end{array}$ & $\begin{array}{l}\text { - Completed charging station design } \\
\text { - Completed station construction } \\
\text { - Demonstrated station's potential to charge } \\
\text { electric vehicles }\end{array}$ \\
\hline $\begin{array}{l}\text { Utilize FCSs and inverters } \\
\text { purchased for the HyTech } \\
\text { Rest Area project }\end{array}$ & Accomplished \\
\hline $\begin{array}{l}\text { Provide opportunity to } \\
\text { expose students to fuel cell } \\
\text { technology }\end{array}$ & Accomplished \\
\hline $\begin{array}{l}\text { Promote the project to the } \\
\text { public }\end{array}$ & Accomplished by students \\
\hline
\end{tabular}

Demonstration of the EVCS was performed using the inverters, with the FCSs left off. Evaluation of the station's performance was based on FCS test results at EnerFuel combined with the results of using the inverters to charge the electric vehicles. The charging station could not be operated as initially intended since no grid power was available to support FCS startup. This is discussed in more detail in section 5.4.

The EVCS design did however successfully incorporate the FCSs and inverters, originally purchased for the HyTech Rest Area project. It also exposed students to the project by allowing several students to assist in the design and construction of the station. 


\subsection{Design}

\subsubsection{EVCS Design}

As previously mentioned the design of the EVCS was configured to utilize the FCSs, inverters and batteries obtained for Phase I. Although intended for backup power, the IdaTech FCSs were determined to be functionally capable of supporting the charging station's intended role. The only prerequisite for their use was the need for grid-power, to startup the FCS fuel processors/reformers. Once started, the FCSs would produce sufficient power for vehicle charging and support of all ancillary loads. However gridpower was not available, due to approval issues associated with connecting the FCSs to the local facility electrical mains. As a result, power for startup would need to come from the EVCS battery system.

In addition to the lack of mains power, permitting issues associated with installation of a 500 gallon methanol tank resulted in reliance on the 55 gallon "day" tanks built into each FCS.

Operation of the EVCS was initially intended to consist of using grid-power to heat up the FCS reformers while the battery system provided power to charge vehicles. Once the reformers reached operating temperature, $\mathrm{MeOH}$ would then be processed to provide the FCS stacks with hydrogen. At this point the FCS would take over the task of vehicle charging, and recharging of the battery system. Since heating of the reformer takes close to 4 hours, the EVCS would be started up early in the morning. It would then operate throughout the day, charging vehicles, until shutdown in the evening. However without grid-power, full operation of the EVCS was not possible.

The original design of the EVCS is shown in Figure 5.4, while Figure 5.5 shows the actual design implemented. Figure 5.6 provides the detailed electrical layout of the asbuilt charging station. Figures 5.7 to 5.10 are pictures of the actual charging station and parking spaces set aside for EV charging. 


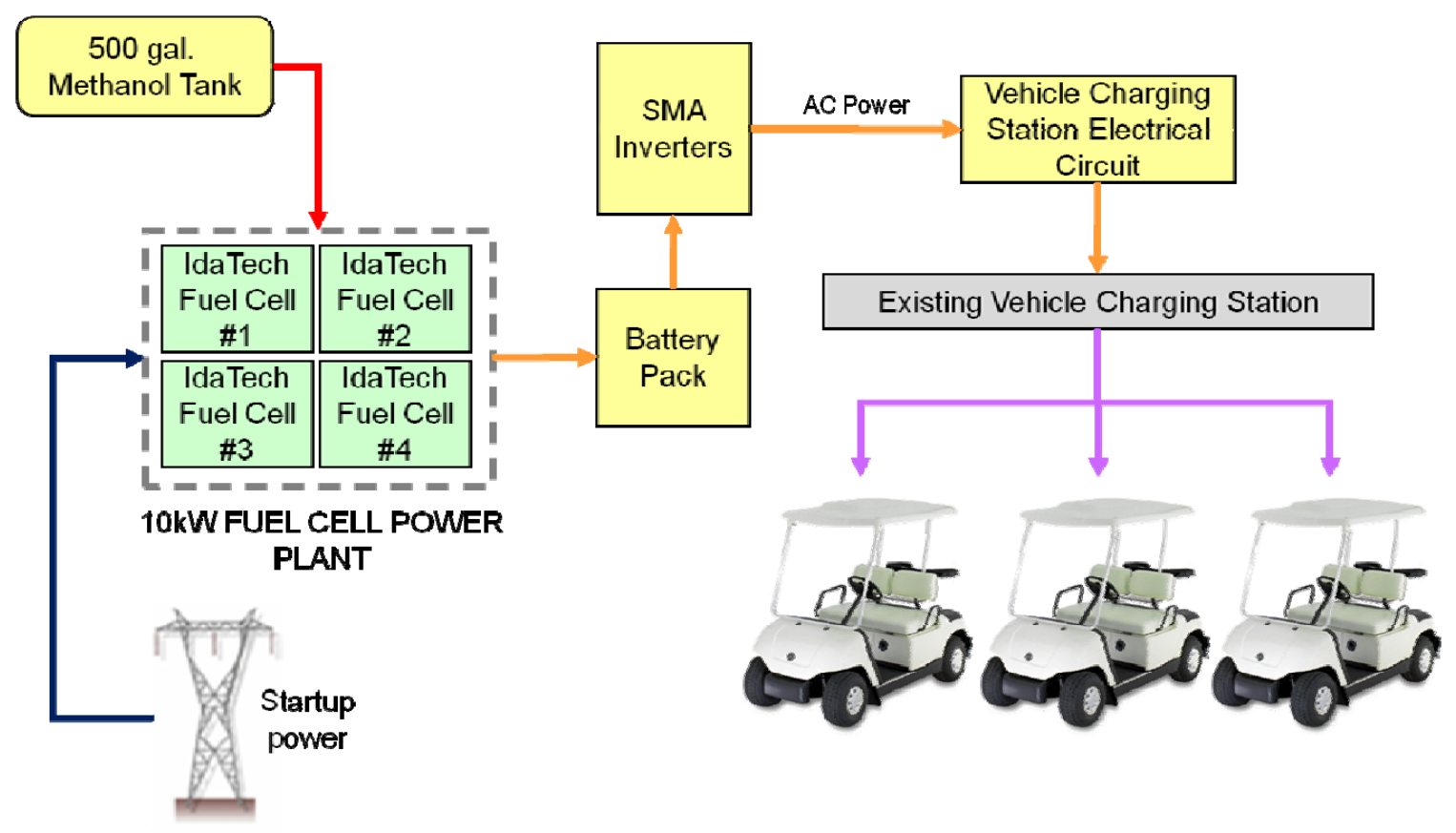

Figure 5.4 Original design of EV charging station

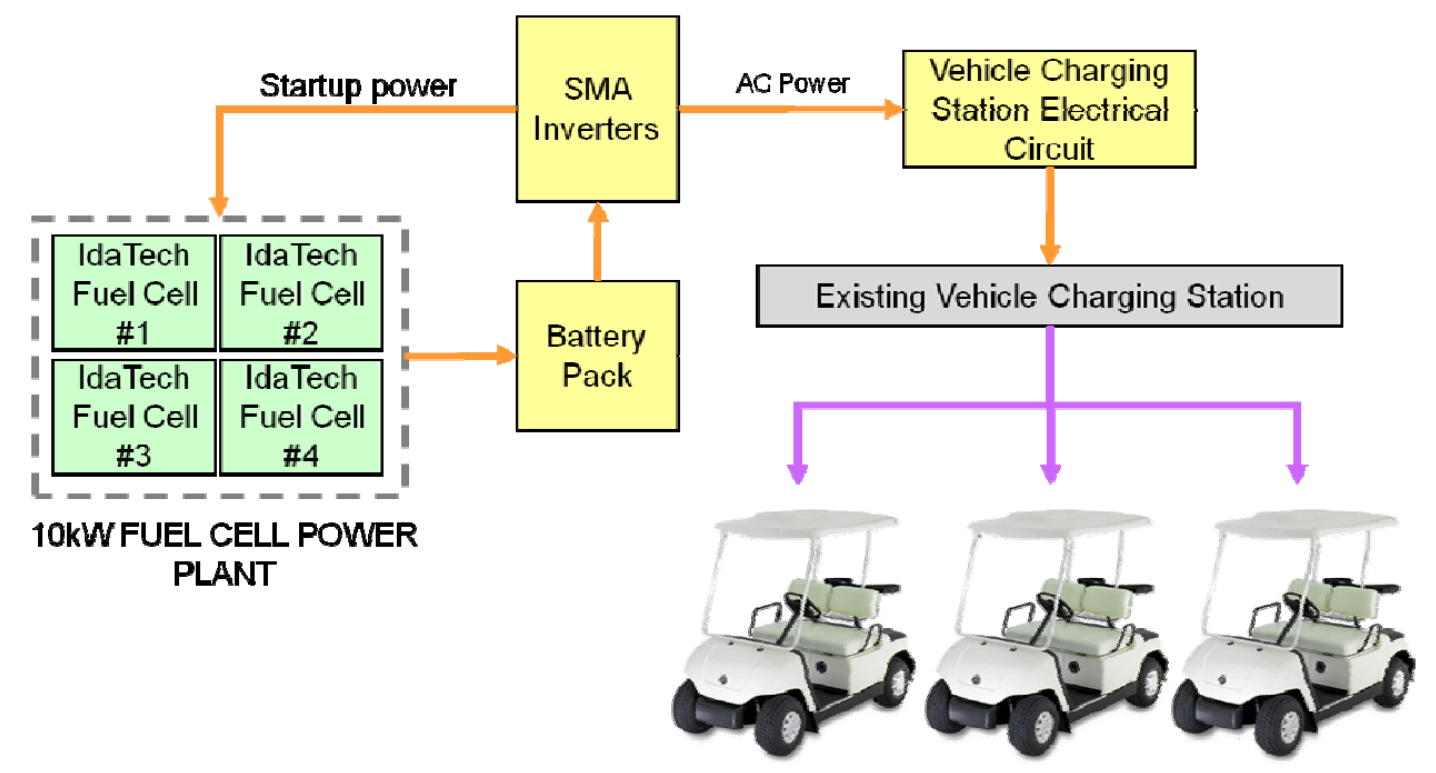

Figure 5.5 Actual EV charging station design implemented 


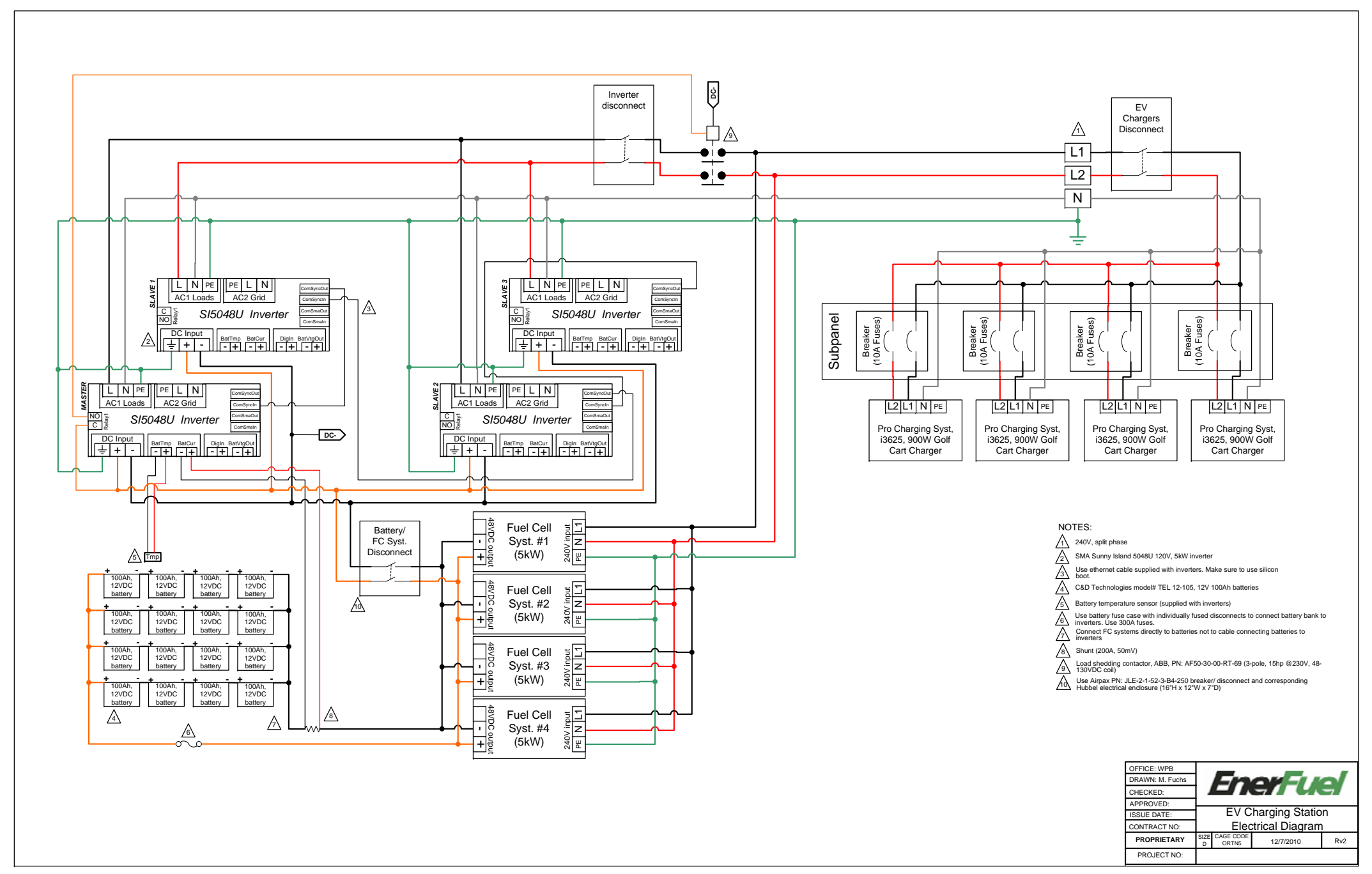

Figure 5.6 Detailed electrical layout of EV charging station, as implemented

ENERFUEL, INC.

Page $\mathbf{2 8}$ of $\mathbf{3 8}$ 


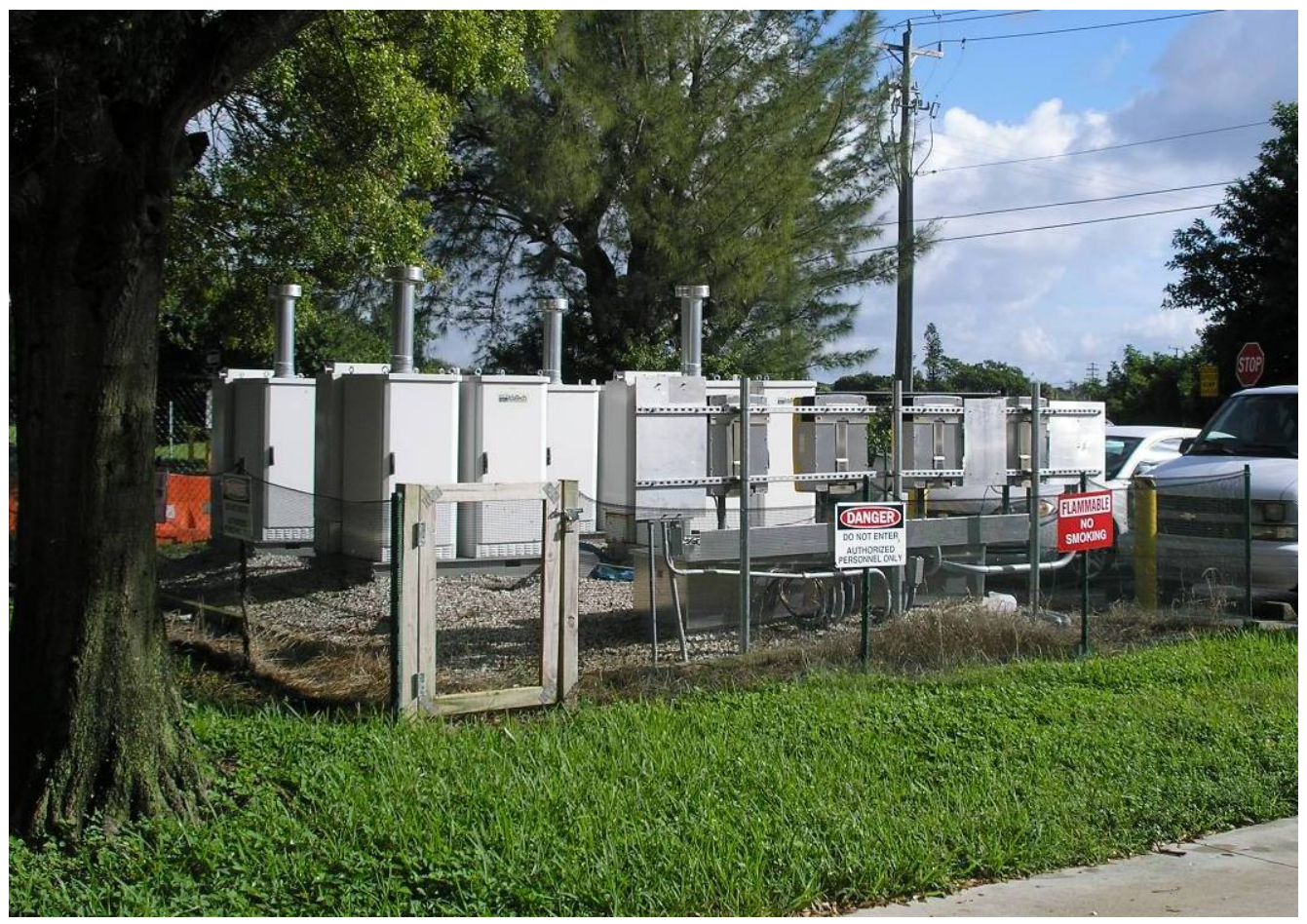

Figure 5.7 EV charging station

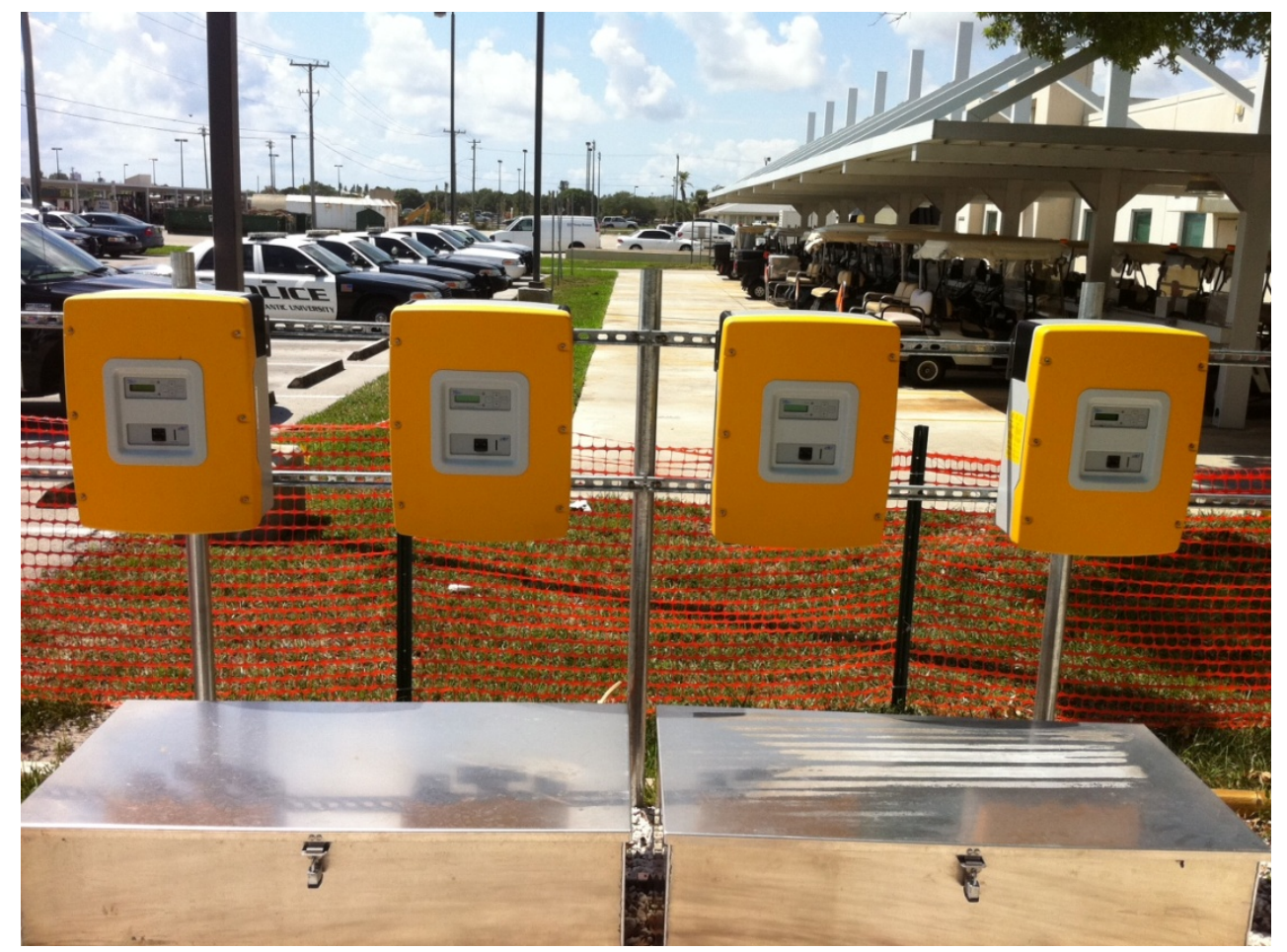

Figure 5.8 EVCS inverters and battery boxes with FAU EV charging complex in background 


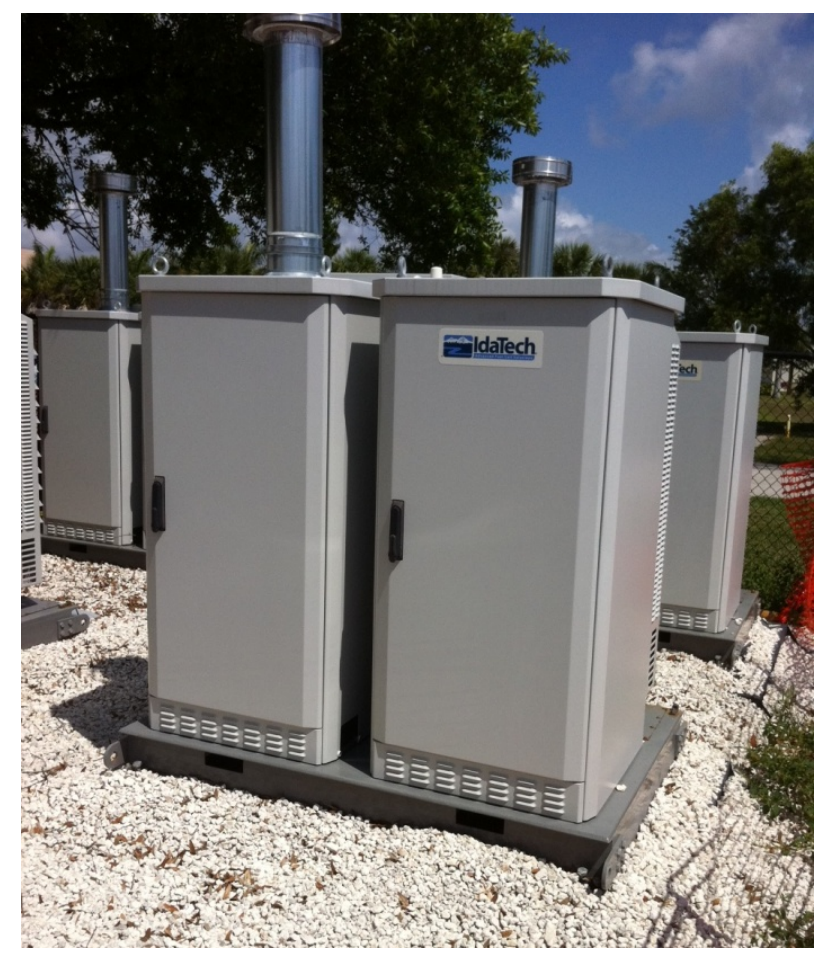

Figure 5.9 Close-up of an EVCS IdaTech FCS

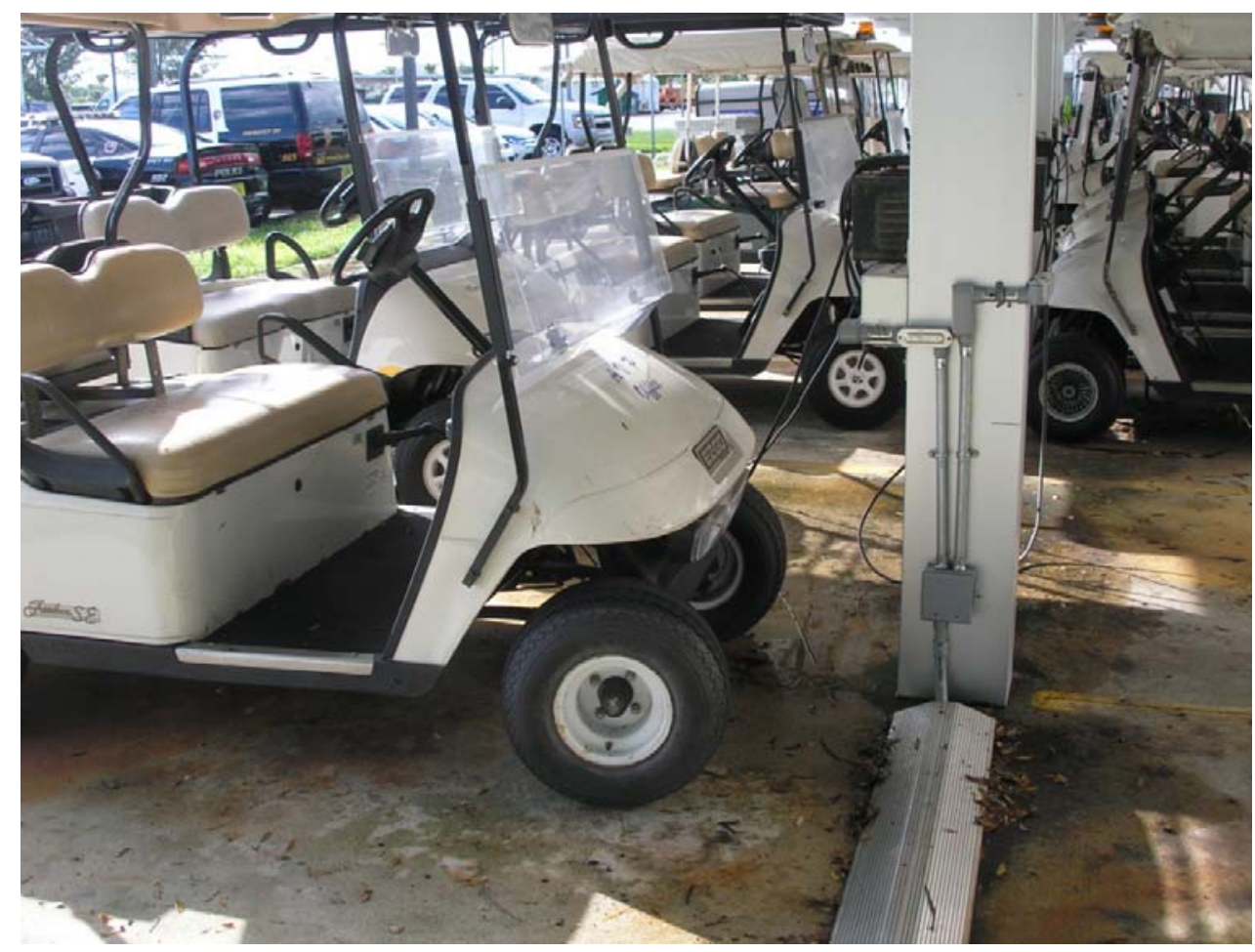

Figure 5.10 Picture of EV charging parking spaces (first two opposing spaces in foreground) 


\subsubsection{EVCS System Modeling}

To assist in design of the EVCS, a Microsoft Excel based analytical model was constructed to simulate the station's performance under a variety of scenarios. The model was configured to utilize user inputs, such as number of FCSs, battery system size, fuel storage size, EV charging load and station hours of operation, to calculate the total number of days the EVCS could be expected to operate on a specific amount of fuel. The model was also designed to estimate the battery system state-of-charge (SOC). Figure 5.11 shows the analytical model.

\section{EV CHARGE STATION SYSTEM MODEL} MF-7/12/2010

\begin{tabular}{|c|c|c|}
\hline \multicolumn{3}{|c|}{ CHARGE STATION OPERATION } \\
\hline Elect. Start=1, Batt. Start $=2$ & 1 & \\
\hline Total hrs of operation /day* & 8 & hrs \\
\hline Vehicle charging hr/day & 4 & hrs \\
\hline \multicolumn{3}{|c|}{ CHARGE STATION LOAD } \\
\hline ClipperCreek charger load & 5760 & Watts \\
\hline Number of ClipperCreeks & 0 & \\
\hline Golf cart charger load & 1380 & Watts \\
\hline Number of Golf cart chargers & 4 & \\
\hline \multicolumn{3}{|l|}{ REF O R M E R } \\
\hline Fuel & Methanol & \\
\hline Energy content & 5.48 & $\mathrm{kWh} / \mathrm{kg}$ \\
\hline Energy content & 16.41 & $\mathrm{kWh} / \mathrm{gal}$ \\
\hline Density & 0.79 & $\mathrm{~kg} / \mathrm{L}$ \\
\hline Number of fuel cell systems & 4 & \\
\hline Heater voltage & 240 & VAC \\
\hline Heater current draw & 9.0 & Amp \\
\hline Startup time & 3.5 & hrs \\
\hline Number of startups & 1 & \\
\hline Standby power & 500 & Watts \\
\hline \multicolumn{3}{|l|}{ FUEL SPECIFICS } \\
\hline Fuel tank size & 720 & gallons \\
\hline Methanol-to-water mix ratio & $62.0 \%$ & Vol\% \\
\hline \multicolumn{3}{|c|}{ B A T TER IES I I NVERTERS } \\
\hline Number of batteries in series & 4 & \\
\hline Number of strings in parallel & 4 & \\
\hline Battery voltage & 12.0 & VDC \\
\hline Battery rated current capacity & 98 & Ah \\
\hline Number of inverters & 4 & \\
\hline Inverter standby power & 4 & Watts \\
\hline Inverter standby duration/ day & 24 & hrs \\
\hline
\end{tabular}

CHARGE STATION PERFORMANCE Total battery voltage

Total battery rated current capacity Total battery rated power capacity Startup power

Battery capacity after startup Battery SOC

Vehicle charging load/ day Battery recharge $\mathrm{Wh} /$ day Reformer standby Wh/ day Inverter standby Wh/ day Total EV Chrg Stn load / day Avg EV Chrg Stn power demand Methanol usage (@23\% EVCS eff.) Time to recharge batteries Fuel tank range

\begin{tabular}{|c|c|}
\hline 48.0 & VDC \\
\hline 392 & $\mathrm{Ah}$ \\
\hline 18816 & $\mathrm{Wh}$ \\
\hline 0 & $\mathrm{Wh}$ \\
\hline 18816 & $\mathrm{Wh}$ \\
\hline $100 \%$ & \\
\hline 22080 & $\mathrm{Wh}$ \\
\hline 0 & $\mathrm{Wh}$ \\
\hline 16000 & $\mathrm{Wh}$ \\
\hline 384 & $\mathrm{Wh}$ \\
\hline 38464 & $\mathrm{Wh}$ \\
\hline 4808 & $\mathrm{Watts}$ \\
\hline 10.2 & gal/day \\
\hline $\mathbf{0 . 0}$ & hrs \\
\hline $\mathbf{4 3 . 8}$ & days \\
\hline
\end{tabular}

Figure 5.11 Microsoft Excel based analytical model (yellow fields denote model inputs while green fields denote outputs)

Model results, assuming grid-power for startup, are shown in Tables 5.3. Simulations showed that the EVCS would be able to operate for well over a month using a 500 gallon methanol tank combined with the built-in tanks on the FCSs. This also assumed the station would be charging vehicles for 4 out of the 8 hours the station is available for 
charging. If the vehicle charging time was increased to 6 hours over the 8-hour day, the charging station would still be expected to operate over a month on a single refueling.

In the case in which the battery system provided for FCS startup, model results showed that the fuel stored in the FCSs would be sufficient to operate the EVCS for roughly 2 weeks (Table 5.4). However, the process of startup would result in either a complete discharge of the batteries or a SOC sufficiently low to cause the inverters to stop outputting power (during startup the inverters would use battery power to provide the FCSs with the necessary 240VAC power for reformer heat-up).

Although the SOC cut-off setting, on the SMA inverters, can be adjusted, the SOC reached by the battery system, as per simulation 2 in Table 5.4, is below the minimum setting. This behavior was observed when attempting to startup one of the FCSs, even with a battery system sized for four FCSs. As per IdaTech installation requirements, each FCS is required to have its own battery system, which, in the EVCS design, is tied in parallel with the other FCS batteries. Increasing the size of the battery system might have resulted in the ability to startup the EVCS, however was not practical both from an integration and battery recharging perspective.

Modeling results did show that the intended EVCS design, i.e. with grid-power startup, would have resulted in a charging station fully meeting project objectives.

Table 5.3 EVCS System Model Results (grid-power used for FCS startup)

\begin{tabular}{|c|c|c|c|c|}
\hline Operating Conditions & $\begin{array}{c}\text { Simulation } \\
1\end{array}$ & $\begin{array}{c}\text { Simulation } \\
2\end{array}$ & $\begin{array}{c}\text { Simulation } \\
3\end{array}$ & $\begin{array}{c}\text { Simulation } \\
4\end{array}$ \\
\hline $\begin{array}{l}\text { Total vehicle charging per } \\
\text { day }\end{array}$ & 4 hours & 4 hours & 4 hours & 6 hours \\
\hline $\begin{array}{l}\text { Total EVCS "on" time per } \\
\text { day }\end{array}$ & 8 hours & 8 hours & 8 hours & 8 hours \\
\hline Number of IdaTech FCSs & 4 & 4 & 2 & 4 \\
\hline $\begin{array}{l}\text { Number of } 900 \text { Watt EV } \\
\text { chargers supported by } \\
\text { EVCS }\end{array}$ & 4 & 4 & 2 & 4 \\
\hline Total MeOH storage & 720 gal.* & 220 gal.** & 720 gal.* & 720 gal.* \\
\hline $\begin{array}{l}\text { Number of days of } \\
\text { operation }\end{array}$ & 43.8 & 13.4 & 86.7 & 34 \\
\hline
\end{tabular}

* 500 gallon tank plus FCS built-in tanks (55 gal. each)

** FCS built-in tanks only (55 gal. each) 


\section{Table 5.4 EVCS System Model Results (Battery system used for FCS startup)}

\begin{tabular}{|c|c|c|}
\hline Operating Conditions & $\underset{1}{\text { Simulation }}$ & $\begin{array}{c}\text { Simulation } \\
2\end{array}$ \\
\hline $\begin{array}{l}\text { Total vehicle charging per } \\
\text { day }\end{array}$ & 4 hours & 4 hours \\
\hline $\begin{array}{l}\text { Total EVCS “on" time per } \\
\text { day }\end{array}$ & 8 hours & 8 hours \\
\hline Number of IdaTech FCSs & 2 & 1 \\
\hline $\begin{array}{l}\text { Number of } 900 \text { Watt EV } \\
\text { chargers supported by } \\
\text { EVCS }\end{array}$ & 2 & 2 \\
\hline Total MeOH storage & 220 gal. ** & 220 gal. $^{* *}$ \\
\hline $\begin{array}{l}\text { Battery system SOC at } \\
\text { end of startup***}\end{array}$ & $0 \%$ & $46 \%$ \\
\hline $\begin{array}{l}\text { Time to recharge battery } \\
\text { system }\end{array}$ & $2.8 \mathrm{hrs}$ & $5.8 \mathrm{hrs}$ \\
\hline $\begin{array}{l}\text { Number of days of } \\
\text { operation }\end{array}$ & 12.4 & 18.8 \\
\hline
\end{tabular}

** FCS built-in tanks only (55 gal. each)

*** Assumes a battery system size designed to support four FCS

\subsection{EVCS Construction}

Construction of the charging station was performed by FAU's mechanical engineering department. Site location, permitting and construction took roughly two years to complete. Although approval for the construction was secured, approval for installation of a 500 gallon, above ground methanol tank could not be obtained. Neither was it possible to connect the FCSs to the adjacent building's mains power. Given these limitations, the EVCS could not be made fully operational.

\subsection{EVCS Testing Results}

Evaluation of the charging station consisted of testing EVCS subsystems independently, i.e. the FCSs, batteries and inverters at EnerFuel, and the inverters, batteries and vehicle chargers at FAU. The data collected at EnerFuel and from vehicle charging at FAU were combined to determine the performance of the EVCS.

Testing at EnerFuel was conducted before the FCSs, inverters and batteries were shipped to FAU for installation. The first set of tests consisted of running each FCS separately to confirm performance at half and full power (5kW). Figure 5.12 shows how each FCS was connected.

The second portion of testing consisted of testing the inverters and batteries connected to one of the FCSs. The purpose of these tests was to verify the physical integration of 
the three components and to program the inverters to properly manage the power from the FCSs during vehicle and battery charging.

Testing at FAU consisted of testing the vehicle charging capabilities of the inverter/battery subsystem. This was achieved by charging two golf carts using the stored energy of the battery system. During this process data was collected from the batteries and inverters, i.e. battery voltage and current into the inverters, and inverter voltage and current into the vehicle chargers. The conversion efficiency of the chargers was obtained from previous measurements made on the chargers themselves.

Figure 5.13 shows the fuel cell stack performance of one of the FCSs. Figure 5.14 and 5.15 provides the typical performance of the FCSs during testing at EnerFuel. Based on the data gathered at FAU and EnerFuel, the overall efficiency of the EVCS was calculated to be $22.5 \%$. This efficiency is based on the energy input of the methanol with respect to the electric power/energy used to charge the vehicle. Table 5.5 summarizes the efficiency steps from conversion of the fuel to the point at which it is delivered to the vehicle.

In comparison with operating a grid powered EV charger, the EVCS's efficiency of $22.5 \%$ is higher. Taking into account the typical losses associated with generating and delivering "grid" electricity (typically $28 \%$ by the time it reaches the receptacle), vehicle charging would occur at an overall efficiency of $19 \%$ to $20 \%$.

Although not able to be fully demonstrated, testing did show that the fuel cell EVCS concept has the ability to charge EVs independent of grid-power, at higher efficiency and using renewable fuels.

Table 5.5 Overall EVCS Efficiency (Fuel-to-EV)

\begin{tabular}{|c|c|c|c|}
\hline $\begin{array}{c}\text { Fuel Cell } \\
\text { System Eff. }\end{array}$ & \multicolumn{1}{|c|}{$\begin{array}{c}\text { Inverter } \\
\text { Eff. }\end{array}$} & $\begin{array}{c}\text { Charger } \\
\text { Eff. }\end{array}$ & $\begin{array}{c}\text { Overall } \\
\text { Eff. }\end{array}$ \\
\hline $35 \%$ & $92 \%$ & $70 \%$ & $\mathbf{2 2 . 5 \%}$ \\
\hline
\end{tabular}




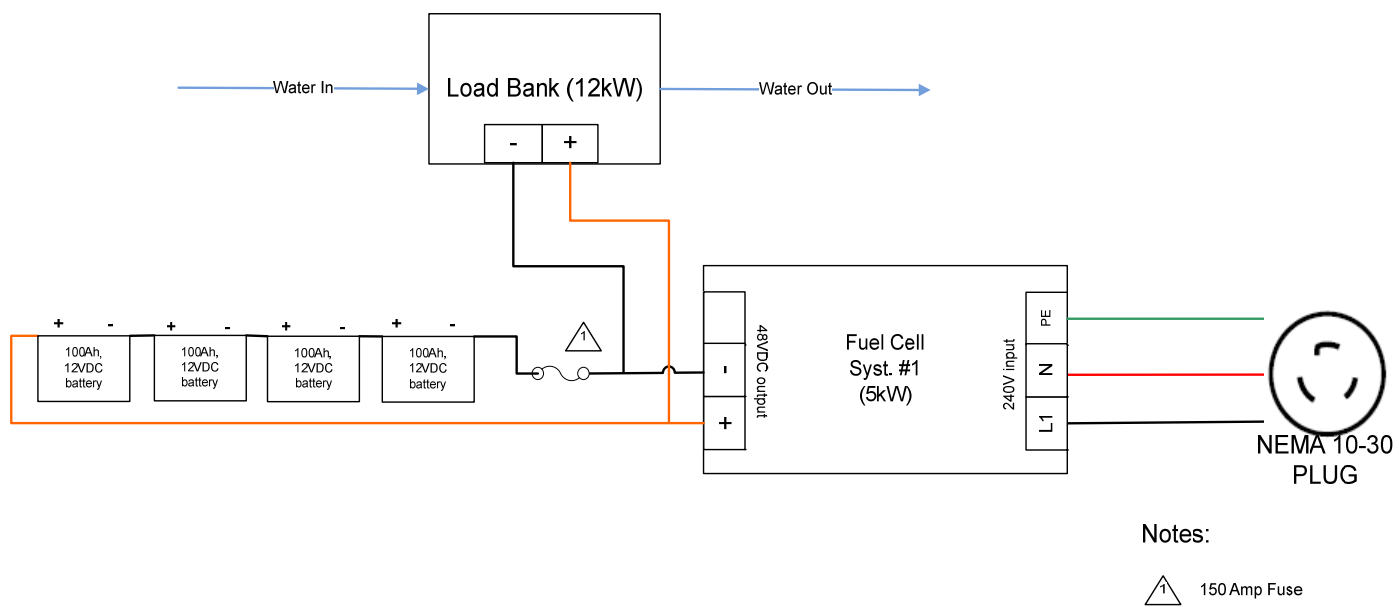

Figure 5.12 Configuration used to test individual fuel cell systems

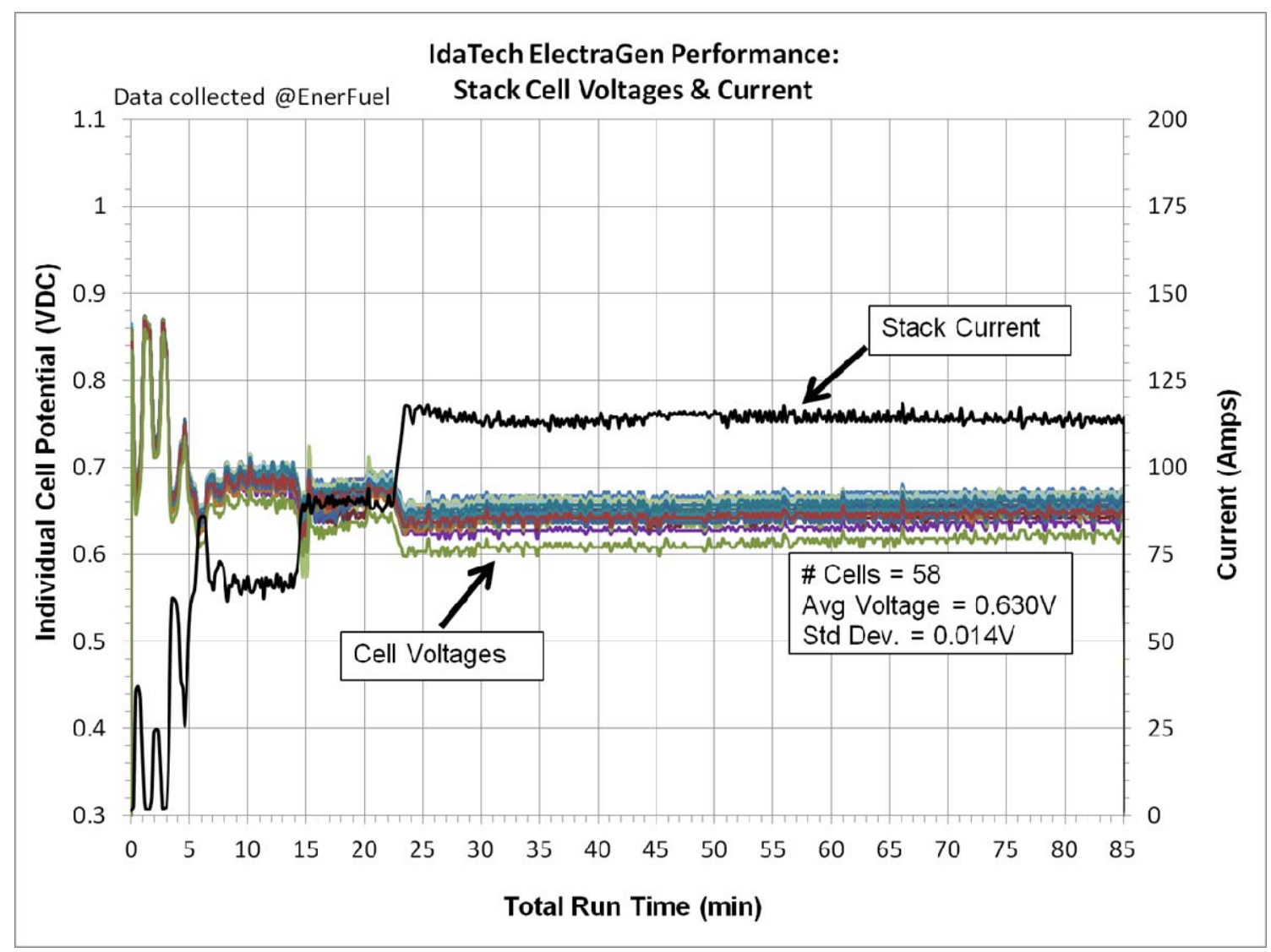

Figure 5.13 Performance of fuel cell system stack during full power operation 


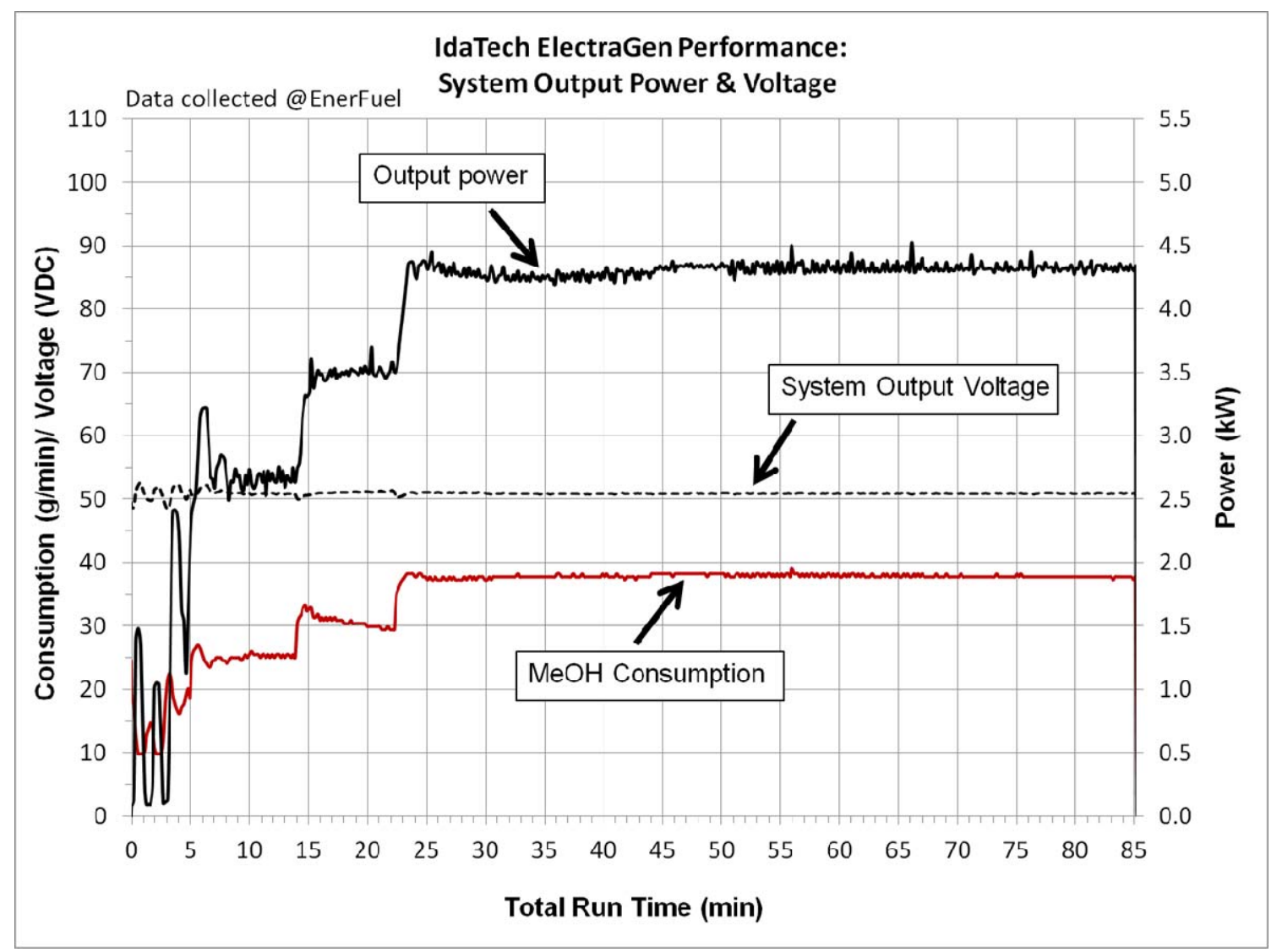

Figure 5.14 Performance of fuel cell system showing net power output, output voltage and fuel consumption 


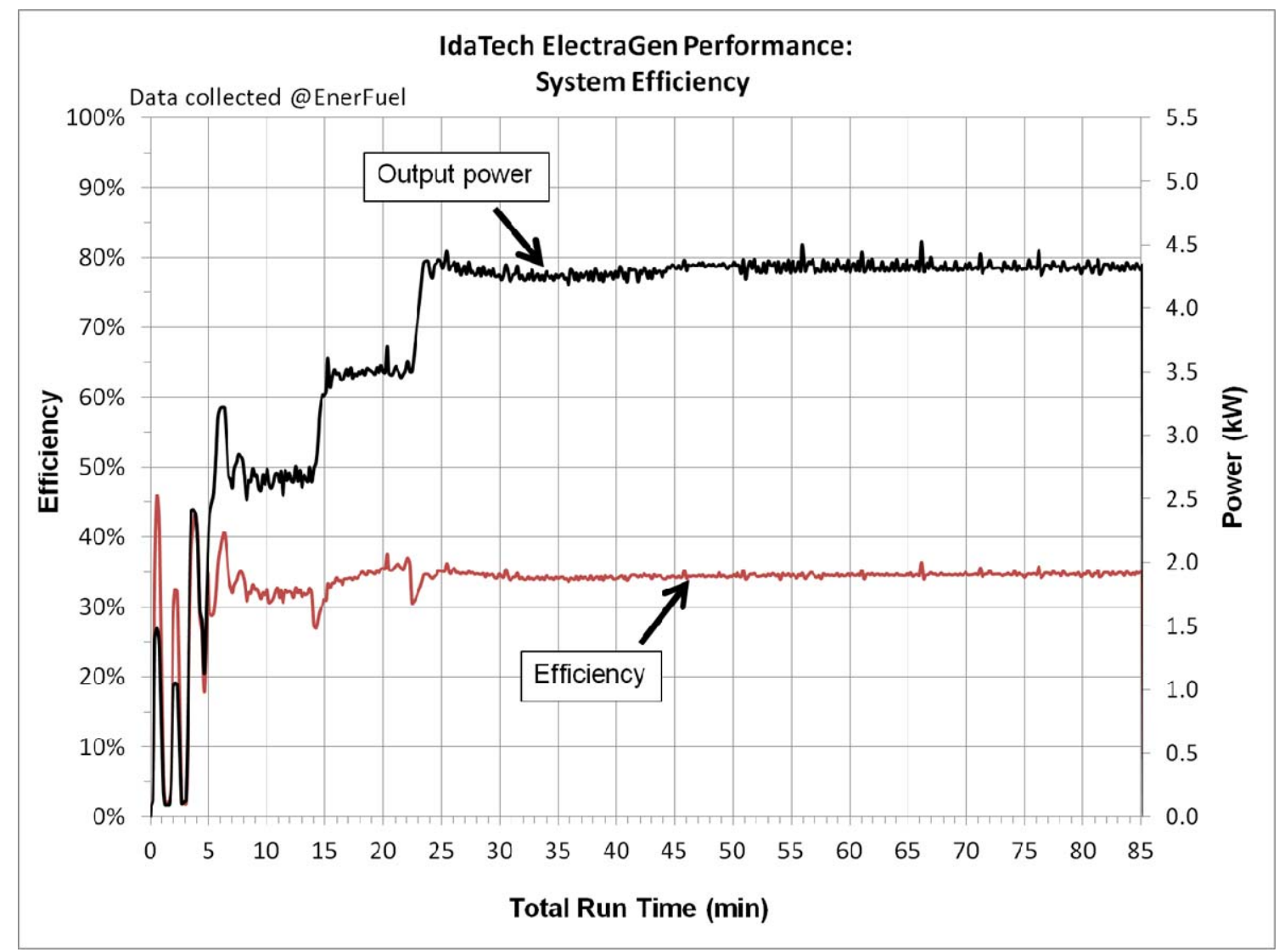

Figure 5.15 Performance of fuel cell system showing net power output and system efficiency

\subsection{EVCS Project Accomplishments}

The project was successful in accomplishing several goals, as well as showing the station's potential to function better than common EV charging methods. Testing showed that the fuel cell EVCS concept has the ability to charge EVs independent of grid-power, at higher efficiency and using renewable fuels.

Project accomplishments are listed below.

- Completed construction of the MeOH fuel cell, EV charging station

- Successfully reused all major components from the HyTech Rest Area project

- Tested charging capabilities with actual electric vehicles

- Assessed overall EVCS performance

- Evaluated performance of fuel cell systems and inverters

- Constructed analytical model of charging station

- Determined overall system efficiency to be greater than $22 \%$, roughly 2 percentage points greater than traditional EV charging methods 


\section{Concluding Remarks}

The Methanol Fuel Cell, Electric Vehicle Charging Station project, although originally a project to demonstrate the generation of electrical power from citrus waste, showcased the use of fuel cell technology in a meaningful way. The charging station showed the advantages of using a fuel cell, i.e. providing grid independent vehicle charging, at high efficiency and with the ability to use renewable fuels. The fuel cell charging station concept could be used at any location that requires a backup to grid charging, that has no access to grid-power or that requires a rapid deployed charging system. Operations that rely on electric vehicles could continue to operate even during a major power outage, such as brought on by "Katrina"/ "Sandy" type events. 


\title{
FLORIDA HYDROGEN INITIATIVE Sub-contractor Finel Report \\ Assessment of Public Understanding of the Hydrogen Economy Through Science
}

\author{
JoAnn Newman \\ Orlando Science Center \\ 777 East Princeton Street \\ Orlando, Florida 32803 \\ inewman@osc.org \\ 407-514-2024 \\ Submitted to: \\ Florida Hydrogen Initiative, Inc. \\ Review done by: \\ David L. Block \\ Florida Solar Energy Center/University of Central Florida \\ 1679 Clearlake Road \\ Cocoa, FL 32922 \\ E-mail: block@fsec.ucf.edu \\ Contract Number: DE-FC36-04GO14225 \\ FHI Report, Task 3
}

Project Start Date: July 31, 2006

Project End Date: April 24, 2009

September 2009 


\title{
Assessment of Public Understanding of the Hydrogen Economy Through Science
}

\author{
Orlando Science Center
}

\section{Executive Summary}

The primary objective of the Assessment of Public Understanding of the Hydrogen Economy Through Science project was to design and build an interactive Science Center exhibit called: " $\mathrm{H}_{2} \mathrm{Now}$ : the Great Hydrogen Xchange". The exhibit would be used to satisfy the following three objectives:

- Create awareness among all science center audiences on hydrogen as a renewable energy source

- Assess current public understanding about hydrogen science and engineering

- Increase public understanding of hydrogen science and engineering

Finding sources of clean, renewable energy is one of today's most pressing issues. However, there is much information - and misinformation- about renewable energy, particularly regarding the use of hydrogen as a new energy source. Increasing the basic knowledge and awareness among the public about hydrogen as a viable energy source, and correcting misconceptions of hydrogen and its properties, is necessary to create accurate and positive opinions about this substance as an important alternative to fossil fuels.

Key to this goal is the accurate and educationally effective translation of technical scientific and engineering concepts into a format that is easily understandable and accessible. The developed and fabricated hydrogen, $\mathrm{H}_{2} \mathrm{Now}$ : the Great Hydrogen Xchange exhibit will allow Orlando Science Center visitors to be able to visualize a community powered entirely by hydrogen, and discover that the renewable energy source is a viable alternative to other energy sources currently in use.

Working with I.d.e.a.s at Disney MGM Studios, the Orlando Science Center (OSC) set out to perform front end evaluation and subsequent design, fabrication, and installation of an interactive exhibition at OSC. Beginning with the schematic design of concepts and educational objectives, the exhibit will undergo design and prototyping of both physical components and interactive software. This exhibition, $\mathrm{H}_{2} \mathrm{Now}$, the Great Hydrogen Xchange, contains two main interactive areas. The first, the $\mathrm{H} 2$ Charging Station, is where the visitors will have the opportunity to express their opinions about alternative energy sources, and then interact with increasing levels of content about hydrogen as a source of fuel. As they answer questions correctly about hydrogen, they can earn Hydrogen Energy Units (HEU's) that they can 'spend' at Hydropolis to power various interactive 'city' elements.

From mid-August through December 2008, software developers at I.d.e.a.s , working with OSC staff, incorporated several changes to the software design and interface based on critical analysis of visitor usage to test for mechanical effectiveness. At the end of that time, it was determined that major modifications were needed to both software design and communication interface. OSC brought in a local multimedia design firm to 
complete this redesign at which time work concentrated on visitor evaluation to determine the extent to which the affective and cognitive goals of the exhibit were being met.

Evaluations with display visitors were conducted in August 2008 and again, after remediation, in February and March 2009. A total number of 65 visitors were evaluated using the observation method and 66 using the interview method for a total of 131 visitors. (Note that not all observed visitors took part in the interview). Since the exhibit was designed for visitors age 12 and up, participants were pulled from this age group and/or if a parent with children under age 12 was interviewed, the questions were posed to the parent. Additionally, children under age 12 were excluded from observation portion of the evaluation. The following two methods were used for the evaluations:

1) Interview: An evaluator approached visitors after they interacted with the exhibit and asked the individual questions and then recorded their responses on the questionnaire. The questionnaire was designed to determine both affective and cognitive objectives.

2) Visitor observation: Visitors were observed using the exhibit, to determine if they could use the exhibit correctly. Correctly was defined as

a. Retrieving a hydrocell at the Supply station

b. Taking hydrocell to one of 4 charging stations, docking the hydrocell, and answering the content questions on their screen to 'charge' their hydrocell

c. Taking the hydrocell to Hydropolis, docking hydrocell and following screen instructions.

There were five learning objectives established for $\mathrm{H}_{2}$ Now. These objectives were done so that after participating in the exhibit, visitors would be able to:

1. Explain what hydrogen is, where it comes from, and some of its possible uses.

2. Distinguish between truth and myth about hydrogen as an alternative energy source, and give specific examples.

3. State a benefit and a challenge of hydrogen as an alternative energy source.

4. Give an example of how hydrogen power is created, and how it can effect everyday life.

5. Comment on a possible impact of hydrogen power on the future state of the world.

The interview/questionnaire method was designed to glean visitor understanding of these five objectives. Clearly, the results indicate that the vast majority of visitors were able to achieve the first learning objective, and most visitors achieved the third learning objective. The results also indicate that, while the 'myth' of hydrogen as an alternative energy source was not specifically measured, it is clear that most visitors came away with a clear understanding about hydrogen's use as a renewable energy source. Additionally, visitors were very clear on the benefits i.e., "clean, renewable, doesn't pollute', and as the results showed, also clear on its current challenges i.e., 'too expensive to produce'. Most visitors were also able to verbalize how hydrogen power is created as it is presented in the exhibit, and the extent that hydrogen can be used to effect everyday life. The only learning objective that was not clearly measured, but can be inferred from the affective questions posed, is the possible impact of hydrogen power on the world's future. 
The $\mathrm{H}_{2}$ Now exhibit has completed software and hardware 'shakedown' that resulted in a streamlined interface and communication and is functioning at $100 \%$ in the Orlando Science Center. In addition, exhibit enhancements have been made including a linear display on additional sources of renewable energy and plans are in the works for an interactive 'video game' on renewable energy to complement the messages of $\mathrm{H}_{2} \mathrm{Now}$. The video game was received from a grant from Progress Energy Foundation.

Evaluations to determine the extent to which $\mathrm{H}_{2} \mathrm{Now}$ meets educational objectives revealed that:

- $77 \%$ of the visitors surveyed either loved the exhibit or liked the exhibit

- $64 \%$ indicated that they 'learned something' or found the exhibit 'informative' in the word choice section of the interview

- $32 \%$ found the exhibit 'interesting' indicating that both cognitive and affective learning has clearly taken place

- $65 \%$ indicated that they found out something they didn't know before. This finding is a clear indication of both cognitive gain and a change of attitude i.e., compare this to the $44 \%$ who, when given the choice to select "I learned something new".

In addition, OSC has enhanced the experience with a short film produced by i.d.e.a.s called "I Am Hydrogen" and is exhibiting a linear display on additional information on renewable energy. 


\section{TABLE OF CONTENTS}

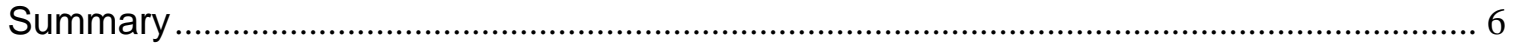

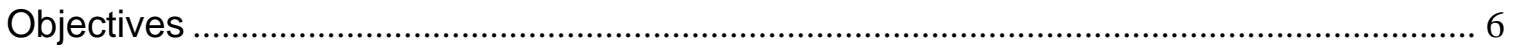

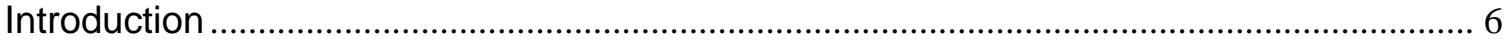

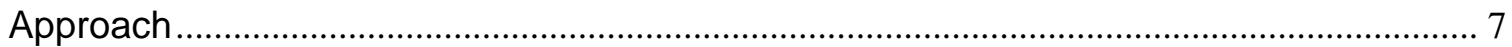

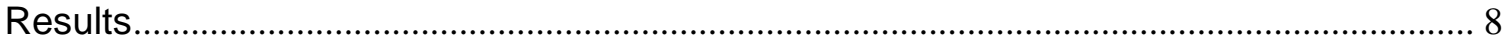

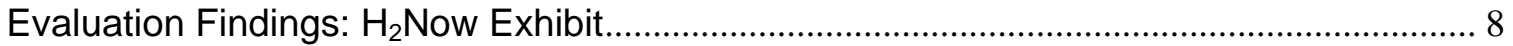

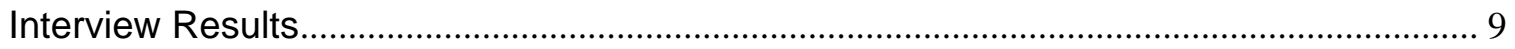

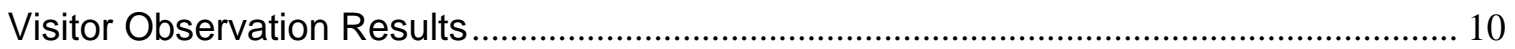

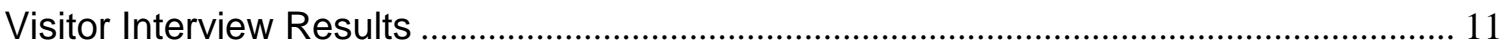

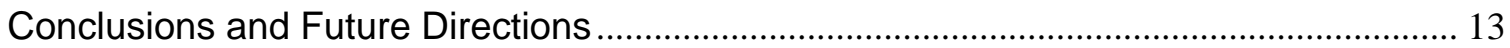

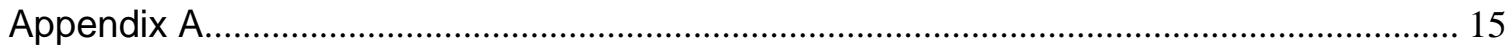




\title{
Assessment of Public Understanding of the Hydrogen Economy Through Science
}

\author{
Orlando Science Center
}

\section{Summary}

The primary objective of this project was to design and build an interactive Science Center exhibit called: " $\mathrm{H}_{2}$ Now: the Great Hydrogen XChange". The exhibit would be used to create awareness among all science center audiences on hydrogen as a renewable energy source, assess current public understanding about hydrogen science and engineering and increase public understanding of hydrogen science and engineering.

Working with I.d.e.a.s at Disney MGM Studios, the Orlando Science Center (OSC) created the $\mathrm{H}_{2}$ Now exhibit. The exhibit has physical components, interactive software and contains two main interactive areas. The first interactive area, the $\mathrm{H} 2$ Charging Station, is where the visitors will have the opportunity to express their opinions about alternative energy sources, and then interact with increasing levels of content about hydrogen as a source of fuel. As they answer questions correctly about hydrogen, they can earn Hydrogen Energy Units (HEU's) that they can 'spend' at Hydropolis to power various interactive 'city' elements which is the second interactive area. Results from visitor evaluations revealed that $77 \%$ of the visitors surveyed either loved or liked the exhibit, $64 \%$ indicated that they 'learned something' or found the exhibit 'informative', $32 \%$ found the exhibit 'interesting' indicating that both cognitive and affective learning has clearly taken place and $65 \%$ indicated that they found out something they did not know before.

\section{Objectives}

The primary objective of this project was to design and build an interactive Science Center exhibit called: " $\mathrm{H}_{2}$ Now: the Great Hydrogen Xchange". The exhibit would be used to satisfy the following objectives:

- Create awareness among all science center audiences on hydrogen as a renewable energy source

- Assess current public understanding about hydrogen science and engineering

- Increase public understanding of hydrogen science and engineering

\section{Introduction}

Finding sources of clean, renewable energy is one of today's most pressing issues. However, there is much information - and misinformation- about renewable energy, particularly regarding the use of hydrogen as a new energy source. Increasing the basic knowledge and awareness among the public about hydrogen as a viable energy source, and correcting misconceptions of hydrogen and its properties, is necessary to create accurate and positive opinions about this substance as an important alternative to fossil fuels. 
Key to this goal is the accurate and educationally effective translation of technical scientific and engineering concepts into a format that is easily understandable and accessible. The developed and fabricated hydrogen, $\mathrm{H}_{2} \mathrm{Now}$ : the Great Hydrogen Xchange exhibit will allow Orlando Science Center visitors to be able to visualize a community powered entirely by hydrogen, and discover that the renewable energy source is a viable alternative to other energy sources currently in use.

\section{Approach}

Working with I.d.e.a.s at Disney MGM Studios, the Orlando Science Center (OSC) set out to perform front end evaluation and subsequent design, fabrication, and installation of an interactive exhibition at OSC. Beginning with the schematic design of concepts and educational objectives, the exhibit will undergo design and prototyping of both physical components and interactive software. This exhibition, $\mathrm{H}_{2} \mathrm{Now}$, the Great Hydrogen Xchange, contains two main interactive areas. The first, the $\mathrm{H} 2$ Charging Station, is where the visitors will have the opportunity to express their opinions about alternative energy sources, and then interact with increasing levels of content about hydrogen as a source of fuel. As they answer questions correctly about hydrogen, they can earn Hydrogen Energy Units (HEU's) that they can 'spend' at Hydropolis to power various interactive 'city' elements.

A sample screen for the $\mathrm{H}_{2}$ Now Charging Stations is shown below:

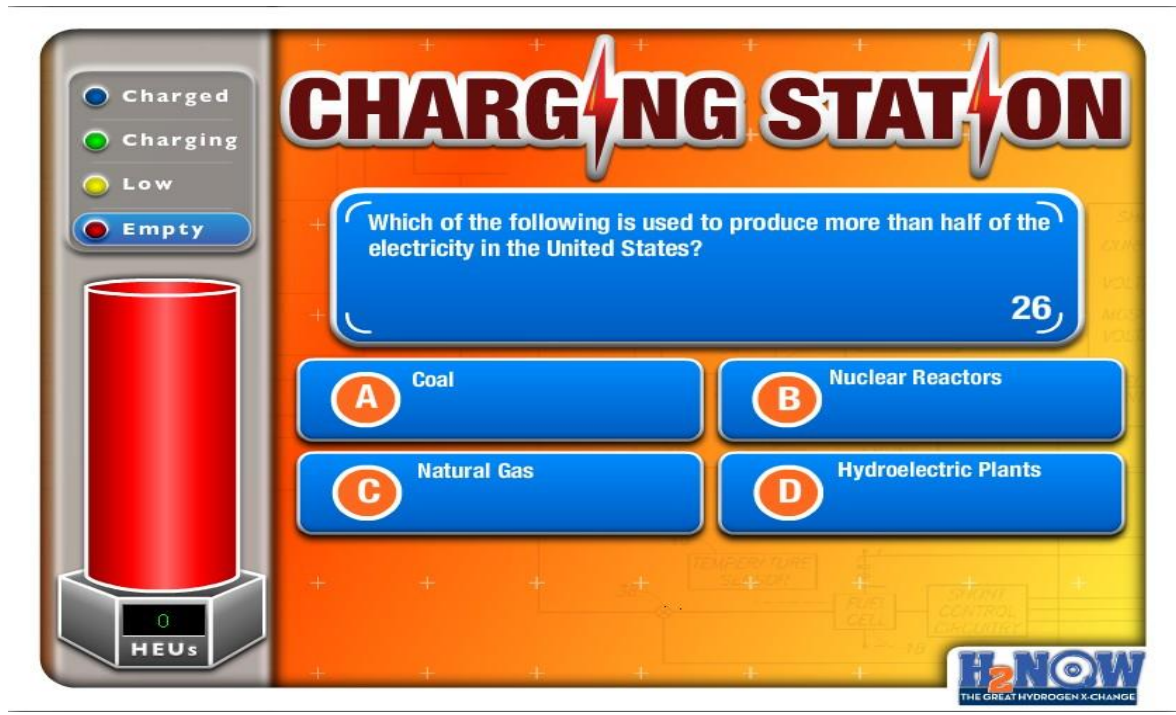

At the above charging station, an individual can use the screen to:

- Obtain options about hydrogen and alternative energy sources

- Increase knowledge level with regard to hydrogen as an energy source

- Collect "Hydrogen Energy Units" that are earned through question and answer prompts. These units are then stored and used in the interactivity city of Hydropolis. 
A sample screen for the interactive city of Hydropolis is shown below:

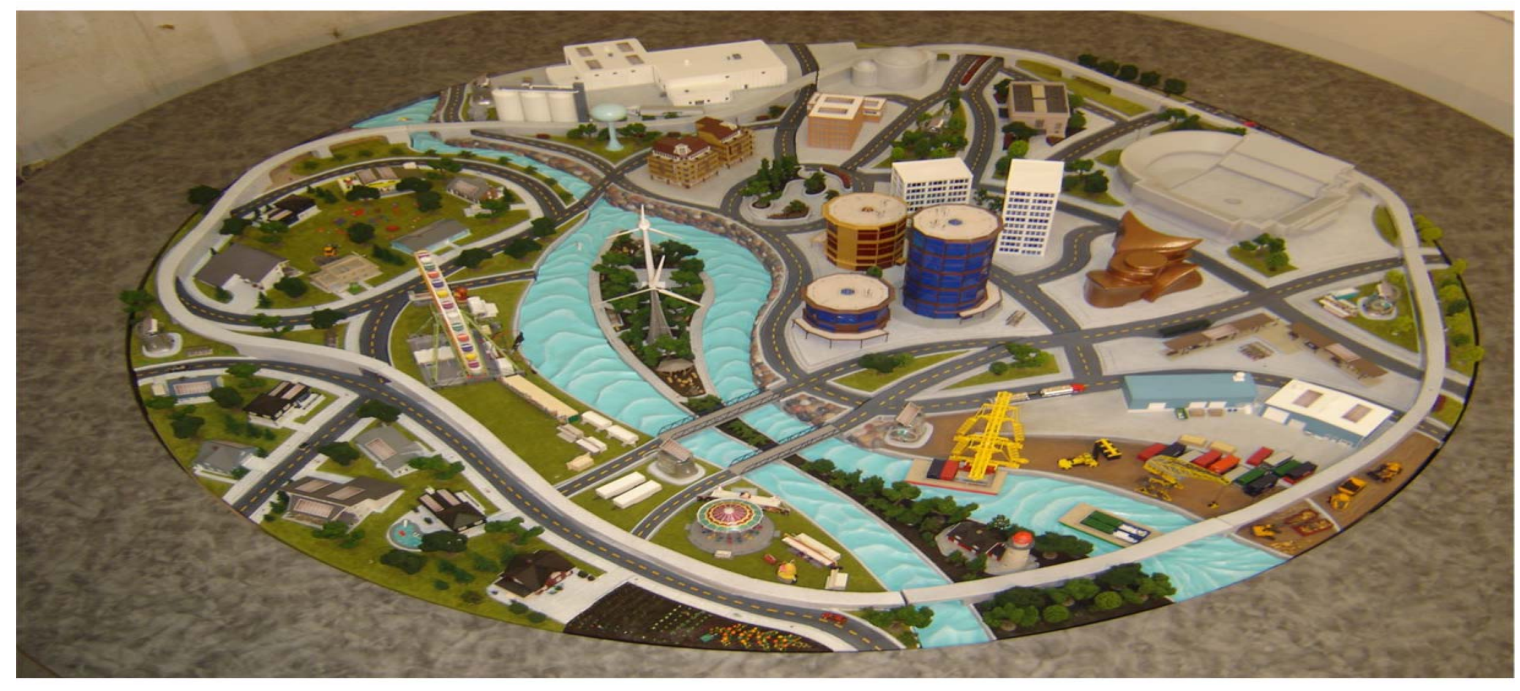

At Hydropolis, an individual can interact at the screen to power various interactive "city" elements with earned Hydrogen Energy Units stored in the hydrocell. Note that each hydrocell is equipped with RFID - radio frequency identification - to transmit its unique "address" to hydropolis. The hydropolis computers access the address to read how many Hydrogen Energy Units the visitor has earned.

Opening of $\mathrm{H}_{2}$ Now: the Great Hydrogen Exchange at the Orlando Science Center was done on August 15, 2009. Following the opening, extensive testing of mechanical and software effectiveness with periods of mechanical and software remediation were done between August 2008 and February 2009. Visitor testing to determine educational effectiveness against educational objectives was completed April 2009.

\section{Results}

From mid-August through December 2008, software developers at I.d.e.a.s, working with OSC staff, incorporated several changes to the software design and interface based on critical analysis of visitor usage to test for mechanical effectiveness. At the end of that time, it was determined that major modifications were needed to both software design and communication interface. OSC brought in a local multimedia design firm to complete this redesign at which time work concentrated on visitor evaluation to determine the extent to which the affective and cognitive goals of the exhibit were being met.

\section{Evaluation Findings: $\mathrm{H}_{2}$ Now Exhibit}

Evaluations with display visitors were conducted in August 2008 and again, after remediation, in February and March 2009. A total number of 65 visitors were evaluated using the observation method and 66 using the interview method for a total of 131 visitors. (Note that not all observed visitors took part in the interview). Since the exhibit was designed for visitors age 12 and up, participants were pulled from this age group 
and/or if a parent with children under age 12 was interviewed, the questions were posed to the parent. Additionally, children under age 12 were excluded from observation portion of the evaluation. See Appendix A for the three evaluation instruments.

Two methods were used:

1) Interview: An evaluator approached visitors after they interacted with the exhibit and asked the individual questions and then recorded their responses on the questionnaire. The questionnaire was designed to determine both affective and cognitive objectives.

2) Visitor observation - Visitors were observed using the exhibit, to determine if they could use the exhibit correctly. Correctly was defined as

a. Retrieving a hydrocell at the Supply station

b. Taking hydrocell to one of 4 charging stations, docking the hydrocell, and answering the content questions on their screen to 'charge' their hydrocell

c. Taking the hydrocell to Hydropolis, docking hydrocell and following screen instructions.

\section{Interview Results}

There were five learning objectives established for $\mathrm{H}_{2}$ Now. These objectives were done so that after participating in the exhibit, visitors would be able to:

1. Explain what hydrogen is, where it comes from, and some of its possible uses.

2. Distinguish between truth and myth about hydrogen as an alternative energy source, and give specific examples.

3. State a benefit and a challenge of hydrogen as an alternative energy source.

4. Give an example of how hydrogen power is created, and how it can effect everyday life.

5. Comment on a possible impact of hydrogen power on the future state of the world.

The interview/questionnaire method was designed to glean visitor understanding of these five objectives. Clearly, the results indicate that the vast majority of visitors were able to achieve the first learning objective, and most visitors achieved the third learning objective. The results also indicate that, while the 'myth' of hydrogen as an alternative energy source was not specifically measured, it is clear that most visitors came away with a clear understanding about hydrogen's use as a renewable energy source. Additionally, visitors were very clear on the benefits i.e., "clean, renewable, doesn't pollute', and as the results showed, also clear on its current challenges i.e., 'too expensive to produce'. Most visitors were also able to verbalize how hydrogen power is created as it is presented in the exhibit, and the extent that hydrogen can be used to effect everyday life.

The only learning objective that was not clearly measured, but can be inferred from the affective questions posed, is the possible impact of hydrogen power on the world's future. This could be due to how the information is presented in the exhibit-it does not specifically address this objective but clearly infers it by communicating the other learning objectives and of course, Hydropolis, the city of the future, meets this learning objective. The fact that $77 \%$ of the visitors surveyed either loved the exhibit or liked the 
exhibit, nearly $64 \%$, in the word choice section of the interview, indicated that they 'learned something' or found the exhibit 'informative'; and nearly 32\% found the exhibit 'interesting' indicates that both cognitive and affective learning has clearly taken place. Additionally, 65\% indicated that they found out something they didn't know before. This finding is a clear indication of both cognitive gain and a change of attitude i.e., compare this to the 44\% who, when given the choice to select "I learned something new". (Research and evaluation studies in museums and science centers has revealed that visitors have a very specific, and narrow definition for the word 'learning' and do not recognize a change in attitude or what is called an "Aha moment" as true learning)

In response to the specific 'test' questions during the interview, most visitors were able to articulate the correct responses to most of the questions. It should be noted, however, that the majority of correct answers were to questions about information presented in the first two of the four levels of information presented in the Charging Stations. As the questions addressed information presented in levels three and four, responses became more vague. Observation revealed that it takes about 15 minutes for a visitor to answer the introductory, level one and level two information on the Charging Stations and as a result, usage drops off. Approximately one third of visitors, mostly those under the age of 12, or parents with young children, don't want to take the additional time to move through the other levels of information. Teens and adults, however, love the exhibit and stick with it through all levels, often not stopping after each level to go to hydropolis but moving through all levels and thus 'racking up' their hydrogen energy units to spend them all at once at hydropolis. Consequently, the amount to which visitors are achieving the higher level learning objectives has more to do with time spent, and not on the quality or interest level of information presented.

Overall, visitors enjoy $\mathrm{H}_{2}$ Now. Observation, both systematic and anecdotal, confirms this-they find the exhibit interesting, engrossing, and fun. In addition to the $36 \%$ interviewed who specifically selected the word 'fun', visitors have been repeatedly overheard using words and phrases such as 'fun' ' neat', 'cool' to describe their experience. Parents enjoy the Charging Stations, and they love to allow their little ones to select the items on hydropolis to power, and letting their children watch the city light up, the ferris wheel turn, etc. The exhibit is so popular that exit intercept surveys that OSC regularly conducts have revealed that visitors do like the exhibit and wish that there was more to it. In response, OSC has added a video presentation produced by the $\mathrm{H}_{2}$ Now exhibit designers, IDEAS, Inc, titled 'I Am Hydrogen" and developed a panel exhibit on other alternative energy resources. Future plans for $\mathrm{H}_{2} \mathrm{Now}$ include developing an interactive instructional video game based on hydrogen and other renewable energy sources.

\section{Visitor Observation Results}

Observation was performed in August of 2008 when $\mathrm{H}_{2} \mathrm{Now}$ first opened. Of the 65 visitors who used the exhibit, 39 used the exhibit correctly, 20 did not use the exhibit correctly. Of those who did not use the exhibit correctly, 14 visitors, mostly older children or young teens, did not pick up a hydrocell but went directly to either a charging station or hydropolis and started tapping repeatedly at the touchscreen. This resulted in the screen response 'locking up'. Twelve visitors were observed taking their hydrocell from the supply station directly to hydropolis and docking, then, since the screen instructions noted their hydrogen energy units as zero and directed them to return to a charging station, would do that. 
The remainder, 4 visitors - again, younger visitors, apparently attracted at once to hydropolis, attempted to use hydropolis without a hydrocell.

Based on the observation results, the touchscreens were augmented with a prominent instruction display directing the user to 'insert hydrocell and tap once". Additionally, a large stanchion sign at the exhibit entrance with prominent instructions - 'what to do' followed by bulleted steps, greatly decreased the number of incorrect users. Finally, the location of the Supply Center was moved closer to the Charging Stations.

\section{Visitor Interview Results}

Interviews with uncued visitors were conducted in February and March, 2009. After a visitor would complete their interaction with $\mathrm{H}_{2} \mathrm{Now}$, and were beginning to leave the exhibit area, the evaluator would approach and ask if they would mind answering questions about their experience with the exhibit.

Question 1: Which face best shows your feelings about this exhibit?

23 visitors chose Love It, 28 visitors chose Like It, 11 visitors chose It's OK, 3 visitors chose "Don't Like It"

0 chose "Hate It"

Question 2: Look at this word list and tell me which words you would apply to this exhibit. Pick as many as you like.

24 chose Learning something

24 chose Fun

21 chose Interesting

18 chose Informative

12 chose Too much to read

12 chose Attractive

6 chose Important

6 chose Impressive

6 chose Useful

6 chose Engaging

3 chose Boring

Question 3: Bearing in mind that there is no right or wrong answer, what do you think this exhibit is all about?

33 visitors responded with answers regarding hydrogen as an alternate or renewable energy source

23 responded with answers regarding what hydrogen is, where it comes from, and how it is made, and how it works

10 responded with more generalized statements such as 'fuel and coal', 'global warming', 'energy is running out', 'teaching about power', or just 'hydrogen'

2 did not answer this question 
Question 4: Did the exhibit tell you something that you didn't know before? If yes, what?

43 responded yes:

It's an energy carrier (11) comes from the sun/where it comes from (10) it can make electricity/renewable energy (9) How hydrogen is made (4) what it can be used for (5). Other responses - didn't know it was energy, didn't know it was on the periodic table, some other things that rely on it

18 responded no

5 didn't answer this question

Question 5: I'm going to ask you a few questions about the information addressed in the exhibit.

What is hydrogen?

A gas received most responses at 29

Renewable energy/renewable energy source/ energy - 18

Used to make electricity/energy - 7

An element, an atom, a molecule -3

It's a fuel cell, 8 didn't answer

Where does it come from?

The sun received the most responses at 24

Water received 16

Air, atmosphere received 9

A lot of things/all over the place -5

Chemical reaction -3

Mined, Big bang

No response -7

What is one way to produce hydrogen?

Water/extract from water; electricity \& water - 31

Fuel cell -2

Chemical reaction/chemistry/separate from water - 17

Cold fusion -1

Solar cells - 1

Get from the sun -3

Didn't know - 11

What is one benefit to using hydrogen as an energy source?

Power cars - 2

Renewable/ clean/doesn't use coal - 32

No exhaust/doesn't pollute - 24

Won't have global warming/no $\mathrm{CO} 2-3$

Didn't know/no answer - 5

Give an example of something that could be powered by hydrogen

City - 6

Anything can be/lots of things - 7

Ships -1

Fuel cells/batteries - 9 
Cars, lights -41

Didn't know/no answer - 2

Is hydrogen a good alternative to fossil fuels? If yes, why? If no, why not?

I don't know/didn't respond - 8

No/not really/too expensive to produce/ other fuels better/not economical - 18

Yes - 2

Running out of coal - 3

Helps pollution/global warming - 9

Clean and renewable/unlimited - 22

Independent from others' control -1

Has unlimited possibilities -1

Question 6: Overall, how do you feel about the topic of hydrogen as it is addressed in this exhibit?

I learned something new -29

I'm comfortable with it-21

I'm uncomfortable with it-3

I don't feel any different - 11

Didn't answer-2

Question 7: Do you think other visitors will understand the information in this exhibit?

Yes - 49

No - 10

Didn't answer -7

\section{Conclusions and Future Directions}

The $\mathrm{H}_{2}$ Now exhibit has completed software and hardware 'shakedown' that resulted in a streamlined interface and communication and is functioning at $100 \%$ in the Orlando Science Center. In addition, exhibit enhancements have been made including a linear display on additional sources of renewable energy and plans are in the works for an interactive 'video game' on renewable energy to complement the messages of $\mathrm{H}_{2} \mathrm{Now}$. The video game was received from a grant from Progress Energy Foundation.

Evaluations to determine the extent to which $\mathrm{H}_{2}$ Now meets educational objectives revealed that:

- $\quad 77 \%$ of the visitors surveyed either loved the exhibit or liked the exhibit

- $64 \%$ indicated that they 'learned something' or found the exhibit 'informative' in the word choice section of the interview

- $32 \%$ found the exhibit 'interesting' indicating that both cognitive and affective learning has clearly taken place

- $65 \%$ indicated that they found out something they didn't know before. This finding is a clear indication of both cognitive gain and a change of attitude i.e., compare this to the $44 \%$ who, when given the choice to select "I learned something new". 
In addition, OSC has enhanced the experience with a short film produced by i.d.e.a.s called "I Am Hydrogen" and is exhibiting a linear display on additional information on renewable energy. 


\section{Appendix A}

\section{Evaluation instruments for $\mathrm{H}_{2} \mathrm{Now} / \mathrm{Hydropolis}$}

Recruiting statement:

Their job is to interact with the exhibit, after which they will be asked questions about their experience. If they agree, tell them they can spend as much time with the exhibit as they wish. If they decline, thank them. Don't ask them why

Part I: Observation (use attached observation sheet)

While the visitor(s) are interacting with the exhibit:

How many were able to accurately determine how to use the exhibit correctly? Correctly is defined as:

1) retrieving a hydrocell,

2) going to recharging stations, answer content questions on the screen to charge their hydrocell,

3) taking the hydrocell to hydropolis, dock hydrocell and following instructions on the screen.

4) repeating the steps

Part II: Interview (use attached interview sheet)

When the visitor finishes interacting with the exhibit, approach, introduce yourself, and explain to visitor that he/they are taking part in helping OSC test a new interactive exhibit, $\mathrm{H}_{2} \mathrm{Now}$, the Hydrogen Exchange. Would they mind answering a few questions. If they agree, thank them, and proceed to questionnaire. Tell the visitor that you will begin the interview. If he has any questions now or during the interview, let him know that you will be happy to answer any questions as soon as the interview is completed.

Read each question exactly as it is written. If the visitor doesn't seem to understand, repeat the question. If he fails to give a response, indicate no response. Do not attempt to reword or explain the question.

Record the visitor's responses, verbatim. 
$\mathrm{H}_{2}$ Now Evaluation Form Date: evaluator's initials

\section{Observation}

How many visitors used the $\mathrm{H}_{2}$ Now exhibit? (used is defined as walked in and made a full stop in front of either the supply center, a charging station, or a hydropolis station)

How many visitors used the exhibit correctly? (correctly is defined as went to supply center first, picked up a hydrocell, docked it in the docking port above, took the hydrocell to a charging station, docked at charging, etc)

Of those who did not use the exhibit correctly, what were they doing wrong? (List actions)

Did you receive any questions from visitors about the exhibit? List below or on back.

$\mathrm{H}_{2}$ Now Evaluation Uncued visitors

Date:

evaluator's initials

Visitor \# Time in:

Time out: Total time:

1. Which face best shows your feelings about this exhibit?

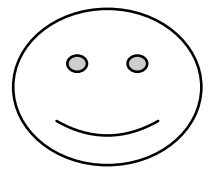

Love It

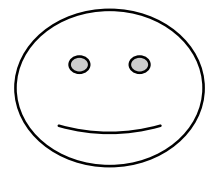

Like it

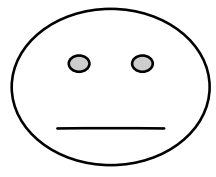

It's OK

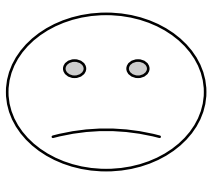

Don't Like it

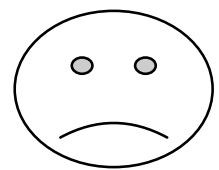

Hate it

2. Look at this word list and tell me which words you would apply to this exhibit. Pick as many as you like.

Attractive

Impressive

Too much to read

Important
Unappealing

Challenging

Fun

Boring

Useful 
Depressing

Engaging

Informative

Learning something

Difficult

Hopeful
Confusing

Unclear

Interesting

(if one or more negative words were chosen)

What was there about the exhibit you didn't care for?

3. Bearing in mind that there is no right or wrong answer, what do you think this exhibit is all about?

4. Did the exhibit tell you something about hydrogen that you didn't know before? _ Y If yes, what?

5. I'm going to ask you a few questions about the information addressed in the exhibit:

a. What is hydrogen?

b. Where does it come from?

c. What is one way to produce hydrogen?

d. What is one benefit to using hydrogen as an energy source?

e. Give an example of something that could be powered by hydrogen.

f. Is hydrogen a good alternative to fossil fuels (Oil, gas, coal)? If yes, why? If no, why not?

6. Overall, how do you feel about the topic of hydrogen as it is addressed in this exhibit?

I'm uncomfortable with it I learned something new
I'm comfortable with it I don't feel any different

7. Do you think other visitors will understand the information in this exhibit? Y $\mathrm{N}$

If no, what do we need to do to make the information in the exhibit more clear or understandable?

8. Anything else you would like to say about the exhibit?

M F Age: 


\title{
FLORIDA HYDROGEN INITIATIVE Sub-contrector Finel Report
}

\section{On-Site Reformation of Diesel Fuel for Hydrogen Fueling Station}

\author{
Dr. Ali Raissi \\ Dr.Nazim Muradov \\ Dr. Karthikeyan Ramasamy \\ Dr. Cunping Huang \\ Dr. Franklyn Smith \\ Florida Solar Energy Center \\ Advanced Energy Research Division \\ E-mail: ali@fsec.ucf.edu \\ (321) 638-1446
}

\author{
Submitted to: \\ David L. Block \\ Florida Solar Energy Center/University of Central Florida \\ 1679 Clearlake Road \\ Cocoa, FL 32922 \\ E-mail: block@fsec.ucf.edu \\ Contract Number: DE-FC36-04GO14225 \\ FSEC Project No.: 20128159
}

Project Start Date: January 21, 2008

Project End Date: December 31, 2009

April 2010

\section{Disclaimer}

The Florida Solar Energy Center/University of Central Florida nor any agency thereof, nor any of their employees, makes any warranty, express or implied, or assumes any legal liability or responsibility for the accuracy, completeness, or usefulness of any information, apparatus, product, or process disclosed, or represents that its use would not infringe privately owned rights. Reference herein to any specific commercial product, process, or service by trade name, trademark, manufacturer, or otherwise does not necessarily constitute or imply its endorsement, recommendation, or favoring by the Florida Solar Energy Center/University of Central Florida or any agency thereof. The views and opinions of authors expressed herein do not necessarily state or reflect those of the Florida Solar Energy Center/University of Central Florida or any agency thereof. 


\title{
On-site Reformation of Diesel Fuel for Hydrogen Fueling Station Applications
}

\author{
Florida Solar Energy Center \\ Advanced Energy Research Division
}

\section{Executive Summary}

The objective of this project was to research options leading to the eventual change from gasoline and diesel as the transportation fuels to that of hydrogen $\left(\mathrm{H}_{2}\right)$. One major research issue regarding the future use of hydrogen is how to deliver $\mathrm{H}_{2}$ fuel within the transportation sector. If $\mathrm{H}_{2}$ is to be derived from organic feedstocks, it may be desirable to deliver the feedstock to the local fueling station and then chemically convert or reform it to $\mathrm{H}_{2}$ on-site. Furthermore, it would be advantageous if the primary feedstock can be used as a fuel in the present engines (e.g., internal combustion engines, ICE, etc.). For example, diesel fuel could be delivered via tanker truck and converted on-site in a small reformer for generating and dispensing hydrogen at the fueling station. There are several advantages to this approach as follows:

- Existing delivery infrastructure (highway to fueling station) could be utilized.

- More energy can be delivered per trip when the tanker is filled with diesel instead of liquid hydrogen.

- The fueling station would be able to service both ICE and fuel cell powered vehicles simultaneously.

- The fueling station operation would be flexible and easily change fuel availability in the form of hydrogen or diesel fuel to meet the customer demand.

Reforming of liquid hydrocarbon fuels to hydrogen has been the focus of intensive worldwide R\&D effort particularly as it relates to automotive transportation. The aim of on-board reforming has been to convert liquid fuels into a hydrogen-rich gas for use in ICE or fuel cell powered vehicles. The drawbacks of on-board reformers include their inherent complexity, weight, high cost, the need for an elaborate purification system to rid hydrogen from impurities that will harm proton exchange membrane fuel cells - although, hydrogen purity requirements of the ICE power plants are much less stringent.

Thus, instead of on-board reforming, the Florida Solar Energy Center (FSEC) jointly with Chevron Technology Ventures (CTV) have developed a process for the on-site reforming of diesel fuel. The approach was to first pre-reform diesel to shorter chain hydrocarbons $\left(\mathrm{C}_{1}-\mathrm{C}_{6}\right)$ before complete treatment in the main reformer unit. The removal of sulfur from the pre-reformate is a critical issue, as the reformer catalyst has limited sulfur tolerance. Thus, another object of this project was to develop a new on-demand forecourt hydrogen production technology by catalytically converting high-sulfur hydrocarbon fuels to an essentially sulfur-free gas. FSEC's responsibilities included building, operating and validating the performance of a diesel pre-reformer as well as developing and demonstrating a small scale desulfurization unit. CTV provided catalysts and know-how for the design and fabrication of the pre-reformer.

In conclusion, a novel process has been developed for converting high-sulfur diesel to a mixture of light molecular weight hydrocarbons that can be readily reformed with steam to fuel cell grade hydrogen gas. The process employs a catalytic pre-reformer coupled with a regenerable $\mathrm{Fe}^{2+} / \mathrm{Fe}^{3+}$ redox/electrolyzer system used for the scrubbing of $\mathrm{H}_{2} \mathrm{~S}$ from the pre-reformer effluent in the form of 
elemental sulfur. Results from laboratory tests showed that electrolysis of acidic $\mathrm{FeSO}_{4}$ aqueous solution was highly efficient with a columbic efficiency of $100 \%$ at a cell voltage of $1.0 \mathrm{~V}$. It was also shown that the electrolytic process can be operated with a Pt-free anode to oxidize ferrous to ferric ions, thereby reducing the cost of the electrolytic system, considerably.

FSEC researchers also developed a robust bi-functional catalyst for accomplishing the pre-reformation of the high-sulfur fuels (sulfur content as high as 5,240 ppmw) to short chain hydrocarbons $\left(\mathrm{C}_{1}-\mathrm{C}_{4}\right.$, mostly propane) at an average yield of $97 \%$. Furthermore, results showed that after100 hours of continuous operation, the combined $\mathrm{Fe}^{2+} / \mathrm{Fe}^{3+}$ redox/electrolyzer $\mathrm{H}_{2} \mathrm{~S}$ scrubber and pre-reformer could achieve desulfurization efficiencies in excess of $95 \%$ and removed sulfur down to less than 5 ppmv in the pre-reformer outlet gas. 


\section{On-Site Reformation of Diesel Fuel for Hydrogen Fueling Station Application \\ Table of Contents}

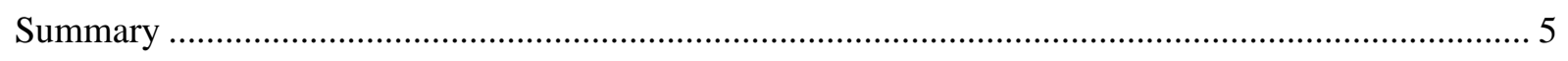

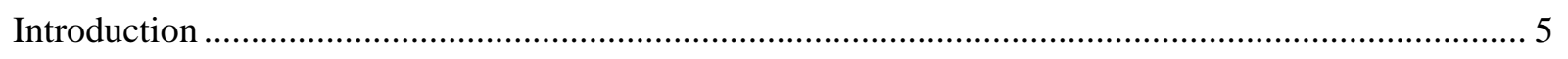

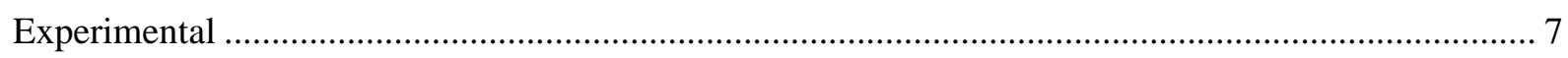

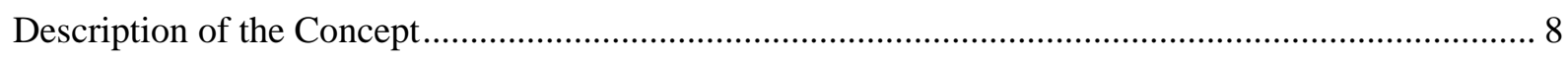

Diesel Pre-reformer Development and Operation ...................................................................... 9

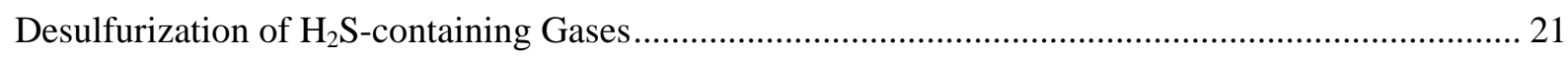

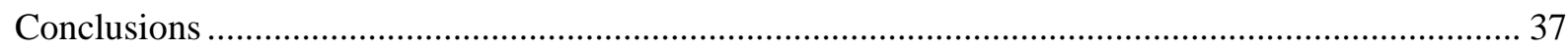

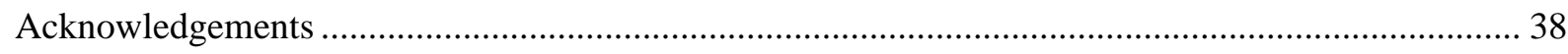

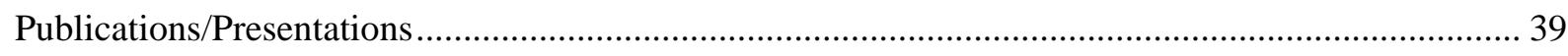

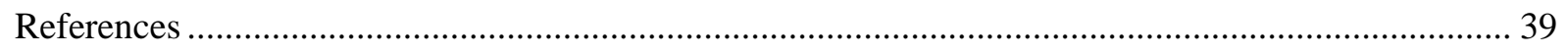




\title{
On-site Reformation of Diesel Fuel for Hydrogen Fueling Station Applications
}

\author{
Florida Solar Energy Center \\ Advanced Energy Research Division
}

\section{Summary}

The objective of the project was to develop a new on-demand forecourt hydrogen production technology by catalytically converting high-sulfur hydrocarbon fuels to an essentially sulfur-free gas. The removal of sulfur from reformate is critical since most catalysts used for the steam reformation have limited sulfur tolerance. The Florida Solar Energy Center's responsibilities included building, operating and validating the performance of a diesel pre-reformer as well as developing and demonstrating a small scale desulfurization unit. Chevron Technology Ventures provided catalysts and know-how for the design and fabrication of thepre-reforming reactor. The results were the development of novel process has for converting high-sulfur diesel to a mixture of light molecular weight hydrocarbons that can be readily reformed with steam to fuel cell grade hydrogen gas. The process employed a catalytic pre-reformer coupled with a regenerable system for the scrubbing of $\mathrm{H}_{2} \mathrm{~S}$. Electrolysis of the resulting acidic $\mathrm{FeSO}_{4}$ aqueous solution was highly efficient and the electrolytic process can be operated with a Pt-free anode, thereby reducing the cost of the electrolytic system, considerably.

\section{Introduction}

Presently, there are many research activities underway in the US aimed at the development of a viable (i.e., cost effective, plentiful, renewable and eco-friendly) alternative to gasoline and diesel as transportation fuels. Hydrogen Economy is viewed by many to present one such alternative for deployment in the future transportation sector. Even at production and usage levels exceeding 12.4 billion SCFD (standard cubic feet per day), the current infrastructure for hydrogen $\left(\mathrm{H}_{2}\right)$ distribution is far below what it needs to be necessary in order to meet market demand for transportation fuels.

One major challenge facing the future Hydrogen Economy is how to deliver $\mathrm{H}_{2}$ fuel within the transportation sector. If hydrogen is to be derived from high energy feedstocks, it may be desirable to transport the feedstock itself to local fueling stations and convert the material on-site. Furthermore, it would be advantageous if the primary feedstock can be used as a fuel in the legacy vehicular power plants (e.g., internal combustion engines, ICE, etc.). For example, diesel fuel could be delivered via tanker truck and converted in a small reformer on site for generating and dispensing hydrogen at the fueling station. There are several advantages to this approach as follows:

- Existing delivery infrastructure (highway to fueling station) could be utilized.

- More energy can be delivered per trip when the tanker is filled with diesel instead of liquid hydrogen.

- The fueling station would be able to service both ICE and fuel cell powered vehicles simultaneously.

- The fueling station operation would be flexible and easily change fuel availability in the form of hydrogen or diesel fuel to meet the customer demand.

Reforming of liquid hydrocarbon fuels to hydrogen has been the focus of intensive worldwide R\&D effort particularly in relation to automotive transportation (e.g., [1-4]). The aim of on-board reforming has been to convert liquid fuels into a hydrogen-rich gas for use in ICE or fuel cell (FC) powered 
vehicles. In many respects, liquid hydrocarbon fuels represent a more attractive means of transporting hydrogen than the compressed or liquefied $\mathrm{H}_{2}$, promising greater vehicle range, shorter refueling times, increased safety, and perhaps most importantly, utilization of the current fuel distribution infrastructure. The drawbacks of on-board reformers include their inherent complexity, weight, high cost, the need for an elaborate purification system to rid hydrogen from impurities (e.g., $\left.\mathrm{CO}, \mathrm{H}_{2} \mathrm{~S}\right)$ that will harm PEMFC (proton exchange membrane fuel cell) performance - although, hydrogen purity requirements of the ICE power plants are much less stringent.

The three main fuel reforming strategies for $\mathrm{H}_{2}$ production from hydrocarbons are: steam reforming (SR), partial oxidation (POx) and autothermal reforming (ATR). A representative chemical reaction for ATR of diesel fuel (empirical formula of $\mathrm{C}_{12} \mathrm{H}_{23}$ ) is given below:

$\mathrm{C}_{12} \mathrm{H}_{23}+5.5 \mathrm{O}_{2}+\mathrm{H}_{2} \mathrm{O} \rightarrow 12 \mathrm{CO}+12.5 \mathrm{H}_{2}$

POx and ATR have a number of advantages over SR, namely, shorter startup time, better transient response, and lighter weight. They can reform a wide range of fuels, including gasoline and diesel fuel. However, the POx and ATR reformers suffer from some shortcomings compared to SR, namely: (i) the reformate from POx and ATR become diluted with $\mathrm{N}_{2}$ (since air is used in the process) resulting in lower FC performance, (ii) $\mathrm{H}_{2}$ in FC, anode exhaust is not easily integrated with the POx or ATR systems, and (iii) due to high exothermicity of the POx process, the reformer may be subject to greater thermal loses.

Different fuels impose different constraints on the reformer design, catalysts used and operating conditions. For example, it is more difficult to reform diesel than gasoline due to a number of factors including: former having to operate at lower $\mathrm{H} / \mathrm{C}$ ratios (thus, the potential for soot formation), higher energy consumption due to lower volatility of diesel fuel, higher temperatures in the reformer, higher sulfur content, etc. The effect of fuel composition on the fuel processor performance was investigated by Borup et al. [5]. It has been shown that short-chain aliphatic hydrocarbons tend to have favorable reforming characteristics for catalytic ATR as opposed to longer-chain and aromatic species. The Argonne National Laboratory researchers have investigated the reactor characteristics and the efficiency of a catalytic autothermal reformer using surrogates of diesel fuel (dodecane and hexadecane) as feedstock [6]. They used $1 \mathrm{wt}$ \% Pt supported on cerium and gadolinium oxides as catalysts and operated the reformer at various oxygen-to-carbon and steam-to-carbon ratios and space velocities that ranged from 10,000 to $100,000 \mathrm{hr}^{-1}$ achieving $\mathrm{H}_{2}$ selectivities as high as $86 \%$.

Reforming high-sulfur (or high-S) hydrocarbon fuels (e.g., diesel, JP-8, etc.) is particularly challenging due to rapid deactivation of conventional reforming catalysts by coke deposits and sulfurous compounds. Moreover, if sulfurous compounds (mostly, $\mathrm{H}_{2} \mathrm{~S}$ ) are not removed from reformate, they readily poison the catalyst used in the anode compartment of FC substantially deteriorating its performance or permanently damaging it. Thus, R\&D efforts in this area have been focused either on desulfurization of liquid hydrocarbon fuels before reforming or on the development of sulfur-tolerant reforming catalysts. Fukunaga et al. have reported an efficient Ni-based adsorbent that lowered sulfur impurities from high-S kerosene down to less than $1 \mathrm{ppm}$ [7]. It has been shown in a number of publications that traditional Ni-based steam reforming catalysts rapidly deactivate during the processing of high-S fuels. Although noble metal based catalysts (e.g., supported Ru, Pd, Rh, etc.) showed improved tolerance toward sulfur poisoning and coking compared to Ni-based catalysts, their long term stability still was not acceptable. For example, McCoy, et al. [8] have reported that supported Rh catalyst initially shows relatively good performance in steam reforming of high-S jet fuel, but over a long period of operation exhibits signs of enhanced deactivation. 
Florida Solar Energy Center (FSEC), in collaboration with the Chevron Technology Ventures (CTV), has developed a new on-demand hydrogen production technology for distributed applications. The objective of this work was to catalytically convert high-S hydrocarbon fuels to an essentially sulfurfree gaseous feedstock for steam reformation, while consuming only a small fraction of hydrogen produced. The removal of sulfur from reformate is critical since most catalysts used for the steam reformation have limited sulfur tolerance. FSEC's responsibilities included building, operating and validating the performance of a pre-reformer unit as well as developing and demonstrating a functioning self-contained desulfurization unit capable of S-scrubbing of the pre-reformer effluent. CTV provided a catalyst for the pre-reforming stage and assisted with the design of the pre-reformer.

\section{Experimental}

Reagents and catalysts. Hexadecane with greater than 98\% purity was obtained from Fisher and used without further purification. Thiophene (99\%) was obtained from Alpha Aesar and used as received. Commercial diesel fuel was purchased from a local Chevron gas station. Since commercial diesel fuel contains very low levels of sulfur (approximately $5.5 \mathrm{ppmw}$ ), the fuel was spiked with thiophene $\left(\mathrm{C}_{4} \mathrm{H}_{8} \mathrm{~S}\right)$ to elevate the sulfur content to $3180-5240 \mathrm{ppmw}$ level. This thiophene-spiked diesel was then used in all experiments as a surrogate high-S fuel. The same treatment was carried out with hexadecane. Alumina-supported Ni-Mo hydroprocessing catalyst in the form of $1 \mathrm{~mm}$ by $5 \mathrm{~mm}$ extruded pellets was provided by Haldor Topsoe. The composition of the Ni-Mo catalyst was (\%w/w): $\mathrm{NiO}(2-5), \mathrm{MoO}_{3}(12-18), \mathrm{Al}_{2} \mathrm{O}_{3}$ (68-80), $\mathrm{AlPO}_{4}$ (5-11). In order to produce catalytically active form of the Ni-Mo catalyst it was sulfided using 6\% dimethyl disulfide (DMDS), $\left(\mathrm{CH}_{3}\right)_{2} \mathrm{~S}_{2}$, in n-heptane as the sulfiding agent according to the following procedure.

The Ni-Mo catalyst (115 g) was placed inside a stainless steel reactor ( $2.5 \mathrm{~cm} \mathrm{OD).} \mathrm{H}_{2}$ was introduced at a pressure of $6.7 \mathrm{~atm}$ for $1 \mathrm{hr}$. Then, 6\% DMDS in heptane was pumped into the reactor at a flow rate of $25 \mathrm{~mL} / \mathrm{hr}$ for $30 \mathrm{~min}$. The catalyst bed temperature was increased to $235^{\circ} \mathrm{C}$ and maintained for $1 \mathrm{hr}$ at that temperature. $\mathrm{H}_{2}$ pressure was increased to $54.5 \mathrm{~atm}$ and catalyst remained under that pressure for another hour. The catalyst bed temperature was then increased to $340^{\circ} \mathrm{C}$ and held there for additional $1 \mathrm{hr}$ before suspending the pumping of the DMDS solution. The catalyst bed temperature was lowered to $175^{\circ} \mathrm{C}$, and $\mathrm{H}_{2}$ flow was maintained overnight at $15 \mathrm{~L} / \mathrm{hr}$ and $54.5 \mathrm{~atm}$ pressure. A proprietary zeolite catalyst in the form of 1.5 by $5 \div 10 \mathrm{~mm}$ extruded pellets was supplied by the CTV for these tests.

Analysis. The analysis of the pre-reformer effluent was carried out by gas chromatography (GC) as follows: the permanent gases, such as $\mathrm{H}_{2}, \mathrm{CO}, \mathrm{CO}_{2}$ and $\mathrm{CH}_{4}$ were analyzed using an SRI-8610A GC (thermal conductivity detector, argon carrier gas, silica gel packed column); gaseous and liquid hydrocarbons were analyzed on a Shimadzu GC-14B (flame ionization detector, helium carrier gas, capillary column). Analysis of sulfurous compounds $\left(\mathrm{H}_{2} \mathrm{~S}\right.$, thiophene, methyl-mercaptan, etc.) was conducted using Perkin Elmer GC (flame photometric detector, capillary column). Additionally, $\mathrm{H}_{2} \mathrm{~S}$ content in the pre-reformate was determined employing a set of Sensidyne ${ }^{\circledR}$ and RAE Systems gas detection tubes in the range of 0.2-5, 1-60 and 25-250 ppmv of $\mathrm{H}_{2} \mathrm{~S}$.

X-ray diffraction (XRD) analysis of the samples of Ni-Mo/alumina catalyst before and after the prereforming experiments was conducted using a Rigaku D-MaxB diffractometer. X-ray photoelectron spectroscopic (XPS) analysis of the catalysts was conducted on a Physical Electronics 5400 XPS instrument applying non-monochromatic $\mathrm{Mg} \mathrm{K}$ alpha X-rays for excitation (pass energy for survey $44.75 \mathrm{eV}$, step size $0.5 \mathrm{eV}$; for high resolution spectra pass energy $35.75 \mathrm{eV}$, step size $0.1 \mathrm{eV}$ was used). The surface morphology of the catalysts before and after pre-reforming were examined using a scanning electron microscopy (JEOL 6400F). 


\section{Description of the Concept}

Figure 1 depicts a simplified block-diagram of the process for conversion of a high-S fuel to $\mathrm{H}_{2}$.

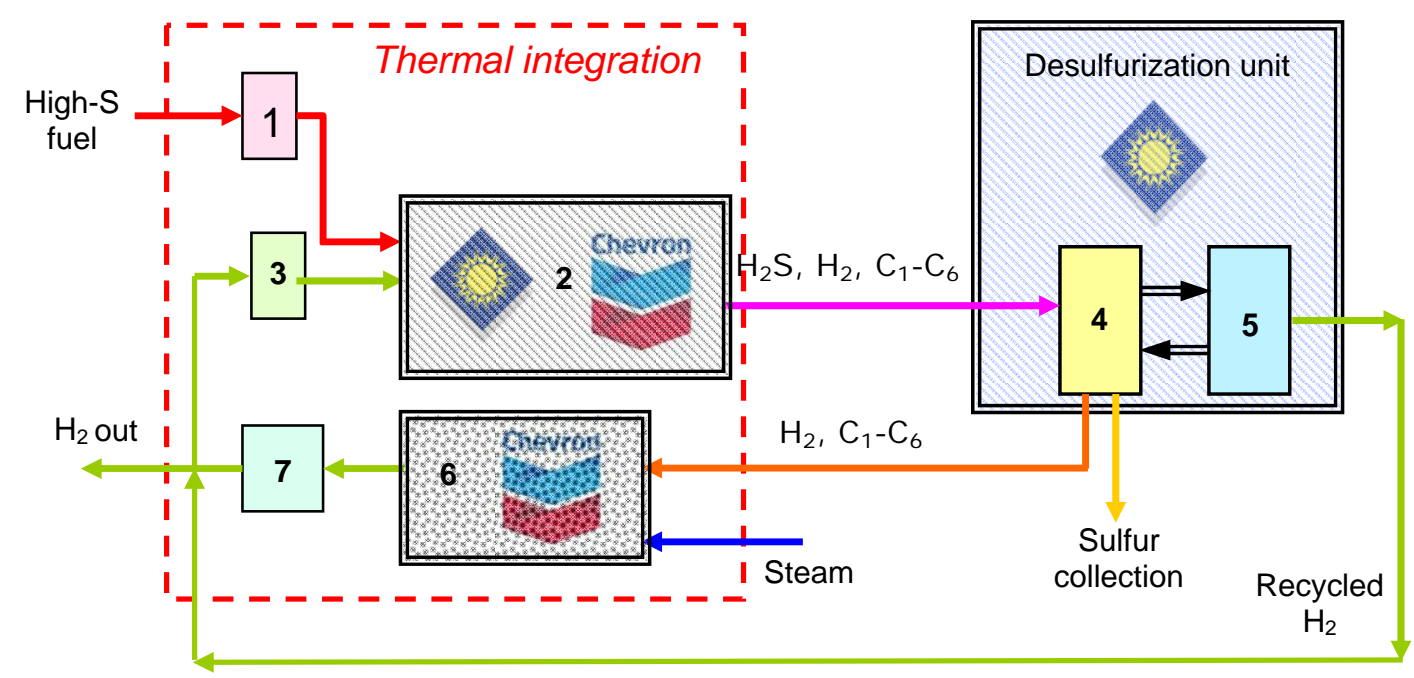

Figure 1. 1- vaporizer, 2- pre-reformer, 3- $\mathrm{H}_{2}$ compressor, 4- $\mathrm{H}_{2} \mathrm{~S}$ stripper, 5- regenerator, 6- steam reforming unit, 7- gas conditioning and $\mathrm{H}_{2}$ purification unit.

A high-S liquid hydrocarbon fuel (e.g., diesel fuel) is vaporized and heated in a pre-heater 1, and catalytically processed in a pre-reformer 2 to shorter chain hydrocarbons $\left(C_{1}-C_{6}\right.$, preferably, $\left.C_{1}-C_{4}\right)$ by hydrogenation (this process is similar to hydro-cracking process, therefore, hereafter we refer to this process "hydro-reforming"). Assuming (for the sake of simplification) the diesel fuel empirical formula of $\mathrm{C}_{12} \mathrm{H}_{23}$ and propane as the main product of the reaction, then, the diesel hydro-reforming can be written as follows:

$\mathrm{C}_{12} \mathrm{H}_{23}+4.5 \mathrm{H}_{2} \stackrel{\text { catalyst }}{\longrightarrow} 4 \mathrm{C}_{3} \mathrm{H}_{8}$

In the pre-reformer, most sulfurous species present in the fuel are catalytically converted to hydrogen sulfide $\left(\mathrm{H}_{2} \mathrm{~S}\right)$ as follows:

$\mathrm{RS}+2 \mathrm{H}_{2} \stackrel{\text { catalyst }}{\longrightarrow} \mathrm{H}_{2} \mathrm{~S}+\mathrm{RH}_{2}$

Where, $\mathrm{R}$ refers to an organic moiety.

The sulfur removal from the pre-reformate is required in order to avoid deactivation of the Ni-based catalyst in steam reformer 6 . Desulfurization of the pre-reformate effluent is carried out in a special regenerative system, which includes a sulfur scrubber 4 coupled with a regenerator 5 . Two types of regenerating systems for the desulfurization unit have been considered in this work: photolytic and electrolytic. The overall reaction is decomposition of $\mathrm{H}_{2} \mathrm{~S}$ to $\mathrm{H}_{2}$ and elemental sulfur according to:

$\mathrm{H}_{2} \mathrm{~S}_{(\mathrm{g})} \rightarrow \mathrm{H}_{2(\mathrm{~g})}+\mathrm{S}_{(\mathrm{s})}$

Desulfurized pre-reformer effluent is fed to the main reformer 6, where it is mixed with steam and processed over a Ni-catalyst to syngas (again, assuming propane as the main product):

$\mathrm{C}_{3} \mathrm{H}_{8}+3 \mathrm{H}_{2} \mathrm{O} \rightarrow 3 \mathrm{CO}+7 \mathrm{H}_{2}$ 
The syngas is further conditioned (i.e., shifted) and purified (using pressure swing adsorption process) to high-purity $\mathrm{H}_{2}$ via conventional steam methane (or naphtha) reforming processes.

A small fraction of the output hydrogen is recycled to the pre-reformer and used to perform hydroreforming of the high-S feedstock. The required amount of recycled hydrogen depends on the composition of the feedstock and hydro-reforming products. For example, assuming an empirical formula of $\mathrm{C}_{12} \mathrm{H}_{23}$ and $\mathrm{C}_{16} \mathrm{H}_{34}$ for diesel, the fraction of hydrogen product needed to carry out prereforming to butane would be about $13 \%$ and 5.7\%, respectively (see Fig. 2).

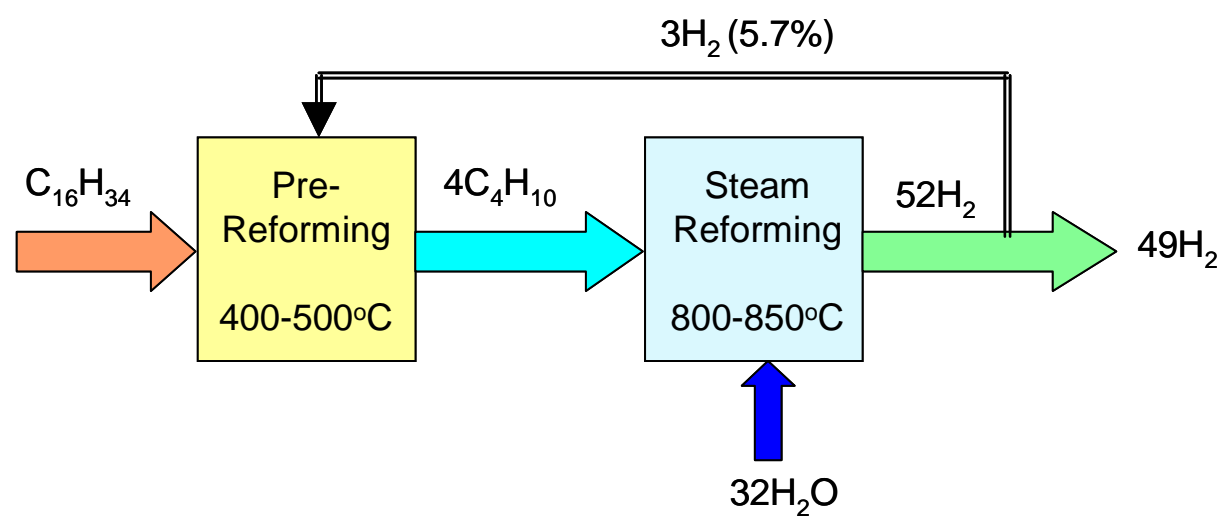

Figure 2. Hydrogen balance of the pre-reforming process coupled with steam reforming.

In the following we present the experimental results related to the development of a novel diesel desulfurization process consisting of pre-reforming and desulfurization sub-units.

\section{Diesel Pre-reformer Development and Operation}

\subsection{Pre-reforming of high-S hexadecane and diesel fuel}

Figure 3 shows a schematic diagram and a photograph of the bench-scale experimental unit employed for pre-reforming and desulfurization of high-S hydrocarbon fuels. In this section, the results of catalytic pre-reforming experiments using hexadecane and commercial diesel fuel spiked with thiophene in the amount corresponding to 3180-5240 ppmw sulfur are described (The data on the desulfurization sub-unit will be presented in the next section). 

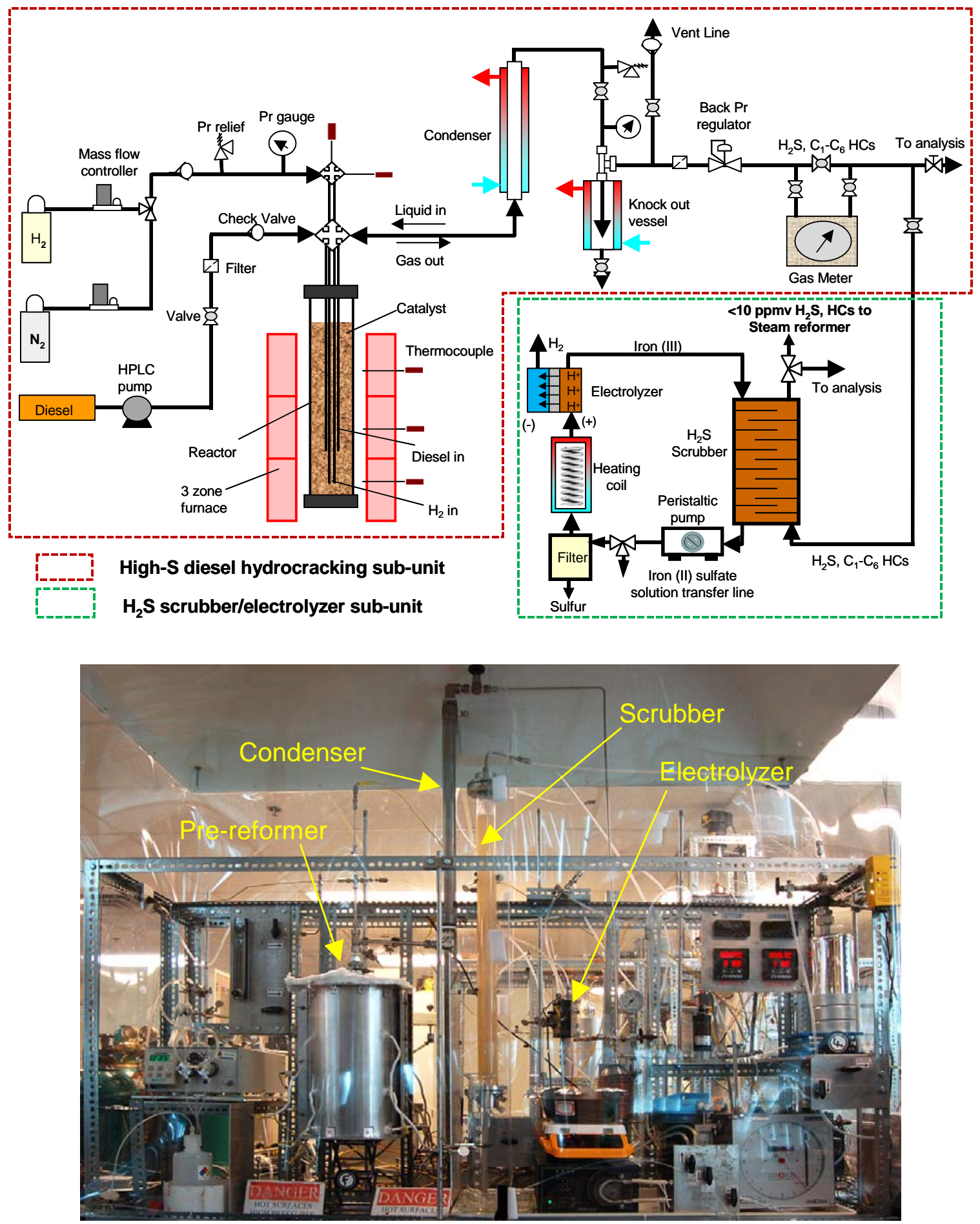

Figure 3. Schematic diagram and photo of the combined diesel pre-reforming-desulfurization unit operating at FSEC.

The pre-reformer sub-unit consists of four sections: 1) fuel metering and delivery section, 2) reactor assembly section, 3 ) products separation section, and 4) products metering and analysis section. In the fuel delivery section, diesel (or hexadecane) is pumped to the reactor at 13.6-14.3 atm pressure using a high performance liquid chromatography pump, HPLC, (Lab Alliance) at flow rates in the range of 
0.2-0.4 $\mathrm{mL} / \mathrm{min}$. Hydrogen flow is controlled by a high-pressure mass flow controller (Parker) and varied in the range of $0.72-3.50 \mathrm{~L} / \mathrm{min}$. The pre-reforming reactor (ID= $25 \mathrm{~mm}$, wall thickness $2 \mathrm{~mm}$, length $45 \mathrm{~cm}$ ) was fabricated out of 316 stainless steel with a maximum pressure rating of $200 \mathrm{~atm}$ at $540^{\circ} \mathrm{C}$. Three quarters of the reactor was filled with catalyst. The hydrogen entered at the bottom of the reactor and the diesel vapor counter flowed from the top. The reactor had an adjustable platform built inside that allowed the height of the catalyst zone be varied and changed, as needed. It also provided a capability to vary the residence time within the reactor.

Fluid pressures and temperatures were monitored, in real time, at the inlet as well as at the outlet of the reactor using a LabView data acquisition system. The temperature in the upper section of the reactor was maintained at $400-450^{\circ} \mathrm{C}$ and in the lower part (where diesel entered into the reaction zone) at $450-500^{\circ} \mathrm{C}$. In the products separation section, the product mixture passed through a shell-and-tube type condenser (kept at a temperature around $15-20^{\circ} \mathrm{C}$ ) where most of $\mathrm{C}_{6}+$ hydrocarbons condensed and refluxed back into the reactor and the gaseous hydrocarbons (predominantly, $\mathrm{C}_{1}-\mathrm{C}_{4}$ ), $\mathrm{H}_{2} \mathrm{~S}$ and excess $\mathrm{H}_{2}$ exited and subsequently transported into a knock-out vessel where the fugitive (from the condenser) heavier hydrocarbons were trapped and collected. The effluent gas flowed through in-line filters that removes any particulates or aerosols that may have been produced in the reaction zone, passing through a back-pressure regulator to reduce pressure to near atmospheric. The pre-reformate then enters into a metering and analysis section wherein most of the gaseous product pass through a gas metering unit (Shinagawa W-NK-2) used to quantify the volume of gas generated.

For safety reasons, several pressure relief valves were installed, and corrosion resistant 316 stainless steel tubing and fittings were used every where in the experimental unit.

Three experiments with different catalytic systems were conducted. They were: (i) zeolite catalyst, (ii) $\mathrm{Ni}-\mathrm{Mo} /$ alumina catalyst and (iii) the mixture of zeolite and Ni-Mo/alumina catalyst. The composition of the effluent gas from hexadecane hydro-reforming over zeolite catalyst at $450^{\circ} \mathrm{C}$ and $14 \mathrm{~atm}$ is depicted in Figure 4. It can be seen that $\mathrm{C}_{1}-\mathrm{C}_{7}$ saturated hydrocarbons (paraffins) were produced in the reaction, with propane being the main component in the mixture followed by butanes.

However, the stability of the zeolite catalyst was not very good, and after about 20-30 hrs of operation there were signs of deactivation evidenced by the appearance of a liquid by-product in the knock-out vessel. Indeed, the zeolite catalyst that was removed from the reactor after the experiment had turned completely black, apparently due to coke deposition on its surfaces. The amount of coke accumulated on the catalyst surface was determined to be about $10 \%$ by weight of the fresh catalyst.

The coked catalyst could be easily reactivated by burning coke off the catalyst surface in air at a temperature of $450^{\circ} \mathrm{C}$ for 4 hrs. 


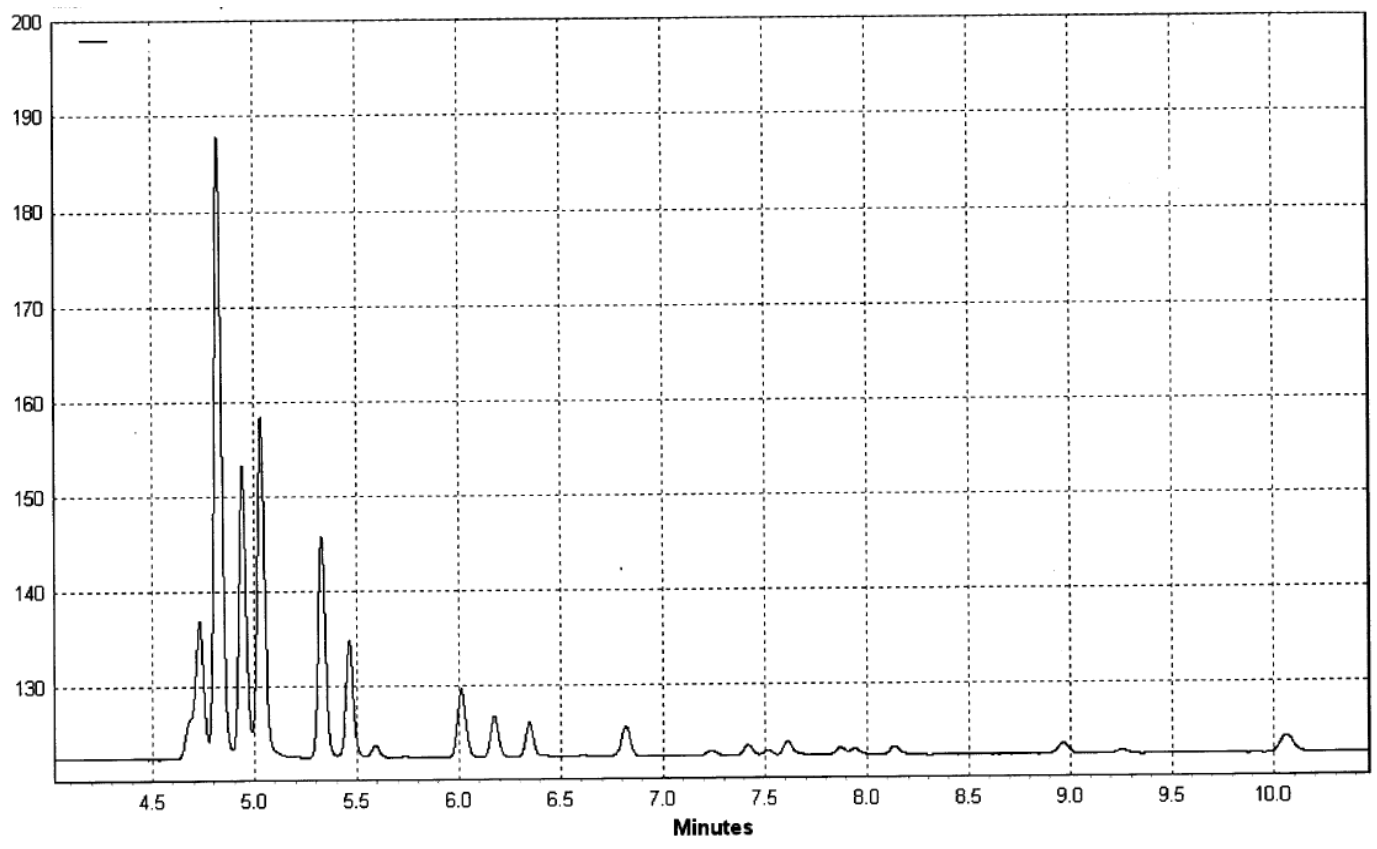

Figure 4. Gas chromatogram of hexadecane pre-reformate; zeolite catalyst, $\mathrm{T}=450^{\circ} \mathrm{C}$.

Figure 5 shows the product slate of hexadecane hydro-reforming over Ni-Mo/alumina catalyst at conditions similar to that used with the zeolite catalyst.

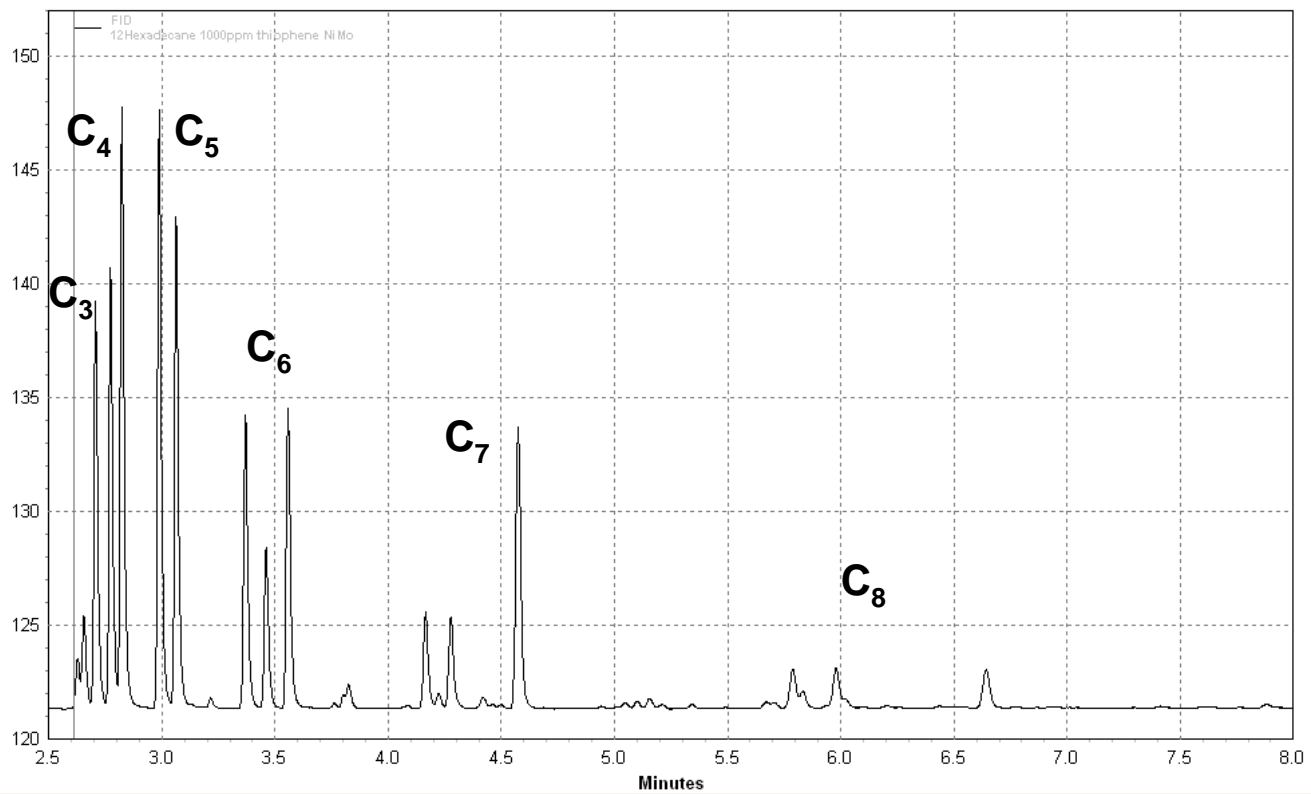

Figure 5. Gas chromatogram of diesel pre-reformate using Ni-Mo catalyst, $\mathrm{T}=450^{\circ} \mathrm{C}$. 
Figure 6 shows the comparison of the products distribution obtained using zeolite catalyst to that of $\mathrm{Ni}$-Mo/alumina under identical experimental conditions.

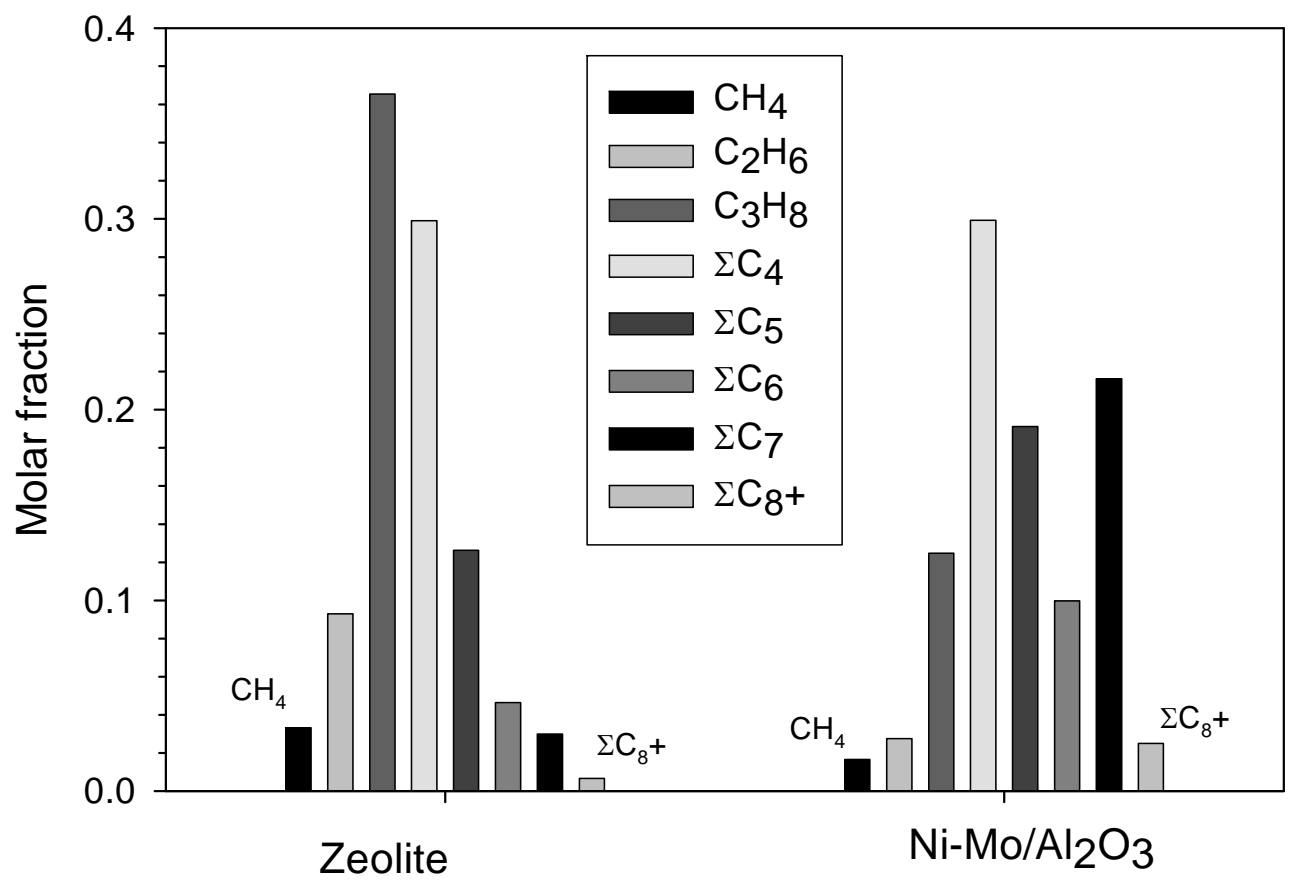

Figure 6. Hexadecane hydro-reforming product distribution with zeolite (left) and Ni-Mo/alumina (right) catalysts; $\mathrm{T}=450^{\circ} \mathrm{C}, \mathrm{P}=14 \mathrm{~atm}$.

In contrast to the zeolite catalyzed hydro-reforming product gas, the product gas from hexadecane hydro-reforming was rich in higher hydrocarbons, in particular, $\mathrm{C}_{4}-\mathrm{C}_{7}$ paraffins, with butanes being the main reaction products, followed by heptanes. Like the zeolite catalyst, the Ni-Mo catalytic activity was not quite satisfactory as it deactivated much faster than the zeolite one. After about 5 hrs of run time, a liquid product appeared in the condenser, indicating the loss of hydro-reforming activity of the Ni-Mo/alumina catalyst. At the end of the experiment, the catalyst color was found to have turned from light green to black.

In the following experiments, a mixture of zeolite and Ni-Mo/alumina catalysts in the weight ratio of 2:1 was tested. The experimental conditions were as follows: temperatures in the upper and lower parts of the reactor were $400^{\circ} \mathrm{C}$ and $470^{\circ} \mathrm{C}$, respectively; reactor pressure of $13.7 \mathrm{~atm}$, and thiophenesupplemented hexadecane input flow rate of $0.3 \mathrm{~mL} / \mathrm{min}$.

The results obtained from this test showed that the composition of the pre-reformate gas changed with time during the first 25-30 hrs of run time, reaching a steady state after about 50 hrs. Figure 7 (a \& b) illustrates the data obtained in a typical experiment. The data has been presented in two formats: (a) the time dependence of the molar fraction of the individual (or the family of) hydrocarbons, and (b) the composition of the product gas after 4, 20, 32 and 56 hrs of operation. Figure 8 depicts the gas chromatogram of the hexadecane pre-reformate gas toward the end of the experimental run discussed above. 

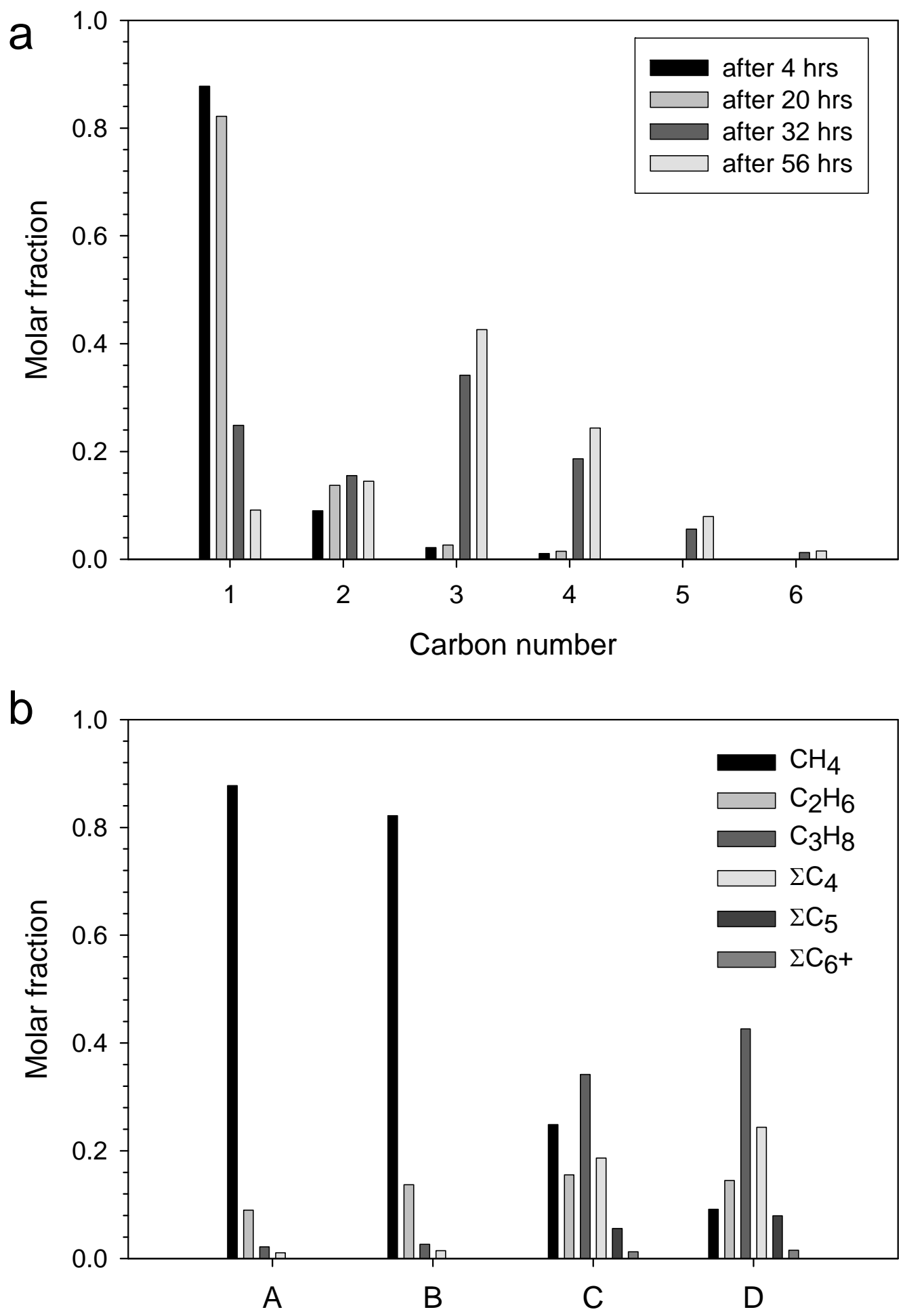

Figure 7. Hexadecane hydro-reforming over zeolite/Ni-Mo catalyst; $\mathrm{T}=400-470^{\circ} \mathrm{C}, \mathrm{P}=13.7 \mathrm{~atm}$. Gas samples were analyzed after: A-4, B-20, C-32 and D- 56 hrs. 


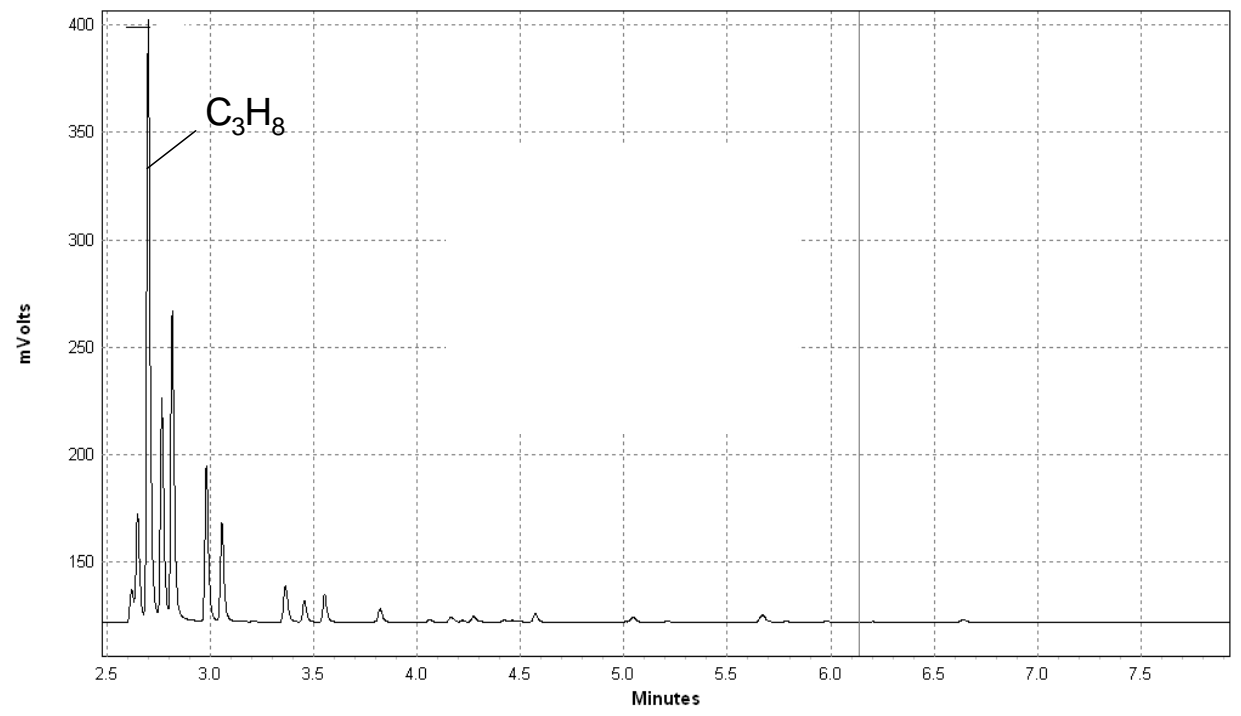

Figure 8. Gas chromatogram of hexadecane hydro-reforming over combined zeolite-Ni-Mo catalyst; $\mathrm{T}=400-470^{\circ} \mathrm{C}, \mathrm{P}=13.7 \mathrm{~atm}$.

It can be seen that at the beginning of the process (while the catalyst is fresh), methane is by far the main component of the pre-reformer effluent, followed by ethane, propane and butanes. There are practically no higher hydrocarbons present. As the experiment progresses, the concentration of methane gradually decreases and the concentration of higher hydrocarbons increase. After about 50 hrs a steady state condition is reached which is characterized by the generation of a pre-reformate consisting of $\mathrm{C}_{1}-\mathrm{C}_{6}$ hydrocarbons only with propane being the main species in the product gas. Overall, the experiment was continued for 80 hrs, and only a small amount of liquid hydrocarbon was found to collect in the knock-out vessel. The overall conversion of hexadecane to gaseous products was $98 \%$.

A similar experiment was conducted by passing the thiophene-containing diesel fuel over mixed zeolite and Ni-Mo/alumina catalysts. The gas chromatogram of the pre-reformate is given in Figure 9.

The reactor operated at two temperature regimes: $450^{\circ} \mathrm{C}$ and $500^{\circ} \mathrm{C}$ (temperature measured in the mid section of the reactor) with other parameters remaining the same as in test using hexadecane as reactant. Again, initially, the pre-reformate was rich with methane; however, after about 12-16 hrs, a steady state condition was reached which prevailed for the duration of the experiment (100 hrs). Average conversion of diesel to gaseous hydrocarbon products in this experiment was $95-97 \%$.

Figure 10 depicts the product distribution of the pre-reformate at $450^{\circ} \mathrm{C}$ (left) and $500^{\circ} \mathrm{C}$ (right) after reaching the steady state condition. It is noteworthy that the distribution of $\mathrm{C}_{1}-\mathrm{C}_{6}$ hydrocarbons from diesel and hexadecane hydro-reforming over the same catalyst and temperature range showed that propane was the main product followed by butanes. In contrast to hexadecane, the diesel-derived gas contained a slightly higher content of heavier hydrocarbons $\left(\mathrm{C}_{6}-\mathrm{C}_{8}+\right)$. It can be seen from Figure 10 that increasing reaction temperature results in an increase in methane concentration in the pre-reformer effluent. 


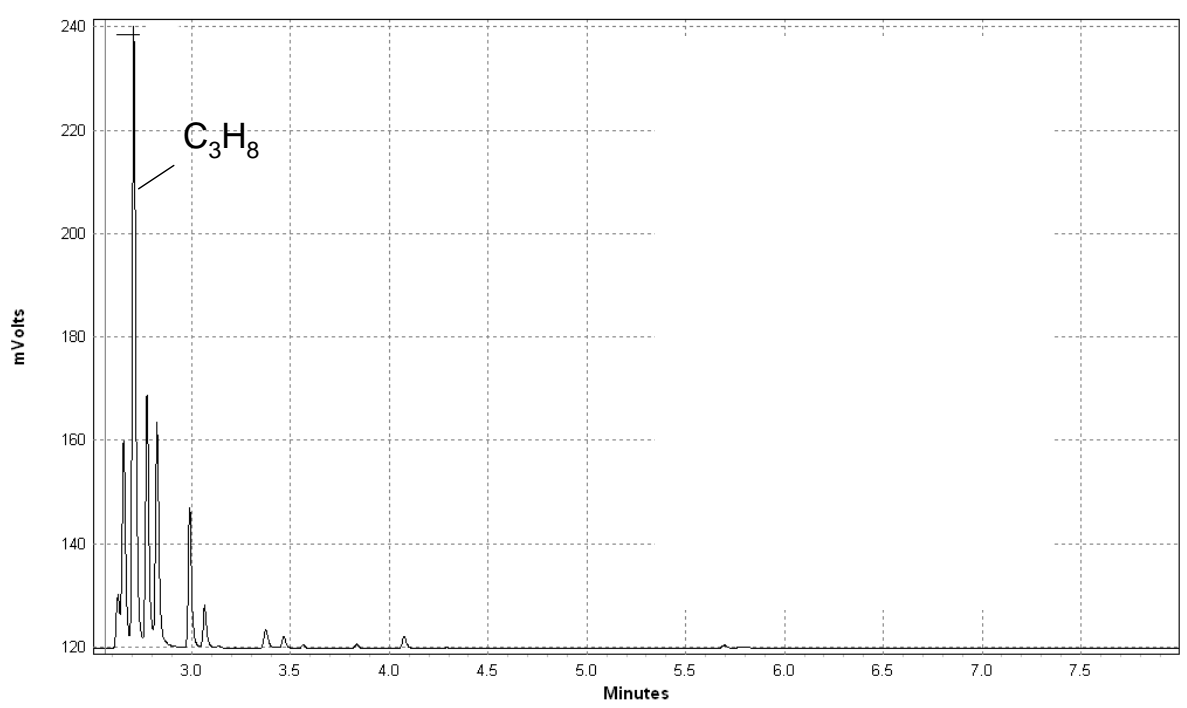

Figure 9. Gas chromatogram of diesel hydro-reforming over combined zeolite-Ni-Mo catalyst. $\mathrm{T}=400-470^{\circ} \mathrm{C}, \mathrm{P}=13.7 \mathrm{~atm}$.

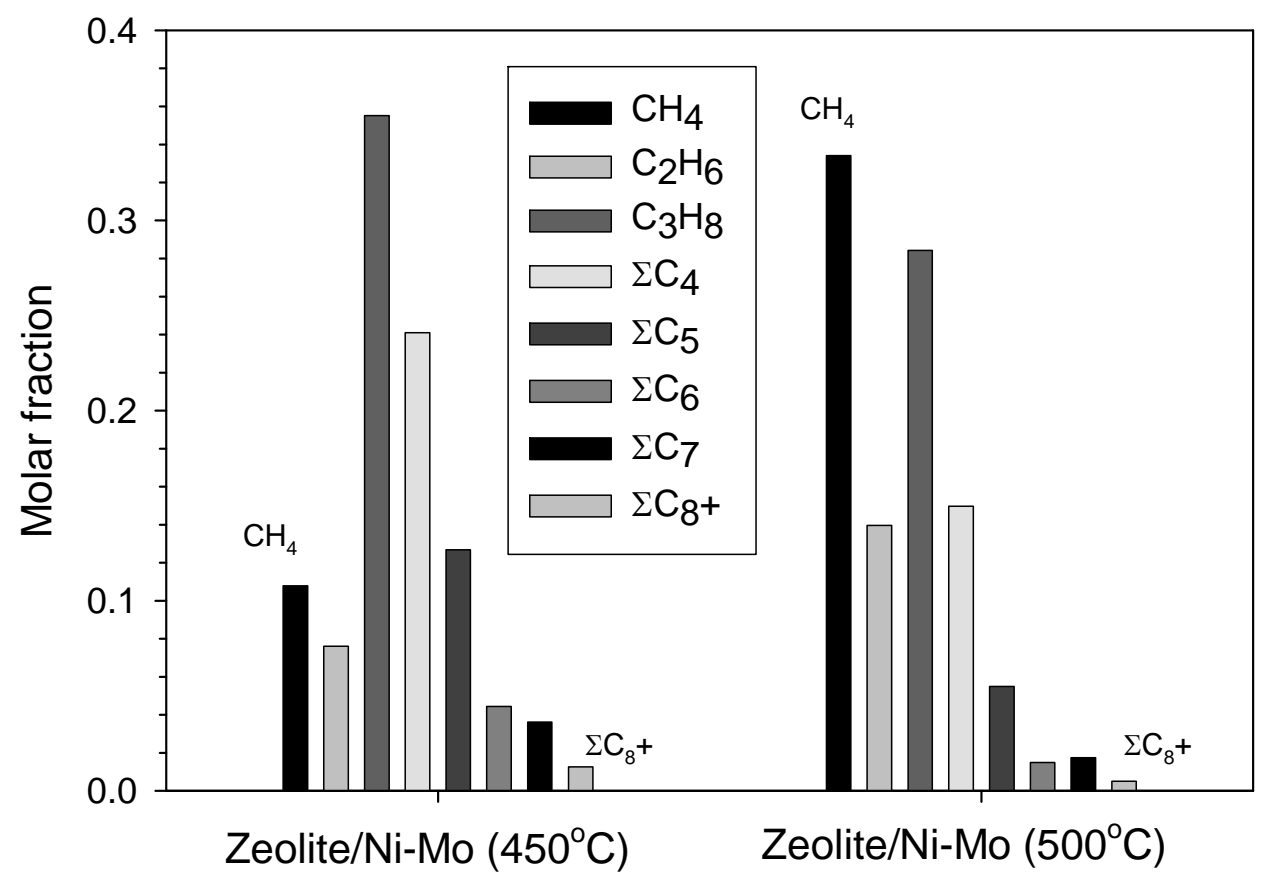

Figure 10. Diesel hydro-reforming over zeolite/Ni-Mo catalyst at $450^{\circ} \mathrm{C}$ (left) and $500^{\circ} \mathrm{C}$ (right); $\mathrm{P}=13.7$ atm.

\subsection{Characterization of pre-reforming catalyst}

Characterization of the zeolite/Ni-Mo catalyst before and after pre-reforming of high-S hexadecane and diesel was carried out by SEM, XPS and XRD analyses. Figure 11 shows the picture of the NiMo/Alumina catalyst: (left) fresh (in oxide form) and (right) after hydro-reforming of high-S diesel (in reduced sulfided form). 


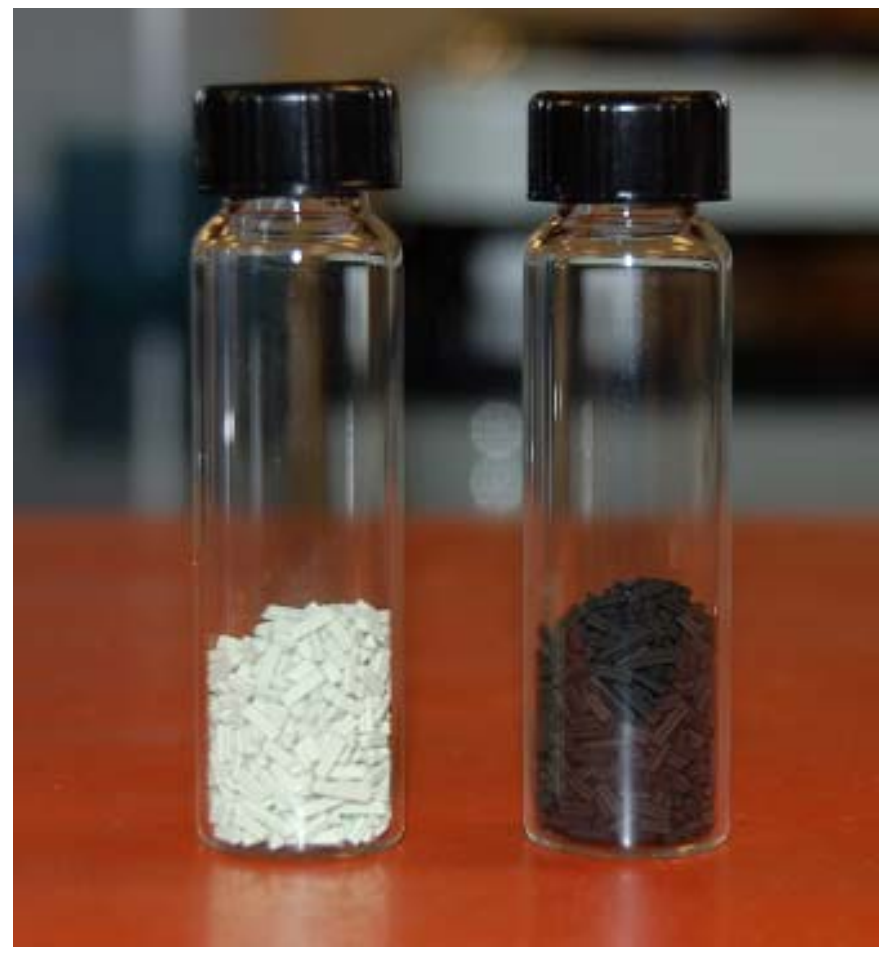

Figure 11. Photograph of Ni-Mo/Alumina catalyst: (left) fresh (in oxide form) (right) after hydroreforming of high-S diesel (in reduced sulfided form).

Figure 12 shows the SEM images of the Ni-Mo/alumina catalyst before (A) and after (B) hydroreforming of high-S diesel. It can be seen that no significant changes in the catalyst surface morphology occurred during the hydro-reforming reaction.
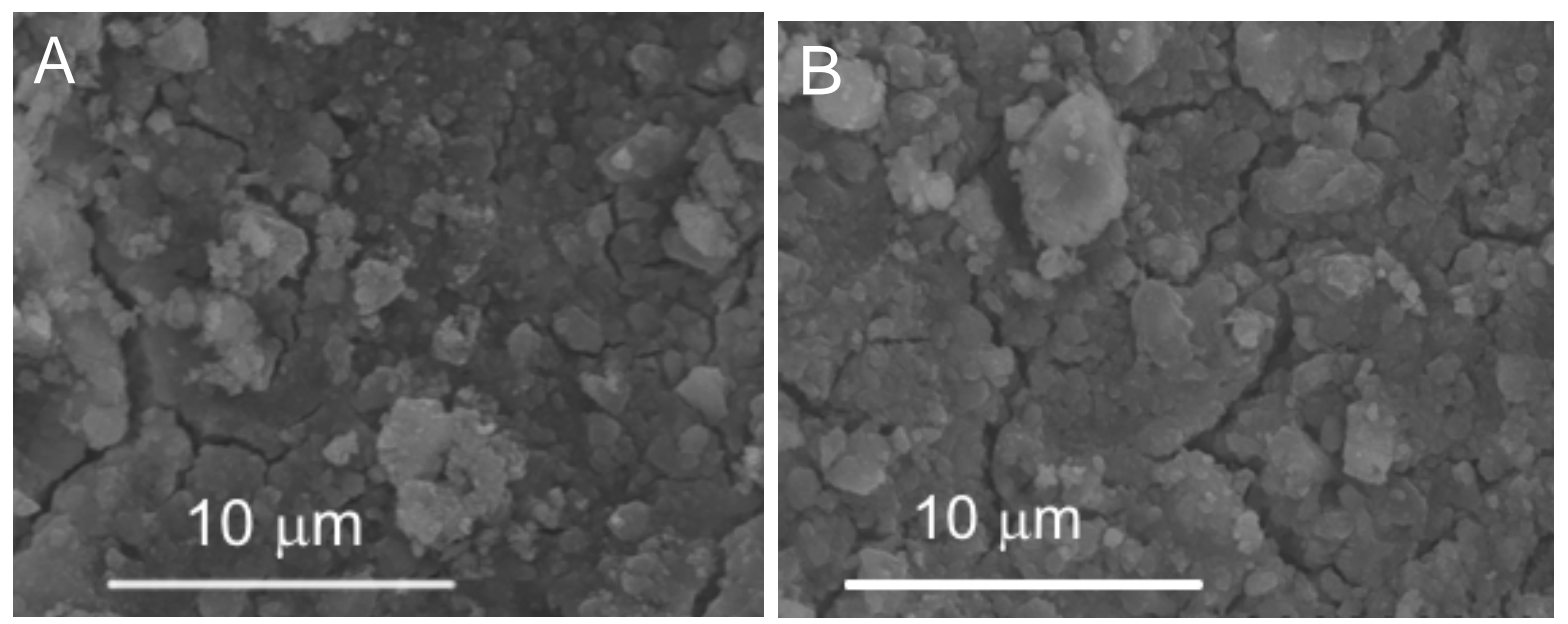

Fig. 12. The SEM images of Ni-Mo/alumina catalyst before (A) and after (B) high-sulfur diesel hydro-reforming.

The XPS spectrum of the original Ni-Mo/alumina pre-reforming catalyst in the region of 0-700 eV is depicted in Figure 13. 


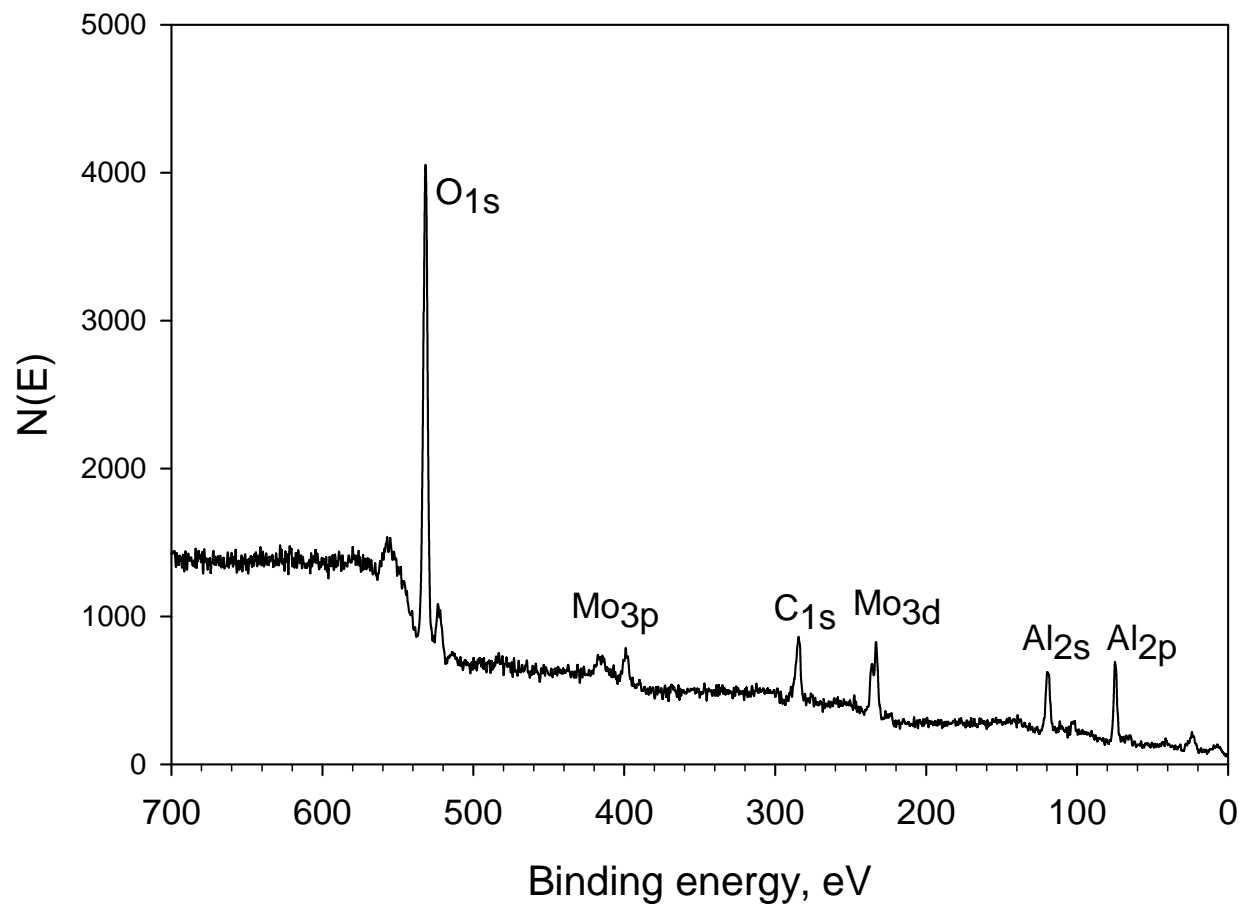

Figure 13. The XPS spectrum of Ni-Mo/alumina catalyst.

The effect of operating conditions during the high-S fuel pre-reforming on the oxidation state of Mo and Ni was investigated. Figure 14 (A \& B) compares the XPS spectra of the oxidized (original) (A) and reduced (after exposure to $\mathrm{H}_{2}$ and high-S diesel fuel) (B) forms of Mo in the Ni-Mo catalyst. The XPS spectrum of the Mo3d $\mathrm{d}_{5 / 2}$ and $\mathrm{Mo3d}_{3 / 2}$ transitions for the oxidized form of catalyst (Figure 14) shows only $\mathrm{Mo}^{6+}$ species, corresponding to binding energies of 233 and $236 \mathrm{eV}$, respectively.

After exposing the catalyst to reducing and sulfiding environment, the reduced $\mathrm{Mo}^{4+}$ species appeared in the XPS spectrum with Mo3d $\mathrm{d}_{5 / 2}$ binding energy of about $229 \mathrm{eV}$ (Figure 14). The peaks at 229$229.6 \mathrm{eV}$ in the sulfided catalyst indicate the presence of $\mathrm{MoS}_{2}$. The spectrum shows that after hydroreforming, a significant amount of Mo in the catalyst remains in its oxidized form, which may point to a strong interaction of oxidized Mo species with the alumina support.

Figure 15 (A \& B) shows the XPS spectra of the oxidized and reduced/sulfided form of the prereforming catalyst, respectively, in the region of 850-866 eV. Interpretation of the Ni2p region of the XPS spectra as it relates to oxidized and reduced forms of the Ni-Mo/alumina catalyst poses some challenges, because of proximity of Ni2p binding energies for different Ni species [9].

Indeed, it is very difficult to distinguish between different $\mathrm{Ni}$ species (e.g., $\mathrm{NiO}, \mathrm{Ni}_{2} \mathrm{O}_{3}, \mathrm{NiAl}_{2} \mathrm{O}_{4}$ ) in the XPS spectrum, as all may appear in the same region. The weak peak at the binding energy of 854 $\mathrm{eV}$ in the reduced/sulfided form of the catalyst may be attributed to NiS. The absence of a peak at a binding energy of $852.4 \mathrm{eV}$ indicates that nickel was not reduced to its metallic state, and most likely, remained in its $\mathrm{Ni}^{2+}$ oxidation state. 


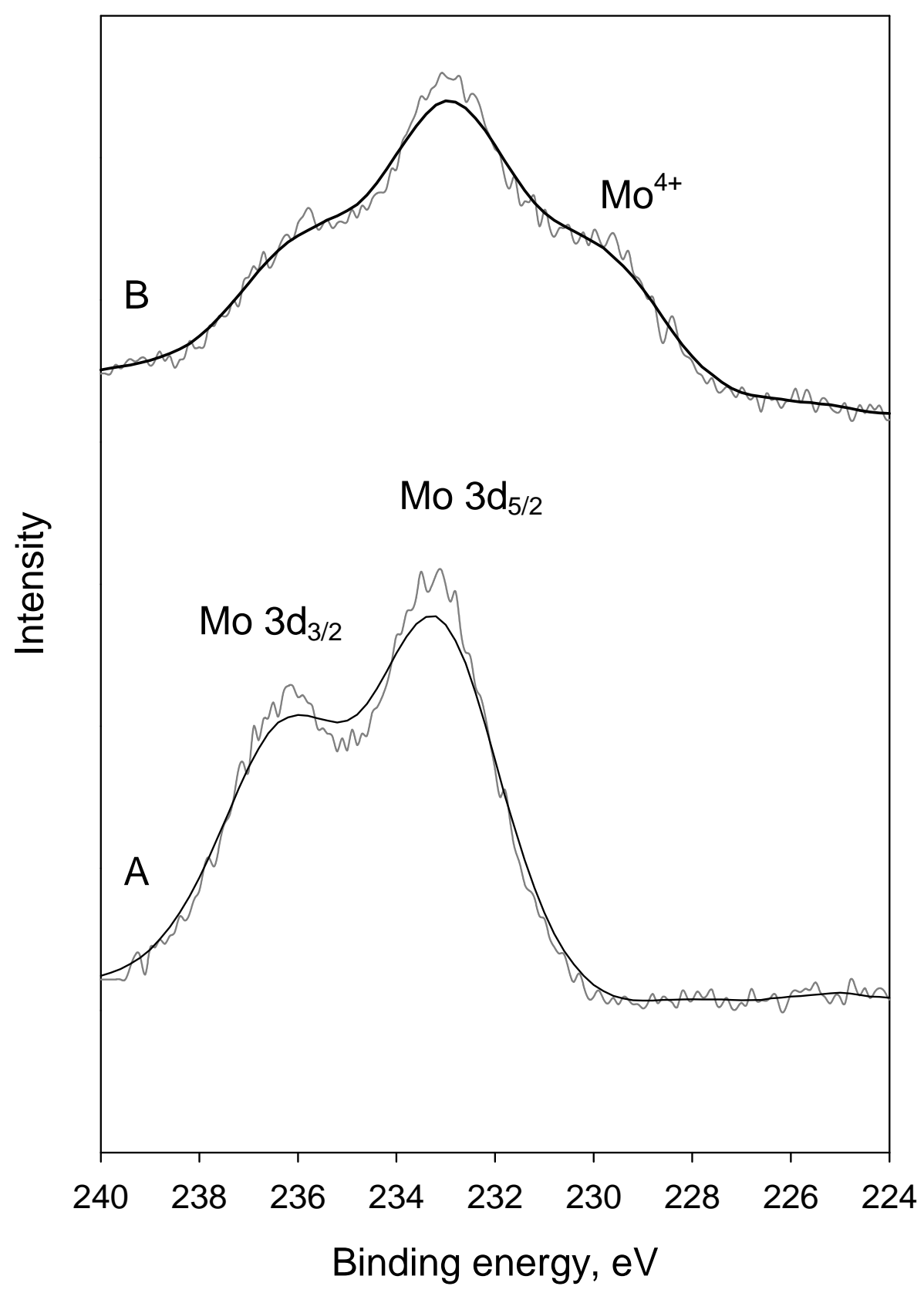

Figure 14. High resolution XPS spectra of Ni-Mo/alumina catalyst before (A) and after (B) high-sulfur diesel hydro-reforming (224-240 eV range). 


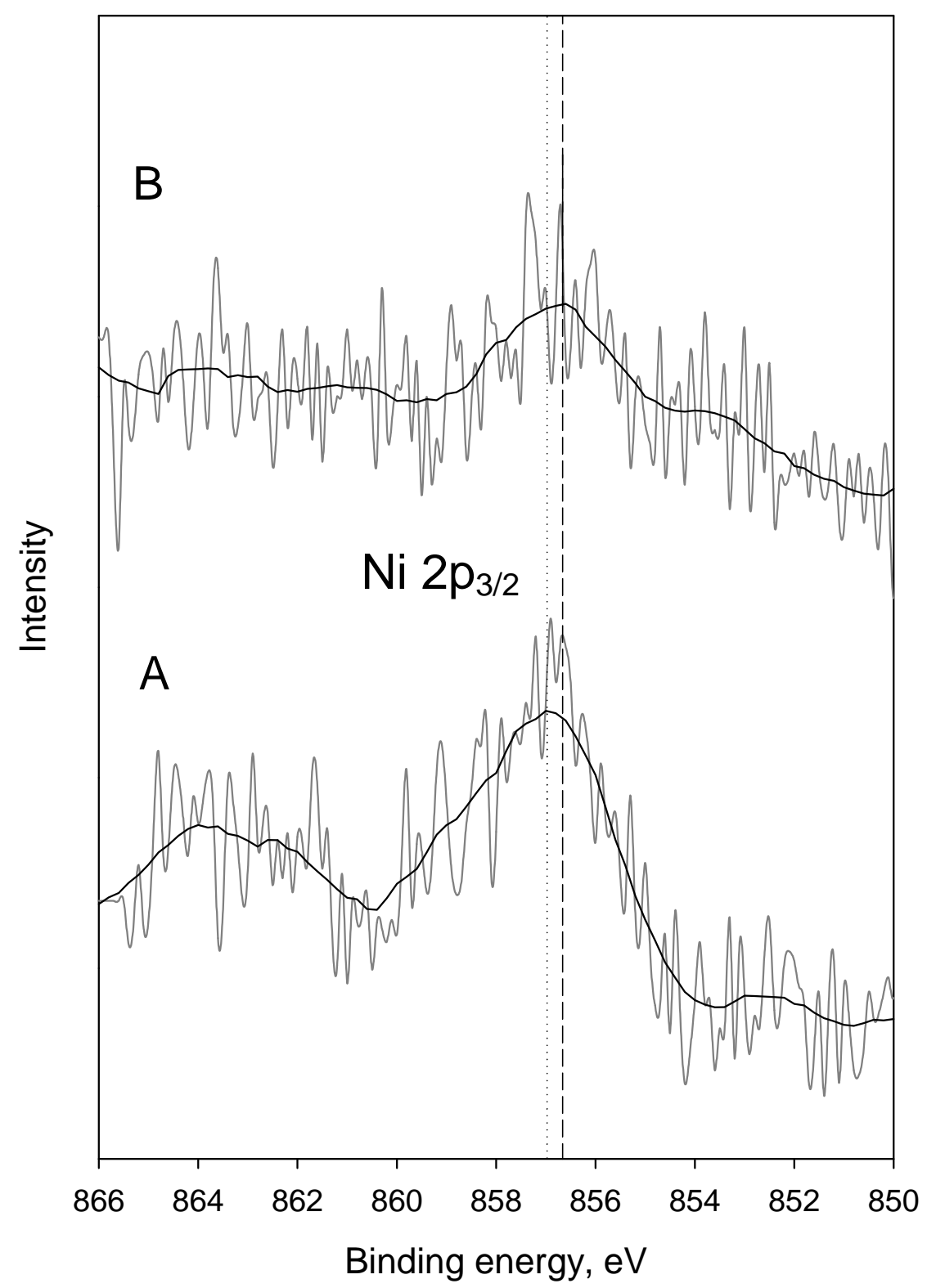

Figure 15. High resolution XPS spectra of Ni-Mo/alumina catalyst before (A) and after (B) high-sulfur diesel hydro-reforming (850-866 eV range).

The XRD pattern of the Ni-Mo/alumina catalyst after high-S diesel pre-reforming is shown in Figure 16. Two most prominent diffraction peaks appearing at $2 \theta$ values of 46 and 67 degrees can be assigned to $\gamma-\mathrm{Al}_{2} \mathrm{O}_{3}$. The XRD pattern does not indicate XRD-observable Ni oxides; however, it is commonly observed that $\mathrm{NiO}$ and $\mathrm{Ni}_{2} \mathrm{O}_{3}$ may also exist in amorphous or microcrystalline phases [10].

Identification of a $\mathrm{NiAl}_{2} \mathrm{O}_{4}$ phase is rather difficult because of proximity of its reflection to that of alumina. Peaks corresponding to Mo compounds are apparently too weak to be distinguishable on the XRD pattern of the catalyst. 


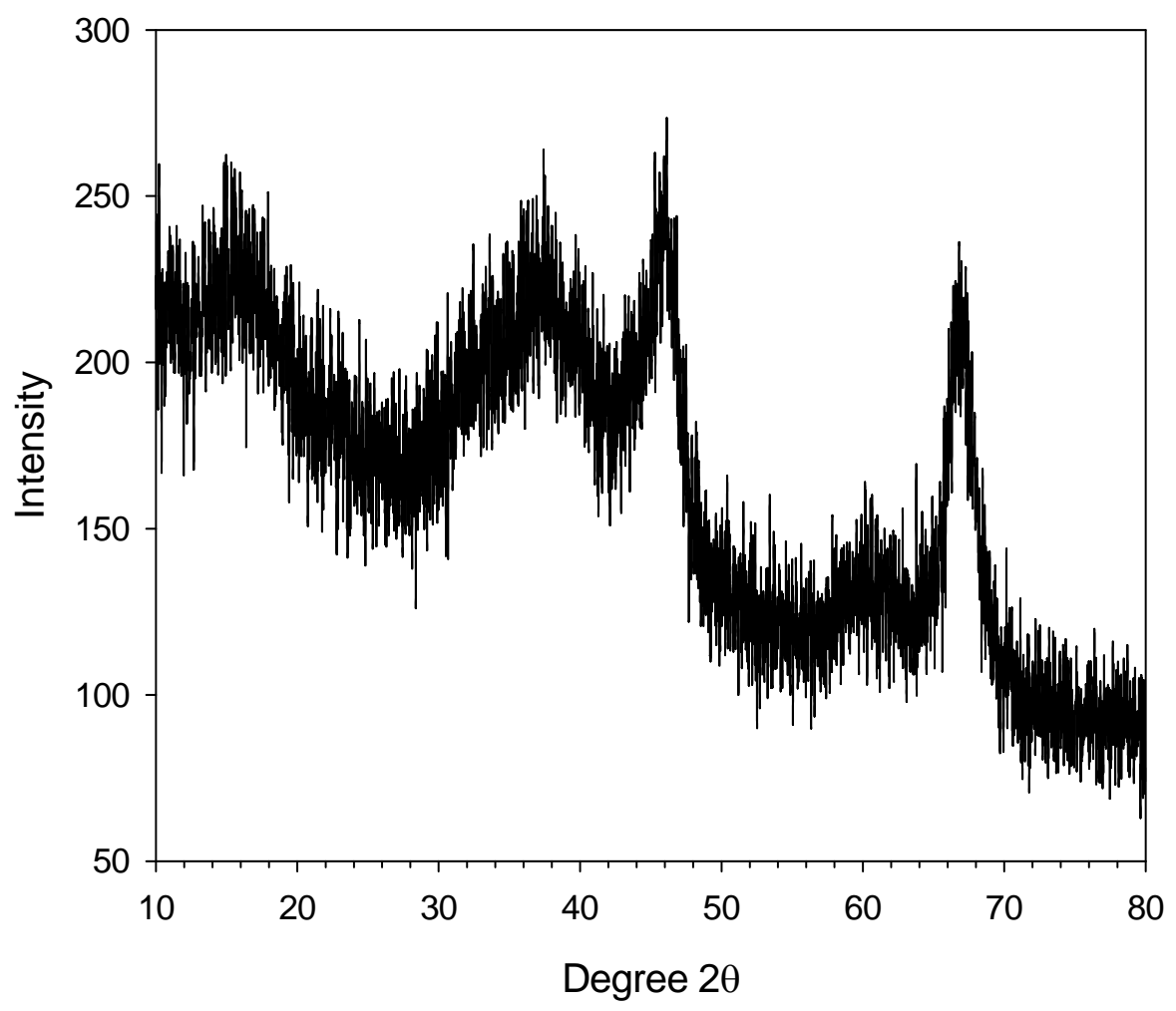

Fig. 16. XRD pattern of Ni-Mo/alumina catalyst after high-sulfur diesel hydro-reforming.

\section{Desulfurization of $\mathbf{H}_{2} \mathrm{~S}$-containing Gases}

\subsection{Photolytic desulfurization system}

In the photolytic desulfurization system, the $\mathrm{H}_{2} \mathrm{~S}$-containing gas is directed to $\mathrm{H}_{2} \mathrm{~S}$ stripper filled with an alkaline, e.g. $\mathrm{NaOH}$ solution, wherein the gas is scrubbed of the remaining $\mathrm{H}_{2} \mathrm{~S}$ down to part per billion (ppb) levels. The details of the photolytic $\mathrm{H}_{2} \mathrm{~S}$ decomposition system are shown in Figure 17. The bisulfide ion, $\mathrm{HS}^{-}$(aq) solution from the $\mathrm{H}_{2} \mathrm{~S}$ stripper 4 , is directed into the photolytic reactor 5 (having an inner sleeve that houses a low-pressure mercury lamp), where it is photolyzed into hydrogen and elemental sulfur.

As the solution is passed through the photoreactor, bisulfide ion is photooxidized to elemental sulfur and water is reduced to hydrogen. The hydrogen gas percolates to the top of the photoreactor where it is collected. The sulfur remains in solution as disulfide ion $\left(\mathrm{S}_{2}{ }^{2-}\right)$. The disulfide solution is returned to the scrubber 4, where it is exposed to fresh $\mathrm{H}_{2} \mathrm{~S}$. Hydrogen sulfide lowers the $\mathrm{pH}$ causing the complexed sulfur to be freed and precipitate inside the scrubber. The sulfide-laden effluent is returned to the photoreactor, while the sulfur precipitate is collected via in-line filters. 


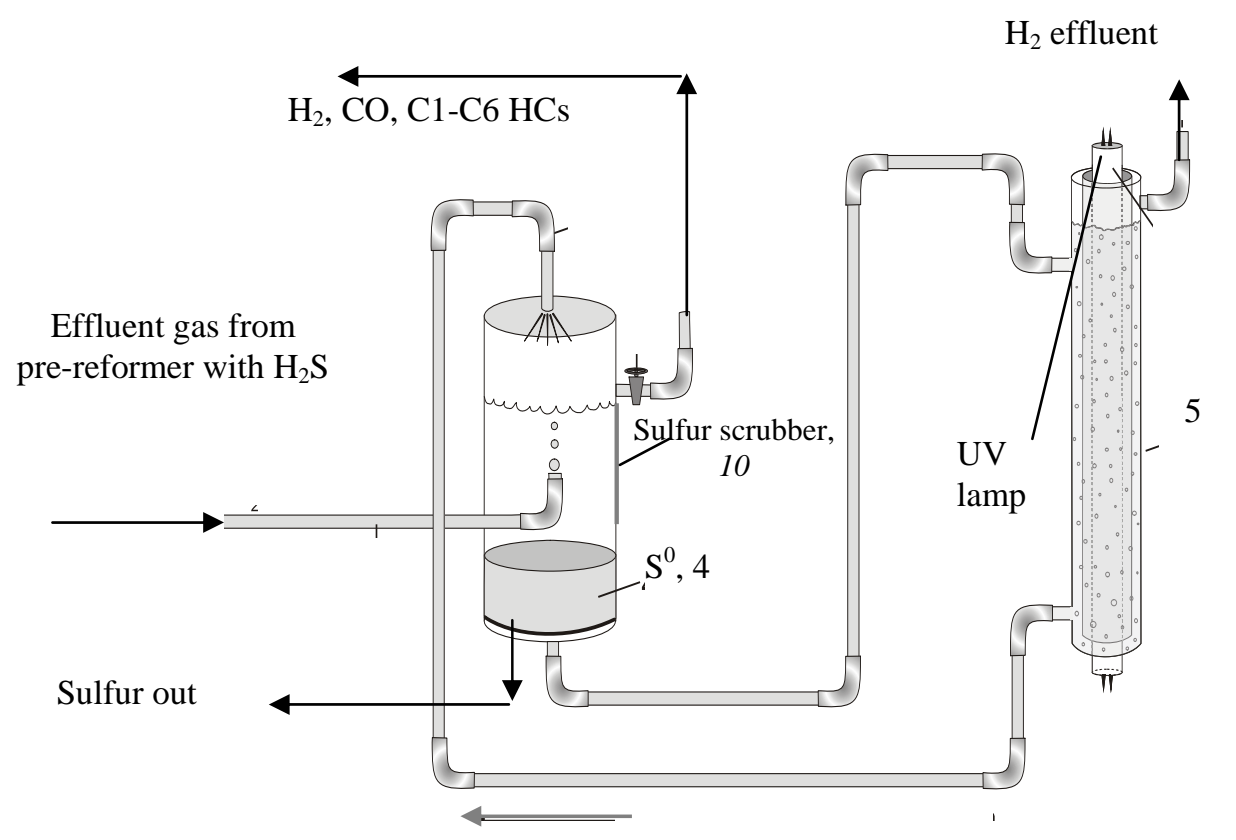

Figure 17. The schematic diagram of FSEC's $\mathrm{H}_{2} \mathrm{~S}$ scrubber photoreactor.

\subsection{Efficiency of UV photolytic scrubber}

Our first task was to determine whether the UV photolytic process could handle the sulfurous effluent from the pre-reformer. $\mathrm{H}_{2} \mathrm{~S}$ is removed from the gas stream via acid dissociation in aqueous solution within a scrubber column:

$\mathrm{H}_{2} \mathrm{~S}(\mathrm{~g})+\mathrm{H}_{2} \mathrm{O} \rightarrow \mathrm{H}^{+}(\mathrm{aq})+\mathrm{HS}^{-}(\mathrm{aq}) \quad \mathrm{pH}>7$

The solvated bisulfide ion then passes to the photoreactor, where it is oxidized to elemental sulfur:

$\mathrm{HS}^{-}(\mathrm{aq})+\mathrm{OH}^{-} \rightarrow \mathrm{S}^{0}+\mathrm{H}_{2} \mathrm{O}+2 \mathrm{e}^{-} \quad$ photooxidation

At the same time, water is reduced in the photoreactor, making $\mathrm{H}_{2}$ :

$2 \mathrm{H}_{2} \mathrm{O}+2 \mathrm{e}^{-} \rightarrow \mathrm{H}_{2}+2 \mathrm{OH}^{-} \quad$ photoreduction

Collecting the sulfur is somewhat complicated, as the colloidal sulfur in the photoreactor quickly reacts with excess bisulfide to make soluble disulfide ion, $\mathrm{S}_{2}{ }^{2-}(\mathrm{aq})$ :

$\mathrm{S}^{\mathrm{o}}+\mathrm{HS}^{-}+\mathrm{H}_{2} \mathrm{O} \rightarrow \mathrm{S}_{2}{ }^{2-}+\mathrm{H}_{2} \mathrm{O}$ disulfide formation

Disulfide ion is then routed back to the scrubber, where the acid equivalents from $\mathrm{H}_{2} \mathrm{~S}$ dissociation force its decomposition, freeing up the sulfur:

$\mathrm{S}_{2}{ }^{2-}(\mathrm{aq})+\mathrm{H}^{+}(\mathrm{aq}) \rightarrow \mathrm{S}^{\mathrm{o}}(\mathrm{s})+\mathrm{HS}^{-}(\mathrm{aq})$

The sulfur is then collected by filtration or sedimentation. 
It was estimated that the gaseous concentration of $\mathrm{H}_{2} \mathrm{~S}$ would be on order of $1300 \mathrm{ppm}$. Furthermore, it was specified that the scrubber needed to reduce $\mathrm{H}_{2} \mathrm{~S}$ to $\leq 50 \mathrm{ppm}$, so that the reformer catalyst would not be deactivated and the $\mathrm{H}_{2}$ coming out of the reformer would be reasonably clean. Our first task then was to attempt sulfur precipitation from disulfide ion under these conditions.

An experimental apparatus was constructed similar to that shown in Figure 17 except that the $\mathrm{H}_{2} \mathrm{~S}$ feed gas was successively diluted with more and more $\mathrm{N}_{2}$, to determine the threshold concentration of $\mathrm{H}_{2} \mathrm{~S}$ that could effect sulfur precipitation. Rather than wait for the photoreactor to build up a background of $\mathrm{S}_{2}{ }^{2-}$ ion, a $0.1 \mathrm{M}$ solution of sodium disulfide, $\mathrm{Na}_{2} \mathrm{~S}_{2}$, was prepared by mixing a stoichiometric amount of sulfur powder with sodium sulfide nona-hydrate, $\mathrm{Na}_{2} \mathrm{~S} \cdot 9 \mathrm{H}_{2} \mathrm{O}$, and stirring until a clear yellow solution was obtained.

A series of curves are shown in Figure 18, where the $\mathrm{pH}$ of the $\mathrm{Na}_{2} \mathrm{~S}_{2}$ solution was monitored as a function of the total amount of $\mathrm{H}_{2} \mathrm{~S}$ fed to it. A reverse sigmoidal plot was obtained in each case, indicative of the acid-base chemistry that was occurring in the scrubber vessel. After numerous trials, it was clear that the threshold $\mathrm{pH}$ below which sulfur precipitation could occur was 7.6. This threshold was achievable for reasonably concentrated $\mathrm{H}_{2} \mathrm{~S}$; the curve for a ten-fold dilution of $\mathrm{H}_{2} \mathrm{~S}$ to 10 volume percent was able to cross the line and produce a turbid solution, indicative of colloidal sulfur formation. However, as $\mathrm{H}_{2} \mathrm{~S}$ became more and more dilute, the $\mathrm{pH}$ curves were unable to cross the precipitation threshold.

As seen in Figure 18, a $0.242 \% \mathrm{H}_{2} \mathrm{~S}$ feed gas (2,420 ppm) could not lower $\mathrm{pH}$ below 9.3, far short of the minimum for sulfur precipitation. Therefore, the estimated $1300 \mathrm{ppm}_{2} \mathrm{~S}$ coming from the prereformer would be unable to effect sulfur precipitation inside the scrubber. Stated from another perspective, the UV photolytic scrubber would be unable to lower the sulfur content of the exit gas from the pre-reformer to $50 \mathrm{ppm}$.

It was of interest to determine the threshold $\mathrm{H}_{2} \mathrm{~S}$ concentration, i.e., what minimum volume percent of $\mathrm{H}_{2} \mathrm{~S}$ could effect sulfur precipitation. In Figure 19, a series of curves are shown where only slight variations of $\mathrm{H}_{2} \mathrm{~S}$ concentration in the feed gas were made. The split between the precipitation threshold was shown to lie between 4.6 and $6.6 \% \mathrm{H}_{2} \mathrm{~S}$. Therefore, we would put the minimum allowable $\mathrm{H}_{2} \mathrm{~S}$ concentration at 5 volume percent, or 50,000 ppm.

\subsection{Pressure effect on sulfur precipitation}

The ability of $\mathrm{H}_{2} \mathrm{~S}$ to lower the $\mathrm{pH}$ of a solution is determined by its dissociation constant and its initial concentration. The higher the background concentration, the more acid gas can be dissociated, lowering $\mathrm{pH}$ and facilitating sulfur precipitation. The concentration of $\mathrm{H}_{2} \mathrm{~S}$ in solution is in turn controlled by the partial pressure of $\mathrm{H}_{2} \mathrm{~S}$ in the vapor phase above the solution. 


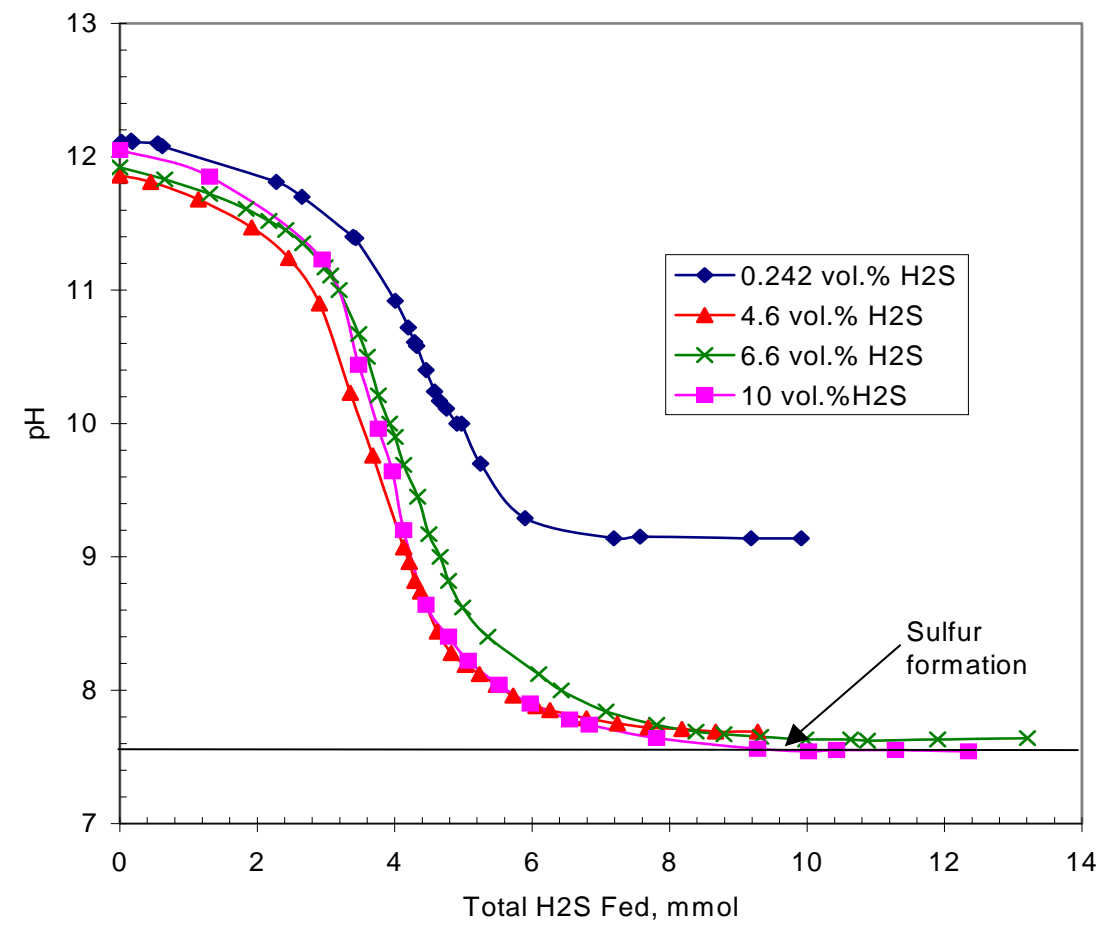

Figure 18. The $\mathrm{pH}$ dependence of $0.1 \mathrm{M} \mathrm{Na}_{2} \mathrm{~S}_{2}$ solution on $\mathrm{H}_{2} \mathrm{~S}$ addition for a series of gas dilutions.

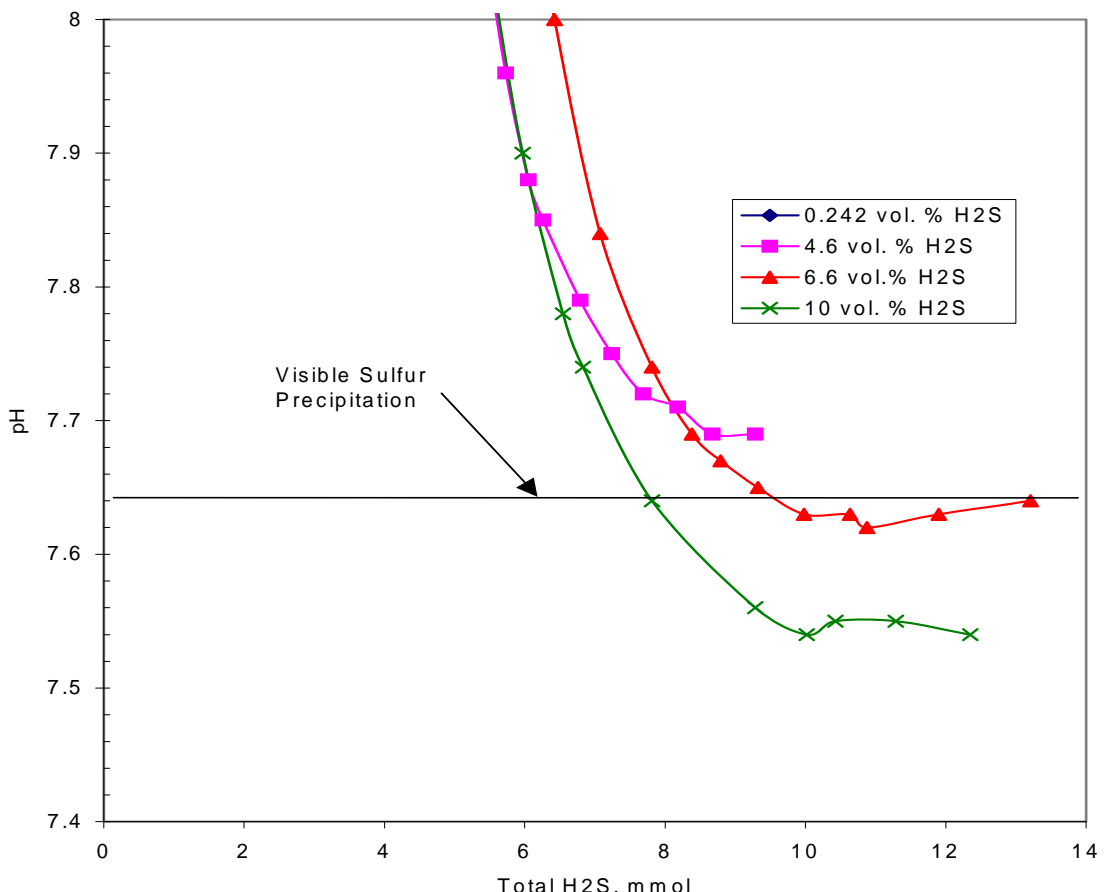

Figure 19. Determining the minimum $\mathrm{H}_{2} \mathrm{~S}$ feed gas concentration to effect sulfur precipitation from $0.1 \mathrm{M} \mathrm{Na}_{2} \mathrm{~S}_{2}$ solution. 
The dependence between gas phase concentration and solution phase concentration is given by the Henry's Law constant for that particular molecular species and solvent. For $\mathrm{H}_{2} \mathrm{~S}$, the Henry's Law constant at room temperature and initially neutral aqueous solution is on the order of $0.1 \mathrm{~mol} / \mathrm{L}$-atm.

As discussed in the previous section, a headspace concentration of 2,500 $\mathrm{ppm}_{2} \mathrm{~S}$ was unable to effect sulfur precipitation. At 1 atmosphere total pressure, that concentration corresponds to a partial pressure of $0.0025 \mathrm{~atm}$. The threshold headspace concentration to effect sulfur precipitation was 5 volume percent, or 50,000 ppm, at 1 atmosphere. This corresponds to a partial pressure of $0.05 \mathrm{~atm}$. Therefore, if the 2,500 $\mathrm{ppm} \mathrm{H}_{2} \mathrm{~S}$ feed is pressurized to $20 \mathrm{~atm}$, the threshold partial pressure could be achieved. The hypothetical 1,300 $\mathrm{ppm}_{2} \mathrm{~S}$ coming from the pre-reformer would require a total pressure of $38.4 \mathrm{~atm}$, something easily achieved in a steel reactor. The Chevron Technology Ventures required that the pre-reformer effluent to be pressurized to $10 \mathrm{~atm}$. Thus, the threshold partial pressure was easily within reach.

An apparatus was put together to test the pressurization effect, as shown in Figure 20 below. The 2,500 $\mathrm{ppm} \mathrm{H}_{2} \mathrm{~S}$, having been obtained as the diluted gas under $\mathrm{N}_{2}$ in a pressurized tank (initially > 2,200 psi), could be delivered under control of a two-stage regulator. The gas was connected to a PARR reactor containing a $\mathrm{Na}_{2} \mathrm{~S}_{2}$ solution. A special pressure-tolerant $\mathrm{pH}$ electrode was inserted to monitor the progress of the reaction. Caution had to be exercised as to how much $\mathrm{H}_{2} \mathrm{~S}$ could be applied to the reaction; too much $\mathrm{H}_{2} \mathrm{~S}$ could blacken the inner walls of the reactor due to sulfiding of the steel alloy. Fortunately, we could obtain the desired partial pressure range without the corrosion chemistry occurring. Nevertheless, a glass liner was inserted into the reactor to help avoid doing corrosion chemistry with the vessel walls.

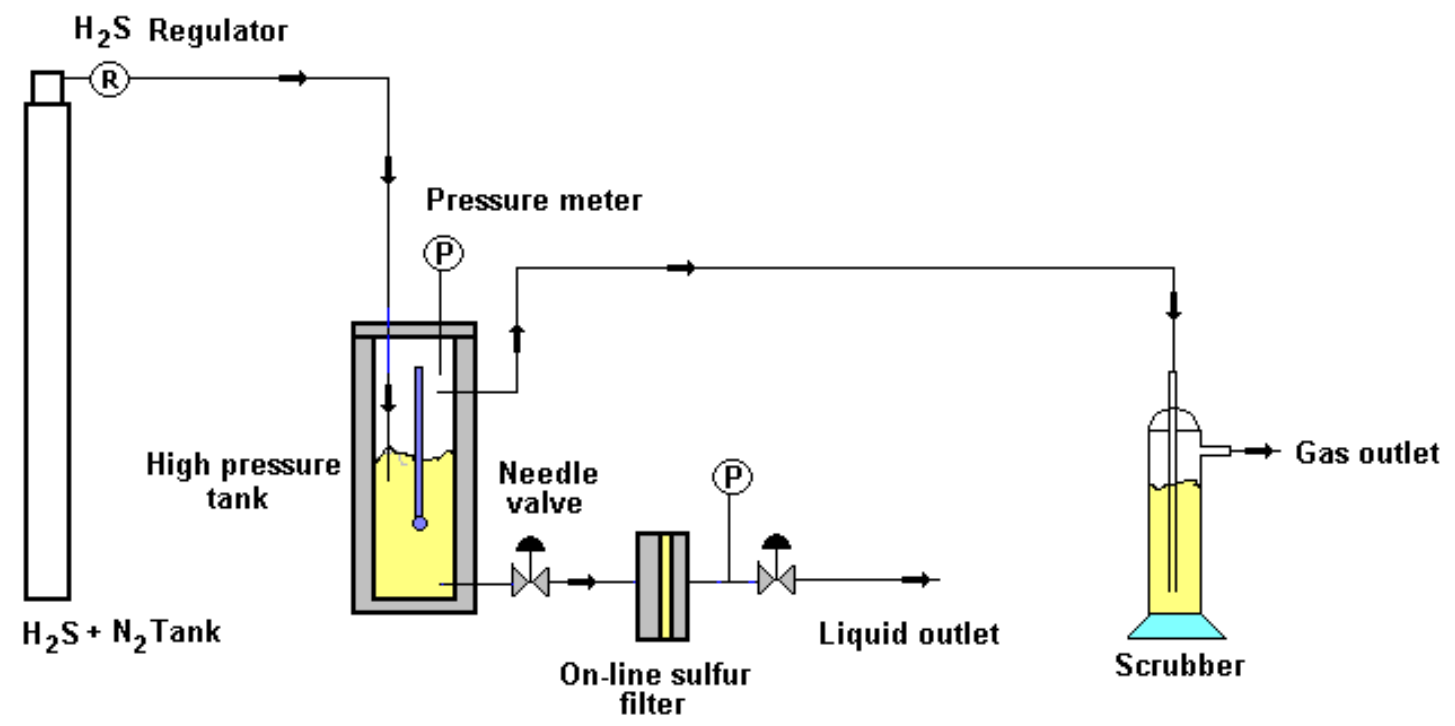

Figure 20. High pressure sulfur precipitation experimental setup.

Gas was first bubbled through the reactor for an hour to achieve equilibrium between the headspace inside the pressure vessel and the solution. A caustic scrubber vessel was attached further down on the purge gas exit line to eliminate sulfur emissions from the fume hood exhaust. At that point, the vessel was sealed off and the pressure was raised to the desired value. The $\mathrm{pH}$ would begin to drop, as shown in Figure below. Once the $\mathrm{pH}$ had leveled off, a needle valve would be opened and the reactor solution forced through an in-line filter. A pressure gauge and needle valve on the back side of the in- 
line filter were installed to collect any precipitated sulfur under pressurized conditions. This was done to ensure that the sulfur precipitate could not slip back into solution due to shifting of the equilibrium as the vessel was opened. Once the solution had been drained out, the filter would be inspected to see if any sulfur had been collected

In the example shown below in Figure 21, total pressure of 2,500 $\mathrm{ppm}_{2} \mathrm{~S}$ was raised to $200 \mathrm{psig}$, corresponding to an $\mathrm{H}_{2} \mathrm{~S}$ partial pressure of $0.034 \mathrm{~atm}$. The $\mathrm{pH}$ dropped from nearly 12 to 7.47, surpassing the $7.6 \mathrm{pH}$ threshold for sulfur precipitation. However, no sulfur was collected using 2,500 ppm $\mathrm{H}_{2} \mathrm{~S}$ in this or any other of several experiments. The fact that pressure was influencing solution $\mathrm{pH}$ became evident at the end of the trial when pressure was released $-\mathrm{pH}$ immediately jumped up to $>10$, indicating that the concentration of hydrogen ions had been increased nearly 1000-fold, but still not enough to effect sulfur precipitation.

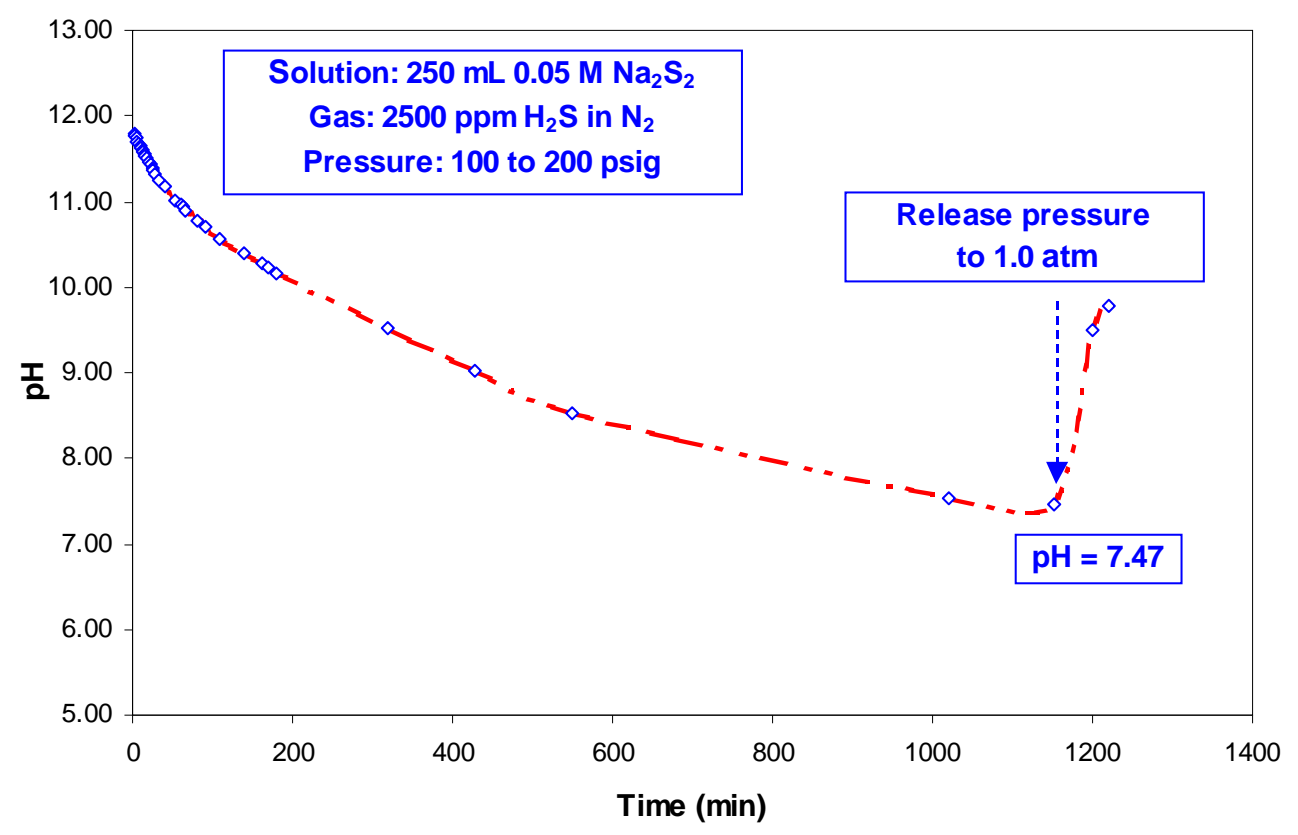

Figure 21. Attempted sulfur precipitation with 2,500 $\mathrm{ppm}_{2} \mathrm{~S}$ sparging at $>10 \mathrm{~atm}$.

\subsection{Photolytic regenerable $\mathrm{Fe}^{3+} / \mathrm{Fe}^{2+}$ system}

We also investigated an alternative UV photolytic system using regenerable ferrous-ferric couple as follows:

$\mathrm{H}_{2} \mathrm{~S}(\mathrm{aq})+2 \mathrm{Fe}^{3+}($ EDTA $) \rightarrow \mathrm{S}(\mathrm{s})+2 \mathrm{Fe}^{2+}($ EDTA $)+2 \mathrm{H}^{+}$

$2 \mathrm{Fe}^{2+}($ EDTA $)+2 \mathrm{H}^{+}+\mathrm{h} v \rightarrow 2 \mathrm{Fe}^{3+}($ EDTA $)+\mathrm{H}_{2}(\mathrm{~g})$

Overall: $\mathrm{H}_{2} \mathrm{~S}(\mathrm{aq})+\mathrm{h} v \rightarrow \mathrm{S}(\mathrm{s})+\mathrm{H}_{2}(\mathrm{~g})$

To keep the iron from hydrolyzing in neutral $\mathrm{pH}$ solution, it was necessary to complex it with a chelating agent, such as EDTA (ethylenediaminetetraacetic acid). Purging 2,500 $\mathrm{ppm} \mathrm{H}_{2} \mathrm{~S} / \mathrm{N}_{2}$ gas through an $85 \% \mathrm{Fe}(\mathrm{III})$ EDTA and 15\% Fe(II)EDTA solution (with total concentration of $0.010 \mathrm{M}$ ), sulfur precipitation was observed after $15 \mathrm{~min}$ at room temperature and pressure. The reduction of 
$\mathrm{Fe}^{3+}$ in the scrubber was accompanied by $\mathrm{Fe}^{2+}$ oxidation and evolution of $\mathrm{H}_{2}$ inside the photoreactor, so that the overall mechanism was the same as that obtained previously by the UV system:

However, it was found that the EDTA chelating agent was itself susceptible to photooxidation. As shown in Figure 22, while the highest amounts of $\mathrm{H}_{2}$ could be made from near stoichiometric amounts of $\mathrm{Fe}^{2+}$ and EDTA, it was also observed that some $\mathrm{H}_{2}$ could be made from EDTA alone, in the absence of iron salts. Solution color changed to light yellow. Subsequent GC analysis showed that $\mathrm{CO}_{2}$ was being produced, i.e., the EDTA was being photooxidized.

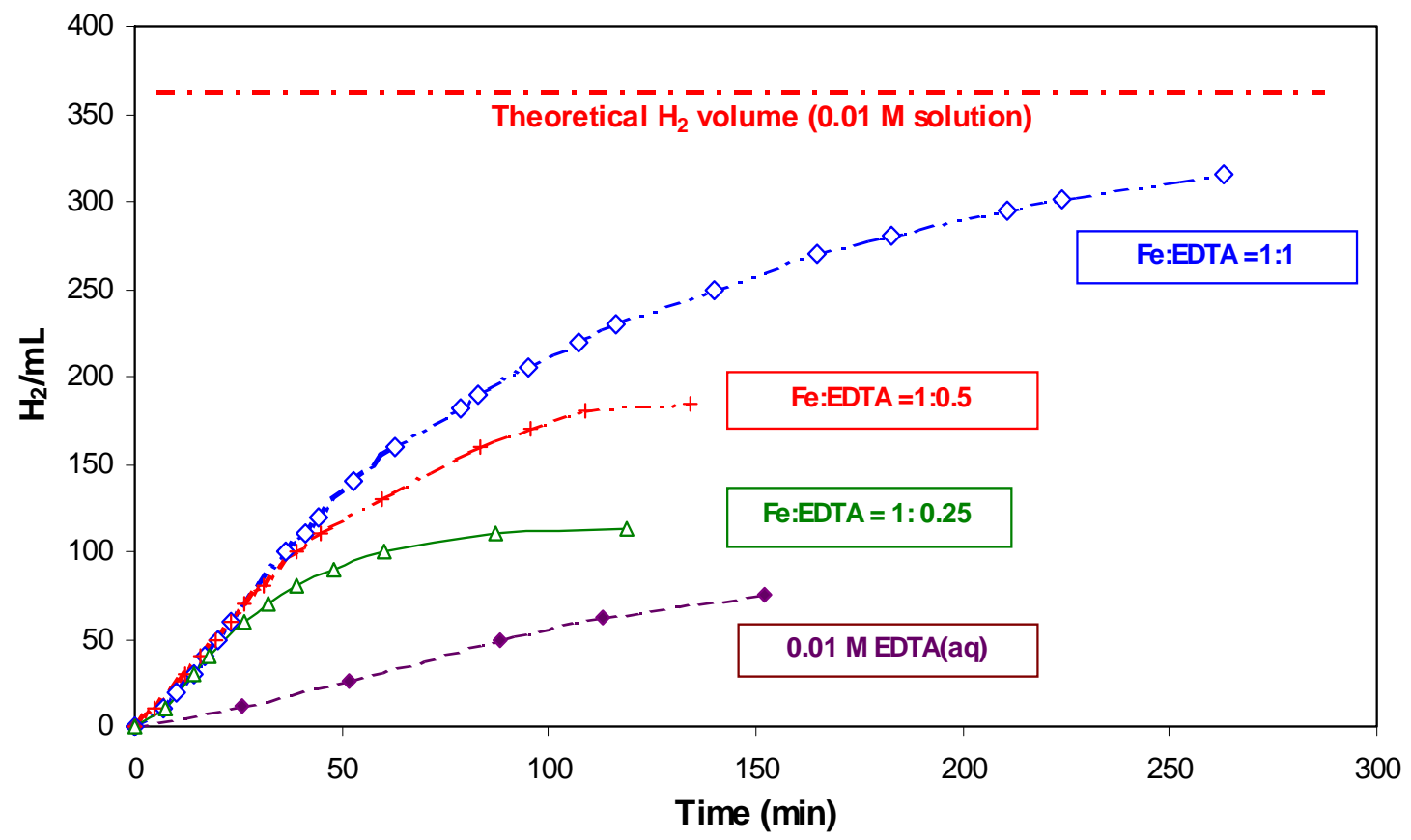

Figure 22. $\mathrm{H}_{2}$ production from photolysis of iron chelate solution.

Thus, UV recycling of the ferrous solution was problematic due to spectral interference of the counterions.

\subsection{Electrolytic desulfurization system}

While UV photolysis of ferrous ion was unsuccessful, we found that ferrous ion could be electrochemically oxidized back to the ferric state. That provided yet another means of completing the sulfur removal process. Hydrogen sulfide would react with ferric sulfate to make sulfuric acid and elemental sulfur. Sulfur would be removed from solution, either by filtration or sedimentation. The solution would then be passed through the anode compartment of an electrochemical cell, where ferrous sulfate would be re-oxidized back to the ferric state. Hydrogen ions in the solution would migrate across the cell to the cathode, where they would be reduced and evolved as hydrogen gas.

Scrubber: $\quad \mathrm{H}_{2} \mathrm{~S}(\mathrm{~g})+\mathrm{Fe}_{2}\left(\mathrm{SO}_{4}\right)_{3}(\mathrm{aq}) \rightarrow 2 \mathrm{FeSO}_{4}(\mathrm{aq})+\mathrm{H}_{2} \mathrm{SO}_{4}(\mathrm{aq})+\mathrm{S}(\mathrm{s})$

Anode: $\quad 2 \mathrm{Fe}^{2+}(\mathrm{aq}) \rightarrow 2 \mathrm{Fe}^{3+}(\mathrm{aq})+2 \mathrm{e}^{-} ; \quad \mathrm{E}=0.77 \mathrm{~V}$ vs NHE

Cathode: $\quad 2 \mathrm{H}^{+}+2 \mathrm{e}^{-} \rightarrow \mathrm{H}_{2}(\mathrm{~g}) ; \quad \mathrm{E}=0.00 \mathrm{~V}$ 
Overall electrolysis: $2 \mathrm{Fe}^{2+}(\mathrm{aq})+2 \mathrm{H}^{+}+\Delta \mathrm{E} \rightarrow \mathrm{H}_{2}(\mathrm{~g})+2 \mathrm{Fe}^{3+}(\mathrm{aq})$

Including the sulfate counter-ions, the overall electrolysis reaction would be

$2 \mathrm{FeSO}_{4}(\mathrm{aq})+\mathrm{H}_{2} \mathrm{SO}_{4}(\mathrm{aq})+\Delta \mathrm{E} \rightarrow \mathrm{H}_{2}(\mathrm{~g})+\mathrm{Fe}_{2}\left(\mathrm{SO}_{4}\right)_{3}(\mathrm{aq})$

Combining this step with the scrubber reaction gives

$\mathrm{H}_{2} \mathrm{~S}(\mathrm{~g})+\Delta \mathrm{E} \rightarrow \mathrm{H}_{2}(\mathrm{~g})+\mathrm{S}(\mathrm{s})$

This is identical to the UV photolytic process except for the source of energy input.

Figure 23 shows the schematic diagram of the electrolytic desulfurization unit developed for this work.

A

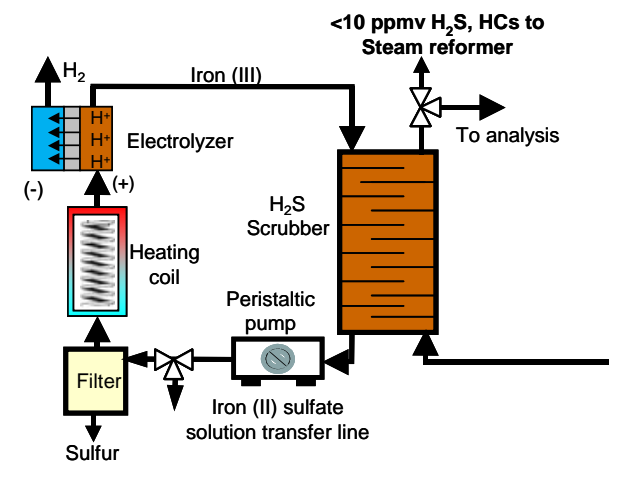

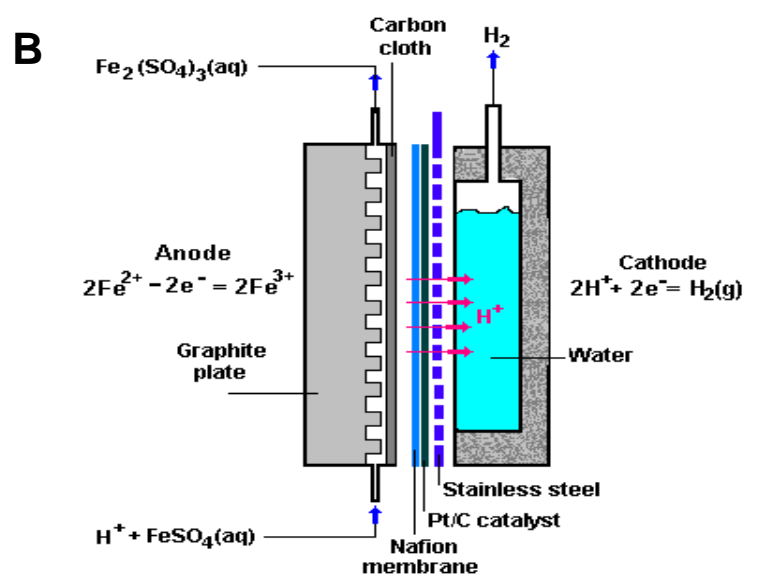

Figure 23. Schematic representation of A- sulfur scrubber coupled with Fe-redox electrolytic regeneration system, and B- electrolyzer used for the regeneration of Fe-redox system.

Alternatively, regeneration of the iron redox couple could be accomplished by reaction with $\mathrm{O}_{2}$ or other oxidizing agent:

$4 \mathrm{Fe}^{2+}(\mathrm{aq})+4 \mathrm{H}^{+}(\mathrm{aq})+\mathrm{O}_{2}(\mathrm{~g}) \rightarrow 4 \mathrm{Fe}^{3+}(\mathrm{aq})+2 \mathrm{H}_{2} \mathrm{O}$

Overall: $2 \mathrm{H}_{2} \mathrm{~S}(\mathrm{~g})+\mathrm{O}_{2}(\mathrm{~g}) \rightarrow 2 \mathrm{H}_{2} \mathrm{O}+2 \mathrm{~S}(\mathrm{~s})$

The overall reaction is the same as the Claus Process before, namely, $\mathrm{H}_{2} \mathrm{~S}$ partial oxidation by $\mathrm{O}_{2}$ to make sulfur and water. While oxidation of $\mathrm{Fe}^{2+}$ by air can be accomplished as shown above, the process is undesirable for our application, as it introduces an oxygen partial pressure into the prereformate.

\subsection{Electrolytic recycling of ferrous iron}

With the basic chemistry established, it was time to begin optimizing some of the process parameters. The first was to determine the best $\mathrm{pH}$ value for the system. At first, optimum $\mathrm{pH}$ values were determined for electrolyzer and scrubber separately from each other. The effect of acidity on the rate of $\mathrm{FeSO}_{4}$ electrolysis is shown in Figure 24 below. 


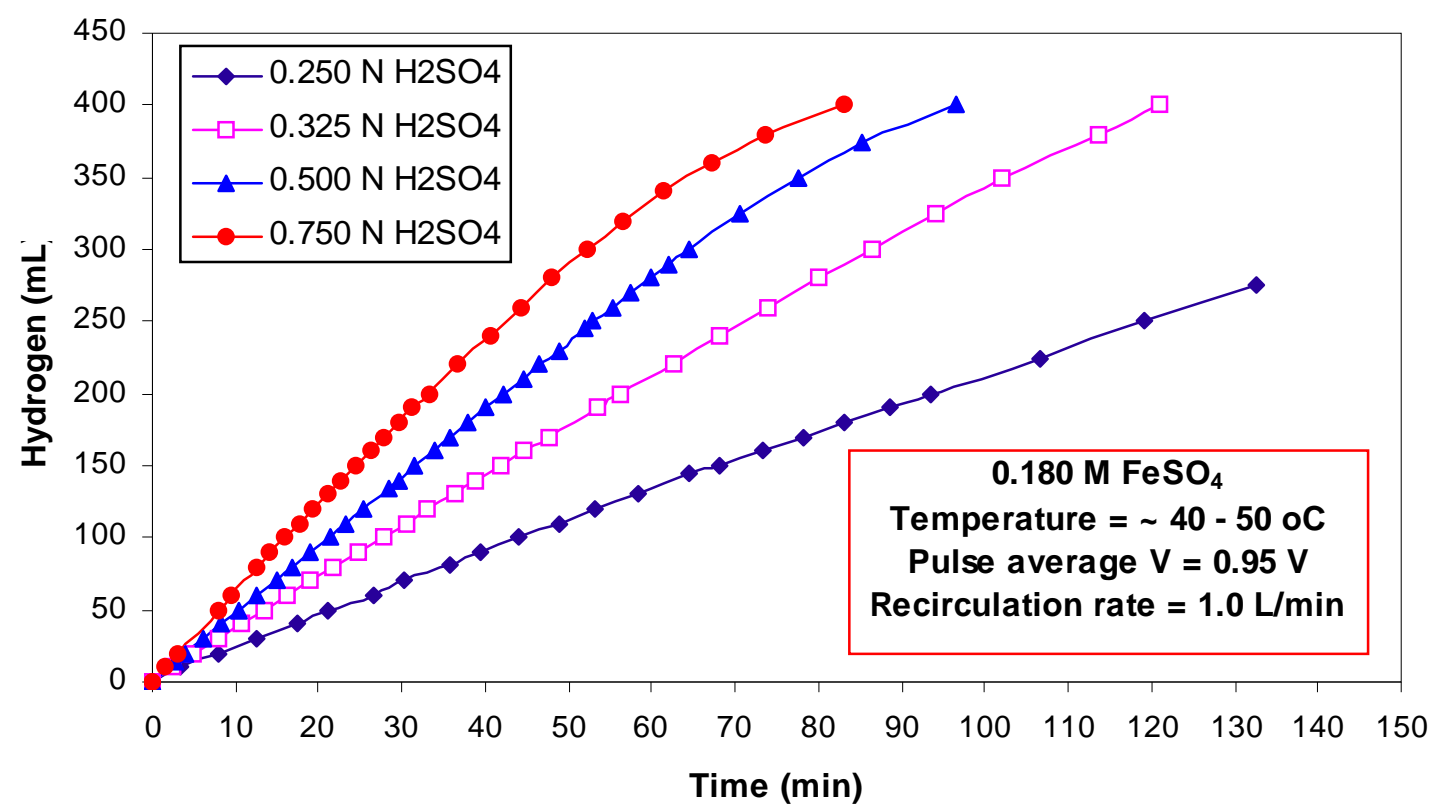

Figure 24. Hydrogen evolution curves for electrolysis of $\mathrm{FeSO}_{4}$ at various $\mathrm{H}_{2} \mathrm{SO}_{4}$ concentrations.

It was observed that each successive increase in acidity resulted in an increase in the rate of $\mathrm{H}_{2}$ evolution from the electrolyzer cathode. Operating the cell at a voltage of $0.95 \mathrm{~V}$ enabled a voltage efficiency of $>70 \%$. The measured $\mathrm{H}_{2}$ evolution correlated with the integrated current within a few percent, indicating high coulombic efficiency. Evidence of some Fe(II) and Fe(III) crossover through the MEA (membrane electrode assembly) was observed, but did not appear to overly compromise electrolytic operation. Temperature enhanced the $\mathrm{H}_{2}$ production rate significantly. The data shown above were obtained at $40-50^{\circ} \mathrm{C}$ temperature range.

Raising $\mathrm{FeSO}_{4}$ concentration also increased the hydrogen evolution rate, although there was a limiting value (>0.2 M) above which the rate began to fall back. This trend is shown in the Figure 25 below. The decay at higher concentration was attributed to polysulfate formation, i.e., aggregation of the iron sulfate species, for which the kinetics of electro-oxidation are much slower than for free ferrous ion. 


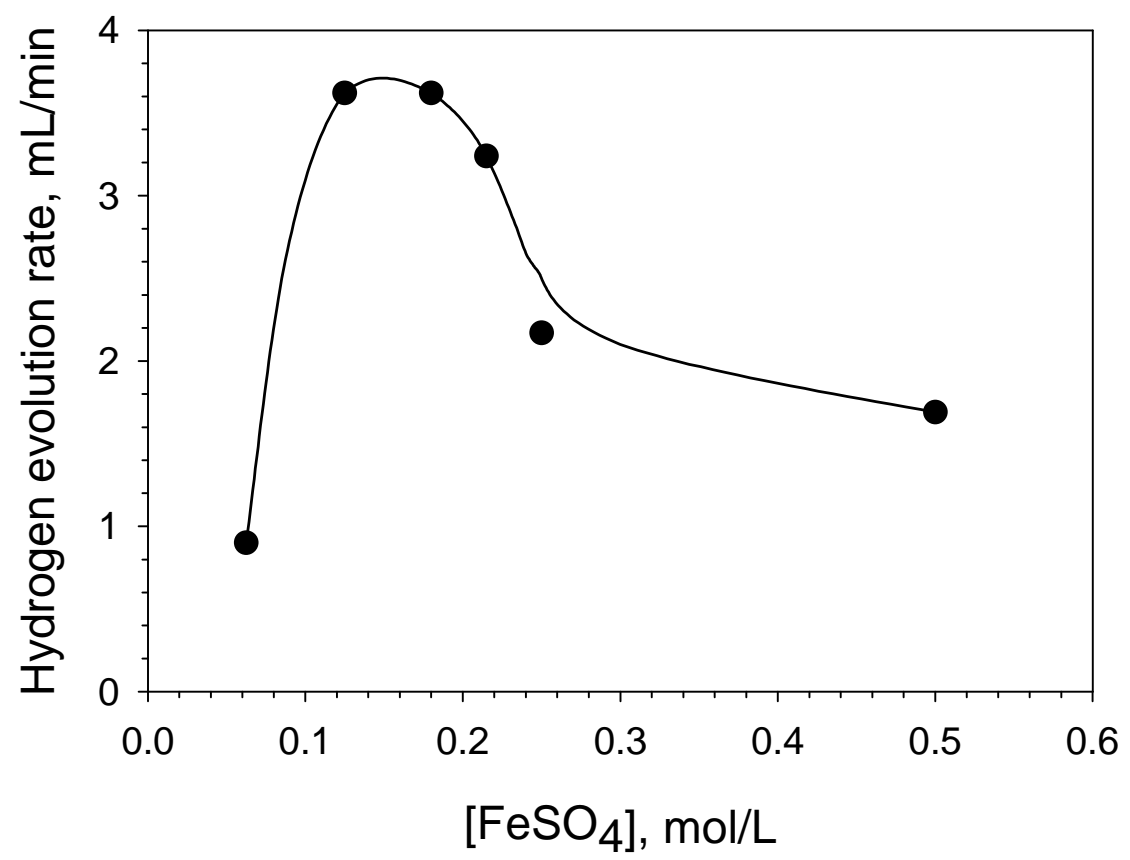

Figure 25. Hydrogen evolution rate as a function of $\mathrm{FeSO}_{4}$ concentration; Pt loading: $1.8 \mathrm{mg} / \mathrm{cm}^{2}, 0.325 \mathrm{~N} \mathrm{H}_{2} \mathrm{SO}_{4}, \mathrm{E}=0.95 \mathrm{~V}$.

Next to determine was the effect of $\mathrm{pH}$ on scrubber efficiency. A 2,500 $\mathrm{ppm} \mathrm{H}_{2} \mathrm{~S} / \mathrm{N}_{2}$ gas stream was bubbled at $300 \mathrm{~mL} / \mathrm{min}$ through a circulating scrubber column containing $2.3 \mathrm{~L}$ of $\mathrm{Fe}_{2}\left(\mathrm{SO}_{4}\right)_{3}$ scrubber solution. The concentration of $\mathrm{H}_{2} \mathrm{SO}_{4}$ was varied so as to produce a $\mathrm{pH}$ range of 0.18 to 1.7 , nearly by order of magnitude. An $\mathrm{H}_{2} \mathrm{~S}$ detector was connected to the scrubber column exit to periodically measure the unreacted $\mathrm{H}_{2} \mathrm{~S}$ concentration. Effluent $\mathrm{H}_{2} \mathrm{~S}$ concentration was measured at various $\mathrm{pH}$ values as a function of time. As long as the scrubber was functioning well, the exit $\mathrm{H}_{2} \mathrm{~S}$ concentration would lie in the single ppm range, but as the scrubber capacity was used up, there would be a sudden jump into the 100's of ppm $\mathrm{H}_{2} \mathrm{~S}$. As shown in Figure 26, the higher the $\mathrm{pH}$, the greater was the scrubber capacity.

The results correlate with equilibrium principles associated with the scrubbing reaction. In the scrubber, two ferric ions oxidize $\mathrm{H}_{2} \mathrm{~S}$ to make sulfur and hydrogen ions:

$2 \mathrm{Fe}^{3+}+\mathrm{H}_{2} \mathrm{~S} \rightarrow 2 \mathrm{Fe}^{2+}+\mathrm{S}^{0}+2 \mathrm{H}^{+}$

Sulfide oxidation results in the creation of solvated hydrogen ions, thus reducing $\mathrm{pH}$. The $\mathrm{H}_{2} \mathrm{SO}_{4}$ added to the scrubber solution already produces an appreciable hydrogen ion background, so that larger amounts of acid tend to limit reaction progress toward the products. The square law dependence of the equilibrium constant on $\mathrm{H}^{+}$concentration implies that an order of magnitude increase in $\left[\mathrm{H}^{+}\right]$ should result in an order of magnitude reduction in ferrous to ferric ratio before breakthrough occurs:

$$
\text { Keq }=\frac{\left[\mathrm{Fe}^{2+}\right]^{2}\left[\mathrm{H}^{+}\right]^{2}}{\left[\mathrm{Fe}^{3+}\right]^{2}\left[\mathrm{H}_{2} \mathrm{~S}\right]}
$$


As a result, a 20-fold increase in hydrogen ion concentration reduced breakthrough time by a similar factor, from 390 minutes to less than 20.

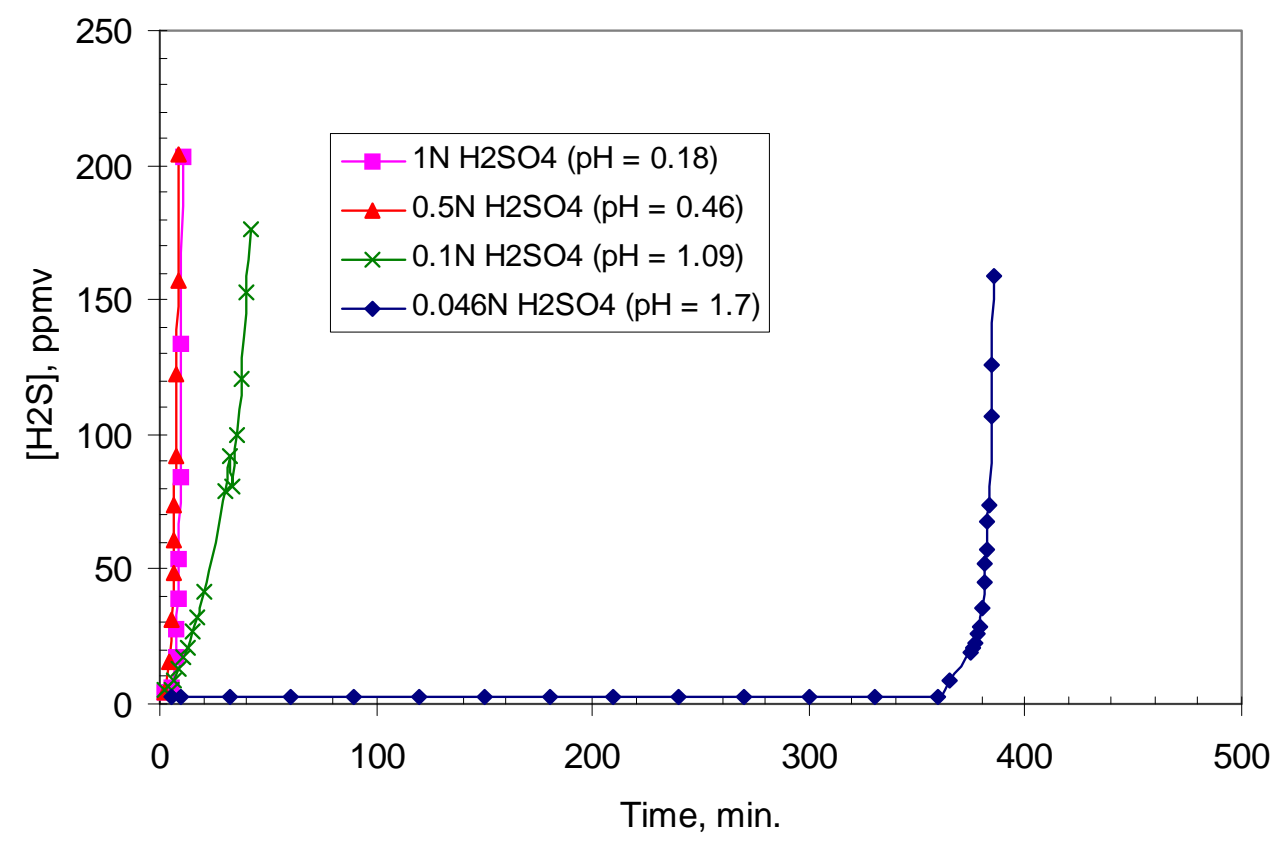

Figure 26. Effect of $\mathrm{pH}$ (acid concentration) on iron scrubber capacity.

Thus optimum conditions for electrolyzer operation are not the same as for scrubber operation. Since the major objective is sulfur removal, our decision was to use a working solution at $\mathrm{pH} 1.7$, where the scrubber operates effectively, and force the electrolyzer to draw enough current under less than optimal conditions to sustain the ferric ion concentration.

\subsection{Electrolytic analogue of Claus Process.}

Alternatively, regeneration of the iron redox couple could be accomplished by reaction with $\mathrm{O}_{2}$ or other oxidizing agent:

$4 \mathrm{Fe}^{2+}(\mathrm{aq})+4 \mathrm{H}^{+}(\mathrm{aq})+\mathrm{O}_{2}(\mathrm{~g}) \rightarrow 4 \mathrm{Fe}^{3+}(\mathrm{aq})+2 \mathrm{H}_{2} \mathrm{O}$

Overall: $2 \mathrm{H}_{2} \mathrm{~S}(\mathrm{~g})+\mathrm{O}_{2}(\mathrm{~g}) \rightarrow 2 \mathrm{H}_{2} \mathrm{O}+2 \mathrm{~S}(\mathrm{~s})$

The overall reaction is the same as the Claus Process before, namely, $\mathrm{H}_{2} \mathrm{~S}$ partial oxidation by $\mathrm{O}_{2}$ to make sulfur and water. While oxidation of $\mathrm{Fe}^{2+}$ by air can be accomplished as shown above, the process is undesirable for our application, as it introduces an oxygen partial pressure into the prereformate.

Figure 27 depicts the schematic diagram of the electrolytic system for indirect oxidation of $\mathrm{H}_{2} \mathrm{~S}$ to elemental sulfur and water (i.e., electrolytic analogue of the Claus process). 


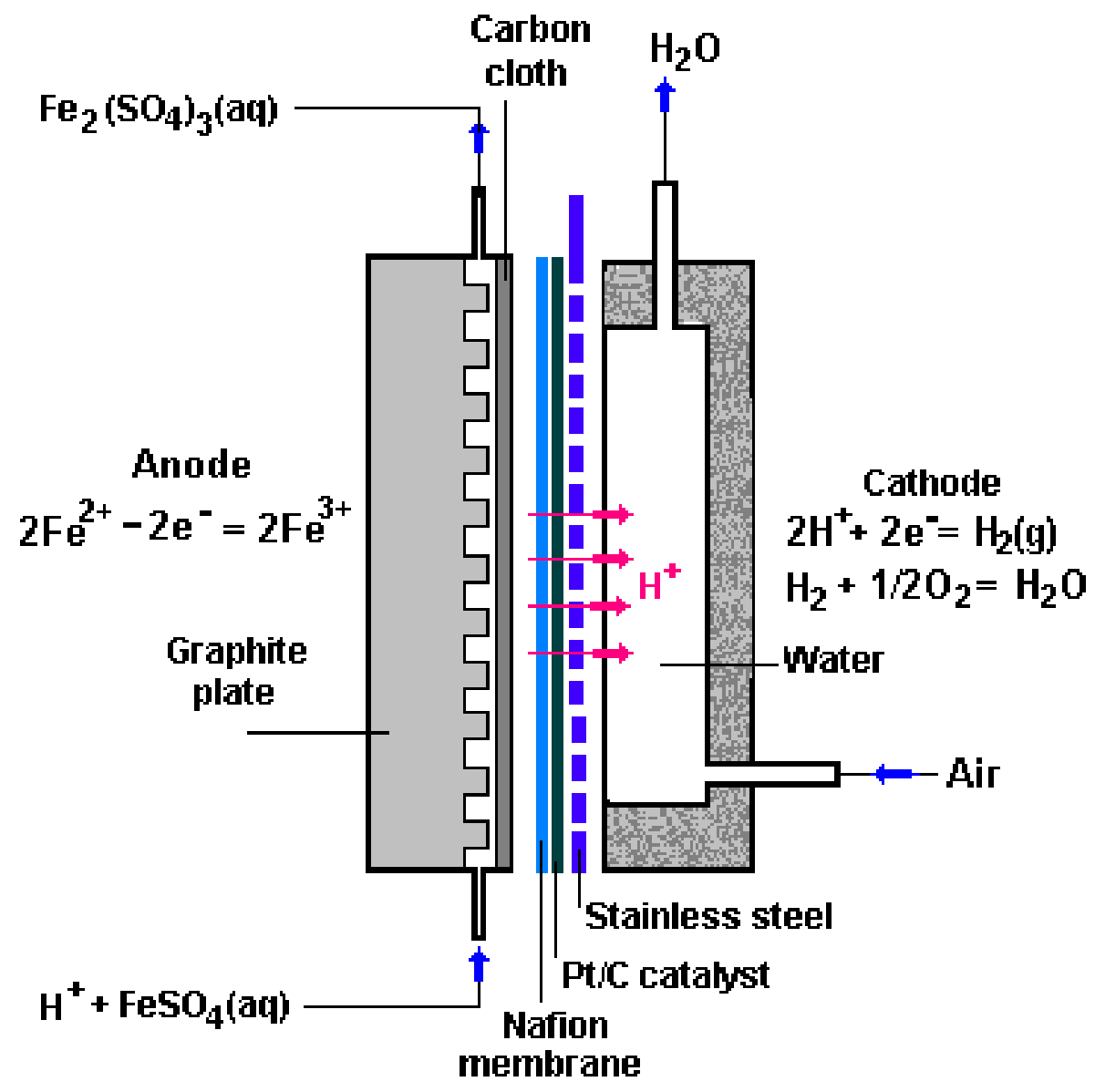

(b) Water generation module

Figure 27. Schematic diagram of the electrolytic system for $\mathrm{H}_{2} \mathrm{~S}$ conversion to elemental sulfur and water using polymer membrane electrolyzer.

Figure 28 compares performance of the electrolytic cell in two regimes: production of hydrogen and water (via reaction 21). One can see that in the case of water production curve, the current steadily increases as voltage of the cell increases reaching the value of $220 \mathrm{~mA}$ at $\mathrm{V}=0.5 \mathrm{~V}$, followed by the gradual decrease in current down to $140 \mathrm{~mA}$. In contrast, the hydrogen production curve shows very low values of current up to the voltage of $0.7 \mathrm{~V}$ after which the current rapidly increases reaching value of $150 \mathrm{~mA}$ at the voltage of about $1.0 \mathrm{~V}$. 


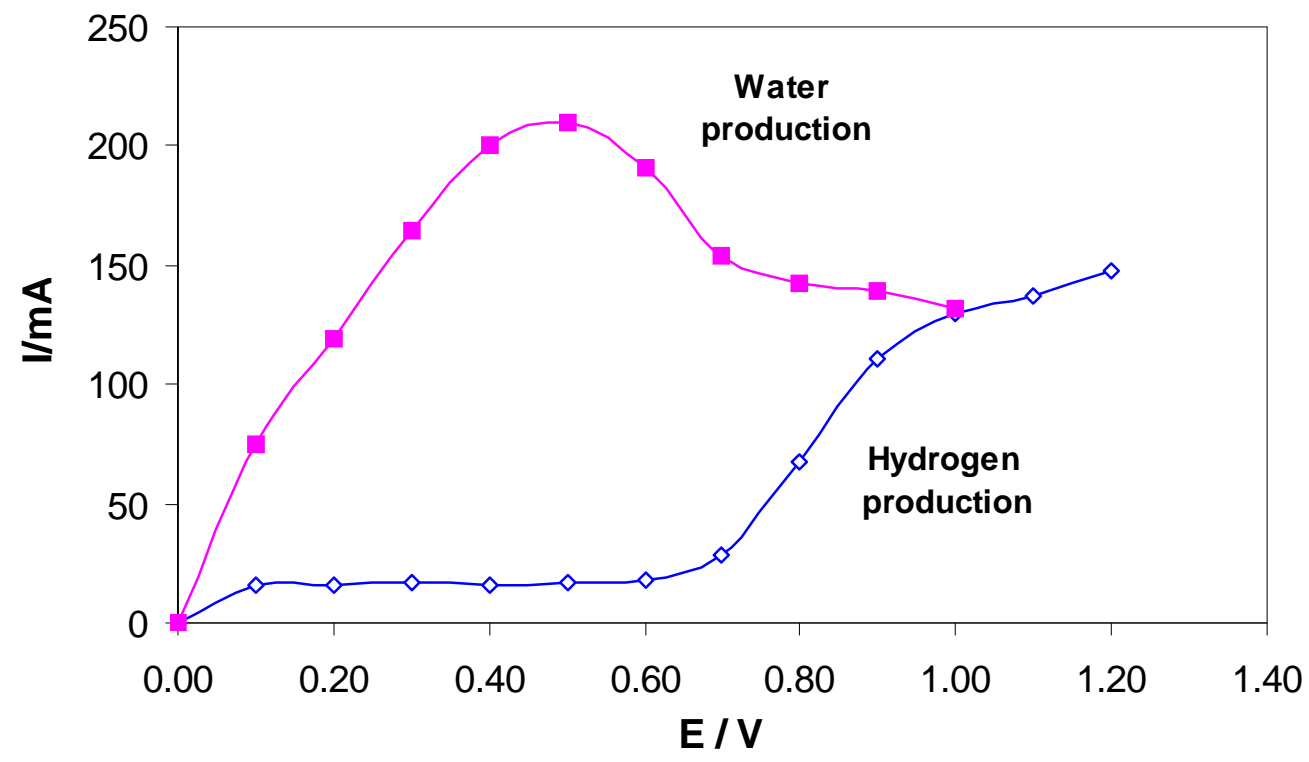

Figure 28. Current-voltage curves for two cases: hydrogen and water production using PEM electrolytic cell.

The comparison of the two options indicates that the $\mathrm{H}_{2} \mathrm{~S}$ oxidation route (with production of water) is more advantageous from the electrochemical performance viewpoint than $\mathrm{H}_{2} \mathrm{~S}$ splitting, but on the other hand it lacks the production of hydrogen as a value-added byproduct.

\subsection{Combined operation of electrolyzer and scrubber}

Having examined operating parameters of electrolyzer and scrubber separately from each other and found overlapping ranges of acceptable function, it was time to try for a combined demonstration, where $\mathrm{H}_{2} \mathrm{~S}$ would be scrubbed, followed by $\mathrm{Fe}(\mathrm{III})$ oxidant regeneration with $\mathrm{H}_{2}$ evolution in the electrolyzer.

As shown in Figure 29. $\mathrm{H}_{2} \mathrm{~S}$ was supplied to the system at a rate of just over $0.5 \mathrm{~mL} / \mathrm{min}$ (total gas flow rate of $200 \mathrm{~mL} / \mathrm{min}$ ). The scrubber solution consisted of a 1:1 ratio of Fe(II) to Fe(III), with $\mathrm{pH}$ adjusted to 1.7 with $\mathrm{H}_{2} \mathrm{SO}_{4}$. The solution was passed through a filter and then into the electrolyzer, containing a carbon cloth anode and dispersed Pt ink cathode affixed to a Nafion ${ }^{\circledR} 112$ membrane. The applied voltage was $1.0 \mathrm{~V}$, enough to draw some $60 \mathrm{~mA}$ at the outset.

Hydrogen evolution was observed from the cathode compartment. The time dependence of $\mathrm{H}_{2}$ evolution is also plotted in Figure 29. The rate of $\mathrm{H}_{2}$ evolution exceeded the rate of $\mathrm{H}_{2} \mathrm{~S}$ influx. In theory they should be equal for steady state operation. The disparity occurs when the electrolysis current is set above or below what is necessary to match the rate of Fe(III) reduction by sulfide in the scrubber. The disparity should eventually disappear, as a prolonged excess or deficit of current would shift the $\mathrm{Fe}(\mathrm{II}) / \mathrm{Fe}(\mathrm{III})$ ratio to match the rate of $\mathrm{H}_{2} \mathrm{~S}$ consumption. Indeed, the $\mathrm{H}_{2}$ evolution curve is seen to be slowly decaying, approaching the $\mathrm{H}_{2} \mathrm{~S}$ input line asymptotically.

The break point, or time at which all the Fe(III) ion would be consumed by the $\mathrm{H}_{2} \mathrm{~S}$ if there was no electrolytic recycling, was calculated to be 15 hrs. The fact that $\mathrm{H}_{2}$ evolution is proceeding at a 
substantial rate past the break point is proof that the system is operating as expected, i.e., we are electrochemically regenerating the iron scrubbing solution and continuously treating the input $\mathrm{H}_{2} \mathrm{~S}$.

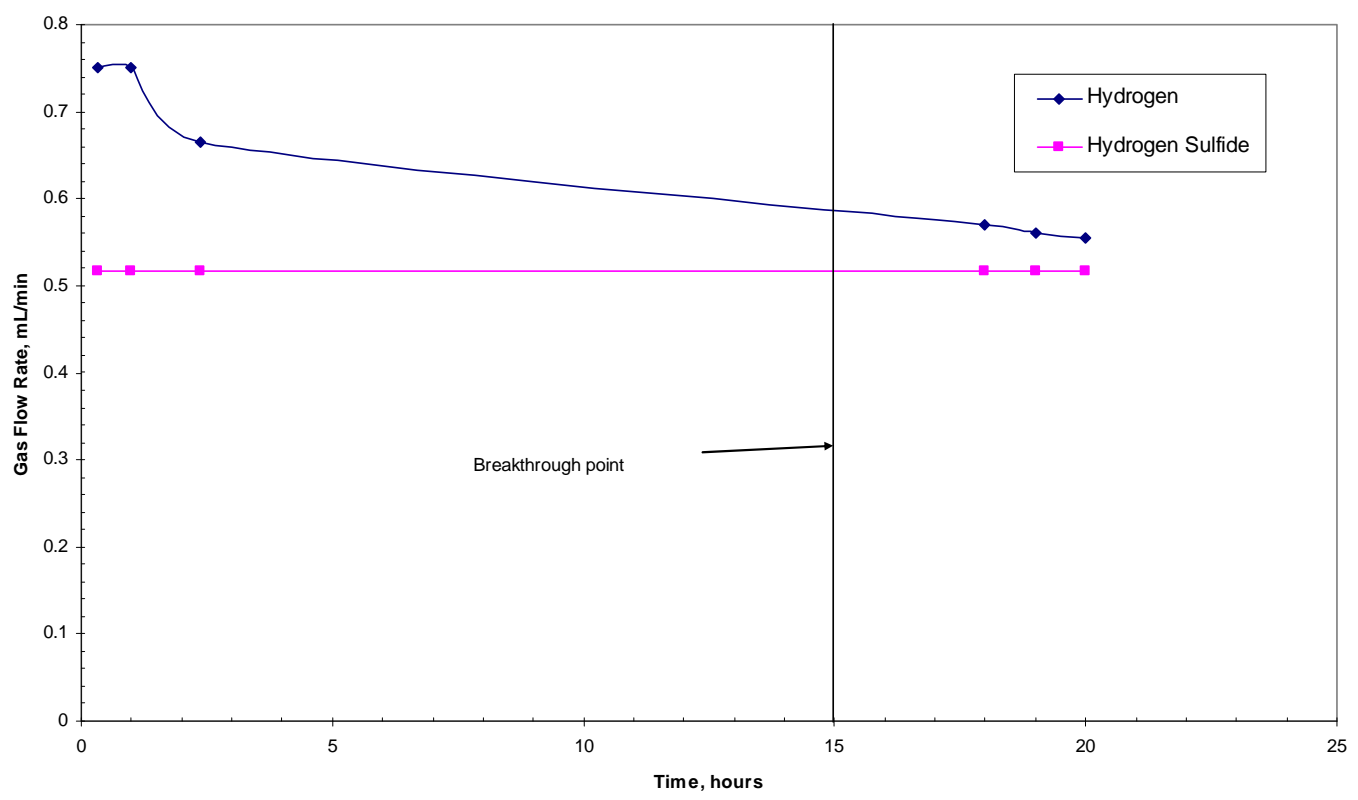

Figure 29. Time dependence of gas uptake and evolution for scrubber/electrolyzer combination.

We have also conducted extended experimental runs to test the stable operation of the scrubberelectrolyzer system. During these experiments, 2,500 $\mathrm{ppm}_{2} \mathrm{~S} / \mathrm{N}_{2}$ gas stream was bubbled at the flow rate of $100 \mathrm{~mL} / \mathrm{min}$ through a scrubber column containing $2.3 \mathrm{~L}$ of iron sulfate scrubber solution. The solution contained $\mathrm{Fe}_{2}\left(\mathrm{SO}_{4}\right)_{3}: \mathrm{FeSO}_{4}$ ratio of $1: 4$ (molar) and $\mathrm{pH}$ of 1.8 adjusted by $5 \mathrm{~N} \mathrm{H}_{2} \mathrm{SO}_{4}$. Voltage applied was in the range of 0.87-1.03 V. The results are presented in Figure 30. The system was continuously operated for 54 hrs.

As in the previous experiment, at the beginning the amount of hydrogen in the effluent gas exceeded that of $\mathrm{H}_{2} \mathrm{~S}$ entering the scrubber, which can be attributed to the excessive amount of $\mathrm{Fe}^{2+}$ in the scrubbing solution. But after several hours there appears a clear trend showing that flowrates of $\mathrm{H}_{2} \mathrm{~S}$ in and $\mathrm{H}_{2}$ out are drawing closer together. Practically no $\mathrm{H}_{2} \mathrm{~S}$ was detected in the effluent gas. This experiment points to a steady state operation of the scrubber coupled with the electrolyzer. 


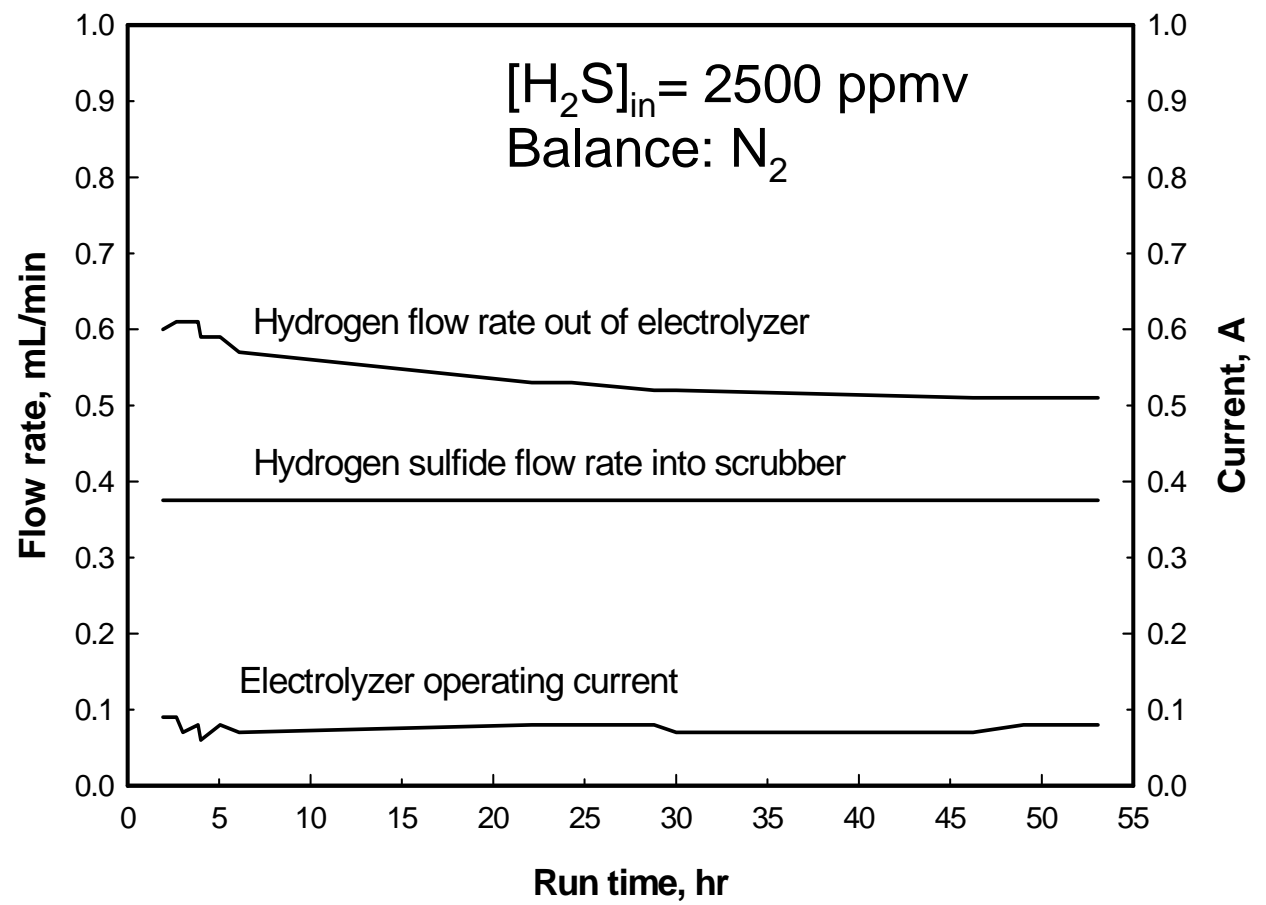

Figure 30. Combined operation of the scrubber and the electrolyzer. Volume of the scrubbing solution: 2.3 L. Potential: 0.87-1.03 V. $\mathrm{pH}=1.8$.

\subsection{Desulfurization of pre-reformer effluent}

The analysis of the pre-reformer effluent produced by the zeolite catalyst revealed that the thiopheneto- $\mathrm{H}_{2} \mathrm{~S}$ conversion yield was very low. On the other hand, the combined zeolite/Ni-Mo catalyst almost quantitatively hydrogenated thiophene to $\mathrm{H}_{2} \mathrm{~S}$ using thiophene-laden hexadecane and diesel feedstocks. The latter pre-reformate gas, containing $\mathrm{H}_{2}, \mathrm{H}_{2} \mathrm{~S}$ and light hydrocarbons $\left(\mathrm{C}_{1}-\mathrm{C}_{6}\right)$, was directed to the desulfurization sub-unit.

Figure 23 (A) depicts a schematic diagram of the desulfurization sub-unit for the continuous removal of hydrogen sulfide from the pre-reformer effluent and its conversion to hydrogen and sulfur. A gaseous mixture from the pre-reformer enters into a scrubbing unit, which includes an absorption column filled with aqueous ferric sulfate solution, $\mathrm{Fe}_{2}\left(\mathrm{SO}_{4}\right)_{3(\mathrm{aq})}$ (total volume of solution $2.3 \mathrm{~L}$ ).

Hydrogen sulfide when dissolved in the aqueous solution is oxidized by ferric ion $\left(\mathrm{Fe}^{3+}\right)$ to elemental sulfur, and simultaneously ferric sulfate is reduced into ferrous sulfate, $\mathrm{FeSO}_{4}$. Elemental sulfur is removed from the solution by a filtration, and the remaining ferrous sulfate solution is fed to an electrolyzer, where ferrous sulfate is oxidized back to ferric sulfate, and protons are reduced to hydrogen gas at the cathode. The regenerated ferric sulfate solution is then fed back to the absorption column and used to scrub more hydrogen sulfide thus, forming a closed cycle with the net reaction being hydrogen sulfide decomposition to elemental sulfur and hydrogen gas.

Electrolysis of acidic $\mathrm{FeSO}_{4}$ aqueous solutions was carried out using a modified proton exchange membrane (PEM) electrolyzer as shown in Figure 23 (B). Platinum catalyst was spray-coated onto the cathode side of a Nafion ${ }^{\circledR}$ film to form a membrane electrode assembly (MEA). The cathode section consisted of a stainless steel plate used as current collector in contact with water where hydrogen evolution takes place. This configuration eliminated the need for a carrier gas to sweep hydrogen from 
the cathode side of the electrolyzer. An electrolyzer potential of 0.80 to 1.03 volts was necessary for the electrochemical process to regenerate the scrubber solution at a rate sufficient to match the $\mathrm{H}_{2} \mathrm{~S}$ flow rate into the scrubber. The electrolytic system can be operated at the range of temperatures from ambient to $90^{\circ} \mathrm{C}$. In our experiments, desulfurization of the pre-reformer effluent occurred optimally at the following process conditions: iron sulfate (total) concentration of $0.1 \mathrm{M}, \mathrm{pH}$ of 1.7-1.8 (adjusted by addition of $5 \mathrm{~N}$ sulfuric acid), and electrolyte temperature of $50^{\circ} \mathrm{C}$.

It should be noted that no Pt catalyst is needed for the oxidation of ferrous ions in the anode side of the electrolyzer. Experimental results shown in Figure 31 imply that oxidation of ferrous to ferric ions is not affected by not having Pt catalyst on the anode. A plain carbon cloth can be used as anode to allow flow of both current and electrolyte. The experimental results demonstrated that concentration of both $\mathrm{H}_{2} \mathrm{SO}_{4}$ and $\mathrm{FeSO}_{4}$ have a significant effect on the electrolytic hydrogen production rate. In particular, it was found that while hydrogen evolution rate increases almost linearly with increase in $\mathrm{H}_{2} \mathrm{SO}_{4}$ concentration (within the range of $\left[\mathrm{H}_{2} \mathrm{SO}_{4}\right]=0.25-0.75 \mathrm{~N}$ ), it is not so for the $\left[\mathrm{FeSO}_{4}\right]$. The hydrogen evolution rate as a function of $\mathrm{FeSO}_{4}$ concentration goes through a maximum at $\left[\mathrm{FeSO}_{4}\right]=0.15 \mathrm{M}$. This is attributed to the generation of polysulfate species at higher $\mathrm{FeSO}_{4}$ concentrations that are less active toward charge transfer at the carbon anode surface.

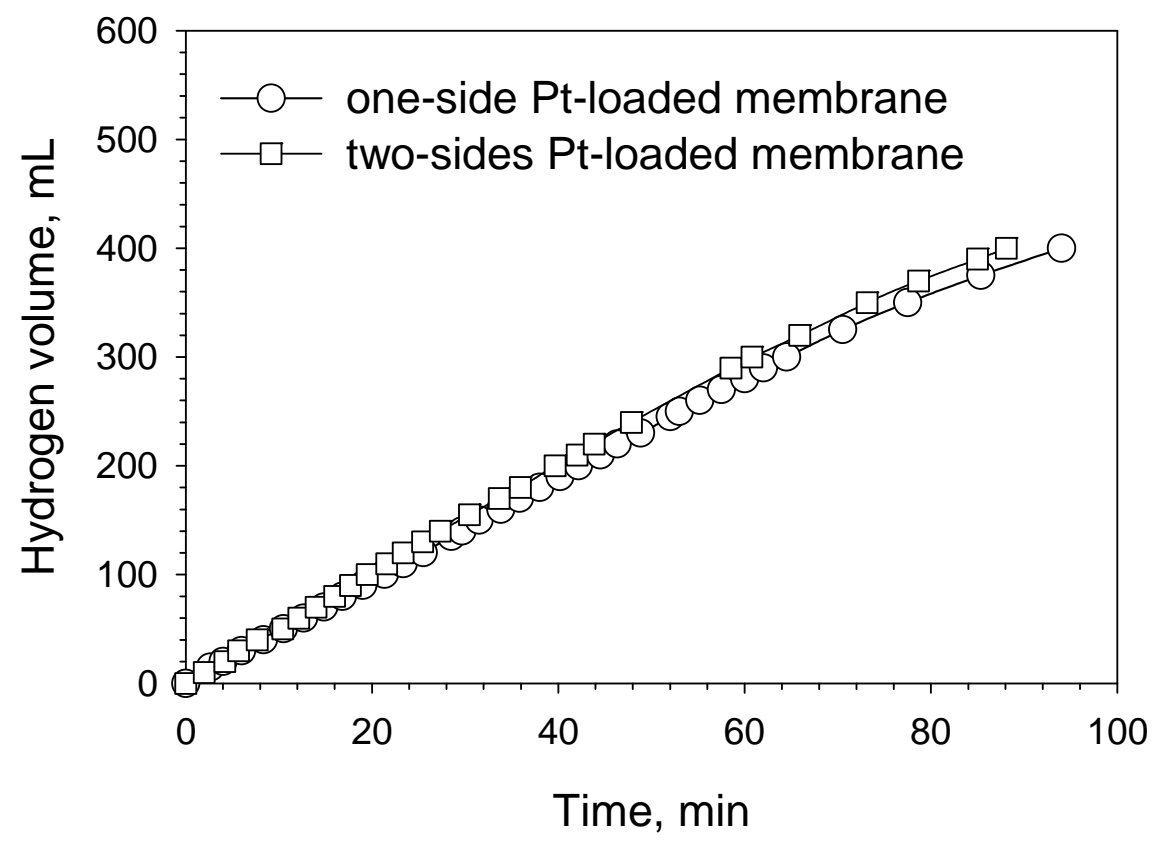

Fig. 31. Electrolysis of acidic $\mathrm{FeSO}_{4}$ solution using one-side and two-sides Pt loaded MEA; Pt loading: $1.8 \mathrm{mg} / \mathrm{cm}^{2}$, current density: $30-50 \mathrm{~mA} / \mathrm{cm}^{2}$, electrolyte: $0.5 \mathrm{~N} \mathrm{H}_{2} \mathrm{SO}_{4}+0.18 \mathrm{M} \mathrm{FeSO}_{4}$, potential $\mathrm{E}=0.95 \mathrm{~V}$.

During initial testing of the combined scrubber-electrolyzer system, the bench-scale desulfurization unit was continuously operated for more than 300 hours using a model $\mathrm{H}_{2} \mathrm{~S}-\mathrm{N}_{2}$ mixture with 2,500 ppmv $\mathrm{H}_{2} \mathrm{~S}$ at the inlet and practically no $\mathrm{H}_{2} \mathrm{~S}$ at the outlet of the scrubber (which corresponded to several turnovers of the $\mathrm{Fe}^{2+} / \mathrm{Fe}^{3+}$-redox system). In the diesel pre-reforming experiment using the zeolite/Ni-Mo catalyst, diesel with 3,180 ppmw of thiophene produced a pre-reformer effluent with $\mathrm{H}_{2} \mathrm{~S}$ content of $185 \mathrm{ppmv}$ (determined by GC-FPD and confirmed by gas-detection tubes). The chromatogram showed the presence of $\mathrm{H}_{2} \mathrm{~S}$ as the only product of hydrogenation of thiophene and no unreacted thiophene or other sulfurous compounds were found (see Figure 32). 


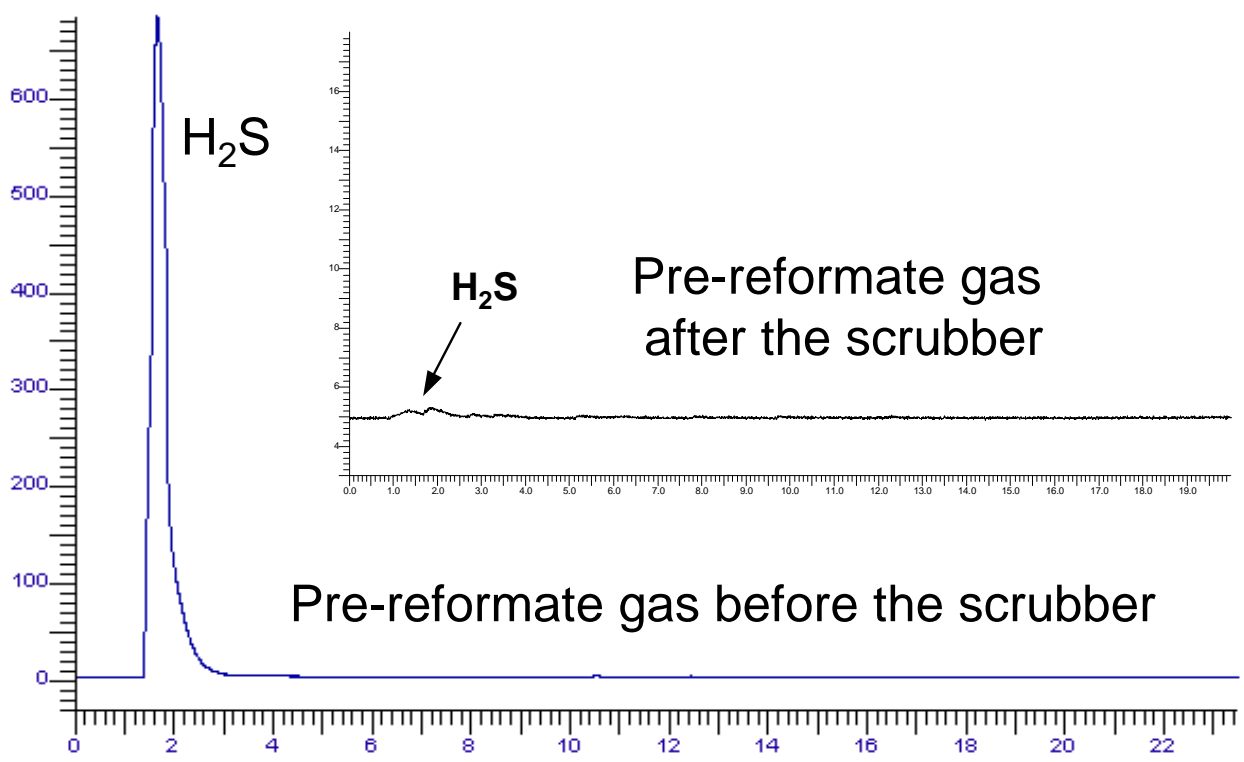

Figure 32. GC-FPD analysis of pre-reformer effluent before and after the scrubber.

On average, about 3 ppmv of $\mathrm{H}_{2} \mathrm{~S}$ was detected exiting the ferric sulfate scrubber during the experiment. A similar experiment using the zeolite-Ni-Mo mixed catalyst and hexadecane containing 5,240 ppmw of thiophene produced largely desulfurized pre-reformate gas with $\mathrm{H}_{2} \mathrm{~S}$ content of less than 5 ppmv. In addition to GC-FPD analysis of $\mathrm{H}_{2} \mathrm{~S}$ in the pre-reformate gas, Sensidyne ${ }^{\mathrm{TM}}$ gas detection tubes were also used for the sulfur analysis (see Figure 33).

Based on the data collected for the high-S diesel and hexadecane pre-reforming-desulfurization tests, the fuel desulfurization yields were found to be in the range of 96-98\% (averaged over a period of several days of operation).

\section{Conclusions}

- A novel process has been developed for converting high-sulfur diesel to a mixture of light molecular weight hydrocarbons that can be readily reformed with steam to fuel cell grade hydrogen gas. The process employs a catalytic pre-reformer coupled with a regenerable $\mathrm{Fe}^{2+} / \mathrm{Fe}^{3+}$ redox/electrolyzer system for the scrubbing of $\mathrm{H}_{2} \mathrm{~S}$ from the pre-reformer effluent and it's conversion to hydrogen gas and elemental sulfur. Electrolysis of acidic $\mathrm{FeSO}_{4}$ aqueous solution was highly efficient with a columbic efficiency of $100 \%$ at a cell voltage of $1.0 \mathrm{~V}$. It was shown that the electrolytic process can be operated with a Pt-free anode to oxidize ferrous to ferric ions, thereby reducing the cost of the electrolytic system, considerably. 


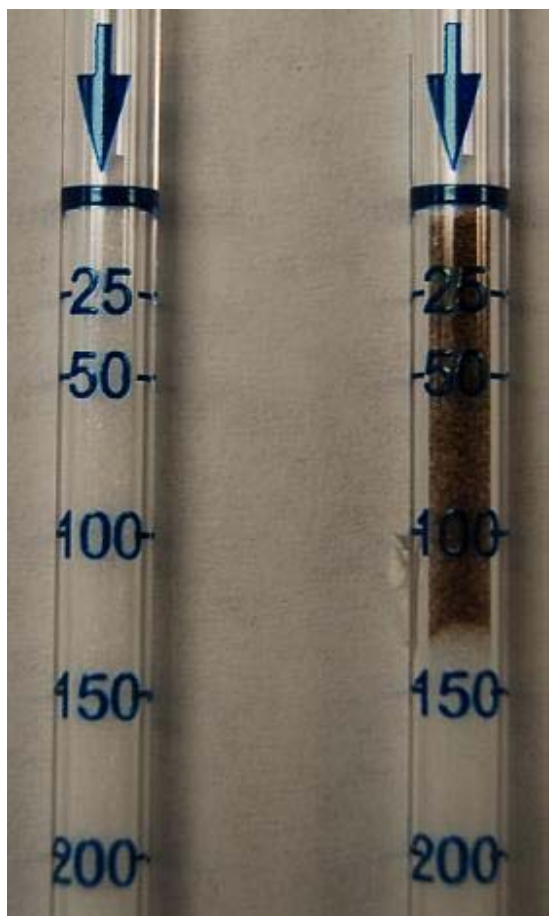

Figure 33. Analysis of $\mathrm{H}_{2} \mathrm{~S}$ in pre-reformer effluent using Sensidyne ${ }^{\mathrm{TM}}$ gas detection tubes.

- A robust bi-functional catalyst was developed for accomplishing the pre-reformation of the high-sulfur fuels (sulfur content as high as $5,240 \mathrm{ppmw}$ ) to short chain hydrocarbons $\left(\mathrm{C}_{1}-\mathrm{C}_{4}\right.$, mostly propane) at an average yield of about $97 \%$.

- $\quad$ The integrated $\mathrm{Fe}^{2+} / \mathrm{Fe}^{3+}$ redox/electrolyzer $\mathrm{H}_{2} \mathrm{~S}$ scrubber and pre-reformer was operated successfully for 100 hrs, continuously, achieving desulfurization efficiencies greater than $95 \%$ and removing sulfur down to less than 5 ppmv in the pre-reformer effluent.

\section{Acknowledgements}

Financial support for this work was provided by the Florida Hydrogen Initiative (FHI) and Chevron Technology Ventures (CTV). Authors thank Haldor Topsoe and CTV for providing the samples of catalysts used in the experiments, and Kirk Scammon (UCF Materials Characterization Facility) for characterization of the catalysts. Authors also wish to acknowledge contributions of Ms. Pam Portwood (FHI), Stephen Adams (FHI) and Dr. David L. Block (FSEC) toward completion of this work.

\section{Special Recognitions \& Awards/Patents Issued}

Huang; C.; Smith; F.; Linkous; C.; Ramasamy; K.; Raissi; Ali T.; Muradov; N., Method and System for Hydrogen Sulfide Removal, U.S. Patent Application No. 20120148465, June 14, 2012.

Huang; C.; Smith; F.; Linkous; C.; Ramasamy; K.; Raissi; Ali T.; Muradov; N., Method and System For Hydrogen Sulfide Removal, U.S. Patent Application No. 20090188164, July 30, 2009 


\section{Publications/Presentations}

Muradov, N.Z., Ramasamy, K.K., Linkous, C.A., Huang, C., Adebiyi, I., Smith, F., T-Raissi, A., Stevens, J. "Combined Pre-Reforming-Desulfurization of High-Sulfur Fuels for Distributed Hydrogen Applications,” J. Fuel 89 (2010) 1221-9.

Muradov, N.Z., Ramasamy, K.K., Huang, C., Adebiyi, I., Smith, F., Linkous, C.A., T-Raissi, A., Stevens, J. "Combined Pre-Reforming-Desulfurization of High-Sulfur Diesel for Hydrogen Fueling Station Applications”, Presented at the 2009 AIChE Spring Meeting, Tampa, Florida (2009).

Muradov, N.Z., Ramasamy, K.K., Huang, C., T-Raissi, A., Stevens, J. “Catalytic Processing of HighSulfur Fuels for Distributed Hydrogen Production”, Paper accepted for presentation at and inclusion in the proceedings of the 18 World Hydrogen Energy Conference, Essen, Germany, May, 2010.

\section{References}

1) C. Thomas, B. James, F. Lomax, I. Kuhn, Fuel options for the fuel cell vehicle: hydrogen, methanol or gasoline, Int. J. Hydrogen Energy 25 (2000) 551-67.

2) A. Avchi, I. Onsan, D. Trimm, On-board fuel conversion for hydrogen fuel cells: comparison of different fuels by computer simulations, Appl. Catal. A: General 216 (2001) 243-56.

3) L. Petterson, R. Westerholm, State of the art of multi-fuel reformers for fuel cell vehicles: problem identification and research needs, Int. J. Hydrogen Energy 26 (2001) 243-64.

4) D. Sopena, A. Melgar, Y. Briceno, R. Navarro, M. Alvarez-Galvan, F. Roza, Diesel fuel processor for hydrogen production for $5 \mathrm{~kW}$ fuel cell application, Int. J. Hydrogen Energy 32 (2007) 1429-36.

5) R. Borup, M. Inbody, T. Semelsberger, J. Tafoya, D. Guidry, Fuel composition effects on transportation fuel cell reforming, Catal. Today 99 (2005) 263-70.

6) D. Liu, T. Kaun, H. Liao, A. Shabir, Characterization of kilowatt-scale autothermal reformer for production of hydrogen from heavy hydrocarbons, Int. J. Hydrogen Energy 29 (2004) 1035-46.

7) T. Fukunaga, H. Katsuno, H. Matsumoto, O. Takahashi, Y. Akai, Development of kerosene fuel processing system for PEFC, Catal. Today 84 (2003) 197-200.

8) A. McCoy, M. Duran, A. Azad, S. Chattopadhyay, M. Abraham, Performance of Sulfur tolerant reforming catalysts for production of hydrogen from jet fuel simulants, Energy \& Fuels, 21 (2007) 3513-9.

9) X. Wang, U. Ozkan, Characterization of active sites over reduced $\mathrm{Ni}-\mathrm{Mo} / \mathrm{Al}_{2} \mathrm{O}_{3}$ catalysts for hydrogenation of linear aldehydes, J. Phys. Chem. B, 109 (2005) 1882-90.

10) F. Liu, S. Xu, L. Cao, Y. Chi, T. Zhang, D. Xue, Comparison of NiMo/ $\mathrm{Al}_{2} \mathrm{O}_{3}$ catalysts prepared by impregnation and co-precipitation methods for hydrodesulfurization of dibenzothiophene, J. Phys. Chem. C 111 (2007) 7396-402. 


\section{Attachment II -- Phase II - Projects Task 7 to 14}

Task 7. Chemochromic Hydrogen Leak Detectors for Safety Monitoring, N. Mohajeri and N. Muradov, Florida Solar Energy Center - April 2010 to December 2012

Task 8. Development of a High Efficiency Low Cost Electrocatalysts for PEM Fuel Cell Applications, M. Rogers, Florida Solar Energy Center - April 2010 to April 2013

Task 9. Understanding Mechanical and Chemical Durability of Fuel Cell Membrane Electrode Assemblies, D. Slattery, Florida Solar Energy Center - April 2010 to June 2012

Task 10. Production of Low Cost Hydrogen from Bio Waste, Robin Parker (HyBrTech ${ }^{\mathrm{TM}}$ ), SRT Group, Inc. - December 2010 to June 2012

Task 11. Development of a Low Cost and High Efficiency 500W Portable PEMFC System, J. Zheng, Florida State University, H. Chen, Bing Energy Inc. - December 2010 to December 2012

Task 12. Development of an Interdisciplinary Hydrogen and Fuel Cell Technology Academic Program, J. Politano, C. Lockyer, M. McCay, and K. Winkelmann, Florida Institute of Technology - December 2010 to March 2013

Task 13. Design and Development of an Advanced Hydrogen Storage System using Novel Materials, E. Stefanakos, D. Goswami, and A. Kumar, University of South Florida - December 2010 to March 2013

Task 14. Advanced HiFoil Bipolar Plates, J. Braun, M. Fuchs, EnerFuel, Inc. - December 2010 to March 2013 


\title{
FLORIDA HYDROGEN INITIATIVE \\ Sub-contrector Finel Report
}

\section{Chemochromic Hydrogen Leak Detectors for Safety Monitoring}

\author{
Dr. Nahid Mohajeri \\ Dr. Nazim Muradov \\ Florida Solar Energy Center \\ Advanced Energy Research Division \\ E-mail: nmohajeri@,fsec.ucf.edu \\ (321) 638-1525
}

\author{
Submitted to: \\ David L. Block \\ Florida Solar Energy Center/University of Central Florida \\ 1679 Clearlake Road \\ Cocoa, FL 32922 \\ E-mail: block@,fsec.ucf.edu
}

Contract Number: DE-FC36-04GO14225

FSEC Project No.: 20126066

Project Start Date: April 8, 2010

Project End Date: December 31, 2012

February 2013

\section{Disclaimer}

The Florida Solar Energy Center/University of Central Florida nor any agency thereof, nor any of their employees, makes any warranty, express or implied, or assumes any legal liability or responsibility for the accuracy, completeness, or usefulness of any information, apparatus, product, or process disclosed, or represents that its use would not infringe privately owned rights. Reference herein to any specific commercial product, process, or service by trade name, trademark, manufacturer, or otherwise does not necessarily constitute or imply its endorsement, recommendation, or favoring by the Florida Solar Energy Center/University of Central Florida or any agency thereof. The views and opinions of authors expressed herein do not necessarily state or reflect those of the Florida Solar Energy Center/University of Central Florida or any agency thereof. 


\title{
Chemochromic Hydrogen Leak Detectors for Safety Monitoring
}

\author{
Florida Solar Energy Center \\ Advanced Energy Research Division
}

\section{Executive Summary}

The ability to detect hydrogen leaks at storage, transmission and usage sites is crucial for its safe handling and use. Hydrogen has high diffusivity that makes it prone to leakage from joints and pipe flanges. Since many hydrogen applications require high $\mathrm{H}_{2}$ gas purity and will not tolerate any additives to the gas, it is of interest to develop methods and materials capable of pinpoint detection of the leak location when it occurs. Presently, the large majority of hydrogen detection research has been focused on the development of electronic sensors. Electronic sensors have several drawbacks including loss of sensitivity in the field due to the environmental effects, costs, and problems associated with power required on-site. Thus, the objective of this project was to develop and demonstrate a cost-effective, high specific chemochromic (visual) hydrogen leak detector for safety monitoring at any facility engaged in handling and use of hydrogen.

The project output is envisioned to lead to a new generation of versatile chemochromic hydrogen detectors that employ "smart" materials that cost less, possess fast discoloration kinetics, are userfriendly, are reliable and have superior field worthiness. The project activities have evaluated the performance (sensitivity) of the sensors at different hydrogen concentrations in air (from 1 to 100 vol\%) and have determined the extent and found no interference with other reducing gases $\left(\mathrm{CO}, \mathrm{NH}_{3}, \mathrm{CH}_{4}, \mathrm{H}_{2} \mathrm{~S}\right)$ at ambient temperatures.

The project research and development activities evaluated two classes of chemochromic hydrogen sensors, one that is irreversible and one that is reversible. The irreversible sensor permanently changes color when exposed to hydrogen and the reversible sensor is one that changes color when exposed to hydrogen, but then after the hydrogen exposure has stopped, it changes back to its original color. The project results that follow are presented for the two cases.

\section{Irreversible Chemochromic Hydrogen Leak Detector - PI: Dr. Nahid Mohajeri, FSEC}

The objectives of the irreversible program was to research, develop, and demonstrate a new generation of versatile chemochromic hydrogen detectors that employ "smart" materials and are fixed to a tape material which is placed on the exposure surface. Table 1 lists the four goals of this research project and summarizes the accomplishments for each goal.

Table 1- Goals vs. Accomplishments

\begin{tabular}{|l|l|}
\hline \multicolumn{1}{|c|}{ Goals } & \multicolumn{1}{c|}{ Accomplishment } \\
\hline $\begin{array}{l}\text { To identify a noble or non-noble metal } \\
\text { dopant to increase the kinetics of coloration } \\
\text { of the irreversible chemochromic sensor in } \\
\text { presence of } \mathrm{H}_{2}\end{array}$ & $\begin{array}{l}\text { Platinum was identified as the dopant. A } \\
\text { sonication technique was developed for } \\
\text { doping purposes. }\end{array}$ \\
\hline $\begin{array}{l}\text { To identify polymer matrices that are } \\
\text { selective toward hydrogen and retard } \mathrm{O}_{2} \\
\text { diffusion and yet simplify the membrane }\end{array}$ & $\begin{array}{l}\text { Dow Corning 734 silicone adhesive was } \\
\text { identified as the pigment encapsulating matrix } \\
\text { of choice. This matrix is fast cure, flowable, }\end{array}$ \\
\hline
\end{tabular}




\begin{tabular}{|l|l|}
\hline casting process and reduce the cost. & and cost effective. \\
\hline $\begin{array}{l}\text { To study the tolerance and selectivity of } \\
\text { irreversible chemochromic sensors toward } \\
\text { other reducing gases. }\end{array}$ & $\begin{array}{l}\text { No color change for encapsulated pigment } \\
\text { when exposed to carbon monoxide gas at } \\
\text { room temperatures for } 1 \mathrm{hr} \text { but bare pigment } \\
\text { discoloration after } 30 \mathrm{~min} \text { of CO exposure. } \\
\text { No sensitivity toward } \mathrm{H}_{2} \mathrm{~S} \text { was observed. }\end{array}$ \\
\hline $\begin{array}{l}\text { To identify the environmental and } \\
\text { operational parameters that might hinder or } \\
\text { reduce the chemochromic sensors' } \\
\text { performance. }\end{array}$ & $\begin{array}{l}\text { Slight UV sensitivity for both titania and } \\
\text { barium sulfate based pigments. } \\
\text { No deterioration when exposed to outdoor } \\
\text { elements such as summer temperatures } \\
\left(\sim 95^{\circ} \mathrm{F}\right), \text { humidity, and rain. }\end{array}$ \\
\hline
\end{tabular}

The first generation of chemochromic hydrogen leak detectors; i.e. sensors, developed at and patented by FSEC, contained titanium dioxide particles doped with PdO encapsulated within a special silicone matrix. The process of coloration is irreversible - i.e. once $\mathrm{PdO}$ pigments were exposed to hydrogen gas, the resulting color change would not revert back to the original color. See Figure 1 below. In the presence of $5 \mathrm{vol} \% \mathrm{H}_{2}$ gas, the color change is discernible within about $5 \mathrm{~min}$. The tape can be used at temperatures as low as $-30{ }^{\circ} \mathrm{C}$ and as high as $50^{\circ} \mathrm{C}$ (higher temperatures have not been tested).

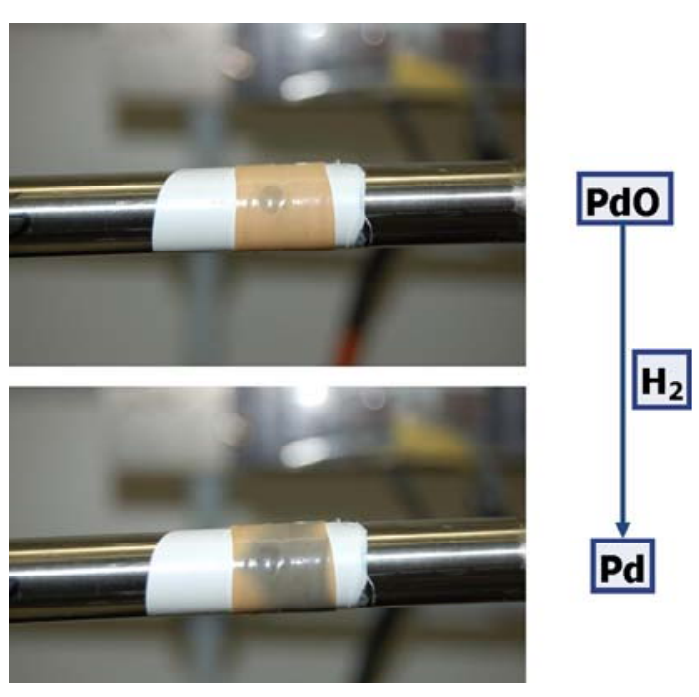

Figure 1. Irreversible Sensor
The focus of the irreversible sensor project was twofold: First, to develop the next generation chemochromic pigments that would have a shorter response time toward hydrogen gas and second, to lower the overall cost of the finished product. Three main approaches were considered to address the two challenges:

1. To study alternative support systems other than $\mathrm{TiO}_{2}$ for $\mathrm{PdO}$ deposition.

2. To dope the pigment with a noble or non-noble metal to improve the reduction kinetics of $\mathrm{PdO}$ particles.

3. To study alternative matrices for pigment encapsulation.

The results were:

1. Alternative support to replace $\mathrm{TiO}_{2}$ for $\mathrm{PdO}$ deposition - One of the most important discoveries of this project was the identification of barium sulfate as a new support for hydrogen chemochromic pigments. This discovery will not only establish this technology as a 100\% US based product, but also will reduce the pigment's cost by $13 \%$. Furthermore, this novel pigment has shown a heightened sensitivity toward hydrogen by reducing the response time by fourfold compared to GEN-I, $\mathrm{TiO}_{2}$ based pigment. Finally, initial environmental testing of the barium sulfate based pigments have shown no effect on the performance when exposed to salt water, fresh water, or humidity. Exposure to sunlight, i.e. UV, has shown minor discoloration similar to $\mathrm{TiO}_{2}$ based pigments.

2. Noble or non-noble metal doping of $\mathrm{PdO} / \mathrm{TiO}_{2}$ pigments to improve the reduction kinetics of PdO particles -- During the course of this project, a new doping technique was developed that has shown to improve the hydrogen response time of the $\mathrm{PdO} / \mathrm{TiO}_{2}$ pigment by eightfold. It is noteworthy to mention that the same doping technique can be used with barium sulfate technology to further enhance the hydrogen sensitivity. Some preliminary results suggest that the Pt doped $\mathrm{PdO} / \mathrm{TiO}_{2}$ pigment may have a great sensitivity toward methane at low temperatures and therefore the doping technique combined with barium sulfate based pigment has the potential for being used as 
methane sensor as well.

3. Alternative matrices for pigment encapsulation - In pursuit of improving the membrane casting technique, a new flowable silicone matrix has been identified that is highly comparable to the original military grade RTV-3145 in terms of hydrogen permeability. The new matrix, Dow Corning 734, has not only simplified the casting process but also has reduced the waste of valuable pigment while casting and reduced curing time. Furthermore, the cost of 734 is about one quarter of the cost of RTV-3145.

The results of the irreversible chemochromic hydrogen sensor technology has been shown to have not only a future in space/spaceport, but also, in industrial operations where hydrogen gas is generated; transported and used (e.g. ammonia plants, oil refineries, semiconductor industries, steel mills, emerging hydrogen economy, etc.). Furthermore, in the "Breakout Session: Fuel Producer/Supplier Environment" reported as part of DOE-sponsored safety workshop, "a leak sensor that is to be installed every mile or so (or at every weld/joint) on a long pipeline, then the response time can be slow (perhaps in the 5 min range) but the cost per sensor has to be very low. If it is a leak checker carried by an operator looking for fugitive gases from small leaks, then you want a very rapid response and recovery time (on the order of 1 second), but you can take a bigger hit on the cost (say \$500). For something like an electrolyzer installed in a remote location and possibly producing significant quantities of hydrogen you may want something in between those two cases". We believe that this new chemochromic pigment technology can easily satisfy these established targets by DOE.

The funding of this research activity has also resulted two filed US patent applications. One based on the new pigments (U.S. Application No. 13/588,779; Doped Palladium Containing Oxidation Catalysts) and the other, the application of chemochromic membrane in PEM fuel cells to check for pinhole and crack defects in PEM before or after fuel cell operation (U.S. Application No. 13/594,163; Chemochromic Membranes for Membrane Defect Detection).

\section{Reversible Chemochromic Hydrogen Leak Detector Sensors -- Dr. N. Muradov, FSEC}

The second part of the chemochromic sensor research was the development of cost-effective, highly selective reversible hydrogen visual sensors that are reversible. Reversible sensors mean that the sensor changes color when exposed to hydrogen, but then after the hydrogen exposure has stopped, the sensor changes back to its original color.

The main objectives of the reversible sensor work have been successfully accomplished in that a series of active, highly selective and durable reversible hydrogen sensors have been developed. The reversible chemochromic pigments are based on the oxo-compounds of molybdenum (Mo) and tungsten (W) that have suitable redox potentials for the reduction reaction in the presence of $\mathrm{H}_{2}$ and bleaching reaction in the presence of air. These sensors show very high selectivity toward hydrogen and no interference with other reducing gases such as $\mathrm{CO}, \mathrm{CH}_{4}, \mathrm{NH}_{3}$. They also exhibited resistance to sulfur poisoning.

By changing the chemical composition of the pigments, they can acquire slow (days-to-weeks) or fast (minutes -to-hours) bleaching characteristics. The developed pigment formulations showed excellent performance and sensitivity at the wide range of $\mathrm{H}_{2}$ concentrations in air (from 1 to 100 vol.\%). Although the pigments (especially, Mo-based pigments) demonstrated some UV sensitivity, they performed well indoor. Long-duration environmental testing of the reversible sensors exposed to the atmospheric elements for 5 months showed that $\mathrm{W}$-based pigments had better durability than Mo-based pigments. More work needs to be done to improve UV-resistance of the reversible pigments. 
The results from the reversible sensor work are summarized as follows:

- Synthesized and tested over 70 novel reversible pigment formulations based on compounds of molybdenum (Mo), tungsten (W) and vanadium (V), including polyoxocompounds, salts, oxides, acids. Among tested formulations, oxo-compounds of Mo and W showed fast (seconds-tominutes) and selective response to hydrogen.

- Studied the effect of different variables (amount of pigment, temperature, activators) on the efficiency of Mo and $\mathrm{W}$ based reversible pigments encapsulated in the silicone matrix. Determined optimum amount of reversible pigments in the chemochromic formulations, i.e., pigment to silicone matrix weight ratio. Different types of silicone matrices were tested; among them RTV-3145 and Dow Corning 734. Both silicones demonstrated excellent performance.

- Studied the effect of different supports, such as, $\mathrm{SiO}_{2}$, different brands of $\mathrm{TiO}_{2}, \mathrm{Al}_{2} \mathrm{O}_{3}$ and ceramic on the performance of both Mo- and W-based pigments. $\mathrm{TiO}_{2}$ and $\mathrm{SiO}_{2}$ supports showed acceptable uses.

- Determined the effect of co-catalysts $(\mathrm{Pd}, \mathrm{Pt}, \mathrm{Rh}, \mathrm{Ru})$ on the rate of coloration in presence of hydrogen. Pt co-catalyst showed the best activity and stability. Pt co-catalysts were used in the form of nano-particles deposited on Mo- and W-pigments or $\mathrm{Pt} / \mathrm{SiO}_{2}$ particles mixed with Moand $\mathrm{W}$-pigments. Both options showed comparable performance.

- Evaluated the performance (sensitivity) of the chemochromic sensors at different $\mathrm{H}_{2}$ concentrations in air (from 1 to 100 vol.\%). It was found that both Mo- and W-based pigments performed adequately under low $\mathrm{H}_{2}$ concentrations.

- Determined the extent of interference with other reducing gases $\left(\mathrm{CO}, \mathrm{NH}_{3}, \mathrm{CH}_{4}, \mathrm{H}_{2} \mathrm{~S}\right)$. Found no interference with either of the reducing gases. It was determined that the developed sensors are highly selective toward hydrogen.

- Studied prolonged exposure of Mo- and W-pigment formulations to UV radiation (black light, wavelength $360 \mathrm{~nm}$ ). Materials, particularly, Mo-based pigments showed UV sensitivity. It was found that UV exposure for 3 months caused slow degradation of performance, especially, with Mo-based pigments. The $\mathrm{H}_{2}$ sensors worked without problems indoor when exposed to daylight and fluorescent bulbs.

- Conducted long-duration environmental testing of Mo- and W-based sensors exposed to elements (sunlight, rain, humidity) for 5 months. Mo-based pigments showed the signs of deterioration, whereas, W-based pigments showed acceptable stability and durability.

- Conducted hydrogen uptake and $\mathrm{H}_{2}$-pigment stoichiometry measurements and mechanistic studies of the pigments coloration and bleaching reactions using TCP system.

- Conducted analysis and material characterization studies of reversible pigments using SEM, TEM, TGA, XPS methods. XPS analysis indicated that in presence of $\mathrm{H}_{2} \mathrm{Mo}$-and W-based pigments were reduced from oxidation state +6 to oxidation states +5 and +4 . During bleaching, both Mo and W-based pigments returned back to their original +6 oxidation state. 


\section{Chemochromic Hydrogen Leak Detectors for Safety Monitoring Table of Contents}

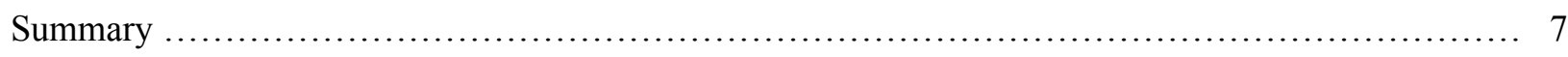

Irreversible Chemochromic Hydrogen Leak Detector (Mohajeri) …............................... 7

Accomplishments/Goals and Objectives Comparison ....................................... 7

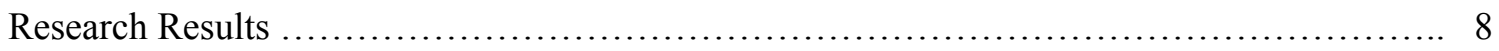

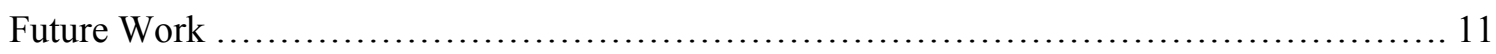

Reversible Chemochromic Hydrogen Leak Detector Sensors (Muradov) …........................... 11

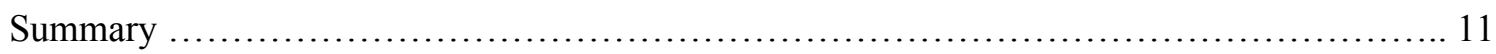

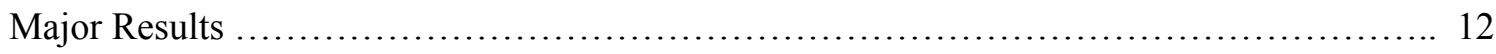

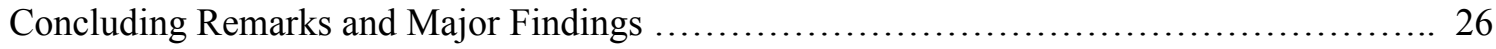

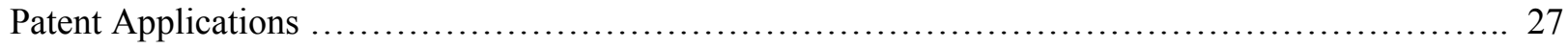

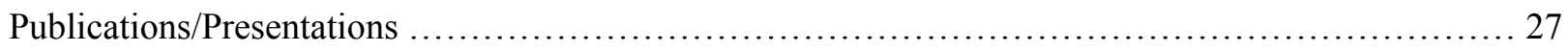




\title{
Chemochromic Hydrogen Leak Detectors for Safety Monitoring
}

\author{
Florida Solar Energy Center \\ Advanced Energy Research Division
}

\section{Summary}

The ability to detect hydrogen leaks at storage, transmission and usage sites is crucial for its safe handling and use. Hydrogen has high diffusivity that makes it prone to leakage from joints and pipe flanges. Since many hydrogen applications require high gas $\mathrm{H}_{2}$ purity and will not tolerate any additives to the gas, it is of interest to develop methods and materials capable of pinpoint detection of the leak location when it occurs. Presently, most hydrogen detection research has been focused on the development of electronic sensors. The objective of this project was to develop and demonstrate a cost-effective, high specific chemochromic (visual) hydrogen leak detector for safety monitoring at any facility engaged in handling and use of hydrogen. The visual sensors use no electrical power. The project results have led to a new generation of versatile chemochromic hydrogen detectors that employ "smart" materials that cost less, possess fast discoloration kinetics, are user-friendly, are reliable and have superior field worthiness. The project research and development activities evaluated two classes of chemochromic hydrogen sensors, one that is irreversible and one that is reversible. The irreversible sensor permanently changes color when exposed to hydrogen and the reversible sensor is one that changes color when exposed to hydrogen, but then after the hydrogen exposure has stopped, it changes back to its original color.

\section{Irreversible Chemochromic Hydrogen Leak Detector -- Dr. Nahid Mohajeri}

The objectives of the irreversible research program was to research, develop, and demonstrate a new generation of versatile chemochromic hydrogen detectors that employ "smart" materials that cost less, possess fast discoloration kinetics, are user-friendly and reliable, and have superior field worthiness.

\section{Accomplishments/Goals and Objectives Comparison}

Table 1 lists the four goals of this research project and summarizes the accomplishments for each goal.

Table 1- Goals vs. Accomplishments

\begin{tabular}{|l|l|}
\hline \multicolumn{1}{|c|}{ Goals } & \multicolumn{1}{c|}{ Accomplishment } \\
\hline $\begin{array}{l}\text { To identify a noble or non-noble metal } \\
\text { dopant to increase the kinetics of coloration } \\
\text { of the irreversible chemochromic sensor in } \\
\text { presence of } \mathrm{H}_{2} .\end{array}$ & $\begin{array}{l}\text { Platinum was identified as the dopant. A } \\
\text { sonication technique was developed for } \\
\text { doping purposes. }\end{array}$ \\
\hline $\begin{array}{l}\text { To identify polymer matrices that are } \\
\text { selective toward hydrogen and retard } \mathrm{O}_{2} \\
\text { diffusion and yet simplify the membrane } \\
\text { casting process and reduce the cost. }\end{array}$ & $\begin{array}{l}\text { Dow Corning 734 silicone adhesive was } \\
\text { identified as the pigment encapsulating matrix } \\
\text { of choice. This matrix is fast cure, flowable, } \\
\text { and cost effective. }\end{array}$ \\
\hline $\begin{array}{l}\text { To study the tolerance and selectivity of } \\
\text { irreversible chemochromic sensors toward } \\
\text { other reducing gases. }\end{array}$ & $\begin{array}{l}\text { No color change for encapsulated pigment } \\
\text { when exposed to carbon monoxide gas at } \\
\text { room temperatures for 1hr but bare pigment } \\
\text { discoloration after 30 min of CO exposure. } \\
\text { No sensitivity toward } \mathrm{H}_{2} \mathrm{~S} \text { was observed. }\end{array}$ \\
\hline $\begin{array}{l}\text { To identify the environmental and } \\
\text { operational parameters that might hinder or } \\
\text { reduce the chemochromic sensors' } \\
\text { performance. }\end{array}$ & $\begin{array}{l}\text { Slight UV sensitivity for both titania and } \\
\text { barium sulfate based pigments. } \\
\text { No deterioration when exposed to outdoor } \\
\text { elements such as summer temperatures }\end{array}$ \\
\hline
\end{tabular}


$\left(\sim 95^{\circ} \mathrm{F}\right)$, humidity, and rain.

\section{Research Results}

The focus of this research project was twofold: First, to develop the next generation chemochromic pigments that would have a shorter response time toward hydrogen gas and second, to lower the overall cost of the finished product. Three main approaches were considered to address the above challenges. The results follow.

1. Alternative support to replace $\mathrm{TiO}_{2}$ for $\mathrm{PdO}$ deposition - Barium sulfate, $\mathrm{BaSO}_{4}$, is an inorganic compound with a white crystalline structure that due to its white opaque appearance is used as a component of white pigment for paints. Furthermore, its low surface area has been utilized in catalyst industry as a support. Therefore, it was hypothesized that barium sulfate could be a candidate to replace the titania support used in first generation chemochromic sensors.

Several commercially available $\mathrm{BaSO}_{4}$ powders were acquired and used as a support for $\mathrm{PdO}$ particles. Of all, one was identified as being the best candidate for chemochromic pigment preparation. Figures 1 depicts the extent of color change when the encapsulated pigment in silicone resin was exposed to $100 \%$ hydrogen gas in comparison to first generation (GEN-1) sensor. Not only is the extent of the color change comparable to GEN-1; i.e. $\Delta \mathrm{E}=30$, but also, the $\mathrm{PdO} / \mathrm{BaSO}_{4}$ pigment response time has shown a significant improvement by a factor of 4-5 compared to GEN-1 in order to display a visible color change. Also, for lower concentrations of hydrogen gas ( $1 \%$ and $10 \%$ in nitrogen), the $\mathrm{PdO} / \mathrm{BaSO}_{4}$ pigment has shown a clear advantage over GEN-1 pigment (Figure 2).

In addition to the mentioned advantages, $\mathrm{BaSO}_{4}$ is approximately four times cheaper than rutile titania used as a support in GEN-1 pigments. This lower price for $\mathrm{BaSO}_{4}$ translates to an approximate $13 \%$ overall cost reduction for the chemochromic pigment.

No color change for encapsulated pigment was observed when exposed to carbon monoxide gas at room temperatures for $1 \mathrm{hr}$. However, the bare pigment discolored after $30 \mathrm{~min}$ of $\mathrm{CO}$ exposure. No sensitivity toward $\mathrm{H}_{2} \mathrm{~S}$ was observed.

Field tests were conducted at FSEC's roof top for three weeks. The barium sulfate based chemochromic hydrogen sensor was slightly darker due to UV exposure and their response time showed an increased response to hydrogen gas compared to the non-exposed sensor. When UV was excluded, no deterioration due to humidity or ambient temperatures was observed. 


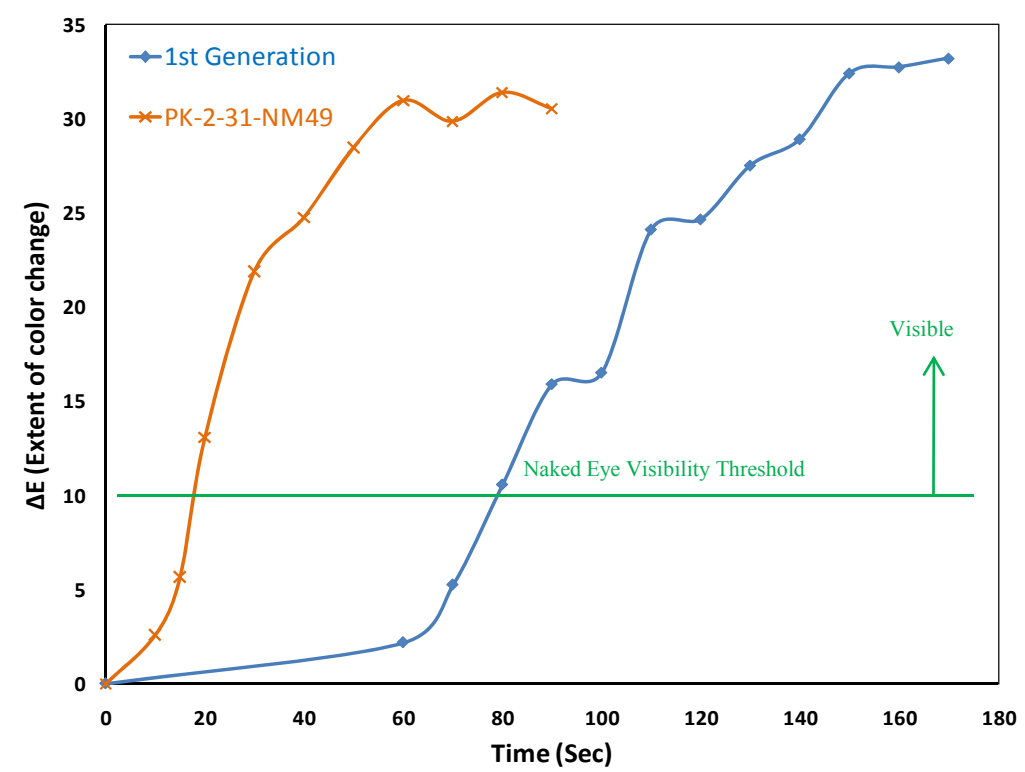

Figure 1- Barium sulfate based pigment vs. GEN-1 when exposed to $100 \% \mathrm{H}_{2}$ gas

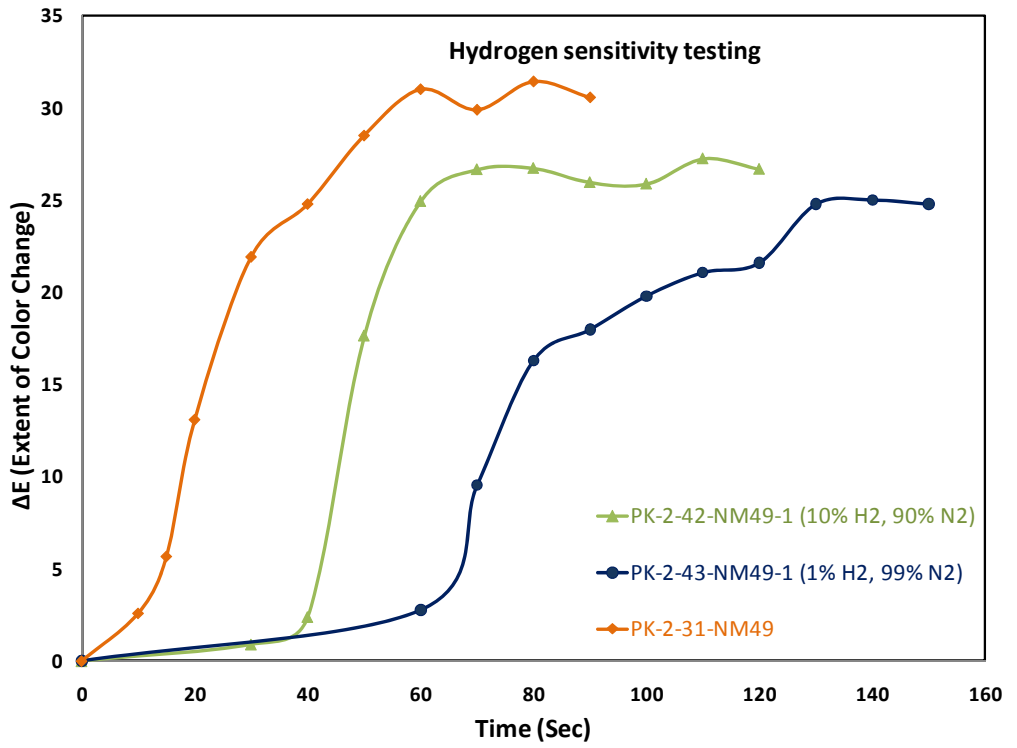

Figure 2-Barium sulfate response profile to $100 \%, 10 \%$, and $1 \%$ hydrogen concentration

2. Noble or non-noble metal doping of $\mathrm{PdO} / \mathrm{TiO}_{2}$ pigments to improve the reduction kinetics of PdO particles - Various metals such as $\mathrm{Ag}, \mathrm{Ru}, \mathrm{Rh}$, and $\mathrm{Pt}$ were selected as dopants for $\mathrm{PdO} / \mathrm{TiO}_{2}$ pigments. Using various doping techniques, it was concluded that a small amount of $\mathrm{Pt}$ added to the $\mathrm{PdO} / \mathrm{TiO}_{2}$ particles, by using an in-house developed sonication technique, can greatly improve the reduction kinetics of $\mathrm{PdO}$ particles. Figure 3 shows the representatives of $0.3 \% \mathrm{Pt}$ doping of $\mathrm{PdO} / \mathrm{TiO}_{2}$ particles using reflux and sonication techniques in various orders. Addition of $0.3 \% \mathrm{Pt}$ to the $\mathrm{PdO} / \mathrm{TiO}_{2}$ particles using a sonication method has been able to reduce the sensor's response time by eightfold compared to GEN-1. However, this addition has shown to have a slight negative impact on maximum color change ( $\Delta \mathrm{E}=25$ for Pt doped pigment vs. $\Delta \mathrm{E}=32$ for GEN-1 pigment). Further optimization resulted in significant reduction of the amount of platinum from $0.3 \%$ to $0.075 \%$. The lower content of platinum was shown to have the same impact on $\mathrm{PdO}$ reduction kinetics and less negative impact on overall maximum color change. 
The superiority of Pt doped pigment over GEN-1 pigment when exposed to $1 \%$ and $10 \%$ hydrogen gas is shown in Figure 4. For example, a visible color change was recorded after only less than 50 seconds of exposure to $1 \%$ hydrogen gas.

Similar to $\mathrm{BaSO}_{4}$ pigments, titania based pigments were field tested for three weeks at FSEC's roof top. This chemochromic hydrogen sensor was slightly darker due to UV exposure and their response time toward hydrogen gas showed an increase compared to the non-exposed sensor. When UV was excluded, no deterioration due to humidity or ambient temperatures was observed.
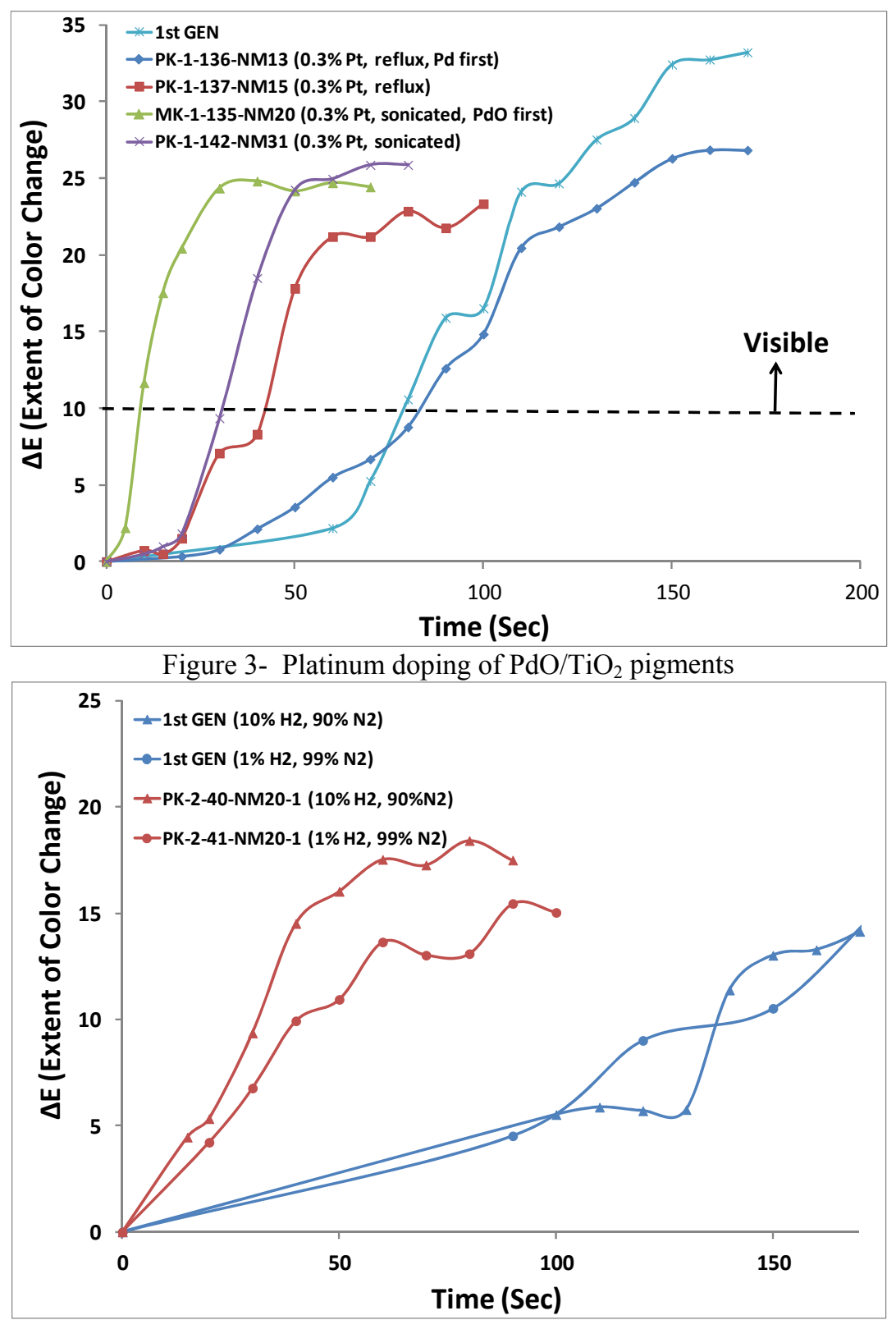

Figure 4-Discoloration of platinum doped $\mathrm{PdO} / \mathrm{TiO}_{2}$ pigments compared to GEN-1 pigments when exposed to $10 \%$ and $1 \% \mathrm{H}_{2}$ gas 
3. Alternative matrices for pigment encapsulation - The Dow Corning RTV-3145 silicone adhesive used for pigment encapsulation of GEN-1 sensor is a highest grade silicone adhesive with great mechanical and durability performance. However its cost ( $\sim 100 /$ cartridge $)$ can greatly impact the abundant use of chemochromic hydrogen sensors. Therefore, finding a comparable but cheaper abundant use encapsulation matrix was highly important. Considering the high permeability of silicone polymers, it was decided to test various cheaper versions of silicone adhesives such as Dow Corning 732, 734, and 737. This study concluded that the flowable Dow Corning 734 has very similar hydrogen permeability characteristics to RTV-3145 but can also greatly enhance the membrane casting process. The cost of Dow Corning 734 is about $\sim \$ 25$ per cartridge and therefore its use can greatly reduce the overall cost of chemochromic sensor.

Two patent applications were filed as a result of this research funding. One based on the new pigments (U.S. Application No. 13/588,779; Doped Palladium Containing Oxidation Catalysts) and the other, the application of chemochromic membrane in PEM fuel cells to check for pinhole and crack defects in PEM before or after fuel cell operation (U.S. Application No. 13/594,163; Chemochromic Membranes for Membrane Defect Detection).

In conclusion, the irreversible sensor work has successfully demonstrated that barium sulfate is a great support candidate for $\mathrm{PdO}$ based chemochromic hydrogen sensor. It was shown that the response time of $\mathrm{PdO} / \mathrm{BaSO}_{4}$ encapsulated pigments to hydrogen is fourfold shorter than $\mathrm{PdO} / \mathrm{TiO}_{2}(\mathrm{GEN}-1)$ pigments . Furthermore, doping the $\mathrm{PdO}$ based pigments with small amounts of platinum, using the novel technique developed under this work, can further enhance the reduction kinetics of $\mathrm{PdO}$ and hence the response time of the sensor. A more cost effective silicone adhesive, Dow Corning 734, was identified for pigment encapsulation. The casting process, due to this matrix flowablility, was shown to be greatly improved and the adhesive also significantly reduced the cure time.

\section{Future Work}

More studies are needed for properly scaling up the pigment preparation procedure and to show their large-scale reproducibility. Further long term field testing is also required to gain a better understanding of sensors stability and performance.

\section{Reversible Chemochromic Hydrogen Leak Detector Sensors -- Dr. Nazim Muradov, FSEC}

\section{Summary}

The second part of the chemochromic sensor research was the development of cost-effective, highly selective hydrogen sensors that are reversible. Reversible sensors mean that the sensor changes color when exposed to hydrogen, but then after the hydrogen exposure has stopped, the sensor changes back to its original color. The main objectives of the reversible sensor work have been successfully accomplished in that a series of active, highly selective and durable reversible hydrogen sensors have been developed. The reversible chemochromic pigments are based on the oxo-compounds of molybdenum (Mo) and tungsten (W) that have suitable redox potentials for the reduction reaction in the presence of $\mathrm{H}_{2}$ and bleaching reaction in the presence of air. These sensors show very high selectivity toward hydrogen and no interference with other reducing gases such as $\mathrm{CO}, \mathrm{CH}_{4}, \mathrm{NH}_{3}$. They also exhibited resistance to sulfur poisoning.

By changing the chemical composition of the pigments, they can acquire slow (days-to-weeks) or fast (minutes-to-hours) bleaching characteristics. The developed pigment formulations showed excellent performance and sensitivity at the wide range of $\mathrm{H}_{2}$ concentrations in air (from 1 to 100 vol.\%). Although the pigments (especially, Mo-based pigments) demonstrated some UV sensitivity, they performed well 
indoor. Long-duration environmental testing of the reversible sensors exposed to the atmospheric elements for 5 months showed that W-based pigments had better durability than Mo- based pigments.

\section{Technical Background and Selection of Materials}

The reversible chemochromic pigments are based on the oxo-compounds of Mo and $\mathrm{W}$ that have suitable redox potentials for the reduction reaction in the presence of $\mathrm{H}_{2}$ and bleaching reaction in the presence of air. The examples of suitable oxo-compounds of Mo and W include polyoxometalates (POM) and oxides. Octahedral moiety with Mo or W at the center and oxygen atoms at the corners is a building block of both POM molecules and oxides.

(see Figure 5).

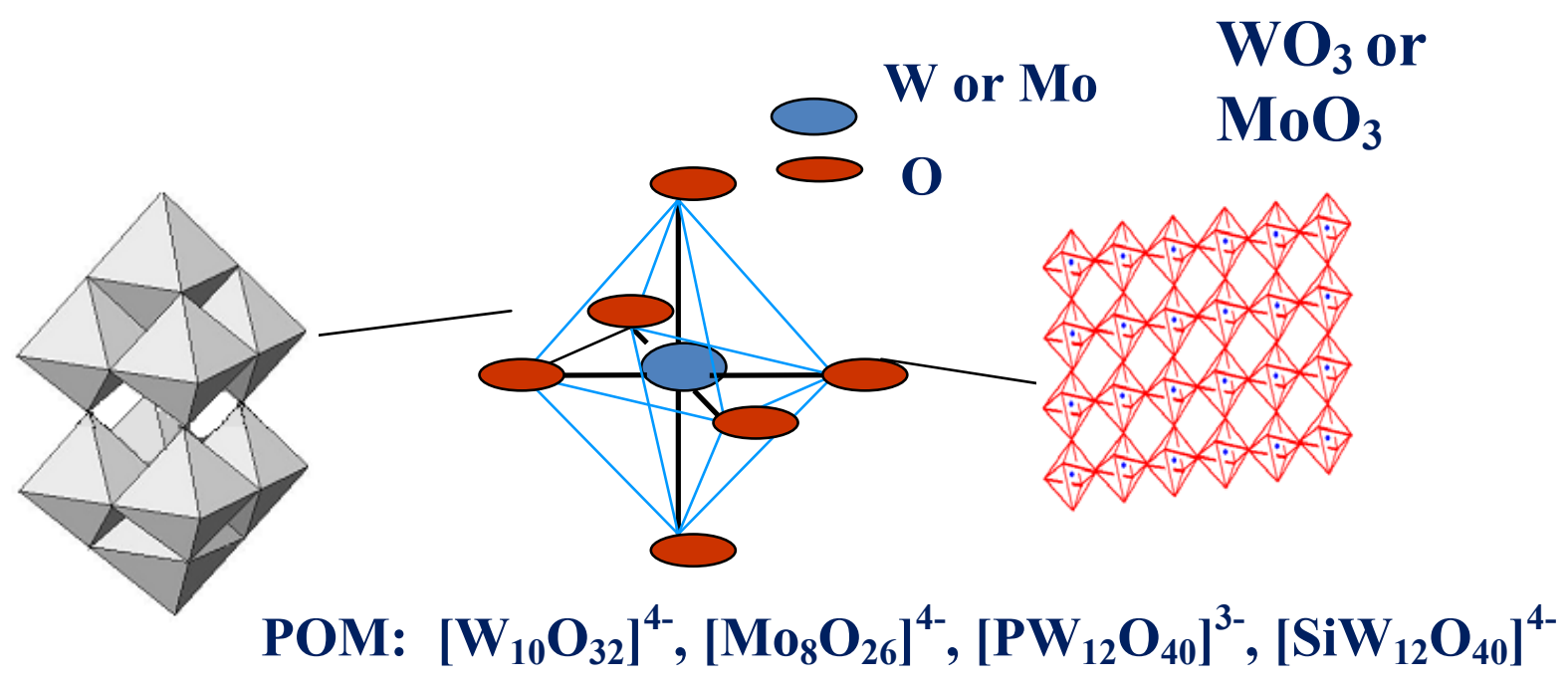

Figure 5. Structure of reversible chemochromic pigments based on oxo-compounds of Mo and W.

In this project, $\mathrm{POM}$ representatives such as silica-tungstic acid $\left(\mathrm{H}_{4} \mathrm{SiW}_{12} \mathrm{O}_{40}\right)$ and phospho-tungstic acid $\mathrm{H}_{3} \mathrm{PW}_{12} \mathrm{O}_{40}$, and oxides of Mo and $\mathrm{W}$ were selected for the testing, because they all possess suitable redox properties in hydrogenation and oxidation reactions. Advantageously, the redox properties of POM can be altered by changing their chemical composition. Oxides of Mo and W were synthesized using precursor compounds such as corresponding ammonia salts, acids, and reagents that were used as received from distributing companies (Fisher, Aldrich).

\section{Major Results}

\section{Reversible Pigments Based on POM Compounds}

The objective of this series of experiments was to performance test POM compounds of Mo and W at hydrogenation and bleaching conditions. Small quantities $(<1 \%)$ of nano-sized Pt particles were added to POM to enhance their coloration kinetics. Figure 6 shows samples of polyoxocompounds of Mo exposed to hydrogen $\left(\mathrm{H}_{2}\right)$ and bleached overnight in air. Figure 7 depicts typical kinetics of coloration and bleaching using Mo-based POM activated with Pt nano-particles. 

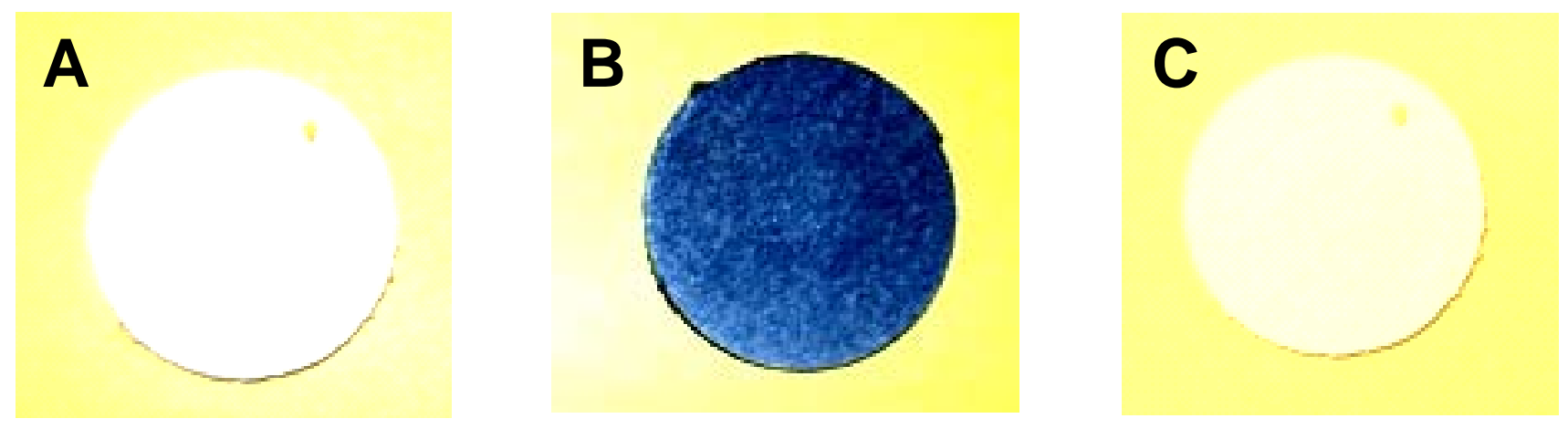

Figure 6. Pigments based on polyoxocompound of Mo. A-original sample, B- the sample exposed to $\mathrm{H}_{2}$, C- sample left overnight in air.
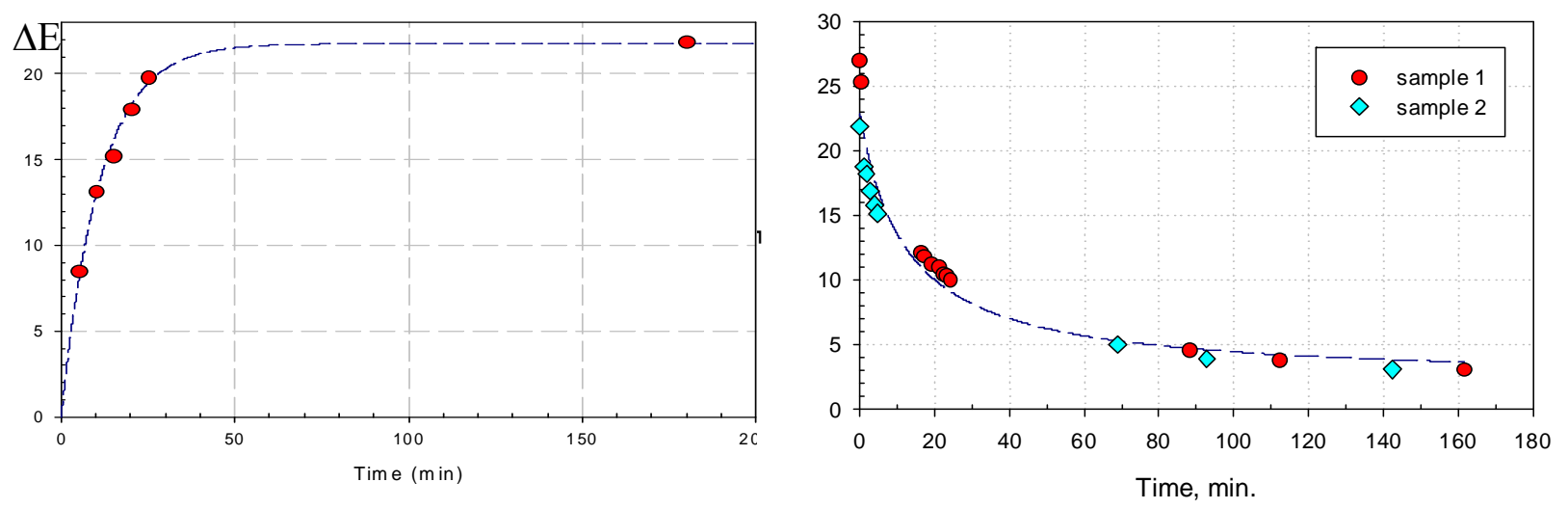

Figure 7. Kinetics of coloration and bleaching of Mo-based polyoxometalate in the presence of $\mathrm{Pt}$ nanoparticles. Figure ordinates are $\Delta \mathrm{E}$ (Extent of Color Change)

In summary, Mo and $\mathrm{W}$ based POM pigments showed excellent kinetics of coloration and bleaching, however, testing showed a very high sensitivity (degradation) following UV radiation and direct sunlight. Therefore, the decision was made to test other oxo-compounds of Mo and W.

\section{Molybdenum-based Slow-bleaching Formulations}

\subsection{Pigments Based on Molybdic Acid (MA) Formulations}

The goal of this series of experiments was to test Pt-activated molybdic acid, $\mathrm{H}_{2} \mathrm{MoO}_{4}$ (MA), as a precursor for reversible pigment formulations. MA-Pt pigments were prepared by baking MA at different temperatures followed by the addition of Pt nanoparticles. Figure 8 shows the color changes and Figure 9 shows the effect of baking temperature on the activity of MA-Pt pigments. 


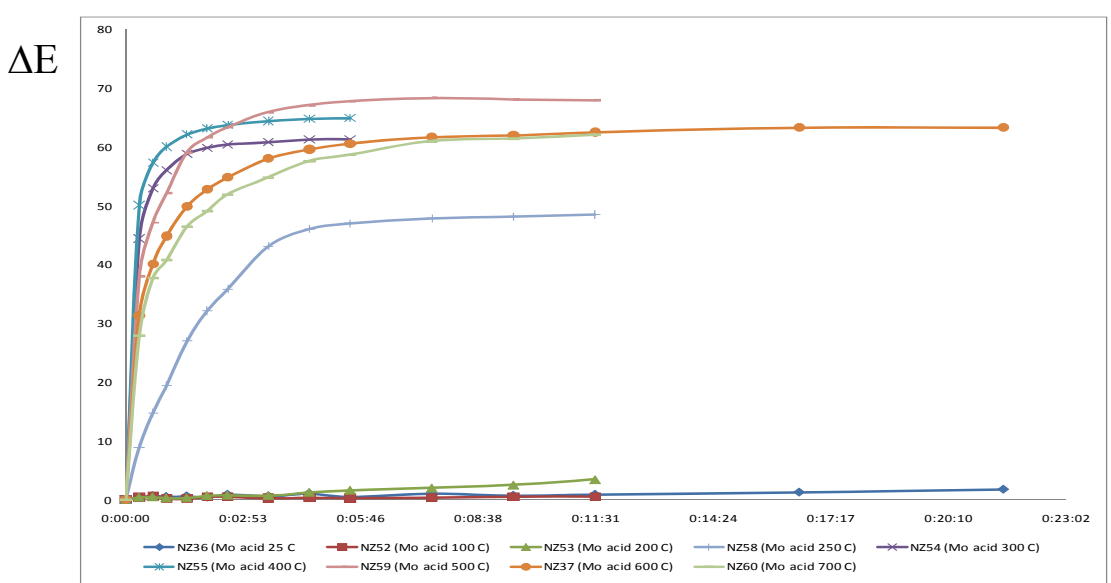

Figure 8. Color Change for MA-Pt pigments at varying baking temperatures. Figure ordinates are $\Delta \mathrm{E}$ (Extent of Color Change) and absicca is time in minutes.

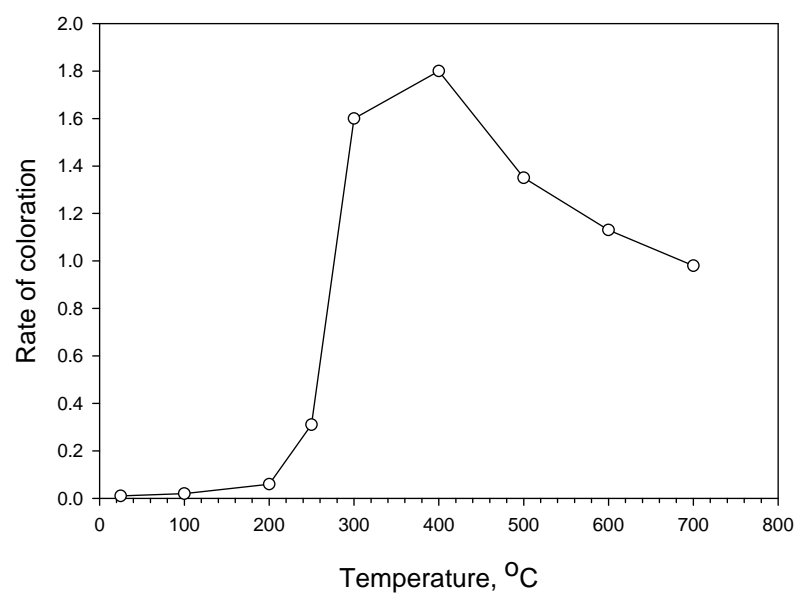

Figure 9. MA-Pt pigment rate of coloration as a function of MA baking temperature.

The results from Figure 9 show that baking MA at $400^{\circ} \mathrm{C}$ provides the fastest kinetics (rate) of coloration. TG/DTA studies of MA were also conducted to determine chemical changes in MA during heating. (see Figure 10). 


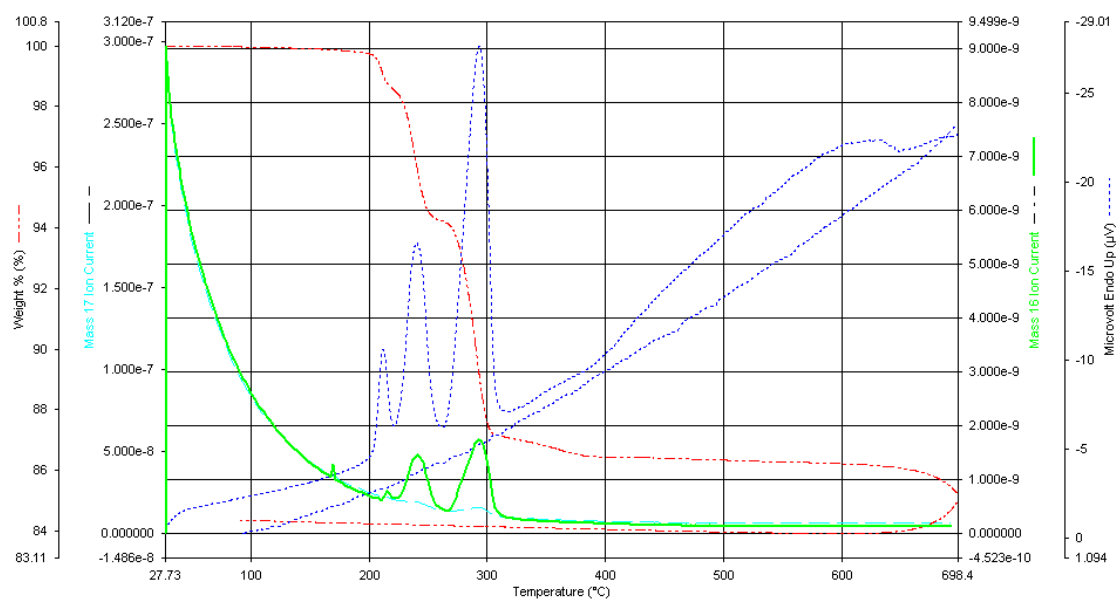

Figure 10. TG/DTA of molybdic acid

TG/DTA data indicated that major structural and chemical changes in MA (most likely, conversion of $\mathrm{MA}$ to $\mathrm{MoO}_{3}$ ) occur at the temperature interval of $250-310^{\circ} \mathrm{C}$. The result is consistent with the data on the efficiency of MA-Pt formulation where the increase in MA-Pt activity occurs in the temperature interval of $250-400^{\circ} \mathrm{C}$.

\subsection{Mo-based Pigments with on Ammonium Molybdate Precursors}

The goal of this series of experiments was to use ammonium molybdate (AM) as a precursor for synthesis of Mo-based pigments. Reversible pigments were synthesized by baking AM at different temperatures, followed by doping them with Pt-nanoparticles. Figure 11 shows the color change and Figure 12 shows the data on the kinetics of coloration process using $\mathrm{AM}$ based formulations baked at the range of temperatures from room temperature to $500^{\circ} \mathrm{C}$ and doped with Pt.

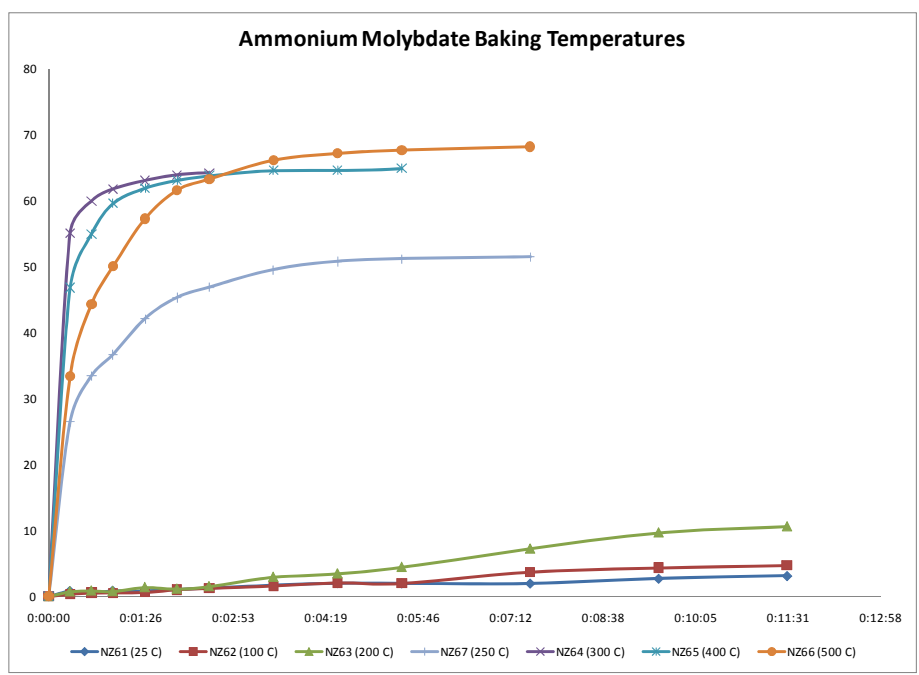

Figure 11. Color change for MA precursor at varying baking temperatures. Figure ordinates are $\Delta \mathrm{E}$ (Extent of Color Change) and absicca is time in minutes. 


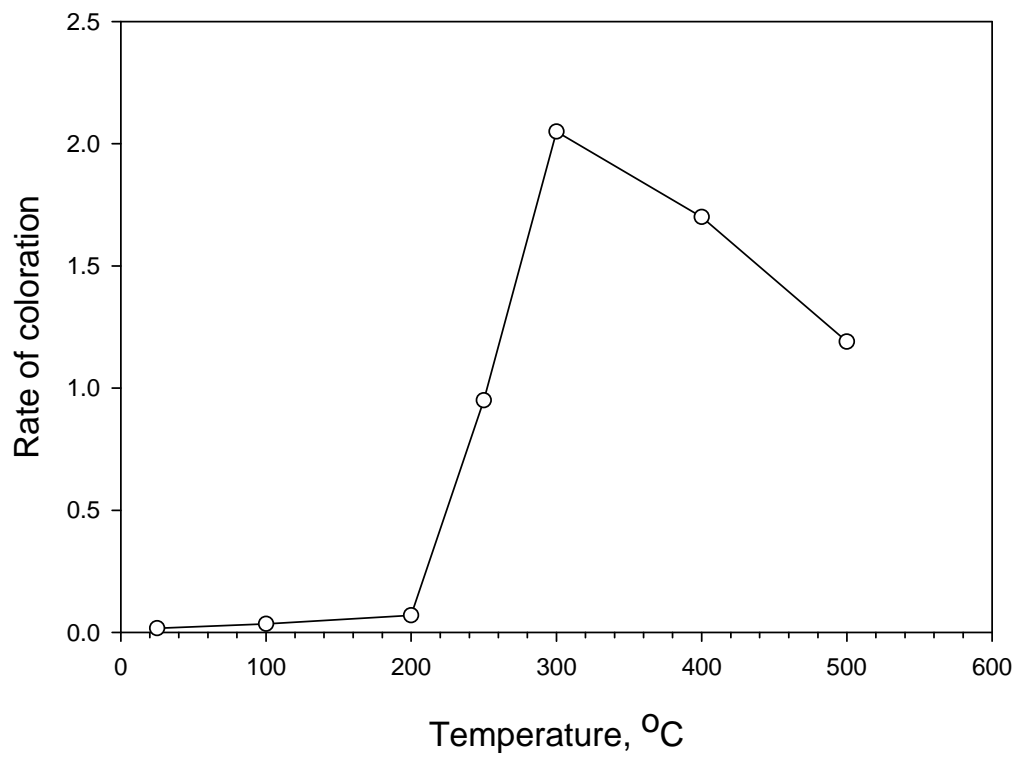

Figure 12. The effect of AM baking temperature on the kinetics of coloration of Mo-based pigments produced from AM and activated with Pt formulations.

The Figure 12 shows that baking AM materials at $300^{\circ} \mathrm{C}$ produces the fastest kinetics of color change. Baking at higher temperatures significantly reduces the efficiency of pigments as does the lower temperature of less than $200^{\circ} \mathrm{C}$. The bleaching test results also indicated that half of the pigment's color intensity was bleached in about 12 days.

TG/DTA studies of thermal decomposition of AM were conducted. The data is shown in Figure 13.

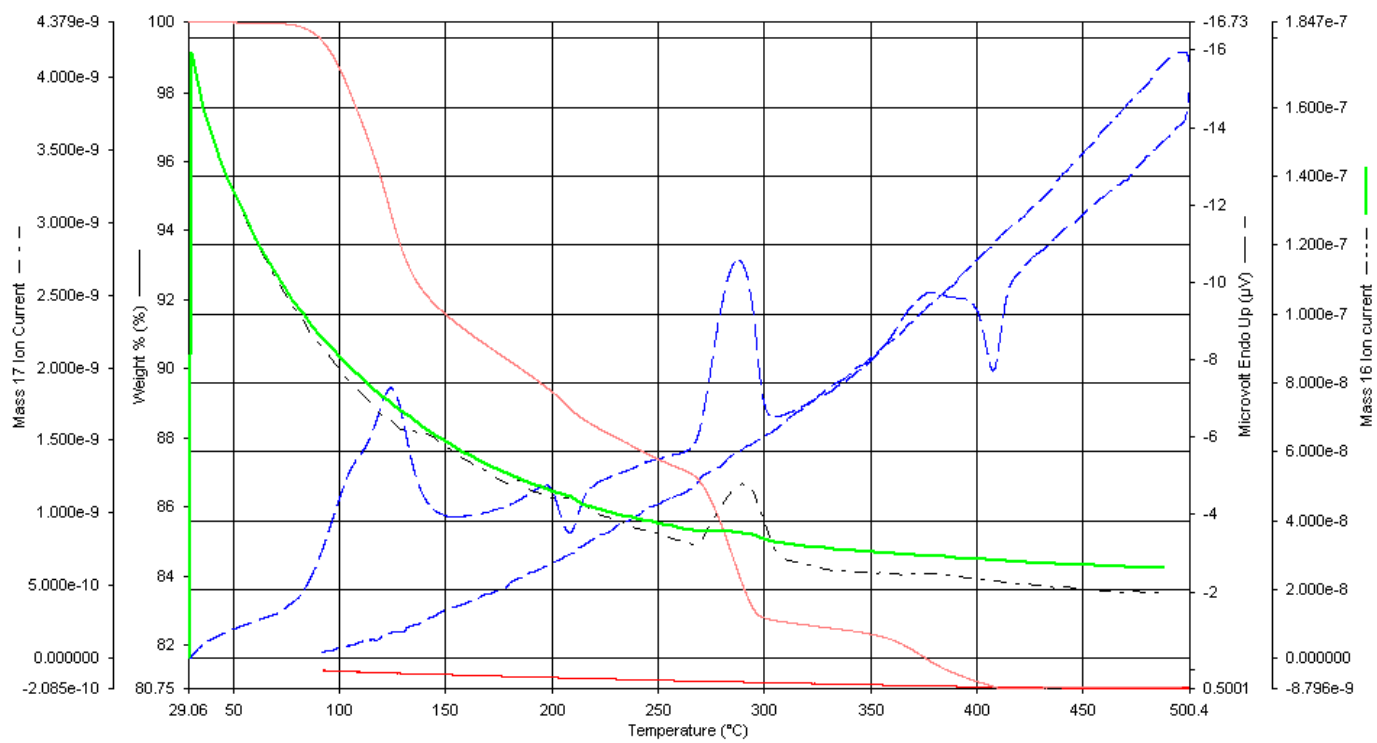

Figure 13. TG/DTA of ammonium molybdate (AM) 
The TG/DTA data show that there are two peaks corresponding to the release of two molecules of $\mathrm{NH}_{3}$ at about $125^{\circ} \mathrm{C}$ and $280^{\circ} \mathrm{C}$. Thus, at about $300^{\circ} \mathrm{C} \mathrm{AM}$ is converted to $\mathrm{MoO}_{3}$, which is consistent with the data presented in Figure 12, where $300^{\circ} \mathrm{C}$ is the optimal temperature for AM-Pt pigment.

\section{Tungsten-based Fast-bleaching Formulations}

The objective of this series of experiments was to develop fast-bleaching reversible formulations based on tungsten compounds, such as ammonium tungstate (AT). A series of pigments were synthesized and tested by baking AT at different temperatures and doping them with Pt. Figures 14 and 15 depict the testing results for AT-Pt based formulations.
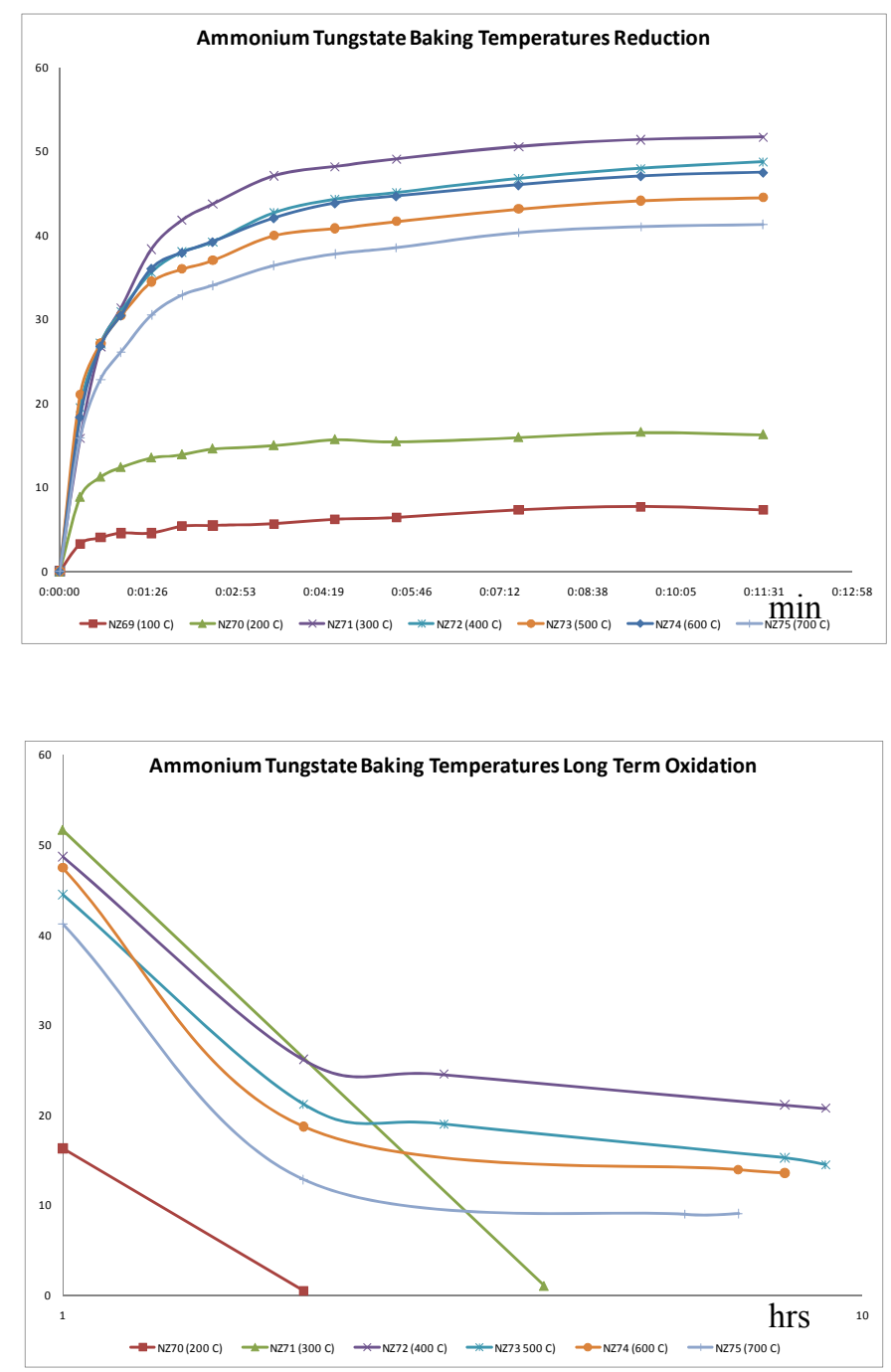

Figure 14. Kinetic curves of coloration (upper) and bleaching (lower) of AT-Pt formulations. Figure ordinates are $\Delta \mathrm{E}$ (Extent of Color Change) and absicca is time in minutes. 


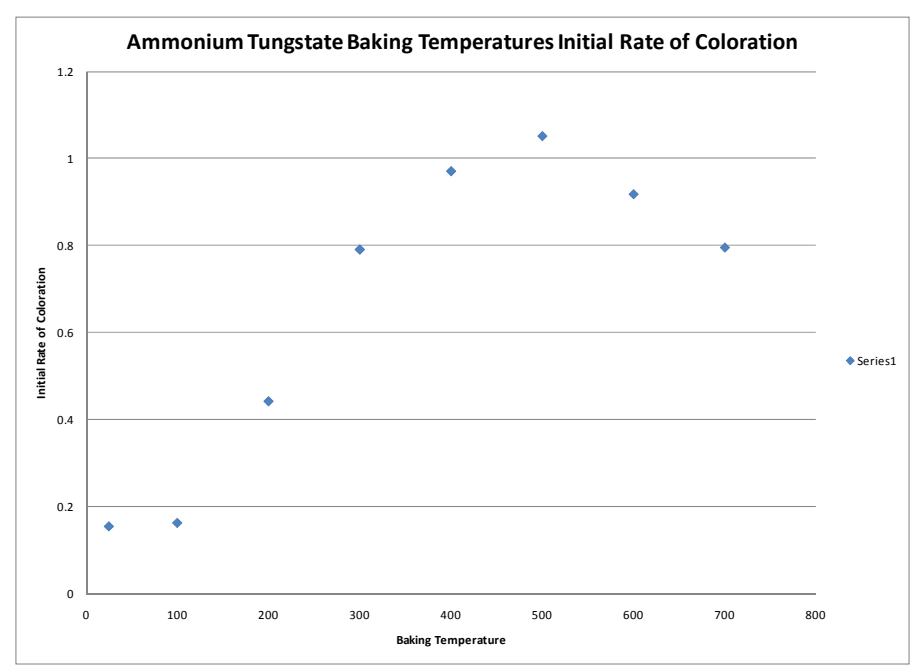

Figure 15. The effect of AT baking temperature on the activity of AT-Pt pigments

Results from the above figures indicate that baking at $500^{\circ} \mathrm{C}$ produces pigment with the fastest rate of coloration at the start of the process. The results of TG/DTA analysis of AT are presented in Figure 16.

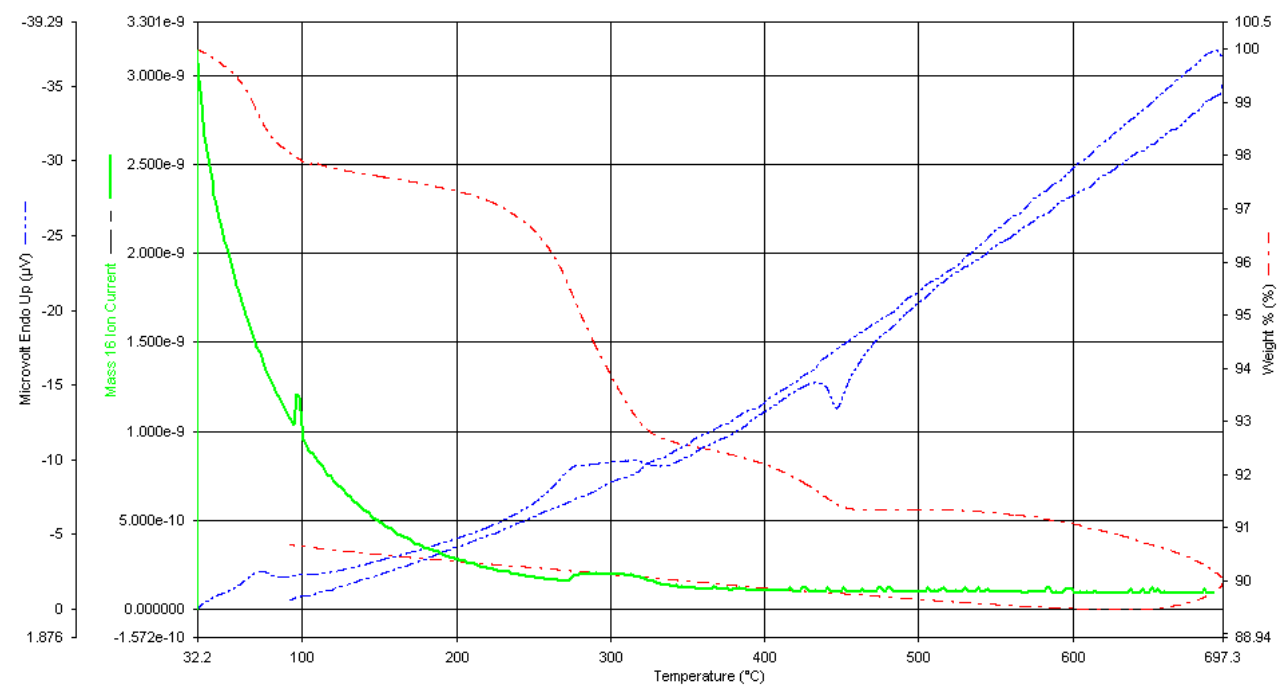

Figure 16. TG/DTA analysis of ammonium tungstate.

Based on Figure 16, AT loses two $\mathrm{NH}_{3}$ molecules at about 300 and $450^{\circ} \mathrm{Cand}$, about $450^{\circ} \mathrm{C}$, AT is converted to $\mathrm{WO}_{3}$, which is an active component of $\mathrm{H}_{2}$ sensor. This result is in agreement with the Figure 15 , which shows that $500^{\circ} \mathrm{C}$ is the optimal baking temperature corresponding to the fastest coloration rate.

It should be emphasized that the kinetics of bleaching of AT-based formulations is about two orders of magnitude faster than the AM based pigments (i.e., few hours instead of several days). Figure 17 shows a typical kinetic curve for bleaching of an AT-Pt pigment. The Figure shows that the AT-Pt pigment is almost completely bleached in about 6 hours. 


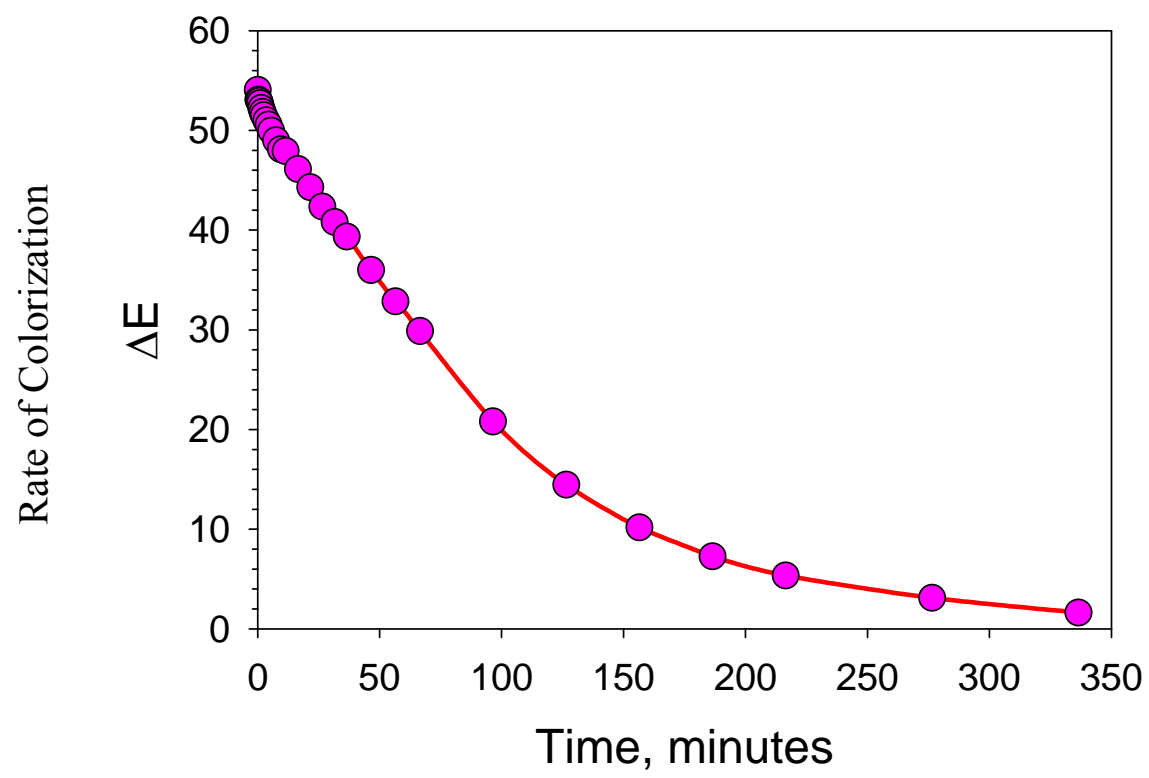

Figure 17. Kinetics of bleaching of AT-Pt $\mathrm{c}_{\mathrm{c}}$ based pigment

Figure 18. shows the photos of original (unexposed) (left), exposed to hydrogen (center) and bleached (right) samples of AT-Pt pigments encapsulated in silicone matrix.
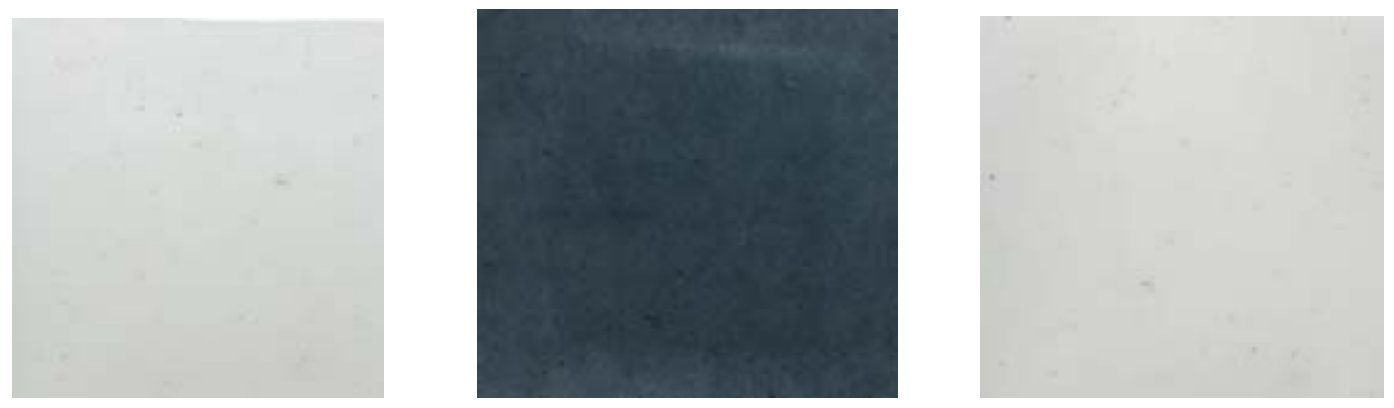

Figure 18. Photos of original (unexposed) (left), exposed to hydrogen (middle) and bleached (6 hrs) (right) samples of AT-Pt pigments.

\section{Environmental Effects on the Performance of Reversible Chemochromic Pigments}

\subsection{Effect of UV Light}

The objective of the environmental research was to study the effect of UV radiation on the kinetics of coloration of both Mo- and W-based pigments. The following graphs show the effect of long-term (for up to 3 months) UV exposure (black light lamp, $365 \mathrm{~nm}$ radiation) on the performance of Mo and W based reversible pigments. It was found that UV radiation had a significant effect on the kinetics and intensity of coloration, depending on the nature of the metal (e.g., Mo vs. W), and also depending on the precursors from which pigment was formed. Figure 19 shows the kinetic curves of coloration and bleaching using 
Mo-based pigment produced by thermal decomposition of ammonium molybdate and doped with $\mathrm{Pt}$ nanoparticles.
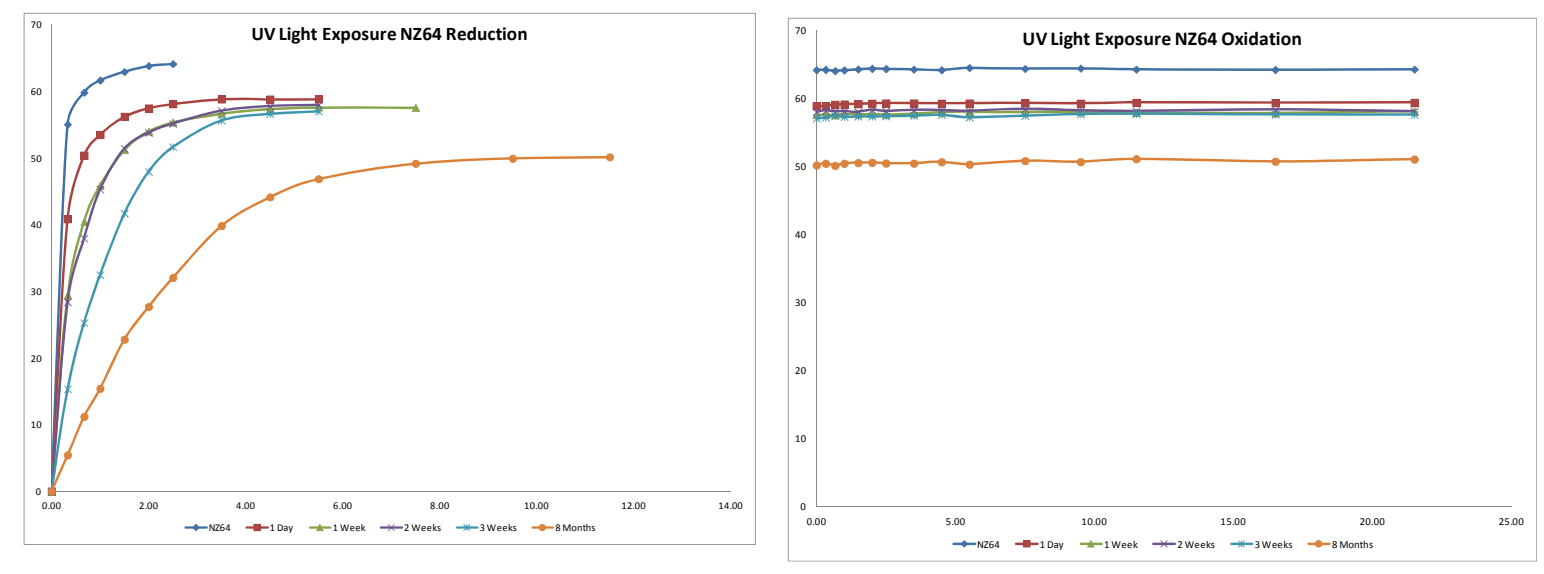

Figure 19. The effect of UV (365 nm wavelength) exposure on the performance of Mo-pigment produced from ammonium molybdate and doped with Pt nanoparticles.

Left- reduction curves; right- bleaching curves. Figure ordinates are $\Delta \mathrm{E}$ (Extent of Color Change).

The results show that the performance of the pigment slowly deteriorated over three months period. The deterioration was in terms of both slower kinetics and lesser intensity of coloration.

Figure 20 depicts the kinetic curves of coloration and bleaching using W-oxide pigments produced from ammonium tungstate and doped with Pt nanoparticles.
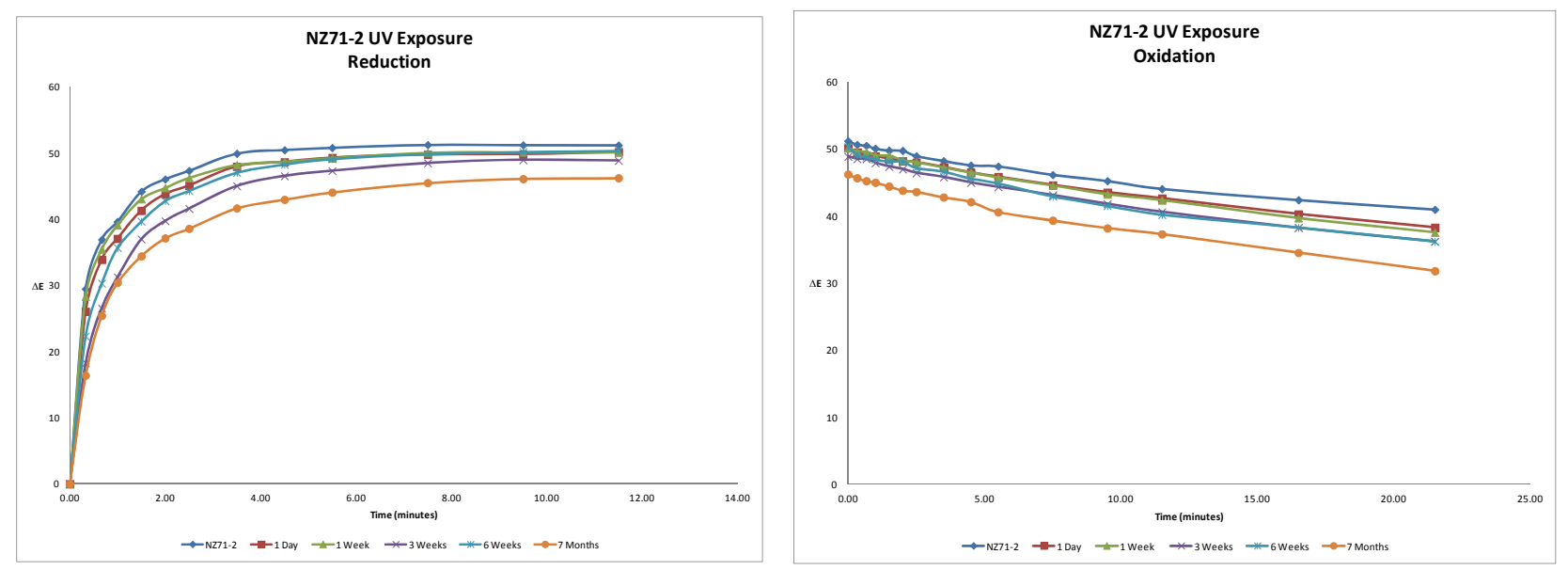

Figure 20. The effect of UV (365 nm wavelength) exposure on the performance of W- based reversible pigment produced by thermal decomposition of ammonium tungstate. Left- reduction curves; rightbleaching curves. Figure ordinates are $\Delta \mathrm{E}$ (Extent of Color Change).

\subsection{Long-term Outdoor Testing}

The main objective of this series of experiments was to perform long-term environmental testing of Mo and $\mathrm{W}$ based reversible pigments encapsulated in silicone membranes. The diagrams below show the 
effect of outside exposure to sun, rain, wind and humidity on kinetics of coloration of 4 pigment formulations produced from Mo-based ( $\mathrm{A}$ and $\mathrm{B})$, and $\mathrm{W}$-based $(\mathrm{C}$ and $\mathrm{D})$ pigments activated by $\mathrm{Pt}$ nanoparticles. All samples were exposed for up to 5 months. The results of testing of Mo- and W-based pigment formulations are shown in Figure 21 (A-D).
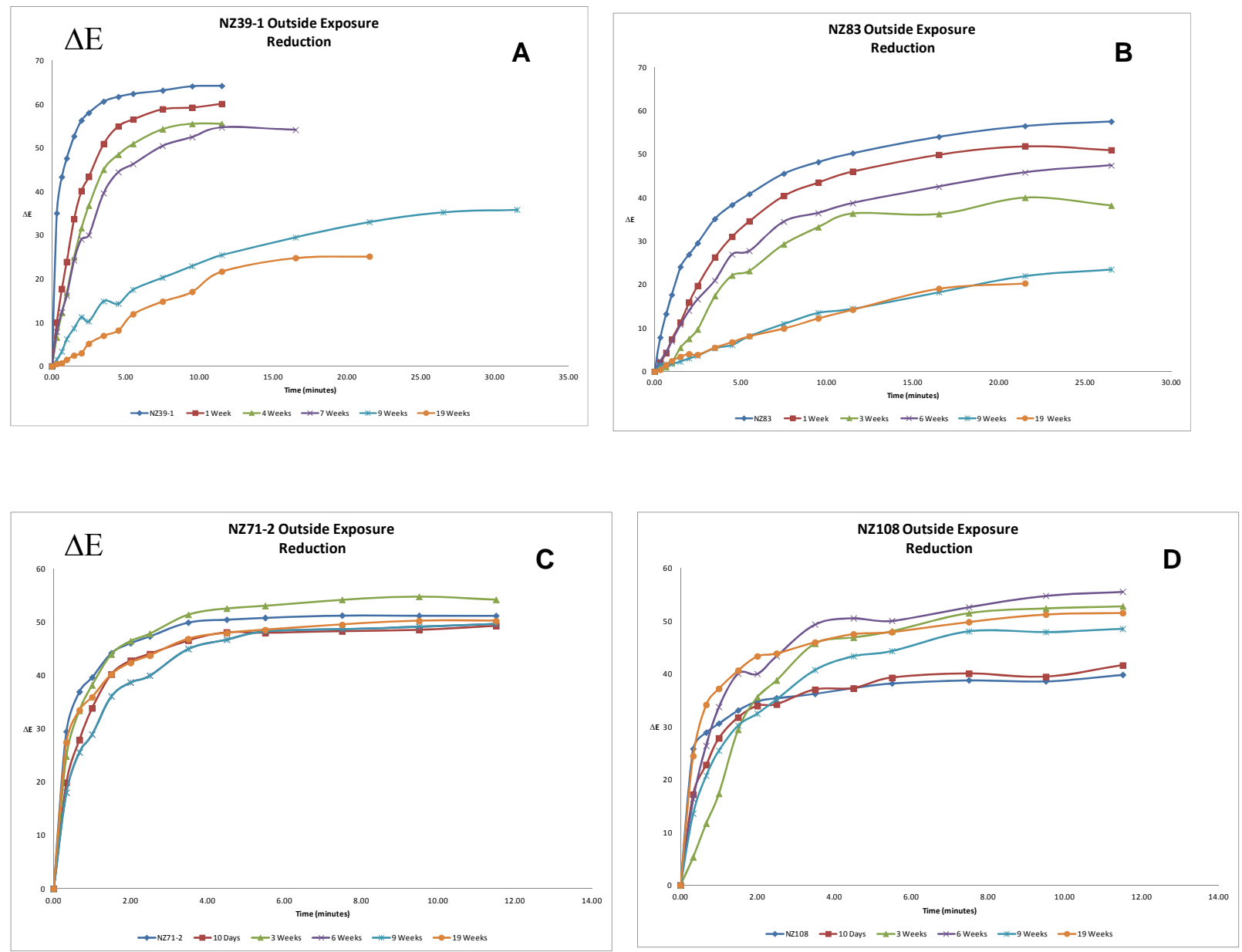

Figure 21. Kinetics of coloration of Mo-oxide/Pt (A), Mo-Si-oxide/Pt (B), W-oxide/Pt (from AT precursor) (C) and $\mathrm{WO}_{3} / \mathrm{Pt}(\mathrm{D})$ exposed to environment over period of 19 weeks. Figure ordinates are $\Delta \mathrm{E}$ (Extent of Color Change) and absicca is time in minutes.

The results showed that Mo-based pigments turned darker the longer they stayed outside. A considerable decrease in delta E values, as well as decreased in coloration kinetics was observed after 9 weeks of outside exposure. The decreased delta $E$ values are likely due to the darkening of the tested pigments exposed to sunlight (before exposure to $\mathrm{H}_{2}$ ). The graphs below show the change in pigments coloration before hydrogen exposure. The tungsten pigments showed less pigment darkening problems compared to the molybdenum pigments tested (Figure 22). 

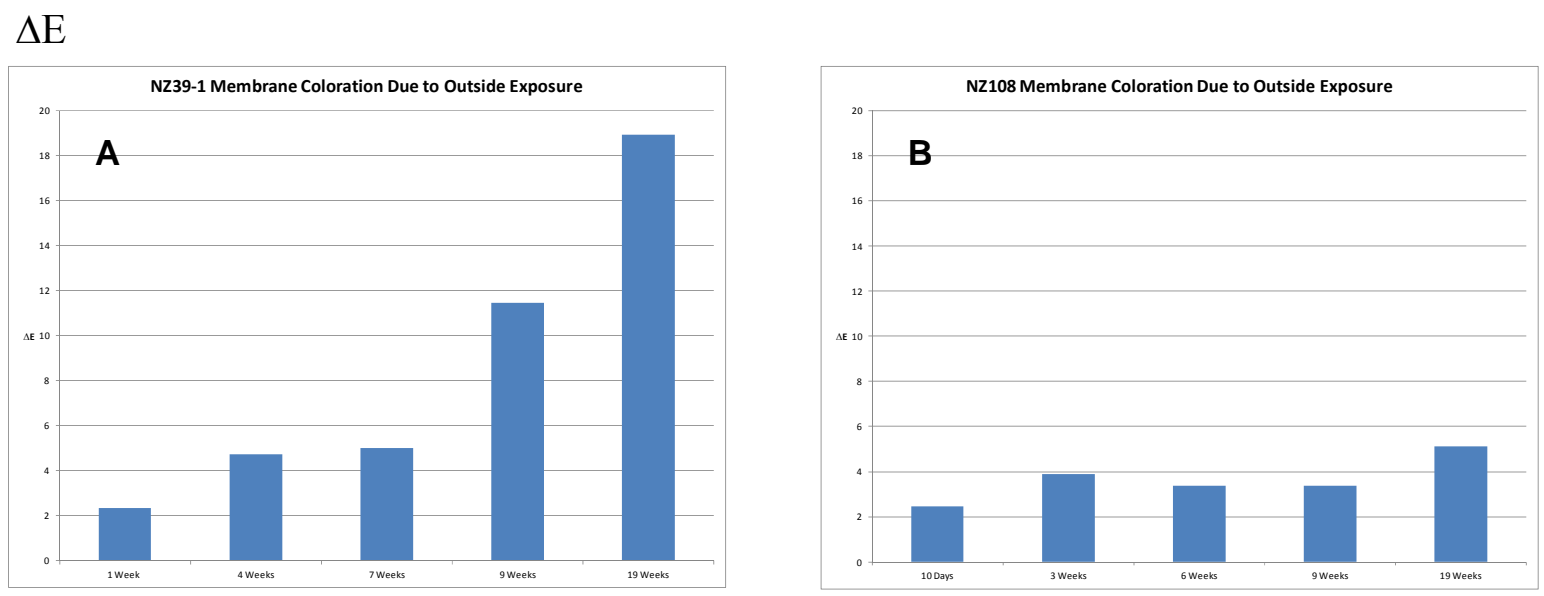

Figure 22. Change in membrane color after exposure to elements for 19 weeks. A- Mo-oxide/Pt, B$\mathrm{WO}_{3} / \mathrm{Pt}$, both in silicone matrix. Figure ordinates are $\Delta \mathrm{E}$ (Extent of Color Change).

\section{Mechanistic Studies}

\section{1. $\mathrm{H}_{2}$ Uptake and Mo-oxide/ $\mathrm{H}_{2}$ Stoichiometry Study}

The objective of this research work was to conduct $\mathrm{H}_{2}$ uptake studies. For these studies, PCT measurements of the $\mathrm{H}_{2}$ uptake by Mo-oxide based pigment were conducted. The results are shown in Figure 23.

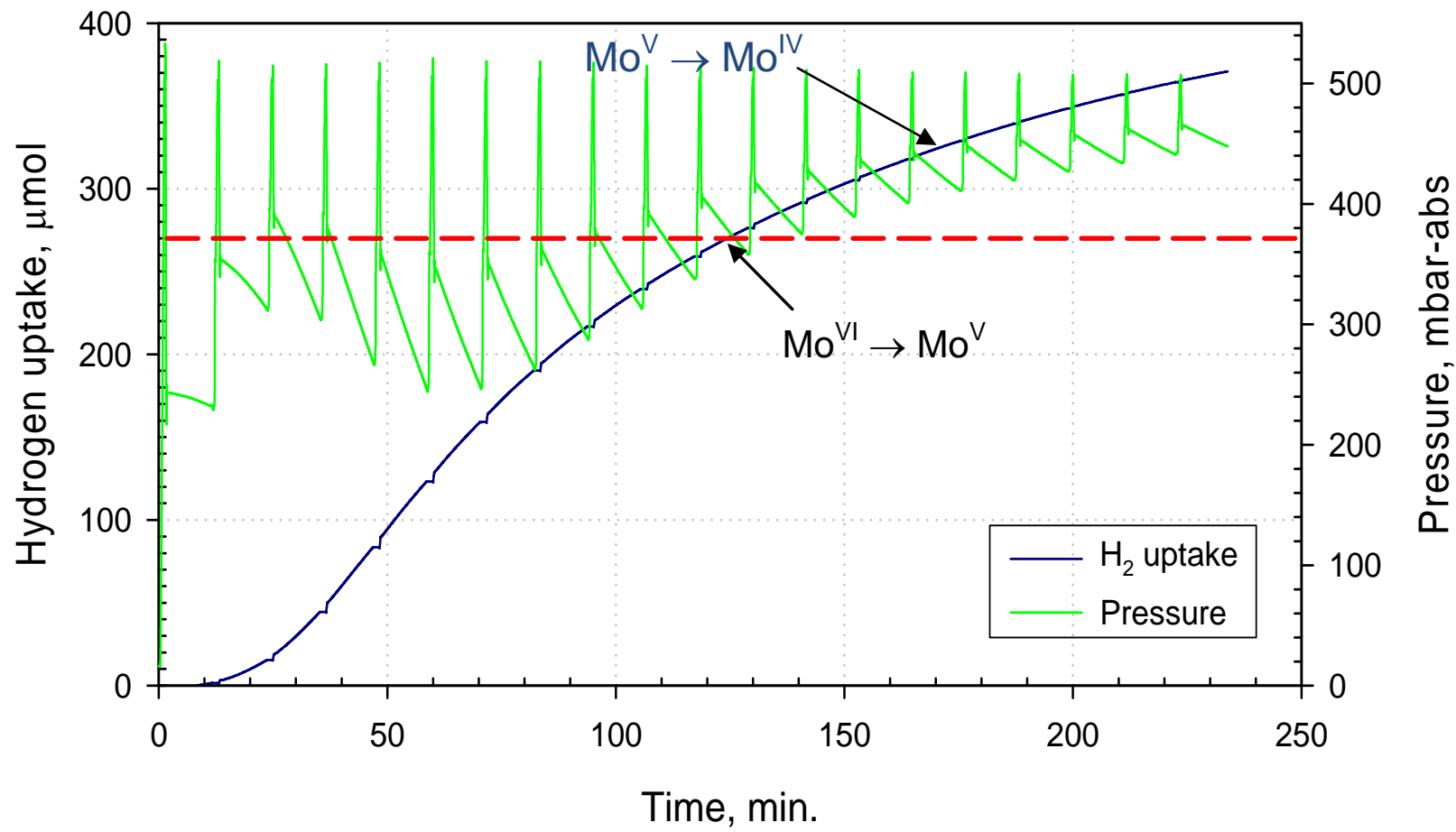

Figure 23. PCT measurements of the $\mathrm{H}_{2}$ uptake by Mo-oxide based pigment. 
The results indicated that upon exposure to $\mathrm{H}_{2}$, Mo was reduced from oxidation state +6 to the oxidation states of +5 and +4 , as shown in Figure 23 .

\subsection{Analysis and Characterization of Reversible Pigments}

\section{XPS Analysis of Mo- and W-based Pigments}

The objective of this work was to determine the oxidation state of Mo-pigment before and after exposure to $\mathrm{H}_{2}$. The work was done by conducting X-ray photoelectron spectroscopic (XPS) analyses of Mo-based pigment (without encapsulation in silicone matrix) before and after exposure to hydrogen. The results are presented in Figure 24 (A and B).
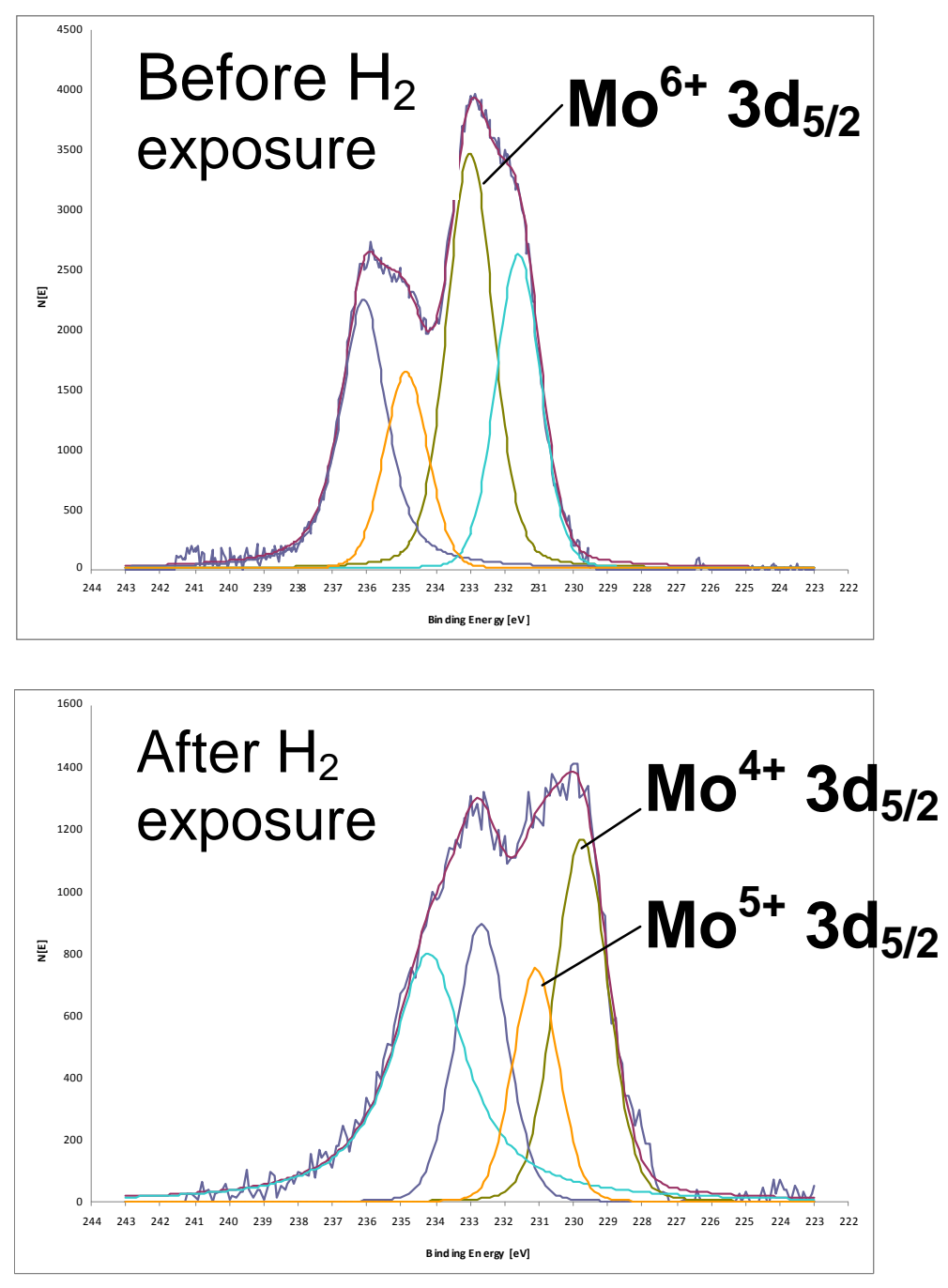

Figure 24. XPS analysis of Mo-based pigment before (A) and after (B) exposure to hydrogen.

The results of the XPS analysis showed that during coloration process molybdenum is reduced from $\mathrm{Mo}^{+6}$ to $\mathrm{Mo}^{+4} / \mathrm{Mo}^{+5}$ oxidation state. 
XPS analysis of W-based pigment before $\mathrm{H}_{2}$ exposure and after the exposure was also conducted (Figure 25).

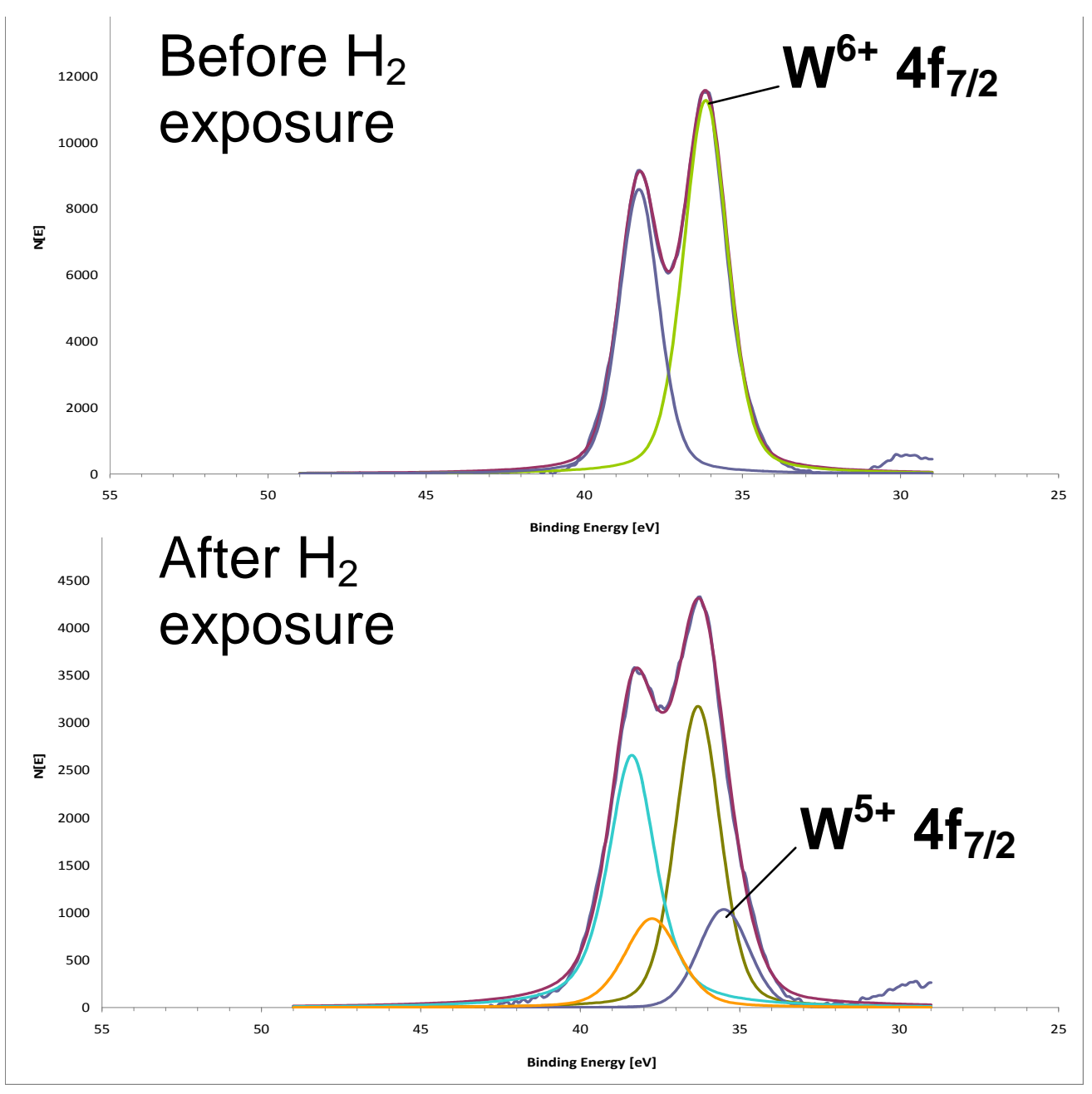

Figure 25. XPS spectra of W-based pigment before (above) and after (below) exposure to hydrogen.

XPS analysis of W-based pigment by monitoring $4 \mathrm{f} 7$ peak indicated conversion of $\mathrm{W}^{6+}$ to $\mathrm{W}^{5+}$ and $\mathrm{W}^{4+}$ after exposure to hydrogen. During this reductive transformation, W-based pigment changes color from yellow to deep blue.

\section{$\underline{\text { TEM of Mo-based Pigment }}$}

TEM analysis of Mo-based reversible formulations was conducted. The TEM image shows the presence of spherical nano-sized Pt particles (about $5 \mathrm{~nm}$ in diameter). See Figure 26. 


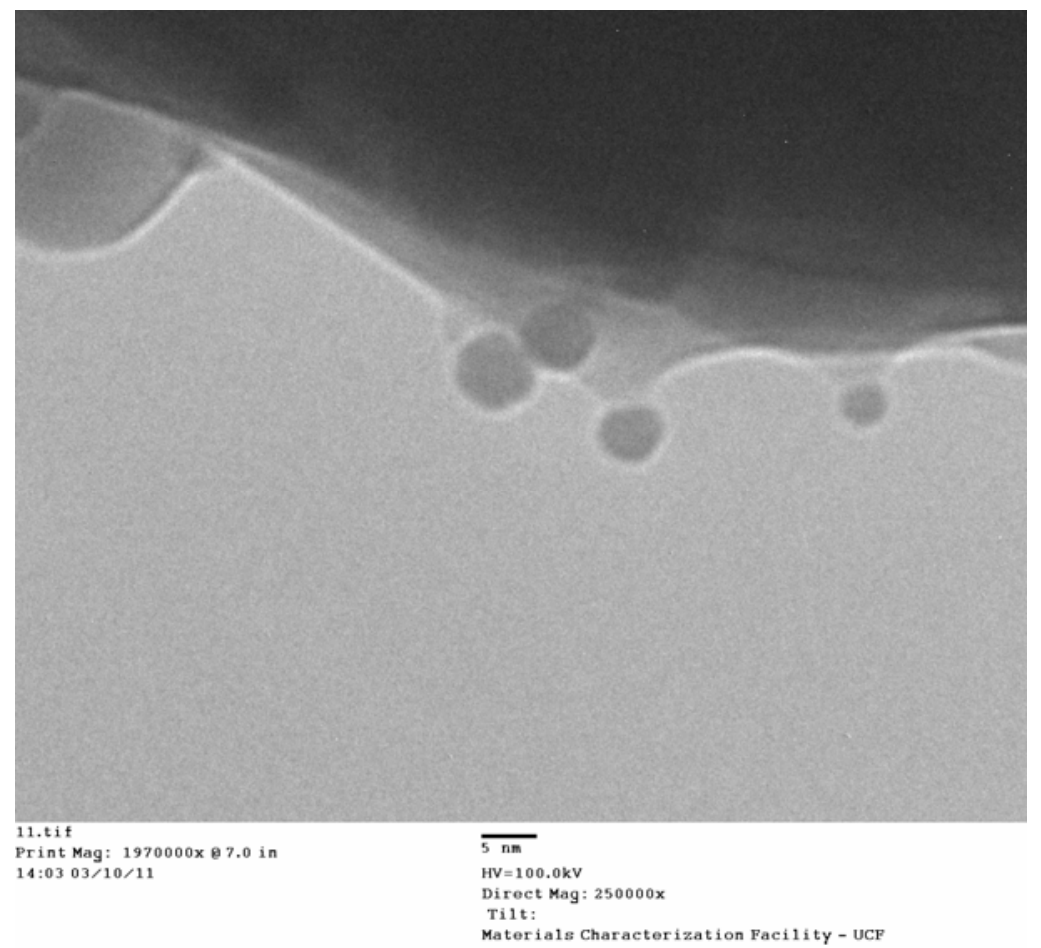

Figure 26. TEM image of Mo-based pigment.

\subsection{Mechanism of Coloration and Bleaching}

Based on the results of the $\mathrm{H}_{2}$-uptake and XPS analysis studies, the following coloration mechanism for Mo-based pigments was proposed:

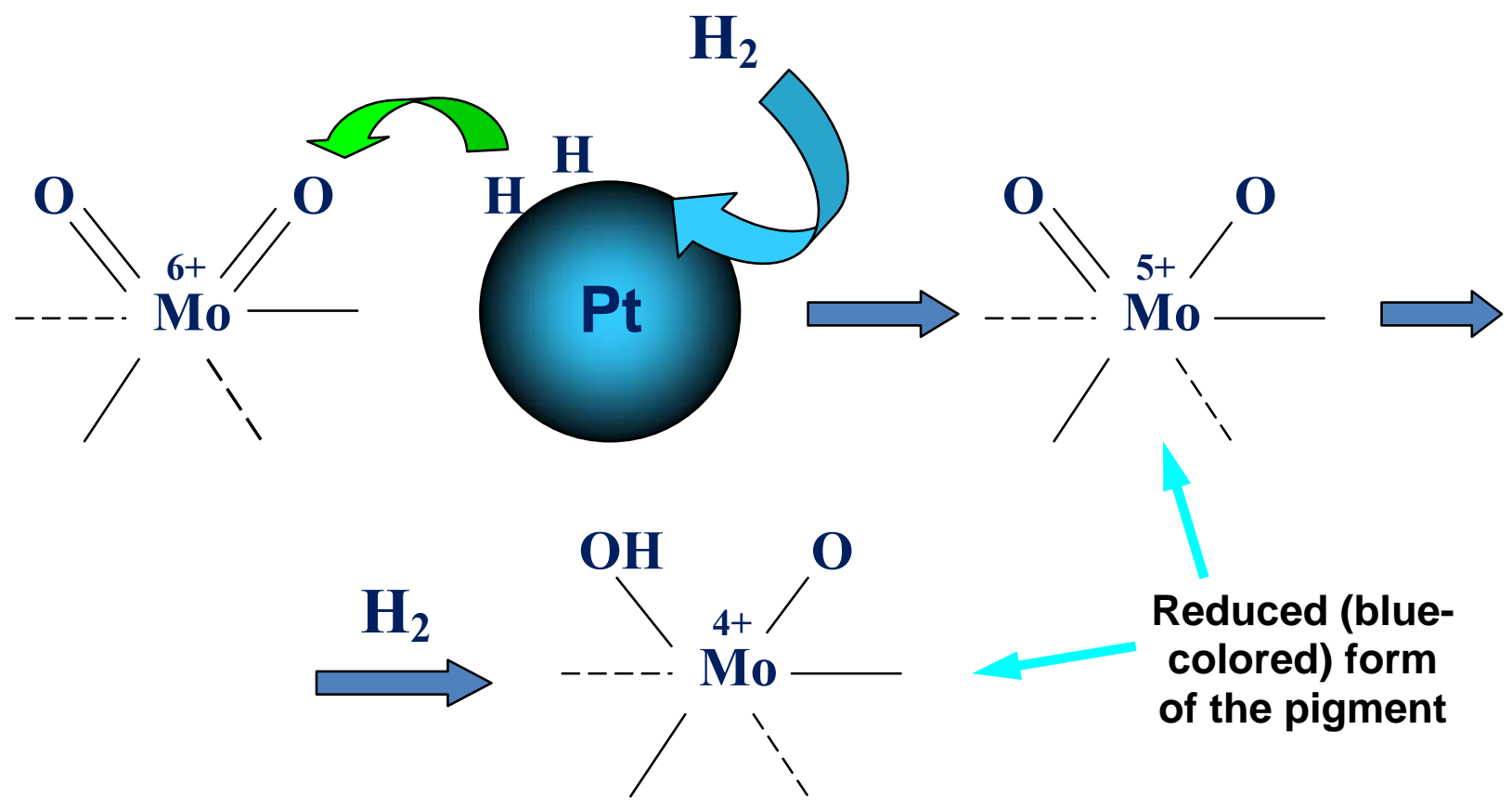


The mechanism of bleaching is as follows:

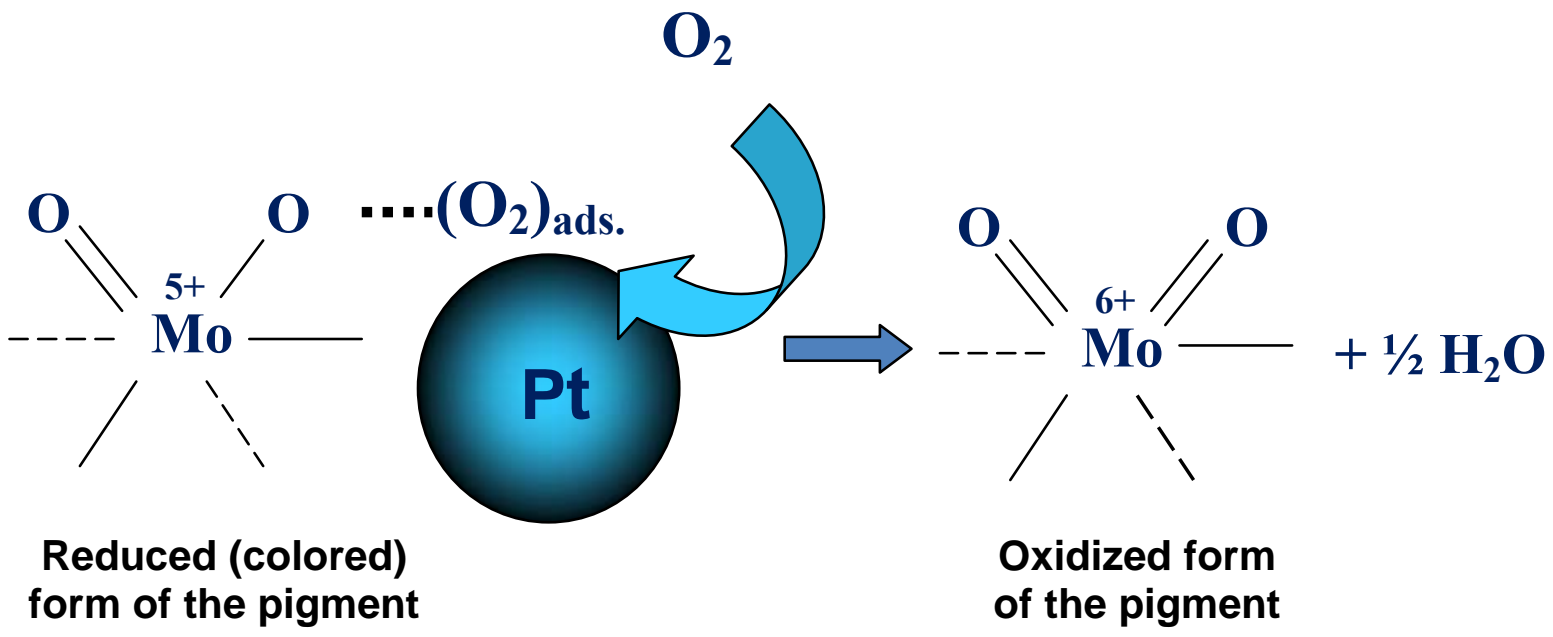

Concluding Remarks and Major Findings:

The main objectives of the reversible sensor work have been successfully accomplished in that a series of active, highly selective and durable reversible hydrogen sensors have been developed. The reversible chemochromic pigments are based on the oxo-compounds of molybdenum (Mo) and tungsten (W) that have suitable redox potentials for the reduction reaction in the presence of $\mathrm{H}_{2}$ and bleaching reaction in the presence of air. The major findings are:

- Synthesized and tested over 70 novel reversible pigment formulations based on compounds of molybdenum (Mo), tungsten (W) and vanadium (V), including polyoxocompounds, salts, oxides, acids. Among tested formulations, oxo-compounds of Mo and W showed fast (seconds-tominutes) and selective response to hydrogen.

- Studied the effect of different variables (amount of pigment, temperature, activators) on the efficiency of Mo and $\mathrm{W}$ based reversible pigments encapsulated in the silicone matrix. Determined optimum amount of reversible pigments in the chemochromic formulations, i.e., pigment to silicone matrix weight ratio. Different types of silicone matrices were tested; among them RTV-3145 and Dow Corning 734. Both silicones demonstrated excellent performance.

- Studied the effect of different supports, such as, $\mathrm{SiO}_{2}$, different brands of $\mathrm{TiO}_{2}, \mathrm{Al}_{2} \mathrm{O}_{3}$ and ceramic on the performance of both Mo- and W-based pigments. $\mathrm{TiO}_{2}$ and $\mathrm{SiO}_{2}$ supports showed acceptable uses.

- Determined the effect of co-catalysts $(\mathrm{Pd}, \mathrm{Pt}, \mathrm{Rh}, \mathrm{Ru})$ on the rate of coloration in presence of hydrogen. Pt co-catalyst showed the best activity and stability. Pt co-catalysts were used in the form of nano-particles deposited on Mo- and W-pigments or $\mathrm{Pt} / \mathrm{SiO}_{2}$ particles mixed with Moand W-pigments. Both options showed comparable performance.

- Evaluated the performance (sensitivity) of the chemochromic sensors at different $\mathrm{H}_{2}$ concentrations in air (from 1 to 100 vol.\%). It was found that both Mo- and W-based pigments performed adequately under low $\mathrm{H}_{2}$ concentrations.

- Determined the extent of interference with other reducing gases $\left(\mathrm{CO}, \mathrm{NH}_{3}, \mathrm{CH}_{4}, \mathrm{H}_{2} \mathrm{~S}\right)$. Found no interference with neither of the reducing gases. It was determined that the developed sensors are highly selective toward hydrogen. 
- Studied prolonged exposure of Mo- and W-pigment formulations to UV radiation (black light, wavelength $360 \mathrm{~nm}$ ). Materials, particularly, Mo-based pigments showed UV sensitivity. It was found that UV exposure for 3 months caused slow degradation of performance, especially, with Mo-based pigments. The $\mathrm{H}_{2}$ sensors worked without problems indoor when exposed to daylight and fluorescent bulbs.

- Conducted long-duration environmental testing of Mo- and W-based reversible sensors exposed to elements (sunlight, rain, humidity) for 5 months. Mo-based pigments showed signs of deterioration, whereas, $\mathrm{W}$-based pigments showed acceptable stability and durability.

- Conducted $\mathrm{H}_{2}$ uptake and $\mathrm{H}_{2}$-pigment stoichiometry measurements and mechanistic studies of the pigments coloration and bleaching reactions using TCP system.

- Conducted analysis and material characterization studies of reversible pigments using SEM, TEM, TGA, XPS methods. XPS analysis indicated that in the presence of $\mathrm{H}_{2}$ Mo-and W-based pigments were reduced from oxidation state +6 to oxidation states +5 and +4 . During bleaching, both Mo and $\mathrm{W}$-based pigments returned back to their original +6 oxidation state.

\section{Patent Applications}

1. N. Mohajeri, "Doped palladium containing oxidation catalysts", U.S. Application No. 13/588,779

2. P. Brooker, N. Mohajeri "Chemochromic membranes for membrane defect detection", U.S. Application No. 13/594,163

\section{Publications/Presentations}

1. N. Mohajeri, “FSEC’s Irreversible Chemochromic Hydrogen Sensor Overview”, Fuel Cell Seminar, Orlando, Fl. November 3, 2011

2. N. Mohajeri, P. Kubiak, "Improved Titania Supported Palladium Oxide Pigments for Irreversible Chemocromic Hydrogen Sensor”, Fuel Cell Seminar, Orlando, Fl. November 3, 2011

3. Nazim Muradov, Ali T-Raissi, Gary Bokerman, Errol Hincamp. Passive Chemochromic Hydrogen Leak Detectors for Safety Monitoring. Proc. $19^{\text {th }}$ World Hydrogen Energy Conference, Toronto, Canada, 2012

4. Nazim Muradov, Ali T-Raissi, Gary Bokerman, Monica Kowalczik, Errol Hincamp. Passive Chemochromic Hydrogen Leak Detectors. International Journal of Hydrogen Energy, in preparation.

5. Nazim Muradov, Nahid Mohajeri, Ali T-Raissi, Gary Bokerman, Errol Hincamp. Chemochromic Hydrogen Leak Detectors for Safety Monitoring. DOE-FSEC FHI Project Progress Review Meeting, October, 2012 


\title{
FLORIDA HYDROGEN INITIATIVE \\ Sub-contractor Finel Report
}

\section{Development of High Efficiency Low Cost Electrocatalyst for PEM Fuel Cell Applications}

\author{
Dr. Marianne Rodgers \\ Florida Solar Energy Center \\ Advanced Energy Research Division \\ E-mail: mrodgers@fsec.ucf.edu \\ (321) 638-1709
}

\author{
Submitted to: \\ David L. Block \\ Florida Solar Energy Center/University of Central Florida \\ 1679 Clearlake Road \\ Cocoa, FL 32922 \\ E-mail: block@fsec.ucf.edu \\ Contract Number: DE-FC36-04GO14225 \\ FSEC Project No.: 20126067
}

Project Start Date: April 8, 2010

Project End Date: June 30, 2013

June 2013

\section{Disclaimer}

The Florida Solar Energy Center/University of Central Florida nor any agency thereof, nor any of their employees, makes any warranty, express or implied, or assumes any legal liability or responsibility for the accuracy, completeness, or usefulness of any information, apparatus, product, or process disclosed, or represents that its use would not infringe privately owned rights. Reference herein to any specific commercial product, process, or service by trade name, trademark, manufacturer, or otherwise does not necessarily constitute or imply its endorsement, recommendation, or favoring by the Florida Solar Energy Center/University of Central Florida or any agency thereof. The views and opinions of authors expressed herein do not necessarily state or reflect those of the Florida Solar Energy Center/University of Central Florida or any agency thereof. 


\title{
Development of High Efficiency Low Cost Electrocatalysts for PEM Fuel Cell Applications
}

\author{
Florida Solar Energy Center \\ Advanced Energy Research Division
}

\section{Executive Summary}

The primary objective of this project was to decrease Pt usage in fuel cells by increasing catalyst activity and broadening the knowledge base of structure-property relationships in catalysts. The potential commercial market has national and international product growth and energy benefits and, thus, is of importance to the public.

Proton exchange membrane fuel cells (PEMFCs) depend on the use of platinum-based electrocatalysts. Due to their high catalytic activity and their stability in the fuel cell environment, platinum or platinum alloys supported on carbon black are presently the catalysts of choice for PEMFCs to catalyze both the anodic hydrogen oxidation and cathodic oxygen reduction reactions. Fuel cell performance is usually limited by the slow kinetics of the cathode oxygen reduction reaction (ORR). A major factor limiting fuel cell commercialization is the cost, and it has been estimated that current $\mathrm{Pt} / \mathrm{C}$ catalysts represent about $40 \%$ of the total cost of fuel cells. The common goal in fuel cell catalyst research is to decrease the cost of fuel cells by reducing platinum loading in the PEMFC catalyst layers while maintaining high performance. This requires an increase in platinum catalyst activity.

Catalytic activity of platinum-based catalysts depends greatly on the dispersion and the size distribution of the platinum crystallites. Not only is small particle size desirable for obtaining large active surface areas, but for all the platinum in a PEMFC catalyst layer to be active, the fuel or oxidant must react at the interfacial region between the PEM and the platinum, known as the "three phase reaction zone". This zone is the three phase interface of catalyst, carbon, and electrolyte that allows effective gas and water diffusion and proton transport and electron transport to and from the catalyst sites.

Because contact between the electrolyte and the catalyst is a critical factor in obtaining high performance, current approaches aimed at lowering the platinum loading focus on localizing the catalyst to where the electrocatalytic reaction takes place. Pulse electrodeposition appears to be the most promising method of catalyst fabrication due to advantages such as controlled particle size, stronger adhesion, uniform electrodeposition, selectivity of hydrogen, and reduction of internal stress. Control of factors such as $t_{\text {off }}$, $t_{o n}$, duty cycle, peak current density, catalyst precursor, co-catalyst, and stabilizing agent allow fine tuning of the catalyst structure. The structure can be further tweaked with the use of methods to change the mass transport rate of the catalysts in the electrodeposition solution.

In this work, catalysts were prepared by electrodeposition on carbon paper that had been loaded with carbon and Nafion ${ }^{\circledR}$, resulting in a three phase reaction zone. Pulse electrodeposition was used with a rotating disk electrode as a first step, taking advantage of the benefits of pulse electrodeposition described above. The rotation of the working electrode in rotating disk experiments brought analyte molecules from the bulk solution towards the electrode, resulting in increased mass transport over diffusion-only experiments. Catalyst activity was also measured using a rotating disk electrode. After achieving high performance, the stability of the electrodes was investigated and the electrodeposition process was scaled up to form an electrode that is a practical size for use in a fuel cell. 
The goal of Task 1 was to establish a standard catalyst synthesis technique. In Task 2, the objective was to evaluate carbon supports used in the gas diffusion electrodes. The aim of Task 3 was the syntheses of Pt alloy based electrocatalysts. In Task 4 a new method for catalyst fabrication was developed. The goal of Task 5 was to evaluate Pt- and Pd-lanthanide based catalysts. In Task 6, the objective was to fabricate and evaluate Pd-based catalysts. In Task 7 scaling up of the electrodes and electrode stability were investigated

In Task 1, a standard catalyst synthesis technique was established. Based on a literature review, pulse electrodeposition was selected as the most promising technique to produce catalysts with controlled structure, high activity, and low loading. For this task, gas diffusion layers (GDLs) were sprayed with carbon and Nafion, followed by Pt electrodeposition, creating a three phase reaction zone. The amount of Nafion and carbon used was controlled so that the electrodes would have the same carbon and Nafion content as the baseline electrodes used at FSEC in fuel cell research ${ }^{[1]}$ Because mass transport was shown to be an important parameter in catalyst electrodeposition, a rotating disk electrode with a standard threeelectrode setup was used for both electrodeposition and electrochemical characterization of the catalysts.

The results of Task 1led to the optimization of several variables (platinum precursor concentration, electrode rotation rate, peak current, number of cycles, on time, off time, stabilizer concentration) during electrodeposition. These values were used in all future electrodepositions. It was evident that electrode morphology impacted performance in that improved performance was generally observed with higher electrode coverage, smaller particle size, and the formation of "feathery" or "flakey" particles.

The goal of Task 2 was to evaluate carbon supports used in the gas diffusion electrodes. Catalyst supports can affect both the activity and the stability of electrodes in fuel cells. Different carbon supports, including XC-72, Cabot Regal GP-47, activated carbon KB-B, and graphene oxide were investigated. Thermal pretreatment of XC-72 carbon had no effect on electrode performance. The performance of electrodes made with XC-72 carbon support was lower than that of Cabot Regal GP-47 and activated carbon KB-B. Graphene is promising as a support due to its high surface area and stability and it was shown that when graphene oxide was reduced, the electrode performance at high voltage was improved over that of the baseline electrode.

In Task 3, three Pt alloy based colloidal particles were synthesized via a polyvinylpyrrolidone (PVP) protected process. The colloidal nano-particles of the Pt ternary system with presence of PVP were stable and the particle size distributions were uniform with an average size of 4 to $5 \mathrm{~nm}$. The nanoparticles were adsorbed onto the carbon electrode through immersion of the sprayed carbon paper into the colloidal solution followed by sonication, but the ORR was very low. This low performance was attributed to the low quantity of particles adsorbed.

It has been made clear that catalyst morphology is very important in electrode performance. In many cases in this project pulse electrodeposition led to less desirable catalyst morphologies, including Pt particle aggregation. To prevent Pt particle aggregation, in Task 4, a new electrodeposition method was developed where the carbon electrodes were seeded with nanosized Pt colloidal particles prior to electrodeposition. This new electrodeposition method improved electrode morphology and, therefore, performance. By seeding the electrodes with catalyst nanoparticles prior to electrodeposition, it was possible to achieve the same performance as a commercial electrode with $1 / 5$ the amount of Pt. The improved performance of the seeded electrodes was attributed to improved catalyst coverage on the electrodes. It was also shown that carrying out successive plating rather than direct plating resulted in improved performance, which was attributed to smaller Pt agglomerate size. 
In Task 5 Pt- and Pd-lanthanide based catalysts were evaluated because lanthanide elements have shown surprising results in terms of catalytic activity and outstanding material properties. Ceria was formed through thermal hydrolysis, adsorbed onto the carbon powder, and deposited onto GDLs. Pd was electrodeposited onto the GDLs. Although differences in performance were observed, SEM showed that the ceria disappeared through the electrodeposition and characterization process. The loss of ceria was attributed to the electrochemical reactions resulting in formation of $\mathrm{Ce}^{3+}$, which is soluble. To prevent loss of ceria, a new procedure was developed whereby the ceria was calcined prior to being exposed to any electrochemical conditions. It was clear that the calcination resulted in improved ceria stability, although the calcined ceria was not shown to affect performance of Pt-based electrodes.

In Task 6, Pd-based electrodes were prepared using catalyst seeding. The best performance was observed when Pt-Pd was used as a seed, while the performance of the electrodes that used Pt-Ru and Pt-Pd-Ru as seeds were quite similar. Although their performances were lower than those of the commercial Pt-based catalyst, when normalized for PGM content, the performance of the Pd electrodes were improved over the commercial catalyst.

In Task 7 scaling up of the electrode and electrode stability were investigated. For scaling up, an electrodeposition flow cell was designed and built and electrodes of $25 \mathrm{~cm}^{2}$ were prepared. This was a 50fold scale up in size compared to the $0.5 \mathrm{~cm}^{2}$ electrodes that were prepared with the rotating disk electrode. Unfortunately, platinum loading using the flow cell was low and, as a consequence, fuel cell performance was low.

An accelerated stress protocol based on potential cycling was developed to investigate the stability of electrodes containing activated carbon and reduced graphene oxide as supports. It was shown that the electrode containing reduced graphene oxide had superior stability and did not degrade during testing. 


\section{Development of High Efficiency Low Cost Electrocatalysts for PEM Fuel Cell Applications}

\section{Table of Contents}

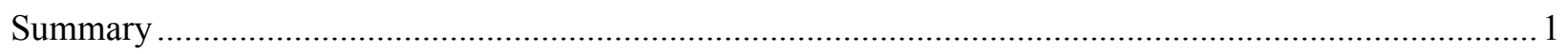

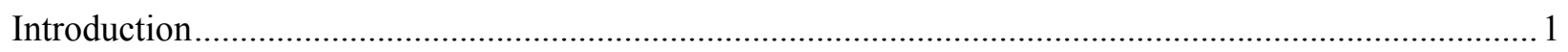

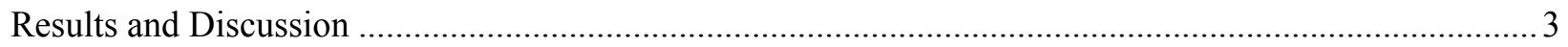

Task 1: Establishing a standard catalyst synthesis technique ...................................................... 3

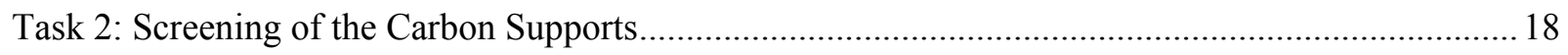

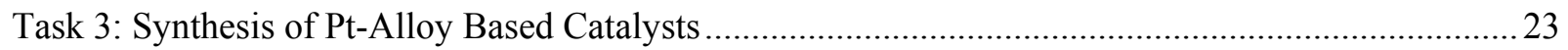

Task 4: Screening of the Pt-metal or Pt-metal oxide based catalysts ..............................................26

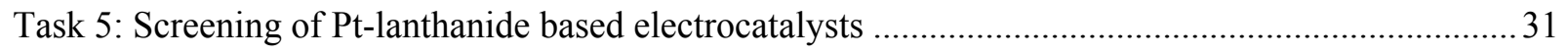

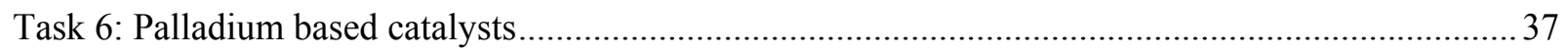

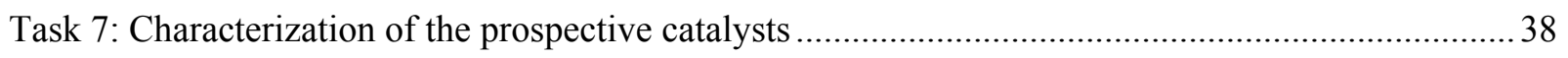

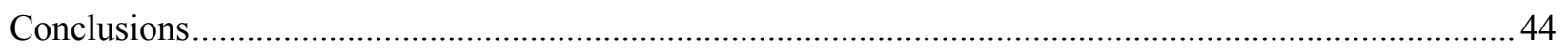

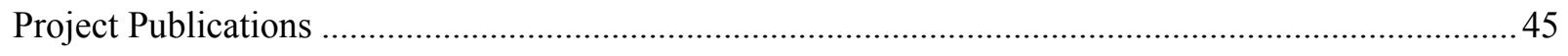

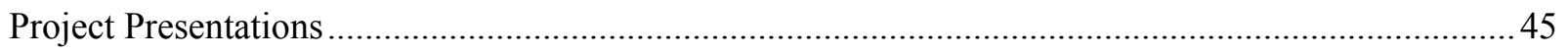

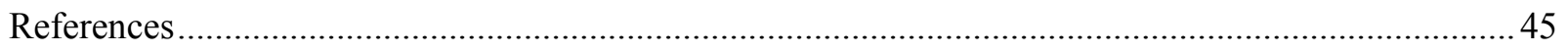




\title{
Development of High Efficiency Low Cost Electrocatalysts for PEM Fuel Cell Applications
}

\author{
Florida Solar Energy Center \\ Advanced Energy Research Division
}

\begin{abstract}
Summary
Experiments were conducted to improve catalyst activity while lowering platinum loading through pulse electrodeposition. Optimum values of several variables during electrodeposition were selected to achieve the highest electrode performance, which was related to catalyst morphology. The type of catalyst support used was found to impact electrode performance, with reduced graphene oxide, Cabot Regal, and activated carbon resulting in the highest performances. A new electrodeposition method was developed where the carbon electrodes were seeded with nanosized Pt colloidal particles prior to electrodeposition. With the new method, it was possible to achieve the same performance as a commercial electrode using $1 / 5$ the amount of Pt. Calcination resulted in improved ceria stability, although the ceria was not shown to affect performance of Pt-based electrodes. Although their performances were lower than those of the commercial Pt-based catalyst, when normalized for PGM content, the performance of Pd electrodes was improved over the commercial catalyst. An electrodeposition flow cell was designed and built, and 25 $\mathrm{cm}^{2}$ electrodes were prepared. This was a 50-fold scale up in size compared to the $0.5 \mathrm{~cm}^{2}$ electrodes that were prepared thus far with the rotating disk electrode. Unfortunately, platinum loading using the flow cell was low and, as a consequence, fuel cell performance was low. An accelerated stress protocol based on potential cycling showed that the electrode containing reduced graphene oxide had superior stability and did not degrade during testing.
\end{abstract}

\section{Introduction}

Proton exchange membrane fuel cells (PEMFCs) depend on the use of platinum-based electrocatalysts. At present, platinum or platinum alloys supported on carbon black $(\mathrm{Pt} / \mathrm{C}$ or $\mathrm{PtM} / \mathrm{C}, \mathrm{M}=$ metal alloyed with platinum) are the catalysts of choice for PEMFCs to catalyze both the anodic hydrogen oxidation $\left(\mathrm{H}_{2} \rightarrow\right.$ $\left.2 \mathrm{H}^{+}+2 \mathrm{e}^{-}\right)$and cathodic oxygen reduction $\left(2 \mathrm{H}^{+}+1 / 2 \mathrm{O}_{2}+2 \mathrm{e}^{-} \rightarrow \mathrm{H}_{2} \mathrm{O}\right)$ reactions due to their high catalytic activity and their stability in the fuel cell environment. ${ }^{[2]}$ Fuel cell performance is usually limited by the slow kinetics of the cathode oxygen reduction reaction (ORR). A major factor limiting fuel cell commercialization is the cost, and it has been estimated that current $\mathrm{Pt} / \mathrm{C}$ catalysts represent about $40 \%$ of the total cost of fuel cells. ${ }^{[3]}$ Two major approaches to decrease cost and improve the efficiency of the ORR include: reducing platinum loading in the PEMFC catalyst layers while maintaining high performance; and exploring non-platinum based metal catalysts that cost much less and yet can achieve the necessary performance level under PEMFC conditions. If the activity of the platinum catalyst can be increased, then the platinum loading in fuel cells can be reduced. For decades, great efforts have been devoted to increasing the activities and reducing the loading of platinum.

Catalytic activity of platinum-based catalysts depends greatly on the dispersion and the size distribution of the platinum crystallites ${ }^{[4,5]}$ Not only is small particle size desirable for obtaining large active surface areas, but for all the platinum in a PEMFC catalyst layer to be active, the fuel or oxidant must react at the interfacial region between the PEM and the platinum, known as the "three phase reaction zone". ${ }^{[6]}$ This zone is the three phase interface of catalyst, carbon, and electrolyte that allows effective gas and water diffusion, as well as proton and electron transport to and from the catalyst sites. ${ }^{[7,8]}$

Alloying Pt with other metals can increase the catalytic activity of platinum. The improvement in the ORR kinetics of Pt-alloys can occur as a result of structural changes caused by alloying - e.g., 
geometrical (decrease of the Pt-Pt bond distance) ${ }^{[9]}$ or electronic (increase of Pt d-electron vacancy) ${ }^{[10]}$ structure of platinum metal. For example, it was recently shown that the oxygen reduction activity of the $\mathrm{Pt}_{3} \mathrm{Ni}$ (111) surface is ten times higher than pure $\mathrm{Pt}(111),{ }^{[11]}$ which the authors attributed to changes in the surface electronic structure.

Methods for fabricating electrodes for PEMFCs include decal transfer, silk screening, brushing, and rolling the catalyst ink that contains solubilized polymer electrolyte binder and carbon supported catalyst onto a gas diffusion layer (GDL) or membrane. ${ }^{[12,13]}$ The catalyst layers in these membrane electrode assemblies (MEAs) have uniform concentration profiles of the catalyst, since the $\mathrm{Pt} / \mathrm{C}$ powder is thoroughly mixed with the Nafion ${ }^{\circledR}$ binder before being applied to the membrane or GDL. However, in electrodes made by these methods, the platinum catalyst is not utilized effectively and inactive catalyst sites exist in the catalyst layer (e.g. Pt particles either completely surrounded by ionomer, or devoid of ionomer) because the electrochemical reaction only occurs at the interface of the electrolyte and the electrode.

Electrodeposition in an aqueous solution offers an effective way to deposit platinum and other noble metal catalysts selectively at desired locations in the electrode with both ionic and electronic access because only in those regions are there electron transport channels and metal ion transport channels. Electrodeposition of noble metal catalysts onto a porous carbon electrode in an aqueous solution is a promising way to increase metal utilization. When compared with standard electrodes, electrodeposited electrodes show higher mass activities. ${ }^{[14]}$

Pulse electrodeposition, compared with direct current electrodeposition, has many advantages in terms of controlled particle size, stronger adhesion, uniform electrodeposition, selectivity of hydrogen, and reduction of internal stress. Pulse deposition provides close contact between the submicrometer particles of platinum and the membrane. It also helps to generate lower grain size and a thin catalyst layer of platinum at the MEA interface. ${ }^{[15]}$ Direct current electrodeposition only has current density as a variable, while pulse electrodeposition has three independent variables, $t_{\text {on }}$ (on-time), $t_{\text {off }}$ (off time) and $i_{p}$ (peak current density). The general concept for the pulse current technique, defining the variables is shown in Figure 1.

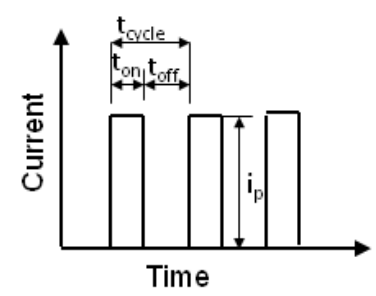

Figure 1. General concept of pulse-current technique

Several researchers have investigated the effects of pulse deposition parameters on performance. Lee et al. ${ }^{[16]}$ pointed out that the peak current density and duty cycle could control the nucleation rate and decrease the catalyst dendritic growth. $\mathrm{Ibl}^{[17]}$ claimed that the duty cycle can be an important factor because the properties of metal deposits can be influenced by both the $t_{o n}$, during which formation of nuclei and growth of existing crystals occur, and the $t_{\text {off }}$, during which desorption of deposited ions takes place. When the $t_{\text {off }}$ is extended, a longer deposition time is required to achieve the same platinum loading, while the overall overpotential of the platinum reduction decreases due to a decrease in the mass transfer overpotential. A longer $t_{\text {off }}$ also results in a decrease in the nucleation rate. Off time plays a more important role than $t_{\text {on }}$ in determining characteristics such as platinum nanoparticle concentration, size, and loading. ${ }^{[14]}$ Short potential pulses favor a more uniform distribution of particles and a general reduction in particle size. By varying the total charge applied for electrodeposition, the amount of platinum loading in the catalyst layer can be controlled. 
Cheh $^{[18]}$ and Countanceau ${ }^{[19]}$ showed that the rate determining step of platinum electrodeposition is mainly controlled by mass transport. Pollet ${ }^{[20]}$ increased mass transport by employing forced convection in the form of ultrasound. Sonication leads to a substantial increase in limiting current attributed to the effects of cavitation and/or micro and macro streaming. PEMFC electrodes prepared sonoelectrochemically gave better performance than those prepared by the galvanostatic pulse method only. ${ }^{[19]}$

Stabilizing agents are used to prevent the agglomeration of nano-sized catalyst metals. For example, polyvinylpyrrolidone (PVP) interacts with PtRu catalyst surface sites ${ }^{[21]}$ ethylenediaminetetraacetic acid (EDTA), tartaric acid, and citric acid have been used as growth inhibitors in silver, copper, zinc, nickel and tin electrodeposition. ${ }^{[22,23,24,25,26]}$ Similar effects are observed in the chemical reduction of Pt and PtRu in aqueous solution containing one or more alcoholic components, especially ethylene glycol, as reducing agent. ${ }^{[27,28,29]}$ Additionally, ethylene glycol addition can prevent particle agglomeration. Sieben et al. ${ }^{[30]}$ found that the particle size of PtRu was three to four times smaller with stabilizers. Ethanol resulted in the most active electrode, followed by ethylene glycol, while the addition of formic acid resulted in lower activity than if there was no stabilizing agent.

In summary, there are many factors that can affect the morphology of Pt particles, and therefore the ORR activity of the electrode:

1. Organic inhibitors can reduce the size of Pt particles. When an organic component is added to a Pt precursor electrolyte, the absorption of the organic component will lead to finer Pt particles.

2. Optimization of pulse plating parameters. Pulse on-time corresponds to particle growth and pulse off-time is responsible for transfer of the Pt ions from bulk electrolyte to the surface of the carbon electrode. Therefore, shorter on-time and longer off-time may result in finer and more uniform Pt particles.

3. Pulse plating current density, electrolyte concentration, and electrode rotation rate. These parameters play an important role for an optimal Pt morphology and Pt particle size. The optimization of these parameters requires experimental validation and trial and error.

4. The uniformity and activity of carbon substrates increase the $\mathrm{Pt}^{4+}$ absorption. When more active carbon sites are available for $\mathrm{Pt}^{4+}$ absorption, $\mathrm{Pt}$ particle formation will be distributed more evenly and the Pt particle growth will slow down, leading to the reduction of particle size and uniformity of Pt particle distributions.

In this work, catalysts were prepared by electrodeposition on carbon paper that had been loaded with a carbon microporous layer and Nafion ${ }^{\circledR}$, resulting in a three phase reaction zone. Pulse electrodeposition was used with a rotating disk electrode, taking advantage of the benefits of pulse electrodeposition described above. The rotation of the working electrode in rotating disk experiments brought analyte molecules from the bulk solution towards the electrode, resulting in mass transport that is faster than diffusion-only experiments. Catalyst activity was also measured using a rotating disk electrode. Parameters such as the $t_{o f f}, t_{o n}$, duty cycle, peak current density, electrode rotation rate, catalyst precursor, stabilizing agent, carbon support were varied and optimized to achieve high performing electrodes. After achieving high performance, the stability of the electrodes was investigated and the electrodeposition process was scaled up to form an electrode that is a practical size to use in a fuel cell.

\section{Results and Discussion}

\section{Task 1: Establishing a standard catalyst synthesis technique}

The goal of Task 1 was to establish a standard catalyst synthesis technique. Based on a literature review, pulse electrodeposition was selected as the most promising technique to produce catalysts with controlled 
structure, high activity, and low loading. For this task, gas diffusion layers (GDLs) were sprayed with carbon and Nafion, creating a three phase reaction zone. The amount of Nafion and carbon used was controlled so that the electrodes would have the same carbon and Nafion content as the baseline electrodes used at FSEC in fuel cell research. Because mass transport was shown to be an important parameter in catalyst electrodeposition, and because rotating disk electrodes can ameliorate mass transport, a rotating disk electrode with a standard three-electrode setup (shown in Figure 2) was used for both electrodeposition and electrochemical characterization of the catalysts. The practical evaluation of the performance of an electrocatalyst is the measurement of a polarization curve carried out within a PEM fuel. This approach is a costly and time consuming process because a large electrode size is needed. Due to large differences in experimental conditions, catalyst performance measured from a rotating disk does not directly correspond to its performance in a PEMFC. However, from the standpoint of catalyst development and screening, a rotating disk electrode can be useful for the expedient evaluation and catalyst screening.

An electrode holder was made in house and is pictured here as the working electrode. A Teflon holder was made to fit onto the end of the working electrode and hold the GDL in place. In establishing the standard catalyst synthesis technique, several parameters, including electrode rotation rate, $t_{o n}, t_{\text {off }}$, peak current, stabilizing agent, and precursor concentration were varied to obtain optimal electrodeposition conditions, which would be used for all other tasks. Scanning electron microscopy (SEM) and ORR activity were used to characterize the catalysts.

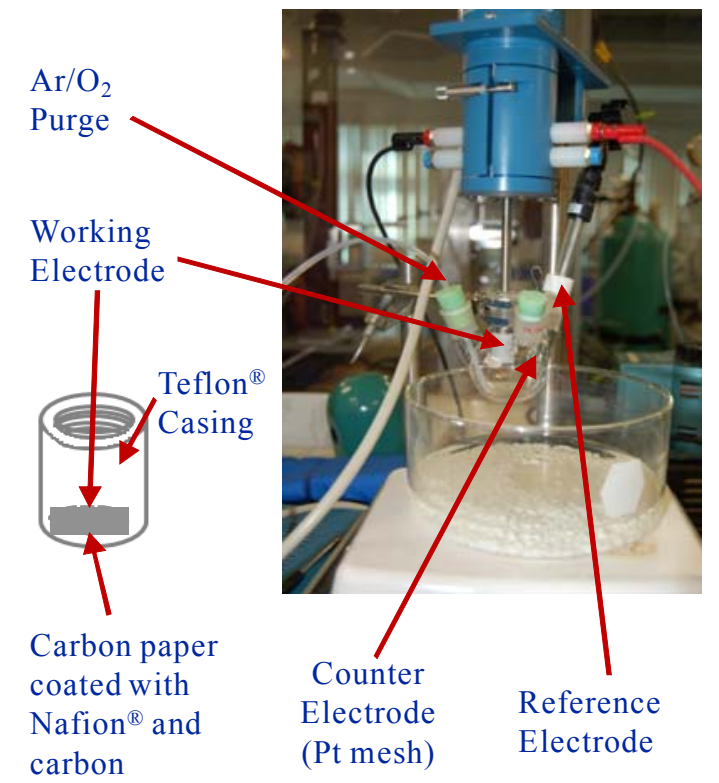

Figure 2. Rotating disk electrode set up that was used for both electrodeposition and electrochemical characterization

\section{Acid Type}

To establish an ORR characterization method, the immersion time of the electrode in oxygen-saturated electrolyte and the type of acid was investigated. In this step, an electrode that was sprayed using a standard FSEC protocol ${ }^{[31]}$ was used.

The two acids that were used for ORR characterization were $0.5 \mathrm{M} \mathrm{H}_{2} \mathrm{SO}_{4}$ and $0.1 \mathrm{M} \mathrm{HClO}_{4}$. There are clear differences between the ORR curves for the two types of acids (see Figure 3 and Figure 4). At high potentials, the $\mathrm{H}_{2} \mathrm{SO}_{4}$ solution showed higher current density, while at lower potentials the $\mathrm{HClO}_{4}$ 
solution showed higher current density. Because the majority of the literature surveyed used $\mathrm{H}_{2} \mathrm{SO}_{4}$ and these curves appeared more well-defined in terms of limiting current (where the current begins to level off as the potential decreases) and onset potentials (the potential at which the ORR curves begin to decrease rapidly as the potential decreases) with $\mathrm{H}_{2} \mathrm{SO}_{4}$, it was decided to use $\mathrm{H}_{2} \mathrm{SO}_{4}$ for characterization in all future studies.

In $\mathrm{H}_{2} \mathrm{SO}_{4}$ the immersion time in oxygen was varied from 20 to 40 minutes (Figure 3), while in $\mathrm{HClO}_{4}$ the immersion time in oxygen was varied from 10 to 30 minutes (Figure 4). It is clear that immersing the sample in oxygen purged acid for longer than 10 minutes does not have a great effect on performance. All further samples were immersed in an oxygen-saturated electrolyte for 15 minutes prior to ORR characterization.

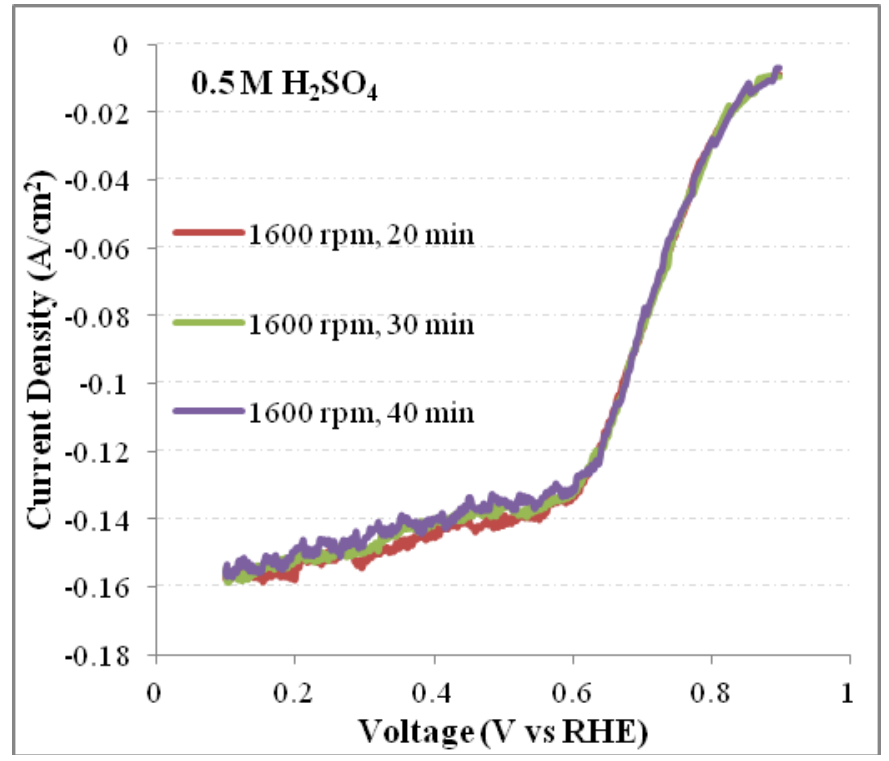

Figure 3. Performance of electrodes with different oxygen immersion times. The plots are the reverse CV curves at $1600 \mathrm{rpm}$. The solution used was $0.5 \mathrm{M} \mathrm{H}_{2} \mathrm{SO}_{4}$ 


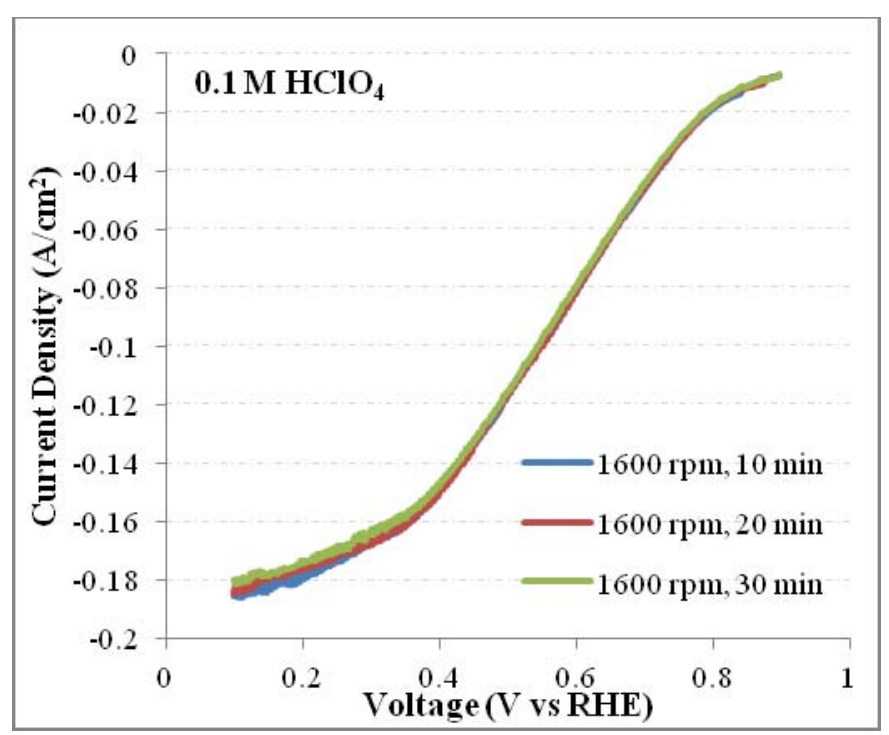

Figure 4. Performance of electrodes with different oxygen immersion times. The plots are the reverse CV curves at $1600 \mathrm{rpm}$. The solution used was $0.1 \mathrm{M} \mathrm{HClO}_{4}$

\section{Precursor Concentration}

The effect of precursor concentration on electrode performance and structure was investigated by varying the concentration of chloroplatinic acid $\left(\mathrm{H}_{2} \mathrm{PtCl}_{6}\right)$ from 1.25 to $10 \mathrm{mM}$. Changing the precursor concentration did not have a large effect on the electrochemical performance of the electrodes, as seen in Figure 5. However, it is clear from the SEM images (Figure 6) that higher concentration of precursor resulted in higher platinum coverage, implying that higher precursor concentration results in lower utilization. Electrodes produced from lower precursor concentration had a "feathery" structure, which would likely have a higher surface area and would thus improve platinum utilization. The feathery structure disappeared as precursor concentration increased.

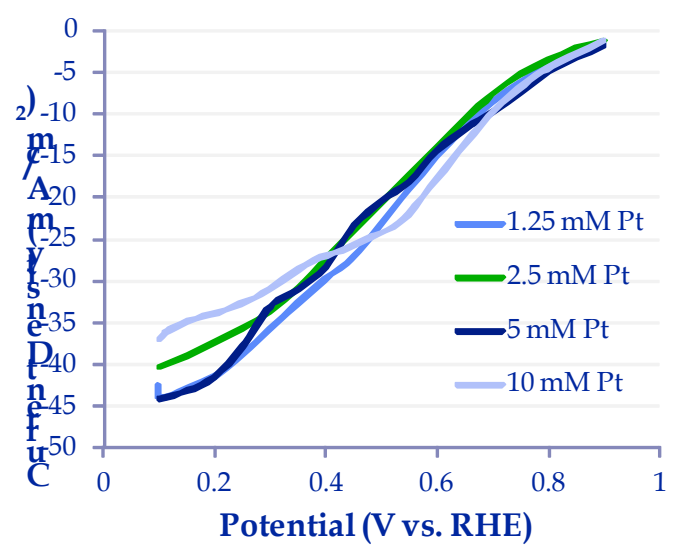

Figure 5. Performance of electrodes with different precursor $\left(\mathrm{H}_{2} \mathrm{PtCl}_{6}\right)$ concentration. The plots are the reverse $\mathrm{CV}$ curves at $2500 \mathrm{rpm}$. The electrodeposition solution contained $0.22 \mathrm{M}$ IPA and $0.5 \mathrm{M} \mathrm{H}_{2} \mathrm{SO}_{4}$. The peak current was $400 \mathrm{~mA} / \mathrm{cm}^{2}$, on/off times were $25 \mathrm{~ms} / 50 \mathrm{~ms}$, rotation rate was $400 \mathrm{rpm}$, and 800 cycles were used. 

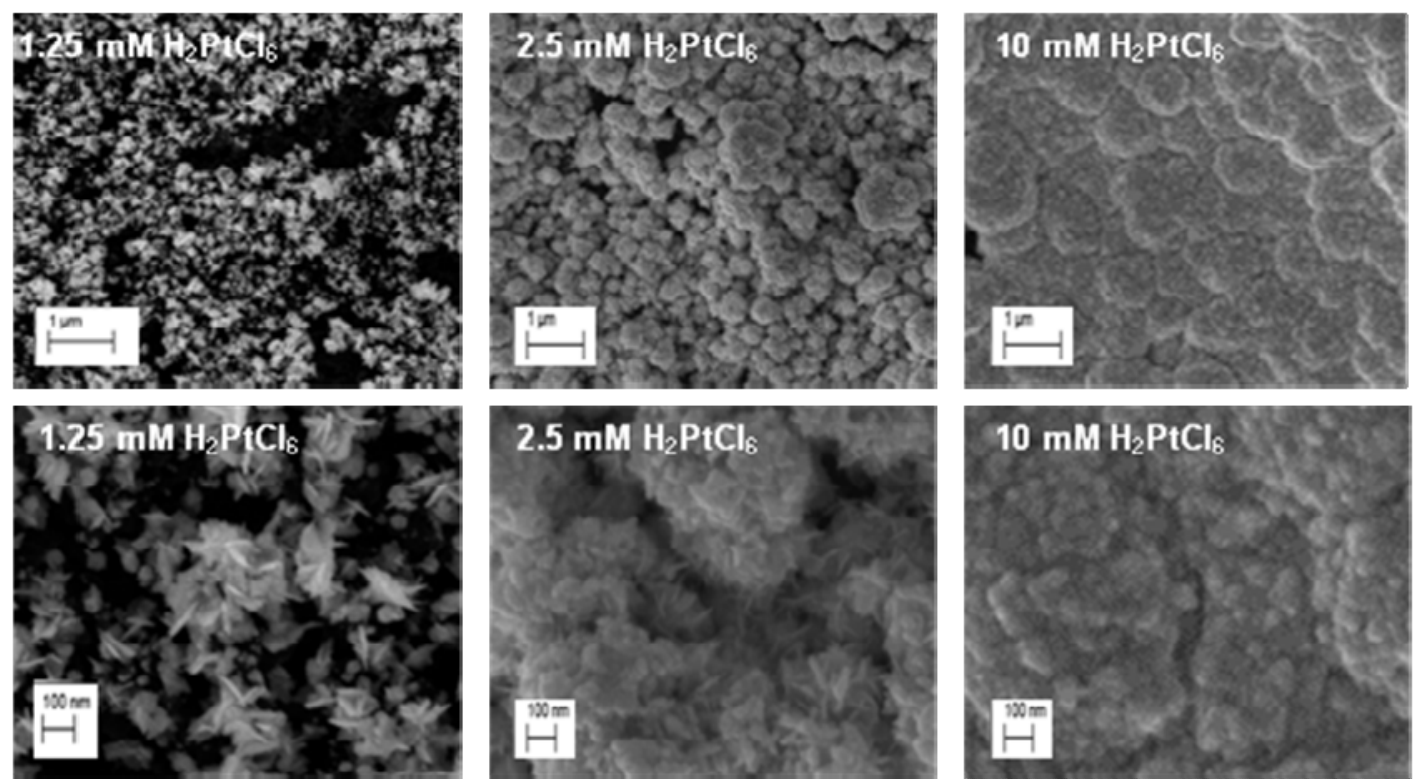

Figure 6. SEM images of electrodes fabricated using 1.25, 2.5, and $10 \mathrm{mM} \mathrm{H}_{2} \mathrm{PtCl}_{6}$

\section{Rotation Rate}

The effect of the electrode rotation rate during electrodeposition on electrode performance and structure was investigated by varying the rotation rate from 100 to $1600 \mathrm{rpm}$. Increasing the rotation rate above 100 rpm greatly improved the performance of the electrodes, as seen in Figure 7. Increasing the rotation rate above $400 \mathrm{rpm}$ did not have a large impact on ORR activity at $0.8 \mathrm{~V}$, although performance increased with increasing rotation rate at low voltage, which is the limiting current density region. Examination of the SEM images (Figure 8) shows no clear relationship between rotation rate and catalyst structure.

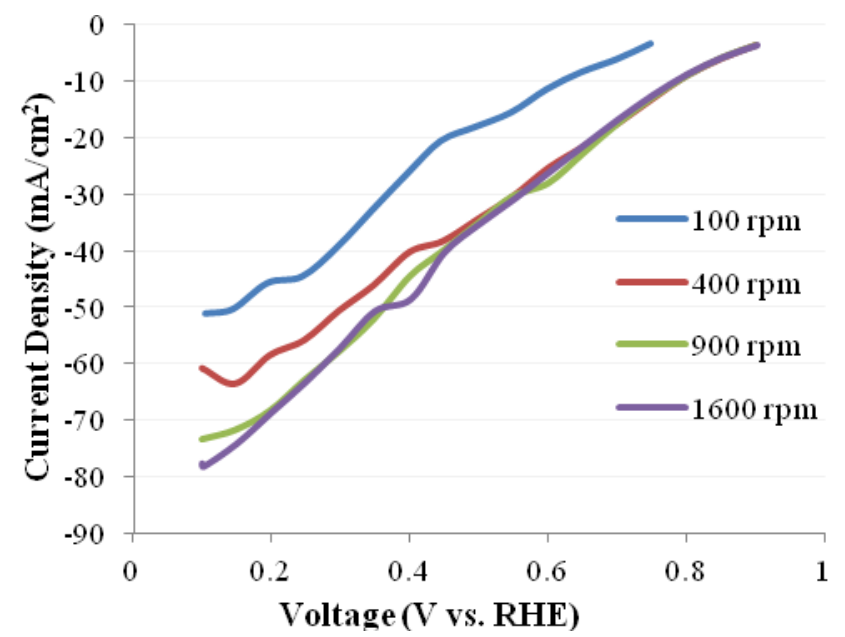

Figure 7. Performance of electrodes at different electrode rotation rates during electrodeposition. The electrodeposition solution contained $0.5 \mathrm{M} \mathrm{H}_{2} \mathrm{SO}_{4}$ and $1.25 \mathrm{mM} \mathrm{H}_{2} \mathrm{PtCl}_{6}$. The plots are the reverse CVs at $2500 \mathrm{rpm}$ in $0.5 \mathrm{M} \mathrm{H}_{2} \mathrm{SO}_{4}$. The peak current was $400 \mathrm{~mA} / \mathrm{cm}^{2}$, on/off times were $25 / 50 \mathrm{~ms}$ and 200 cycles were used. 


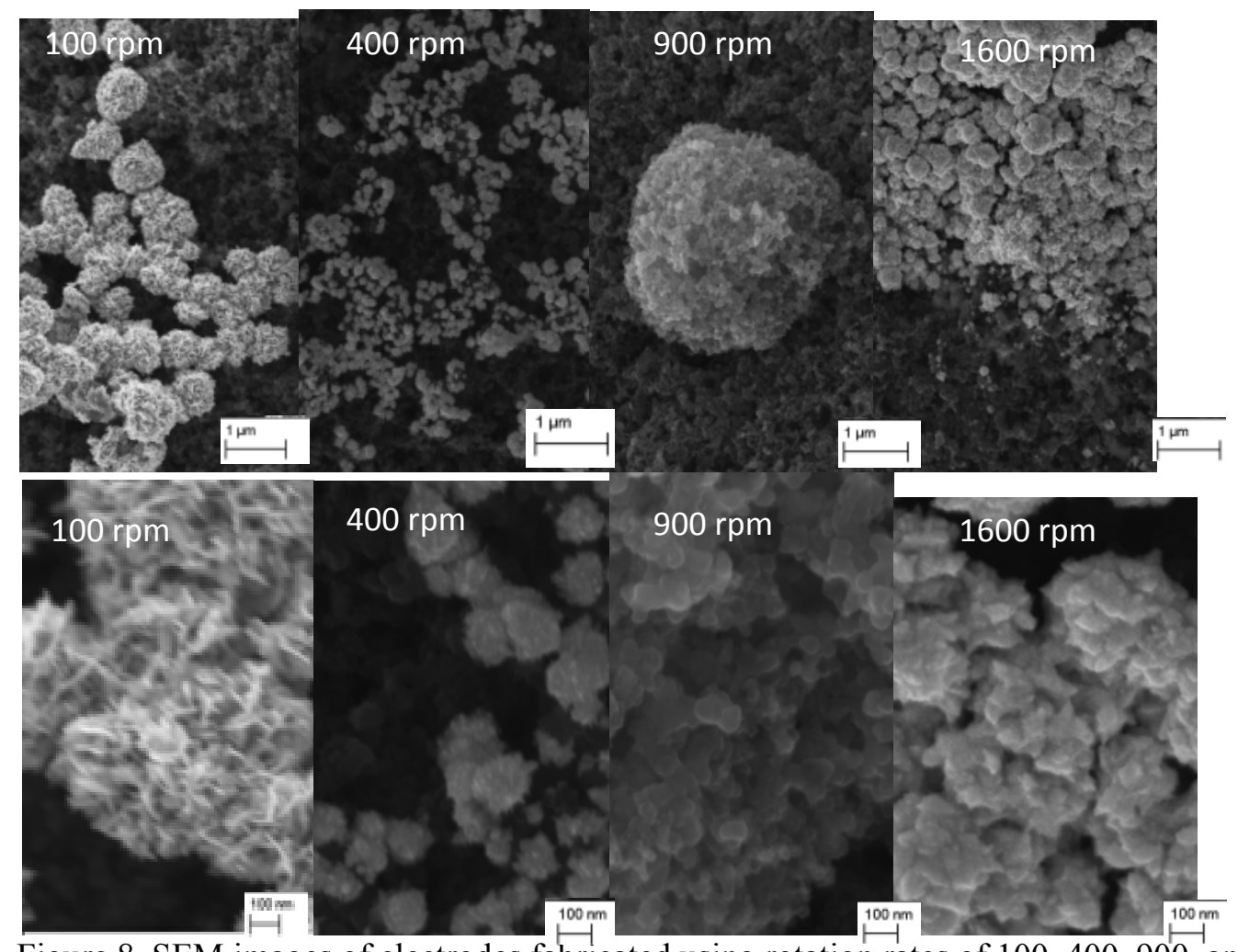

Figure 8. SEM images of electrodes fabricated using rotation rates of $100,400,900$, and $1600 \mathrm{rpm}$

\section{Peak Current}

An advantage to pulse electrodeposition compared to direct current deposition is that pulse electrodeposition can raise the limiting current density considerably in comparison with direct current electrodeposition because the depletion of metal ions near the cathode surface can be re-supplied from the bulk solution during the off time of the pulse. This prevents the deposited metal ions near the cathode from becoming exhausted and forming dendritic crystals. Using different peak currents, but keeping the number of deposition cycles the same changes the total charge applied for electrodeposition. By varying the total charge applied for electrodeposition, the amount of platinum loading in the catalyst layer can be controlled. In Figure 9, it is clear that increasing the peak current from 50 to $400 \mathrm{~mA} / \mathrm{cm}^{2}$ increases the performance of the electrodes. However, when normalized for mass of platinum (Figure 10) the electrode that was prepared with a peak current of $400 \mathrm{~mA} / \mathrm{cm}^{2}$ had the lowest performance, while that prepared with a peak current of $50 \mathrm{~mA} / \mathrm{cm}^{2}$ had the highest performance. SEM images for electrodes that were plated with peak currents of 50,100, and $400 \mathrm{~mA} / \mathrm{cm}^{2}$ are shown in Figure 11. From these images it appears that increasing the peak current results in smaller Pt agglomerate size. Although the improved performance with increasing peak current can be attributed to increased platinum loading, it is clear that the Pt has lower utilization as peak current density increases. 


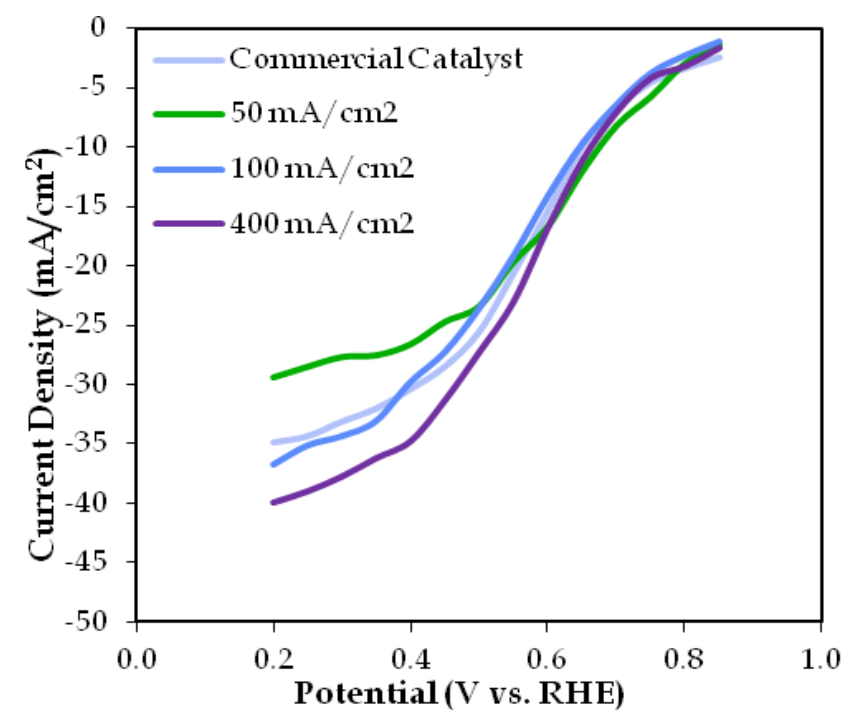

Figure 9. Performance of electrodes for different peak current densities during electrodeposition. The electrodeposition solution contained $0.5 \mathrm{M} \mathrm{H}_{2} \mathrm{SO}_{4}$ and $1.25 \mathrm{mM} \mathrm{H}_{2} \mathrm{PtCl}_{6}$. The plots are the reverse CVs at $2500 \mathrm{rpm}$ in $0.5 \mathrm{M} \mathrm{H}_{2} \mathrm{SO}_{4}$. The on/off times were $25 / 50 \mathrm{~ms}$, rotation rate was $400 \mathrm{rpm}$, and 200 cycles were used. The commercial catalyst is a Pt catalyst coated carbon cloth from E-TEK with a Pt loading of $0.5 \mathrm{mg} / \mathrm{cm}^{2}$.

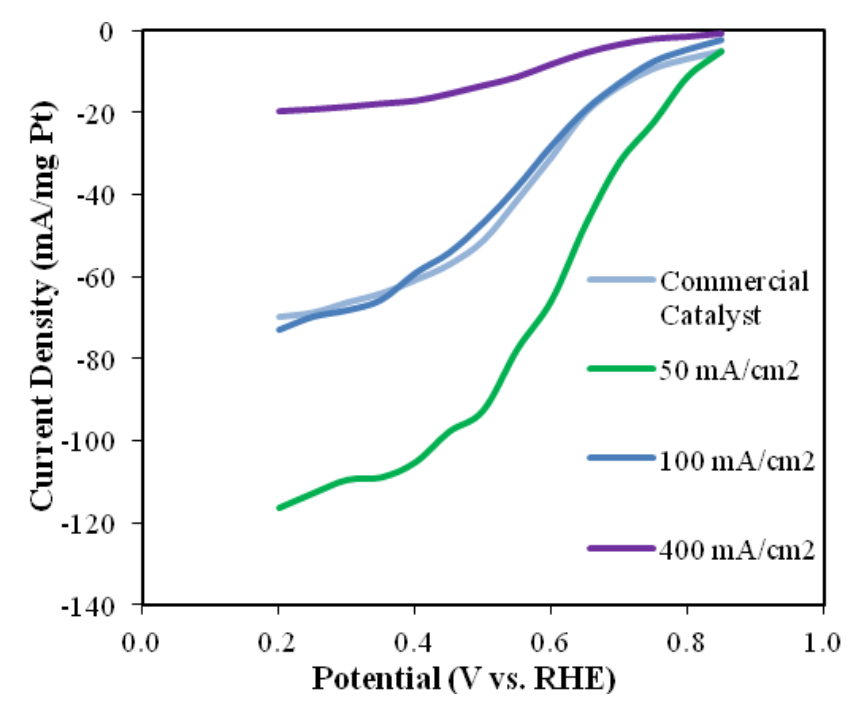

Figure 10. Performance of electrodes for different peak current densities during electrodeposition normalized by mass of Pt deposited 


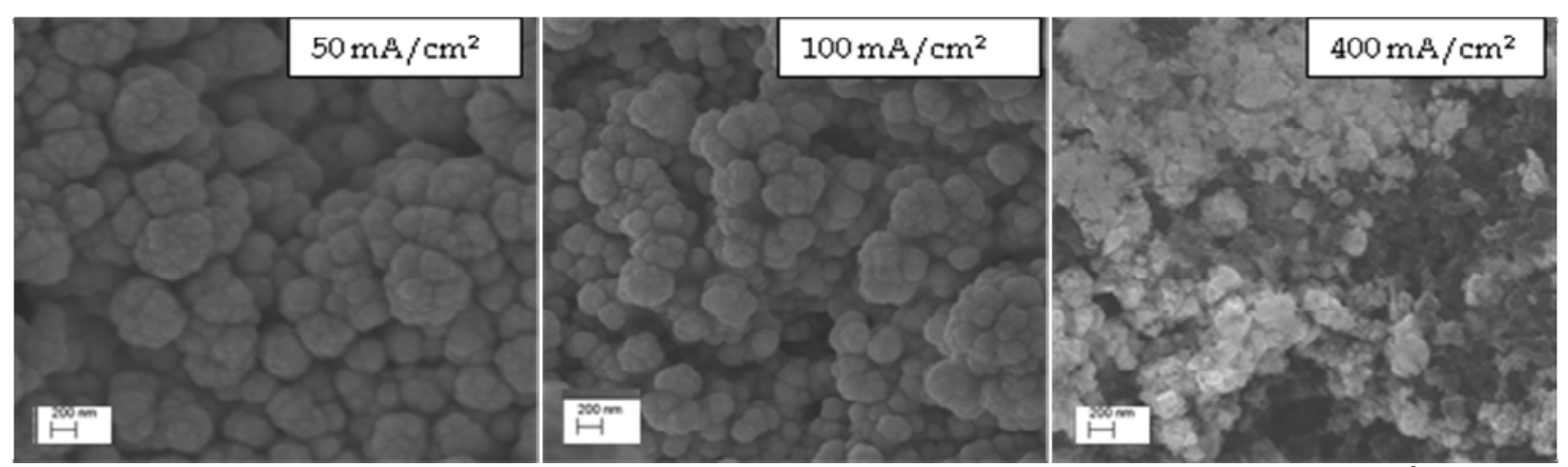

Figure 11. SEM images of electrodes fabricated using peak currents of 50,100 , and $400 \mathrm{~mA} / \mathrm{cm}^{2}$ during electrodeposition

\section{Number of Cycles}

Another way to increase the total charge, and therefore the amount of Pt deposited, is by increasing the number of cycles. As shown in Figure 12, although performance is increased when the number of cycles is increased from 400 to 600 , when the number of cycles is increased further, there is no effect on performance. The performance was normalized for the mass of Pt on the electrode (Figure 13) and in this case it is clear that performance decreases with increasing number of cycles. This means that with lower cycles, the Pt is used more efficiently. From the SEM images (Figure 14), the Pt particle size increases from 0.04 to $0.5 \mathrm{~m}$ for 400 cycles to 0.1 to $0.9 \mathrm{~m}$ for 1000 cycles. The Pt particles also change from feathery or flakey in shape to round as the number of electrodeposition cycles increases. This higher surface area despite lower Pt loading for the electrode with 400 cycles explains why the Pt is used more efficiently with lower cycle numbers. TEM images show that, at high magnification, the flakey structure can still be observed even with 3200 cycles (Figure 15).

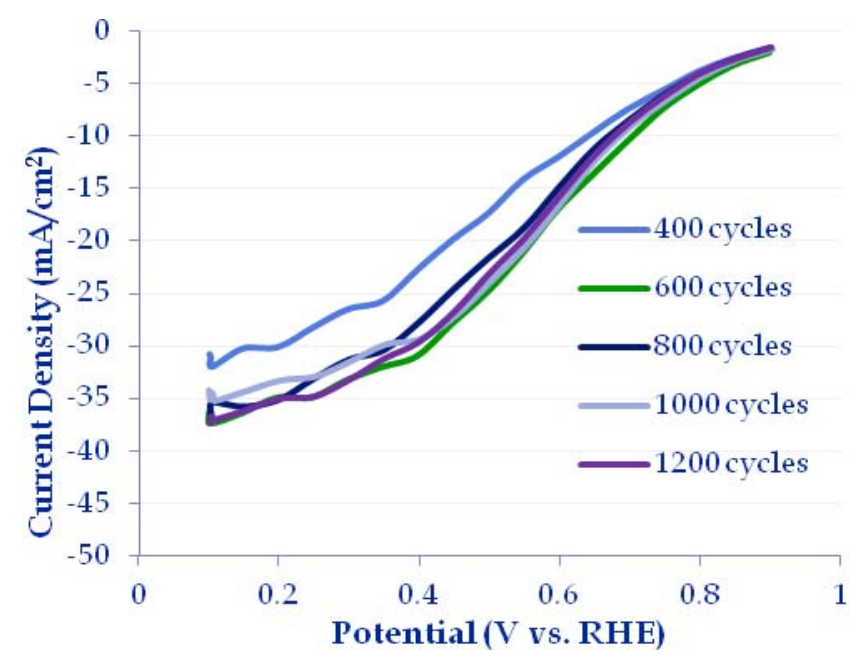

Figure 12. Performance of electrodes prepared using different numbers of electrodeposition cycles. The electrodeposition solution contained $0.22 \mathrm{M}$ IPA, $0.5 \mathrm{M} \mathrm{H}_{2} \mathrm{SO}_{4}$, and $1.25 \mathrm{mM} \mathrm{H}_{2} \mathrm{PtCl}_{6}$. The plots are the reverse CVs at $2500 \mathrm{rpm}$ in $0.5 \mathrm{M} \mathrm{H}_{2} \mathrm{SO}_{4}$. The peak current was $400 \mathrm{~mA} / \mathrm{cm}^{2}$, rotation rate was $400 \mathrm{rpm}$, and on/off times were $25 / 50 \mathrm{~ms}$. 


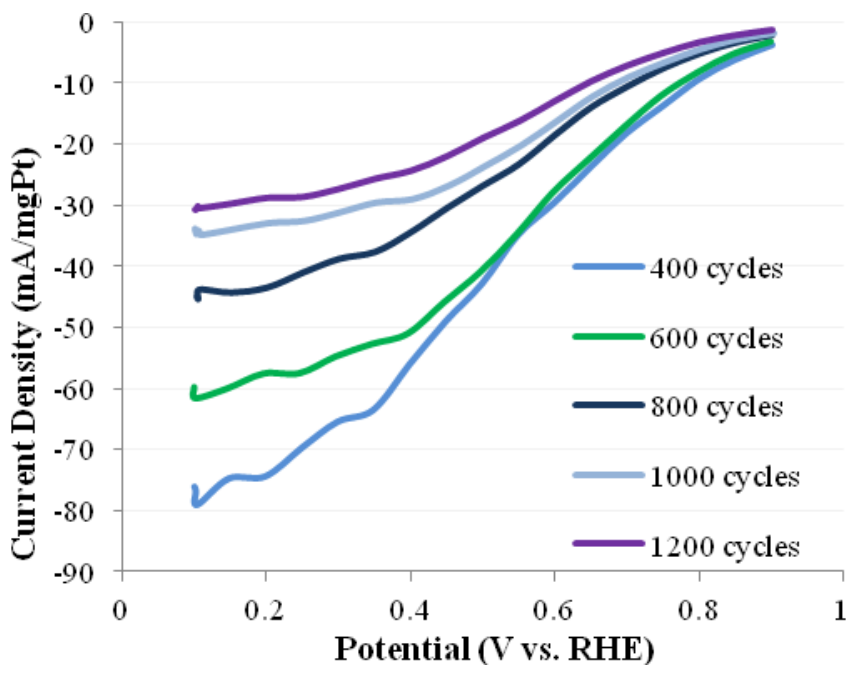

Figure 13. Performance of electrodes prepared using different numbers of electrodeposition cycles normalized for mass of platinum on the electrode
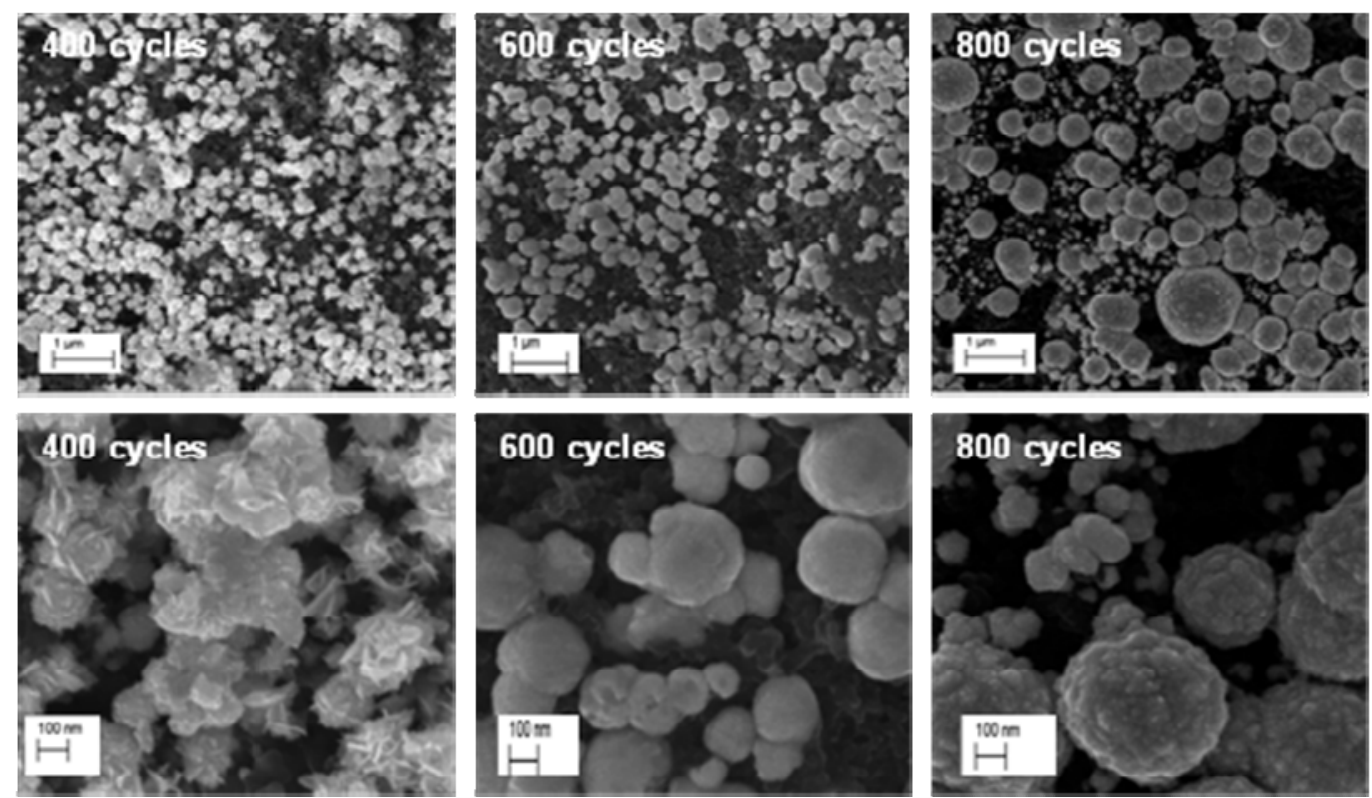

Figure 14. SEM images of electrodes fabricated using 400, 600, and 800 electrodeposition cycles 


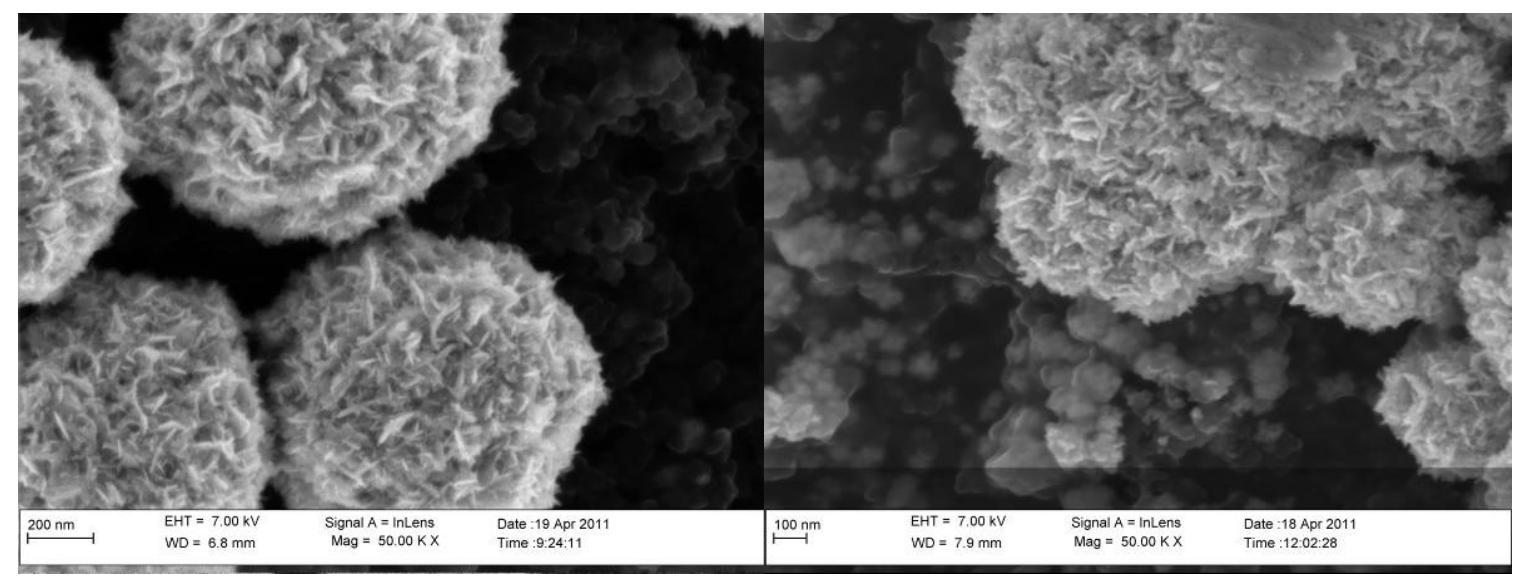

Figure 15. TEM images of particles generated by 3200 cycles

\section{Off time}

The effect of the off time during electrodeposition on electrode performance and structure was investigated by varying the off time from 10 to $300 \mathrm{~ms}$. From the ORR curves (Figure 16), it is clear that performance was the worst when the off time was $10 \mathrm{~ms}$ and it was the best when the off time was $50 \mathrm{~ms}$. Examination of the SEM images (Figure 17) shows that the average particle size of the Pt increases as off time increases.

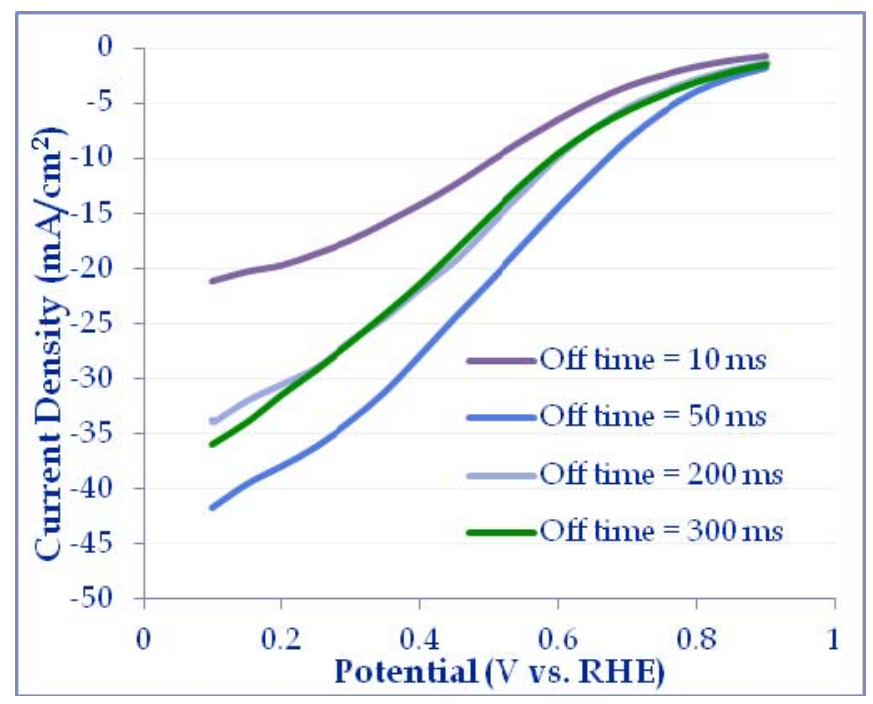

Figure 16. Performance of electrodes as a function of off time. The electrodeposition solution contained $0.22 \mathrm{M}$ IPA, $0.5 \mathrm{M} \mathrm{H}_{2} \mathrm{SO}_{4}$, and $1.25 \mathrm{mM} \mathrm{H}_{2} \mathrm{PtCl}_{6}$. The plots are the reverse CVs at $2500 \mathrm{rpm}$ in $0.5 \mathrm{M}$ $\mathrm{H}_{2} \mathrm{SO}_{4}$. The peak current was $400 \mathrm{~mA} / \mathrm{cm}^{2}$, rotation rate was $400 \mathrm{rpm}$, and on time was $25 \mathrm{~ms}$. 

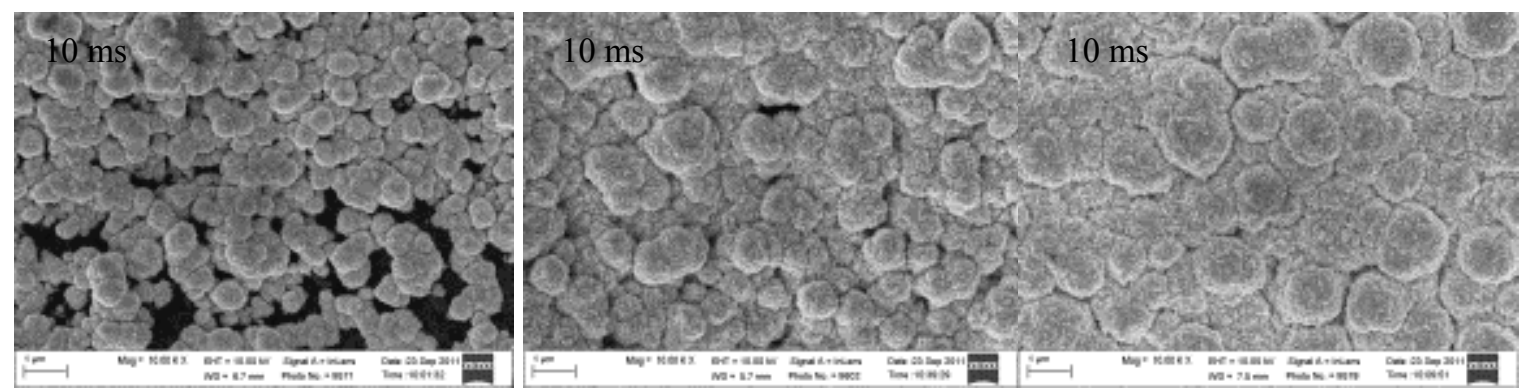

Figure 17. SEM images of electrodes fabricated using off times of 10, 50, and $200 \mathrm{~ms}$.

\section{On time}

The effect of the on time during electrodeposition on electrode performance and structure was investigated by varying the on time from 5 to $50 \mathrm{~ms}$. From the ORR curves (Figure 18), it is clear that performance was the worst when the on time was $5 \mathrm{~ms}$ and it was the best when the on time was $25 \mathrm{~ms}$. Examination of the SEM images (Figure 19) shows that the average particle size of the Pt and number of "3-D" features did not change as the on time changed.

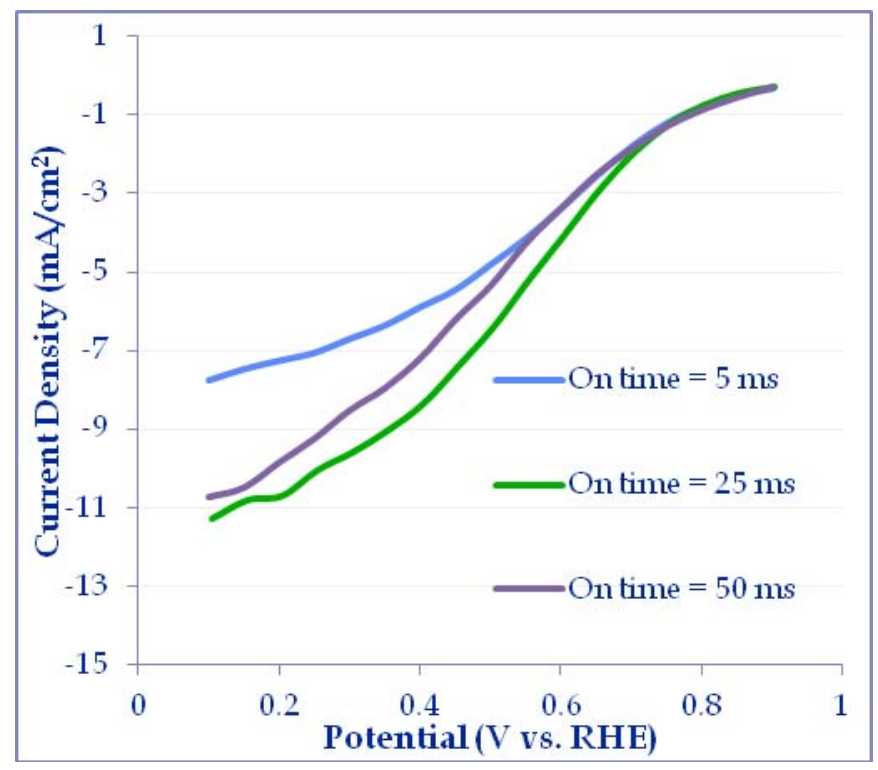

Figure 18. Performance of electrodes as a function of on time. The electrodeposition solution contained $0.22 \mathrm{M}$ IPA, $0.5 \mathrm{M} \mathrm{H}_{2} \mathrm{SO}_{4}$, and $1.25 \mathrm{mM} \mathrm{H}_{2} \mathrm{PtCl}_{6}$. The plots are the reverse CVs at $2500 \mathrm{rpm}$ in $0.5 \mathrm{M}$ $\mathrm{H}_{2} \mathrm{SO}_{4}$. The peak current was $400 \mathrm{~mA} / \mathrm{cm}^{2}$, rotation rate was $400 \mathrm{rpm}$, and off time was $10 \mathrm{~ms}$. 


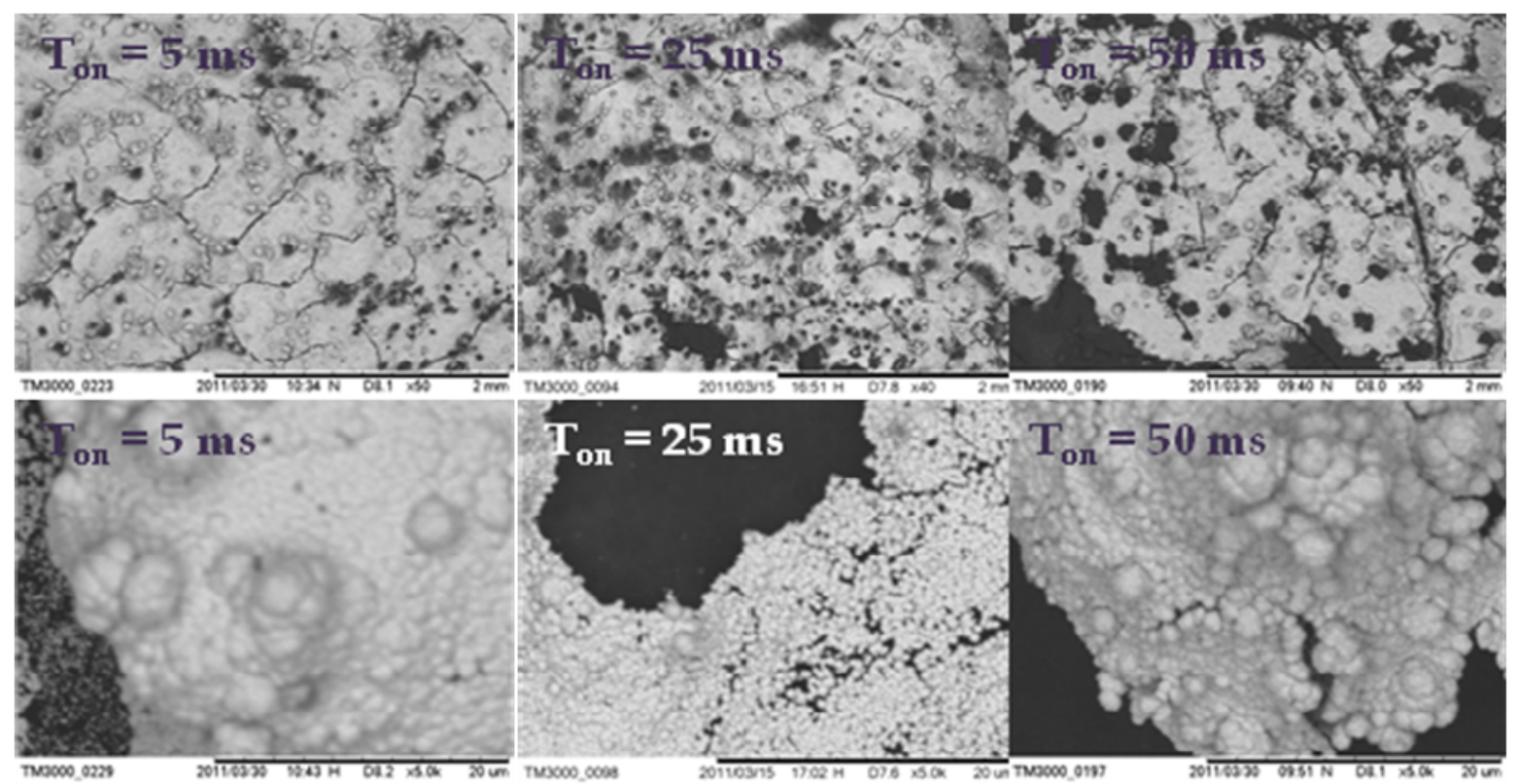

Figure 19. SEM images of electrodes fabricated using on times of 5, 25, and $50 \mathrm{~ms}$.

\section{Stabilizing Agent}

Previously FSEC and others showed decreased catalyst particle size due to stabilization from additives. In this work, methanol (MeOH), glycerol, isopropyl alcohol (IPA), and polyvinylpyrrolidone (PVP) were used as additives.

\section{Methanol}

Addition of methanol resulted in improved performance (Figure 20) and decreasing particle size (Figure 21) with increasing methanol concentration. Increased performance with increasing methanol concentration was attributed to increased active surface area of the platinum.

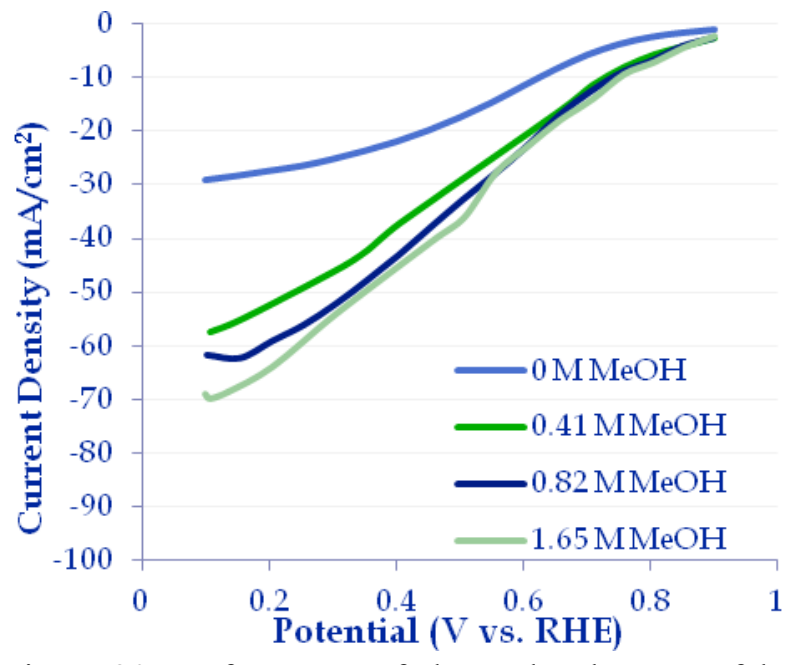

Figure 20. Performance of electrodes that were fabricated with different methanol $(\mathrm{MeOH})$ concentrations during electrodeposition. The plots are the reverse $\mathrm{CV}$ curves taken at $2500 \mathrm{rpm}$ in $0.5 \mathrm{M} \mathrm{H}_{2} \mathrm{SO}_{4}$. Platinum was deposited from a solution containing $10 \mathrm{mM} \mathrm{H}_{2} \mathrm{PtCl}_{6}$ and $0.5 \mathrm{M} \mathrm{H}_{2} \mathrm{SO}_{4}$, with a peak current of $400 \mathrm{~mA} / \mathrm{cm} 2$ ?, on/off times of $25 \mathrm{~ms} / 50 \mathrm{~ms}$, rotation rate was $400 \mathrm{rpm}$, and 800 cycles. 


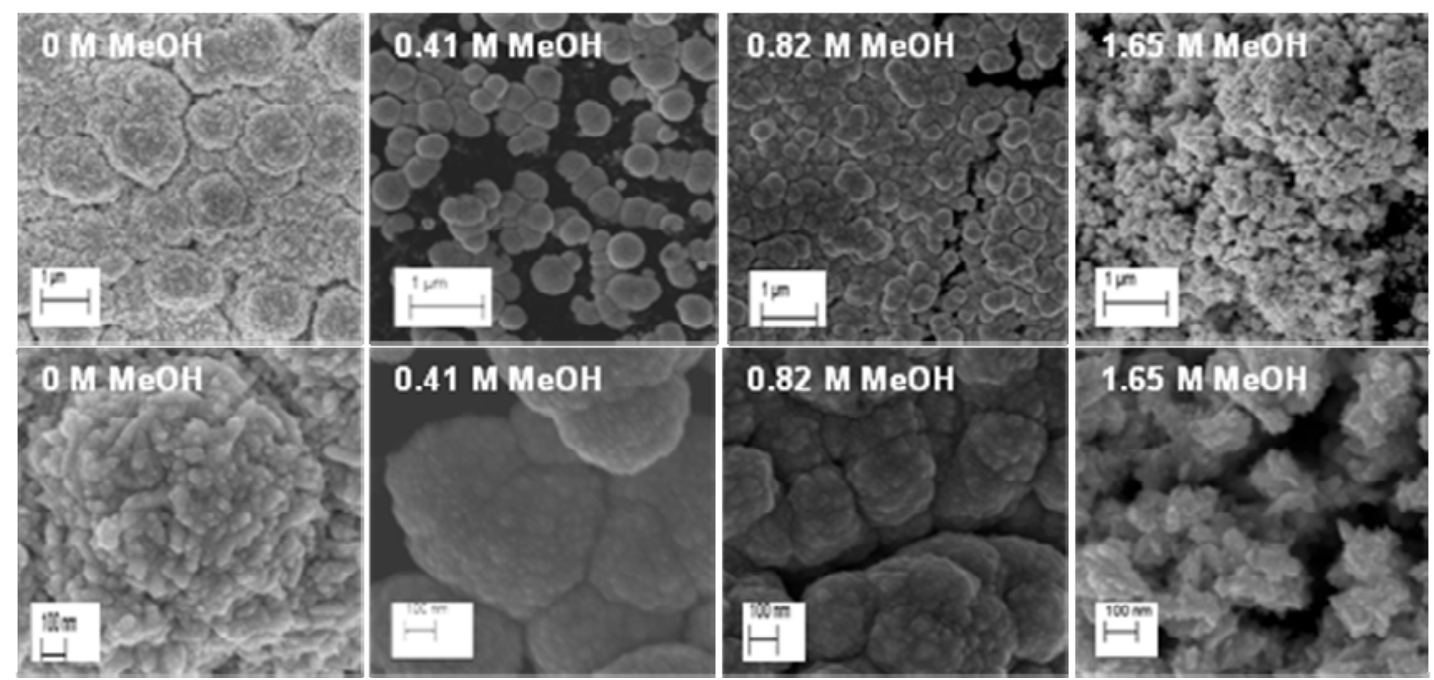

Figure 21. SEM images of electrodes with 0, 0.41, 0.82, and 1.65 $\mathrm{M}$ methanol $(\mathrm{MeOH})$ during electrodeposition

\section{Isopropanol}

Addition of isopropanol (IPA) resulted in improved performance (Figure 22) and decreasing particle size (Figure 23) with increasing IPA concentration. Like with the methanol, increased performance with increasing IPA concentration was attributed to increased active surface area of the platinum.

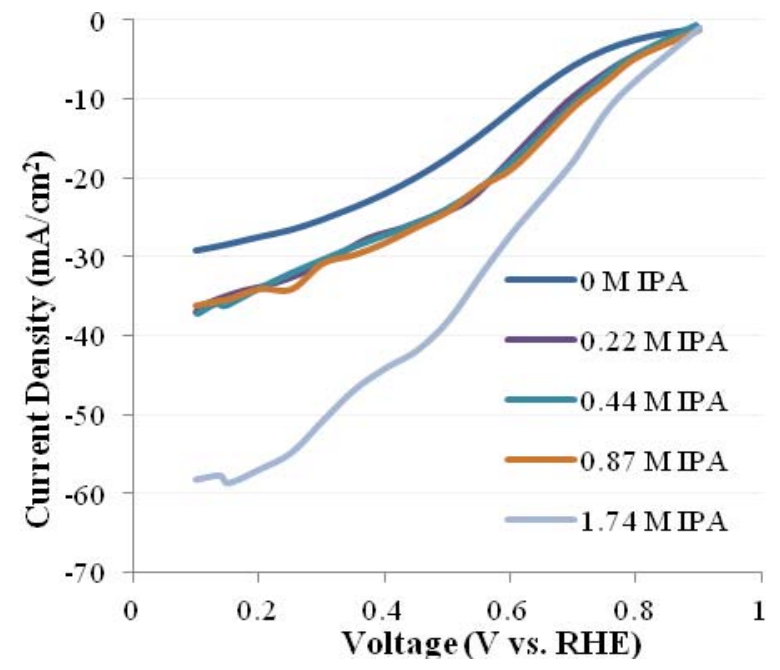

Figure 22. Performance of electrodes that were fabricated with different isopropanol (IPA) concentrations during electrodeposition. The plots are the reverse $\mathrm{CV}$ curves taken at $2500 \mathrm{rpm}$ in $0.5 \mathrm{M} \mathrm{H}_{2} \mathrm{SO}_{4}$. Platinum was deposited from a solution containing $10 \mathrm{mM} \mathrm{H}_{2} \mathrm{PtCl}_{6}$ and $0.5 \mathrm{M} \mathrm{H}_{2} \mathrm{SO}_{4}$, with a peak current of $400 \mathrm{~mA} / \mathrm{cm}^{2}$, on/off times of $25 \mathrm{~ms} / 50 \mathrm{~ms}$, rotation rate was $400 \mathrm{rpm}$, and 800 cycles. 

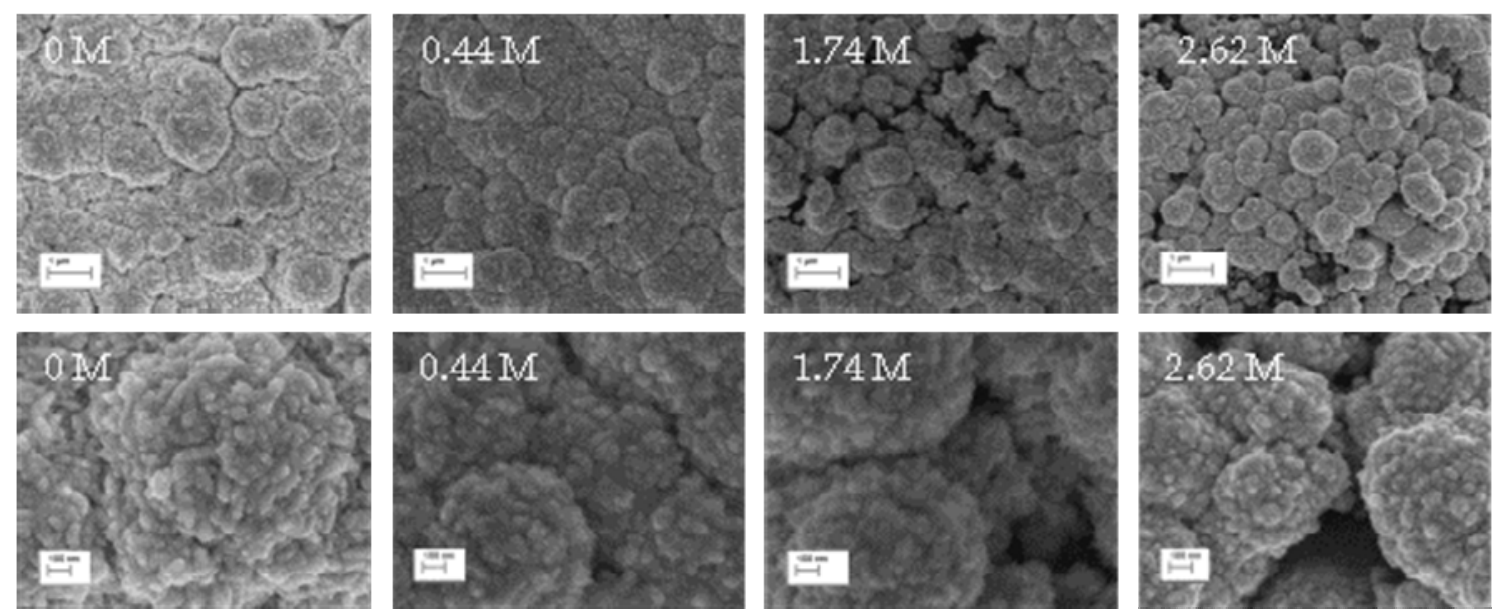

Figure 23. SEM images of electrodes with $0,0.44,1.74$, and $2.62 \mathrm{M}$ IPA during electrodeposition

\section{Glycerol}

Addition of $0.46 \mathrm{M}$ glycerol to the electrodeposition solution resulted in no change in performance (Figure 24). However, higher concentrations of glycerol showed improved performance. Platinum coverage was very low in the electrode that had $0.46 \mathrm{M}$ glycerol in the electrodeposition solution (Figure 25), which could explain the lower performance for this electrode. Addition of glycerol to the electrodeposition solution resulted in smaller Pt particle sizes, which likely resulted in the improved performance for the electrodes that were deposited from solutions containing higher concentrations of glycerol.

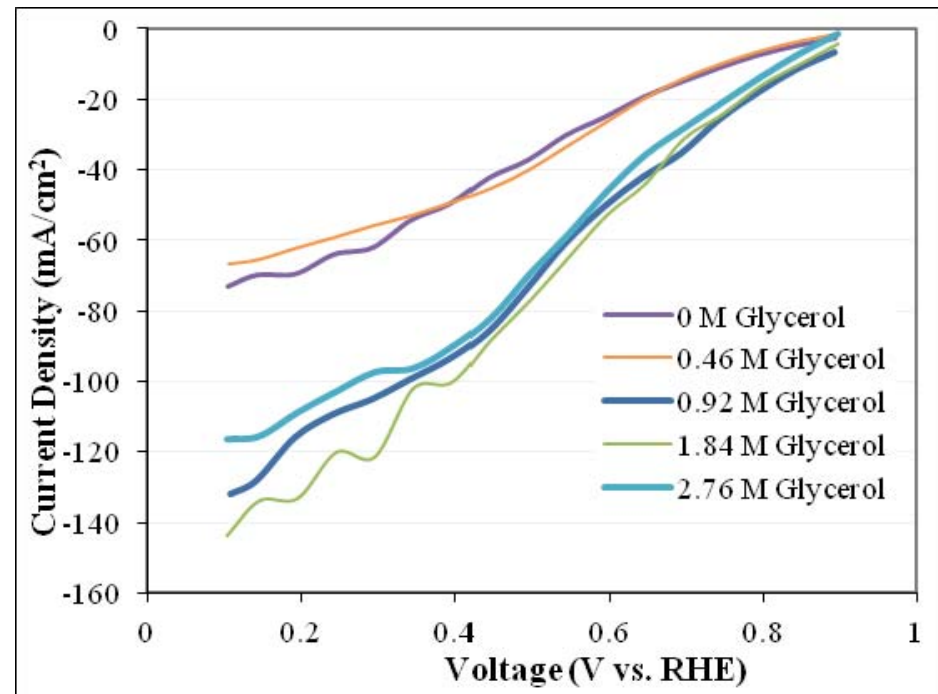

Figure 24. Performance of electrodes that contained different glycerol concentration during electrodeposition. The plots are the reverse $\mathrm{CV}$ curves taken at $2500 \mathrm{rpm}$ in $0.5 \mathrm{M} \mathrm{H}_{2} \mathrm{SO}_{4}$. Platinum was deposited from a solution containing $10 \mathrm{mM} \mathrm{H}_{2} \mathrm{PtCl}_{6}$ and $0.5 \mathrm{M} \mathrm{H}_{2} \mathrm{SO}_{4}$, with a peak current of 400 $\mathrm{mA} / \mathrm{cm}^{2}$, on/off times of $25 \mathrm{~ms} / 50 \mathrm{~ms}$, rotation rate was $400 \mathrm{rpm}$, and 800 cycles 

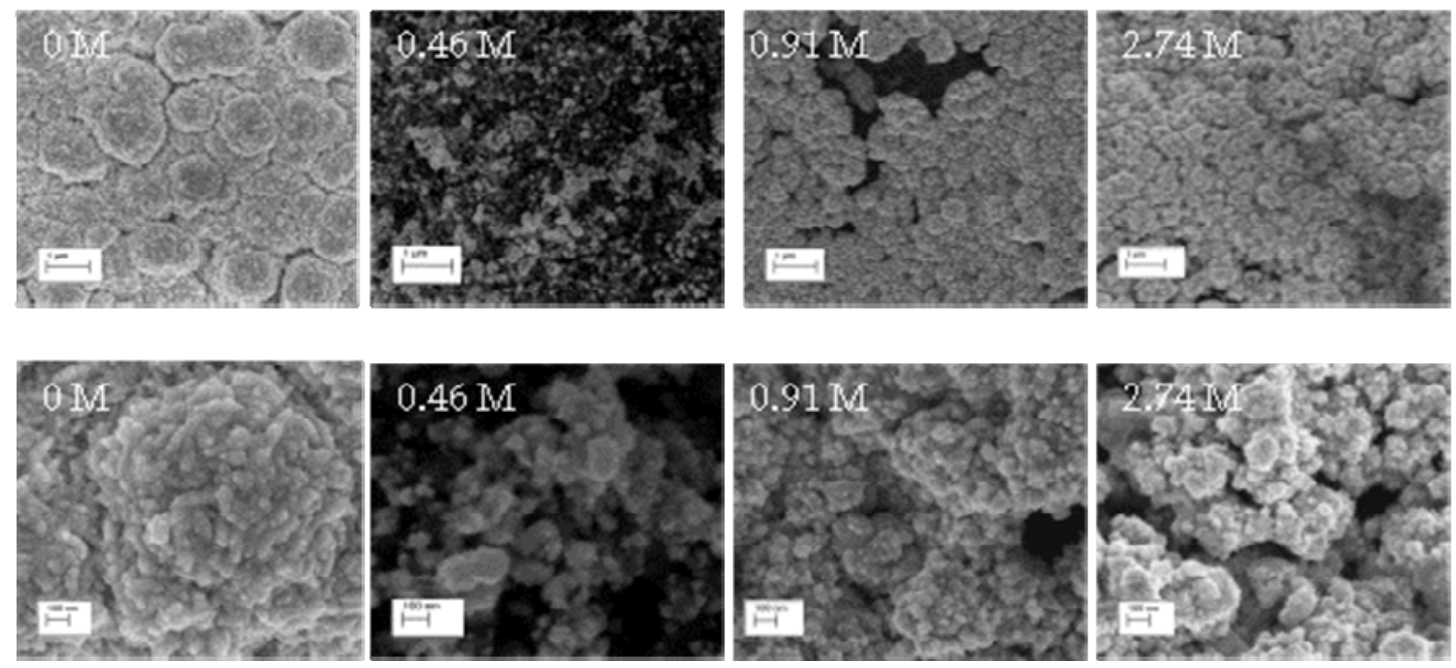

Figure 25. SEM images of electrodes that contained 0, 0.46, 0.91, and 2.74 $\mathrm{M}$ glycerol in the electrodeposition solution.

\section{$P V P$}

Addition of polyvinylpyrrolidone (PVP) resulted in improved performance (Figure 26) and increasing particle size and coverage (Figure 27) with increasing PVP concentration. The improved performance with increased PVP concentration is attributed to increased catalyst coverage of the electrode.

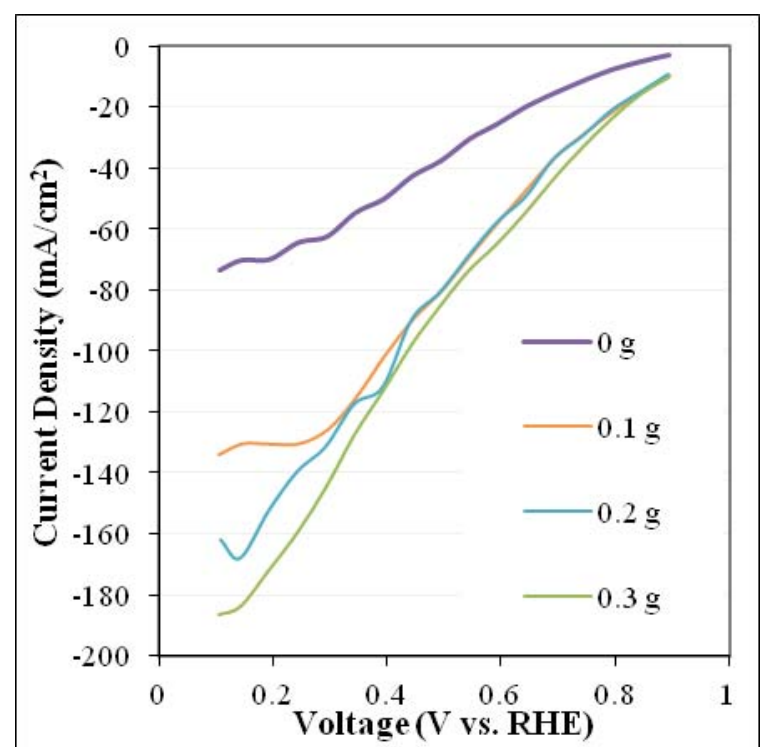

Figure 26. Performance of electrodes that were deposited using different polyvinylpyrrolidone (PVP) concentration during electrodeposition. The plots are the reverse CV curves taken at $2500 \mathrm{rpm}$ in $0.5 \mathrm{M}$ $\mathrm{H}_{2} \mathrm{SO}_{4}$. Platinum was deposited from a solution containing $1.25 \mathrm{mM} \mathrm{H}_{2} \mathrm{PtCl}_{6}$ and $0.5 \mathrm{M} \mathrm{H}_{2} \mathrm{SO}_{4}$, with a peak current of $400 \mathrm{~mA} / \mathrm{cm}^{2}$, on/off times of $25 \mathrm{~ms} / 50 \mathrm{~ms}$, rotation rate was $400 \mathrm{rpm}$, and 800 cycles. 

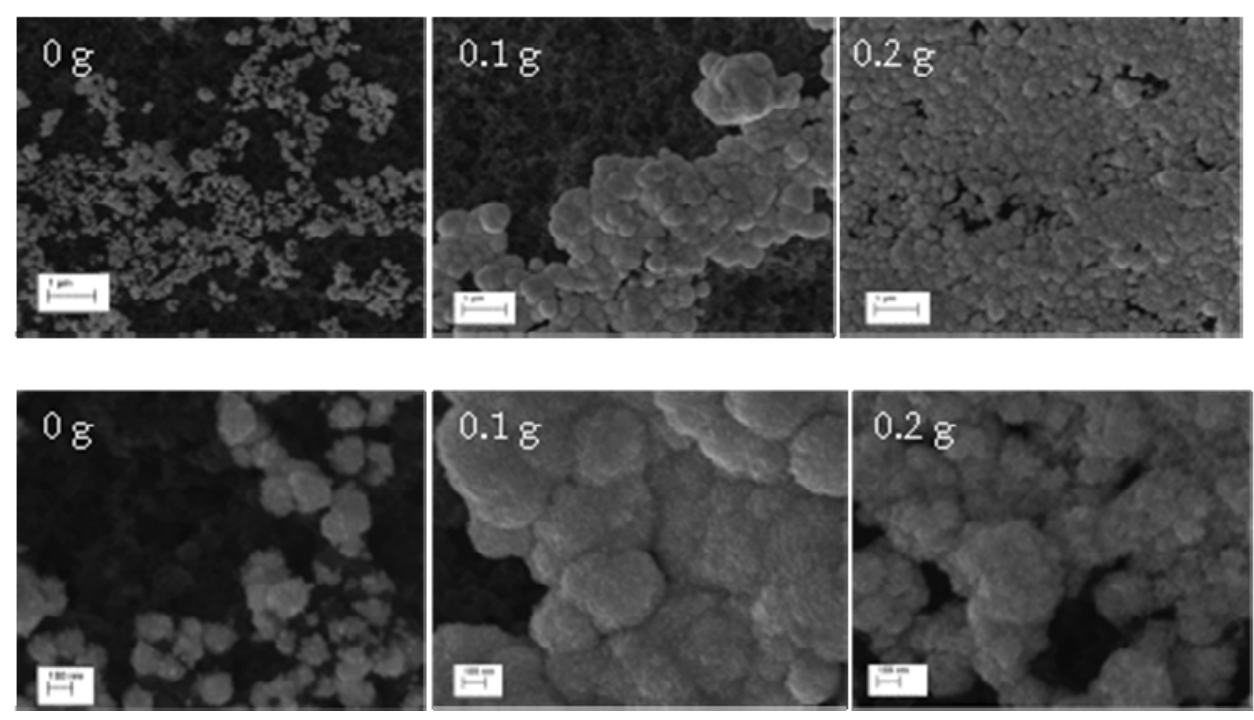

Figure 27. SEM images of electrodes with $0,0.1$, and $0.2 \mathrm{~g}$ PVP during electrodeposition. The plots are the reverse $\mathrm{CV}$ curves taken at $2500 \mathrm{rpm}$ in $0.5 \mathrm{M} \mathrm{H}_{2} \mathrm{SO}_{4}$.

\section{Summary}

In Task 1, optimum values of several variables (platinum precursor concentration, electrode rotation rate, peak current, number of cycles, on time, off time, stabilizer concentration) during electrodeposition were selected to achieve the highest electrode performance. These values were used in all future electrodepositions, except where noted. It was evident that electrode morphology impacted performance in that improved performance was generally observed with higher electrode coverage, smaller particle size, and the formation of "feathery" or "flakey" particles.

\section{Task 2: Screening of the Carbon Supports}

The goal of Task 2 was to evaluate carbon supports used in the gas diffusion electrodes. Pt can be well dispersed on a high surface area substrate to attain high utilization of noble metals. However, catalyst supports can affect both the activity and the stability of electrodes in fuel cells.

\section{Pretreatment}

Thermal pre-treatment of carbon under inert atmosphere at high temperature has been shown to efficiently remove the impurities present on the carbon surfaces, leading to an increase in the active surface area of the carbon. The effect of thermally treating Vulcan XC-72 carbon at $600{ }^{\circ} \mathrm{C}$ for 2 hours on electrode performance was investigated. As shown in Figure 28, the thermal treatment had little effect on ORR activity. 


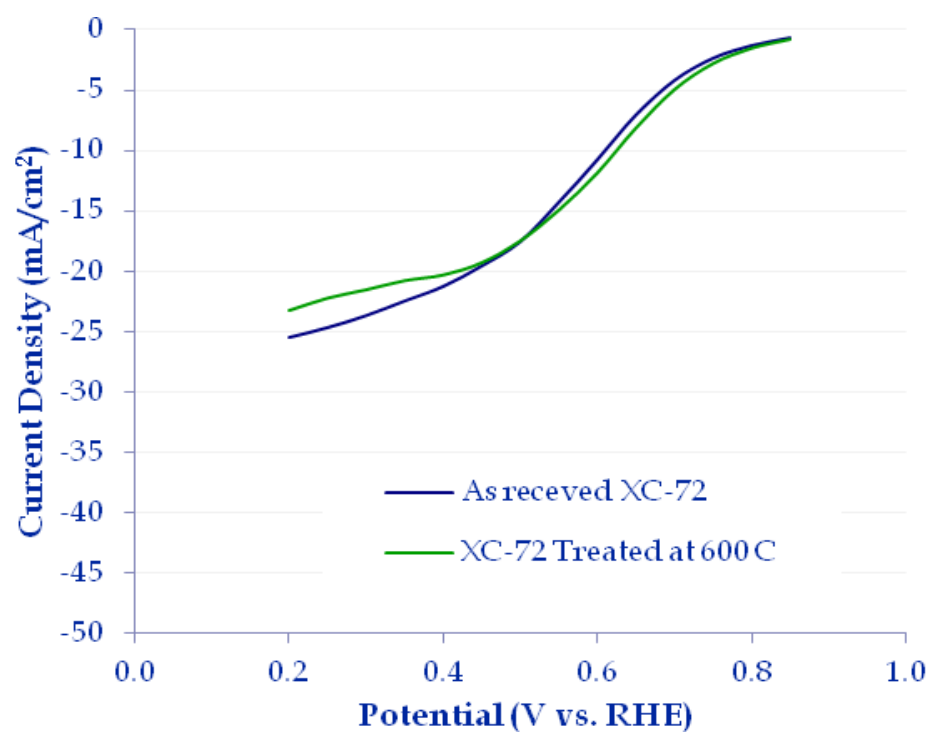

Figure 28. Performance of electrodes containing as received XC-72 carbon and thermally treated XC-72 carbon as supports. The electrodeposition solution contained $0.5 \mathrm{M} \mathrm{H}_{2} \mathrm{SO}_{4}$ and $1.25 \mathrm{mM} \mathrm{H}_{2} \mathrm{PtCl}_{6}$. The plots are the reverse CVs at $2500 \mathrm{rpm}$ in $0.5 \mathrm{M} \mathrm{H}_{2} \mathrm{SO}_{4}$. The on/off times were $25 / 50 \mathrm{~ms}$, peak current was $400 \mathrm{~mA} / \mathrm{cm}^{2}$, rotation rate was $400 \mathrm{rpm}$, and 200 cycles were used.

\section{Carbon Type}

Activated carbon KB-B, Cabot Regal GR-47, and Vulcan XC-72 were used as supports and compared to a commercial Pt catalyst carbon cloth from E-TEK (loading $=0.5 \mathrm{mg} / \mathrm{cm}^{2}$ ), as shown in Figure 29. All of these supports have both a high surface area and adequate electrical conductivity to act as a path for electron flow. Vulcan XC-72R carbon is widely used as a support for Pt catalysts in low-temperature fuel cells due to its low cost and high availability. The electrode with Vulcan XC-72 as a support had the lowest performance. There was little difference in performance for the electrodes containing the other two types of carbon as supports. All of the prepared electrodes had lower performance than the commercial electrode. 


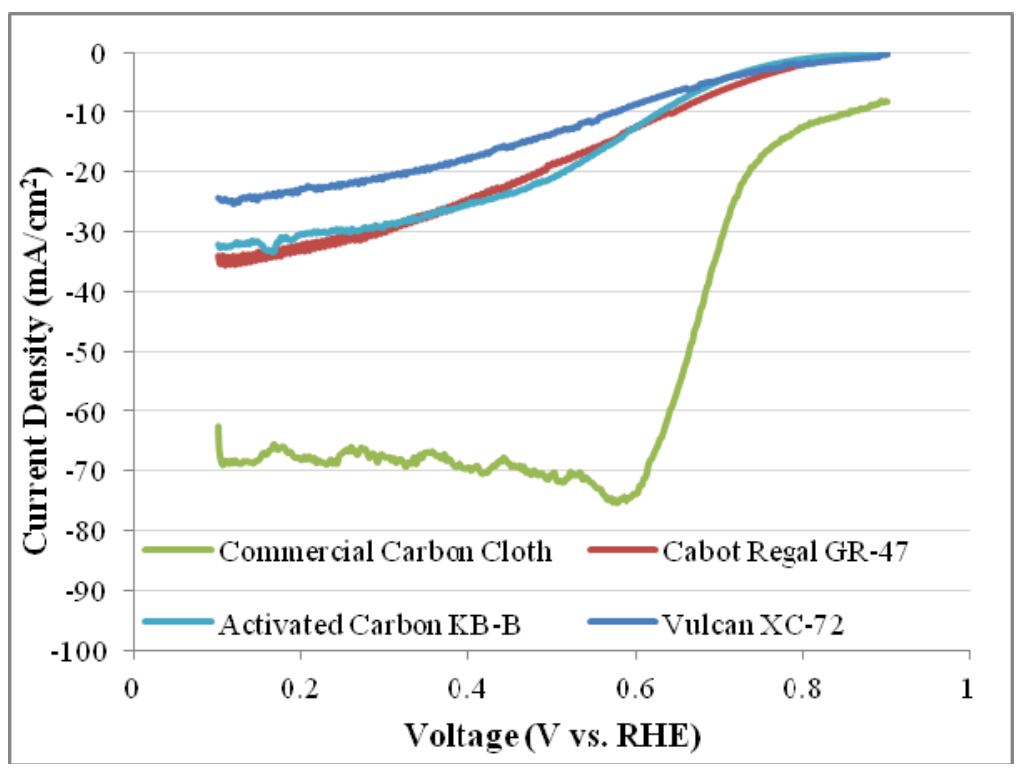

Figure 29. Performance of electrodes containing activated carbon and Cabot Regal as carbon supports. The electrodeposition solution contained $0.5 \mathrm{M} \mathrm{H}_{2} \mathrm{SO}_{4}$ and $10 \mathrm{mM} \mathrm{H}_{2} \mathrm{PtCl}_{6}$. The plots are the reverse CVs at $2500 \mathrm{rpm}$ in $0.5 \mathrm{M} \mathrm{H}_{2} \mathrm{SO}_{4}$. The on/off times were $25 / 50 \mathrm{~ms}$, peak current was $400 \mathrm{~mA} / \mathrm{cm}^{2}$, rotation rate was $400 \mathrm{rpm}$, and 800 cycles were used. The commercial catalyst is a Pt catalyst coated carbon cloth from E-TEK with a Pt loading of $0.5 \mathrm{mg} / \mathrm{cm}^{2}$.

\section{Graphene Oxide}

In the final year of the project, graphene oxide, which is promising as a support due to its high surface area and high stability was made available, so it was also investigated as a carbon support. Initially, SEM images of the graphene oxide were obtained to determine what it looks like on a microscopic level, as shown in Figure 30. In this image, the graphene oxide appears to be porous and uniform.

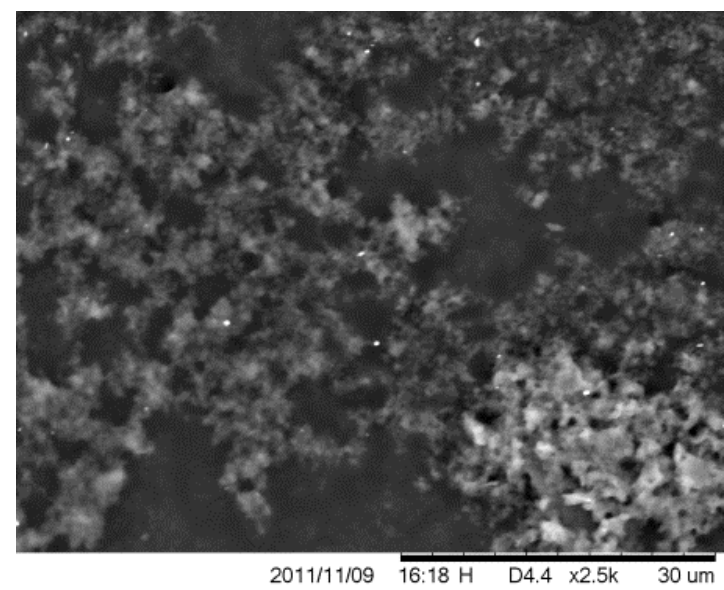

Figure 30. Graphene oxide on carbon tape

Because graphene rather than graphene oxide has been shown to have increased surface area and stability, a procedure was developed and carried out to reduce graphene oxide to graphene. To reduce graphene oxide, it was exposed to $4 \% \mathrm{H}_{2}$ in argon at $450{ }^{\circ} \mathrm{C}$ for five hours. 
The TGA results from a sample exposed to $4 \% \mathrm{H}_{2}$ in $\mathrm{Ar}$, ramping at $10{ }^{\circ} \mathrm{C} / \mathrm{min}$ from $30{ }^{\circ} \mathrm{C}$ to $550{ }^{\circ} \mathrm{C}$, then holding $550{ }^{\circ} \mathrm{C}$ for 30 minutes are shown in Figure 31. An initial drop in weight between $30{ }^{\circ} \mathrm{C}$ and 100 ${ }^{\circ} \mathrm{C}$ may be attributable to adsorbed water, and accounts for about $1 \%$ of the total weight. The remainder of the weight change (approximately 7\%) is attributable to graphene oxide reduction to graphene. This is likely accomplished through oxidation of $\mathrm{OH}$ groups to $\mathrm{H}_{2} \mathrm{O}$.

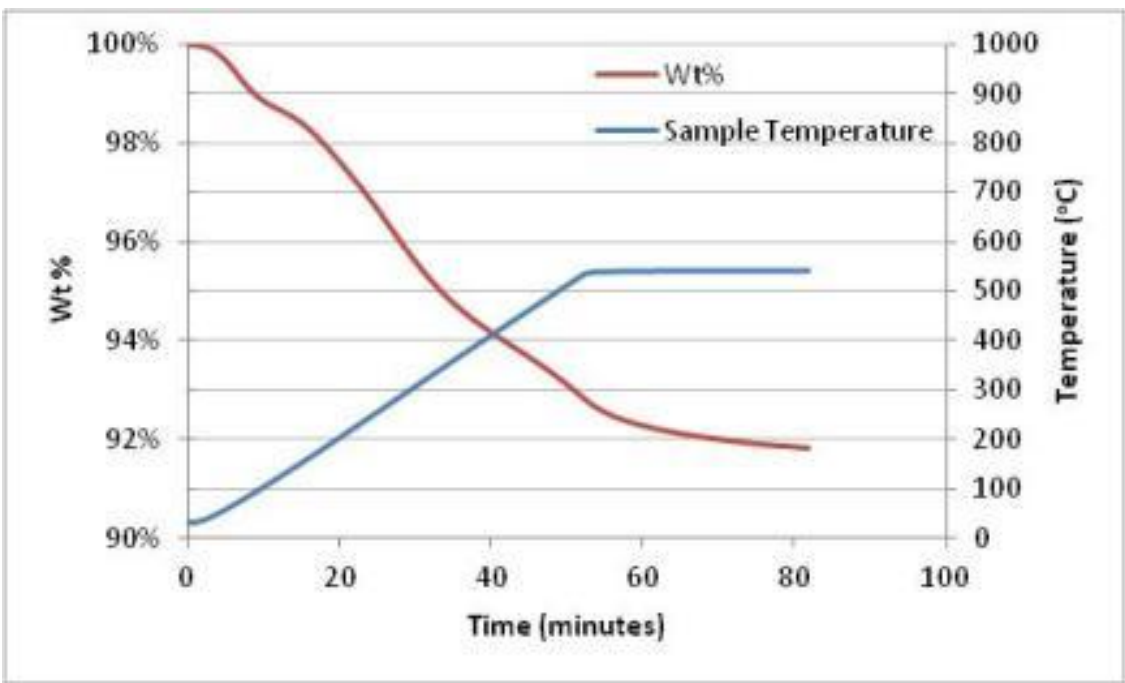

Figure 31. TGA data of reducing graphene oxide in $4 \% \mathrm{H}_{2} / \mathrm{Ar}$ atmosphere.

XPS spectra of the initial graphene oxide and reduced graphene oxide is shown in Figure 32. A small difference is observed near 285 and $288 \mathrm{eV}$, which represent $\mathrm{C}=\mathrm{C} / \mathrm{C}-\mathrm{C}$ in aromatic rings and $\mathrm{C}=\mathrm{O} / \mathrm{COOH}$ groups, respectively. This analysis shows that the as-received $\mathrm{GO}$ had $94 \% \mathrm{C}, 6 \% \mathrm{O}$, while the reduced GO had $96 \%$ C, 4\% O. Evidently, while the reduction procedure did reduce the $\mathrm{OH}$ surface concentration, it may have also reduced the aromaticity of the graphene.

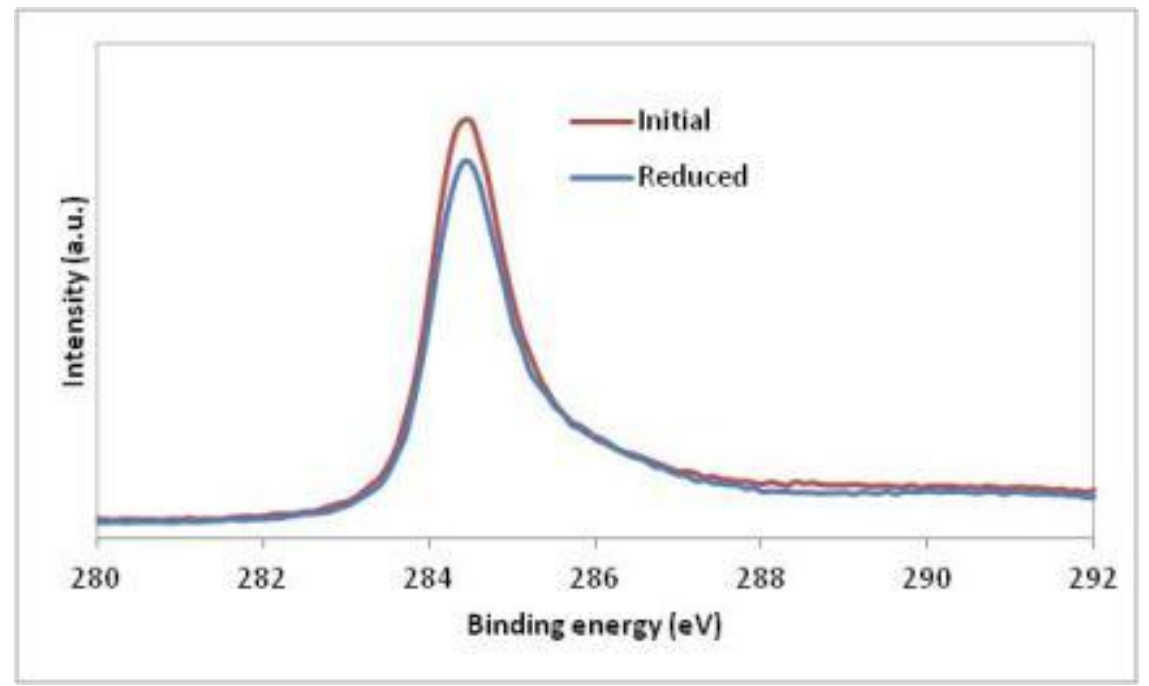

Figure 32. XPS data of graphene oxide before and after thermal reduction.

To examine the effect of using graphene oxide as support, eight gas diffusion electrodes (GDEs) were prepared, using either untreated or treated (reduced) graphene oxide and with and without Pt-Pd-Ru seeding (see Task 4 for an explanation of seeding), as in Table 1. The graphene or graphene oxide was mixed with Nafion ${ }^{\circledR}$ and dropped onto the surface of SGL 10BC gas diffusion layers (GDLs). The coated 
GDLs were then subjected to electrodeposition to deposit Pt onto their surface, with SEM images obtained at each step.

Table 1. Samples prepared with graphene oxide

\begin{tabular}{|l|l|l|}
\hline $\begin{array}{l}\text { Sample } \\
\text { name }\end{array}$ & $\begin{array}{l}\text { State of GO (oxidized or } \\
\text { reduced) }\end{array}$ & Seeded or Unseeded \\
\hline GO1-a & Oxidized & Unseeded \\
\hline GO1-b & Oxidized & Unseeded \\
\hline GO1-c & Oxidized & Seeded \\
\hline GO1-d & Oxidized & Seeded \\
\hline GO2-a & Reduced & Unseeded \\
\hline GO2-b & Reduced & Unseeded \\
\hline GO2-c & Reduced & Seeded \\
\hline GO2-d & Reduced & Seeded \\
\hline
\end{tabular}

The average ORR curves for all the samples are shown in Figure 33. Although the baseline electrode (using XC-72 carbon) showed the highest performance at low voltage, at high voltage the electrodes with reduced graphene oxide (GO) show superior performance.

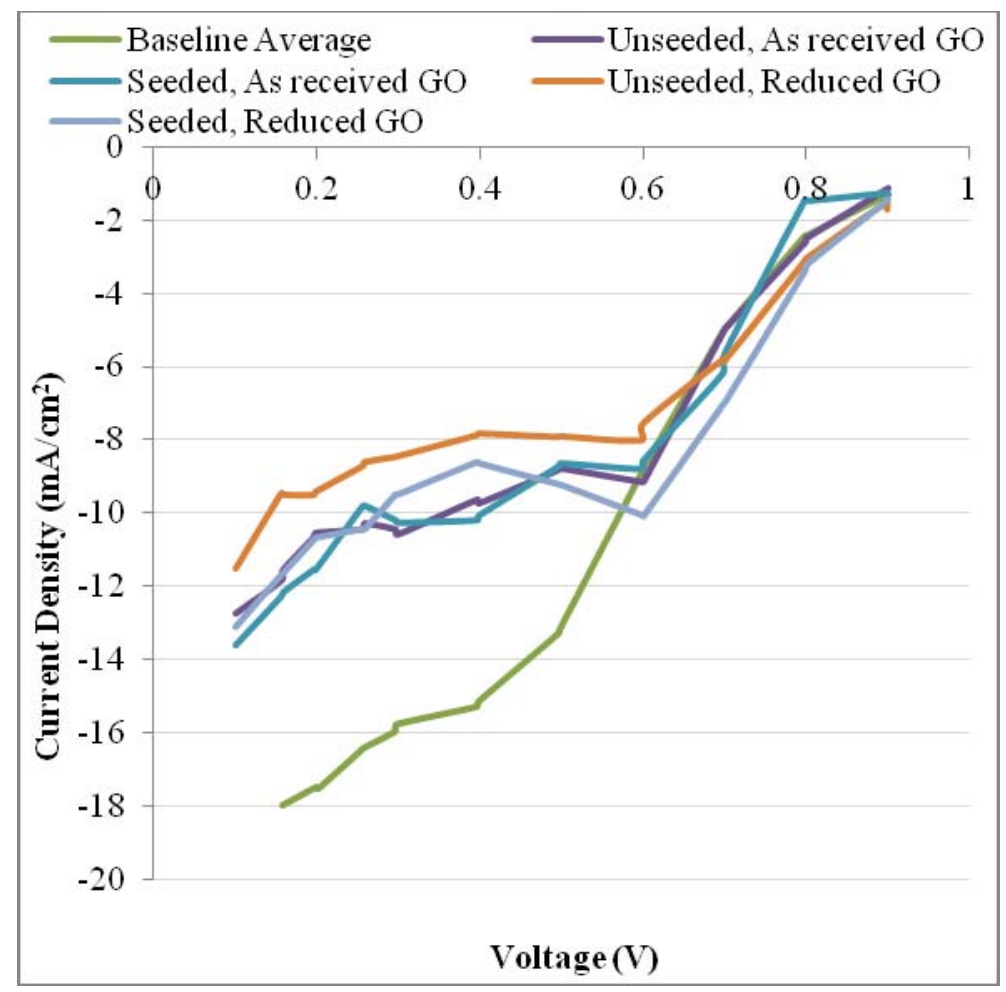

Figure 33. ORR of gas diffusion electrodes (GDEs) using graphene oxide as a support at $1600 \mathrm{rpm}$, after 200 electrodeposition cycles.

SEM images were taken of each type of electrode at each stage in the electrodeposition process to monitor any changes in surface characteristics. In general, the only change in surface was observed after electrodeposition of $\mathrm{Pt}$, which is expected, and this surface was maintained after successive plating and ORR. Typical SEM results are shown in Figure 34. 

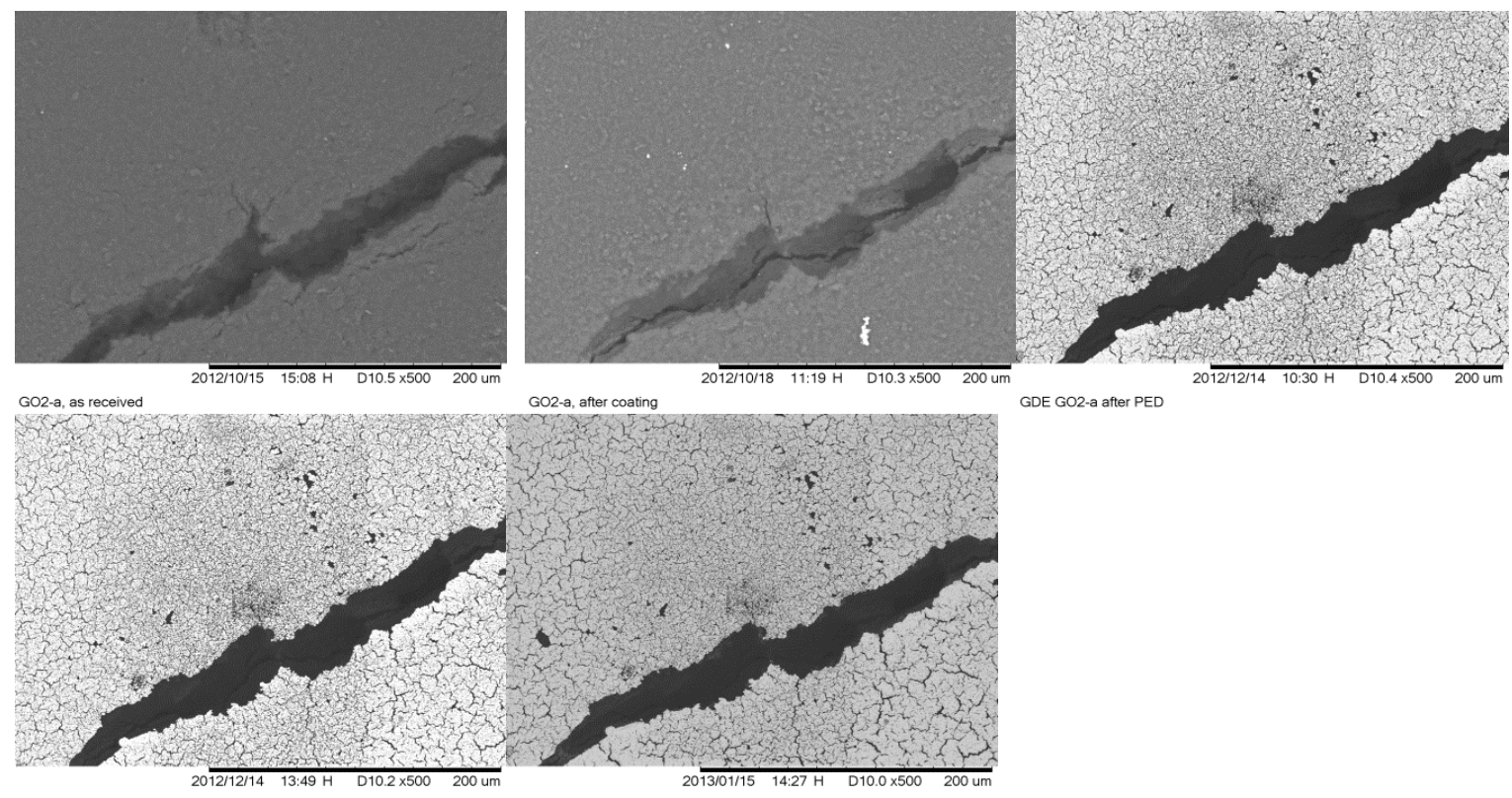

GO2-a, after coating GDE GO2-a after PED

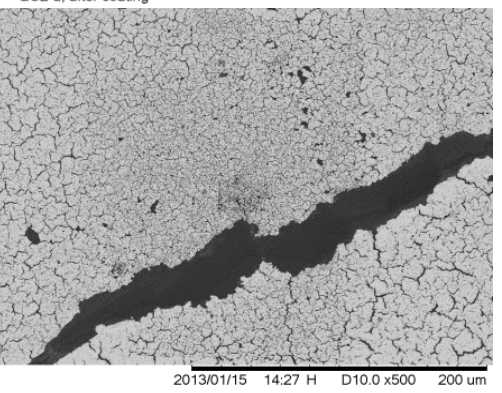

Figure 34. SEM images of reduced graphene oxide GDE, from as received to complete

\section{Summary}

In Task 2 different carbon supports were investigated. Thermal pretreatment of XC-72 carbon had no effect on electrode performance. The performance of electrodes made with XC-72 carbon support was lower than that of Cabot Regal GP-47 and activated carbon KB-B. Graphene is promising as a support due to its high surface area and stability and it was shown that when graphene oxide was reduced, the electrode performance at high voltage was improved over that of the baseline electrode.

\section{Task 3: Synthesis of Pt-Alloy Based Catalysts}

In this task, three Pt alloy based colloidal particles (Pt-Pd, Pt-Ru, and Pt-Pd-Ru) were synthesized via a polyvinylpyrrolidone (PVP) protected process. As shown in Figure 35 and Figure 36, the colloidal nanoparticles of the Pt-Pd-Ru ternary system with presence of PVP were stable and the particle size distributions were uniform with an average size of 4 to $5 \mathrm{~nm}$.
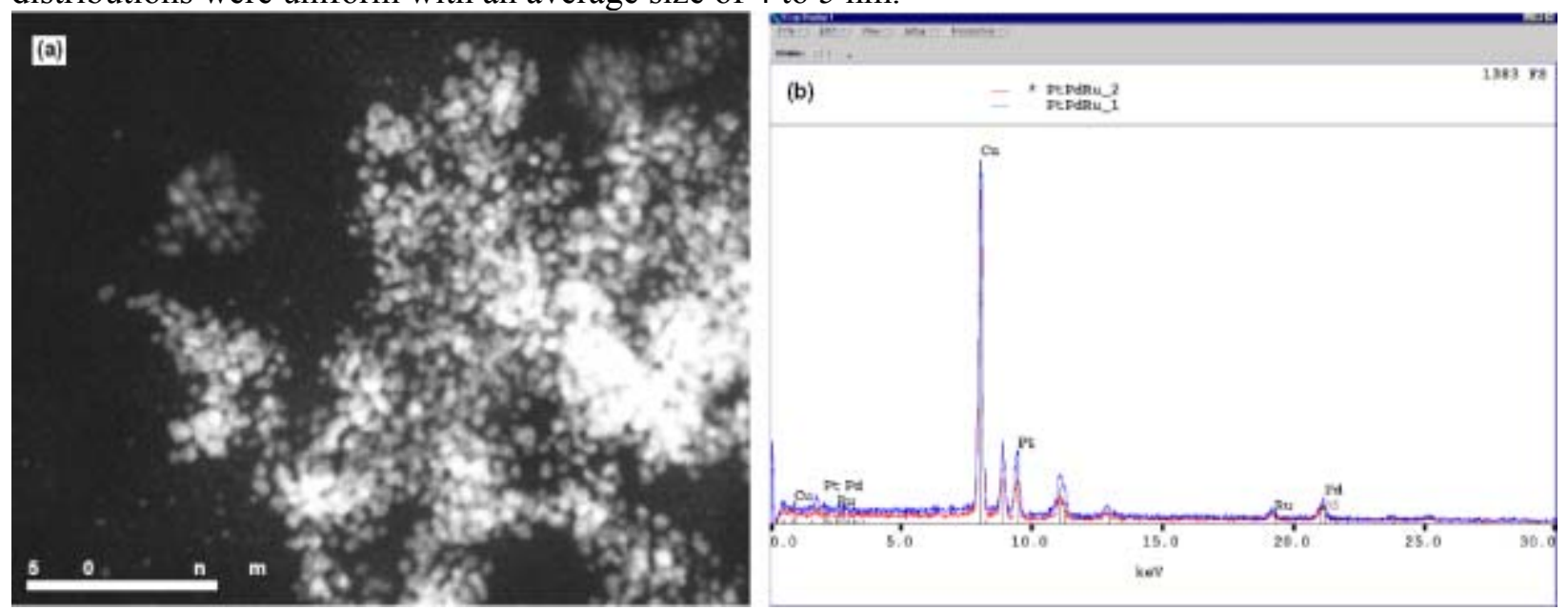

Figure 35. (a) STEM Z-contrast image of the Pt-Pd-Ru particles, and (b) EDS from the large areas showing Pt, Pd and Ru elements. 


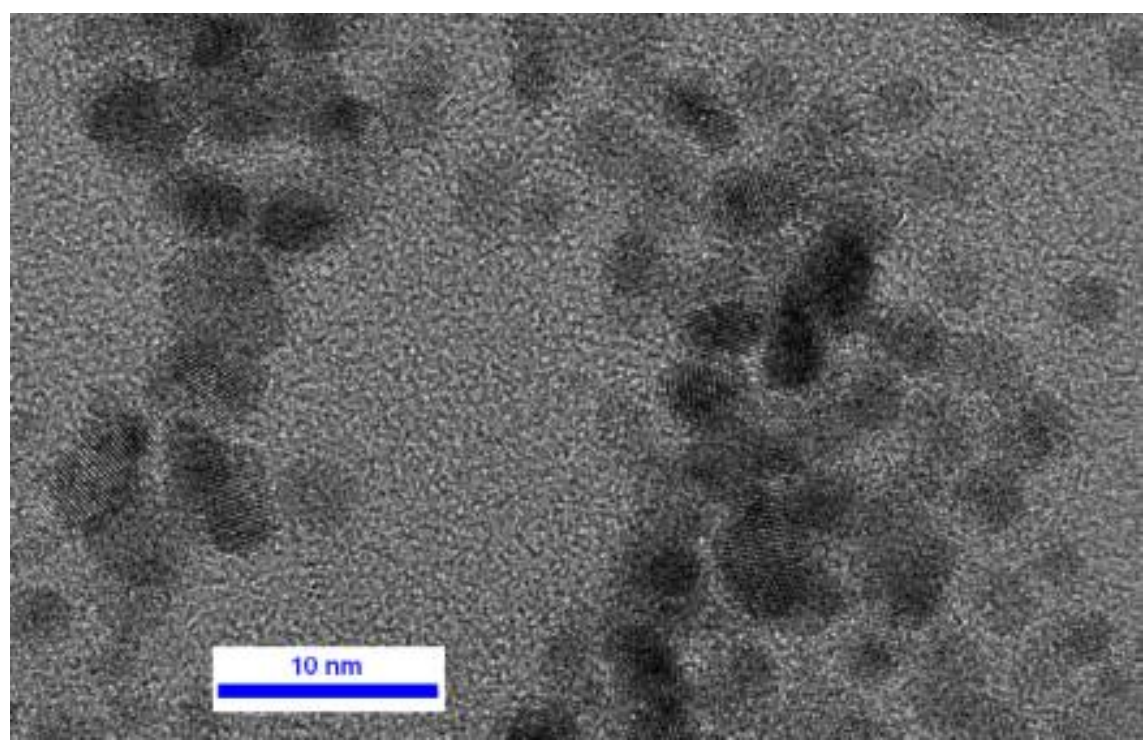

Figure 36. High resolution image of particle size distribution

Atomic resolution STEM images (Figure 37) indicate that the ternary system of Pt-Pd-Ru is an alloy with defined crystal structures.
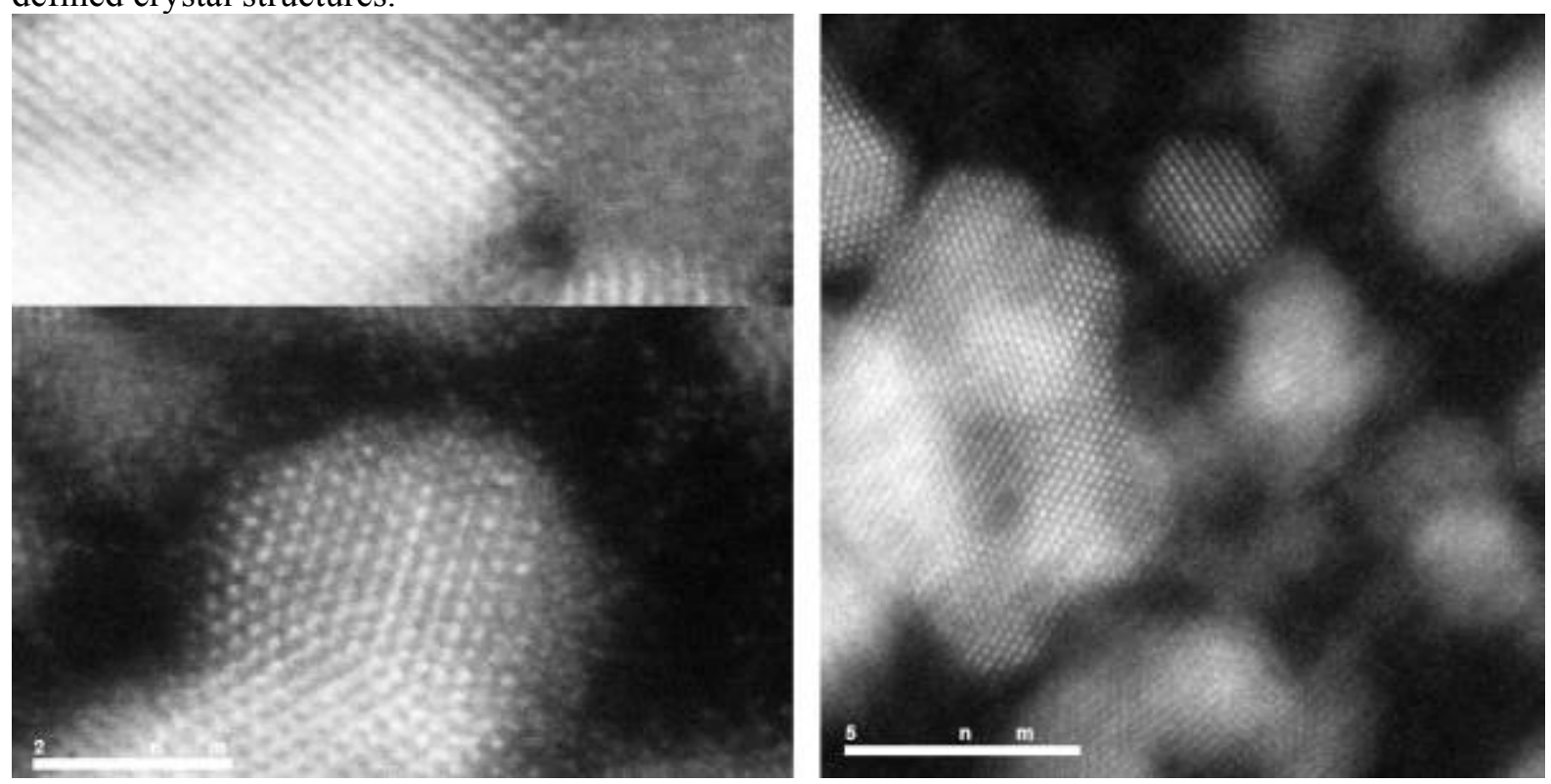

Figure 37. Atomic resolution STEM Z-contrast images

Figure 38 shows the UV-Vis spectra of Pt and Pt alloy based colloidal solutions. Four samples show very similar trends of UV-Vis absorption characters with peaking at $200 \mathrm{~nm}$. The spectra of $\mathrm{Pt}$ and $\mathrm{Pt}$ alloy samples (Pt-Ru and Pt-Pd-Ru) are overlapped at the $200 \mathrm{~nm}$ peak absorption. Although showing a slightly higher absorption, the spectrum of Pt-Pd sample also shows an absorption peak at $200 \mathrm{~nm}$. These characterizations indicate that the UV-Vis spectra of both Pt and Pt alloys result in major Pt colloidal absorption. The effect of single Pd or Ru colloidal particle on the UV-Vis light absorption can be neglected. This may indicate that, on average, the interaction between $\mathrm{Pt}$ and $\mathrm{Pd}$ and $\mathrm{Pt}$ and $\mathrm{Ru}$ may be at a minimum level. 


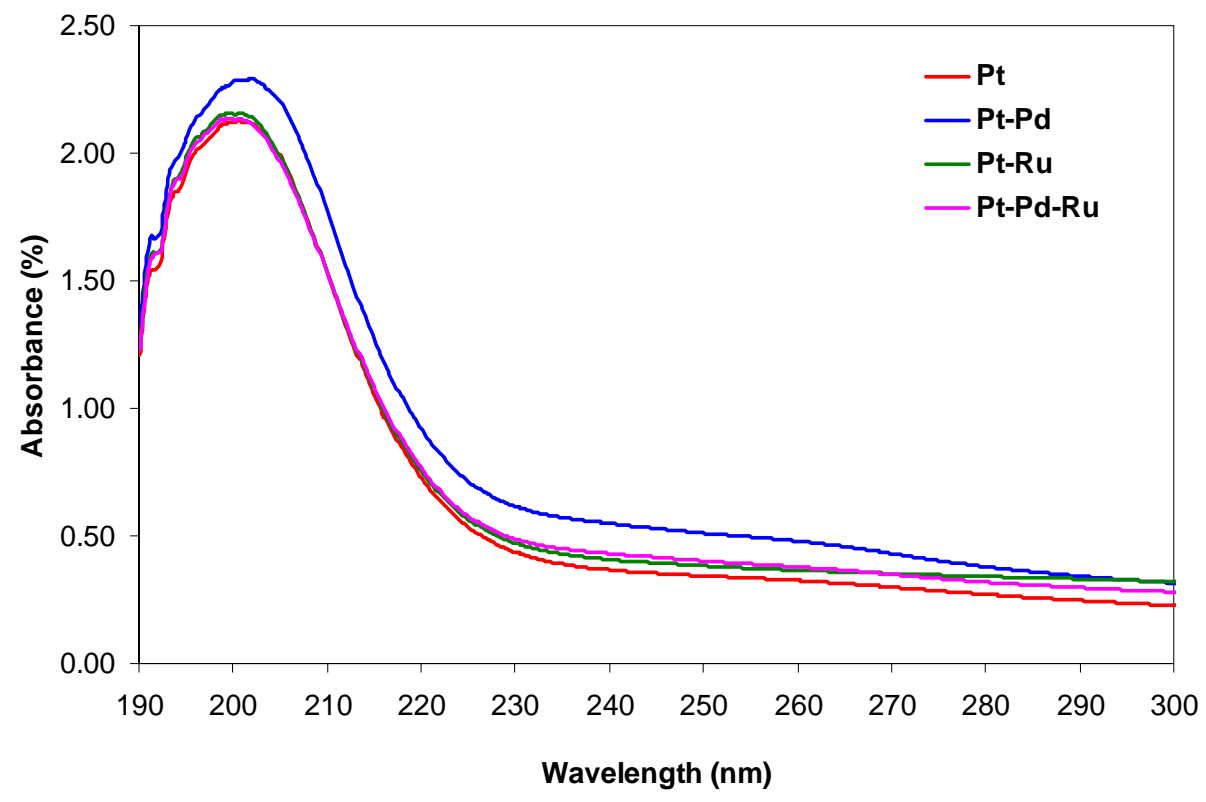

Figure 38. UV-Vis spectra of Pt and Pt alloy colloidal solutions

The nanoparticles were adsorbed onto the carbon electrode, through immersion of the sprayed carbon paper into the colloidal solution and sonication. When weighed before and after adsorption, the difference in mass was immeasurable on a microbalance, indicating low adsorption. Due to the low quantity of particles adsorbed, the ORR was very low (Figure 39). This is likely due to the low quantity of particles adsorbed.

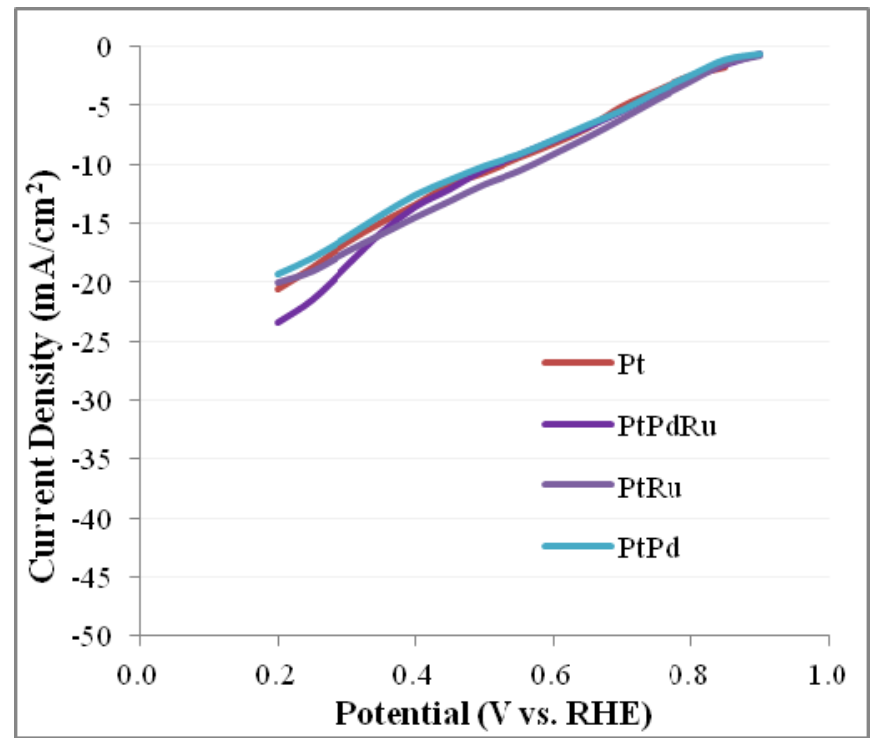

Figure 39. Performance of electrodes prepared by adsorption of catalyst nanoparticles onto GDLs.

\section{Summary}

In Task 3, three Pt alloy based colloidal particles were synthesized via a polyvinylpyrrolidone (PVP) 
protected process. The colloidal nano-particles of the Pt ternary system with presence of PVP were stable and the particle size distributions were uniform with an average size of 4 to $5 \mathrm{~nm}$. The nanoparticles were adsorbed onto the carbon electrode, through immersion of the sprayed carbon paper into the colloidal solution and sonication, but the ORR was very low (Figure 39). This is likely due to the low quantity of particles adsorbed.

\section{Task 4: Screening of the Pt-metal or Pt-metal oxide based catalysts}

It has been made clear that catalyst morphology is very important in electrode performance. In many cases in this project pulse electrodeposition led to less desirable catalyst morphologies. For example, as shown in Figure 40, sometimes the Pt particles severely aggregated on the electrode surface.

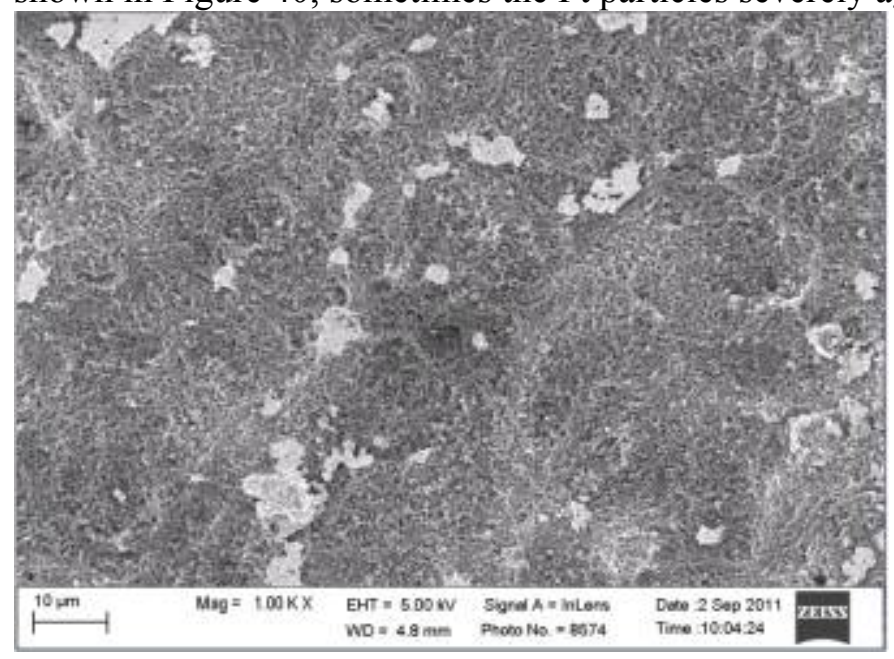

Figure 40. SEM image of an electrode fabricated by pulse electrodeposition showing severe Pt particle aggregation

To prevent Pt particle aggregation, a new electrodeposition method was developed. This method consists of three steps:

Step I: Synthesize Pt particles with optimal size $(\sim 3 \mathrm{~nm})$ and uniform size distribution.

Step II: Pre-load nanosized Pt colloidal particles to the surface of carbon electrodes.

Step III: Electroplate pre-loaded carbon electrodes with Pt using pulse electrodeposition.

This approach can enhance the performance of electrocatalysts while requiring much lower Pt loading. By pre-loading the sample with nanoparticles, the nucleation sites are uniformly incorporated onto the surface, where platinum particle growth will be promoted, creating a more homogeneous platinum deposition. Nanoparticles were prepared as described in Task 3. Figure 41 shows TEM images of Pt colloidal particles with a narrow size distribution. The average particle size is $\sim 3 \mathrm{~nm}$, which is in the optimal size range for the oxygen reduction reaction in a PEM fuel cell. Figure 42 shows highly defined nanostructured Pt colloidal particles $=$. The nanotwin structures in Pt alloy nanoparticles are also observed using atomic level microcopy analyses. These structures promote the electron transfers in oxygen reduction reactions and lead to higher performance of electrocatalysts. 


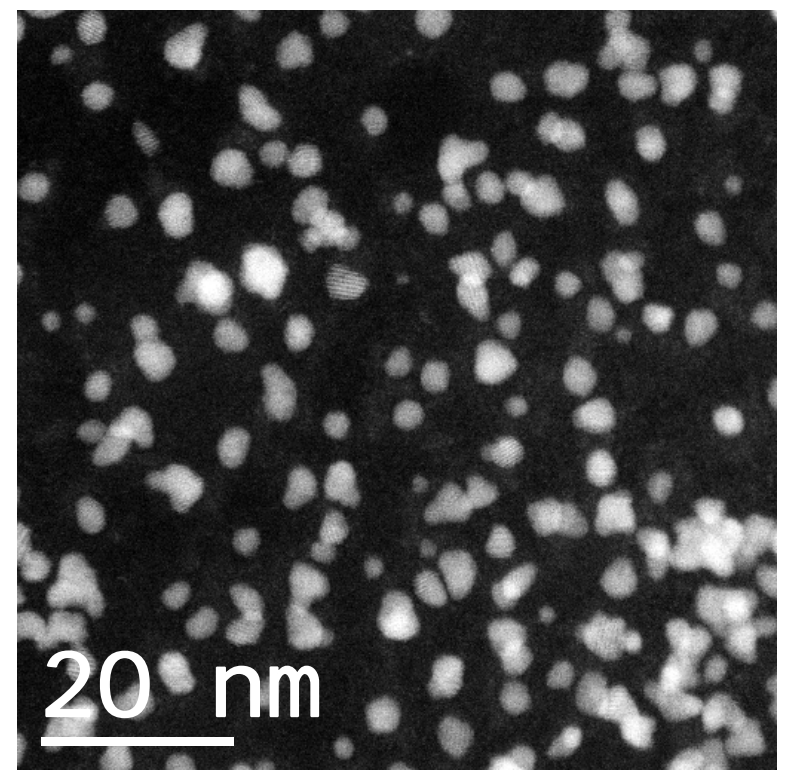

Figure 41. Nanosized Pt colloidal particles with average size of $3.0 \mathrm{~nm}$
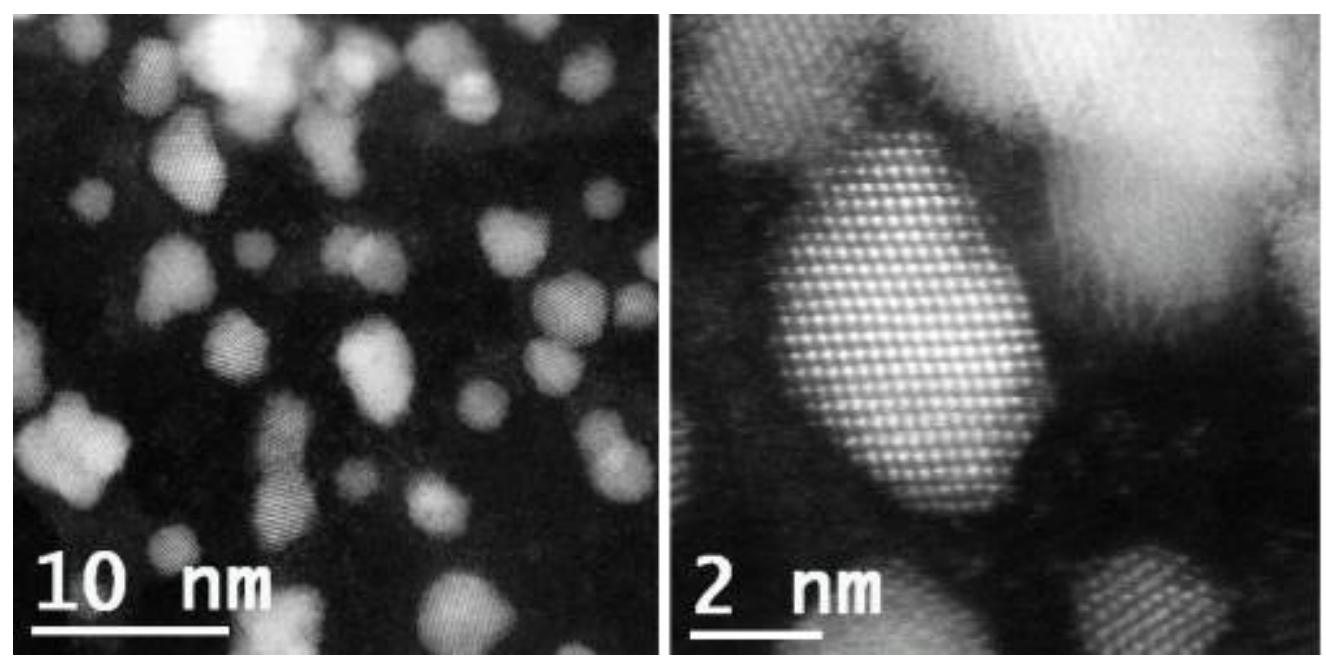

Figure 42. Atomic level STEM of Nanosized Pt colloidal particles

The nanoparticles were adsorbed onto the carbon electrode, through immersion of the sprayed carbon paper into the colloidal solution followed by sonication, and then pulse electrodeposition was carried out as before. As shown in Figure 43, without seeding, 300 deposition cycles were needed to reach the performance of the commercial electrode. After seeding, the performance of the commercial electrode was achieved after only 100 cycles (Figure 44). Based on the pulse electroplating parameters, the Pt density can be calculated from Faraday's Law. It is determined that 100 plating cycles in this experiment correspond to less than $0.10 \mathrm{mg} / \mathrm{cm}^{2}$ platinum loading onto a carbon electrode, which was confirmed by weight changes before and after electroplating. This means that same performance as the commercial catalyst was achieved with $1 / 5$ the loading. 


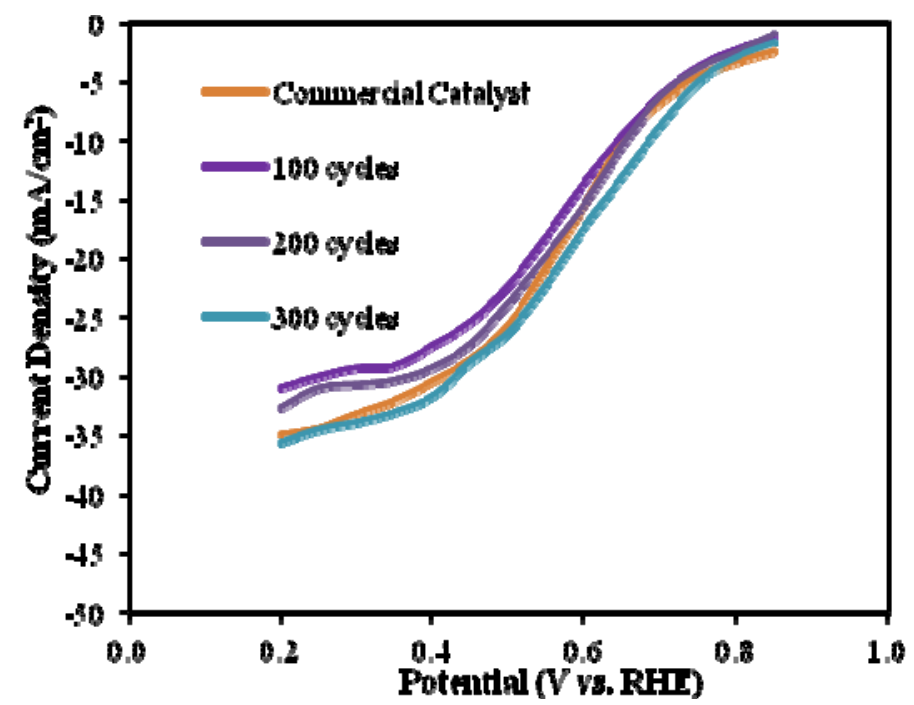

Figure 43. Performance of baseline electrodes prepared with no catalyst seeding prior to pulse electrodeposition. The electrodeposition solution contained $0.5 \mathrm{M} \mathrm{H}_{2} \mathrm{SO}_{4}$ and $1.25 \mathrm{mM} \mathrm{H}_{2} \mathrm{PtCl}_{6}$. The plots are the reverse CVs at $2500 \mathrm{rpm}$ in $0.5 \mathrm{M} \mathrm{H}_{2} \mathrm{SO}_{4}$. The on/off times were $25 / 50 \mathrm{~ms}$, peak current was $400 \mathrm{~mA} / \mathrm{cm}^{2}$, and rotation rate was $400 \mathrm{rpm}$.

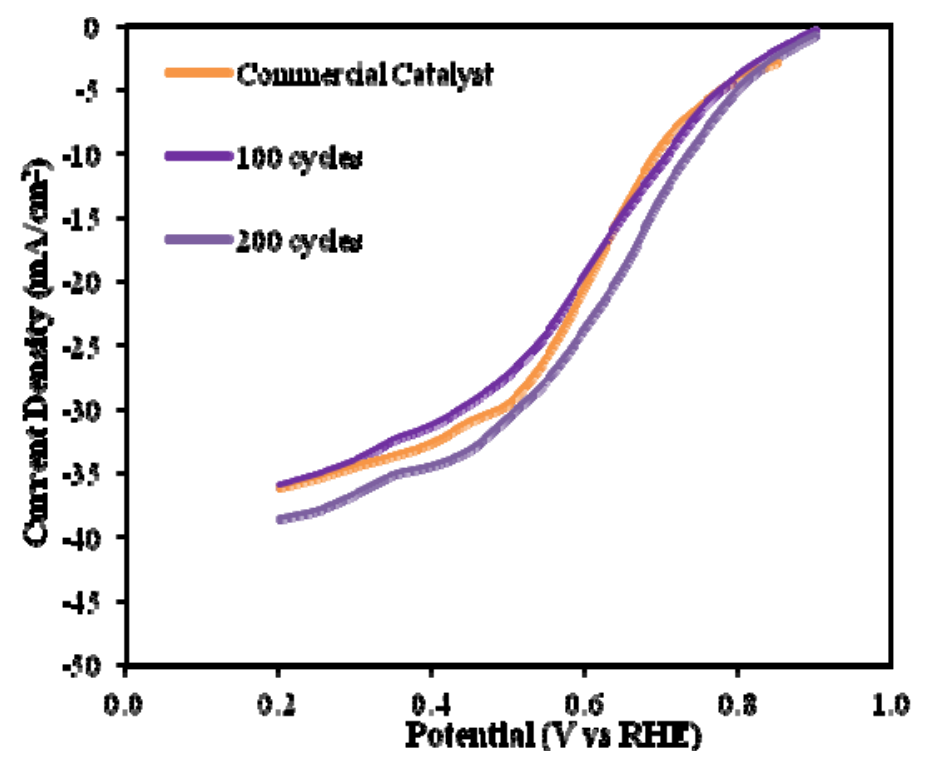

Figure 44. Performance of electrodes prepared with catalyst seeding prior to pulse electrodeposition. The electrodeposition solution contained $0.5 \mathrm{M} \mathrm{H}_{2} \mathrm{SO}_{4}$ and $1.25 \mathrm{mM} \mathrm{H}_{2} \mathrm{PtCl}_{6}$. The plots are the reverse CVs at $2500 \mathrm{rpm}$ in $0.5 \mathrm{M} \mathrm{H}_{2} \mathrm{SO}_{4}$. The on/off times were $25 / 50 \mathrm{~ms}$, peak current was $400 \mathrm{~mA} / \mathrm{cm}^{2}$, and rotation rate was $400 \mathrm{rpm}$.

Electrodes were prepared by seeding with each of the different types of catalyst nanoparticles. In looking at all the different types of nanoparticle seeding, it was clear that, except for PtRu, the performance improvement did not depend on the type of nanoparticle. 


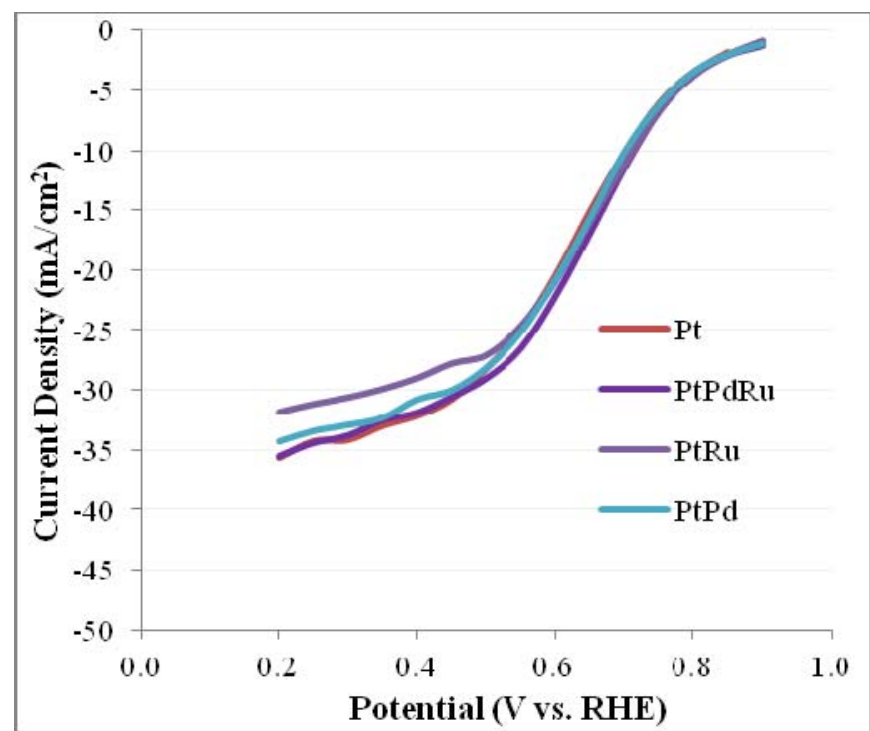

Figure 45. Performance of electrodes prepared with different types of catalyst seeding prior to pulse electrodeposition. The electrodeposition solution contained $0.5 \mathrm{M} \mathrm{H}_{2} \mathrm{SO}_{4}$ and $1.25 \mathrm{mM} \mathrm{H}_{2} \mathrm{PtCl}_{6}$. The plots are the reverse CVs at $2500 \mathrm{rpm}$ in $0.5 \mathrm{M} \mathrm{H}_{2} \mathrm{SO}_{4}$. The on/off times were $25 / 50 \mathrm{~ms}$, peak current was $400 \mathrm{~mA} / \mathrm{cm}^{2}$, rotation rate was $400 \mathrm{rpm}$, and 200 cycles were used.

Electrodes prepared by pulse electrodeposition onto electrodes prepared with the different types of catalyst nanoparticles were examined under SEM to view the effect of seeding nanoparticle type on electrode morphology, as shown in Figure 46. Upon examination of the SEM images, it is clear that using different types of seeds results in differences in catalyst morphology. Electrodes seeded with Pt appeared the largest and most textured, whereas those seeded with Pt-Ru were the smallest and least textured.
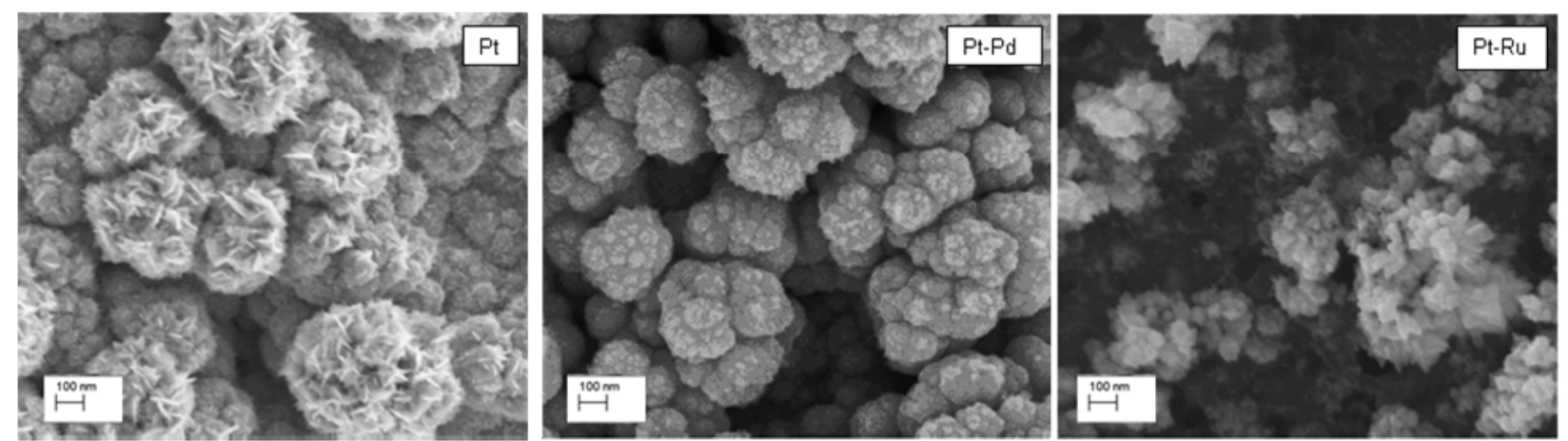

Figure 46. SEM images of electrodes prepared by electrodepositing Pt onto electrodes that have been seeded with Pt, Pt-Pd, and Pt-Ru nanoparticles.

In some cases catalysts were fabricated by directly electroplating the catalyst onto the carbon electrode. In other cases, the ORR was measured after every 25 to 100 plating cycles, which was termed "successive" plating. The effect of plating 100 cycles directly onto the carbon electrodes vs. successively depositing the $\mathrm{Pt}$ was investigated, was shown in Figure 47. From this figure, it is clear that successively depositing the Pt resulted in improved performance. 


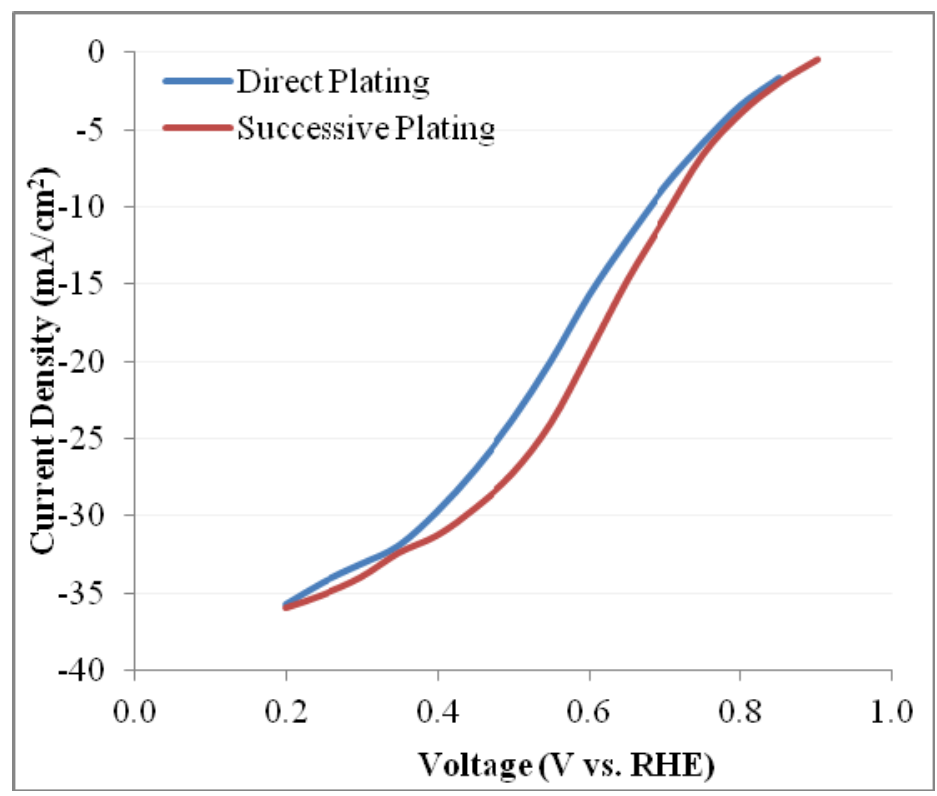

Figure 47. Comparison of ORR activity for 100 plating cycles of successive Pt deposition vs. direct Pt deposition

SEM images of electrodes that were direct plated and successively plated are shown with different magnifications in Figure 48, Figure 49, and Figure 50. The image at low magnification (Figure 48) shows that directly plating the Pt onto the electrode results in many cracks in the electrode surface. Higher magnification images (Figure 49 and Figure 50) show that successive plating results in smaller Pt agglomerate size. This smaller agglomerate size would result in higher surface area available for reaction and explains why the successive deposition results in improved performance.
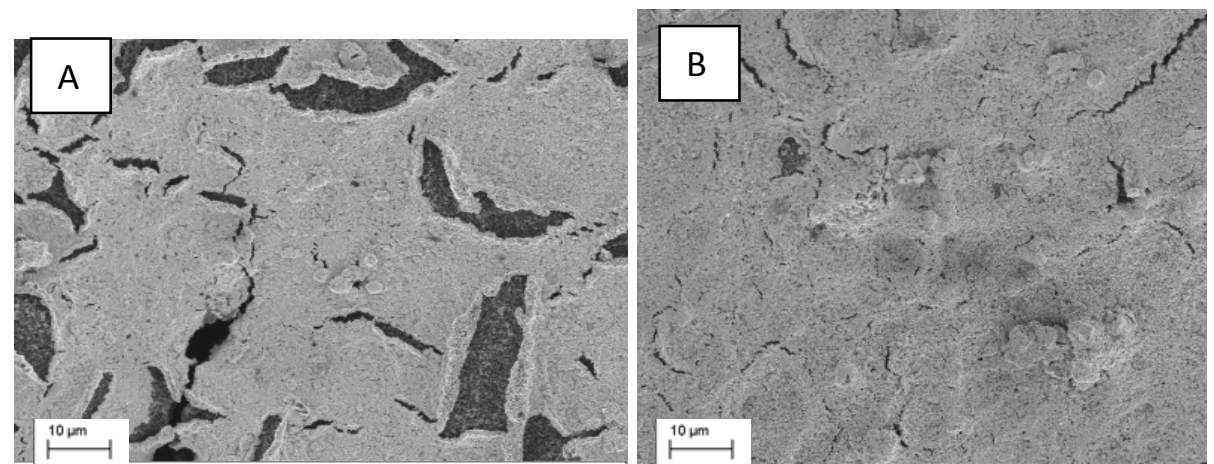

Figure 48. SEM images of electrocatalyst surfaces at 1000 magnification. (A) direct plating, 200 cycles; (B) successive plating, 200 cycles 

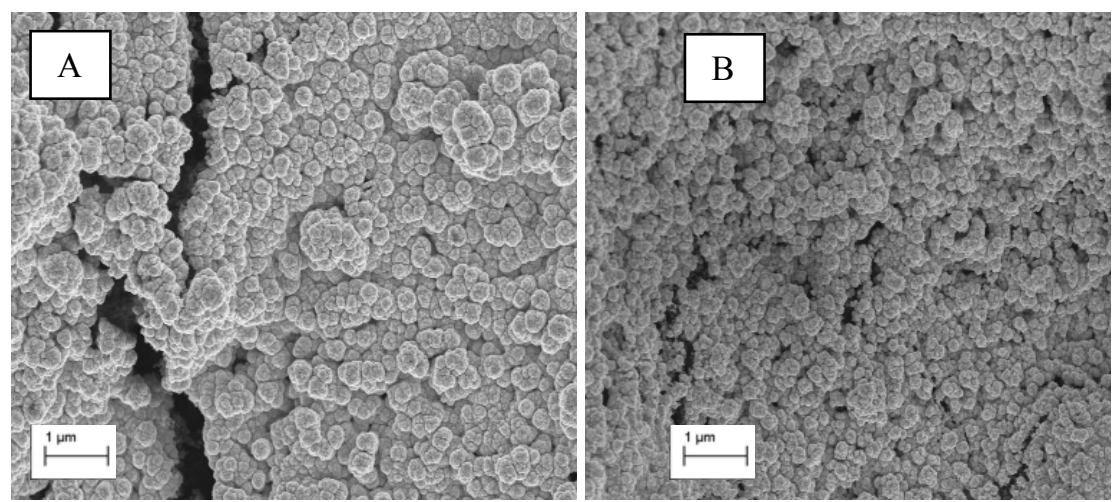

Figure 49. SEM images of electrocatalyst surfaces at 10000 magnification. (A) direct plating, 200 cycles, (B) successive plating, 200 cycles

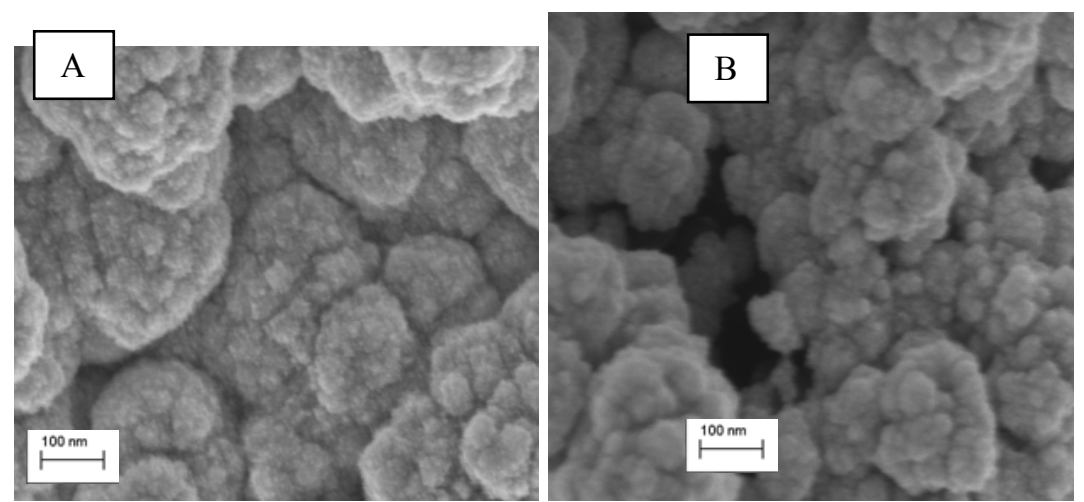

Figure 50. SEM images of electrocatalyst surfaces at 100000 magnification. (A) direct plating, 200 cycles, (B) successive plating, 200 cycles

\section{Summary}

In this task a new electrodeposition method was developed to improve electrode morphology, and therefore, performance. By seeding the electrodes with catalyst nanoparticles prior to electrodeposition, it was possible to achieve the same performance as a commercial electrode with $1 / 5$ the amount of Pt. The improved performance of the seeded electrodes was attributed to improved catalyst coverage on the electrodes. It was shown that carrying out successive plating rather than direct plating resulted in improved performance, which was attributed to smaller Pt agglomerate size.

\section{Task 5: Screening of Pt-lanthanide based electrocatalysts}

Because lanthanide elements have been shown to promote ORR and typically exhibit outstanding material properties, Task 5 was to evaluate Pt-lanthanide based catalysts. A procedure was developed to deposit ceria $\left(\mathrm{CeO}_{2}\right)$ onto the surface of GDLs.

For unsprayed GDLs, it was observed that a substantially higher amount of $\mathrm{CeO}_{2}$ is deposited when the $\mathrm{CeO}_{2}$ precursor solvent is 50/50 water/IPA mixture. This was due to the hydrophobicity of the GDL, preventing water from effectively wetting the surface and depositing $\mathrm{Ce}^{4+}$ ions. In the case of sprayed GDLs, the ionomer within the catalyst layer is hydrophilic, which caused absorption of the water and $\mathrm{Ce}^{4+}$ ions. Therefore, the solvent for the $\mathrm{CeO}_{2}$ precursor was pure water, which targeted $\mathrm{CeO}_{2}$ deposition to within the catalyst layer. In both cases, $\mathrm{CeO}_{2}$ was also found to be deposited on the back of the GDL (i.e. on the plain carbon paper). 
Palladium has been reported to experience improved ORR in the presence of $\mathrm{CeO}_{2}$. Therefore, $\mathrm{Pd}$ was deposited onto $\mathrm{CeO}_{2}$-treated samples in the same manner as done for $\mathrm{Pt}$ (i.e. pulse current of RDE in solution of Pd-precursor in $\mathrm{H}_{2} \mathrm{SO}_{4}$ ). The polarization curves for ORR activity are shown in Figure 51. When water was used as a solvent for the $\mathrm{CeO}_{2}$ precursor, the performance was improved at high voltage, but decreased at low voltage compared to that of the electrode without ceria. When a 50/50 water/isopropyl alcohol mixture was used as a solvent for the $\mathrm{CeO}_{2}$ precursor, the performance was greatly improved.

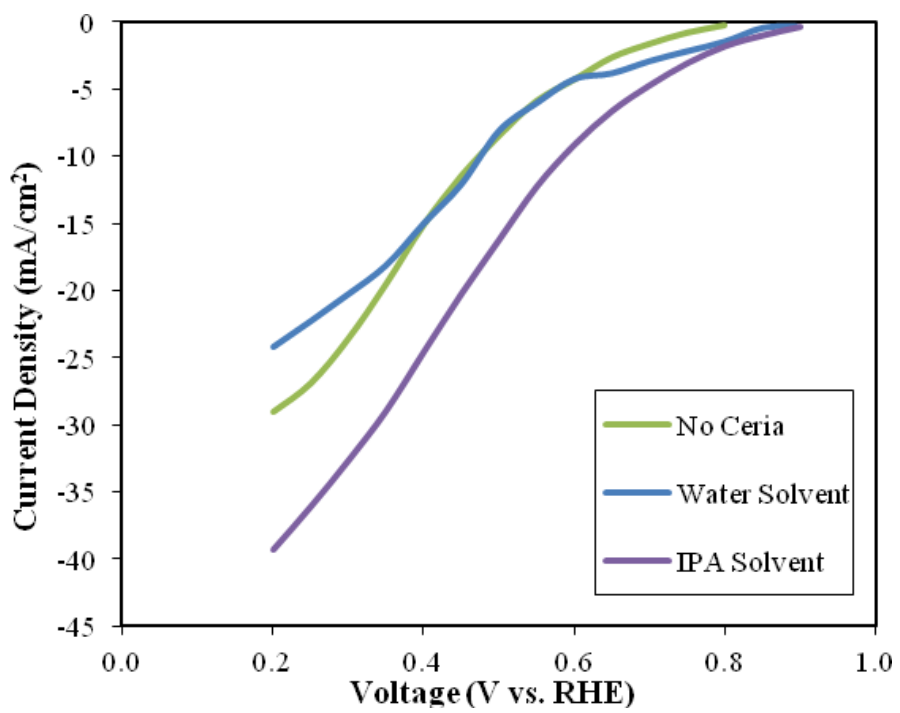

Figure 51. Curves for ORR of a Pd electrode without ceria and with ceria that was formed using water and a 50/50 water/IPA solvent mixture. The electrodeposition solution contained $0.5 \mathrm{M} \mathrm{H}_{2} \mathrm{SO}_{4}$ and 2.5 $\mathrm{mM} \mathrm{PdCl}_{2}$. The plots are the reverse CVs at $2500 \mathrm{rpm}$ in $0.5 \mathrm{M} \mathrm{H}_{2} \mathrm{SO}_{4}$. The on/off times were $25 / 50 \mathrm{~ms}$, peak current was $400 \mathrm{~mA} / \mathrm{cm}^{2}$, rotation rate was $400 \mathrm{rpm}$, and 100 cycles were used.

When examined under SEM, no $\mathrm{CeO}_{2}$ was observed on the GDL surface. For example, Figure 52 shows the GDL surface before and after electroplating. In the image on the left, the $\mathrm{CeO}_{2}$ is evident as the white substance present on the GDL surface. After electrodeposition, there is no white substance present (see the area indicated by the yellow circle). Note that for these images, the view is for the same region of the same GDL.

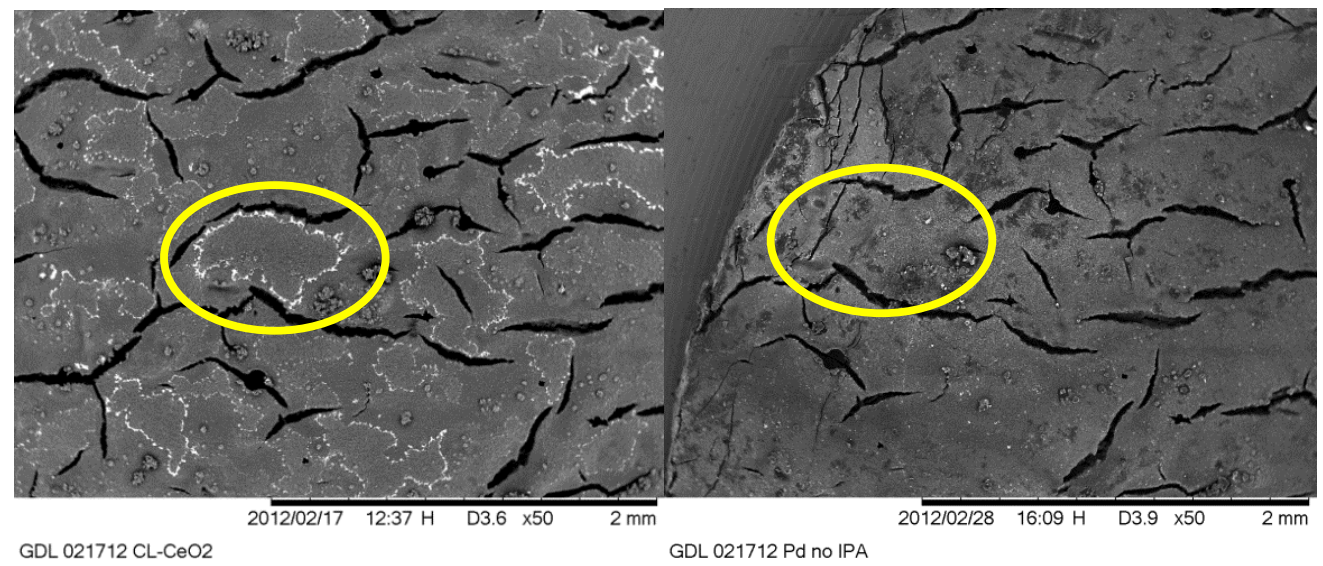

Figure 52. SEM images of a GDL before (left) and after (right) Pd electrodeposition

Since the $\mathrm{CeO}_{2}$ disappears, it is difficult to ascribe any changes in ORR to the presence of $\mathrm{CeO}_{2}$. It is 
theorized that the $\mathrm{CeO}_{2}$ is unstable within one of the steps of the electrodeposition process. To investigate which step causes the loss of $\mathrm{CeO}_{2}$, a GDL sample was investigated at each step throughout the electrodeposition process, i.e. after soaking in $0.5 \mathrm{~N} \mathrm{H}_{2} \mathrm{SO}_{4}$, after a cleaning $\mathrm{CV}$, and after electrodeposition. The following figures show the GDL sample at each step in the process. From Figure 53 and Figure 54, it can be seen that only a small amount (if any) cerium is lost during the $\mathrm{H}_{2} \mathrm{SO}_{4}$ soaking step. A far greater amount is lost after the CV (as determined from Figure 55 and Figure 56), while all of the cerium is lost after Pd electrodeposition (Figure 57). The reasons for this is likely related to the potential that the GDL experiences during the CV (e.g. from -0.1 to $0.9 \mathrm{~V}$ ) and electrodeposition (e.g. from 0 to $>5 \mathrm{~V}$ ). These potentials are within the region of the Pourbaix diagram for cerium where $\mathrm{Ce}^{3+}$ is more stable. ${ }^{[32]}$
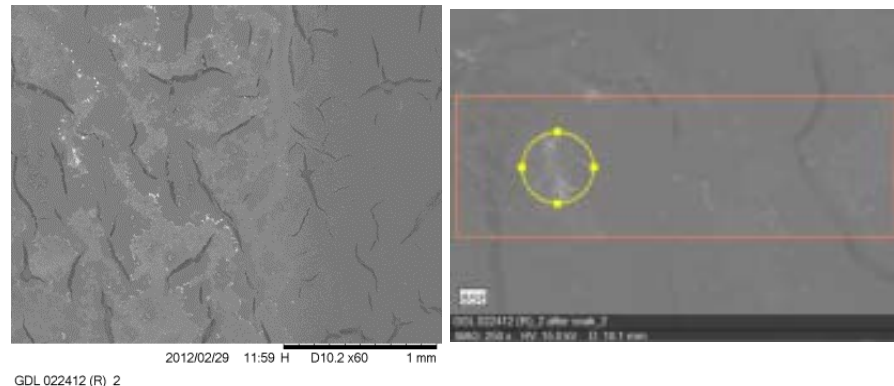

\begin{tabular}{||l|c|c||}
\hline Element & $\begin{array}{c}\text { Norm. C } \\
\text { [wt.\%] }\end{array}$ & $\begin{array}{c}\text { Atom. C } \\
\text { [at.\%] }\end{array}$ \\
\hline Palladium & 0.27 & 0.05 \\
\hline Carbon & 53.44 & 83.27 \\
\hline Cerium & 31.55 & 4.21 \\
\hline Fluorine & 9.63 & 9.49 \\
\hline Sulfur & 5.11 & 2.98 \\
\hline
\end{tabular}

Figure 53. SEM image (left) and elemental analysis from EDX (right) of the circled portion (middle) of a ceria-containing GDL before soaking in $0.5 \mathrm{~N} \mathrm{H}_{2} \mathrm{SO}_{4}$

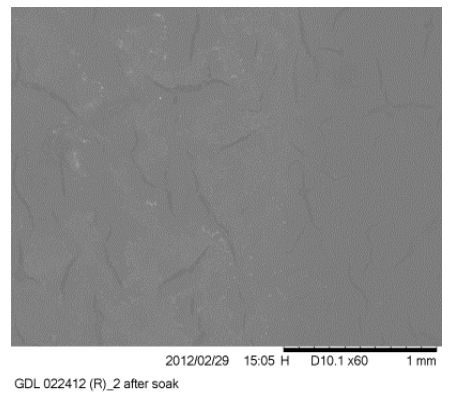

\begin{tabular}{|l|c|c||}
\hline Element & $\begin{array}{c}\text { Norm. C } \\
\text { [wt.\%] }\end{array}$ & $\begin{array}{c}\text { Atom. C } \\
{[\text { at.\%] }}\end{array}$ \\
\hline Palladium & 1.11 & 0.19 \\
\hline Carbon & 54.45 & 83.51 \\
\hline Cerium & 28.59 & 3.76 \\
\hline Fluorine & 8.71 & 8.45 \\
\hline Sulfur & 7.14 & 4.10 \\
\hline
\end{tabular}

Figure 54. SEM image (left) and elemental analysis from EDX (right) of the circled portion (middle) of a ceria-containing GDL after soaking in $0.5 \mathrm{~N} \mathrm{H}_{2} \mathrm{SO}_{4}$
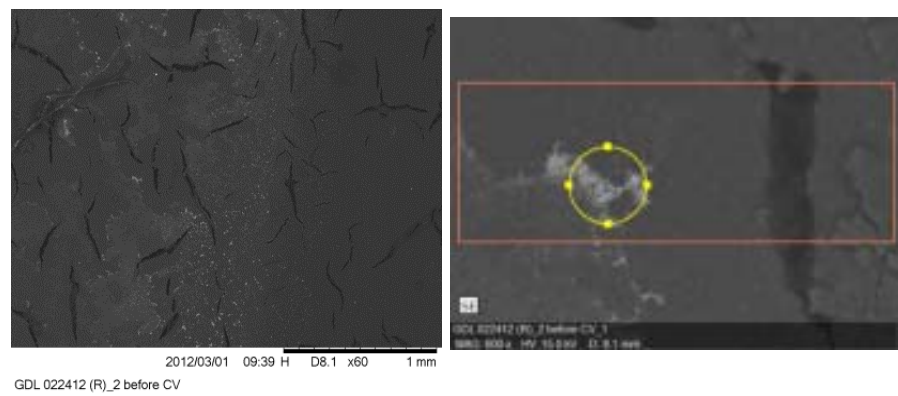

\begin{tabular}{|l|c|c|}
\hline Element & $\begin{array}{c}\text { Norm. C } \\
{[\text { wt. } \%]}\end{array}$ & $\begin{array}{c}\text { Atom. C } \\
{[\text { at.\% }]}\end{array}$ \\
\hline Palladium & 0.00 & 0.00 \\
\hline Carbon & 62.44 & 85.26 \\
\hline Cerium & 21.20 & 2.48 \\
\hline Fluorine & 11.06 & 9.55 \\
\hline Sulfur & 5.30 & 2.71 \\
\hline
\end{tabular}

Figure 55. SEM image (left) and elemental analysis from EDX (right) of the circled portion (middle) of a ceria-containing GDL before cleaning CV 

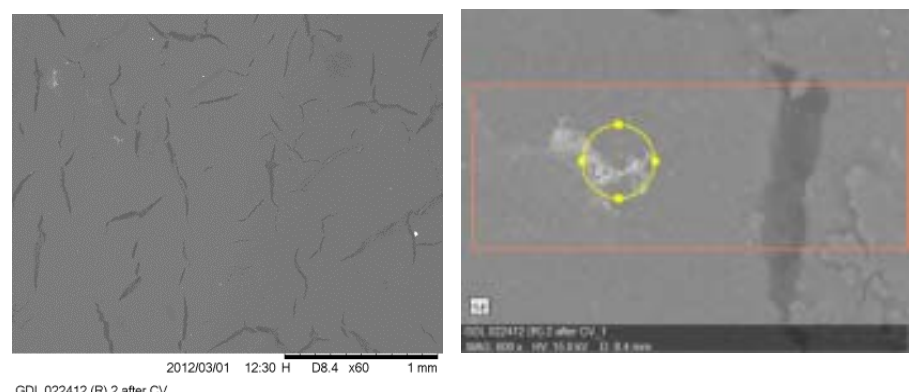

\begin{tabular}{||l|c|c||}
\hline Element & $\begin{array}{c}\text { Norm. C } \\
\text { [wt.\%] }\end{array}$ & $\begin{array}{c}\text { Atom. C } \\
\text { [at.\%] }\end{array}$ \\
\hline Palladium & 0.53 & 0.07 \\
\hline Carbon & 72.46 & 87.91 \\
\hline Cerium & 11.65 & 1.21 \\
\hline Fluorine & 12.22 & 9.38 \\
\hline Sulfur & 3.14 & 1.42 \\
\hline
\end{tabular}

Figure 56. SEM image (left) and elemental analysis from EDX (right) of the circled portion (middle) of a ceria-containing GDL after cleaning $\mathrm{CV}$
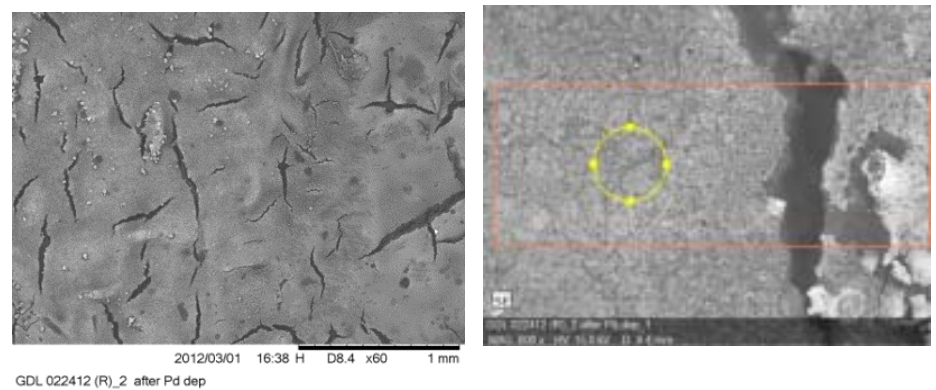

\begin{tabular}{|l|c|c|}
\hline Element & $\begin{array}{c}\text { Norm. C } \\
\text { [wt.\%] }\end{array}$ & $\begin{array}{c}\text { Atom. C } \\
\text { [at.\%] }\end{array}$ \\
\hline Palladium & 62.19 & 16.69 \\
\hline Carbon & 31.41 & 74.67 \\
\hline Cerium & 0.00 & 7.21 \\
\hline Fluorine & 4.80 & 7.21 \\
\hline Sulfur & 1.60 & 1.43 \\
\hline
\end{tabular}

Figure 57. SEM image (left) and elemental analysis from EDX (right) of the circled portion (middle) of a ceria-containing GDL after Pd electrodeposition

Because literature has shown that heat treatment (calcinations) can stabilize ceria, a new protocol was developed with the aim of improving ceria stability and electrode activity. To do this, ceria was synthesized and calcined at $300{ }^{\circ} \mathrm{C}$ and mixed in with the carbon/Nafion ink and applied to the carbon paper GDL. The coated GDLs were then subjected to electrodeposition to deposit Pt onto their surface, with SEM images obtained at each step. Pt was chosen at this step instead of Pd because we had more experience with Pt than Pd. To investigate the effect of heat-treating the ceria on stability and performance, a systematic series of tests was proposed and carried out.

1. $600{ }^{\circ} \mathrm{C}$ pre-treatment of XC-72R carbon, soaked in $50 \mathrm{~mL}$ IPA overnight, $300{ }^{\circ} \mathrm{C}$ calcined

2. $600{ }^{\circ} \mathrm{C}$ pre-treatment of $\mathrm{XC}-72 \mathrm{R}$ carbon, soaked in $50 \mathrm{~mL}\left(\mathrm{NH}_{4}\right)_{2} \mathrm{Ce}\left(\mathrm{NO}_{3}\right)_{3}$ in IPA overnight

3. $600{ }^{\circ} \mathrm{C}$ pre-treatment of XC-72R carbon, soaked in $50 \mathrm{~mL}\left(\mathrm{NH}_{4}\right)_{2} \mathrm{Ce}\left(\mathrm{NO}_{3}\right)_{3}$ in IPA overnight, $300{ }^{\circ} \mathrm{C}$ calcined

Addition of ceria did not affect performance of the Pt electrodes, as shown in Figure 58. Surprisingly, when ceria was present, seeding also did not affect performance. Unfortunately, no SEM images of the morphology are available to correlate this structure to the results. 


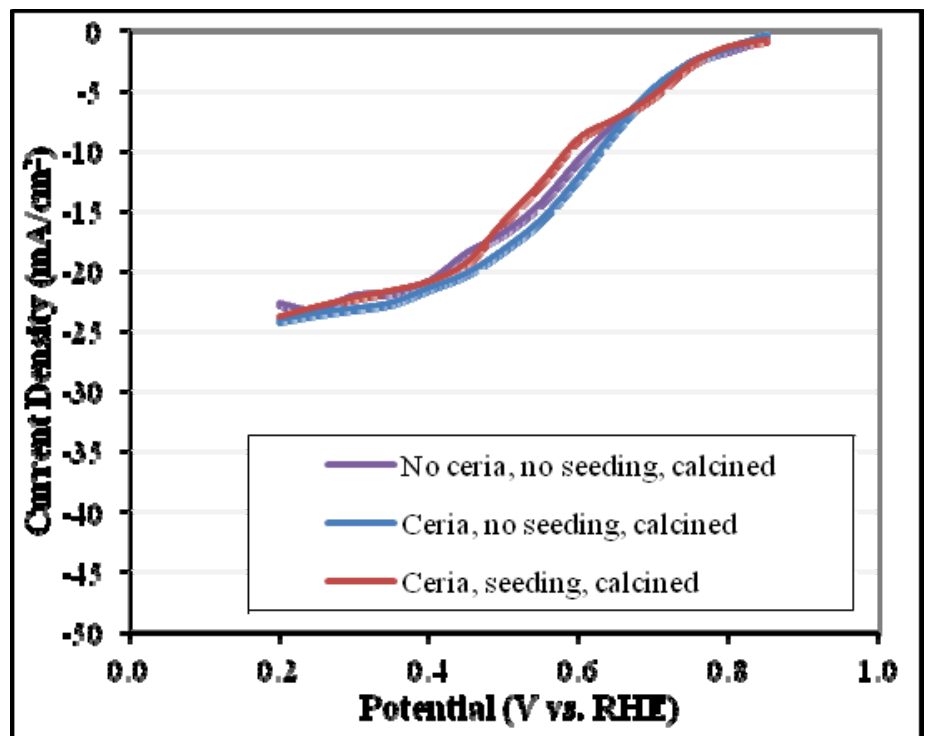

Figure 58. Curves for ORR of a Pt electrode without ceria or seeding, but calcined; with ceria and calcined, but no seeding; and with ceria, seeding and calcined. The electrodeposition solution contained $0.5 \mathrm{M} \mathrm{H}_{2} \mathrm{SO}_{4}$ and $1.25 \mathrm{mM} \mathrm{PdCl}_{2}$. The plots are the reverse CVs at $2500 \mathrm{rpm}$ in $0.5 \mathrm{M} \mathrm{H}_{2} \mathrm{SO}_{4}$. The on/off times were $25 / 50 \mathrm{~ms}$, peak current was $400 \mathrm{~mA} / \mathrm{cm}^{2}$, rotation rate was $400 \mathrm{rpm}$, and 100 cycles were used.

To directly examine the effect of calcinations on ceria stability, SEM images were again taken at each stage of electrodeposition and characterization. Figure 59 shows the electrode that has been coated with carbon that was treated at $600{ }^{\circ} \mathrm{C}$ under Ar, soaked in ceria/propanol overnight, but was not calcined. On this electrode, white structures that have been identified as containing cerium are present on the electrode surface. These structures disappear after performing electrochemistry on the electrode. Locations from where the cerium containing particles have disappeared are circled in yellow.
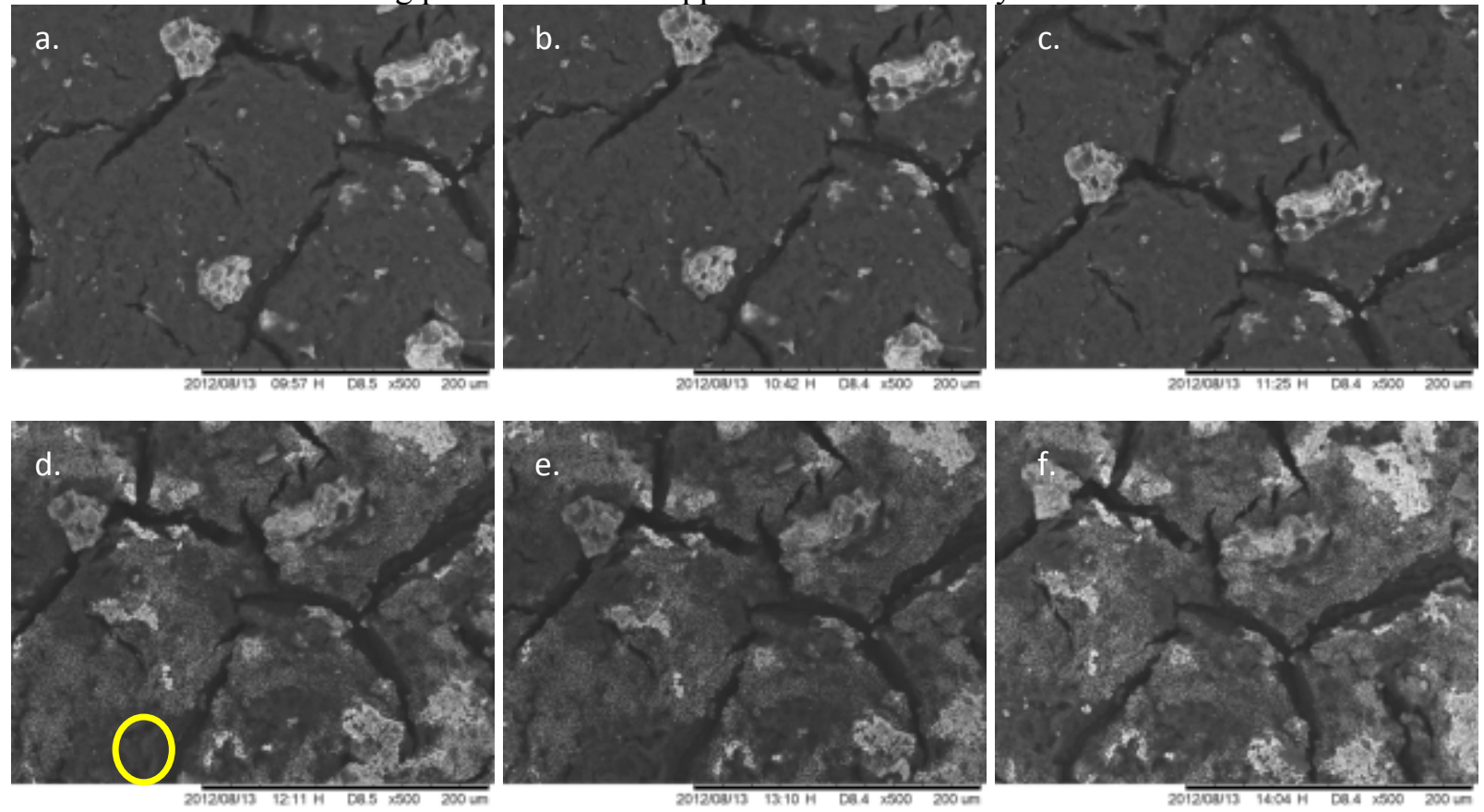


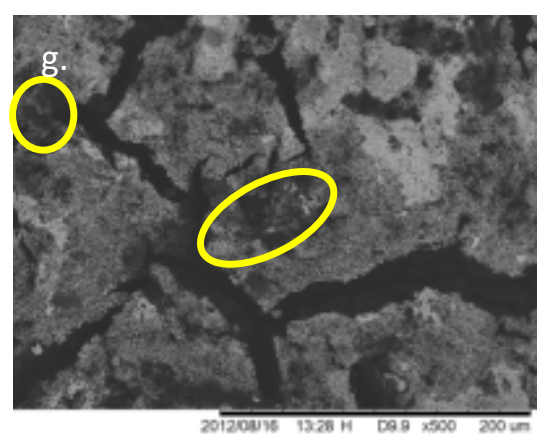

COE 0 131312 4.h aner OARSO

Figure 59. SEM images of GDL of Sample 5 (a) after coating with Nafion/XC-72 carbon mixture, (b) after soaking in $\mathrm{H}_{2} \mathrm{SO}_{4}$, (c) after a cleaning $\mathrm{CV}$, (d) after performing 50 cycles of pulse electrodeposition, (e) after performing ORR characterization, (f) after performing 200 cycles of pulse electrodeposition, (g) after ORR characterization. Yellow circles denote areas from which ceria particles have disappeared.

Figure 60 shows the electrode that has been coated with carbon that was treated at $600{ }^{\circ} \mathrm{C}$ under $\mathrm{Ar}$, soaked in ceria/propanol overnight, and was calcined at $300{ }^{\circ} \mathrm{C}$. On this electrode, white structures that have been identified as containing cerium are present on the electrode surface. These structures remain after performing electrochemistry on the electrode, which shows that the calcinations step improved stability of the ceria particles, as desired.
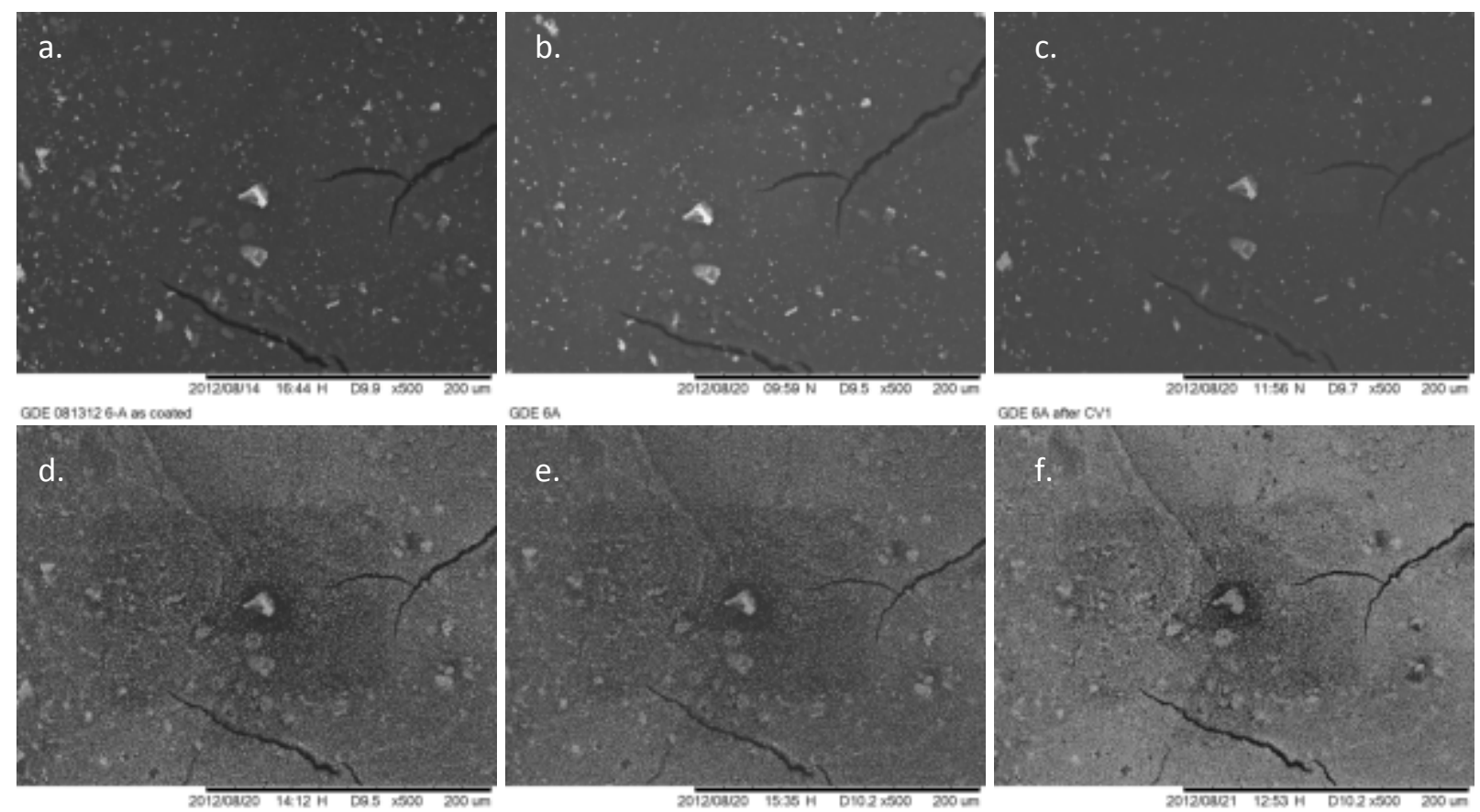

COE GS Ane PEDSO

COE Grane CY2

COE GM une PEDQNOC.0PE

Figure 60. SEM images of GDL of Sample 6 (a) after coating with Nafion/XC-72 carbon mixture, (b) after soaking in $\mathrm{H}_{2} \mathrm{SO}_{4}$, (c) after a cleaning $\mathrm{CV}$, (d) after performing 50 cycles of pulse electrodeposition, (e) after performing ORR characterization, (f) after performing 200 cycles of pulse electrodeposition and ORR characterization.

\section{Summary}

In this task Pt- and Pd-lanthanide based catalysts were evaluated because lanthanide elements have shown surprising results in terms of catalytic activity and outstanding material properties. Ceria was formed 
through thermal hydrolysis, adsorbed onto the carbon powder, and deposited onto GDLs. Pd was electrodeposited onto the GDLs. Although differences in performance were observed, SEM showed that the ceria disappeared through the electrodeposition and characterization process. The loss of ceria was attributed to the electrochemical reactions resulting in formation of $\mathrm{Ce}^{3+}$, which is soluble. To prevent loss of ceria, a new procedure was developed whereby the ceria was calcined prior to being exposed to any electrochemical conditions. It was clear that the calcination resulted in improved stability, although the ceria was not shown to affect performance of Pt-based electrodes. Unfortunately, we were unable to run the performance curves with $\mathrm{Pd}$ and the stabilized $\mathrm{CeO}_{2}$ due to time constraints.

\section{Task 6: Palladium based catalysts}

The goal of Task 6 was to evaluate Pd-based catalysts. Pd was electrodeposited from a $2.5 \mathrm{mM} \mathrm{PdCl}_{2}$ solution. No electrodeposition parameters were optimized. The colloidal solutions of Pt-Pd-Ru, Pt-Ru, and Pt-Pd were used to seed the carbon electrodes prior to Pd electrodeposition. Although the performance of the electrodes seeded with Pt-Ru and Pt-Pd-Ru were quite similar, the performance of the Pt-Pd seeded electrode is much higher (Figure 61). When normalized for platinum group metal (PGM) mass, the performances of the Pd electrodes are improved over the commercial electrode, especially at low voltages (Figure 62). Because Pt costs $2.5 \mathrm{x} \mathrm{Pd}$, on a cost basis, this electrode is quite promising. It is clear from the SEM images (Figure 63) that the different types of seeds result in very different catalyst morphologies. Because these seeds are covered with the same electrodeposited layer, it is likely that differences in performance can be attributed to these different morphologies.

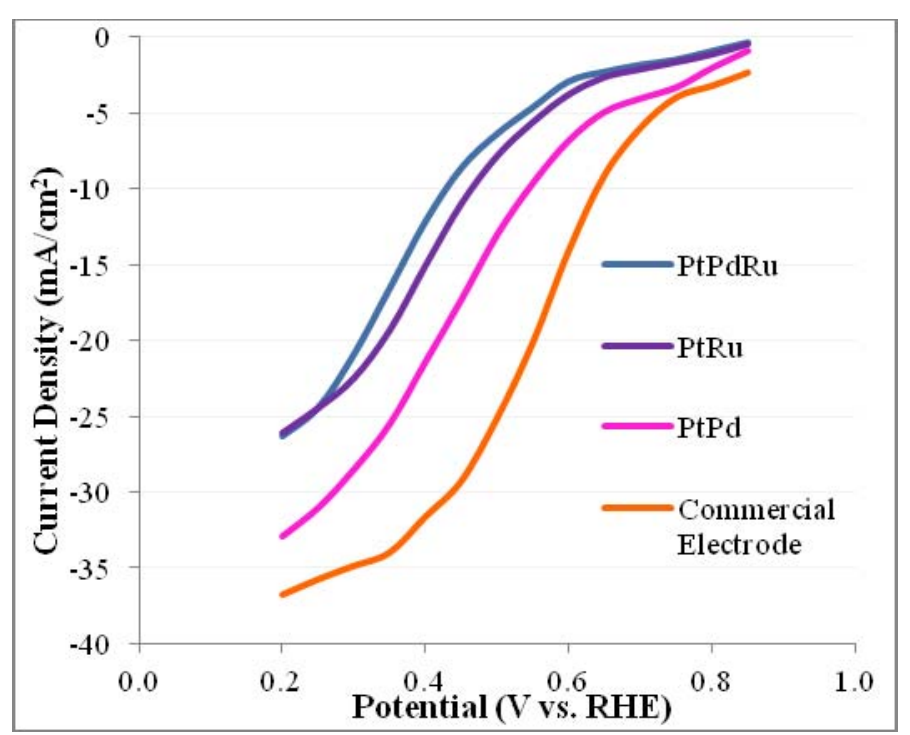

Figure 61. Curves for ORR of Pd electrodes that have been seeded. The electrodeposition solution contained $0.5 \mathrm{M} \mathrm{H}_{2} \mathrm{SO}_{4}$ and $2.5 \mathrm{mM} \mathrm{PdCl}_{2}$. The plots are the reverse CVs at $2500 \mathrm{rpm}$ in $0.5 \mathrm{M} \mathrm{H}_{2} \mathrm{SO}_{4}$. The on/off times were $25 / 50 \mathrm{~ms}$, peak current was $400 \mathrm{~mA} / \mathrm{cm}^{2}$, rotation rate was $400 \mathrm{rpm}$, and 100 cycles were used. 


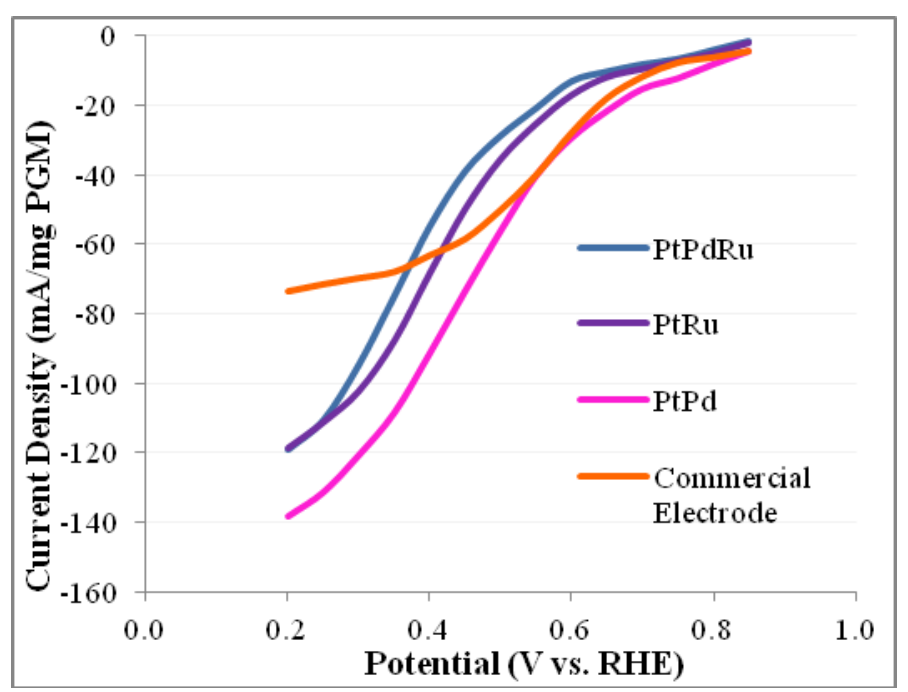

Figure 62. Curves for ORR of Pd electrodes that have been seeded, normalized for platinum group metal concentration. The electrodeposition solution contained $0.5 \mathrm{M} \mathrm{H}_{2} \mathrm{SO}_{4}$ and $2.5 \mathrm{mM} \mathrm{PdCl}_{2}$. The plots are the reverse CVs at $2500 \mathrm{rpm}$ in $0.5 \mathrm{M} \mathrm{H}_{2} \mathrm{SO}_{4}$. The on/off times were $25 / 50 \mathrm{~ms}$, peak current was 400 $\mathrm{mA} / \mathrm{cm}^{2}$, rotation rate was $400 \mathrm{rpm}$, and 100 cycles were used.
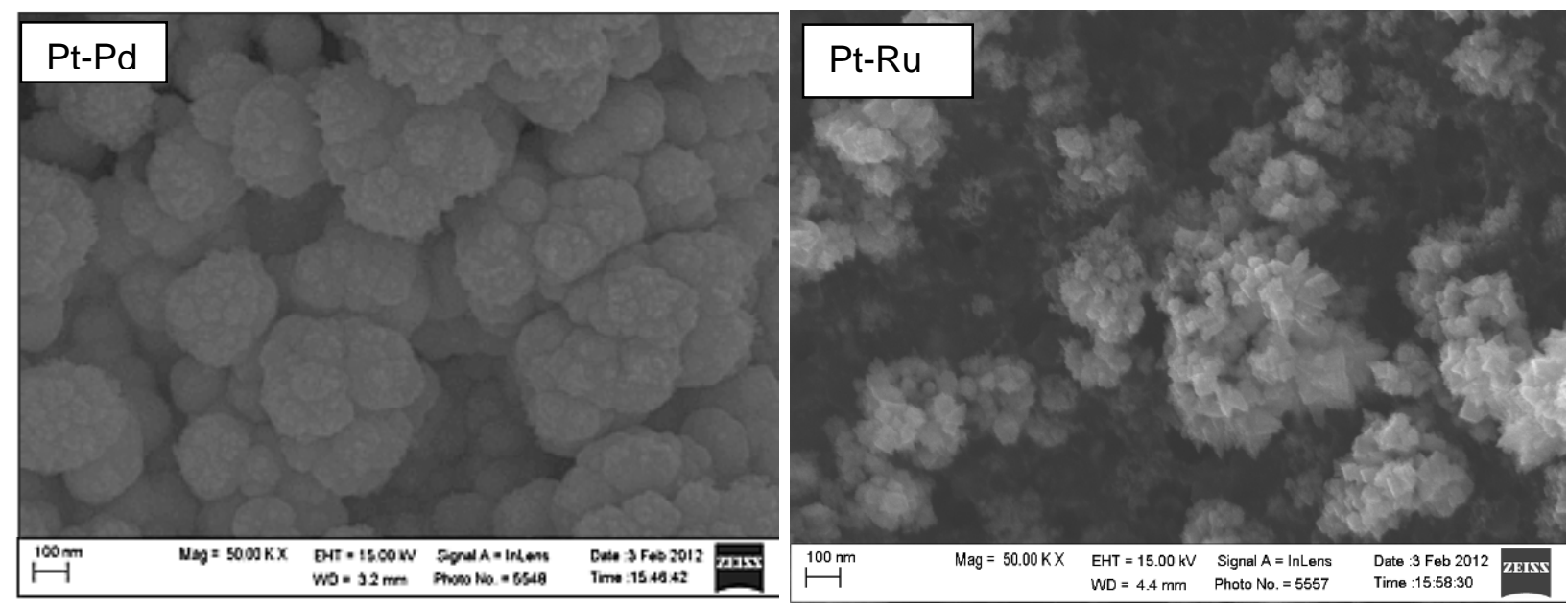

Figure 63. SEM images for Pt electrodeposited at 200 plating cycles using Pt-Pd and Pt-Ru as seeds.

\section{Summary}

In summary, Pd-based electrodes were prepared using catalyst seeding. The best performance was observed when Pt-Pd was used as a seed, while the performance of the electrodes that used Pt-Ru and Pt$\mathrm{Pd}-\mathrm{Ru}$ as seeds were quite similar. Although their performances were lower than that of the commercial Pt-based catalyst, when normalized for PGM content, the performance of the Pd electrodes were improved over the commercial catalyst.

\section{Task 7: Characterization of the prospective catalysts}

Characterization of the catalysts has been described in each of the previous tasks. In this section, additional tests, beyond the statement of work were carried out, including scaling up of the electrodes and stability of the electrodes. 


\section{Scale Up}

For these electrodes to be usable in fuel cells, they must be scaled up. Unfortunately, the rotating disk deposition method that was used thus far in the project does not scale up directly. With this in mind, a new electrodeposition cell was designed (Figure 64). In this cell, flow of solution entered and exited from the same side of the cell, while a cavity was formed within the cell by the Teflon gaskets. The flow of the electrodeposition solution was intended to mimic the electrode rotation that has occurred in the electrodepositions thus far. The GDE was slightly oversized relative to the cavity dimensions, allowing it to be held in place by the gaskets. The counter electrode was formed by spraying a $\mathrm{Pt} / \mathrm{C}$ electrode onto a carbon plate, then drilling bolt and solution feed holes. The reference electrode ( $\mathrm{Ag} / \mathrm{AgCl}$, not shown) was placed in a T-junction at the flow-in tubing, just exterior to the cell.

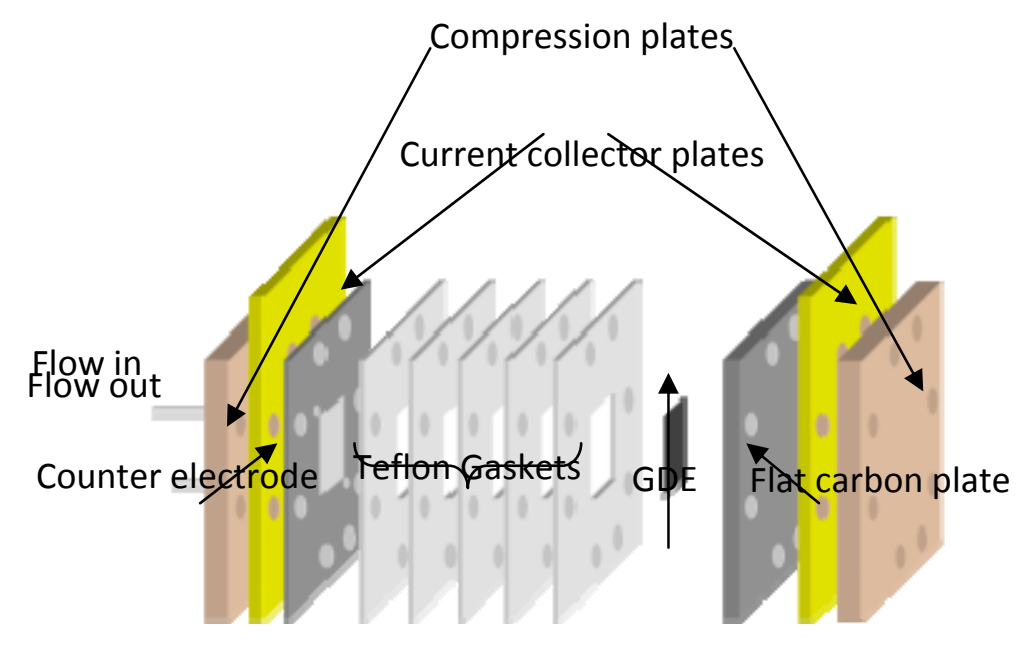

Figure 64. Schematic of scale-up PED cell

The cell was connected to a pump, allowing solutions to be pumped through the system. Initially, the solution was $1 \mathrm{M} \mathrm{H}_{2} \mathrm{SO}_{4}$, to clean the system of impurities. Then, a platinum precursor solution, made from $\mathrm{H}_{2} \mathrm{PtCl}_{6}, \mathrm{H}_{2} \mathrm{SO}_{4}$, and isopropanol, was pumped through the cell, with the reservoir being purged with argon to remove oxygen. After pumping for ten minutes, PED of platinum was accomplished (2 A ( 80 $\mathrm{mA} / \mathrm{cm}^{2}$ ) current, $25 \mathrm{~ms} / 50 \mathrm{~ms}$ on/off) for 50, 50, 100 then 200 cycles, successively. Following PED, a 1 $\mathrm{M} \mathrm{H}_{2} \mathrm{SO}_{4}$ solution was again pumped through the cell, after which it was disassembled. Figure 65a shows an SEM image of a GDE prepared from an XC-72R sprayed GDL after PED, clearly indicating that platinum was deposited uniformly over the surface of the GDE. The weight change of the GDE before and after PED determined a loading of $0.076 \mathrm{mg} \mathrm{Pt} / \mathrm{cm}^{2}$. Pieces of the GDE were removed for ORR and fuel cell characterization (Figure 66). The ORR (Figure 65b) shows that the performance of the scaled up GDE was lower than that prepared through an RDE method, indicating that the scale up method still must be optimized. 

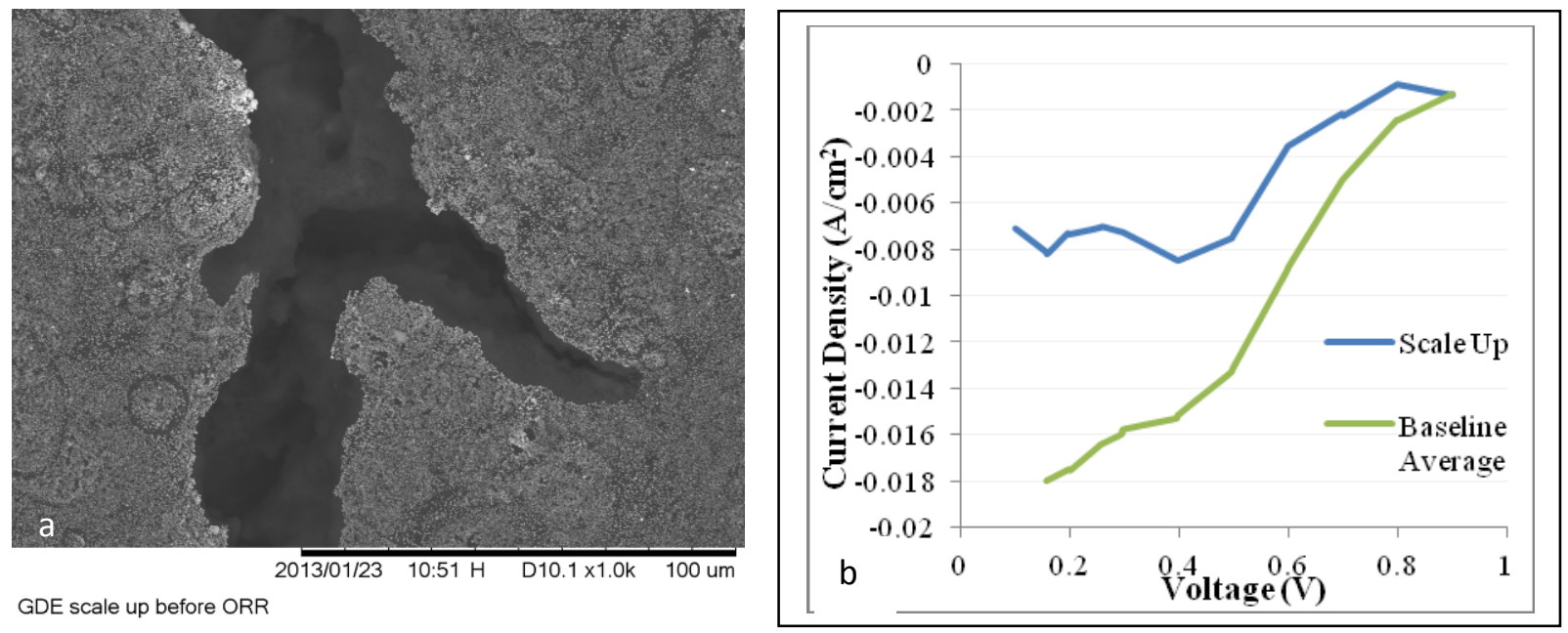

Figure 65. (a) SEM image and (b) ORR of the scaled-up PED XC-72R GDE

Inlet region

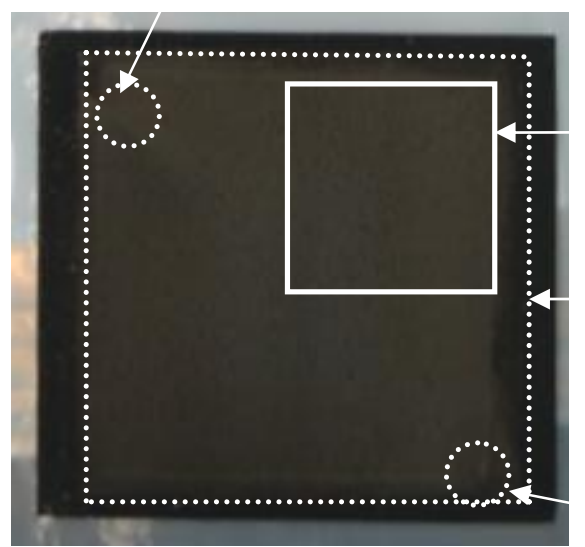

Section used for $5 \mathrm{~cm}^{2}$ cell testing

Sprayed region

Outlet region

Figure 66. Scaled up GDE after PED

A $5 \mathrm{~cm}^{2}$ section of the GDE was cut and tested in a fuel cell cathode, with a $32 \% 1100 \mathrm{EW} \mathrm{Pt} / \mathrm{C}$ decal transfer as the anode. The cell was tested at $80{ }^{\circ} \mathrm{C} / 100 \% \mathrm{RH}$ (ambient and 7 psig pressure), as well as at $95^{\circ} \mathrm{C} / 83 \% \mathrm{RH}$ and $120^{\circ} \mathrm{C} / 35 \% \mathrm{RH}$, both at $7 \mathrm{psig}$. At all conditions, performance was low, which was attributed to poor interfacial connectivity, as well as low platinum loading at the cathode. Performance at $80{ }^{\circ} \mathrm{C} / 100 \% \mathrm{RH}$ and 7 psig with air and oxygen is plotted in Figure 67, along with data from a typical cell prepared via the catalyst coated membrane (CCM) method. Clearly, the CCM method out-performs the PED approach. 


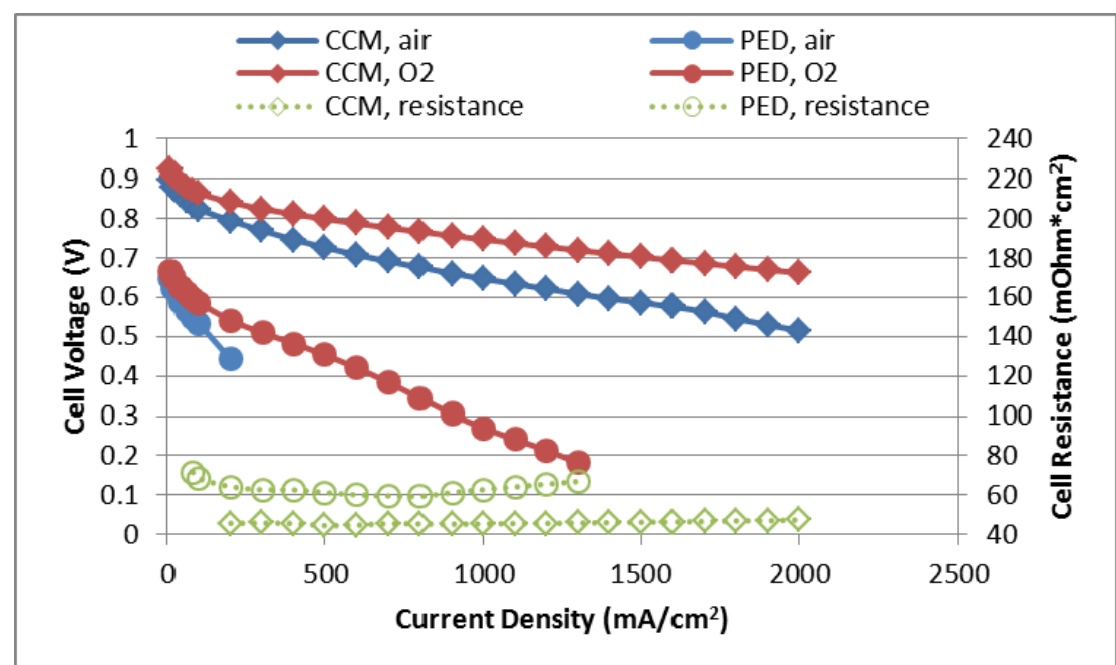

Figure 67. Fuel cell performance for PED and CCM cells at $80{ }^{\circ} \mathrm{C} / 100 \% \mathrm{RH}$ and $7 \mathrm{psig}$.

Because improved activity was observed at high voltage when reduced graphene oxide was used as a carbon support, a scaled-up electrode was also investigated with reduced graphene oxide support. The GDL was prepared as above, except that the ink contained graphene oxide. The GDE was fabricated, again using a flow cell, as described above.

Figure 68 shows the GDE after PED, clearly indicating that platinum was deposited uniformly over the surface of the GDE, but in very small quantities when compared to an XC-72R-GDL prepared previously (Figure 65a). The weight change of the RGO-GDL before and after PED determined a loading of 0.016 $\mathrm{mg} \mathrm{Pt} / \mathrm{cm}^{2}$. For comparison, the Pt-loading of the Pt-XC72R-GDL in Figure 65b was $0.076 \mathrm{mg} \mathrm{Pt} / \mathrm{cm}^{2}$.

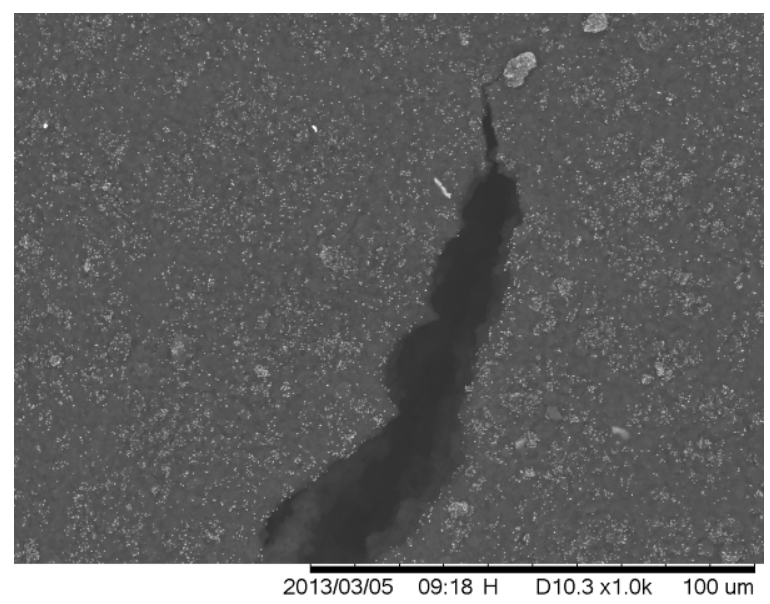

Figure 68. SEM image of Pt-RGO-GDL after PED of platinum

A higher applied overpotential was also observed for the RGO-GDL PED than for the XC72R-GDL PED (see Figure 69). It is hypothesized that the higher potential for the RGO-GDL PED is a direct result of lower electrical conductivity of the catalyst substrate. A lower electrical conductivity would increase the required overpotential for platinum deposition on the surface of the catalyst support, manifesting itself as a higher potential of deposition. In consequence of the greater applied overpotential, the extent of hydrogen evolution from the working potential also increased, thereby reducing the efficiency of the PED. Hydrogen evolution from carbon is known to occur around $-0.6 \mathrm{~V}$ vs. NHE, which is close to the value observed during PED of the RGO-GDL. By evolving hydrogen from the surface of the RGO-GDL, 
less platinum was deposited, even though the same current was applied.

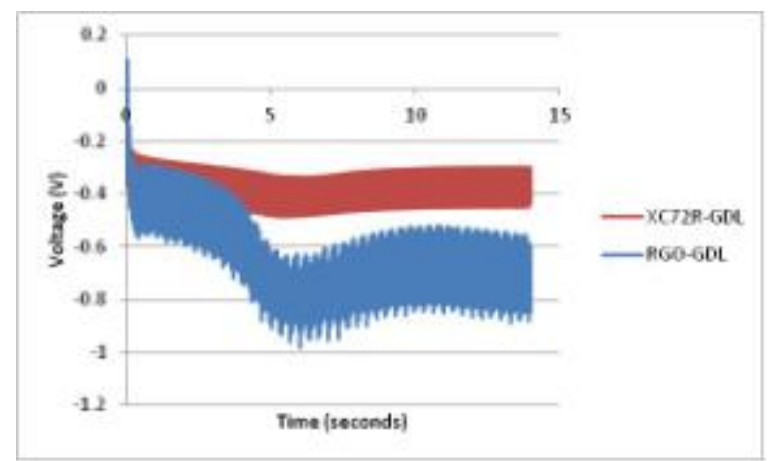

Figure 69. PED profile for XC72R-GDL and RGO-GDL. Conditions: $80 \mathrm{~mA} / \mathrm{cm}^{2}, 25 / 50 \mathrm{~ms}$ on/off, 200 cycles

A piece of the Pt-RGO-GDL was cut out and used in a $5 \mathrm{~cm}^{2}$ fuel cell test (see Figure 70). The same procedure was used for fuel cell assembly and testing as described above.

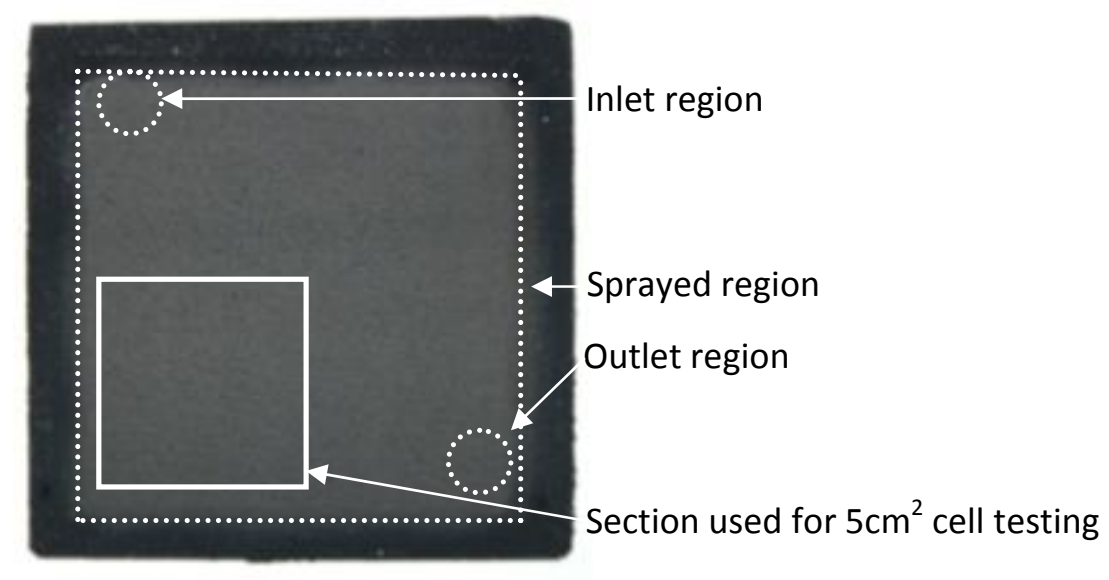

Figure 70. GDE after PED

Although the membrane integrity was confirmed to be adequate, performance at $80{ }^{\circ} \mathrm{C} / 100 \% \mathrm{RH}$ was very poor, with a maximum current of $20 \mathrm{~mA} / \mathrm{cm}^{2}$ at $0.207 \mathrm{~V}$ with oxygen at 7 psig. Given that this is a very low current density at a very low voltage, all at conditions that typically provide the best performance, the cell was considered unable to achieve reasonable currents. Therefore, no performance curves were obtained. The reason for the poor performance is probably due in large part to the very little platinum that was deposited on the surface.

\section{Stability}

Because of the importance of durability to increased fuel cell commercialization, durability studies were carried out on selected electrodes. An accelerated stress protocol was developed based on the literature catalyst durability protocols. ${ }^{[33]}$ In this protocol the voltage was cycled from 0.8 to $1.6 \mathrm{~V}$ at $50 \mathrm{mV} / \mathrm{s}$ and 0 rpm for 2100 cycles. The ORR was measured every 300 cycles, following the same procedures as used in previous tasks. Electrodes containing XC-72 and reduced graphene oxide were investigated in this task to observe whether the graphene oxide shows enhanced stability. 
Typical ORR plots for one electrode containing XC-72 as a support at each measuring point $(1=0$ cycles, $2=300$ cycles, $3=600$ cycles, etc.) are shown in Figure 71. In this figure it is evident that performance at high voltage decreases with increasing cycles, while performance at low voltage increases with increasing cycles. Because ORR activity is typically measured at high voltages, the current density for each type of electrode at $0.8 \mathrm{~V}$ was averaged and plotted in Figure 72. In this figure, it is clear that, while the activity of the electrode containing XC-72 decreases with increasing cycles, the activity of the electrode containing reduced graphene oxide remains relatively constant. Known degradation modes of platinum on carbon include increased Pt particle size due to support corrosion. As the particle size increases, the activity will decrease, as observed in Figure 72. This demonstrates that the reduced graphene oxide has improved the stability of platinum, due to a decreased oxidation of the graphitic support.

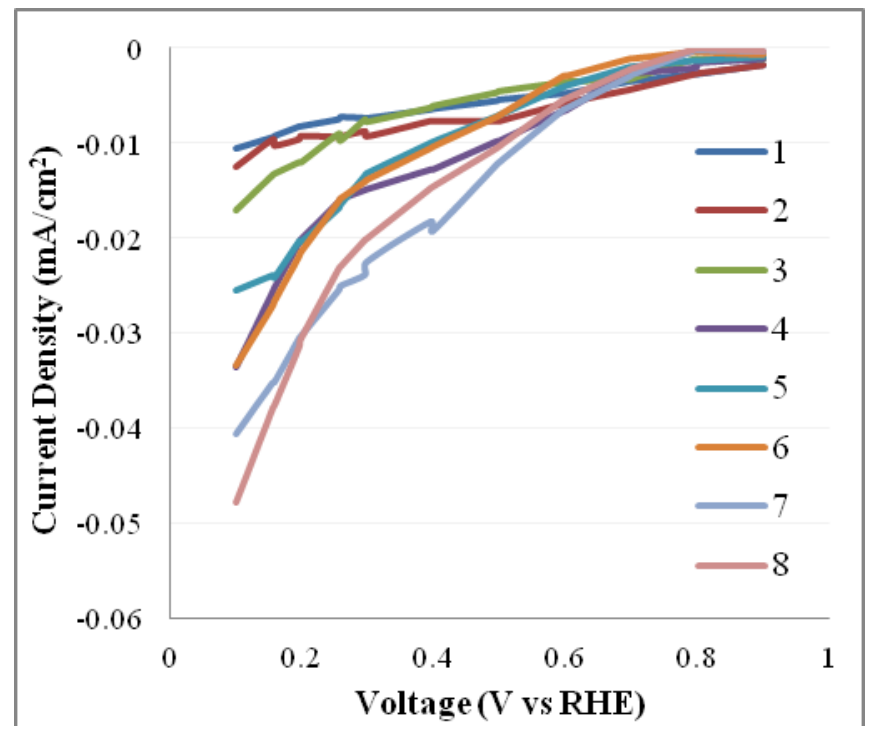

Figure 71. ORR Curves Pt electrodes that contain XC-72 as a support that have undergone different amounts of cycles. $1=0$ cycles, $2=300$ cycles, $3=600$ cycles, etc. The plots are the reverse CVs at 2500 rpm in $0.5 \mathrm{M} \mathrm{H}_{2} \mathrm{SO}_{4}$.

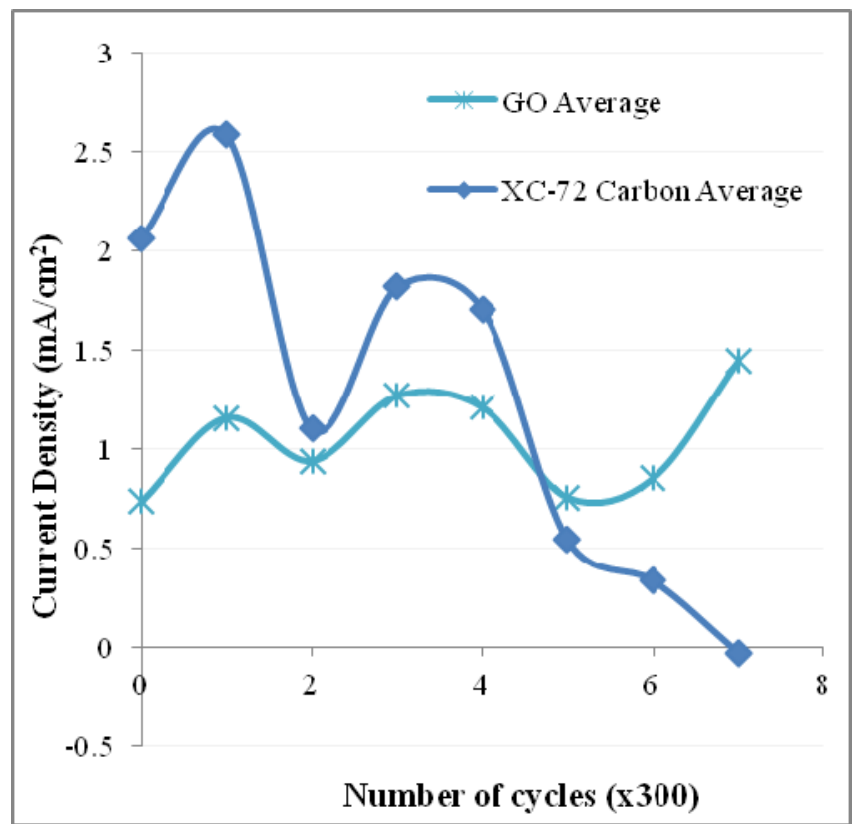

Figure 72. Current densities at $0.8 \mathrm{~V}$ for each type of electrode as a function of number of cycles 


\section{Summary}

In Task 7 scaling up of the electrode and electrode stability were investigated. For scaling up, an electrodeposition flow cell was designed and built and electrodes of $25 \mathrm{~cm}^{2}$ were prepared. This was a 50 fold scale up in size compared to the $0.5 \mathrm{~cm}^{2}$ electrodes that were prepared with the rotating disk electrode. Unfortunately, platinum loading using the scale-up cell was low and, as a consequence, fuel cell performance was low.

An accelerated stress protocol based on potential cycling was developed to investigate the stability of electrodes containing XC-72 and reduced graphene oxide as supports. It was shown that the electrode containing reduced graphene oxide had superior stability and did not degrade during testing.

\section{Conclusions}

With a goal of decreasing Pt loading in fuel cell electrodes while improving ORR activity, a study of catalyst preparation was conducted. The program was divided into seven tasks: 1) Establish a standard catalyst synthesis technique, 2) Evaluate carbon supports, 3) Synthesize Pt alloy based electrocatalysts, 4) Evaluate Pt or Pt-alloy based catalysts, 5) Evaluate Pt-lanthanide based electrocatalysts, 6) Evaluate Pd based catalysts, 7) Characterize catalysts.

The key results for the projects are as follows

- Optimum values of several variables (platinum precursor concentration, electrode rotation rate, peak current, number of cycles, on time, off time, stabilizer concentration) during electrodeposition were selected to achieve the highest electrode performance.

- It was evident that electrode morphology impacted performance in that improved performance was generally observed with higher electrode coverage, smaller particle size, and the formation of "feathery" or "flakey" particles.

- Thermal pretreatment of XC-72 carbon had no effect on electrode performance.

- The performance of electrodes made with XC-72 carbon support was lower than that of Cabot Regal GP-47 and activated carbon KB-B.

- Reduced graphene oxide had improved performance at high voltage over that of the baseline electrode.

- A new electrodeposition method was developed where the carbon electrodes were seeded with nanosized Pt colloidal particles prior to electrodeposition. With the new electrodeposition method, it was possible to achieve performance equivalent to a commercial electrode, but with $1 / 5$ the amount of Pt.

- Carrying out successive plating rather than direct plating resulted in improved performance, which was attributed to smaller Pt agglomerate size.

- Calcination of ceria improved its electrochemical stability, although the ceria was not shown to affect performance of Pt-based electrodes.

- Although their performances were lower than those of the commercial Pt-based catalyst, when normalized for PGM content the performance of the Pd electrodes was improved over the commercial catalyst.

- An electrodeposition scale-up flow cell was designed and built and electrodes of $25 \mathrm{~cm}^{2}$ were prepared. This was a 50-fold scale up in size compared to the $0.5 \mathrm{~cm}^{2}$ electrodes that were prepared with the rotating disk electrode. Unfortunately, platinum loading using the flow cell was low and, as a consequence, fuel cell performance was low.

- It was shown through a potential cycling accelerated stress test that the electrode containing reduced graphene oxide had superior stability and did not degrade during testing. 


\section{Project Publications:}

* C. Odetola, Master's Thesis, 2012, Development of High Efficiency, Low Cost Electrocatalysts for Proton Exchange Membrane Fuel Cell Applications, Florida Institute of Technology

* C. Huang, C. Odetola, M.P. Rodgers, Nanoparticle modified electroplating for the preparation of low Pt loading high performance electrocatalysts, submitted to Chemical Communications, 2012.

* C. Huang, C. Odetola, M.P. Rodgers, Seed-mediated pulse electrodeposition of Pt electrocatalysts for oxygen reduction reaction, submitted to Chemistry of Materials, 2012.

\section{Project Presentations}

* October 2011: M.P. Rodgers, C. Huang, C. Odetola, Development of electrocatalysts for PEM fuel cells by electrodeposition, Electrochemical Society Meeting, Boston, Massachusetts, USA.

* September 2011: C. Huang, Preparation and Characterization of Nanophase Pt and Pt-Alloy Cocatalysts for Solar Hydrogen Production, NanoFlorida, Miami, Florida

$>$ Winner of outstanding oral presentation award

* September 2010: M.P. Rodgers, C. Huang, Electrodeposition of Catalyst for use in Proton Exchange Membrane Fuel Cells, Florida Energy Systems Consortium Summit, Orlando, Florida, USA

\section{References}

${ }^{1}$ M. P. Rodgers, B. P. Pearman, N. Mohajeri, L. J. Bonville and D. K. Slattery, Electrochim. Acta, 100, 180 (2013).

${ }^{2}$ M. Gangeri, S. Perathoner, G. Centi, Inorganica Chimica Acta 359 (2006) 4828.

${ }^{3}$ H. Tsuchiya, O. Kobayashi, Int. J. Hydrogen Energy 29 (2004) 985.

${ }^{4}$ G.S. Chai, S.B. Yoon, J.S. Yu, J.H. Choi, Y.E. Sung, J. Phys Chem B, 108 (2004) 7074.

${ }^{5}$ S.H. Joo, S.J. Choi, I. Oh, J. Kwak, Z. Liu, O. Terasaki, R. Ryoo, Nature, 412 (2001) 169.

${ }^{6}$ S.D. Thompson, L.R. Jordan, M. Forsyth, Electrochim. Acta 46 (2001) 1657.

${ }^{7}$ Z.D. Wei, H.B. Ran, X.A. Liu, Y. Liu, C.X. Sun, S.H. Chan, P.K. Shen, Electrochim. Acta 51 (2006) 3091.

${ }^{8}$ G. Lister, G. McLean, J. Power Sources 130 (2004) 61.

${ }^{9}$ V. Jalan, E.J. Taylor, J. Electrochem. Soc. 130 (1983) 2299.

${ }^{10}$ T. Toda, H. Igarashi, H. Uchida, M. Watanabe, J. Electrochem. Soc. 146 (1999) 3750.

${ }^{11}$ V. R. Stamenkovic, B. Fowler, B. S. Mun, G. Wang, P. N. Ross, C. A. Lucas, N. M. Marković. Science 315 (2007) 493.

${ }^{12}$ A.J. Dickinson, L.P.L. Carrette, J.A. Collins, K.A. Friedrich, U. Stimming, Electrochim. Acta 47 (2002) 3733

${ }^{13}$ S. Wasmus, A. Kuver, J. Electroanal. Chem. 461 (1999) 14.

${ }^{14}$ L. Ji, X. Zhang Mater. Lett. 62 (2008) 4

${ }^{15}$ S.M. Ayyadurai,Y.-Seok. Choi, P. Ganesan, S.P. Kumaraguru, B.N. Popov, J.Electrochem. Soc. 154(10) (2007) B1063.

${ }^{16}$ J. Lee, J. Seo, K. Han and H. Kim, Journal of Power Sources, 163, 349 (2006).

${ }^{17}$ N. Ibl, Surf. Technol. 10 (1980) 81.

${ }^{18}$ H. Y. Cheh, Journal of The Electrochemical Society, 118, 1132 (1971).

${ }^{19}$ C. Coutanceau, A. F. RakotondrainibÃ $@$, A. Lima, E. Garnier, S. Pronier, J. M. LÃ@ger and C. Lamy, Journal of Applied Electrochemistry, 34, 61 (2004)

${ }^{20}$ B.G. Pollet Electrochem. Comm. 11 (2009) 1445.

${ }^{21}$ A. Dalmia, C.L. Lineken, R.F. Savinell, J. Colloid Interface Sci. 205 (1998) 535.

${ }^{22}$ M.D. Obradović, R.M Stevanović, A.R. Despić, J Electroanal. Chem. 552 (2003) 185.

${ }^{23}$ O. Younes, L. Zhu Langmuir 17 (2001) 8270. 
${ }^{24}$ E. Guaus, J. Torrent-Burgués J. Electroanal Chem 575 (2005) 301.

${ }^{25}$ G.M. Zarkadas, A. Steriou, G. Papanastasiou J. Appl. Electrochem. 34 (2001) 1251.

${ }^{26}$ G.M. Zarkadas, A. Steriou, G. Papanastasiou Electrochim Acta 50 (2005) 5022.

${ }^{27}$ Y. Mu, H. Liang J. Phys. Chem. B 109 (2005) 22212.

${ }^{28}$ V. Lordi, N. Yao, J. Wei Chem. Mater. 13 (2001) 733.

${ }^{29}$ T. Matsumoto, T. Komatsu Catal. Today 90 (2004) 277.

${ }^{30}$ J. Sieben, M. Duarte, C. Mayer and J. BazÃ $i n$, Journal of Applied Electrochemistry, 39, 1045 (2009).

${ }^{31}$ M. P. Rodgers, R. P. Brooker, N. Mohajeri, L. J. Bonville, H. R. Kunz, D. K. Slattery and J. M. Fenton, J. Electrochem. Soc., 159, F338 (2012).

${ }^{32}$ Figure 4 in Journal of the Electrochemical Society 153 (1) C74-C79 2006

${ }^{33}$ V.A.T. Dam, F.A. de Bruijn J. Electrochem. Soc., 154 (2007), pp. B494-B499 


\title{
FLORIDA HYDROGEN INITIATIVE \\ Sub-contrector Finel Report
}

\section{Understanding Mechanical and Chemical Durability of Fuel Cell Membrane Electrode Assemblies}

\author{
Dr. Darlene Slattery \\ Dr.Leonard Bonville \\ Dr. Nahid Mohajeri \\ Dr. Marianne Rogers \\ Florida Solar Energy Center \\ Advanced Energy Research Division \\ dkslatt@fsec.ucf.edu \\ (321) 638-1449
}

\author{
Submitted to: \\ David L. Block \\ Florida Solar Energy Center/University of Central Florida \\ 1679 Clearlake Road \\ Cocoa, FL 32922 \\ E-mail: block@fsec.ucf.edu \\ Contract Number: DE-FC36-04GO14225 \\ FSEC Project No.: 20126068 \\ Project Start Date: April 8, 2010 \\ Project End Date: June 30, 2012
}

October 2012

\section{Disclaimer}

The Florida Solar Energy Center/University of Central Florida nor any agency thereof, nor any of their employees, makes any warranty, express or implied, or assumes any legal liability or responsibility for the accuracy, completeness, or usefulness of any information, apparatus, product, or process disclosed, or represents that its use would not infringe privately owned rights. Reference herein to any specific commercial product, process, or service by trade name, trademark, manufacturer, or otherwise does not necessarily constitute or imply its endorsement, recommendation, or favoring by the Florida Solar Energy Center/University of Central Florida or any agency thereof. The views and opinions of authors expressed herein do not necessarily state or reflect those of the Florida Solar Energy Center/University of Central Florida or any agency 


\title{
Understanding Mechanical and Chemical Durability of Fuel Cell Membrane Electrode Assemblies
}

\author{
Florida Solar Energy Center \\ Advanced Energy Research Division
}

\section{Executive Summary}

The useable life of the current proton exchange membrane fuel cell (PEMFC) falls short of durability requirements for commercialization, primarily due to combined chemical and mechanical degradation of the membrane. The objective of this project was to increase PEM membrane useable life through a better understanding of the degradation mechanisms and their mitigation. Durability targets for membranes in automotive and stationary fuel cell applications have been established by the U.S. Department of Energy (DOE) to be 5000 hours for automotive and 40,000 hours for stationary applications. The current fuel cell membrane useable life (i.e. 2,500 hrs, as of 2011) falls short of both DOE durability targets.

One of the main sources of membrane degradation is hydroxyl radical attack of the membrane polymer. These hydroxyls are formed where platinum, hydrogen, and oxygen are present. Once formed, the radicals diffuse into the membrane, and attack both the main- and side-chains of the membrane polymer, leading to pinhole and crack formation. This loss of membrane integrity results in large-scale hydrogen crossover, shortening the cell life and generating large performance losses. Mitigation of chemical degradation will significantly enhance the lifetime of membranes, making fuel cells more economical. However, in order to decrease chemical degradation, the chemical processes that contribute to membrane failure must be better understood.

With this understanding of the basics of membrane degradation, the project was developed with four major tasks: 1) the chemical mitigation of membrane degradation; 2) the evaluation of platinum band formation; 3) the mitigation of platinum band formation; and 4) radical scavenging and platinum band reduction. The objectives for Task 1 were to identify five suitable additives for the scavenging radicals in the membrane and then to characterize the membranes containing additives and determine the platinum particle size as a function of additive. Task 2 objectives were to locate and characterize the platinum band in the membranes and to investigate the impact of cell components and operation parameters on platinum band formation and membrane degradation. The goals of Task 3 were to select some heteropolyacids to be used for mitigation of platinum band formation and to incorporate these into a sublayer between the membrane and the electrode. And, finally, Task 4 was to combine the best performing additive from Task 1 with the most effective heteropolyacid from Task 3 to achieve a more durable cell.

The Task 1 objective was to identify additives for scavenging radicals. The results from experimentation established ceria's ability to mitigate membrane degradation through radical scavenging. Key results are as follows:

1) Five additives for scavenging radicals were identified, down selected to two candidates through ex-situ gas and liquid Fenton testing; and then the best candidate, cerium oxide, was evaluated insitu for both automotive and stationary applications.

2) Ex-situ testing on ceria's ability to stabilize a PEM membrane demonstrated that:

- The ceria particles reduced fluoride emission by up to one order of magnitude due to the cerium's ability to scavenge hydroxyl and hydroperoxyl radicals;

- The degradation mitigation was found to increase with increasing additive concentration, 
though it was independent of ceria formulation and therefore of particle size;

- End-group unzipping was confirmed as the main degradation mechanism for the liquid Fenton test by reaction product analysis;

- Additionally, the gas Fenton test verified a reduced level of side-chain attack versus testing without cerium.

3) Experiments showed that cerium oxide acts as a radical scavenger and successively protects the membrane, reducing both the OCV decay and fluoride emissions dramatically.

4) The analysis of the platinum particles in the membrane showed that Pt band formation is also influenced by the presence of this cerium additive. Cerium oxide incorporation resulted in the formation of fewer and larger particles platinum, with less platinum precipitation overall. The result implies that the potential profile through the membrane is shifted to the cathode interface by the presence of the ceria, resulting in an altered, more diffuse Pt band. The more diffuse platinum band reduces the extent of localized degradation (weak spots) as well as reduces the total amount of membrane degradation.

5) In 500 hour tests, the addition of $1.0 \mathrm{wt} \%$ of commercial ceria resulted in sevenfold decrease in the OCV decay rate versus the baseline while losing less than $1 \%$ of its fluorine inventory. It also showed no change in performance or hydrogen crossover, resulting in an essentially unchanged MEA that appears capable of exceeding the required durability targets set by DOE.

The results from Task 2, experiments showed relative humidity ( $\mathrm{RH})$, equivalent weight (EW), catalyst type, and amount of platinum deposited in the membrane each influenced degradation in varying degrees.

For RH, degradation was higher for the 20 and 30\% RH and lower for 10 and 100\% RH. At high RH, decreased degradation is attributed to dilution of radicals, higher oxide coverage on Pt, and lower partial pressures of reactant gases. At low $\mathrm{RH}$, decreased degradation is attributed to reduced Pt band formation due to low mobility of Pt in low RH conditions.

With regard to the influence of EW, $950 \mathrm{EW}$ polymers exhibited the lowest durability. This has been explained in terms of degree of crystallinity, which increases with increasing EW and decreasing side chain length, and which in turn impacts the mechanical stability of the membrane.

The third parameter investigated, catalyst type, showed that fluoride emission was three times higher for $\mathrm{Pt} / \mathrm{C}$ than for $\mathrm{PtCo} / \mathrm{C}$ and the OCV decay was nearly twice as great for the $\mathrm{Pt} / \mathrm{C}$ as that for the $\mathrm{PtCo} / \mathrm{C}$. Transmission electron microscopy images showed that platinum particles were dispersed throughout the membrane of the catalyst coated membrane (CCM) containing PtCo/C, while the $\mathrm{CCM}$ containing Pt/C showed formation of a platinum band. The platinum band in the cell containing $\mathrm{Pt} / \mathrm{C}$ is cited as the cause for the increased degradation compared to the cell containing PtCo/C.

When Pt was incorporated into the membrane, the highest degradation was observed with the membrane containing $10 \mathrm{~mol} \% \mathrm{Pt}$, while membranes containing 0, 30, and $50 \mathrm{~mol} \%$ Pt resulted in very low fluoride emission. Membrane degradation was only accelerated when the Pt particles were small and close together (average particle size $=2.4 \mathrm{~nm}$, average particle distance $=10 \mathrm{~nm}$ for $10 \mathrm{~mol} \%$ Pt after testing). When the Pt particles in the membrane were larger and further apart, very little degradation was observed.

In Task 3, the addition of heteropoly acids (HPAs) to sublayers was investigated to determine their effect on platinum band formation. These results showed HPAs reduce the fluoride emission rate (FER) by as much as half, with phosophotungstic acid (PTA) showing the greatest reduction. The OCV loss with HPAs was significantly reduced, again with PTA showing the greatest improvement. Modeling of the voltage profile through the membrane and sublayers indicated that only PTA was capable of reducing platinum ions. The PTA-1V and STA-1V HPAs resulted in sublayer voltage profiles that were too high for platinum reduction. For all HPAs investigated, HPA mobility within the cell proved problematic, with 
the concentration of HPA in the sublayers decreasing after testing. Tethering the HPA to the surface of the carbon support through covalent bonding is possible, and could be employed in future efforts.

The Task 4 objective was to combine the best additive to a membrane (from Task 1 ) and combine it with a sublayer containing the most effective HPA (from Task 3). Using the experimental results from Task 1 and 3, $1 \mathrm{wt} \%$ ceria was added to the membrane and PTA to a sublayer. The resulting system demonstrated a 5-fold reduction in degradation compared to a cell without either membrane additive or PTA sublayer. Additional improvements in performance and durability are expected by incorporating the PTA directly into the electrode.

While the primary objective of this project was to increase PEM membrane life, increasing the knowledge base of the degradation mechanisms for membranes, the development path of reducing membrane degradation is at the very heart of the PEM fuel cell technology. The potential commercial market has national and international product growth and energy benefits and, thus, is of importance to the public. 


\section{Understanding Mechanical and Chemical Durability of Fuel Cell Membrane Electrode Assemblies}

\section{Table of Contents}

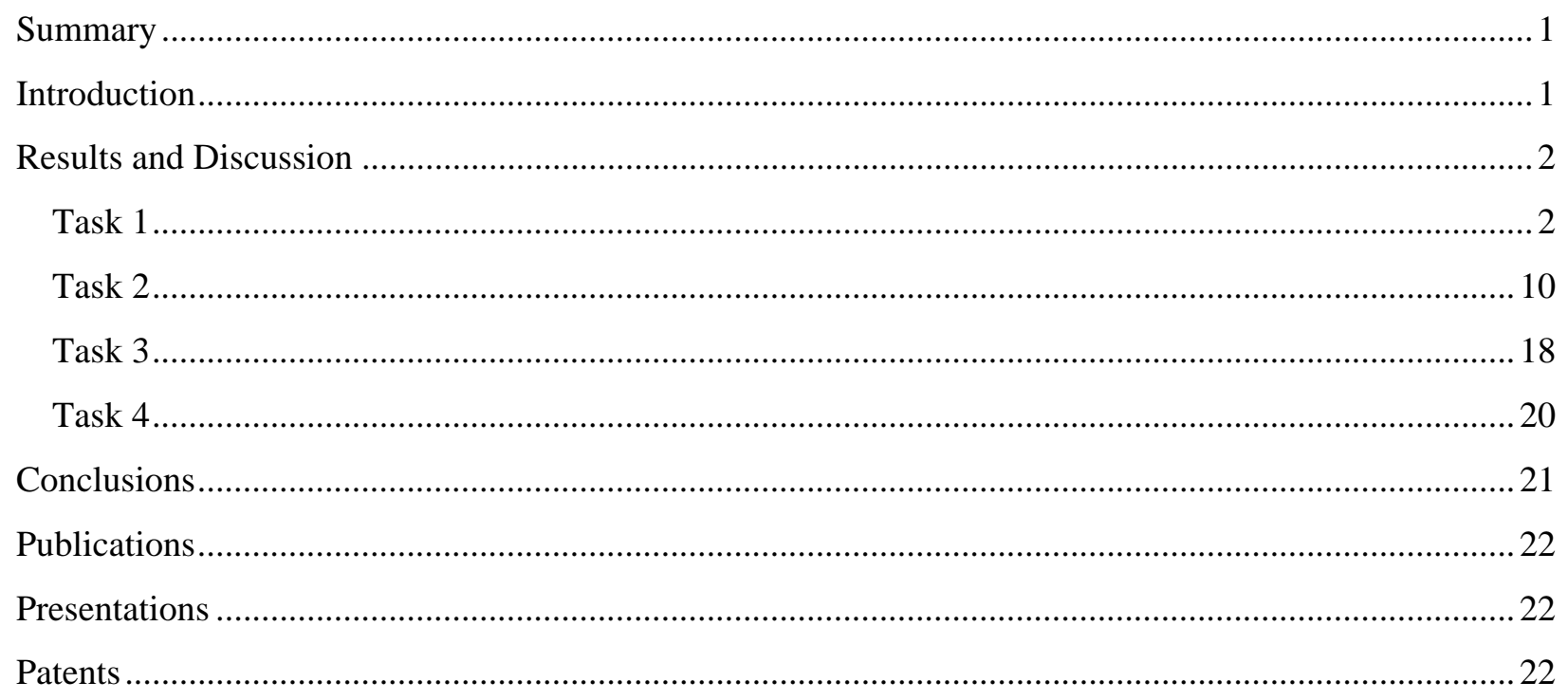




\title{
Understanding Mechanical and Chemical Durability of Fuel Cell Membrane Electrode Assemblies
}

\author{
Florida Solar Energy Center \\ Advanced Energy Research Division
}

\begin{abstract}
Summary
Experiments were conducted to determine methods for the mitigation of degradation in a fuel cell membrane. Ceria was added to a membrane to scavenge radicals that are known to have a negative effect on membrane integrity. This was found to be very effective. Additionally, heteropoly acids were added to a sublayer of carbon that was placed between the membrane and the catalyst layer in an effort to reduce platinum ions before they could reach the membrane. This also worked but it was determined that to increase performance, it would be better to add the heteropoly acid directly to the catalyst layer. An additional task was performed to characterize the platinum band that is known to form in membranes from the migration of the platinum ions from the catalyst layer. The influence of parameters such as relative humidity $(\mathrm{RH})$, equivalent weight $(\mathrm{EW})$, catalyst type, and amount of platinum deposited in the membrane were investigated and found to influence degradation.
\end{abstract}

\section{Introduction}

Radical attack is the dominant source of chemical degradation of the membrane. In one mechanism, these radicals form in-situ, after peroxide is generated through the $2 \mathrm{e}^{-}$reduction of oxygen on platinum, as described in Equation 1:

$$
\mathrm{O}_{2}+2 \mathrm{H}^{+}+2 e^{-} \rightarrow \mathrm{H}_{2} \mathrm{O}_{2} \quad \mathrm{E}^{0}=0.695 \mathrm{~V}_{\mathrm{RHE}}
$$

Peroxide can then be decomposed through a Fenton reaction (Eq. 2), forming hydroxyl radicals:

$$
\mathrm{H}_{2} \mathrm{O}_{2}+\mathrm{M}^{2+} \rightarrow \mathrm{M}^{3+}+{ }^{\bullet} \mathrm{OH}+\mathrm{OH}^{-}
$$

where $\mathrm{M}^{2+}$ represents trace metals (iron, cobalt, etc) present as contaminants within the membrane. Other mechanisms for radical formation have been proposed, where radicals are formed following Pt-OH and $\mathrm{Pt}-\mathrm{OOH}$ species generation. ${ }^{1,2}$ In either case, it has been shown that membrane degradation requires the presence of $\mathrm{H}_{2}, \mathrm{O}_{2}$ and platinum. ${ }^{2}$

The conditions for radical formation are generally met at the anode and cathode, where any reactants diffusing through the membrane can react on the surface of the platinum catalyst. In addition, high potentials at the cathode can result in platinum ion formation. These ions can then migrate into the membrane, until they are reduced by hydrogen. Over time, the number and size of platinum particles increase within the membrane, forming a platinum band. At this location, the potential is low and since this band forms within the membrane, oxygen concentrations can be higher than at the anode. These conditions lead to significant generation of hydroxyl radicals, resulting in severe membrane degradation.

Membrane degradation is characterized by several methods, but commonly for perfluorosulfonic acidtype membranes, the release of fluoride ions, or fluoride emission rate (FER), is used. Although several compounds are released during membrane degradation, such as $\mathrm{CO}_{2}, \mathrm{SO}$, and $\mathrm{SO}_{2}$, fluoride ions can be easily measured in the effluent water, providing a convenient measurement technique. It has been observed that FER will gradually increase at the beginning of testing, which may be a measure of platinum band growth. ${ }^{3}$ Further, FER appears to change with relative humidity, having higher FER values 
at $50 \% \mathrm{RH}$ than at $10 \% \mathrm{RH}^{4}$. The dependence of FER on RH may depend on the ability of platinum to dissolve and move into the membrane. However, increasing relative humidity to $100 \%$ decreases FER, most probably due to a passivating effect of water on the platinum surface. It is known that high relative humidity conditions result in more oxide coverage on platinum, and this oxide layer can protect the platinum from dissolution.

\section{Results and Discussion}

\section{Task 1}

The goals of Task 1 were to quantify the effects of inorganic chemical mitigators on PFSA membranes durability. Cerium oxide (ceria) was identified as a possible radical scavenger because of its ability to act as a redox couple, facilely switching between the $3+$ and $4+$ oxidation states. Two kinds of ceria were utilized, an in-house synthesized material and one that was commercially available. Each was incorporated into a composite membrane cast from an 1100 EW PFSA ionomer and a porous PFTE support. Table 1 lists the MEAs measured for accelerated durability testing (94 h OCV hold and 500h OCV hold).

Table 1- List of MEAs and OCV hold conditions

\begin{tabular}{ccc}
\multicolumn{3}{c}{ 94 h OCV Hold } \\
\hline \multicolumn{1}{c}{ MEA } & Ceria & \# of cells \\
\hline Baseline & None & 3 \\
Synthesized ceria & 0.5 wt $\%$ & 2 \\
Synthesized ceria & $1.0 w t \%$ & 2 \\
Synthesized ceria & $2.0 w t \%$ & 2 \\
Commercial ceria & $0.5 w t \%$ & 2 \\
Commercial ceria & $1.0 w t \%$ & 2 \\
Commercial ceria & $2.0 w t \%$ & 2 \\
\hline
\end{tabular}

$500 \mathrm{~h}$ OCV Hold

\begin{tabular}{llc}
\hline \multicolumn{1}{c}{ MEA } & Ceria & \# of cells \\
\hline Baseline & None & 1 \\
Synthesized ceria & $1.0 \mathrm{wt} \%$ & 1 \\
Commercial ceria & $1.0 \mathrm{wt} \%$ & 1 \\
\hline
\end{tabular}

OCV Hold Test Conditions

\begin{tabular}{lcccc}
\hline & Type & Flow & RH & Pressure \\
\hline $94 \mathrm{~h}$ OCV hold anode fuel & $\mathrm{H}_{2}$ & $200 \mathrm{~cm}^{3} \mathrm{~min}^{-1}$ & $30 \%$ & Ambient \\
$94 \mathrm{~h}$ OCV hold cathode fuel & Air & $200 \mathrm{~cm}^{3} \mathrm{~min}^{-1}$ & $30 \%$ & Ambient \\
& & & & \\
& $\mathrm{H}_{2}$ & $350 \mathrm{~cm}^{3} \mathrm{~min}^{-1}$ & $30 \%$ & $150 \mathrm{kPa}$ \\
$500 \mathrm{~h}$ OCV hold anode fuel & Air & $830 \mathrm{~cm}^{3} \mathrm{~min}^{-1}$ & $30 \%$ & $150 \mathrm{kPa}$ \\
\hline $500 \mathrm{~h}$ OCV hold cathode fuel & & &
\end{tabular}

\section{4 h OCV Hold Durability Testing}

Two fuel cells with MEAs containing ceria concentrations of $0.5,1.0$ and $2.0 \mathrm{wt} \%$ for each ceria formulation, as well as three baselines, were built and tested. Figure 1 shows the OCV decay rate of the cells over 94 hours as a function of the ceria concentration in the membranes. The addition of ceria reduced the decay rate by approximately $50 \%$ compared to the baseline though changes in the concentration of ceria had no significant effect. Further, the OCV decay rate mitigation was independent of additive formulation and therefore of particle size. 


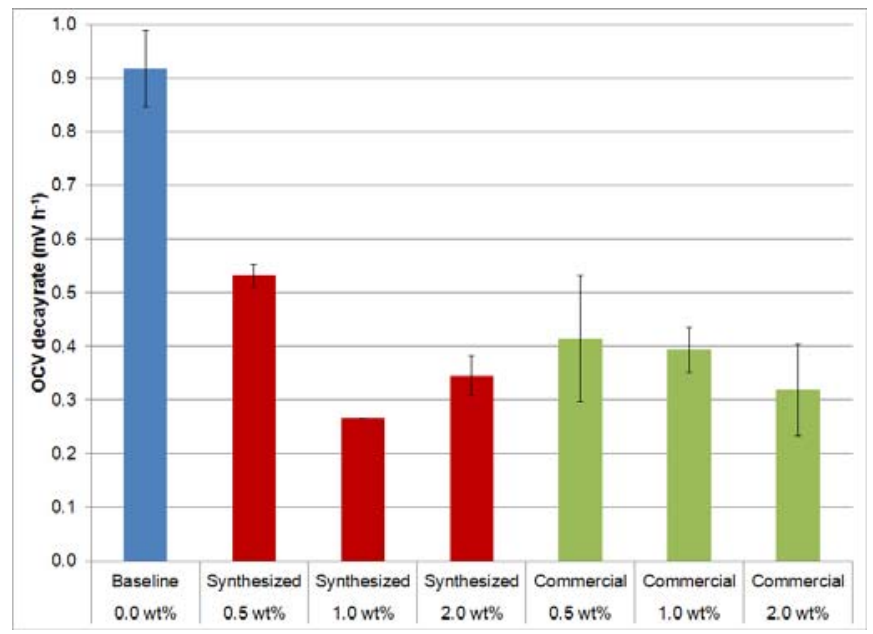

Figure 1. Average OCV decay rate of MEAs in $94 \mathrm{~h}$ OCV hold test

Figure 2a-c show representative SEM images of cross-sections of MEAs demonstrating the formation of a distinct band of particles in the membrane. The nature of these particles, found at a distance of 3.5 $4.5 \mu \mathrm{m}$ from the cathode, was confirmed by EDS analysis (Figure 2d) to be platinum. Reports of Pt band particles in the literature have shown that they can be either faceted or dendritic, though the precise mechanism of growth is not well known. ${ }^{5,6}$ We observed both structures, sometimes even within the same sample. Representative high magnification images of faceted and dendritic particles are given Figure 3c and $\mathrm{d}$, respectively, showing the crystal structure of both.
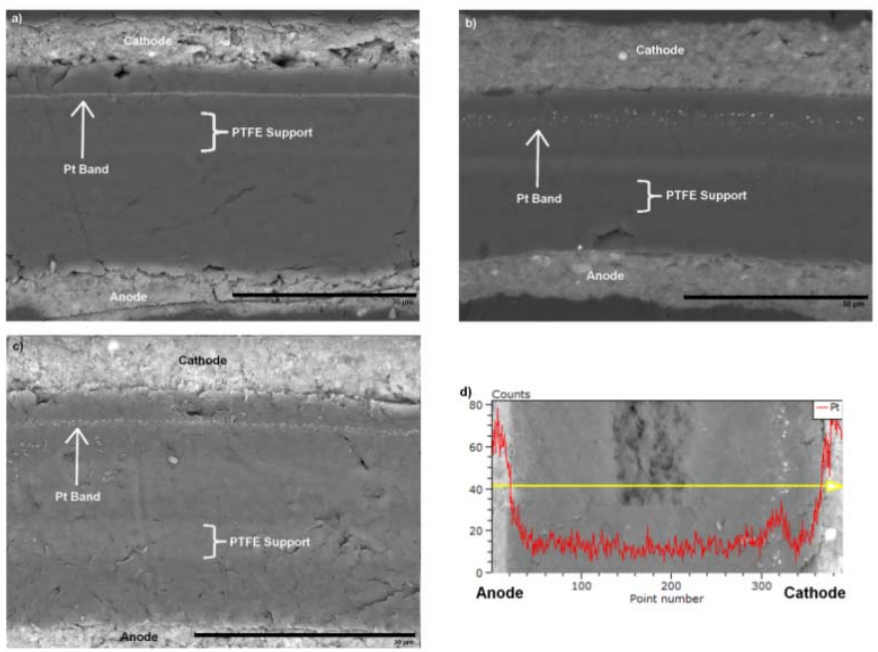

Figure 2. SEM images of $94 \mathrm{~h}$ OCV hold tested CCM cross-sections: a) baseline, b) synthesized ceria 2.0 wt\%, c) commercial ceria $2.0 \mathrm{wt} \%$ and d) baseline with platinum EDS spectrum overlay 


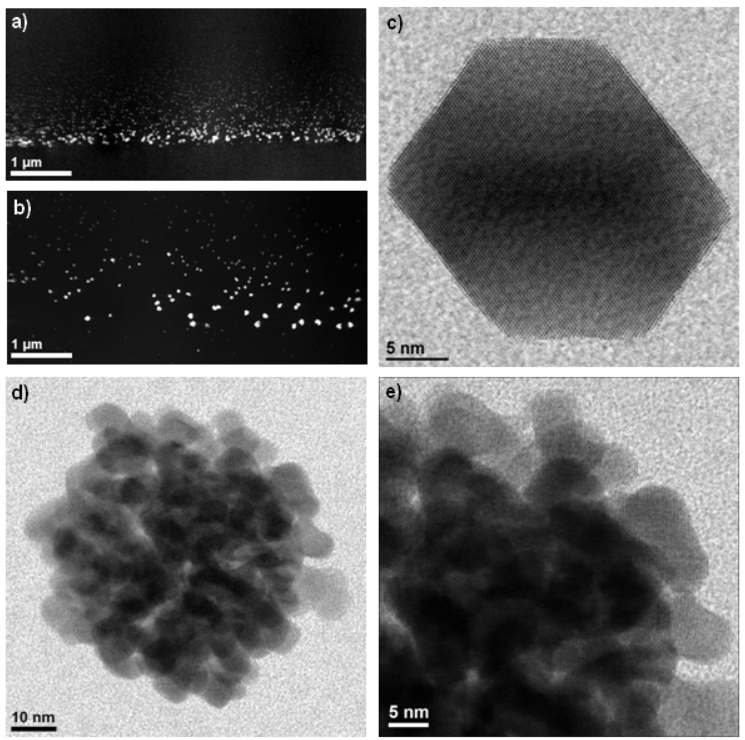

Figure 3. STEM images of cross-sections of $94 \mathrm{~h}$ OCV hold tested MEAs: a) Pt band in a baseline MEA (cathode at bottom of image), b) Pt band in a commercial $1.0 \mathrm{wt} \%$ MEA (cathode at bottom of image), c) high magnification image of a faceted Pt particle, d) a dendritic Pt particle and e) high magnification image of a dendritic Pt particle

The inclusion of ceria into membranes had no effect on the types of Pt particles formed. However, SEM (Figure 2) and TEM images (Figure 3a and b) indicated that fewer particles were present in ceriacontaining MEAs. Size and distribution of the Pt particles were determined from STEM images for five MEAs: a baseline, a synthesized ceria $1.0 \mathrm{wt} \%$ and $2.0 \mathrm{wt} \%$ and a commercial ceria $1.0 \mathrm{wt} \%$ and $2.0 \mathrm{wt} \%$ MEA.

As listed in Table 2, the average Pt particle size for the baseline MEA was two to four times smaller than for the ceria-containing MEAs, though it should be noted that there was a significant variation in particle size within each sample. Figures $3 a$ and $b$ clearly shows the fading of the Pt band, from many large particles to fewer and smaller particles, with increasing distance from the cathode, a common observation for Pt bands in OCV hold tested membranes. ${ }^{7,8,9,10}$

On the other hand, the number of particles per area in the baseline was at least one order of magnitude higher than in the ceria-containing MEAs. The combination of more but smaller particles means that the total area covered by the particles in the baseline was at least three times as high, demonstrating that less Pt has been deposited in the ceria-containing MEAs.

Table 2- Average particle size, relative particle counts and area coverage Baseline Synthesized

Commercial

1.0 wt $\% \quad 2.0$ wt $\% \quad 1.0$ wt $\% \quad 2.0$ wt $\%$

\begin{tabular}{cccccc}
\hline Average Particle Size $(\mathrm{nm})$ & $19.0 \pm 10.1$ & $31.9 \pm 26.8$ & $71.3 \pm 51.6$ & $50.1 \pm 30.1$ & $35.3 \pm 21.7$ \\
Particle Counts per $\mu \mathrm{m}^{2}$ & 253 & 8 & 2 & 1 & 24 \\
Area Coverage & $9.3 \%$ & $1.8 \%$ & $1.3 \%$ & $0.7 \%$ & $3.2 \%$ \\
\hline
\end{tabular}

The decrease in the number of particles in the Pt bands of ceria-containing membranes demonstrates that ceria influences the behavior of dissolved Pt ions. As all MEAs showed a similar decrease in ECA, it is unlikely that ceria prevents catalyst dissolution. The observation that particles extend further into the 
membrane, sometimes even all the way to the anode, suggests that the presence of ceria changes the potential profile. Brooker et al. ${ }^{11}$ have shown that the inclusion of redox-active heteropolyacids in a sublayer between the catalyst and the membrane, perturbs the potential profile resulting in the deposition of the metal in said sublayer. It is proposed here that a similar mechanism is at play. It is considered that the ceria particles, to some extent, influence the point at which the potential decreases to zero volts, thereby broadening the band.

\section{0 h OCV Hold Durability Testing}

To ascertain cerium oxide's radical scavenging ability over longer periods of time, $500 \mathrm{~h}$ OCV hold tests were performed on a baseline, a synthesized $1.0 \mathrm{wt} \%$ and commercial $1.0 \mathrm{wt} \% \mathrm{MEA}$. The test conditions, shown in Table 1, were based on DOE specifications ${ }^{12}$ which involved higher flow rates than the 94 hour OCV experiment, and were performed under pressure to increase gas crossover and thereby accelerate degradation. The pre-test hydrogen crossover, platinum ECA and performance (Figure 4) were found to be comparable to the $94 \mathrm{~h}$ tested MEAs.

One issue encountered during testing was that the synthesized $1.0 \mathrm{wt} \%$ MEA developed a defect after about $350 \mathrm{~h}$, which lead to a very large increase in hydrogen crossover. As explained below, the nature of the defect was determined to be localized, as opposed to a general failure of the MEA.

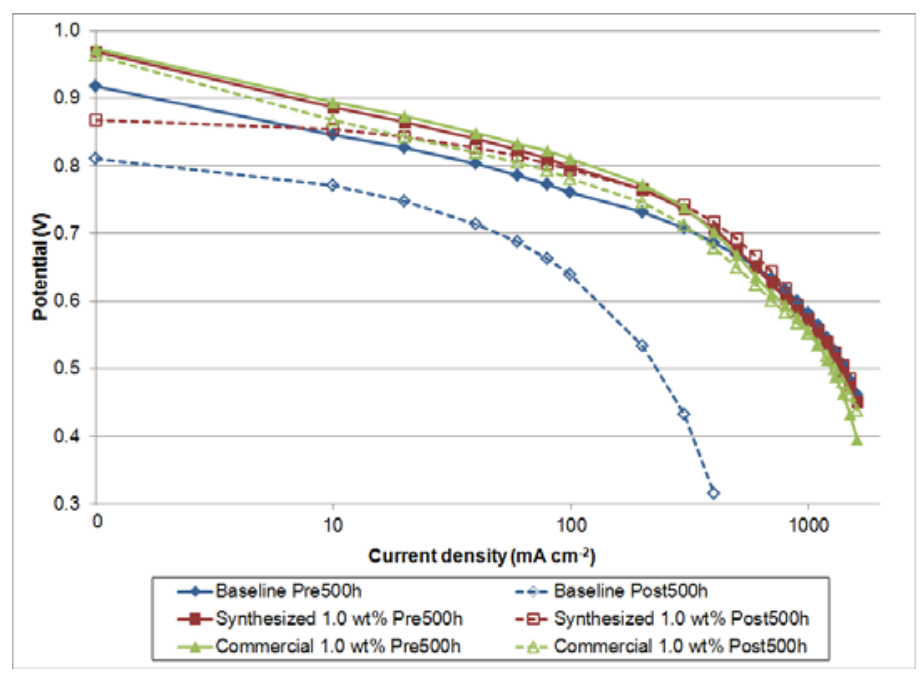

Figure 4. Pre- and post-500 h test performance curves

The baseline cell degraded severely, losing over half its total fluorine content during the first $100 \mathrm{~h}$ of measurement, which was expected, as discussed in the introduction. The MEA remained intact by virtue of the structural integrity provided by the PTFE support. The SEM images in Figure 5 a and b show that the membrane thinned considerably, from $\sim 25 \mu \mathrm{m}$ to 8-10 $\mu \mathrm{m}$, whereby the membrane on the cathode side was completely degraded, leaving the PTFE support in direct contact with the electrode. One consequence of the membrane thinning was the development of an electrical short, most likely due to the penetration of carbon fibers through the membrane, which influenced the linear sweep voltammetry measurements. The 25/25/25 LSV data given in Table 3 for the baseline MEA includes the decreased resistance due to the short and, though it does not present accurate values for the hydrogen crossover, it clearly shows increased gas permeability. This is further supported by IR images that the MEA developed pinholes, which are visible as intense red spots see Figure 6. 

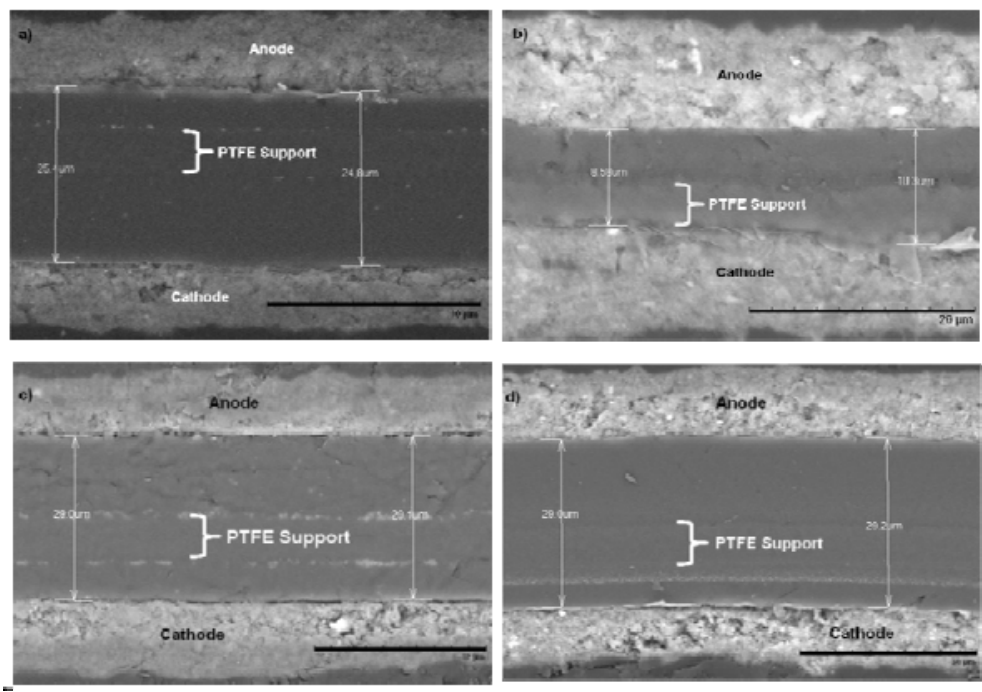

Figure 5. SEM images of CCM cross-sections: baseline a) before and b) after 500h OCV hold test; commercial $1.0 \mathrm{wt} \%$ ceria c) before and d) after 500h OCV hold test
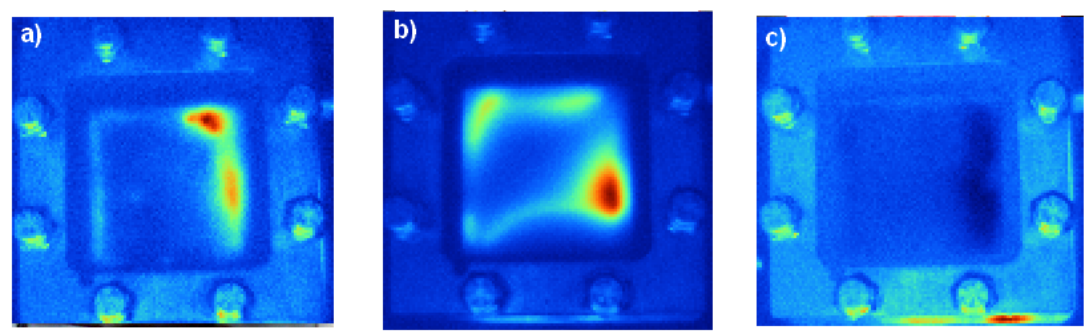

Figure 6. IR images of CCMs after $500 \mathrm{~h}$ OCV hold test: a) baseline, b) synthesized $1.0 \mathrm{wt} \% \mathrm{~h}$ and c) commercial ceria $1.0 \mathrm{wt} \%$

Table 3- Total fluoride emission, $\mathrm{H}_{2}$ crossover, OCV decay rate and resistance change for $500 \mathrm{~h}$ test Baseline Synthesized Commercial

\begin{tabular}{|c|c|c|c|}
\hline & & 1.0 wt $\%$ & 1.0 wt $\%$ \\
\hline Total fluoride emission ( $\mu \mathrm{mol})$ & 3800 & 340 & 33 \\
\hline Membrane fluorine inventory loss & $89 \%$ & $8 \%$ & $0.9 \%$ \\
\hline Pre500h $\mathrm{H}_{2}$ crossover \& shorting current density $\left(\mathrm{mA} \mathrm{cm}{ }^{-2}\right)$ & 0.73 & 0.93 & 1.00 \\
\hline Post500h $\mathrm{H}_{2}$ crossover \& shorting current density $\left(\mathrm{mA} \mathrm{cm}{ }^{-2}\right)$ & 21.49 & 10.04 & 0.80 \\
\hline OCV decay rate $\left(\mathrm{mV} \mathrm{h}^{-1}\right)$ & 0.847 & 0.363 & 0.120 \\
\hline OCV decay rate $\left(\mathrm{mV} \mathrm{h}^{-1}\right)$ until pinhole formation & & 0.151 & 0.116 \\
\hline Pre500h resistance @ $400 \mathrm{~mA} \mathrm{~cm}{ }^{-2}(\mathrm{~m} \Omega \mathrm{cm})$ & 67 & 72 & 78 \\
\hline Post500h resistance @ $400 \mathrm{~mA} \mathrm{~cm}{ }^{-2}(\mathrm{~m} \Omega \mathrm{cm})$ & 469 & 70 & 85 \\
\hline
\end{tabular}

The potential plot for the baseline MEA is also given in Figure 7 and shows a very fast decay in the first $\sim 40$ h due to the large degradation which slows, presumably due the membrane compacting. The overall OCV decay rate was seven times higher than for the ceria-containing MEAs (Table 3). 


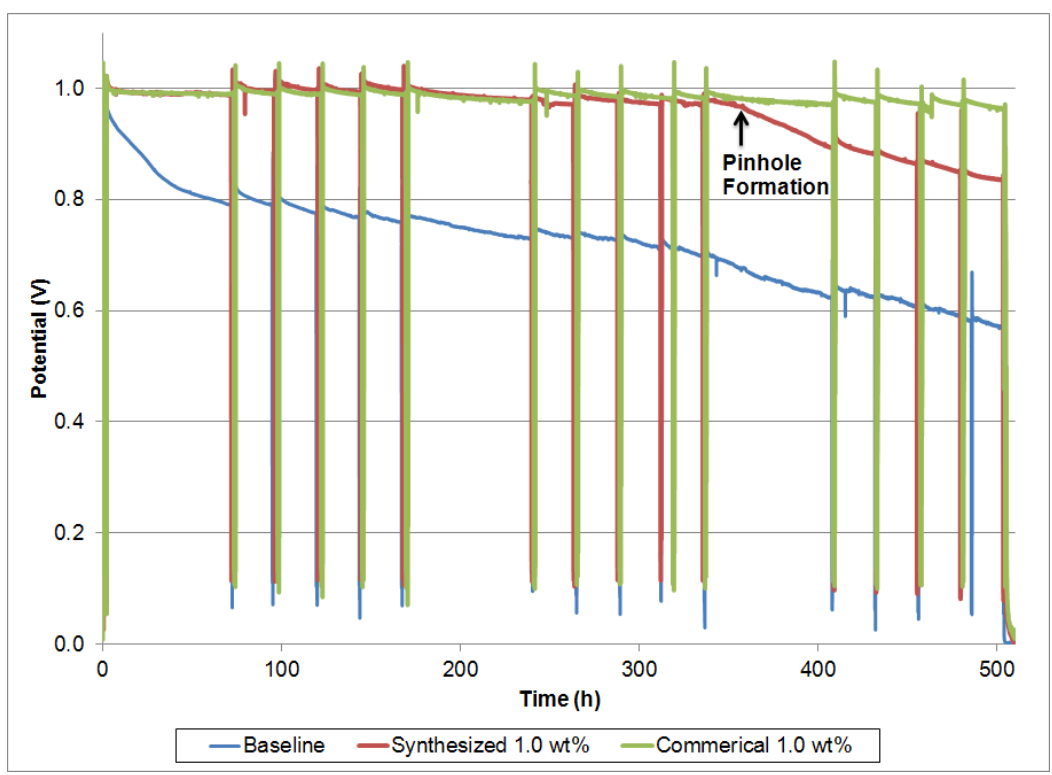

Figure 7. OCV decay for $500 \mathrm{~h} \mathrm{OCV} \mathrm{hold} \mathrm{(spikes} \mathrm{in} \mathrm{potential} \mathrm{show} \mathrm{breaks} \mathrm{in} \mathrm{the} \mathrm{experiment} \mathrm{to} \mathrm{perform}$ electrochemical measurements)

The membrane degradation had a very large effect on the both the performance (Figure 4) and resistance, which increased seven-fold (Table 3), of the baseline MEA. The performance, which pre-tests had shown to be very similar to the ceria-containing MEAs, decreased dramatically and did not even pass $400 \mathrm{~mA} \mathrm{~cm}^{-2}$. The fact that any performance could be measured was once again due to the PTFE support. The pinhole in the synthesized $1.0 \mathrm{wt} \%$ MEA's only affected its performance in the low current density regions $\left(<20 \mathrm{~mA} \mathrm{~cm}^{-2}\right.$ ) where the hydrogen crossover has a significant impact on the potential. At higher currents, both the synthesized and commercial ceria-containing MEAs were unchanged with regard to their pre-test performance. The commercial $1.0 \mathrm{wt} \%$ MEA showed a slight increase in resistance, which is considered to be within the error of the experiment. Otherwise, no significant change in any parameters that would indicate a lowering in proton conductivity was measured. This result provides little reason to believe that cerium oxide is being reduced to $\mathrm{Ce}^{3+}$, however, more work in this area is needed.

\section{Membrane Ex-Situ Analysis}

Proton Conductivity: For ceria-containing membranes, the proton conductivity was found to slowly but continually decrease over time, with a concurrent decrease in membrane opacity. To determine the cause of this phenomenon, the proton conductivity was measured while holding the membranes at $80^{\circ} \mathrm{C}$ and $70 \%$ RH for up to four days, as plotted in Figure 8. Over 30 hours of measurement, no significant change in the conductivity of the baseline material is observed, yielding a typical value of $35 \mathrm{mS} \mathrm{cm}^{-1}$ for PFSA membranes ${ }^{12}$. Both ceria-containing membranes, on the other hand, show a greater than three-fold decrease in proton conductivity and did not reach a minimum even after 18 and 90 hours of testing for the synthesized and commercial ceria, respectively.

Figure 9a and c show SEM images of the cross sections of the synthesized ceria-containing membrane before and after 18 hours of measurements at $80^{\circ} \mathrm{C}$ and $70 \% \mathrm{RH}$, respectively. Before testing, the precipitation of ceria onto the PTFE support is clearly visible as an intermittent band of white nanoparticles. These agglomerates, confirmed by EDS analysis (Figure 9b) to contain cerium, are no longer observable after conductivity testing (Figure 9c). 


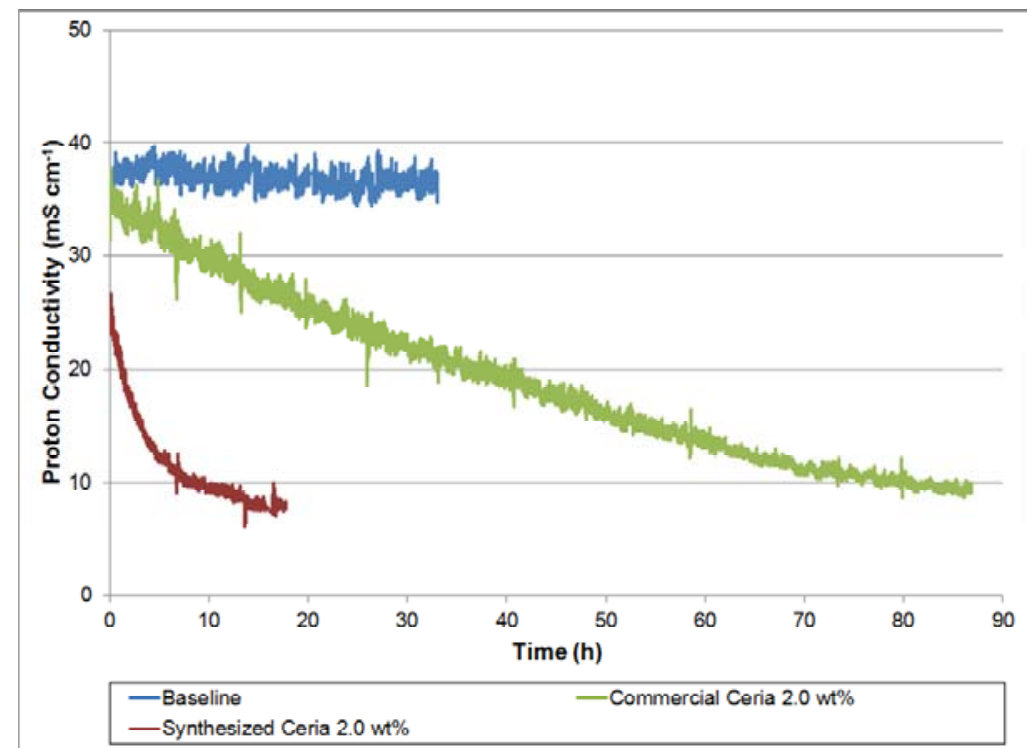

Figure 8. In-plane proton conductivity of various membranes held at $80{ }^{\circ} \mathrm{C}$ and $70 \% \mathrm{RH}$
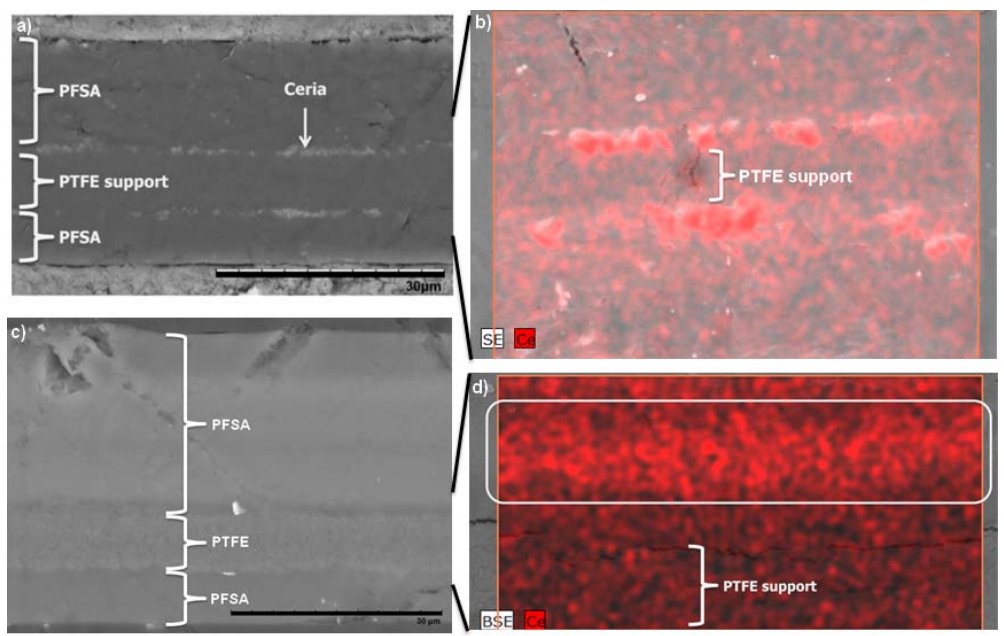

Figure 9. SEM images of cross-sections of a $2.0 \mathrm{wt} \%$ synthesized ceria-containing membrane a) before proton conductivity testing, b) before proton conductivity testing with an EDS cerium map overlay, c) after $18 \mathrm{~h}$ of proton conductivity testing and c) after six hours of proton conductivity testing with an EDS cerium map overlay (intense cerium band highlighted by box).

However, EDS mapping of a six hour tested membrane, shown in Figure 9d, clearly demonstrates the presence of cerium, as seen by the intense band highlighted by the box. The ceria particles were found to be dispersing which was considered as one of the causes leading to the decrease in opacity.

To probe any changes in the chemical nature of the ceria and further understand the complete loss of opacity, UV/Vis spectroscopy measurements were performed. Ce(III) and Ce(IV) absorb strongly in the ultraviolet spectrum; 252 and $298 \mathrm{~nm}$ for ionic solutions, respectively. Figure 10 shows the UV/Vis spectra of $2.0 \mathrm{wt} \%$ ceria-containing membranes, before and after proton conductivity measurement. Prior to testing, both synthesized and commercial ceria membranes absorb over a broad range from 225 to $400 \mathrm{~nm}$. After conductivity measurements, a change in the spectra is observed with the appearance of a strong peak around $255 \mathrm{~nm}$, an absorbance characteristic of $\mathrm{Ce}^{3+}$. A baseline membrane that was ionexchanged with $\mathrm{Ce}^{3+}$ yielded a UV/Vis spectrum that mirrored the absorbance of the tested membranes, 
indicating the conversion of cerium oxide to $\mathrm{Ce}^{3+}$ ions.

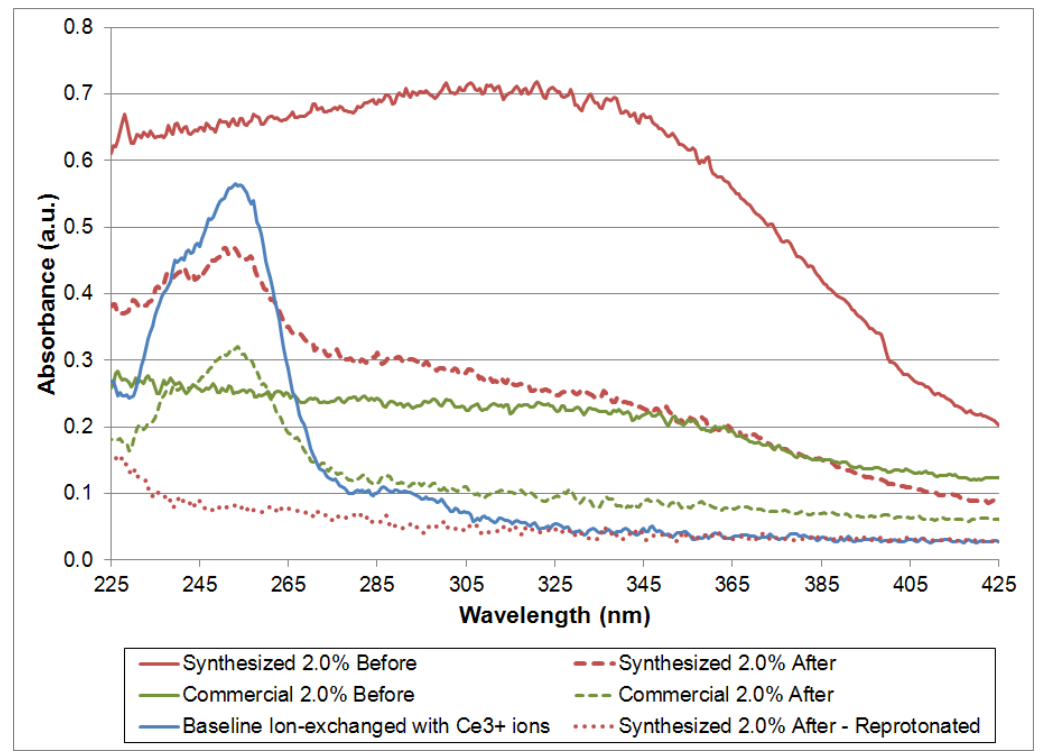

Figure 10. UV/Vis spectra of $2.0 \mathrm{wt} \%$ ceria-containing membranes before and after conductivity measurements

Fenton Testing: In order to study the ceria loading effect on membrane durability, series of gas and liquid Fenton test were conducted. Figure 11 and Figure 12 show the results for $\mathrm{Fe}^{2}+$ ion-exchanged membranes exposed to liquid and gaseous hydrogen peroxide. In both tests, ceria provides a large decrease in the FE, a degradation mitigation effect that increases with increasing additive concentration up to an order of magnitude for the $2.0 \mathrm{wt} \%$ ceria-containing membranes. The durability improvement is independent of the ceria formulation and therefore particle size. However, for the LF, the initial FE reduction is significantly more pronounced than for the GF. It is thought that this difference is a consequence of the different reaction mechanisms that are effective in the different phases.

In solution, the majority of hydroxyl radicals that are formed react with the large amounts of available hydrogen peroxide much faster than with the low concentrations of vulnerable polymer groups. This reaction, given in Eq.(3), produces the less reactive HOO'. In the vapor phase of the GF on the other hand, the $\mathrm{HO} \cdot$ that is formed is not in close contact with many other $\mathrm{H}_{2} \mathrm{O}_{2}$ molecules, giving it a longer lifetime. Its kinetics of membrane degradation are also considerably quicker and thus higher concentrations of ceria increase the likelihood of quenching and have a greater impact on the fluoride emission than in the LF tests.

$\mathrm{HO} \cdot+\mathrm{H}_{2} \mathrm{O}_{2} \rightarrow \mathrm{HOO} \cdot+\mathrm{H}_{2} \mathrm{O}$ 


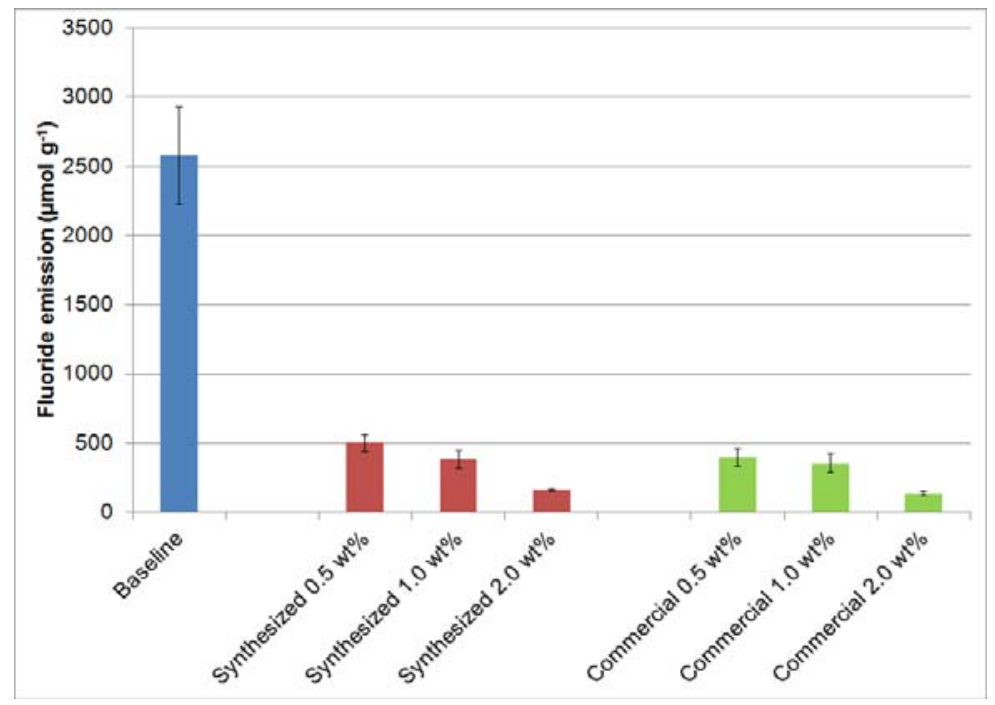

Figure 11. Normalized total fluoride emission after $48 \mathrm{~h}$ for the liquid Fenton test

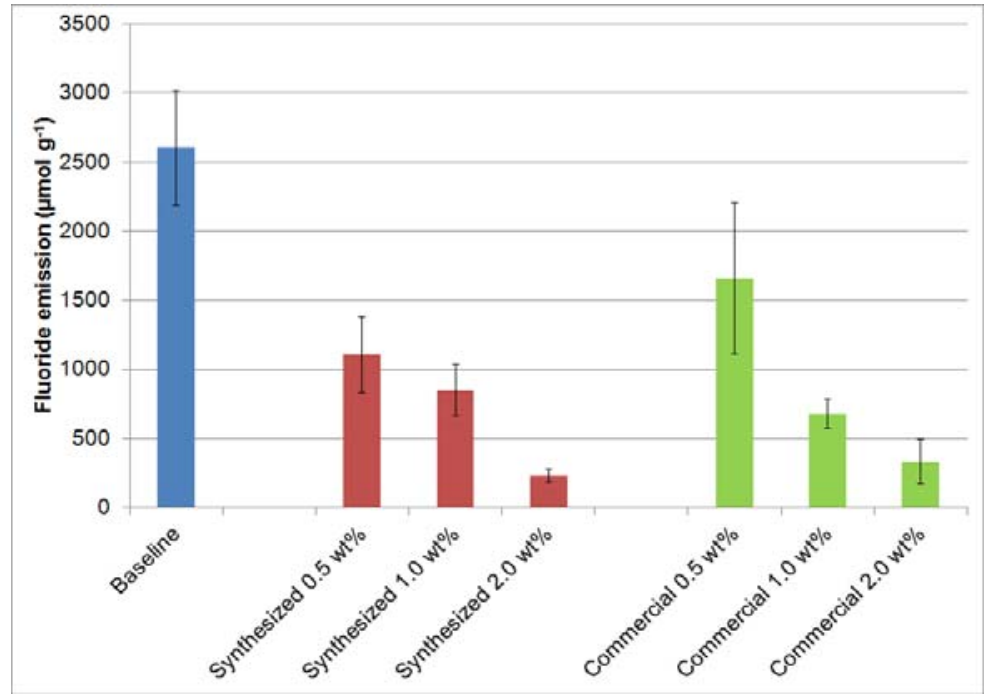

Figure 12. Normalized total fluoride emission after $48 \mathrm{~h}$ for the gaseous Fenton test

\section{Task 2}

The goals of Task 2 were to evaluate platinum band formation through (a) Examining factors that affect Pt migration through the ionomer, and (b) Examining the effect of Pt properties within the membrane on FER. This information was to be correlated with durability data to determine the relationships between the test parameters/MEA components, Pt band, and durability.

\section{Examine factors that affect Pt migration through the ionomer}

Even though previous research has resulted in greater understanding of the mechanism through which the Pt band forms and the effect the Pt band has on membrane degradation, there are many areas that are unknown. Varying membrane electrode assembly (MEA) components such as the membrane or catalyst layer and varying test parameters such as relative humidity $(\mathrm{RH})$ and temperature have been shown to 
have a large effect on membrane degradation, but there are no clear relationships between the fuel cell components, the test parameters, and the Pt band formation. Understanding these relationships would increase our ability to mitigate Pt band formation and/or its effect on membrane durability and therefore improve fuel cell durability.

Critical components that may define Pt band characteristics are the ease with which the Pt can be solubilized and the ease with which it can migrate through the ionomer. If the Pt can easily be solubilized and migrate through the ionomer, it is likely that larger Pt bands will form and they will form more quickly. Factors such as amount of water in the ionomer, the size of the hydrophilic channels in the ionomer, and whether the Pt is alloyed with another metal can impact the rate and size of Pt band formation. Task 2a examined the effect of three variables: (1) relative humidity, (2) ionomer equivalent weight, and (3) using Pt vs PtCo catalysts on Pt band formation while testing under an OCV hold.

The approach in Task 2a was to prepare four types of catalyst coated membranes (CCMs): a) $1100 \mathrm{EW}$ membrane with $\mathrm{PtCo} / \mathrm{C}$ in the electrode, b) $1100 \mathrm{EW}$ membrane with $\mathrm{Pt} / \mathrm{C}$ in the electrode, c) $950 \mathrm{EW}$ membrane with $\mathrm{Pt} / \mathrm{C}$ in the electrode, d) $750 \mathrm{EW}$ membrane with $\mathrm{Pt} / \mathrm{C}$ in the electrode. These CCMs were tested under an accelerated durability protocol that held the cells at open circuit voltage (OCV) at $90{ }^{\circ} \mathrm{C}$ for $100 \mathrm{~h}$ at 10, 20, 30 and 100\% RH The durability of the CCMs was measured by comparing the electrochemically active surface areas, levels of hydrogen crossover, and polarization curves before and after testing. During OCV testing, voltage and FER was monitored. Cross-sections of MEAs were examined by scanning electron microscopy (SEM) and transmission electron microscopy (TEM) before and after testing to discern whether there were any visual differences in the MEAs as a result of the accelerated stress test and to locate and characterize the Pt bands. This approach was carried out as planned, with no departures from the planned methodology.

\section{Pt/C vs. PtCo/C}

Pt alloys show promise as catalyst candidates for fuel cells to achieve high performance and to reduce PEMFC cost. Although extensive research has been conducted in the areas of alloy development and characterization, ${ }^{13,14,15}$ there has been little research on the effect of using Pt alloys on membrane durability. The hypothesis in the current work was that because the PtCo/C catalyst is more stable in fuel cells than Pt/C, it will lead to lower membrane degradation because less Pt will be deposited inside the membrane.

Compared to the CCM containing the Pt/C electrode, the CCM containing the PtCo/C electrode had lower OCV losses (Figure 13) and fluoride emission during testing (Figure 14) as well as lower performance losses before and after testing at OCV for $100 \mathrm{~h}$ at $90^{\circ} \mathrm{C} / 30 \% \mathrm{RH}$. Both the Pt/C and PtCo/C cells contained the same amount of Pt in their electrodes prior to testing. TEM results (Figure 15) showed that Pt particles were present in the membranes from both cells after testing. The Pt particles were localized within a narrow platinum band after testing the cell containing $\mathrm{Pt} / \mathrm{C}$, while after testing the cell containing $\mathrm{PtCo} / \mathrm{C}$, platinum particles were distributed throughout the membrane. The Pt particles in the PtCo/C cells were also smaller and closer together than those from the cells containing Pt/C. The differences in durability that were observed are attributed to the differences in Pt particle size and distribution. The Pt band in the membrane results in higher degradation because the location of the Pt band was the same place where $\mathrm{OH}$ radical formation is favored, resulting in a strong, localized radical attack on the polymeric membrane. The hypothesis in the present study was only partially supported by this work. Although using $\mathrm{PtCo} / \mathrm{C}$ rather than $\mathrm{Pt} / \mathrm{C}$ resulted in improved durability, it was not because there was less Pt deposited in the membrane. Rather, the improved durability with the PtCo/C cell was due to favorable size and distribution of the Pt particles. Based on this work, it can be recommended that using PtCo/C rather than $\mathrm{Pt} / \mathrm{C}$ in fuel cell electrodes will result in improved fuel cell durability. 


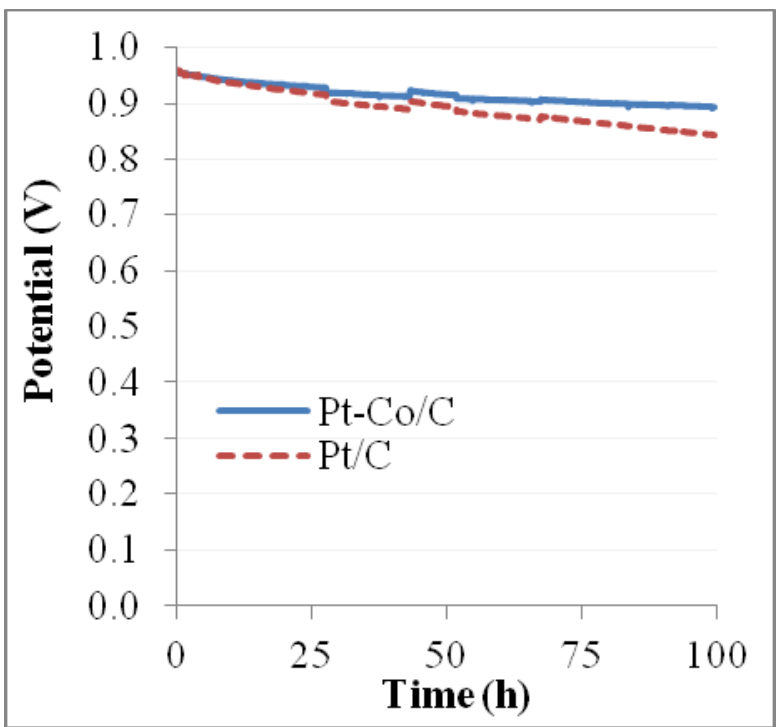

Figure 13. OCV during testing at $90{ }^{\circ} \mathrm{C} / 30 \% \mathrm{RH}, \mathrm{H}_{2} /$ air for cells containing $\mathrm{Pt}-\mathrm{Co} / \mathrm{C}$ and $\mathrm{Pt} / \mathrm{C}$ in their electrodes

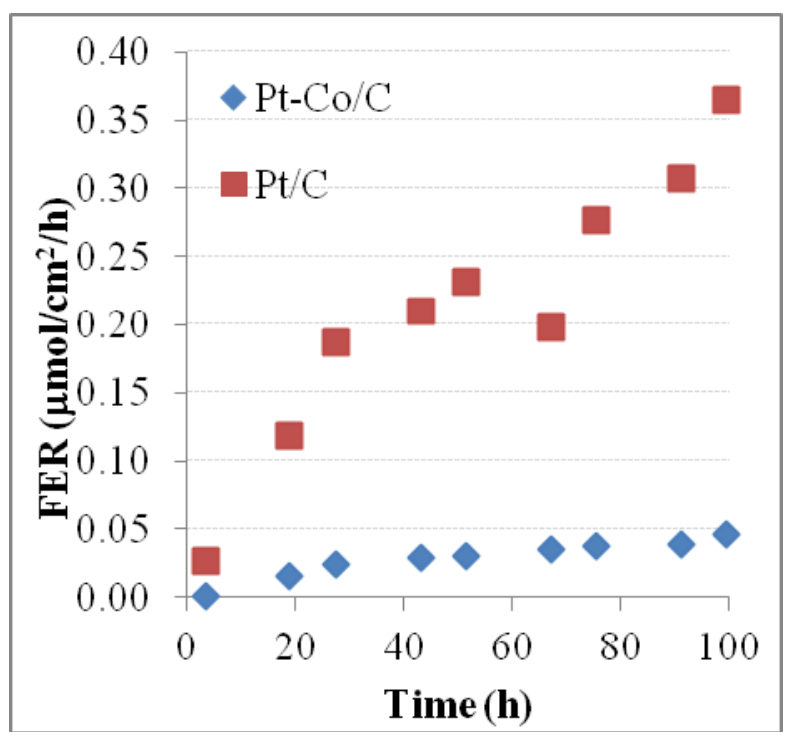

Figure 14. FER during testing at $90{ }^{\circ} \mathrm{C} / 30 \% \mathrm{RH}, \mathrm{H}_{2} /$ air for cells containing $\mathrm{PtCo} / \mathrm{C}$ and $\mathrm{Pt} / \mathrm{C}$ in their electrodes 


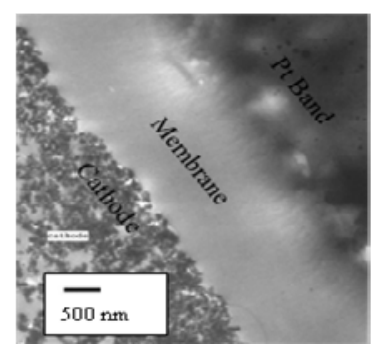

a

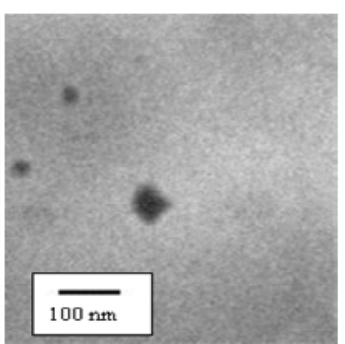

b
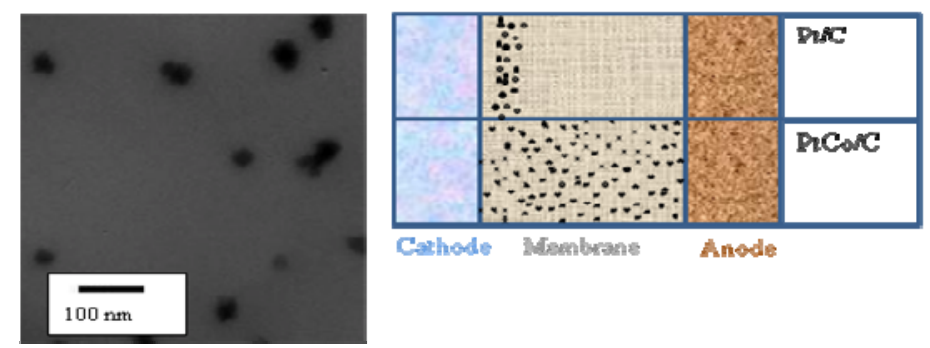

C

d

Figure 15. TEM images of CCMs containing ( $a$ and b) $\mathrm{Pt} / \mathrm{C}$ in the electrode and c) $\mathrm{PtCo} / \mathrm{C}$ in the electrode after OCV testing at 90 C/30\% RH for 100 h. d) Schematic of Pt distributions in the CCMs after testing.

\section{Equivalent Weight}

The equivalent weight (EW) of membranes has a large effect on their properties and can impact performance and durability. For example, for perfluorosulfonic acid membranes, crystallinity and water content increase with increases in EW, while glass transition temperature decreases with increases in EW. ${ }^{16,17}$ The length of the sulfonic acid side chain also impacts membrane properties. It has been shown that perfluorosulfonic acid membranes with shorter sulfonic acid side chains result in higher crystallinity, higher glass transition temperature, similar gas permeability, lower water content, and lower proton conductivity than membranes with longer sulfonic acid side chains for a given EW. ${ }^{18,19,20}$ Although many reports have shown improved cell performance for membranes with low EW and shorter side chains, the impact of EW and side chain length on cell durability is not well understood. Because side chain attack by radicals formed during fuel cell operation is a major source of membrane degradation, the hypothesis in the present work is that membranes with lower EW and, therefore, more sulfonic acid side chains, would have lower durability.

This study evaluates membrane degradation for cells containing PFSA membranes with 750, 950, and $1100 \mathrm{EW}$. The $750 \mathrm{EW}$ membrane contained short sulfonic acid side-chains while the 950 and $1100 \mathrm{EW}$ membranes were Nafion ${ }^{\circledR}$-based with long sulfonic acid side chains. After testing at OCV for $100 \mathrm{~h}$ at 90 ${ }^{\circ} \mathrm{C} / 30 \% \mathrm{RH}$, the $950 \mathrm{EW}$ cell had the highest voltage decay (Figure 16), fluoride emission (Figure 17), loss in ECA, and loss in cell performance. The $1100 \mathrm{EW}$ cell showed the lowest degradation. This has been explained in terms of degree of crystallinity, which impacts the mechanical stability of the membrane. The hypothesis was supported in this work. The $950 \mathrm{EW}$ membrane had lower durability than the $1100 \mathrm{EW}$ membrane. The $750 \mathrm{EW}$ membrane had lower EW than the others, but because it had shorter side chains, which impart higher crystallinity, durability was improved. 


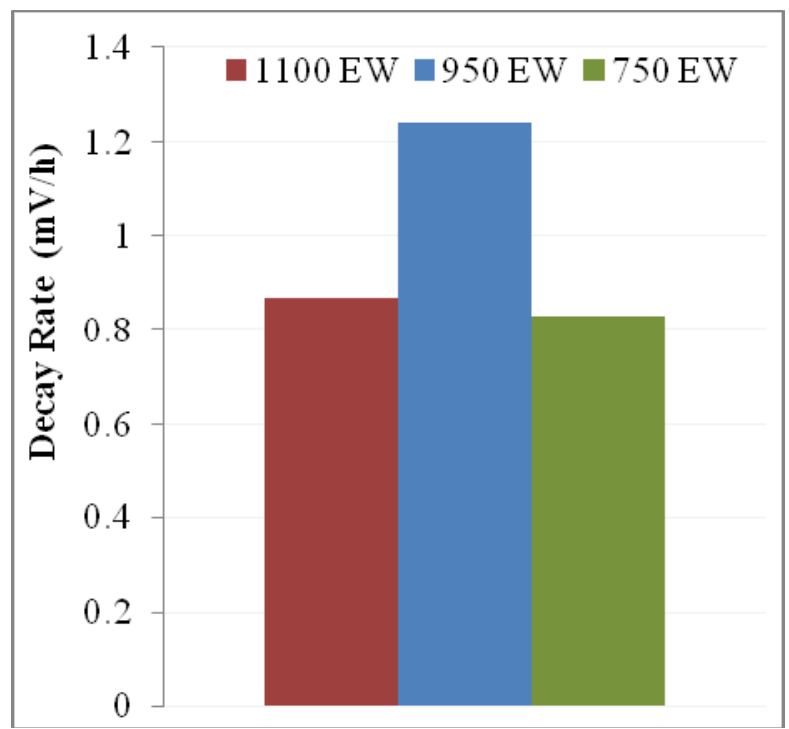

Figure 16. OCV decay rate during testing for $100 \mathrm{~h}$ at $90{ }^{\circ} \mathrm{C} / 30 \% \mathrm{RH}, \mathrm{H}_{2} /$ air for cells containing membranes with 1100, 950, and $750 \mathrm{EW}$

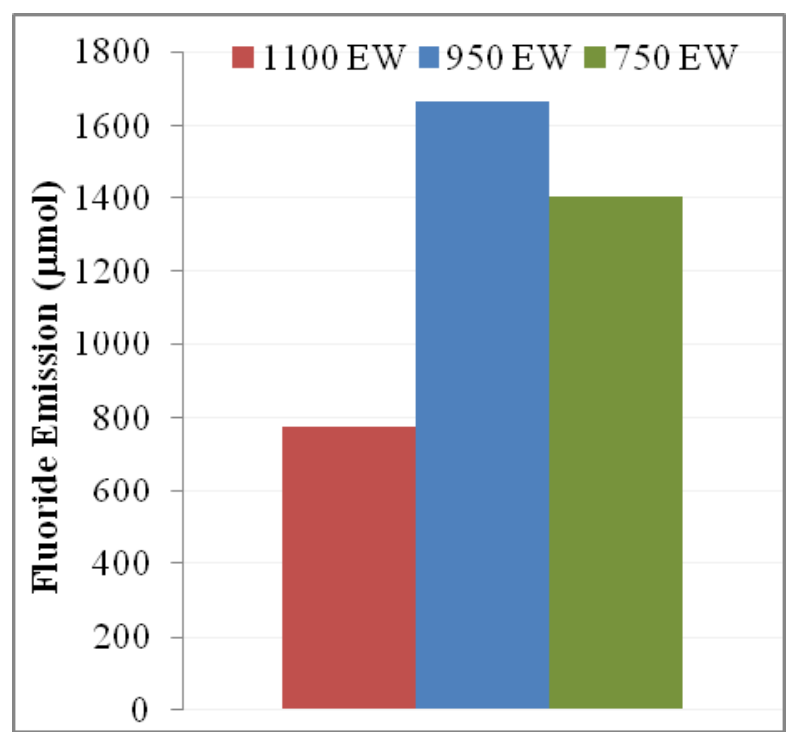

Figure 17. Total fluoride emission after OCV testing for $100 \mathrm{~h}$ at $90{ }^{\circ} \mathrm{C} / 30 \% \mathrm{RH}, \mathrm{H}_{2} /$ air for cells containing membranes with 1100, 950, and $750 \mathrm{EW}$

\section{Relative Humidity}

Previous studies have shown that RH is one of the most important factors influencing the membrane degradation rate. Most studies have shown that the membrane degradation rate increases with a decrease in RH. However, these tests were only conducted down to a RH of 30\%. Recent reports suggest that upon further decrease in $\mathrm{RH}$ (to $20 \%$ or even $0 \%$ ), ${ }^{4}$ the membrane degradation rate may decrease. Depending on the cell design and the operating conditions, the RH the membrane experiences in the cell is expected to vary from $0-100 \%$. Thus, it is important to understand the impact of a range of RHs on membrane degradation rate. 
The hypothesis in the present study was that lower degradation would be observed at low and high RH (10 and 100\%, respectively) than at medium-low RH (20 and 30\% RH). At high RH, decreased degradation is observed due to dilution of radicals, higher oxide coverage on Pt, and lower partial pressures of reactant gases. At low RH, the hypothesis is that decreased degradation will result from reduced Pt band formation due to low mobility of Pt in low RH conditions.

After OCV testing for $100 \mathrm{~h}$ at $90^{\circ} \mathrm{C}$ and 10 to $100 \% \mathrm{RH}$, the cells that were tested at 10 and $100 \% \mathrm{RH}$ showed the lowest fluoride emission (Figure 18) and voltage decay (Figure 19), while 20 and 30\% RH resulted in much higher degradation. The SEM and TEM images (Figure 20) show Pt bands only in the CCMs that were tested at 30 and $100 \% \mathrm{RH}$. Considerable thinning was observed in the CCM that was tested at $20 \% \mathrm{RH}$. Because lower degradation and no Pt band is observed in the CCM that was tested at $10 \% \mathrm{RH}$, the hypothesis for this study was supported.

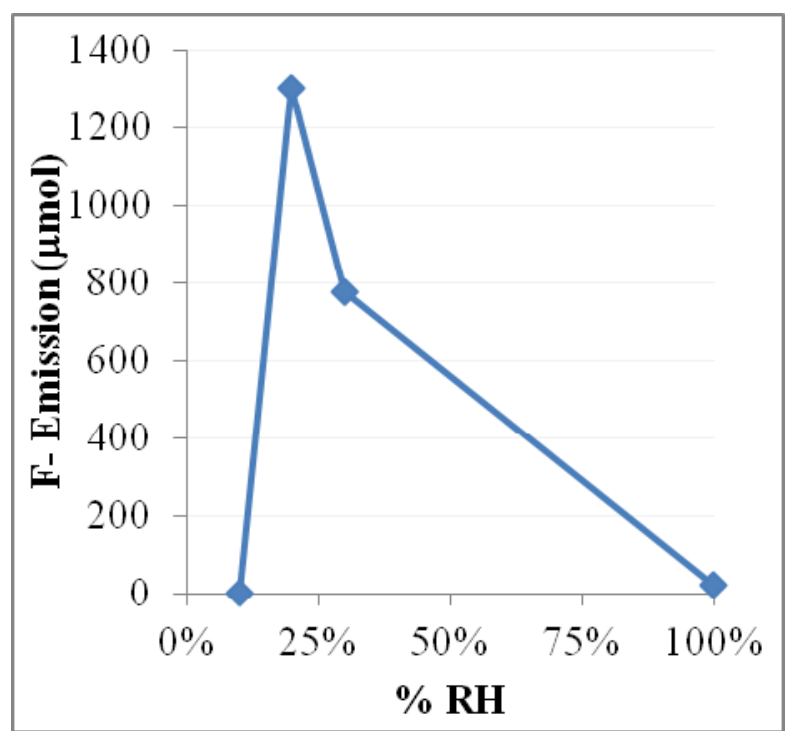

Figure 18. Total fluoride emission after OCV testing for $100 \mathrm{~h}$ at $90{ }^{\circ} \mathrm{C}$ and 10 to $100 \% \mathrm{RH}, \mathrm{H}_{2}$ /air for cells containing CCMs with $1100 \mathrm{EW}$ and Pt/C catalysts 


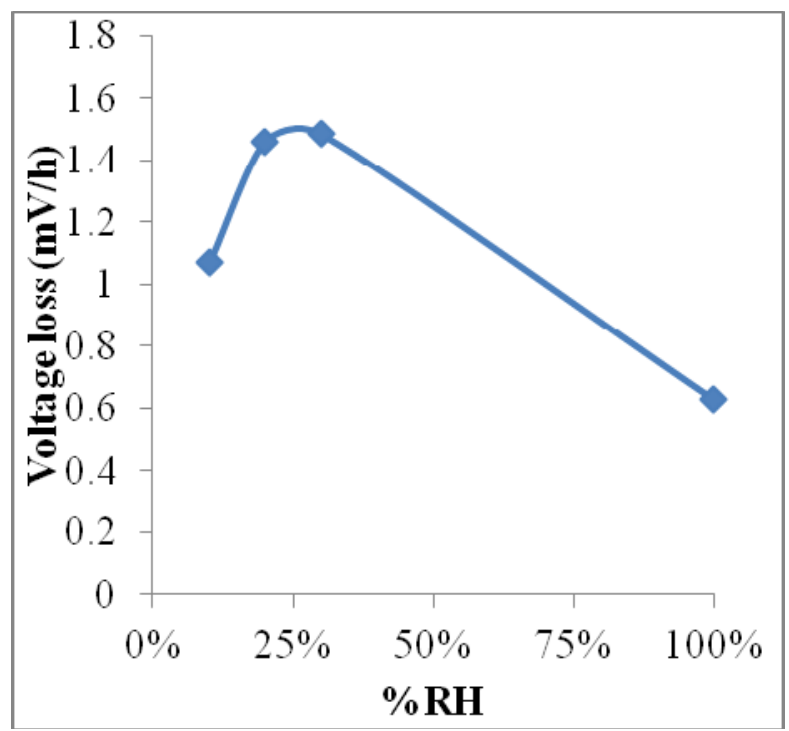

Figure 19. Average voltage decay during OCV testing for $100 \mathrm{~h}$ at $90{ }^{\circ} \mathrm{C}$ and 10 to $100 \% \mathrm{RH}, \mathrm{H}_{2} /$ air for cells containing CCMs with $1100 \mathrm{EW}$ and Pt/C catalysts
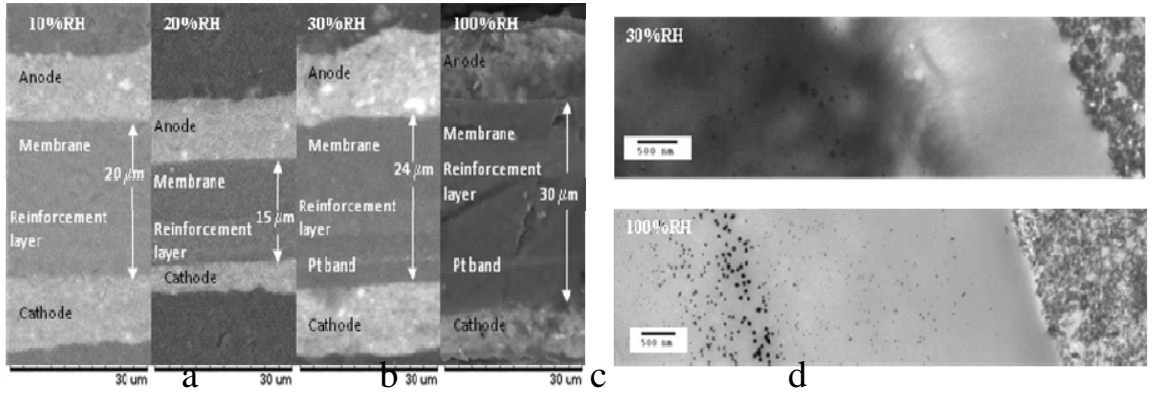

Figure 20. SEM images (a-d) of CCMs after testing at (a) 10, (b) 20, (c) 30, and (d) 100\% RH; and TEM images (e-f) of CCMs after testing at (e) 30 and (f) 100\% RH. CCMs contain 1100 EW membranes and $\mathrm{Pt} / \mathrm{C}$ in the catalyst layer. Accelerated test conditions: OCV, $100 \mathrm{~h}$, and $90{ }^{\circ} \mathrm{C}$.

\section{Examine the effect of Pt properties within the membrane on FER}

The presence of Pt particles in membranes generates contradictory results with regards to membrane durability. ${ }^{21,22,23,24,25}$ For example, it was reported that Pt impregnated membranes have been used as selfhumidifying membranes in fuel cells; the Pt particles catalyze the oxidation of crossover hydrogen with oxygen to generate water and the fuel cell shows exceptionally stable and high performance. ${ }^{21,22}$ However, other researchers have shown that Pt bands increase degradation due to formation of radicals on the catalyst surface. ${ }^{23,24,25}$ These differences in the effect of Pt on degradation have been explained through modeling by differences in platinum particle size, location, and distribution., ${ }^{9,26}$

The approach in Task 2b was to impregnate membranes with Pt to better control the size, amount and distribution. These membranes were assembled into a fuel cell and exposed to air on one side, and hydrogen on the other for $100 \mathrm{~h}$ at $90^{\circ} \mathrm{C}$ and $30 \% \mathrm{RH}$. No catalysts were applied to these membranes to remove complicating effects. This approach was carried out as planned, with no departures from the planned methodology. The hypothesis in this work was that membrane durability would be affected by $\mathrm{Pt}$ size, shape, and distribution and that Pt in the membrane would accelerate membrane degradation. 
Preliminary results have shown that the highest degradation was observed with the membrane containing $10 \mathrm{~mol} \% \mathrm{Pt}$, with fluoride emissions of the same magnitude of those seen with catalyst coated CCMs containing Pt/C. Membranes containing 0, 30, and 50 mol\% Pt resulted in very low fluoride emission. TEM images (Figure 22, taken at Oak Ridge National Lab through the ShaRE program) show that although Pt bands formed with 30 and 50 mol\% Pt, no Pt band formed with 10 mol\% Pt. The membrane containing $10 \mathrm{~mol} \% \mathrm{Pt}$ had the smallest particle distance and size and the greatest overall Pt coverage. The fact that the membrane with $10 \mathrm{~mol} \%$ Pt had the highest degradation is attributed to a higher concentration of small particles, which has been shown to result in increased degradation. ${ }^{9}$ The hypothesis in this work was partially supported. Although it is clear that membrane durability is affected by Pt size, shape, and distribution, it is not always true that Pt in the membrane accelerates degradation. In the present study, membrane degradation was only accelerated when the Pt particles were small and close together (average particle size $=2.4 \mathrm{~nm}$, average particle distance $=10 \mathrm{~nm}$ for $10 \mathrm{~mol} \% \mathrm{Pt}$ after testing). When the Pt particles in the membrane were larger and further apart, very little degradation was observed.

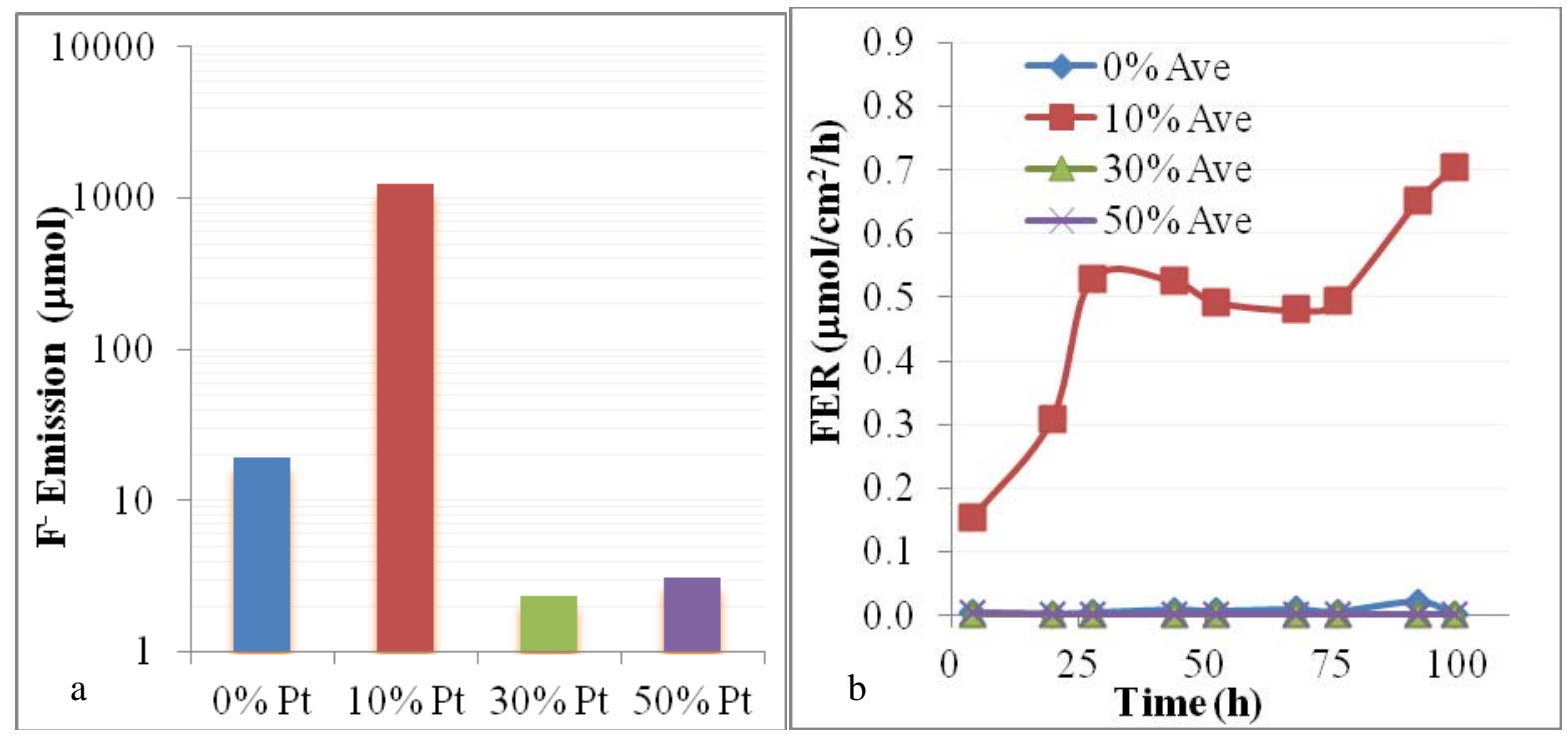

Figure 21. Total fluoride emission (a) and fluoride emission rate (b) for membranes impregnated with 0 , 10, 30, and $50 \mathrm{~mol} \% \mathrm{Pt}$ and tested for $100 \mathrm{~h}$ at $90^{\circ} \mathrm{C} / 30 \% \mathrm{RH}$.
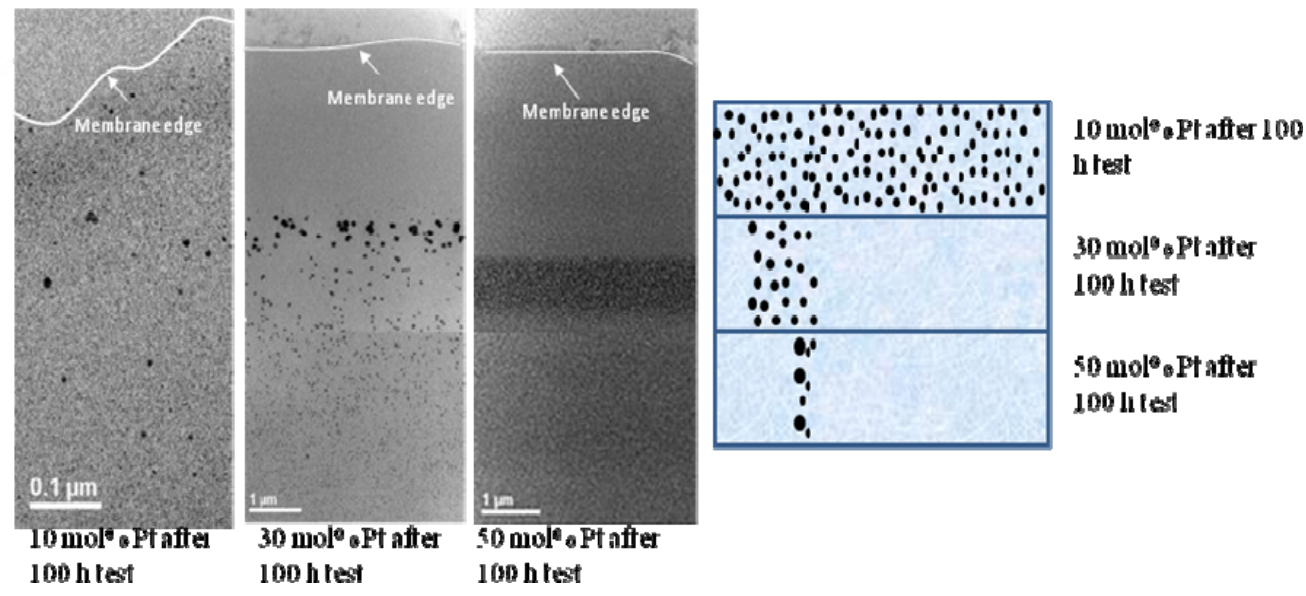

Figure 22. TEM images of membranes containing (a) 10, (b) 30, and (c) $50 \mathrm{~mol} \% \mathrm{Pt}$ and a schematic of the Pt distribution for each membrane after testing for $100 \mathrm{~h}$ at $90{ }^{\circ} \mathrm{C} / 30 \% \mathrm{RH}$ under $\mathrm{H}_{2} /$ air. 


\section{Task 3}

Precipitation of platinum ions within the membrane and subsequent formation of the platinum band has been implicated in membrane degradation. The focus of Task 3 was to evaluate the ability of heteropolyacids to precipitate platinum ions in a sublayer away from the membrane (see Figure 3-1). The purpose of the sub-layer was to provide a region away from the electrode where platinum ions may be precipitated and visualized, as well as for investigating HPA mobility. Therefore, the sub-layer configuration is considered to be a tool for this study, and would not be incorporated into future fuel cell designs.

Heteropolyacids (HPAs) are inorganic acids, commonly used as industrial catalysts due to their high stability and strong oxidizing abilities. However, these molecules have multiple reduction peaks, allowing them to have the ability to reduce different compounds at different potentials. An example of the multiple reduction peaks for one heteropolyacid, phosphotungstic acid, is shown in Figure 23. By selecting the appropriate heteropolyacid, the platinum ions migrating from the cathode may be reduced by the heteropolyacid, which in turn is reduced by hydrogen crossover. By precipitating the platinum within the sublayer, the membrane degradation would be decreased, and subsequent fuel cell lifetime would be increased.

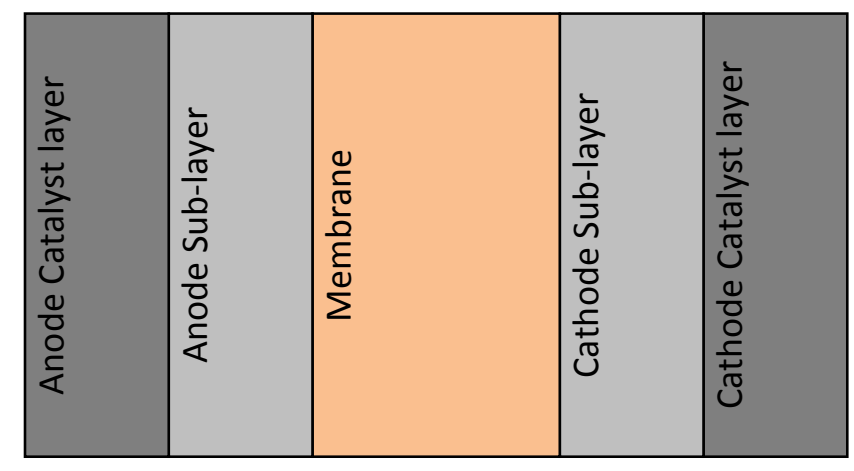

Figure 23. Schematic of sub-layers for fuel cells in Task 3

Three different HPAs were selected, based on their reduction potentials, and adsorbed onto high surface area carbon. These HPAs were phosphotungstic acid (PTA, $\mathrm{H}_{3} \mathrm{PW}_{12} \mathrm{O}_{40}$ ), silico-vanadotungstic acid (STA-1V, $\mathrm{H}_{5} \mathrm{SiW}_{11} \mathrm{VO}_{40}$ ), and phospho-vanadotungstic acid (PTA-1V, $\mathrm{H}_{4} \mathrm{PW}_{11} \mathrm{VO}_{40}$ ). Once adsorbed onto the carbon support, the HPA/C was sprayed onto an NRE211 membrane, followed by application of the platinum catalyst layer, also applied by spraying.

These catalyst coated membranes were then assembled into fuel cell hardware and subjected to an accelerated stress test, wherein the cell was held at open circuit voltage (OCV) for 100 hours at 90 ${ }^{\circ} \mathrm{C} / 30 \% \mathrm{RH}$ with $\mathrm{H}_{2} /$ air. During the stress test, the OCV was monitored, and the effluent water was collected and analyzed for fluoride. The OCV loss and total fluoride released for the three different HPA/C sublayers is shown in Figure 24. Also included is a cell where no HPAs were added to the sublayer. 


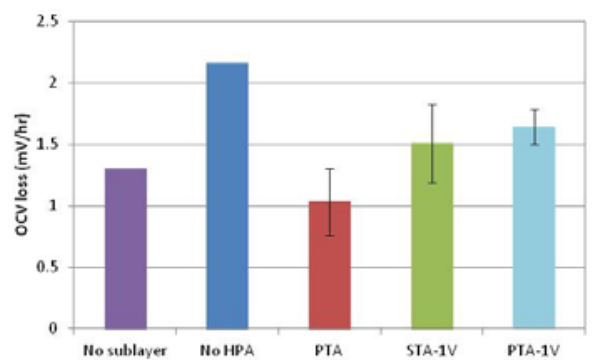

(b)

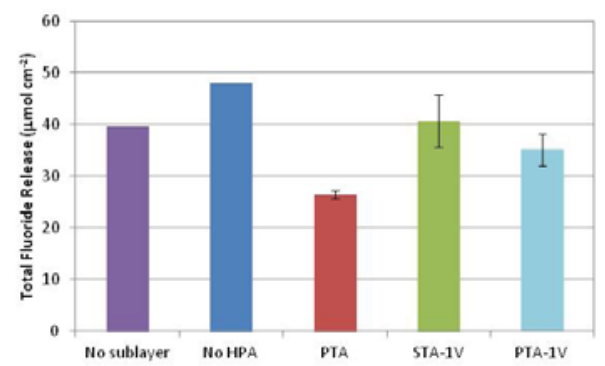

Figure 24. (a) OCV loss rates and (b) total fluoride release during the stress test.

As can be seen, the addition of HPAs to the sublayer has decreased both the OCV loss rates as well as the fluoride emission, both of which indicate improved membrane durability. To further ascertain the ability of the HPA to reduce membrane degradation, the cross-section of the tested cells were analyzed under SEM (Figure 25). Clearly, those cells containing HPAs in the sublayer showed much thicker membranes after the stress test than those cells without HPAs.

It was also observed that the cell with the PTA sublayer showed a bright band in the cathode sub-layer. This band was determined to be from tungsten, and indicates significant HPA mobility within the cell. The other cells did not show any bright regions after the stress test, although bright areas were evident before testing. Again, these bright areas before testing were identified as tungsten, and their absence after testing indicates that much of the tungsten has left the cell. Indeed, elemental analysis with EDX showed that the concentration of tungsten within the sub-layers for the STA-1V and PTA-1V cells was lower after testing. Platinum was not identified in high concentrations within the sublayers, although mathematical modeling predicts that the potential profile through the sublayer should be sufficient to reduce the ions to metals. From the modeling results, the best HPA is the PTA, as it caused the greatest reduction in voltage through the sublayer. Indeed this follows with the results from the OCV and fluoride emission measurements, that the PTA sublayer had the greatest impact on membrane durability.

The results of Task 3 show that the addition of HPAs to sub-layers in cells can reduce membrane degradation, and that the PTA showed the greatest impact. 

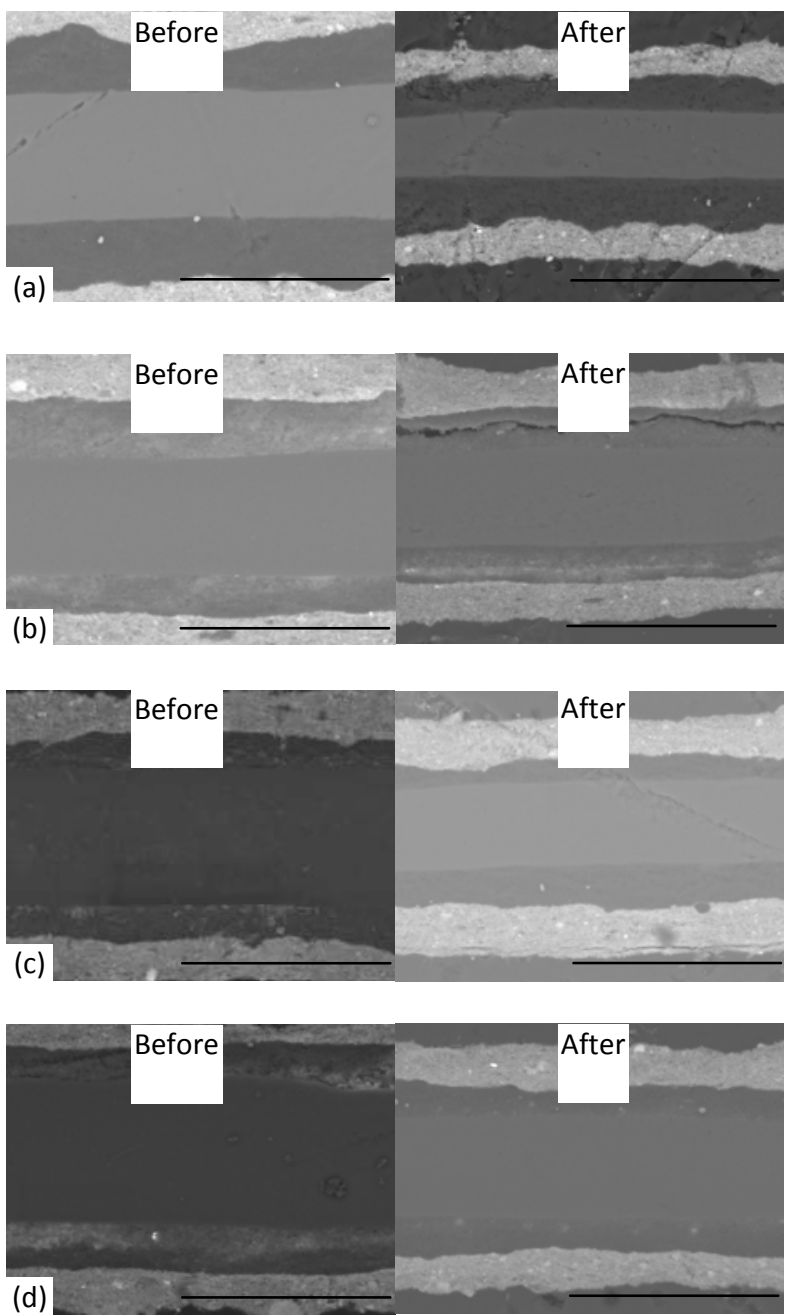

Anode Pt/C electrode

XC-72R Sub-layer

Membrane

XC-72R Sub-layer

Cathode Pt/C

Anode Pt/C electrode

PTA Sub-layer

Membrane

PTA Sub-layer

Cathode Pt/C

Anode Pt/C electrode

STA-1V Sub-layer

Membrane

STA-1V Sub-layer

Cathode Pt/C

Anode Pt/C electrode

PTA-1V Sub-layer

Membrane

PTA-1V Sub-layer

Cathode Pt/C

${ }^{\ulcorner}$Figure 25. SEM images of sublayer cells before and after stress test. The bars represent $50 \mu \mathrm{m}$.

The goal of Task 4 was to combine the results from Task 1 with the results from Task 3 and develop an "ultra-durable" cell. For this effort, the membrane selected was a $1 \mathrm{wt} \% \mathrm{CeO}_{2}$ membrane with mechanical reinforcement and 1100EW ionomer, combined with a PTA/C sublayer. Two other types of cells were also evaluated, both with no HPAs in the sublayer, but one without $\mathrm{CeO}_{2}$, the other with $1 \mathrm{wt} \% \mathrm{CeO}_{2}$. In this fashion, the effect of the sublayer may be evaluated by comparing the sublayer cells to cells from Task 1 . In addition, the ability of the HPA to increase membrane durability with $\mathrm{CeO}_{2}$-containing membranes may also be evaluated. The results of the OCV and fluoride losses (in percent) are shown in Figure 26. 


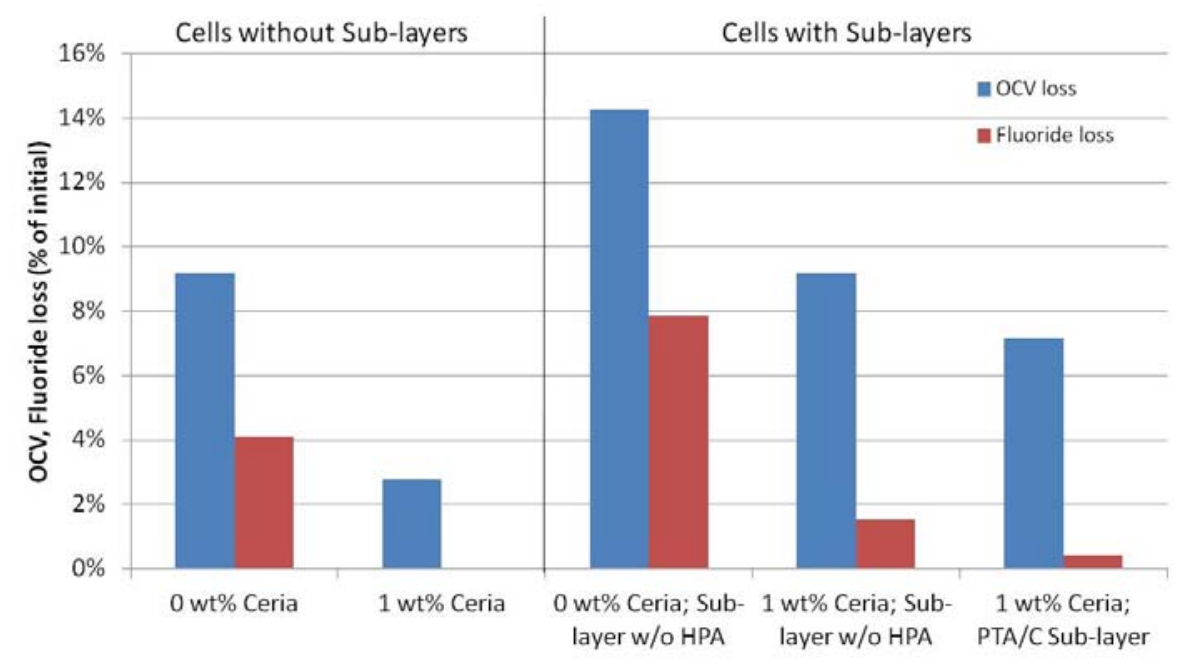

Figure 26. OCV and fluoride \% losses for several cells, subjected to stress tests

From Figure 26, it can be seen that the addition of PTA to the sublayer of the $1 \mathrm{wt} \%$ ceria cell significantly decreased FER, again showing the improvement that PTA can provide to membrane durability. The cell containing 1wt\% Ceria with PTA/C sub-layer showed the best FER of any of the sublayer containing cells from Tasks 3 and 4 . However, as can also be seen from Figure 26, the addition of a sub-layer to a $0 \mathrm{wt} \%$ and a $1 \mathrm{wt} \% \mathrm{CeO}_{2}$ membrane caused significant increases in fluoride emission. This indicates that while sublayers have been used as characterization tools for this study, future designs of fuel cell electrodes should incorporate the PTA directly within the electrode.

\section{Conclusions}

With a goal of decreasing the degradation of fuel cell membranes, a study of platinum band formation and impact was conducted. The program was divided into four tasks: 1) identify suitable additives for the scavenging radicals in the membrane and characterizing the membranes containing additives; 2) locate and characterize the platinum band in the membranes and investigate the impact of cell components and operation parameters on platinum band formation and membrane degradation; 3) select some heteropolyacids to be used for mitigation of platinum band formation and to incorporate these into a sublayer between the membrane and the electrode; and 4) to combine the best performing additive from Task 1 with the most effective heteropolyacid from Task 3 to achieve a more durable cell.

The key results for the project are as follows:

- cerium oxide acts as a radical scavenger and protects the membrane, reducing both the OCV decay and fluoride emissions dramatically, independent of concentration and particle size.

- relative humidity, equivalent weight, catalyst type, and amount of Pt dispersed in the membrane were all found to influence degradation.

- the addition of HPAs to sublayers was found to reduce FER, with PTA showing the greatest reduction.

- the resulting system with ceria in the membrane and PTA in the sublayer showed a $90 \%$ reduction in FER over a baseline cell without either additive. 


\section{Collaborations:}

- David Cullen at Oak Ridge National Labs through the ShaRE program

\section{Publications}

- $\quad$ M.P. Rodgers, L.J. Bonville, D.K. Slattery, Evaluation of the Durability of Polymer Electrolyte Membranes in Fuel Cells Containing Pt/C and Pt-Co/C Catalysts under Accelerated Testing, Electrochemical Society Transactions, 41(1) 1461, 2011.

- M.P. Rodgers, N. Mohajeri, L.J. Bonville, D.K. Slattery, Accelerated testing of polymer electrolyte membranes in fuel cells containing $\mathrm{Pt} / \mathrm{C}$ and $\mathrm{PtCo} / \mathrm{C}$ catalysts, Journal of the Electrochemical Society, 159, B564, 2012.

- $\quad$ R.P. Brooker, L.J. Bonville and D.K. Slattery, Decreasing Membrane Degradation through Heteropolyacid Sublayers, Journal of the Electrochemical Society, accepted 2012

- M.P. Rodgers, B.P. Pearman, N. Mohajeri, L.J. Bonville, D.K. Slattery, Effect of Membrane Equivalent Weight on Degradation Under Accelerated Stress Conditions, submitted to Electrochimica Acta, 2012.

\section{Presentations}

- $\quad$ March 2011, M.P. Rodgers, Fuel Cell Research, Women's International Research Engineering Summit (WIRES), Cocoa, Florida, USA.

- March 2011, M.P. Rodgers, L.J. Bonville, D.K. Slattery, Evaluation of the Durability of Polymer Electrolyte Membrane Fuel Cells containing Pt/C and Pt-Co/C Catalysts under Accelerated Testing, 2011 Annual Joint Symposium \& Exhibition of the Florida Chapter of the AVS Science and Technology Society, Orlando, Florida, USA.

- October 2011, R.P. Brooker, D.K. Slattery, L.J. Bonville and J.M. Fenton, Platinum Band Formation Mitigation through Heteropolyacid Sublayers, Presentation \# LRD42-1, Fuel Cell Seminar, Orlando, Florida, USA.

- October 2011, R.P. Brooker, D.K. Slattery, L.J. Bonville and J.M. Fenton, Platinum Band Formation Mitigation through Heteropolyacid Sublayers, Electrochemical Society Meeting, Boston, Massachusetts, USA.

- October 2011, M.P. Rodgers, L.J. Bonville, D.K. Slattery, Evaluation of the Durability of Polymer Electrolyte Membranes in Fuel Cells Containing Pt/C and Pt-Co/C Catalysts under Accelerated Testing, Electrochemical Society Meeting, Boston, Massachusetts, USA.

- $\quad$ September 2012, M.P. Rodgers, B.P. Pearman, D. Cullen, L.J. Bonville, D.K. Slattery, The effect of Pt deposited in the membrane on membrane degradation, Carisma Medium and High Temperature Fuel Cell Conference, Copenhagen, Denmark.

- $\quad$ September 2012: M.P. Rodgers, B.P. Pearman, N. Mohajeri, L.J. Bonville, D.K. Slattery, Effect of membrane equivalent weight on degradation under accelerated stress conditions, Carisma Medium and High Temperature Fuel Cell Conference, Copenhagen, Denmark.

\section{Patents}

- R. Paul Brooker and Leonard J. Bonville. "Electroactive species in layer between electrode and membrane for fuel cells", (2012) US Patent Application 20130089805

- B. P. Pearman, M. Rodgers, N. Mohajeri, R. P. Brooker, D. K. Slattery, "Polymer Membranes with Rare Earth or Transition Metal Modifier”, (2012) U.S. Application No. 61/682,833. 


\footnotetext{
${ }^{1}$ Mittal, V.O.; Kunz, H.R.; Fenton, J.M. ECS Trans. 2006, 1, 275.

${ }^{2}$ Liu, H.; Gasteiger, H.A.; Laconti, A.; Zhang, J. ECS Trans. 2006, 1, 283.

${ }^{3}$ Inaba, M.; Yamada, H.; Umebayashi, R.; Sugishita, M.; Tasaka, A. Electrochemistry 2007, 75, 207.

${ }^{4}$ Rodgers, M.P.,; Agarwal, R.; Pearman, B.P.; Li, B.; Slattery, D.K.; Bonville, L.J.; Kunz, H.R.; Fenton, J.M., ECS Trans. 2009, 25, 1861.

${ }^{5}$ Akita, T.; Taniguchi, A.; Maekawa, J.; Siroma, Z.; Tanaka, K.; Kohyama, M.; Yasuda, K. J. Power Sources, 159 (2006) 7.

${ }^{6}$ Ferreira, P.J.; Shao-Horn, Y.; Electrochem. Solid-State Lett., 10 (2007) 4.

${ }^{7}$ Cipollini, N.E., Mater. Res. Soc. Symp. Proc., 885 (2006) 33-44.

${ }^{8}$ Cipollini, N.E., ECS Trans., 11 (2007) 1071-1082.

${ }^{9}$ Gummalla, M.; Atrazhev, V.; Condit, D.; Cipollini, N.; Madden, T.; Kuzminyh, N.Y.; Weiss, D.; Burlatsky, S.; J. Electrochem. Soc., 157 (2010) 7.

${ }^{10}$ Burlatsky, S.F.; Atrazhev, V.; Cipollini, N.; Condit, D.; Erikhman, N.; ECS Trans., 1 (2006) 239-246.

${ }^{11}$ Brooker, R.P.; Bonville, L.J.; Slattery, D.K.; Journal of the Electrochemical Society (accepted), (2012).

${ }^{12}$ Mench, M.M.; Kumbur, E.C.; Veziroglu, T.N.; Editors, Polymer Electrolyte Fuel Cell Degradation, 1st ed., Elsevier Ltd., 2012.

${ }^{13}$ N.M. Markovic, B.N. Grgur, P.N. Ross, J. Phys. Chem. B 101 (1997) 5405.

${ }^{14}$ M. Watanabe, K. Tsurumi, T. Mizukami, T. Nakamura, P. Stonehart, J. Electrochem. Soc. 141 (1994) 2659.

${ }^{15}$ S. Mukerjee, S. Srinivasan, J. Electroanal. Chem. 357 (1993) 201.

${ }^{16}$ H.W. Starkweather, Jr., Macromolecules, 15 (1982) 320.

${ }^{17}$ S. Gottesfeld and T.A. Zawodzinski, Adv. Electrochem. Sci. Eng., 5 (1997) 195.

${ }^{18}$ A. Ghielmi, P. Vaccarono, C. Troglia and V. Arcella, J. Power Sources, 145 (2005) 108.

${ }^{19}$ R.B. Moore and C.R. Martin, Macromolecules, 22 (1989) 3594.

${ }^{20}$ G.A. Eisman, Proc. - Electrochem. Soc., 86(13) (1986) 156.

${ }^{21}$ H. Hagihara, H. Uchida, M. Watanabe. Electrochim. Acta, 51 (2006) 3979.

${ }^{22}$ E. Endoh, S. Hommura, S. Terazono, H. Widjaja, J. Anzai, J. ECS Trans., 11 (2007) 1083.

${ }^{23}$ V.V. Atrazhev, N.S. Erikhman, S.F. Burlatsky, J. Electroanal. Chem., 601 (2007) 251.

${ }^{24}$ S. Stucki, G.G. Scherer, S. Schlagowski, E. Fischer, J. Appl. Electrochem., 28 (1998) 1041.

${ }^{25}$ D. Zhao, B.L. Yi, H.M. Zhang, M. Liu, J. Power Sources, 195 (2010) 4606.

${ }^{26}$ Bonakdarpour, A.; Dahn, T. R.; Atanasoski, R. T.; Debe, M. K.; Dahn, J. R. Electrochem. Solid-State Lett., 11 (2008) B208.
} 


\title{
FLORIDA HYDROGEN INITIATIVE Sub-contrecter Finel Report
}

\section{Production of Low-Cost Hydrogen from Biowaste}

\author{
Dr. Robin Z. Parker, rzpsrt@gmail.com, 305-321-3677 \\ Dr. Melahn L. Parker, mlpsrt@gmail.com \\ SRT Group, Inc. \\ P. O. Box 330985 \\ Miami, FL 33233
}

\author{
Submitted to: \\ David L. Block \\ Florida Solar Energy Center/University of Central Florida \\ 1679 Clearlake Road \\ Cocoa, FL 32922 \\ E-mail: block@fsec.ucf.edu
}

Contract Number: DE-FC36-04GO14225

FSEC Project No.: 20126050 (PO No. 214802)

Project Start Date:

Project End Date:

August 29, 2012

Copywright @2012 SRT Group, Inc. All Rights Reserved 


\title{
Production of Low-cost Hydrogen from Biowaste
}

\author{
SRT Group, Inc. \\ August 29, 2012
}

\section{Executive Summary}

This project uses the SRT HyBrTec ${ }^{\mathrm{TM}}$ process to solve problems associated with conventional biowaste-to-fuel processing using anaerobic digester by exploiting two thermochemical advantages that reduce both the cost and energy of converting waste-to-fuel. The first advantage is that the SRT process occurs at moderate temperatures and pressures giving fast chemical reactions, high product yields, and the release of significant thermal energy. This advantage minimizes the size of equipment and allows for use of the co-produced heat. Second, the intermediate hydrogen carrier chemical bonds are weak, requiring less energy than water electrolysis to free hydrogen from the bromine solution.

This report presents the results from SRT's biowaste-to-hydrogen process $\left(\mathrm{HyBrTec}^{\mathrm{TM}}\right)$ that was developed and tested from December 2010 through June 2012. The final report presents the work undertaken, the results obtained, and the recommended next steps to further advance the technology. The work is considered a success by verifying cellulose to $\mathrm{H}_{2}$ conversion efficiency of $85 \%$. The objective to establish ash-treatment procedures and confirm $99.9 \% \mathrm{Br}_{2}$ recovery rates will be met in future work.

In the described work, a demonstration reactor and electrolysis vessel was designed, built and operated. Data was collected from bromination experiments at temperatures up to $250^{\circ} \mathrm{C}$, as well as electrolysis and joint bromination-electrolysis runs up to $200^{\circ} \mathrm{C}$. Learning occurred throughout this process to better define the requirements for a prototype system. An economic model was also created to estimate the costs for a demonstration system.

Pure cellulose was used for bromination. While full conversion was expected at $200^{\circ} \mathrm{C}$, it was found that only $150^{\circ} \mathrm{C}$ was required to achieve $100 \%$ conversion of cellulose to hydrobromic acid $(\mathrm{HBr})$ and carbon dioxide $\left(\mathrm{CO}_{2}\right)$. It was also found that $80 \%$ of the cellulose was converted at the significantly lower temperature of $75^{\circ} \mathrm{C}$. Both these results were surprising, and suggest a glass reactor can be used instead of the high pressure metal one currently used to meet anticipated safety concerns.

The electrolysis experiments showed open circuit voltages below 1 volt at most conditions considered. For instance, a $20 \mathrm{wt} \% \mathrm{HBr}$ solution at $150^{\circ} \mathrm{C}$ yielded an open circuit voltage of 0.6 volts. Combination bromination and electrolysis runs were undertaken in which it was shown that hydrogen was produced as cellulose was brominated. This supports a process in which cellulose and electricity inputs are continuously converted to hydrogen. 
The electrolysis of $\mathrm{HBr}$ solution inside a single reactor vessel was found to be inefficient and, thus, future work will separate bromination and electrolysis reactors to allow each to be optimized to its specific requirements and characteristics.

The results presented herein are very favorable for further development of the process. Next steps are to continue high temperature $\mathrm{HBr}$ electrolysis, perform more detailed analysis of ash remaining after bromination, and integrate a prototype system to operate continuously. Further economic details will follow in parallel to the above experimental work.

In addition, SRT acknowledges the participation provided by subcontractor, Electrolytic Technologies Corporation, North Miami Beach, FL, whose support contributed to the success of the program. 


\section{Contents}

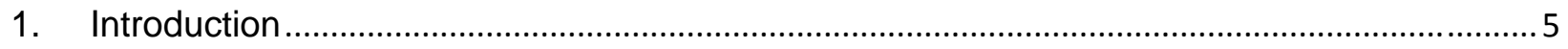

Figure 1. Simplified process flow of HyBrTec process........................................................ 5

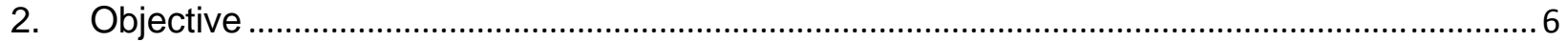

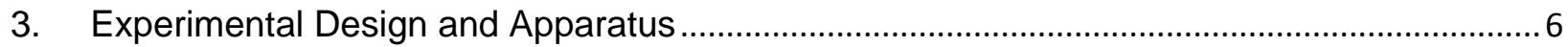

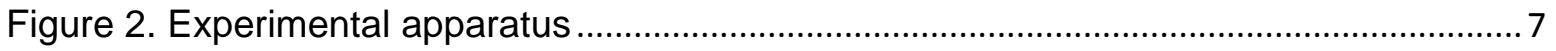

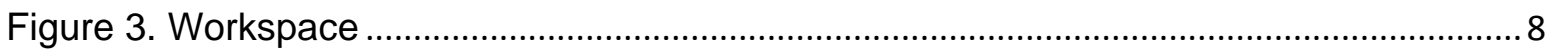

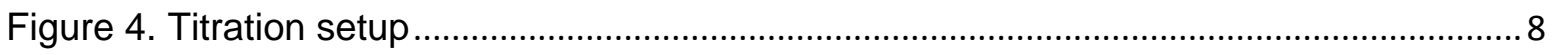

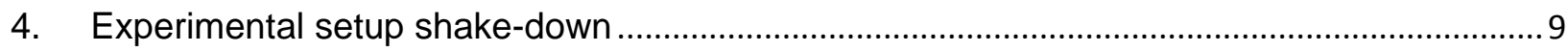

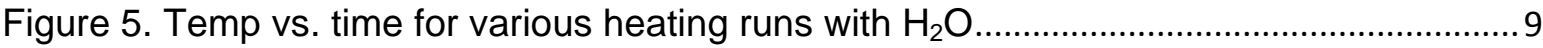

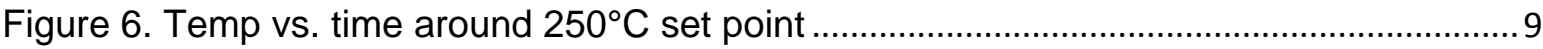

Figure 7. Pressure rise and drop during test run ............................................................... 10

Figure 8. Temperature of run with water quenching and jacket for water cooling.................10

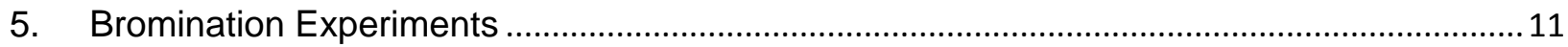

Figure 9. Bromine vapors above and liquid in solution (13.9 wt $\left.\% \mathrm{HBr}, 14.7 \mathrm{wt} \% \mathrm{Br}_{2}\right) \ldots \ldots . . .11$

Figure 10. Opened reactor, reactant, and product solution .................................................. 12

Figure 11. Temperature and pressure of runs with and without cellulose ............................ 12

Figure 12. Bromine conversion to $\mathrm{HBr}$ vs. temperature and duration ................................... 13

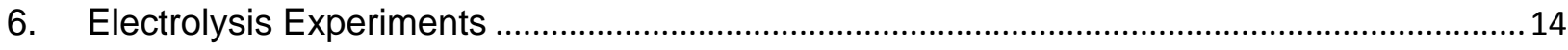

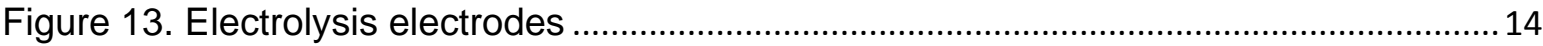

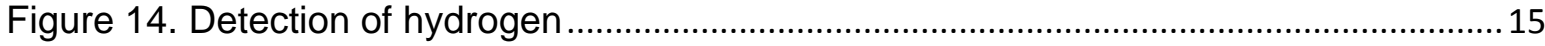

Figure 15. Increased bromine color at each sample time ................................................. 15

Figure 16. Open circuit voltage vs. temperature and $\mathrm{HBr}$ concentration .............................. 16

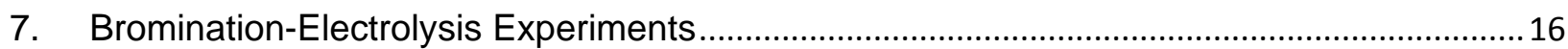

Figure 17. Detection of hydrogen in bromination-electrolysis run ........................................ 16

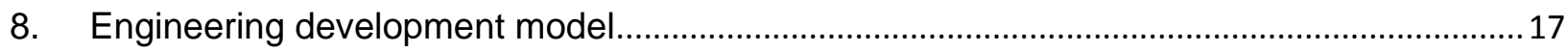

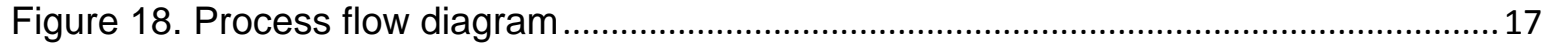

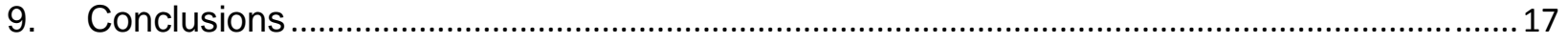




\title{
Production of Low-Cost Hydrogen from Biowaste
}

\author{
SRT Group, Inc.
}

\section{Introduction}

SRT's biowaste-to-hydrogen process $\left(\mathrm{HyBrTec}^{\mathrm{TM}}\right)$ is a paradigm-shift over other biomass-tohydrogen processes. The process produces hydrogen, carbon dioxide, thermal energy and a micronutrient ash from wet biowaste as found in sewage, manure, agricultural residuals, paper, and municipal solid waste. Biowaste is a rich source of stored solar energy, but is also an ubiquitous environmental liability. Quite the opposite of cultivating or purchasing feedstock, $\mathrm{HyBrTec}^{\mathrm{TM}}$ uses negative-value biowaste that is subject to increased disposal costs ( $\$ 50$ 350/ton) and environmental regulations. Processing wet cellulosic-biowaste into hydrogen will reduce: pollution, disposal-costs, fossil-fuel use, oil-dependence, trade-deficit, and establish new employment opportunities.

$\mathrm{HyBrTec}^{\mathrm{TM}}$ begins with the bromination of biowaste. Bromination is an exothermic reaction analogous to burning wood with oxygen from air, but since bromine $\left(\mathrm{Br}_{2}\right)$ is the oxidizer, weaklybonded hydrogen bromide $(\mathrm{HBr})$ is produced instead of more-stable water. The $\mathrm{HBr}$ forms hydrobromic acid $\left(\mathrm{HBr}_{\mathrm{aq}}\right)$ with unreacted water. The carbon in the wet-biowaste will combine with oxygen from water to produce carbon dioxide $\left(\mathrm{CO}_{2}\right)$, and additional thermal energy. As illustrated in the process-flow diagram below, the $\mathrm{Br}_{2}$ reagent is continuously recycled back to the reactor. Consequently only a minimum amount of elemental bromine is in the system.

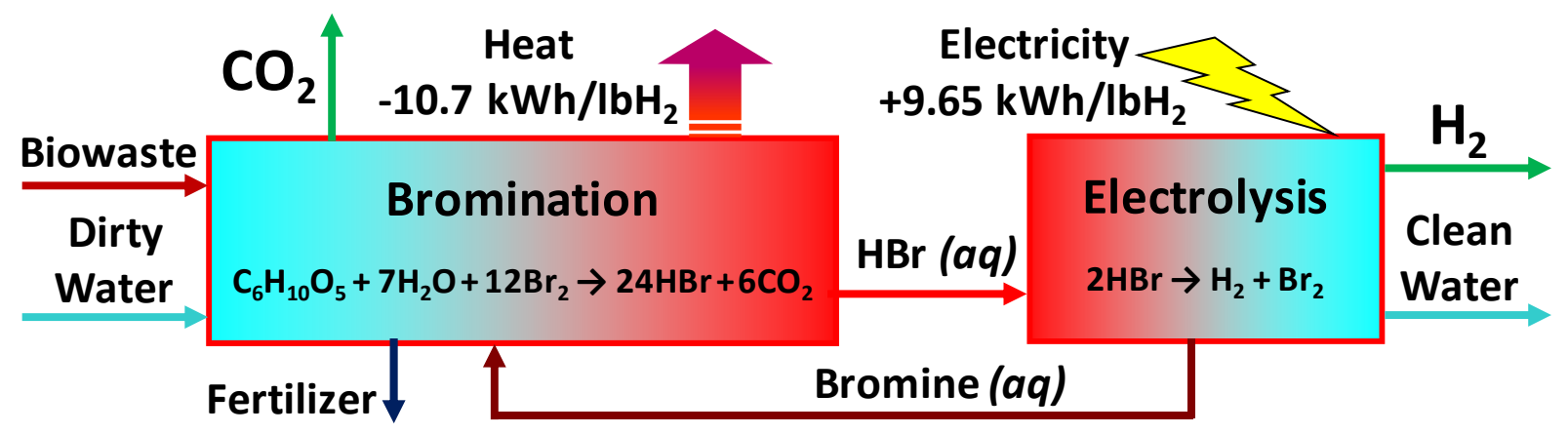

Figure 1. Simplified process flow of $\mathrm{HyBrTec}$ process

Two thermodynamic shortcuts reduce the energy and capital for producing hydrogen. First, at moderate temperatures and pressures the chemical reactions are fast, the product yields are high, and significant thermal energy is released. This minimizes the size of equipment and allows use of the co-produced heat. Second, the chemical bonds requiring energy to free hydrogen are weak, requiring less energy than the hydrogen will produce as a fuel when burned with oxygen from air.

These 'shortcuts' provide a biowaste-to-hydrogen energy efficiency of $70 \%$ that exceeds $170 \%$ when omitting the energy content of the negative-value biowaste feedstock. Despite the theoretical potential significant steps must be taken to achieve a commercial product. 


\section{Objective}

The DOE Multi-Year Research, Development and Demonstration Plan: Planned Program Activities for 2005-2015, includes a goal to develop low-cost, highly efficient hydrogen production technologies. The DOE production objective is to reduce the cost of hydrogen to $\$ 2.00-\$ 3.00$ gge at the pump, independent of the technology pathway. The cost and timeframe to achieve the distributed production and delivery of hydrogen from biomass-derived resources is $\$ 3.80 / g g e$, by 2012 . The SRT Group, Inc. in this project has conducted research and development of a novel production process that promises to produce distributed hydrogen for less than $\$ 2.00$ gge from wet biowaste.

\section{Experimental Design and Apparatus}

To evaluate the described process a systems was designed to run bromination, electrolysis, and joint bromination-electrolysis experiments. The joint experiments are to evaluate the opportunity of continuously running the two step process in one vessel. Initial work designed the apparatus, procured parts from suppliers, developed an engineering development model, and fabricated the system. The following images illustrate the equipment assembled: 

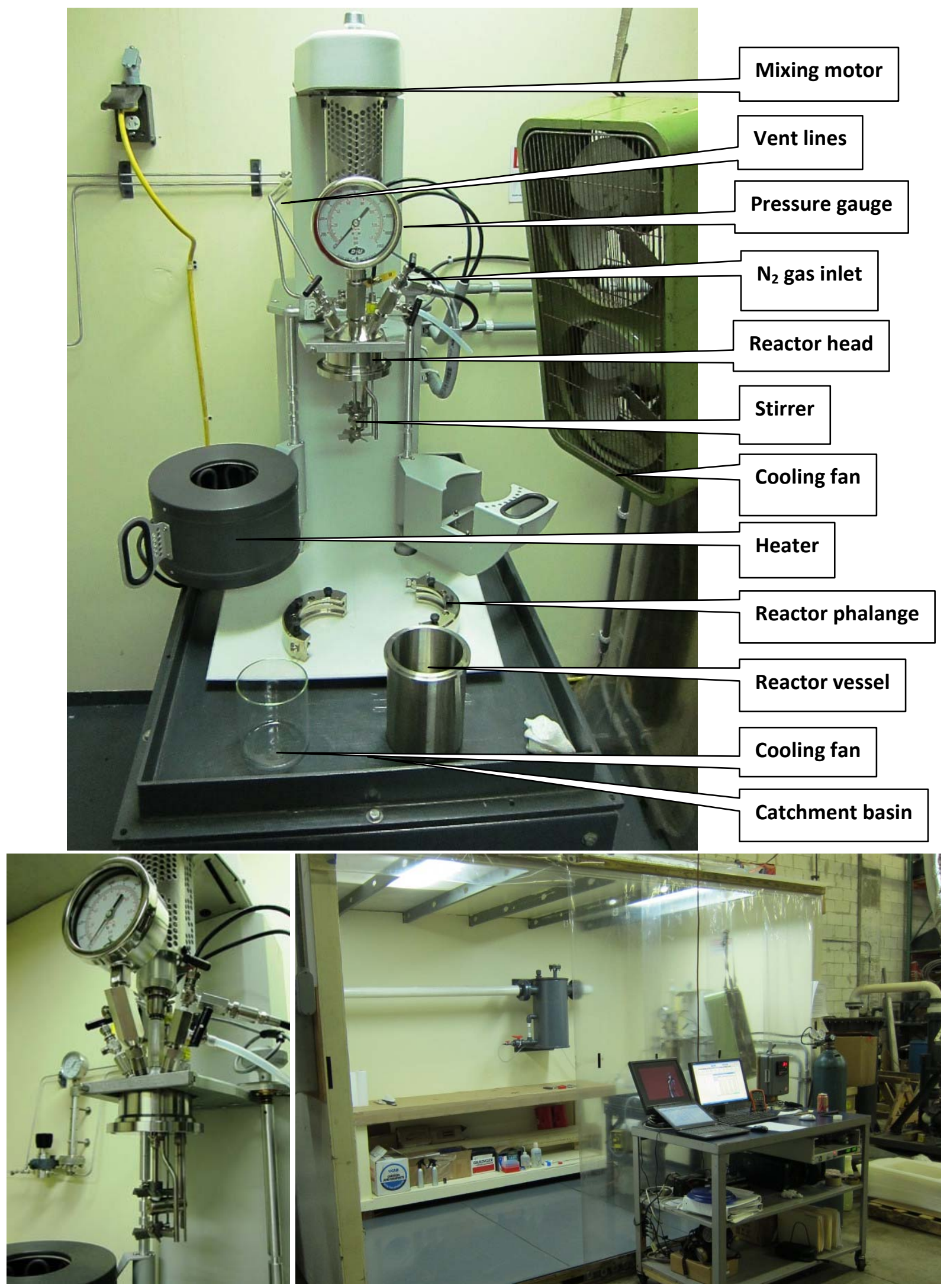

Figure 2. Experimental apparatus 


\section{DAQs and infrastructure}

A Campbell Scientific CR10 data logger was set up to record the power supply voltage and current signal at the millivolt level. The air intake for the fume room was placed in the corner closest to the reactor to draw in potential fumes and minimize leakage from nearby openings. The clear plastic panes were weighted to form a more complete seal to the external environment. The wire bundles were ported through the panes to reduce leakage as shown:

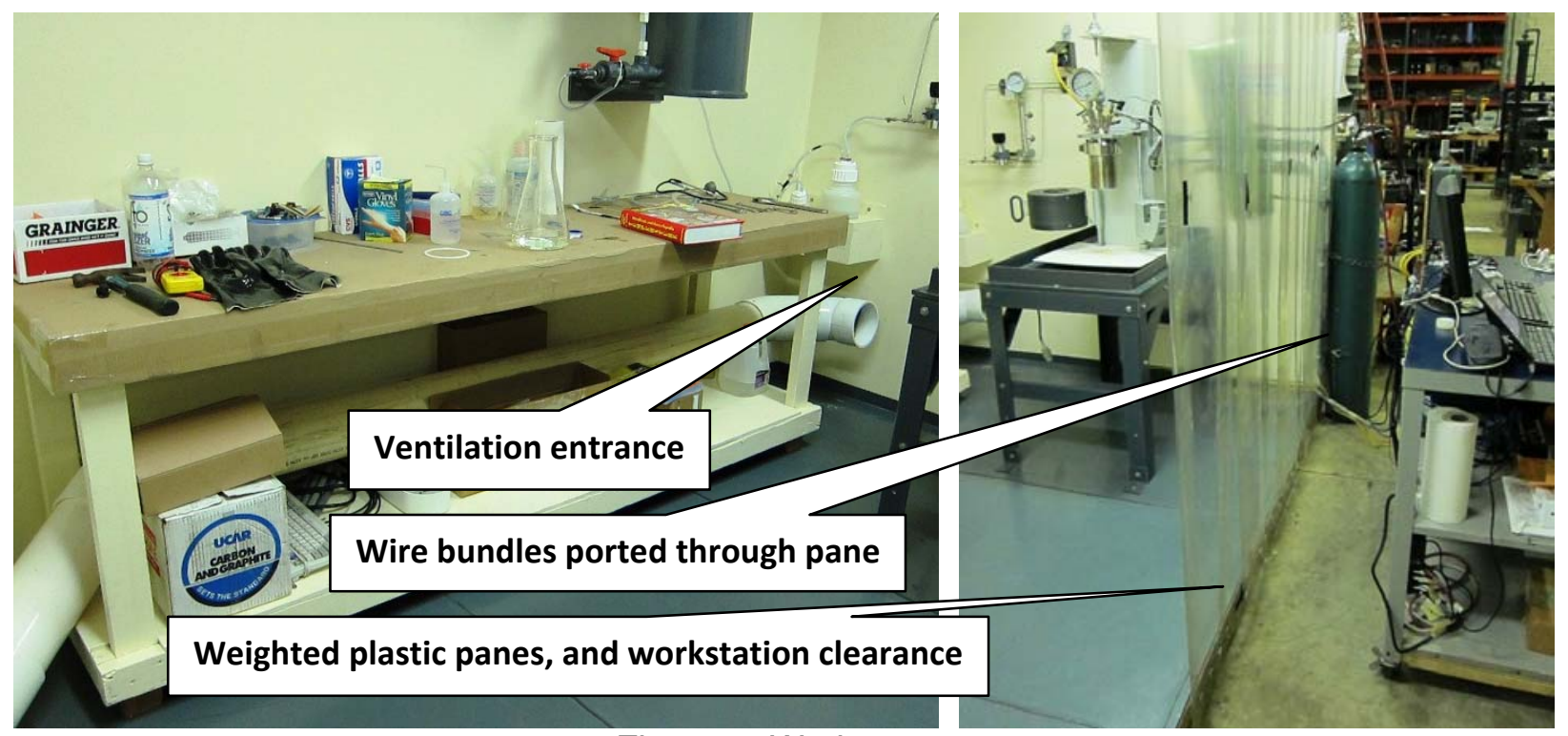

Figure 3. Workspace

Each experiment involves chemical titrations to determine the solution bromine and $\mathrm{HBr}$ content. All results were reproducible with $+/-3 \%$ precision. The following shows the titration set up used:

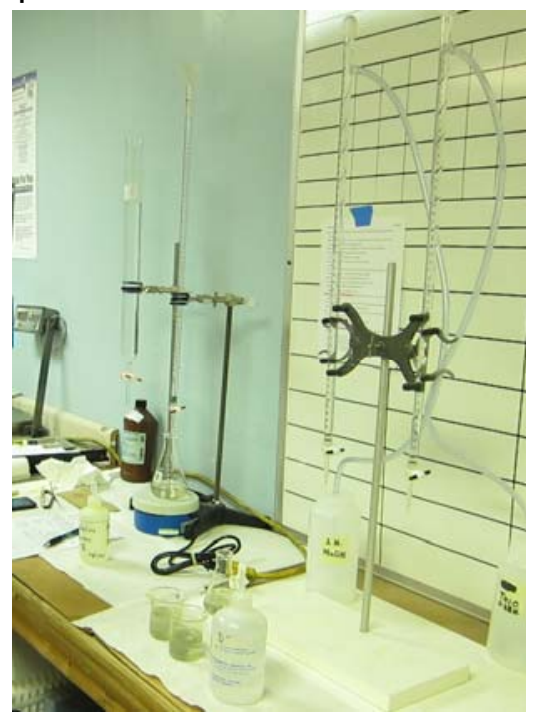

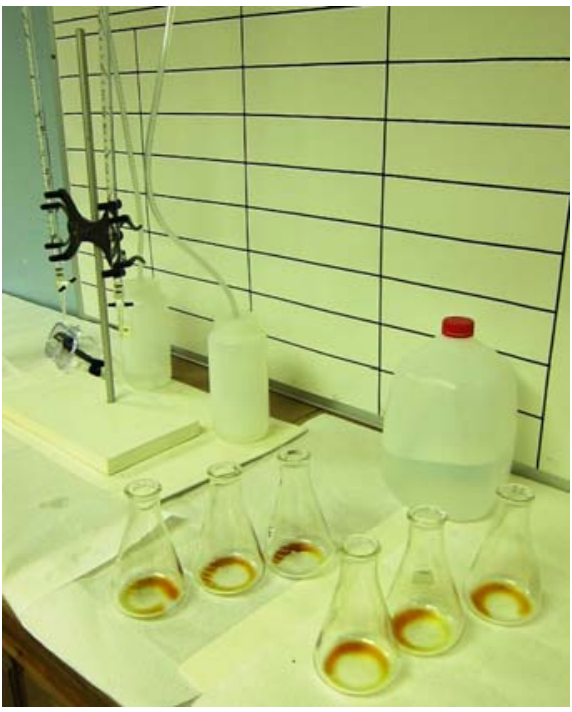

Figure 4. Titration setup

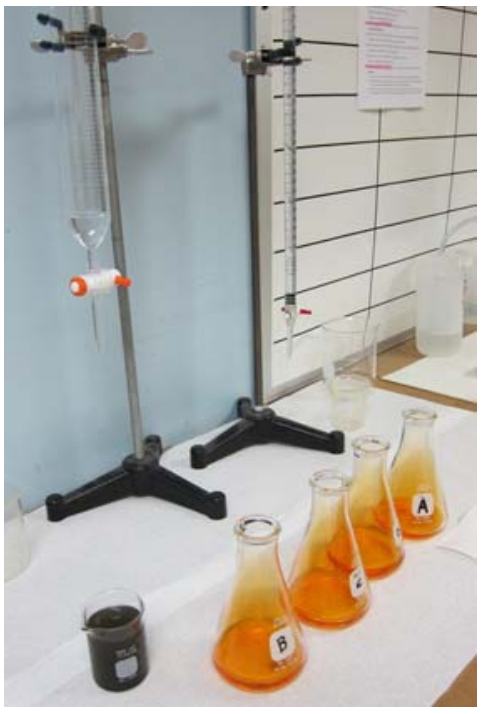




\section{Experimental setup shake-down}

To understand the heating \& cooling characteristics, and debug data acquisition equipment heating runs were performed with $500 \mathrm{ml}$ distilled water. The data from three runs is shown below. The thermal behavior depends on the reactor loading (amount inside) and whether the heater is left in place or not.

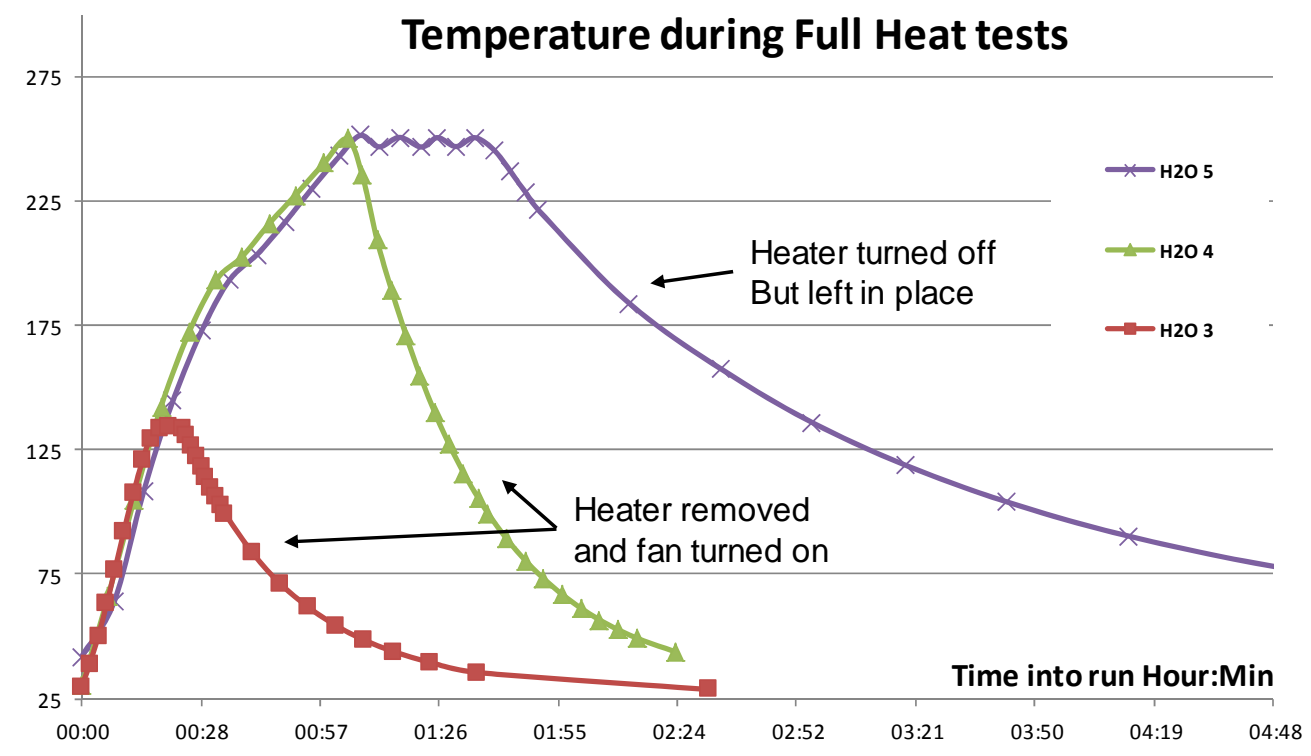

Figure 5. Temp vs. time for various heating runs with $\mathrm{H}_{2} \mathrm{O}$

\section{Temperature control}

Full on/off heating was used to minimize the time to heat. In the runs performed it was confirmed that temperature overshoot was not significant at higher temperatures $\left(<3^{\circ} \mathrm{C}\right.$ above $\left.175^{\circ} \mathrm{C}\right)$. Overshoot was larger at low temperatures $\left(135^{\circ} \mathrm{C}\right.$ achieved with set point of $\left.120^{\circ} \mathrm{C}\right)$. The figure below shows the stable temperature profile from on/off control around a set point of $250^{\circ} \mathrm{C}$ which varied between $247-251^{\circ} \mathrm{C}$.

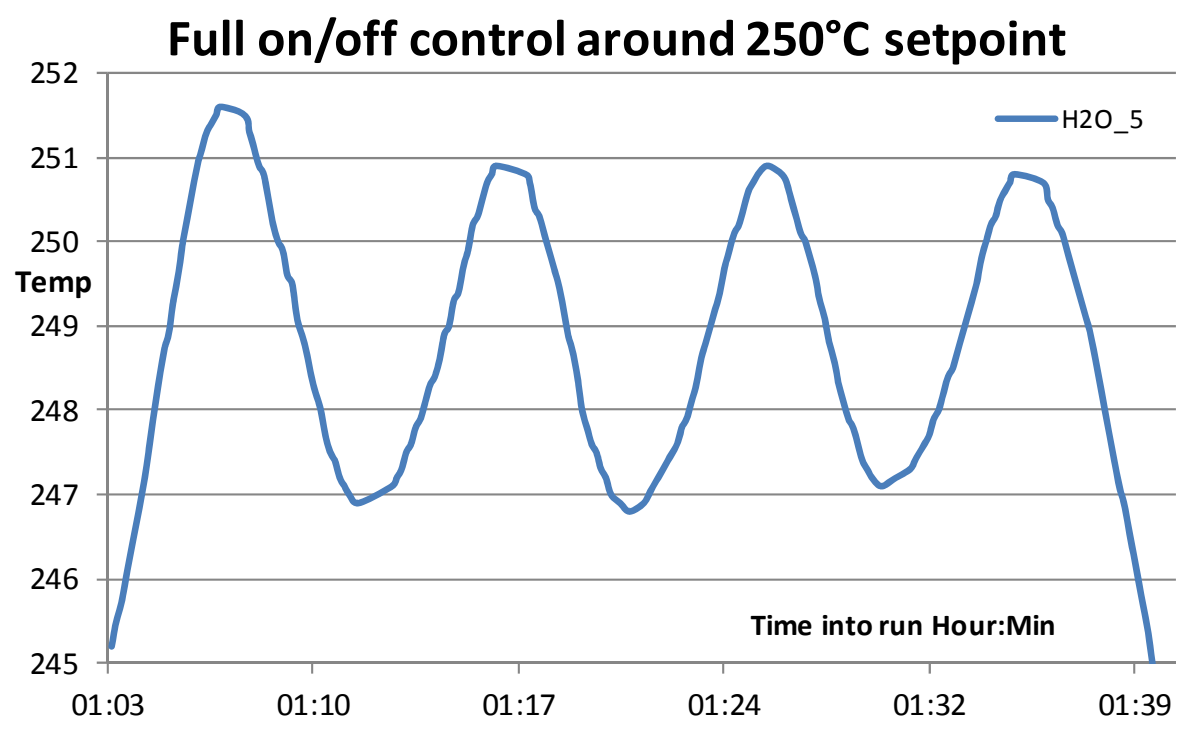

Figure 6. Temp vs. time around $250^{\circ} \mathrm{C}$ set point 


\section{Pressure - calculated vs. measured}

The measured pressure was consistently less than expected for the recorded temperature, and this deficiency was greater during the heat up than the cooling down as shown in the following figure. This behavior is explained by the thermocouple recording a higher temperature initially because it heats up faster than the reactor contents, and likewise, when the heat is turned off, the thermocouple cools faster than the reactor contents.

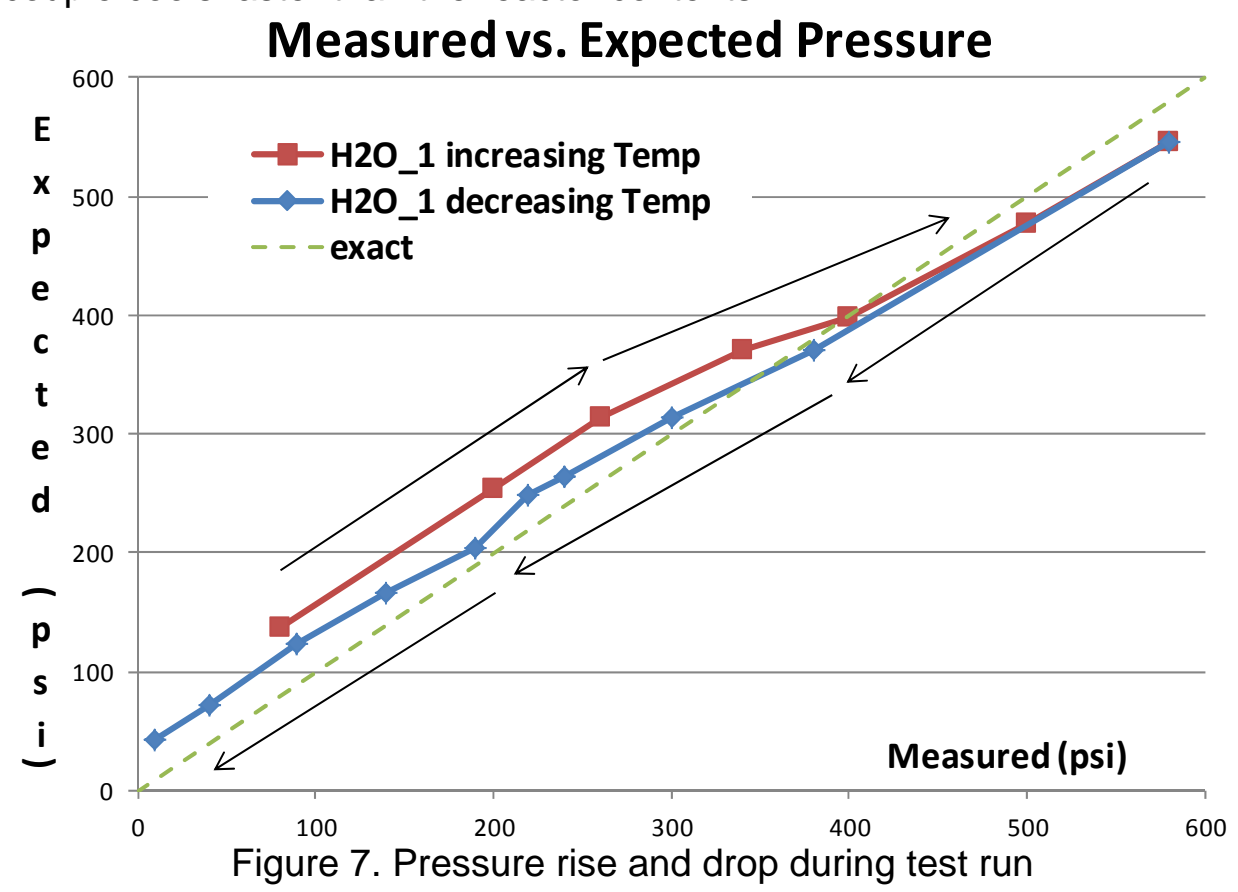

\section{Rapid quenching with water to drop temperature}

To accelerate the cooling process the vessel is put in a bucket with recirculating chilled water. This allowed experimental runs to be performed every 1-2 hours, and allowed greater control of reaction time at the set point. The rapid temperature decrease compared to fan-only cooling is shown below.
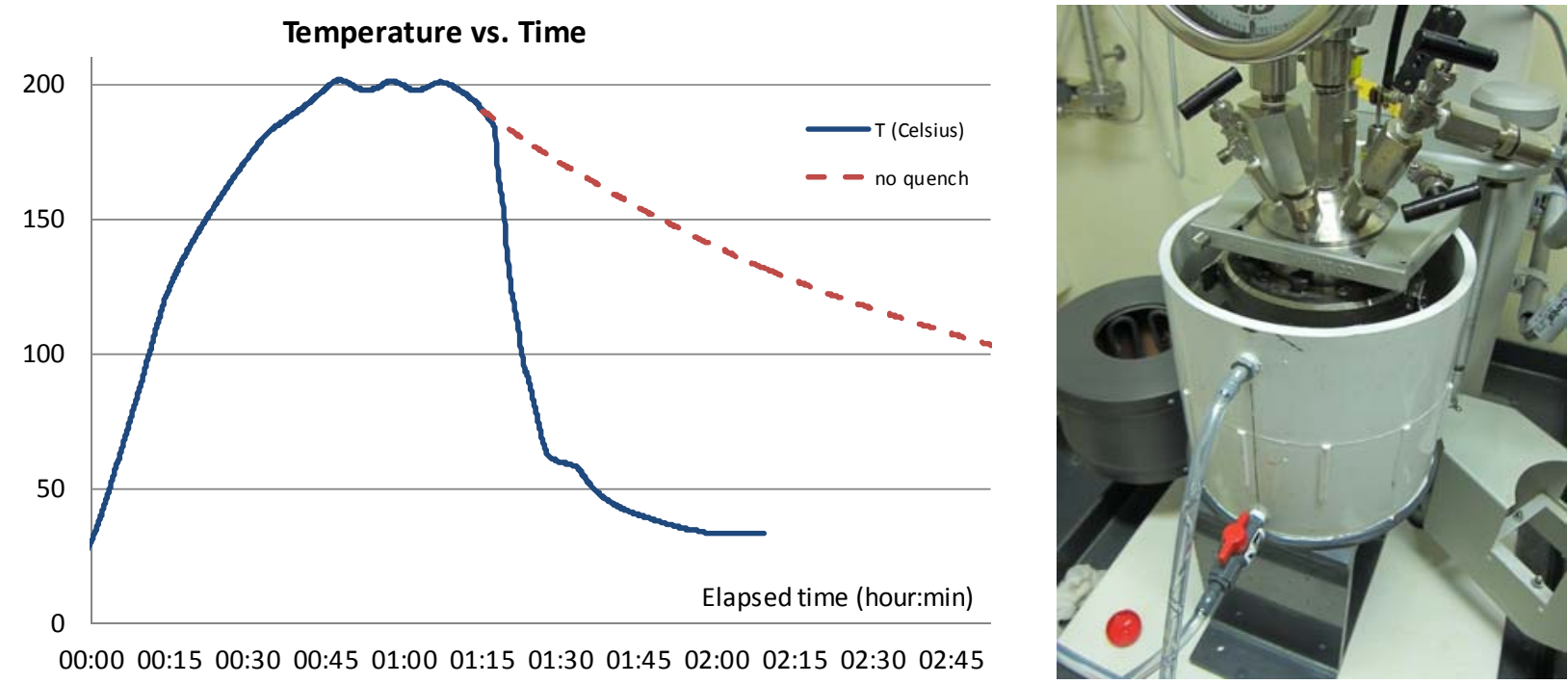

Figure 8. Temperature of run with water quenching and jacket for water cooling 


\section{Bromination Experiments}

The reactors heating and cooling behavior were understood following several heating runs using $\mathrm{HBr}$ solutions to confirm the vessel pressures. These results were as expected at the reaction temperatures, thus, the bromination experiments commenced.

\section{Bromine - $\mathrm{HBr}$ mixture}

Three bromine $-\mathrm{HBr}$ mixtures were prepared by using a two $\mathrm{kg}$ capacity scale with accuracy to 0.01 grams. This allowed the relatively accurate addition of $\mathrm{HBr}$ solution and liquid bromine by mass. No difference was found between the three solutions' reaction yields and rates:
1) $14.7 \mathrm{wt} \% \mathrm{Br}_{2}, 13.9 \mathrm{wt} \% \mathrm{HBr}$
2) $6.4 \mathrm{wt} \% \mathrm{Br}_{2}, 17.8 \mathrm{wt} \% \mathrm{HBr}$
3) $8.8 \mathrm{wt} \% \mathrm{Br}_{2}, 17.9 \mathrm{wt} \% \mathrm{HBr}$

It was noted that the first mixture had high bromine volatility causing it to readily fume bromine vapors. The images below show visibly heavy bromine vapors and bromine being poured into the container.
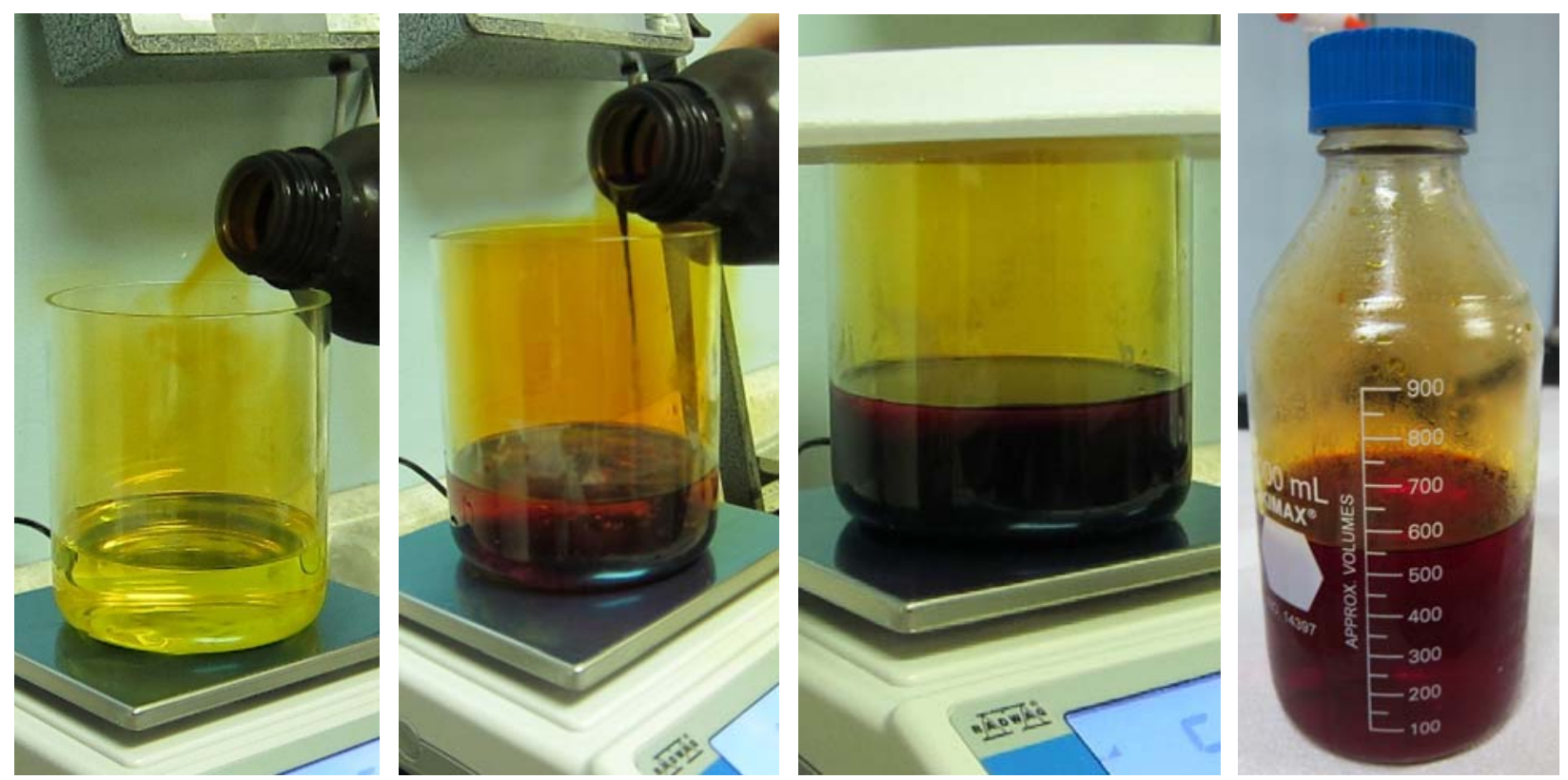

Figure 9. Bromine vapors above and liquid in solution (13.9 wt\% $\mathrm{HBr}, 14.7 \mathrm{wt} \% \mathrm{Br}_{2}$ )

To reduce the vapor pressure (not done in above images) the solutions are refrigerated prior to mixing to lower their temperature. Once mixed the solution is kept in a closed container.

\section{Bromine - $\mathrm{HBr}$ - cellulose runs}

Runs were performed with cellulose and bromine- $\mathrm{HBr}$ solution at up to $200^{\circ} \mathrm{C}$. For most runs, $25 \%$ Excess cellulose was used, but several runs were performed with excess bromine to confirm its continued presence at the end of reaction. Before and after each reaction, samples were taken for titration, and the total mass of reactants and products were measured. The 
following shows the initial solution, the pure white cellulose reactant used, and the solution produced after an example run.
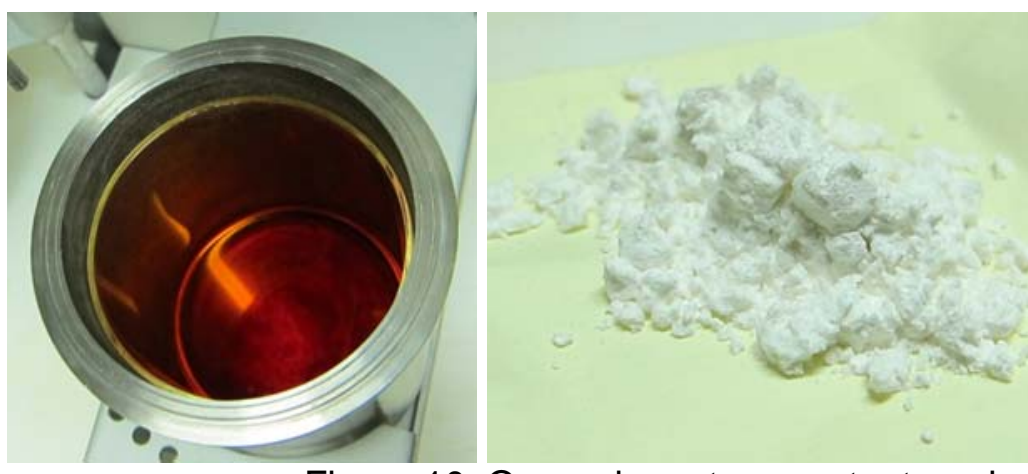

Figure 10. Opened reactor, reactant, and product solution

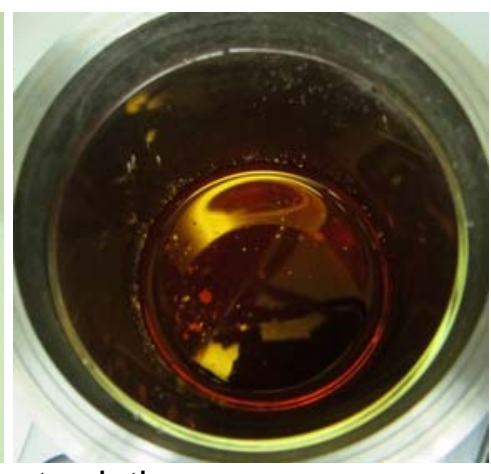

Even at low temperatures of $150^{\circ} \mathrm{C}$ the product $\mathrm{HBr}$ content corresponded to a bromine to $\mathrm{HBr}$ conversion of $100 \%$. This was more than expected at this relatively low temperature, but may be explained by the high surface area of cellulose particles used, and hence its 'eagerness' to react.

In addition to measuring increased $\mathrm{HBr}$ concentrations after reaction two other signs of reaction were noted. The first was the presence of residual pressure remaining in the reactor after reaction corresponding to generated carbon dioxide. The second was a faster heat up of reactor signaling the release of heat from the bromination reaction. This later effect is illustrated below:

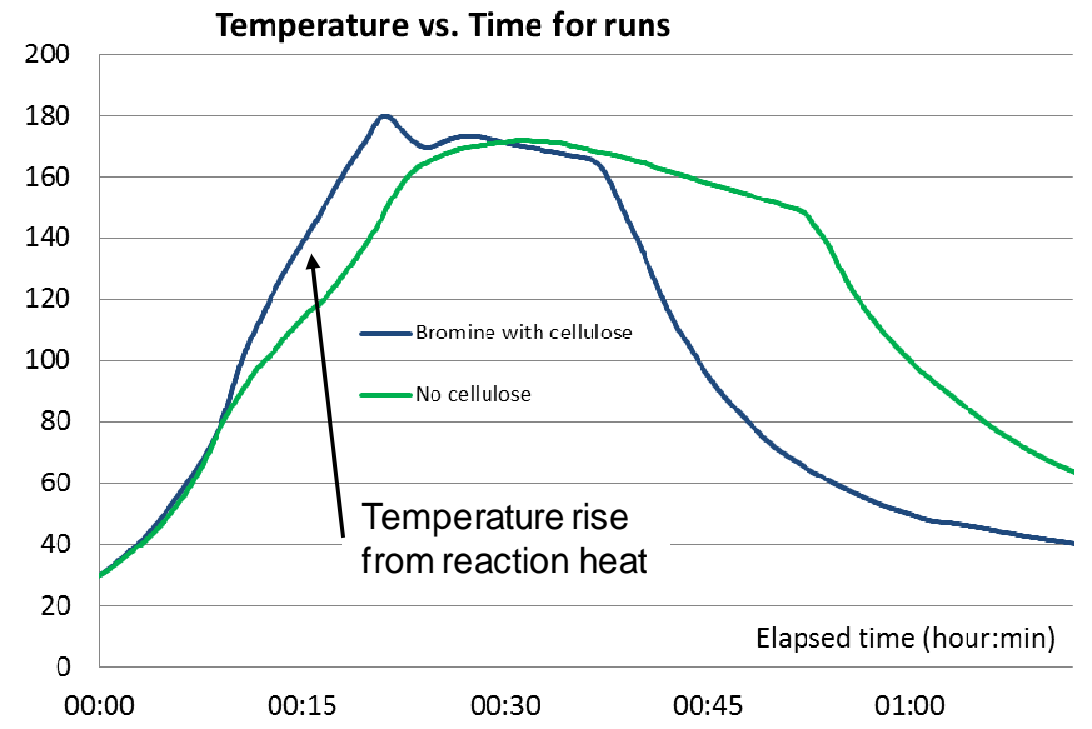

Figure 11. Temperature and pressure of runs with and without cellulose

In the above runs the temperature was set to $150^{\circ} \mathrm{C}$ and the heater was left in place until being removed just before quenching with water. In the no cellulose run above the heater was left in place longer. The interesting rise in temperature with cellulose is a result of the bromination heat of reaction. 
Experiments were run at different temperatures up to $200^{\circ} \mathrm{C}$, and durations up to 30 mins. The higher temperatures runs were not performed at longer reaction durations because it was shown that the reaction was fully complete at the lower temperatures. Hence the longer duration experimental results were anticipated from shorter duration experimental runs.

The 0 min reaction time corresponds to the reactor being taken to temperature, and then immediately quenched. For 10 min runs the temperature is held constant for 10 mins at the set point, and then quenched. In both cases some reaction occurs in the period before temperature set point is reached when the temperature is above $100^{\circ} \mathrm{C}$ (roughly $0-15$ mins). This amount of reaction in this preheating stage is estimated from the conversion found at the lower temperatures.

\section{Analysis of results}

Initial and final titrations were performed along with mass measurements to quantify the initial and final solutions. Three effects were considered in these calculations:

1. Cellulose and water reactants are converted to $\mathrm{CO}_{2}$ which leaves as an unmeasured gas

2. The reaction of water results in a small increase in solution concentrations

3. The collection of samples reduces the amount of solution available in future runs

The following image shows the \% of bromine converted to $\mathrm{HBr}$ when reacted with excess cellulose for 0 and 10 min durations:

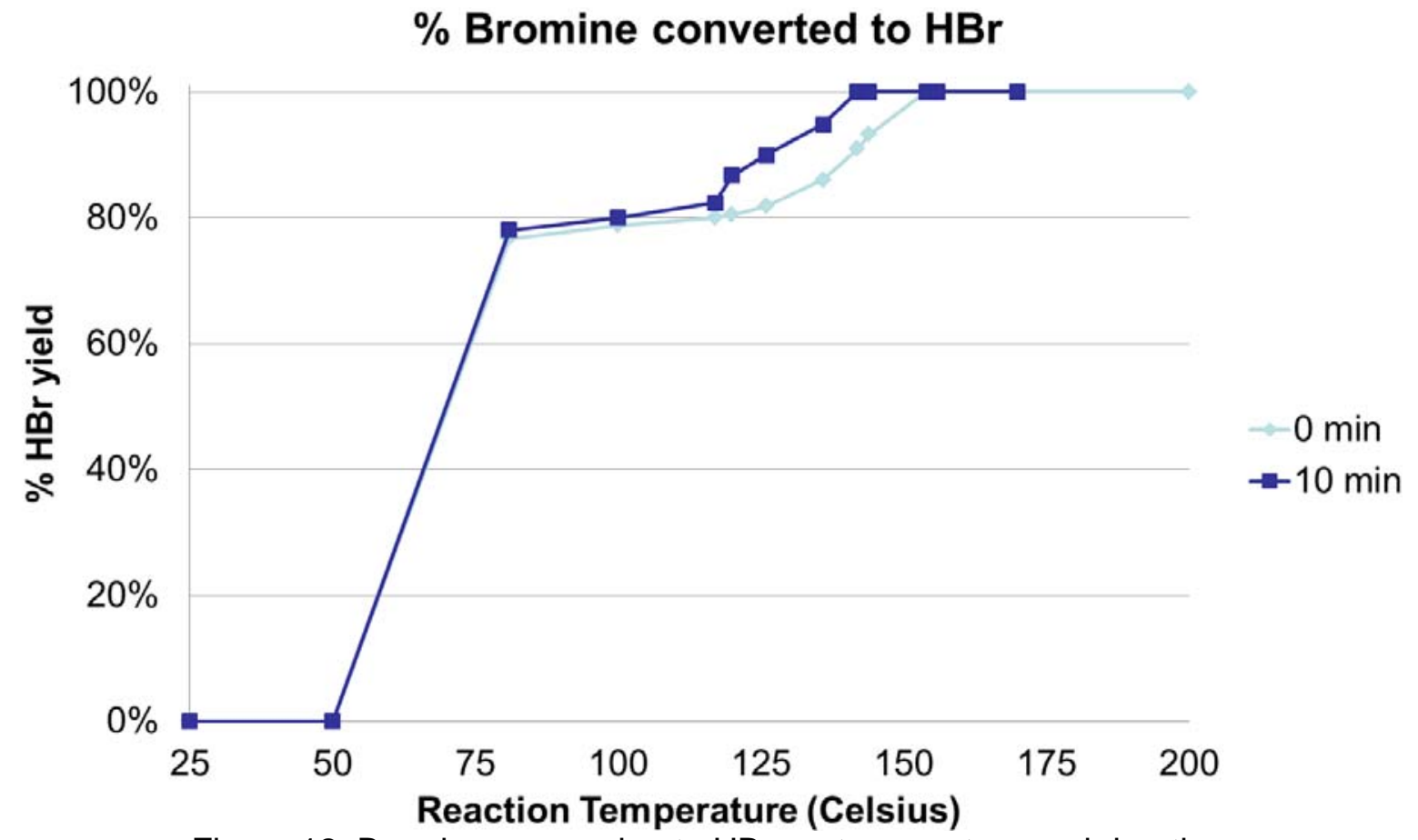

Figure 12. Bromine conversion to HBr vs. temperature and duration 
Uncertainty in values comes from errors in titrating and weighing samples. Of particular interest are:

1. Almost all the $\mathrm{HBr}$ expected from $100 \%$ bromine conversion is being detected after reaction

2. Change in solution mass can largely be accounted for by expected $\mathrm{CO}_{2}$ production

3. Overall mass balance is almost closed ( $<2 \%$ unaccounted)

Overall there was great satisfaction with the bromination results as they confirmed high bromination yields, at fast rates, and lower than expected temperatures.

\section{Electrolysis Experiments}

Electrolysis experiments started with Hastelloy electrodes, but were soon replaced with graphite cylinders to increase their active area and provide greater resistance to corrosion at high temperatures. A cylinder of graphite was machined, fitted with threads, and attached to the Hastelloy anode. A gap of about $1 / 2$ " was allowed between this new anode and the thermocouple well, and is shown below next to a picture before the electrode was attached (picture of two Hastelloy electrodes not available).

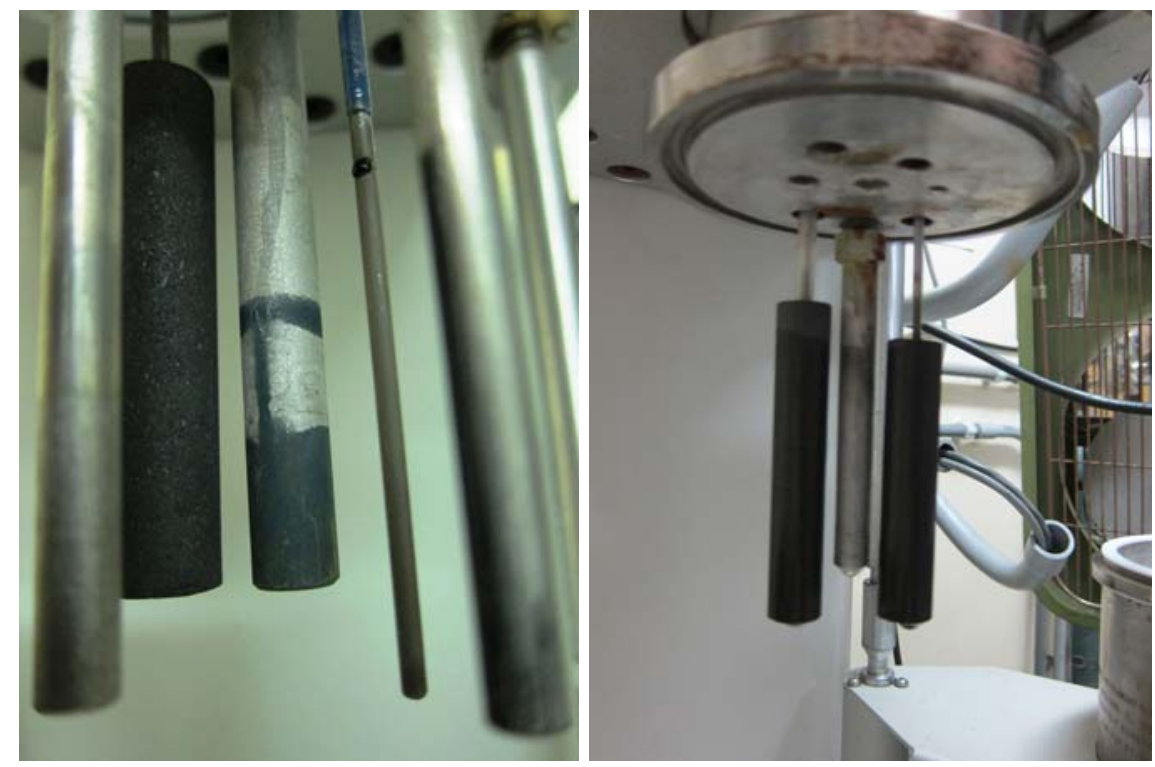

Figure 13. Electrolysis electrodes

During electrolysis runs nitrogen is passed through the reactor to carry away hydrogen, and prevent its build up and the associated dangers. To confirm hydrogen was being produced a visual detection method was utilized. The image below shows the detection ampule registering some $0.5-1 \%$ hydrogen, compared to an ampule exposed to atmosphere that does not record any hydrogen. This confirmed that hydrogen was being produced during application of voltage. The concentration is low due to the nitrogen carrier gas passed through the vessel during electrolysis. 


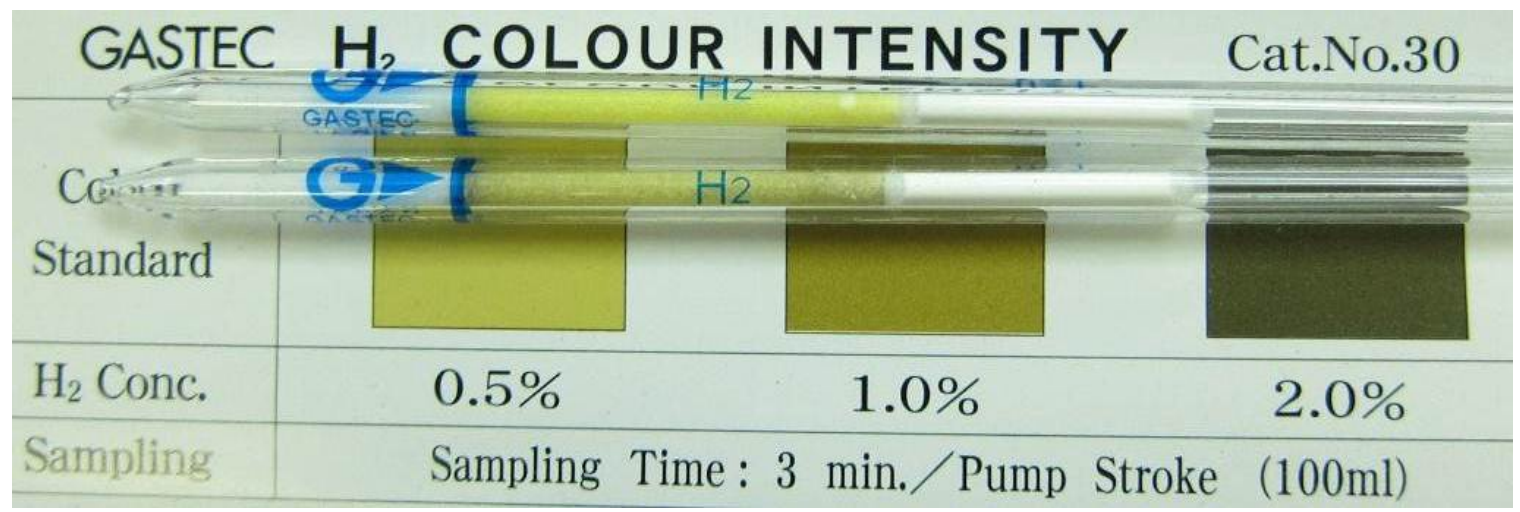

Figure 14. Detection of hydrogen

A solution of $11.5 \mathrm{wt} \% \mathrm{HBr}$ was made and $200 \mathrm{ml}$ of it was put in the reactor, and nitrogen carrier gas was flowed through the system to remove generated hydrogen. Over the course of four days it was electrolyzed at 1.2-1.6 volts and currents from 0.1-0.3 Amps. Samples were withdrawn from the reactor after $\sim 20 \mathrm{hrs}, \sim 44 \mathrm{hrs}$, and $\sim 90 \mathrm{hrs}$ for titration. The images below show the darkening color of the solution as bromine concentration increased over the electrolysis run. A final concentration of $3.5 \mathrm{wt} \%$ Bromine was measured which was less than expected. This is explained by re-reaction of bromine with hydrogen to form $\mathrm{HBr}$ in the absence of proper separation of the two product species.
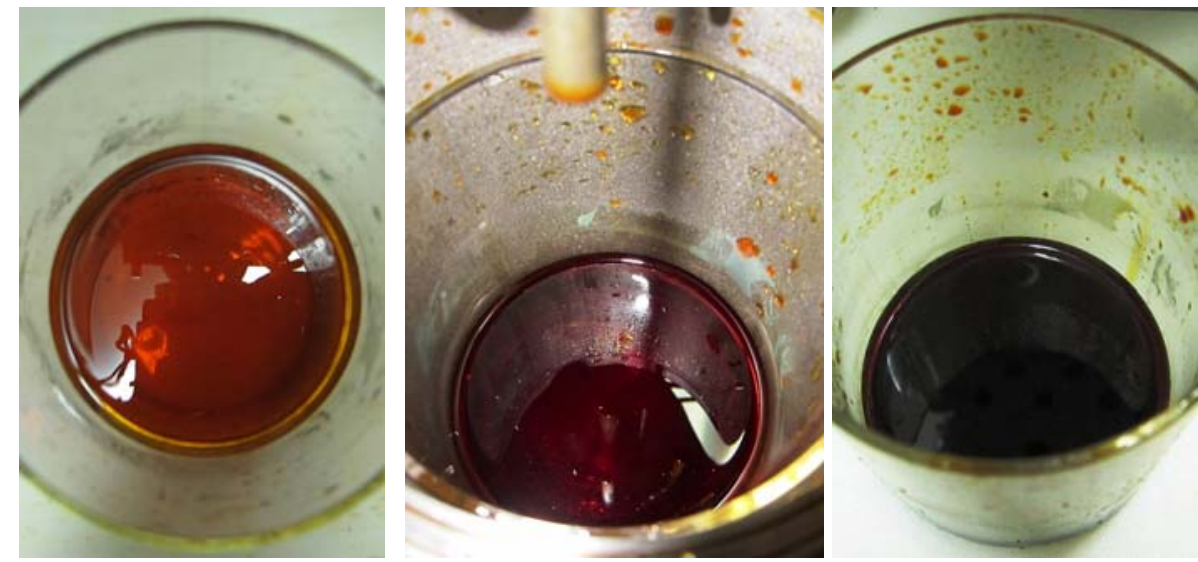

Figure 15. Increased bromine color at each sample time

Open circuit electrolysis voltages were measured for five $\mathrm{HBr}$ concentrations at different temperatures up to $200^{\circ} \mathrm{C}$ by applying the minimum voltage that yielded a measureable voltage of 0.01 amps. For each run, the temperature was cycled up to $200^{\circ} \mathrm{C}$ and then back, and voltage was measured continuously. The results below show the very low voltages achieved at high temperature. These voltages are extremely favorable for a process that produces hydrogen from electricity. 


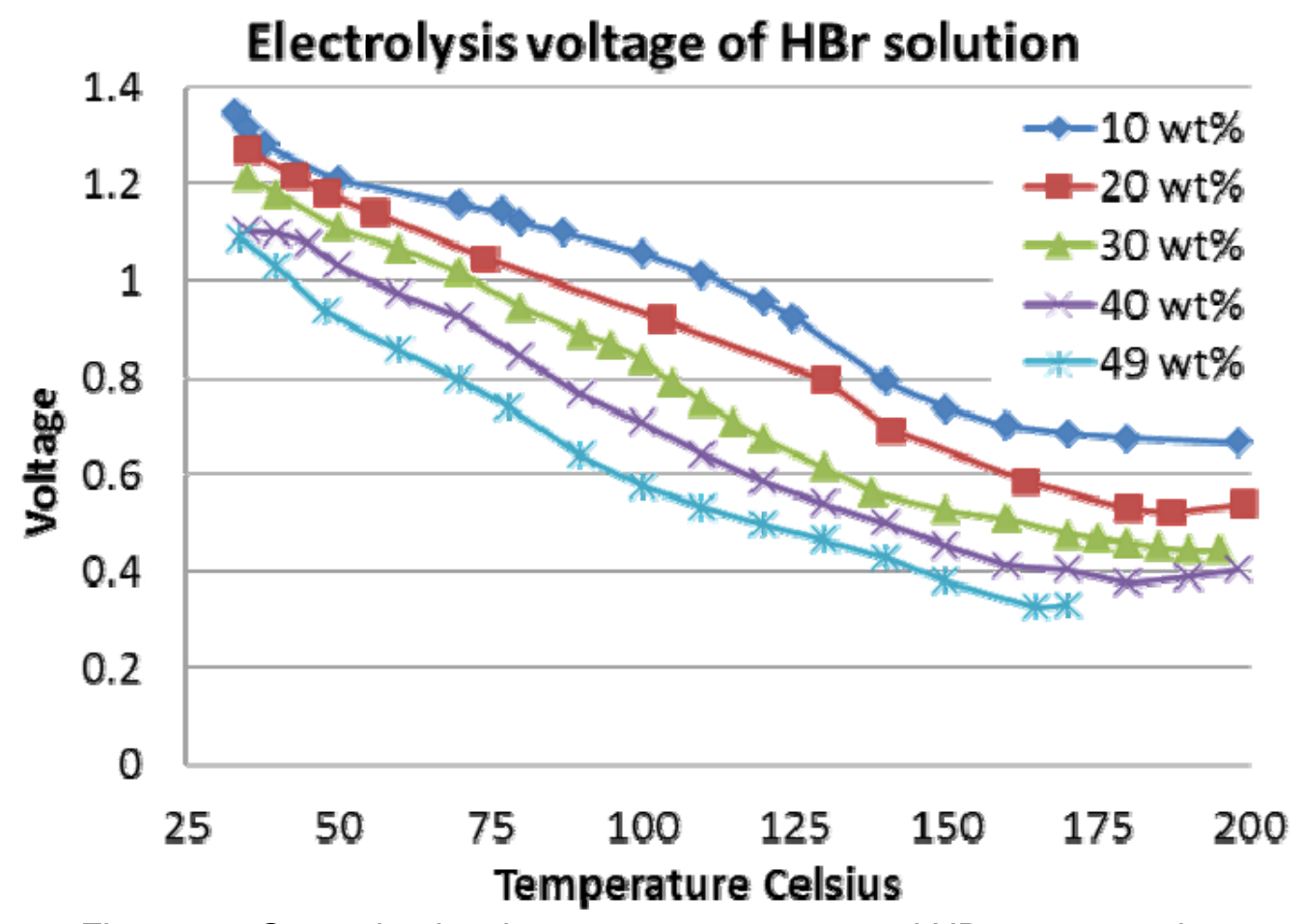

Figure 16. Open circuit voltage vs. temperature and $\mathrm{HBr}$ concentration

\section{Bromination-Electrolysis Experiments}

A run was undertaken to confirm bromination and electrolysis could be performed in parallel. An initial solution of 3.8 Molar $\mathrm{HBr}$ solution with no bromine was mixed with 7.75 grams of cellulose and heated to $200^{\circ} \mathrm{C}$. Nitrogen carrier gas was set flowing through the reactor to carry away expected hydrogen. At $150^{\circ} \mathrm{C}$ a $0.5 \mathrm{Amp}$ current was applied to the graphite electrodes, and at $200^{\circ} \mathrm{C}$ a sample was taken from the venting gas. The reactor was then cooled down through water quenching. The image below shows the detection of hydrogen in the venting carrier gas at $>1$ vol\%.

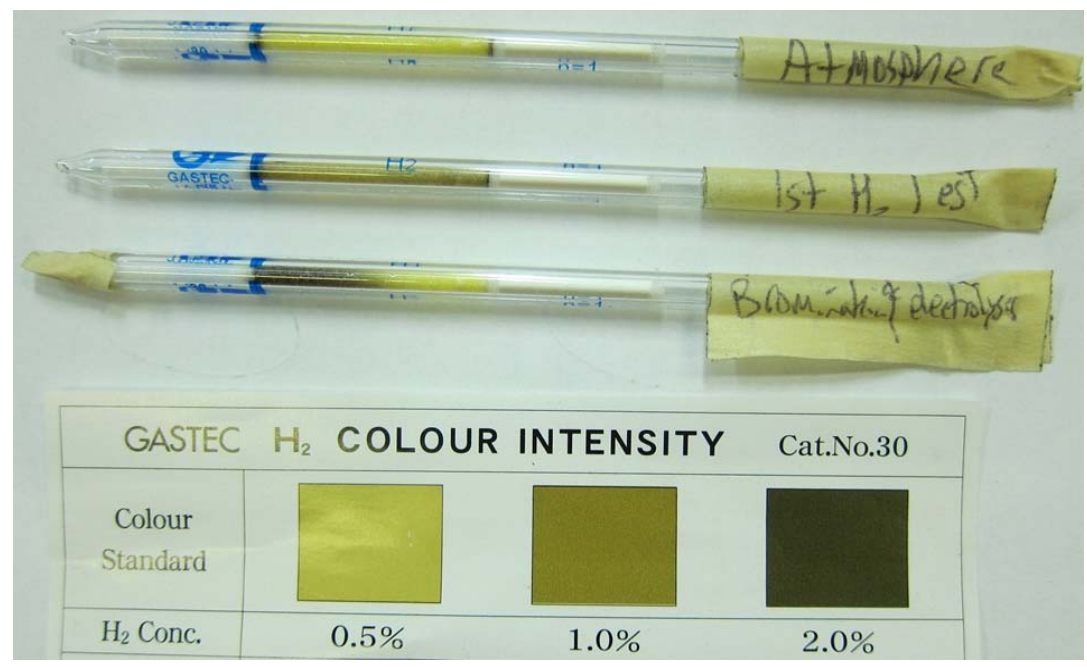

Figure 17. Detection of hydrogen in bromination-electrolysis run 
The final solution concentration was 5.1 Molar $\mathrm{HBr}$ with no bromine which suggests cellulose and water were converted to $\mathrm{HBr}$ in the absence of initial bromine. The bromine is hypothesized to come from the electrolysis of $\mathrm{HBr}$, and thus this demonstrates that bromination and electrolysis can occur in parallel.

\section{Engineering development model}

The following process flow diagram was put together for the next prototype intended to continuously brominate cellulose and produce $1 \mathrm{~kg}$ of hydrogen per day. Such a system will allow further validation of the above mentioned results and the debugging required for a commercial system.

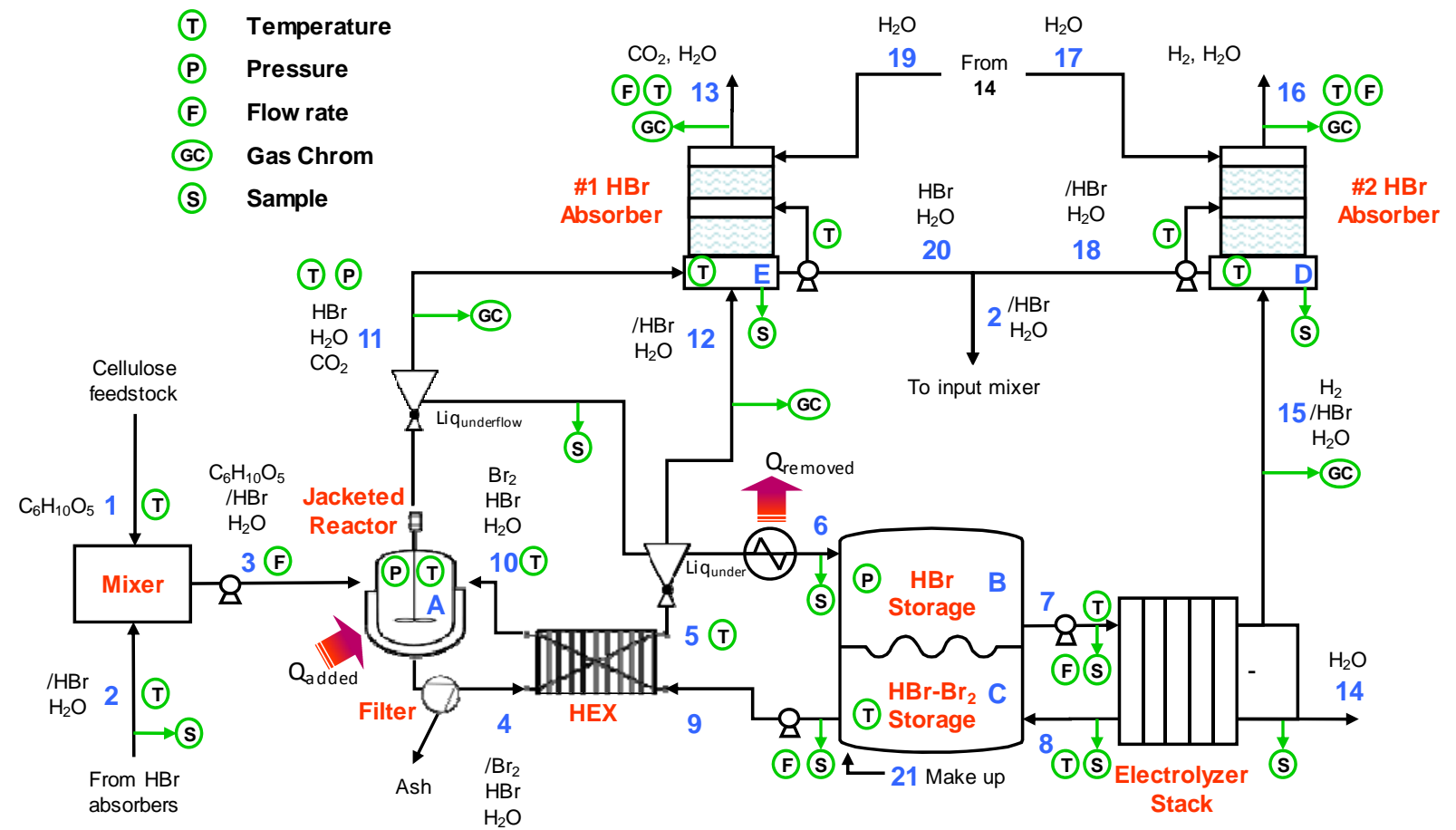

Figure 18. Process flow diagram

\section{Conclusions}

The following report documents work undertaken to understand the bromination of cellulose and water to form hydrobromic acid. It is considered a success. Work performed and data collected are as follows:

- $\quad$ Pressure vs. temperature of 48 wt\% $\mathrm{HBr}$ confirmation

- Temperature ramp time for experimental set up

- Room temperature electrolysis of $\mathrm{HBr}$ solution

- Initial bromination of cellulose

- Conversion of cellulose vs. temperature

- Electrolysis voltage vs. temperature and $\mathrm{HBr}$ concentration

- Confirmation of combined bromination-electrolysis 
The electrolysis of $\mathrm{HBr}$ solution inside the same container that generates $\mathrm{HBr}$ from the bromination of cellulose, while interesting, has two inherent inefficiency flaws and has been discontinued.

These two flaws are:

1. The bromination reactor is small relative to the $\mathrm{HBr}$ it can produce, and does not afford enough area to electrolyze product $\mathrm{HBr}$. For example:

a. If we covered the inside of our reactor with a maximum of $300 \mathrm{~cm}^{2}$ of electrode active area, and operated at a high current density of $400 \mathrm{~mA} / \mathrm{cm}^{2}$ with $100 \%$ current efficiency it would take 40 minutes to convert a third of a $48 \mathrm{wt} \% \mathrm{HBr}$ solution into bromine.

b. By comparison it should take $<10$ minutes to make the same $\mathrm{HBr}$ from cellulose reactants, and this dichotomy will be exacerbated as the reactor gets larger and there is less external area per unit of internal volume.

2. The high temperature required for the bromination reaction poses challenges to preventing the hydrogen from re-reacting with bromine after electrolysis.

Future project work will seek to further understand these reactions by producing an integrated prototype demonstration with separate bromination and electrolysis vessels to allow each to be optimized to its specific requirements and characteristics. 


\title{
FLORIDA HYDROGEN INITIATIVE Sub-contrecter Finel Report
}

\section{Development of a Low-Cost and High-Efficiency 500W Portable PEMFC System}

\author{
Dr. James Zheng \\ Florida State University \\ Electical \& Computer Engineering \\ zheng@eng.fsu.edu \\ (850) 410-6464 \\ in partner with \\ Bing Energy International
}

\author{
Submitted to: \\ David L. Block \\ Florida Solar Energy Center/University of Central Florida \\ 1679 Clearlake Road \\ Cocoa, FL 32922 \\ E-mail: block@,fsec.ucf.edu
}

DOE Contract Number: DE-FC36-04GO14225

UCF Account No. 20126050 (PO No. 215066)

FSU Project No.: 028327

Project Start Date: December 1, 2010

Project End Date: December 31, 2012

February 2013 


\title{
Development of a Low-Cost and High-Efficiency 500W Portable PEMFC System
}

\author{
Florida State University and \\ Bing Energy International
}

\section{Executive Summary}

The objective of the "Development of a Low-Cost and High Efficiency 500W Portable PEMFC System" was to research and develop a proton exchange membrane fuel cell (PEMFC) in order to improve membrane manufacturing processes and improve cell efficiencies and lifetimes. PEMFC has been selected as the leading near-term technology candidate for fuel cell vehicles because of its high power density, quick start capability, and technology maturity. Advancements in fuel cell technology have reduced the size, weight and cost of fuel cell electric vehicles. Fuel cell electric vehicles have been produced with "a driving range of more than 250 miles between refueling". They can be refueled in less than 5 minutes. Deployed fuel cell buses have a 40\% higher fuel economy than diesel buses. However, high cost and relative low performance of PEM fuel cell are still major barriers to hinder the widespread application of PEMFC power source for autos and the military.

Electrode structures and catalysts are critical for the determining performance of PEMFC and their products. Particularly, catalyst activity and utilization efficiency of platinum group metal (PGM) based electrocatalyst electrode technology will directly determine the cost. To reduce PGM usage and improve cell performance, new technologies based on novel electrode materials and structures, understanding of the electrode structure-performance relationships and optimization of catalyst-support interactions need to be explored, characterized and demonstrated. This project has made technical breakthrough on catalyst usage and life, and cell power density by using the mixed carbon nanotubes (CNT) and carbon nanofibers (CNF) free-standing papers (buckypaper) as supporting network of catalytic electrodes.

In this project, a research and development team from Bing Energy International (BEI) have explored and demonstrated effective and scalable techniques to fabricate affordable Pt/buckypaper electrode with gradient-structured ready for fuel cell stack. The team has systematically investigated the effect of electrode microstructure on the performance of resultant fuel cells and optimized the electrode structure to greatly improve the catalyst efficiency and cell durability. A product prototype of a fuel cell stack used for portable power applications has been demonstrated.

The project results determined that Pt/double-layered buckypaper (DLBP) with tailored gradient structure demonstrated promising Pt utilization and stability of supports in spite of a relatively large Pt particle size. By applying this Pt/DLBP as a cathode catalyst layer, the MEA exhibited excellent power performance with a relative low Pt loading. The rated powers of $1.5 \mathrm{~W} / \mathrm{cm}^{2}$ and $0.8 \mathrm{~W} / \mathrm{cm}^{2}$ at $0.65 \mathrm{~V}$ were achieved with a cathode Pt loading of $0.2 \mathrm{mgPt} / \mathrm{cm}^{2}$ for oxygen and air, respectively. The Pt utilization was better than the current state-of-the-art value $\left(>0.4 \mathrm{mgPt} / \mathrm{cm}^{2}\right)$ achieved by the conventional $\mathrm{Pt} / \mathrm{C}$ catalyst at a comparable cell $\left(0.8-1.0 \mathrm{~W} / \mathrm{cm}^{2}\right)$ output.

The research also evaluated the durability of catalyst support for Pt/DLBP in a MEA according to DOE's suggested protocol. The SWNT/CNF buckypaper-based Pt catalyst has shown good durability under an accelerated degradation test in a mimic cathode environment. This was due to the high corrosion resistance of CNF and its high graphitization degree.

Florida State University and Bing Energy also developed an 11 cell stack. The MEA size of each cell is $50 \mathrm{~cm}^{2}$. The Pt loading is $0.2 \mathrm{mg} / \mathrm{cm}^{2}$ and $0.05 \mathrm{mg} / \mathrm{cm}^{2}$ at cathode and anode, respectively. The fuel cell 
stack was characterized using hydrogen at anode and oxygen or air at cathode. The rated powers $(0.65$ $\mathrm{V} /$ cell) of 670 and $358 \mathrm{~W}$ were obtained when oxygen and air were used, respectively. 


\section{Development of a Low-Cost and High-Efficiency 500W Portable PEMFC System}

Table of Contents

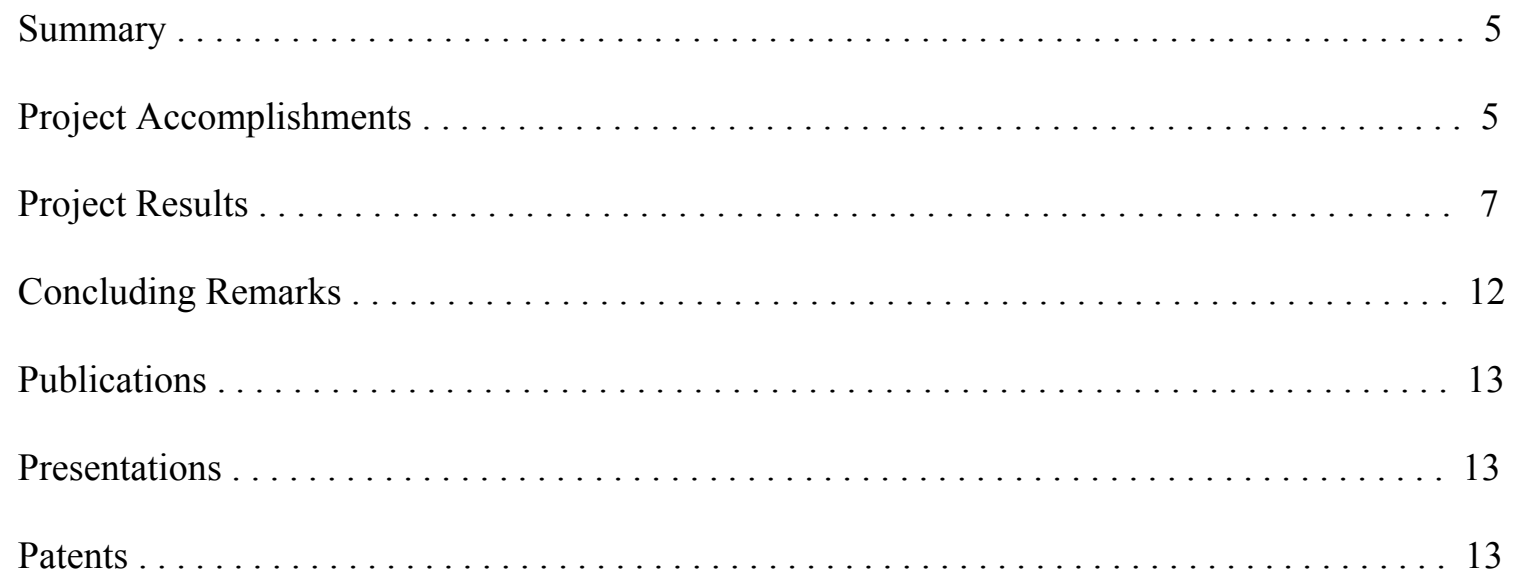




\title{
Development of a Low-Cost and High-Efficiency 500W Portable PEMFC System
}

\author{
Florida State University and \\ Bing Energy International
}

\section{Summary}

The objective of the "Development of a Low-Cost and High Efficiency 500W Portable PEMFC System" was to research and develop a proton exchange membrane fuel cell (PEMFC) in order to improve membrane manufacturing processes and improve cell efficiencies and lifetimes. The project results have shown breakthroughs on catalyst usage and life, and cell power density by using the mixed carbon nanotubes (CNT) and carbon nanofibers (CNF) free-standing papers (buckypaper) as supporting network of catalytic electrodes. The Florida State University (FSU) and Bing Energy International (BEI) teams have explored and demonstrated effective and scalable techniques to fabricate affordable Pt/buckypaper electrode with gradient-structured ready for fuel cell stack. The team has systematically investigated the effect of electro microstructure on the performance of resultant fuel cells and optimized the electrode structure to greatly improve the catalyst efficiency and cell durability. A product prototype of a fuel cell stack used for portable power applications has been demonstrated.

\section{Project Accomplishments}

For the project, six tasks were successfully completed. In the results presented below, each task description is first given followed by the accomplishments.

Task 1 - Design and optimize the gradient structures to facilitate electrochemical reactions for improving cell performance. The research team developed analytical models based on electrochemistry and catalyst theories of PEMFC to reveal the effects of catalyst deposition and nanoscale porous structures of electrodes on cell performance. The results provided essential knowledge to design optimized and gradient porous structures of high-performance electrodes.

Accomplishments - The FSU team developed analytical models based on electrochemistry and catalyst theories of PEMFC to reveal the effects of catalyst deposition and nanoscale porous structures of electrodes on cell performance. The developed model is based on stochastic microstructure reconstruction and direct numerical simulation of the catalytic electride and fuel cell performance.

Task 2 - Develop an effective technique for large size of gradient-structured buckypaper. The research team will demonstrate scalable buckypaper fabrication based on FSU's patented filtration process. Large-sized (4" $\times 4 ")$ buckypaper with gradient structure will be delivered for the fuel cell application. We will also demonstrate low-cost buckypaper without SWNTs. This can further bring cost down.

Accomplishments - The team has developed an effective technique for scalable buckypaper fabrication based on our patented filtration process. Large-sized (4" $\times 4 ")$ buckypaper with gradient structure was fabricated for the fuel cell application.

Task 3 - Investigate and develop surface coating techniques to optimize catalyst deposition on 16$\mathbf{c m}^{2}$ buckypaper and increase catalyst efficiency. The research team will develop the protocols of $\mathrm{Pt}$ deposition process to realize good deposition quality, with ability to tailor Pt loading and distribution along the thickness of buckypaper. We will systemically characterize deposition quality using SEM, TEM 
and electrochemical techniques. The results will be used to optimize protocols of deposition to increase catalyst efficiency to meet the DOE's targets.

Accomplishments - A supercritical fluid deposition method for Pt deposition to realize good deposition quality, with ability to tailor Pt loading and distribution along the thickness of buckypaper was developed. A Pt utilization of as low as $0.125 \mathrm{~g} / \mathrm{kW}$ was achieved; however, due to the low loading of Pt in the electrode, the power densities are $400 \mathrm{~mW} / \mathrm{cm}^{2}$ and $830 \mathrm{~mW} / \mathrm{cm}^{2}$ at voltage of $0.65 \mathrm{~V}$ and at peak power, respectively.

Task 4 - Fabrication and characterization of $16-\mathbf{c m}^{2}$ MEAs. The research team will fabricate $4 \times 4 \mathrm{~cm}^{2}$ MEAs by hot press where Pt deposited buckypaper served as the cathode catalyst layer and commercial $\mathrm{Pt} / \mathrm{C}$ as anode catalyst. We will systemically study the effects of electrode material and microstructure on the performance and durability of resultant fuel cells in order to achieve an optimized buckypaper structure to meet or exceed the major 2015 DOE targets for electrocatalyst and MEAs including the Pt utilization better than $0.2 \mathrm{~g}_{\mathrm{P}} / \mathrm{kW}$, power density greater than $1,000 \mathrm{~mW} / \mathrm{cm}^{2}$ at rated voltage $0.65 \mathrm{~V}$, and durability with cycling better than 3,000 hours.

Accomplishments - The team further optimized the porosity gradient by reducing the thickness of the multi-wall carbon nanotubes (MWNTs) layer in buckypapers as the support for the catalytic electrode. By doing that, the power density has been significantly increased. This work successfully developed high quality MEA at a size of $50 \mathrm{~cm}^{2}$ at power density greater than $1.5 \mathrm{~W} / \mathrm{cm}^{2}$ with $\mathrm{O}_{2}$ and $0.75 \mathrm{~W} / \mathrm{cm}^{2}$ with air and $\mathrm{Pt}$ utilization of $0.17 \mathrm{~g} / \mathrm{kW}\left(\mathrm{O}_{2}\right)$ and $0.33 \mathrm{~g} / \mathrm{kW}$ (air).

Task 5 - Assemble and test a short stack. Collaborating with BEI, the FSU team will demonstrate a 5cell short stack based on the optimized single cells with buckypaper supported catalyst. Bing Energy will conduct the stack design, modeling, fixture, and tooling to accomplish the manufacture of MEA, components, and stack. We will test the performance and durability of the stack per DOE's testing protocols to optimize the stack design.

Accomplishments - FSU worked with BEI on the optimized single cells with buckypaper supported catalyst. Bing Energy has focused on the stack design, modeling, fixture, and tooling to accomplish the manufacture of MEA, components, and stack. FSU has tested the performance and durability of the single cell per DOE's testing protocols to optimize the design. The table below shows 2017 DOE Targets and Current Results Achieved in FSU for Electrocatalyst and MEAs

\begin{tabular}{|l|l|l|l|}
\hline Characteristic & Units & $\begin{array}{l}\text { DOE 2017 } \\
\text { Targets }\end{array}$ & $\begin{array}{l}\text { 2011 MEA Status } \\
\text { at BEI }\end{array}$ \\
\hline $\begin{array}{l}\text { PGM total content (both } \\
\text { electrodes) }\end{array}$ & $\mathrm{g} / \mathrm{kW}$ (rated) & 0.125 & 0.34 \\
\hline PGM total loading & $\begin{array}{l}\mathrm{mg} \text { PGM } / \mathrm{cm}^{2} \text { electrode } \\
\text { area }\end{array}$ & 0.125 & 0.25 \\
\hline $\begin{array}{l}\text { Performance @ rated power } \\
\text { (MEA) }\end{array}$ & $\mathrm{mW} / \mathrm{cm}^{2}$ & 1,000 & 720 \\
\hline Mass activity & $\mathrm{A} / \mathrm{mg}^{2} \mathrm{Pt}$, 900 $\mathrm{mV}_{\text {iR-free }}$ & 0.44 & 0.07 \\
\hline Specific activity & $\mu \mathrm{A} / \mathrm{cm}^{2} @ 900 \mathrm{mV}_{\text {iR-free }}$ & 720 & 230 \\
\hline Electrochemical area loss & $\%$ after 30K cycles & $<40$ & 24 \\
\hline Mass activity loss & $\%$ after 30K cycles & $<60$ & 33 \\
\hline Electrochemical area loss & $\%$ after 400 hours @1.2V & $<40$ & 33 \\
\hline Mass activity loss & $\%$ after 400 hours @1.2V & $<60$ & 45 \\
\hline
\end{tabular}


Task 6 - Develop and demonstrate product prototype. BEI will conduct the analysis of prototype development and commercialization feasibility study. At end of the project, we will deliver $500 \mathrm{~W}$ stack prototype based on the optimized Pt/buckypaper electrode. The cost of assembly shall meet or exceed DOE's 2015 targets. The cost/performance of the prototype will be systematically analyzed based on current market status and future market trends. This program establishes a team to make MEAs and stack products to meet DOE goals.

Accomplishments - FSU and Bing developed an 11 cell stack. The MEA size of each cell is $50 \mathrm{~cm}^{2}$. The Pt loading is $0.2 \mathrm{mg} / \mathrm{cm}^{2}$ and $0.05 \mathrm{mg} / \mathrm{cm}^{2}$ at cathode and anode, respectively. The fuel cell stack was characterized using hydrogen at anode and oxygen or air at cathode. The rated powers $(0.65 \mathrm{~V} / \mathrm{cell})$ of 670 and $358 \mathrm{~W}$ were obtained when oxygen and air were used, respectively, which correspond to power densities of 1.22 and $0.65 \mathrm{~W} / \mathrm{cm}^{2}$.

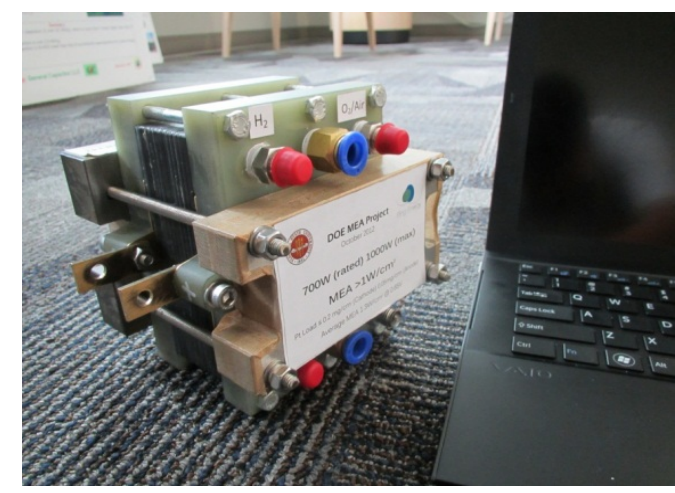

\section{Project Results}

\section{Preparation and Characterization of Double-Layered Buckypaper}

A double-layered buckypaper was prepared by filtering the single SWNT/CNF mixture and the CNF suspension sequentially under full vacuum. SWNTs of 0.8-1.2 nm in diameter and 100-1000 nm in length were purchased from Carbon Nanotechnologies, Inc and CNFs of 100-200 nm in diameter and 30-100 $\mu \mathrm{m}$ long from Applied Sciences, Inc. All materials were used as received without further purification. The mono-layered buckypapers were prepared in the same way by filtrating only one type of suspension. The surface analyses were performed on the mono-layered buckypaper. A Tristar 3000 and AutoPore 9520 (Micromeritics) were used to characterize the Brunauer-Emmett-Teller (BET) surface areas and pore sizes of the buckypapers.

\section{Preparation and Characterization of Buckypaper-Supported Pt Electrocatalysts}

Pt nanoparticles were deposited onto the buckypaper using a pulse electrodeposition technique from a mixture solution of $10 \mathrm{mM} \mathrm{H}_{2} \mathrm{PtCl}_{6}, 0.1 \mathrm{M} \mathrm{H}_{2} \mathrm{SO}_{4}$, and $0.5 \mathrm{M}$ ethylene glycol with $\mathrm{N}_{2}$ bubbling. The blank buckypaper working electrode was loaded on a locally produced sample holder coupled with a hydrophobic carbon fiber paper as a current collector. A saturated calomel electrode (SCE) was used as the reference electrode and Pt gauze as the counter electrode. The electrodeposited side of the buckypaper was $5 \mathrm{~cm}^{2}$ placed on the window side of the sample holder exposed to the electrolyte. The applied potential increased from $0.3 \mathrm{~V}$ to $-0.35 \mathrm{~V}$ (vs. SCE) with a pulse width of $4 \mathrm{~s}$ and a pulse duty cycle of $25 \%$. The pulse was repeated until it reached the desired Pt loading, which was determined by weighing the mass difference before and after the deposition. The Pt loading was also confirmed by the inductively coupled plasma mass spectrometry (ICP-MS) analysis. 
The surface and cross-section morphology of the Pt/DLBP was characterized using a scanning electron microscope (SEM, JEOL JSM 7401F). The cross-section sample was prepared by a triple ion-beam miller (Leica EM TIC020). The elemental mapping of platinum was performed on the cross-section of Pt/DLBP by an energy-dispersive X-ray spectrometer (EDS) attached to the JSM 7401F microscope. The detailed morphologies of $\mathrm{Pt}$ were characterized using a transmission electron microscope (TEM, JEM-2010, JEOL). The Pt size distributions were obtained from the TEM image by analyzing 150 randomly selected Pt particles.

The electrochemical surface area of Pt/DLBP catalysts was characterized by using cyclic voltammetry $(\mathrm{CV})$ in a three-electrode/one-compartment cell. To prepare the working electrode, a piece of Pt/DLBP was attached to the top of a glassy carbon (GC) electrode $\left(0.196 \mathrm{~cm}^{2}\right)$ using a drop of $0.5 \%$ Nafion solution. The electrolyte solution was $0.5 \mathrm{M} \mathrm{H}_{2} \mathrm{SO}_{4}$, which was thoroughly deaerated by bubbling $\mathrm{N}_{2}$ gas for 30 minutes. $\mathrm{A} \mathrm{N}_{2}$ atmosphere was maintained over the solution throughout the test. The potential ranged from $-0.25 \mathrm{~V}$ to $+1.1 \mathrm{~V}$ (vs. SCE) at a scan rate of $50 \mathrm{mV} / \mathrm{s}$. Oxygen reduction reaction (ORR) activities of Pt/DLBP were measured by a rotating disk electrode (RDE) in $\mathrm{O}_{2}$-saturated $0.1 \mathrm{M} \mathrm{HClO}_{4}$ at room temperature. The linear voltammograms were recorded in the range of $0-0.75 \mathrm{~V}$ (vs. SCE) at a scan rate of $5 \mathrm{mV} / \mathrm{s}$ with the rotation speed between 400 and $1600 \mathrm{rpm}$.

\section{Fabrication and Characterization of MEA}

A two-layer structure was used as the gas diffusion layer (GDL) at both cathode and anode sides: the outer layers were teflonized (30 wt.\% Teflon in cathode, $10 \mathrm{wt} . \%$ in anode) carbon paper (TGPH-090, Toray), and inner layers (between the carbon paper and catalyst layer) were prepared by spraying an isopropanol mixture of the carbon black (Vulcan XC-72, Cabot) and a $30 \mathrm{wt} . \%$ or $10 \mathrm{wt} . \%$ Teflon emulsion (Aldrich) onto the carbon paper, which was then sintered at $340^{\circ} \mathrm{C}$ for 1 hour. The anode catalyst layer was prepared by a conventional ink process. The proper amount of the $\mathrm{Pt} / \mathrm{C}$ catalyst $(20 \% \mathrm{Pt}$ on Vulcan XC-72, E-Tek) was mixed with $10 \mathrm{wt} . \%$ Nafion in isopropanol and then air-sprayed onto the inter GDL to constitute the anode catalyst layer with a Pt loading of $0.05 \mathrm{mg} / \mathrm{cm}^{2}$. A thin layer of Nafion solution $\left(0.5 \mathrm{mg} / \mathrm{cm}^{2}\right)$ was then sprayed onto the surface of the anode catalyst layer. Pt/buckypaper was impregnated with 5\% Nafion solution (Aldrich) under a vacuum. After drying at $80^{\circ} \mathrm{C}$, it was placed on the cathode GDL to serve as a catalyst layer where the selected side of the buckypaper was exposed. The membrane electrode assembly was formed by sandwiching the electrolyte membrane (Nafion NRE211, Dupont) between the anode and cathode and hot-pressing the assembly at $130^{\circ} \mathrm{C}$ for 3 minutes under 30 $\mathrm{kg} / \mathrm{cm}^{2}$ of pressure. The MEA was operated by a fuel cell testing system (Fuel Cell Technologies) with humidified $\mathrm{H}_{2}$ as the fuel and humidified $\mathrm{O}_{2}$ or air as the oxidant. The cell temperature was $80^{\circ} \mathrm{C}$, the relative humidity $(\mathrm{RH})$ is $100 \%$ for both anode and cathode and the back pressures were 20 psi on both sides of the cell. The flow rates were set at a stoichiometric of 2 for hydrogen and 2 for oxygen or 3 for air. The cell performance was recorded by electronic load assembled in the testing system. The electrochemical impedance spectra (EIS) for the cathodic reactions were measured using a Solartron 1280B electrochemical workstation (Solartron) in the frequency range from $0.1 \mathrm{~Hz}$ to $10 \mathrm{kHz}$ in galvanostatic mode. The anode was used as the reference electrode.

\section{$\underline{\text { 4. Durability test of catalyst support }}$}

The accelerated stress test of Pt/DLBP was performed in an MEA at $95^{\circ} \mathrm{C}$ by feeding hydrogen and nitrogen in the anode and cathode respectively. The relative humidity of gases was set to $80 \%$ and back pressures were kept at $20 \mathrm{psi}$ on both sides of the cell. The cell voltage was held at $1.2 \mathrm{~V}$ for 200 hours. The polarization curves were recorded every 24 hours at $80{ }^{\circ} \mathrm{C}$ per protocol listed in the previous section. 

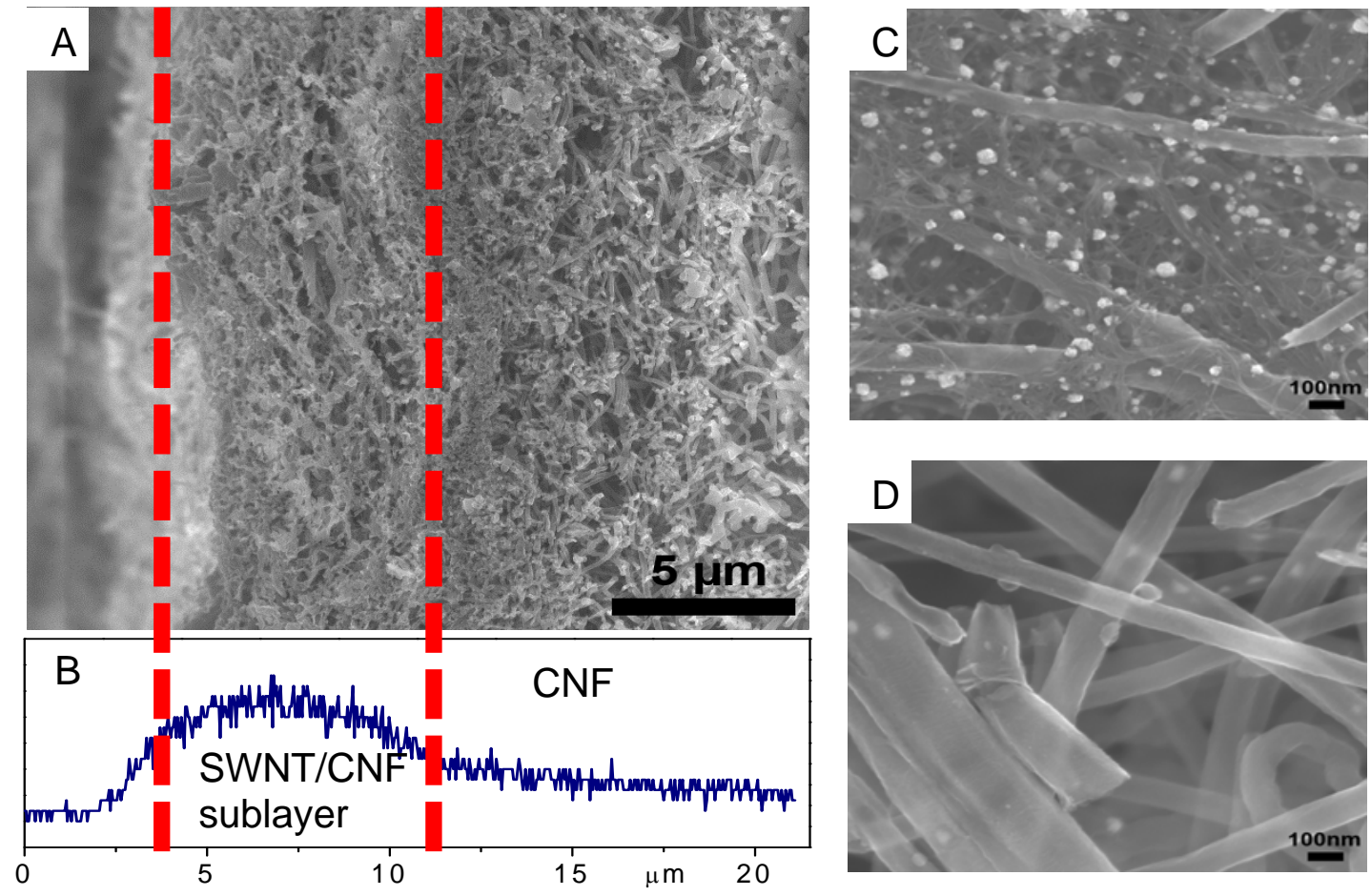

Figure 1. A) SEM image of cross-section of Pt/DLBP buckypaper, which was etched by ion beams; the left side of buckypaper in the image is SWNT/CNF sublayer and the right side is CNF sublayer; B) Pt element distribution along the cross-section of Pt/DLBP obtained by EDS analysis; SEM image of surfaces of C) SWNT/CNF sublayer and D) CNF sublayer.

As shown in Fig. 1A, large-sized CNFs entangled randomly forming a highly porous sublayer with a porosity of $90.8 \%$ and an average pore size of $85 \mathrm{~nm}$, while fewer and smaller pores were formed in the SWNT/CNF sublayer by adding 25 wt. \% smaller SWNTs. As a result, the SWNT/CNF sublayer had a much larger specific surface area $\left(105 \mathrm{~m}^{2} / \mathrm{g}\right)$ than the CNF sublayer $\left(24 \mathrm{~m}^{2} / \mathrm{g}\right)$ because of the higher specific surface area of SWNT ( 400 $\left.\mathrm{m}^{2} / \mathrm{g}\right)$. After depositing Pt on the buckypaper by electrochemical deposition, an EDS analysis showed an obvious gradient distribution of $\mathrm{Pt}$ (Fig. 2B); over $70 \% \mathrm{Pt}$ was distributed in the 7-micron-thick SWNT/CNF sublayer. A large amount of Pt deposited on the surface of the SWNT/CNF sublayer (Fig. 1C), while only a small amount deposited on the surface of the CNF sublayer (Fig. 1D). This could provide an indication of how Pt was distributed inside the buckypaper since the Pt distribution was quite uniform within each sublayer. The pictures of surface morphology showed the Pt preferred to grow on the surface of SWNT instead of CNF. More detail could be seen in TEM image of Pt/DLBP catalyst as shown in Fig. 2A. Although, the mechanism of selective deposition of Pt on SWNT/CNF buckypaper is not fully understood, the most possible reasons are: 1) SWNT has much higher surface area and more surface defects resulting in more anchor sites for Pt nucleation; and 2) a higher deposition current on SWNTs because of their higher conductivity. 

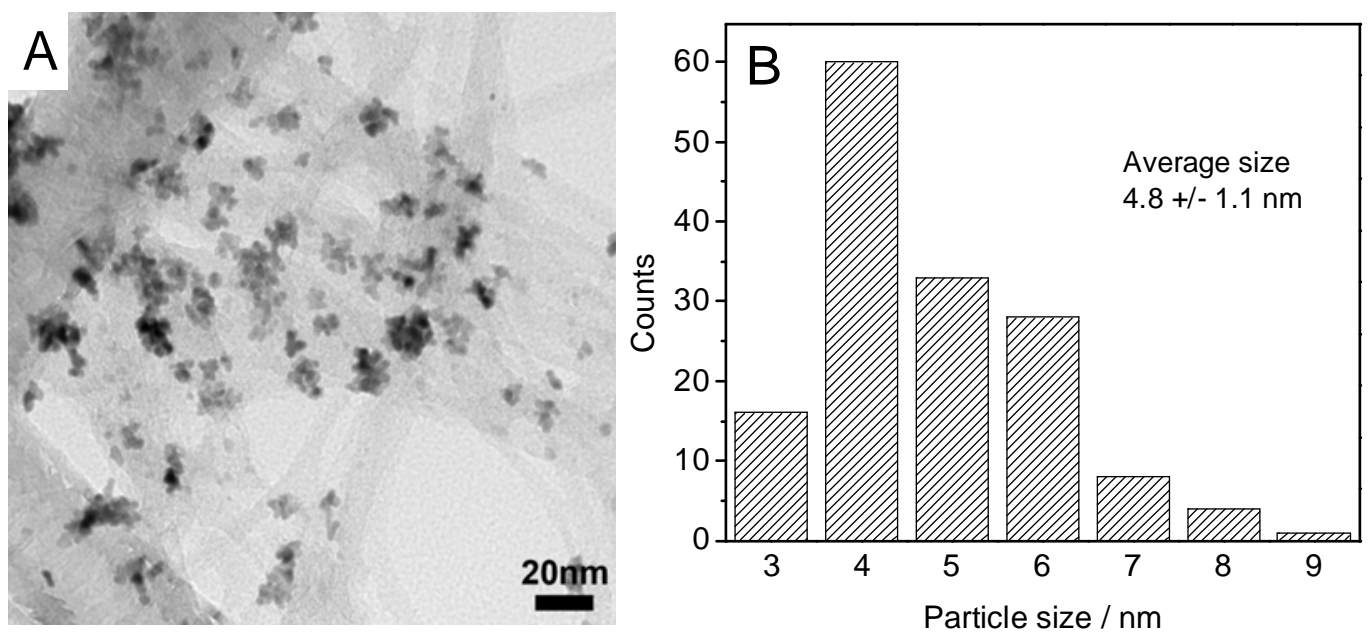

Figure 2. A) TEM image of Pt/DLBP; B) Pt particle size distribution in Pt/DLBP determined by the image analysis of 150 randomly selected particles in the TEM picture.
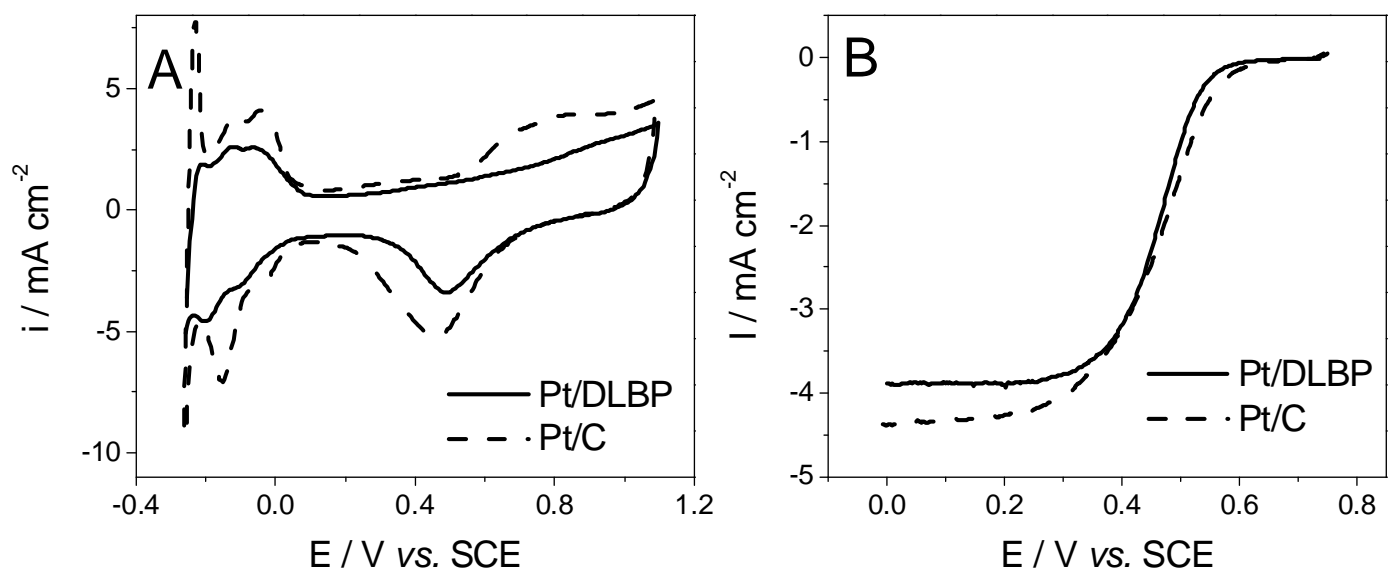

Figure 3. Comparison of the electrochemical characteristics of Pt/DLBP and the commercial $20 \% \mathrm{Pt} / \mathrm{C}$ (E-Tek). The Pt loading is $0.1 \mathrm{mg} / \mathrm{cm}^{2}$ for each sample. A) Cyclic voltammograms obtained in $\mathrm{N}_{2^{-}}$ saturated $0.5 \mathrm{M} \mathrm{H}_{2} \mathrm{SO}_{4}$ at a scan rate of $50 \mathrm{mV} / \mathrm{s}$; B) Potentiodynamic oxygen reduction current densities obtained in $0.1 \mathrm{M}$ oxygen-saturated $\mathrm{HClO}_{4}$ at room temperature. The data were obtained at a scan rate of $5 \mathrm{mV} / \mathrm{s}$ and a rotation speed of $1600 \mathrm{rpm}$.

The Pt size distribution obtained from the image analysis of 150 randomly selected Pt particles from TEM image is shown in Fig. 2B. The average Pt particle size is $4.8 \mathrm{~nm}$ with a relative wide range due to the agglomeration of Pt nanoparticles. The electrochemical surface area (ECSA) of Pt/DLBP was obtained by the integration of the $\mathrm{H}_{2}$-adsorption peak in the hydrogen region $(-0.2$ to $0.15 \mathrm{~V}$ vs. SCE) of the cyclic voltammogram (Fig. 3A) with subtraction of the current due to double layer charging. The calculated ECSA for the Pt/DLBP electrocatalyst was $56.0 \mathrm{~m}^{2} / \mathrm{g}$, which is less than that achieved in the commercial $\mathrm{Pt} / \mathrm{C}\left(70.1 \mathrm{~m}^{2} / \mathrm{g}\right)$ due to the relatively large Pt particles in the Pt/DLBP compared with average Pt size of $2.2 \mathrm{~nm}$ in Pt/C. Accordingly, Pt/DLBP catalyst showed smaller limit current than that of Pt/C (Fig, 3B) in the RDE measurement indicating less catalytic activity towards ORR. However, the catalyst utilization in the Pt/DLBP electrode was relatively high considering such large Pt particle size. 
Before applied to the MEA, Pt/buckypaper was impregnated with 5\% Nafion solution under a vacuum followed by drying at $80^{\circ} \mathrm{C}$ to introduce the proton-conducting phase. Since the Nafion loading is estimated as $\sim 0.2 \mathrm{~g} / \mathrm{cm}^{3}$ in the double-layered buckypaper and $0.29 \mathrm{~g} / \mathrm{cm}^{3}$ in a single-layered SWNT/CNF (1:3 wt. /wt.) buckypaper under the same preparation conditions, we believed a gradient distribution of Nafion occurred along the buckypaper's thickness. More Nafion distributed in the SWNT/CNF layer may be due to the smaller pores facilitating the adsorption of Nafion solution by the effect of capillary force.

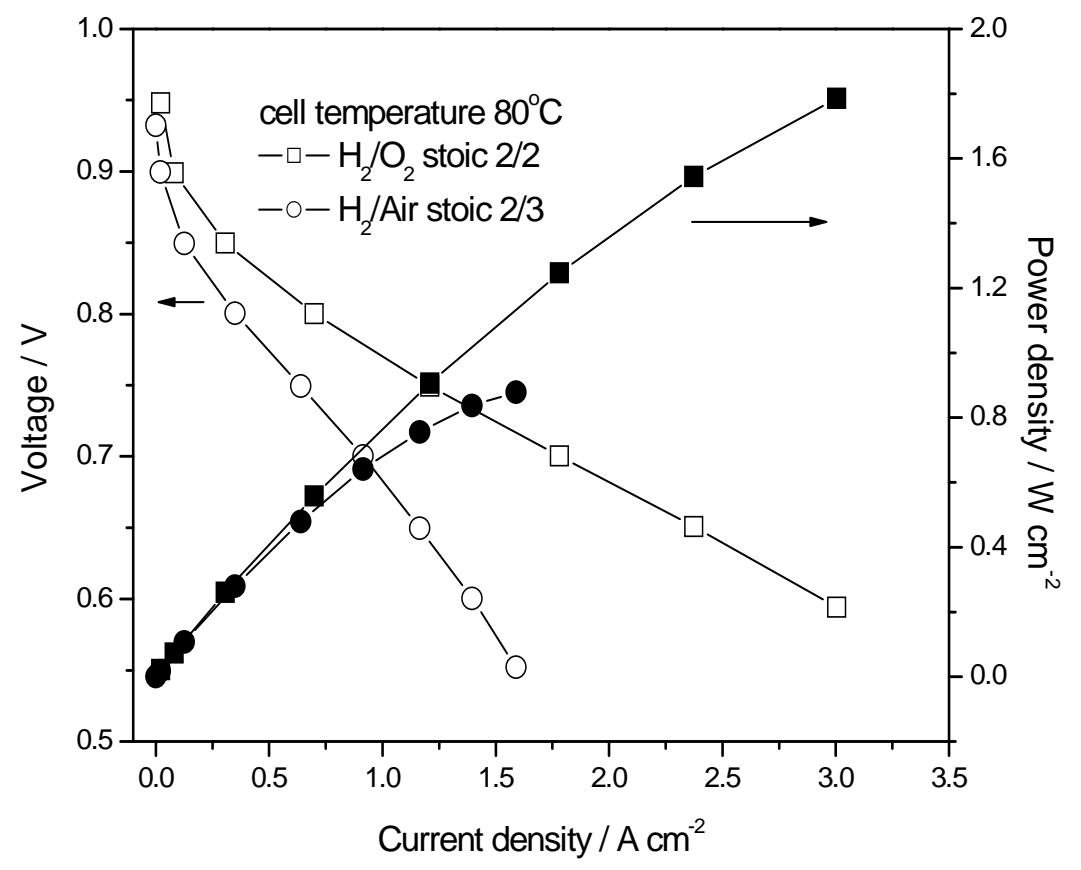

Figure 4. Cell potentials and power densities as a function of current density for the $50 \mathrm{~cm}^{2}$ MEAs with fully humidified oxygen and air at a back pressure of 20 psi.

By applying this double-layered buckypaper as a cathode catalyst layer, the MEA exhibited excellent power performance with a relative low Pt loading. As shown in Figure 4 rated power of $1.54 \mathrm{~W} / \mathrm{cm}^{2}$ and $0.76 \mathrm{~W} / \mathrm{cm}^{2}$ (at $0.65 \mathrm{~V}$ ) were achieved in oxygen and air, respectively. The cathode $\mathrm{Pt}$ loading is 0.2 $\mathrm{mg} / \mathrm{cm}^{2}$ resulting in a total Pt utilization of $0.33 \mathrm{~g}_{\mathrm{Pt}} / \mathrm{kW}$ (cathode and anode) for air. Considering the fact that ORR activity showed negligible improvement by using Pt/double-layered buckypaper, such high cell performance is believed to benefit from the optimized microstructure of a catalyst layer. The gradient distribution of $\mathrm{Pt}$ is considered a key issue for achieving the relatively high mass activity in Pt/DLBP since most of Pt located within a thin layer close to the membrane greatly reduces the possibility of Pt being out of reach by the protons. Therefore, the transport limitations of protons and reactants in the Pt/DLBP catalyst layers were greatly improved by the optimized microstructure with the functional grade resulting in remarkable catalyst efficiency. 


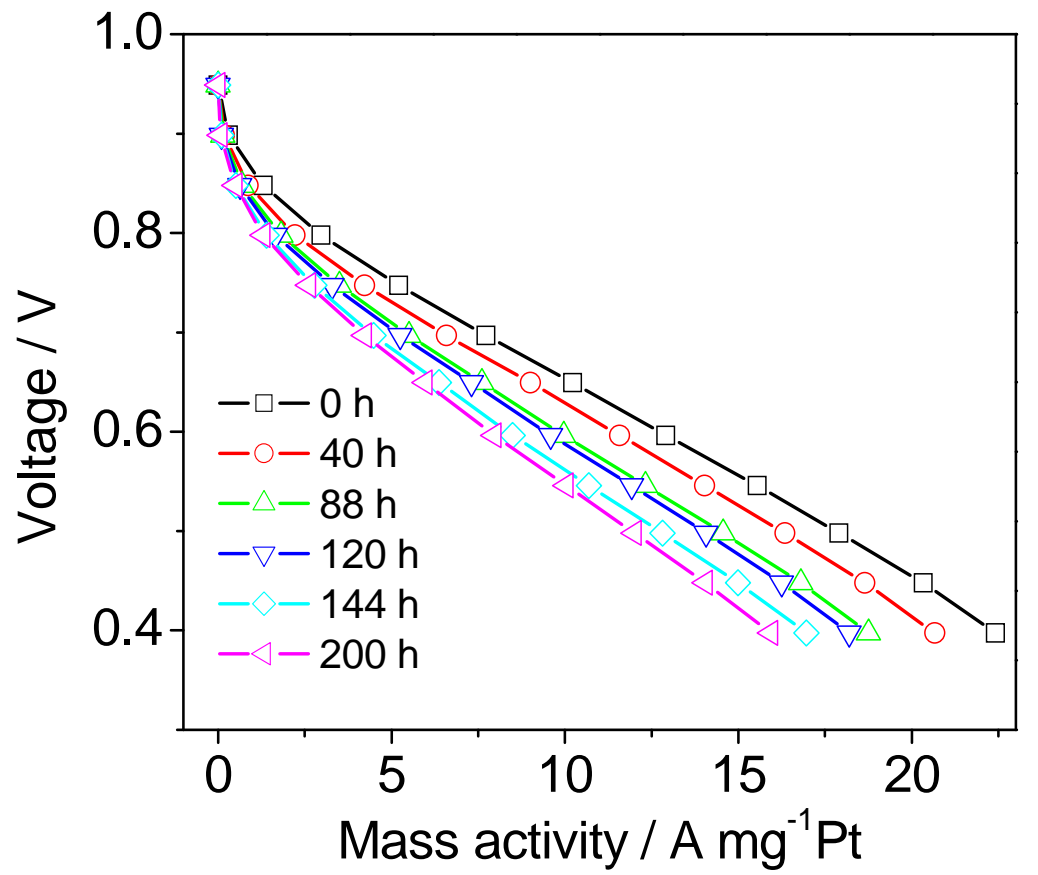

Figure 5. Impact of accelerated carbon support corrosion on the cell performance of a MEA with Pt/DLBP cathode catalyst layer.

The SWNT/CNF buckypaper-based Pt catalyst has already shown good durability under an accelerated degradation test in a mimic cathode environment in our previous study (14). This was due to the high corrosion resistance of CNF and its high graphitization degree. Hence, we evaluated the durability of catalyst support for Pt/DLBP in a MEA per DOE's test protocol (3). Figure 5 shows the polarization curves at different time intervals during the 200-hour durability test. The mass activity measured at 900 $\mathrm{mV}$ lost only $57.6 \%$ of initial activity after a 200 -hour operation, which is much better than that obtained in conventional $\mathrm{Pt} / \mathrm{C}$ (90\% loss of initial activity) and approaching the DOE's 2015 target ( $\leq 60 \%$ loss of initial activity). The result indicates that the DLBP is potentially a good candidate for the catalyst support to achieve a highly stable electrode although some characteristics are still under the DOE's requirement, e.g., the loss of cell voltage at $1.5 \mathrm{~A} / \mathrm{cm}^{2}$ after 120 -hour test is $60 \mathrm{mV}$ while DOE targets a $30 \mathrm{mV}$ of voltage loss after 100-hour operation.

\section{Concluding Remarks}

The Pt/double-layered buckypaper (DLBP) with tailored gradient structure demonstrated promising Pt utilization and stability of supports in spite of a relatively large Pt particle size. By applying this Pt/DLBP as a cathode catalyst layer, the MEA exhibited excellent power performance with a relative low $\mathrm{Pt}$ loading. The rated powers of $1.5 \mathrm{~W} / \mathrm{cm}^{2}$ and $0.8 \mathrm{~W} / \mathrm{cm}^{2}$ at $0.65 \mathrm{~V}$ were achieved with a cathode $\mathrm{Pt}$ loading of $0.2 \mathrm{mg}_{\mathrm{Pt}} / \mathrm{cm}^{2}$ for oxygen and air, respectively. The Pt utilization was better than the current state-of-the-art value $\left(>0.4 \mathrm{mg}_{\mathrm{P}} / \mathrm{cm}^{2}\right)$ achieved by the conventional $\mathrm{Pt} / \mathrm{C}$ catalyst at a comparable cell $\left(0.8-1.0 \mathrm{~W} / \mathrm{cm}^{2}\right)$ output. The research evaluated the durability of catalyst support for Pt/DLBP in a MEA according to DOE's suggested protocol. The SWNT/CNF buckypaper-based Pt catalyst has shown good durability under an accelerated degradation test in a mimic cathode environment. This was due to the high corrosion resistance of CNF and its high graphitization degree. FSU and Bing also developed an 11 cell 
stack. The MEA size of each cell is $50 \mathrm{~cm}^{2}$. The Pt loading is $0.2 \mathrm{mg} / \mathrm{cm}^{2}$ and $0.05 \mathrm{mg} / \mathrm{cm}^{2}$ at cathode and anode, respectively. The fuel cell stack was characterized using hydrogen at anode and oxygen or air at cathode. The rated powers $(0.65 \mathrm{~V} /$ cell $)$ of 670 and $358 \mathrm{~W}$ were obtained when oxygen and air were used, respectively.

Future work should focus on (1) improving catalytic activity towards ORR by reducing Pt particle size or replacing Pt with Pt-alloy; (2) further optimizing the microstructure; and (3) improving the stability of buckypaper by surface modification.

\section{Publications}

1. W. Zhu, C. Zeng, J.P. Zheng, R. Liang, C. Zhang, and B. Wang, "Preparation of Buckypaper Supported Pt Catalyst for PEMFC Using Supercritical Fluid Method", Electrochem. and Solid-State Lett. 14, B81 (2011).

2. W. Zhu, R. Liang, and J.P. Zheng, "A 3-D Catalytic Electrode Structure for Ultra-low Platinum Loading and High Performance PEMFCs", accepted by ECS Transactions.

\section{Presentations}

1. W. Zhu and J.P. Zheng, "Ultra-Low Platinum Loading High-Performance PEMFCs Using Buckypaper-Supported Electrodes”, Small Fuel Cells 2011, Boston MA, Jun 9-10, 2011.

2. J.P. Zheng, W. Zhu, R. Liang, C. Zhang, and B. Wang, "A 3-D Catalytic Electrode Structure for Ultra-low Platinum Loading and High Performance PEMFCs", The 2012 Villa Conference on Energy and and Environmental Research, Olando, FL, April 16-20, 2012.

3. J.P. Zheng, W. Zhu, R. Liang, C. Zhang, and B. Wang, "A 3-D Catalytic Electrode Structure for High Performance and Los Cost PEMFCs", the 243rd American Chemical Society National Meeting, San Diego, California, March 27, 2012.

4. J.P. Zheng, "Transformation of University Research to Commercialization", Florida Energy Summit Orlando, Florida, August 17, 2012. (Panel Speaker)

5. J.P. Zheng, W.J. Cao, G.Q. Zhang, W. Zhu, P. Andrei, R1 Liang, and M. Zhang, "Carbon Nanotube composite electrodes for energy storage devices and fuel cells", IUPAC $8^{\text {th }}$ International Conference on Novel Materials and Synthesis (NMS-VIII) \& $22^{\text {nd }}$ International Symposium on Fine Chemistry and Functional Polymers (FCFP-XXII), Xi'An, China, October 16, 2012, (Keynote Speaker).

\section{Patents}

1. J.P. Zheng, W. Zhu, C. Zeng, Z.Y. Liang, B. Wang, C. Zhang, and W. Zhu, "A Method of making catalytic electrodes for fuel cells", U.S. Provisional Patent Application was filed. 


\title{
FLORIDA HYDROGEN IN|TIATIVE Sub-contrecker Fincl Report \\ Develop an Interdisciplinary Hydrogen and Fuel Cell Technology Academic Program
}

\author{
Dr. Mary Helen McCay and Dr. Kurt Winkelmann \\ Florida Institute of Technology \\ College of Engineering \\ mmccay@fit.edu \\ (321) 674-8803
}

\author{
Submitted to: \\ David L. Block \\ Florida Solar Energy Center/University of Central Florida \\ 1679 Clearlake Road \\ Cocoa, FL 32922 \\ E-mail: block@fsec.ucf.edu
}

DOE Contract Number: DE-FC36-04GO14225

UCF Account No. 20126050 (PO No. 214261)

Project Start Date: December 2010

Project End Date: March 2013

March 2013 


\title{
Develop an Interdisciplinary Hydrogen and Fuel Cell Technology Academic Program
}

\author{
Florida Institute of Technology \\ College of Engineering
}

\section{Executive Summary}

For hydrogen and fuel cells to become viable, an interdisciplinary approach to curriculum development is needed. This project provides public and private sectors with skilled employees and researchers in hydrogen technology by: developing undergraduate modules, general chemistry laboratory experiments, and a graduate area of specialization academic program; growing research and development in the arena of hydrogen and fuel cell technology; applying assessment tools to determine the effectiveness of the laboratory curriculum.

\section{Task 1.0 Develop Master's Degree Area of Specialization under Existing MS Programs in Hydrogen and Fuel Cell Technologies.}

The Master of Science with an area of specialization in Hydrogen or Fuel Cell Technology (HFCT) requires a bachelor of science or the equivalent in engineering, applied mathematics, chemistry or physics for entrance.

A list of core courses was developed that comprise the academic elements necessary for this area of specialization. The student, in consultation with his or her advisor, selects three of the courses which constitute required courses for the specialization (typically comprising nine credit hours). Six credit hours are required of any 5000-level courses with a MTH prefix along with nine credit hours of elective courses at the 5000- or 6000-level with an MAE (for the Mechanical Engineering specialization) of CHE prefix (for the Chemical Engineering specialization). Courses from other departments may be used with department head approval. The remaining program requirements are met with thesis, six credit hours (minimum) of XXX5999 (depending on the department). Requirements for the non-thesis option are the same as for the thesis option except the thesis credits are replaced with two additional elective courses for a total of 15 elective credits.

New graduate courses were developed. CHE 5250: Hydrogen Technology Presents the fundamental knowledge of hydrogen and the potential future development of hydrogen science and technology. Investigates the use of hydrogen as a fuel, its properties, production and storage. Discusses hydrogen technology applications; MAE 5330: Principles of Fuel Cells

The program specializations and new courses were approved by the Florida Tech College of Engineering Council and the Florida Tech Graduate Council. Changes were made to the School Catalog to reflect the new specializations and to include the new courses.

\section{Task 2.0 Develop Modules for Core and Elective Courses.}

This project identified and developed modules to bring hydrogen and fuel cell technology into the traditional mechanical engineering and chemistry engineering undergraduate curriculum. Following module development, a select number of undergraduate engineering and sciences courses were identified to include the developed hydrogen and fuel cell related modules. 
Undergraduate courses which included hydrogen modules are: Engineering Thermo-dynamics; MAE 3191, Introduction to Sustainability, ISC 1500; Applied Sustainability, ISC 4000; Introduction to Electrical Power Systems, ECE 4861; Power System Operation, ECE 5683; Physical Chemistry Laboratory I, CHM 3011; Introduction to Chemical Engineering, CHE 1101; Materials Science and Engineering, CHE 3260; Nanotechnology, CHE 5567/4592 and the Materials Characterization Lab.

\section{Task 3.0 Senior Design Students.}

Graduating seniors in the fields of engineering and science are required to undertake a capstone project which may take the form of a design or research project. These are guided by the relevant faculty. Students prepare and exhibit these capstones at the end of the spring term along with a written report.

One project is the Metal Hydride Hydrogen/Thermal Energy Storage Senior Design Project. Its objective is to design, build, and investigate a metal hydride hydrogen/ thermal energy storage system. A graduate level project titled Hydrogen-based Residential Emergency Power Supply Capstone Project was undertaken with the objective being to develop final cost estimates for a hydrogen based residential emergency power supply.

\section{Task 4.0 Develop Laboratory Experiments for General Chemistry Courses.}

This task is unique within the DOE education program. Dr. Winkelmann implemented hydrogen-themed, laboratory experiments within the General Chemistry II lab curriculum in order to:1) implement a set of general chemistry laboratory experiments that focus on issues related to hydrogen fuel research, and 2) measure students' gains in knowledge of hydrogen as a fuel source and their interest in and appreciation of chemistry as a subject. Students began performing these experiments in spring 2012 and will continue to perform them for the foreseeable future. In spring 2013, Dr. Winkelmann created video tutorials were implemented to help students understand the laboratory experiment procedures.

Students completed an attitudinal survey created at Florida Tech and the U.S. Department of Energy's Hydrogen Knowledge and Opinion Survey (HKOS). These surveys measured students' attitudes about chemistry and their understanding of issues related to hydrogen fuel technology. Students enrolled in General Chemistry II lab completed both surveys at the beginning and end of each semester of 2011 and 2012. An analysis of the surveys shows no change in students' attitudes about chemistry lab before or after adding the new hydrogen-themed experiments. This result was disappointing since it was hoped that the hydrogen-themed experiments would spark students' interest in chemistry. Students were more favorably inclined towards hydrogen technology and demonstrated more knowledge of hydrogen after completing the new experiments. This result shows that students can not only learn about chemistry topics in their lab course but can also learn about other important issues, such as hydrogen fuel technology.

Dr. Winkelmann supported two students to perform an extensive web and literature search to find other sources of hydrogen curriculum materials which other schools have created. The purpose of this search is to keep up to date with the growth of hydrogen technology in science and engineering education. The web and literature search were completed in December 2012. 


\section{Develop an Interdisciplinary Hydrogen and \\ Fuel Cell Technology Academic Program}

\section{Table of Contents}

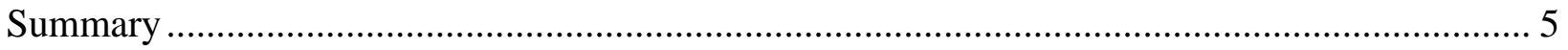

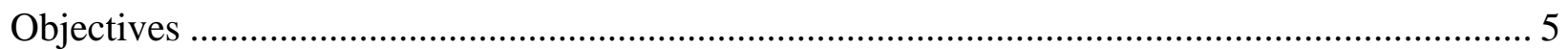

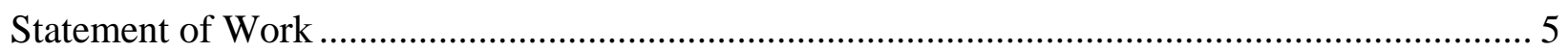

Task 1.0 Develop Masters Degree Area of Specialization Under Existing MS Programs in Hydrogen and Fuel Cell Technologies. ...................................................................................... 5

Task 2.0 Develop Modules for Core and Elective Undergraduate Engineering and Science

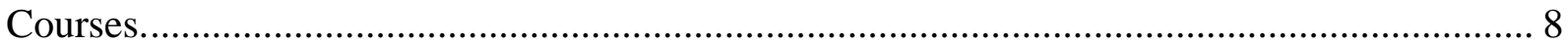

Task 3.0 Senior Design Students. ........................................................................................ 9

Task 4.0 Develop Inquiry Based Laboratory Experiments for General Chemistry Courses.... 11

Task 5.0 Project Management and Reporting........................................................................... 12

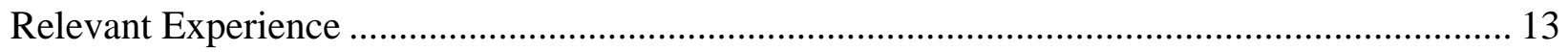




\title{
Develop an Interdisciplinary Hydrogen and Fuel Cell Technology Academic Program
}

\author{
Florida Institute of Technology \\ College of Engineering
}

\begin{abstract}
Summary
This project will provide public and private sectors with skilled employees and researchers in hydrogen technology. The development of undergraduate modules, enquiry-based laboratory experiments, and a graduate area of specialization academic program will enable the growth of research and development in the arena of hydrogen and fuel cell technology. Assessment tools are also included that determine the effectiveness of the laboratory curriculum.
\end{abstract}

\section{Objectives}

For hydrogen and fuel cells to become viable, an interdisciplinary approach to curriculum development is needed. In other words, new curricula which cross the standard academic lines must be developed and implemented - quickly - if both our academic institutions and our industrial/research organizations are to be prepared for the future hydrogen economy.

The program described in this final report enables students to:

1. Follow the technology as it evolves from introduction to long-term applications.

2. Obtain a basic understanding of the fundamentals of the field.

3. Redirect their current technology focus as a means for new career options.

4. Measure students' gains in knowledge of hydrogen as a fuel source.

5. Satisfy the current need for hydrogen technology graduates in government, industry and academia.

With regard to Florida Institute of Technology (FIT), the university program develops a high caliber and national recognized hydrogen and fuel cell academic program that operates at both the undergraduate and graduate levels. The academic program was developed by designing and implementing course modules that were be inserted into existing courses or by developing new courses. The program has become a permanent part of the FIT academic programs.

\section{Statement of Work}

The statement of work (SOW) and tasks for this program are as follows:

Task 1.0 Develop Masters Degree Area of Specialization Under Existing MS Programs in Hydrogen and Fuel Cell Technologies.

The objective is to prepare students for careers in, or conduct research related to, hydrogen and fuel cell technology. Hydrogen is used in many engineering applications including chemical processing, aircraft and rocket propellants, fuel cells, shock tubes and hydrogen vehicles. It is believed by many that hydrogen will be the dominant alternative fuel in the future, with the potential to also be a renewable fuel when produced by energy sources such as wind or solar power, thus limiting the use of fossil fuels. 
The Master of Science with an area of specialization in Hydrogen or Fuel Cell Technology (HFCT) requires a bachelor of science or the equivalent in engineering, applied

mathematics, chemistry or physics for entrance. Additionally, it requires a minimum of 30 credit hours of approved graduate study, nine credit hours of core courses, six credit hours of mathematics and 15 credits of electives (including six credit hours of thesis).

\section{Task 1.1 Development of the Courses and Curriculum.}

A list of core specialization courses was developed that comprise the academic elements necessary for this area of specialization.

- MAE 5130-Viscous Flows

- MAE 5220-Convective Heat Transfer

- MAE 5330-Principles of Fuel Cells (New Course)

- CHE 5250-Hydrogen Technology (New Course)

- CHE 5150-Transport Phenomena 1

- CHE 5567-Nanotechnology

- SYS 5367-Decision and Risk Analysis OR

- SYS 5385-System Life Cycle Cost Estimation

Required texts are left to the discretion of the instructor.

The student, in consultation with his or her advisor, selects three of the above courses which constitute required courses for the specialization (typically comprising nine credit hours). Six credit hours are required of any 5000-level courses with a MTH prefix along with nine credit hours of elective courses at the 5000- or 6000-level with an MAE (for the Mechanical Engineering specialization) of CHE prefix (for the Chemical Engineering specialization). Courses from other departments may be used with department head approval. The remaining program requirements are met with thesis, six credit hours (minimum) of XXX5999. Requirements for the non-thesis option are the same as for the thesis option except the thesis credits are replaced with two additional elective courses for a total of 15 elective credits.

Task 1.2 Plan for Introducing and Implementing the MS Program.

Dr. Archambault prepared the documents to be presented before the Florida Tech Graduate Council to obtain approval for the new area of specialization. The Academic Proposal Segments that were required are:

1. Program Relevance

2. Program Demand

a. What is the market for this program?

b. How many new students are expected to enroll in the first year?

c. What other universities offer this program?

d. Are there current trends or forecasts for interest in this program?

e. Will this program be offered to a non-traditional audience?

f. What are employment opportunities after graduation?

g. If this is an undergraduate program, what are the graduate program opportunities?

h. Is internship part of the program?

3. Competition

a. Do other competitive universities offer this program?

b. How is this program different, if different?

4. Academic Requirements 
5. Financial Resources and Budget Plan

6. Administrative Processes

A copy of the Academic Proposal was provided with an earlier quarterly report.

\section{Task 1.3 Offering of New Courses.}

In this task, new graduate courses were developed. It should be noted that these courses must meet the normal FIT requirements (time, length, number of students, etc. They are:

\section{CHE 5250: Hydrogen Technology}

Presents the fundamental knowledge of hydrogen and the potential future development of hydrogen science and technology. Investigates the use of hydrogen as a fuel, its properties, production and storage. Discusses hydrogen technology applications.

\section{MAE 5330: Principles of Fuel Cells}

Presents the fundamentals of fuel cell technology, including basic operating principles, thermodynamics, reaction kinetics, charge and mass transport, and modeling, with emphasis on hydrogen fuel cells.

Discusses types of fuel cells, fuel cell stacks, thermal management, fuel delivery, and power management.

Course content for MAE 5330 is given below:

MAE 5330: Principles of Fuel Cells, Dr. Mark Archambault

Topics: (75-Minute Lectures)

Introduction 1

Types of Fuel Cells 1

Fuel Cell Thermodynamics 4

Reaction Kinetics 5

Charge and Mass Transport 6

Modeling 4

Fuel Cell Characterization 4

Fuel Cell Systems 3

Examinations 2

Total: 30 lectures

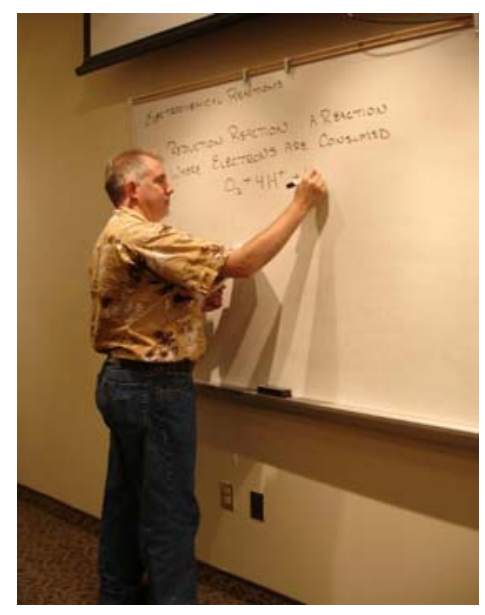


The program specializations and new courses were approved by the Florida Tech College of Engineering Council and the Florida Tech Graduate Council. Changes were made to the School Catalog to reflect the new specializations and to include the new courses.

\section{Task 2.0 Develop Modules for Core and Elective Undergraduate Engineering and Science Courses.}

The goal of this project is to identify and/or develop modules that bring hydrogen and fuel cell technology into the traditional mechanical engineering and chemistry engineering undergraduate curriculum.

Following module development, a select number of undergraduate engineering and sciences courses will be identified to include the developed hydrogen and fuel cell related modules.

Task 2.1 Identification of Modules for Development and Implementation into Existing Courses.

The curriculum files and papers were mainly obtained from the websites of U.S. Department of Energy (DOE), Michigan Technology University (MTU) and UC Berkeley, UC Davis, and American Chemical Society Division of Energy \& Fuels, etc. They were categorized into folders.

\section{- Folder: curriculum modules}

Several curriculum modules merged together to let the faculty have easy access to these modules

- Folder: development and future plan of $\mathrm{H} 2$ and fuel cells Collected strategic plan and report for the research, development, and demonstration of hydrogen and fuel cell technologies

- Folder: lab procedures Notes, introductory and manuals for lab procedure

- Folder: journal papers on fuel cells

Recently published journal papers focused on hydrogen and fuel cell technologies or related technologies

\section{- Folder: reference books}

Books used as reference for the modules or the journal papers

- Folder: websites and selected research departments

Colleges and universities with fuel cell and hydrogen-specific courses or research programs

The objective of the modules is to give the students an exposure to hydrogen and fuel cell technologies and how they relate to the broader topics of a course. Courses considered for these modules relate to heat transfer, computational methods, chemistry, fluid mechanics, systems engineering and sustainability engineering.

Dr. Archambault assembled the necessary documentation to seek and receive approval for the module insertions into the undergraduate curriculum, while Dr. Hsu and his student identified and categorized the modules, and provided relevant faculty with a CD containing the module folders.

Task 2.2 Offering of Courses Which Include Lecture Modules and Laboratory Modules.

A few examples will be given of undergraduate courses that included hydrogen modules.

In Engineering Thermodynamics, MAE 3191, Dr. Hsu included Module 1: Equation of State for Hydrogen Fuel. In this module, the Peng-Robinson and Van der Waals non-ideal equations of state are 
used for hydrogen to estimate the effect of high pressure on hydrogen gas density. Also included was Module 2: Fuel Cell Efficiency. In this module, Gibbs free energy, enthalpy and efficiency of hydrogen will be defined and solved.

Within the Undergraduate Minor for Sustainability, Dr. Ken Lindeman added hydrogen fuel modules to Introduction to Sustainability, ISC 1500, and Applied Sustainability, ISC 4000. He also conducted the DOE survey (see later sections) in ISC 1500.

In the Power Engineering Curriculum, Dr. Robert Sullivan added a hydrogen fuel cell module to Introduction to Electrical Power Systems, ECE 4861, and hydrogen-related content to his graduate course Power System Operation, ECE 5683.

Physical Chemistry Laboratory I, CHM 3011, is required of all chemical engineering as well as chemistry and biochemistry undergraduates. Dr. Clayton Baum developed a four hour lab experiment consisting of two parts:

Part 1: Electrolysis of water to produce hydrogen and oxygen. Students measure voltage, current and volume of hydrogen produced. They calculate the chemical energy and the electrical energy to determine the efficiency and heat loss.

Part 2: Use the fuel cell to produce electricity to lift a known mass a known distance. Students will calculate the electrical energy and the efficiency of the fuel cell and compare the electrical energy required to produce the hydrogen and oxygen with that produced by the fuel cell.

Dr. James Brenner inserted hydrogen modules into the chemical engineering curriculum: Introduction to Chemical Engineering, CHE 1101, Materials Science and Engineering, CHE 3260, Nanotechnology, CHE 5567/4592 and the Materials Characterization Lab.

\section{Task 3.0 Senior Design Students.}

Graduating seniors in the fields of engineering and science are currently required to undertake a capstone project. This project may take the form of a design or research project.

The protocol for Senior Design is as follows. An advising professor reviews a list of possible topics to be presented as options for the class. The Florida Tech National Hydrogen Research Center, under the sponsorship of this program, prepared a short presentation of possible hydrogen related projects. Projects are subsequently selected by groups of students and usually have different timing schemes. Most of the projects include a junior design semester during which the project is proposed, and one year of dedicated work. (This presents a problem for long and complex hydrogen related projects.)

\section{Task 3.1 Development of Potential Capstone Project Descriptions}

A list of potential projects was developed by key project faculty in consultation with other faculty members. This list was made available to students so that they could select a topic for their capstone project. The students may also suggest additional topics, subject to approval.

\section{$\underline{\text { Task 3.2 Capstone Project Completion }}$}

Selected student capstone projects are guided by the participating relevant faculty. Students prepare and exhibit these capstones at the end of the spring term along with a written report. 
One project recently selected and currently underway is the Metal Hydride Hydrogen/Thermal Energy Storage Senior Design Project. Its objective is to design, build, and investigate a metal hydride hydrogen/ thermal energy storage system.

- To facilitate complete hydrogen storage and release, efficient heat transfer is important.

- This project is designing and building a baseline thermochemical energy storage system container/bed with a metal hydride storage material in order to enhance its storage performance using different heat exchange configurations. An example is to add a centrally located radial heat exchanger to the bed and bed with fins on the radial heat exchanger.

- Design considerations include heat exchanger size, fin thickness, spacing between fins and size of metal hydride bed for required thermal energy storage.

- Performance is being measured by the amount of heat absorbed in the material and hydrogen released from the tank as a function of time for the enhanced configurations compared to the baseline configuration.

- The figure below shows the current student designs.

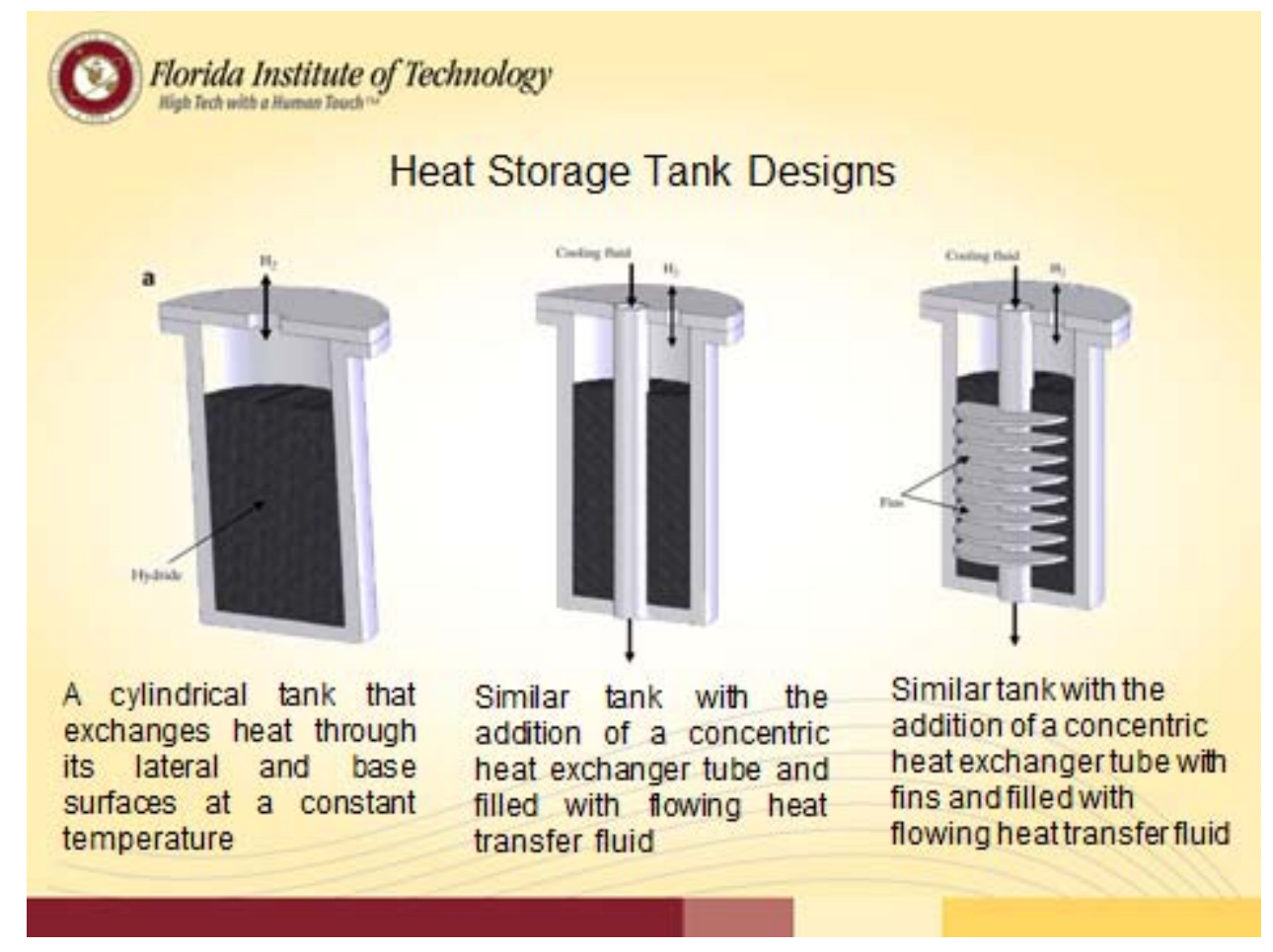

A graduate level project titled Hydrogen-based Residential Emergency Power Supply Capstone Project was undertaken with the objective being to develop final cost estimates for a hydrogen based residential emergency power supply based on:

- Holistic modeling

- Parametric modeling

- Activity-based modeling

This included providing cost estimate spreadsheets for adjusting cost estimates and providing an integrated MS Project master schedule that combines everything. 


\section{Task 4.0 Develop Inquiry Based Laboratory Experiments for General Chemistry Courses.}

This task is a unique task within the DOE education program. For this task, students in the General Chemistry laboratory study the chemical reactions related to many aspects of hydrogen and fuel cell technologies. The chemistry laboratory course is populated by students in all areas of science and engineering. Their appreciation for hydrogen as a fuel source will be enhanced by the inclusion of inquiry based laboratory experiments and periodic use of assessment tools, including the 2004 Hydrogen Knowledge and Opinion Survey (HKOS) developed by the U.S. Department of Energy. Assessment will take place starting in the year prior to the introduction of new experiments and continue throughout the duration of this project.

Hydrogen-themed, inquiry based laboratory experiments are incorporated within the General Chemistry II lab curriculum. The objectives of this project are to: 1) implement a set of inquiry-based, general chemistry laboratory experiments that focus on issues related to hydrogen fuel research, and 2) measure students' gains in knowledge of hydrogen as a fuel source and their interest in and appreciation of chemistry as a subject, with a particular focus on the laboratory course. Subtasks for developing a hydrogen-themed, inquiry based laboratory experiments for general chemistry laboratory courses are described below in roughly chronological order. Minor revisions to hydrogen-themed general chemistry lab experiment procedures were made based on student and lab instructor feedback from spring 2012.

\section{Task 4.1 Prepare Surveys for General Chemistry II Students}

An attitudinal survey (SPiCE) used in a previously NSF-funded redesign of Florida Tech's General Chemistry I lab course was adopted for this project. The 2004 student version of HKOS was used to reveal students' understanding of issues related to hydrogen fuel technology. Deliverables: Survey tools that measure (1) students' preexisting attitudes about chemistry and (2) students' knowledge of hydrogen fuels and fuel cell technologies were used beginning in the spring 2011 General Chemistry II lab course.

Task 4.2 - Administer surveys (Spring 2011 - Fall 2012)

Students enrolled in General Chemistry II lab completed the SPiCE and HKOS surveys at the beginning and end of each semester during the 2011 and 2012 calendar years (four semesters total).

\section{Task 4.3 Modify Published Hydrogen-Themed Experiments}

Dr. Winkelmann and his research students modified published experiments in order to fit within the framework of this project. When possible, Dr. Winkelmann rewrote the experiments to be consistent with inquiry pedagogy and prepared background information that explained the important features of current hydrogen and fuel technology that relate to each laboratory activity. Finally, Dr. Winkelmann prepared questions for pre-lab quizzes and grading rubrics for each experiment's lab report. Deliverables: Dr. Winkelmann created a set of hydrogen and fuel cell themed, laboratory experiments to be offered in the General Chemistry II laboratory course.

\section{Task 4.4: Analyze assessment data collected during spring and fall 2011 (January 2012)}

Drs. Winkelmann and Marcinkowski analyzed SPiCE and HKOS data collected in the General Chemistry II lab during the spring 2011 and spring 2012 semesters. This analysis of the SPiCE survey shows no change in students' attitudes about chemistry lab during the spring 2011 semester. This result is expected since students were performing the "cookbook" lab experiments which were later replaced with the hydrogen-themed curriculum. After completing the new lab experiments in spring 2012, students still 
showed no statistically significant change in their attitudes about chemistry, which is unexpected. Students became more favorably inclined towards hydrogen technology and demonstrated more knowledge of hydrogen by the end of the spring 2012 semester. This is expected since they had completed the hydrogen-themed lab experiments. A science education article describing these findings in more detail is planned.

\section{Task 4.5: Organize methods for feedback about new experiments (November 2011)}

Dr. Winkelmann met with the teaching assistants during the spring 2012 and fall 2012 semesters each week to get their feedback about the new experiments. Based on their comments, Dr. Winkelmann created new video tutorials to help students understand the laboratory experiment procedures. These are now available to students in the General Chemistry II lab course.

Task 4.6: Implement new experiments (Spring and Fall 2012)

Dr. Winkelmann incorporated new hydrogen-themed experiments into the General Chemistry II lab course during the spring and fall 2012 semesters. Students continue to perform these experiments during future semesters also.

Task 4.7: Analyze assessment data collected during fall and spring 2012 (January 2013)

This task is completed and described in Task 4.4.

Task 4.8: Collect information about other schools' efforts to develop curriculum materials related to hydrogen fuel cell and related technology for science and engineering disciplines

This new task was created during this report period. Dr. Winkelmann supported two students to perform an extensive web and literature search to find other sources of hydrogen curriculum materials which other schools have created. The purpose of this search is to keep up to date with the growth of hydrogen technology in science and engineering education. The web and literature search were completed in December 2012. A review article describing the current state of college-level hydrogen energy education is planned for the near future.

\section{Task 5.0 Project Management and Reporting.}

The period of performance was 27 months, during which the courses and modules were developed and implemented, and interim and final reports prepared. Upon insertion into the curriculum, the proposed course modules and Masters Degree curriculum became part of the programs Florida Tech offers to potential and existing students and therefore supported by the University after the DOE funding ends. The Masters Degree area of specialization in Hydrogen Technology provides the opportunity for individuals from other fields to redirect their career paths into the energy arena.

Participants in the project traveled to attend meetings, conferences and to visit the UCF facility. Additional course development funding will be provided during the summer time period. Both software and laboratory equipment (e.g. experimental fuel cells) will be procured to support the theoretical and experimental sides of the educational initiative.

The Principal Investigators on this project are Dr. Mary Helen McCay, Dr. Mark Archambault, Dr. Kurt Winkelmann, and Dr. Pei-feng Hsu. Dr. Marcinkowski served as the assessment analyst. 
Dr. Mary Helen McCay, Director of Florida Tech's National Center for Hydrogen Research will served as the Project Director and was responsible for the daily administrative and programmatic support of the project.

Dr. Pei-feng Hsu chaired the Curriculum Development Committee. Its role is to develop the undergraduate and master's curriculum across the academic disciplines at Florida Tech. Dr. Hsu was also responsible for interacting with the Department Heads of the applicable Departments to procure course development from their faculty.

Dr. Mark Archambault was the liaison between the Curriculum Development Committee and the Undergraduate Curriculum Committee and the Graduate Council. These two committees ensure that the curriculum satisfies the requirements for the introduction of new degrees and programs.

Dr. Kurt Winkelmann implemented all chemistry experiments related to this project, administered the surveys to students and collected the survey data. He was the contact person to Florida Tech's Office of Institutional Research when coordinating the student focus groups and he supervised the undergraduate and graduate research assistants related to the chemistry experiments.

Dr. Thomas Marcinkowski served as the assessment analyst. He obtained the "raw" survey data and performed item and content analysis, as appropriate, and reported his findings to Dr. Winkelmann.

The team of Investigators had periodic meetings to ensure progress on the project. At that time, staffing and resource allocations were made to ensure that the objectives were achieved on schedule and within the planned expenditure. Monthly meetings were be held with the Research Office to review and expenditures and identify future needs or concerns. Deliverables: Quarterly reports that document the above mentioned deliverables and Final Report.

\section{$\underline{\text { Results }}$}

The results are:

- A strong curriculum on hydrogen and fuel cell technology that will assist undergraduate students in furthering their understanding of hydrogen and fuel cell technology and offer graduate students a career path into renewable energy.

- Student and faculty interaction with industrial/research organizations within the energy community.

- Students prepared for entry into research and other positions related to hydrogen technology within government, industry and academia.

- The strengthening of Florida as a cornerstone of the southeastern fuel cell and hydrogen hub.

\section{Relevant Experience}

Florida Tech is an accredited coeducational independently controlled and supported university. It is committed to the pursuit of excellence in teaching and research in the sciences, engineering, technology, business and related disciplines.

The University has the National Center for Hydrogen Research (NCHR), established through a congressional earmark. In addition, the participating faculty have been involved in other interdisciplinary 
projects for the development of experiments and curriculum on relevant new areas such as nuclear technology and biomedical engineering. This will ensure the success of the proposed curriculum development. 


\title{
FLORIDA HYDROGEN INITIATIVE Sub-contracter Find Report
}

\section{Design and Development of an Advanced Hydrogen Storage System using Novel Materials}

\author{
Dr. Elias K. Stefanakos (PI) \\ Dr. Yogi Goswami (Co-PI) \\ Dr. Ashok Kumar \\ Dr. Ram Manoj \\ Dr. Chand Jotshi \\ University of South Florida \\ Clean Energy Research Center \\ Dr. Sesha Srinivasan \\ Tuskegee University \\ stefanak@eng.usf.edu \\ (813) 974-4413
}

\author{
Submitted to: \\ David L. Block \\ Florida Solar Energy Center/University of Central Florida \\ 1679 Clearlake Road \\ Cocoa, FL 32922 \\ E-mail: block@fsec.ucf.edu \\ DOE Contract Number: DE-FC36-04GO14225 \\ UCF Account No. 20126050 (PO No.214912) \\ Project Start Date: December 1, 2010 \\ Project End Date: March 31, 2013
}

\begin{abstract}
April 2013
Disclaimer

The Clean Energy Research Center/University of South Florida nor any agency thereof, nor any of their employees, makes any warranty, express or implied, or assumes any legal liability or responsibility for the accuracy, completeness, or usefulness of any information, apparatus, product, or process disclosed, or represents that its use would not infringe privately owned rights. Reference herein to any specific commercial product, process, or service by trade name, trademark, manufacturer, or otherwise does not necessarily constitute or imply its endorsement, recommendation, or favoring by the Clean Energy Research Center/University of South Florida or any agency thereof. The views and opinions of authors expressed herein do not necessarily state or reflect those of the Clean Energy Research Center/University of South Florida or any agency thereof.
\end{abstract}




\title{
Design and Development of an Advanced Hydrogen Storage System using Novel Materials
}

\author{
University of South Florida \\ Clean Energy Research Center
}

\section{Executive Summary}

Depletion of fossil fuels, rising environmental concerns due to global warming, and necessity of a secure energy supply have created a worldwide interest in renewable energy technologies. Among many forms of renewable energy, hydrogen has attracted attention mainly as an energy carrier due to its potential for the replacement of oil in stationary and mobile applications. However, hydrogen storage continues to be the weakest link in the realization of a hydrogen economy despite the intense research efforts within the last decade. Among the many goals set by the US Department of Energy (DOE), gravimetric/volumetric density and reversibility near ambient conditions are the most stringent ones. Currently, the conventional storage methods of compressed and liquefied hydrogen are the only well-established options; however their drawbacks have prevented their widespread application as a storage media for mobile applications. As of now, there is no single material capable of attaining the desired set of targets designated by DOE.

The goal of this project was to design and develop novel conducting polymeric nanomaterials for on-board hydrogen storage with a system gravimetric capacity of $5.5 \mathrm{wt} \%$ or greater and completed reversible hydrogen storage characteristics at moderate temperature $\left(<100^{\circ} \mathrm{C}\right)$. The project approach was to examine synthesis of polyanitine (PANI) solid state hydrogen storage materials and to modify the synthesis parameters for optimized storage capabilities. The major challenge in conducting the research was to develop polymer nanostructures that can store hydrogen at room temperature, and be reversible for many cycles. The preliminary work conducted at the University of South Florida (USF) used PANI nanostructures and studied the morphological effects of $\mathrm{H}_{2}$ cycling on PANI NF-ES.

Several novel materials such as polyaniline (emeraldine salt), ion doped polyaniline, carbon nanotube doped polyaniline, transition metal doped polyaniline, and graphene doped polyaniline, electrospun polyaniline, and hyper cross-linked polystyrene material were examined. The spillover enhancement for high surface area polymeric materials (i.e., hypercrosslinked polystyrene) was investigated for the first time. Spillover enhancement attracted the attention of the hydrogen storage community due to the very promising results reported (i.e. $\approx 5 \mathrm{wt} . \%$ at room temperature and $10 \mathrm{MPa}$ ). However, spillover enhancement continues to be a disputable subject due to contradictory results reported by different research groups. The results reported in this report showed that spillover enhancement in high surface area polymers has little practical value (i.e., $0.32 \mathrm{wt} . \%$. at room temperature and $10 \mathrm{MPa}$ ) for mobile applications.

The original tasks of the projects were based on the promising results of previous USF work on electrospun polyaniline, and its modification by carbon nanostructures, which showed 3-10 wt. \% storage capacity at $25^{\circ} \mathrm{C}-125^{\circ} \mathrm{C}$. However, the promising results of the electrospun polyaniline were not repeatable. The actual hydrogen storage capacity of the electrospun polyaniline was found to be less than 0.1 wt. $\%$ at room temperature which could not support further design work since the system level design utilizing a $0.1 \mathrm{wt}$. \% storage capacity material has no practical outcome.

Following this initial materials research, the project objectives were modified to address the difficulties associated with the hydrogen storage measurements, and shifted our research direction to develop novel porous, high surface area polymeric materials and to explore the spillover phenomenon in the hypercrosslinked polystyrene. The revised tasks and the proposed work therein are as follows: 
- Task 1: Development of discrepancies in experimental results among various research groups because of inaccuracies in measuring procedures and equipment (amount of material measured, etc.).

- Task 2: Pursue the development of a modified list of polymeric materials that may provide improved gravimetric densities.

- Task 3: Continue testing to understand the hydrogen storage physisorption and chemisorption processes in conducting polymers (i.e., polyaniline).

- Task 4: Conduct education and outreach.

In the first task, USF research presented an uncertainty analysis of hydrogen storage measurements and discussed the most important error sources. The errors in hydrogen sorption measurements are of major concern in the hydrogen storage community which impede the down selection process of candidate materials and set back progress in the field. Therefore, the design stage uncertainty analysis of the volumetric hydrogen storage measurements was studied in detail and guidelines are given for high accuracy hydrogen storage measurements. For instance, sample loading in volumetric hydrogen storage measurements are shown to be an important parameter that needs to be considered. The research presented in this study will help the experimentalists to spot the major error sources in volumetric hydrogen storage measurements and to improve the accuracy of their measurements.

In task two, the spillover enhancement in hypercrosslinked polystyrene was investigated and the results found that the bridge building technique is better than the wet impregnation method. However, the results showed that the hydrogen storage capacity of the hypercrosslinked polystyrene is around $0.32 \mathrm{wt} . \%$ at room temperature and 100 bar pressure which is not useful for practical applications. Contrary to other works published on spillover enhancement in activated carbon and metal organic frameworks which claimed a 10 fold increase in the hydrogen storage capacity at room temperature and $10 \mathrm{MPa}$ pressure, USF researchers did not observe significant enhancement, but only an increase of $10 \%$ in the hydrogen storage capacity of the hypercrosslinked polystyrene as compared to the base material. Thus, the conclusion was that, spillover enhancement in hydrogen storage materials continues to be a highly disputable subject.

More fundamental studies are required to elucidate the spillover enhancement mechanism and more collaborative work (i.e., round robin tests) are required to address the discrepancies in the hydrogen storage measurements among the different labs. Other work in this task successfully synthesized nitrogen enriched high surface area polymers by Yamamato coupling; however, synthesized boron enriched high surface area polymers were not achieved.

In task three, polyemeraldine base, polyemeraldine salt, polyleucoemerldine and polypernigraniline materials were synthesized. However, the experimental investigations did not show more than $0.1 \mathrm{wt}$. \% hydrogen storage capacity. Finally in task 4 on education, USF pursued the development of an energy course in materials for hydrogen and fuel cells, organized a seminar on hydrogen storage and fuel cells in order to educate researchers and students within USF and regularly held seminars on energy related research. 


\section{Table of Contents}

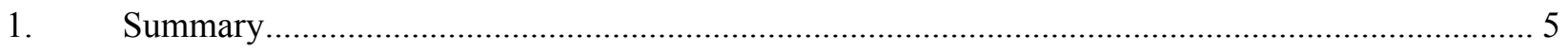

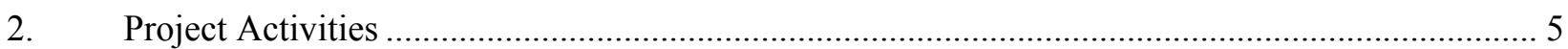

2.1. Task 1 - Development of a research paper that addresses discrepancies in experimental results among various research groups because of inaccuracies in measuring procedures and equipment (amount of material measured, etc.)............................................................................................ 5

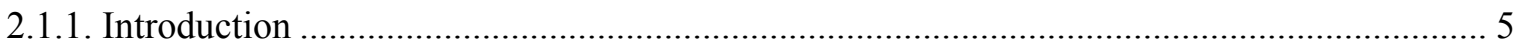

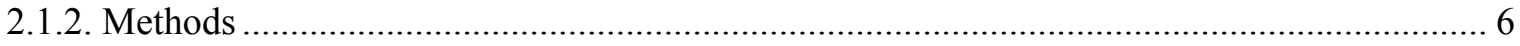

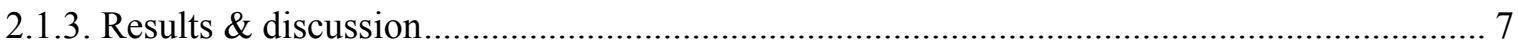

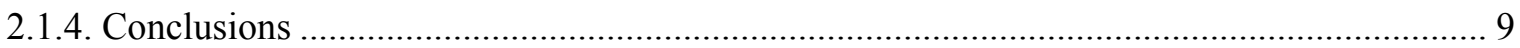

2.2. Task 2 - Development of a modified list of polymeric materials that may provide improved

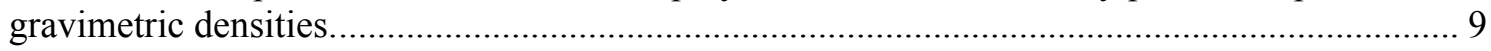

2.2.1. Investigation of the spillover enhancement in hypercrosslinked polystyrene ...................... 9

2.2.2. Nitrogen $(\mathrm{N})$ and boron $(\mathrm{B})$ doped high surface area polymers ....................................... 13

2.3. Task 3 - Understanding of the hydrogen storage physisorption and chemisorption processes in

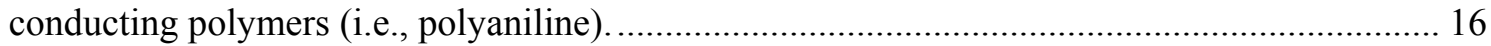

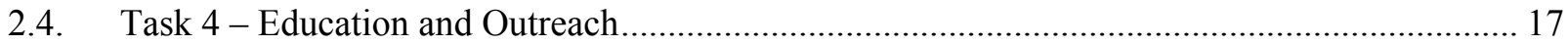

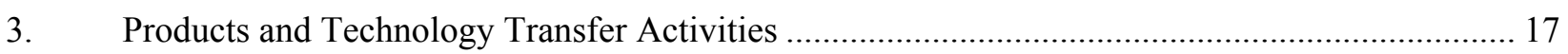

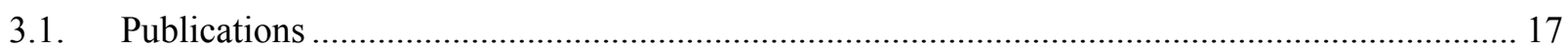

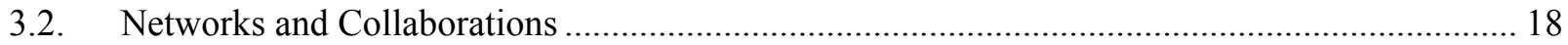

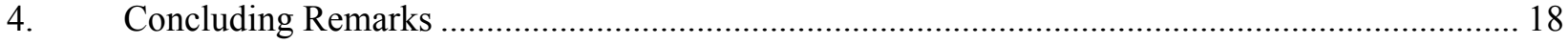

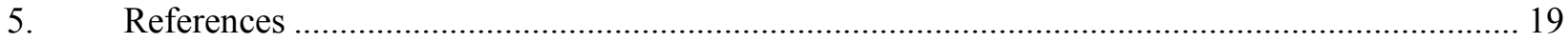




\title{
Design and Development of an Advanced Hydrogen Storage System using Novel Materials
}

\author{
University of South Florida \\ Clean Energy Research Center
}

\section{Summary}

The main objective of this project is the design and development of novel conducting polymeric nanomaterials for on-board hydrogen storage. The project approach was to examine the synthesis of polyanitine (PANI) solid state hydrogen storage materials and to modify the synthesis parameters for optimized storage capabilities. The major challenge in conducting the research has been the development of polymer nanostructures that can store hydrogen at room temperature, and be reversible for many cycles. The preliminary work conducted at the University of South Florida (USF) used PANI nanostructures and studied the morphological effects of $\mathrm{H}_{2}$ cycling on PANI NF-ES.

Published work shows discrepancies in the experimental results among various research groups because of inaccuracies in the measuring procedures and equipment (amount of material measured, etc.). The development of a modified list of polymeric materials that may provide improved gravimetric densities, procedures to avoid errors in these measurements, and recommendations for continued testing to understand the hydrogen storage physisorption and chemisorption processes in conducting polymers (i.e., polyaniline) aresome of the contributions resulting from work carried out in this project.

\section{Project Activities}

\subsection{Task 1 - Development of a research paper that addresses discrepancies in experimental results among various research groups because of inaccuracies in measuring procedures and equipment (amount of material measured, etc.).}

Scope and the key findings of this task are summarized below. Refer to the published article [1] for more details (i.e., real gas equation of state, uncertainty calculations and the derivations).

\subsubsection{Introduction}

The errors in hydrogen sorption measurements are of major concern in the hydrogen storage community which impede the down selection process of candidate materials and set back progress in the field. Hydrogen storage measurements are prone to various sources of errors since there are only a few methods available to detect the hydrogen absorption and desorption directly ${ }^{1}$ (i.e., thermal desorption mass spectroscopy [2,3] and neutron scattering [4]), and these methods either require sophisticated and expensive tools or can only be utilized for specific types of materials under certain conditions (i.e., cryogenic temperatures, ultra high vacuum, etc.). Therefore, the vast majority of the hydrogen storage measurements reported in the literature make use of indirect methods by utilizing volumetric (Sievert's type) and/or gravimetric apparatuses since these techniques are much more affordable, easier to fabricate and operate, and better suited to the rapid screening of samples.

The measurement errors are especially problematic and harder to realize for the materials that are based on physisorption, since the theoretical upper limit is not known unambiguously as compared to complex metal hydrides. In addition, for such materials, the storage capacity is typically low (i.e., less than $1 \mathrm{wt}$ \%,

\footnotetext{
1 "Directly" refers to the determination of the gas species unambiguously using spectroscopic tools as opposed to "indirectly" where the quantity of the absorbed or desorbed hydrogen is calculated from pressure and temperature measurements using a real gas equation of state (i.e., volumetric apparatus) or weight change using a high sensitivity balance (i.e., gravimetric apparatus) assuming all the absorbed or desorbed species are hydrogen.
} 
which is about an order of magnitude less than that of well-known hydrides such as $\mathrm{MgH}_{2}$ and $\mathrm{LiNH}_{2}+\mathrm{MgH}_{2}$ ) at room temperature and high pressure (i.e., $300 \mathrm{~K}$ and $10 \mathrm{MPa}$ ). Physisorption based materials can adsorb significant amount of hydrogen at $77 \mathrm{~K}$ and high pressure [5]; however the most erroneous measurements reported in the literature are performed at room temperature and high pressure where their capacity is typically less than $1 \mathrm{wt}$. \%. In other words, for any material, the hydrogen sorption measurements are most cumbersome at a point (i.e., at a specific P and T) where the sample's hydrogen storage capacity is the lowest.

In this task, our aim is twofold. First, the design of a volumetric apparatus is investigated by an uncertainty analysis to determine the uncertainties in the high pressure hydrogen sorption measurements. Guidelines are presented on the selection of the reservoir and sample cell volumes to minimize the uncertainty in the measurements. In addition, the effect of the sample mass loading on the measurement uncertainty is studied based on the sample's estimated hydrogen storage capacity and the design of a volumetric apparatus. Secondly, the effect of the thermal equilibration time (i.e., the time required for thermal equilibrium when hydrogen gas is admitted into a reservoir volume at a temperature different than that of the instrument enclosure temperature, and when there is a temperature gradient within the instrument enclosure) on the accuracy of the measurements is investigated.

\subsubsection{Methods}

In a volumetric apparatus, the hydrogen uptake of a material is calculated by using an appropriate real gas equation of state from measurements of the pressure drop in a volume calibrated reservoir and sample cell, the temperature of the reservoir and sample cell volumes, and the density of the material which is usually determined by a helium expansion test. The schematic of a volumetric apparatus is shown in Figure 1.

The operation of a volumetric apparatus is as follows: initially the isolation valve is closed and hydrogen is fed into the reservoir volume; after the system reaches thermal equilibrium, the pressure and temperature of the hydrogen are recorded, and then the isolation valve is opened. After the pressure change in the system levels off, the pressure and temperature of the hydrogen in the system are recorded again. Then the sample's hydrogen uptake can be calculated by subtracting the initial hydrogen content in the reservoir from the final hydrogen content in the system. 


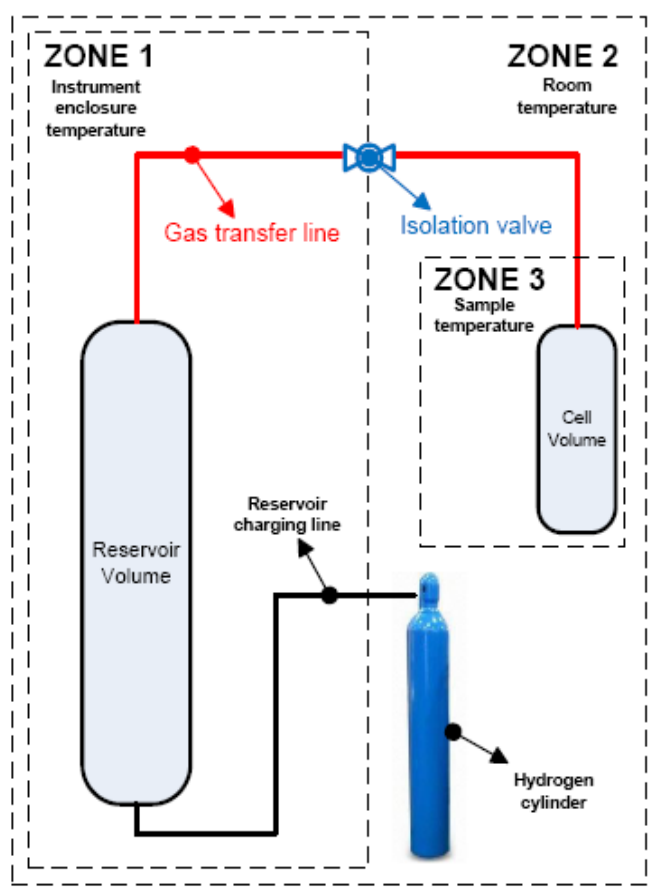

Figure 1. Schematic of a volumetric apparatus.

\subsubsection{Results \& discussion}

Sample mass loading is an important parameter that needs to be considered in hydrogen storage measurements. Figure 2 shows that measurements of a small sample (i.e., less than $0.5 \mathrm{~g}$ ) always result in very high uncertainty when using a $10 \mathrm{cc}$ reservoir volume and should be avoided in high pressure sorption measurements. In other words, optimum size of the sample mass loading depends on the size of the reservoir volume and the sample's intrinsic storage capacity, and regardless of the size of the reservoir volume, greater sample loading is always better. Nevertheless, the selection of the reservoir volume size also depends on the purpose and the type of the experiment.

For instance, for single step desorption kinetics measurements selecting a large volume is preferable to keep the back pressure as close as possible to vacuum conditions, or for single step absorption kinetics measurements a larger volume is required to keep the charging pressure relatively constant over time, and to keep the system pressure (i.e., charging pressure) close to the initial (i.e., before opening the isolation valve) reservoir pressure when the intention is to charge the sample with high pressure. On the other hand, in PCT measurements (i.e., charging sample with multiple doses) using a smaller volume is desirable due to reasons outlined in this work. 


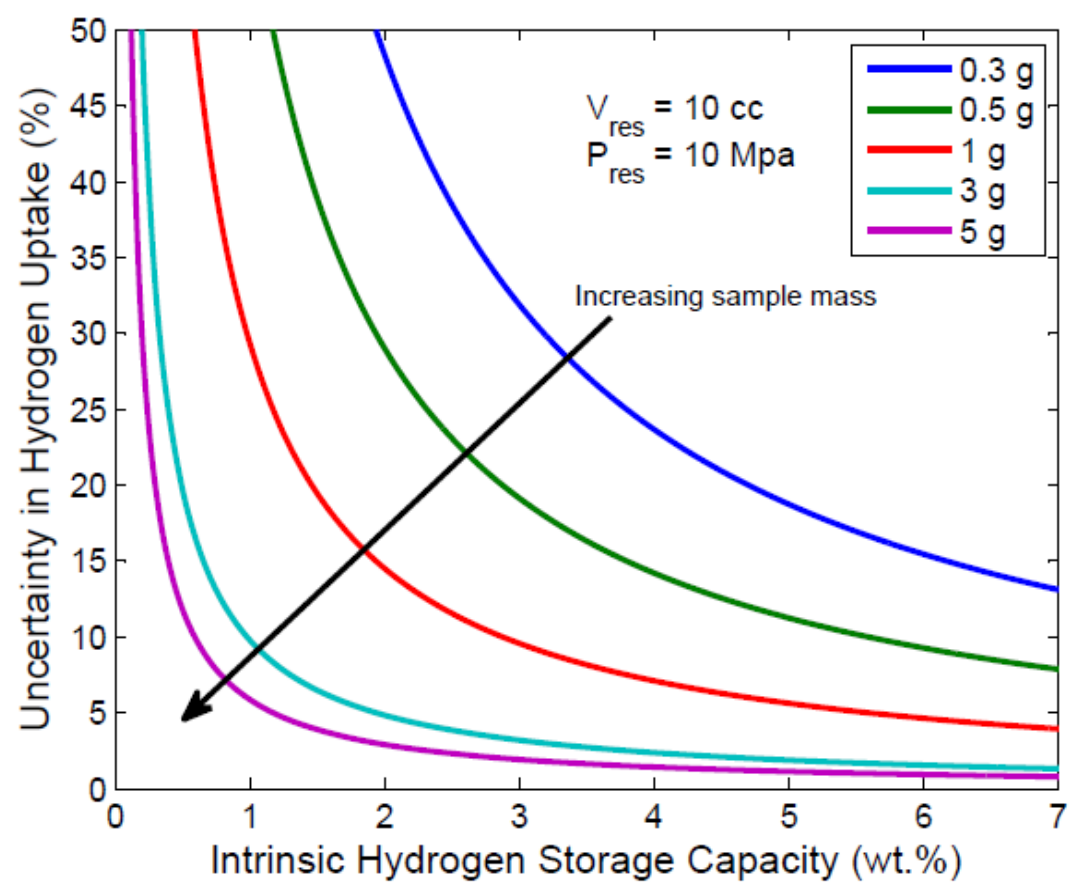

Figure 2. The effect of the sample mass loading in a sample's hydrogen uptake uncertainty.

The thermal equilibration time is one of the critical factors to be considered for accurate hydrogen storage measurements in a volumetric apparatus. Considering the simplified schematic of a volumetric apparatus given in Figure 1, if the instrument enclosure temperature (Zone 1) is different than the room temperature (Zone 2) or there is a temperature gradient inside the instrument enclosure, the thermal equilibration time must be considered to minimize errors.

The thermal equilibration time is an important parameter to be considered at the start of the measurements (i.e., before opening the isolation valve in Figure 1). Since the temperature reading is taken from the external surface of the reservoir volume, the actual gas temperature inside the reservoir volume might be different. Therefore, if the measurements (i.e., P and T) start before thermal equilibration is reached, the change in gas temperature will result in an increase or decrease of the gas pressure inside the reservoir volume which in turn will cause erroneous hydrogen uptake values. The effect of the temperature difference between the thermocouple reading and the actual gas temperature (i.e., $\Delta \mathrm{T}$ ) is studied for $\Delta \mathrm{T}=$ $1 \mathrm{~K}, 3 \mathrm{~K}$, and $5 \mathrm{~K}$ using the real gas equation of state given in Eq. (1),

$Z(p, T)=\frac{p}{\rho R T}=1+\sum_{i=1}^{p} a_{i}\left(\frac{100 K}{T}\right)^{h_{i}}\left(\frac{p}{1 M P \alpha}\right)^{Q_{i}}$

where $\mathrm{Z}$ is the compressibility factor, $p$ is the pressure in $\mathrm{MPa}, \rho$ is the density in $\mathrm{g} / \mathrm{mol}, \mathrm{R}$ is the universal gas constant in $\mathrm{J} /(\mathrm{mol} \cdot \mathrm{K}), \mathrm{T}$ is the absolute temperature in $\mathrm{K}$ and the constants $\mathrm{a}_{\mathrm{i}}, \mathrm{b}_{\mathrm{i}}$ and $\mathrm{c}_{\mathrm{i}}$ are taken from Lemmon et al. [6]

Figure 3 shows the pseudo hydrogen sorption due to changes in the actual hydrogen temperature (i.e., $\left.\Delta \mathrm{I}^{\prime}=\mathrm{I}^{\prime}\left(\mathrm{t}_{\alpha}\right) \mathrm{I}^{\prime} \mathrm{t}_{\mathrm{th}}\right)^{2}$ for three different calibrated volumes of PCTPro 2000 [7] at $100 \mathrm{~atm}$. The

\footnotetext{
${ }^{2} T\left(t_{0}\right)$ is the temperature of the hydrogen initially (at $\left.t=0\right), T\left(t_{t h}\right)$ is the temperature of the hydrogen when it is in thermal equilibrium with the instrument enclosure (i.e., when actual gas temperature is equal to the thermocouple reading and assuming instrument enclosure is isothermal).
} 
larger the calibrated volume the larger the error in the measurements, since the quantity of hydrogen gas in the larger volume (i.e., large reservoir) is considerably bigger than the one in the small volume (i.e. manifold volume). The inset in Figure 3 shows that even small temperature differences $(\Delta \mathrm{T}=\mathrm{G} .1 \mathrm{~K})$ can cause significant pseudo sorption as the reservoir volume increases. This outcome is also in agreement with the uncertainty analysis which favors using a reservoir volume as small as possible; however, the selection of the reservoir volume size also needs to be compatible with the goals of the experiment.

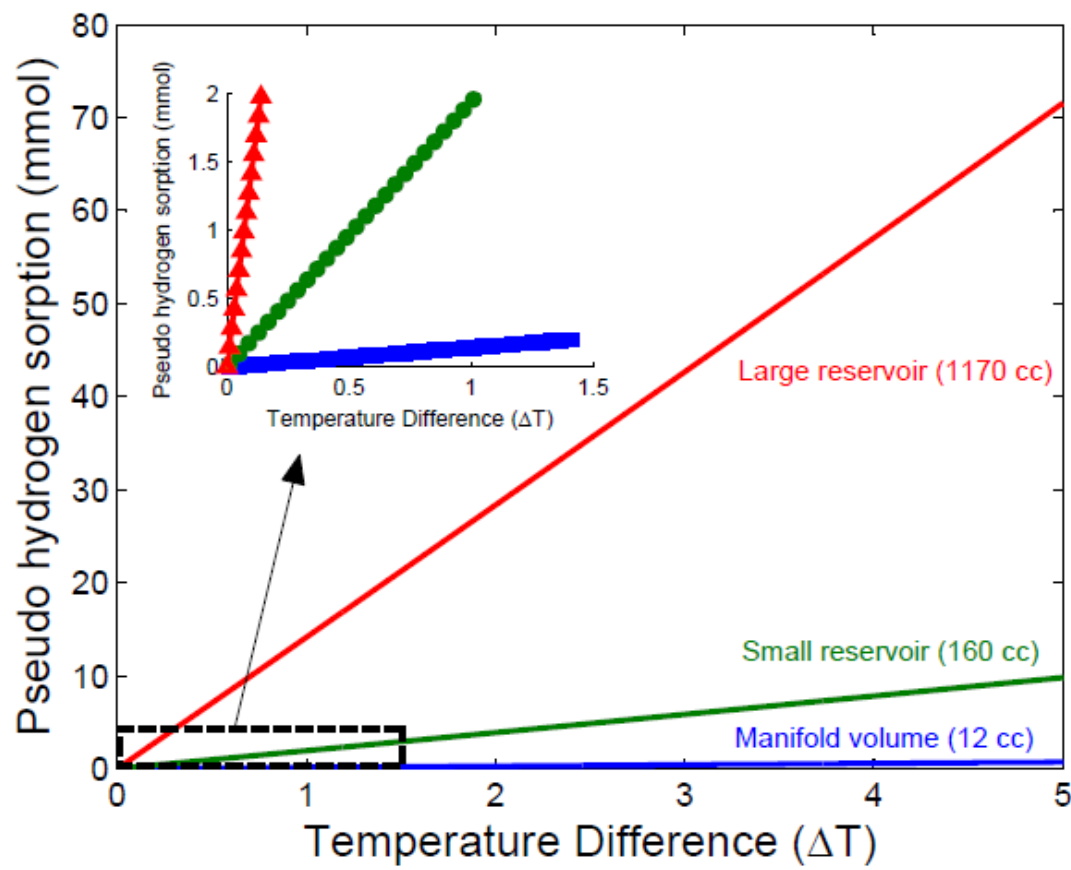

Figure 3. Pseudo hydrogen sorption as a function of $\Delta \mathrm{T}$ at $100 \mathrm{~atm}$.

\subsubsection{Conclusions}

The sample mass loading is one of the most important parameters to be considered regardless of the characteristics of the system and the sample. In general, the higher the sample mass loading the lower the uncertainty in measurements, the optimal loading being a function of the sample's intrinsic storage capacity. For highest accuracy, the system should not contain significantly more gas than the sample can absorb since the amount of hydrogen in the sample at a specific $\mathrm{P}$ and $\mathrm{T}$ is a fixed amount, and the error in measuring this amount will depend on how large or small this amount is compared to the hydrogen in the gas phase. Moreover, the effect of the thermal equilibration time on the hydrogen storage measurements is investigated. If there is a possibility (i.e., when the instrument enclosure temperature and the room temperature are not the same or there is a temperature gradient within the instrument enclosure) that the actual gas temperature inside the reservoir volume could be different than the ambient temperature, then the thermal equilibration time needs to be taken into account. Depending on the size and shape of the reservoir volume, a temperature difference as small as $0.1 \mathrm{~K}$ can cause significant errors.

\subsection{Task 2 - Development of a modified list of polymeric materials that may provide improved gravimetric densities.}

\subsubsection{Investigation of the spillover enhancement in hypercrosslinked polystyrene}

Scope and the key findings of this task are summarized below. Refer to the published article [8] for more details. 


\subsubsection{Introduction}

The high surface area porous materials have drawn interest in the hydrogen storage challenge due to their fast sorption kinetics, reversibility, and promising gravimetric capacity. High surface area porous materials can achieve significant hydrogen uptake by physisorption at $77 \mathrm{~K}$, however, their uptake at room temperature (RT) is usually less than $1 \mathrm{wt} . \%$ at moderate pressures (i.e., $100 \mathrm{~atm}$.) [9]. In addition, adsorption enthalpy of these materials is low ( $<6 \mathrm{~kJ} / \mathrm{mol})$, which needs to be improved (20-40 kJ/mol) [10] for significant hydrogen uptake at RT. The tuning of the adsorption enthalpy and/or higher hydrogen uptake at RT can be realized via multiple modification methods such as optimizing the pore size/volume [11], enhancing the specific surface area (SSA), and doping with impurity atoms (B, N, alkali/transition metals) [12].

Incorporation of transition metals can enhance the hydrogen binding via Kubas type interactions due to the empty d-orbitals [13] and/or by hydrogen spillover (i.e., Pt, Ru, Pd, and Ni) [14]. The hydrogen spillover phenomenon has been well-known in catalysis for over three decades and various literature reviews have been published [15]. Briefly, spillover is the dissociation of the hydrogen molecules into atoms by transition metals and subsequent diffusion of these atoms to the host material, in which hydrogen atoms can hydrogenate the unsaturated C-C bonds (i.e., activated carbon, graphite and SWCNT) [16] and/or the benzene ring (i.e., metal organic frameworks (MOFs), covalent organic frameworks (COFs,) polymers etc.) [17]. Among the various steps of spillover, dissociation of hydrogen molecules and diffusion of hydrogen atoms are considered as instantaneous and barrier-less, respectively; however, hydrogen atoms need to overcome high energy barriers for migration to the host material which makes migration the rate limiting step. Therefore, minimum clustering and fine molecular level dispersion of transition metals within the host material is desirable to make the migration path as short as possible.

Hydrogen spillover enhancement in carbon nanostructures [18], MOFs [19], and COFs [20] was extensively investigated both theoretically and experimentally. However, spillover studies on polymeric materials are rather scarce [21] and transition metal doping of the polymers were studied mostly within the context of catalysis research [22]. Additionally, high pressure hydrogen uptake of transition metal doped polymers at RT has never been reported to the best of our knowledge.

In this task, commercially available hypercrosslinked polystyrene (HPS) was investigated in detail to study the effects of Pt doping/hydrogen spillover on hydrogen uptake enhancement at RT. This has been realized by utilizing two different synthetic methods, wet impregnation and bridge building technique, to elucidate the effectiveness of these widely applied methods for the spillover enhancement on hydrogen storage.

\subsubsection{Experimental details}

As received HPS (i.e., commercial name MN270) beads had a 0.5-1 mm diameter on average. To facilitate the Pt dispersion within the polymer matrix and sample characterization, MN270 was ball milled with Fritsch Pulverisette 6 for $2 \mathrm{~h}$ at 200 revolutions per minute (RPM) before any modification.

Due to the larger sample masses $(\approx 1.5 \mathrm{~g})$ used in high pressure hydrogen measurements, the doping procedure was scaled-up to prepare MN270 with a $6 \mathrm{wt}$. \% Pt loading. Typically, $2 \mathrm{~g}$ of MN270 was degassed in vacuum at least $12 \mathrm{~h}$ at $393 \mathrm{~K}$. The dried sample was dispersed in $150 \mathrm{ml}$ of acetone and stirred for $30 \mathrm{~min}$. A $20 \mathrm{ml}$ acetone solution containing $324 \mathrm{mg}\left(6 \mathrm{wt}\right.$. \%) of $\mathrm{H}_{2} \mathrm{PtCl}_{6} \cdot 6 \mathrm{H}_{2} \mathrm{O}$ was added to the MN270 solution over 90 min using a syringe pump. After sonication $(100 \mathrm{~W}, 42 \mathrm{kHz})$ for $1 \mathrm{~h}$, the solution was then stirred for another $24 \mathrm{~h}$. The sample was dried in an oven at $323 \mathrm{~K}$ overnight and then loaded into a tube type furnace in a quartz boat for activation. The sample was further dried in helium $(300 \mathrm{ml} / \mathrm{min}$ ) for $2 \mathrm{~h}$ at $473 \mathrm{~K}$, then activated in hydrogen flow for $5 \mathrm{~h}$ at the same temperature/flow rate 
and cooled to RT in hydrogen flow. Finally, the sample was passivated in helium for $5 \mathrm{~h}$ at RT and stored in an argon-filled glove-box until further analysis. The synthesized sample was named MN270-6wt\%Pt.

For the bridge building treatment, a previously reported procedure was followed [23]. A $2 \mathrm{~g}$ of dry MN270 was mixed with $5 \mathrm{wt} . \%$ Pt/activated carbon (Pt/AC) and D-glucose with a weight ratio of 8:1:1 (MN270: Pt/AC: D-glucose) and ball milled for $1 \mathrm{~h}$ at $200 \mathrm{RPM}$. This mixture was then loaded in a quartz boat and placed in a tube type furnace. The mixture was heated to $453 \mathrm{~K}$ at $5 \mathrm{~K} / \mathrm{min}$ under $100 \mathrm{ml} / \mathrm{min}$ helium flow and kept at $453 \mathrm{~K}$ for $3 \mathrm{~h}$ and then temperature ramped at $5 \mathrm{~K} / \mathrm{min}$ to $523 \mathrm{~K}$ and maintained for an additional $12 \mathrm{~h}$. After cooling to RT, the sample was stored in an argon-filled glove-box until further analysis. This sample was named MN270-Bridged.

\subsubsection{Results \& discussion}

Hydrogen uptake of the MN270, MN270-Bridged, MN270-6wt\%Pt, and Pt/AC (i.e., catalyst used in the bridge building method) at $294 \mathrm{~K}$ and up to $100 \mathrm{~atm}$. is shown in Figure 4. All the isotherms showed repeatability between successive measurements after evacuation at $150^{\circ} \mathrm{C}$ for at least $12 \mathrm{~h}$, and error bars based on three measurements are plotted in Figure 4.

Typical sorption kinetics data is also given in Figure 5 for the best performing material, MN270-Bridged. The kinetics data shows that equilibrium has been reached for each pressure-concentration data point shown in Figure 5. The initial (i.e., 0-10 atm.) steep rise in the hydrogen uptake of the Pt doped samples seen in Figure 4 can be attributed to the chemisorption of the hydrogen at low pressure. As seen in Figure 4, the MN270-Bridged has the highest uptake of $0.36 \mathrm{wt}$. \% at $294 \mathrm{~K} / 100 \mathrm{~atm}$.; however, this is only $10 \%$ higher than the MN270 base material and of insignificant value for RT hydrogen uptake enhancement purposes. The MN270-6wt\%Pt did not show any enhancement in the hydrogen uptake compared to the base material MN270 despite its higher Pt content and smaller Pt particle size as compared to the MN270Bridged. The Pt content appeared to be of minimal effect but the dispersion/particle size of the Pt was considered important for spillover enhancement [24]. However, this alone cannot explain the better performance of the MN270-Bridged over the MN270-6wt\%Pt, because the MN270-Bridged has bigger Pt particles on average. Nevertheless, it shows the effectiveness of the bridge building technique on the hydrogen uptake by enabling closer contacts between the polymer matrix and the Pt. 


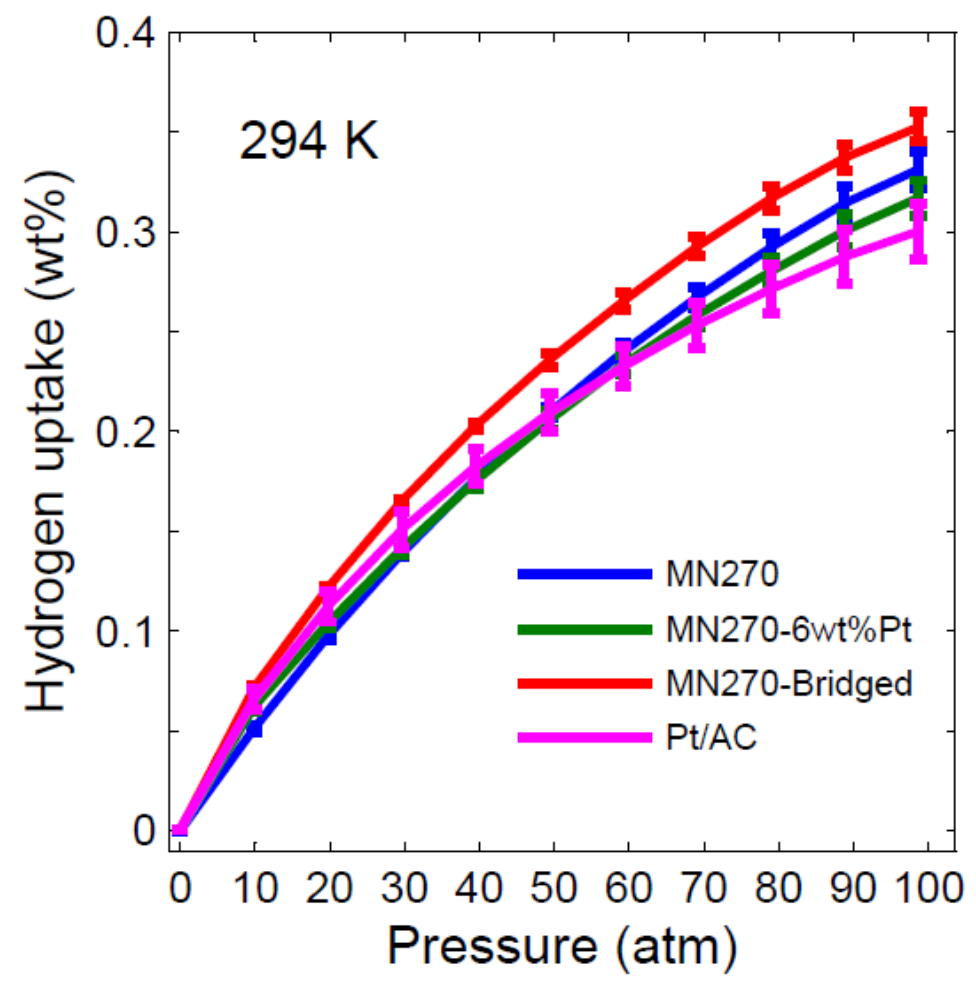

Figure 4. Hydrogen uptake of MN270, MN270-Bridged, MN270-6wt\%Pt, and Pt/AC at $294 \mathrm{~K} / 100 \mathrm{~atm}$.

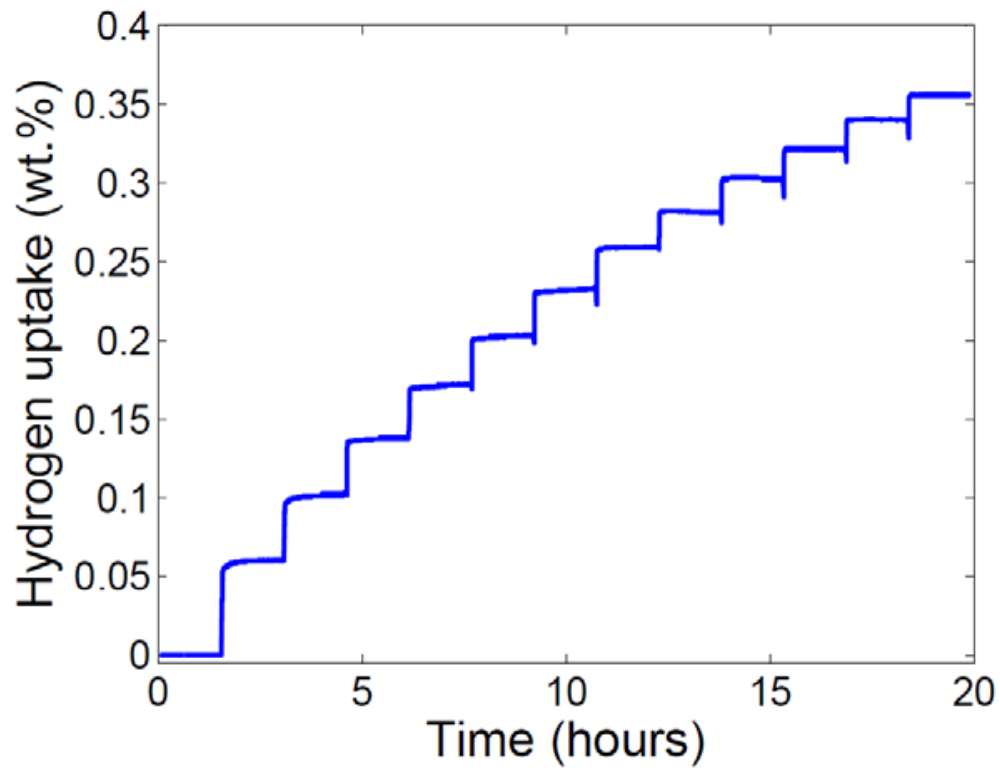

Figure 5. Kinetic hydrogen uptake of MN270-Bridged 


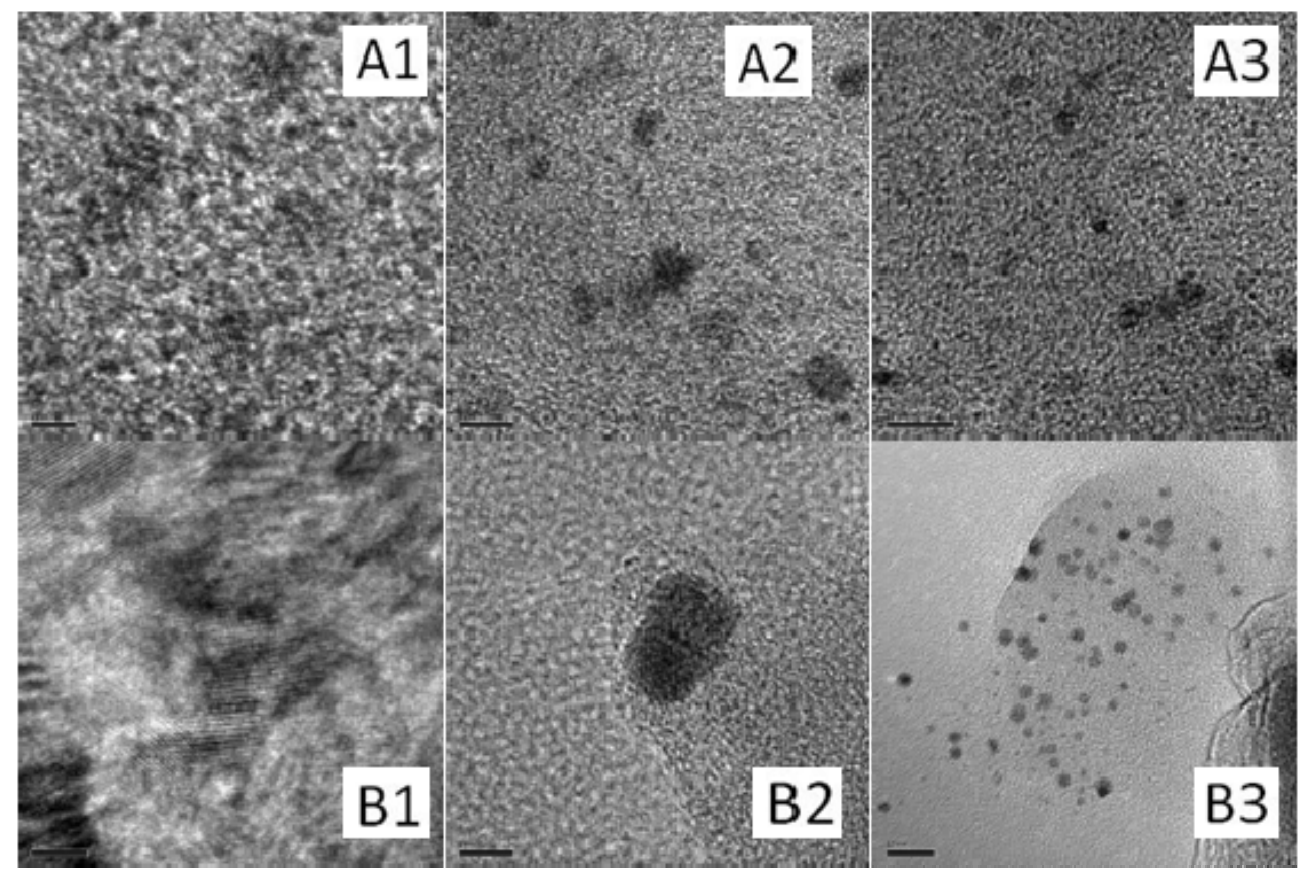

Figure 6. (A1-A3): TEM images of MN270-6wt\%Pt; scale bar in (A1), (A2) and (A3) corresponds to 2nm, 5nm and 10nm, respectively. (B1-B3): TEM images of MN270-Bridged; scale bar in (B1), (B2) and (B3) corresponds to $5 \mathrm{~nm}, 5 \mathrm{~nm}$ and $10 \mathrm{~nm}$, respectively.

The effectiveness of the bridge building technique is also evident from the TEM images given in Figure 6. The MN270-6wt\%Pt has a narrow Pt particle size distribution (2-5nm), whereas the MN270-Bridged has a wide Pt particle size distribution (2-15nm). Although the Pt particles in the MN270-6wt\% Pt are smaller on average compared to the MN270-Bridged, the MN270-6wt\%Pt did not show higher hydrogen uptake at RT. On the contrary, the MN270-Bridged outperformed the MN270-6wt\%Pt as seen from Figure 4. Therefore design of the optimal Pt/AC catalyst needs to be further explored to fully exploit the potential of the bridge building technique.

\subsubsection{Conclusions}

The spillover enhancement by Pt doping was investigated by utilizing two different synthesis methods; wet impregnation (i.e., MN270-6wt\%Pt) and the bridge building technique (MN270-Bridged). The MN270-Bridged showed $10 \%$ increase in hydrogen uptake at $294 \mathrm{~K} / 100$ atm compared to the MN270 base material, whereas the MN270-6wt\%Pt did not show any enhancement. However, such enhancement factor (EF) has insignificant value for practical applications and is considerably lower compared to other materials studied (i.e., for MOFs, $\mathrm{EF} \approx 2-9$; for $\mathrm{AC}, \mathrm{EF} \approx 1-5$ ) [25]. The spillover enhancement for hydrogen storage continues to be a highly disputable topic due to conflicting results for the same materials (i.e., MOFs, AC). Since there is no spillover enhancement study for polymers, we are not able to compare our results with those of other groups, yet considering the similarity of the organic building block of the HPS with MOFs/COFs (i.e., benzene ring), the reasons underlying the small spillover enhancement for hydrogen storage in polymers needs to be further investigated.

\subsubsection{Nitrogen $(\mathrm{N})$ and boron $(\mathrm{B})$ doped high surface area polymers}

In this task, high surface area polymers were synthesized with increasing $\mathrm{N}$ content to understand if $\mathrm{N}$ doping is favorable or not for hydrogen storage capacity of the porous organic polymers. The high surface 
area polymers were synthesized using the Yamamato coupling method. The starting monomers are given in Figure 7.<smiles>c1ccc(N(c2ccccc2)c2ccccc2)cc1</smiles>

1<smiles>c1ccc(-c2nc(-c3ccccc3)nc(-c3ccccc3)n2)cc1</smiles>

2<smiles>c1c[nH]c(-c2nc(-c3ccc[nH]3)nc(-c3ccc[nH]3)n2)c1</smiles>

3<smiles>c1ccc(-c2cc(-c3ccc[nH]3)nc(-c3ccc[nH]3)c2)cc1</smiles>

4<smiles>c1c[nH]c(-c2nc(-c3ccc[nH]3)nc(-c3ccc[nH]3)n2)c1</smiles>

5<smiles></smiles>

6<smiles>c1ccc(N(c2ccccc2)c2ccc(N(c3ccccc3)c3ccccc3)cc2)cc1</smiles>

7

Figure 7. $\mathrm{N}$ enriched monomers.

The polymer \#1 (P\#1) and polymer \#2 (P\#2) were synthesized by the Yamamato coupling method using literature methods [26]. The nitrogen adsorption and desorption isotherms, and hydrogen uptake at $77 \mathrm{~K}$ and $1 \mathrm{~atm}$ for $\mathrm{P} \# 1$ and $\mathrm{P} \# 2$ are given in Figs. 8 and 9, respectively.

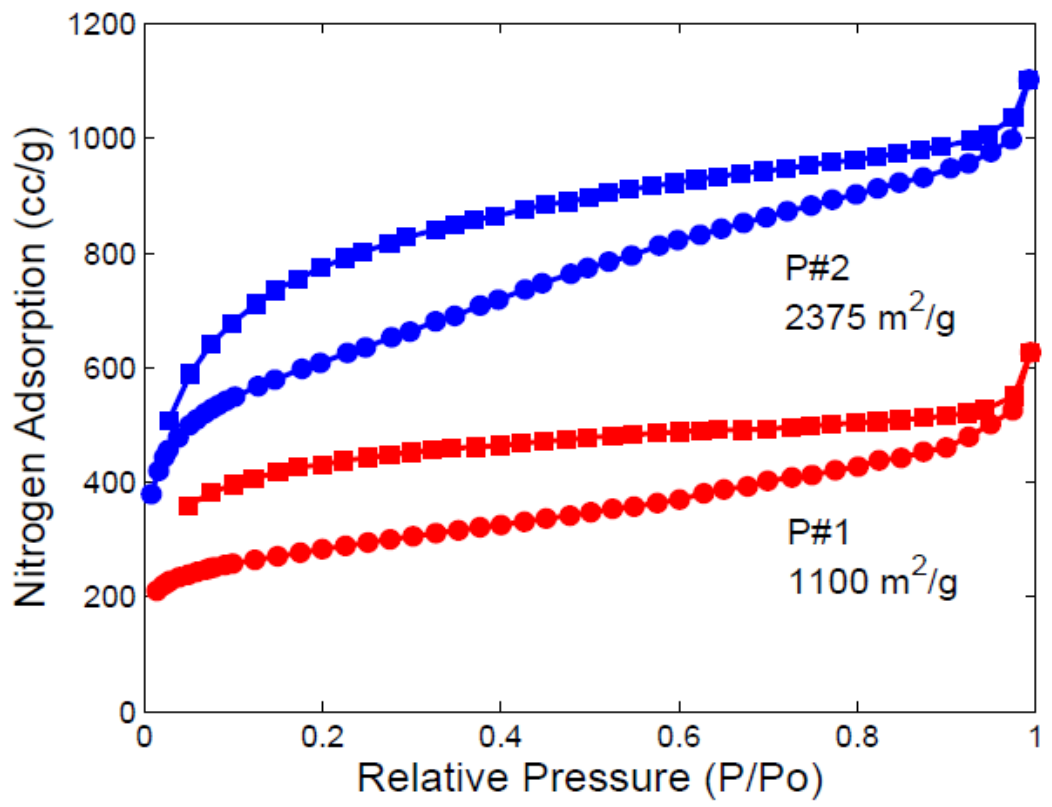

Figure 8. Nitrogen adsorption isotherms of $\mathrm{P} \# 1$ and $\mathrm{P} \# 2$. Circles and squares represent the adsorption and desorption parts of the isotherms, respectively. 


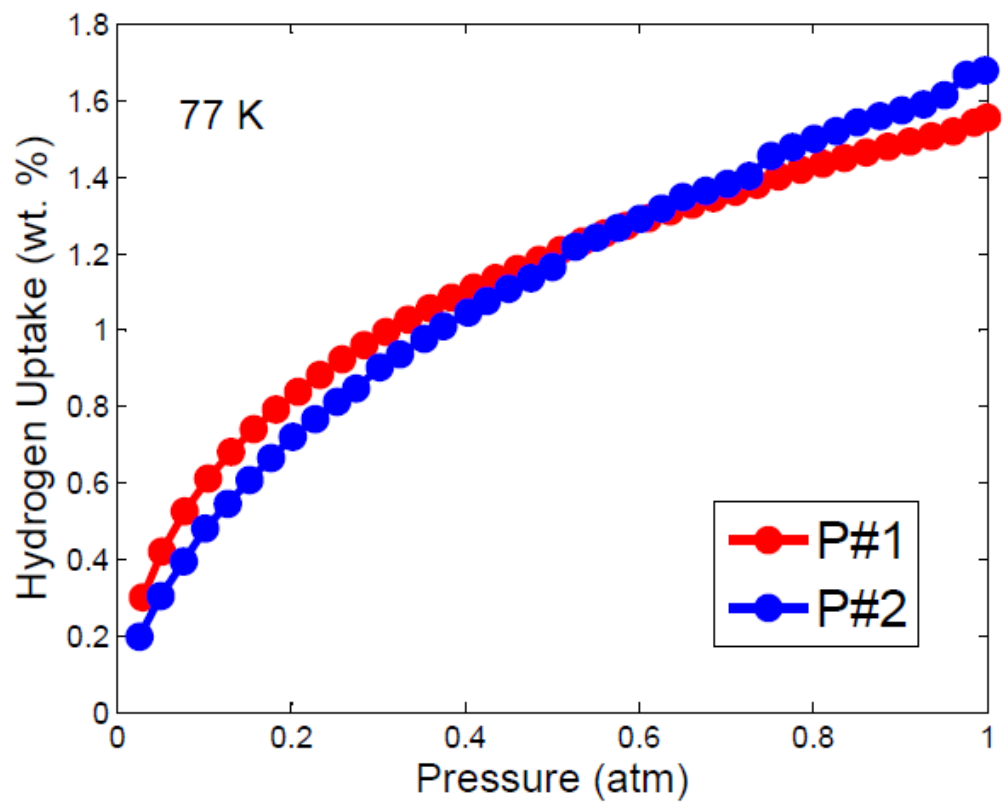

Figure 9. Hydrogen uptake of $\mathrm{P} \# 1$ and $\mathrm{P} \# 2$ at $77 \mathrm{~K}$.

Although P\#2 (i.e., $2375 \mathrm{~m}^{2} / \mathrm{g}$ ) has more than two times the surface area of P\#1 (i.e., $1100 \mathrm{~m}^{2} / \mathrm{g}$ ), they have similar hydrogen uptake at $77 \mathrm{~K}$ and $1 \mathrm{~atm}$. However, both P\#1 and P\#2 did not reach saturation at 1 atm.; therefore they can store more hydrogen at higher pressures. The high pressure sorption measurements at $77 \mathrm{~K}$ and $298 \mathrm{~K}$ are underway. The room temperature hydrogen uptake of $\mathrm{P} \# 1$ is given in Figure 10.

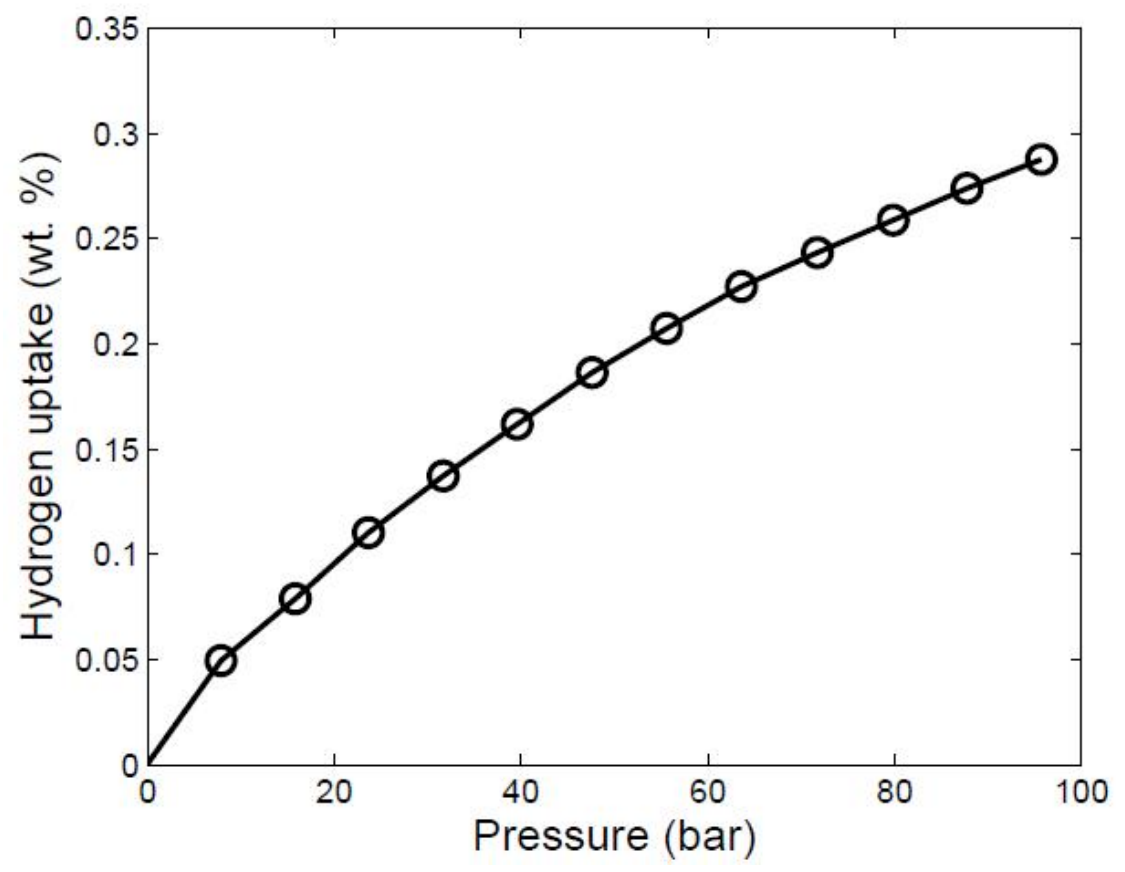

Figure 10. Hydrogen uptake of $\mathrm{P} \# 1$ at 298K. 


\subsection{Task 3 - Understanding of the hydrogen storage physisorption and chemisorption processes in conducting polymers (i.e., polyaniline).}

We have synthesized Polyemeraldine base, Polyemeraldine salt, polyleucoemeraldine and polypernigraniline materials for room temperature hydrogen storage. Figure 11 shows the schematic of the state change of emeraldine base to emeraldine salt with protonation. Figure 12 shows the hydrogen uptake of polyanilines at $298 \mathrm{~K}$. However, the experimental investigation did not show more than $0.1 \mathrm{wt} \%$.

(a)

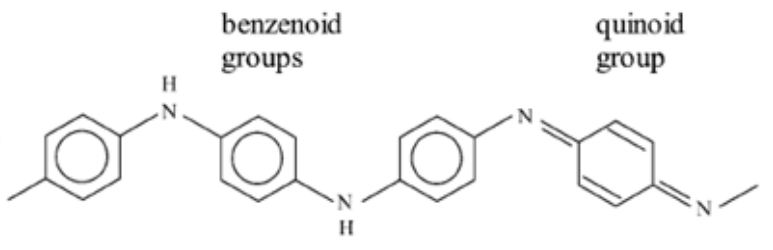

(b)

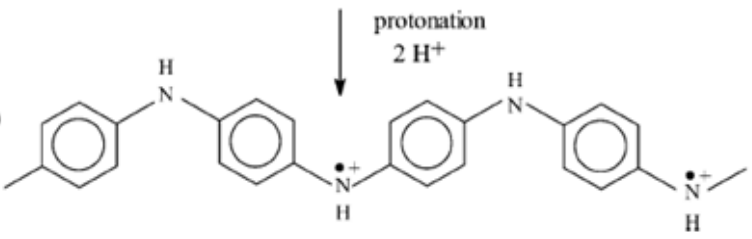

Figure 11. Scheme of protonation of the (a) emeraldine base form of (b) emeraldine salt

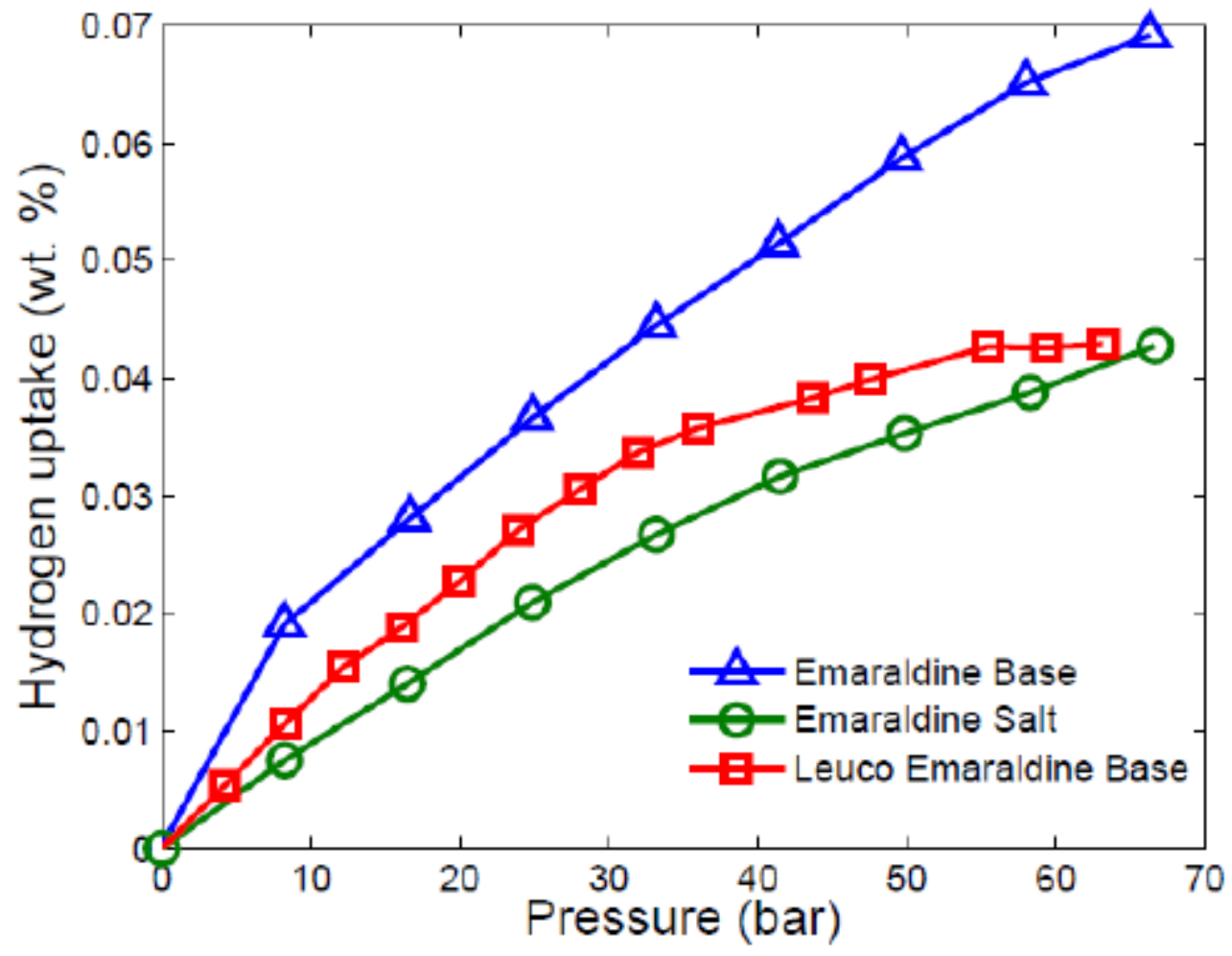

Figure 12. Hydrogen uptake of polyanilines at $298 \mathrm{~K}$. 


\subsection{Task 4 - Education and Outreach}

In this task, we integrated hydrogen storage and energy in a materials course. We organized seminars on hydrogen storage and fuel cells in order to educate researchers within USF and we also organized seminars in the energy related field regularly.

The research collaborators at Tuskegee University have designed a cohesive educational plan around the continuing research with three major objectives: (i) course development and hands-on training to students, (ii) outreach, and (iii) research laboratory development. The continuation of research on developing novel materials such as polymeric nanostructures will have significant impact on the educational, research activities and infrastructure capacity building at the Tuskegee University. It has enabled broadening of the laboratory component of our existing curriculum in Science and Engineering. The continuation of research studies will also be critical in training new and promising researchers by exposing them to the state-of-art technology and techniques. An integral component of the research is to disseminate the reversible, solid state hydrogen storage technology to the Tuskegee city and Macon county schools via outreach programs. In the on-going DOE-FHI project, the collaborating PI and their undergraduate research students have established a good relation with Tuskegee Public Elementary School System by visiting and demonstrating sustainable energy and environmental technologies to the school children (see pictures below).
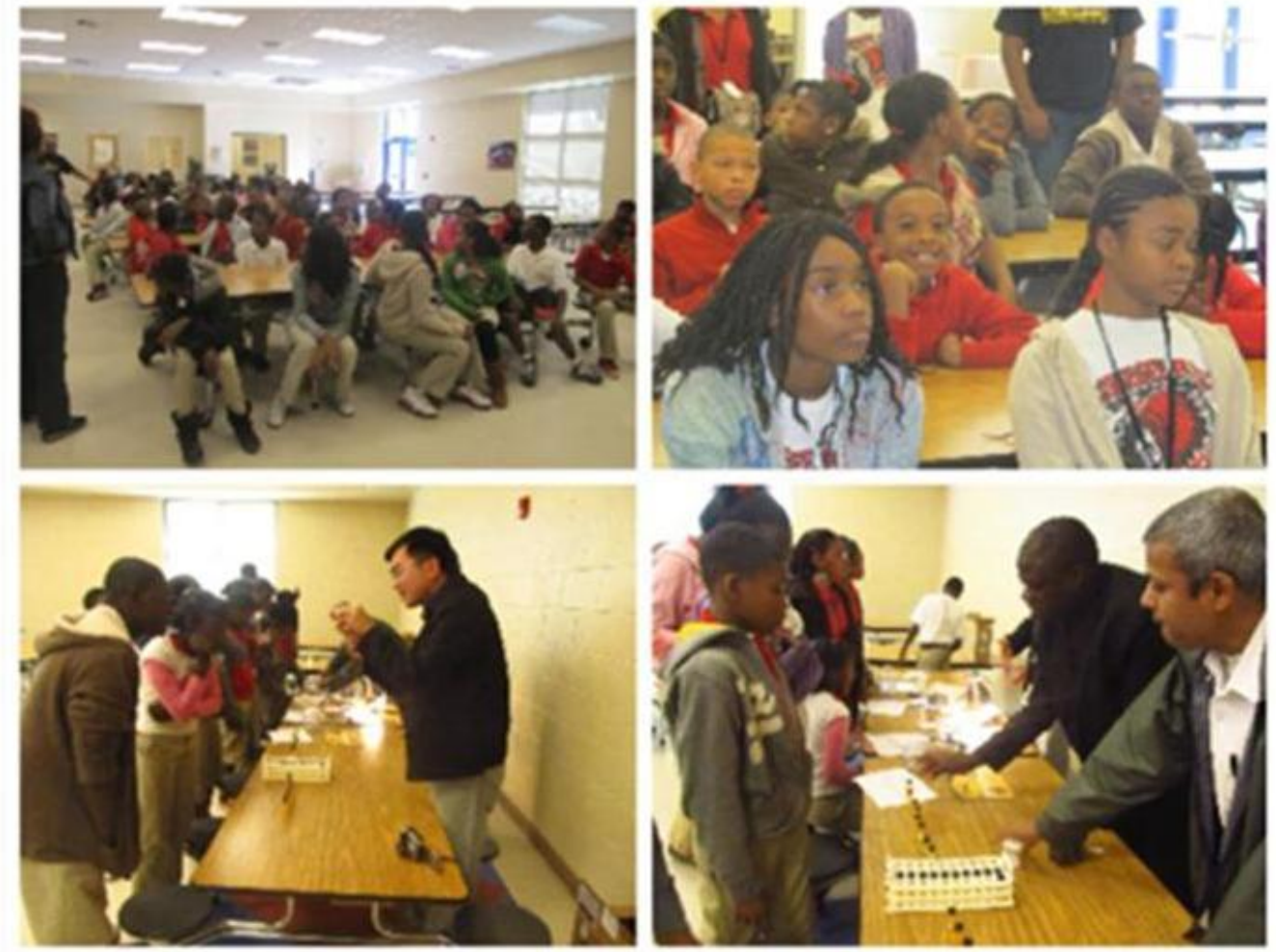

Figure 13. Education and Outreach activities at the Tuskegee Public Elementary School

\section{Products and Technology Transfer Activities}

\subsection{Resultant Publications}

- DE Demirocak, PhD. Thesis on Hydrogen Storage Materials (Hydrogen storage in hypercrosslinked polystyrene and Li-Mg-N-H complex hydride), University of South Florida (Submission Date: 04/02/2013) 
- A D'angelo, MSc. Thesis on Hydrogen Storage Materials (Investigation and Synthesis of Novel Graphene-Based Nanocomposites for Hydrogen Storage), University of South Florida (Submission Date: 03/22/2012)

- DE Demirocak, S Kuravi, MK Ram, CK Jotshi, SS Srinivasan, A Kumar, DY Goswami, EK Stefanakos, Investigation of Polyaniline Nanocomposites and Cross-Linked Polyaniline for Hydrogen Storage, Advanced Materials Research, 2012, 445, p. 571-6.

- DE Demirocak, MK Ram, SS Srinivasan, A Kumar, DY Goswami, EK Stefanakos, Spillover enhancement for hydrogen storage by Pt doped hypercrosslinked polystyrene, International Journal of Hydrogen Energy, 2012, 37(12), p. 12402-10.

- DE Demirocak, SS Srinivasan, MK Ram, DY Goswami, EK Stefanakos, Volumetric Hydrogen Sorption Measurements - Uncertainty Error Analysis and the Importance of Thermal Equilibration Time, International Journal of Hydrogen Energy, 2012, 38(3), p. 1469-77.

- DE Demirocak, MK Ram, SS Srinivasan, DY Goswami, EK Stefanakos, Hydrogen and Carbon Dioxide and Ammonia Adsorption in N doped High Surface Area Polymers. (in preparation)

- SS Srinivasan, R Ratnadurai, AR Phani, PC Sharma, DY Goswami, EK Stefanakos, Study of the growth of PANI nanofibers by various methods and its effect on Hydrogen Storage, Tatvanveshan, Volume 1, Issue 1, May-October 2012, Page 12-19, ISSN: 2319-5827.

\subsection{Networks and Collaborations}

Dr. Sesha S Srinivasan, Department of Physics, College of Arts and Sciences, Tuskegee University, Tuskegee, AL 36088, USA

\section{Concluding Remarks}

In this project, hydrogen storage in polymeric materials (spillover enhancement in hypercrosslinked polystyrene, nitrogen rich high surface area polymers, polyaniline and its composites) and error sources in hydrogen storage measurements were studied.

The spillover enhancement in the hypercrosslinked polystyrene was studied for the first time. The results showed that the bridge building technique is effective in enhancing the hydrogen storage capacity of the hypercrosslinked polystyrene compared to the wet impregnation method via spillover; however, the storage capacity $(0.36 \mathrm{wt} . \%)$ is too low for practical applications. More fundamental research is required to understand the different results reported in the literature as discussed in previous sections.

Nitrogen rich high surface area polymers were synthesized successfully. The room temperature storage capacity of these porous polymers is below $0.5 \mathrm{wt}$. \% based on their moderate surface areas $\left(\approx 2000 \mathrm{~m}^{2} / \mathrm{g}\right)$ and pore volumes.

Polyaniline and its derivatives (polyemeraldine base, polyemeraldine salt, polyleucoemeraldine and polypernigraniline) were synthesized; however, their storage capacity is below $0.1 \mathrm{wt} . \%$.

Volumetric hydrogen storage measurements were investigated via uncertainty analysis and guidelines were developed to improve the accuracy of the volumetric hydrogen storage measurements. The sample mass loading is one of the most important parameters to be considered regardless of the characteristics of the system and the sample. In general, the higher the sample mass loading the lower the uncertainty in measurements, the optimal loading being a function of the sample's intrinsic storage capacity. For physisorption based materials (porous high surface area materials) sample mass loading should be around $3 \mathrm{~g}$ to limit the uncertainty when using standard pressure and temperature transducers available in the market. Moreover, the effect of the thermal equilibration time on the hydrogen storage measurements has been investigated. If there is a possibility (i.e., when the instrument enclosure temperature and the room 
temperature are not the same or there is a temperature gradient within the instrument enclosure) that the actual gas temperature inside the reservoir volume could be different than the ambient temperature, then the thermal equilibration time needs to be taken into account. Depending on the size and shape of the reservoir volume, a temperature difference as small as $0.1 \mathrm{~K}$ can cause significant errors.

\section{References}

[1] Demirocak DE, Srinivasan SS, Ram MK, Goswami DY, Stefanakos EK. Volumetric hydrogen sorption measurements-Uncertainty error analysis and the importance of thermal equilibration time. International Journal of Hydrogen Energy 2013;38:1469-77.

[2] Von Zeppelin F, Haluska M, Hirscher M. Thermal desorption spectroscopy as a quantitative tool to determine the hydrogen content in solids. Thermochimica acta 2003;404:251-8.

[3] Miller MA, Wang CY, Merrill GN. Experimental and theoretical investigation into hydrogen storage via spillover in IRMOF-8. Journal of Physical Chemistry C 2009;113:3222-31.

[4] Tsao CS, Liu Y, Li M, Zhang Y, Leao JB, Chang HW, Yu MS, Chen SH. Neutron scattering methodology for absolute measurement of room-temperature hydrogen storage capacity and evidence for spillover effect in a Pt-doped activated carbon. Journal of Physical Chemistry Letters 2010;1:1569-73.

[5] Murray LJ, Dincă M, Long JR. Hydrogen storage in metal-organic frameworks. Chemical Society Reviews 2009;38:1294-314.

[6] Lemmon EW, Huber ML, Leachman JW. Revised standardized equation for hydrogen gas densities for fuel consumption applications. Journal of Research of the National Institute of Standards and Technology 2008;113:341-50.

[7] Setaram Instrumentation, [cited 2013 March 30]; Available from: http://www.setaram.com/PCTPro-E-E.htm.

[8] Demirocak DE, Ram MK, Srinivasan SS, Kumar A, Goswami DY, Stefanakos EK. Spillover enhancement for hydrogen storage by Pt doped hypercrosslinked polystyrene. International Journal of Hydrogen Energy 2012;37:12402-10.

[9] Kajiura H, Tsutsui S, Kadono K, Kakuta M, Ata M, Murakami Y. Hydrogen storage capacity of commercially available carbon materials at room temperature. Applied physics letters 2003;82:1105-7.

[10] Jhi SH, Ihm J. Developing high-capacity hydrogen storage materials via quantum simulations. MRS Bulletin 2011;36:198-204.

[11] Bhatia SK, Myers AL. Optimum conditions for adsorptive storage. Langmuir 2006;22:1688-700.

[12] Chung TCM, Jeong Y, Chen Q, Kleinhammes A, Wu Y. Synthesis of microporous boronsubstituted carbon $(\mathrm{B} / \mathrm{C})$ materials using polymeric precursors for hydrogen physisorption. Journal of the American Chemical Society 2008;130:6668-9.

[13] Kubas GJ. Fundamentals of $\mathrm{H} 2$ binding and reactivity on transition metals underlying hydrogenase function and H2 production and storage. Chemical Reviews 2007;107:4152-205.

[14] Prins R. Hydrogen spillover. Facts and fiction. Chemical Reviews 2012;112:2714-38. [15] Sermon P, Bond G. Hydrogen spillover. Catalysis Reviews - Science and Engineering 1974;8:211-39.

[16] Nikitin A, Ogasawara H, Mann D, Denecke R, Zhang Z, Dai H, Cho K, Nilsson A. Hydrogenation of single-walled carbon nanotubes. Physical Review Letters 2005;95:225507. [17] Ganz E, Dornfeld M. Storage capacity of metal-organic and covalent-organic frameworks by hydrogen spillover. Journal of Physical Chemistry C 2012;116:3661-6. 
[18] Li Y, Yang RT. Hydrogen storage on platinum nanoparticles doped on superactivated carbon. Journal of Physical Chemistry C 2007;111:11086-94.

[19] Tsao CS, Yu MS, Wang CY, Liao PY, Chen HL, Jeng US, Tzeng YR, Chung TY, Wu HC. Nanostructure and hydrogen spillover of bridged metal-organic frameworks. Journal of the American Chemical Society 2009;131:1404-6.

[20] Li Y, Yang RT. Hydrogen storage in metal-organic and covalent-organic frameworks by spillover. AIChE Journal 2008;54:269-79.

[21] Hasell T, Wood CD, Clowes R, Jones JTA, Khimyak YZ, Adams DJ, Cooper AI. Palladium nanoparticle incorporation in conjugated microporous polymers by supercritical fluid processing. Chemistry of Materials 2009;22:557-64.

[22] Sidorov S, Bronstein L, Davankov V, Tsyurupa M, Solodovnikov S, Valetsky P, Wilder E, Spontak R. Cobalt nanoparticle formation in the pores of hyper-cross-linked polystyrene: Control of nanoparticle growth and morphology. Chemistry of Materials 1999;11:3210-5.

[23] Lachawiec Jr AJ, Qi G, Yang RT. Hydrogen storage in nanostructured carbons by spillover: bridge-building enhancement. Langmuir 2005;21:11418-24.

[24] Tsao CS, Tzeng YR, Yu MS, Wang CY, Tseng HH, Chung TY, Wu HC, Yamamoto T, Kaneko K, Chen SH. Effect of catalyst size on hydrogen storage capacity of Pt-impregnated active carbon via spillover. Journal of Physical Chemistry Letters 2010;1:1060-3.

[25] Wang L, Yang RT. Hydrogen storage on carbon-based adsorbents and storage at ambient temperature by hydrogen spillover. Catalysis Reviews - Science and Engineering 2010;52:41161.

[26] Ben T, Ren H, Ma S, Cao D, Lan J, Jing X, Wang W, Xu J, Deng F, Simmons JM. Targeted synthesis of a porous aromatic framework with high stability and exceptionally high surface area. Angewandte Chemie 2009;121:9621-4. 


\title{
Florida Hydrogen Initiative: Advanced HiFoil ${ }^{\mathrm{TM}}$ Bipolar Plates
}

Final Report

April 30, 2013

Principal Investigator:

James Braun

james.braun@enerfuel.com, 561-868-6720, ext 227

Project Manager:

Dr. Michel Fuchs

michel.fuchs@enerfuel.com, 561-868-6720, ext 239

Project Period: December 1, 2010 to April 30, 2013

Delivered to:

\author{
Dr. David Block \\ Florida Solar Energy Center \\ University of Central Florida \\ 1679 Clearlake Road \\ Cocoa, Florida 32922
}

Contract No. 20126050, PO: 231090

This report does not contain any proprietary, confidential or otherwise restricted information

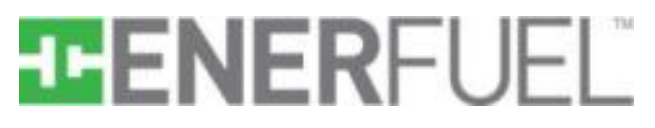

1501 Northpoint Pkwy, Suite 101

West Palm Beach, Florida 33407

561-868-6720

www.enerfuel.com 
Final Report: Advanced HiFoil ${ }^{\mathrm{TM}}$ Bipolar Plates

April 30, 2013

\section{Final Report Advanced HiFoil ${ }^{\mathrm{TM}}$ Bipolar Plates}

UCF Contract Number: 20126050, PO: 231090

April 30, 2013

\section{Executive Summary}

The Advanced HiFoil Bipolar Plates project is a comprehensive R\&D effort aimed at advancing renewable energy technologies in Florida. The goal of the Advanced HiFoil ${ }^{\mathrm{TM}}$ Bipolar Plates, or simply the "HiFoil" project, is to provide a durable, low cost bipolar plate for use in advanced high temperature proton exchange membrane (HTPEM) fuel cells for stationary, vehicle auxiliary power units and electric vehicle range extender (EVRE) applications. As a result of this project, EnerFuel successfully developed and demonstrated a mass producible bipolar plate with unparalleled performance and physical attributes.

EnerFuel's patent pending HiFoil ${ }^{\mathrm{TM}}$ laminate technology was used as the starting point for this development. HiFoil ${ }^{\mathrm{TM}}$ laminates consist of two layers of flexible graphite that are bonded to a thin metal foil using a proprietary high temperature conductive adhesive. As a baseline, these laminates provide the following attributes:

- Two orders of magnitude lower cost than gold coated stainless steel

- Greater corrosion resistance

- Excellent performance in 4-cell testing (using corrugated flow field inserts)

- HTPEM stack tested for 1,000 hours with no failure

- Ex-situ thermal cycled from room temperature to $200^{\circ} \mathrm{C}$ over 10,000 times with no delamination

Unfortunately these laminates were only capable of being formed into corrugations. They were used as flow field inserts in a complex air-cooled fuel cell that required eleven layers of materials to function as the bipolar plate. In addition, the corrugated laminate flow field design did not accomodate the use of a liquid coolant for recovery of fuel cell waste heat. Yet, effective heat recovery dramatically improves energy efficiency and lowers the operating cost for HTPEM fuel cell systems, particularly for stationary combined heat and power (CHP) applications.

In order to accomplish the project goal of providing a durable, low cost bipolar plate for a wide range of HTPEM applications, and based on EnerFuel's existing technology, two generations of advanced bipolar plates were developed. The first generation bipolar plate (Gen1 HiFoil ${ }^{\mathrm{TM}}$ ) involved the use of a laminate with thicker and more thermally conductive flexible graphite outer layers. The intent was to emboss the flow field channels into these outer layers, and to use 
these layers to conduct heat to the outer edges of the cell. The thin metal layer at the core of the laminate was also modified to improve conductivity through the z-axis of the bonded layers.

However, the Gen1 efforts revealed that the advanced flexible graphite was already provided at near the maximum theoretical density, thus the material did not allow for embossing the required reactant channels. The flexible graphite vendor was willing to provide the material in a less dense form, yet this approach has additional drawbacks. Lowering the density of the foil, while allowing the channels to be imprinted, would have resulted in low and high density regions within the bipolar plate, resulting in high acid up-take from the MEA and low structural integrity at these low density regions. In addition, the modification to the metal core layer necessarily contained small amounts of thallium, a toxic substance. As a result of the shortcomings in the Gen1 approach, the design was reevaluated and an alternate approach was selected.

Although not quite as low in material cost as the Gen1 approach, the new Gen2 HiFoil ${ }^{\mathrm{TM}}$ approach is less challenging from a manufacturing standpoint, and is better suited for high volume bipolar plate production. The Gen 2 approach maintains the thicker layers of advanced flexible graphite from the previous generation, but instead of laminating the flexible graphite to a metal foil core, the flexible graphite is laminated between two outer layers of moldable graphite composite. In this arrangement, the flexible graphite's high in-plane thermal conductivity can still be exploited for conducting heat out to the edge of the cell, without the need to emboss any flow channels in this difficult to form material. Due to the moldable nature of the outer graphite composite material, the flow fields can be easily molded into the outer layers of the bipolar plate. In addition, the polymer binder in the outer composite layer acts as an effective barrier to acid, thereby mitigating issues associated with acid up-take from the MEA.

In situ evaluation of the Gen2 laminate bipolar plates consisted of 4, 36 and 132-cell fuel cell stack testing, as well as validation in a $3 \mathrm{~kW}$ net stationary power system. Electrical performance of the fuel cell stacks matched very closely with MEA manufacture performance curves. In addition, stack testing revealed a typical cell-to-cell temperature spread between $7^{\circ} \mathrm{C}$ and $10^{\circ} \mathrm{C}$. In system testing this spread increased, but still remained below $14^{\circ} \mathrm{C}$. Such temperature uniformity is critical in maintaining optimal fuel cell performance. This is especially important when utilizing a fuel processor, since cells operating at lower temperature are more susceptible to the carbon monoxide contained in the hydrogen rich stream.

Along with the MEA, the bipolar plate makes up the core of the HTPEM fuel cell power system, and the cost of these two components comprise the bulk of the fuel cell system cost. Cost quotes for molded HiFoil ${ }^{\text {TM }}$ bipolar plates yield a bipolar plate cost of $\$ 840$ per $\mathrm{kW}$ of net electrical power, at production volumes of 2000 systems per year. In yearly system volumes of 50,000 , this cost would be expected to drop well below $\$ 500$ per $\mathrm{kW}$, in line with DOE overall cost targets for stationary CHP fuel cell systems. The overall objectives of the program were successfully achieved and are highlighted below in Table 1.

UCF Contract Number: 20126050, PO: 231090 
Table 1. Accomplished Objectives vs. HiFoil Technology Generation

\begin{tabular}{|c|c|c|}
\hline \multirow[b]{2}{*}{ Objective } & \multicolumn{2}{|c|}{ Objective Accomplished? } \\
\hline & Gen1 HiFoil & Gen2 HiFoil \\
\hline 1. Moldable Plate & No & Yes \\
\hline 2. Integral Seal & No & Yes \\
\hline 3. Stack Validation & No & Yes \\
\hline 4. Meet DOE Targets & No & Yes $^{*}$ \\
\hline
\end{tabular}

*Supports DOE Table 3.4.5 Technical Targets: 1-10 kW Residential Combined Heat and Power and Distributed Generation Fuel Cell Systems Operating on Natural Gas (see http://www1.eere.energy.gov/hydrogenandfuelcells/mypp/pdfs/fuel_cells.pdf) by virtue of successful demonstration in a comparable system, and by plate cost projection (extrapolation of vendor quotes) to high volumes (50,000 systems per year).

The development efforts supported by this program have been remarkably successful. EnerFuel's HiFoil ${ }^{\mathrm{TM}}$ bipolar plate and related fuel cell stack technology is currently patent pending under U.S. Patent Applications 13/566347, 13/566406, 13/566531, 13/566551, $13 / 566585$, and $13 / 566629$. This technology is being deployed in production ready 36 -cell and 132-cell HTPEM fuel cell stacks. The 36-cell stacks are integrated into EnerFuel's commercially available 1.5kW Fuel Cell Power System (FCPS); while the 132-cell stacks are integrated into EnerFuel's advanced 3kW stand-alone stationary power system (currently available to selected partners). More information is available at http://www.enerfuel.com/solutions/. 


\section{Table of Contents}

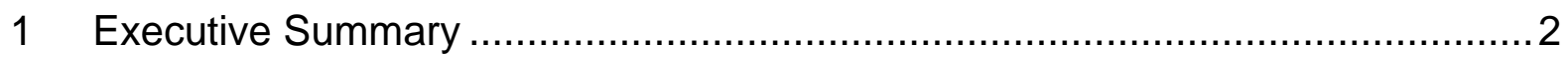

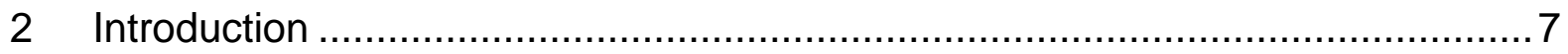

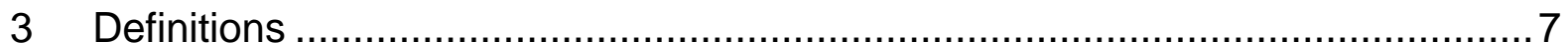

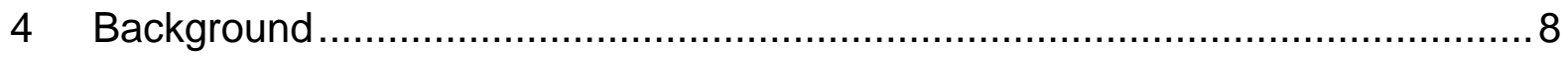

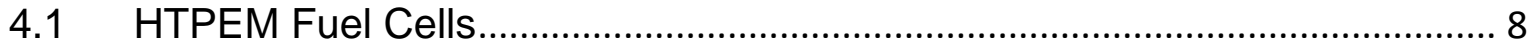

4.2 Advanced Plate Materials .............................................................................. 10

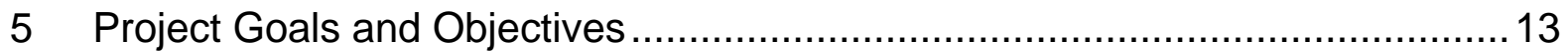

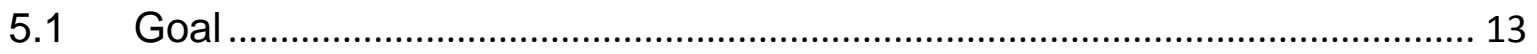

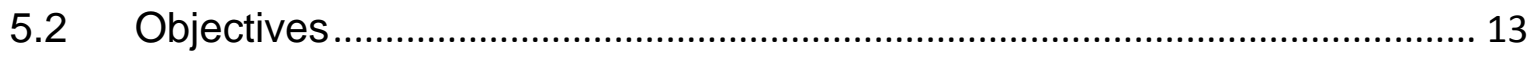

5.2.1 Initial Objectives .............................................................................. 13

5.2.2 Modified Objectives......................................................................... 13

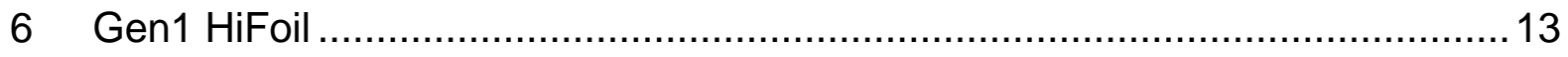

6.1 Gen1 HiFoil Approach ............................................................................... 13

6.2 Gen1 HiFoil Results .............................................................................. 14

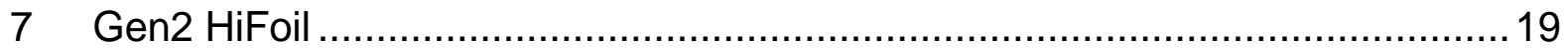

7.1 Gen2 HiFoil Approach ........................................................................... 19

7.2 Gen2 HiFoil 4-Cell Stack Test Results .......................................................... 21

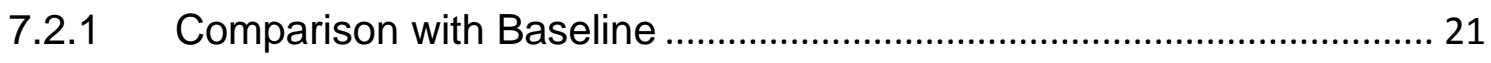

7.2.2 Seal Geometry Optimization and New Stack Compression Hardware 23

7.2.3 Flow Field Geometry Optimization ........................................................ 24

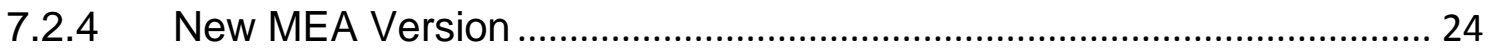

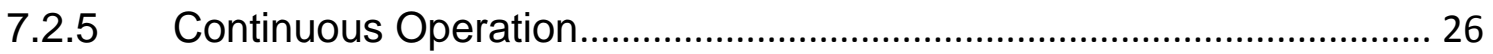

7.3 Gen2 HiFoil 36-Cell Module Test Results ...................................................... 28

7.4 Gen2 HiFoil Laminate Bipolar Plate Costs...................................................... 35 
Final Report: Advanced HiFoil ${ }^{\mathrm{TM}}$ Bipolar Plates

April 30, 2013

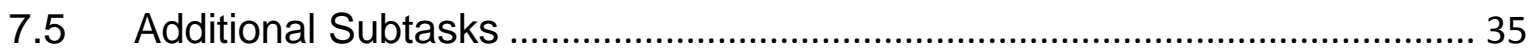

7.6 Gen2 HiFoil 132-Cell Stand-Alone System Test Results ............................. 36

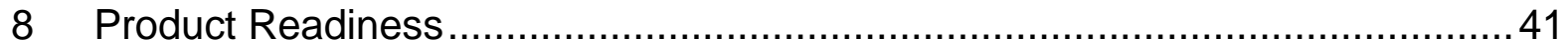

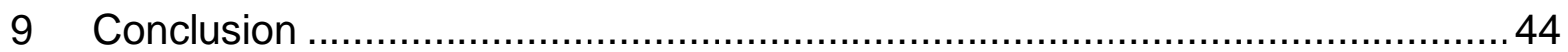

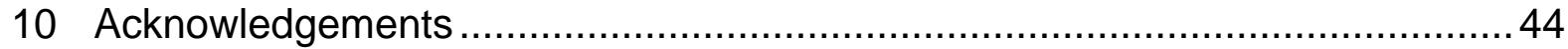




\section{Introduction}

The goal of the Advanced HiFoil Bipolar Plates, or simply the "HiFoil" project, was to develop durable, low cost bipolar plates for use in advanced high temperature proton exchange membrane (HT-PEM) fuel cell stacks. Using EnerFuel's patent pending HiFoil ${ }^{\mathrm{TM}}$ laminated technology, EnerFuel successfully developed and demonstrated a mass producible bipolar plate with unparallel performance and physical attributes. Capitalizing on the moldability of graphite composite materials and the high, in-plane thermal conductivity of graphite foil, the bipolar plate allows for well characterized manufacturing processes, such as compression molding or embossing, and also implementation of edge cooling strategies. The ability to edge cool the fuel cell stack enabled the design of the fuel cell stack hardware to be much more compact and lower part count, In addition, a wider range of coolants can be exploited, since the fluid flows external to the electrical active area in a separate heat exchanger. Cost quotes for molded HiFoil ${ }^{\mathrm{TM}}$ bipolar plates show a stationary system bipolar plate cost of $\$ 840$ per $\mathrm{kW}$ of net electrical power, at production volumes of 2000 systems per year. In yearly system volumes of 50,000 , this cost would be expected to drop well below $\$ 500$ per $\mathrm{kW}$.

The bipolar plates developed during the course of this effort were the result of two design iterations. The first approach, Gen1, utilized EnerFuel's patent pending HiFoil ${ }^{\mathrm{TM}}$ Laminate technology (a lightweight, corrugated laminate consisting of a very thin, coated stainless steel sheet laminated between two flexible graphite layers) with the aim of embossing flow field channels into the flexible graphite layers. However this approached revealed that the graphite foil's densification did not allow for reactant channels to be molded or embossed. Lowering the density of the foil, although allowing channels to be imprinted, would have lead to high acid take up from the MEA and low structural integrity of the plate structure. The corrugated laminate design also included a high part count per cell, difficulty achieving adequate reactant flow uniformity and the inability to use a liquid coolant for recovery of fuel cell waste heat.In addition, the necessary coating applied to the stainless steel contained thallium, a toxic substance.

As a result of these shortcomings, the design was reevaluated and an alternate approach selected based on another EnerFuel bipolar plate concept. Although not quite as low cost as the Gen1 approach, the new "Gen2" design was substantially less technically challenging and still well suited for volume manufacturing processes.

The Gen 2 approach takes advantage of the same graphite foil but instead of using it to laminate a stainless steel sheet it is laminated between layers of graphite composite material. In this arrangement, the foil's high in-plane thermo-conductivity can still be exploited without limitations in molding flow fields. Due to the moldable nature of the graphite composite material, the flow fields can easily be molded into the plates. In addition, the high polymer content of the 


\section{Final Report: Advanced HiFoil ${ }^{\mathrm{TM}}$ Bipolar Plates}

April 30, 2013

composite layer acts as an effective barrier to acid, thereby mitigating issues associated with absorption of acid from the MEA.

The Gen2 HiFoil concept eventually lead to the final bipolar plate design adopted for use in EnerFuel's fuel cell stacks. Description of the evolution of the design and its validation in 4, 36 and 132-cell stack testing, as well as validation in a $3 \mathrm{~kW}$ net stationary power system are discussed in this report.

\section{Definitions}

ABBA: Gen2 HiFoil ${ }^{\mathrm{TM}}$ bipolar plate configuration with A and B layers

CHP: Combined heat and power

DOE: US Department of Energy

FCPS: Fuel cell power system

HTPEM: High temperature, proton exchange membrane

LHV: Lower heating value

LPG: Liquefied petroleum gas

MEA: Membrane electrode assembly

SR: Stoichiometric ratio

UCF: University of Central Florida

\section{Background}

\subsection{HTPEM Fuel Cells}

HTPEM fuel cells have specific characteristics that make them unique with respect to other types of fuel cells:

- Membrane based on high temperature polymer and phosphoric acid

- Operating temperatures between $120^{\circ} \mathrm{C}$ to $180^{\circ} \mathrm{C}$

- High tolerance to carbon monoxide impurity in the fuel stream

- Humidification of reactants is not required

- Long term stability

EnerFuel has been developing HTPEM fuel cells since 2007, and has specifically demonstrated their utility in applications ranging from electric vehicle range extension, vehicle auxiliary power unit, stationary distributed generation, combined heat and power (CHP), and back-up power systems. A primary benefit of HTPEM systems includes easy adaptation for use with either hydrogen or readily available fuels (i.e. natural gas, propane, gasoline, diesel, biogas, ethanol and methanol). Another major advantage is the operating temperature range, which is high 
enough to make efficient use of the waste heat, but low enough to provide for rapid startup and to make use lower cost materials of construction.

However, since HTPEM fuel cells are a relatively new technology, there are only a limited number of fuel cell component suppliers who offer materials designed for the elevated temperature range and corrosive environment. Prior to this program, EnerFuel had developed an air-cooled HTPEM stack using gold coated stainless steel bipolar plates (Fig.1), and later evaluated an experimental grade of an expanded graphite/resin composite (Fig.2). This composite has since been discontinued due to very high manufacturing costs.

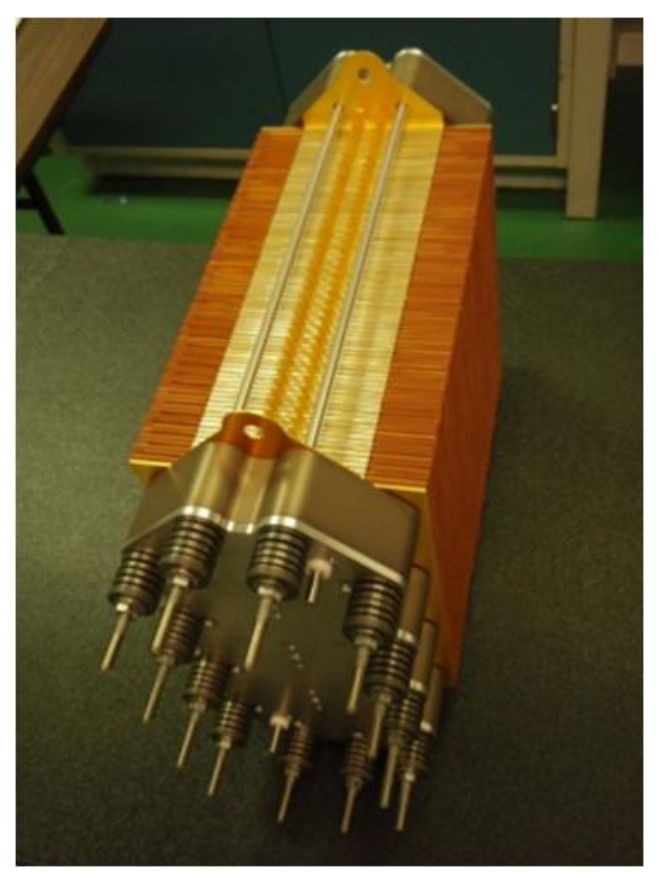

Figure 1. One of EnerFuel's early air-cooled HTPEM stacks with gold coated stainless steel bipolar plates. 
April 30, 2013

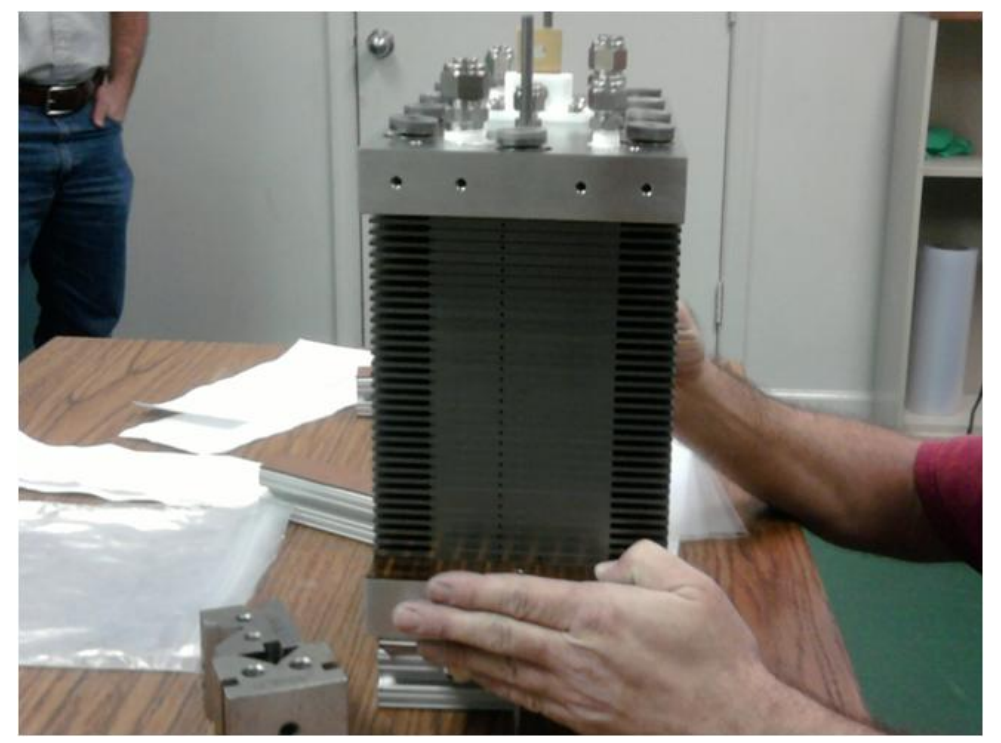

Figure 2. Another of EnerFuel's early air-cooled HTPEM stacks, this time with an experimental expanded graphite/resin composite material that requires machined flow fields. This material has since been discontinued by the supplier due to high manufacturing costs.

\subsection{Advanced Plate Materials}

After some internal development, EnerFuel created a replacement for the earlier gold coated stainless steel. Due to its much higher thermal conductivity, this replacement material became known as the HiFoil ${ }^{\mathrm{TM}}$ Laminate. The HiFoil ${ }^{\mathrm{TM}}$ Laminate consisted of a very thin metal core with modified surface structure, that was laminated between two sheets of flexible graphite using a proprietary high temperature conductive adhesive (Fig.3).
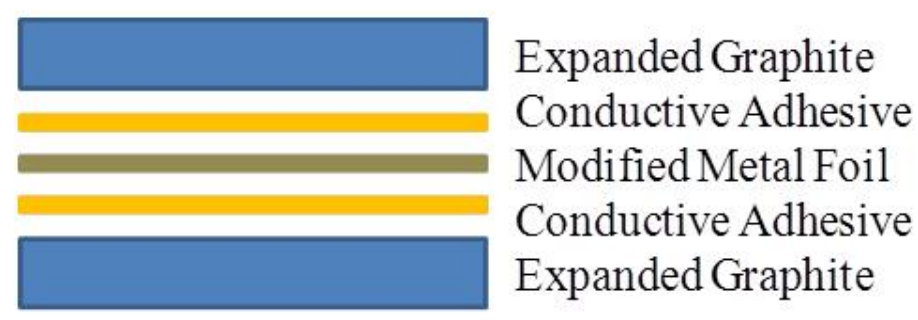

Figure 3. Sketch of the HiFoil ${ }^{\mathrm{TM}}$ Laminate configuration.

The laminate was corrugated with the same equipment used to form the gold plated stainless steel corrugations (Fig.4), and was tested in a 4-cell stack for comparison with the gold coated stainless steel technology (Fig.5-6). The laminates were estimated to provide about 30 times higher heat dissipation from the MEA, enabling operation at higher current density. While the 
gold coated stainless steel plates had become perforated after 1,000 hours of operation, the HiFoil $^{\mathrm{TM}}$ Laminates showed no visible signs of deterioration over the same period.

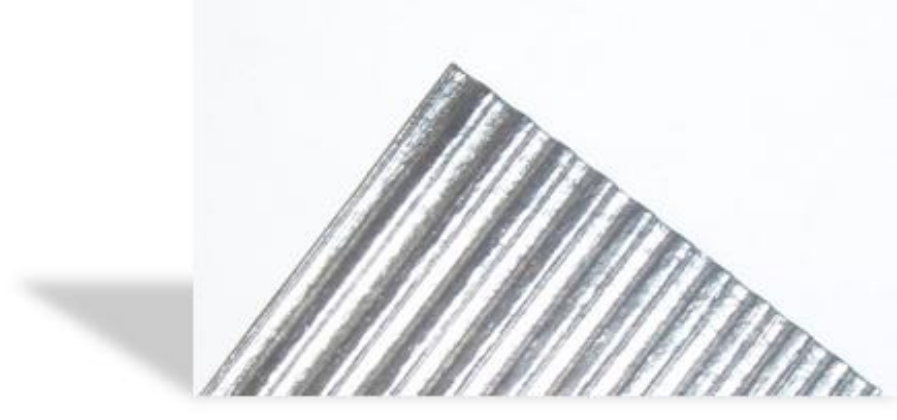

Figure 4. Picture of a corrugated flow field insert formed from the HiFoil ${ }^{\mathrm{TM}}$ Laminate.

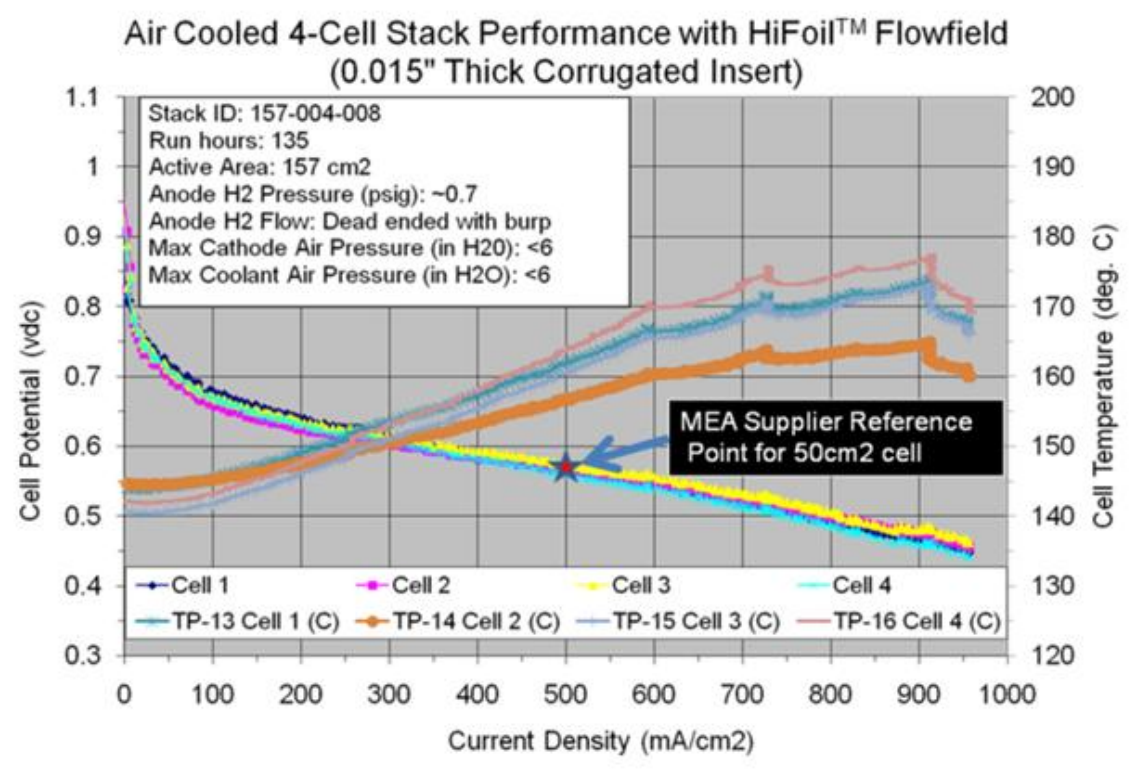

Figure 5. Polarization curve for an air-cooled 4-cell stack with corrugated HiFoil ${ }^{\mathrm{TM}}$ Laminate flow field inserts. A current density of over $950 \mathrm{~mA} / \mathrm{cm}^{2}$ was obtained with adequate cell cooling. 


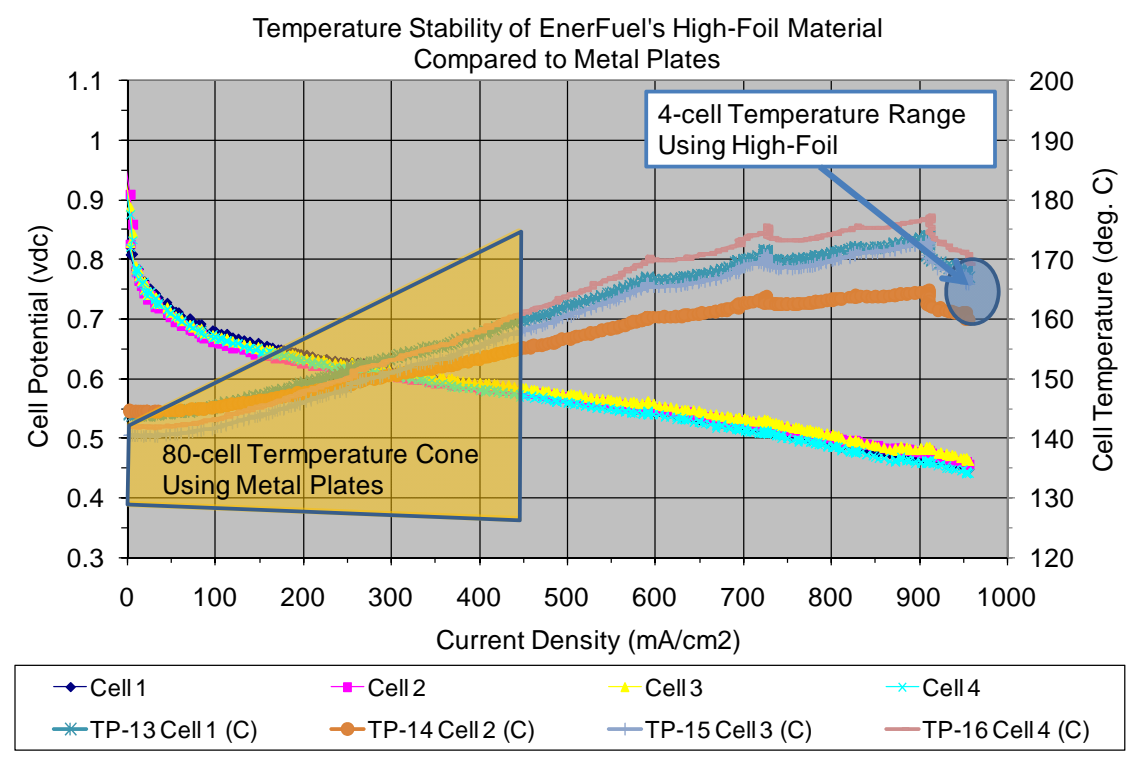

Figure 6. Overlay of the temperature spread for an air-cooled stack using gold coated stainless steel flow field inserts (depicted by the gold-colored overlay) onto the polarization data for the stack with HiFoil ${ }^{\mathrm{TM}}$ Laminate flow field inserts.

In ex-situ testing, the HiFoil TM Laminates were thermally cycled from room temperature to over $200^{\circ} \mathrm{C}$ for $>10,000$ cycles with no delamination.

In summary, the HiFoil ${ }^{\mathrm{TM}}$ Laminate technology demonstrated the following attributes:

- Two orders of magnitude lower cost than gold coated stainless steel

- Greater corrosion resistance

- Excellent performance in 4-cell testing (using corrugated flow field inserts)

- HTPEM stack tested for 1,000 hours with no failure

- Ex-situ thermal cycled from room temperature to $200^{\circ} \mathrm{C}$ over 10,000 times with no delamination

However, the cons of a fuel cell design using corrugated flow field inserts include a high part count per cell, difficulty achieving adequate reactant flow uniformity and the inability to use a liquid coolant for recovery of the waste heat. Consequently, EnerFuel proposed the current project to investigate forming low cost traditional flow fields in the HiFoil ${ }^{\mathrm{TM}}$ Laminate material in order to create a bipolar plate. 
Final Report: Advanced HiFoil ${ }^{\mathrm{TM}}$ Bipolar Plates

April 30, 2013

$5 \quad$ Project Goals and Objectives

\subsection{Goal}

To provide a durable, low cost bipolar plate for use in High Temperature PEM fuel cells.

\subsection{Objectives}

\subsubsection{Initial Objectives}

1. Double-sided Plate - Achieve two-sided flow field formation in HiFoil laminates using a lab-scale embossing process.

2. Integral Seal - Achieve a robust seal against porosity around fluid ports and plate edges using a dispensed sealant or coating.

3. Stack Validation - Obtain performance data in an air-cooled, reformate capable HTPEM fuel cell stack.

4. Meet DOE Targets - Meet or exceed DOE bipolar plate technical targets.

\subsubsection{Modified Objectives}

1. Moldable Plate - Develop a multi-layer bipolar plate configuration that permits molding of the reactant flow field channels and enables edge heat conduction.

2. Integral Seal - Achieve a robust seal against porosity around fluid ports and plate edges using a dispensed sealant or coating.

3. Stack Validation - Obtain performance data in a liquid-cooled, reformate capable HTPEM fuel cell stack with $1 \mathrm{~kW} \underline{\text { entput. }}$

4. Meet DOE Targets - Meet or exceed DOE bipolar plate technical targets.

\section{Gen1 HiFoil}

\subsection{Gen1 HiFoil Approach}

The Gen1 HiFoil ${ }^{\mathrm{TM}}$ bipolar plate approach included increasing the thickness of the flexible graphite layer, such that the flow fields could be embossed using a suitable die set (Fig.7). The conduction of the cell heat to the edge of the plate had already been demonstrated with an experimental grade expanded graphite composite bipolar plate, so there was no need to incorporate cooling channels into the plate design. This new approach would use low cost, commercially available expanded graphite and include a secondary sealing operation around the plate edges. 


\section{HiFoil $^{\mathrm{TM}}$ Laminate Bipolar Plate}

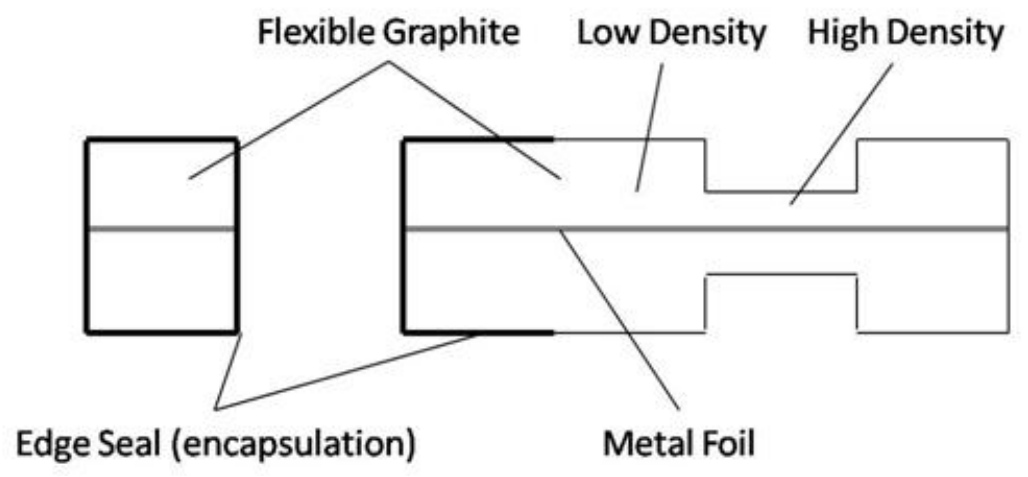

Figure 7. Sketch of the proposed Gen1 HiFoil TM bipolar plate configuration (edge view) showing the embossed flow field channels and encapsulated edges.

\subsection{Gen1 HiFoil Results}

The metal foil layer was modified to dramatically increase its surface area by electrolytic deposition of a nodular nickel coating (Fig.8). This coating had a typical thickness of 10 microns. A high temperature conductive coating comprising graphite and polymer was applied over the nodular nickel coating for the purpose of corrosion protection and to promote adhesion to the flexible graphite layers. 
April 30, 2013

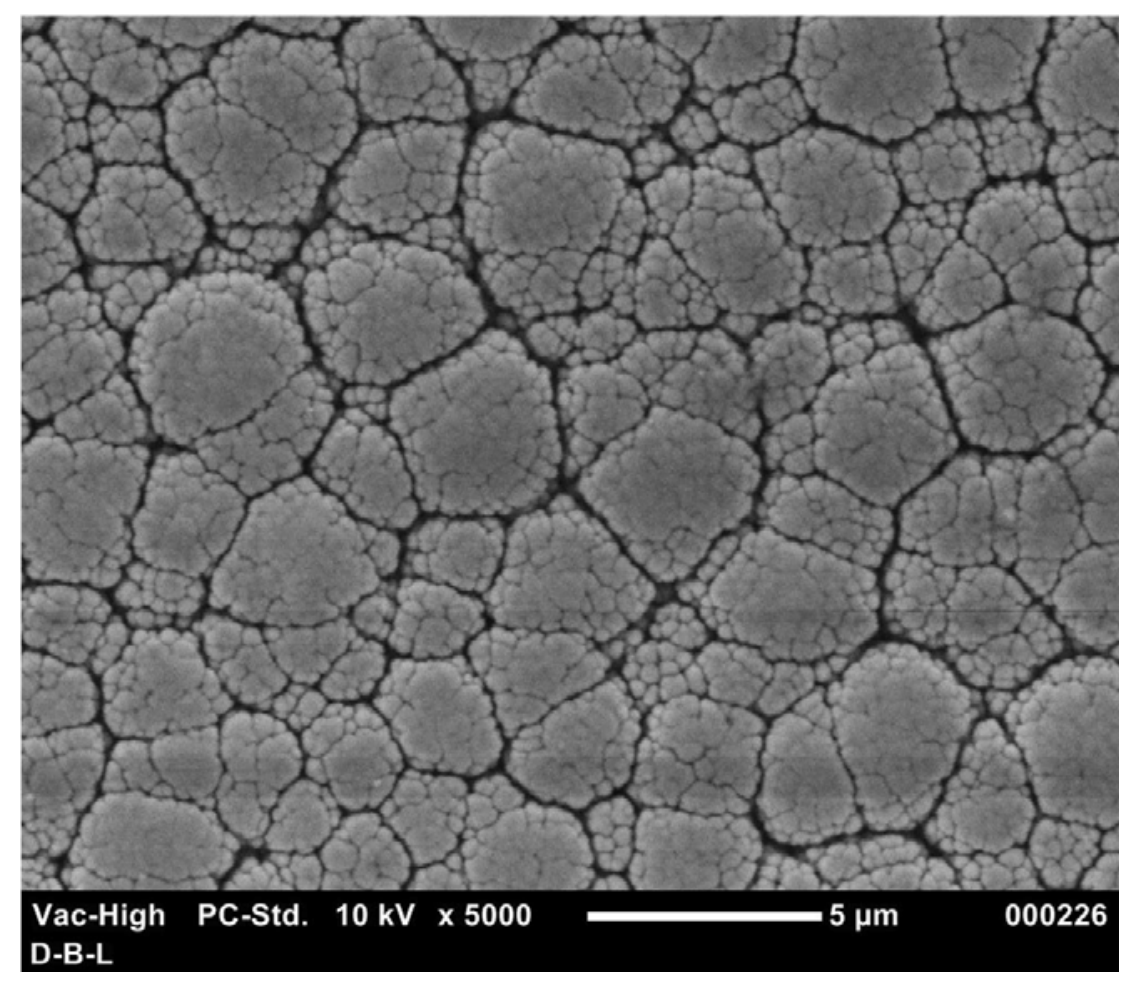

Figure 8. Photomicrograph showing the nodular nickel layer electrodeposited onto the surface of the stainless steel foil.

EnerFuel continued to develop its proprietary high temperature conductive adhesive to enable process scale, up while providing more uniform coverage of the laminate surfaces. Initially a slurry process and several types of dry powder application were evaluated. Powder coating using commercially available powder coating equipment resulted in very high electrical resistance due to preferential deposit of the polymer component. Subsequently, EnerFuel developed a proprietary method of applying the conductive coating, using existing in-house equipment (Fig.9-11), resulting in a flexible highly conductive coating (Fig.12-14). 
April 30, 2013

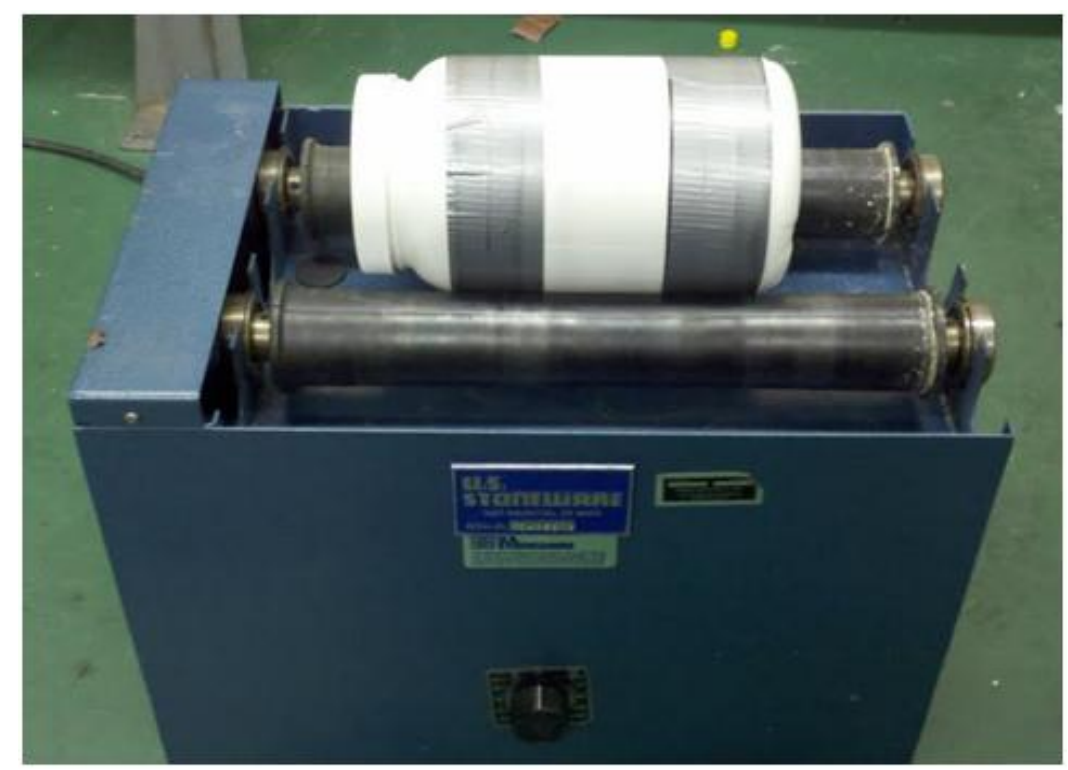

Figure 9. Roll mill used to blend the components of the conductive coating before application to the metal foil.

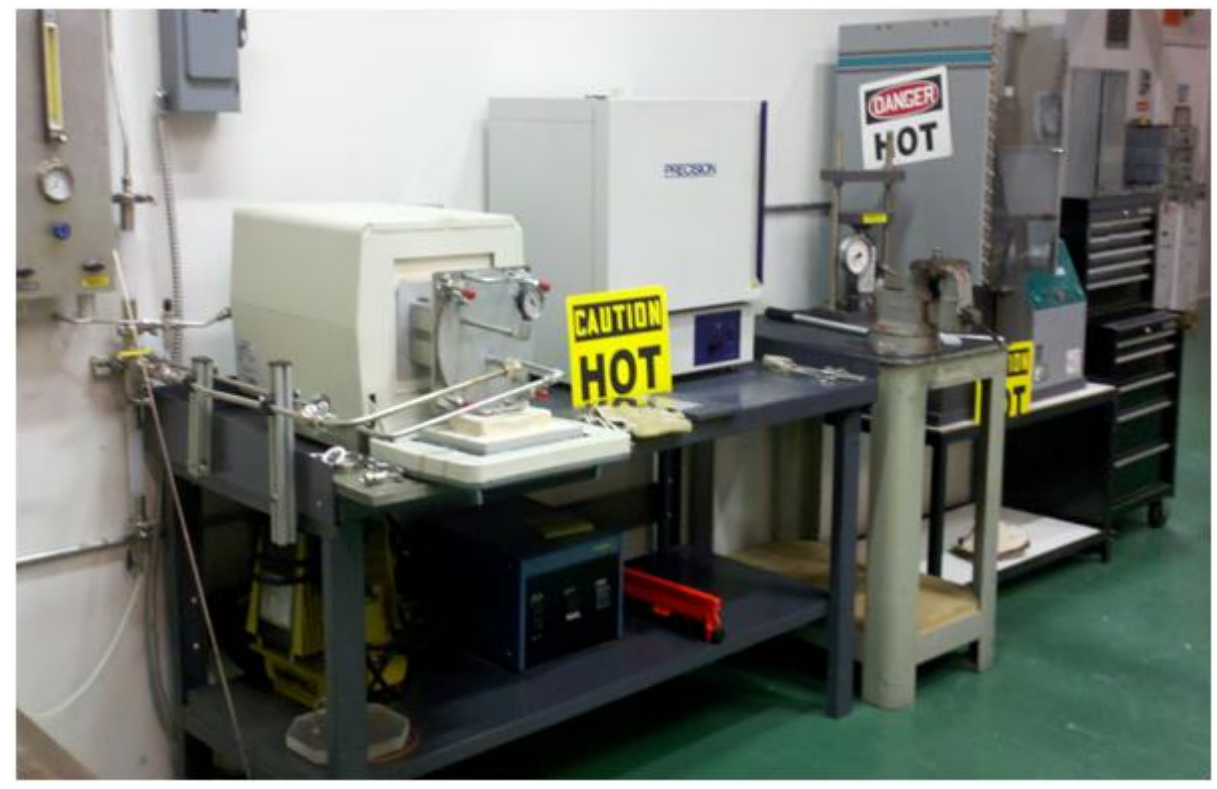

Figure 10. Controlled atmosphere furnace used to bake the conductive coating after application to the metal foil. 
April 30, 2013

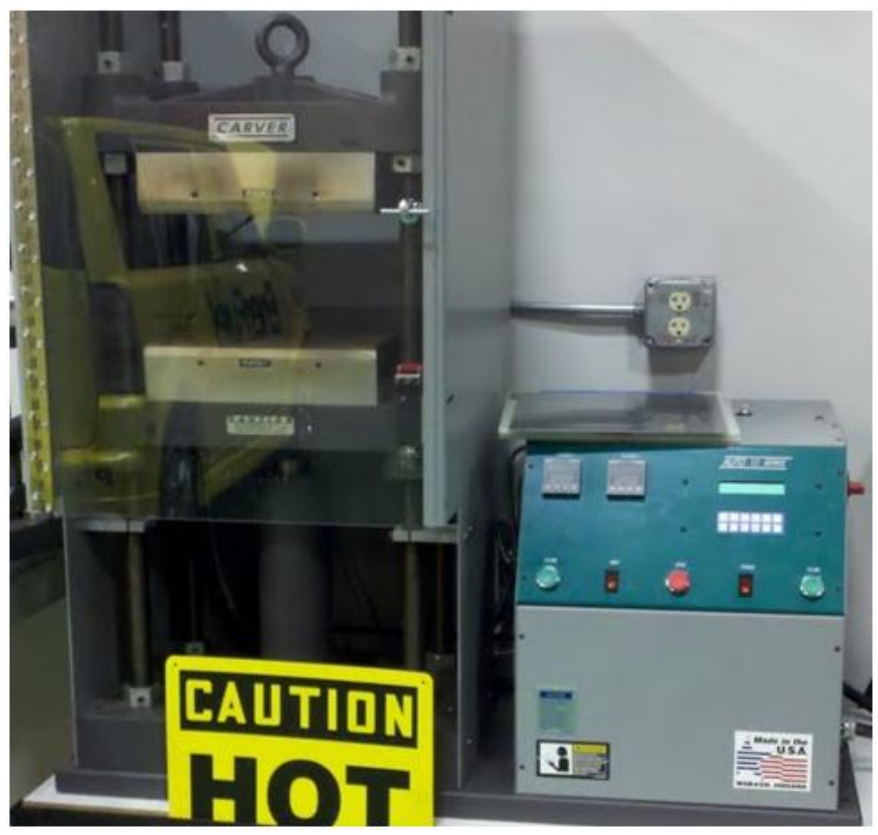

Figure 11. Heated press used to form the HiFoil ${ }^{\mathrm{TM}}$ laminates.

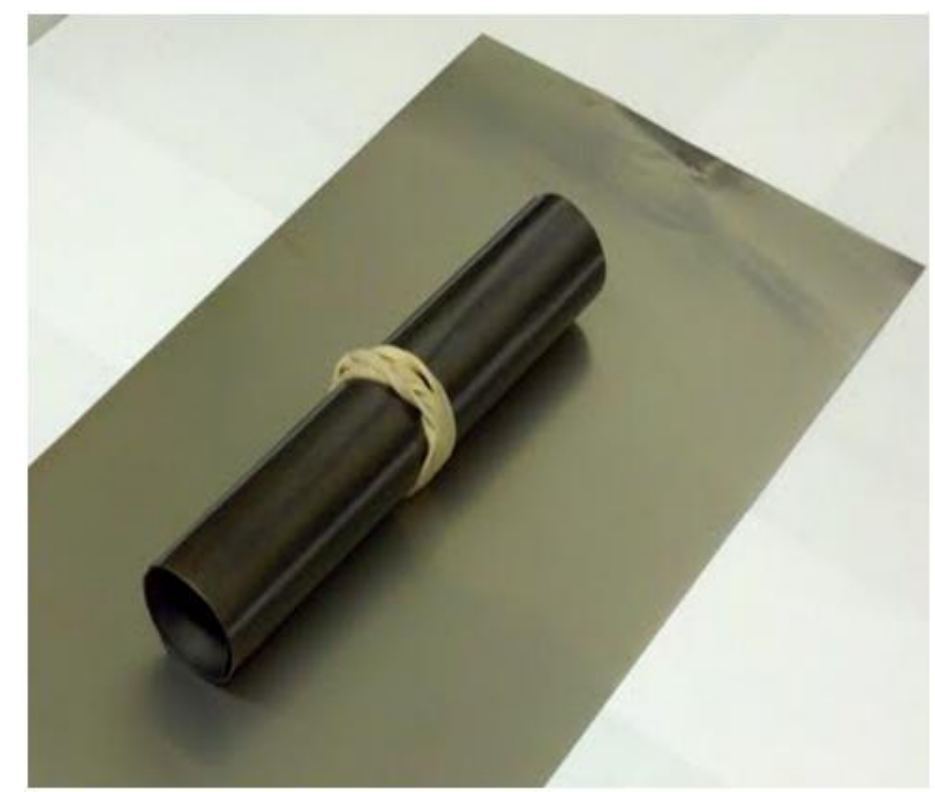

Figure 12. EnerFuel's proprietary high temperature conductive coating comprising graphite and polymer applied to the modified metal foil, and rolled up to demonstrate excellent flexibility. 
April 30, 2013

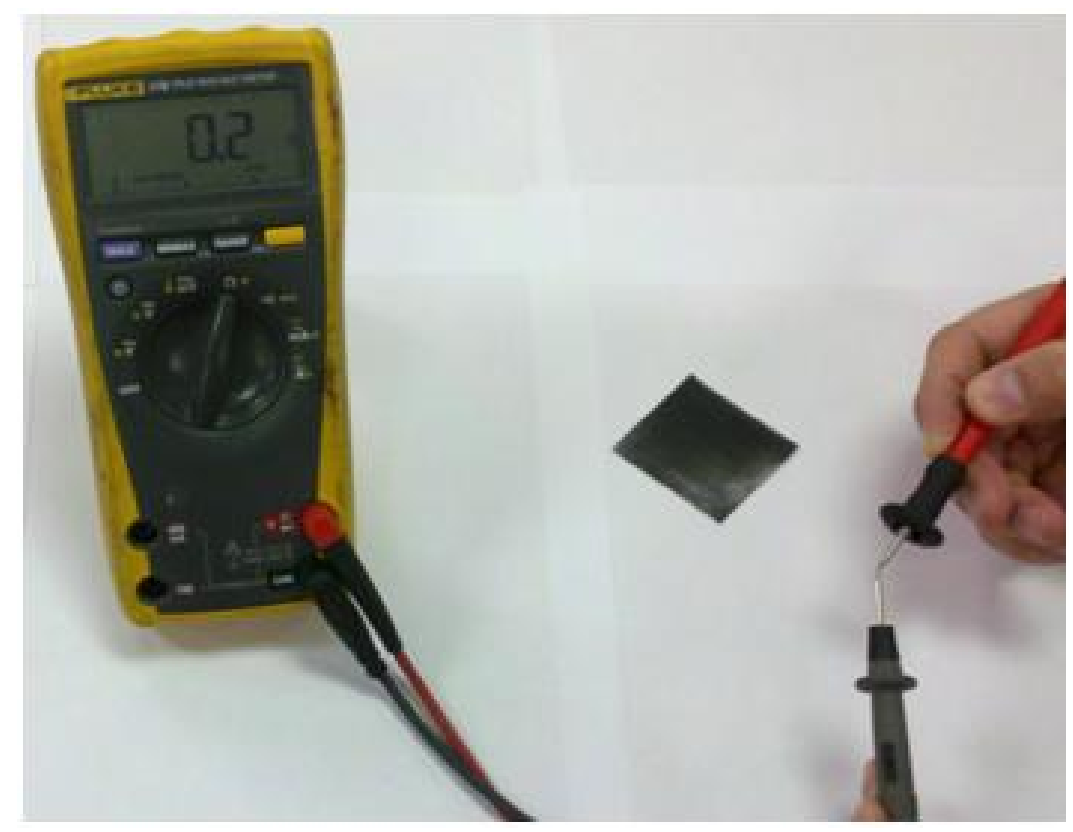

Figure 13. Photo showing the baseline electrical resistance of the probes in contact with each other.

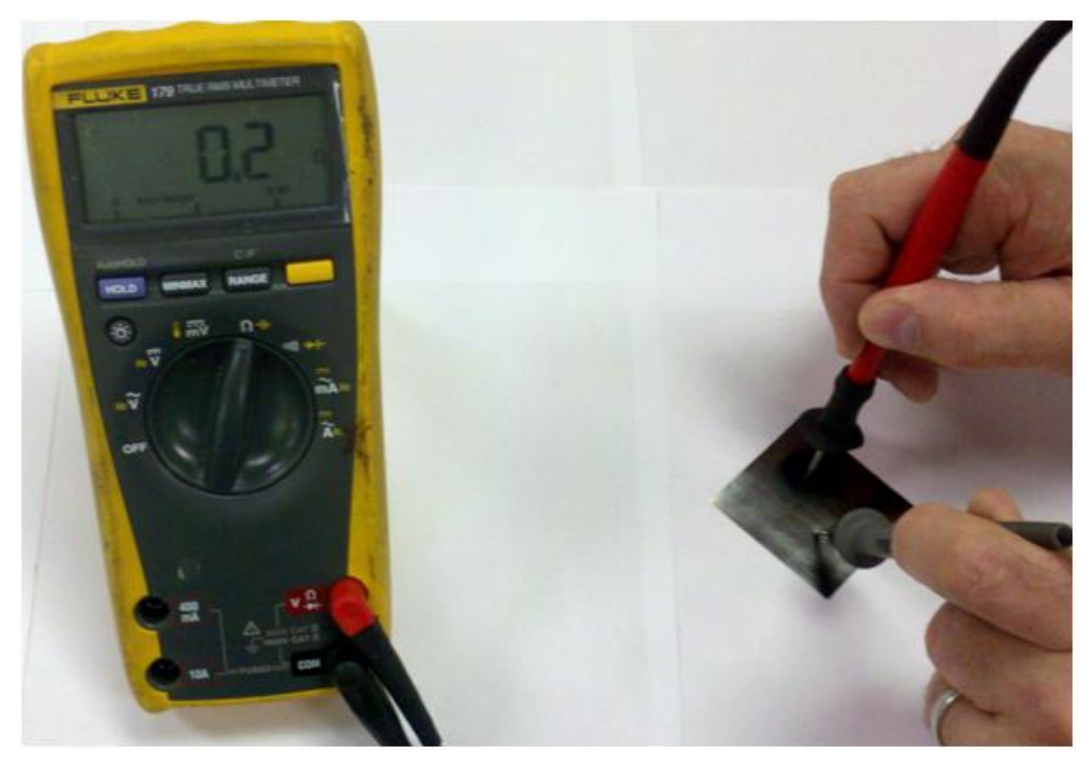

Figure 14. Photo showing the negligible electrical resistance of the proprietary coating.

While the coating and laminating process was being optimized, two major technical problems were encountered: 
1. The advanced flexible graphite is supplied at nearly its maximum density and limited in thickness to less than $1 \mathrm{~mm}$, making it impractical to shape the flow fields using an embossing process. If the material were to be supplied at lower density prior to embossing, the walls of the channels would remain at lower density after embossing, leading to high acid uptake from the MEA and poor integrity of the low density land regions which provide the mechanical support to the MEA, and are also responsible for the conduction of the MEA's heat and electricity to the external loads. Therefore, the original proposal of forming the channels in the expanded graphite was abandoned.

2. The technology used to modify the metal foil core (for promoting adhesion within the laminate), was discovered to have trace amounts of thallium in its microstructure. Thallium is a toxic substance.

\section{Gen2 HiFoil}

\subsection{Gen2 HiFoil Approach}

The solution to the problems identified above was embodied in the Gen2 HiFoil approach, and was provided in two steps. The first step was to introduce a moldable graphite composite at the outer layers of the bipolar plate, such that the flow field features could be readily molded. The second step was to eliminate the metal foil core, as the new outer layers of the graphite composite now provide sufficient mechanical integrity and permeation resistance. The advanced expanded graphite layers are retained in the center portion of the bipolar plate assembly to conduct the cell heat out to the edges of the plate for external cooling (Fig.15-16).

The new Gen2 HiFoil ${ }^{\mathrm{TM}}$ bipolar plate configuration affords some significant benefits:

- The configuration uses commercially available materials with well characterized manufacturing processes.

- The bipolar plate layers combine to conduct heat from the cell to the outer edge of the plate assembly - therefore no coolant (or coolant flow field) is required in the electrically active region.

- The bipolar plate layers, seals and MEA can be formed into a unitized cell (Fig.17). 
April 30, 2013

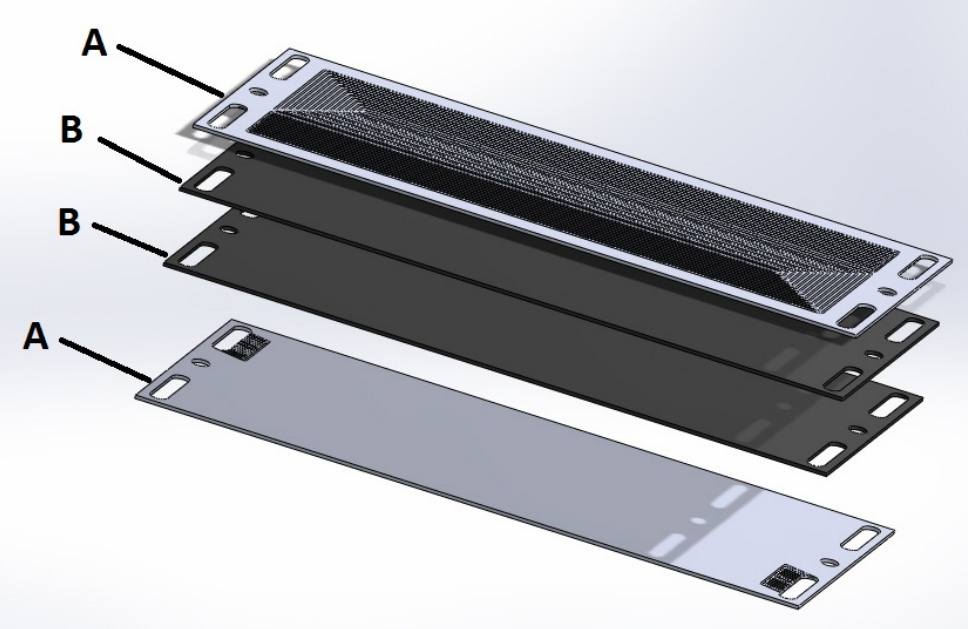

Figure 15. Depiction of the patent pending Gen2 HiFoil ${ }^{\mathrm{TM}}$ configuration, showing the outer moldable graphite composite layers (A) and the inner advanced flexible graphite layers (B).

Figure 16. Depiction of the Gen2 HiFoil ${ }^{\mathrm{TM}}$ as an assembled bipolar plate. 
April 30, 2013

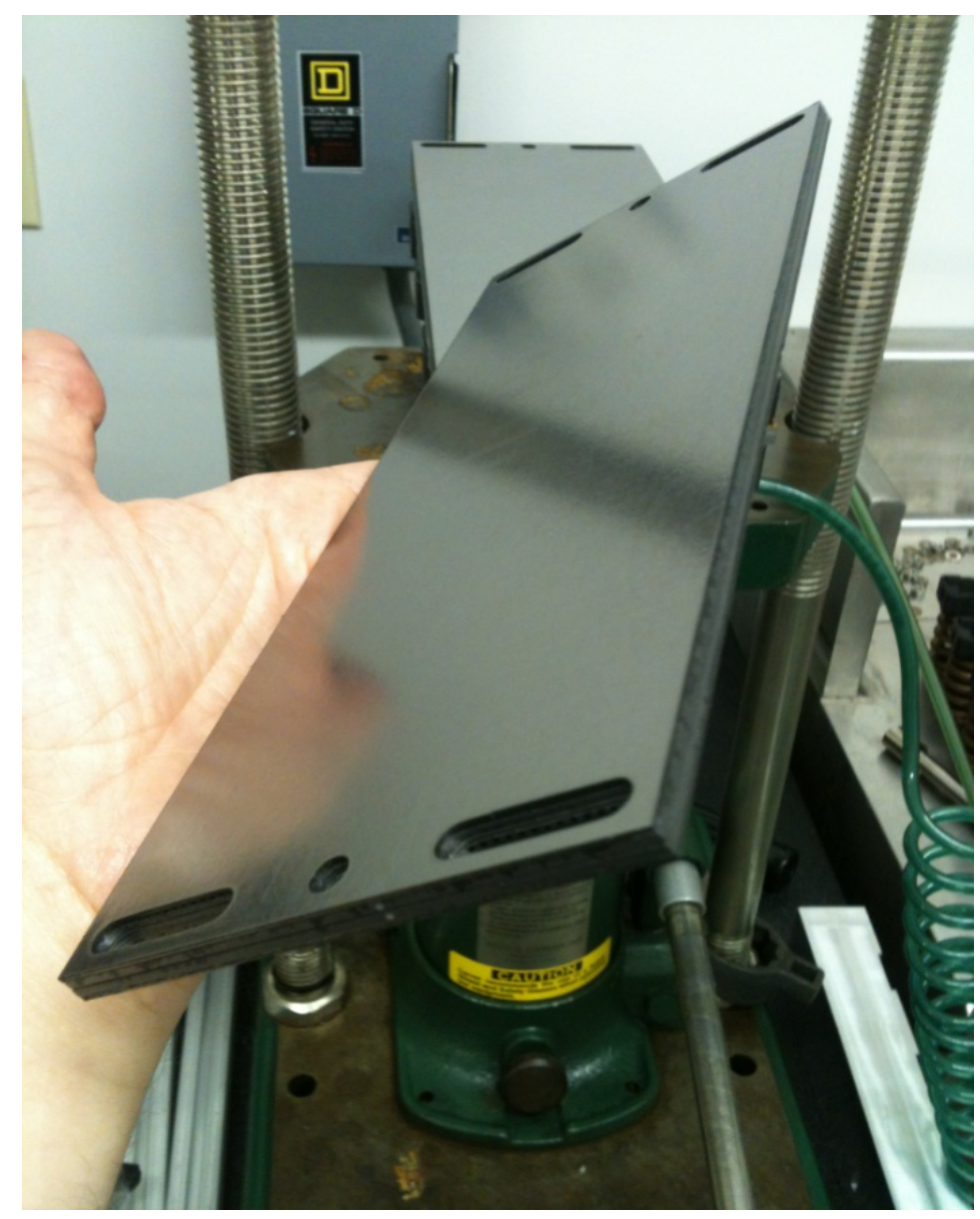

Figure 17. Photo of a unitized cell formed by pressing the MEA between inner moldable graphite composite layers and surrounded by outer layers of advanced flexible graphite.

\subsection{Gen2 HiFoil 4-Cell Stack Test Results}

\subsubsection{Comparison with Baseline}

The first test of the Gen2 HiFoil TM bipolar plates was very successful. Compared to baseline testing, cell uniformity was significantly improved, i.e. the individual cell voltages were nearly identical to each other, as were the individual plate temperatures (Fig.18-19). This had not previously been seen in HTPEM stacks; although the high in-plane thermal conductivity of the advanced flexible graphite was expected to help spread the heat across the cell and reduce the potential for developing MEA hot spots. 
Final Report: Advanced HiFoil ${ }^{\mathrm{TM}}$ Bipolar Plates

April 30, 2013

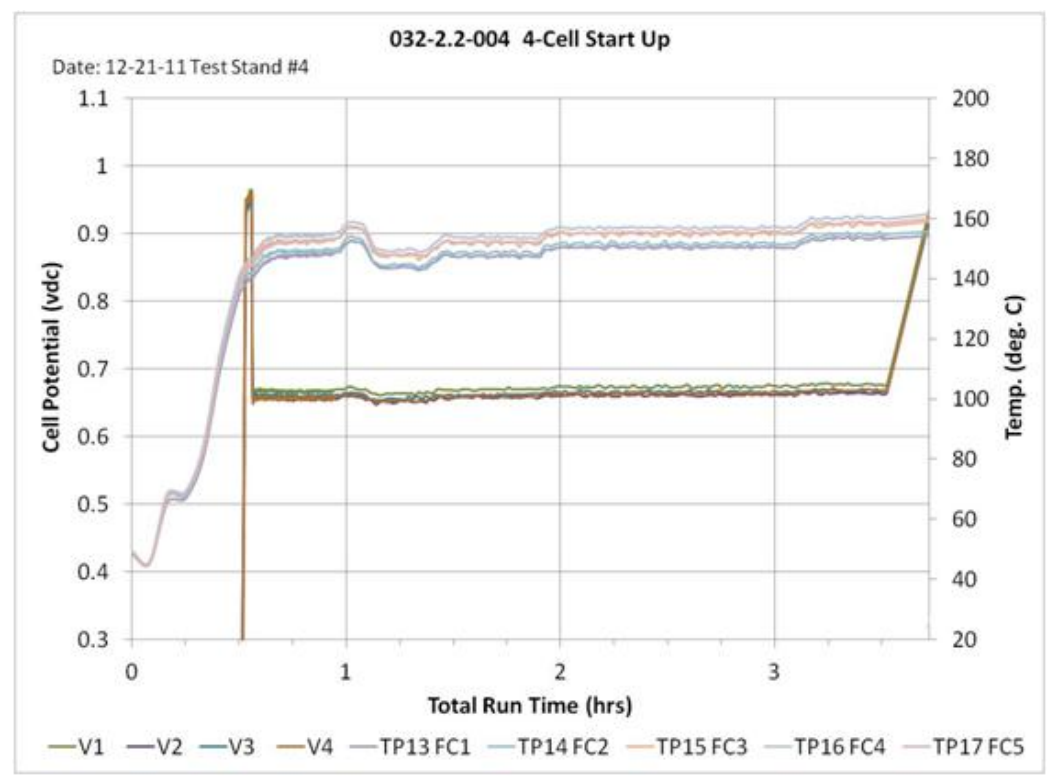

Figure 18. Baseline test data showing the voltage and temperature of each cell in EnerFuel's baseline 4-cell stack (with pre-HiFoil ${ }^{\mathrm{TM}}$ bipolar plates comprising an experimental expanded graphite/resin composite). The cell active area is $157 \mathrm{~cm}^{2}$ and the current density is $200 \mathrm{~mA} / \mathrm{cm}^{2}$.

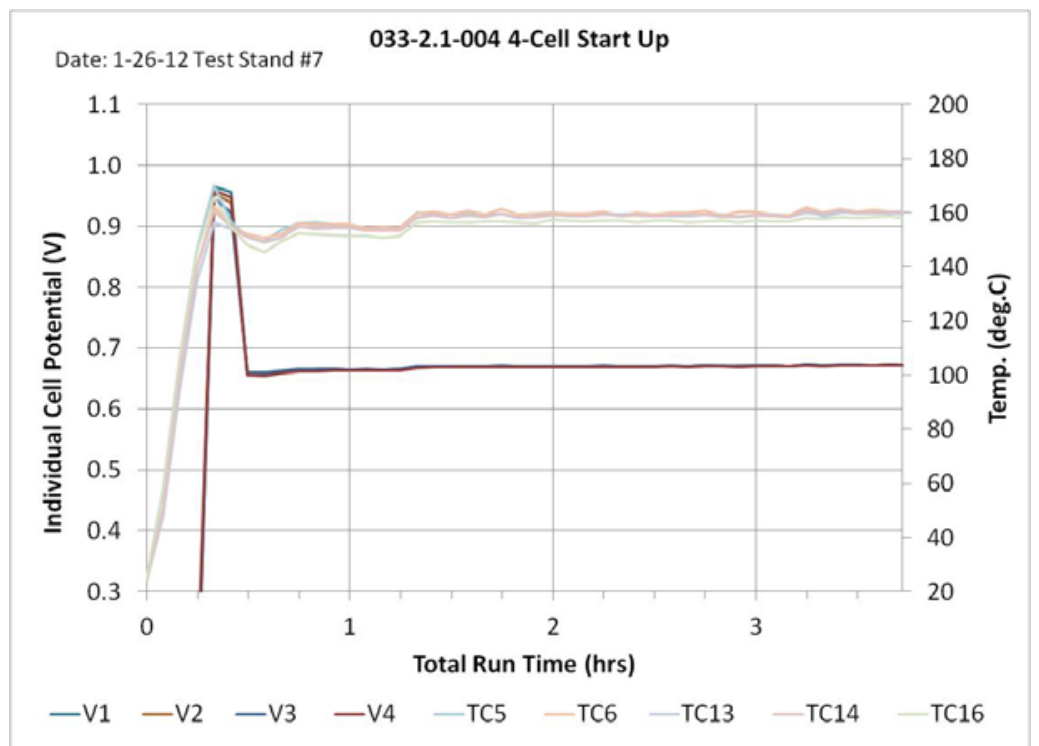

Figure 19. Test data for EnerFuel's first 4-cell stack with the Gen2 HiFoil ${ }^{\mathrm{TM}}$ bipolar plates showing the excellent uniformity of the individual cell voltage and temperature measurements. 


\subsubsection{Seal Geometry Optimization and New Stack Compression Hardware}

Plate geometry was modified to eliminate separate MEA hardstops, and to increase cell seal effectiveness at lower compressive loads. In addition, new endplates were developed that reduce the stack compression hardware weight by $50 \%$, and also provide for significant cost reduction. Whereas EnerFuel stacks employing the old endplates and compression hardware are referred to either as Gen 2.0 or Gen 2.1 (depending on the number and location of tie-rods), the stacks with the new endplate and compression hardware are referred to as Gen 2.2.

The Gen 2.2 4-cell stack was operated without separate MEA hardstops, and at a $40 \%$ reduction in stack compressive load (Fig.20). The stack thermal insulation was not optimized, and this stack did not demonstrate effective sealing of reactants (possibly due to leaks at the under-compressed interfaces between the HiFoil ${ }^{\mathrm{TM}}$ bipolar plate layers). Prior to operation, the internal $\mathrm{N}_{2}$ pressure decay went from 2.0 to 1.9 psig in about 40 seconds. This is generally considered unacceptable. However, this stack also did not use any dispensed seals at these interfaces. The stack was operable for a short period of time, and provided a baseline for which to compare the effects of adding the dispensed seals.

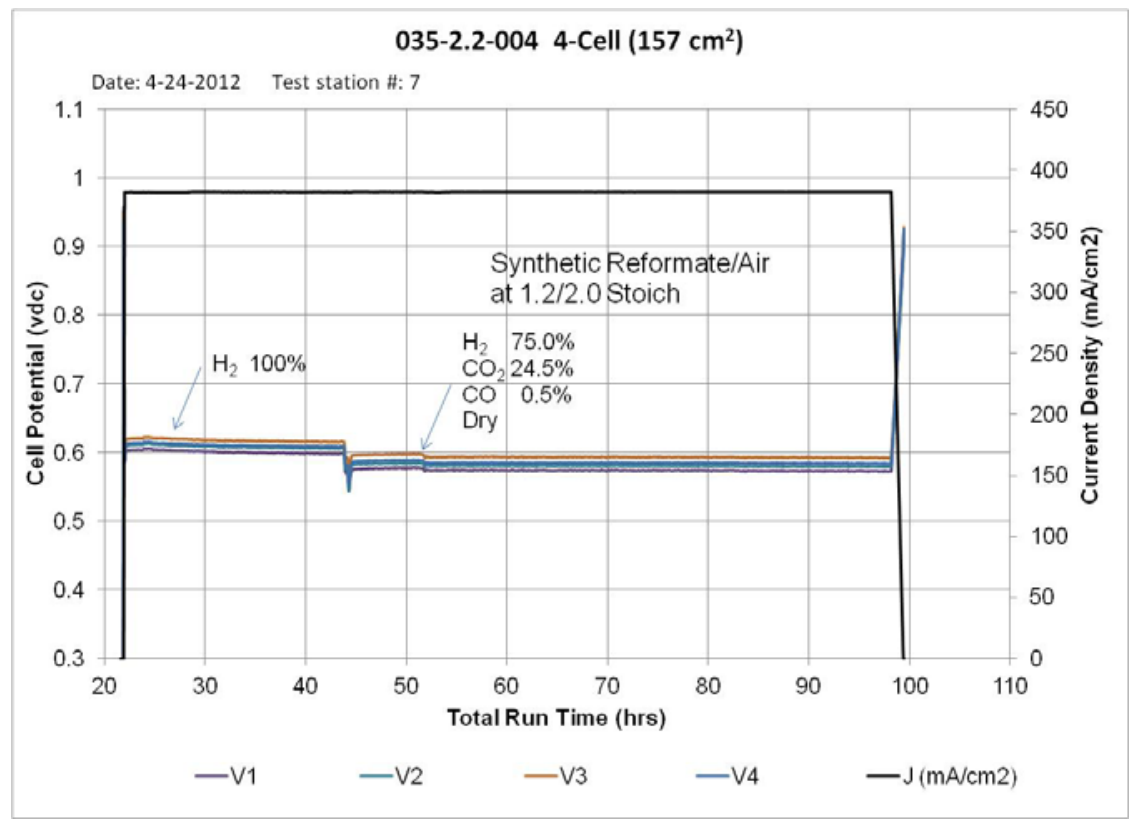

Figure 20. 4-cell stack test data with modified HiFoil ${ }^{\mathrm{TM}}$ bipolar plates and Gen 2.2 hardware. 


\subsubsection{Flow Field Geometry Optimization}

The reactant flow fields were redesigned to reduce the reactant pressure drops. In the case of the cathode flow field, this pressure drop significantly affects the air compressor power requirement, and is a major contributor to the total parasitic power draw in a fuel cell system. By changing the flow field geometry (but without increasing either the plate thickness or the amount of plate material required) we were able to reduce the cathode pressure drop by $33 \%$. This stack also ran well at the targeted low reactant stoics (Fig.21).

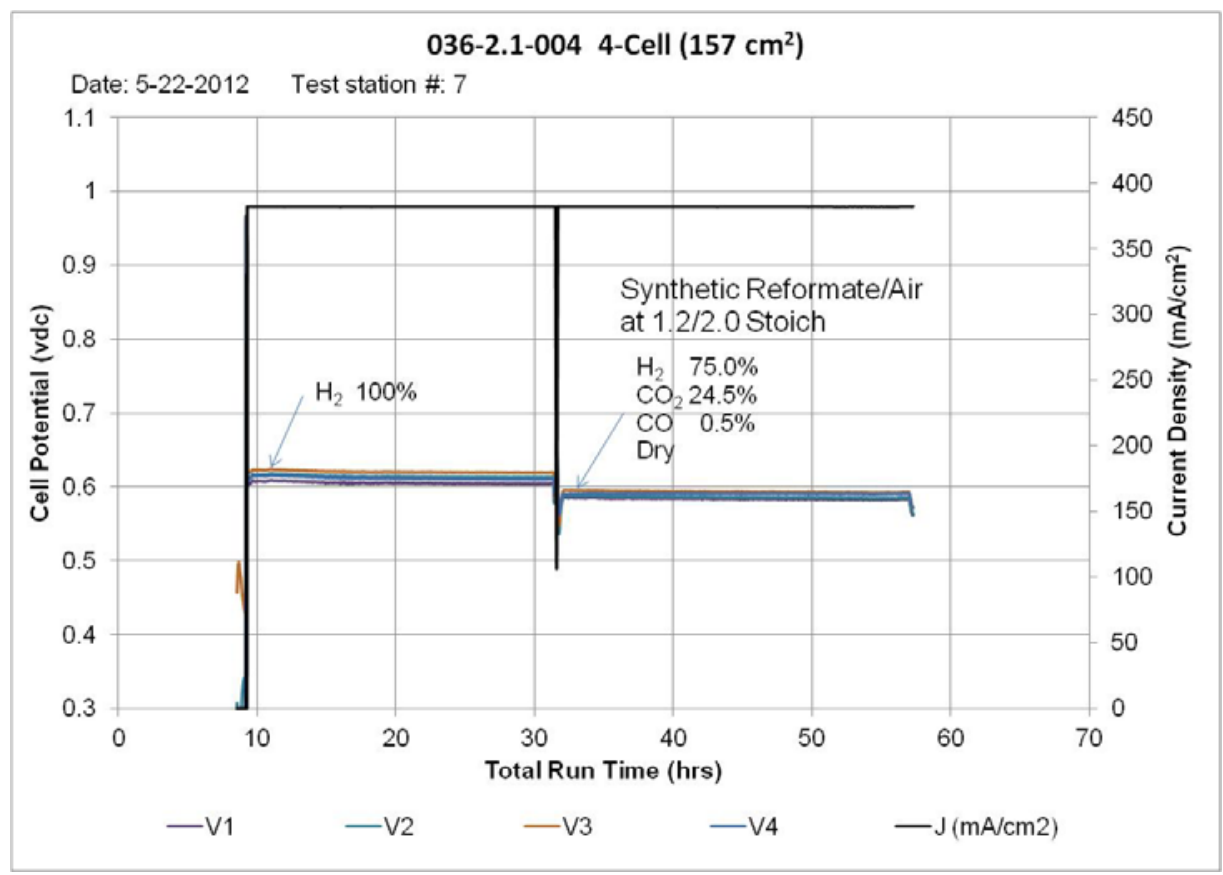

Figure 21. 4-cell stack test data with modified HiFoil ${ }^{\mathrm{TM}}$ bipolar plate flow fields that provide for a $33 \%$ drop in cathode pressure drop.

\subsubsection{New MEA Version}

In order to further reduce the fuel cell cost, a new version of MEA with improved manufacturing potential was tested in a 4-cell stack, and demonstrated approximately $3 \%$ greater cell electrical efficiency (Fig.22-23). This stack was tested for over 200 hours and produced 145 watts at 60 amps. EnerFuel's MEA supplier indicated that the new version of MEA has significantly improved coating uniformity, and that the new MEA coating process enables manufacturing scale up to support higher production quantities and lower cost. 
Final Report: Advanced HiFoil ${ }^{\mathrm{TM}}$ Bipolar Plates

April 30, 2013

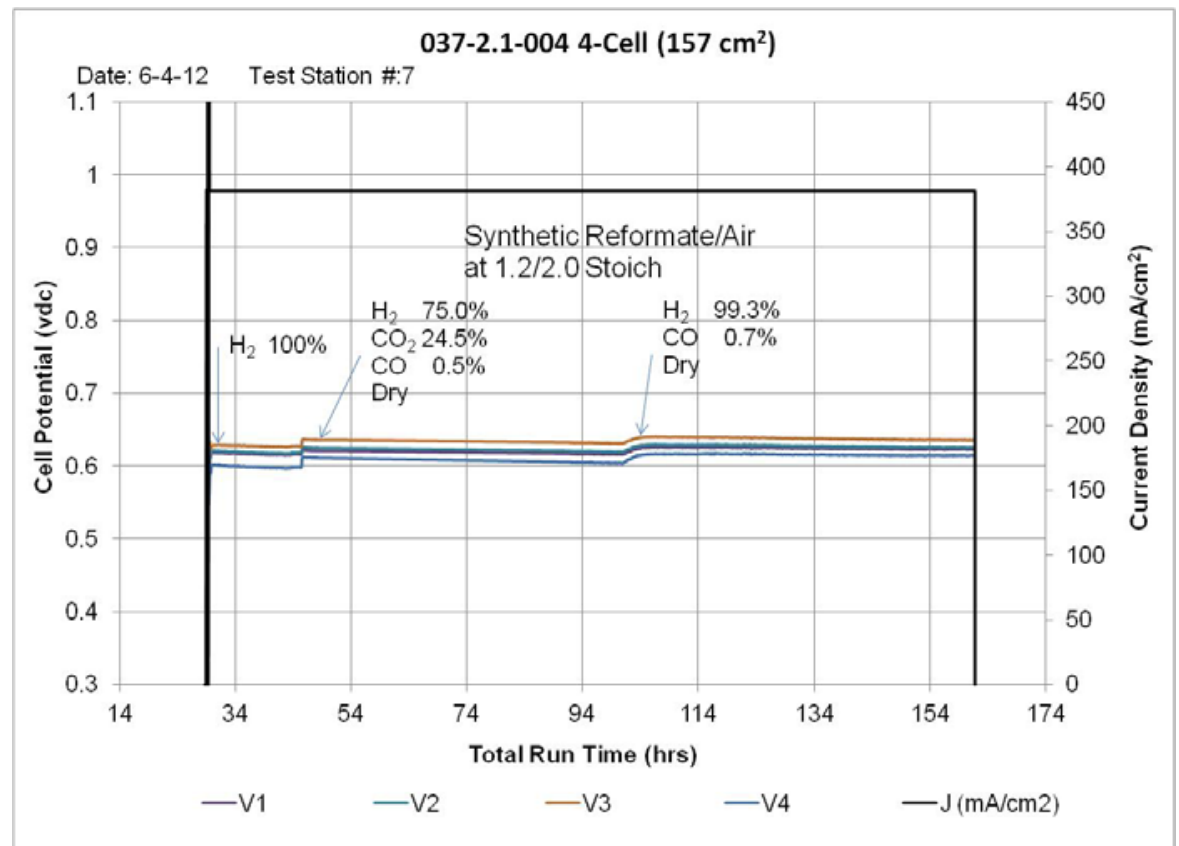

Figure 22. Test data showing performance of 4-cell stack with new MEA version and HiFoil ${ }^{\mathrm{TM}}$ bipolar plates. The new MEA provides approximately $3 \%$ greater cell electrical efficiency.

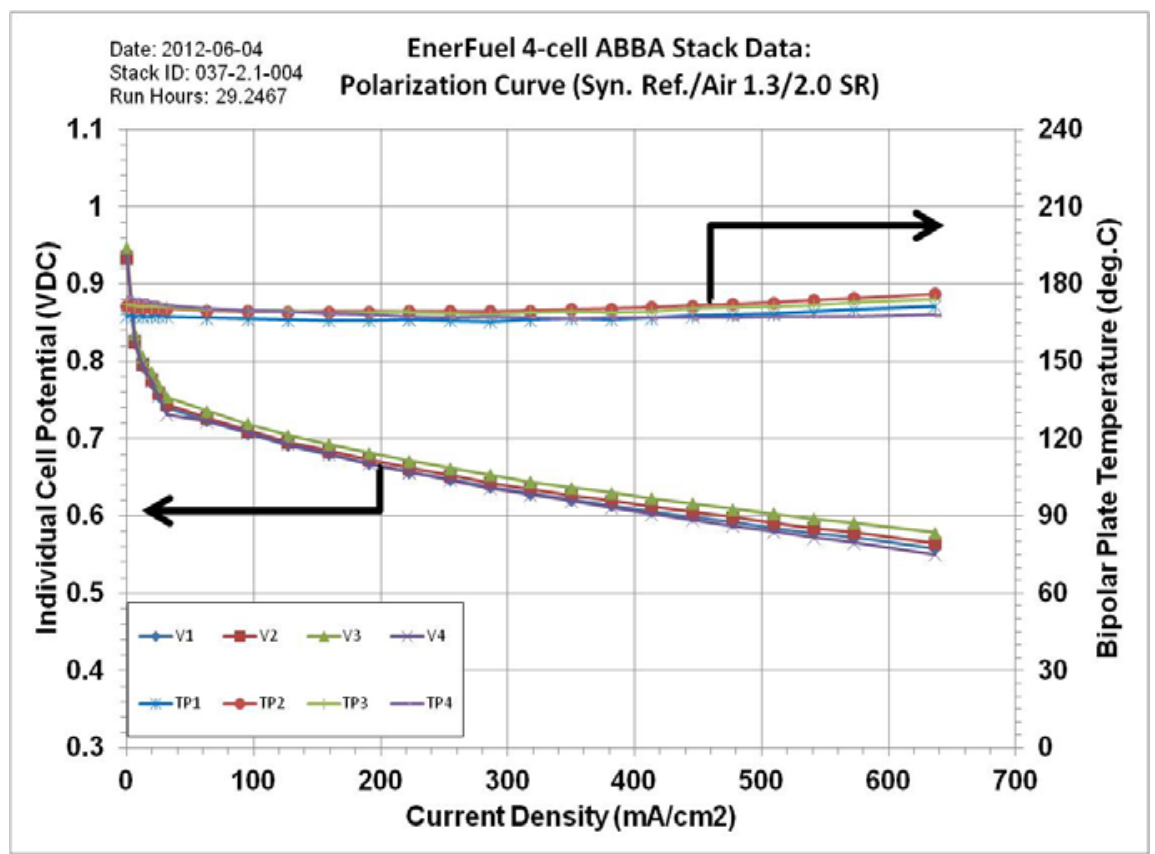

Figure 23. Test data showing a reformate polarization curve for the new MEA version. 


\subsubsection{Continuous Operation}

A 4-cell stack containing the Gen2 HiFoil ${ }^{\mathrm{TM}}$ bipolar plates (ABBA configuration), new Gen 2.0 compression hardware and dispensed seals was operated continuously to observe the voltage degradation rate at constant current with synthetic reformate fuel. Initially the voltage degradation was rather low, however after about 100 hours, the changes to the operating conditions began a downward slope which resulted in $>12 \%$ decrease in power over a 400 hour period (Fig.24).

The operating conditions during the total 500 hour run are plotted in the following charts. The individual cell potentials are plotted on the left axis in all charts. Current density and electrical power are shown in Figure 25. The bipolar plate temperatures and the fuel feed and dew point temperature are shown in Figure 25. Reactant flow rates are shown in Figure 26. The reactant stoichiometric ratios are shown in Figure 27. EnerFuel is currently building new test stations to provide better stack thermal management and more precise control of fuel humidification levels.

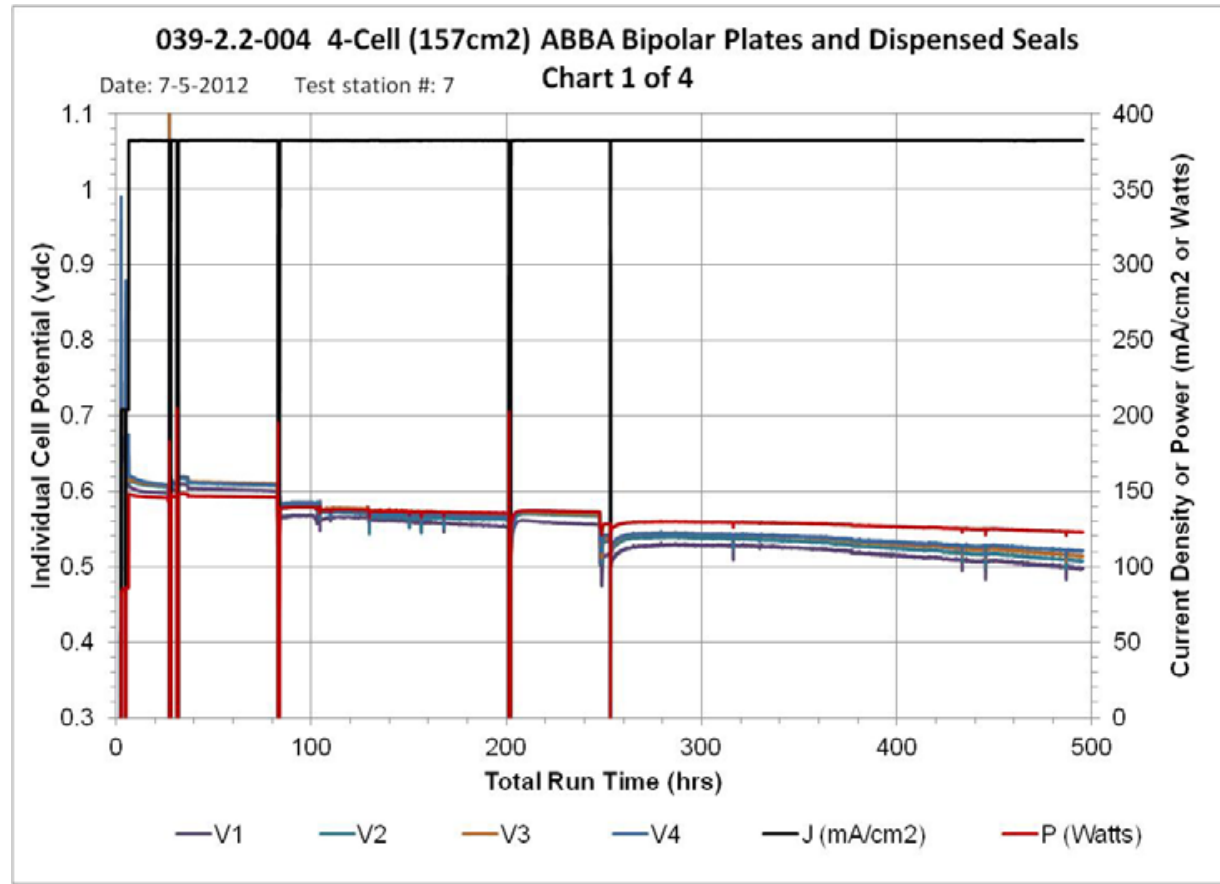

Figure 24. 4-cell stack test data at constant current showing the individual cell voltages, current density and electrical power. The electrical power output decreased by over $12 \%$ during the last 400 hours of operation. 
Final Report: Advanced HiFoil ${ }^{\mathrm{TM}}$ Bipolar Plates

April 30, 2013

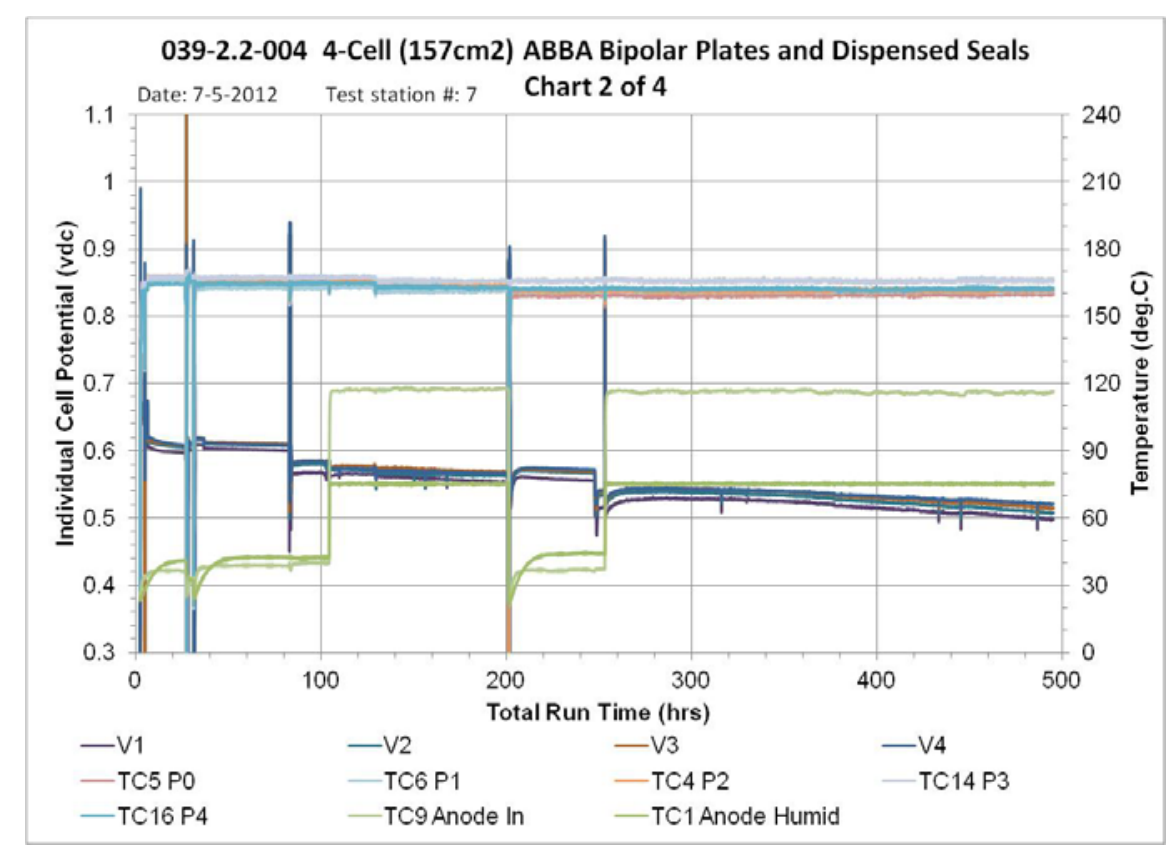

Figure 25. 4-cell stack test data at constant current showing the bipolar plate temperatures and the fuel feed and dew point temperatures.

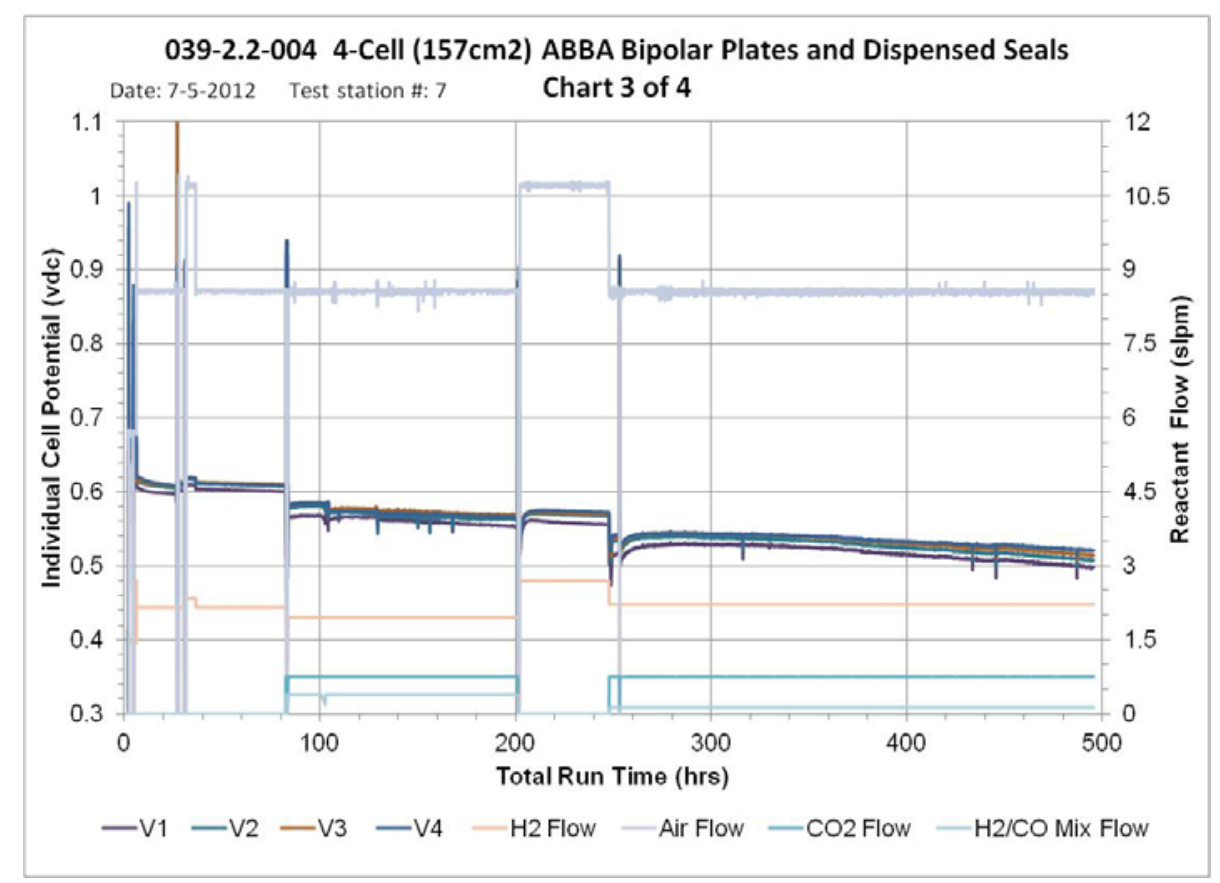

Figure 26. 4-cell stack test data at constant current showing the reactant flow rates. 


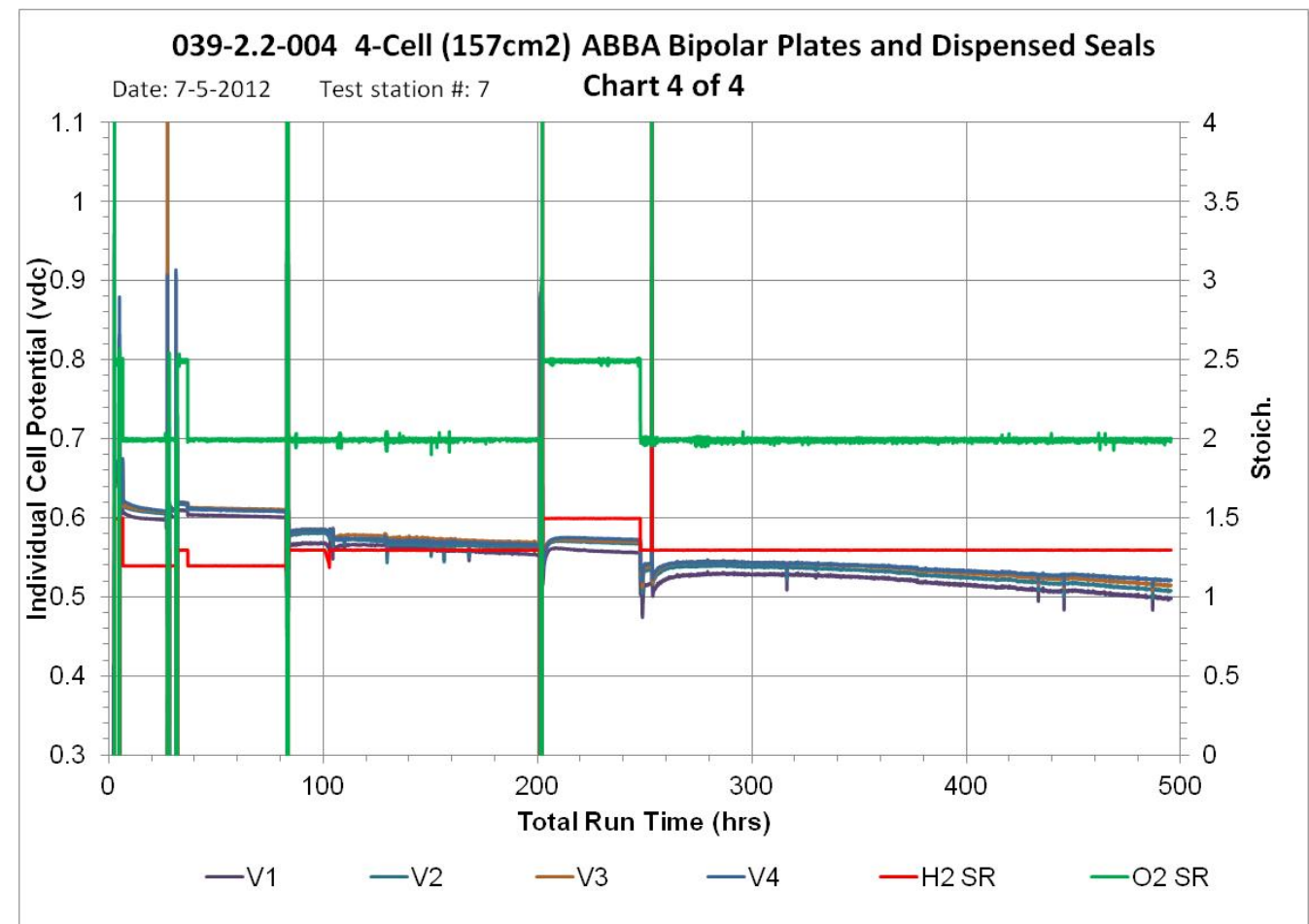

Figure 27. 4-cell stack test data at constant current showing the reactant stoichiometric ratios.

\subsection{Gen2 HiFoil 36-Cell Module Test Results}

The limited amount of heat produced by the 4-cell stack was largely absorbed by the stack compression hardware and plumbing, and then radiated/convected into the testing room. This was evidenced by the fact that without active cooling, the electric heaters would cycle on and off in order to maintain the stack at the desired temperature, even when the stack was being operated at nominal power.

Although the previous 4-cell testing went a long way toward vetting the Gen2 HiFoil ${ }^{\mathrm{TM}}$ concept, not all of the plate features could be effectively demonstrated on a smaller scale. Since the bipolar plates were designed to spread the cell heat out to the edge of the plate and conduct it to an external cooling circuit, a more thorough test of the concept required a larger size stack, one capable of producing a substantial amount of heat. A 36-cell stack was constructed with the Gen2 HiFoil ${ }^{\text {TM }}$ bipolar plates without dispensed seals. A custom liquid cooling circuit was attached to the outer edges of the stack (Fig.28). 
April 30, 2013

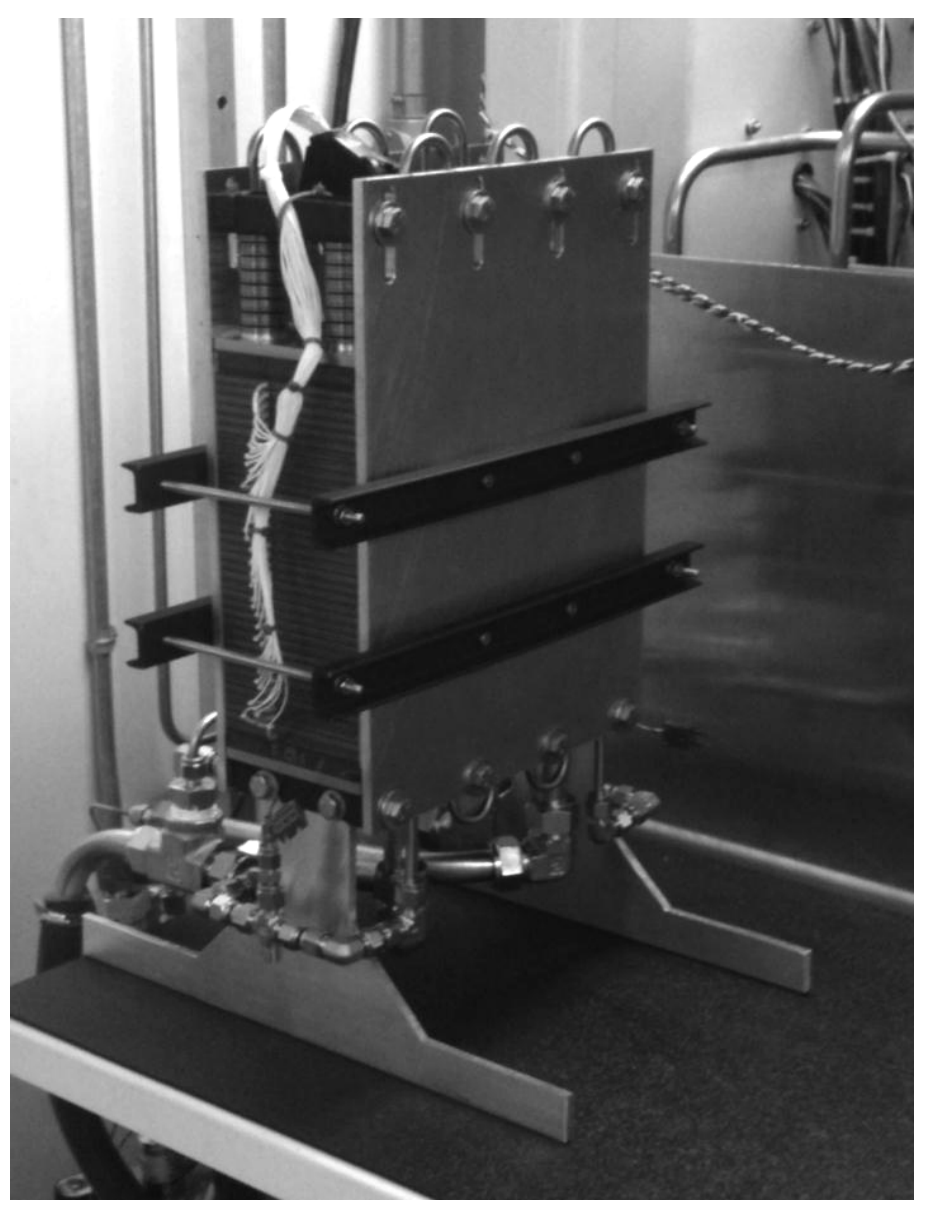

Figure 28. Photo of EnerFuel's 36-cell stack with Gen2 HiFoil ${ }^{\text {TM }}$ bipolar plates and external liquid cooling system. This stack was operated without dispensed seals. Initial testing was also performed without any insulation, although HTPEM systems typically require the stack to be insulated in order to recover as much of the waste heat as possible.

The 36-cell stack was operated at $1.25 \mathrm{~kW}$ electric power using oil as the coolant (Fig.29). In this test the individual cell temperatures are very closely grouped. The 1.2/2.0 anode/cathode stoichiometric ratio was quite low, contributing to the moderate spread in cell voltages.

The same stack was tested with 2-phase water cooling (Fig.30). During this test the anode/cathode stoichiometric ratio or $1.5 / 2.5$ helped tighten the voltage spread (Fig.31). Of significance is that the 2-phase coolant is exiting at $\sim 160^{\circ} \mathrm{C}$ and provides tight control of cell temperatures (Fig.32). 
Final Report: Advanced HiFoil ${ }^{\mathrm{TM}}$ Bipolar Plates

April 30, 2013

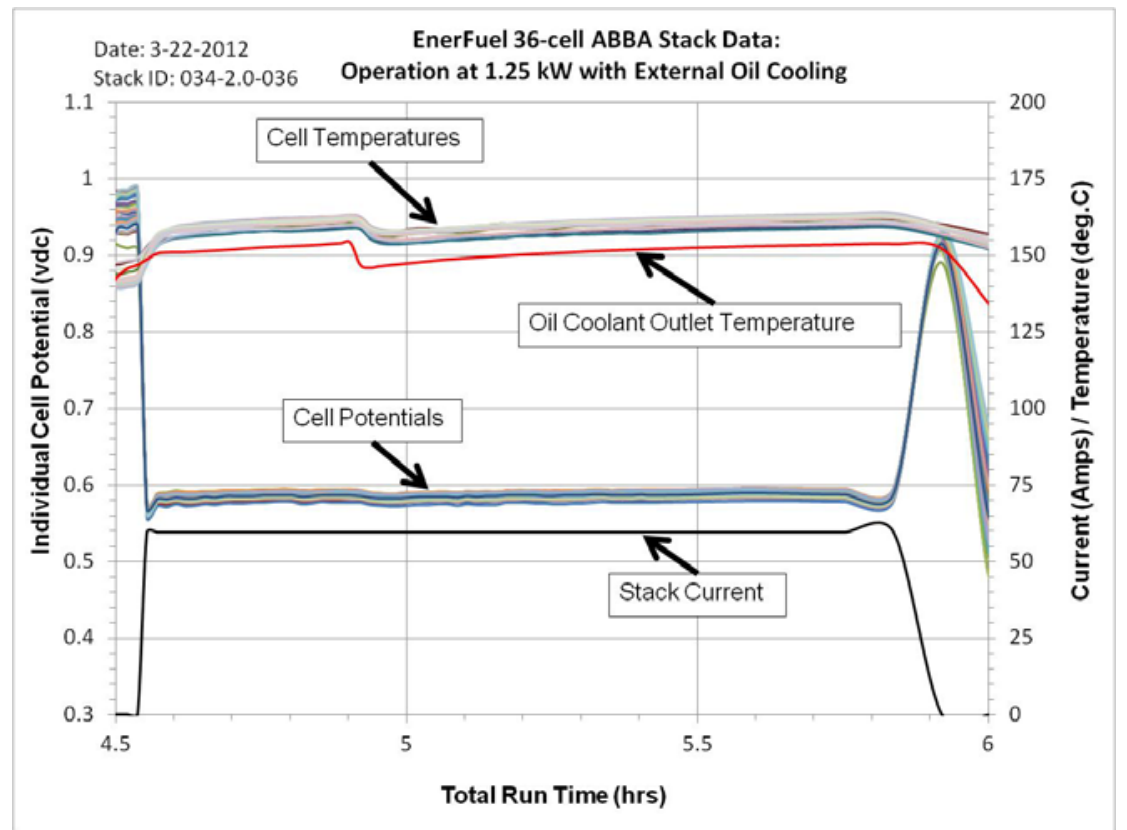

Figure 29. 36-cell stack test data with Gen 2.0 HiFoil ${ }^{\mathrm{TM}}$ bipolar plates with external oil cooling at 1.2/2.0 SR $\mathrm{H}_{2} /$ Air; showing excellent cell temperature uniformity $\left(\sim 7^{\circ} \mathrm{C}\right.$ spread).

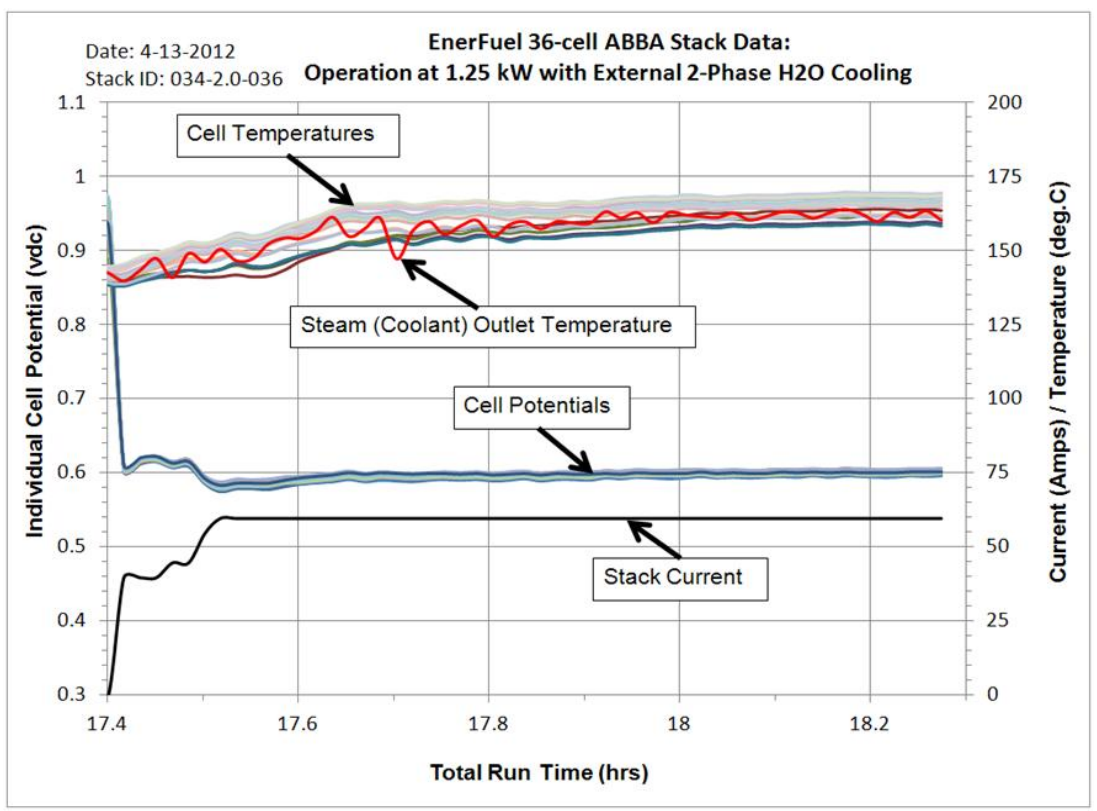

Figure 30. 36-cell stack test data with Gen $2.0 \mathrm{HiFoil}^{\mathrm{TM}}$ bipolar plates with 2-phase cooling at 1.5/2.5 SR $\mathrm{H}_{2} /$ Air; showing tight cell voltage uniformity with $\sim 160^{\circ} \mathrm{C}$ coolant temperature. 


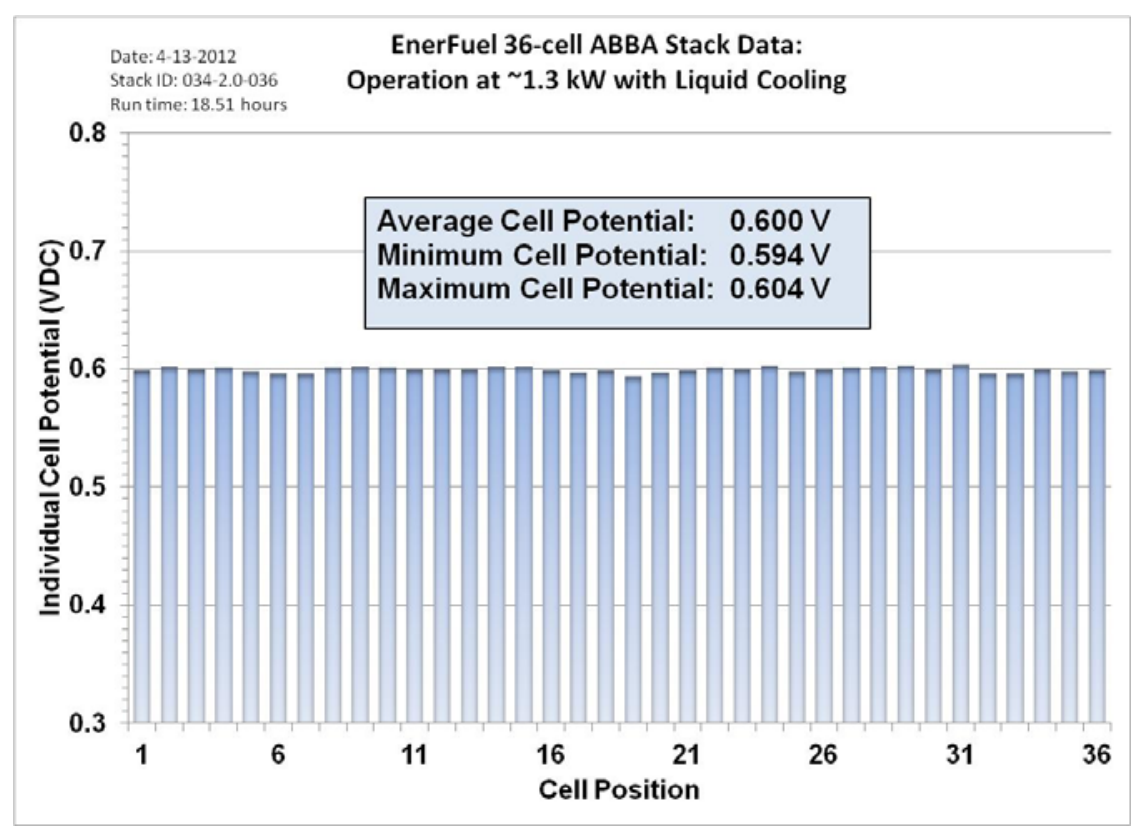

Figure 31. 36-cell stack test data with Gen 2.0 HiFoil $^{\mathrm{TM}}$ bipolar plates and 2-phase cooling showing $10 \mathrm{mV}$ spread in cell voltages.

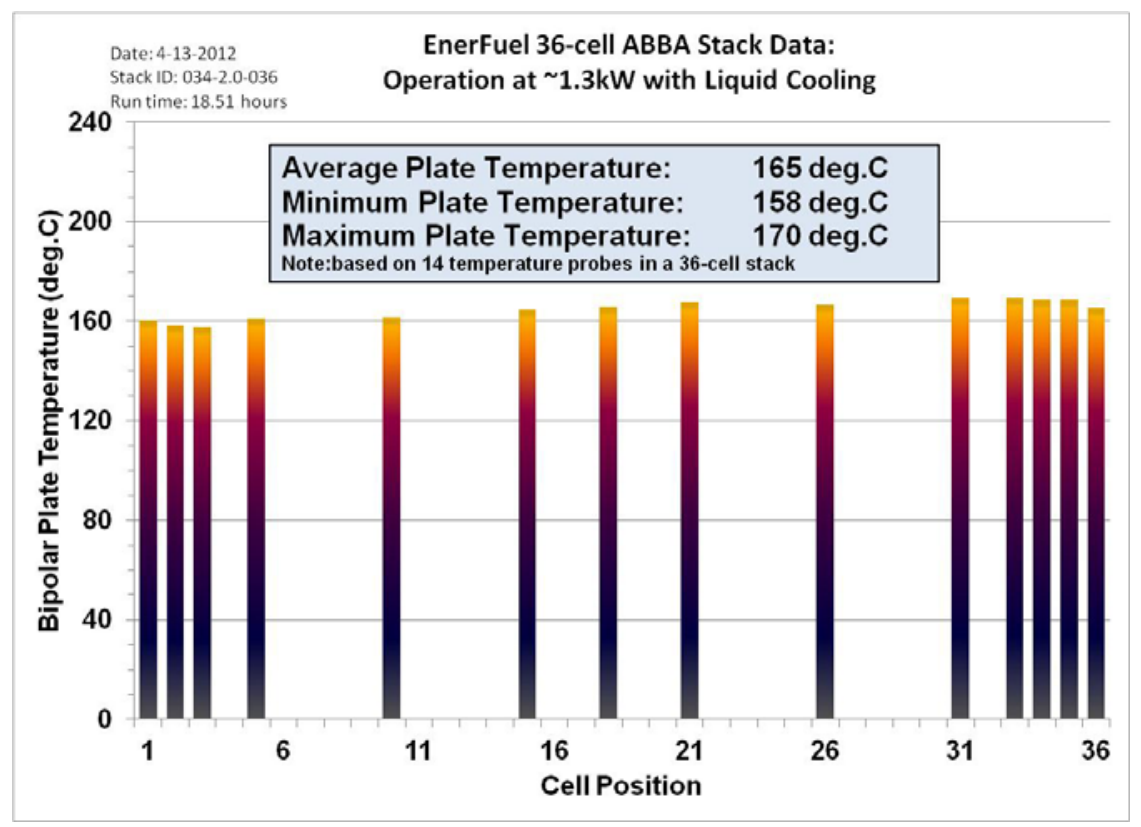

Figure 32. 36-cell stack test data with Gen $2.0 \mathrm{HiFoil}^{\mathrm{TM}}$ bipolar plates and 2-phase cooling showing $12^{\circ} \mathrm{C}$ spread in cell temperatures. 
A second 36-cell stack with Gen 2.0 HiFoil TM bipolar plates and dispensed seals was constructed in order to validate that the dispensed seals could be incorporated into a large stack. These seals were dispensed onto the plates by EnerFuel using proprietary manufacturing steps and custom dispensing equipment (Fig.33).

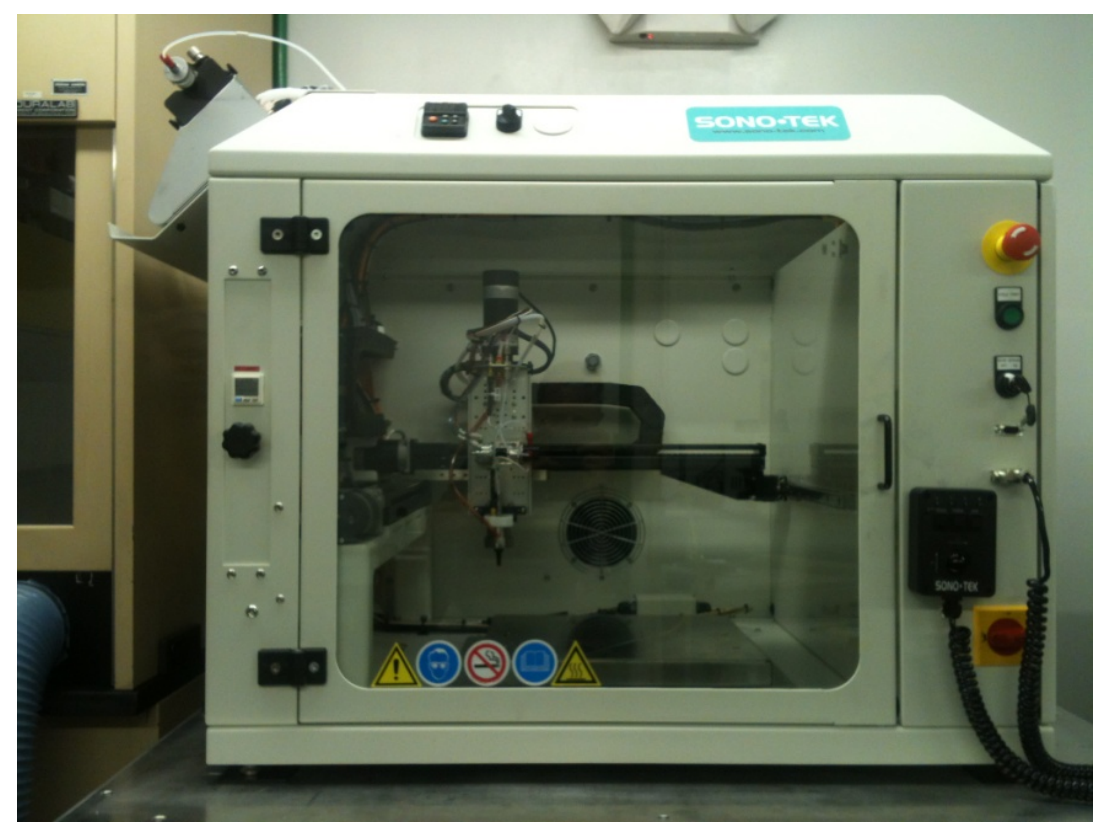

Figure 33. EnerFuel purchased customized equipment for automated dispensing of elastomeric seals onto bipolar plates.

Operational data from the 36-cell stack with the Gen2 HiFoil ${ }^{\mathrm{TM}}$ bipolar plates and dispensed seals are shown below in Figures 34-37. The 36-cell stack produced $>1.2$ kilowatts at 60 amps with $\mathrm{H}_{2} /$ Air at 1.5/2.5 stoichiometric ratio with microprocessor controlled thermal management system using 2-phase water cooling. The cell potentials are plotted on the left axis in all charts. Excluding data the lowest performing cell, the individual cell voltage spread was $-30 \mathrm{mV}$. The cell temperature spread was $<20^{\circ} \mathrm{C}$. Stack current density and electrical power are shown in Figure 34. The bipolar plate temperatures are shown in Figure 35. Reactant flow rates are shown in Figure 36. The reactant stoichiometric ratios are shown in Figure 37. 
Final Report: Advanced HiFoil ${ }^{\mathrm{TM}}$ Bipolar Plates

April 30, 2013

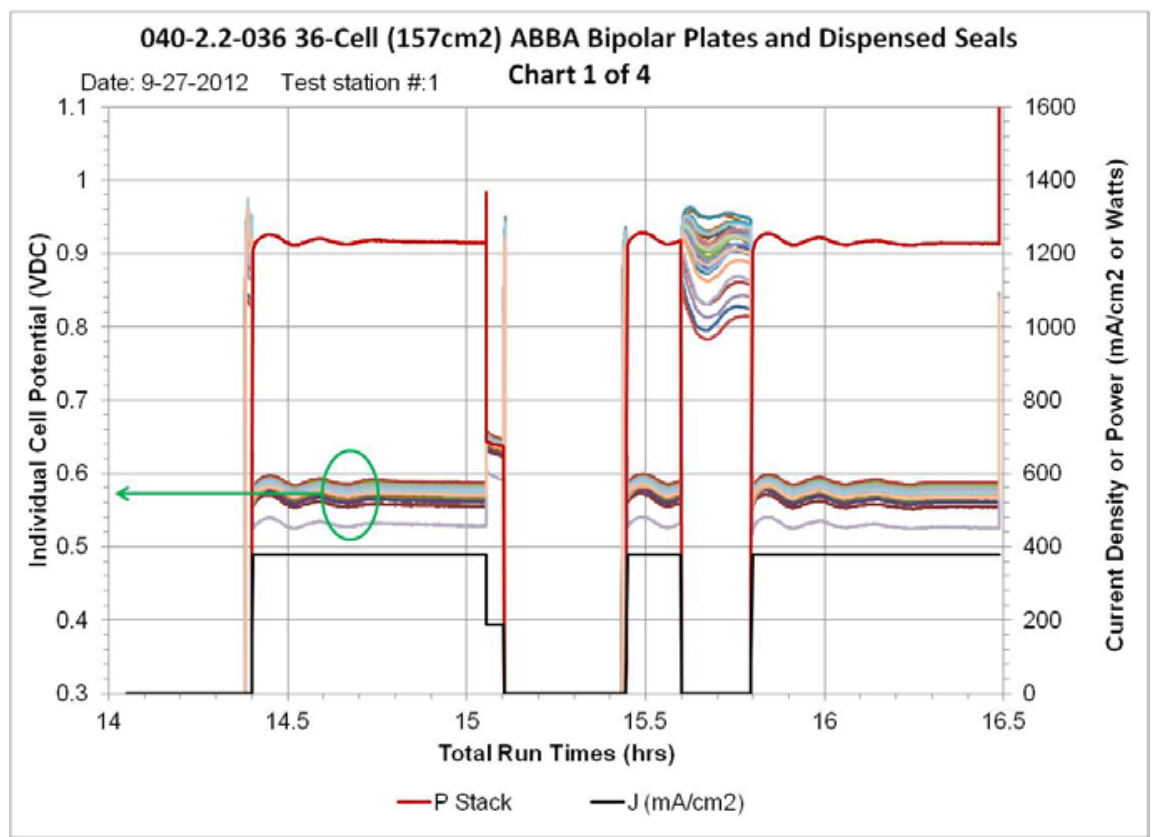

Figure 34. 36-cell stack test data at constant current with 2-phase cooling showing the individual cell voltages, current density and electrical power.

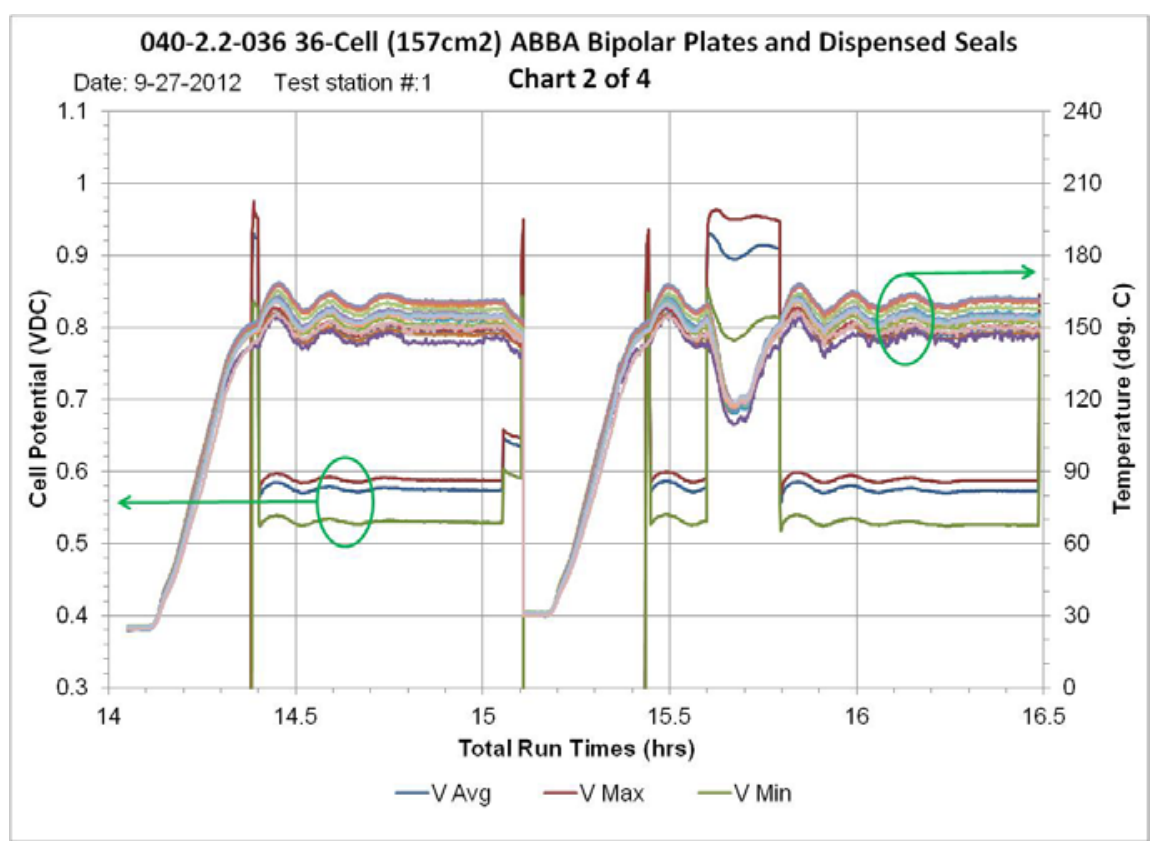

Figure 35. 36-cell stack test data at constant current with 2-phase cooling showing the bipolar plate temperatures. 
Final Report: Advanced HiFoil ${ }^{\mathrm{TM}}$ Bipolar Plates

April 30, 2013

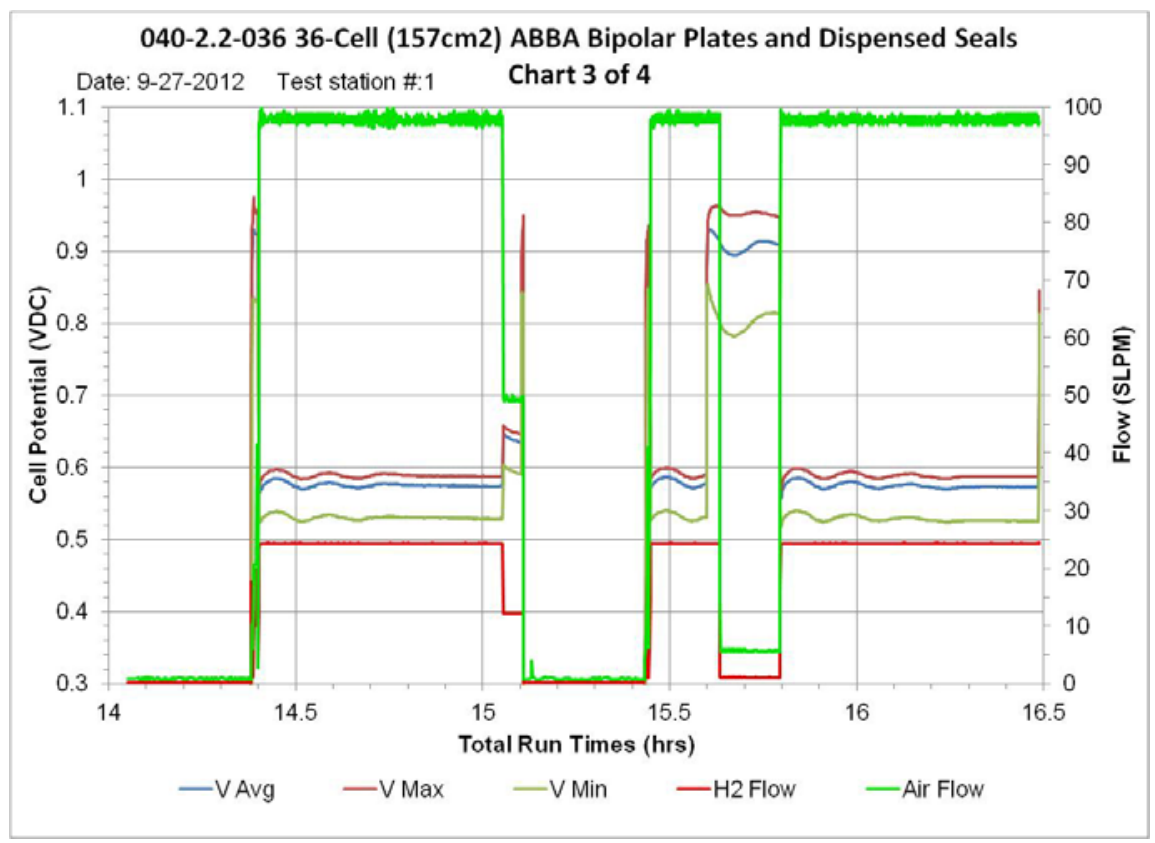

Figure 36. 36-cell stack test data at constant current with 2-phase cooling showing the reactant flow rates.

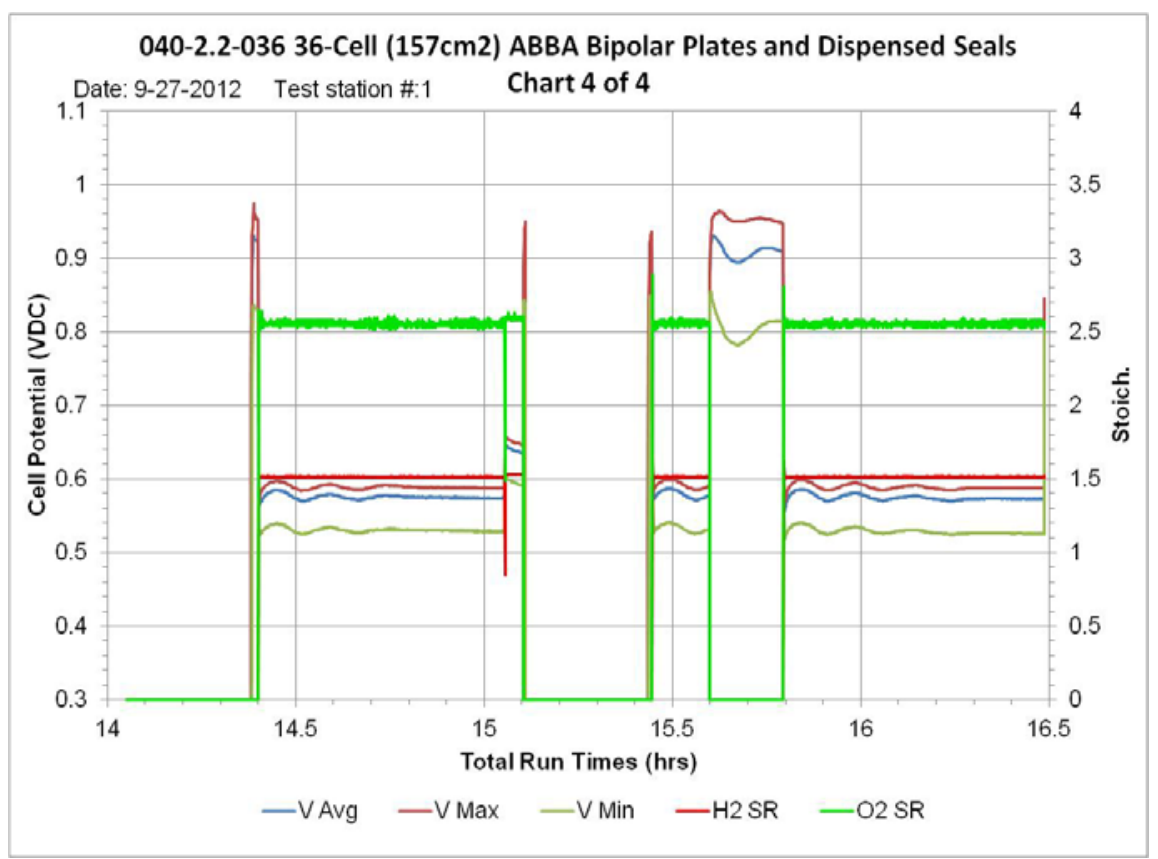

Figure 37. 36-cell stack test data at constant current with 2-phase cooling showing the reactant stoichiometric ratios. 


\subsection{Gen2 HiFoil Laminate Bipolar Plate Costs}

The Gen2 HiFoil laminate bipolar plates developed as through this program has shown many advantages over existing technologies. In addition, to chemical and thermal enhancements the plates are compatible with well established manufacturing processes. Quotes received for molding the Gen2 bipolar plates show a bipolar plate cost of $\$ 840$ per $\mathrm{kW}$ of net electrical power, at production volumes of 2000 fuel cell systems per year. In volumes of 50,000 systems per year, this cost would be expected to drop well below $\$ 500$ per $\mathrm{kW}$. These are in line with DOE cost targets for stationary systems (Figure 38).

\begin{tabular}{|c|c|c|c|}
\hline Characteristic & 2011 Status & 2015 Targets & 2020 Targets \\
\hline Electrical efficiency at rated power ${ }^{b}$ & $34-40 \%$ & $42.5 \%$ & $>45 \%^{\circ}$ \\
\hline $\mathrm{CHP}_{\text {energy efficiency }}{ }^{\mathrm{d}}$ & $80-90 \%$ & $87.5 \%$ & $90 \%$ \\
\hline Equipment cost ${ }^{\mathrm{e}}, 2-\mathrm{kW}$ avg $^{f}$ system & NA & $\$ 1,200 / \mathrm{kW}_{\text {avg }}$ & $\$ 1,000 / \mathrm{kW}_{\text {avg }}$ \\
\hline Equipment cost ${ }^{e}, 5-k W_{\text {avg }}$ system & $\begin{array}{c}\$ 2,300- \\
\$ 4,000 / \mathrm{kW}^{9}\end{array}$ & $\$ 1,700 / \mathrm{kW}_{\text {avg }}$ & $\$ 1,500 / \mathrm{kW}_{\text {avg }}$ \\
\hline Equipment cost $\mathrm{t}^{\mathrm{e}}, 10-\mathrm{kW}_{\text {avg }}$ system & NA & $\$ 1,900 / \mathrm{kW}_{\text {avg }}$ & $\$ 1,700 / \mathrm{kW}_{\text {avg }}$ \\
\hline $\begin{array}{l}\text { Transient response (10 - } 90 \% \text { rated } \\
\text { power) }\end{array}$ & $5 \mathrm{~min}$ & $3 \mathrm{~min}$ & $2 \mathrm{~min}$ \\
\hline $\begin{array}{l}\text { Start-up time from } 20^{\circ} \mathrm{C} \text { ambient } \\
\text { temperature }\end{array}$ & $<30 \min$ & $30 \mathrm{~min}$ & $20 \mathrm{~min}$ \\
\hline Degradation with cycling ${ }^{\text {h }}$ & $<2 \% / 1,000 \mathrm{~h}$ & $0.5 \% / 1,000 \mathrm{~h}$ & $0.3 \% / 1,000 \mathrm{~h}$ \\
\hline Operating lifetime ${ }^{i}$ & $12,000 \mathrm{~h}$ & $40,000 \mathrm{~h}$ & $60,000 \mathrm{~h}$ \\
\hline System availability & $97 \%$ & $98 \%$ & $99 \%$ \\
\hline
\end{tabular}

Figure 38. DOE Table 3.4.5 Technical Targets: 1-10 kW Residential Combined Heat and Power and Distributed Generation Fuel Cell Systems Operating on Natural Gas (see http://www1.eere.energy.gov/hydrogenandfuelcells/mypp/pdfs/fuel_cells.pdf).

\subsection{Additional Subtasks}

\section{Additional Goal}

Leverage EnerFuel's product development efforts to perform a scaled up demonstration of the HiFoil $^{\text {TM }}$ bipolar plates, unitized cells and advanced stack hardware that was developed under the current DOE/UCF grant. 
- Scale the advanced stack hardware from 36 to 132 cells

- Integrate with EnerFuel's 3kW HTPEM stationary power system

- Test for stand-alone operation using propane fuel

\section{Additional Subtasks}

1. Build a 132-cell stack with the advanced plates and dispensed seals

2. Modify the test station for 132-cell advanced heating and cooling

3. Test the 132-cell stack on the test station

4. Integrate the 132-cell stack into the stationary power system

5. Test the stationary power system

\subsection{Gen2 HiFoil 132-Cell Stand-Alone System Test Results}

EnerFuel's stationary power system is a highly advanced power generator characterized by:

- fully automated stand-alone operation

- LPG-fueled and water-neutral

- $3 \mathrm{~kW}_{\text {net }}$ continuous electric power output

- waste heat recovery

- onboard energy storage

- wireless data transmission

The externally cooled 132 -cell stack with Gen2 HiFoil ${ }^{\mathrm{TM}}$ bipolar plates was integrated into the advanced power system and tested in the laboratory (Fig.39). Data compiled during six different days of operation from 1-31-2013 to 2-28-2013 is shown below in Figure 40 and Table 2. The net system electrical efficiency was $\sim 36 \%$ (net electrical power out divided by LHV propane in) with $\mathrm{a}<15^{\circ} \mathrm{C}$ cell temperature spread. 


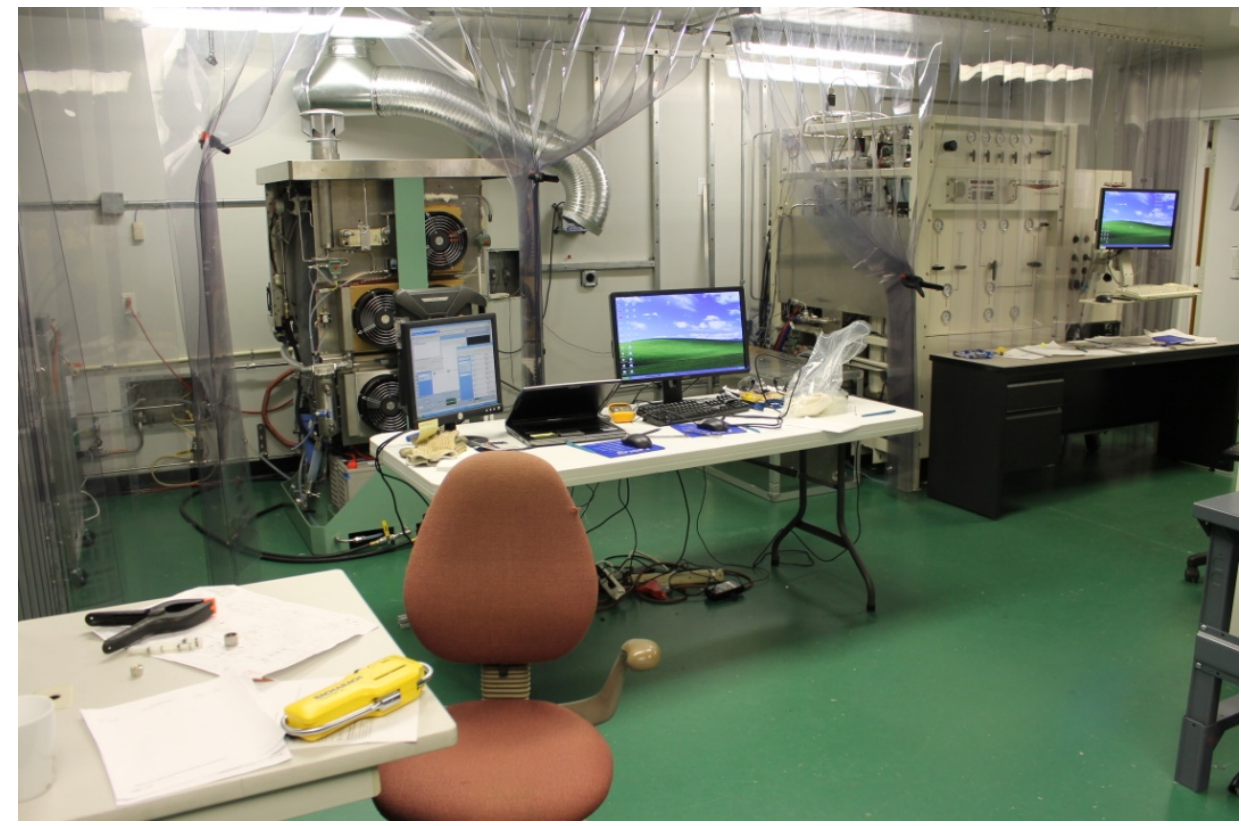

Figure 39. EnerFuel's LPG stationary power system at upper left (comprising the Gen2 HiFoil bipolar plate stack) during testing and debugging in the laboratory.

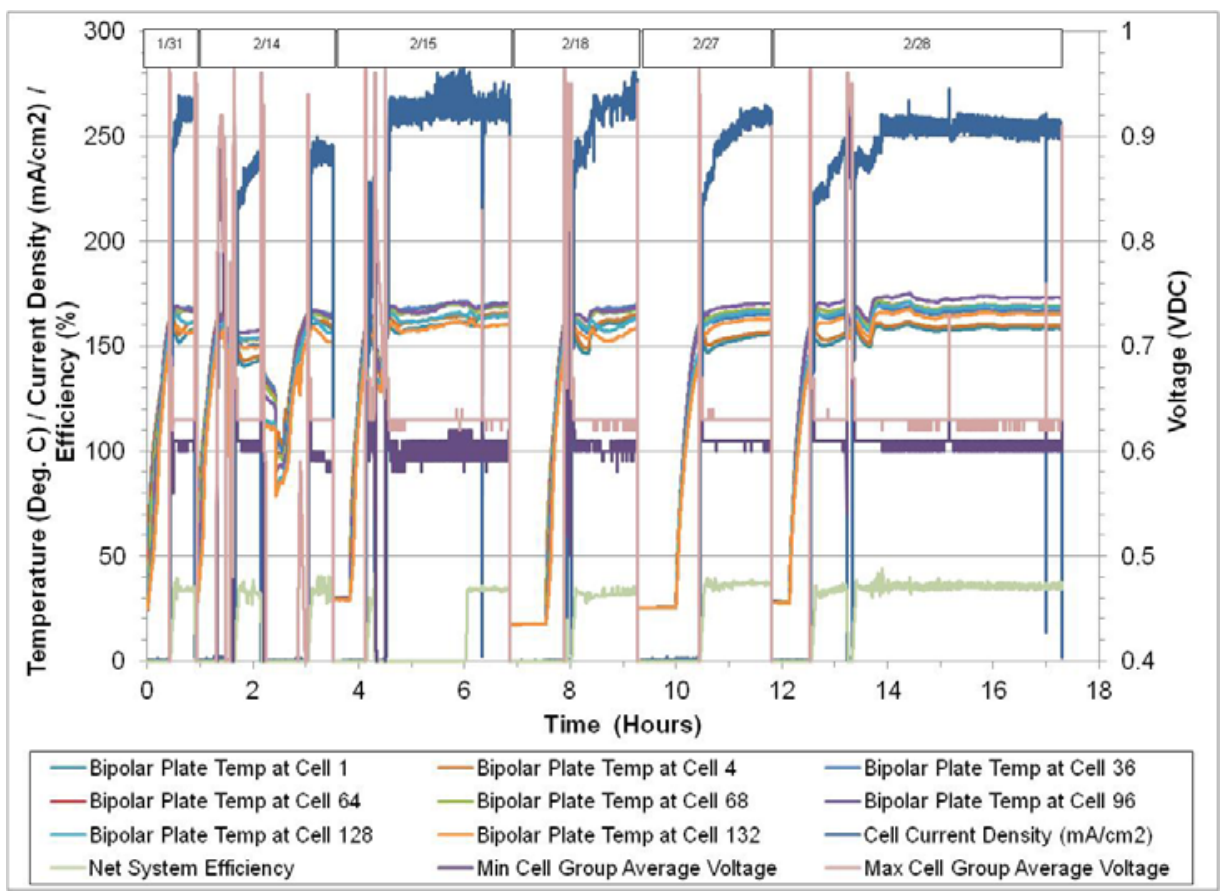

Figure 40. System test data with 132 -cell stack showing $<15^{\circ} \mathrm{C}$ cell temperature spread and a net system electrical efficiency of $\sim 36 \%$ (net electrical power out divided by LHV propane in). 
Table 2. Analysis of fuel cell temperature spread during system operation with LPG.

\begin{tabular}{|c|c|c|c|c|c|c|}
\hline Date & $\begin{array}{l}\text { Run } \\
\text { Hour }\end{array}$ & $\begin{array}{l}\text { Current } \\
\text { Density } \\
\text { (mA/cm2) }\end{array}$ & $\begin{array}{l}\text { Max } \\
\text { Temp } \\
\text { (deg.C) }\end{array}$ & $\begin{array}{l}\text { Min Temp } \\
\text { (deg.C) }\end{array}$ & $\begin{array}{l}\text { Min Temp } \\
\text { Position }\end{array}$ & $\begin{array}{l}\text { Stack } \\
\text { Temp } \\
\text { Spread } \\
\text { (deg.C) }\end{array}$ \\
\hline $1-31$ & .8939 & 267.5 & 168.1 & 157.6 & TC99-08 & 10.5 \\
\hline $2-15$ & 5.11 & 260 & 168 & 157.3 & ТC99-08 & 10.7 \\
\hline $2-15$ & 6.50 & 266 & 170.3 & 159.8 & TC99-08 & 10.5 \\
\hline $2-18$ & 9.27 & 270 & 170 & 158.8 & TC99-08 & 11.2 \\
\hline $2-27$ & 11.66 & 260 & 170 & 155.2 & TC99-01 & $14.8^{*}$ \\
\hline $2-28$ & 17.01 & 252 & 173.1 & 158.4 & TC99-01 & $14.7^{*}$ \\
\hline
\end{tabular}

* The increase in cell temperature spread observed during the last two runs is the result of a change to the stack's external cooling circuit. The circuit was modified to include both a primary and secondary cooling path for enhancing the reformer steam generation.

The stack's external thermal management subsystem uses only propane fuel as the source of heat for start-up, and enables the stack to deliver $90 \%$ of rated power in less than 30 minutes when heating from room temperature (Fig.41). The gross stack electrical power is $-3.25 \mathrm{~kW}$ while operating (Fig.42), which results in $\sim 3 \mathrm{~kW}$ electric power output for powering the external load and charging the internal batteries. The primary coolant outlet temperature is maintained above $165^{\circ} \mathrm{C}$ during operation (Fig.43), enabling high quality heat to be used for cogeneration. 


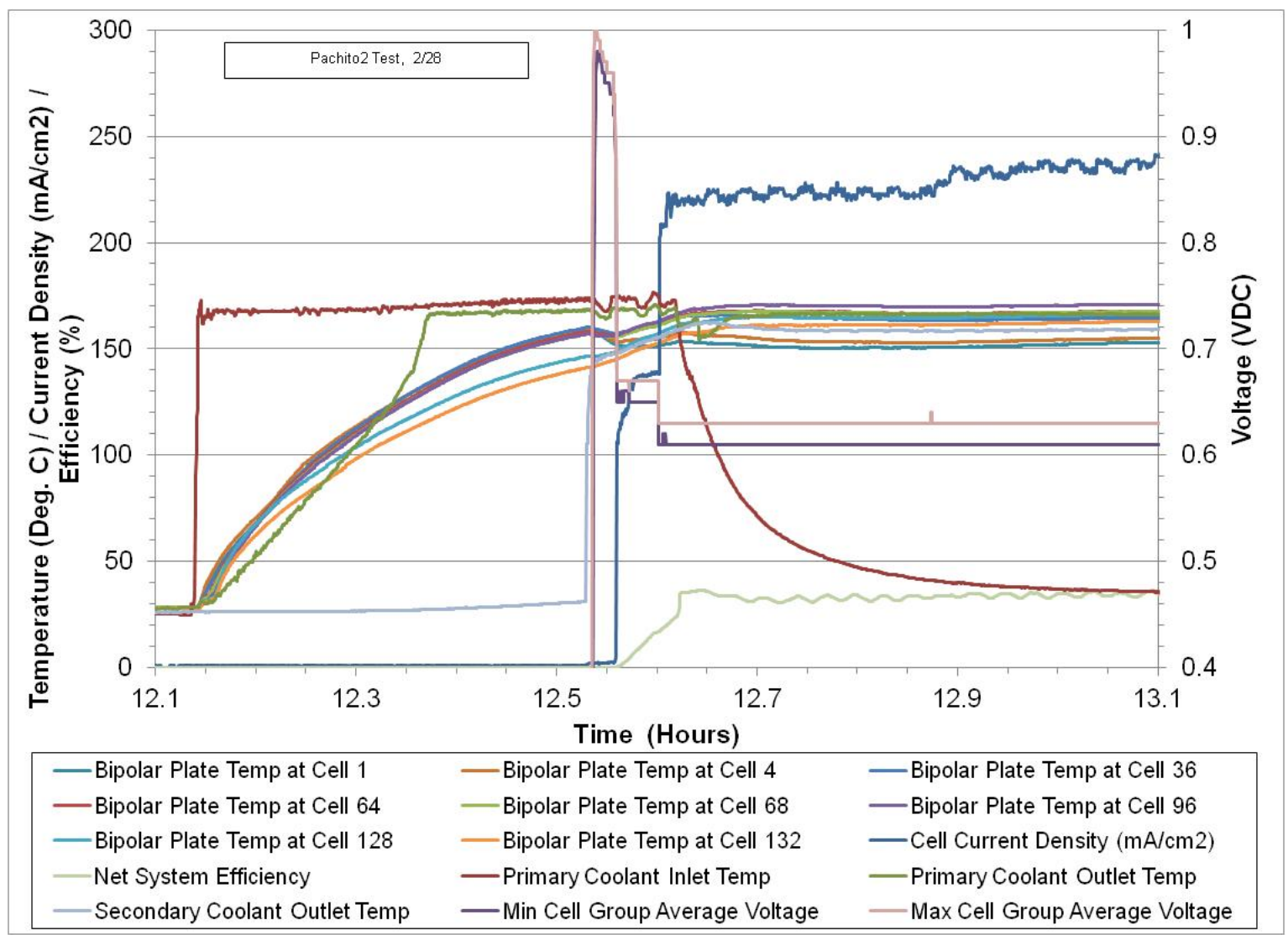

Figure 41. Test data showing the heat up of the 132-cell stack in the advanced power system using only propane fuel as the source of heat. The stack heats up and delivers $90 \%$ of rated power in less than 30 minutes from room temperature. 


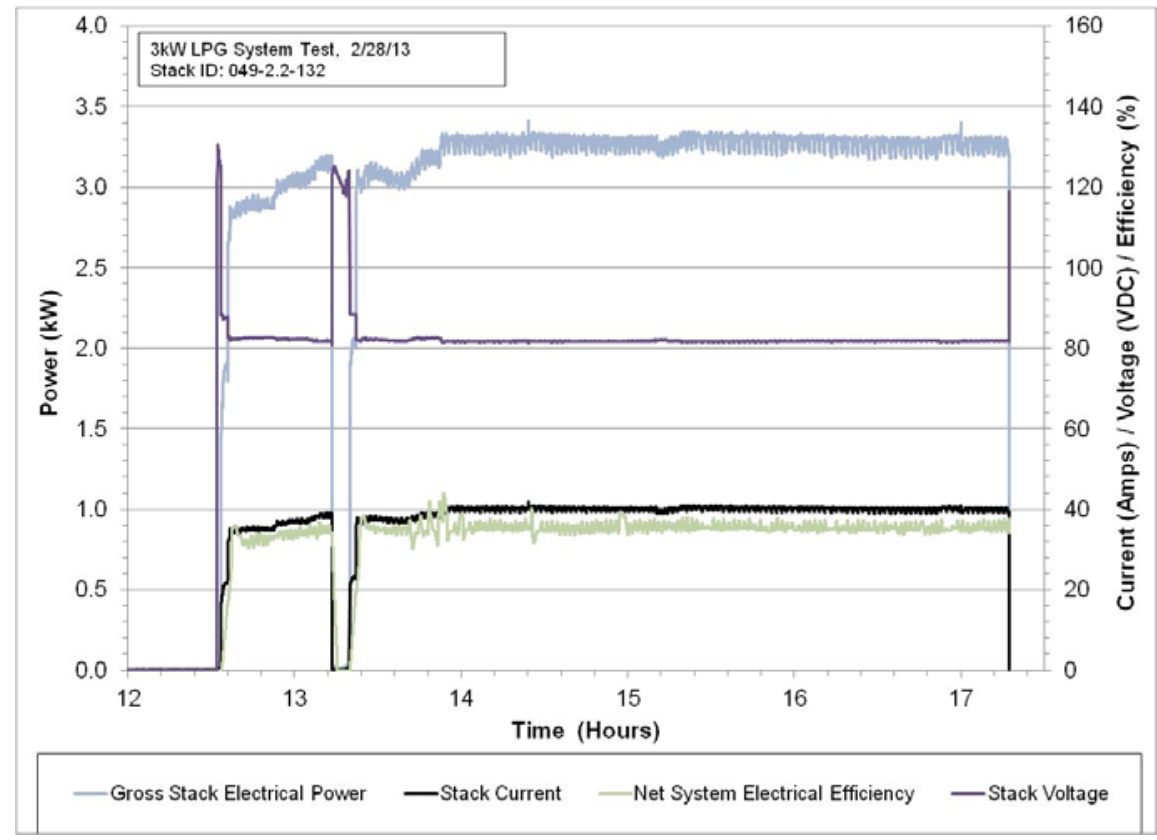

Figure 42. Test data showing the stack current, voltage and gross power during the entire run for the system test on 2-28-2013.

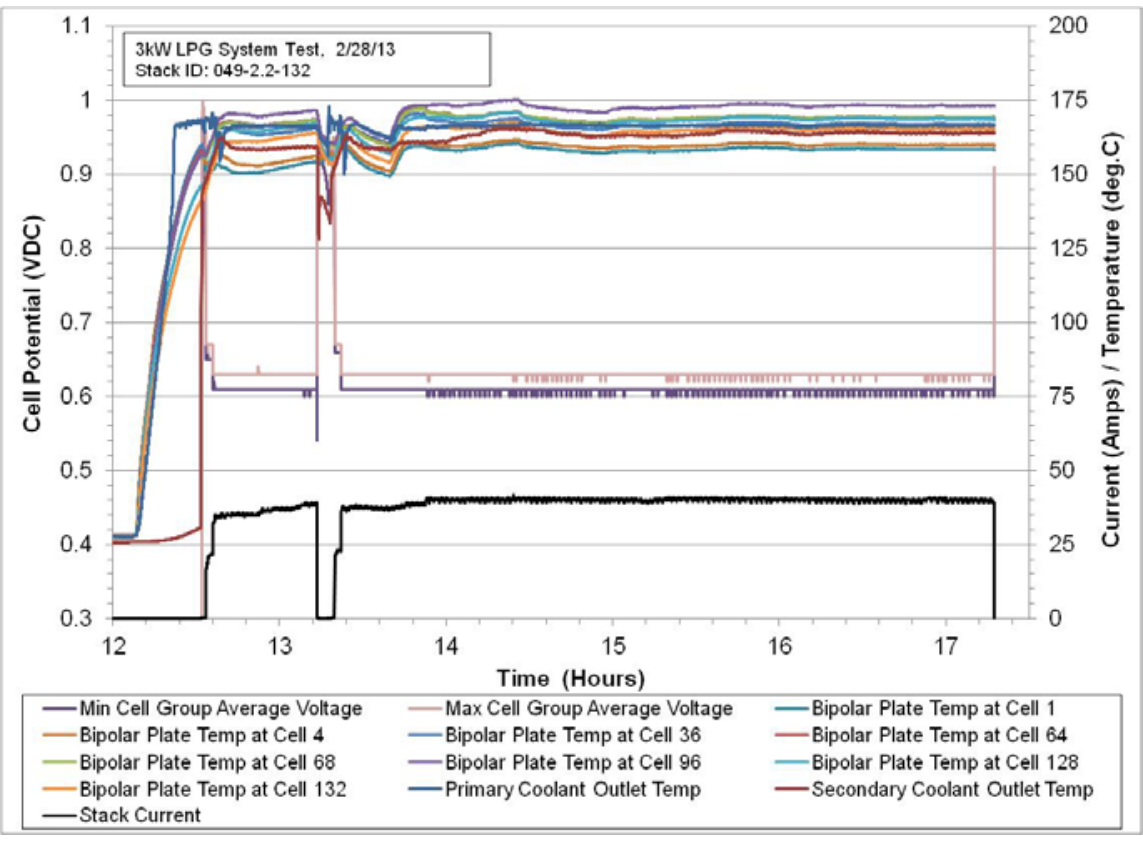

Figure 43. Test data showing the bipolar plate and coolant temperatures during the entire run for the system test on 2-28-2013. 
April 30, 2013

\section{Product Readiness}

The development efforts supported by this program have been remarkably successful. Along with the MEA, the bipolar plate makes up the core of the HTPEM fuel cell power system. EnerFuel's HiFoil ${ }^{\mathrm{TM}}$ bipolar plate and stack technology is currently patent pending under U.S. Patent Applications 13/566347, 13/566406, 13/566531, 13/566551, 13/566585, and 13/566629.

This technology is deployed in production ready 36-cell and 132-cell HTPEM fuel cell stacks (Fig.44). The 36-cell stacks are integrated into EnerFuel's commercially available 1.5kW Fuel Cell Power System (FCPS); while 132-cell stacks are integrated into EnerFuel's advanced 3kW stand-alone stationary power system (currently available to selected partners) (Fig.45). More information is available at http://www.enerfuel.com/solutions/.

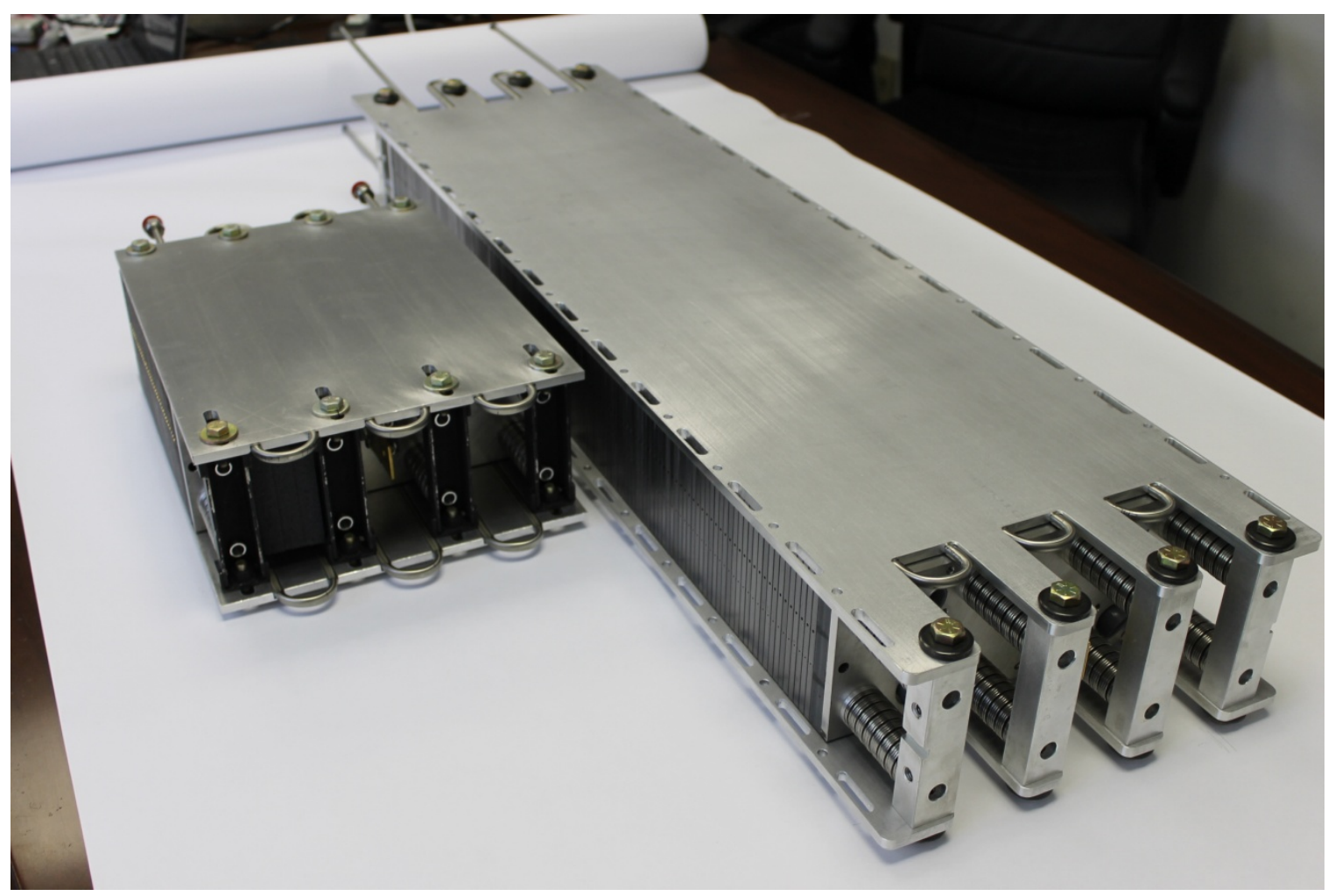

Figure 44. EnerFuel's 36-cell and 132-cell HTPEM stacks containing HiFoil ${ }^{\mathrm{TM}}$ bipolar plates and structurally integrated external cooling circuits. 

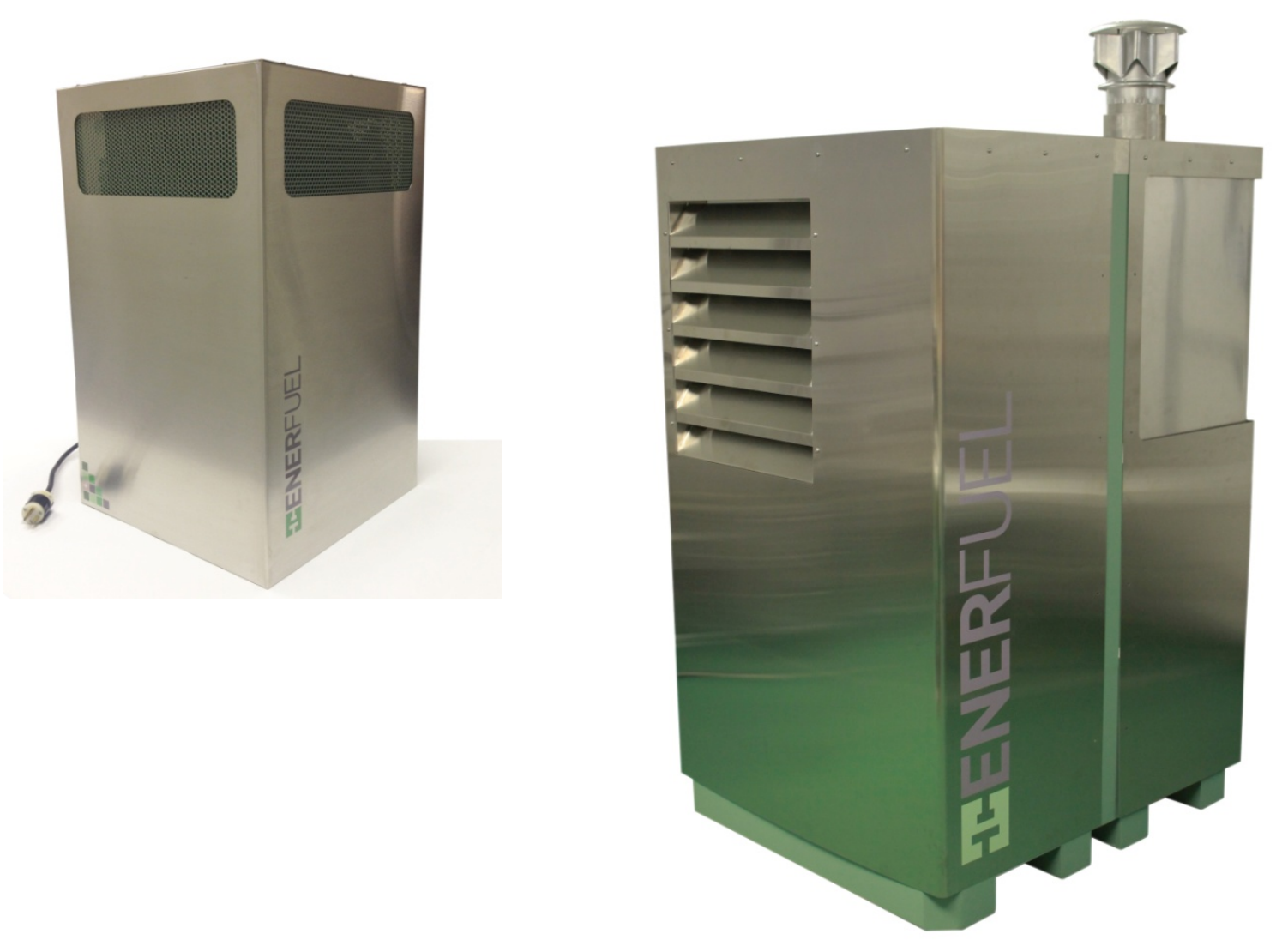

Figure 45. Commercially available 1.5kW FLEXYS 036 Plug n' Play HTPEM Fuel Cell Power System (left) and advanced 3kW stand-alone CHP stationary power system (right).

The stand-alone CHP stationary power system was unveiled in early 2013 at the EnerFuel facility, in West Palm Beach, FL, during a gathering of top level management, investors, employees and family (Fig.46-47). This system will be deployed to selected partners in 2013 for performance and durability testing. EnerFuel is actively engaged with customers to provide power solutions using the HiFoil ${ }^{\mathrm{TM}}$ bipolar plates in applications such as back-up power, distributed generation and combined heat and power where high reliability, high efficiency and low emissions are critical. 
April 30, 2013

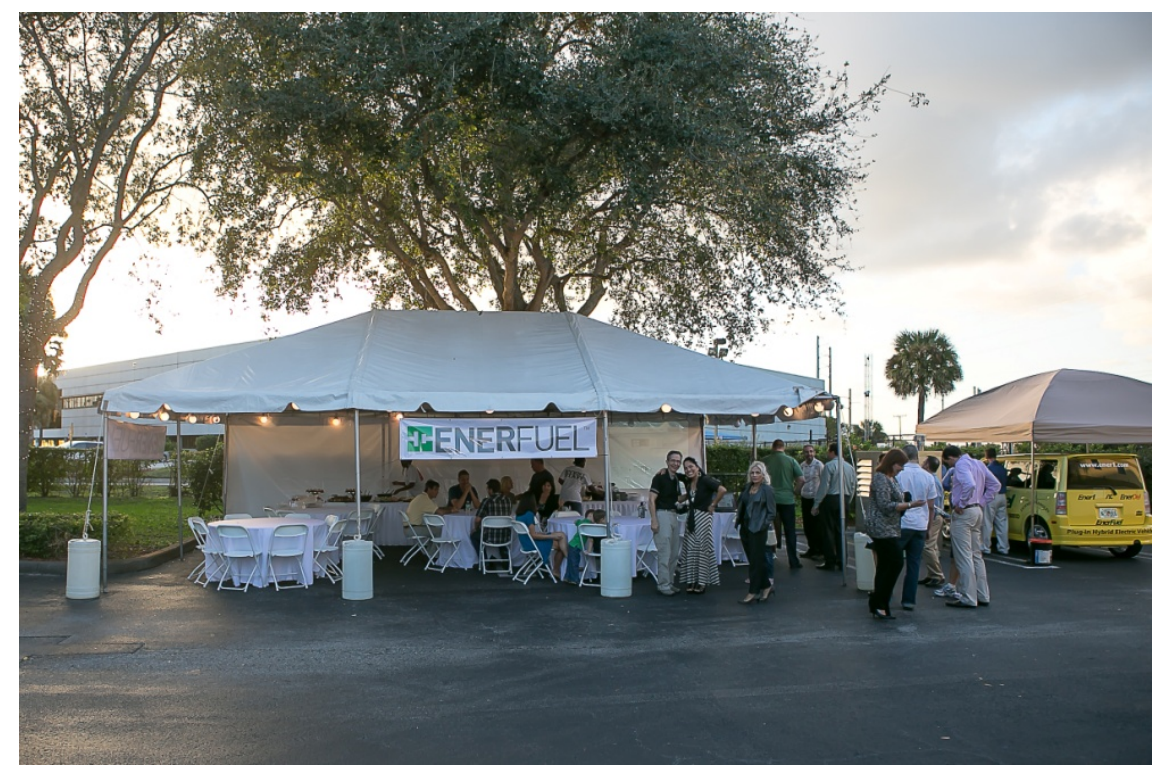

Figure 46. EnerFuel unveils its stand-alone power system (located between the two tents) at a gathering of top level management, investors, employees and family. The CHP system provided all power for the event including running lights, laptops and projectors, making espressos and charging an electric vehicle (which provided some very peppy rides for all the guests).

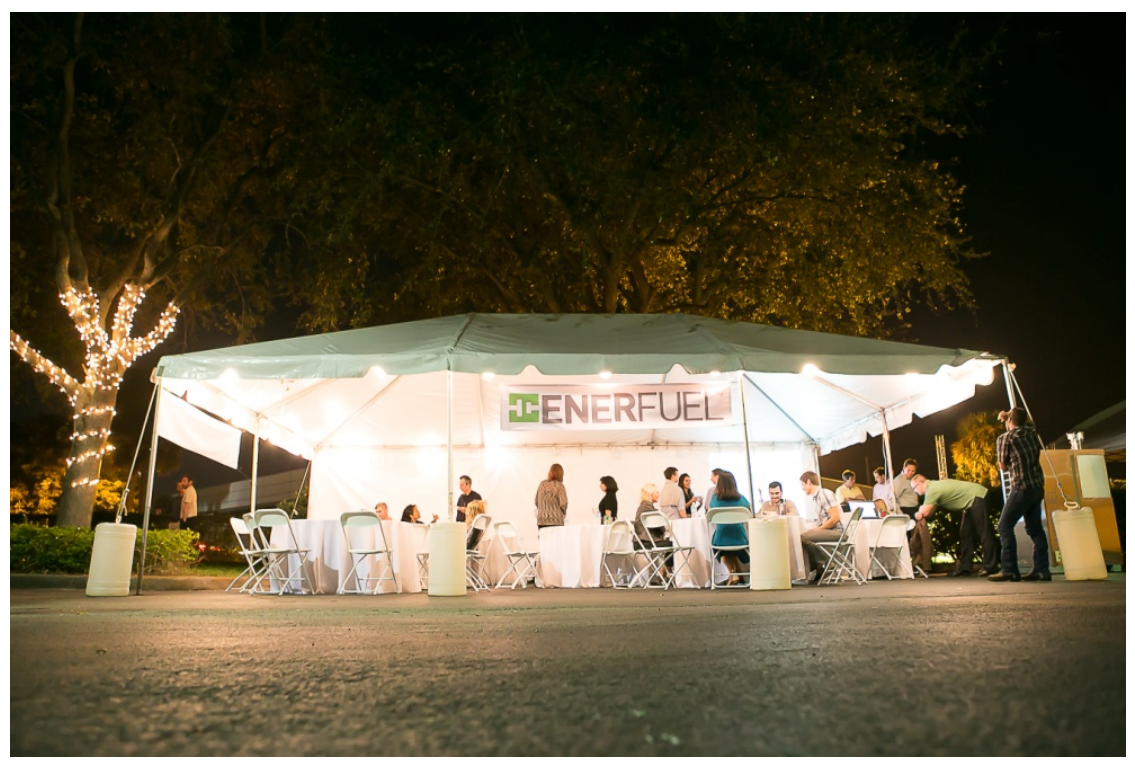

Figure 47. The full automated fuel cell system ran flawlessly all day and well into the evening on a bottle of LPG, producing virtually no noise or harmful emissions. 


\section{Conclusion}

A durable, low cost bipolar plate for use in HTPEM fuel cell systems was developed, having very high in-plane thermal conductivity. Stacks and systems using the advanced plate were operated in stand-alone, fully automated operation using propane from a portable tank. All of the heat generated by the fuel cell was conducted through the edges of the bipolar plate and to an external 2-phase cooling circuit. Some of this heat was used to generate steam for use inside the fuel processor, while the additional waste heat is provided at $-165^{\circ} \mathrm{C}$ to be put to work in combined heat and power applications. The net system efficiency was $36 \%$ at $3 \mathrm{~kW}$ electric power output.

When employed in a stationary CHP power system, the bipolar plate technology is estimated to cost less than $\$ 500 / \mathrm{kW}$ at 50,000 units per year production volume, supporting the 2015 DOE target cost of $\$ 1,700 / \mathrm{kW}$ for the overall system.

\section{Acknowledgements}

The EnerFuel would like to acknowledge the US DOE for supporting this effort via award number DE-FC36-04GO14225. Special thanks are also extended to Dr. David Block, of UCF, for his support and guidance in the execution of the project. 